

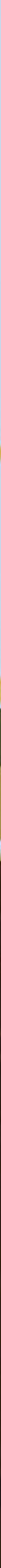




\section{VOLUME 3}

\section{RECARBONIZING \\ GLOBAL SOILS \\ A technical manual \\ of recommended \\ management practices}

\section{CROPLAND, GRASSLAND, INTEGRATED SYSTEMS AND FARMING APPROACHES}

Food and Agriculture Organization of the United Nations Rome, 2021 


\section{Required citation:}

FAO and ITPS. 2021. Recarbonizing global soils: A technical manual of recommended management practices. Volume 3: Cropland, Grassland, Integrated systems and farmingapproaches - Practicesoverview. Rome, FAO.

https://doi.org/10.4060/cb6595en

The designations employed and the presentation of material in this information product do not imply the expression of any opinion whatsoever on the part of the Food and Agriculture Organization of the United Nations (FAO) concerning the legal or development status of any country, territory, city or area or of its authorities, or concerning the delimitation of its frontiers or boundaries. The mention of specific companies or products of manufacturers, whether or not these have been patented, does not imply that these have been endorsed or recommended by $\mathrm{FAO}$ in preference to others of a similar nature that are not mentioned.

The views expressed in this information product are those of the author(s) and do not necessarily reflect the views or policies of FAO.

ISBN 978-92-5-134893-2

(C) FAO, 2021

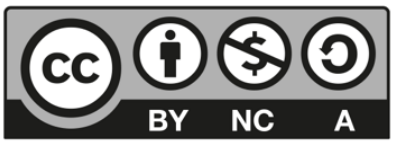

Some rights reserved. This work is made available under the Creative Commons Attribution-NonCommercial-ShareAlike 3.0 IGO licence (CC BY-NC-SA 3.0 IGO; https://creativecommons.org/licenses/by-ncsa/3.0/igo/legalcode/legalcode).

Under the terms of this licence, this work may be copied, redistributed and adapted for non-commercial purposes, provided that the work is appropriately cited. In any use of this work, there should be no suggestion that FAO endorses any specific organization, products or services. The use of the FAO logo is not permitted. If the work is adapted, then it must be licensed under the same or equivalent Creative Commons licence. If a translation of this work is created, it must include the following disclaimer along with the required citation: "This translation was not created by the Food and Agriculture Organization of the United Nations (FAO). FAO is not responsible for the content or accuracy of this translation. The original [Language] edition shall be the authoritative edition.”

Disputes arising under the licence that cannot be settled amicably will be resolved by mediation and arbitration as described in Article 8 of the licence except as otherwise provided herein. The applicable mediation rules will be the mediation rules of the World Intellectual Property Organization http://www.wipo.int/amc/en/mediation/rules and any arbitration will be conducted in accordance with the Arbitration Rules of the United Nations Commission on International Trade Law (UNCITRAL).

Third-party materials. Users wishing to reuse material from this work that is attributed to a third party, such as tables, figures or images, are responsible for determining whether permission is needed for that reuse and for obtaining permission from the copyright holder. The risk of claims resulting from infringement of any third-party-owned component in the work rests solely with the user.

Sales, rights and licensing. FAO information products are available on the FAO website (www.fao.org/publications) and can be purchased through publications-sales@fao.org. Requests for commercial use should be submitted via: www.fao.org/contact-us/licence-request. Queries regarding rights and licensing should be submitted to: copyright@fao.org 


\section{Contents}

\section{Cropland}

Soil organic cover

1. Cover cropping

2. Organic mulch

Crop diversification

3. Crop rotations

4. Intercropping: Multiple cropping

5. Intercropping: Strip cropping

Tillage

6. No-till

7. Conservation, reduced and superficial tillage

8. Strip, precision, zone tillage

9. Non-inversion tillage

Nutrient management

Organic matter additions

10. Manure additions

11. Digestate application

12. Compost application

13. Sewage sludge additions

Chemical and mineral fertilization

14. Mineral fertilization (macro, secondary and micronutrients)

15. Fertigation

Integrated nutrient management

16. Integrated soil fertility management 
Biochar

17. Biochar

Addition of living organisms

18. Biofertilizers application

19. Earthworm inoculation

20. Dung burial by beetles

Chemical amendments

21. Liming Acidic Soils

22. Application of gypsum on sodic soils

Soil and water conservation techniques

Erosion control techniques

23. Terraces

24. Check dams

25. Shelterbelts

26. Hedges and Buffer Strips

27. Avoiding improper earth movements before planting tree crops

Appropriate soil water management

28. Adequate irrigation practices

Limited soil disturbance

29. Controlled traffic farming

\section{Grasslands}


Grazing management

34. Improved pasture management

35. Grazing exclusion and rotational grazing

36. Pastoralism

General Conclusions

\section{Integrated systems and farming approaches}

37. Integrated crop-livestock systems

38. Agroforestry 1: Agrisilvicultural systems

39. Agroforestry 2: Sylvopastoral systems

40. Agroforestry 3: Agrosilvopastoral systems

41. Syntropic Agriculture

42. Conservation agriculture

43. Permaculture

44. Zero Budget Natural Farming

45. Agroecological farming

46. Climate-smart agriculture

47. Regenerative agriculture

48. Precision agriculture

608

49. Organic agriculture 


\section{Tables}

Table 1. Global and regional potential of additional SOC storage 3

Table 2. Soil threats 4

Table 3. Soil threats $\quad 6$

Table 4. Potential barriers to adoption $\quad 7$

Table 5. Related cases studies available in volumes 3 and 5

Table 6. Mulch management experiments reporting changes in SOC stocks in $\begin{array}{ll}\text { four different pedoclimatic conditions } & 16\end{array}$

Table 7. Soil threats $\quad 18$

Table 8. Potential barriers to adoption 21

Table 9. Related cases studies available in volumes 3 and $5 \quad 22$

Table 10. Crop rotation experiments reporting changes in SOC stocks in three different climate zones 29

Table 11. Soil threats $\quad 31$

Table 12. Potential barriers to adoption 33

Table 13. Related cases studies available in volumes 3 and 5

Table 14. Overview of the main features of intercropping studies reporting effects
on carbon storage

Table 15. Soil threats $\quad 40$

Table 16. Potential barriers to adoption $\quad 42$

Table 17. Related cases studies available in volumes 3 and 5

Table 18. Evolution of SOC stocks with application of strip cropping 49

Table 19. Soil threats $\quad 50$

Table 20. Potential barriers to adoption $\quad 52$

Table 21. Related cases studies available in volumes 3 and $5 \quad 52$

Table 22. Reviews of no-till effects on soil carbon $\quad 57$

Table 23. Soil threats $\quad 59$

$\begin{array}{ll}\text { Table 24. Soil threats } & 60\end{array}$

Table 25. Potential barriers to adoption 63

Table 26. Related cases studies available in volumes 3 and $5 \quad 66$

Table 27. Evolution of SOC stocks under conservation, reduced or superficial tillage 74

$\begin{array}{ll}\text { Table 28. Soil threats } & 75\end{array}$

$\begin{array}{ll}\text { Table 29. Soil threats } & 77\end{array}$

$\begin{array}{ll}\text { Table 30. Potential barriers to adoption } & 78\end{array}$

Table 31. Related cases studies available in volumes 3 and $5 \quad 80$ 
Table 32. Evolution of SOC stocks after application of strip tillage vs. conventional tillage (moldboard plough) in two studies in the United States of America

Table 33. Soil threats

Table 34. Potential barriers to adoption

Table 35. Related cases studies available in volumes 3 and 5

Table 36. Evolution of SOC stocks in non-inversion tillage systems 96

Table 37. Soil threats $\quad 97$

Table 38. Soil threats 99

Table 39. Potential barriers to adoption $\quad 100$

Table 40. General features of research about manure addition on soil organic $\begin{array}{ll}\text { carbon storage } & 106\end{array}$

$\begin{array}{ll}\text { Table 41. Soil threats } & 107\end{array}$

$\begin{array}{lr}\text { Table 42. Soil threats } & 109\end{array}$

Table 43. Potential barriers to adoption 110

Table 44. Related cases studies available in volumes 3 and $5 \quad 112$

Table 45. Effect of digestate in comparison to synthetic fertilizers on SOC in experiments that lasted at least 2 years

Table 46. Soil threats $\quad 120$

Table 47. Soil threats $\quad 121$

Table 48. Potential barriers to adoption 122

Table 49. Evolution of SOC stocks after compost application 129

Table 50. Soil threats 130

Table 51. Soil threats 132

Table 52. Potential barriers to adoption 133

Table 53. Related cases studies available in volumes 3 and $5 \quad 135$

Table 54. General features of research about sewage sludge addition on soil $\begin{array}{lr}\text { organic carbon storage } & 140\end{array}$

Table 55. Soil threats $\quad 141$

Table 56. Soil threats $\quad 142$

Table 57. Potential barriers to adoption 144

Table 58. Related cases studies available in volumes 3 and $5 \quad 146$

Table 59. Effects of soil nutrient amendments with mineral fertilizers on soil organic carbon sequestration $\quad 153$

Table 60. Soil threats $\quad 159$

Table 61. Effect of balanced and unbalanced fertilizers on yields of various crops in China, India and Kenya $\quad 161$

Table 62. Soil threats 164

Table 63. Potential barriers to adoption 167 
Table 64. Related cases studies available in volumes 3 and 5

Table 65. Evaluation of SOC stocks ${ }^{\S}$ under fertigation management

Table 66. Soil threats

Table 67. Soil threats

Table 68. Potential barriers to adoption

Table 69. Related cases studies available in volumes 3 and 5

Table 70. Soil carbon sequestration potential rates associated to ISFM

Table 71. Soil threats

Table 72. Summary of effect of ISFM on crop production

Table 73. Potential barriers to adoption

Table 74. Related cases studies available in volumes 3 and 5

Table 75. Overview of changes in soil organic carbon stocks reported with Biochar applications

Table 76. Soil threats

Table 77. Soil threats

Table 78. Potential barriers for adoption

Table 79. Related cases studies available in volumes 3 and 5

Table 80. Dominant biofertilizer organism classes by taxonomy or function

Table 81. Studies reporting significant positive effects on SOC storage

Table 82. Soil threats

Table 83. Soil threats

Table 84. Potential barriers to adoption

Table 85. Related cases studies available in volumes 3 and 5

Table 86. Measured effects of earthworm inoculation on soil organic carbon stocks

Table 87. Soil threats

Table 88. Soil threats

Table 89. Potential barriers to adoption

Table 90. Related cases studies available in volumes 3 and 5

Table 91. Evolution of SOC stocks after dung beetle burial

Table 92. A carbon budget for soil cores (gC per soil core section) (about $40 \mathrm{~cm}$ deep) demonstrating the effects of burial of cattle dung by B. bison on mean soil carbon levels $(g \pm S D)$ after 10 months in the field in a warm temperate dry IPCC climate zone in southern Australia

Table 93. Soil threats

Table 94. Potential barriers to adoption

Table 95. Measured effects of liming acidic soils on soil organic carbon stocks 
Table 97. Soil threats

Table 98. Potential barriers to adoption 280

Table 99. Related cases studies available in volumes 3 and $5 \quad 281$

Table 100. Soil threats 290

$\begin{array}{ll}\text { Table 101. Soil threats } & 291\end{array}$

Table 102. Potential barriers to adoption 292

Table 103. Styles of terracing 296

$\begin{array}{ll}\text { Table 104. Soil threats } & 298\end{array}$

$\begin{array}{ll}\text { Table 105. Soil threats } & 299\end{array}$

Table 106. Potential barriers to adoption 300

Table 107. Related cases studies available in volumes 3 and $5 \quad 303$

$\begin{array}{ll}\text { Table 108. Soil threats } & 307\end{array}$

Table 109. Soil threats 309

Table 1 10. Potential barriers to adoption 310

Table 111. Evolution of SOC stocks with shelterbelts 314

Table 112. Soil threats $\quad 315$

Table 113. Potential barriers to adoption 317

Table 114. Effect of hedgerows and buffer strips on rate of soil carbon storage as computed from available literature 323

Table 115. Soil threats 325

Table 116. Soil threats 328

Table 1 17. Emission of greenhouse gases and estimated net global warming potential under shelterbelts compared with adjacent cropped fields $\quad 328$

Table 1 18. Potential barriers to adoption 330

Table 1 19. Related cases studies available in volumes 3 and 5

Table 120. Evolution of SOC stocks (topsoil and subsoil) thanks to minimized land leveling operations and no scalping and/or topsoil re-spreading 337

Table 121. Soil threats 338

Table 122. Potential barriers to adoption $\quad 341$

Table 123. SOC sequestration potential of irrigated croplands 346

Table 124. Soil threats 347

Table 125. Soil threats $\quad 349$

Table 126. Greenhouse gas emission from soil under different irrigation practices 350

Table 127. Potential barriers to adoption 352

Table 128. Related cases studies available in volumes 3 and 5

Table 129. Evolution of SOC stocks after application of

Controlled Traffic Farming 
Table 130. Soil threats

Table 131. Soil threats 361

Table 132. Potential barriers to adoption 363

Table 133. SOC sequestration in conserved grassland at 0-30 cm depth 378

Table 134. Soil threats $\quad 379$

Table 135. Potential barriers to adoption 383

Table 136. Evolution of SOC stocks with grassland diversification 391

Table 137. Soil threats $\quad 392$

Table 138. Related cases studies available in volumes 3 and 5

Table 139. Evolution of Soc stocks after restoration of degraded grassland 399

Table 140. Soil threats $\quad 400$

Table 141. Soil threats $\quad 401$

Table 142. Potential barriers to adoption 402

Table 143.Related cases studies available in volumes 3 and 5

Table 144. Evolution of SOC stocks after conversion from cropland to grassland 407

Table 145. Soil threats 408

Table 146. Soil threats 410

Table 147. Potential barriers to adoption 411

Table 148. Related cases studies available in volumes 3 and 5

Table 149. Evolution of SOC stocks with improved pasture

Table 150. Soil threats $\quad 421$

Table 151. Soil threats $\quad 423$

Table 152. Potential barriers to adoption $\quad 425$

Table 153. Related cases studies available in volumes 3 and $5 \quad 427$

Table 154. Evolution of SOC stocks with grazing exclusion of rotational grazing 435

Table 155. Soil threats $\quad 436$

Table 156. Potential barriers to adoption 439

Table 157. Related cases studies available in volumes 3 and $5 \quad 440$

Table 158. Evolution of SOC stocks with pastoralism 446

Table 159. Soil threats 447

Table 160. Potential barriers to adoption 449

Table 161. Estimates of soil organic C sequestration with integrated
crop-livestock systems around the world

Table 162. Soil threats 459

Table 163. Soil threats $\quad 461$

Table 164. Potential barriers to adoption 464 
Table 165. Related cases studies available in volumes 3 and 5

Table 166. Examples of observed soil organic carbon (SOC) sequestration rates of selected agrisilvicultural systems

Table 167. Soil threats 476

Table 168. Soil threats $\quad 478$

Table 169. Potential barriers to adoption 479

Table 170. Related cases studies available in volumes 3 and $5 \quad 481$

Table 171. Examples of soil organic carbon sequestration or loss rates of selected silvopastoral systems

$\begin{array}{ll}\text { Table 172. Soil threats } & 489\end{array}$

Table 173. Soil threats $\quad 490$

Table 174. Potential barriers to adoption 492

Table 175. Related cases studies available in volumes 3 and 5

Table 176. Examples of SOC sequestration rates of selected $\begin{array}{ll}\text { agrosilvopastoral systems } & 501\end{array}$

$\begin{array}{ll}\text { Table 177. Soil threats } & 502\end{array}$

Table 178. Soil threats $\quad 504$

Table 179. Potential barriers to adoption 505

Table 180. Related cases studies available in volumes 3 and 5

Table 181. Soil properties in relation to soil threats $\quad 514$

Table 182. Barriers to adoption $\quad 517$

Table 183. Related cases studies available in volumes 3 and 5

Table 184. Evolution of SOC stocks under Conservation Agriculture 528

Table 185. Soil threats $\quad 530$

Table 186. Soil threats 534

Table 187. Potential barriers to adoption $\quad 537$

Table 188. Related cases studies available in volumes 3 and 5

Table 189. Soil threats $\quad 549$

Table 190. Potential barriers to adoption $\quad 552$

Table 191. Related cases studies available in volumes 3 and 5

Table 192. Calculation of approximate carbon (C) content of jiwamrita and beejamrita $\quad 559$

Table 193. Impact of Acchadana (mulching) on soil carbon (C) content in India 562

Table 194. Soil threats 563

Table 195. Potential barriers to adoption 567

Table 196. Evolution of soil organic carbon (SOC) stocks under different
agroecological systems over time

Table 197. Soil threats $\quad 576$ 
Table 198. Soil threats

Table 199. Potential barriers to adoption

Table 200. Global evolution of SOC stocks after application of reduced tillage, cover crop, and biochar as an example of CSA Practices

Table 201. Soil threats 590

Table 202. Soil threats $\quad 592$

Table 203. Potential barriers to adoption $\quad 594$

Table 204. Examples of practices that lead to incremental regenerative outcomes 600

Table 205. Potential barriers to adoption $\quad 602$

Table 206. Impact on soil organic carbon stocks $\quad 610$

$\begin{array}{ll}\text { Table 207. Soil threats } & 611\end{array}$

Table 208. Potential barriers to adoption $\quad 614$

Table 209. Evolution of SOC stocks in organic agriculture $\quad 622$

Table 210. Soil threats $\quad 623$

Table 211. Soil threats $\quad 624$

Table 212. Potential barriers to adoption 626

Table 213. Related cases studies available in volumes 3 and $5 \quad 628$ 


\section{Figures}

Figure 1. Soil organic C content (a), soil organic N content (b) and $\mathrm{C} / \mathrm{N}$ ratio (c) across a $1 \mathrm{~m}$ deep soil profile, averaged over intercrop systems and sole crop rotations with the same species in July 2010, after 7 years in the long-term experiment. (Cong et al., 2015)

Figure 2. Graphical representation of the strip-tillage management, where only the seed row is processed, while the area between the rows is covered with crop residues and remains unprocessed

Figure 3. World Bank global estimates of quantities and the extent of fertilizer use per hectare of cropland in 2015

Figure 4. Effect of ISFM along with Varity different in North West India (Naresh, Singh and Kumar, 2013).

Figure 5. Worldwide origin of 145 studies reviewed for this document

Figure 6. Evolution of rate of studies published on the topic

Figure 7. Calculated change (delta) in soil organic carbon found by a selection of studies (those with statistically significant positive results)

Figure 8. Sum of biofertilizer types encountered among the studies utilized in the meta-analysis above

Figure 9. Sum of crop types encountered among the studies utilized in the meta-analysis above

Figure 10. A functional analysis of dung burial by beetles

(Adapted from Doube, 1990)

Figure 11. Schematic figure demonstrating the aggregate dispersion induced by $\mathrm{Na}$ ions and subsequent sealing and crust formation due to the rearrangement of dispersed clays

Figure 12. Schemes of alternatives to reduce soil compaction by agricultural traffic. a) One wheeled tramline (round and round),

b) Controlled traffic farming.

Figure 13. Global share of land area used for pastures, measured as the percentage of total land area, 1961-2014 (a), and livestock production systems (b). Source: FAO

Figure 14. Succession scheme proposed by Ernst Götsch that illustrates the intervals of successional consortia occupation (placenta, secondary, climax and transitional) between disturbances (clearings) under natural conditions

Figure 15. Strata occupation proposed by Ernst Götsch with approximately 20 percent cover of emergent layer, 40 percent canopy, 60 percent medium, 80 percent low and 15-20 percent ground layer

Figure 16. Historical development of Agroecology

(Adapted from Wezel et al. 2020) 


\section{Photos}

Photo 1. Direct seeding of cover crop (peas) into maize stubbles,

Middle Ebro Valley (Zaragoza, Spain), November 2018

Photo 2. Olive groves with spontaneous resident vegetation adopted to protect soils from erosion and increase soil organic carbon content, Jaén (Andalusia, Spain), 2016

Photo 3. Winter cereal cultivation under a mulched soil in a Mediterranean semiarid area of Catalonia (NE Spain)

Photo 4. Rainfed field in the Northeastern Spain (Barcelona) where crop rotation includes canola, field pea, wheat, and barley

Photo 5. Wheat/maize strip intercropping in Gansu Province, China

Photo 6. Durum wheat/lentil mixed intercropping in France

Photo 7. Corn under no-till, after wheat. Spring 2019, Santa Fe, Argentina

Photo 8. Seeding pastures under no-till in a cattle farm. Autumn 2019,

Santa Fe, Argentina

Photo 9. Reduced tillage in rainfed almond fields in Andalusia, Spain

Photo 10. Non-inversion tillage operation in the Alhagüeces farm (Zarzadilla de Totana, Murcia, Spain) under rainfed organic almonds

Photo 11. Traditional periurban maize crop system based on cattle manure addition and animal tillage (Mexico city - Mexico)

Photo 12. Digestion of food waste and animal slurry contributes to sustainable electricity

Photo 13. (Top) Processing vermicompost, Mexico.

(Bottom) Compost windrow building, India

Photo 14. a) Treated sewage sludge, the solid byproduct of wastewater treatment, is often applied to farmland as a soil amendment in the United States, and b) Shallow injection of digestate into the grassland.

Photo 15. Farmers harvesting greengrams from APNI/AGRA Fertilizer

System Project in Makueni county, Kenya in February 2019

Photo 16. Farmer applying a fertilizer blend containing macronutrients, secondary nutrients and micro-nutrients in a APNI/OCP/AGRA nutrient omission trial in Meru, county Kenya in October 2019

Photo 17. Subsurface drip irrigation system with the filter and control station installed on a cement pad, and manifolds, flow meters, and air injectors that supply irrigation to each management zone

Photo 18. Components of an Integrated Soil Fertility program for Teff and Maize production

Photo 19. Thermochemical Biochar production diagram

Photo 20. Carbonization pilot Plant. (“Carbon”, Pantent IT1429282)

RE-CORD (Renewable Energy Consortium for Research and

Demonstration), in Florence, Italy, 2019 
Photo 21. Biochar distribution in vineyard. Poggio Torselli Estate,

Chianti Classico DOP 2018

Photo 22. Biochar distribution in Orchard. Frescobaldi Estate,

Le Sieci, Florence, Italy, 2020

Photo 23. An example of production and application of a biofertilizer

Photo 24. Inoculation of Pontoscolex corethrurus in cereal crops in Madagascar)

Photo 25. Bubas bison

Photo 26. B. bison released onto Australia (top) and three weeks later (bottom)

Photo 27. Soil from 25-50 cm deep without dung beetles (top), soil core with dung beetles showing vine roots entering soil core (after 10 months) (middle) and soil from $25-50 \mathrm{~cm}$ deep showing vine roots and buried dung remains (after 10 months (bottom)

Photo 28. Liming acidic Ferralsol under grass pasture and annual crop in Brazil

Photo 29. Examples of terraces

Photo 30. Examples of Check dams

Photo 31. A criss-cross patern of shelterbelts in North Dakota,

United States of America

Photo 32. Aerial view of different types of hedge rows and buffer strips

Photo 33. Hedgerows

Photo 34. Effects of a business-as-usual strategy before planting a vineyard

Photo 35. Alternative strategy for topsoil conservation before planting

a new vineyard

Photo 36. Center pivot sprinkler irrigation for field crops

Photo 37. Controlled traffic farming in a field of Argentina

Photo 38. One-wheeled tramline for multiple machinery widths method

Photo 39. Permanent grassland and land mosaic with permanent grassland field in France

Photo 40. Showing grass growth difference between diversified (left) vs. monocultured (right) grasslands.

Photo 41. Showing degraded (left) and restored (Right) grasslands

Photo 42. Conversion of cropland (top) to grassland (bottom)

Photo 43. Example of different grazing management practices and intensities when grazed by sheep grazing (top), continuously grazing by cattle (middle) and strip-grazed by cattle (bottom)

Photo 44. Example of moderate and overgrazed area (left) and grazing exclusion (right)

Photo 45. Nomadic pastoralism in Mongolia (Khövsgöl Province), milking of yaks (2017)

Photo 46. Free livestock movements in Africa 
Photo 47. Conceptual model for decoupling and (re)coupling of crop and livestock production across a range of possible specialization/ diversification scenarios

Photo 48. Urochloa forage intercropped with maize in the Federal District of Brazil, July 2015

Photo 49. Nelore cattle grazing Urochloa forage under Eucalyptus silvopasture in the Federal District of Brazil, July 2015

Photo 50. Cattle grazing winter annual forages prior to maize production in Parana, Brazil, August 2017

Photo 51. Cattle grazing winter annual forage prior to rice cultivation in Rio Grande do Sul, Brazil, September 2012

Photo 52. Sorghum under Faidherbia albida in Napak district, North-Eastern Uganda

Photo 53. Silvopastoral system with Acacia hokii in Karamoja, Uganda

Photo 54. Grazing of livestock in a field after the harvest of sorghum in an Acacia-dominated parkland in Karamoja, northeastern Uganda

Photo 55. Stratified system at Fazenda da Toca, Brazil. Eucalypts, banana, citrus and grass

Photo 56. Scheme for grains/vegetables cultivation between tree-lines

Photo 57. Typology of CA-based systems

Photo 58. Some recent trends incorporated in advanced CA-based systems design

Photo 59. Two pictures taken on the same day (Feb. 10, 2016)

during the midst of a drought in Malawi, Africa

Photo 60. (Left) Both pictures taken on the same day (Jan 21, 2016) from fields about 50 meters apart

Photo 61. (Right) Two contrasting agricultural systems photographed on the same day (Jan. 08, 2018) about 100 meters apart from each other during Malawi's 2018 drought

Photo 62. Production of Azolla pinnata for live mulching with rice.

Photo 63. Agroecological farming:

Photo 64. Syrah Vineyard in Drama, Greece

Photo 65. Measurement of soil electrical conductivity (a) to produce a map (b) and based on this information delineate management zones (c) in a Syrah Vineyard in Drama, Greece

Photo 66. Measurement of vineyard side canopy to map their vigor using the Normalized Difference Vegetation Index (NDVI) in a Syrah Vineyard in Drama, Greece

Photo 67. Organic cereals intercropped with legumes and grass;

Northern Germany (Schleswig-Holstein), June 2017

Photo 68. Shaded organic coffee, with soil cover and intercropped;

South Mexico (Chiapas), December 2018 


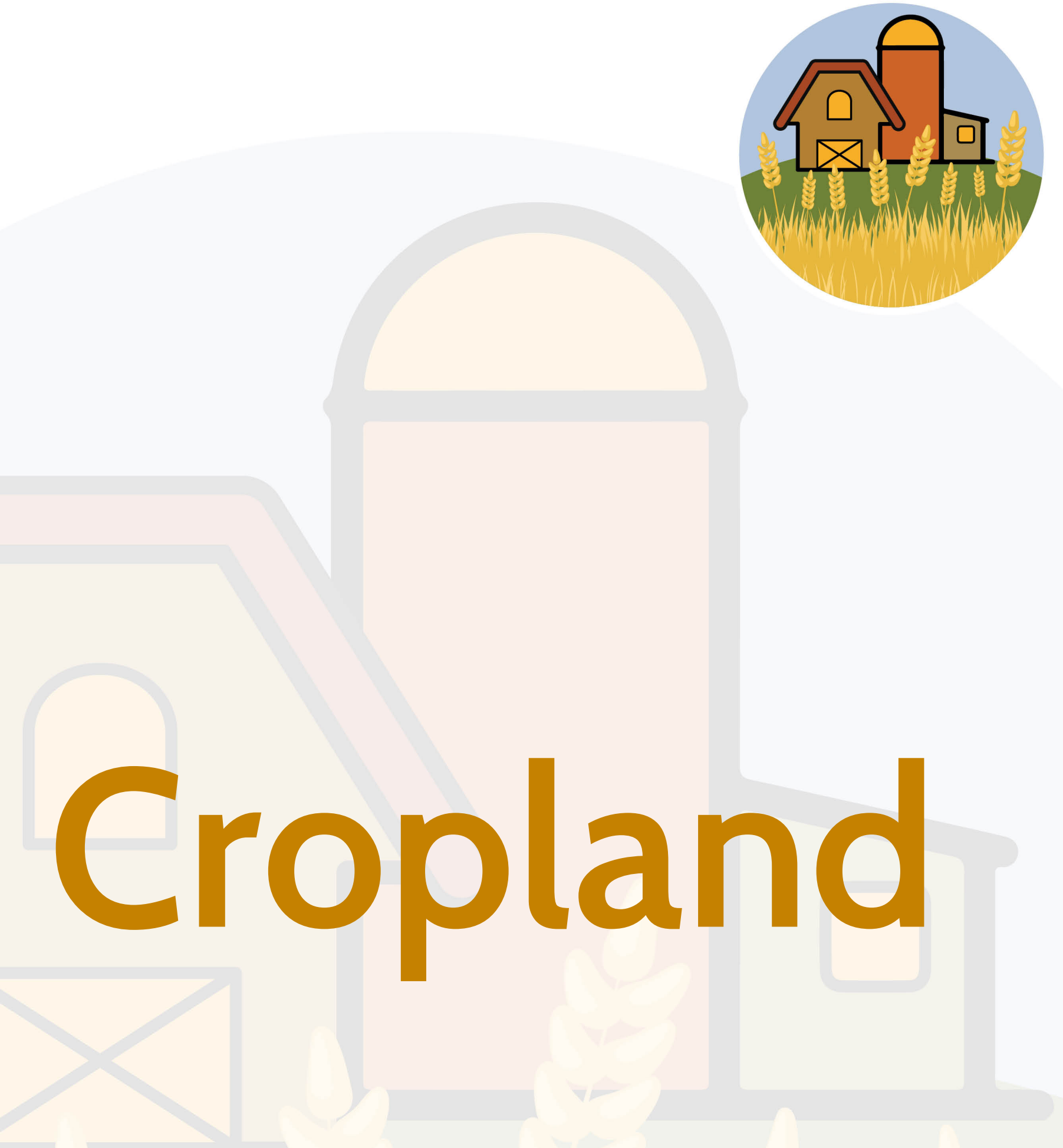


CROPLAND

SOIL ORGANIC COVER

\section{Cover cropping}

\section{Rosa Francaviglia', José Luis Vicente-Vicente²}

${ }^{1}$ Council for Agricultural Research and Economics, Research Centre for Agriculture and Environment

(CREA-AA), Rome, Italy

${ }^{2}$ Agricultural Landscape Systems, working group on Land Use Decisions in the Spatial and System Context, Leibniz Centre for Agricultural Landscape Research (ZALF), Müncheberg, Germany

\section{Description of the practice}

Cover crops are defined as a "close-growing crop that provides soil protection, seeding protection, and soil improvement between periods of normal crop production, or between trees in orchards and vines in vineyards. When plowed under and incorporated into the soil, cover crops may be referred to as green manure crops" (SSSA, 2008). Cover crops are also found called "Living mulch" or "Green manure". In some cases, cover crops can remain permanently on the soil, which constitutes a living soil cover (also see factsheets Nos. 4 and 5 on intercropping, this volume).

Typically, cover crops (CCs) are grasses (e.g. ryegrass), legumes (e.g. vetch), brassicas (e.g. rapeseed) or mixtures of two or more species (Jian et al., 2020). In arable cropping systems, cover crops are cultivated in monocultures or crop rotations to avoid long periods of bare soil and are ploughed into the soil (as green manure) before the next main crop is sowed (Photo 1). In woody crops are mostly intercropped as herbaceous crops in the inter-rows (Photo 2) to protect soil from erosion (perennial seeded species, spontaneous natural vegetation, or annual covers) and are removed and used as green manure, mulch or forage (Hartwig and Ammon, 2002; Vicente-Vicente et al., 2016). They are grown to improve soil health, which benefits crop yield in the medium and long term, are commonly used to suppress weeds, manage soil erosion, help build and improve soil fertility and quality, control diseases and pests, and promote biodiversity (Carlson and Stockweel, 2013). Cover crops have been used since antiquity in China (more than 3000 years ago), Japan $\left(9^{\text {th }}\right.$ century AD) and by the Greeks, Romans (e.g. Cato and Columella) and Aztecs (Ortiz Ceballos et al., 2012; Lal, 2015). 


\section{Range of applicability}

Cover cropping (CC) can be applied worldwide, but there is not a cover crop that fits every farming situation and potential benefits vary with climate, soil type and plant species. In detail, CCs can better fit in humid and subhumid regions than in semiarid regions where precipitation is limited (Unger and Vigil, 1998). The possible competition of CCs for available soil water in semiarid regions can limit the adoption of the practice (Unger and Vigil, 1998; Nielsen et al,, 2015). A different approach to agricultural management is required for arable and woody crops.

\section{Impact on soil organic carbon stocks}

The $\mathrm{C}$ storage potential of $\mathrm{CCs}$ is quite variable at regional level and with land use, ranging from 0.27 to 1.03 $\mathrm{tC} / \mathrm{ha} / \mathrm{yr}$ in the examined meta-analyses (Table 1). A higher potential was found in woody crops in warm temperate dry climates (about $1.00 \mathrm{tC} / \mathrm{ha} / \mathrm{yr}$ ), and lower values in arable crops most for temperate and tropical climates $(0.32-0.56 \mathrm{tC} / \mathrm{ha} / \mathrm{yr})$. C storage potential in arable systems was $0.71,0.51$ and $0.46 \mathrm{tC} / \mathrm{ha} / \mathrm{yr}$ in tropical, temperate and arid climates respectively (Jian et al., 2020). Data from a literature review (BlancoCanqui et al. 2015) reported that CCs increase soil organic C stocks by $0.1-1 \mathrm{t} / \mathrm{ha} / \mathrm{yr}$. Soil C sequestration rate is highest during the first years after CCs implementation and progressively decreases as $\mathrm{C}$ stocks approach equilibrium.

Table 1. Global and regional potential of additional SOC storage

\begin{tabular}{|c|c|c|c|c|c|c|c|}
\hline Location & $\begin{array}{l}\text { Climate } \\
\text { zone }\end{array}$ & $\begin{array}{l}\text { Soil } \\
\text { type }\end{array}$ & $\begin{array}{l}\text { Baseline } \\
\text { C stock } \\
\text { (tC/ha) }\end{array}$ & $\begin{array}{l}\text { Additional C } \\
\text { storage } \\
\text { (tC/ha/yr) }\end{array}$ & $\begin{array}{l}\text { Duration } \\
\text { (years) }\end{array}$ & $\begin{array}{l}\text { More } \\
\text { information }\end{array}$ & Reference \\
\hline Regional & $\begin{array}{l}\text { Warm } \\
\text { temperate } \\
\text { dry }\end{array}$ & NA & NA & 0.27 & 10.6 & $M A(A+W)$ & $\begin{array}{l}\text { Aguilera et al. } \\
\text { (2013) }\end{array}$ \\
\hline Global & $\begin{array}{l}\text { Arid, snow, } \\
\text { temperate, } \\
\text { tropical }\end{array}$ & NA & NA & 0.56 & 8.5 & $M A(A)$ & Jian et al. (2020) \\
\hline \multirow{2}{*}{ Regional } & \multirow{2}{*}{$\begin{array}{l}\text { Warm } \\
\text { temperate } \\
\text { dry }\end{array}$} & NA & NA & 0.43 & 5.6 & $M A(A C+W)$ & \multirow{2}{*}{$\begin{array}{l}\text { Morugán- } \\
\text { Coronado et al. } \\
(2020)\end{array}$} \\
\hline & & NA & NA & 1.01 & 6.7 & $M A(P C+W)$ & \\
\hline Global & $\begin{array}{l}\text { Temperate, } \\
\text { tropical }\end{array}$ & NA & NA & 0.32 & 11.9 & $M A(A)$ & $\begin{array}{l}\text { Poeplau and } \\
\text { Don (2015) }\end{array}$ \\
\hline $\begin{array}{l}\text { Mediterranean } \\
\text { basin }\end{array}$ & $\begin{array}{l}\text { Warm } \\
\text { temperate } \\
\text { dry }\end{array}$ & NA & NA & 1.03 & 7.7 & $M A(W)$ & $\begin{array}{l}\text { Vicente-Vicente } \\
\text { et al. (2016) }\end{array}$ \\
\hline
\end{tabular}

Note: $\mathrm{MA}=$ meta-analysis; $\mathrm{A}=$ arable crops; $\mathrm{W}$ = woody crops; $\mathrm{AC}=$ annual cover crops; $\mathrm{PC}$ = permanent cover crops.

Duration has been recalculated from the data if available in the meta-analyses or from the references' supplementary materials. 


\section{Other benefits of the practice}

\subsection{Improvement of soil properties}

CCs decrease bulk density and compaction in the long-term (Blanco-Canqui et al., 2015), increase soil porosity and water retained at field capacity (Basche and DeLonge, 2017), residues left on the soil surface reduce soil evaporation and increase soil water content (Kaspar and Singer, 2011).

\subsection{Minimization of threats to soil functions}

Table 2. Soil threats

\begin{tabular}{|c|c|}
\hline Soil threats & \\
\hline Soil erosion & $\begin{array}{l}\text { Minimize the impact of rainfall (Kaspar and Singer, 2011). Rapidly } \\
\text { increase aggregate stability and macro-porosity because soil } \\
\text { aggregates are larger and more stable compared to soils without } \\
\text { CCs. Increase water infiltration and decrease water runoff (Blanco- } \\
\text { Canqui et al., 2015). }\end{array}$ \\
\hline $\begin{array}{l}\text { Nutrient imbalance } \\
\text { and cycles }\end{array}$ & $\begin{array}{l}\text { Increase } \mathrm{N} \text { content in } \mathrm{N} \text {-fixing species, reduce } \mathrm{N} \text { leaching and } \\
\text { nutrient erosion by runoff (Kaspar and Singer, 2011). }\end{array}$ \\
\hline Soil biodiversity loss & Increase soil microbial parameters (Kim et al., 2020). \\
\hline Soil compaction & $\begin{array}{l}\text { Limited change in soil penetration resistance in the short-term ( } 3 \\
\text { years) (Aldridge et al., 2019). Cover crops with deep taproots such as } \\
\text { brassicas can reduce soil compaction by penetrating compact layers } \\
\text { (Blanco-Canqui et al., 2015). }\end{array}$ \\
\hline $\begin{array}{l}\text { Soil water } \\
\text { management }\end{array}$ & $\begin{array}{l}\text { Increase water infiltration and decrease water runoff (Blanco- } \\
\text { Canqui et al., 2015). }\end{array}$ \\
\hline
\end{tabular}




\subsection{Increases in production (e.g. food/fuel/feed/timber/fibre)}

In semiarid regions, CCs may reduce cash crops yields under conventional tillage but not under no-tillage management because evapotranspiration is lower and soil water storage is higher (Pittelkow et al., 2015). High$\mathrm{N}_{2}$-fixing (i.e., legumes) CCs can have faster and higher effects on increasing crop yields than species with low or no $\mathrm{N}_{2}$-fixing capacity. Specifically, summer legumes are more effective than winter legumes due to the higher potential biomass and $\mathrm{N}$ inputs. CCs may provide forage for livestock and feedstock for cellulosic biofuel production (Blanco-Canqui et al., 2015).

\subsection{Mitigation of and adaptation to climate change}

Generally, studies on $\mathrm{CO}_{2}$ and $\mathrm{CH}_{4}$ emissions found no negative effects of CCs (Liebig et al., 2010; SanzCobena et al., 2014). $\mathrm{N}_{2} \mathrm{O}$ emissions are reduced during the CC cycle because soil $\mathrm{N}$ availability is lower (Davidson et al., 2000), however their residues can lose $\mathrm{N}$ through emissions of $\mathrm{NH}_{3}, \mathrm{~N}_{2} \mathrm{O}$, $\mathrm{NO}$, or $\mathrm{N}_{2}$ (Kaspar and Singer, 2011).

\subsection{Socio-economic benefits}

CCs can decrease inorganic fertilization requirements, increase crop yields, reduce soil erosion by water and wind by physically protecting the soil surface, reduce soil evaporation during fallow periods and improve rainfall interception (Lal, 2015). The few data available about the reduction of fertilization requirements suggest that overall farm margins can decrease in the short term, and there is no clear evidence that the integration of CCs in the system has a positive effect on $\mathrm{N}$ fertility management and economics.

\subsection{Other benefits of the practice}

CCs compete with weeds, can release allelochemicals to inhibit weed growth and interrupt the cycles of pests and, through the emission of phytotoxic chemicals, may be able to suppress soilborne diseases (Ortiz Ceballos et al., 2012; O'Connell et al., 2015).

\section{Potential drawbacks to the practice}

\subsection{Tradeoffs with other threats to soil functions}




\begin{tabular}{|l|l|}
\hline Soil threats & \\
\hline Soil erosion & $\begin{array}{l}\text { CCs combined with conventional tillage in semiarid sites } \\
\text { (Blanco-Canqui et al., 2015). }\end{array}$ \\
\hline $\begin{array}{l}\text { Nutrient imbalance and } \\
\text { cycles }\end{array}$ & Possible competition for nutrient uptake (Lal, 2015). \\
\hline Soil water management & $\begin{array}{l}\text { Possible competition for available soil water in semiarid regions } \\
\text { (Blanco-Canqui et al., 2015). }\end{array}$ \\
\hline
\end{tabular}

\subsection{Increases in greenhouse gas emissions}

Field studies in semiarid environments (Sanz-Cobena et al., 2014), reported that $\mathrm{N}_{2} \mathrm{O}$ fluxes were close to zero during the CC period under barley (Hordeum vulgare L.) and rape (Brassica napus L.), while were significantly higher in vetch (Vicia villosa L.) compared to the fallow plot ( 0.16 vs. $0.04 \mathrm{~kg} \mathrm{~N}_{2} \mathrm{O}-\mathrm{N} / \mathrm{ha}$, i.e. $48 \mathrm{vs} .12 \mathrm{~kg} / \mathrm{ha}$ $\mathrm{CO}_{2} \mathrm{eq}$ ). During the following maize (Zea mays L.) crop, the incorporation of barley and rape residues increased $\mathrm{N}_{2} \mathrm{O}$ emissions compared with the treatment where residues were removed from the field, due to the combined effect of $\mathrm{N}$ fertilization, but decreased with the incorporation of vetch residues. Total $\mathrm{N}_{2} \mathrm{O}$ emissions for the whole cropping period when CC residues where removed amounted to 98,48 and $72 \mathrm{~kg} / \mathrm{ha} \mathrm{CO} \mathrm{C}_{2} \mathrm{eq}$ in vetch, barley and rape respectively and to 86,80 , and $86 \mathrm{~kg} / \mathrm{ha} \mathrm{CO} \mathrm{CO}_{2} \mathrm{eq}$ when residues were incorporated. So, the three species of CCs were equivalent with residue incorporation, as confirmed by Basche et al. (2014).

Results from a meta-analysis (Basche et al., 2014) indicated that legumes showed positive response ratios (RR, given by ratio between the $\mathrm{N}_{2} \mathrm{O}$ flux with a cover crop treatment to $\mathrm{N}_{2} \mathrm{O}$ flux without a cover crop), while the RRs for nonlegume species were close to zero. The same meta-analysis also reported that in legume CCs there was a pronounced increase in RRs with an increase of total precipitation. In addition, legume CCs had higher relative $\mathrm{N}_{2} \mathrm{O}$ emissions at low $\mathrm{N}$ fertilization rates and lower emissions at high $\mathrm{N}$ rates, whereas $\mathrm{N}_{2} \mathrm{O}$ emissions of nonlegume CCs increased as $\mathrm{N}$ fertilization rate increased.

\subsection{Conflict with other practice(s)}

Conventional tillage might decrease the benefits of CCs (Blanco-Canqui et al., 2015). In fact, tillage breaks down soil aggregate and accelerates SOC mineralization and can reduce the soil benefits of CCs compared to no-till or reduced tillage management (Sainju, Whitehead and Singh, 2003). 


\subsection{Decreases in production (e.g. food/fuel/feed/timber/fibre)}

Higher yield variance using legumes could increase the economic risk, because CCs alone may not supply sufficient N. So, combining CCs with inorganic fertilization can be an alternative to increase crop yields (Blanco-Canqui et al, 2015). Generally, CCs do not have market value, unless CCs with an economic function, like livestock fodder are selected, or CCs are used to graze livestock directly.

\section{Recommendations before implementing the practice}

The environmental benefits of CCs are widely recognized but adoption levels are still quite low. A major weakness is that few farmers are experts in CCs management, and thus technical assistance to farmers should be strengthened (e.g., plant species suited to the local pedoclimatic conditions and best management). For example, the potential adverse effects on plant-available water and cash crop yields often limit the adoption of CCs in semiarid regions (Unger and Vigil 1998; Nielsen et al., 2015).

Possible recommendations include reducing seeding rates and the early termination of the CC, as well as mulching of CC residues on the soil surface and adoption of no-tillage management in the cash crop that contribute to store water in the soil. Additionally, CCs can be directly seeded into the residues of the cash crop

\section{Potential barriers to adoption}

Table 4. Potential barriers to adoption

\begin{tabular}{|l|l|l|}
\hline Barrier & YES/NO & \\
\hline Biophysical & Yes & $\begin{array}{l}\text { Potential benefits vary with climate, soil type and plant } \\
\text { species (Lal, 2015). }\end{array}$ \\
\hline Cultural & Yes & $\begin{array}{l}\text { Reluctance to change from traditional management practices } \\
\text { to alternatives (O'Connell et al., 2015). }\end{array}$ \\
\hline Social & Yes & $\begin{array}{l}\text { Age of farmers is an index that determines attitudes, so older } \\
\text { farmers are less prone to participate in pilot studies or } \\
\text { demonstration projects, particularly in more developed } \\
\text { countries (O'Connell et al., 2015). }\end{array}$ \\
\hline Economic & Yes & $\begin{array}{l}\text { Equipment for incorporation of residues, or CC termination } \\
\text { (O'Connell et al., 2O15). }\end{array}$ \\
\hline
\end{tabular}




\begin{tabular}{|l|l|l|}
\hline Barrier & YES/NO & Les \\
\hline Institutional & Yes & $\begin{array}{l}\text { Limited knowledge of the benefits of the measure among key } \\
\text { decision makers/administrators or poor knowledge transfer } \\
\text { to users/ limited dissemination/limited promotion of the } \\
\text { measure/limited training/education (Roesch-McNally et al., } \\
\text { 2018). }\end{array}$ \\
\hline Legal (Right & Yes & $\begin{array}{l}\text { There is evidence that farmers are more likely to expect the } \\
\text { long-term benefits associated with CCS in long-term rental } \\
\text { arrangements, that is more uncertainties are associated with } \\
\text { short-term rentals (Nadella et al., 2O14). }\end{array}$ \\
\hline Knowledge & Yes & $\begin{array}{l}\text { Planting the right CC and incorporation of residues (O'Connell } \\
\text { et al., 2O15). }\end{array}$ \\
\hline
\end{tabular}

\section{Photos of the practice}

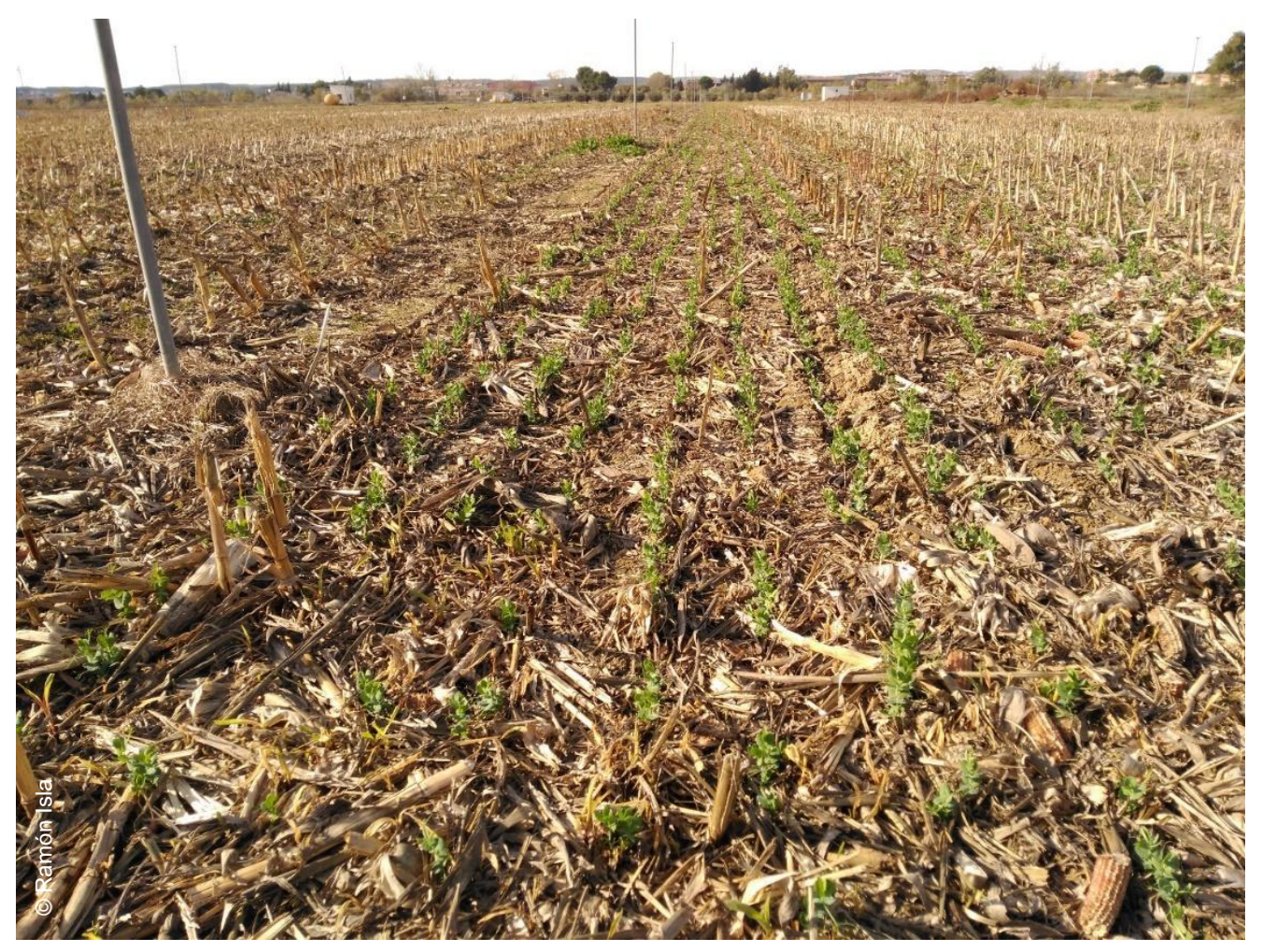

Photo 1. Direct seeding of cover crop (peas) into maize stubbles, Middle Ebro Valley (Zaragoza, Spain), November 2018 


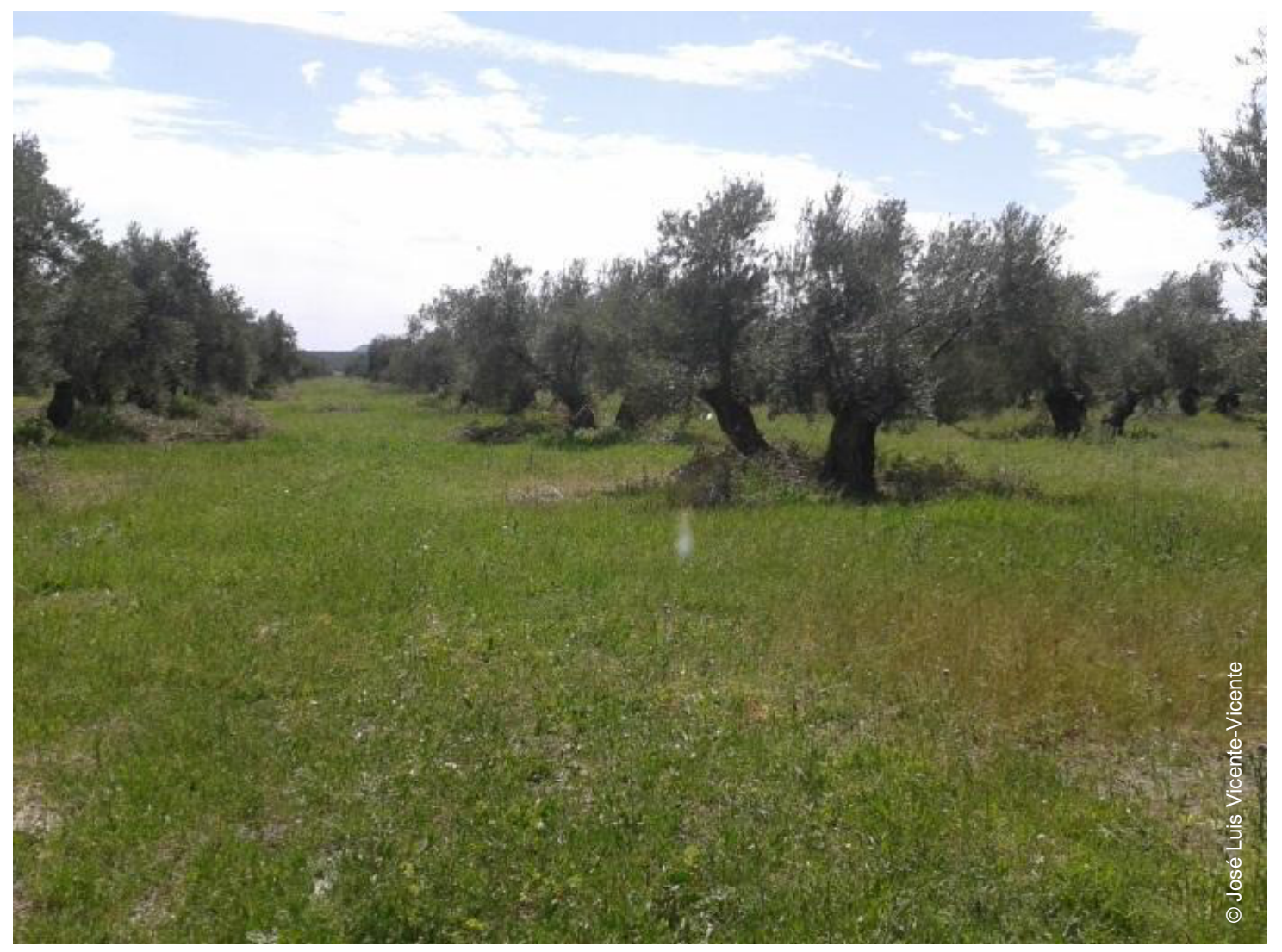

Photo 2. Olive groves with spontaneous resident vegetation adopted to protect soils from erosion and increase soil organic carbon content, Jaén (Andalusia, Spain), 2016

Table 5. Related cases studies available in volumes 3 and 5

\begin{tabular}{|l|l|c|c|c|}
\hline Title & Region & $\begin{array}{l}\text { Duration } \\
\text { of study } \\
\text { (Years) }\end{array}$ & Volume & $\begin{array}{l}\text { Case- } \\
\text { study } \\
\text { No. }\end{array}$ \\
\hline $\begin{array}{l}\text { Short-time effects of no-tillage in olive } \\
\text { orchards in Lebanon }\end{array}$ & NENA & 5 & 3 & 1 \\
\hline $\begin{array}{l}\text { Mediterranean olive orchard subjected to } \\
\text { sustainable management in Matera, } \\
\text { Basilicata, Italy }\end{array}$ & Europe & 20 & 3 & 16 \\
\hline $\begin{array}{l}\text { Cover cropping in olive and vineyards } \\
\text { (woody crops) in Spain }\end{array}$ & Europe & 2 to 4 & 3 & 18 \\
\hline
\end{tabular}




\begin{tabular}{|c|c|c|c|c|}
\hline Title & Region & $\begin{array}{l}\text { Duration } \\
\text { of study } \\
\text { (Years) }\end{array}$ & Volume & $\begin{array}{l}\text { Case- } \\
\text { study } \\
\text { No. }\end{array}$ \\
\hline $\begin{array}{l}\text { Application of mulching in subtropical } \\
\text { orchards in Granada, Spain }\end{array}$ & Europe & 5 & 3 & 20 \\
\hline $\begin{array}{l}\text { Reduced tillage frequency and no-till to } \\
\text { allow ground covers and seeding cover } \\
\text { crops in rainfed almond fields, Spain }\end{array}$ & Europe & 10 & 3 & 21 \\
\hline $\begin{array}{l}\text { Interrow organic management to restore } \\
\text { soil functionality of vineyards }\end{array}$ & $\begin{array}{l}\text { Europe and } \\
\text { Eurasia }\end{array}$ & 2 & 3 & 27 \\
\hline $\begin{array}{l}\text { Cover crops, organic amendments and } \\
\text { combined management practices in } \\
\text { Mediterranean woody crops }\end{array}$ & $\begin{array}{l}\text { Europe, NENA, } \\
\text { Eurasia, North } \\
\text { America }\end{array}$ & $<30$ & 3 & 28 \\
\hline $\begin{array}{l}\text { Increasing carbon inputs in agricultural } \\
\text { lands in Argentina: fertilizer use, } \\
\text { inclusion of cover crops and integration } \\
\text { of perennial pastures in crop rotations }\end{array}$ & $\begin{array}{l}\text { Latin America } \\
\text { and the } \\
\text { Caribbean }\end{array}$ & 2 to 23 & 3 & 29 \\
\hline $\begin{array}{l}\text { No tillage and cover crops in the } \\
\text { Pampas, Argentina }\end{array}$ & $\begin{array}{l}\text { Latin America } \\
\text { and the } \\
\text { Caribbean }\end{array}$ & 2 to 8 & 3 & 31 \\
\hline $\begin{array}{l}\text { Long-term no-tillage maize in Kentucky, } \\
\text { United States of America }\end{array}$ & North America & $\begin{array}{l}48 \text { and } \\
79\end{array}$ & 3 & 45 \\
\hline
\end{tabular}




\section{References}

Aguilera, E., Lassaletta, L., Gattinger, A. \& Gimeno, B.S. 2013. Managing soil carbon for climate change mitigation and adaptation in Mediterranean cropping systems: A meta-analysis. Agriculture Ecosystems \& Environment, 168: 25-36. https://doi.org/10.1016/j.agee.2013.02.003

Aldridge, C.A., Baker, B.H. \& Omer, A.R. 2019. Investigation of short-term effects of winter cover crops on compaction and total soil carbon in a long-term no-till agricultural system. Journal of Soil and Water Conservation, 74(1): 77-84. https://doi.org/10.2489/jswc.74.1.77

Basche, A.D., Miguez, F.E., Kaspar, T.C. \& Castellano, M.J. 2014. Do cover crops increase or decrease nitrous oxide emissions? A meta-analysis. Journal of Soil and Water Conservation, 69(6): 471-482. https://doi.org/10.2489/jswc.69.6.471

Basche, A. \& DeLonge, M. 2017. The impact of continuous living cover on soil hydrologic properties: A meta-analysis. Soil Science Society of America Journal, 81(5): 1179-1190.

https://doi.org/10.2136/sssaj2017.03.0077

Blanco-Canqui, H., Shaver, T.M., Lindquist, J.L., Shapiro, C.A., Elmore, R.W., Francis, C.A. \& Hergert, G.W. 2015. Cover crops and ecosystems services: insights from studies in temperate soils. Agronomy Journal, 107: 2449-2474. https://doi.org/10.2134/agronj15.0086

Carlson, S. \& Stockwell, R. 2013. Research priorities for advancing adoption of cover crops in agricultureintensive regions. Journal of Agriculture, Food Systems, and Community Development, 3(4): 125-129. https://doi.org/10.5304/jafscd.2013.034.017

Davidson, E.A., Keller, M., Erickson, H.E., Verchot, L.V. \& Veldkamp, E. 2000. Testing a conceptual model of soil emissions of nitrous and nitric oxides. Bioscience, 50: 667-680. https://doi.org/10.1641/0006-3568(2000)050[0667:TACMOS]2.0.CO;2

Jian, J., Du, X., Reiter, M.S. \& Stewart, R.D. 2020. A meta-analysis of global cropland soil carbon changes due to cover cropping. Soil Biology and Biochemistry, 143: 107735.

https://doi.org/10.1016/j.soilbio.2020.107735

Hartwig, N.L. \& Ammon, H.U. 2002. Cover crops and living mulches. Weed Science, 50: 688-699. https://www.jstor.org/stable/4046641

Kaspar, T.C. \& Singer, J.W. 2011. The Use of Cover Crops to Manage Soil. In Hatfield, J.L. \& Sauer, T.J. (Eds.), Soil management: Building a stable base for agriculture, pp. 321-337. American Society of Agronomy and Soil Science Society of America, Madison, WI, USA.

Kim, N., Zabaloy, M.C., Guan, K. \& Villamil, M.B. 2020. Do cover crops benefit soil microbiome? A meta-analysis of current research. Soil Biology and Biochemistry, 142: 107701.

https://doi.org/10.1016/j.soilbio.2019.107701

Lal, R. 2015. Soil carbon sequestration and aggregation by cover cropping. Journal of Soil and Water Conservation, 70(6): 329-339. https://doi.org/10.2489/jswc.70.6.329 
Liebig, M.A., Tanaka, D.L. \& Gross, J.R. 2010. Fallow effects on soil carbon and greenhouse gas flux in central North Dakota. Soil Science Society of America Journal, 74: 358-365.

https://doi.org/10.2136/sssaj2008.0368

Morugán-Coronado, A., Linares, C., Gómez-López, M.D., Faz, Á. \& Zornoza, R. 2020. The impact of intercropping, tillage and fertilizer type on soil and crop yield in fruit orchards under Mediterranean conditions: A meta-analysis of field studies. Agricultural Systems, 178: 102736.

https://doi.org/10.1016/j.agsy.2019.102736

Nadella, K., Deaton, B., Lawley, C. \& Weersink, A. 2014. Do farmers treat rented land differently than the land they own? A fixed effects model of farmer's decision to adopt conservation practices on owned and rented land. Selected Paper prepared for presentation at the Agricultural \& Applied Economics Association's Annual Meeting, Minneapolis, MN. (also available at:

http://ageconsearch.umn.edu/record/170633/files/AAEA_submission.pdf)

Nielsen, D.C., Lyon, D.J., Hergert, G.W., Higgins, R.K., Calderón, F.J. \& Vigil, M.F. 2015. Cover crop mixtures do not use water differently than single-species plantings. Agronomy Journal, 107: 1025-1038. https://doi.org/10.2134/agronj14.0504

O'Connell, S., Grossman, J.M., Hoyt, G.D., Shi, W., Bowen, S., Marticorena, D.C., Fager, K.L. \& Creamer, N.G. 2015. A survey of cover crop practices and perceptions of sustainable farmers in North Carolina and the surrounding region. Renewable Agriculture and Food Systems, 30(06): 550-562. https://doi.org/10.1017/S1742170514000398

Ortiz Ceballos, Á.I., Aguirre Rivera, J.R., Osorio Arce, M.M. \& Peña Valdivia, C. 2012. Velvet Bean (Mucuna pruriens var. utilis) a cover crop as bioherbicide to preserve the environmental services of soil. [online]. [Cited 4 August 2020]. http://www.intechopen.com/books/herbicides-environmental-impactstudies-and-managementapproaches/velvetbean-biological-herbicide-to-conserve-soil-environmentalservices

Pittelkow, C.M., Linquist, B.A., Lundy, M.E., Liang, X., van Groenigen, K.J., Lee, J., van Gestel, N., Six, J., Venterea, R. \& van Kessel, C. 2015. When does no-till yield more? A global meta-analysis. Field Crop Research, 183: 156-168. https://doi.org/10.1016/j.fcr.2015.07.020

Poeplau, C. \& Don, A. 2015. Carbon sequestration in agricultural soils via cultivation of cover crops-A meta-analysis. Agriculture Ecosystems \& Environment, 200: 33-41.

https://doi.org/10.1016/j.agee.2014.10.024

Roesch-McNally, G.E., Basche, A.D., Arbuckle, J.G., Tyndall, J.C., Miguez, F.E., Bowman, T. \& Clay, R. 2018. The trouble with cover crops: Farmers' experiences with overcoming barriers to adoption. Renewable Agriculture and Food Systems, 33(4): 322-333. https://doi.org/10.1017/S1742170517000096

Sainju, U.M., Whitehead, W.F. \& Singh, B.P. 2003. Cover crops and nitrogen fertilization effects on soil aggregation and carbon and nitrogen pools. Canadian Journal of Soil Science, 83: 155-165.

https://doi.org/10.4141/S02-056 
Sanz-Cobena, A., Garcia-Marco, A., Quemada, M., Gabriel, J.L., Almendros, P. \& Vallejo, A. 2014.

Do cover crops enhance $\mathrm{N}_{2} \mathrm{O}, \mathrm{CO}_{2}$ or $\mathrm{CH}_{4}$ emissions from soil in Mediterranean arable systems? Science of the Total Environment, 466-467: 164-174. https://doi.org/10.1016/j.scitotenv.2013.07.023

Soil Science Society of America (SSSA). 2008. Glossary of soil science terms. [online]. [Cited 19 February 2020]. https://www.soils.org/publications/soils-glossary

Unger, P.W. \& Vigil, M.F. 1998. Cover crop effects on soil water relationships. Journal of Soil and Water Conservation, 53: 200-207.

Vicente-Vicente, J.L., García-Ruiz, R., Francaviglia, R., Aguilera, E. \& Smith, P. 2016. Soil carbon sequestration rates under Mediterranean woody crops using recommended management practices: A metaanalysis. Agriculture Ecosystems \& Environment, 235: 204-214.

https://doi.org/10.1016/j.agee.2016.10.024 
CROPLAND

SOIL ORGANIC COVER

\section{Organic mulch}

\section{Daniel Plaza-Bonilla, Genís Simon-Miquel}

Crop and Forest Sciences Dpt., Agrotecnio, University of Lleida, Lleida, Spain

\section{Description of the practice}

A mulch can be defined as a material which is applied to the soil surface in order to reduce water loss and soil erosion, suppress weeds, reduce fruit splashing, modify soil temperatures and generally improve crop productivity (FAO, 2004). Organic mulches would entail any material such as straw, leaves, loose soil, etc. that is spread or formed upon the surface of the soil to protect the soil and/or plant roots from the effects of raindrops, soil crusting, freezing, evaporation, etc. (SSSA, 2020). Farming with the presence of a mulch on the soil surface has been used for centuries by smallholders. For instance, the "tapado" or slash-and-mulch system of Central America is based on placing crop seeds on the soil below a dense stand of vegetation after first rains and cutting the vegetation allowing it to dry out (Kearney et al., 2019). The maintenance of a soil cover or mulch based on crop residues as well as a range of other materials is common in many types of agricultural productions (field crops, tree crops, horticultural crops, etc.). When organic mulches decompose they provide nutrients for the crops and, depending on the material, can release allelopathic substances (i.e. secondary metabolites) which combat biotic stresses such as weed infestations, insect pests and disease pathogens (Farooq et al., 2011); in some conditions, such as cool wet climates, these allelochemicals can be detrimental to crop growth (Jabran et al., 2015).

\section{Range of applicability}

The use of mulches based on crop residues is applicable to any type of pedoclimatic context. Agroecosystems with greater net primary productivity pose fewer limitations to the availability of enough crop residues to keep the soil completely covered with crop residues. In contrast, low productivity areas face limitations in terms of soil coverage by mulches (Plaza-Bonilla et al, 2015). For instance, semiarid and arid regions of West and Southeastern Africa as well as of the Mediterranean basin present low levels of biomass production. Besides this, in these and other regions the competition for crop residues for other purposes such as fodder, fuel and/or construction material further limits the potential to cover soils (German Agency for Technical Cooperation, 1998; Lahmar et al., 2012). 
In another different context, current demand for cellulosic-based fuels leads to the harvest of a significant proportion of crop residues in some developed countries, which hinders the sequestration of SOC (Miner et al., 2013). Furthermore, to reduce the risk of soil erosion removal of crop residues for bioenergy must leave a minimum soil cover of 30 percent.

\section{Impact on soil organic carbon stocks}

The maintenance of crop residues as dead mulches implies a $\mathrm{C}$ input to the soil. Depending on the local conditions, this $\mathrm{C}$ will be either converted to SOC or released from the soil, mainly, as carbon dioxide $\left(\mathrm{CO}_{2}\right)$. Therefore, these mulches might have a direct effect on the SOC stocks. Some research has been done in order to estimate the crop residues' potential on SOC changes, with great differences depending on pedoclimatic conditions and cropping systems. For instance, Page et al. (2020) found that crop residue mulch mitigated SOC losses up to $0.1 \mathrm{tC} / \mathrm{ha} / \mathrm{yr}$ of SOC under no-tillage (Table 6). Native areas tend to have higher SOC stocks than cultivated soils (Post and Kwon, 2000). Furthermore, warm-temperate areas of Australia have medium to low yields, which implies low carbon inputs and hence, low sequestration potentials. In contrast, Tenelli et al. (2019), when working in a highly productive and intensively tilled system, found that maintaining crop residues on soil surface was a key strategy to either start sequestering $\mathrm{C}$ or reducing C losses 10 -fold (Table 6 ). It is worth to consider that in many situations SOC losses are observed under an agricultural management in different pedoclimatic conditions (Table 6). For instance, Page et al. (2020) point out that a net decline in SOC over time is often observed in semiarid environments as a result of low net primary productivity and use of fallow periods to store soil water. Furthermore, the last authors stress the importance carrying out a diachronic approach, where the effects of treatments are quantified throughout time instead than using a business as usual treatment (e.g. ploughing, residue removal, etc.) as baseline in the same sense to what was previously elegantly recommended by Olson (2013) and Dimassi et al. (2014).

According to the literature (e.g. Page et al., 2019), crop residue mulches contribute to SOC sequestration, or at least might mitigate the losses. However, the magnitude of SOC sequestration depends highly on the local conditions, the previous land use, the practice's duration, and the sampling moment and methodology (Table 6). It is worth stressing that accurate and robust studies are needed to estimate crop residue's mulches sequestration potential accurately. 
Table 6. Mulch management experiments reporting changes in SOC stocks in four different pedoclimatic conditions

\begin{tabular}{|c|c|c|c|c|c|c|c|c|c|c|c|c|}
\hline \multirow{2}{*}{ Location } & \multicolumn{3}{|l|}{ Climate zone } & \multicolumn{3}{|l|}{ Soil type } & \multirow{2}{*}{$\begin{array}{l}\text { Baseline } \\
\text { C stock } \\
\text { (tC/ha) }\end{array}$} & \multirow{2}{*}{ Duration } & \multirow{2}{*}{$\begin{array}{l}\text { Additional C } \\
\text { storage } \\
\text { (tC/ha/yr) }\end{array}$} & \multicolumn{2}{|c|}{ More information } & \multirow{2}{*}{ Reference } \\
\hline & Zone & $\begin{array}{l}\text { MAP } \\
(\mathrm{mm})\end{array}$ & $\begin{array}{l}\text { MAT } \\
\left({ }^{\circ} \mathrm{C}\right)\end{array}$ & Soil type ${ }^{b}$ & Texture & $\mathrm{pH}$ & & & & Treatments & $\begin{array}{l}\text { Depth } \\
\text { (cm) }\end{array}$ & \\
\hline \multirow{2}{*}{$\begin{array}{l}\text { Queeensland, } \\
\text { Australia }\end{array}$} & \multirow{2}{*}{$\begin{array}{l}\text { Warm } \\
\text { Temperate } \\
\text { Dry }\end{array}$} & \multirow{2}{*}{685} & \multirow{2}{*}{17.5} & \multirow{2}{*}{ Vertisol } & \multirow{2}{*}{ Clay } & \multirow{2}{*}{7.6} & $73,1^{c}$ & \multirow{2}{*}{34} & -0.35 & CRR & \multirow{2}{*}{40} & \multirow{2}{*}{$\begin{array}{l}\text { Page et al. } \\
(2020)\end{array}$} \\
\hline & & & & & & & 72,6 & & -0.25 & CRL & & \\
\hline Various $^{\mathrm{a}}$ & $\begin{array}{l}\text { Warm } \\
\text { Temperate } \\
\text { moist mainly }\end{array}$ & NA & NA & $\begin{array}{l}74 \\
\text { experiments }\end{array}$ & Loam & NA & $N A^{d}$ & $\begin{array}{l}10 \text { yr av. } \\
(3->20)\end{array}$ & $0,41 \pm 0.16$ & 272 pairs $^{\mathrm{e}}$ & $\begin{array}{l}30 \text { on } \\
\text { av. }\end{array}$ & $\begin{array}{l}\text { Xu et al. } \\
(2019)\end{array}$ \\
\hline \multirow{2}{*}{ Quatà, Brazil } & \multirow{2}{*}{ Tropical moist } & \multirow{2}{*}{1254} & \multirow{2}{*}{20.8} & \multirow{2}{*}{$\begin{array}{l}\text { arenic } \\
\text { Kandiudult } \\
\text { soil }\end{array}$} & \multirow{2}{*}{$\begin{array}{l}\text { Sandy } \\
\text { loam }\end{array}$} & \multirow{2}{*}{4.8} & \multirow{2}{*}{21,2} & \multirow{4}{*}{5} & -0.62 & CRR & \multirow{4}{*}{40} & \multirow{4}{*}{$\begin{array}{l}\text { Tenelli et al. } \\
\text { (2019) }\end{array}$} \\
\hline & & & & & & & & & 0.23 & CRL & & \\
\hline \multirow{2}{*}{$\begin{array}{l}\text { Quirinópolis, } \\
\text { Brazil }\end{array}$} & \multirow{2}{*}{ Tropical moist } & \multirow{2}{*}{1520} & \multirow{2}{*}{22.5} & \multirow{2}{*}{$\begin{array}{l}\text { Rhodic } \\
\text { Eutrudox }\end{array}$} & \multirow{2}{*}{ Clayey } & \multirow{2}{*}{5.6} & \multirow{2}{*}{94} & & -0.5 & CRR & & \\
\hline & & & & & & & & & -0.05 & CRL & & \\
\hline
\end{tabular}

aMeta-analysis including several references from different areas, so that it is not possible to characterize either the climate or the soil further than the general classification given.

bSoil Survey Staff.

‘Unless it is specified, tO approach is used to characterize the baseline.

${ }^{d}$ since this review compiles several experiments and the baseline of each one is different, it is not possible to show a representative value for all of them. Nevertheless, it is mentioned that a BAU approach is used to characterize the baseline.

e272 paired points between left and removed corn crop residues.

Mulch treatments (CRL: crop residue left on the surface; CRR: crop residue removed, either burned or retired from the soil surface). 


\section{Other benefits of the practice}

The use of a mulch based on the maintenance of crop residues spread on the soil surface entails a range of direct and indirect benefits due to the concomitant input of $\mathrm{C}$ to the soil, the provision of a source of energy to the soil fauna, and the changes in energy balance between the soil surface and the atmosphere.

\subsection{Improvement of soil properties}

Mulched systems present improved soil physical properties because of greater soil surface structural stability (Plaza-Bonilla et al., 2013). The presence of a mulch reduces raindrop impacts and/or irrigation, which mitigates the dispersion of the finest soil particles and the creation of soil crusts (Pareja-Sánchez et al., 2017). This crusting process is common in silty soils and soils with presence of surface salts. The input of $\mathrm{C}$ that represents the use of crop residues as a mulch enhances the stability of soil aggregates, with a greater proportion of macroaggregates enriched with C. Consequently, the pores between aggregates become more continuous and stable which eases the infiltration of water into the soil and allows a greater diffusion of gases, providing aeration to the roots and soil biology (Ball, 2013). In this regard, Mulumba and Lal (2008) measured an increase (18-35 percent) in available water capacity, total soil porosity (35-46 percent) and soil moisture retention (29-70 percent) using samples from an Alfisol from central Ohio to which different mulch rates were imposed ( $2,4,8$ and $16 \mathrm{t} / \mathrm{ha} / \mathrm{yr})$ compared to the control without mulch.

The use of organic mulches provides substrates for soil macro- and microfauna. For instance, the addition of composted cotton gin trash to a soil cropped with organic tomato led to an increase in soil microbial biomass $\mathrm{C}$ and activity ranging between 103 and 151 percent and between 88 and 170 percent, respectively, compared to a control based on synthetic fertilizer ( $\mathrm{Tu}$, Ristaino and $\mathrm{Hu}$, 2006). Furthermore, potential mineralizable N (i.e. the amount of this nutrient that could be provided by the soil under non-limiting conditions) was increased by a 182 to 285 percent compared to the control (Tu, Ristaino and $\mathrm{Hu}, 2006)$. Mulches have also been reported to increase the activity of different types of enzymes such as arylsulfatases (Deng and Tabatabai, 1997). These are involved in sulphur cycling, an essential nutrient for all plants, which is especially important for crucifers.

Mulches can also affect soil chemical properties. For instance, working in a sandy Ultisol in Nigeria, Hulugalle, Lal and Gichuru (1990) found higher levels of exchangeable calcium, magnesium and potassium and lower acid saturation when using a $12 \mathrm{t} / \mathrm{ha}$ mulch of Chromolaena odorata L. compared to the non-mulched treatment. However, it must be taken into account that the impact of mulches on soil chemical properties are not ubiquitous and are more common in infertile soils such as the one reported by Hulugalle, Lal and Gichuru (1990). 


\subsection{Minimization of threats to soil functions}

Table 7. Soil threats

\begin{tabular}{|c|c|}
\hline Soil threats & \\
\hline Soil erosion & $\begin{array}{l}\text { The use of a mulch cover has been long known to be one of the } \\
\text { best low-cost strategies to reduce soil erosion (Smets, Poesen } \\
\text { and Knapen, 2008). }\end{array}$ \\
\hline $\begin{array}{l}\text { Nutrient imbalance and } \\
\text { cycles }\end{array}$ & $\begin{array}{l}\text { Organic mulches are a source of nutrients. When maintained on } \\
\text { the soil surface their release is slower, avoiding high losses (Al- } \\
\text { Kaisi and Guzman, 2013). }\end{array}$ \\
\hline $\begin{array}{l}\text { Soil salinization and } \\
\text { alkalinization }\end{array}$ & $\begin{array}{l}\text { Evaporation and soil temperature are reduced when using } \\
\text { mulches which marginally could reduce the risks of salinization. }\end{array}$ \\
\hline Soil acidification & Byproducts of decomposition may affect acidity. \\
\hline Soil biodiversity loss & $\begin{array}{l}\text { Mulching with organic sources helps sustain soil micro-organisms } \\
\text { and earthworms (Turbé et al., 2010). }\end{array}$ \\
\hline Soil compaction & $\begin{array}{l}\text { Mulches reduce soil surface compaction from the impact of water } \\
\text { drops (Pareja-Sánchez et al., 2017). }\end{array}$ \\
\hline
\end{tabular}

\subsection{Increases in production (e.g. food/fuel/feed/timber/fibre)}

The maintenance of a cover on soil surface is key to store water, especially in dryland areas (Montenegro et al., 2013). Where there is no availability of irrigation, soil water storage is key to maintain and increase crop production (e.g. Lampurlanés et al., 2016). Soil water storage also buffers precipitation shortages during key crop stages such as flowering. For instance, Page et al. (2019) found grain yield increases when wheat stubble was retained on the soil surface instead of being burned (2.51 vs $2.41 \mathrm{t} / \mathrm{ha}$, respectively).

Furthermore, the presence of a mulch on the soil surface inhibits the presence of weeds acting as a physical barrier for seedling emergence, lower light transmittance and soil surface temperature (Mohler and Teasdale, 1993). The release of allelopathic substances when some mulches (e.g. rye) decompose is another key mechanism of biotic stresses control (Farooq et al., 2011), as well as the different temperatures between a mulched soil and a bare soil. In this regard, Ranaivoson et al. (2019) studied the impact of conservation agriculture practices on rice production in Madagascar following smallholder-based cropping practices. The authors observed a significant decrease in weed pressure when using conservation agriculture with mulch which led to a 40 percent increase in rice yield. 


\subsection{Mitigation of and adaptation to climate change}

Mulches can play a major role in climate change mitigation. For instance, in rice cultivation under flooded conditions, the maintenance of mulches on the soil surface reduces $\mathrm{CH}_{4}$ emissions compared to their incorporation into the soil with tillage (Fangueiro et al., 2017), which accelerates their anaerobic decomposition and, consequently, leading to a release of $\mathrm{CH}_{4}$ by methanogenic bacteria (Also see factsheet No.17, Volume 5 on "Straw residue management in rice paddies"). Maintenance of crop residues on soil surface can also increase the amount of solar radiation reflected back into space compared to bare ground, through albedo modification, in areas with dark soils (Carrer et al., 2018). Adaptation to climate change is also a positive consequence of the use of mulches through reduced moisture loss, increased water infiltration and lower erosion risk.

\subsection{Other benefits of the practice}

In horticulture, the presence of mulches in the soil surface keeps the fruits clean (Tyagi et al., 2018).

\section{Potential drawbacks to the practice}

\subsection{Increases in greenhouse gas emissions}

The emission of nitrous oxide $\left(\mathrm{N}_{2} \mathrm{O}\right)$, a potent greenhouse gas with a global warming potential almost 300 higher than carbon dioxide $\left(\mathrm{CO}_{2}\right)$, is affected by the use of organic mulches. Mulches with low $\mathrm{C}: \mathrm{N}$ ratio, such as the ones of legumes, decompose faster than cereals and provide mineral $\mathrm{N}$ to the soil which can act as a substrate for $\mathrm{N}_{2} \mathrm{O}$ emission. For instance, when comparing different rates of vetch and wheat mulches in a subtropical soil (Schmatz et al., 2020) a 50 percent higher emission of $\mathrm{N}_{2} \mathrm{O}$ was found under vetch compared to wheat mulches. However, the emission factor (i.e. calculated as $\mathrm{N}_{2} \mathrm{O}$ emissions of the amended crop residue plots subtracting control plots divided by the quantity of residue total $\mathrm{N}$ added) was higher for wheat than for vetch.

\subsection{Conflict with other practice(s)}

The use of mulches is commonly linked to the implementation of no-tillage practices. Under that context, weed control is restricted to other practices different than tillage, which sometime can create dependence on synthetic products. 


\subsection{Decreases in production (e.g. food/fuel/feed/timber/fibre)}

An excessive amount of crop residues in specific pedoclimatic conditions can be counterproductive for grain yield. A relationship has been found between rainfed maize grain yield and annual rainfall in a meta-analysis performed by Rusinamhodzi et al. (2011). The last authors reported lower yield of maize when using mulch cover in high annual rainfall areas $(>1000 \mathrm{~mm})$, due to waterlogging. Mulches can lead to allelopathy that reduces crop growth, lower soil temperatures that impede fast establishment of crops (e.g. Venterea, Maharjan and Dolan, 2011), as well as exacerbation of frost damage (Snyder and de Melo-Abreu, 2005).

\subsection{Other conflicts}

Mulching with crop residues competes with other uses in smallholder farming. For instance, as pointed out by Giller et al. (2009) crop residues provide valuable fodder for livestock in sub-Saharan Africa. Livestock is key in smallholder farms, as a source of food, manure, traction, etc. (Giller et al., 2009). In other socioeconomic contexts, stubble is sometimes removed and sold for bioenergetic purposes, bedding, etc., which also compromises the maintenance of an adequate soil cover.

\section{Recommendations before implementing the practice}

In field crop production the presence of an excessive amount of mulch can pose difficulties to poorly adapted sowing and planting machines. When using sowing machines with disk openers care must be taken to avoid humid mulches, which instead of being cut are easily pressed into the slot compromising the viability of seeds. When tine openers are used excessive mulches can create straw clumping, which leads to problems in seed placement and seedling emergence. 


\section{Potential barriers to adoption}

Table 8. Potential barriers to adoption

\begin{tabular}{|l|c|l|}
\hline Barrier & YES/NO & \\
\hline Social & Yes & Mindset. \\
\hline Economic & Yes & $\begin{array}{l}\text { Competition for other purposes (e.g. fodder, fuel, construction } \\
\text { material, cellulosic-based fuels, etc.) (Lahmar et al., 2012; Miner } \\
\text { et al., 2013). Purchase of adequate drilling machines entail higher } \\
\text { costs than conventional ones (Epplin, 2008). }\end{array}$ \\
\hline Knowledge & Yes & $\begin{array}{l}\text { Lack of enough technical knowledge to establish the following } \\
\text { crop in the presence of crop residues (Bechini et al., 2015). }\end{array}$ \\
\hline
\end{tabular}

\section{Photo of the practice}

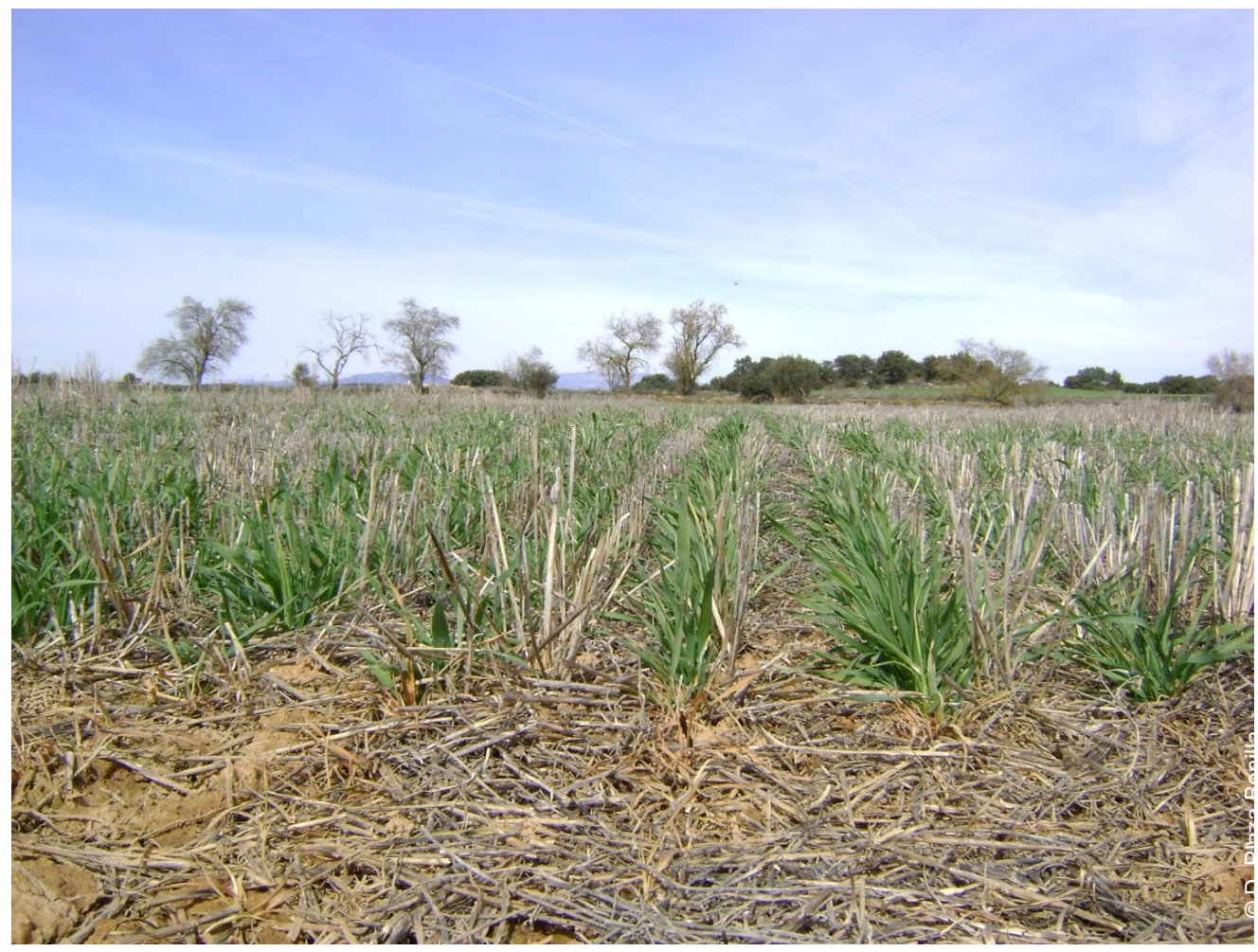

Photo 3. Winter cereal cultivation under a mulched soil in a Mediterranean semiarid area of Catalonia (NE Spain) 
Table 9. Related cases studies available in volumes 3 and 5

\begin{tabular}{|c|c|c|c|c|}
\hline Title & Region & $\begin{array}{l}\text { Duration } \\
\text { of study } \\
\text { (Years) }\end{array}$ & Volume & $\begin{array}{l}\text { Case- } \\
\text { study } \\
\text { No. }\end{array}$ \\
\hline Conservation Agriculture in Mozambique & Africa & 2 & 3 & 4 \\
\hline $\begin{array}{l}16 \text { years of no tillage and residue cover on } \\
\text { continuous maize in a Black soil of China }\end{array}$ & Asia & 16 & 3 & 10 \\
\hline $\begin{array}{l}\text { Rice straw mulching, charcoal, and no-tillage on } \\
\text { maize in Lopburi, Thailand }\end{array}$ & Asia & 4 & 3 & 11 \\
\hline $\begin{array}{l}\text { Long-term experiment of manure treatments on } \\
\text { a sandy soil, Germany }\end{array}$ & Europe & 29 & 3 & 12 \\
\hline $\begin{array}{l}\text { Mediterranean olive orchard subjected to } \\
\text { sustainable management in Matera, Basilicata, } \\
\text { Italy }\end{array}$ & Europe & 20 & 3 & 16 \\
\hline $\begin{array}{l}\text { Application of mulching in subtropical orchards in } \\
\text { Granada, Spain }\end{array}$ & Europe & 5 & 3 & 20 \\
\hline $\begin{array}{l}\text { Interrow organic management to restore soil } \\
\text { functionality of vineyards }\end{array}$ & $\begin{array}{l}\text { Europe and } \\
\text { Eurasia }\end{array}$ & 2 & 3 & 27 \\
\hline $\begin{array}{l}\text { Cover crops, organic amendments and combined } \\
\text { management practices in Mediterranean woody } \\
\text { crops }\end{array}$ & $\begin{array}{l}\text { Europe, NENA, } \\
\text { Eurasia, North } \\
\text { America }\end{array}$ & $<30$ & 3 & 28 \\
\hline $\begin{array}{l}30 \text { years of conservation agriculture practices on } \\
\text { Vertisols in central Mexico }\end{array}$ & $\begin{array}{l}\text { Latin America } \\
\text { and the } \\
\text { Caribbean }\end{array}$ & 30 & 3 & 37 \\
\hline $\begin{array}{l}\text { Whole orchard recycling as a practice to build soil } \\
\text { organic carbon in the San Joaquin Valley, } \\
\text { California, United States of America }\end{array}$ & North America & 9 & 3 & 47 \\
\hline $\begin{array}{l}\text { Straw mulch and biochar application in recently } \\
\text { burned areas of Algarve (Portugal) and Andalusia } \\
\text { (Spain) }\end{array}$ & Europe & 1 & 5 & 10 \\
\hline $\begin{array}{l}\text { Management of Common Reed (Phragmites } \\
\text { australis) in Mediterranean wetlands, Spain }\end{array}$ & Europe & Unknown & 5 & 18 \\
\hline $\begin{array}{l}\text { Water and residues management on a golf course, } \\
\text { Nebraska, United States }\end{array}$ & North America & 4 & 5 & 30 \\
\hline
\end{tabular}




\section{References}

Al-Kaisi, M.M. \& Guzman, J.G. 2013. Effects of tillage and nitrogen rate on decomposition of transgenic Bt and near-isogenic non-Bt maize residue. Soil and Tillage research, 129: 32-39.

https://doi.org/10.1016/j.still.2013.01.004

Ball, B.C. 2013. Soil structure and greenhouse gas emissions: a synthesis of 20 years of experimentation. European Journal of Soil Science, 64(3): 357-373. https://doi.org/10.1111/ejss.12013

Bechini, L., Costamagna, C., Zavattaro, L., Grignani, C., Bijttebier, J. \& Ruysschaert, G. 2015. Barriers and drivers towards the incorporation of crop residue in the soil. Analysis of Italian farmers' opinion with the theory of planned behaviors. Italian Journal of Agronomy, 10(4): 663.

https://doi.org/10.4081/ija.2015.663

Carrer, D., Pique, G., Ferlicoq, M., Ceamanos, X. \& Ceschia, E. 2018. What is the potential of cropland albedo management in the fight against global warming? A case study based on the use of cover crops. Environmental Research Letters, 13: 044030. https://doi.org/10.1088/1748-9326/aab650

Deng, S.P. \& Tabatabai, M.A. 1997. Effect of tillage and residue management on enzyme activities in soils: III. Phosphatases and arylsulfatase. Biology and Fertility of Soils, 24: 141-146. https://doi.org/10.1007/s003740050222

Dimassi, B., Mary, B., Wylleman, R., Labreuche, J., Couture, D., Piraux, F. \& Cohan, J-P. 2014. Longterm effect of contrasted tillage and crop management on soil carbon dynamics during 41 years. Agriculture, Ecosystems and Environment, 188: 134-146. https://doi.org/10.1016/j.agee.2014.02.014

Epplin, F.M. 2008. Economics: No-till versus Conventional Tillage. In Malone, J. No-till Cropping Systems in Oklahoma. E-996. Oklahoma Cooperative Extension Service. Division of Agricultural Sciences and Natural Resources. Oklahoma State University.

Fangueiro, D., Becerra, D., Albarrán, A., Peña, D., Sánchez-Llerena, J., Rato-Nunes, J.M. \& LópezPiñeiro, A. 2017. Effect of tillage and water management on GHG emissions from Mediterranean rice growing ecosystems. Atmospheric Environment, 150: 303-312. https://doi.org/10.1016/j.atmosenv.2016.11.020

FAO. 2004. Manual. Hot pepper seed and crop production in the Bahamas. Rome, Italy. 39 pp.

Farooq, M., Jabran, K., Cheema, Z.A., Wahid, A. \& Siddique, K.H.M. 2011. The role of allelopathy in agricultural pest management. Pest Management Science, 67: 493-506. https://doi.org/10.1002/ps.2091

German Agency for Technical Cooperation. 1998. Conserving natural resources and enhancing food security by adopting no-tillage: an assessment of the potential for soil-conserving production systems in various agro-ecological zones of Africa. TOV Publ. TOB F-5/e. GTZ, Eschborn, Germany.

Giller, K.E., Witter, E., Corbeels, M. \& Tittonell, P. 2009. Conservation agriculture and smallholder farming in Africa: The heretics' view. Field Crops Research, 114: 23-34.

https://doi.org/10.1016/j.fcr.2009.06.017

Hulugalle, N.R., Lal, R. \& Gichuru, M. 1990. Effect of five years of no-tillage and mulch on soil properties and tuber yield of cassava on an acid Ultisol in south-eastern Nigeria. Experimental Agriculture, 26, 235-240. https://doi.org/10.1017/S001447970001824X 
Jabran, K., Mahajan, G., Sardana, V. \& Chauhan, B.S. 2015. Allelopathy for weed control in agricultural systems. Crop Protection, 72: 57-65. https://doi.org/10.1016/j.cropro.2015.03.004

Kearney, S.P., Fonte, S.J., García, E., Siles, P., Chan, K.M.A. \& Smukler, S.M. 2019. Evaluating ecosystem service trade-offs and synergies from slash-and-mulch agroforestry systems in El Salvador.

Ecological Indicators, 105: 264-278. https://doi.org/10.1016/j.ecolind.2017.08.032

Lampurlanés, J., Plaza-Bonilla, D., Álvaro-Fuentes, J. \& Cantero-Martínez, C. 2016. Long-term analysis of soil water conservation and crop yield under different tillage systems in Mediterranean rainfed conditions. Field Crops Research, 189: 59-67. https://doi.org/10.1016/j.fcr.2016.02.010

Lahmar, R., Bationo, B.A., Lamso, N.D., Guéro,Y. \& Tittonell, P. 2012. Tailoring conservation agriculture technologies to West Africa semi-arid zones: building on traditional local practices for soil restoration. Field Crop Research, 132: 158-167. https://doi.org/10.1016/j.fcr.2011.09.013

Miner, G.L., Hansen, N.C., Inman, D., Sherrod, L.A. \& Peterson, G.A. 2013. Constraints of no-till dryland agroecosystems as bioenergy production systems. Agronomy Journal, 105: 364-376.

https://doi.org/10.2134/agronj2012.0243

Mohler, C.L. \& Teasdale, J.R. 1993. Response of weed emergence to rate of Vicia villosa Roth and Secale cereale L. residue. Weed Research, 33: 487-499. https://doi.org/10.1111/j.1365-3180.1993.tb01965.x

Montenegro, A.A.A., Abrantes, J., de Lima, J., Singh, V.P. \& Santos, T.E.M. 2013. Impact of mulching on soil and water dynamics under intermittent simulated rainfall. Catena, 109: 139-149.

https://doi.org/10.1016/j.catena.2013.03.018

Mulumba, L.N. \& Lal, R. 2008. Mulching effects on selected soil physical properties. Soil and Tillage Research, 98: 106-111.https://doi.org/10.1016/j.still.2007.10.011

Olson, K.R. 2013. Soil organic carbon sequestration, storage, retention and loss in U.S. croplands: Issues paper for protocol development. Geoderma, 195-196: 201-206.

https://doi.org/10.1016/j.geoderma.2012.12.004

Page, K.L., Dang, Y.P., Dalal, R.C., Reeves, S., Thomas, G., Wang, W. \& Thompson, J.P. 2019. Changes in soil water storage with no-tillage and crop residue retention on a Vertisol: Impact on productivity and profitability over a 50 year period. Soil Tillage Res., 194: 104319.

https://doi.org/10.1016/j.still.2019.104319

Page, K.L., Dalal, R.C., Reeves, S.H., Wang, W.J., Jayaraman, S. \& Dang, Y.P. 2020. Changes in soil organic carbon and nitrogen after 47 years with different tillage, stubble and fertiliser management in a Vertisol of north-eastern Australia. Soil Res., 58: 346-355. https://doi.org/10.1071/SR19314

Pareja-Sánchez, E., Plaza-Bonilla, D., Ramos, M.C., Lampurlanés, J., Álvaro-Fuentes, J. \& CanteroMartínez, C. 2017. Long-term no-till as a means to maintain soil Surface structure in an agroecosystem transformed into irrigation. Soil and Tillage Research, 174: 221-230.

https://doi.org/10.1016/j.still.2017.07.012

Plaza-Bonilla, D., Arrúe, J.L., Cantero-Martínez, C., Fanlo, R., Iglesias, A. \& Álvaro-Fuentes, J. 2015. Carbon management in dryland agricultural systems: a review. Agronomy for Sustainable Development, 35 : 1319-1334. https://doi.org/10.1007/s13593-015-0326-x

Plaza-Bonilla, D., Ávaro-Fuentes, J. \& Cantero-Martinez, C. 2013. Soil aggregate stability as affected by fertilization type under semiarid no-tillage conditions. Soil Science Society of America Journal, 77: 284-292. https://doi.org/10.2136/sssaj2012.0258 
Post, W.M. \& Kwon, K.C. 2000. Soil carbon sequestration and land-use change: processes and potential. Global Change Biology, 6: 317-327. https://doi.org/10.1046/j.1365-2486.2000.00308.x

Ranaivoson, L., Naudin, K., Ripoche, A., Rabeharisoa, L. \& Corbeels, M. 2019. Effectiveness of conservation agriculture in increasing crop productivity in low-input rainfed rice cropping systems under humid subtropical climate. Field Crops Research, 239: 104-113.

https://doi.org/10.1016/j.fcr.2019.05.002

Rusinamhodzi, L., Corbeels, M., van Wijk, M.T., Rufino, M.C., Nyamangara, J. \& Giller, K.E. 2011. A meta-analysis of long-term effects of conservation agriculture on maize grain yield under rain-fed conditions. Agronomy for Sustainable Development, 31: 657-673. https://doi.org/10.1007/s13593-011-0040-2

Schmatz, R., Recous, S., Weiler, D.A., Pilecco, G.E., Schu, A.L., Giovelli, R.L. \& Giacomini, S.J. 2020. How the mass and quality of wheat and vetch mulches affect drivers of soil $\mathrm{N}_{2} \mathrm{O}$ emissions. Geoderma, 372: 114395. https://doi.org/10.1016/j.geoderma.2020.114395

Smets, T., Poesen, J. \& Knapen, A. 2008. Spatial scale effects on the effectiveness of organic mulches in reducing soil erosion by water. Earth-Science Reviews, 89: 1-12.

https://doi.org/10.1016/j.earscirev.2008.04.001

Snyder, R.L. \& de Melo-Abreu, J.P. 2005. Frost Protection: fundamentals, practice and economics. Volume 1. Environmental and Natural Resources Series 10. FAO, Rome. ISBN: 92-5-105328-6.

Soil Science Society of America. 2020. Glossary of Soil Science Terms [online]. [Cited 24 September 2020]. https://www.soils.org/publications/soils-glossary\#

Tenelli, S., Oliveira Bordonal, R., Barbosa, L.C. \& Carvalho, J.L.N. 2019. Can reduced tillage sustain sugarcane yield and soil carbon if straw is removed? Bioenergy Research, 12(4): 764-777.

https://doi.org/10.1007/s12155-019-09996-3

Tu, C., Ristaino, J.B. \& Hu, S. 2006. Soil microbial biomass and activity in organic tomato farming systems: Effects of organic inputs and straw mulching. Soil Biology and Biochemistry, 38: 247-255.

https://doi.org/10.1016/j.soilbio.2005.05.002

Turbé, A., De Toni, A., Benito, P., Lavelle, P., Lavelle, P., Ruiz, N., Van der Putten, W.H., Labouze, E. \& Mudgal, S. 2010. Soil biodiversity: functions, threats and tools for policy makers. Bio Intelligence Service, IRD, and NIOO, Report for European Commission (DG Environment).

Tyagi, S., Singh, A., Sahay, S. \& Kumar, N. 2018. Mulching for comercial fruit production. In Kumar Ojha, P. (Ed.). Dimensions of agricultural science. Kalyani Publishers, Ludhiana. ISBN: 978-93-272-8337-2.

Venterea, R., Maharjan, B. \& Dolan, M.S. 2011. Fertilizer source and tillage effects on yield-scaled nitrous oxide emissions in a corn cropping system. Journal of Environmental Quality, 40: 1521-1531.

https://doi.org/10.2134/jeq2011.0039

Xu, H., Sieverding, H., Kwon, H., Clay, D., Stewart, C., Johnson, J.M.J., Qin, Z., Karlen, D.L. \&

Wang, M. 2019. A global meta-analysis of soil organic carbon response to corn stover removal. $G C B$ Bioenergy, 11(10): 1215-1233. https://doi.org/10.1111/gcbb.12631 
CROPLAND

\title{
CROP DIVERSIFICATION
}

\section{Crop rotations}

\author{
Genís Simon-Miquel, Daniel Plaza-Bonilla
}

Crop and Forest Sciences Dpt., Agrotecnio, University of Lleida, Lleida, Spain

\section{Description of the practice}

Crop rotation consists of the repetitive growing of an ordered succession of crops (or crops and fallow) on the same land, where one cycle often takes several seasons or years to complete (Francis, 1989). It is also one of the oldest agronomical practices and dates back more than 3000 years ago, to the Han dynasty of China and the Roman Empire eras (Farina et al. 2017). At that time, growers already knew that using different species (mainly legumes) as the precedents of the main crop (most often winter cereals) resulted in some increases in grain yields. Nowadays, crop rotations are still present in the cropping systems and, in some socioeconomic contexts is encouraged by agricultural policies (for example, in the European Union).

Over the years, scientific research has revealed that the benefits of this ancient management practice are due to improved resources use efficiency, increased $\mathrm{N}$ supply by legumes (from biological nitrogen fixation), and the breaking of pest cycles (Ryan et al., 2008). However, there is not a general conclusion on soil organic carbon (SOC) sequestration potential of crop rotations in the literature, since either increases, decreases or no effects on SOC sequestration have been observed (McDaniel et al., 2014). Therefore, care must be taken when assessing the potential of this management practice on SOC sequestration.

\section{Range of applicability}

The use of crop rotations in permanent croplands can be ubiquitous, although a high degree of crop diversity is usually hindered by pedo-climatic and socio-economic limitations. The climatic water balance (i.e. the difference between annual precipitation and potential evapotranspiration) is one key limiting factor for the implementation of diverse crop rotations. For instance, in many regions of the Mediterranean basin, crop rotations are mainly based on winter season crops (e.g. winter cereals, pulses, etc.) since the lack of available soil water impedes profitable crop cultivation during summer months (Photo 4). 
In other cases, such in northern latitudes, low temperatures and snowfall during autumn and winter months restricts crop cultivation until spring. In turn, different soil characteristics can limit the range of crops to be chosen for rotation. A clear example is the role played by soil $\mathrm{pH}$ on some crops. For instance, lupins are well adapted in slightly acidic to neutral soils whereas they grow poorly in alkaline soils.

As stated, socio-economic factors also play a major role in the use of diverse crop rotations. Since the advent of industrialized agriculture and the availability of synthetic fertilizers, cereals have been dominant in the globe, with just four crops (i.e. wheat, maize, rice and barley) representing more than 50 percent of the global cropland (Leff, Ramankutty and Foley, 2004). Cereals are highly demanding in N fertilizer which has led to different negative environmental consequences. Consequently, an adequately designed crop rotation including $\mathrm{N}_{2}$-fixing legumes not only increases subsequent cereal yields but also reduces the losses of reactive $\mathrm{N}$ to the environment (Plaza-Bonilla et al, 2015, 2017). The specialization of industrial farms to only a few commodities and the lack of financial support to invest in different machinery also impede adoption by many farmers. Similarly, the lack of appropriate markets for alternative products represents a major limitation for the implementation of diverse crop rotations in many areas of the globe.

\section{Impact on soil organic carbon stocks}

Many factors affect SOC stocks and its dynamic, (i.e. annual precipitation and temperature, soil type, different management practices, etc.). Moreover, its distribution throughout soil profile varies greatly with depth, with larger amounts of SOC near the surface. Therefore, quantifying SOC sequestration potential of a given crop sequence and management practice in a specific agricultural environment requires considering key criteria. Strict criteria of selection were followed to assure robustness in the data presented in Table 10 (following Olson (2013)). First, the description of each treatment was examined to characterize both the monoculture and the crop rotation, making sure that both were set up in an agricultural land use. Therefore, experiments comparing crop rotations with wild forest or non-cultivated land were discarded, considering that the effect of crop rotation on SOC sequestration was not evaluated per se. Also, any information regarding the previous management of the land had to be taken into account, since the SOC content does not change quickly and is highly dependent on previous land use and management practices. Second, the sampling methodology was carefully examined and the selected studies followed the subsequent criteria: (i) only studies reporting SOC stocks at the beginning of the comparison to adequately characterize the baseline, (ii) studies with different sampling times, (iii) studies covering greater depth than the plough layer (in order to include $\mathrm{C}$ inputs buried by tillage implements), and (iv) studies reporting the results in SOC stocks. Studies using an equivalent soil mass were also promoted to ensure that the real mass sampled was the same between rotations independently of soil bulk density (Ellert and Bettany, 1995). Unfortunately, the number of studies fulfilling these criteria was very low.

From the selected literature, it can be concluded that crop rotations have the capacity to store or at least mitigate SOC losses in a range of pedoclimates from tropical to dry and cool temperate areas under a range of different crop sequences (Table 10). For instance, in tropical conditions, Fugisaki et al. (2018) found that crop rotations led to an average increase of $830 \mathrm{~kg} \mathrm{C} / \mathrm{ha} / \mathrm{yr}$ in the SOC storage potential compared to monoculture. In warm and cool conditions, two studies showed different impacts of crop rotations on SOC stocks. 
On the one hand, Chan et al. (2011) found that crop rotation mitigated SOC losses from 278 to $176 \mathrm{~kg} \mathrm{C} / \mathrm{ha} / \mathrm{yr}$ (Table 10). This result was the consequence of a previous management practice that had sequestered large amounts of SOC during years; importantly the crop rotation mitigated this loss. On the other hand, Börjesson et al. (2018) compared a wheat monoculture to a 3-year ley of grass-clover (Festuca pratensis, Phleum pratense L. and Trifolium pratense L.) where the biomass was removed twice per year. Under wheat monoculture SOC was lost at a rate of $380 \mathrm{~kg} \mathrm{C} / \mathrm{ha} / \mathrm{yr}$ while the introduction of the 3 -year ley reverted the losses and led to a SOC sequestration rate of $470 \mathrm{~kg} \mathrm{C} / \mathrm{ha} / \mathrm{yr}$ (Table 10). 
Table 10. Crop rotation experiments reporting changes in SOC stocks in three different climate zones

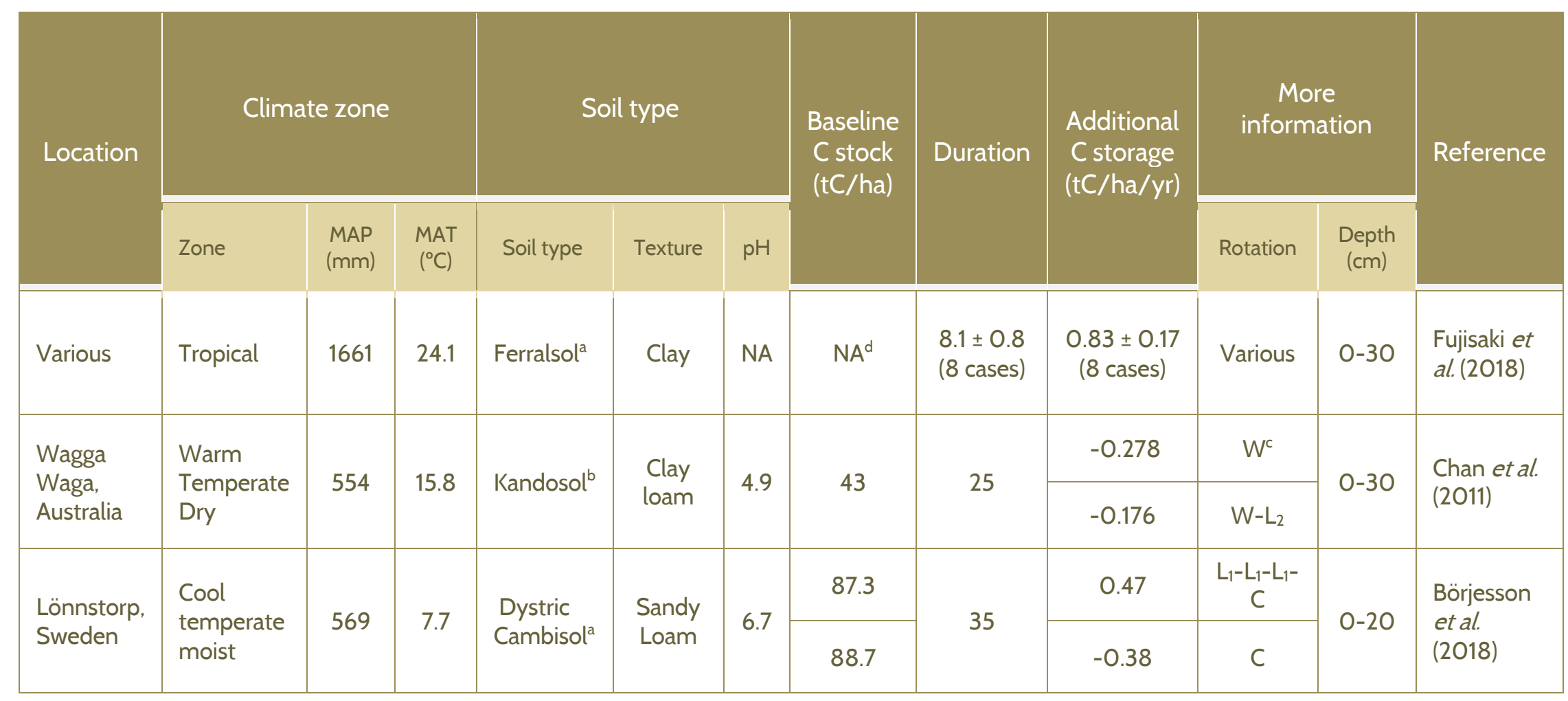

${ }^{a}$ WRB;

${ }^{\mathrm{b}}$ Australian Soil Classification;

'Different crops in the rotation (C: winter cereal; L1: grass-clover ley; L2: lupin; W: wheat);

${ }^{d}$ since this review compiles several experiments and the baseline of each one is different, it is not possible to show a representative value for all of them. 


\section{Other benefits of the practice}

\subsection{Improvement of soil properties}

The implementation of crop rotations provides several benefits in terms of soil properties. Soil physical properties improve significantly when using diverse crops through a variety of mechanisms, which impact on soil structure. The more stable the structural aggregates, the more resistant the soil structure is to different degradation processes such as erosion, compaction, soil crusting, etc. Crops differ in their root systems. Cereals such as wheat or barley present a fibrous root system which mostly develops at the soil surface. Differently, other crops such as crucifers (e.g. rapeseed) tend to have a vertical root system, which enhances soil vertical porosity and oxygen, water and nutrient transport to deeper layers when decomposed. In turn, some perennials such as alfalfa have a tap-root able to grow to very deep soil layers, which enhances the use of remnant soil water and nutrients, reducing drainage and $\mathrm{N}$ leaching. Moreover, legumes present higher activity in the rhizosphere (the environment close to the roots where most of soil biological activity occurs) and greater root exudation (Kumar et al., 2019). This last process entails the release of organic products from roots to ease the uptake of different nutrients. Similarly, many crops promote symbiosis between roots and arbuscular fungi. These fungi produce Glycoproteins, which are a major binding agent for soil aggregates. Glycoproteins, root exudates and soil particles enmeshment by roots and fungi play a major role in binding soil mineral particles and enhancing aggregate stability (Wright and Anderson, 2000). As a consequence of the introduction of legumes in crop rotations, the need for soil tillage is reduced (Preissel et al., 2015).

Crop rotations also lead to improved soil biological properties. In many cases, the changes in soil organic matter resulting from the use of different crop species and the different quality of their residues (e.g. $\mathrm{C} / \mathrm{N}$ and lignin/N ratios) are the main levers for enhanced soil biological activity. Cereal/legume rotations decrease the number of plant-parasitic nematodes and increase P availability in P-depleted African soils of Niger and Burkina Faso (Alvey et al.,2011). Moreover, in some instances, the benefits of crop rotations can be synergic to those of other practices such as reduced- or no-tillage. For instance, different soil biological indicators such as organic matter content, active $\mathrm{C}$, respiration and protein were enhanced when using no-tillage in combination with a crop rotation of maize and perennial grass and cover crops in a temperate area of north-east United States of America (Nunes et al., 2018). 


\subsection{Minimization of threats to soil functions}

Table 11. Soil threats

\begin{tabular}{|c|c|}
\hline Soil threats & \\
\hline Soil erosion & $\begin{array}{l}\text { The use of rotations reduces soil erosion through different root system } \\
\text { and greater soil structure stability. }\end{array}$ \\
\hline $\begin{array}{l}\text { Nutrient imbalance } \\
\text { and cycles }\end{array}$ & $\begin{array}{l}\text { Reduction in root diseases when using crop rotations increases } \\
\text { nitrogen use efficiency (Angus et al., 1998), while different rooting } \\
\text { patterns increase nutrient use efficiencies (Thorup-Kristensen, 2006). } \\
\text { P cycling is increased with root exudates and mycorrhizal activity } \\
\text { (Grant et al., 2002). }\end{array}$ \\
\hline $\begin{array}{l}\text { Soil biodiversity } \\
\text { loss }\end{array}$ & $\begin{array}{l}\text { Crop rotations increase soil microbial richness and diversity (Venter, } \\
\text { Jacobs and Hawkins, 2016) }\end{array}$ \\
\hline $\begin{array}{l}\text { Soil water } \\
\text { management }\end{array}$ & Crop rotation increases water-use efficiency (Ryan et al., 2008) \\
\hline
\end{tabular}

\section{3. (Increases in production (e.g. food/fuel/feed/timber/fibre)}

The benefits of crop rotations in terms of an increase in productivity for subsequent crops have been known for more than 3000 years (Karlen et al., 1994). For instance, the use of a preceding break-crop before wheat increases wheat yield from $0.5 \mathrm{t} / \mathrm{ha}$ to $1.2 \mathrm{t} / \mathrm{ha}$, with the greatest increases after grain legumes such as field peas, faba bean, chickpeas, lentils or lupins (Angus et al., 2015). According to these authors, additional yield increases (20-60 percent) are also found in the second year of wheat after a break-crop.

\subsection{Mitigation of and adaptation to climate change}

Nitrogen fertilization is the agricultural management practice that leads to the greatest soil nitrous oxide $\left(\mathrm{N}_{2} \mathrm{O}\right)$ emissions to the atmosphere (Bouwman et al., 2013), as this gas is a powerful greenhouse gas with a global warming potential 265 times that of carbon dioxide. Thanks to their capacity to fix atmospheric $\mathrm{N}$ in symbiosis with bacteria, crop diversification with legumes entails a reduction in the needs of $\mathrm{N}$ fertilizer at the crop and the rotation scale, minimizing the emission of $\mathrm{N}_{2} \mathrm{O}$. For instance, the mitigation of $\mathrm{N}_{2} \mathrm{O}$ when cropping legumes instead of fertilized cereals or oilseeds has been reported in the Northern Great Plains of North America (Lemke et al., 2002), France (Jeauffroy et al., 2013), and Australia (Schwenke et al., 2015). 


\subsection{Socio-economic benefits}

Crop rotations present different socio-economic benefits. Besides the diversification of revenues that takes place when establishing complex crop rotations compared to the reliance on a single commodity, crop rotations also spread labour needs, reduces equipment costs and peak labour demand, buffers price changes in the marketplace and increases the interaction with the local community for labour (Francis, 2005).

\section{Potential drawbacks to the practice}

\subsection{Tradeoffs with other threats to soil functions}

Soil threats are very uncommon when using diverse crop rotations if basic agronomic rules are followed.

\subsection{Increases in greenhouse gas emissions}

The implementation of some crop rotations can entail GHG emissions to the atmosphere in comparison to the traditional cropping systems of a given area. For instance, Plaza-Bonilla et al. (2018) quantified the C footprint (i.e. all direct and indirect emissions of GHG) of innovative crop 3-year rotations based on the introduction of grain legumes in comparison to the traditional rotation based on cereals and sunflower in south-west France. The authors observed a greater C footprint of the rotations, including grain legumes, when cover crops were not used as a result of SOC losses. However, as stated, that process is finite until SOC reaches a new equilibrium while significant amounts of GHG emissions are avoided thanks to the N-fertilizer savings when adopting legumes (Plaza-Bonilla et al., 2018).

\section{Recommendations before implementing the practice}

Before establishing any crop rotation, the pedoclimatic limitations to the different crops must be analyzed and taken into account. Potential markets must be explored and, if possible, secured before establishing alternative or neglected crops. These last should be implemented in small fractions of the available land. 


\section{Potential barriers to adoption}

Table 12. Potential barriers to adoption

\begin{tabular}{|l|l|l|}
\hline Barrier & YES/NO & \\
\hline Biophysical & No & No barriers if adequate crops are to be established. \\
\hline Cultural & No & Ancient and accepted agricultural practice. \\
\hline Economic & Yes & Lack of market for alternative crops. \\
\hline Institutional & No & $\begin{array}{l}\text { Crop rotation is encouraged in different areas (e.g. European } \\
\text { Union). }\end{array}$ \\
\hline Knowledge & Yes & Lack of knowledge on managing alternative crops. \\
\hline
\end{tabular}

\section{Photo of the practice}

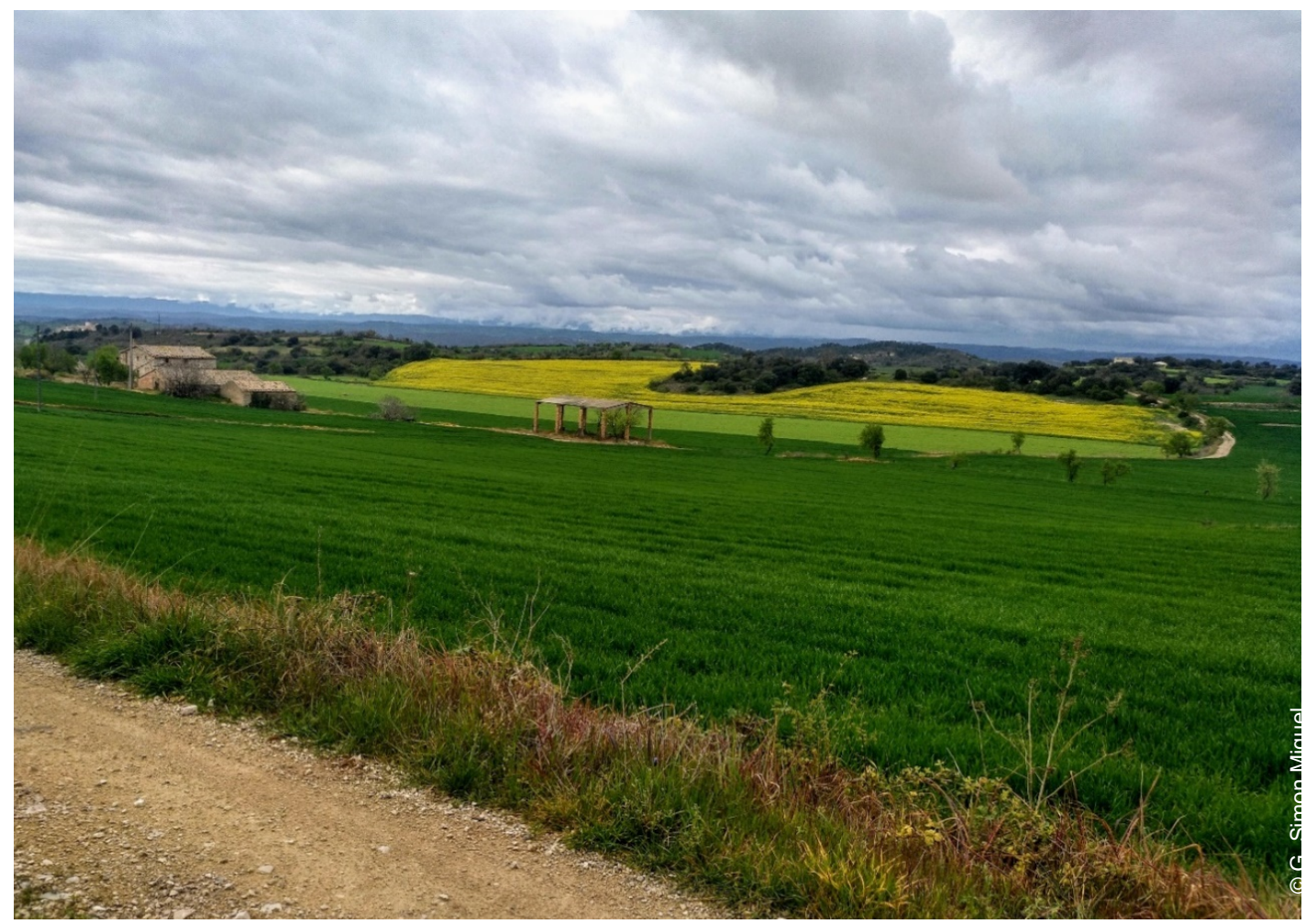

Photo 4. Rainfed field in the Northeastern Spain (Barcelona) where crop rotation includes canola, field pea, wheat, and barley 
Table 13. Related cases studies available in volumes 3 and 5

\begin{tabular}{|c|c|c|c|c|}
\hline Title & Region & $\begin{array}{l}\text { Duration } \\
\text { of study } \\
\text { (Years) }\end{array}$ & Volume & $\begin{array}{l}\text { Case- } \\
\text { study } \\
\text { No. }\end{array}$ \\
\hline Conservation Agriculture in South Africa & Africa & 6 & 3 & 5 \\
\hline $\begin{array}{l}\text { Irrigated cotton cropping systems in } \\
\text { Australian Vertisols under minimum } \\
\text { tillage }\end{array}$ & $\begin{array}{l}\text { Southwest } \\
\text { Pacific }\end{array}$ & 4 to 20 & 3 & 8 \\
\hline $\begin{array}{l}\text { Irrigation and SOC sequestration in the } \\
\text { region of Navarre in Spain }\end{array}$ & Europe & 6 to 20 & 3 & 19 \\
\hline $\begin{array}{l}\text { Irrigated Wheat-Maize-Cotton in the } \\
\text { Harran Plain, Southeast Turkey }\end{array}$ & Eurasia & 30 & 3 & 25 \\
\hline $\begin{array}{l}30 \text { years of conservation agriculture } \\
\text { practices on Vertisols in central Mexico }\end{array}$ & $\begin{array}{l}\text { Latin } \\
\text { America and } \\
\text { the } \\
\text { Caribbean }\end{array}$ & 30 & 3 & 37 \\
\hline $\begin{array}{l}\text { Rehabilitation of hardened neo-volcanic } \\
\text { soils in Mexico }\end{array}$ & $\begin{array}{l}\text { Latin } \\
\text { America and } \\
\text { the } \\
\text { Caribbean }\end{array}$ & $\begin{array}{l}10,50 \\
\text { and } 60\end{array}$ & 3 & 38 \\
\hline $\begin{array}{l}\text { Response of soil carbon to various } \\
\text { combinations of management practices } \\
\text { (annual-perennial rotation system, } \\
\text { animal manure application, reduced } \\
\text { tillage) in Quebec, Canada }\end{array}$ & $\begin{array}{l}\text { North } \\
\text { America }\end{array}$ & 21 & 3 & 43 \\
\hline $\begin{array}{l}\text { Long term fertilization in a subtropical } \\
\text { floodplain soil in Bangladesh }\end{array}$ & Asia & 42 & 5 & 13 \\
\hline $\begin{array}{l}\text { Organic rice cultivation with internal } \\
\text { nutrient cycling in Japanese Andosols }\end{array}$ & Asia & $\begin{array}{l}4,8 \text { and } \\
12\end{array}$ & 5 & 14 \\
\hline $\begin{array}{l}\text { Urban agriculture on rooftops in Paris, } \\
\text { France - the T4P research project (Pilot } \\
\text { Project of Parisian Productive Rooftops) }\end{array}$ & Europe & 5 & 5 & 23 \\
\hline
\end{tabular}




\section{References}

Alvey, S., Bagayoko, M., Neumann, G. \& Buerkert, A. 2001. Cereal/legume rotations affect chemical properties and biological activities in two West African soils. Plant and Soil, 231: 45-54.

https://doi.org/10.1023/A:1010386800937

Angus, J.F., Kirkegaard, J.A., Hunt, J.R., Ryan, M.H., Ohlander, L. \& Peoples, M.B. 2015. Break crops and rotations for wheat. Crop and Pasture Science, 66: 523-552. https://doi.org/10.1071/CP14252

Angus, F.E., Peoples, M.B. \& van Herwaarden, A.F. 1998. Water and nitrogen in crop and pasture systems in southern Australia. In Management of nutrients and water in rainfed arid and semi-arid areas: Proceedings of a consultants meeting organized by the Joint FAO/IAEA Division of Nuclear Techniques in Food and Agriculture. Vienna, 26-29 May 1997, IAEA-TECDOC No.1026, Vienna

Börjesson, G., Bolinder, M.A., Kirchmann, H. \& Kätterer, T. 2018. Organic carbon stocks in topsoil and subsoil in long-term ley and cereal monoculture rotations. Biology and Fertility of Soils, 54: 549-558.

https://doi.org/10.1007/s00374-018-1281-x

Bouwman, A.F., Beusen A.H.W., Griffioen, J., Van Groenigen, J.W., Hefting, M.M., Oenema, O., Van Puijenbroek, P.J.T.M., Seitzinger, S., Slomp, C.P. \& Stehfest, E. 2013. Global trends and uncertainties in terrestrial denitrification and $\mathrm{N}_{2} \mathrm{O}$ emissions. Philosophical Transactions of the Royal Society B-Biological Sciences, 368: 1621. https://doi.org/10.1098/rstb.2013.0112

Chan, K.Y., Conyers, M.K., Li, G.D., Helyar, K.R., Poile, G., Oates, A. \& Barchia, I.M. 2011. Soil carbon dynamics under different cropping and pasture management in temperate Australia: Results of three long-term experiments. Soil Research, 49(4): 320-328. https://doi.org/10.1071/SR10185

Ellert, B.H. \& Bettany. J.R. 1995. Calculation of organic matter and nutrients stored in soils under contrasting management regimes. Canadian Journal of Soil Sciences, 75: 529-538.

https://doi.org/10.4141//jss95-075

Farina, R., Di Bene, C., Piccini, C., Marchetti, A., Troccoli, A. \& Francaviglia, R. 2017. Do Crop Rotations Improve the adaptation of agricultural systems to Climate Change? A modelling approach to predict the effect of durum wheat-based rotations on Soil Organic Carbon and Nitrogen. In Munoz, M. \& Zornoza, R. (Eds.). Soil Management and Climate Change. Effects on Organic Carbon, Nitrogen Dynamics, and Greenhouse Gas Emissions. Chapter: 15, Elsevier.

Francis, C.A. 1989. Biological efficiencies in multiple-cropping systems. Advances in Agronomy, 42: 1-42. https://doi.org/10.1016/S0065-2113(08)60522-2

Francis, C.A. 2005. Crop rotations. In Hillel, D., Rosenzweig, C., Powlson, D., Scow, K., Singer, M., Sparks, D. (Eds.). Encyclopedia of Soils in the Environment. pp. 318-322. Academic Press.

Fujisaki, K., Chevallier, T., Chapuis-Lardy, L., Albrecht, A., Razafimbelo, T., Masse, D., Ndour, Y.B. \& Chotte, J.-L. 2018. Soil carbon stock changes in tropical croplands are mainly driven by carbon inputs: A synthesis. Agriculture, Ecosystems \& Environment, 259: 147-158.

https://doi.org/10.1016/j.agee.2017.12.008 
Grant, C.A., Peterson, G.A. \& Campbell, C.A. 2002. Nutrient considerations for diversified cropping systems in the Northern Great Plains. Agronomy Journal, 94: 186-198.

https://doi.org/10.2134/agronj2002.1860

Jeuffroy, M.H., Baranger, E., Carrouée, B., de Chezelles, E., Gosme, M., Hénault, C., Schneider, A. \& Cellier, P. 2013. Nitrous oxide emissions from crop rotations including wheat, oilseed rape and dry peas. Biogeosciences 10: 1787-1797. https://doi.org/10.5194/bg-10-1787-2013

Karlen, D.L., Varvel, G.E., Bullock, D.G. \& Cruse, R.M. 1994. Crop rotations for the $21^{\text {st }}$ Century. Advances in Agronomy, 53: 1-45. https://doi.org/10.1016/S0065-2113(08)60611-2

Kumar, N., Nath, C.P., Hazra, K.K., Das, K., Venkatesh, M.S., Singh, M.K., Singh, S.S., Praharaj, C.S. \& Singh, N.P. 2019. Impact of zero-till residue management and crop diversification with legumes on soil aggregation and carbon sequestration. Soil and Tillage Research, 189: 158-167.

https://doi.org/10.1016/j.still.2019.02.001

Leff, B., Ramankutty, N. \& Foley, J.A. 2004. Geographic distribution of major crops across the world. Global Biogeochemicalcycles, 18(1). https://doi.org/10.1029/2003GB002108

Lemke, R.L., Goddard, T.G., Selles, F. \&Zentner, R.P. 2002. Nitrous oxide emissions from wheat-pulse rotations on the Canadian prairies. In Proc. of the 4 th Annual Canadian Pulse Res. Workshop. 8-10 Dec. 2002, Edmonton, AB, Canada. p. 95-98.

McDaniel, M.D., Tiemann, L.K. \& Grandy, A.S. 2014. Does agricultural crop diversity enhance soil microbial biomass and organic matter dynamics? A meta-analysis. Ecological Applications, 24(3): 560-570. https://doi.org/10.1890/13-0616.1

Nunes, M.R., van Es, H.M., Schindelbeck, R., Ristow, A.J. \& Ryan, M. 2018. No-till and cropping system diversification improve soil health and crop yield. Geoderma, 328: 30-43.

https://doi.org/10.1016/j.geoderma.2018.04.031

Olson, K.R. 2013. Soil organic carbon sequestration, storage, retention and loss in U.S. croplands: Issues paper for protocol development. Geoderma, 195-196: 201-206.

https://doi.org/10.1016/j.geoderma.2012.12.004

Plaza-Bonilla, D., Nogué-Serra, I., Raffaillac, D., Cantero-Martínez, C. \& Justes, E. 2018. Carbon footprint of cropping systems with grain legumes and cover crops: A case-study in SW France. Agricultural Systems, 167: 92-102. https://doi.org/10.1016/j.agsy.2018.09.004

Plaza-Bonilla, D., Nolot, J-M., Raffaillac, D. \& Justes, E. 2015. Cover crops mitigate nitrate leaching in cropping systems including grain legumes: Field evidence and model simulations. Agriculture, Ecosystems and Environment, 212: 1-12. https://doi.org/10.1016/j.agee.2015.06.014

Plaza-Bonilla, D., Nolot, J-M., Raffaillac, D. \& Justes, E. 2017. Innovative cropping systems to reduce N inputs and maintain wheat yields by inserting grain legumes and cover crops in southwestern France.

European Journal of Agronomy, 82: 331-341. https://doi.org/10.1016/j.eja.2016.05.010 
Preissel, S., Reckling, M., Schläfke, N. \& Zander, P. 2015. Magnitude and farm-economic value of grain legume pre-crop benefits in Europe: A review. Field Crops Research, 175: 64-79.

https://doi.org/10.1016/j.fcr.2015.01.012

Schwenke, G.D., Herridge, D.F., Scheer, C., Rowlings, D.W., Haigh, B.M. \& McMullen, K.G. 2015.

Soil $\mathrm{N}_{2} \mathrm{O}$ emissions under $\mathrm{N}_{2}$-fixing legumes and $\mathrm{N}$-fertilised canola: a reappraisal of emissions factor calculations. Agriculture, Ecosystems and Environment, 202: 232-242.

https://doi.org/10.1016/j.agee.2015.01.017

Ryan, J., Pala, M., Masri, S., Singh, M. \& Harris, H. 2008. Rainfed wheat-based rotations under Mediterranean conditions: crop sequences, nitrogen fertilization, and stubble grazing in relation to grain and straw quality. European Journal of Agronomy, 28: 111-118. https://doi.org/10.1016/j.eja.2007.05.008

Thorup-Kristensen, K. 2006. Effect of deep and shallow root systems on the dynamics of soil inorganic N during 3-year crop rotations. Plant Soil, 288: 233-248. https://doi.org/10.1007/s11104-006-9110-7

Venter, Z.S., Jacobs, K. \& Hawkins, H-J. 2016. The impact of crop rotation on soil microbial diversity: A meta-analysis. Pedobiologia, 59: 215-223. https://doi.org/10.1016/j.pedobi.2016.04.001

Wright, S.F. \& Anderson, R.L. 2000. Aggregate stability and glomalin in alternative crop rotations for the central Great Plains. Biology and Fertility of Soils, 31(3): 249-253.

https://doi.org/10.1007/s003740050653 


\title{
4. Intercropping: Multiple cropping
}

\author{
Wen-Feng Cong', Wopke van der Werf², Fusuo Zhang' \\ ${ }^{1}$ College of Resources and Environmental Sciences, National Academy of Agriculture Green Development, \\ China Agricultural University, Beijing, China.
}

${ }^{2}$ Wageningen University, Plant Sciences, Crop Systems Analysis Group, Wageningen, The Netherlands

\section{Description of the practice}

Intercropping is the cultivation of multiple crop species on a single piece of land with biologically significant interaction between individual plants belonging to different species (Brooker et al., 2015). Intercropping can be divided into row intercropping, strip intercropping and fully mixed intercropping. Row intercropping implies either that crop species are grown in alternate rows or (more rarely) that species are grown fully mixed within an overall row arrangement. Strip intercropping means species are arranged in strips that comprise multiple crop rows. Mixed intercropping means that there is no distinct row arrangement. Furthermore, distinction is made between simultaneous intercrops that consist of species with the same growing period and relay-intercrops that comprise species with different growing periods, but still a significant period of co-growth during which they interact.

Global meta-analyses show that intercropping increases crop yields (Li et al., 2020; Yu et al., 2015, MartinGuay et al., 2018) and it also provides several ecological services such as pest, disease and weed control and it has positive effects on nutrient use efficiency and soil quality (Cong et al., 2015; Xu et al., 2020; Li et al., 2020). Intercropping can suppress weeds since mixed crops take away "niche space" that would have allowed weeds to grow (Liebman and Dyck, 1993). Furthermore, pest and disease pressure are reduced due to the dilution of suitable hosts (Boudreau, 2013). Long-term intercropping helps to enhance soil organic carbon concentration partly through enhanced belowground carbon inputs. Therefore, intercropping is a good option in the toolbox for sustainable soil management. 


\section{Range of applicability}

Intercropping has a long history of practice in smallholder farming in tropical and temperate regions of Africa, Asia, Europe and the Americas for centuries (Li et al., 2020). Currently, intercropping is not widely used in modern mechanized farming, except for fully mixed systems of small grains and legumes which can be harvested simultaneously with grain separation after harvest if required (Bedoussac et al., 2015).

\section{Impact on soil organic carbon stocks}

So far, six studies have looked at the effect of intercropping on soil C sequestration. Most of the studies are short-term, with the longest one covering an intercropping period of 7 years. Intercropping generally increases soil carbon sequestration compared to monoculture, with the changes in $\mathrm{C}$ storage ranging from - 0.020 to $0.184 \mathrm{tC} / \mathrm{ha} / \mathrm{yr}$ (Table 14). The effect of intercropping on soil $\mathrm{C}$ storage is most pronounced in the topsoil (Cong et al., 2015).

Table 14. Overview of the main features of intercropping studies reporting effects on carbon storage

\begin{tabular}{|c|c|c|c|c|c|c|c|}
\hline Location & $\begin{array}{l}\text { Climate } \\
\text { zone }\end{array}$ & Soil type & $\begin{array}{l}\text { Baseline } \\
\text { C stock } \\
\text { (tC/ha) }\end{array}$ & $\begin{array}{l}\text { Additional } \\
\text { C storage } \\
\text { (tC/ha/yr) }\end{array}$ & $\begin{array}{l}\text { Duration } \\
\text { (Years) }\end{array}$ & $\begin{array}{l}\text { Depth } \\
(\mathrm{cm})\end{array}$ & Reference \\
\hline $\begin{array}{l}\text { Costa Rica in La } \\
\text { Conquista, } \\
\text { Sarapiqui }\end{array}$ & NA & NA & 33.2 & -0.020 & 2 & $0-10$ & $\begin{array}{l}\text { Reeves et al. } \\
\text { (1997) }\end{array}$ \\
\hline $\begin{array}{l}\text { Bhopal Madhya } \\
\text { Pradesh, India }\end{array}$ & $\begin{array}{l}\text { Hot sub } \\
\text { humid }\end{array}$ & Vertisol & 15.1 & 0.042 & 2 & $0-15$ & $\begin{array}{l}\text { Singh et al. } \\
(2014)\end{array}$ \\
\hline $\begin{array}{l}\text { the rolling Pampa, } \\
\text { Balcarce, Argentina }\end{array}$ & NA & $\begin{array}{l}\text { Luvic } \\
\text { Phaozem }\end{array}$ & 33.7 & -0.006 & 2 & $\begin{array}{c}0- \\
120\end{array}$ & $\begin{array}{l}\text { Oelbermann } \\
\text { and Echarte } \\
\text { (2011) }\end{array}$ \\
\hline Guangzhou, China & $\begin{array}{l}\text { Subtropical } \\
\text { monsoon }\end{array}$ & $\begin{array}{l}\text { Crimson } \\
\text { soil }\end{array}$ & 26.8 & 0.166 & 4 & $0-30$ & Jianwu (2017) \\
\hline $\begin{array}{l}\text { Barangay Upi, } \\
\text { Gamu, Isabela, } \\
\text { Philippines }\end{array}$ & $\begin{array}{l}\text { Tropical } \\
\text { monsoon }\end{array}$ & Red soil & 19.3 & 0.014 & 5 & $0-5$ & $\begin{array}{l}\text { Ocampo and } \\
\text { Zamora } \\
(2016)\end{array}$ \\
\hline $\begin{array}{l}\text { Gansu province, } \\
\text { Northwest China }\end{array}$ & $\begin{array}{l}\text { Temperate } \\
\text { continental }\end{array}$ & NA & 34.5 & 0.184 & 7 & $0-20$ & $\begin{array}{l}\text { Cong et al. } \\
\text { (2015) }\end{array}$ \\
\hline
\end{tabular}




\section{Other benefits of the practice}

\subsection{Improvement of soil properties}

Intercropping increases soil macro-aggregates contents and soil microbial activity compared to corresponding monocropping (Tian et al., 2019). Importantly, intercropping enhances organic soil nitrogen, presumably due to better retention of nitrogen in the soil-plant system due to species complementarities (Cong et al., 2015)

(Figure 1).
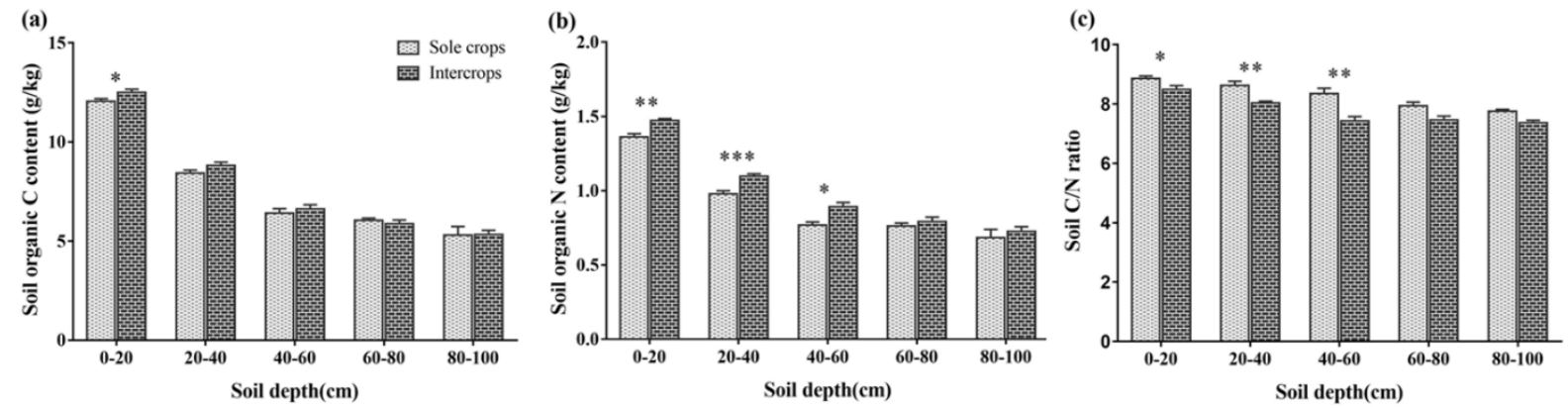

Figure 1. Soil organic C content (a), soil organic $\mathrm{N}$ content (b) and $\mathrm{C} / \mathrm{N}$ ratio (c) across a $1 \mathrm{~m}$ deep soil profile, averaged over intercrop systems and sole crop rotations with the same species in July 2010, after 7 years in the long-term experiment. (Cong et al., 2015)

Data are means $\pm \mathrm{SEM}, \mathrm{N}=9$

Asterisks refer to significant differences between sole crops and intercrops per depth: ${ }^{* * *} \mathrm{P}<0.001 ;{ }^{* *} \mathrm{P}<0.01 ;{ }^{*} \mathrm{P}<0.05$

\subsection{Minimization of threats to soil functions}

Table 15. Soil threats

\section{Soil threats}

\begin{tabular}{|l|l|}
\hline Soil erosion & $\begin{array}{l}\text { Reduced soil erosion through prolonged growing period of intercropped } \\
\text { species (Chen et al., 2010). }\end{array}$ \\
\hline $\begin{array}{l}\text { Nutrient imbalance } \\
\text { and cycles }\end{array}$ & $\begin{array}{l}\text { Intercropping can efficiently capture nutrients through temporal and } \\
\text { spatial niche differentiation as well as complementary use of different } \\
\text { nutrient sources (e.g. biological fixed N and soil mineral N) (Tsialtas et al., } \\
2018) .\end{array}$ \\
\hline Soil biodiversity loss & $\begin{array}{l}\text { Intercropping can promote soil biodiversity through continuously } \\
\text { providing more diverse root exudates for soil food web throughout the } \\
\text { growing season (Zhong and Zeng, 2019). }\end{array}$ \\
\hline
\end{tabular}




\begin{tabular}{|l|l|}
\hline Soil threats & $\begin{array}{l}\text { Intercropping may help improve soil structure through promoting root } \\
\text { growth and increasing soil organic carbon (Cong et al., 2015). }\end{array}$ \\
\hline $\begin{array}{l}\text { Soil water } \\
\text { management }\end{array}$ & $\begin{array}{l}\text { Intercropping can efficiently capture water through temporal and spatial } \\
\text { niche differentiation (Miyazawa et al., 2010). }\end{array}$ \\
\hline
\end{tabular}

\section{3. (Increases in production (e.g. food/fuel/feed/timber/fibre)}

Intercropping can increase crop yield through temporal differentiation of crop growth (Dong et al., 2018) or improvement of $\mathrm{N}$ utilization efficiency (Chen et al., 2019). More importantly, intercropping can also enhance yield stability, which is especially obvious in cereal-grain legume intercropping (Raseduzzaman and Jensen, 2017).

\subsection{Mitigation of and adaptation to climate change}

A few studies showed that intercropping can mitigate climate change by reducing $\mathrm{CO}_{2}$ and $\mathrm{N}_{2} \mathrm{O}$ emission compared to monocropping (Chai et al., 2014; Huang et al., 2019).

\subsection{Socio-economic benefits}

Intercropping maximizes cultural and regulation services compared with a single crop (Alcon et al., 2020).

\subsection{Other benefits of the practice}

Intercropping also suppresses diseases and weeds (Lv et al., 2018; Silberg, Richardson and Lopez, 2020).

\section{Potential drawbacks to the practice}

\subsection{Tradeoffs with other threats to soil functions}

No tradeoffs recorded. 


\subsection{Increases in greenhouse gas emissions}

In some cases, intercrop may increase $\mathrm{N}_{2} \mathrm{O}$ emissions compared with a single crop, which may be partly due to crop cultivars (Pappa et al., 2011).

\subsection{Decreases in production (e.g. food/fuel/feed/timber/fibre)}

When the competition between the two crops is strong, the yield advantage of intercropping is strong only when the proportion of the more competitive crops is large (Zhang, Yang and Dong, 2011). At this time, the yield advantage of intercropping is at the expense of the yield of another crop.

\section{Recommendations before implementing the practice}

- Include legume into intercrops to reduce $\mathrm{N}$ fertilizer and improve soil fertility.

- For regions where light and heat resources are more than enough to grow for one season but not enough for two seasons, relay-intercropping with appropriate fertilization can increase crop yield and enhance soil organic carbon.

- For humid area, such as southwest of China, maize/potato and rice/rice variety mixture will inhibit soil-borne diseases.

- Deep-rooting species with shallow-rooting species can complementarily take up water and nutrients.

- Food and oil combination (e.g. maize and peanut) achieve yield and farmer's profitability.

\section{Potential barriers to adoption}

Table 16. Potential barriers to adoption

\begin{tabular}{|l|c|l|}
\hline Barrier & YES/NO & \\
\hline Cultural & Yes & $\begin{array}{l}\text { The Western mindset is not yet geared towards exploiting diversity (Li et } \\
\text { al., 2O2O; Tilman, 202O). }\end{array}$ \\
\hline Social & Yes & Labor shortage. \\
\hline Economic & Yes & Labor cost is high if mechanization is unavailable. \\
\hline
\end{tabular}




\begin{tabular}{|l|c|l|}
\hline Barrier & YES/NO & \\
\hline Institutional & Yes & $\begin{array}{l}\text { There is no current market for tailored mechanization for modern } \\
\text { intercropping (Li et al., 202O; Stomph et al., 2020). }\end{array}$ \\
\hline Knowledge & Yes & $\begin{array}{l}\text { Western stakeholders have insufficient knowledge of the advantages of } \\
\text { intercropping (Tilman, 202O). }\end{array}$ \\
\hline Other & Yes & It is difficult to realize scale mechanization. \\
\hline
\end{tabular}

\section{Photos of the practice}

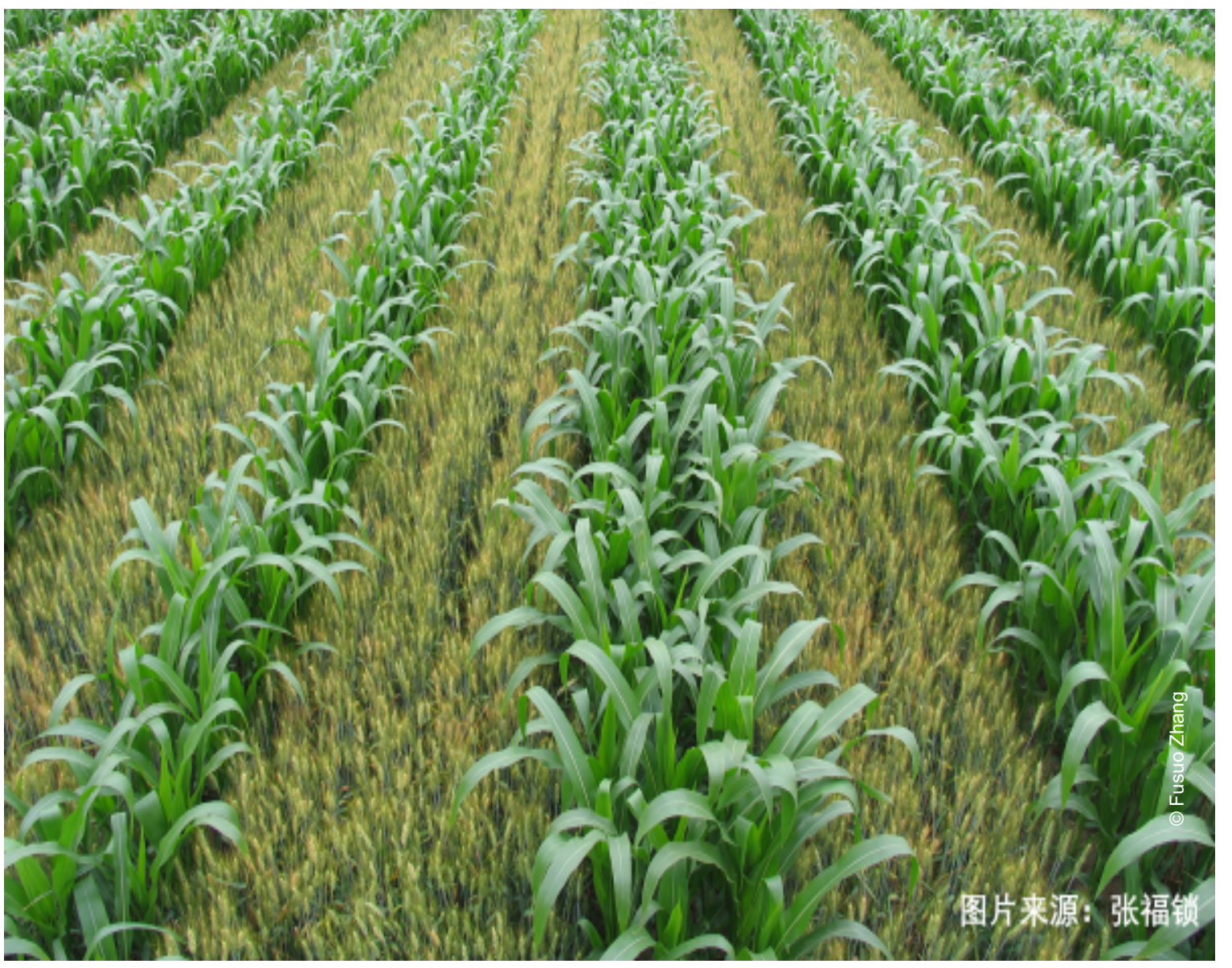

Photo 5. Wheat/maize strip intercropping in Gansu Province, China 


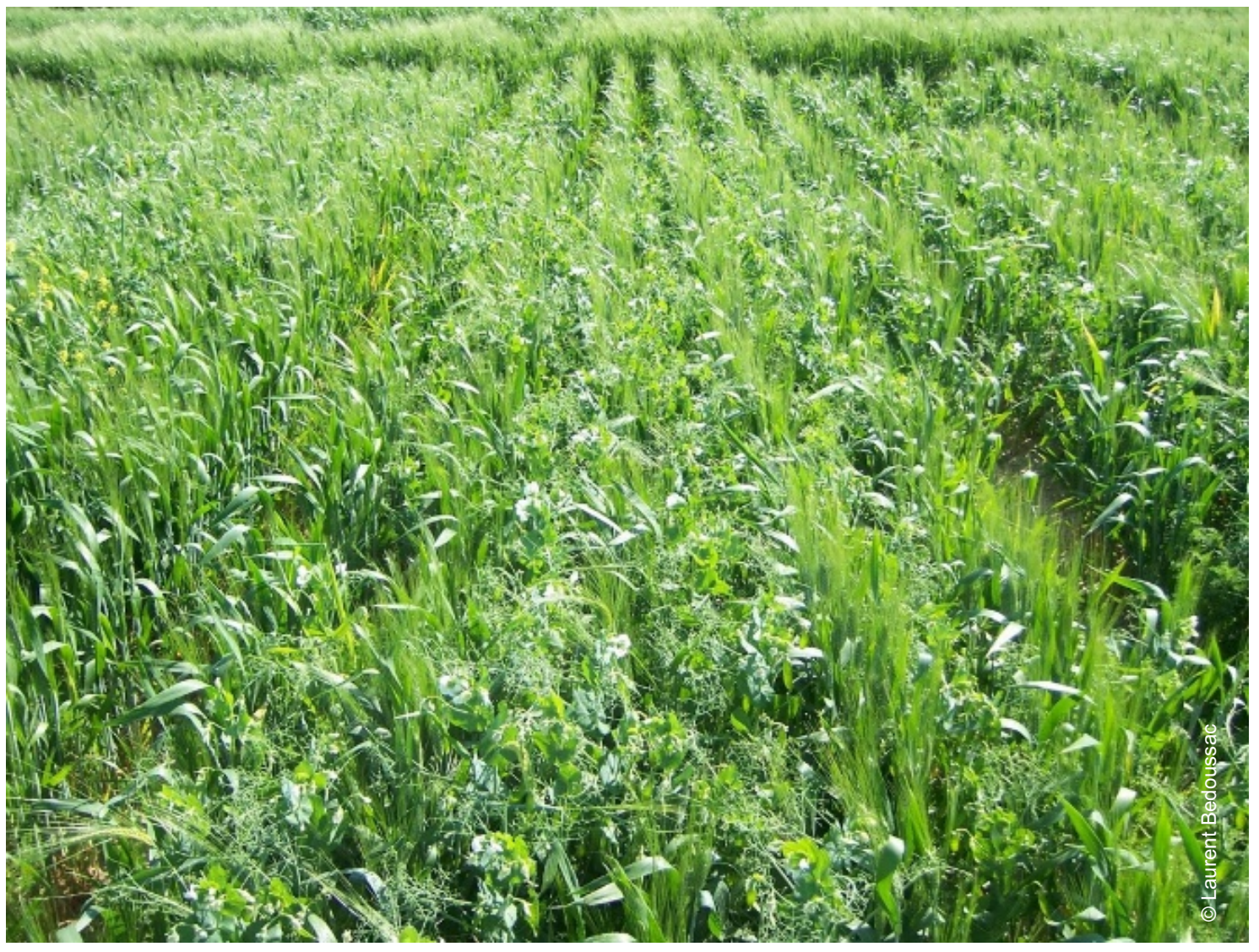

Photo 6. Durum wheat/lentil mixed intercropping in France

Table 17. Related cases studies available in volumes 3 and 5

\begin{tabular}{|l|l|c|c|c|}
\hline Title & Region & $\begin{array}{l}\text { Duration } \\
\text { of study } \\
\text { (Years) }\end{array}$ & Volume & $\begin{array}{l}\text { Case- } \\
\text { study } \\
\text { No. }\end{array}$ \\
\hline Conservation Agriculture in Mozambique & Africa & 2 & 3 & 4 \\
\hline Conservation Agriculture in South Africa & Africa & 6 & 3 & 5 \\
\hline $\begin{array}{l}\text { Intercropping grain legumes and cereals in } \\
\text { Africa }\end{array}$ & Africa & 2 to 11 & 3 & 6 \\
\hline $\begin{array}{l}\text { Interrow organic management to restore } \\
\text { soil functionality of vineyards }\end{array}$ & $\begin{array}{l}\text { Europe and } \\
\text { Eurasia }\end{array}$ & 2 & 3 & 27 \\
\hline $\begin{array}{l}\text { Rehabilitation of hardened neo-volcanic } \\
\text { soils in Mexico }\end{array}$ & $\begin{array}{l}\text { Latin America } \\
\text { and the } \\
\text { Caribbean }\end{array}$ & $\begin{array}{l}10,50 \\
\text { and } 60\end{array}$ & 3 & 38 \\
\hline
\end{tabular}




\section{References}

Alcon, F., Marín-Miñano, C., Zabala, J.A., de-Miguel, M.D. \& Martínez-Paz, J.M. 2020. Valuing diversification benefits through intercropping in Mediterranean agroecosystems: A choice experiment approach. Ecological Economics, 171: 106593. https://doi.org/10.1016/j.ecolecon.2020.106593

Bedoussac, L., Journet, E.-P., Hauggaard-Nielsen, H., Naudin, C., Corre-Hellou, G., Jensen, E.S., Prieur, L. \& Justes, E. 2015. Ecological principles underlying the increase of productivity achieved by cereal-grain legume intercrops in organic farming. A review. Agronomy for Sustainable Development, 35(3): 911-935. https://doi.org/10.1007/s13593-014-0277-7

Boudreau, M.A. 2013. Diseases in intercropping systems. Annual review of phytopathology, 51: 499-519. https://doi.org/10.1146/annurev-phyto-082712-102246

Brooker, R.W., Bennett, A.E., Cong, W.F., Daniell, T.J., George, T.S., Hallett, P.D., Hawes, C., Iannetta, P.P.M., Jones, H.G., Karley, A.J., Li, L., Mckenzie, B.M., Pakeman, R.J., Paterson, E., Schöb, C., Shen, J., Squire, G., Watson, C.A., Zhang, C., Zhang, F., Zhang, J. \& White, P.J. 2015. Improving intercropping: A synthesis of research in agronomy, plant physiology and ecology. New Phytologist, 206(1): 107-117. https://doi.org/10.1111/nph.13132

Chai, Q., Qin, A., Gan, Y. \& Yu, A. 2014. Higher yield and lower carbon emission by intercropping maize with rape, pea, and wheat in arid irrigation areas. Agronomy for Sustainable Development, 34(2): 535-543. https://doi.org/10.1007/s13593-013-0161-x

Chen, P., Song, C., Liu, X., Zhou, L., Yang, H., Zhang, X., Zhou, Y., Du, Q., Pang, T., Fu, Z., Wang, X., Liu, W., Yang, F., Shu, K., Du, J., Liu, J., Yang, W. \& Yong, T. 2019. Yield advantage and nitrogen fate in an additive maize-soybean relay intercropping system. Science of The Total Environment, 657: 987999. https://doi.org/10.1016/j.scitotenv.2018.11.376

Chen, Z., Cui, H., Wu, P., Zhao, Y. \& Sun, Y. 2010. Study on the optimal intercropping width to control wind erosion in North China. Soil and Tillage Research, 110(2): 230-235.

https://doi.org/10.1016/j.still.2010.07.014

Cong, W.-F., Hoffland, E., Li, L., Six, J., Sun, J.-H., Bao, X.-G., Zhang, F.-S. \& Werf, W.V.D. 2015. Intercropping enhances soil carbon and nitrogen. Global Change Biology, 21(4): 1715-1726.

https://doi.org/10.1111/gcb.12738

Dong, N., Tang, M.M., Zhang, W.P., Bao, X.G., Wang, Y., Christie, P. \& Li, L. 2018. Temporal Differentiation of Crop Growth as One of the Drivers of Intercropping Yield Advantage. Scientific Reports, 8(1): 1-11. https://doi.org/10.1038/s41598-018-21414-w

Huang, J., Sui, P., Gao, W. \& Chen, Y. 2019. Effects of Maize-Soybean Intercropping on Nitrous Oxide Emissions from a Silt Loam Soil in the North China Plain. Pedosphere, 29(6): 764-772.

https://doi.org/10.1016/S1002-0160(17)60389-8

Jianwu, W. 2017. Effects of reduced nitrogen application rates and soybean intercropping on sugarcane fields in Southern China. Agroecology in China: Science, Practice, and Sustainable Management, 24(2011): 61-81. 
Li, C., Hoffland, E., Kuyper, T.W., Yu, Y., Zhang, C., Li, H., Zhang, F. \& van der Werf, W. 2020. Syndromes of production in intercropping impact yield gains. Nature Plants, 6(6): 653-660. https://doi.org/10.1038/s41477-020-0680-9

Liebman, M. \& Dyck, E. 1993. Crop Rotation and Intercropping Strategies for Weed Management. Ecological Applications, 3(1): 92-122. https://doi.org/10.2307/1941795

Lv, H., Cao, H., Nawaz, M.A., Sohail, H., Huang, Y., Cheng, F., Kong, Q. \& Bie, Z. 2018. Wheat intercropping enhances the resistance of watermelon to fusarium wilt. Frontiers in Plant Science, 9: 1-15. https://doi.org/10.3389/fpls.2018.00696

Martin-Guay, M.O., Paquette, A., Dupras, J. \& Rivest, D. 2018. The new green revolution: sustainable intensification of agriculture by intercropping. Science of the Total Environment, 615: 767-772. https://doi.org/10.1016/j.scitotenv.2017.10.024

Miyazawa, K., Murakami, T., Takeda, M. \& Murayama, T. 2010. Intercropping green manure cropseffects on rooting patterns. Plant and Soil, 331(1): 231-239. https://doi.org/10.1007/s11104-009$0248-y$

Ocampo, N.P. \& Zamora, O.B. 2016. Carbon Storage of Corn-based Cropping Systems in Isabela, Philippines. Philippine Journal of Crop Science, 41(3): 20-29.

Oelbermann, M. \& Echarte, L. 2011. Evaluating soil carbon and nitrogen dynamics in recently established maize-soyabean inter-cropping systems. European Journal of Soil Science, 62(1): 35-41.

https://doi.org/10.1111/j.1365-2389.2010.01317.x

Pappa, V.A., Rees, R.M., Walker, R.L., Baddeley, J.A. \& Watson, C.A. 2011. Nitrous oxide emissions and nitrate leaching in an arable rotation resulting from the presence of an intercrop. Agriculture, Ecosystems and Environment, 141(1-2): 153-161. https://doi.org/10.1016/j.agee.2011.02.025

Raseduzzaman, M. \& Jensen, E.S. 2017. Does intercropping enhance yield stability in arable crop production? A meta-analysis. European Journal of Agronomy, 91: 25-33.

https://doi.org/10.1016/j.eja.2017.09.009

Reeves, M., Lal, R., Logan, T. \& Sigaran, J. 1997. Soil nitrogen and carbon response to maize cropping system, nitrogen source, and tillage. Journal of Food Agriculture and Environment, 61: 1387-1392. https://doi.org/10.2136/sssaj1997.03615995006100050015x

Silberg, T.R., Richardson, R.B. \& Lopez, M.C. 2020. Maize farmer preferences for intercropping systems to reduce Striga in Malawi. Food Security, 12(2): 269-283. https://doi.org/10.1007/s12571-020-

01013-2

Singh, R., Chaudhary, R., Somasundaram, J., Rashmi, I., Hati, K., Sinha, N. \& Rao, A. 2014. Impact of crop covers on soil properties, runoff, soil-nutrients losses and crop productivity in Vertisols of central India. Indian Journal of Soil Conservation, 42(3): 268-275.

Stomph, T.J., Dordas, C., Baranger, A., de Rijk, J., Dong, B., Evers, J., Gu, C., Li, L., Simon, J., Jensen, E.S., Wang, Q., Wang, Y., Wang, Z., Xu, H., Zhang, C., Zhang, L., Zhang, W.P., Bedoussac, L. \& van der Werf, W. 2020. Designing intercrops for high yield, yield stability and efficient use of resources: Are there principles? First edition. Elsevier Inc. 1-50 pp.

http://dx.doi.org/10.1016/bs.agron.2019.10.002 
Tian, X., Wang, C., Bao, X., Wang, P., Li, X., Yang, S., Ding, G., Christie, P. \& Li, L. 2019. Crop diversity facilitates soil aggregation in relation to soil microbial community composition driven by intercropping. Plant and Soil, 436(1-2): 173-192. https://doi.org/10.1007/s11104-018-03924-8

Tilman, D. 2020. Benefits of intensive agricultural intercropping. Nature Plants, 6(6): 604-605. https://doi.org/10.1038/s41477-020-0677-4

Tsialtas, I.T., Baxevanos, D., Vlachostergios, D.N., Dordas, C. \& Lithourgidis, A. 2018. Cultivar complementarity for symbiotic nitrogen fixation and water use efficiency in pea-oat intercrops and its effect on forage yield and quality. Field Crops Research, 226: 28-37. https://doi.org/10.1016/j.fcr.2018.07.005

Xu, Z., Li, C., Zhang, C., Yu, Y., van der Werf, W. \& Zhang, F. 2020. Intercropping maize and soybean increases efficiency of land and fertilizer nitrogen use; A meta-analysis. Field Crops Research, 246: 107661. https://doi.org/10.1016/j.fcr.2019.107661

Yu, Y., Stomph, T. J., Makowski, D. \& van der Werf, W. 2015. Temporal niche differentiation increases the land equivalent ratio of annual intercrops: a meta-analysis. Field Crops Research, 184: 133-144.

https://doi.org/10.1016/j.fcr.2015.09.010

Zhang, G., Yang, Z. \& Dong, S. 2011. Interspecific competitiveness affects the total biomass yield in an alfalfa and corn intercropping system. Field Crops Research, 124(1): 66-73.

https://doi.org/10.1016/j.fcr.2011.06.006

Zhong, S. \& Zeng, H.-C. 2019. Effect of peanut (Arachis hypogaea L.)/cowpea (Vigna unquiculata L.) intercropping combined with organic mature application on soil microfauna. Geoderma, 354: 113863. https://doi.org/10.1016/j.geoderma.2019.07.021 


\title{
5. Intercropping: strip cropping
}

\author{
Claudia Pozzi Jantalia, Segundo Urquiaga, Robert Michael Boddey, \\ Bruno José Rodrigues Alves
}

Embrapa Agrobiologia, Seropédica, RJ, Brasil

\section{Description of the practice}

Strip cropping is the growing of crops in alternating long, narrow strips, generally used in sloping areas as a measure of preventing soil erosion (Vandermeer, 1989; Wojtkowsk, 2008). This is commonly adopted to grain/fiber production using a plant species to block run-off or wind erosion. For instance, strips seeded to cereals alternated with strips of a sod-forming crop (such as hay) arranged to follow an approximate contour of the land are a recommendation to minimize losses by wind or rain (USDA-NRCS, 1995). In fact, this practice is an ancient recommendation for soil conservation, as reported by Kell and Brown (1938) of USDA in a technical bulletin in 1938. These authors defined three types of systematic arrangement of strips according to farmland slope, which were denominated contour, field, and wind strip cropping, briefly detailed here. Contour strip cropping is recommended in areas of variable land slope, where seeding obeys the natural land relief by establishing the plant rows on contour lines. Strips are relatively narrow but width can be variable. In these systems, dense erosion-control crops alternate with clean-tilled or erosion-permitting crops placed crosswise of the line of slope, approximately on the contour. Field strip cropping is possible where crops can be set up in more or less uniform strips laid out crosswise of the general slope but not obeying the true contour, and is a modified form of contour strip cropping. This is applicable to uniform gradual slopes of soils resistant to erosion, connecting water ways where the true relief contour cannot be easily followed. Wind strip cropping is recommended in flat lands. Different strip crops, relatively narrow and straight, are placed parallel to the direction of the prevailing wind without regard to the contour of the land. Actually, the combination with other soil conservation practices, such as no-tillage, are also recommended, considering that wind strip cropping does not promote significant soil water gains or run-off control. 


\section{Range of applicability}

Strip cropping has been used worldwide on sloping lands where agricultural mechanization is possible. This practice performs better when high yields are expected, and planting and harvesting are performed by machinery. It is recommended to protect against erosion (wind and water), except for non-mechanizable steep hillsides (Wojtkowsk, 2008; USDA-NRCS, 1995).

\section{Impact on soil organic carbon stocks}

Academic (Exner et al., 1999; Baumhardt and Blanco-Canqui, 2014; Soni et al., 2013) and technical (e.g USDA-NRCS reports 2020) literature suggest that strip cropping is strategic to reduce soil erosion and to sustain or improve soil quality. Other articles affirmed this practice supports the provision of ecosystem services such as biodiversity conservation (Vandermeer, 1989; Hauggaard-Nielsen, 2010). However, the absence of published research to allow reasonable global coverage of the impact on soil carbon sequestration evidences a scientific gap (

Table 18).

Table 18. Evolution of SOC stocks with application of strip cropping

\begin{tabular}{|l|l|l|l|l|l|l|l|}
\hline Location & $\begin{array}{l}\text { Climate } \\
\text { Zone }\end{array}$ & $\begin{array}{l}\text { Soil } \\
\text { type }\end{array}$ & $\begin{array}{l}\text { Additional } \\
\text { C storage } \\
\text { (tC/ha/yr) }\end{array}$ & $\begin{array}{l}\text { Duration } \\
\text { (Years) }\end{array}$ & $\begin{array}{l}\text { Depth } \\
\text { (cm) }\end{array}$ & More information & Reference \\
\hline $\begin{array}{l}\text { Regional, } \\
\text { hot arid } \\
\text { western } \\
\begin{array}{l}\text { Rajasthan, } \\
\text { India }\end{array}\end{array}$ & $\begin{array}{l}\text { Warm } \\
\text { temperate } \\
\text { dry }\end{array}$ & Sand & 0.06 & 2 & $0-10$ & $\begin{array}{l}\text { Measured soil } \\
\text { erosion and SOC } \\
\text { under C. ciliaris grass } \\
\text { sole and based } \\
\text { strip-cropping in hot } \\
\text { arid western } \\
\text { Rajasthan }\end{array}$ & $\begin{array}{l}\text { Soni et al. } \\
\text { (2013) }\end{array}$ \\
\hline $\begin{array}{l}\text { Cropping } \\
\text { areas of } \\
\begin{array}{l}\text { United } \\
\text { States of } \\
\text { America }\end{array}\end{array}$ & Various & - & $\begin{array}{l}\text { (0 to } \\
0.16)\end{array}$ & 20 & $0-20$ & $\begin{array}{l}\text { Data based on the } \\
\text { use of dense grasses } \\
\text { or legumes, hay } \\
\text { crops or other } \\
\text { perennial cover in } \\
\text { strips. }\end{array}$ & $\begin{array}{l}\text { Swan et al. } \\
\text { (2015); } \\
\text { Chambers, } \\
\text { Lal and } \\
\text { Paustian, } \\
\text { (2016) }\end{array}$ \\
\hline
\end{tabular}

Baseline stocks are not provided in any of the two studies presented. 


\section{Other benefits of the practice}

\subsection{Improvement of soil properties}

Increased water infiltration owing to the run-off contention by the strips (USDA-NRCS, 1995) explains the reduction of soil erosion and nutrient loss. In addition, wind erosion and its impacts on crops can be controlled by this practice (Swan et al., 2015). Because different crops are combined under strip cropping, the biodiversity of the agroecosystem is enhanced and the nature of crop type and field arrangement favors the accumulation of soil organic carbon (Baumhardt and Blanco-Canqui, 2014).

\subsection{Minimization of threats to soil functions}

Table 19. Soil threats

\begin{tabular}{|c|c|}
\hline Soil threats & \\
\hline Soil erosion & $\begin{array}{l}\text { Crop management strategy that reduces soil erosion (USDA-NRCS, } \\
\text { 1995). }\end{array}$ \\
\hline $\begin{array}{l}\text { Nutrient imbalance } \\
\text { and cycles }\end{array}$ & Benefits in nutrients cycling (Baumhardt and Blanco-Canqui, 2014). \\
\hline Soil biodiversity loss & $\begin{array}{l}\text { Increase plant diversification in the same crop season (Exner et al., } \\
\text { 1999). }\end{array}$ \\
\hline Soil compaction & The root diversity reduces soil compaction (Olson et al., 2017). \\
\hline $\begin{array}{l}\text { Soil water } \\
\text { management }\end{array}$ & $\begin{array}{l}\text { Increase water infiltration because strips act as a barrier (USDA-NRCS, } \\
\text { 1995). }\end{array}$ \\
\hline
\end{tabular}

\subsection{Increases in production (e.g. food/fuel/feed/timber/fibre)}

This practice could offer low to moderate levels of protection against crop-eating insects and plant diseases by the increase in plant diversification (Wojtkowski, 2008; NWRM, 2005). In addition, yield and plant residues inputs can be enhanced in comparison to individual crops owing to the increase in efficiency of water use (Maruthi, Reddy and Pankaj, 2017), and the use of soil nutrients (Hauggaard-Nielsen, Ambus and Jensen, 2001; Li et al, 2004). 


\subsection{Mitigation of and adaptation to climate change}

Literature suggests that strip cropping is a crop management strategy to increase the resilience of agroecosystems to excess rainfall, drought or warming (Maruthi et al., 2017). In addition, the capacity to improve $\mathrm{C}$ and $\mathrm{N}$ cycling in soil along with the reduction in $\mathrm{N}_{2} \mathrm{O}$ emissions due to the reduction of $\mathrm{N}$ fertilization makes it a greenhouse gas mitigation strategy (Chambers et al., 2016).

\subsection{Socio-economic benefits}

Strip cropping is a conservation agricultural practice that helps to preserve soil fertility and enables land use with higher yields and economic returns where constraints to conventional cropping exist such as variable sloping and extreme weather events. As the yield was increased ( Hauggaard-Nielsen, Ambus and Jensen, 2001; Li et al., 2004), the farmers have an economic return with this practice, because the costs are similar with sole crops.

\subsection{Other benefits of the practice}

If this practice is implemented with other conservation practices and selecting plant species that offer the benefits to the wildlife, as food or flowers, can be enhanced by providing habitat for pollinators, wildlife, and desired organisms (Exner et al., 1999; USDA-NSCS, 2017). The weed control in grain crops using mechanical operation was more efficient for strip cropping than for monocropping (Głowacka, 2014).

\section{Potential drawbacks to the practice}

\subsection{Increases in greenhouse gas emissions}

The use of legume strips could increase $\mathrm{N}$ uptake by gramineous strips, reducing the available $\mathrm{N}$ to losses as nitrate (indirect $\mathrm{N}_{2} \mathrm{O}$ emissions) or directly as nitrous oxide (Hauggaard-Nielsen et al., 2001; HauggaardNielsen, 2010)

\subsection{Decreases in production (e.g. food/fuel/feed/timber/fibre)}

When strips are planted to non-commercial crops alternately with strips of commercial crops, the production on a hectare basis will be negatively impacted compared to the commercial crop and consequently the farm income will be also affected. 


\section{Recommendations before implementing the practice}

The Natural Resources Conservation Service of US Department of Agriculture considers strip cropping as a conservation practice standard and provides recommendations about implementation (USDA-NRCS, 2017). Briefly, for mechanized farms, the cropping area must be suitable for setting the strips (allowing extended, unbroken strips). The farmer needs to know that strip cropping should be combined with other conservation practices to reach resource management objectives.

\section{Potential barriers for adoption}

Table 20. Potential barriers to adoption

\begin{tabular}{|l|l|l|}
\hline Barrier & YES/NO & \\
\hline Biophysical & Yes & $\begin{array}{l}\text { Slopes over 50 percent and the cropping area are not strip suitable } \\
\text { (USDA-NSCS, 2017). }\end{array}$ \\
\hline Cultural & Yes & $\begin{array}{l}\text { Communication strategies to help farmers to test strip cropping and } \\
\text { supporting them in choosing appropriate systems (NWRM, 2005). }\end{array}$ \\
\hline Social & Yes & $\begin{array}{l}\text { If implemented only on individual will and at field scale, the measure } \\
\text { will not be sufficient to impact on flood risk reduction (NWRM, }\end{array}$ \\
\hline Economic & Yes & $\begin{array}{l}\text { Machinery acquisition (NWRM, 2005). } \\
\text { Knowledge }\end{array}$ \\
\hline Yes & $\begin{array}{l}\text { Strips should be designed to facilitate operation of machinery, and } \\
\text { need know do the corrected soil contour to prevent effectiveness } \\
\text { soil loss (USDA-NSCS, 2017). }\end{array}$ \\
\hline
\end{tabular}

Table 21. Related cases studies available in volumes 3 and 5

\begin{tabular}{|l|c|c|c|c|}
\hline Title & Region & $\begin{array}{l}\text { Duration } \\
\text { of study } \\
\text { (Years) }\end{array}$ & Volume & $\begin{array}{l}\text { Case- } \\
\text { study } \\
\text { No. }\end{array}$ \\
\hline $\begin{array}{l}\text { Cover cropping in olive and vineyards (woody } \\
\text { crops) in Spain }\end{array}$ & Europe & 2 to 4 & 3 & 18 \\
\hline
\end{tabular}




\section{References}

Baumhardt, R.L. \& Blanco-Canqui, H. 2014. Soil: Conservation Practices. In N.K. Van Alfen (Ed.) Encyclopedia of Agriculture and Food Systems. pp. 153-165. Cambridge, US. Academic Press.

Chambers, A., Lal, R. \& Paustian, K. 2016. Soil carbon sequestration potential of US croplands and grasslands: implementing the 4 per thousand initiative. Journal of Soil Water Conservation, 71: 68A-74A. https://doi.org/10.2489/jswc.71.3.68A

Exner D.N., Davidson, D.G, Ghaffarzadeh, M. \& Cruse, R.M. 1999. Yields and returns from strip intercropping on six Iowa farms. American Journal of Alternative Agriculture, 14(2): 69-77.

https://doi.org/10.1017/S0889189300008092

Glowacka, A. 2014. The effects of strip cropping and weed control methods on yields of dent maize, narrowleafed lupin and oats. International Journal of Plant Production, 8(4): 505-529.

Hauggaard-Nielsen, H. 2010. Strip cropping system for sustainable food and energy production. Technical University of Denmark. (also available at: https://orgprints.org/18961/4/18961.pdf)

Hauggaard-Nielsen, H., Ambus, P., \& Jensen, E.S. 2001. Interspecific competition, N use and interference with weeds in pea-barley intercropping. Field Crops Research, 70: 101-109.

https://doi.org/10.1016/S0378-4290(01)00126-5

Kell, W.V. \& Brown, G.F. 1938. Strip cropping for soil conservation. Farmers bulletin No.1776. Washington, USDA.

Li, L., Sun, J.H., Zhang, F.S., Li, X.L., Yang, S.C. \& Rengel, Z. 2001. Wheat/maize or wheat/soybean strip intercropping. I. Yield advantage and interspecific interactions on nutrients. Field Crops Research, 71: 123-137. https://doi.org/10.1016/S0378-4290(01)00156-3

Maruthi, V., Reddy, K.S. \& Pankaj, P.K. 2017. Strip cropping system as a climate adaptation strategy in semi-arid Alfisols of South Central India. Indian Journal of Agricultural Sciences, 87(9): 1238-45.

Natural Water Retention Measures (NWRM). 2005. A4: Strip cropping along contours. (also available at: http://nwrm.eu/sites/default/files/nwrm_ressources/a4___strip_cropping_along_contours.pdf)

Olson, K.R., Al-Kaisi, M., Lal, R. \& Morton, L.W. 2017. Soil ecosystem services and intensified cropping systems. Journal of Soil and Water Conservation, 72(3): 64A-69A.

https://doi.org/10.2489/jswc.72.3.64A

Soni, M.L., Yadava, N.D., Beniwal, R.K., Singh, J.P., Kumar, S. \& Birbal. 2013. Grass based strip cropping systems for controlling soil erosion and enhancing system productivity under drought situations of hot arid western Rajasthan. International Journal of Agricultural and Statistical Sciences, 9(2): 685-692.

Swan, A., Williams, S.A., Brown, K., Chambers, A., Creque, J., Wick, J. \& Paustian, K. 2015. COMET-Planner. Carbon and greenhouse gas evaluation for NRCS conservation practice planning. (also available at: http://comet-planner.nrel.colostate.edu/COMET-Planner_Report_Final.pdf) 
United States Department of Agriculture -Natural Resources Conservation Service (USDA-NRCS). 1995. A Historical Study of Soil Conservation: Northern Mississippi Valley Working Paper No. 10. [online] [Cited 10 July 2020]. https://www.nres.usda.gov/wps/portal/nres/detail/?cid=nres143_014204)

United States Department of Agriculture-Natural Resources Conservation Service (USDA-NRCS). 2017. CONSERVATION PRACTICE. [online] [Cited 10 July 2020].

https://www.nrcs.usda.gov/wps/portal/nres/detailfull/national/technical/cp/ncps/?cid=nrcs143_02684 9

United States Department of Agriculture -Natural Resources Conservation Service (USDA-NRCS). 2020. NRCS Conservation Programs. [online] [Cited 16 July 2020].

http://www.nrcs.usda.gov/Internet/NRCS_RCA/reports/fb08_cp_crp.html

Vandermeer, J.H, ed. 1989. The Ecology of Intercropping. Cambridge, US. University Press.

Wojtkowski, P.A, ed. 2008. Agroecological Economics. Cambridge, US. Academic Press. 


\section{CROPLAND}

TILLAGE

\section{No-till}

\section{Gonzalo Berhongaray}

ICIAgro Litoral, Consejo Nacional de Investigaciones Científicas y Técnicas (UNL-CONICET),

Esperanza, Argentina

\section{Description of the practice}

The need for tillage has been questioned since the dustbowls in the mid-west United States of America in the 1930s. In the decades that followed no-till and other forms of soil cover were developed as practices for soil erosion protection. No-till is a system where a crop is planted directly into a seedbed that has not been tilled since harvest of the previous crop. It is also called zero tillage and it is used in conservation agriculture. The notill operation consists of a one-pass planting and fertilizer operation in which the soil and the surface residues are minimally disturbed (Parr et al., 1990). No-tillage systems eliminate all mechanical seedbed preparation before seeding except for the opening of a narrow (2-3 cm wide) strip or small hole in the ground for seed placement to ensure adequate seed/soil contact. The entire soil surface is covered by crop residue, mulch or sod. The surface residues of such a system are of critical importance for soil and water conservation. Weed control is generally achieved with herbicides or in some cases with cover crops and crop rotation.

\section{Range of applicability}

No-till can be applied in all row crops and in all countries. The greatest adoption is in South America where continuous no-till is being used on nearly 100 percent of the cropland in Argentina and Paraguay and approximately 70 percent of the arable land in Brazil (Kassam et al., 2015). It is currently used in agriculture under dry conditions (300 mm/yr in the Plurinational State of Bolivia) to very humid (2000 mm/yr in Brazil).

A review of tillage studies in Nigeria (Opara-Nadi, 1990) shows that no-tillage with residue mulch is appropriate for Luvisols in the humid tropics. No-tillage is used in mechanized wheat farming in the northern United Republic of Tanzania and for some perennial crops (Antapa and Angen, 1990; de Leijster et al, 2019). Several studies have reported the success of no-tillage systems in many parts of the United States of America (Smika and Unger, 1986; Unger, Langdale, and Papendick, 1988; Parr et al., 1990). Though the use of no-till is increasing, adoption has been slow in many parts of the world. 


\section{Impact on soil organic carbon stocks}

In Table 22 is a summary of information from studies on the effect of no-till on soil organic carbon (SOC) sequestration. Conversion to no-till is usually associated with increased SOC stocks in comparison to conventional tillage. However, in most studies SOC content is significantly greater only in the surface soil layers. A number of studies have shown that this effect is sometimes partly or completely offset by greater SOC content near the bottom of the plow layer under conventional tillage. For that reason, SOC stock changes have to be measured to at least $30 \mathrm{~cm}$ depth. Moreover, SOC stocks need to be expressed in an equivalent soil mass. Calculating stocks based on fixed depth layers, and without consideration of the equivalent soil mass, results in an overestimation of the increase ins SOC under no-till. Increases in SOC storage induced by no-till conversion seem to be largely related to increases of crop C inputs. Overall, this difference in favor of no-till increased significantly with the duration of the experiment, so long-term experiments are necessary for evaluation of SOC changes. Most of the studies of no-till effects on SOC are from North America and Europe; Oceania, Central and South America are less represented, and information from the other continents (Asia and Africa) is scarce or lacking. 
Table 22. Reviews of no-till effects on soil carbon

\begin{tabular}{|c|c|c|c|c|c|c|c|c|}
\hline Location & $\begin{array}{l}\text { Climate } \\
\text { zone }\end{array}$ & Soil type & $\begin{array}{l}\text { Baseline } \\
\text { C stock } \\
\text { (tC/ha) }\end{array}$ & $\begin{array}{l}\text { Additional C } \\
\text { storage } \pm \mathrm{SE} \\
\text { (tC/ha/yr) }\end{array}$ & $\begin{array}{l}\text { Duration } \\
\text { (Years) }\end{array}$ & $\begin{array}{l}\text { Depth } \\
\text { (cm) }\end{array}$ & $\begin{array}{l}\text { Methodology; } \\
\text { Main crops }\end{array}$ & Reference \\
\hline \multicolumn{9}{|l|}{ Global studies } \\
\hline $\begin{array}{l}\text { Europe, North and South } \\
\text { America }\end{array}$ & \multirow{4}{*}{ Various } & \multirow{4}{*}{ Various } & 95.4 & $0.30^{1}$ & $>5($ mean: 16) & $\begin{array}{l}\geq 30 \mathrm{~cm} \\
(0-30 \text { to } \\
0-100)\end{array}$ & $\begin{array}{l}\text { MA; Various crops (mainly } \\
\text { maize, wheat, soybean). } \\
\text { Baseline is the average value of } \\
\text { full inversion tillage }\end{array}$ & $\begin{array}{l}\text { Angers and } \\
\text { Eriksen-Hamel } \\
(2008)\end{array}$ \\
\hline $\begin{array}{l}\text { Africa, Europe, Oceania, North } \\
\text { and South America }\end{array}$ & & & NA & ns & $>4(4-41)$ & $\begin{array}{l}\geq 40 \mathrm{~cm} \\
(0-40 \text { to } \\
0-120)\end{array}$ & MA & $\begin{array}{l}\text { Luo, Wang and } \\
\text { Sun (2010) }\end{array}$ \\
\hline Americas and Europe & & & 62.6 & $0.23 \pm 0.08$ & $>5$ (mean: 15) & $0-30$ & $\begin{array}{l}\text { MA; Various crops } \\
\text { Baseline is the average of } \\
\text { inversion tillage treatment }\end{array}$ & Virto et al. (2012) \\
\hline $\begin{array}{l}\text { Boreo-temperate regions from } \\
\text { Europe, Oceania, North and } \\
\text { South America }\end{array}$ & & & NA & $0.13 \pm 0.09$ & $>5$ (mean: 17.6) & $\begin{array}{l}0-30 \text { and } \\
0-60\end{array}$ & MA; mainly annual crops & $\begin{array}{l}\text { Meurer et al. } \\
(2018)\end{array}$ \\
\hline \multicolumn{9}{|l|}{ Regional meta-analysis or reviews } \\
\hline $\begin{array}{l}\text { Mediterranean croplands } \\
\text { (Mediterranean basin, } \\
\text { California, Chile, South Africa, } \\
\text { Australia) }\end{array}$ & $\begin{array}{l}\text { Warm } \\
\text { Temperate Dry }\end{array}$ & Various & NA & 0.48 & $\stackrel{>3}{(\text { mean: 11.7) }}$ & $0-33.8$ & $\begin{array}{l}\text { MA; } \\
\text { Cereals, horticulture, woody } \\
\text { crops }\end{array}$ & $\begin{array}{l}\text { Aguilera et al. } \\
\text { (2013) }\end{array}$ \\
\hline \multicolumn{9}{|l|}{ National studies } \\
\hline Brazil & Tropical moist & $\begin{array}{l}\text { Sand and clay } \\
\text { soils }\end{array}$ & NA & $0.41 \pm 0.06$ & Various & $0-20$ and $0-30$ & $\begin{array}{l}\mathrm{R} ; \\
\text { Soybean and maize }\end{array}$ & $\begin{array}{l}\text { La Scala Júnior, De } \\
\text { Figueiredo and } \\
\text { Panosso (2012) }\end{array}$ \\
\hline
\end{tabular}

${ }^{1}$ Total C stocks difference between full inversion tillage (FIT) and no-till (NT) = +4.9 tC/ha ( 95.4 tC/ha under FIT and $100.3 \mathrm{tC} /$ ha under NT divided by the average duration, i.e. 16 years). 


\begin{tabular}{|c|c|c|c|c|c|c|c|c|}
\hline Location & $\begin{array}{l}\text { Climate } \\
\text { zone }\end{array}$ & Soil type & $\begin{array}{l}\text { Baseline } \\
\text { C stock } \\
\text { (tC/ha) }\end{array}$ & $\begin{array}{l}\text { Additional C } \\
\text { storage } \pm \mathrm{SE} \\
\text { (tC/ha/yr) }\end{array}$ & $\begin{array}{l}\text { Duration } \\
\text { (Years) }\end{array}$ & $\begin{array}{l}\text { Depth } \\
\text { (cm) }\end{array}$ & $\begin{array}{l}\text { Methodology; } \\
\text { Main crops }\end{array}$ & Reference \\
\hline United States of America & Various & Various & NA & $0.04 \pm 0.6 \mathrm{~ns}$ & 13.5 & $0-60$ & $\begin{array}{l}\mathrm{R} ; \\
\text { Various crops }\end{array}$ & $\begin{array}{l}\text { Blanco-Canqui } \\
\text { and Lal (2008) }\end{array}$ \\
\hline China & Various & Various & NA & $0.14 \pm 0.12$ & $>3$ (mean: 6.5) & $0-30$ & $\begin{array}{l}\text { MA; } \\
\text { Various crops (mainly maize, } \\
\text { wheat, rice, soybean) }\end{array}$ & Du et al. (2017) \\
\hline \multicolumn{9}{|l|}{ Local studies } \\
\hline \multirow{2}{*}{ Pampean region, Argentina } & $\begin{array}{l}\text { Warm } \\
\text { Temperate } \\
\text { Moist }\end{array}$ & Typic Argiudoll & 46.7 & $0.48 \pm 0.11$ & $\begin{array}{l}1 \text { to } 20 \text { (mean: } \\
8.2 \text { ) }\end{array}$ & $0-20$ & $\begin{array}{l}\text { MA; Annual crops (Corn, wheat, } \\
\text { soybean) }\end{array}$ & \multirow{2}{*}{$\begin{array}{l}\text { Steinbach and } \\
\text { Alvarez (2006) }\end{array}$} \\
\hline & $\begin{array}{l}\text { Warm } \\
\text { Temperate Dry }\end{array}$ & Entic Haplustoll & 39.0 & $0.32 \pm 0.18$ & 5 to 8 (mean: 6 ) & $0-20$ & $\begin{array}{l}\text { MA; Annual crops (Wheat, } \\
\text { sunflower) }\end{array}$ & \\
\hline Northern France & $\begin{array}{l}\text { Warm } \\
\text { Temperate } \\
\text { Moist }\end{array}$ & Haplic Luvisol & 44.2 & 0.02 & 41 & $0-28$ & Annual crops in rotation & $\begin{array}{l}\text { Dimassi et al. } \\
\text { (2014) }\end{array}$ \\
\hline Eastern Cape, South-Africa & Semi-arid & Haplic Cambisol & 29.8 & 1.71 & 3 & $0-20$ & Maize, soybean, wheat & $\begin{array}{l}\text { Mtyobile, } \\
\text { Muzangwa and } \\
\text { Mnkeni (2019) }\end{array}$ \\
\hline Buffelsvlei, South-Africa & Cold Arid & Chromic Lixisol & 19.7 & $0 ; n s$ & 8 & $0-30$ & $\begin{array}{l}\text { Conservation Agriculture; } \\
\text { Millet, sunflower, maize }\end{array}$ & $\begin{array}{l}\text { Swanepoel et al. } \\
(2018)^{2}\end{array}$ \\
\hline Tripura, India & Tropical Moist & Typic Kandiudults & 19.1 & 0.18 & 4 & $0-30$ & $\begin{array}{l}\text { Conservation Agriculture; } \\
\text { Rice, rapeseed, cowpea }\end{array}$ & Yadav et al. (2019) \\
\hline
\end{tabular}

2 Also see case study No.5, Volume 4

MA: Meta-analysis; R: Review; NA: not applicable; ns: not significant 


\section{Other benefits of the practice}

\subsection{Improvement of soil properties}

No-till can often increase soil carbon, soil quality and function, and reduce $\mathrm{CO}_{2}$ emissions when compared to conventional tilling practices (Karlen et al., 1994; Kladivko, 2001; Bolliger et al., 2006). Soil microbial biomass increases $(+37$ percent), including both fungal $(+31$ percent) and bacterial biomass $(+11$ percent), in top 20-cm soils under no-till agro-ecosystems, but not in sandy soils (Chen et al., 2020). No-till increased wet aggregate stability by 1 to 97 percent, water infiltration by 17 to 86 percent, and available water by 44 percent (Blanco-Canqui and Ruis 2018). However, no-till benefits largely depend on crop rotations (Mtyobile et al, 2019). In some studies, however, no changes in SOC, aggregate stability or water infiltration have been found (Alvarez et al., 2009; Swanepoel et al., 2018).

\subsection{Minimization of threats to soil functions}

\section{Table 23. Soil threats}

\begin{tabular}{|l|l|}
\hline Soil threats & \\
\hline Soil erosion & $\begin{array}{l}\text { Surface crop residue prevent from wind erosion by reducing wind speed in the } \\
\text { soil surface and water erosion by absorbing the energy of raindrop impact } \\
\text { (Langdale et al., 1979). }\end{array}$ \\
\hline $\begin{array}{l}\text { Soil biodiversity } \\
\text { loss }\end{array}$ & No-till increase soil biodiversity (Soane et al., 2012). \\
\hline $\begin{array}{l}\text { Soil water } \\
\text { management }\end{array}$ & $\begin{array}{l}\text { Surface crop residue decreases soil temperature and soil water evaporation } \\
\text { (Dardanelli, 1998). }\end{array}$ \\
\hline
\end{tabular}

\subsection{Increases in production (e.g. food/fuel/feed/timber/fibre)}

In Europe, reduction of 5 percent in yield have been reported for no-till crops, and yields tend to approach or exceed those after ploughing as the rainfall decreases from northern to southwestern Europe (Soane et al. 2012). In Argentina, a review by Alvarez and Steinbach (2009) indicates soybean yield was not significantly different between plow tillage and no-till.

In unfertilized situations wheat and corn yields were in average 9-12 percent significantly lower under no-till. Yield was not affected by tillage management when nitrogen was not a limiting resource (Alvarez and Steinbach, 2009). A global meta-analysis showed that overall no-till reduces yields, but this response is variable (Pittelkow 
et al., 2014). When combined with residue retention and crop rotation no-till can produce equivalent or greater yields than conventional tillage. Moreover, in dry areas no-till significantly increases rainfed crop yields.

\subsection{Mitigation of and adaptation to climate change}

No-till farming reduces the rapid oxidation of organic matter to $\mathrm{CO}_{2}$ which is induced by tillage (Alvarez et al., 1995). Limited $\mathrm{C}$ inputs, ranging between 0.1 and $1 \mathrm{~g} \mathrm{C} / \mathrm{kg}$ soil/yr, are likely to be the major bottleneck for $\mathrm{C}$ increase (Virto et al., 2012: Powlson et al, 2014; VandenBygaart, 2016). The presence of a mulch at the soil surface decreases soil water evaporation (Chakraborty et al., 2008; Verhulst et al., 2011; Balwinder et al., 2011), and hence no-till may become an important climate-change adaptation strategy for ever-drier regions of the world (Pittelkow et al., 2014).

\subsection{Socio-economic benefits}

No-till facilitates seeding of crops in soils where seed bed preparations is not easy. Moreover, surveys among European farmers indicated that reduced working time and lower costs were the dominant reasons for adopting no-till. The reductions of labour and mechanization costs with no-till represent 46 euros per hectare, while an increase of herbicide costs of 5 euros per hectare (Soane et al. 2012). Australian no-till farmers recognized the soil benefits of no-till, but it was not an important factor in explaining the no-till adoption. Shorter-term crop production benefits, such as weed management and the ability to sow crops earlier on less rainfall, were influential ( D’Emden, Llewellyn and Burton, 2008). In India, the main driver of adoption was found to be a significant, immediate and recurring "cost saving effect", reduced tractor time and fuel for land preparation and wheat establishment led to around 15 percent saving in operating costs (Erenstein et al., 2012). Profit increase of 800-2200 Rs/ha/yr was attributed to cost savings under no-till in India (Sidhu, Vatta, and Dhaliwal, 2010).

\section{Potential drawbacks to the practice}

\subsection{Tradeoffs with other threats to soil functions}

Table 24. Soil threats

\begin{tabular}{|l|l|}
\hline Soil threats & \\
\hline $\begin{array}{l}\text { Nutrient imbalance } \\
\text { and cycles }\end{array}$ & $\begin{array}{l}\text { Available } \mathrm{P} \text { and } \mathrm{K} \text { tend to become highly stratified near the soil surface. } \\
\text { Soil temperature is lower under no-till slowing down nutrient release } \\
\text { from organic matter ( } \mathrm{N} \text { and } \mathrm{S}) .\end{array}$ \\
\hline $\begin{array}{l}\text { Soil salinization and } \\
\text { alkalinization }\end{array}$ & Crop production in marginal soils can increase soil salinization risks. \\
\hline
\end{tabular}




\begin{tabular}{|c|c|}
\hline Soil threats & \\
\hline $\begin{array}{l}\text { Soil contamination } \\
\text { /pollution }\end{array}$ & $\begin{array}{l}\text { No-till might increase herbicide persistence in soil (Mickelson et al., } \\
\text { 2001). }\end{array}$ \\
\hline Soil acidification & $\begin{array}{l}\text { Concentration of SOC in the first upper layer decreases the pH } \\
\text { (Limousin and Tessier, 2007). Increases of acidity in surface layers of } \\
\text { soils under no-till have been associated with the acidifying effect of } \\
\text { nitrification of ammoniacal fertilizers and the decomposition of crop } \\
\text { residues (Soane et al., 2012). }\end{array}$ \\
\hline Soil compaction & $\begin{array}{l}\text { No-till without proper management tend to increase soil compaction } \\
\text { (Blanco-Canqui and Lal, 2008). Wheel traffic of heavy machinery over } \\
\text { moist soils, especially at harvest can cause substantial compaction to a } \\
\text { depth of } 20-30 \mathrm{~cm} \text { and sometimes deeper (Botta et al., 2018). }\end{array}$ \\
\hline
\end{tabular}

\subsection{Increases in greenhouse gas emissions}

No-till generally increased $\mathrm{N}_{2} \mathrm{O}$ emissions in poorly aerated soils but was neutral in soils with good and medium aeration (Rochette, 2008). A meta-analysis comparing soil $\mathrm{N}_{2} \mathrm{O}$ emissions from no-till and conventional tillage showed that emissions were significantly higher under no-till in the tropical climate (74.1 percent) and warm temperate climate (17.0 percent), but not in the cool temperate climate (Mei et al., 2018).

This trace gas has a large impact on mitigation potential because $1 \mathrm{~kg} \mathrm{~N} \mathrm{~N}_{2} \mathrm{O}-\mathrm{N}$ produces the warming effect of $120 \mathrm{~kg} \mathrm{CO}-\mathrm{C}$ (Houghton et al., 2001) and this might reduce the mitigation potential of no-till (Smith et al., 2000; Guenet et al., 2021). However, increased cropping frequency and crop diversity, such as double crops rotation, significantly reduce $\mathrm{CH}_{4}$ uptake by 18.4 percent, $\mathrm{N}_{2} \mathrm{O}$ emission by 21.0 percent, and overall global warming potential by 20.8 percent compared to the single crop monoculture system as revealed by a recent review (Feng et al., 2018).

\subsection{Conflict with other practice(s)}

No-till has a major influence in the vertical distribution of weed seedbank (Swanton et al,, 2000). Weed species which germination is stimulated by exposure to light become more prevalent under no-till. The performance of herbicides, particularly for soil active herbicides, is reduced under no-till. In summary, no till has effect on the weed ecology and care need to be taken with weed control practice (Chauhan, Gill and Preston, 2006). 


\subsection{Decreases in production (e.g. food/fuel/feed/timber/fibre)}

In cold climates, the presence of crop residues on the surface generally results in wetter and cooler conditions, thus favoring disease and pests, and pathogens also multiply with an additional source of energy (Reicosky, 2008).

\subsection{Other conflicts}

The large adoption of no-till is in regions characterized by large-scale mechanized monocropping of corn, soybeans, wheat, and other row crops. The adoption of NT farming is practically negligible by poor small land holders of sub-Saharan Africa (SSA), South and Southeast Asia, Central America, the Caribbean, and the Pacific Islands. These are also the regions where the potential benefits of NT farming are probably the highest (Lal, 2007).

\section{Recommendations before implementing the practice}

A list of top critical factors for no-tillage adoption has been prepared by Derpsch (2008):

- Improve your knowledge about the system, especially in weed control and plan for the change to permanent no-tillage at least 1 year in advance.

- Analyze your soil (aim for a balanced nutrient and $\mathrm{pH}$ status).

- Avoid soils with poor drainage or invest in an adequate drainage system before starting no-tillage. Notillage does not work on poorly drained soils.

- Level the soil surface. An uneven soil surface is a very unfavorable condition for seeding at an even depth.

- Eliminate soil compaction issues before starting no-till. When plow pan compaction is present it needs to be removed before going into a no-till system. Use a chisel or subsoiler.

- A special no-till seeding machine is needed. Buy or find one to rent.

- Start on 10 percent of your farm

- Use crop rotation and green manure cover crops to produce the largest possible amount of mulch cover. This is the best way to avoid soil compaction and to reduce $\mathrm{N}_{2} \mathrm{O}$ emissions.

- Be prepared to learn constantly and watch for new developments. 


\section{Potential barriers for adoption}

\section{Table 25. Potential barriers to adoption}

\begin{tabular}{|c|c|c|}
\hline Barrier & YES/NO & \\
\hline Biophysical & Yes & $\begin{array}{l}\text { In cold climate sites, problems have been found when cereal crops } \\
\text { were drilled in the presence of crop residues on the surface. Crops } \\
\text { which require much traffic of heavy harvesting machinery, may } \\
\text { cause difficulties for no-till establishment of the following crop. Soils } \\
\text { with imperfect drainage and weak structure are unfavorable for no- } \\
\text { till (Soane et al., 2012). In dry climate, there are also risks of residue } \\
\text { fire. }\end{array}$ \\
\hline Cultural & Yes & $\begin{array}{l}\text { Farmers have been plowing for weed control and seedbed } \\
\text { preparation for many millennia (Lal, Reicosky, and Hanson, 2007). }\end{array}$ \\
\hline Social & Yes & $\begin{array}{l}\text { Social conflicts are associated with an increase of the use of } \\
\text { pesticides under no-till (Levidow, 2007). }\end{array}$ \\
\hline Economic & Yes & $\begin{array}{l}\text { No-till requires a significant investment in new machinery for their } \\
\text { effective implementation (Trigo et al., 2009). A profitability analysis } \\
\text { in the U.S. suggests that about } 10 \text { years after implementation are } \\
\text { needed to recuperate the initial expense of no-till implementation, } \\
\text { with the probability of higher relative profit increasing with longevity } \\
\text { (Cusser et al., 2019). }\end{array}$ \\
\hline Institutional & Yes & $\begin{array}{l}\text { In Argentina, the no-till association (AAPRESID) as a consolidated } \\
\text { network, brought together all relevant stakeholders to share } \\
\text { technical and economic information and to promote the benefits of } \\
\text { the no-till and cover crops technology. During the 1990's and along } \\
\text { with farmer associations with similar objectives in Brazil, Mexico, } \\
\text { Paraguay, and Uruguay, these organization later coalesced into the } \\
\text { American Confederation of No-Till Farmers Associations (CAAPAS, } \\
\text { www.caapas.org). }\end{array}$ \\
\hline Knowledge & Yes & $\begin{array}{l}\text { No-till substantially change crop management (weeds pest control, } \\
\text { fertilization). New knowledge needs to be created locally to adopt } \\
\text { this practice. }\end{array}$ \\
\hline
\end{tabular}




\section{Photos of the practice}

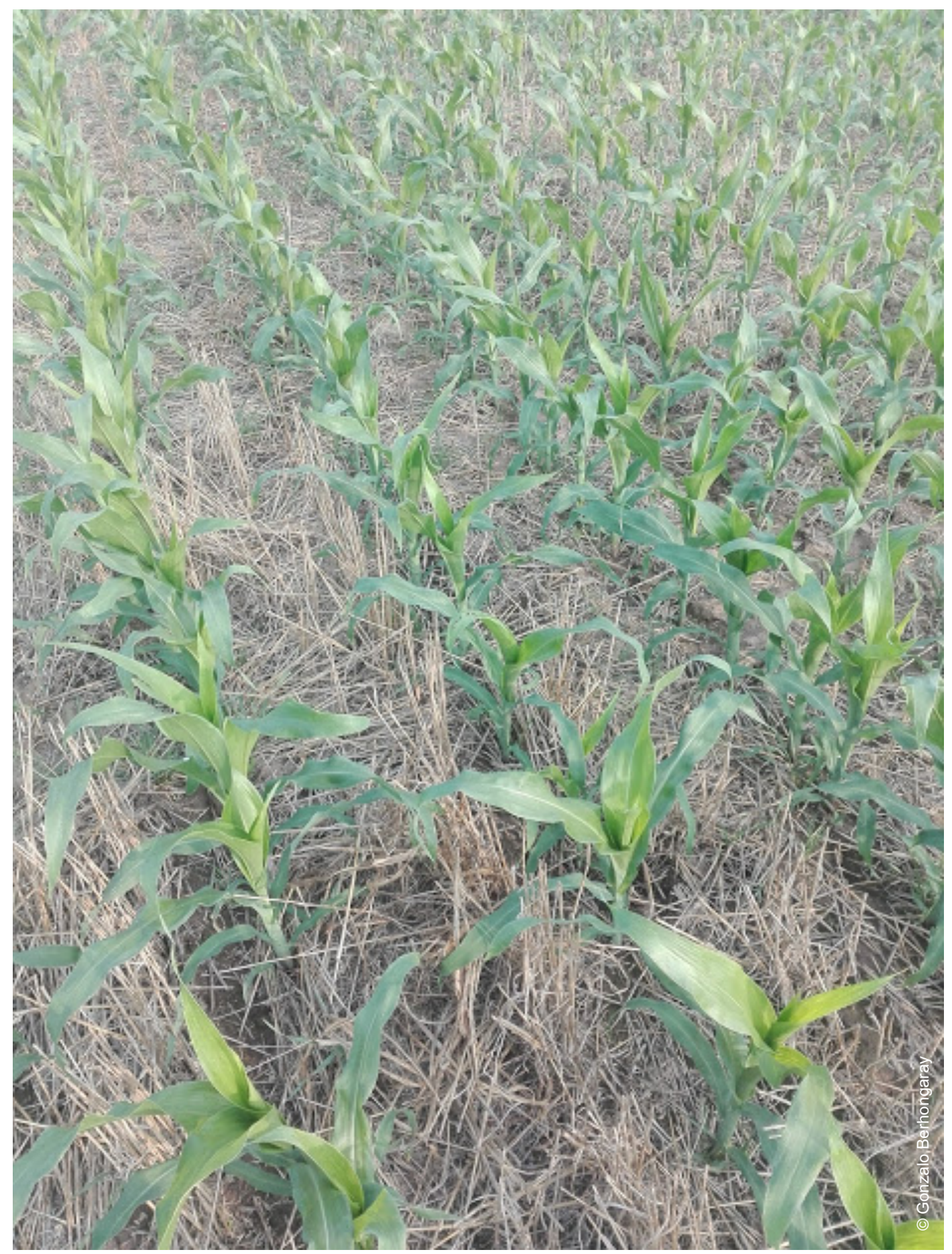

Photo 7. Corn under no-till, after wheat. Spring 2019, Santa Fe, Argentina 


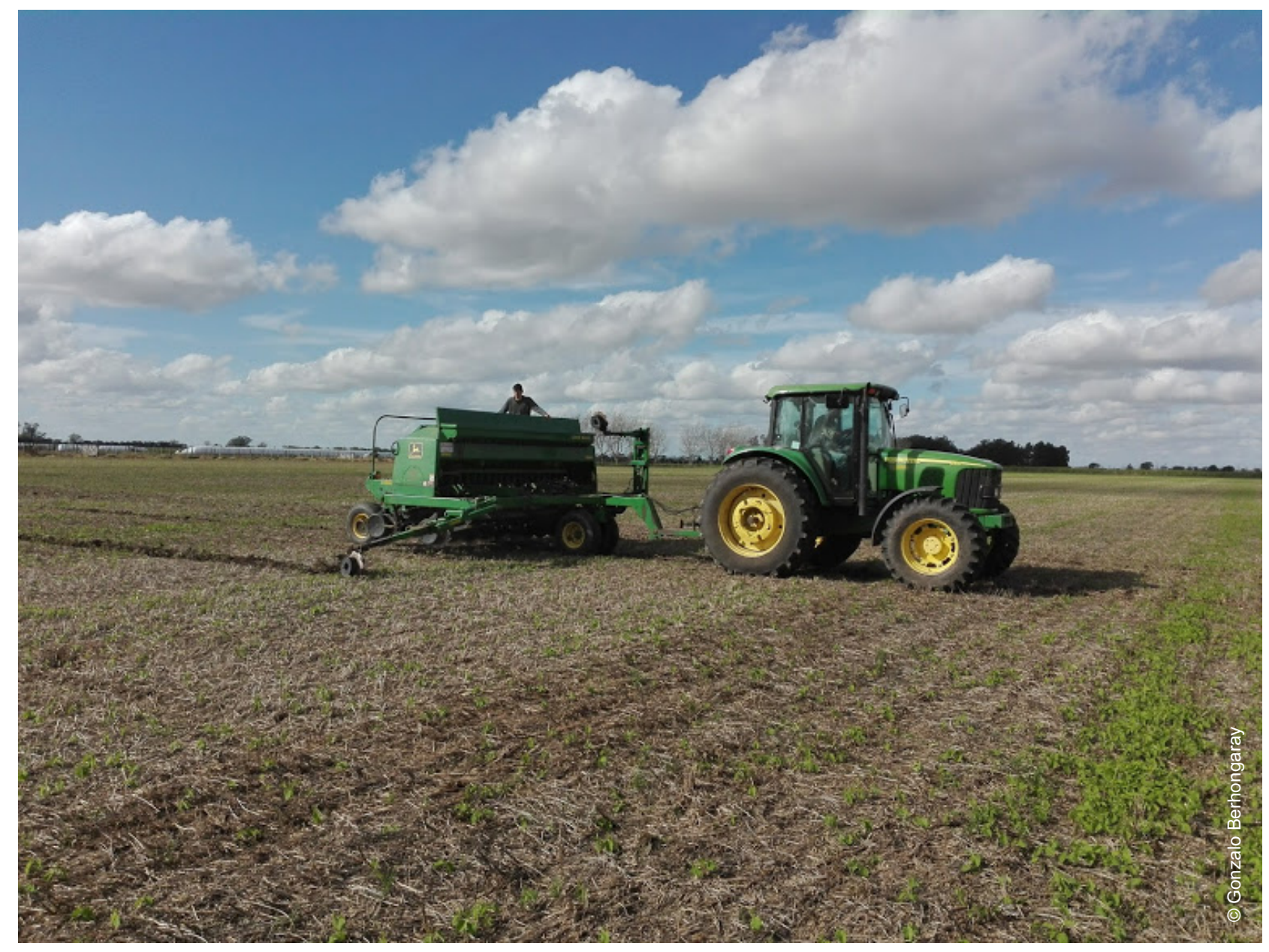

Photo 8. Seeding pastures under no-till in a cattle farm. Autumn 2019, Santa Fe, Argentina 
Table 26. Related cases studies available in volumes 3 and 5

\begin{tabular}{|c|c|c|c|c|}
\hline Title & Region & $\begin{array}{l}\text { Duration } \\
\text { of study } \\
\text { (Years) }\end{array}$ & Volume & $\begin{array}{l}\text { Case- } \\
\text { study } \\
\text { No. }\end{array}$ \\
\hline $\begin{array}{l}\text { Short-time effects of no-tillage in olive } \\
\text { orchards in Lebanon }\end{array}$ & NENA & 5 & 3 & 1 \\
\hline $\begin{array}{l}16 \text { years of no tillage and residue cover on } \\
\text { continuous maize in a Black soil of China }\end{array}$ & Asia & 16 & 3 & 10 \\
\hline $\begin{array}{l}\text { Rice straw mulching, charcoal, and no-tillage } \\
\text { on maize in Lopburi, Thailand }\end{array}$ & Asia & 4 & 3 & 11 \\
\hline $\begin{array}{l}\text { Mediterranean olive orchard subjected to } \\
\text { sustainable management in Matera, Basilicata, } \\
\text { Italy }\end{array}$ & Europe & 20 & 3 & 16 \\
\hline $\begin{array}{l}\text { Application of mulching in subtropical } \\
\text { orchards in Granada, Spain }\end{array}$ & Europe & 5 & 3 & 20 \\
\hline $\begin{array}{l}\text { Reduced tillage frequency and no-till to allow } \\
\text { ground covers and seeding cover crops in } \\
\text { rainfed almond fields, Spain }\end{array}$ & Europe & 10 & 3 & 21 \\
\hline $\begin{array}{l}\text { No tillage and cover crops in the Pampas, } \\
\text { Argentina }\end{array}$ & $\begin{array}{l}\text { Latin America } \\
\text { and the } \\
\text { Caribbean }\end{array}$ & 2 to 8 & 3 & 31 \\
\hline $\begin{array}{l}\text { Increasing Yield and Carbon Sequestration in a } \\
\text { Signalgrass Pasture by Liming and Fertilization } \\
\text { in Sao Carlos (SP, Brazil) }\end{array}$ & $\begin{array}{l}\text { Latin America } \\
\text { and the } \\
\text { Caribbean }\end{array}$ & 6 & 3 & 32 \\
\hline $\begin{array}{l}\text { Crop-pasture rotation on Black Soils of } \\
\text { Uruguay and Argentine }\end{array}$ & $\begin{array}{l}\text { Latin America } \\
\text { and the } \\
\text { Caribbean }\end{array}$ & $\begin{array}{l}10 \text { to } \\
48\end{array}$ & 3 & 39 \\
\hline $\begin{array}{l}\text { Zone Tillage of a Clay Loam in Southwestern } \\
\text { Ontario, Canada }\end{array}$ & North America & 13 & 3 & 44 \\
\hline $\begin{array}{l}\text { Long-term no-tillage maize in Kentucky, } \\
\text { United States of America }\end{array}$ & North America & $\begin{array}{l}48 \text { and } \\
79\end{array}$ & 3 & 45 \\
\hline $\begin{array}{l}\text { Deficit irrigation scenarios using sprinkle } \\
\text { irrigation system in western Kansas, United } \\
\text { States of America }\end{array}$ & North America & 5 and 8 & 3 & 46 \\
\hline
\end{tabular}




\section{References}

Aguilera, E., Lassaletta, L., Gattinger, A. \& Gimeno, B.S. 2013. Managing soil carbon for climate change mitigation and adaptation in Mediterranean cropping systems: A meta-analysis. Agriculture, Ecosystems \& Environment, 168: 25-36. https://doi.org/10.1016/j.agee.2013.02.003

Alvarez, C.R., Taboada, M.A., Gutiérrez Boem, F.H., Bono, A., Fernández, P.L. \& Prystupa, P. 2009. Topsoil properties as affected by tillage systems in the Rolling Pampa region of Argentina. Soil Science Society of America Journal, 73: 1242-1250. https://doi.org/10.2136/sssaj2008.0246

Alvarez, R. \& Steinbach, H.S. 2009. A review of the effects of tillage systems on some soil physical properties, water content, nitrate availability and crops yield in the Argentine Pampas. Soil \& Till. Res., 104: 1-15. https://doi.org/10.1016/j.still.2009.02.005

Alvarez, R., Diaz, R.A., Barbero, N., Santanatoglia, O.J. \& Blotta, L. 1995. Soil organic carbon, microbial biomass and $\mathrm{CO}_{2}$-C production from three tillage systems. Soil and Tillage Research, 33: 17-28. https://doi.org/10.1016/0167-1987(94)00432-E

Angers, D.A. \& Eriksen-Hamel, N.S. 2008. Full-inversion tillage and organic carbon distribution in soil profiles: a meta-analysis. Soil Science Society of America Journal, 72(5): 1370-1374.

https://doi.org/10.2136/sssaj2007.0342

Antapa, P.L. \& Angen, T.V. 1990. Tillage practices and residue management in Tanzania. In Organicmatter Management and Tillage in Humid and Sub-humid Africa. Proceedings, 10: 49-57. Bangkok, IBSRAM.

Balwinder, S., Eberbach, P.L., Humphreys, E. \& Kukal, S.S. 2011. The effect of rice straw mulch on evapotranspiration, transpiration and soil evaporation of irrigated wheat in Punjab, India. Agricultural Water Management, 98: 1847-1855. https://doi.org/10.1016/j.agwat.2011.07.002

Blanco-Canqui, H. \& Lal, R. 2008. No-tillage and soil-profile carbon sequestration: an on-farm assessment. Soil Science Society of America Journal, 72: 693-701. https://doi.org/10.2136/sssaj2007.0233

Blanco-Canqui, H. \& Ruis, S. J. 2018. No-tillage and soil physical environment. Geoderma, 326: 164-200. https://doi.org/10.1016/j.geoderma.2018.03.011

Bolliger, A., Magid, J., Amado, J.C. \& Neto, F.S., dos Santos Ribeiro, M.D., Calegari, A., Ralisch, R. \& de Neergaard, A. 2006. Taking stock of the Brazilian "Zero-Till Revolution": A Review of Landmark Research and Farmers' Practice. Advances in Agronomy, 91:47-110. https://doi.org/10.1016/S00652113(06)91002-5

Botta, G.F., Tolón-Becerra, A., Bienvenido, F., Rivero, D., Laureda, D.A., Ezquerra-Canalejo, A. \& Contessotto, E.E. 2018. Sunflower (Helianthus annuus L.) harvest: Tractor and grain chaser traffic effects on soil compaction and crop yields. Land degradation and development, 29: 4252-4261.

https://doi.org/10.1002/ldr.3181

Chakraborty, D., Nagarajan, S., Aggarwal, P., Gupta, V.K., Tomar, R.K., Garg, R.N., Sahoo, R.N., Sarkar, A., Chopra, U.K., Sarma, K.S.S. \& Kalra, N. 2008. Effect of mulching on soil and plant water status, and the growth and yield of wheat (Triticum aestivum L.) in a semi-arid environment. Agricultural Water Management, 95: 1323-1334. https://doi.org/10.1016/j.agwat.2008.06.001 
Chauhan, B.S., Gill, G.S., \& Preston, C. 2006. Tillage system effects on weed ecology, herbicide activity and persistence: a review. Australian Journal of Experimental Agriculture, 46: 1557-1570.

https://doi.org/10.1071/EA05291

Chen, H., Dai, Z., Veach, A.M., Zheng, J., Xu, J. \& Schadt, C.W. 2020. Global meta-analyses show that conservation tillage practices promote soil fungal and bacterial biomass. Agriculture, Ecosystems \&

Environment, 293: 106841. https://doi.org/10.1016/j.agee.2020.106841

Cusser, S., Bahlai, C., Swinton, S.M., Robertson, G.P. \& Haddad, N.M. 2019. Long-term research needed to avoid spurious and misleading trends in sustainability attributes of no-till. bioRxiv, 788240 .

https://doi.org/10.1101/788240

D'Emden, F.H., Llewellyn, R.S. \& Burton, M.P. 2008. Factors influencing adoption of conservation tillage in Australian cropping regions. Australian Journal of Agricultural and Resource Economics, 52(2): 169-182. https://doi.org/10.1111/j.1467-8489.2008.00409.x

Dardanelli, J. 1998. Eficiencia del uso del agua según sistemas de labranza. In J.L. Panigatti, et al. (Eds.) Siembra Directa. Hemisferio Sur, Buenos Aires. 107-115.

De Leijster, V., Santos, M.J., Wassen, M.J., Ramos-Font, M.E., Robles, A.B., Díaz, M., Staal, M. \& Verweij, P.A. 2019. Agroecological management improves ecosystem services in almond orchards within one year. Ecosystem Services, 38: 100948. https://doi.org/10.1016/j.ecoser.2019.100948

Derpsch, R. 2008, Critical Steps to No-till Adoption, In Goddard, T., Zoebisch, M.A., Gan, Y., Ellis, W., Watson, A. \& Sombatpanit, S. (Eds.) No-till Farming Systems. WASWC. pp. $479-495$.

https://doi.org/10.1016/j.still.2007.02.002.

Dimassi, B., Mary, B., Wylleman, R., Labreuche, J., Couture, D., Piraux, F. \& Cohan, J.P. 2014. Longterm effect of contrasted tillage and crop management on soil carbon dynamics during 41 years. Agriculture, Ecosystems \& Environment, 188: 134-146. https://doi.org/10.1016/j.agee.2014.02.014

Du, Z., Angers, D.A., Ren, T., Zhang, Q. \& Li, G. 2017. The effect of no-till on organic C storage in Chinese soils should not be overemphasized: A meta-analysis. Agriculture, Ecosystems \& Environment, 236: 1-11. https://doi.org/10.1016/j.agee.2016.11.007

Erenstein, O., Farooq, U., Malik, R.K. \& Sharif, M. 2008. On-farm impacts of zero tillage wheat in South Asia's rice-wheat systems. Field Crops Research, 105(3): 240-252.

https://doi.org/10.1016/j.fcr.2007.10.010

Feng, J., Li, F., Zhou, X., Xu, C., Ji, L., Chen, Z. \& Fang, F. 2018. Impact of agronomy practices on the effects of reduced tillage systems on $\mathrm{CH}_{4}$ and $\mathrm{N}_{2} \mathrm{O}$ emissions from agricultural fields: a global meta-analysis. PloS one, 13 e0196703. https://doi.org/10.1371/journal.pone.0196703

Guenet, B., Gabrielle, B., Chenu, C., Arrouays, D., Balesdent, J., Bernoux, M., Bruni, E., Caliman, J.P., Cardinael, R., Chen, S., Ciais, P., Desbois, D., Fouche, J., Frank, S., Henault, C., Lugato, E., Naipal, V., Nesme, T., Obersteiner, M., Pellerin, S., Powlson, D.S., Rasse, D.P., Rees, F., Soussana, J.F., Su, Y., Tian, H., Valin, H. \& Zhou, F. 2021. Can $\mathrm{N}_{2} \mathrm{O}$ emissions offset the benefits from soil organic carbon storage? Global Change Biology, 27(2): 237-256. https://doi.org/10.1111/gcb.15342 
Karlen, D.L., Wollenhaupt, N.C., Erbach, D.C., Berry, E.C., Swan, J.B., Eash, N.S. \& Jordahl, J.L. 1994. Long-term tillage effects on soil quality. Soil and Tillage Research, 32: 313-27.

https://doi.org/10.1016/0167-1987(94)00427-G

Kassam, A., Friedrich, T., Derpsch, R. \& Kienzle, J. 2015. Overview of the Worldwide Spread of Conservation Agriculture. Field Actions Science Reports. The journal of field actions, 8. (also available at http://journals.openedition.org/factsreports/3966).

Kladivko, E.J. 2001. Tillage systems and soil ecology. Soil and Tillage Research, 61: 61-76.

https://doi.org/10.1016/S0167-1987(01)00179-9

La Scala Júnior, N., De Figueiredo, E.B. \& Panosso, A.R. 2012. A review on soil carbon accumulation due to the management change of major Brazilian agricultural activities. Brazilian Journal of Biology, 72(3): 775785. https://doi.org/10.1590/S1519-69842012000400012

Lal, R. 2007. Constraints to adopting no-till farming in developing countries. Soil and Tillage Research, 94(1): 1-3. https://doi.org/10.1016/j.still.2007.02.002

Lal, R., Reicosky, D.C. \& Hanson, J.D. 2007. Evolution of the plow over 10,000 years and the rationale for no-till farming. Soil and Tillage Research, 93: 1-12. https://doi.org/10.1016/j.still.2006.11.004

Langdale, G.W., Barnett, A.P., Leonard, R.A. \& Fleming, W.G. 1979. Reduction of soil erosion by the no-till system in the Southern Piedmont. Transactions of the ASAE, 22: 82-0086.

Levidow, L. 2007. Governing conflicts over sustainability: agricultural biotechnology in Europe. In Vaughan, H. \& Geoffrey, L. (Eds.) Agricultural Governance. Routledge. London. pp. 114-133.

Limousin, G. \& Tessier, D. 2007. Effects of no-tillage on chemical gradients and topsoil acidification. Soil and Tillage Research, 92: 167-174. https://doi.org/10.1016/j.still.2006.02.003

Luo, Z., Wang, E. \& Sun, O.J. 2010. Can no-tillage stimulate carbon sequestration in agricultural soils? A meta-analysis of paired experiments. Agriculture, Ecosystems \& Environment, 139(1-2): 224-231.

https://doi.org/10.1016/j.agee.2010.08.006

Mei, K., Wang, Z., Huang, H., Zhang, C., Shang, X., Dahlgren, R.A., Zhang, M. \& Xia, F. 2018.

Stimulation of $\mathrm{N}_{2} \mathrm{O}$ emission by conservation tillage management in agricultural lands: A meta-analysis. Soil and Tillage Research, 182: 86-93. https://doi.org/10.1016/j.still.2018.05.006

Meurer, K.H.E., Haddaway, N.R., Bolinder, M.A. \& Kätterer, T, 2018. Tillage intensity affects total SOC stocks in boreo-temperate regions only in the topsoil-A systematic review using an ESM approach. EarthScience Reviews, 177: 613-622. https://doi.org/10.1016/j.earscirev.2017.12.015

Mickelson, S.K., Boyd, P., Baker, J.L. \& Ahmed, S.I. 2001. Tillage and herbicide incorporation effects on residue cover, runoff, erosion, and herbicide loss. Soil and Tillage Research, 60: 55-66.

https://doi.org/10.1016/S0167-1987(01)00170-2

Mtyobile, M., Muzangwa, L. \& Mnkeni, P.N.S. 2019. Tillage and crop rotation effects on soil carbon and selected soil physical properties in a Haplic Cambisol in Eastern Cape, South Africa. Soil and Water Research, 15: 47-54. https://doi.org/10.17221/176/2018-SWR 
Parr, J.F., Papendick, R.I., Hornick, S.B. \& Meyer, R.E. 1990. The use of cover crops, mulches and tillage for soil water conservation and weed control. In Organic-matter Management and Tillage in Humid and Sub-humid Africa. pp. 246-261. Proceedings No.10. Bangkok, IBSRAM.

Pittelkow, C.M., Liang, X., Linquist, B.A., van Groenigen, K.J., Lee, J., Lundy, M.E., van Gestel, N., Six, J., Venterea, R.T. \& van Kessel, C. 2014. Productivity limits and potentials of the principles of conservation agriculture. Nature, 517: 365-367. https://doi.org/10.1038/nature13809

Powlson, D.S., Stirling, C.M., Jat, M.L., Gerard, B.G., Palm, C.A., Sanchez, P.A. \& Cassman, K.G. 2014. Limited potential of no-till agriculture for climate change mitigation. Nature Climate Change, 4: 678683. https://doi.org/10.1038/nclimate2292

Reicosky, D.C. 2008. Carbon sequestration and environmental benefits from no-till systems. No-till farming systems, 3, pp. 43-58. (also available at: https://naldc.nal.usda.gov/download/39383/PDF)

Rochette, P. 2008. No-till only increases $\mathrm{N}_{2} \mathrm{O}$ emissions in poorly-aerated soils. Soil and Tillage Research, 101: 97-100. https://doi.org/10.1016/j.still.2008.07.011

Sidhu, R.S., Vatta, K. \& Dhaliwal, H.S. 2010. Conservation Agriculture in Punjab - economic implications of technologies and practices. Indian Journal of Agricultural Economics, 65: 413-427.

https://doi.org/10.22004/ag.econ.204693

Smika, D.E. \& Unger, P.W. 1986. Effect of Surface Residues on Soil Water Storage. In Stewart B.A. (eds) Advances in Soil Science. Advances in Soil Science, vol 5. Springer, New York, NY.

https://doi.org/10.1007/978-1-4613-8660-5_2

Smith, P., Goulding, K.W.T., Smith, K.A., Powlson, D.S., Smith, J.U., Falloon, P. \& Coleman, K. 2000. Including trace gas fluxes in estimates of the carbon mitigation potential of UK agricultural land. Soil Use and Management, 16: 251-259. https://doi.org/10.1111/j.1475-2743.2000.tb00204.x

Soane, B.D., Ball, B.C., Arvidsson, J., Basch, G., Moreno, F. \& Roger-Estrade, J. 2012. No-till in northern, western and south-western Europe: a review of problems and opportunities for crop production and the environment. Soil and Tillage Research, 118: 66-87. https://doi.org/10.1016/j.still.2011.10.015

Steinbach, H.S. \& Alvarez, R. 2006. Changes in soil organic carbon contents and nitrous oxide emissions after introduction of no-till in Pampean agroecosystems. Journal of Environment Quality, 35: 3-13.

https://doi.org/10.2134/jeq2005.0050

Swanepoel, C.M., Rötter, R.P., Van der Laan, M., Annandale, J.G., Beukes, D.J., du Preez, C.C., Swanepoel, L.H., van der Merwe, A. \& Hoffmann, M.P. 2018. The benefits of conservation agriculture on soil organic carbon and yield in southern Africa are site-specific. Soil and Tillage Research, 183: 72-82. https://doi.org/10.1016/j.still.2018.05.016

Swanton, C.J., Shrestha, A., Knezevic, S.Z., Roy, R.C. \& Ball-Coelho, B.R. 2000. Influence of tillage type on vertical weed seedbank distribution in a sandy soil. Canadian Journal of Plant Science, 80: 455-457. https://doi.org/10.4141/P99-020

Trigo, E., Cap, E., Malach, V. \& Villareal, F. 2009. The Case of Zero-Tillage Technology in Argentina. IFPRI Discussion Paper 00915. 40 pp. (also available at: http://ebrary.ifpri.org/utils/getfile/collection/p15738coll2/id/29503/filename/29504.pdf) 
Unger, P.W., Langdale, G.W. \& Papendick, R.I. 1988. Role of crop residues - improving water conservation and use. In Hargrove, W.L. (Ed.) Cropping Strategies for Efficient Use of Water and Nitrogen. pp. 69-100. American Society of Agronomy Special Publication No.51.

VandenBygaart, A.J. 2016. The myth that no-till can mitigate global climate change. Agriculture, Ecosystems and Environment, 216: 98-99. https://doi.org/10.1016/j.agee.2015.09.013

Verhulst, N., Nelissen, V., Jespers, N., Haven, H., Sayre, K.D., Raes, D., Deckers, J. \& Govaerts, B. 2011. Soil water content, maize yield and its stability as affected by tillage and crop residue management in rainfed semi-arid highlands. Plant and Soil, 344: 73-85. https://doi.org/10.1007/s11104-011-0728-8

Virto, I., Barré, P., Burlot, A. \& Chenu, C. 2012. Carbon input differences as the main factor explaining the variability in soil organic C storage in no-tilled compared to inversion tilled agrosystems. Biogeochemistry, 108: 17-26. https://doi.org/10.1007/s10533-011-9600-4

Yadav, G.S., Das, A., Lal, R., Babu, S., Datta, M., Meena, R.S., Patil, S.B. \& Singh, R. 2019. Impact of no-till and mulching on soil carbon sequestration under rice (Oryza sativa L.)-rapeseed (Brassica campestris L. var. rapeseed) cropping system in hilly agro-ecosystem of the Eastern Himalayas, India. Agriculture,

Ecosystems \& Environment, 275: 81-92. https://doi.org/10.1016/j.agee.2019.02.001 


\title{
7. Conservation, reduced and superficial tillage
}

\author{
María Martínez-Mena', María Almagro1,2 \\ ${ }^{1}$ CEBAS-CSIC, Murcia, Spain \\ ${ }^{2} \mathrm{BC} 3$, Leioa, Spain
}

\section{Description of the practice}

According to the Soil Science Society of America Glossary of Terms (SSSA, 2008):

\section{Conservation tillage}

Is "a method of tillage that consists on reducing the ploughing depth occasionally or continuously, replacing mouldboard ploughing by shallower tillage with other implements and/or reducing the intensity of seedbed preparation. The aim of this practice is to minimize soil disturbance as well as to reduce losses of soil and water, for which $\geq 30$ percent soil surface is covered by crop residues".

\section{Reduced tillage}

Is "a tillage practice in which the total number of tillage operations preparatory for seed planting (in herbaceous crops) or for soil aeration and decompaction (in perennial crops) is reduced from that normally used under conventional (intensive) tillage on that particular field or soil. This practice is also called minimum tillage."

\section{Superficial tillage}

Is performed at the upper soil layer, which is annually or periodically loosened.

In this section, conservation, reduced, and superficial tillage will be addressed together since they share common features and benefits. Conservation tillage normally includes the use of organic amendments such as manure, compost, agro-industry by-products (Vicente-Vicente et al, 2016) or improvements in N management, when the use of mineral fertilizers is adopted, in order to decrease $\mathrm{N}_{2} \mathrm{O}$ emissions either per ha or per tonne of grain (Powlson et al, 2016). Reduced tillage practices are used in conservation agriculture (also see factsheet No.42 on Conservation agriculture, this volume). 


\section{Range of applicability}

Based on many different authors (see all references cited in Section 3), this agricultural practice is applied worldwide under a wide variety of climate conditions (e.g. temperate, boreal and tropical), soil types (e.g. Calcisol, Fluvisol, Cambisol, Regosol, etc.) and crops (e.g. woody orchards, cereals and any other kind of herbaceous crops, including horticultural crops). However, potential benefits and drawbacks vary with climate, soil and crop type (arable vs woody), and therefore this practice has to be locally adapted or combined with other practices to become more cost-effective. Nevertheless, and despite the fact that conservation tillage has several environmental positive effects, the increase of organic carbon in soils is limited in time depending on the carbon saturation level, and after a certain point the rate of accumulation slows down towards a plateau level depending on the soil type, length of growing period, and climatic conditions (Gonzalez-Sánchez et al., 2019).

\section{Impact on soil organic carbon stocks}

Although in theory reduced tillage practices (reduced, superficial and conservation) differ from no-tillage, in the literature reduced tillage practices and no-tillage are often considered under the same umbrella. It is therefore challenging to individualize the specific effects of reduced, conservation or superficial tillage under their strict definition. Table 27 shows results from several meta-analyses and modelling efforts assessing the changes in SOC stocks when shifting from conventional (intensive) tillage systems to reduced tillage and notillage. Reducing tillage intensity (frequency and depth) promotes SOC sequestration worldwide. However, SOC sequestration rates differ among studies depending on the climate conditions, soil characteristics, initial SOC levels, crop type (arable vs woody cropping systems), management (rainfed vs irrigated), and the duration of experiments. From this literature review, we noticed that the baseline SOC stocks are rarely reported, and therefore we encourage the scientific community to do so in future studies. 
Table 27. Evolution of SOC stocks under conservation, reduced or superficial tillage

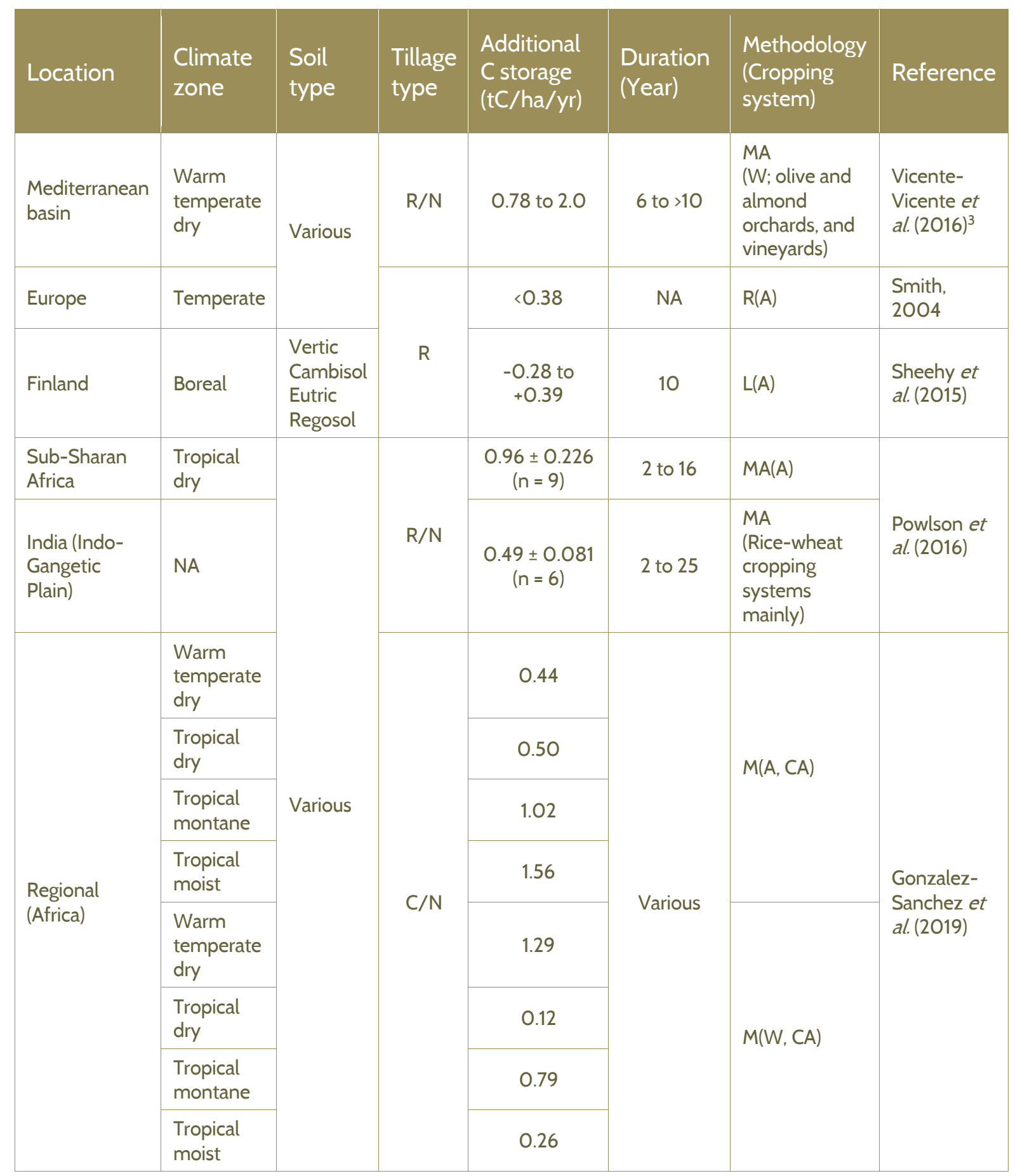

MA: meta-analysis, R: review, L: local study, C: Conservation tillage; R: Reduced tillage; S: Superficial tillage; N: No tillage; M: modelling, A: arable crops, W: woody crops, CA: Conservation agriculture, NA: Not available

\footnotetext{
${ }^{3}$ Also see case study No.28 "Cover crops, organic amendments and combined management practices in Mediterranean woody crops", Volume 4.
} 


\section{Other benefits of the practice}

\subsection{Improvement of soil properties}

Improvements of soil physical, chemical, and biological properties have been observed worldwide when shifting from conventional (intensive) tillage to reduced and no tillage (e.g. Stavi, Bel and Zaady , 2016; Blanco-Canqui and Francis, 2016), such as: i) soil water infiltration capacity and water availability for crops (Almagro et al., 2017); ii) aggregate stability (Almagro, Garcia-Franco and Martinez-Mena, , 2017; Martínez-Mena et al., 2020); and iii) increased soil resilience to temperature and moisture fluctuations (Almagro et al., 2017).

\subsection{Minimization of threats to soil functions}

Table 28. Soil threats

\begin{tabular}{|c|c|}
\hline Soil threats & \\
\hline Soil erosion & $\begin{array}{l}\text { Reductions in runoff and erosion with decreasing tillage intensity is due, in part, to } \\
\text { the development of a vegetation cover. Reductions in runoff between } 30 \text { percent } \\
\text { and } 65 \text { percent and in erosion between } 63 \text { and } 80 \text { percent have been observed } \\
\text { worldwide (Matínez-Mena et al., 2020; Biddoccu et al., 2017; Preiti et al., 2017). }\end{array}$ \\
\hline $\begin{array}{l}\text { Nutrient imbalance } \\
\text { and cycles }\end{array}$ & $\begin{array}{l}\text { Improve nitrogen availability (Martínez-Mena et al., 2021; Paredes et al., 2015) and } \\
\text { reduced nutrient losses by erosion (Martínez-Mena et al., 2020). }\end{array}$ \\
\hline $\begin{array}{l}\text { Soil contamination } \\
\text { /pollution }\end{array}$ & $\begin{array}{l}\text { Only under organic/low input agricultural systems, when mineral fertilizers and } \\
\text { pesticides use is reduced ( Stavi, Bel and Zaady, 2016). }\end{array}$ \\
\hline Soil acidification & $\begin{array}{l}\text { Reductions of } \mathrm{pH} \text { between } 1.7 \text { percent and } 3 \text { percent have been reported (Chen et } \\
\text { al., 2020; Li et al., 2019). However, increases of } \mathrm{pH} \text { in acidic soils have been also } \\
\text { reported (Husson et al., 2018). Observations depend on previous soil condition, the } \\
\text { chemical quality of crop residues and the duration of the experiments. }\end{array}$ \\
\hline Soil biodiversity loss & $\begin{array}{l}\text { The presence of a vegetation cover due to reduced tillage increase soil biodiversity } \\
\text { and can provide habitat for arthropod predators and parasitoids, which promote } \\
\text { biological control by feeding on pests and microorganisms (Paredes et al., 2015; } \\
\text { Stavi et al., 2016; Li et al., 2019). }\end{array}$ \\
\hline $\begin{array}{l}\text { Soil water } \\
\text { management }\end{array}$ & $\begin{array}{l}\text { Improvement of soil water retention and infiltration; reduction of runoff (Stavi et al., } \\
\text { 2016; Martínez-Mena et al., 2020; Biddoccu et al., 2017; Preiti et al., 2017). }\end{array}$ \\
\hline
\end{tabular}




\section{3. (Increases in production (e.g. food/fuel/feed/timber/fibre)}

Conservation tillage generally has a positive impact on crop yields because the enhancement of organic matter inputs into the system improves water infiltration and storage capacity and the availability of nutrients in soils (Morugan-Colorado et al., 2020). However, the magnitude of the impacts of conservation tillage on crop yields depends on annual climatic conditions (Van den Putte et al., 2010), soil condition, crop type (arable or woody) and management conditions (e.g. rotations, irrigated vs rainfed systems, and fertilizer application; Alvarez and Steinbach, 2009).

\subsection{Mitigation of and adaptation to climate change}

In general, agricultural systems under conservation, reduced, and superficial tillage reduce $\mathrm{CO}_{2}$ emissions compared to conventional (intensive) tillage systems. First, because reducing the number of passes per year mitigates annual $\mathrm{CO}_{2}$ emissions by tillage machinery, but also because the peaks of $\mathrm{CO}_{2}$ emissions from soils after tillage operations are also reduced (Almagro et al., 2017). On the other hand, those practices can modulate the response of soil $\mathrm{CO}_{2}$ flux to soil temperature and moisture making soils more resilient to extreme rainfall events, droughts and warming (Almagro et al., 2017). Conservation tillage usually includes improvements in N management, leading to decreases in total soil $\mathrm{N}_{2} \mathrm{O}$ emissions per ha, or at least to a decrease in yield-scaled $\mathrm{N}_{2} \mathrm{O}$ emissions (Bhatia et al., 2012; Chauhan et al., 2012). Furthermore, the effectiveness of these agricultural practices in controlling soil erosion and carbon and nutrient losses is higher during extreme erosive events (Martínez-Mena et al., 2020), which are forecasted to increase under climate change scenarios (Eekhout and de Vente, 2019).

\subsection{Socio-economic benefits}

Although the assessment of direct economic costs and benefits from these practices is complicated as they are simultaneously affected by a wide range of local, national and global factors. It has been proved that they have multiple on-site and off-site socio-economic benefits, such as: i) improving crop yields in the long-term; ii) reducing soil organic matter and nutrient losses (indirect costs) by preventing soil erosion; iii) maintaining soil fertility, biodiversity and health condition; iv) fuel, fertilizer and pesticides savings; v) making agroecosystems more resilient against the impacts of climate change, vi) reducing floods and associated damages, vii) favouring preservation of cultural landscapes and viii) improving local economy an retaining population in rural areas (Stavi, Bel and Zaady, 2016; Sanz et al., 2017).

\subsection{Other benefits of the practice}

The benefits of these practices increase when they are combined with other practices (such as inter-cropping, wise crop rotation, crop residue retention, green and organic manure addition). The combination of these practices promoted an increase of ca. 50 percent of SOC levels compared to conventional management in Mediterranean cropping systems (Aguilera et al., 2015; Corbeels et al., 2019). 


\section{Potential drawbacks to the practice}

\subsection{Tradeoffs with other threats to soil functions}

Table 29. Soil threats

\begin{tabular}{|l|l|}
\hline Soil threats & \multicolumn{1}{|c|}{$\begin{array}{l}\text { Although soil nutrient balance and cycles can improve, competition for } \\
\text { nutrient imbalance } \\
\text { nutrients with the main crop can also occur (Martinez-Mena et al., } \\
2013) .\end{array}$} \\
\hline $\begin{array}{l}\text { Soil contamination } \\
\text { /pollution }\end{array}$ & $\begin{array}{l}\text { If pesticides are applied to combat weeds and pathogens (Stavi et al., } \\
\text { 2016). }\end{array}$ \\
\hline $\begin{array}{l}\text { Soil acidification } \\
\text { Soil compaction }\end{array}$ & $\begin{array}{l}\text { See section 4.2. } \\
\text { Increase of bulk density and soil penetration resistance (Li et al., 2019) }\end{array}$ \\
\hline $\begin{array}{l}\text { Soil water } \\
\text { management }\end{array}$ & $\begin{array}{l}\text { Competition by water with the main crop can be promoted. This effect } \\
\text { is more accused in high aridity and warm temperatures climates } \\
\text { (Morugán-Coronado et al., 2O2O). }\end{array}$ \\
\hline
\end{tabular}

\subsection{Increases in greenhouse gas emissions}

It is not possible to estimate an overall net GHG balance for these practices since the GHG balance will depend very much on the local pedo-climatic conditions, crop type (woody vs arable), management (irrigated vs rainfed systems; type and dose of fertilizers), and whether these practices are adopted alone or in combination with other practices (e.g. if fertilizer or manure is applied, intercropping, green manure, crop residue retention, etc.). It is also important to note that the positive impacts of reducing tillage operations (i.e. fuel saving results in less $\mathrm{CO}_{2}$ emissions, soil carbon sequestration is improved) can be counterbalanced with the increase in soil $\mathrm{N}_{2} \mathrm{O}$ emissions if the fertilizer dose has to be increased, as pointed out in a recent meta-analysis (Guenet et al., 2020). However, results highly vary depending on the duration of the experiments and management (e.g. use and dose of fertilizers, use and type of cover crops, and crop residue management) and therefore no general conclusions can be drawn. 


\subsection{Conflict with other practice(s)}

As above mentioned, the positive impacts of reducing tillage operations (i.e. fuel saving results in less $\mathrm{CO}_{2}$ emissions, soil carbon sequestration is improved) can be counterbalanced with increments in soil $\mathrm{N}_{2} \mathrm{O}$ emissions if fertilizers are applied or doses are increased (Guenet et al., 2020).

\subsection{Decreases in production (e.g. food/fuel/feed/timber/fibre)}

The evaluation of direct economic costs and benefits from these practices is rather complicated as they are simultaneously affected by a wide range of local, national and global factors (Stavi, Bel and Zaady, 2016). Generally, yields are reduced in the short-term, but this trend can be reversed in the long-term, especially if reduced tillage is adopted in combination with other practices (e.g. organic or green manure addition (Pittelkow et al., 2015).

\section{Recommendations before implementing the practice}

The benefits of these practices increase when they are combined with other practices (such as inter-cropping; wise crop rotation; crop residue retention; green and organic manure addition; Malobane et al., 2020; Lujan Soto et al., 2021).

\section{Potential barriers to adoption}

Table 30. Potential barriers to adoption

\begin{tabular}{|c|c|c|}
\hline Barrier & YES/NO & \\
\hline Biophysical & Yes & $\begin{array}{l}\text { Farm type; in water-limited areas the adoption of these practices } \\
\text { may be hampered because of competition problems for water and } \\
\text { nutrient between the ground covers and the main crop (Cooper et } \\
\text { al., 2016). Also in water-logged and heavy clay soils, such in rice } \\
\text { fields, reduced tillage is hampered. }\end{array}$ \\
\hline Cultural & Yes & $\begin{array}{l}\text { Farmer perception regarding the traditional belief that a "clean" } \\
\text { and "tidy" orchard must always be free of vegetation except for the } \\
\text { trees (Ramos et al., 2010). }\end{array}$ \\
\hline Social & Yes & $\begin{array}{l}\text { Peer pressure; degree of autonomy in choosing and implementing } \\
\text { results; and community support (Borgström, Zachrisson and } \\
\text { Eckerberg, 2016; Runhaar et al., 2017). }\end{array}$ \\
\hline
\end{tabular}




\begin{tabular}{|c|c|c|}
\hline Barrier & YES/NO & \\
\hline Economic & Yes & $\begin{array}{l}\text { Yield reduction in the short-term; limited finance and access to } \\
\text { capital for implementation; lack of access to appropriate } \\
\text { technologies, practices, or equipment is a major barrier in many } \\
\text { countries (Sanz et al., 2017). }\end{array}$ \\
\hline Institutional & Yes & $\begin{array}{l}\text { Lack of economic incentives and support from governments, } \\
\text { including subsidies (Runhaar et al., 2017). }\end{array}$ \\
\hline $\begin{array}{l}\text { Legal (Right to } \\
\text { soil) }\end{array}$ & Yes & $\begin{array}{l}\text { Lack of strictness of legislation and standards (Ahnström et al., } \\
\text { 2009). }\end{array}$ \\
\hline Knowledge & Yes & $\begin{array}{l}\text { Lack of: i) awareness among farmers, ii) community feeling, iii) } \\
\text { innovativeness, and iv) understanding of the agroecosystem } \\
\text { (Ferwerda 2015; (Schoonhoven and Runhaar, 2018). }\end{array}$ \\
\hline Other & Yes & Lack of motivation. \\
\hline
\end{tabular}

\section{Photo of the practice}

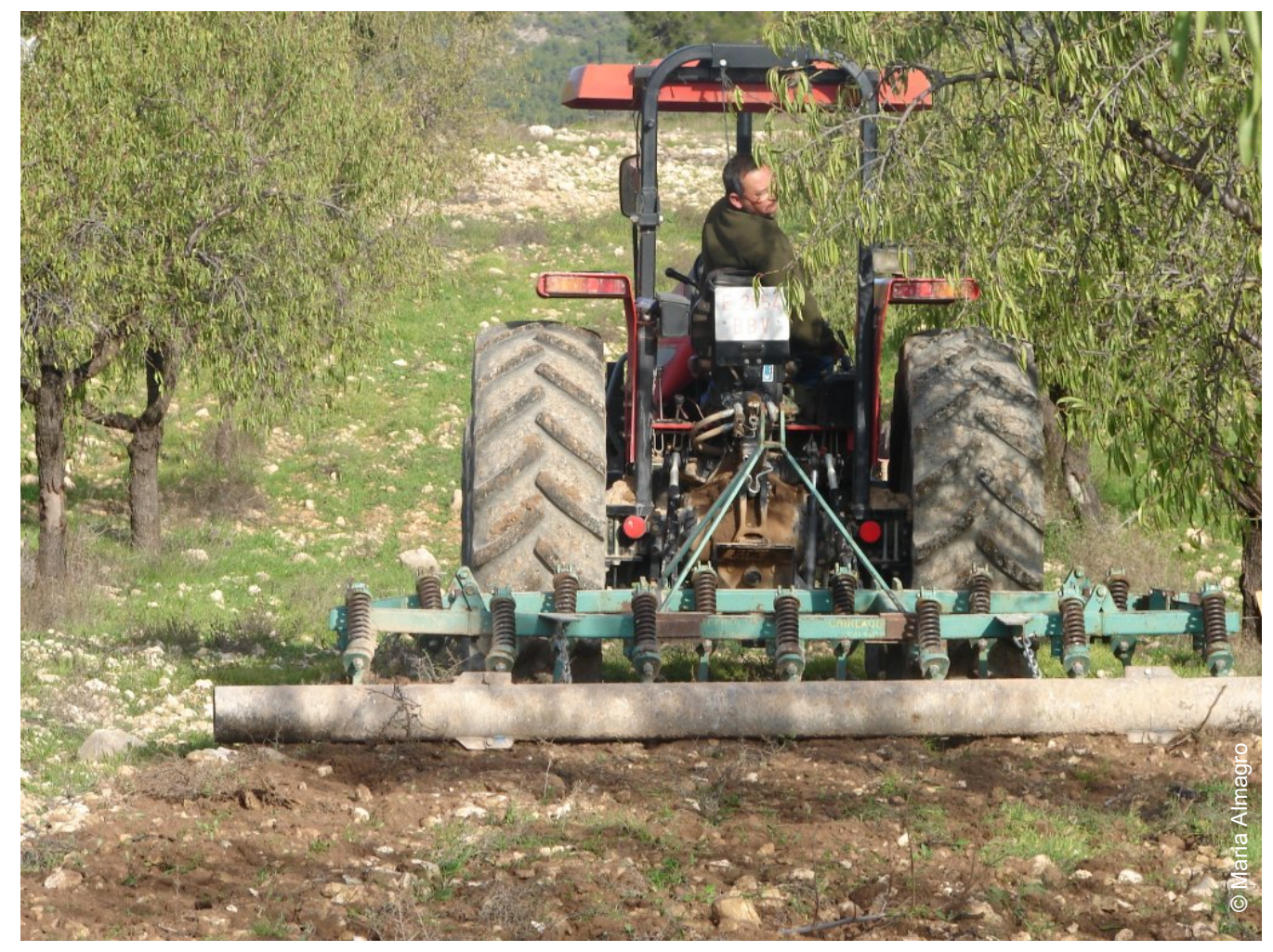

Photo 9. Reduced tillage in rainfed almond fields in Andalusia, Spain 
Table 31. Related cases studies available in volumes 3 and 5

\begin{tabular}{|c|c|c|c|c|}
\hline Title & Region & $\begin{array}{l}\text { Duration } \\
\text { of study } \\
\text { (Years) }\end{array}$ & Volume & $\begin{array}{l}\text { Case- } \\
\text { study } \\
\text { No. }\end{array}$ \\
\hline Conservation Agriculture in Mozambique & Africa & 2 & 3 & 4 \\
\hline Conservation Agriculture in South Africa & Africa & 6 & 3 & 5 \\
\hline $\begin{array}{l}\text { Irrigated cotton cropping systems in } \\
\text { Australian Vertisols under minimum tillage }\end{array}$ & $\begin{array}{l}\text { Southwest } \\
\text { Pacific }\end{array}$ & 4 to 20 & 3 & 8 \\
\hline $\begin{array}{l}\text { Reduced tillage frequency and no-till to } \\
\text { allow ground covers and seeding cover } \\
\text { crops in rainfed almond fields, Spain }\end{array}$ & Europe & 10 & 3 & 21 \\
\hline $\begin{array}{l}30 \text { years of conservation agriculture } \\
\text { practices on Vertisols in central Mexico }\end{array}$ & $\begin{array}{l}\text { Latin America } \\
\text { and the } \\
\text { Caribbean }\end{array}$ & 30 & 3 & 37 \\
\hline $\begin{array}{l}\text { Response of soil carbon to various } \\
\text { combinations of management practices } \\
\text { (annual-perennial rotation system, animal } \\
\text { manure application, reduced tillage) in } \\
\text { Quebec, Canada }\end{array}$ & North America & 21 & 3 & 43 \\
\hline $\begin{array}{l}\text { Conservation tillage to tackle smog issue } \\
\text { and improve carbon sequestration in rice- } \\
\text { wheat cropping system in Pakistan }\end{array}$ & Asia & 1 & 5 & 15 \\
\hline
\end{tabular}




\section{References}

Aguilera, E., Guzmán, G. \& Alonso, A. 2015. Greenhouse gas emissions from conventional and organic cropping systems in Spain. I. Herbaceous crops. Agronomy for Sustainable Development, 35(2): 713-724. https://doi.org/10.1007/s13593-014-0267-9

Ahnström, J., Höckert, J., Bergeå, H.L., Francis, C.A., Skelton, P. \& Hallgren, L. 2009. Farmers and nature conservation: What is known about attitudes, context factors and actions affecting conservation? Renewable Agriculture and Food Systems, 24(1): 38-47. https://doi.org/10.1017/S1742170508002391

Almagro, M., Garcia-Franco, N. \& Martínez-Mena, M. 2017. The potential of reducing tillage frequency and incorporating plant residues as a strategy for climate change mitigation in semiarid Mediterranean agroecosystems. Agriculture, Ecosystems \& Environment, 246: 210-220.

https://doi.org/10.1016/j.agee.2017.05.016

Alvarez, R. \& Steinbach, H.S. 2009. A review of the effects of tillage systems on some soil physical properties, water content, nitrate availability and crops yield in the Argentine Pampas. Soil and tillage research, 104(1): 1-15. https://doi.org/10.1016/j.still.2009.02.005

Bhatia, A., Pathak, H., Jain, N., Singh, P.K. \& Tomer, R. 2012. Greenhouse gas mitigation in rice-wheat system with leaf color chart-based urea application. Environmental Monitoring and Assessment, 184(5):

3095-3107. https://doi.org/10.1007/s10661-011-2174-8

Biddoccu, M., Ferraris, S., Pitacco, A. \& Cavallo, E. 2017. Temporal variability of soil management effects on soil hydrological properties, runoff and erosion at the field scale in a hillslope vineyard, North-West Italy. Soil and Tillage Research, 165: 46-58. https://doi.org/10.1016/j.still.2016.07.017

Blanco-Canqui, H. \& Francis, C.A. 2016. Building resilient soils through agroecosystem redesign under fluctuating climatic regimes. Journal of Soil and Water Conservation, 71(6): 127A-133A.

https://doi.org/10.2489/jswc.71.6.127A

Borgström, S., Zachrisson, A. \& Eckerberg, K. 2016. Funding ecological restoration policy in practicepatterns of short-termism and regional biases. Land Use Policy, 52: 439-453.

https://doi.org/10.1016/j.landusepol.2016.01.004

Chauhan, B.S., Mahajan, G., Sardana, V., Timsina, J. \& Jat, M.L. 2012. Productivity and sustainability of the rice-wheat cropping system in the Indo-Gangetic Plains of the Indian subcontinent: problems, opportunities, and strategies. Advances in Agronomy, 117: 315-369. https://doi.org/10.1016/B978-0$12-394278-4.00006-4$

Chen, H., Dai, Z., Veach, A.M., Zheng, J., Xu, J. \& Schadt, C.W. 2020. Global meta-analyses show that conservation tillage practices promote soil fungal and bacterial biomass. Agriculture, Ecosystems \& Environment, 293: 106841. https://doi.org/10.1016/j.agee.2020.106841

Cooper, J., Baranski, M., Stewart, G., Nobel-de Lange, M., Bàrberi, P., Fließbach, A., Peigné, J., Berner, A., Brock, C. \& Casagrande, M. 2016. Shallow non-inversion tillage in organic farming maintains crop yields and increases soil C stocks: a meta-analysis. Agronomy for Sustainable Development, 36(1): 22. https://doi.org/10.1007/s13593-016-0354-1 
Corbeels, M., Cardinael, R., Naudin, K., Guibert, H. \& Torquebiau, E. 2019. The 4 per 1000 goal and soil carbon storage under agroforestry and conservation agriculture systems in sub-Saharan Africa. Soil and Tillage Research, 188: 16-26. https://doi.org/10.1016/j.still.2018.02.015

Eekhout, J.P.C. \& de Vente, J. 2019. Assessing the effectiveness of Sustainable Land Management for large-scale climate change adaptation. Science of the Total Environment, 654: 85-93.

https://doi.org/10.1016/j.scitotenv.2018.10.350

Gonzalez-Sanchez, E.J., Veroz-Gonzalez, O., Conway, G., Moreno-Garcia, M., Kassam, A., Mkomwa, S., Ordoñez-Fernandez, R., Triviño-Tarradas, P. \& Carbonell-Bojollo, R. 2019. Meta-analysis on carbon sequestration through Conservation Agriculture in Africa. Soil and Tillage Research, 190: 22-30. https://doi.org/10.1016/j.still.2019.02.020

Guenet, B., Gabrielle, B., Chenu, C., Arrouays, D., Balesdent, J., Bernoux, M., Bruni, E., Caliman, J.P., Cardinael, R., Chen, S., Ciais, P., Desbois, D., Fouche, J., Frank, S., Henault, C., Lugato, E., Naipal, V., Nesme, T., Obersteiner, M., Pellerin, S., Powlson, D.S., Rasse, D.P., Rees, F., Soussana, J.F., Su, Y., Tian, H., Valin, H. \& Zhou, F. 2020. Can $\mathrm{N}_{2} \mathrm{O}$ emissions offset the benefits from soil organic carbon storage? Global Change Biology. https://doi.org/10.1111/gcb.15342

Husson, O., Brunet, A., Babre, D., Charpentier, H., Durand, M. \& Sarthou, J.-P. 2018. Conservation agriculture systems alter the electrical characteristics (Eh, pH and EC) of four soil types in France. Soil and Tillage Research, 176: 57-68. https://doi.org/10.1016/j.still.2017.11.005

Li, Y., Li, Z., Cui, S., Jagadamma, S. \& Zhang, Q. 2019. Residue retention and minimum tillage improve physical environment of the soil in croplands: A global meta-analysis. Soil and Tillage Research, 194: 104292. https://doi.org/10.1016/j.still.2019.06.009

Luján Soto, R., Martínez-Mena, M., Cuéllar Padilla, M. \& de Vente, J. 2021. Restoring soil quality of woody agroecosystems in Mediterranean drylands through regenerative agriculture. Agriculture, Ecosystems \& Environment, 306: 107191. https://doi.org/10.1016/j.agee.2020.107191

Malobane, M.E., Nciizah, A.D., Mudau, F.N. \& Wakindiki, I.I. 2020. Tillage, Crop Rotation and Crop Residue Management Effects on Nutrient Availability in a Sweet Sorghum-Based Cropping System in Marginal Soils of South Africa. Agronomy, 10(6): 776. https://doi.org/10.3390/agronomy10060776

Martínez-Mena, M., Garcia-Franco, N., Almagro, M., Ruiz-Navarro, A., Albaladejo, J., de Aguilar, J.M., Gonzalez, D. \& Querejeta, J.I. 2013. Decreased foliar nitrogen and crop yield in organic rainfed almond trees during transition from reduced tillage to no-tillage in a dryland farming system. European Journal of Agronomy, 49: 149-157. https://doi.org/10.1016/j.eja.2013.04.006

Martínez-Mena, M., Carrillo-López, E., Boix-Fayos, C., Almagro, M., García Franco, N., Díaz-Pereira, E., Montoya, I. \& de Vente, J. 2020. Long-term effectiveness of sustainable land management practices to control runoff, soil erosion, and nutrient loss and the role of rainfall intensity in Mediterranean rainfed agroecosystems. Catena, 187. https://doi.org/10.1016/j.catena.2019.104352

Martínez-Mena, M., Perez, M., Almagro, M., Garcia-Franco, N. \& Díaz-Pereira, E. 2021. Long-term effects of sustainable management practices on soil properties and crop yields in rainfed Mediterranean almond agroecosystems. European Journal of Agronomy, 123: 126207.

https://doi.org/10.1016/j.eja.2020.126207 
Morugán-Coronado, A., Linares, C., Gómez-López, M.D., Faz, Á. \& Zornoza, R. 2020. The impact of intercropping, tillage and fertilizer type on soil and crop yield in fruit orchards under Mediterranean conditions: A meta-analysis of field studies. Agricultural Systems, 178: 102736.

https://doi.org/10.1016/j.agsy.2019.102736

Paredes, D., Cayuela, L., Gurr, G.M. \& Campos, M. 2015. Is ground cover vegetation an effective biological control enhancement strategy against olive pests? PLoS One, 10(2).

https://doi.org/10.1371/journal.pone.0117265

Pittelkow, C.M., Liang, X., Linquist, B.A., Van Groenigen, K.J., Lee, J., Lundy, M.E., Van Gestel, N., Six, J., Venterea, R.T. \& Van Kessel, C. 2015. Productivity limits and potentials of the principles of conservation agriculture. Nature, 517(7534): 365-368. https://doi.org/10.1038/nature13809

Powlson, D.S., Stirling, C.M., Thierfelder, C., White, R.P. \& Jat, M.L. 2016. Does conservation agriculture deliver climate change mitigation through soil carbon sequestration in tropical agro-ecosystems? Agriculture, Ecosystems \& Environment, 220: 164-174.

Preiti, G., Romeo, M., Bacchi, M. \& Monti, M. 2017. Soil loss measure from Mediterranean arable cropping systems: Effects of rotation and tillage system on C-factor. Soil and Tillage Research, 170: 85-93. https://doi.org/10.1016/j.still.2017.03.006

Ramos, M.E., Benítez, E., García, P.A. \& Robles, A.B. 2010. Cover crops under different managements vs. frequent tillage in almond orchards in semiarid conditions: Effects on soil quality. Applied Soil Ecology, 44(1): 6-14. https://doi.org/10.1016/j.apsoil.2009.08.005

Runhaar, H.A.C., Melman, Th.C.P., Boonstra, F.G., Erisman, J.W., Horlings, L.G., de Snoo, G.R., Termeer, C.J.A.M., Wassen, M.J., Westerink, J. \& Arts, B.J.M. 2017. Promoting nature conservation by Dutch farmers: a governance perspective. International Journal of Agricultural Sustainability, 15(3): 264281. https://doi.org/10.1080/14735903.2016.1232015

Soil Science Society of America (SSSA). 2008. Glossary of Soil Science Terms 2008. Madison, WI, Soil Science Society of America Inc

Sanz, M.J., De Vente, J.L., Chotte, J.-L., Bernoux, M., Kust, G., Ruiz, I., Almagro, M., Alloza, J.A., Vallejo, R., Castillo, V., Hebel, A. \& Akhar-Schuster, M. 2017. Sustainable land management contribution to successful land-based climate change adaptation and mitigation: a report of the Science-Policy Interface. United Nations Convention to Combat Desertification (UNCCD), Bonn, Germany. (also available at: https://www.unccd.int/sites/default/files/documents/2017-09/UNCCD_Report_SLM.pdf)

Schoonhoven, Y. \& Runhaar, H. 2018. Conditions for the adoption of agro-ecological farming practices: a holistic framework illustrated with the case of almond farming in Andalusia. International Journal of Agricultural Sustainability, 16(6): 442-454. https://doi.org/10.1080/14735903.2018.1537664

Sheehy, J., Regina, K., Alakukku, L. \& Six, J. 2015. Impact of no-till and reduced tillage on aggregation and aggregate-associated carbon in Northern European agroecosystems. Soil and Tillage Research, 150: 107-113. https://doi.org/10.1016/j.still.2015.01.015

Smith, P. 2004. Carbon sequestration in croplands: the potential in Europe and the global context. European journal of agronomy, 20(3): 229-236. https://doi.org/10.1016/j.eja.2003.08.002 
Stavi, I., Bel, G. \& Zaady, E. 2016. Soil functions and ecosystem services in conventional, conservation, and integrated agricultural systems. A review. Agronomy for Sustainable Development, 36(2): 1-12.

https://doi.org/10.1007/s13593-016-0368-8

Vicente-Vicente, J.L., García-Ruiz, R., Francaviglia, R., Aguilera, E. \& Smith, P. 2016. Soil carbon sequestration rates under Mediterranean woody crops using recommended management practices: A metaanalysis. Agriculture, Ecosystems \& Environment, 235: 204-214.

https://doi.org/10.1016/j.agee.2016.10.024

Van den Putte, A., Govers, G., Diels, J., Gillijns, K. \& Demuzere, M. 2010. Assessing the effect of soil tillage on crop growth: A meta-regression analysis on European crop yields under conservation agriculture. European Journal of Agronomy, 33(3): 231-241.

https://doi.org/10.1016/j.eja.2010.05.008 


\title{
8. Strip, precision, zone tillage
}

\author{
Noelia Garcia-Franco'1, María Almagro 2,3 \\ ${ }^{1}$ Chair of Soil Science, TUM School of Life Sciences Weihenstephan, Technical University of Munich, \\ Freising, Germany \\ ${ }^{2}$ CEBAS-CSIC, Murcia, Spain \\ ${ }^{3} \mathrm{BC}$, Leioa, Spain
}

\section{Description of the practice}

Strip-tillage is a tillage practice in which soil disturbance is limited to the crop row while the rest of the soil remains undisturbed (Figure 2; Haramoto and Brainard, 2012). Strip-tillage is one of the conservation tillage methods aimed at soil loss prevention while maintaining crop sustainability by limiting excessive tilling (Idowu and Flynn, 2013). This kind of tillage was developed as an alternative technique to solve the problems associated with conventional tillage or direct seeding methods (Gil Domínguez, 2019; Lahmar, 2010). Strip-till was developed a few decades ago in the United States of America and today it is commonly used throughout the Coastal Plains region of the southeastern United States of America (with the aim of breaking up the compacted soil layers that frequently form in that area) for a wide variety of crops such as cotton, maize, peanuts, beans, soybean, melon, tomato, zucchini, transplanted broccoli and cauliflower. Strip-tillage started in Europe around the year 2005 (Gil Domínguez, 2019).

There are several terms that are synonymous with strip tillage, including: precision tillage zone tillage, sitespecific tillage, row clearing" and deep zoning. The seedbed is divided into a seedling zone and a soil management zone. The seedling zone $(5-10 \mathrm{~cm}$ wide) is mechanically tilled to optimize the soil and microclimate environment for germination and seedling establishment. The interrow zone is left undisturbed and protected by mulch (Figure 2). Strip-tillage can also be achieved by chiseling in the row zone to assist water infiltration and root proliferation. In addition, strip tillage can benefit from using a Global Positioning System (GPS) guidance equipment (Nowatzki et al., 2011). 


\section{Range of applicability}

Strip-tillage can be applied worldwide in arable crops, preferably on relatively flat land with poorly drained soils (Al-Kaisi and Yin, 2005; Haramoto and Brainard, 2012; Nowatzki et al., 2011). To date, and to our knowledge, there are no studies on strip-tillage carried out in woody crops.

\section{Impact on soil organic carbon stocks}

Even though there are many studies on the benefits of strip-tillage, those are mainly focused on the improvements in physical, chemical and biological soil properties, and to date, there is a lack of long-term studies on the impacts of strip-tillage on SOC stocks and its potential for SOC sequestration (Table 32).

Table 32. Evolution of SOC stocks after application of strip tillage vs. conventional tillage (moldboard plough) in two studies in the United States of America

\begin{tabular}{|c|c|c|c|c|c|c|c|}
\hline Location & $\begin{array}{l}\text { Climate } \\
\text { zone }\end{array}$ & Soil type & $\begin{array}{l}\text { Baseline } \\
\text { OC } \\
\text { stock } \\
\text { (tC/ha) }\end{array}$ & $\begin{array}{l}\text { Additional } \\
\text { OC } \\
\text { storage } \\
\text { (tC/ha/yr) }\end{array}$ & $\begin{array}{l}\text { Duration } \\
\text { (Years) }\end{array}$ & $\begin{array}{l}\text { More } \\
\text { information }\end{array}$ & Reference \\
\hline $\begin{array}{l}\text { United } \\
\text { States of } \\
\text { America } \\
\text { (Illinois) }\end{array}$ & $\begin{array}{l}\text { Cool } \\
\text { temperate } \\
\text { moist }\end{array}$ & $\begin{array}{l}\text { Typic } \\
\text { Haplaquolss }\end{array}$ & 39.9 & 4.36 & 3 & $\begin{array}{l}\mathrm{L}(\mathrm{A}) ; 0-15 \mathrm{~cm} \\
\text { depth }\end{array}$ & $\begin{array}{l}\text { Al-Kaisi } \\
\text { and Yin } \\
(2005)\end{array}$ \\
\hline $\begin{array}{l}\text { United } \\
\text { States of } \\
\text { America } \\
\text { (Alabama) }\end{array}$ & $\begin{array}{l}\text { Subtropical } \\
\text { with no dry } \\
\text { season }\end{array}$ & $\begin{array}{l}\text { Plinthic } \\
\text { Paleudult }\end{array}$ & NA & None & 2 & $\begin{array}{l}\mathrm{L}(\mathrm{A}) ; 0-10 \mathrm{~cm} \\
\text { depth }\end{array}$ & $\begin{array}{l}\text { Kingery, } \\
\text { Wood } \\
\text { and } \\
\text { Williams } \\
\text { (1996) }\end{array}$ \\
\hline
\end{tabular}

MA: meta-analysis, R: review, L: local study, M: modelling, A: arable crops, W: woody crops, NA: non available 


\section{Other benefits of the practice}

\subsection{Improvement of soil properties}

Strip tillage reduces bulk density and soil resistance to root growth while increasing the amount of biopores and soil water filtration rate (Laufer et al., 2016). It also improves soil aggregate stability (Garcia-Franco et al., 2018), which all together makes soils less prone to erosion (Dick and Gregorich, 2004; Fernández, Sorenson and Villamil, 2015). Strip-tillage contributes to protect the soil surface from nutrient losses by erosion through crop residue retention (Unger et al., 1991). It increases soil carbon sequestration (Balesdent, Chenu and Balabane, 2000), and reduces the impact of high solar radiation that promotes excessive soil temperatures during the summer season (Haramoto and Brainard, 2012). In a recent study carried out in Western Nebraska, it was demonstrated how applying strip-tillage in a sugar beet cropping system can overcome some of the main concerns regarding soil quality without compromising crop yields (Mikha et al., 2020). Finally, under semi-arid conditions, strip-tillage increases total bacteria and fungi biomass, arbuscular mycorrhizae fungi and total saprophytes, compared to conventional tillage (Idowu et al., 2019).

\subsection{Minimization of threats to soil functions}

\section{Table 33. Soil threats}

\begin{tabular}{|l|l|}
\hline Soil threats & \multicolumn{1}{|c|}{$\begin{array}{l}\text { Strip tillage reduces surface runoff and soil loss (Haramoto and Brainard, 2012; } \\
\text { Laufer et al., 2016; Tarkalson and King, 2017). }\end{array}$} \\
\hline $\begin{array}{l}\text { Nutrient } \\
\text { imbalance and } \\
\text { cycles }\end{array}$ & $\begin{array}{l}\text { Increases in soil organic carbon and nitrogen (Jahiruddin et al., 2017; Mikha et } \\
\text { al., 202O) and reductions in soil temperature and N mineralization (Haramoto } \\
\text { and Brainard, 2012). }\end{array}$ \\
\hline $\begin{array}{l}\text { Soil biodiversity } \\
\text { loss }\end{array}$ & $\begin{array}{l}\text { Strip tillage could positively impact microbial community activity (Karlen et al., } \\
\text { 1994) and diversity (Idowu et al., 2019) in the no-tilled zones. }\end{array}$ \\
\hline Soil compaction & $\begin{array}{l}\text { Bulk density decreases after tillage (Basso et al., 2003); increases in the } \\
\text { amount of biopores in the non-tilled zones (Francis and Knight, 1993). }\end{array}$ \\
\hline $\begin{array}{l}\text { Soil water } \\
\text { management }\end{array}$ & $\begin{array}{l}\text { Enhances soil moisture because of crop residue between the strips reduces } \\
\text { evaporation from the soil and increase soil water-holding capacity (Nowatzki } \\
\text { et al., 2011; Overstreet et al., 2007). In addition, it increases the particulate } \\
\text { organic matter (POM) which offsets water evaporation (Mikha et al., 202O). }\end{array}$ \\
\hline
\end{tabular}




\subsection{Increases in production (e.g. food/fuel/feed/timber/fibre)}

Strip-till could reduce variability in plant growth and their yield within a field, especially in adverse environmental conditions, such as was demonstrated by (Jaskulska, Gałązka and Jaskulski, 2019) with grain yield of winter barley cultivated on Cambisol in a region of Scandinavia with low rainfall. Although crop yields are not generally affected by strip tillage, many authors have reported higher yields under this practice compared to conventional tillage (Wiatrak et al, 2002; Temesgen et al., 2012; Olson, Falk and Aiken, 2007).

\subsection{Mitigation of and adaptation to climate change}

Strip tillage is an effective measure to mitigate drought stress and improve the resilience of crops under climate change conditions (Schneider et al., 2017). In addition, the adoption of strip tillage reduces soil $\mathrm{CO}_{2} \mathrm{emissions}$ compared to conventional tillage while maintains higher levels of soil organic carbon (Faaborg et al., 2005; Reicosky, 2001).

\subsection{Socio-economic benefits}

Costs are substantially reduced by this tillage practice (Haramoto and Brainard, 2012). Average unit fuel consumption and unit cost are lower compared to conventional tillage (Basso et al., 2003). According to several tillage guides, such as the "Upper Midwest Tillage Guide" from the University of Minnesota Extension (DeJong-Hughes and Daigh, 2017), the cost per hectare is similar to chisel plow, however, chisel plow systems need an extra pass for broadcasting fertilizer and an additional tillage pass for fertilizer incorporation and seedbed preparation. Besides savings in fuel and $\mathrm{CO}_{2}$ emissions, if strip tillage is adopted precisely the use of fertilizers and pesticides can be reduced, and therefore air, water and soil contamination will be prevented.

In semi-arid agroecosystems, especially in developing countries such as Ethiopia, compared to conventional tillage, the adoption of strip-tillage that involved subsoiling for smallholder farmers resulted in the least surface runoff, the highest plant transpiration and the highest grain yield, followed by the strip tillage system without subsoiling (Temesgen et al., 2012).

\subsection{Other benefits of the practice}

The agronomic and environmental benefits of strip-tillage increase when is combined with organic amendments, cover crops, crop residue retention, and addition of organic/inorganic fertilizers (Haramoto and Brainard, 2012; Farmaha et al., 2011; Laufer et al., 2016) and when automated equipment such as GPS is used (Nowatzki et al., 2011). 


\section{Potential drawbacks to the practice}

\subsection{Tradeoffs with other threats to soil functions}

No tradeoffs are recorded for this practice, except risk of soil contamination if pesticides and herbicides are applied to combat weeds and pathogens.

\subsection{Increases in greenhouse gas emissions}

Strip-tillage releases less carbon to the atmosphere than other tillage practices due to labor reduction and maintains higher contents of soil organic matter.

\subsection{Decreases in production (e.g. food/fuel/feed/timber/fibre)}

No negative impacts on crop yields have been reported. Shifting cropping systems from conventional tillage to strip-tillage may negatively impact crop yields, especially in the early years of transition (Hughes et al., 1992; Idowu et al., 2019; López and Arrúe, 1997; Salem et al., 2015).

\subsection{Other conflicts}

There is likely to be higher use of herbicides and pesticides to control weeds (Li et al., 2020; Mikha et al., 2020). In addition, an investment of money is required at the beginning when strip-tillage is adopted that is relatively high for small farmers (Rodriguez et al., 2009) if there is no financial support, suitable subsidies and/or credits must be available (Lahmar, 2010).

\section{Recommendations before implementing the practice}

Although combining strip-tillage with the application of fertilizers (N, P, and K) can save time, it is important to check whether soil environmental conditions are appropriate for both practices (e.g. when soil conditions are adequate for strip-tillage operations, soil temperatures are usually too warm to apply nitrogen). 


\section{Potential barriers to adoption}

\section{Table 34. Potential barriers to adoption}

\begin{tabular}{|c|c|c|}
\hline Barrier & YES/NO & \\
\hline Biophysical & Yes & $\begin{array}{l}\text { Topography and soil conditions: Sloping terrain and poorly drained soils } \\
\text { are not adequate (Claassen et al., 2018); } \\
\text { Farm size (smallholder farmers cannot invest easily); } \\
\text { In semiarid climates, and especially in poor countries, the difficulties in } \\
\text { maintaining the soil cover due to low rainfall and communal grazing } \\
\text { and because of high costs of herbicides for smallholder farmers } \\
\text { (Temesgen et al., 2012). }\end{array}$ \\
\hline Cultural & Yes & $\begin{array}{l}\text { Lack of acceptance of/Fear to change to new tillage systems } \\
\text { (Temesgen et al., 2012). }\end{array}$ \\
\hline Social & Yes & $\begin{array}{l}\text { Peer pressure and lack of marketing infrastructure for sustainable } \\
\text { products and farmers' age preconditions the adoption of a new practice } \\
\text { (Foley, 2013). }\end{array}$ \\
\hline Economic & Yes & $\begin{array}{l}\text { Initial investment in new machinery and uncertainty of profitability and } \\
\text { high risk (Rodriguez et al., 2009); } \\
\text { Absence of training and support, suitable subsidies and credits (Lahmar, } \\
\text { 2010). }\end{array}$ \\
\hline Institutional & Yes & $\begin{array}{l}\text { Lack of economic incentives and support from governments, including } \\
\text { subsidies (Runhaar et al., 2017). }\end{array}$ \\
\hline $\begin{array}{l}\text { Legal (Right } \\
\text { to soil) }\end{array}$ & Yes & Lack of strictness of legislation and standards (Ahnström et al., 2009). \\
\hline Knowledge & Yes & $\begin{array}{l}\text { The complexity of the practice can discourage its adoption (Foley, } \\
\text { 2013). }\end{array}$ \\
\hline Other & Yes & $\begin{array}{l}\text { No appropriate machinery available; lack of access to technical } \\
\text { information (Rodriguez et al., 2009). }\end{array}$ \\
\hline
\end{tabular}




\section{Representation of the practice}

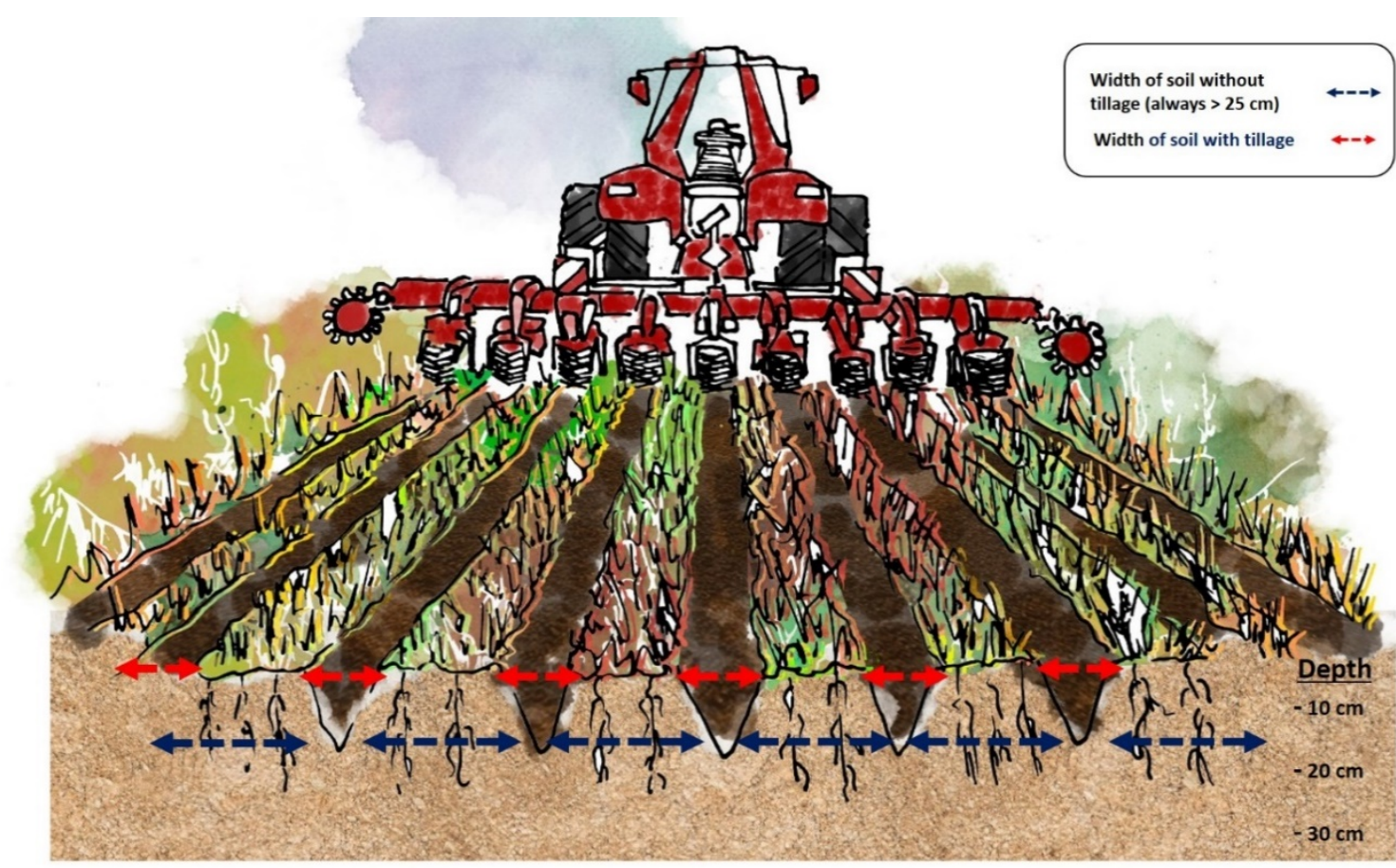

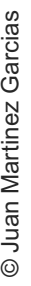

Figure 2. Graphical representation of the strip-tillage management, where only the seed row is processed, while the area between the rows is covered with crop residues and remains unprocessed

Table 35. Related cases studies available in volumes 3 and 5

\begin{tabular}{|l|l|l|c|c|}
\hline Title & Region & $\begin{array}{l}\text { Duration } \\
\text { of study } \\
\text { (Years) }\end{array}$ & Volume & $\begin{array}{l}\text { Case- } \\
\text { study } \\
\text { No. }\end{array}$ \\
\hline $\begin{array}{l}\text { Zone Tillage of a Clay Loam in } \\
\text { Southwestern Ontario, Canada }\end{array}$ & $\begin{array}{l}\text { North } \\
\text { America }\end{array}$ & 13 & 3 & 44 \\
\hline $\begin{array}{l}\text { Conservation Agriculture in intensive } \\
\text { rice-based cropping systems in the } \\
\text { Eastern Gangetic Plain }\end{array}$ & Asia & 5 & 5 & 12 \\
\hline
\end{tabular}




\section{References}

Ahnström, J., Höckert, J., Bergeå, H.L., Francis, C.A., Skelton, P. \& Hallgren, L. 2009. Farmers and nature conservation: What is known about attitudes, context factors and actions affecting conservation? Renewable Agriculture and Food Systems, 24(1): 38-47. https://doi.org/10.1017/S1742170508002391

Al-Kaisi, M.M. \& Yin, X. 2005. Tillage and crop residue effects on soil carbon and carbon dioxide emission in corn-soybean rotations. Journal of Environmental Quality, 34(2): 437-445.

https://doi.org/10.2134/jeq2005.0437

Alliaume, F., Rossing, W.A.H., Tittonell, P., Jorge, G. \& Dogliotti, S. 2014. Reduced tillage and cover crops improve water capture and reduce erosion of fine textured soils in raised bed tomato systems. Agriculture, Ecosystems \& Environment, 183: 127-137. https://doi.org/10.1016/j.agee.2013.11.001

Balesdent, J., Chenu, C. \& Balabane, M. 2000. Relationship of soil organic matter dynamics to physical protection and tillage. Soil and Tillage Research, 53(3): 215-230. https://doi.org/10.1016/S01671987(99)00107-5

Basso, B., Sartori, L., Bertocco, M. \& Oliviero, G. 2003. Evaluation of variable depth tillage: economic aspects and simulation of long term effects on soil organic matter and soil physical properties. Precision agriculture: Papers from the 4th European Conference on Precision Agriculture, Berlin, Germany, 15-19 June 2003. pp. 61-67. (also available at https://www.cabdirect.org/cabdirect/abstract/20033117611).

Claassen, R., Bowman, M., McFadden, J., Smith, D. \& Wallander, S. 2018. Tillage Intensity and Conservation Cropping in the United States. Economic Information Bulletin No. (EIB-197). 27 pp. (also available at: https://www.ers.usda.gov/webdocs/publications/90201/eib-197.pdf?v=3818.1)

DeJong-Hughes, J. \& Daigh, A.L.M. 2017. Tillage implements, purpose, and ideal use. In Upper Midwest tillage guide. Part, 2. (also available at: https://www.researchgate.net/profile/Aaron_Daigh

Dick, W.A. \& Gregorich, E.G. 2004. Developing and maintaining soil organic matter levels. In Schjønning, P., Elmholt, S. \& Christensen, B.T. (Eds.) Managing soil quality: challenges in modern agriculture, pp. 103120. Wallingford, CABI. (also available at http://www.cabi.org/cabebooks/ebook/20033208657).

Faaborg, R., Wente, C., De Jong-Hughes, J.M. \& Reicosky, D.C. 2005. A comparison of soil $\mathrm{CO}_{2}$ emissions following moldboard plowing, disk ripping and strip tilling. USDA-ARS research update.

Farmaha, B.S., Fernández, F.G. \& Nafziger, E.D. 2011. No-till and strip-till soybean production with surface and subsurface phosphorus and potassium fertilization. Agronomy Journal, 103(6): 1862-1869. https://doi.org/10.2134/agronj2011.0149

Fernández, F.G., Sorensen, B.A. \& Villamil, M.B. 2015. A comparison of soil properties after five years of no-till and strip-till. Agronomy Journal, 107(4): 1339-1346. https://doi.org/10.2134/agronj14.0549

Foley, K.M. 2013. Examining the voluntary adoption of agricultural conservation practices in northern Malheur County, Oregon. MSc thesis.

Francis, G.S. \& Knight, T.L. 1993. Long-term effects of conventional and no-tillage on selected soil properties and crop yields in Canterbury, New Zealand. Soil and Tillage Research, 26(3): 193-210. https://doi.org/10.1016/0167-1987(93)90044-P 
Garcia-Franco, N., Hobley, E., Hübner, R. \& Wiesmeier, M. 2018. Chapter 23 - Climate-Smart Soil Management in Semiarid Regions. In M.Á. Muñoz \& R. Zornoza (Eds.) Soil Management and Climate Change, pp. 349-368. Academic Press. (also available at http://www.sciencedirect.com/science/article/pii/B9780128121283000239).

Gil Domínguez, B. 2019. Análisis de la técnica de laboreo en bandas (Strip Till) en ensayos de campo reales. Paper presented at X Congreso Ibérico de Agroingeniería, 3 September 2019, Huesca, Spain. (also available at https://zaguan.unizar.es/record/84532).

Haramoto, E.R. \& Brainard, D.C. 2012. Strip Tillage and Oat Cover Crops Increase Soil Moisture and Influence N Mineralization Patterns in Cabbage. HortScience, 47(11): 1596-1602.

https://doi.org/10.21273/HORTSCI.47.11.1596

Hughes, K.A., Horne, D.J., Ross, C.W. \& Julian, J.F. 1992. A 10-year maize/oats rotation under three tillage systems. 2. Plant population, root distribution and forage yields. Soil and Tillage Research, 22(1-2): 145-157. https://doi.org/10.1016/0167-1987(92)90028-A

Idowu, J. \& Flynn, R. 2013. Understanding Soil Health for Production Agriculture in New Mexico. NM State University, Cooperative Extension Service. (also available at: https://aces.nmsu.edu/pubs/_a/A148.pdf)

Idowu, O.J., Sultana, S., Darapuneni, M., Beck, L. \& Steiner, R. 2019. Short-term Conservation Tillage Effects on Corn Silage Yield and Soil Quality in an Irrigated, Arid Agroecosystem. Agronomy, 9(8): 455. https://doi.org/10.3390/agronomy9080455

Jahiruddin, M., Hossain, R., Paul, T., Islam, M.R., Kader, M.A., Haque, M.E. \& Bell, R.W. 2017. Strip tillage with residue retention increases soil organic carbon, nitrogen requirement and system productivity of a rice-wheat-mungbean cropping system. Paper presented at 2nd Conference on Conservation Agriculture for Smallholders (CASH-II), 14-16 February 2017, Mymensingh, Bangladesh. (also available at: https://researchrepository.murdoch.edu.au/id/eprint/36820/1/strip-tillage-with-residue-retention.pdf)

Jaskulska, I., Gałązka, A. \& Jaskulski, D. 2019. Strip-till as a means of decreasing spatial variability of winter barley within a field scale. Acta Agriculturae Scandinavica, Section B-Soil \& Plant Science, 69(6): 516-527. https://doi.org/10.1080/09064710.2019.1616812

Karlen, D.L., Wollenhaupt, N.C., Erbach, D.C., Berry, E.C., Swan, J.B., Eash, N.S. \& Jordahl, J.L. 1994. Crop residue effects on soil quality following 10-years of no-till corn. Soil and Tillage Research, 31(23): 149-167. https://doi.org/10.1016/0167-1987(94)90077-9

Kingery, W.L., Wood, C.W. \& Williams, J.C. 1996. Tillage and amendment effects on soil carbon and nitrogen mineralization and phosphorus release. Soil and Tillage Research, 37(4), 239-250. https://doi.org/10.1016/0167-1987(96)01009-4

Lahmar, R. 2010. Adoption of conservation agriculture in Europe: lessons of the KASSA project. Land use policy, 27(1): 4-10. https://doi.org/10.1016/j.landusepol.2008.02.001

Laufer, D., Loibl, B., Märländer, B. \& Koch, H.-J. 2016. Soil erosion and surface runoff under strip tillage for sugar beet (Beta vulgaris L.) in Central Europe. Soil and Tillage Research, 162: 1-7.

https://doi.org/10.1016/j.still.2016.04.007 
López, M.V. \& Arrúe, J.L. 1997. Growth, yield and water use efficiency of winter barley in response to conservation tillage in a semi-arid region of Spain. Soil and Tillage Research, 44(1-2): 35-54.

https://doi.org/10.1016/S0167-1987(97)00030-5

Mikha, M.M., Hergert, G.W., Qiao, X. \& Maharjan, B. 2020. Soil chemical properties after 12 years of tillage and crop rotation. Agronomy Journal, 112(5): 4395-4406. https://doi.org/10.1002/agj2.20281

Nowatzki, J., Endres, G., Dejong-Hughes, J. \& Aakre, D. 2011. Strip till for field crop production. NDSU Extension Service. (also available at: https:/ / www.ag.ndsu.edu/publications/crops/strip-till-for-field-cropproduction/ae1370.pdf)

Olson, B., Falk, J. \& Aiken, R. 2007. Sunflower yield as affected by strip-till. Paper presented at National Sunflower Association 2007 Research Forum.

Overstreet, L.F., Franzen, D., Cattanach, N.R. \& Gegner, S. 2007. Strip-tillage in sugarbeet rotations. In 2007 Sugarbeet Research and Extension Reports. Vol. 38.

Reicosky, D.C. 2001. Effects of conservation tillage on soil organic carbon dynamics: field experiments in the US corn belt. Sustaining the global farm. Selected paper from the 10th International Soil Conservation Organization Meeting held May 24-29, 1999 at Purdue University and the USDA-ARS National Soil Erosion Research Laboratory.

Runhaar, H.A.C., Melman, T.C.P., Boonstra, F.G., Erisman, J.W., Horlings, L.G., Snoo, G.R. de, Termeer, C.J.A.M., Wassen, M.J., Westerink, J. \& Arts, B.J.M. 2017. Promoting nature conservation by Dutch farmers: a governance perspective. International Journal of Agricultural Sustainability, 15(3): 264281. https://doi.org/10.1080/14735903.2016.1232015

Salem, H.M., Valero, C., Muñoz, M.Á., Rodríguez, M.G. \& Silva, L.L. 2015. Short-term effects of four tillage practices on soil physical properties, soil water potential, and maize yield. Geoderma, 237-238: 6070. https://doi.org/10.1016/j.geoderma.2014.08.014

Tarkalson, D.D. \& King, B.A. 2017. Effects of tillage and irrigation management on sugarbeet production. Agronomy Journal, 109(5): 2396-2406. https://doi.org/10.2134/agronj2016.09.0530

Temesgen, M., Savenije, H.H.G., Rockström, J. \& Hoogmoed, W.B. 2012. Assessment of strip tillage systems for maize production in semi-arid Ethiopia: Effects on grain yield, water balance and water productivity. Physics and Chemistry of the Earth, Parts A/B/C, 47-48: 156-165.

https://doi.org/10.1016/j.pce.2011.07.046

Unger, P.W., Stewart, B.A., Parr, J.F. \& Singh, R.P. 1991. Crop residue management and tillage methods for conserving soil and water in semi-arid regions. Soil and Tillage Research, 20(2): 219-240.

https://doi.org/10.1016/0167-1987(91)90041-U

Wiatrak, P.J., Wright, D.L., Pudelko, J., Koziara, W. 2002. Influence of nitrogen and tillage on cotton. In Van Santen, E. (Ed.) Making Conservation Tillage Conventional: Building a Future on 25 Years of Research. Proc. of 25th Annual Southern Conservation Tillage Conference for Sustainable Agriculture. Auburn, AL 24-26 June 2002. Special Report no. 1. Alabama Agric. Expt. Stn. and Auburn University, AL 36849. USA. 


\title{
9. Non-inversion tillage
}

\author{
Carolina Boix-Fayos ${ }^{1}$, María Almagro ${ }^{1,2}$
}

${ }^{1}$ CEBAS-CSIC, Murcia, Spain

${ }^{2} \mathrm{BC}$, Leioa, Spain

\section{Description of the practice}

Non-inversion tillage (also known as non-inversion seedbed preparation) involves tillage operations which do not mix (or minimizes the mixing of) soil horizons or do not vertically mix soil within a horizon (SSSA, 2020). It is based on the use of tine and disc implements that do not invert the soil and often includes a cultivation system involving fewer passes than conventional tillage, with implements working very often at shallow depths $(5-10 \mathrm{~cm})$ or a bit deeper $(15-25 \mathrm{~cm})$ whereby crop residues are mixed into the topsoil but leave a proportion on the soil surface (Morris et al, 2010; Cooper et al., 2016).

The objective of non-inversion tillage is to limit the mechanical disturbance of the soil to that required for seed placement, and to create a soil physical environment that, in particular, mechanical impedance and aeration do not restrict root growth and function, and therefore crop yield (Cannell, 1985).

\section{Range of applicability}

Based on many different authors (see references cited at the end of this chapter), this technique is applied under a wide variety of climate conditions, soil types and crops. Results are reported for climatic zones such as warm temperate dry, warm temperate humid, cool temperate moist, and tropical wet. It is applied in soils with textural classes of clay, clay-loam, loam, loamy sand, sandy clay loam, sandy loam, silt loam, and silty clay loam. It is applied in arable soils cultivating a great range of crops: legume ley, legume cover crop, non-legume cover crop, leaf vegetable, root vegetable winter small-grain cereal/oilseed, spring small-grain cereal/oilseed, peas/beans, maize sorghum, and woody crops. It can be potentially applied to all classes of soils, climatic areas and crops. 


\section{Impact on soil organic carbon stocks}

In Table 36, some examples of changes in SOC stocks or concentrations are given (only information related to studies in which non-inversion tillage is specifically mentioned have been included in order to avoid overlapping with other practices, such as reduced tillage, already described in the Manual). Although there are other tillage systems that do not involve soil inversion (e.g. reduced tillage, minimum tillage), results on those tillage practices are given in their specific factsheet.

Table 36. Evolution of SOC stocks in non-inversion tillage systems

\begin{tabular}{|c|c|c|c|c|c|c|c|}
\hline Location & Climate zone & Soil type & $\begin{array}{l}\text { Baseline } \\
\mathrm{C} \text { stock } \\
\text { (tC/ha) }\end{array}$ & $\begin{array}{l}\text { Additional } \\
\text { C storage or } \\
\text { content }\end{array}$ & $\begin{array}{l}\text { Duration } \\
\text { (Years) }\end{array}$ & $\begin{array}{l}\text { More } \\
\text { information }\end{array}$ & Reference \\
\hline Global & $\begin{array}{l}\text { Humid oceanic, } \\
\text { Humid } \\
\text { continental, } \\
\text { Mediterranean }\end{array}$ & Various & NA & $\begin{array}{l}\text { Net effect of } \\
\text { SOC } \\
\text { change: } \\
1.43 \mathrm{tC} / \mathrm{ha}\end{array}$ & $\begin{array}{c}\text { From } 3 \text { to } \\
10\end{array}$ & $\begin{array}{l}\text { Averaged } \\
\text { value from } \\
\text { meta-analysis } \\
\text { ( } n=184) \\
\text { Deep } \\
\text { inversion } \\
\text { tillage vs } \\
\text { shallow non- } \\
\text { inversion } \\
\text { tillage }\end{array}$ & $\begin{array}{l}\text { Cooper et al. } \\
(2016)^{*}\end{array}$ \\
\hline $\begin{array}{l}\text { Rock Springs, } \\
\text { PA, United } \\
\text { States of } \\
\text { America }\end{array}$ & $\begin{array}{l}\text { Humid } \\
\text { continental }\end{array}$ & Silt loam & & $\sim 15^{* *}$ & 3 & Labile OC & $\begin{array}{l}\text { Lewis et al. } \\
\text { (2011) }\end{array}$ \\
\hline $\begin{array}{l}\text { Mainz, } \\
\text { Germany }\end{array}$ & & $\begin{array}{l}\text { Silty clay } \\
\text { loam }\end{array}$ & & $\sim 5.2^{* *}$ & 4 & $\begin{array}{l}\text { Soil organic } \\
\text { matter at O- } \\
25 \mathrm{~cm} \text { depth }\end{array}$ & $\begin{array}{l}\text { Emmerling, } \\
\text { (2007) }\end{array}$ \\
\hline UK & & $\begin{array}{l}\text { Clay } \\
\text { loam to } \\
\text { sandy } \\
\text { clay } \\
\text { loam }\end{array}$ & 18 & $\begin{array}{l}1.18 \\
\mathrm{tC} / \mathrm{ha} / \mathrm{yr}\end{array}$ & 22 months & - & $\begin{array}{l}\text { Cooper et al. } \\
\text { (2017) }\end{array}$ \\
\hline $\begin{array}{l}\text { Lelystad, } \\
\text { Netherlands }\end{array}$ & Humid oceanic & $\begin{array}{l}\text { Clay } \\
\text { loam }\end{array}$ & 10.03 & $\begin{array}{l}0.23 \\
\text { tC/ha/yr } \\
0.22 \\
\text { tC/ha/yr } \\
\\
-0.4 \\
\text { tC/ha/yr } \\
0.03 \\
\text { tC/ha/yr }\end{array}$ & 4 & $\begin{array}{l}\text { At } 0-15 \mathrm{~cm} \\
\text { ( } 2 \text { organic } \\
\text { farms) } \\
\text { At } 20-30 \mathrm{~cm} \\
\text { (1 organic } \\
\text { farm) } \\
\text { At } 30-40 \mathrm{~cm} \\
(1 \text { organic } \\
\text { farm) } \\
\text { At } 40-50 \mathrm{~cm}\end{array}$ & $\begin{array}{l}\text { Crittenden } \\
\text { et al. (2015) }\end{array}$ \\
\hline
\end{tabular}




\begin{tabular}{|c|c|c|c|c|c|c|c|}
\hline Location & Climate zone & Soil type & $\begin{array}{l}\text { Baseline } \\
\text { C stock } \\
\text { (tC/ha) }\end{array}$ & $\begin{array}{l}\text { Additional } \\
\text { C storage or } \\
\text { content }\end{array}$ & $\begin{array}{l}\text { Duration } \\
\text { (Years) }\end{array}$ & $\begin{array}{l}\text { More } \\
\text { information }\end{array}$ & Reference \\
\hline & & & & & & $\begin{array}{l}\text { (1 organic } \\
\text { farm) }\end{array}$ & \\
\hline Across Europe & $\begin{array}{l}\text { Humid oceanic, } \\
\text { Humid } \\
\text { continental, } \\
\text { Mediterranean, } \\
\text { Boreal. }\end{array}$ & All types & NA & $\begin{array}{l}\text { Increase of } 5 \\
* \star\end{array}$ & At least 10 & At $0-30 \mathrm{~cm}$ & $\begin{array}{l}\text { Sandén et } \\
\text { al. (2018) }\end{array}$ \\
\hline
\end{tabular}

*Average increase in soil organic carbon stocks comparing deep inversion tillage and reduced tillage intensity (including non-inversion tillage at different depths) in a subset of 184 observations worldwide.

${ }^{* *}$ Increase in SOC concentration (mg/g)

\section{Other benefits of the practice}

\subsection{Improvement of soil properties}

Studies have shown that non-inversion tillage systems have improved both soil water retentionand soil aggregate stability at 0-10 cm and 10-20 cm layers (Crittenden et al., 2015) As well as increases of total $\mathrm{N}$ and available $\mathrm{K}$ by 7 percent and 43 percent, respectively (Sandén et al., 2018). Surface accumulation of $\mathrm{N}$ and $\mathrm{K}$ can be advantageous for early crop growth under favorable moist conditions (Neugschwandtner et al., 2014). General improvement of many physical, chemical and biological soil properties compared to conventional tillage (Holland, 2004; Sandén et al., 2018) have been observed For instance Sandén et al. (2018) observed an increase in earthworm numbers (33 percent) and biomass (68 percent) . Some specific long-term experiments demonstrated that several types of reduced tillage treatments (including non-inversion tillage at $15 \mathrm{~cm}$ depth) resulted in soils with higher earthworm species richness compared to conventional tillage (Ernst and Emmerling, 2009).

\subsection{Minimization of threats to soil functions}

\section{Table 37. Soil threats}

\section{Soil threats}

Soil erosion

Decrease soil erosion (Lahmar, 2010), decrease sediment yield up to 63 percent (Sandén et al., 2018). 


\section{Soil threats}

\begin{tabular}{|l|l|}
\hline $\begin{array}{l}\text { Nutrient } \\
\text { imbalance and } \\
\text { cycles }\end{array}$ & $\begin{array}{l}\text { Higher available P and K at O-10 cm soil depth explained by the } \\
\text { shallow incorporation of crop residues (Abdollahi and Munkholm, } \\
2014) .\end{array}$ \\
\hline $\begin{array}{l}\text { Soil biodiversity } \\
\text { loss }\end{array}$ & $\begin{array}{l}\text { When shallow, it increases earthworm number and microbial biomass } \\
\text { by } 30 \text { percent (D'Hose et al., 2018). }\end{array}$ \\
\hline Soil compaction & $\begin{array}{l}\text { Reduced soil compaction, especially when used with sub-soiling and } \\
\text { cover crops (Holland, 2004). }\end{array}$ \\
\hline $\begin{array}{l}\text { Soil water } \\
\text { management }\end{array}$ & $\begin{array}{l}\text { Increased infiltration capacity and reduction of runoff between } 15 \text { and } \\
89 \text { percent (Holland, 2004; Sandén et al., 2018) }\end{array}$ \\
\hline
\end{tabular}

\subsection{Increases in production (e.g. food/fuel/feed/timber/fibre)}

Shallow non-inversion tillage $(<25 \mathrm{~cm})$ results in non-significant reductions in crop yields compared to deep inversion tillage (Cooper et al., 2016; Crittenden et al., 2015; Alarcón et al., 2018). Increased crop yields of mixture wheat/faba beans were found applying non-inversion tillage compared to conventional tillage (Crittenden et al., 2015). However other authors found contradictory results on the influence of tillage systems on crop yields, depending on the crop type. For example, conventional tillage had significant lower oat yields than non-inversion tillage systems but the opposite occurred for barley yields in Croatia (Bogunovic et al., 2020). Nevertheless, non-inversion tillage is less labour intensive, implies lower fuel use and reduced tillage costs (Bijttebier et al., 2018).

\subsection{Mitigation of and adaptation to climate change}

It saves $\mathrm{CO}_{2}$ emissions in machinery (Holland, 2004). Soil $\mathrm{CO}_{2}$ emissions were significantly higher in conventional tillage than in non-inversion tillage systems in an experiment carried out during two years in Croatia (Bogunovic et al., 2020).

\subsection{Socio-economic benefits}

Saving costs in fuel, machinery and inputs. Saving time to dedicate to other agricultural activities. More flexibility and improved timeliness for operations (Lahmar, 2010). 


\subsection{Other benefits of the practice}

A reduction in the loss of sediments by erosion implies an improvement in water quality and aquatic wildlife (Holland, 2004). Positive effects on micro-, meso- and macro-fauna (Holland, 2004).

\section{Potential drawbacks to the practice}

\subsection{Tradeoffs with other threats to soil functions}

Table 38. Soil threats

\begin{tabular}{|l|l|}
\hline Soil threats & $\begin{array}{l}\text { Less effective for soil conservation in erodible soils (Bijttebier et al., 2018). } \\
\text { Under dry conditions soil nutrients can be lost by soil erosion (Sandén et } \\
\text { al., 2018). }\end{array}$ \\
\hline $\begin{array}{l}\text { Nutrient } \\
\text { imbalance and } \\
\text { cycles }\end{array}$ & $\begin{array}{l}\text { Non-inversion seedbed preparation is not effective in reducing N and P } \\
\text { losses by leaching when applied alone (without cover crops; Cooper et al. } \\
\text { (2017). Under dry conditions nutrients can be inaccessible for plant uptake } \\
\text { (Sandén et al., 2O18). }\end{array}$ \\
\hline $\begin{array}{l}\text { Soil biodiversity } \\
\text { loss }\end{array}$ & $\begin{array}{l}\text { Decrease of soil microbial biomass by 14 percent at 10-30 cm depth with } \\
\text { shallow non-inversion tillage, compared to conventional tillage (D'Hose et } \\
\text { al., 2018). }\end{array}$ \\
\hline Soil compaction & $\begin{array}{l}\text { Higher penetration resistance (Crittenden et al., 2015). Possibility of } \\
\text { increased soil compaction at long term in some cases (Holland, 2004). }\end{array}$ \\
\hline $\begin{array}{l}\text { Soil water } \\
\text { management }\end{array}$ & $\begin{array}{l}\text { Difficulties for soil drying (Bijttebier et al., 2018). Lower field saturated } \\
\text { hydraulic conductivity (Crittenden et al., 2015) }\end{array}$ \\
\hline
\end{tabular}

\subsection{Increases in greenhouse gas emissions}

Insignificant average decreases of $\mathrm{CO}_{2}$ and $\mathrm{N}_{2} \mathrm{O}$ compared to conventional tillage (Sandén et al., 2018). 


\subsection{Decreases in production (e.g. food/fuel/feed/timber/fibre)}

Different values have been given regarding the impact of non-inversion tillage on productivity. Non-inversion tillage decreased yields by 4 percent, $N$ uptake by 9 percent, and nitrogen use efficiency by 10 percent (Sandén et al., 2018). Furthermore deep-inversion tillage resulted in averaged yield reductions of 11.6 percent (Cooper et al., 2016).

\subsection{Other conflicts}

Problems can occur with weeds (because of increases in weed incidence and density) and crop residue management (Holland, 2004). It can resultin an increase of 75 percent of weed incidence (Cooper et al., 2016). It can increase the risk of leaching, particularly of herbicides when combating weeds. During long-term applications phosphate can accumulate in the soil surface increasing loss via runoff (Holland, 2004).

\section{Recommendations before implementing the practice}

Increasing crop varieties by crop rotation diversification and inter-cropping in order to enhance biodiversity and functionality before implementing non-inverse tillage has been prove effective to manage weeds. In this regard, the use of "strategic tillage" at critical stages in the rotation is recommended to managed pernicious weeds or control residue-borne crop diseases (Dang et al., 2015), but also to avoid soil biota damages (Cooper et al., 2016). For example, by placing tillage operations in dry periods, when vertically burrowing earthworms move to the subsoil, negative impacts on earthworms can be further reduced. Likewise, cover crops adoption and the use of mechanical methods (e.g. roller crimpers) instead of herbicides to terminate them is highly recommended to suppress weeds.

\section{Potential barriers to adoption}

Table 39. Potential barriers to adoption

\begin{tabular}{|l|l|l|}
\hline Barrier & YES/NO & \\
\hline Biophysical & Yes & $\begin{array}{l}\text { Experience of not functioning properly when applied to clay soils, } \\
\text { specific crops, time of sowing and harvest of particular crops (Bijttebier } \\
\text { et al., 2018). Increase biomass and density of annual and perennial } \\
\text { weeds (Crowley et al. 2010). }\end{array}$ \\
\hline Cultural & Yes & $\begin{array}{l}\text { Perception of less beautiful fields and increase in weeds, pests and } \\
\text { diseases. Good results obtained with conventional ploughing, } \\
\text { demotivate adoption of non-inversion tillage (Bijttebier et al., 2018) }\end{array}$ \\
\hline
\end{tabular}




\begin{tabular}{|l|c|l|}
\hline Barrier & YES/NO & \\
\hline Social & Yes & $\begin{array}{l}\text { Not frequently applied by farmers (Bijttebier et al., 2018). Lack of } \\
\text { leadership by farmers organizations (Lahmar, 2010). }\end{array}$ \\
\hline Economic & Yes & $\begin{array}{l}\text { Not clear positive effects on yields and other productivity indicators, } \\
\text { and even perception of lower yields (Alarcón et al., 2018; Bijttebier et } \\
\text { al., 2018; Sandén et al., 2018). }\end{array}$ \\
\hline Institutional & Yes & $\begin{array}{l}\text { Many conditions to obtain subsidies (Bijttebier et al., 2018). Absence of } \\
\text { training and support, suitable subsidies and credits (Lahmar, 2010). }\end{array}$ \\
\hline Knowledge & Yes & $\begin{array}{l}\text { Not technical knowledge, no/little experience (Lahmar, 2010; Bijttebier } \\
\text { et al., 2018). }\end{array}$ \\
\hline Other & Yes & Not appropriate machinery available (Bijttebier et al., 2018). \\
\hline
\end{tabular}

\section{Photo of the practice}

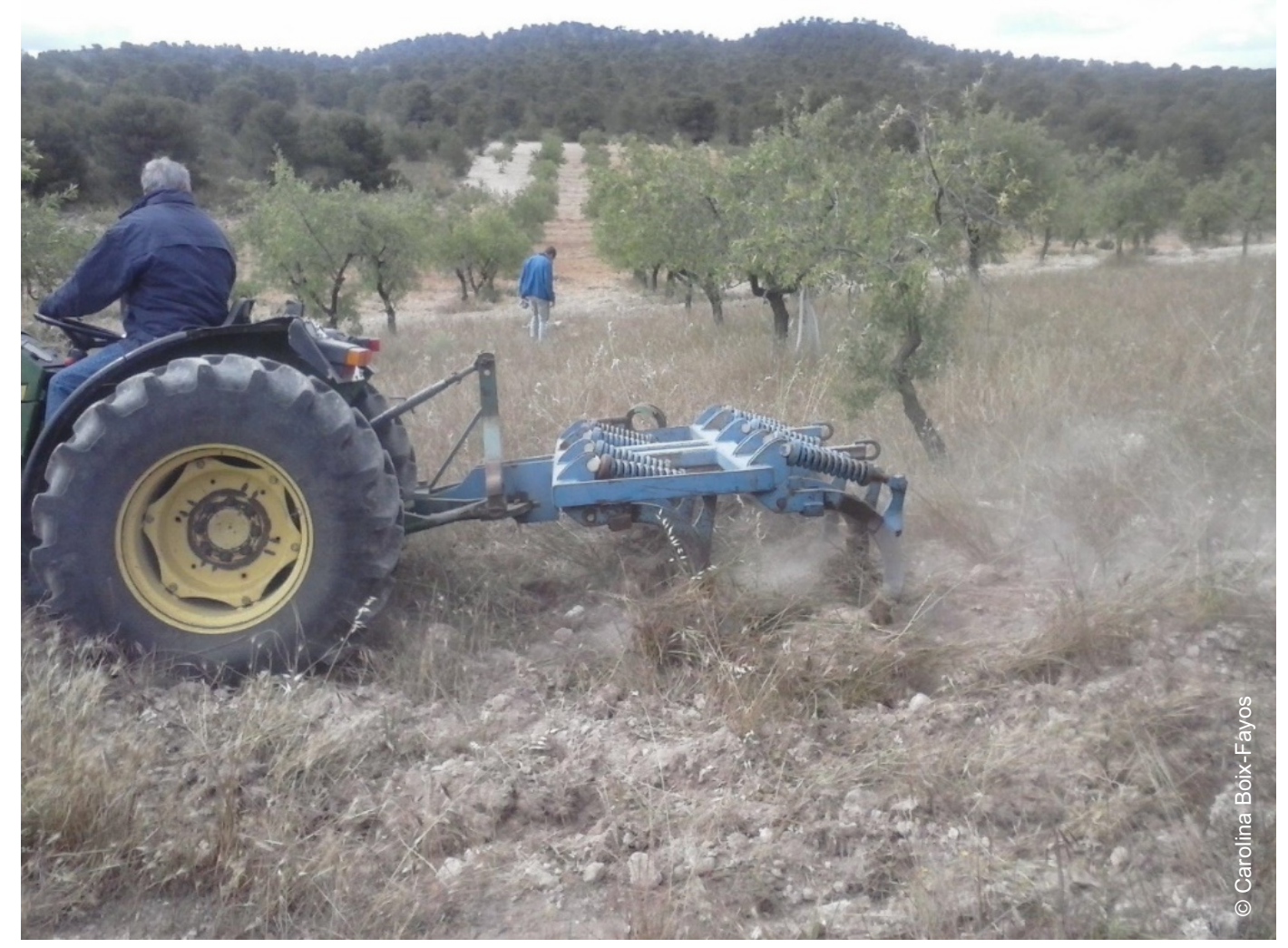

Photo 10. Non-inversion tillage operation in the Alhagüeces farm (Zarzadilla de Totana, Murcia, Spain) under rainfed organic almonds 


\section{References}

Abdollahi, L. \& Munkholm, L.J. 2014. Tillage System and Cover Crop Effects on Soil Quality: I. Chemical, Mechanical, and Biological Properties. Soil Science Society of America Journal, 78(1): 262-270.

https://doi.org/10.2136/sssaj2013.07.0301

Alarcón, R., Hernández-Plaza, E., Navarrete, L., Sánchez, M.J., Escudero, A., Hernanz, J.L., SánchezGiron, V. \& Sánchez, A.M. 2018. Effects of no-tillage and non-inversion tillage on weed community diversity and crop yield over nine years in a Mediterranean cereal-legume cropland. Soil and Tillage Research, 179: 54-62. https://doi.org/10.1016/j.still.2018.01.014

Bijttebier, J., Ruysschaert, G., Hijbeek, R., Werner, M., Pronk, A.A., Zavattaro, L., Bechini, L., Grignani, C., ten Berge, H., Marchand, F. \& Wauters, E. 2018. Adoption of non-inversion tillage across Europe: Use of a behavioural approach in understanding decision making of farmers. Land Use Policy, 78: 460-471. https://doi.org/10.1016/j.landusepol.2018.05.044

Bogunovic, I., Pereira, P., Galic, M., Bilandzija, D. \& Kisic, I. 2020. Tillage system and farmyard manure impact on soil physical properties, $\mathrm{CO}_{2}$ emissions, and crop yield in an organic farm located in a Mediterranean environment (Croatia). Environmental Earth Sciences, 79(3): 70.

https://doi.org/10.1007/s12665-020-8813-Z

Cannell, R.Q. 1985. Reduced tillage in north-west Europe-A review. Soil and Tillage Research, 5(2): 129177. https://doi.org/10.1016/0167-1987(85)90028-5

Cooper, J., Baranski, M., Stewart, G., Nobel-de Lange, M., Bàrberi, P., Fließbach, A., Peigné, J., Berner, A., Brock, C., Casagrande, M., Crowley, O., David, C., De Vliegher, A., Döring, T.F., Dupont, A., Entz, M., Grosse, M., Haase, T., Halde, C., Hammerl, V., Huiting, H., Leithold, G., Messmer, M., Schloter, M., Sukkel, W., van der Heijden, M.G.A., Willekens, K., Wittwer, R. \& Mäder, P. 2016. Shallow non-inversion tillage in organic farming maintains crop yields and increases soil C stocks: a metaanalysis. Agronomy for Sustainable Development, 36(1): 22. https://doi.org/10.1007/s13593-016-03541

Cooper, R.J., Hama-Aziz, Z., Hiscock, K.M., Lovett, A.A., Dugdale, S.J., Sünnenberg, G., Noble, L., Beamish, J. \& Hovesen, P. 2017. Assessing the farm-scale impacts of cover crops and non-inversion tillage regimes on nutrient losses from an arable catchment. Agriculture, Ecosystems \& Environment, 237: 181193. https://doi.org/10.1016/j.agee.2016.12.034

Crittenden, S.J., Poot, N., Heinen, M., van Balen, D.J.M. \& Pulleman, M.M. 2015. Soil physical quality in contrasting tillage systems in organic and conventional farming. Soil and Tillage Research, 154: 136-144. https://doi.org/10.1016/j.still.2015.06.018

Crowley, O., Showering, J. 2010. Saving fuel with non-inversion tillage. ORC Bulletin December 2010. (also available at: https://orgprints.org/19879/1/2010.Crowley_et_al.NIT.Bulletin103.pdf)

Dang, Y.P., Moody, P.W., Bell, M.J., Seymour, N.P., Dalal, R.C., Freebairn, D.M. \& Walker, S.R. 2015. Strategic tillage in no-till farming systems in Australia's northern grains-growing regions: II. Implications for agronomy, soil and environment. Soil and Tillage Research, 152: 115-123.

https://doi.org/10.1016/j.still.2014.12.013 
D'Hose, T., Molendijk, L., Van Vooren, L., van den Berg, W., Hoek, H., Runia, W., van Evert, F., ten Berge, H., Spiegel, H., Sandèn, T., Grignani, C. \& Ruysschaert, G. 2018. Responses of soil biota to noninversion tillage and organic amendments: An analysis on European multiyear field experiments.

Pedobiologia, 66: 18-28. https://doi.org/10.1016/j.pedobi.2017.12.003

Emmerling, C. 2007. Reduced and Conservation Tillage Effects on Soil Ecological Properties in an Organic Farming System. Biological Agriculture \& Horticulture, 24(4): 363-377.

https://doi.org/10.1080/01448765.2007.9755033

Ernst, G. \& Emmerling, C. 2009. Impact of five different tillage systems on soil organic carbon content and the density, biomass, and community composition of earthworms after a ten year period. European Journal of Soil Biology, 45(3): 247-251. https://doi.org/10.1016/j.ejsobi.2009.02.002

Holland, J.M. 2004. The environmental consequences of adopting conservation tillage in Europe: reviewing the evidence. Agriculture, Ecosystems \& Environment, 103(1): 1-25.

https://doi.org/10.1016/j.agee.2003.12.018

Lahmar, R. 2010. Adoption of conservation agriculture in Europe: Lessons of the KASSA project. Land Use Policy, 27(1): 4-10. https://doi.org/10.1016/j.landusepol.2008.02.001

Lewis, D.B., Kaye, J.P., Jabbour, R. \& Barbercheck, M.E. 2011. Labile carbon and other soil quality indicators in two tillage systems during transition to organic agriculture. Renewable Agriculture and Food Systems, 26(4): 342-353. https://doi.org/10.1017/S1742170511000147

Morris, N.L., Miller, P.C.H., J.H.Orson \& Froud-Williams, R.J. 2010. The adoption of non-inversion tillage systems in the United Kingdom and the agronomic impact on soil, crops and the environment-A review. Soil and Tillage Research, 108(1): 1-15. https://doi.org/10.1016/j.still.2010.03.004.

Neugschwandtner, R.W., Liebhard, P., Kaul, H.-P. \& Wagentristl, H. 2014. Soil chemical properties as affected by tillage and crop rotation in a long-term field experiment. Plant, Soil and Environment, 60: 57-62. https://doi.org/10.17221/879/2013-PSE

Sandén, T., Spiegel, H., Stüger, H.-P., Schlatter, N., Haslmayr, H.-P., Zavattaro, L., Grignani, C., Bechini, L., D’Hose, T., Molendijk, L., Pecio, A., Jarosz, Z., Guzmán, G., Vanderlinden, K., Giráldez, J.V., Mallast, J. \& Berge, H. ten. 2018. European long-term field experiments: knowledge gained about alternative management practices. Soil Use and Management, 34(2): 167-176.

https://doi.org/10.1111/sum.12421

SSSA, Soil Science Society of America 2020. Glossary of terms. [Online]. [Cited 27 November 2020]. https://www.soils.org/publications/soils-glossary\# 


\title{
10. Manure additions
}

\author{
Ofelia I. Beltrán-Paz, , Nadia E. Nava-Arsola, ${ }^{1,2}$, Bruno M. Chávez-Vergara1,2 \\ 'Institute of Geology, National Autonomous University of Mexico, Mexico \\ ${ }^{2}$ Laboratorio Nacional de Geoquímica y Mineralogía, México
}

\section{Description of the practice}

Manure includes the excreta of animals raised for meat or other products whose chemical composition depends on the diet and the type of animal from which it originates (e.g., poultry, cows, sheep, horses, rabbits, etc.) and may also include the plant material (straw) used as bedding for animals (Rasouli-Sadaghiani and Moradi, 2014). Manure can be found in liquid (liquid manure or slurry) or solid (solid manure) form. Animal manure is a valuable resource as part of integrated nutrient management strategies for sustainable soil management. It is used more efficiently in combination with other sustainable practices such as crop rotation, cover crops, green manures, and liming. In organic production, manure is commonly applied to the soil as raw manure (fresh or dry) or as composted manure (Kuepper, 2003). Manure can add essential plant nutrients (nitrogen, potassium, and phosphorus, collectively known as NPK) to the soil and improve soil quality. While partial substitutions of mineral fertilizers with manure can enhance crop yields, the complete replacement of mineral fertilization with manures can have detrimental effects on crop yields (Zhang et al., 2020).

Nevertheless, a recent meta-analysis by Du et al. (2020) shows a mean increase in crop yields of 7.6 percent when using manure compared to mineral fertilizers. Composting raw manure while adding other natural materials and animal waste improves decomposition and produces a humus-rich end-product with minor or noneasily leachable $\mathrm{N}$ forms (Franco-Otero et al., 2012), which will improve soil fertility (Evanylo et al., 2008). If manure is applied and managed correctly, it can be an effective way of improving soil quality and crop nutrition. Still, there are important aspects of soil health and food security to consider when used as organic fertilizer in agroecological systems (Rasouli-Sadaghiani and Moradi, 2014). 


\section{Range of applicability}

The use of manure is a widely spread practice among different climates, soil types, crop types, and in conjunction with other techniques such as the addition of synthetic fertilizers, type of tillage, and irrigation (Maillard and Angers, 2014).

\section{Impact on soil organic carbon stocks}

In the available research on increases in SOC concentration $(\mathrm{g} / \mathrm{kg})($ Table 40) the increment in SOC is sensitive to manure type, doses, time of application, and parallel agricultural practices (Liu et al., 2020). Although this information is very useful for observing the beneficial impact of manure addition on SOC, it is challenging to compare SOC sequestration rate or increments $(\mathrm{Mg} / \mathrm{ha})$ across studies because of additional data needs (e.g. sampling depth, bulk density, stoniness) that are highly variable among agricultural systems. 
Table 40. General features of research about manure addition on soil organic carbon storage

\begin{tabular}{|c|c|c|c|c|c|c|c|c|}
\hline Location & Climate zone & Soil type & $\begin{array}{l}\text { Baseline C } \\
\text { stock } \\
\text { (tC/ha) }\end{array}$ & $\begin{array}{l}\text { Additional } \\
\text { C storage }+/- \\
\text { SE } \\
(\mathrm{tC} / \mathrm{ha} / \mathrm{yr})\end{array}$ & $\begin{array}{l}\text { Duration } \\
\text { (Years) }\end{array}$ & $\begin{array}{l}\text { Depth } \\
\text { (cm) }\end{array}$ & More information & Reference \\
\hline \multirow{2}{*}{ Global } & \multirow{2}{*}{ Various } & \multirow{2}{*}{ Various } & NA & 3.1 & 3 to 82 & $0-30$ & $\begin{array}{l}\text { Meta-analysis; } \\
\text { Liquid and solid manures of pig, cattle, } \\
\text { poultry and goat }\end{array}$ & $\begin{array}{l}\text { Maillard and } \\
\text { Angers (2014) }\end{array}$ \\
\hline & & & NA & $27 \pm 4.2$ & 1 to $>5$ & NA & $\begin{array}{l}\text { Meta-analysis of diverse manure } \\
\text { types: farmayard, cattle, pig and } \\
\text { poultry. }\end{array}$ & $\begin{array}{l}\text { Liu et al. } \\
(2020)\end{array}$ \\
\hline $\begin{array}{l}\text { North China } \\
\text { Plain }\end{array}$ & $\begin{array}{l}\text { Warm temperate } \\
\text { semi-humid } \\
\text { monsoon }\end{array}$ & $\begin{array}{l}\text { Fluvi-Aquic } \\
\text { (Aquept) }\end{array}$ & $6.57^{\star}$ & $0.30^{*}$ & 32 & $0-20$ & $\begin{array}{l}\text { Cattle manure } \\
\text { Also positive impacts on soil fungal } \\
\text { diversity }\end{array}$ & $\begin{array}{l}\text { Wen et al. } \\
(2020)\end{array}$ \\
\hline $\begin{array}{l}\text { Central } \\
\text { Germany }\end{array}$ & Dry cold climate & Haplic Chernozem & $160^{*}$ & $0.45^{\star}$ & 110 & $0-20$ & Farmyard manure & $\begin{array}{l}\text { Francioli et al. } \\
\text { (2016) }\end{array}$ \\
\hline $\begin{array}{l}\text { Copenhague, } \\
\text { Denmark }\end{array}$ & NA & $\begin{array}{l}\text { Luvisol (Sandy } \\
\text { loam) }\end{array}$ & $15.4^{\star}$ & $0.53^{*}$ & 12 & $0-20$ & Cattle farmyard manure & $\begin{array}{l}\text { Lemming et } \\
\text { al. (2019) }\end{array}$ \\
\hline \multirow{3}{*}{$\begin{array}{l}\text { Southern } \\
\text { Chile }\end{array}$} & \multirow{3}{*}{ Humid climate } & $\begin{array}{l}\text { Acrudoxic } \\
\text { Hapludands (Loam) }\end{array}$ & 105 & $0.92^{*}$ & \multirow{3}{*}{5} & $0-5$ & $\begin{array}{l}\text { One site; } \\
\text { Composted poultry manure }\end{array}$ & \multirow{3}{*}{$\begin{array}{l}\text { Poblete- } \\
\text { Grant et al. } \\
(2020)\end{array}$} \\
\hline & & $\begin{array}{l}\text { Acrudoxic } \\
\text { fulvudands } \\
\text { (Sandy loam) }\end{array}$ & $99.4-141^{*}$ & $1.32-6.24^{*}$ & & $\begin{array}{l}0-5 \\
0-10\end{array}$ & $\begin{array}{l}\text { Two sites; } \\
\text { Composted poultry manure }\end{array}$ & \\
\hline & & $\begin{array}{l}\text { Typic Durudands } \\
\text { (Loamy silty clay) }\end{array}$ & $86.6^{*}$ & $11.2^{*}$ & & $0-10$ & $\begin{array}{l}\text { One site; } \\
\text { Composted poultry manure }\end{array}$ & \\
\hline $\begin{array}{l}\text { Tarnab, } \\
\text { Pakistan }\end{array}$ & Semiarid & Clay loam & 0.75 & 7.78 & 2 & $0-20$ & Farmyard manure & $\begin{array}{l}\text { Shehzadi, } \\
\text { Shah and } \\
\text { Mohammad, } \\
\text { (2017) }\end{array}$ \\
\hline
\end{tabular}

SE: Standard error of the mean; NA: No information; ${ }^{*}$ SOC concentration rate in $\mathrm{g} / \mathrm{kg} / \mathrm{yr}$ because SOC stock data is not available 


\section{Other benefits of the practice}

\subsection{Improvement of soil properties}

Manure application decreases soil bulk density, improves aggregate stability, and increases organic matter contents in soils (Thangarajan et al., 2016; Chen et al., 2020; Lemming et al., 2020; Yadav et al., 2020). However, in some instances, these positive effects of manures on soil quality are not always significantly different than control (Chang et al., 2014; Chen et al., 2020). Field capacity and soil moisture are also increased (Hargreaves, Adl and Warman, 2008; Thangarajan et al., 2016). The increase in soil organic matter due to the addition of organic amendments can increase the concentrations of dissolved organic carbon, which improves soil microbial activity (Bai et al., 2020; Ma et al., 2020).

The addition of animal manure increases the activity of various exoenzymes that contribute to depolymerization processes such as urease, protease, cellulose, $\beta$-glucosidase, $\mathrm{N}$-acetylglucosaminidase, and xylanase (Chang, Chung and Tsai, 2007; Bastida et al., 2008; Ros et al, 2008; Chakraborty et al., 2011; Reeve et al., 2012; Thangarajan et al., 2013; Francioli et al., 2016; Ma et al., 2020) and poultry manure promotes the activity of acid phosphatase (Acosta- Martínez et al., 2011). Other processes that are stimulated are soil respiration and carbon and nitrogen immobilization in microbial biomass, compared to soils to which cow dung was not added (Bastida et al., 2008; Ros et al., 2008; Luo et al., 2010; Francioli et al., 2016; Wang et al., 2018; Ma et al., 2020).

\subsection{Minimization of threats to soil functions}

Table 41. Soil threats

\begin{tabular}{|c|c|}
\hline Soil threats & \\
\hline Soil erosion & $\begin{array}{l}\text { The application of cow and poultry manure improves soil structure and avoids } \\
\text { soil erosion (Annabi et al., 2011). }\end{array}$ \\
\hline $\begin{array}{l}\text { Nutrient } \\
\text { imbalance and } \\
\text { cycles }\end{array}$ & $\begin{array}{l}\text { The addition of poultry and cow manure increase the total and available N, P, } \\
\text { and } \mathrm{K} \text { for enhanced microbial activity; the concentration of } \mathrm{P} \text { increases and the } \\
\text { adsorption by soil decreases (Guppy et al., 2005; Ayaga, Todd and Brookes, } \\
\text { 2006; Odlare et al., 2008; Medina et al., 2012; Francioli et al., 2016; Jing et al., } \\
\text { 2018; Wang et al., 2018; Chen et al., 2019; Wen et al., 2020; Poblete-Grant et } \\
\text { al., 2020; Krauss et al, 2020; Ma et al., 2020; Lemming et al., 2020). }\end{array}$ \\
\hline $\begin{array}{l}\text { Soil salinization } \\
\text { and alkalinization }\end{array}$ & $\begin{array}{l}\text { In saline soils, manure additions decreased soil salinity compared to control } \\
\text { soils; however, in non-saline soils, the addition of poultry manure can increase } \\
\text { the total soluble solids and particularly } \mathrm{Na}^{+}, \mathrm{K}^{+}, \mathrm{Mg}^{2+}, \mathrm{SO}_{4}{ }^{2} \text {, and } \mathrm{Cl}^{-}(\mathrm{Li} \text {-Xian et } \\
\text { al., 2007; Ding et al., 202O; Goldberg et al., 2020). }\end{array}$ \\
\hline
\end{tabular}




\begin{tabular}{|c|c|}
\hline Soil threats & \\
\hline $\begin{array}{l}\text { Soil } \\
\text { contamination/ } \\
\text { pollution }\end{array}$ & $\begin{array}{l}\text { It has been found that the addition of cow manure in soils contaminated with } \\
\text { heavy metals (HM) could reduce the mobility and availability of HM compared } \\
\text { to the control (Baker, White and Pierzynski, 2011). }\end{array}$ \\
\hline Soil acidification & $\begin{array}{l}\text { The soil pH soil is slightly modified by adding organic amendments such as cow } \\
\text { and chicken manure. Results showed an ambiguous response: slightly } \\
\text { acidification or alkalization of the soil. (Odlare, Pell and Svensson, 2008; } \\
\text { Franco-Otero et al., 2012; Francioli et al., 2016; Lemming et al., 2020; Poblete- } \\
\text { Grant et al., 2020; Wen et al., 2020). }\end{array}$ \\
\hline $\begin{array}{l}\text { Soil biodiversity } \\
\text { loss }\end{array}$ & $\begin{array}{l}\text { The addition of cow manure promotes fungal and bacterial diversity and } \\
\text { microbial community structure (Wen et al., 2010; Bastida et al., 2008; Ros et } \\
\text { al., 2008; Baker, White and Pierzynski, 2011; Chakraborty et al., 2011; Francioli } \\
\text { et al., 2016). }\end{array}$ \\
\hline Soil compaction & $\begin{array}{l}\text { The addition of chicken and cow manure for more than a year decreases the } \\
\text { compaction of the soil, which causes an increase in porosity and field capacity } \\
\text { (Annabi } \text { et al., 2011; Eden et al., 2011; Chang et al., 2014; Yadav et al., 2020). }\end{array}$ \\
\hline
\end{tabular}

\subsection{Increases in production (e.g. food/fuel/feed/timber/fibre)}

The addition of fresh or composted manure promotes the growth and yield of vegetables, grains, and forage and the positive response in yellow-poplar seedlings (Chang, Chung and Tsai, 2007; Reeve et al, 2012; Han et al, 2016; Jing et al., 2018). However, the addition of fresh manure should come with a caution because fast decomposition of fresh manure could produce soil warming and result in damage to plant roots.

\subsection{Mitigation of and adaptation to climate change}

Although $\mathrm{CO}_{2}$ emissions increased after manure addition on non-paddy soils, no increase in $\mathrm{CH}_{4}$ and/or $\mathrm{NOx}$ was observed. The addition of manure improves the physical conditions and availability of organic carbon support microbial processes that regulate nitrification and methanogenesis (Thangarajan et al., 2013; Xia et al., 2017).

\subsection{Socio-economic benefits}

The adequate use, as related to quantity and frequency, of livestock manure in agroecosystems, can improve food production, reduce $\mathrm{N}$ losses, and increases SOC storage together with a better final disposition of farm 
wastes (Xia et al., 2017). The better results are observed in SOC-poor soils, while in the SOC-rich soils, results may not be evident and, in some cases, maybe adverse by limiting access to other nutrients (Xia et al., 2017). Recycling of animal wastes is a component of circular economy, which can be combined with anaerobic digestion and biogas production (Yazan et al., 2018).

\section{Potential drawbacks to the practice}

\subsection{Tradeoffs with other threats to soil functions}

Table 42. Soil threats

\begin{tabular}{|l|l|}
\hline Soil threats & Nutrient imbalance and \\
cycles & $\begin{array}{l}\text { The excess in the manure addition may generate nutrient imbalance and risk } \\
\text { of toxicity for nutrients excess (Xia et al., 2017). }\end{array}$ \\
\hline $\begin{array}{l}\text { Soil salinization and } \\
\text { alkalinization }\end{array}$ & $\begin{array}{l}\text { Some manure types, such as poultry or pig manure, may cause an excess of } \\
\text { soluble salts and sodium (Lemming et al., 202O). }\end{array}$ \\
\hline $\begin{array}{l}\text { Soil } \\
\text { contamination/pollution }\end{array}$ & $\begin{array}{l}\text { Manures may contain heavy metals, and their long-term application may } \\
\text { result in accumulation which could be a potential threat to human health (Xia } \\
\text { et al., 2017). }\end{array}$ \\
\hline Soil water management & $\begin{array}{l}\text { The excess of manure, especially the uncontrolled application of liquid pig } \\
\text { manure (pig slurry) or in general of liquid manures with low C/N ratios can be } \\
\text { the cause of N pollution in groundwaters, may promote the runoff and } \\
\text { lixiviation of nutrients and in general encourage eutrophication of aquifers } \\
\text { and water bodies (Thangarajan et al., 2O13; Xia et al, 2017) }\end{array}$ \\
\hline
\end{tabular}

\subsection{Increases in greenhouse gas emissions}

Most GHGs are not directly emitted from soils; the principal emission of $\mathrm{CH}_{4}$ and $\mathrm{NOx}$ occurs along in the livestock production (e.g. breeding, fattening, and milking) and during manure storage under anaerobic conditions (Thangarajan et al., 2013; Xia et al., 2017). 


\subsection{Other conflicts}

The manure could contain pharmaceuticals because of the therapies applied to livestock. Therefore, the application of manure could increase the pharmaceutical residues and the diversity and abundance of microbes with antibiotic-resistance genes, which represent a risk to wildlife, livestock, and human health (Thangarajan et al., 2013; Xia et al., 2017).

\section{Recommendations before implementing the practice}

Preliminary characterization of the manure to determine the concentration and availability of nutrients can be more beneficial before the application according to the time of cultivation. This can reduce the risk of nutrient and $\mathrm{C}$ losses to the environment.

\section{Potential barriers for adoption}

Table 43. Potential barriers to adoption

\begin{tabular}{|c|c|l|}
\hline Barrier & YES/NO & \\
\hline Economic & Yes & $\begin{array}{l}\text { Livestock and agricultural production systems do not always coincide } \\
\text { in time and space, so manure may not be accessible where and when } \\
\text { needed. }\end{array}$ \\
\hline Institutional & Yes & $\begin{array}{l}\text { Lack of communication between soil scientists, society and } \\
\text { government institutions (Keesstra et al., 2016) }\end{array}$ \\
\hline Legal & Yes & $\begin{array}{l}\text { Some legislations in some countries restrict the application of some } \\
\text { types of manures, as pig slurries, in order to avoid groundwater } \\
\text { pollution. }\end{array}$ \\
\hline Knowledge & Yes & $\begin{array}{l}\text { The determination of the maximum amount that can be applied, for } \\
\text { a given type of soil and manure without harming the environment is } \\
\text { needed. }\end{array}$ \\
\hline
\end{tabular}




\section{Photos of the practice}

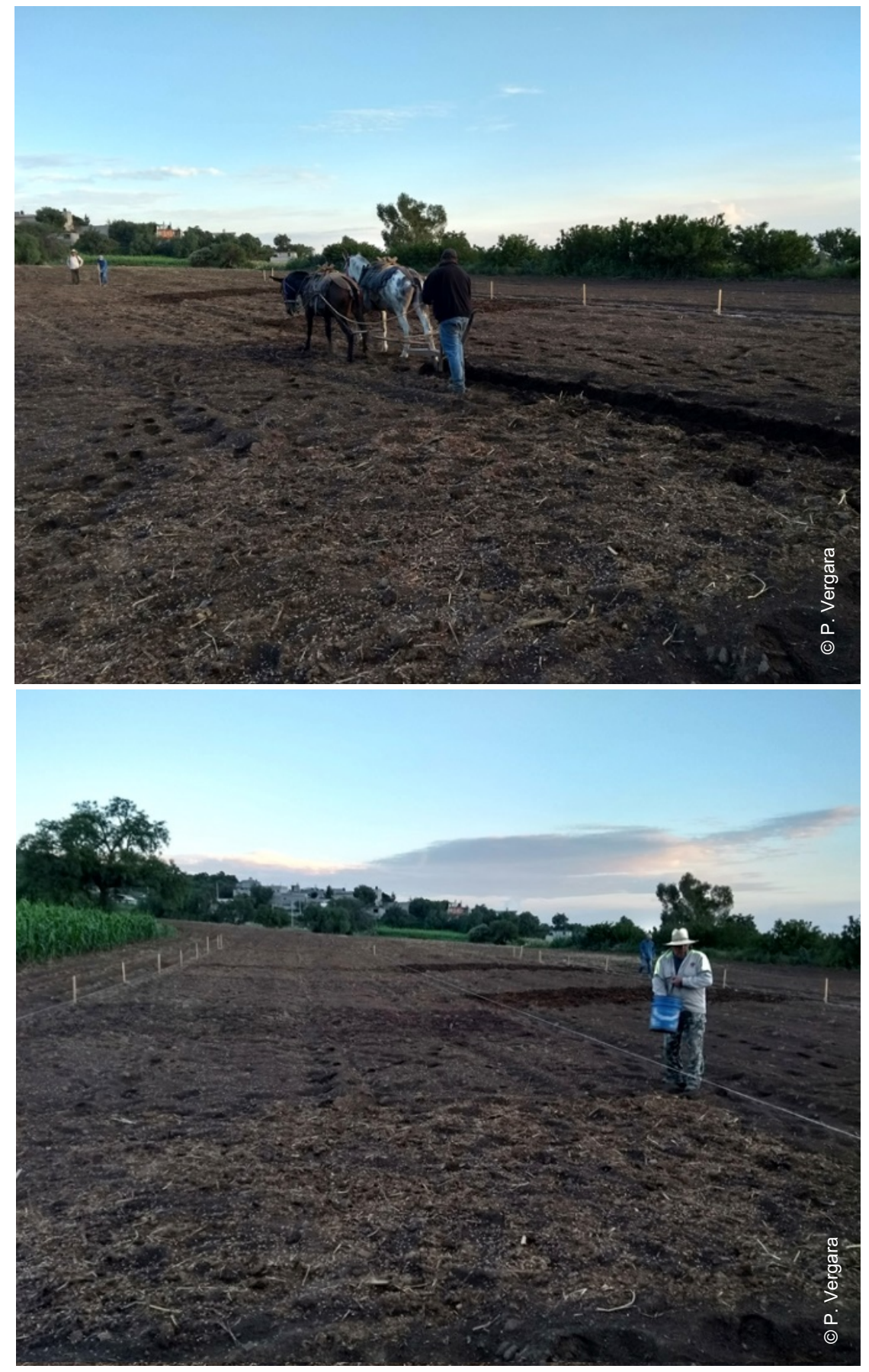

Photo 11. Traditional periurban maize crop system based on cattle manure addition and animal tillage (Mexico city - Mexico).

This management is endangered by the preference of producers for chemical fertilization and inaccessibility of cattle manure. ChávezVergara et al., in preparation 
Table 44. Related cases studies available in volumes 3 and 5

\begin{tabular}{|c|c|c|c|c|}
\hline Title & Region & $\begin{array}{l}\text { Duratio } \\
\mathrm{n} \text { of } \\
\text { study } \\
\text { (Years) }\end{array}$ & $\begin{array}{l}\text { Volum } \\
\text { e }\end{array}$ & $\begin{array}{l}\text { Case- } \\
\text { study } \\
\text { No. }\end{array}$ \\
\hline $\begin{array}{l}\text { Agricultural practices for the restoration of } \\
\text { Soil Ecological Functions in Madagascar }\end{array}$ & Africa & 2 & 3 & 2 \\
\hline $\begin{array}{l}\text { Long-term experiment of manure treatments } \\
\text { on a sandy soil, Germany }\end{array}$ & Europe & 29 & 3 & 12 \\
\hline $\begin{array}{l}\text { Pickle Melon (Cucumis melo) production in } \\
\text { Karapınar, Central Turkey }\end{array}$ & Eurasia & 60 & 3 & 24 \\
\hline $\begin{array}{l}\text { Organo-mineral fertilization on a Ukrainian } \\
\text { black soil }\end{array}$ & Europe & 5 & 3 & 26 \\
\hline $\begin{array}{l}\text { Cover crops, organic amendments and } \\
\text { combined management practices in } \\
\text { Mediterranean woody crops }\end{array}$ & $\begin{array}{l}\text { Europe, } \\
\text { NENA, } \\
\text { Eurasia, } \\
\text { North } \\
\text { America }\end{array}$ & $<30$ & 3 & 28 \\
\hline $\begin{array}{l}\text { Application of swine and cattle manure } \\
\text { through injection and broadcast systems in a } \\
\text { black soil of the Pampas, Argentina }\end{array}$ & $\begin{array}{l}\text { Latin } \\
\text { America } \\
\text { and the } \\
\text { Caribbean }\end{array}$ & 1 & 3 & 30 \\
\hline $\begin{array}{l}\text { Rehabilitation of hardened neo-volcanic soils } \\
\text { in Mexico }\end{array}$ & $\begin{array}{l}\text { Latin } \\
\text { America } \\
\text { and the } \\
\text { Caribbean }\end{array}$ & $\begin{array}{l}10,50 \\
\text { and } 60\end{array}$ & 3 & 38 \\
\hline $\begin{array}{l}\text { Biochar as a Soil Amendment for Carbon } \\
\text { Sequestration in Canada }\end{array}$ & $\begin{array}{l}\text { North } \\
\text { America }\end{array}$ & 1 and 3 & 3 & 41 \\
\hline $\begin{array}{l}\text { Response of soil carbon to various } \\
\text { combinations of management practices } \\
\text { (annual-perennial rotation system, animal } \\
\text { manure application, reduced tillage) in } \\
\text { Quebec, Canada }\end{array}$ & $\begin{array}{l}\text { North } \\
\text { America }\end{array}$ & 21 & 3 & 43 \\
\hline $\begin{array}{l}\text { Long term fertilization in a subtropical } \\
\text { floodplain soil in Bangladesh }\end{array}$ & Asia & 42 & 5 & 13 \\
\hline
\end{tabular}




\section{References}

Annabi, M., Le Bissonnais, Y., Le Villio-Poitrenaud, M. \& Houot, S. 2011. Improvement of soil aggregate stability by repeated applications of organic amendments to a cultivated silty loam soil. Agriculture, Ecosystems \& Environment, 144(1): 382-389. https://doi.org/10.1016/j.agee.2011.07.005

Acosta-Martinez, V., Mikha, M.M., Sistani, K.R., Stahlman, P.W., Benjamin, J.G., Vigil, M.F. \& Erickson, R. 2011. Multi-Location Study of Soil Enzyme Activities as Affected by Types and Rates of Manure Application and Tillage Practices. Agriculture, 1(1): 4-21. https://doi.org/10.3390/agriculture1010004

Ayaga, G., Todd, A. \& Brookes, P.C. 2006. Enhanced biological cycling of phosphorus increases its availability to crops in low-input sub-Saharan farming systems. Soil Biology and Biochemistry, 38(1): 81-90. https://doi.org/10.1016/j.soilbio.2005.04.019

Bai, L., Deng, Y., Li, J., Ji, M. \& Ruan, W. 2020. Role of the proportion of cattle manure and biogas residue on the degradation of lignocellulose and humification during composting. Bioresource Technology, 307: 122941. https://doi.org/10.1016/j.biortech.2020.122941

Baker, L.R., White, P.M. \& Pierzynski, G.M. 2011. Changes in microbial properties after manure, lime, and bentonite application to a heavy metal-contaminated mine waste. Applied Soil Ecology, 48(1): 1-10. https://doi.org/10.1016/j.apsoil.2011.02.007

Bastida, F., Kandeler, E., Moreno, J.L., Ros, M., García, C. \& Hernández, T. 2008. Application of fresh and composted organic wastes modifies structure, size and activity of soil microbial community under semiarid climate. Applied Soil Ecology, 40(2): 318-329. https://doi.org/10.1016/j.apsoil.2008.05.007

Chakraborty, A., Chakrabarti, K., Chakraborty, A. \& Ghosh, S. 2011. Effect of long-term fertilizers and manure application on microbial biomass and microbial activity of a tropical agricultural soil. Biology and Fertility of Soils, 47(2): 227-233. https://doi.org/10.1007/s00374-010-0509-1

Chang, E.-H., Chung, R.-S. \& Tsai, Y.-H. 2007. Effect of different application rates of organic fertilizer on soil enzyme activity and microbial population. Soil Science \& Plant Nutrition, 53(2): 132-140.

https://doi.org/10.1111/j.174.7-0765.2007.00122.x

Chang, E.-H., Wang, C.-H., Chen, C.-L. \& Chung, R.-S. 2014. Effects of long-term treatments of different organic fertilizers complemented with chemical $\mathrm{N}$ fertilizer on the chemical and biological properties of soils. Soil Science and Plant Nutrition, 60(4): 499-511.

https://doi.org/10.1080/00380768.2014.917333

Chen, S., Zhang, X., Shao, L., Sun, H., Niu, J. \& Liu, X. 2020. Effects of straw and manure management on soil and crop performance in North China Plain. Catena, 187: 104359.

https://doi.org/10.1016/j.catena.2019.104359

Du, Y., Cui, B., Zhang, Q., Wang, Z., Sun, J. \& Niu, W. 2020. Effects of manure fertilizer on crop yield and soil properties in China: A meta-analysis. Catena, 193: 104617.

https://doi.org/10.1016/j.catena.2020.104617 
Ding, Z., Kheir, A.M.S., Ali, M.G.M., Ali, O.A.M., Abdelaal, A.I.N., Lin, X., Zhou, Z., Wang, B., Liu, B. \& He, Z. 2020. The integrated effect of salinity, organic amendments, phosphorus fertilizers, and deficit irrigation on soil properties, phosphorus fractionation and wheat productivity. Scientific Reports, 10(1): 2736. https://doi.org/10.1038/s41598-020-59650-8

Eden, M., Schjønning, P., Moldrup, P. \& Jonge, L.W.D. 2011. Compaction and rotovation effects on soil pore characteristics of a loamy sand soil with contrasting organic matter content. Soil Use and Management, 27(3): 340-349. https://doi.org/10.1111/j.1475-2743.2011.00344.x

Evanylo, G., Sherony, C., Spargo, J., Starner, D., Brosius, M. \& Haering, K. 2008. Soil and water environmental effects of fertilizer-, manure-, and compost-based fertility practices in an organic vegetable cropping system. Agriculture, Ecosystems \& Environment, 127(1): 50-58.

https://doi.org/10.1016/j.agee.2008.02.014

Franco-Otero, V.G., Soler-Rovira, P., Hernández, D., López-de-Sá, E.G. \& Plaza, C. 2012. Short-term effects of organic municipal wastes on wheat yield, microbial biomass, microbial activity, and chemical properties of soil. Biology and Fertility of Soils, 48(2): 205-216. https://doi.org/10.1007/s00374-011$0620-\mathrm{y}$

Francioli, D., Schulz, E., Lentendu, G., Wubet, T., Buscot, F. \& Reitz, T. 2016. Mineral vs. Organic Amendments: Microbial Community Structure, Activity and Abundance of Agriculturally Relevant Microbes Are Driven by Long-Term Fertilization Strategies. Frontiers in Microbiology, 7.

https://doi.org/10.3389/fmicb.2016.01446

Goldberg, N., Nachshon, U., Argaman, E. \& Ben-Hur, M. 2020. Short Term Effects of Livestock Manures on Soil Structure Stability, Runoff and Soil Erosion in Semi-Arid Soils under Simulated Rainfall. Geosciences, 10(6): 213. https://doi.org/10.3390/geosciences10060213

Guppy, C.N., Menzies, N.W., Moody, P.W. \& Blamey, F.P.C. 2005. Competitive sorption reactions between phosphorus and organic matter in soil: a review. Soil Research, 43(2): 189-202.

https://doi.org/10.1071/SR04049

Han, S.H., An, J.Y., Hwang, J., Kim, S.B. \& Park, B.B. 2016. The effects of organic manure and chemical fertilizer on the growth and nutrient concentrations of yellow poplar (Liriodendron tulipifera Lin.) in a nursery system. Forest Science and Technology, 12(3): 137-143.

https://doi.org/10.1080/21580103.2015.1135827

Hargreaves, J.C., Adl, M.S. \& Warman, P.R. 2008. A review of the use of composted municipal solid waste in agriculture. Agriculture, Ecosystems \& Environment, 123(1): 1-14.

https://doi.org/10.1016/j.agee.2007.07.004

Jing, J., Christensen, J.T., Sørensen, P., Christensen, B.T. \& Rubak, G.H. 2019. Long-term effects of animal manure and mineral fertilizers on phosphorus availability and silage maize growth. Soil Use and Management, 35(2): 323-333. https://doi.org/10.1111/sum.12477

Krauss, M., Berner, A., Perrochet, F., Frei, R., Niggli, U. \& Mäder, P. 2020. Enhanced soil quality with reduced tillage and solid manures in organic farming-a synthesis of 15 years. Scientific Reports, 10(1): 1-12. https://doi.org/10.1038/s41598-020-61320-8 
Keesstra, S.D., Bouma, J., Wallinga, J., Tittonell, P., Smith, P., Cerdà, A., Montanarella, L., Quinton, J.N., Pachepsky, Y., van der Putten, W.H., Bardgett, R.D., Moolenaar, S., Mol, G., Jansen, B. \& Fresco, L.O. 2016. The significance of soils and soil science towards realization of the United Nations Sustainable Development Goals. SOIL, 2(2): 111-128. https://doi.org/10.5194/soil-2-111-2016

Kuepper, G. 2003. Manures for Organic Crop Production: soil systems guide. NCAT/ATTRA Publication. (also available at:

http://citeseerx.ist.psu.edu/viewdoc/download?doi=10.1.1.474.205\&rep=rep1\&type=pdf)

Lemming, C., Oberson, A., Magid, J., Bruun, S., Scheutz, C., Frossard, E. \& Jensen, L.S. 2019. Residual phosphorus availability after long-term soil application of organic waste. Agriculture, Ecosystems \& Environment, 270-271: 65-75. https://doi.org/10.1016/j.agee.2018.10.009

Li-Xian, Y., Guo-Liang, L., Shi-Hua, T., Gavin, S. \&Zhao-Huan, H. 2007. Salinity of animal manure and potential risk of secondary soil salinization through successive manure application. Science of The Total Environment, 383(1): 106-114. https://doi.org/10.1016/j.scitotenv.2007.05.027

Liu, S., Wang, J., Pu, S., Blagodatskaya, E., Kuzyakov, Y., Razavi, B. 2020. Impact of manure on soil biochemical properties: A global synthesis. Science of the Total Environment, 745: 141003.

https://doi.org/10.1016/j.scitotenv.2020.141003

Ma, Q., Wen, Y., Wang, D., Sun, X., Hill, P.W., Macdonald, A., Chadwick, D.R., Wu, L. \& Jones, D.L. 2020. Farmyard manure applications stimulate soil carbon and nitrogen cycling by boosting microbial biomass rather than changing its community composition. Soil Biology and Biochemistry, 144: 107760. https://doi.org/10.1016/j.soilbio.2020.107760

Maillard, É. \& Angers, D.A. 2014. Animal manure application and soil organic carbon stocks: a metaanalysis. Global Change Biology, 20(2): 666-679. https://doi.org/10.1111/gcb.12438

Medina, E., Paredes, C., Bustamante, M.A., Moral, R. \& Moreno-Caselles, J. 2012. Relationships between soil physico-chemical, chemical and biological properties in a soil amended with spent mushroom substrate. Geoderma, 173-174: 152-161. https://doi.org/10.1016/j.geoderma.2011.12.011

Odlare, M., Pell, M. \& Svensson, K. 2008. Changes in soil chemical and microbiological properties during 4 years of application of various organic residues. Waste Management, 28(7): 1246-1253.

https://doi.org/10.1016/j.wasman.2007.06.005

Poblete-Grant, P., Suazo-Hernández, J., Condron, L., Rumpel, C., Demanet, R., Malone, S.L. \&

Mora, M. de L.L. 2020. Soil available P, soil organic carbon and aggregation as affected by long-term poultry manure application to Andisols under pastures in Southern Chile. Geoderma Regional, 21: e00271.

https://doi.org/10.1016/j.geodrs.2020.e00271

Rasouli-Sadaghiani, M.H. \& Moradi, N. 2014. Effect of poultry, cattle, sheep manures and sewage sludge on N mineralisation. Chemistry and Ecology, 30(7): 666-675.

https://doi.org/10.1080/02757540.2014.889122

Reeve, J.R., Endelman, J.B., Miller, B.E. \& Hole, D.J. 2012. Residual Effects of Compost on Soil Quality and Dryland Wheat Yield Sixteen Years after Compost Application. Soil Science Society of America Journal, 76(1): 278-285. https://doi.org/10.2136/sssaj2011.0123 
Ros, M., Pascual, J.A., Garcia, C., Hernandez, M.T. \& Insam, H. 2006. Hydrolase activities, microbial biomass and bacterial community in a soil after long-term amendment with different composts. Soil Biology and Biochemistry, 38(12): 3443-3452. https://doi.org/10.1016/j.soilbio.2006.05.017

Shehzadi, S., Shah, Z. \& Mohammad, W. 2017. Impact of organic amendments on soil carbon sequestration, water use efficiency and yield of irrigated wheat. BASE, 21(1) : 36-49.

https://doi.org/10.25518/1780-4507.13435

Thangarajan, R., Bolan, N.S., Tian, G., Naidu, R. \& Kunhikrishnan, A. 2013. Role of organic amendment application on greenhouse gas emission from soil. Science of The Total Environment, 465: 7296. https://doi.org/10.1016/j.scitotenv.2013.01.031

Wang, K., Chu, C., Li, X., Wang, W. \& Ren, N. 2018. Succession of bacterial community function in cow manure composing. Bioresource Technology, 267: 63-70.

https://doi.org/10.1016/j.biortech.2018.06.028

Wen, Y.-C., Li, H.-Y., Lin, Z.-A., Zhao, B.-Q., Sun, Z.-B., Yuan, L., Xu, J.-K. \& Li, Y.-Q. 2020. Longterm fertilization alters soil properties and fungal community composition in fluvo-aquic soil of the North China Plain. Scientific Reports, 10(1): 7198. https://doi.org/10.1038/s41598-020-64227-6

Xia, L., Lam, S.K., Yan, X. \& Chen, D. 2017. How Does Recycling of Livestock Manure in Agroecosystems Affect Crop Productivity, Reactive Nitrogen Losses, and Soil Carbon Balance? Environmental Science \& Technology, 51(13): 7450-7457. https://doi.org/10.1021/acs.est.6b06470

Yadav, S.S., Guzman, J.G., Meena, R.S., Lal, R. \& Yadav, G.S. 2020. Long term crop management effects on soil organic carbon, structure, and water retention in a cropland soil in central Ohio, USA. Journal of Plant Nutrition and Soil Science, 183(2): 200-207. https://doi.org/10.1002/jpln.201900430

Yazan, D.M., Cafagna, D., Fraccascia, L., Mes, M., Pontrandolfo, P. \& Zijm, H. 2018. Economic sustainability of biogas production from animal manure: a regional circular economy model. Management Research Review, 41(5): 605-624. https://doi.org/10.1108/MRR-02-2018-0053

Zhang, X., Fang, Q., Zhang, T., Ma, W., Velthof, G.L., Hou, Y., Oenema, O. \& Zhang, F. 2020. Benefits and trade-offs of replacing synthetic fertilizers by animal manures in crop production in China: A meta-analysis. Global Change Biology, 26(2): 888-900. https://doi.org/10.1111/gcb.14826 


\title{
11. Digestate application
}

\author{
Rima Porre, René Rietra, Jan Peter Lesschen
}

Wageningen Environmental Research, The Netherlands

\section{Description of the practice}

Digestate and biogas are the main products of the process called anaerobic digestion (Möller, 2015). Anaerobic digestion occurs in an oxygen free environment where specific bacteria degrade organic materials and produce biogas, primarily consisting of $\mathrm{CO}_{2}, \mathrm{CH}_{4}$ as well as the by-product digestate (also referred to as bioslurry) which is used as a fertilizer (Möller, 2015). The biogas is used as a renewable source of energy and development has been stimulated by governments and various organizations (Scarlat, Dallemand and Fahl, 2018; Surendra et al., 2014; Vasco-Correa et al., 2018).

A digester can be fed with one or with many different organic materials: animal manure, crops, waste products, and sewage sludge resulting in different digestates. The use of crops, animal slurry and biowaste for digestion is considered a sustainable practice in contrast to fossil energy. However, the use of crops for biogas production, such as maize in Germany, potentially creates competition with crops for food consumption (Herrmann, 2013). The biogas production from various organic materials varies strongly: the energy from $1 \mathrm{~m}^{3}$ manure is much lower than $1 \mathrm{~m}^{3}$ oil-rich waste (Braguglia et al., 2018). In smallholders farms, especially in Asia, it is considered as a technique to provide a cheap and clean source of energy, and also to be less dependent on wood or fossil fuel (Quinn et al. 2018). Digestates retain most of the original nutrients (NPK) contained in the input materials while also increasing the mineral (plant-available) fraction of these nutrients (Insam, Gómez-Brandón and Ascher, 2015) Digestates have a high water content (90-95 percent) and in order to facilitate transport are often separated in a liquid and a solid fraction (Valentinuzzi et al., 2020); The liquid fraction typically contains a significant amount of plant available nitrogen, phosphorus and potassium, and can be used as a nitrogen fertiliser. It is, however, low in carbon. The solid fraction has a high dry matter content, is rich in phosphorus and organic carbon, and has the potential to be used as a $\mathrm{P}$ fertiliser and can stimulate soil organic matter buildup (Egene et al., 2020; Valentinuzzi et al., 2020). 


\section{Range of applicability}

There is no clear data on the amount of digestate that is produced in each country, whereas the amount of biogas produced is well known (although part of this biogas can also originate from landfills). In 201850 percent of the global biogas production was in the EU (Scarlat, Dallemand and Fahl, 2018), 32 percent in Asia and 17 percent in the America's (WBA, 2020). The production of biogas grew from 12.4 to 59.3 billion $\mathrm{m}^{3}$ between 2000 and 2018. Germany is the largest producer of biogas in the EU and the average input in 2010 in biogas plant on the basis of fresh matter was 46 percent crops, 45 percent animal slurry, and 7 percent biowaste (Herrmann, 2013). Small-scale digesters are most numerous in Asia (Chen et al., 2012; Mittal, Ahlgren and Shukla, 2018), but have recently also been introduced in various African countries (Roopnarain and Adeleke, 2017).

In many cases digestate cannot be directly used as a fertilizer and needs to be stored. Similar to manure $\mathrm{N}_{2} \mathrm{O}$, $\mathrm{H}_{2} \mathrm{~S}, \mathrm{NH}_{3}, \mathrm{CH}_{4}$ gases can be released in open systems although losses can be higher compared to untreated animal slurry and therefore digestate is often stored in closed systems (Vasco-Correa et al., 2018). As a fertilizer digestate is often compared to animal slurry and can thus be used in a similar way. The question does still remain if different input materials for digestate production will result in very different fertilizer potential (Coelho et al., 2020b).

\section{Impact on soil organic carbon stocks}

There is general consensus that more than 10 years of modest applications of fertilizers are necessary to achieve an effect on soil organic matter in typical arable soils (Smith, 2004; Thomas et al., 2019). Due to the relative recent development of anaerobic digestion long term data ( $>10$ years), similar to synthetic fertilizer and animal manure (Körschens et al., 2014), are not available for digestate. Only a few field studies have determined SOC after at least three years after addition of digestate (Table 45). These studies show that it is possible, in some cases, to increase SOC using digestate.

It is however not possible to make accurate calculations about the amount of $\mathrm{C}$ which is mineralized to $\mathrm{CO}_{2}$ and the amount of $\mathrm{C}$ that results in the increase in soil C. Long term experiments ( $>100$ years) using farmyard manure in comparison to synthetic fertilizers show an increase of soil carbon (SOC) during the first 20 years yet no further increase in the time after that. This means that there is no further SOC sequestration after approximately 20 years (Körschens et al, 2014). A higher SOC content due to the addition of exogenous $\mathrm{C}$ is therefore not equal to $\mathrm{C}$ sequestration. The impact of anaerobic digestion on SOC sequestration depends on the reference situation or system boundaries and is a typical question for life cycle analysis (LCA). Part of such LCA is the effect of digestate on SOC on the long term in comparison to other treatments. There is a concern that digestate from animal manure will result in less SOC than animal manure (Insam, Gómez-Brandón and Ascher, 2015). This concern arises due to the $\mathrm{CO}_{2}$ loss during the anaerobic digestion, which results in a digestate low in organic C. A Laboratory experiment determined the amount of $\mathrm{C}$ that remained in soil when using untreated feed, feed after anaerobically digestion as well as animal manure. In all cases 12-14 percent of $\mathrm{C}$ from the original feed was present in the soil after 240 days (Thomsen et al., 2013). Another study with maize (digestate) similarly showed positive $\mathrm{C}$ sequestration effects after application of the digestate (Béghin-Tanneau et al., 2019). It can be concluded from the laboratory experiments that the $\mathrm{CO}_{2}$ loss during digestion does not 
have to result in less SOC due to preservation of the recalcitrant $\mathrm{C}$ during digestion, and even can results in more SOC.

Table 45. Effect of digestate in comparison to synthetic fertilizers on SOC in experiments that lasted at least 2 years

\begin{tabular}{|c|c|c|c|c|c|c|c|c|}
\hline Location & $\begin{array}{l}\text { Soil } \\
\text { type }\end{array}$ & $\begin{array}{l}\text { C } \\
\text { added } \\
\text { t C/ha }\end{array}$ & $\begin{array}{l}\text { Baseline } \\
\text { (To) soil } \\
\mathrm{C} \\
\mathrm{g} \mathrm{C} / \mathrm{kg}\end{array}$ & $\begin{array}{l}\text { Soil C } \\
\text { treatment } \\
\mathrm{g} \mathrm{C/kg}\end{array}$ & $\begin{array}{l}\text { Duration } \\
\text { (Years) }\end{array}$ & $\begin{array}{l}\text { Depth } \\
(\mathrm{cm})\end{array}$ & $\begin{array}{l}\text { More } \\
\text { information }\end{array}$ & Reference \\
\hline \multirow{2}{*}{ Italy } & NA & $\begin{array}{l}1.9 \\
1.6\end{array}$ & 14.76 & $\begin{array}{l}17.02 \\
14.99\end{array}$ & 3 & 40 & \multirow{2}{*}{$\begin{array}{l}\text { WFF'1 } \\
\text { WWW }^{1}\end{array}$} & \multirow{2}{*}{$\begin{array}{l}\text { Montemurro } \\
\text { et al. (2010) }\end{array}$} \\
\hline & & $\begin{array}{l}1.2 \\
1.0\end{array}$ & 14.12 & $\begin{array}{l}10.59 \\
13.35\end{array}$ & & & & \\
\hline England & $\begin{array}{l}\text { Flinty } \\
\text { clay } \\
\text { loam }\end{array}$ & 3.5 & 15 & 19 & 5 & 30 & $A D^{1}$ & $\begin{array}{l}\text { Thomas et } \\
\text { al. (2019) }\end{array}$ \\
\hline Germany & $\begin{array}{l}\text { Loamy } \\
\text { sand }\end{array}$ & $\begin{array}{c}9.9 \\
9 \\
9 \\
7.2\end{array}$ & 0.95 & $\begin{array}{c}1.2 \\
1.05 \\
1.1 \\
1.1\end{array}$ & 3 & 20 & $\begin{array}{l}\text { Agglomerate } \\
\text { Pellets } \\
\text { Fine fraction } \\
\text { Course fract. }\end{array}$ & $\begin{array}{l}\text { Roß et al. } \\
2018^{2}\end{array}$ \\
\hline $\begin{array}{l}\text { Czech } \\
\text { Republic }\end{array}$ & $\begin{array}{l}\text { Orthic } \\
\text { Luvisol } \\
\text { - Clay } \\
\text { loam }\end{array}$ & $\begin{array}{c}1.17 \mathrm{t} \\
\text { ha }^{-1} \\
\text { Dry } \\
\text { matter. } \\
4\end{array}$ & 15.6 & 15.6 & 4 & 30 & $\begin{array}{l}\text { Digestate } \\
\text { input: cattle } \\
\text { slurry, maize } \\
\text { silage, haylage }\end{array}$ & $\begin{array}{l}\text { Barłóg, } \\
\text { Hlisnikovský } \\
\text { and } \\
\text { Kunzová } \\
\text { (2020) }\end{array}$ \\
\hline
\end{tabular}

${ }^{1}$ digestate from frozen food processing (WFF) and wine production waste (WWW), vegetable waste (AD). ${ }^{2}$ various dried products from digestate. ${ }^{3}$ cumulative addition in years. ${ }^{4}$ The $\mathrm{C}$ content of the digestate was not mentioned

\section{Other benefits of the practice}

\subsection{Improvement of soil properties}

Results of meta-analysis of long-term studies worldwide shows that the application of organic amendments in comparison to chemical fertilizers, only results in additional crop yield in specific soils: low fertility, sand structure, near neutral $\mathrm{pH}$, under tropical climate (Chen et al., 2018). The application of organic amendments, such as animal manure and also digestates, provides advantages if applied carefully and at application rates to maximize the nutrient use efficiency and minimize unwanted effects on environment (Chen et al., 2018).

The liquid fraction of digested has a high nutrient use efficiency for $\mathrm{N}$ giving it the potential to substitute synthetic N fertilizer (Sigurnjak et al, 2017). In comparison to synthetic fertilizers or unfertilized controls, digestate resulted in more microbial activity (Bachmann, Gropp and Eichler-Löbermann, 2014; Möller, 2015), yet microbial activity was lower compared to after manure application. Further studies have shown more 
earthworms (Frøseth et al., 2014; Koblenz et al., 2015) and a higher soil aggregate stability after digestate application (Frøseth et al., 2014).

\subsection{Minimization of threats to soil functions}

Table 46. Soil threats

\section{Soil threats}

\begin{tabular}{|l|l|} 
Soil erosion & $\begin{array}{l}\text { Digestate application to soil has been shown increase aggregate stability } \\
\text { (Frøseth et al., 2014) and might thus reduce wind erosion. }\end{array}$ \\
$\begin{array}{l}\text { Nutrient imbalance } \\
\text { and cycles }\end{array}$ & $\begin{array}{l}\text { The liquid fraction of digestate supplies large amounts of plant available } \\
\mathrm{N}, \mathrm{P} \text { and K (Valentinuzzi et al., 2020). }\end{array}$ \\
$\begin{array}{l}\text { Soil biodiversity } \\
\text { loss }\end{array}$ & $\begin{array}{l}\text { Few studies reported a positive, although short-lived, effect of digestate } \\
\text { on soil microbial diversity (Coelho et al., 202Oa; Johansen et al., 2013). }\end{array}$
\end{tabular}

\subsection{Increases in production (e.g. food/fuel/feed/timber/fibre)}

The addition of digestates, compared to other fertilizers such as cattle slurry or chemical fertilizers, performs similarly well or better in terms of crop yield (Coelho et al., 2020b; Nkoa, 2014).

\subsection{Mitigation of and adaptation to climate change}

The implementation of anaerobic digestion has the potential to reduce GHG emissions on a farm-scale level. Various factors can cause a lower emission of GHG due to the introduction of anaerobic digestion (Lijó et al., 2014). It however strongly varies depending on the types of organic materials used for digestion as well as on proper handling and storage of the digestate (Möller, 2015). Mono-digestion of animal manure results in lower GHG emissions (when considering the whole-farm budget) but offers only little energy (De Vries et al, 2012). The positive effect is related to the avoidance of $\mathrm{CH}_{4}$ emissions from stored manure. Digestate application to soil can result in GHG emissions, just like with other (organic) manures. Upon field spreading $\mathrm{NH}_{3}, \mathrm{CO}_{2}$ and $\mathrm{N}_{2} \mathrm{O}$ emissions can occur, yet it remains unclear how this compares to other organic manures (Baral et al, 2017; Buchen-Tschiskale, Hagemann and Augustin, 2020).

\subsection{Socio-economic benefits}

The energy from anaerobic digestion can be important as a clean and sustainable source of energy, and is therefore supported by various governments and organizations such as the CleanCookingAlliance. 


\subsection{Other benefits of the practice}

Anaerobic digestion can result in less pathogens in comparison to raw manure and result in less emission of odours (Insam, Gómez-Brandón and Ascher, 2015).

\section{Potential drawbacks to the practice}

\subsection{Tradeoffs with other threats to soil functions}

Table 47. Soil threats

\begin{tabular}{|c|c|}
\hline Soil threats & \\
\hline $\begin{array}{l}\text { Soil salinization and } \\
\text { alkalinization }\end{array}$ & $\begin{array}{l}\text { The salt concentration is sometimes high compared to animal manure } \\
\text { due to easily degradable additives that give more energy, and acid and } \\
\text { bases used in the processes. }\end{array}$ \\
\hline $\begin{array}{l}\text { Soil contamination } \\
\text { / pollution }\end{array}$ & $\begin{array}{l}\text { The energy from oil rich wastes is often high in comparison to animal } \\
\text { manure. A rather large amount of environmental rules and control by } \\
\text { government is necessary in the Netherlands to protect against the use } \\
\text { of energy-rich waste containing pollutants (Oenema, Velthof and } \\
\text { Commissie Deskundigen, 2015). Also in other countries there are various } \\
\text { types of control, for example a certification in Germany, in necessary for } \\
\text { soil protection. }\end{array}$ \\
\hline
\end{tabular}

\subsection{Increases in greenhouse gas emissions}

Leakage during biogas production or improper storage of digestate pose a risk for GHG emissions (Nkoa, 2014), these can be avoided by proper design of biogas plants and manure stores. Characteristic of digestate is that digestion of animal manure results in more $\mathrm{NH}_{4}$ and less organic $\mathrm{N}$ in comparison to animal manure (Möller, 2015). In combination with a higher $\mathrm{pH}$ of digestate this results in a fertilizer with more risk of gaseous loss of $\mathrm{N}$ in the form $\mathrm{NH}_{3}$ if not applied using low emission techniques. Effects of digestate application to $\mathrm{N}_{2} \mathrm{O}$ and $\mathrm{NH}_{3}$ emissions from soil remain unclear with studies reporting negligible effects on $\mathrm{N}_{2} \mathrm{O}$ emissions (Johansen et al, 2013; Möller, 2015) or increased $\mathrm{N}_{2} \mathrm{O}$ emissions (Buchen-Tschiskale, Hagemann and Augustin, 2020; Dietrich, Fongen and Foereid, 2020; Viaene et al., 2017). 


\section{Recommendations before implementing the practice}

In order to avoid ammonia loss through volatilization it is strongly recommended that digestates are applied on the field through injection. To date there have only been a limited number of studies that study the effect of digestate on soil quality and more importantly field studies are lacking. Considering the many different possible input materials that can be used to create biogas and digestates more studies are needed in order to quantify the effect of digestate application on soil.

\section{Potential barriers for adoption}

Table 48. Potential barriers to adoption

\begin{tabular}{|l|l|l|}
\hline Barrier & YES/NO & \\
\hline Biophysical & Yes & $\begin{array}{l}\text { The salt concentration is sometimes high compared to animal } \\
\text { manure due to easily degradable additives that give more energy, } \\
\text { and acid and bases used in the processes. }\end{array}$ \\
\hline Cultural & Yes & $\begin{array}{l}\text { Some farmers/citizens/political parties relate digestion to industrial } \\
\text { type of farming, which they object to, }\end{array}$ \\
\hline Knowledge & Yes & $\begin{array}{l}\text { Some farmers worry about the loss of carbon during digestion } \\
\text { process, and the net effect digestion has on soil. The net effect of } \\
\text { digesters on SOC depends strongly on the system that is considered, } \\
\text { and without this rather different conclusions can be drawn (Insam, } \\
\text { Gómez-Brandón and Ascher, 2015; Riding et al., 2015). }\end{array}$ \\
\hline
\end{tabular}




\section{Photo of the practice}

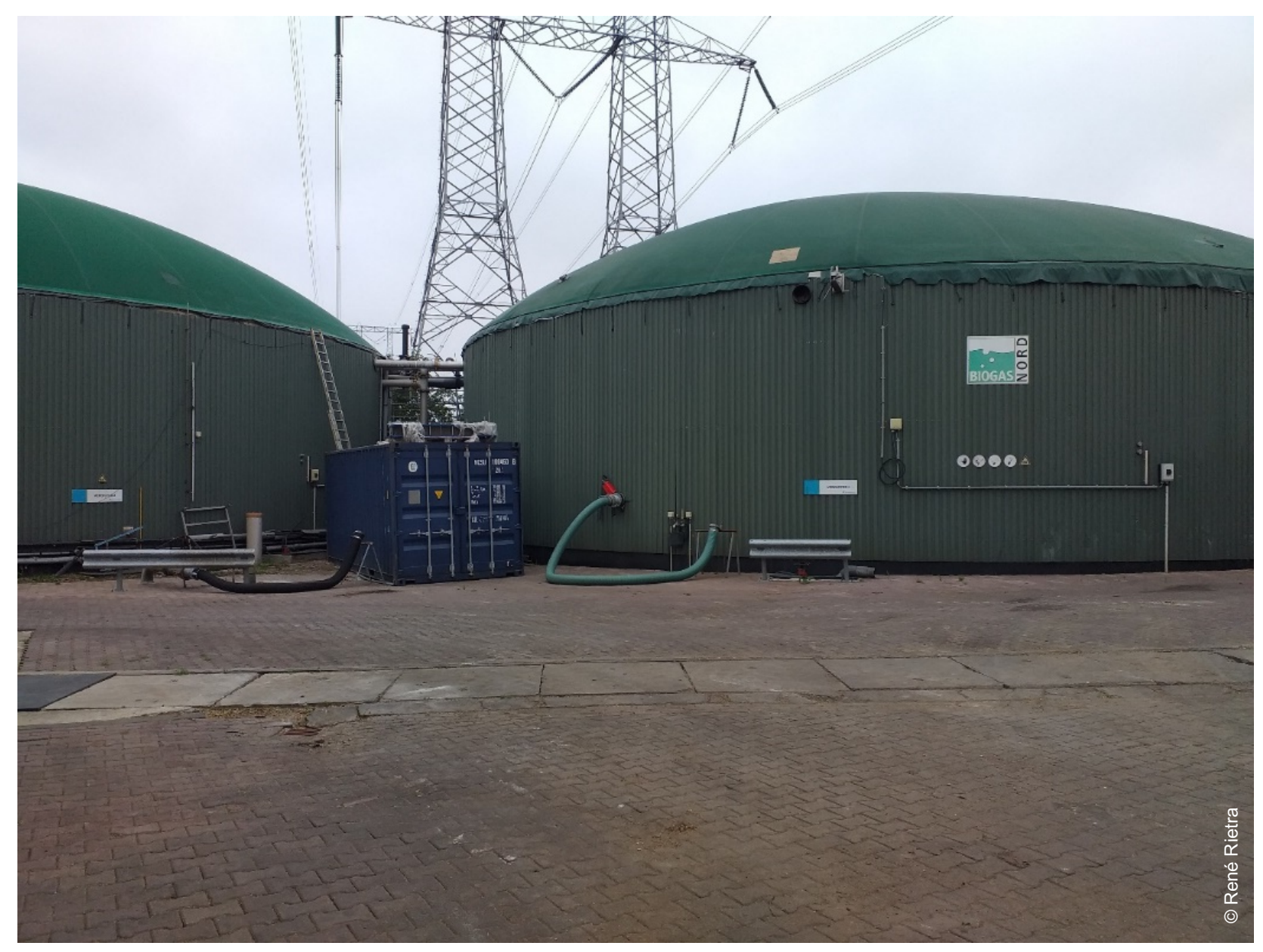

Photo 12. Digestion of food waste and animal slurry contributes to sustainable electricity.

Photo taken at digester, on 8 September 2020, Oirschot, The Netherlands 


\section{References}

Bachmann, S., Gropp, M. \& Eichler-Löbermann, B. 2014. Phosphorus availability and soil microbial activity in a 3 year field experiment amended with digested dairy slurry. Biomass and Bioenergy, 70: 429439. https://doi.org/10.1016/j.biombioe.2014.08.004

Baral, K.R., Labouriau, R., Olesen, J.E. \& Petersen, S.O. 2017. Nitrous oxide emissions and nitrogen use efficiency of manure and digestates applied to spring barley. Agriculture, Ecosystems \& Environment, 239: 188-198. https://doi.org/https://doi.org/10.1016/j.agee.2017.01.012

Barłóg, P., Hlisnikovský, L. \& Kunzová, E. 2020. Effect of digestate on soil organic carbon and plantavailable nutrient content compared to cattle slurry and mineral fertilization. Agronomy, 10(3). https://doi.org/10.3390/agronomy10030379

Béghin-Tanneau, R., Guérin, F., Guiresse, M., Kleiber, D. \& Scheiner, J.D. 2019. Carbon sequestration in soil amended with anaerobic digested matter. Soil and Tillage Research, 192: 87-94.

https://doi.org/10.1016/j.still.2019.04.024

Braguglia, C.M., Gallipoli, A., Gianico, A. \& Pagliaccia, P. 2018. Anaerobic bioconversion of food waste into energy: A critical review. Bioresource Technology, 248: 37-56.

https://doi.org/10.1016/j.biortech.2017.06.145

Buchen-Tschiskale, C., Hagemann, U. \& Augustin, J. 2020. Soil incubation study showed biogas digestate to cause higher and more variable short-term $\mathrm{N}_{2} \mathrm{O}$ and $\mathrm{N}_{2}$ fluxes than mineral-N. Journal of Plant Nutrition and SoilScience, 183(2): 208-219. https://doi.org/https://doi.org/10.1002/jpln.201900075

Chen, L., Zhao, L., Ren, C. \& Wang, F. 2012. The progress and prospects of rural biogas production in China. Energy Policy, 51: 58-63. https://doi.org/10.1016/j.enpol.2012.05.052

Chen, Y., Camps-Arbestain, M., Shen, Q., Singh, B. \& Cayuela, M.L. 2018. The long-term role of organic amendments in building soil nutrient fertility: a meta-analysis and review. Nutrient Cycling in Agroecosystems, 111(2-3): 103-125. https://doi.org/10.1007/s10705-017-9903-5

Coelho, J.J., Hennessy, A., Casey, I., Bragança, C.R.S., Woodcock, T. \& Kennedy, N. 2020a. Biofertilisation with anaerobic digestates: A field study of effects on soil microbial abundance and diversity. Applied Soil Ecology, 147. https://doi.org/10.1016/j.apsoil.2019.103403

Coelho, J.J., Hennessy, A., Casey, I., Woodcock, T. \& Kennedy, N. 2020b. Biofertilisation with Anaerobic Digestates: Effects on the Productive Traits of Ryegrass and Soil Nutrients. Journal of Soil Science and Plant Nutrition, 20(4): 1665-1678. https://doi.org/10.1007/s42729-020-00237-7

Dietrich, M., Fongen, M. \& Foereid, B. 2020. Greenhouse gas emissions from digestate in soil. International Journal of Recycling of Organic Waste in Agriculture, 9(1): 1-19. https://doi.org/10.30486/IJROWA.2020.1885341.1005

Egene, C.E., Sigurnjak, I., Regelink, I.C., Schoumans, O.F., Adani, F., Michels, E., Sleutel, S., Tack, F.M.G. \& Meers, E. 2020. Solid fraction of separated digestate as soil improver: implications for soil fertility and carbon sequestration. Journal of Soils and Sediments. https://doi.org/10.1007/s11368-020-02792-Z 
Frøseth, R.B., Bakken, A.K., Bleken, M.A., Riley, H., Pommeresche, R., Thorup-Kristensen, K. \& Hansen, S. 2014. Effects of green manure herbage management and its digestate from biogas production on barley yield, N recovery, soil structure and earthworm populations. European Journal of Agronomy, 52: 90102. https://doi.org/10.1016/j.eja.2013.10.006

Herrmann, A. 2013. Biogas Production from Maize: Current State, Challenges and Prospects. 2. Agronomic and Environmental Aspects. Bioenergy Research, 6(1): 372-387. https://doi.org/10.1007/s12155-012$9227-\mathrm{x}$

Insam, H., Gómez-Brandón, M. \& Ascher, J. 2015. Manure-based biogas fermentation residues - Friend or foe of soil fertility? Soil Biology and Biochemistry, 84: 1-14.

https://doi.org/10.1016/j.soilbio.2015.02.006

Johansen, A., Carter, M.S., Jensen, E.S., Hauggard-Nielsen, H. \& Ambus, P. 2013. Effects of digestate from anaerobically digested cattle slurry and plant materials on soil microbial community and emission of $\mathrm{CO}_{2}$ and $\mathrm{N}_{2} \mathrm{O}$. Applied Soil Ecology, 63: 36-44. https://doi.org/10.1016/j.apsoil.2012.09.003

Koblenz, B., Tischer, S., Rücknagel, J. \& Christen, O. 2015. Influence of biogas digestate on density, biomass and community composition of earthworms. Industrial Crops and Products, 66: 206-209.

https://doi.org/10.1016/j.indcrop.2014.12.024

Körschens, M., Albert, E., Baumecker, M., Ellmer, F., Grunert, M., Hoffmann, S., Kismanyoky, T., Kubat, J., Kunzova, E., Marx, M., Rogasik, J., Rinklebe, J., Rühlmann, J., Schilli, C., Schröter, H., Schroetter, S., Schweizer, K., Toth, Z., Zimmer, J. \& Zorn, W. 2014. Humus and climate change - results of 15 long-term experiments. Archives of Agronomy and Soil Science, 60(11): 1485-1517.

https://doi.org/10.1080/03650340.2014.892204

Lijó, L., González-Garciá, S., Bacenetti, J., Fiala, M., Feijoo, G., Lema, J.M., Moreira, M.T. 2014. Life Cycle Assessment of electricity production in Italy from anaerobic co-digestion of pig slurry and energy crops. Renewable Energy, 68: 625-635. https://doi.org/10.1016/j.renene.2014.03.005

Mittal, S., Ahlgren, E.O. \& Shukla, P.R. 2018. Barriers to biogas dissemination in India: A review. Energy Policy, 112: 361-370. https://doi.org/https://doi.org/10.1016/j.enpol.2017.10.027

Möller, K. 2015. Effects of anaerobic digestion on soil carbon and nitrogen turnover, N emissions, and soil biological activity. A review. Agronomy for Sustainable Development, 35(3): 1021-1041.

https://doi.org/10.1007/s13593-015-0284-3

Montemurro, F., Vitti, C., Diacono, M., Canali, S., Tittarelli, F. \& Ferri, D. 2010. A three-year field anaerobic digestates application: Effects on fodder crops performance and soil properties. Fresenius Environmental Bulletin, 19(9 B): 2087-2093. (also available at https://www.scopus.com/inward/record.uri?eid=2-s2.078649878844\&partnerID=40\&md5=b7e62b52c577bc8fa0764062d3760300).

Nkoa, R. 2014. Agricultural benefits and environmental risks of soil fertilization with anaerobic digestates: a review. Agronomy for Sustainable Development, 34(2): 473-492. https://doi.org/10.1007/s13593-0130196-Z 
Oenema, O., Velthof, G.L. \& Commissie Deskundigen, M. 2015. Nut en risico's van covergisting: syntheserapport. Wageningen, Wettelijke Onderzoekstaken Natuur \& Milieu. (also available at https://edepot.wur.nl/339376).

Quinn, A.K., Bruce, N., Puzzolo, E., Dickinson, K., Sturke, R., Jack, D.W., Mehta, S., Shankar, A., Sherr, K. \& Rosenthal, J.P. 2018. An analysis of efforts to scale up clean household energy for cooking around the world. Energy for Sustainable Development, 46: 1-10.

https://doi.org/https://doi.org/10.1016/j.esd.2018.06.011

Riding, M.J., Herbert, B.M.J., Ricketts, L., Dodd, I., Ostle, N. \& Semple, K.T. 2015. Harmonising conflicts between science, regulation, perception and environmental impact: The case of soil conditioners from bioenergy. Environment International, 75: 52-67. https://doi.org/10.1016/j.envint.2014.10.025

Roopnarain, A. \& Adeleke, R. 2017. Current status, hurdles and future prospects of biogas digestion technology in Africa. Renewable and Sustainable Energy Reviews, 67: 1162-1179.

https://doi.org/10.1016/j.rser.2016.09.087

Roß, C.L., Sensel-Gunke, K., Wilken, V., Muskolus, A., Krause, G., Tschuikowa, S., Hübner, A., Fiebrich, V. \& Ellmer, F. 2018. Composted biowaste digestates as fertilizers: Effects on soil chemistry and plant development in a three-year field trial. Journal fur Kulturpflanzen, 70(6): 196-210.

https://doi.org/10.1399/JFK.2018.06.03

Scarlat, N., Dallemand, J.-F. \& Fahl, F. 2018. Biogas: Developments and perspectives in Europe. Renewable energy, 129: 457-472. https://doi.org/10.1016/j.renene.2018.03.006

Sigurnjak, I., Vaneeckhaute, C., Michels, E., Ryckaert, B., Ghekiere, G., Tack, F.M.G. \& Meers, E. 2017. Fertilizer performance of liquid fraction of digestate as synthetic nitrogen substitute in silage maize cultivation for three consecutive years. Science of the Total Environment, 599-600: 1885-1894.

https://doi.org/10.1016/j.scitotenv.2017.05.120

Smith, P. 2004. How long before a change in soil organic carbon can be detected? Global Change Biology, 10(11): 1878-1883. https://doi.org/10.1111/j.1365-2486.2004.00854.x

Surendra, K.C., Takara, D., Hashimoto, A.G. \& Khanal, S.K. 2014. Biogas as a sustainable energy source for developing countries: Opportunities and challenges. Renewable and Sustainable Energy Reviews, 31: 846-859. https://doi.org/10.1016/j.rser.2013.12.015

Thomas, C.L., Acquah, G.E., Whitmore, A.P., McGrath, S.P. \& Haefele, S.M. 2019. The effect of different organic fertilizers on yield and soil and crop nutrient concentrations. Agronomy, 9(12). https://doi.org/10.3390/agronomy9120776

Thomsen, I.K., Olesen, J.E., Møller, H.B., Sørensen, P. \& Christensen, B.T. 2013. Carbon dynamics and retention in soil after anaerobic digestion of dairy cattle feed and faeces. Soil Biology and Biochemistry, 58: 82-87. https://doi.org/10.1016/j.soilbio.2012.11.006

Valentinuzzi, F., Cavani, L., Porfido, C., Terzano, R., Pii, Y., Cesco, S., Marzadori, C. \& Mimmo, T. 2020. The fertilising potential of manure-based biogas fermentation residues: pelleted vs. liquid digestate. Heliyon, 6(2): e03325. https://doi.org/https://doi.org/10.1016/j.heliyon.2020.e03325 
Vasco-Correa, J., Khanal, S., Manandhar, A. \& Shah, A. 2018. Anaerobic digestion for bioenergy production: Global status, environmental and techno-economic implications, and government policies. Bioresource Technology, 247: 1015-1026. https://doi.org/10.1016/j.biortech.2017.09.004

Viaene, J., Agneessens, L., Capito, C., Ameloot, N., Reubens, B., Willekens, K., Vandecasteele, B. \& DeNeve, S. 2017. Co-ensiling, co-composting and anaerobic co-digestion of vegetable crop residues: Product stability and effect on soil carbon and nitrogen dynamics. Scientia Horticulturae, 220: 214-225. https://doi.org/https://doi.org/10.1016/j.scienta.2017.03.015

DeVries, J.W., Vinken, T.M.W.J., Hamelin, L. \& De Boer, I.J.M. 2012. Comparing environmental consequences of anaerobic mono- and co-digestion of pig manure to produce bio-energy - A life cycle perspective. Bioresource Technology, 125: 239-248. https://doi.org/10.1016/j.biortech.2012.08.124

WBA. 2020. Global Bioenergy Statistics 2020. Stockholm, World Bioenergy Association. (also available at: https://worldbioenergy.org/global-bioenergy-statistics) 


\title{
12. Compost application
}

\author{
Rainer Nerger, Tobias Bandel
}

Soil \& More Impacts, Germany

\section{Description of the practice}

\begin{abstract}
"Composting is the biological decomposition of organic materials by microorganisms under controlled, aerobic conditions to a relatively stable humus-like material called compost" ${ }^{4}$. Thus, the application of compost on the soil can increase soil organic carbon (SOC). Well-prepared compost features a humous structure of stable aggregates and clay-humus complexes which improve the soil structure (Misra et al., 2003). Through composting biomass found on-farm can be 'recycled' and re-used, thus potentially avoid rotting and GHG emissions from crop residues, manure, leaves, etc. Compost can be made out of very different ingredients (manure, crop residues, biowaste, kitchen waste, etc.) and it is widely used among farmers, especially smallholder farmers. Different composting methods exist, mainly aerobic and anaerobic composting (Misra et al, 2003). Globally, aerobic thermophilic composting is the most common method and preferable to avoid methane emissions (FAO, 2015), which will potentially make composting a carbon source instead of a carbon sink. Vermicomposting uses earthworms for the aerobic composting process. The quality of the compost and its carbon sequestration potential increases with the quality of preparation and the variety of input material. There are different phases in thermophilic composting, starting with a mesophilic and thermophilic phase where the biomass of the ingredients is decomposed, and pathogens destroyed (FAO, 2015). Each part of a compost pile or windrow should heat up to $>65^{\circ} \mathrm{C}$ for approx. 3 days (Misra et al., 2003). This is similar in every region in the world and the main reason why a pile or windrow must be turned 2-4 times in these early phases. Afterwards, the cooling and maturation phase takes place for several weeks and stable aggregates are formed. In this time, the pile or windrow should not be turned, except if oxygen levels fall. After a total duration of 10-12 weeks the compost is then ready to be applied on the field. This should be preferably done by incorporating the compost into the topsoil.
\end{abstract}

\footnotetext{
${ }^{4}$ http://www.omafra.gov.on.ca/english/engineer/facts/05-023.htm
} 


\section{Range of applicability}

The practice can be applied in any climatic region except in extreme cold or arid environments. Compost can be applied best before sowing or planting, but important is not to apply it on the peaks of seasonal climatic extremes (dry time, rain time, frost).

\section{Impact on soil organic carbon stocks}

The impact on SOC sequestration shown in the Table 49 depends on the amount of applied compost, but also on the soil texture and the original SOC concentration. For example, poorer sandy soils have a high potential of SOC sequestration, while more clayey soils with perhaps already higher SOC concentrations have a lower potential.

It also depends on the SOC concentration of the compost and thus, on the ingredient composition of the compost.

For Vermicompost there is much less data available on SOC sequestration. A study of Ngo et al. (2012) shows no SOC sequestration after one year of vermicompost application, however, one year is a very short time.

Table 49. Evolution of SOC stocks after compost application

\begin{tabular}{|c|c|c|c|c|c|c|c|}
\hline Location & $\begin{array}{l}\text { Soil } \\
\text { type }\end{array}$ & $\begin{array}{l}\text { Type of } \\
\text { application }\end{array}$ & $\begin{array}{l}\text { Additional } \\
\text { C storage } \\
\text { (tC/ha/yr) }\end{array}$ & $\begin{array}{l}\text { Duration } \\
\text { (Years) }\end{array}$ & $\begin{array}{l}\text { Depth } \\
\text { (cm) }\end{array}$ & Method & Reference \\
\hline $\begin{array}{l}\text { Global } \\
\text { (India, Brazil, } \\
\text { Madagascar, } \\
\text { Niger, } \\
\text { Zimbabwe, } \\
\text { Mexico, etc.) }\end{array}$ & Various & $\begin{array}{l}\text { Manure or } \\
\text { compost } \\
\text { applications } \\
\text { (different } \\
\text { rates) }\end{array}$ & 0.51 & Various & \multirow{3}{*}{$0-30$} & $\begin{array}{l}\text { Review } \\
\text { of } \\
\text { sampled } \\
\text { values }\end{array}$ & $\begin{array}{l}\text { Fujisaki et } \\
\text { al. (2018) }\end{array}$ \\
\hline $\begin{array}{l}\text { Italy } \\
\text { (Campania } \\
\text { region) }\end{array}$ & $\begin{array}{l}\text { Sandy } \\
\text { loam }\end{array}$ & $\begin{array}{l}\text { Biowaste } \\
\text { compost (15- } \\
30 \text { t/ha/yr) }\end{array}$ & 3.3 & 5 & & \multirow{4}{*}{ Sampled } & $\begin{array}{l}\text { Baiano } \\
\text { and Morra } \\
(2017)\end{array}$ \\
\hline $\begin{array}{l}\text { Canada } \\
\text { (Ontario } \\
\text { region) }\end{array}$ & $\begin{array}{l}\text { Clay } \\
\text { loam }\end{array}$ & $\begin{array}{l}\text { Yard waste } \\
\text { and biowaste } \\
\text { compost ( } 75 \\
\text { t/ha/yr each) }\end{array}$ & $\begin{array}{l}0.9 \text { (yard } \\
\text { waste } \\
\text { compost); } \\
\text { O (bio } \\
\text { waste } \\
\text { compost); }\end{array}$ & 10 & & & $\begin{array}{l}\text { Yang et } \\
\text { al. (2014) }\end{array}$ \\
\hline $\begin{array}{l}\text { Canada } \\
\text { (Quebec } \\
\text { region) }\end{array}$ & $\begin{array}{l}\text { Sandy } \\
\text { loam }\end{array}$ & $\begin{array}{l}\text { Composted } \\
\text { cattle manure } \\
\text { (5-15 t/ha/yr) }\end{array}$ & $1.35-2.02$ & NA & $0-15$ & & $\begin{array}{l}\text { Whalen } \\
\text { et al. } \\
(2008)\end{array}$ \\
\hline $\begin{array}{l}\text { India } \\
\text { (Odisha region) }\end{array}$ & $\begin{array}{l}\text { Sandy } \\
\text { loam }\end{array}$ & $\begin{array}{l}\text { Composted } \\
\text { cattle manure } \\
(5 \mathrm{t} / \mathrm{ha} / \mathrm{yr})\end{array}$ & 0.27 & 20 & $0-45$ & & $\begin{array}{l}\text { Nayak et } \\
\text { al. (2009) }\end{array}$ \\
\hline
\end{tabular}




\begin{tabular}{|c|c|c|c|c|c|c|c|}
\hline Location & $\begin{array}{l}\text { Soil } \\
\text { type }\end{array}$ & $\begin{array}{l}\text { Type of } \\
\text { application }\end{array}$ & $\begin{array}{l}\text { Additional } \\
\text { C storage } \\
\text { (tC/ha/yr) }\end{array}$ & $\begin{array}{l}\text { Duration } \\
\text { (Years) }\end{array}$ & $\begin{array}{l}\text { Depth } \\
\text { (cm) }\end{array}$ & Method & Reference \\
\hline $\begin{array}{l}\text { Egypt } \\
\text { (Nile delta } \\
\text { border and } \\
\text { close to Suez } \\
\text { channel) }\end{array}$ & $\begin{array}{l}\text { Reclai- } \\
\text { med } \\
\text { desert }\end{array}$ & $\begin{array}{l}\text { Mixed } \\
\text { compost (of } \\
\text { plant and } \\
\text { manure) } \\
\left(47 \mathrm{~m}^{3} / \mathrm{ha} / \mathrm{yr}\right)\end{array}$ & $\begin{array}{l}0.9 \\
\text { (most of } \\
\text { the } \\
\text { increase in } \\
\text { the first } \\
\text { years) }\end{array}$ & 30 & & & $\begin{array}{l}\text { Luske and } \\
\text { van der } \\
\text { Kamp } \\
\text { (2009) }\end{array}$ \\
\hline $\begin{array}{l}\text { South } \\
\text { Democratic } \\
\text { People's } \\
\text { Republic of } \\
\text { Korea } \\
\text { (Southeast } \\
\text { part) }\end{array}$ & Paddy & $\begin{array}{l}\text { Rice straw } \\
\text { compost (10 } \\
\text { t/ha/yr) }\end{array}$ & 0.29 & 42 & $0-30$ & & $\begin{array}{l}\text { Lee et al. } \\
\text { (2013) }\end{array}$ \\
\hline
\end{tabular}

\section{Other benefits of the practice}

\subsection{Improvement of soil properties}

Compost improves the soil structure through the formation of stable humous aggregates. Likewise, it reduces the risk for erosion, regulates soil moisture and increases the microbial and faunistic diversity of soils. All these effects help to preserve SOC in the soil (FAO, 2015).

\subsection{Minimization of threats to soil functions}

Table 50. Soil threats

\section{Soil threats}

\begin{tabular}{|l|l|}
\hline Soil erosion & $\begin{array}{l}\text { Better soil structure of more stable soil aggregates which is more resistant } \\
\text { (FAO, 2015). }\end{array}$ \\
\hline $\begin{array}{l}\text { Nutrient imbalance } \\
\text { and cycles }\end{array}$ & $\begin{array}{l}\text { Compost provides nutrients but most importantly it increases nutrient } \\
\text { holding capacity and enhances biological cycling through a better soil } \\
\text { structure (FAO, 2015). }\end{array}$ \\
\hline Soil acidification & $\begin{array}{l}\text { Enhancing soil buffer properties and improving cation exchange capacity } \\
\text { (Amlinger et al., 2007; FAO, 2015). }\end{array}$ \\
\hline
\end{tabular}




\begin{tabular}{|l|l|}
\hline Soil threats & Soil biodiversity \\
loss & $\begin{array}{l}\text { Compost provides bacteria, fungi and carbon which allow an improved } \\
\text { soil fauna and microbiology (FAO, 2015). Good quality compost has a } \\
\text { phytosanitary effect (Amlinger et al., 2007). }\end{array}$ \\
\hline $\begin{array}{l}\text { Soil water } \\
\text { management }\end{array}$ & $\begin{array}{l}\text { Better soil structure helps to increase meso pores and thus water-holding } \\
\text { capacity. Surface-applied compost keeps the soil surface moist (FAO, } \\
\text { 2015). }\end{array}$
\end{tabular}

\subsection{Increases in production (e.g. food/fuel/feed/timber/fibre)}

Compared to unfertilized controls, fields treated frequently with compost showed higher yield levels (Erhart $e t$ al., 2005; Reynolds et al., 2015).

\subsection{Mitigation of and adaptation to climate change}

The complete use/composting of available farm biomass (e.g. crop residues, green waste, manure) avoids the rotting and thus GHG emissions. Using a good quality compost regularly (e.g. annually or for each cropping season) can reduce the need for application of chemical fertilizers. On the climate change mitigation side, composting can be used in emission reduction projects, e.g. avoidance of methane in compost production (UNFCCC methodologies as AMS-III.-F: "Avoidance of methane production from decay of biomass through composting”) to generate carbon credits for small-holder farmers (Deiters, 2013). Likewise, compost can promote soil carbon sequestration, also in carbon standard projects (Verra methodologies). On the adaptation side, compost helps to regulate soil temperature and water and thus, increases the topsoil's resilience against climate change impacts.

\subsection{Socio-economic benefits}

Compost can save money (reduced erosion risk, nutrient supply, healthier and more productive stable soils on the long-term), the costs of compost making are counterbalanced in the mid-term to long-term scale. 


\section{Potential drawbacks to the practice}

\subsection{Tradeoffs with other threats to soil functions}

Table 51. Soil threats

\section{Soil threats}

\begin{tabular}{|l|l|}
\hline $\begin{array}{l}\text { Soil contamination / } \\
\text { pollution }\end{array}$ & $\begin{array}{l}\text { Depending on the source of the composting ingredients (e.g. municipal } \\
\text { waste), there can be a significant input of heavy metals, pesticides or } \\
\text { organic pollutants. Likewise, the quality of the compost (degree of } \\
\text { maturation) determines the mobility of heavy metal mobility (Amlinger } \\
\text { et al., 2007). }\end{array}$ \\
\hline Soil biodiversity loss & $\begin{array}{l}\text { If compost making was not adequate (e.g. no heat phase in thermophilic } \\
\text { compost), then the phytosanitary effect will be the opposite and can } \\
\text { degrade soil microbiology. }\end{array}$
\end{tabular}

\subsection{Increases in greenhouse gas emissions}

Compost can feature significant GHG emissions, especially if the process of compost making was not adequate. This is the case if the process was not fully aerobic, thus methane can form and emit. To avoid methane formation, compost must be turned fully several times in the initial heat phase. Proper compost making results in certain $\mathrm{CO}_{2}$ emissions which however do not necessarily counterbalance the soil carbon sequestration achieved through compost, especially in the first 20 years of application (Hillier et al, 2011, for the model "Cool Farm Tool" ${ }^{5}$ ). Nigussie et al. (2012) investigated the greenhouse gas emissions of vermicompost and concluded that "vermicompost decreased $\mathrm{N}_{2} \mathrm{O}$ emissions by 25-36 percent and $\mathrm{CH}_{4}$ emissions by 22-26 percent" compared to thermophilic composting.

\subsection{Decreases in production (e.g. food/fuel/feed/timber/fibre)}

Just in case compost replaces chemical fertilizers, the yield can decrease in the first years. This is because the nutrients in applied compost are mainly not directly plant-available but will be released during the following years. However, there are some studies suggesting the profitability of composting as part of organic farm management (Forster et al., 2013; Adamtey et al., 2016). Amongst others this could be related to the reduction in nutrient losses from erosion and leaching.

\footnotetext{
5 https://coolfarmtool.org/
} 


\section{Recommendations before implementing the practice}

It is important that composting is implemented in an aerobic form, otherwise methane can emit to the air (FAO, 2015), thus avoiding that composting becomes a carbon source instead of a carbon sink.

\section{Potential barriers for adoption}

Table 52. Potential barriers to adoption

\begin{tabular}{|c|c|c|}
\hline Barrier & YES/NO & \\
\hline Biophysical & Yes & $\begin{array}{l}\text { If there is not enough biomass for composting (crop residues, manure, } \\
\text { kitchen waste, biowaste) then this could be a barrier for compost } \\
\text { production. }\end{array}$ \\
\hline Economic & Yes & $\begin{array}{l}\text { Proper composting requires knowledge, time and eventually money for } \\
\text { labour force. Industrial compost making costs } 25 \text { (10-40) USD/tonne } \\
\text { on small scale (e.g. for } 30 \text { ha and } 5 \text { t/ha application) and on large scale } \\
\text { (e.g. for } 3000 \text { ha and } 10 \text { t/ha application), not considering special } \\
\text { technical legal regulations in industrialized countries (source: SMI). } \\
\text { Compost making means higher short-term costs which will be } \\
\text { profitable on the long-term (Viaene et al., 2016). }\end{array}$ \\
\hline Institutional & Yes & $\begin{array}{l}\text { In industrialized countries, especially in the EU, there are legal } \\
\text { restrictions, e.g. through the European Nitrate Directive, which can be a } \\
\text { barrier for compost making. }\end{array}$ \\
\hline $\begin{array}{l}\text { Legal (Right } \\
\text { to soil) }\end{array}$ & Yes & $\begin{array}{l}\text { In many countries there are farmers who don't own their land and thus } \\
\text { don't want to invest in it (e.g. in compost). }\end{array}$ \\
\hline Knowledge & Yes & $\begin{array}{l}\text { Often there is a lack of knowledge, thus the available biomass will not } \\
\text { be composted but rots or is to be sold. }\end{array}$ \\
\hline
\end{tabular}




\section{Photos of the practice}

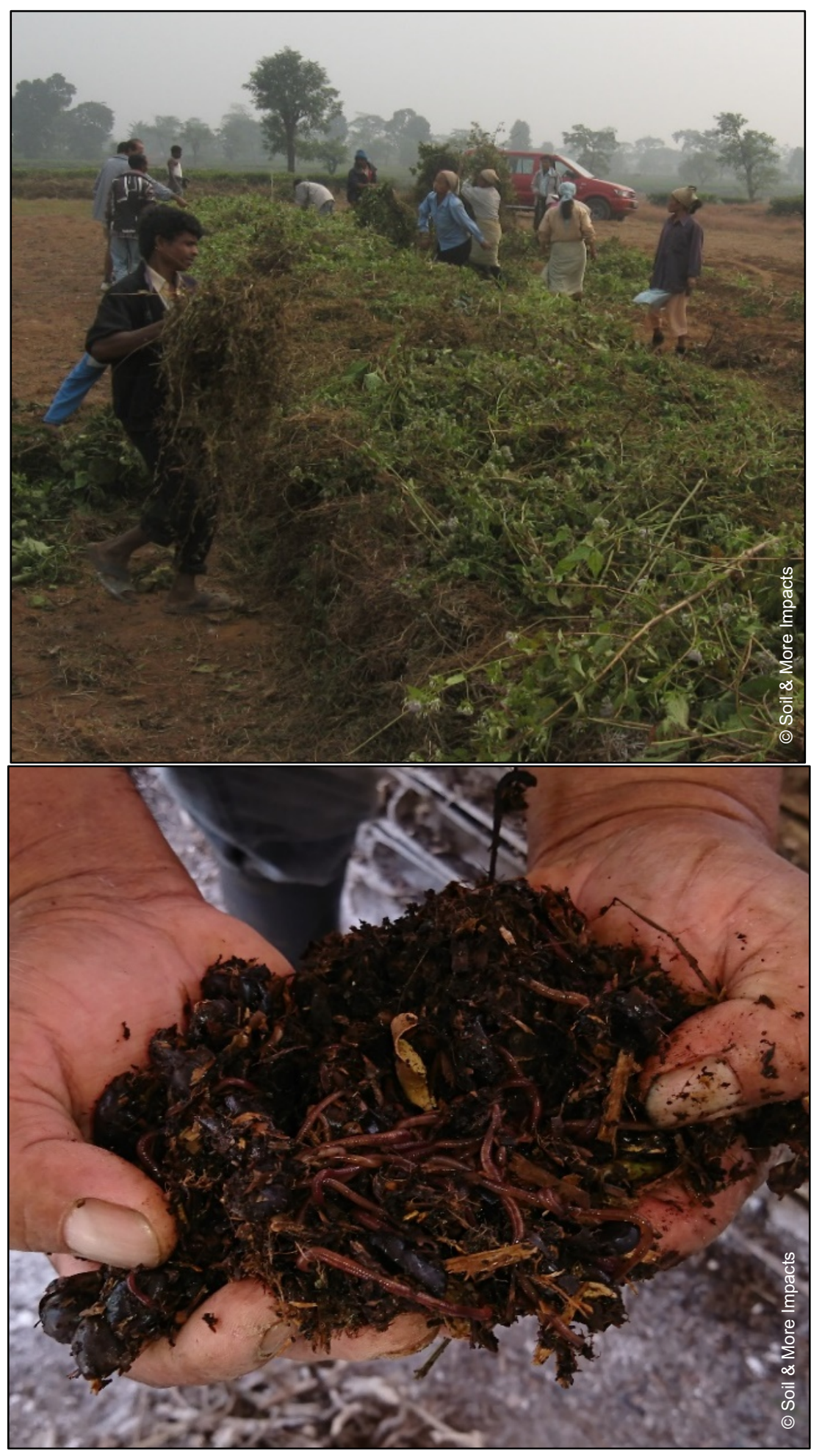

Photo 13. (Top) Processing vermicompost, Mexico. (Bottom) Compost windrow building, India 
Table 53. Related cases studies available in volumes 3 and 5

\begin{tabular}{|c|c|c|c|c|}
\hline Title & Region & $\begin{array}{l}\text { Duration } \\
\text { of study } \\
\text { (Years) }\end{array}$ & Volume & $\begin{array}{l}\text { Case- } \\
\text { study } \\
\text { No. }\end{array}$ \\
\hline $\begin{array}{l}\text { Agricultural practices for the } \\
\text { restoration of Soil Ecological Functions } \\
\text { in Madagascar }\end{array}$ & Africa & 2 & 3 & 2 \\
\hline $\begin{array}{l}\text { Biochar and compost application in an } \\
\text { olive orchard, Spain }\end{array}$ & Europe & 4 & 3 & 22 \\
\hline $\begin{array}{l}\text { Interrow organic management to } \\
\text { restore soil functionality of vineyards }\end{array}$ & $\begin{array}{l}\text { Europe and } \\
\text { Eurasia }\end{array}$ & 2 & 3 & 27 \\
\hline $\begin{array}{l}\text { Cover crops, organic amendments and } \\
\text { combined management practices in } \\
\text { Mediterranean woody crops }\end{array}$ & $\begin{array}{l}\text { Europe, } \\
\text { NENA, } \\
\text { Eurasia, North } \\
\text { America }\end{array}$ & $<30$ & 3 & 28 \\
\hline $\begin{array}{l}\text { Carbon storage in soils built from } \\
\text { waste for tree plantation in Angers, } \\
\text { France }\end{array}$ & Europe & 3 & 5 & 22 \\
\hline $\begin{array}{l}\text { Urban agriculture on rooftops in Paris, } \\
\text { France - the T4P research project } \\
\text { (Pilot Project of Parisian Productive } \\
\text { Rooftops) }\end{array}$ & Europe & 5 & 5 & 23 \\
\hline $\begin{array}{l}\text { Compost application to restore post- } \\
\text { disturbance soil health in Montgomery } \\
\text { county, Virginia, United States }\end{array}$ & $\begin{array}{l}\text { North } \\
\text { America }\end{array}$ & 4 & 5 & 28 \\
\hline
\end{tabular}




\section{References}

Adamtey, N., Musyoka, M.W.,Zundel, C., Cobo, J.G., Karanja, E., Fiaboe, K.K.M., Muriuki, A., Mucheru-Muna, M., Vanlauwe, B., Berset, E., Messmer, M.M., Gattinger, A., Bhullar, G.S., Cadisch, G., Fliessbach, A., Mäder, P., Niggli, U. \& Foster, D. 2016. Productivity, profitability and partial nutrient balance in maize-based conventional and organic farming systems in Kenya. Agriculture, Ecosystems \& Environment, 235: 61-79. https://doi.org/10.1016/j.agee.2016.10.001

Amlinger, F., Peyr, S., Geszti, J., Dreher, P., Karlheinz, W. \& Nortcliff, S. 2007. Beneficial effects of compost application on fertility and productivity of soils. Federal Ministry for Agricultural and Forestry, Environment and Water Management.

Baiano, S. \& Morra, L. 2017. Changes in soil organic carbon after five years of biowaste compost application in a Mediterranean vegetable cropping system. Pedosphere, 27(2): 328-337.

https://doi.org/10.1016/S1002-0160(17)60320-5

Deiters, T. 2013. Composting systems in the developing world. In Ecology \& Farming, 16-18.

Erhart, E., Hartl, W. \& Putz, B. 2005. Biowaste compost affects yield, nitrogen supply during the vegetation period and crop quality of agricultural crops. European Journal of Agronomy, 23(3): 305-314. https://doi.org/10.1016/j.eja.2005.01.002

FAO. 2015. Farmer's Compost Handbook - Experiences in Latin America. Food and Agriculture Organization of the United Nations Regional Office for Latin America and the Caribbean Santiago, 2015.

Forster, D., Andres, C., Verma, R., Zundel, C., Messmer, M.M. \& Mäder, P. 2013. Yield and economic performance of organic and conventional cotton-based farming systems-results from a field trial in India. PloS one, 8(12). https://doi.org/10.1371/journal.pone.0081039

Fujisaki, K., Chevallier, T., Chapuis-Lardy, L., Albrecht, A., Razafimbelo, T., Masse, D., Ndour, Y.B. \& Chotte, J.L. 2018. Soil carbon stock changes in tropical croplands are mainly driven by carbon inputs: a synthesis. Agriculture, Ecosystems \& Environment, 259: 147-158.

https://doi.org/10.1016/j.agee.2017.12.008

Hillier, J., Walter, C., Malin, D., Garcia-Suarez, T., Mila-i-Canals, L. \& Smith, P. 2011. A farm-focused calculator for emissions from crop and livestock production. Environmental Modelling \& Software, 26(9): 1070-1078.

Lee, C.H., Jung, K.Y., Kang, S.S., Kim, M.S., Kim, Y.H. \& Kim, P.J., 2013. Effect of long term fertilization on soil carbon and nitrogen pools in paddy soil. Korean J. Soil Sci. Fert., 46: 216-222. https://doi.org/10.7745/KJSSF.2013.46.3.216

Luske, B. \& van der Kamp, J. 2009. Carbon sequestration potential of reclaimed desert soils in Egypt. Louis Bolk Instituut \& Soil \& More International.

Misra, R.V., Roy, R.N. \& Hiraoka, H. 2003. On-farm composting methods. Rome, Italy: UN-FAO. (also available at: http://www.fao.org/docrep/007/y5104e/y5104e00.htm\#Contents) 
Nayak, P., Patel, D., Ramakrishnan, B., Mishra, A.K. \& Samantaray, R.N. 2009. Long-term application effects of chemical fertilizer and compost on soil carbon under intensive rice-rice cultivation. Nutrient Cycling in Agroecosystems, 83(3): 259-269. https://doi.org/10.1007/s10705-008-9217-8

Ngo, P.T., Rumpel, C., Doan, T.T. \& Jouquet, P. 2012. The effect of earthworms on carbon storage and soil organic matter composition in tropical soil amended with compost and vermicompost. Soil Biology and Biochemistry, 50: 214-220. https://doi.org/10.1016/j.soilbio.2012.02.037

Nigussie, A., Kuyper, T.W., Bruun, S. \& de Neergaard, A. 2016. Vermicomposting as a technology for reducing nitrogen losses and greenhouse gas emissions from small-scale composting. Journal of Cleaner Production, 139: 429-439. https://doi.org/10.1016/j.jclepro.2016.08.058

Reynolds, W.D., Drury, C.F., Tan, C.S. \& Yang, X.M. 2015. Temporal effects of food waste compost on soil physical quality and productivity. Canadian Journal of Soil Science, 95(3): 251-268.

https://doi.org/10.4141/cjss-2014-114

Viaene, J., Van Lancker, J., Vandecasteele, B., Willekens, K., Bijttebier, J., Ruysschaert, G., De Neve, S. \& Reubens, B. 2016. Opportunities and barriers to on-farm composting and compost application: A case study from northwestern Europe. Waste Management, 48, 181-192.

https://doi.org/10.1016/j.wasman.2015.09.021

Whalen, J.K., Benslim, H., Jiao, Y. \& Sey, B.K. 2008. Soil organic carbon and nitrogen pools as affected by compost applications to a sandy-loam soil in Quebec. Canadian Journal of Soil Science, 88(4): 443-450. https://doi.org/10.4141/CJSS07077

Yang, X., Reynolds, W.D., Drury, C.F., Fleming, R., Tan, C.S., Denholm, K. \& Yang, J. 2014. Organic carbon and nitrogen stocks in a clay loam soil 10 years after a single compost application. Canadian Journal of Soil Science, 94: 357-363. https://doi.org/10.4141/cjss2013-076 


\title{
13. Sewage sludge additions
}

\author{
Nadia E. Nava-Arsola ${ }^{1,2}$, Ofelia I. Beltrán-Paz ${ }^{1,2}$, Bruno M. Chávez-Vergara ${ }^{1,2}$ \\ ${ }^{1}$ Institute of Geology, National Autonomous University of Mexico, Mexico \\ ${ }^{2}$ Laboratorio Nacional de Geoquímica y Mineralogía, México
}

(Also refers to: biosolids, organic wastes additions)

\section{Description of the practice}

The disposal of products derived from the treatment of industrial, domestic, or farm residues is an environmental challenge. Their use as soil improvers is an attractive alternative; an example of this is sewage sludge. Sewage sludge, a type of biosolid, is the semi-solid residual material produced as a by-product during sewage treatment of industrial or municipal wastewater. These products are mainly composed of water and organic compounds. Two types are distinguished based on their dry matter (DM) content: if DM $<15$ percent are considered a liquid but if $>15$ percent DM is considered solid (Alvarenga et al., 2015, Barłóg et al., 2020; Delibacak et al., 2020).

The addition of sewage sludge increases the concentration of $\mathrm{C}, \mathrm{N}, \mathrm{P}$, and $\mathrm{K}$ (total and available), wherein the liquid is a higher concentration of inorganic forms of $\mathrm{N}$ and $\mathrm{P}$. This practice not only optimizes the disposal of these residues but also mitigates the incorporation of synthetic fertilizers (Alvarenga et al., 2015; SorianoDisla, Navarro-Pedreño and Gómez, 2010). However, it has been observed that these organic materials can also pose a risk to the environment and human health because, depending on the origin and the process that produces them, they can contain compounds at concentrations over certain limits that pose a risk to the environment, as heavy metals ( $\mathrm{Fe}, \mathrm{Cr}, \mathrm{Mn}, \mathrm{Zn}, \mathrm{Hg}, \mathrm{Pb}, \mathrm{Ni}, \mathrm{Cd}$, and $\mathrm{Cu}$ ), pathogens and organic pollutants (Delibacak et al., 2020; Hamdi et al., 2019; Kumar et al., 2017) or ammonium $\left(\mathrm{NH}_{4}^{+}\right)$that may quickly transform to nitrates $\left(\mathrm{NO}_{3}{ }^{-}\right)$representing a contamination risk for waterbodies (Barłóg et al., 2020; Rigby and Smith, 2013). 


\section{Range of applicability}

As commonly reported, sewage sludge may contain pathogens, metals, and organic pollutants (Delibacak et al., 2020; Hamdi et al., 2019; Kumar, Chopra and Kumar, 2017) so the application of these as organic amendments or as fertilizers is restricted according to the type of soil or environmental condition. For example, application to acidic soils can increase the bioavailability of heavy metals, or in sandy soils, can increase the risk of groundwater contamination. The usage of these organic amendments is usually focused on agricultural soils whose fertility needs to be improved but is also used in mine soil rehabilitation. The doses applied to soils are commonly $5-10 \mathrm{~L} / \mathrm{m}^{2}$. Given its application it can have adverse effects on the environment and human health, and thus its use must be regulated (Kumar, Chopra and Kumar, 2017). The benefits of addition of sewage sludge depend on the physicochemical characteristics of the waste, as well as the initial conditions of the soil where it will be applied (Alvarenga et al., 2015; Cattin et al., 2019).

\section{Impact on soil organic carbon stocks}

The addition of sewage sludge increases the organic carbon content of the soil with possible positive consequences for SOC sequestration by chemical and physical stability (Roig et al., 2012) but there still exists a lack in SOC storage information to be more conclusive (Fernández et al., 2009; Haller et al., 2010; Roig et al., 2012; Kalisz et al., 2017; Table 54). 
Table 54. General features of research about sewage sludge addition on soil organic carbon storage

\begin{tabular}{|c|c|c|c|c|c|c|c|c|}
\hline Location & Climate zone & Soil type & $\begin{array}{l}\text { Baseline C stock } \\
\text { (tC/ha) }\end{array}$ & $\begin{array}{l}\text { Additional C } \\
\text { storage } \\
\text { (tC/ha/yr) }\end{array}$ & $\begin{array}{l}\text { Duration } \\
\text { (Years) }\end{array}$ & Depth $(\mathrm{cm})$ & More information & Reference \\
\hline \multicolumn{9}{|c|}{ National studies } \\
\hline Spain & Warm temperate dry & NA & NA & $3.4^{\star}$ & 0.6 & $0-30$ & Experiment ex situ & $\begin{array}{l}\text { Soriano-Disla et al. } \\
(2010)\end{array}$ \\
\hline \multicolumn{9}{|c|}{ Regional studies } \\
\hline $\begin{array}{l}\text { Southern } \\
\text { Sweden }\end{array}$ & $\begin{array}{l}\text { Cold } \\
\text { temperate }\end{array}$ & $\begin{array}{l}\text { Loam and sandy } \\
\text { loam }\end{array}$ & 77.3 & 0.3 & 30 & $0-20$ & Experiment in situ & $\begin{array}{l}\text { Börjesson and } \\
\text { Kätterer (2018) }\end{array}$ \\
\hline $\begin{array}{l}\text { North-East } \\
\text { Poland }\end{array}$ & $\begin{array}{l}\text { Cold temperate dry } \\
\text { and moist }\end{array}$ & Arenosol & 17.9 & 0.1 & 3 & $0-10$ & Non-significant increase & Kalisz et al. (2017) \\
\hline $\begin{array}{l}\text { Pamplona, } \\
\text { Spain }\end{array}$ & Warm temperate dry & $\begin{array}{l}\text { Calcaric Fluvisol } \\
\text { (FAO) }\end{array}$ & $9.8^{*}$ & $0.2^{*}$ & 16 & $0-25$ & Microbial activity & Roig et al. (2012) \\
\hline $\begin{array}{l}\text { Illinois, United } \\
\text { States of } \\
\text { America }\end{array}$ & $\begin{array}{l}\text { Warm temperate } \\
\text { moist }\end{array}$ & $\begin{array}{l}\text { Mine spoil soil and } \\
\text { nonmined soil }\end{array}$ & NA & $2.2-4.9$ & 34 & $0-15$ & $\begin{array}{l}\text { Biosolids additions; Experiment } \\
\text { in situ }\end{array}$ & Tian et al. (2009) \\
\hline \multicolumn{9}{|l|}{ Local studies } \\
\hline $\begin{array}{l}\text { Campinas, São } \\
\text { Paulo, Brazil }\end{array}$ & Tropical moist & $\begin{array}{l}\text { Haplic Ferralsol } \\
\text { (FAO) }\end{array}$ & 58.8 & $1.7-3.0$ & 8 & $0-40$ & GHG flux & Pitombo et al. (2015) \\
\hline Madrid, Spain & Mediterranean & $\begin{array}{l}\text { Calcareous } \\
\text { Mediterranean soil }\end{array}$ & $13^{*}$ & $1-11^{*}$ & 2 & $0-20$ & Organic C fractions & $\begin{array}{l}\text { González-Ubierna et } \\
\text { al. (2013) }\end{array}$ \\
\hline Toledo, Spain & Semiarid continental & $\begin{array}{l}\text { Typic Haploxeralf } \\
\text { (USDA) }\end{array}$ & $7.2^{*}$ & $1.5-2.7^{\star}$ & 3 & $0-20$ & Microbial activity & $\begin{array}{l}\text { Fernández et al. } \\
\text { (2009) }\end{array}$ \\
\hline
\end{tabular}

NA: Not available, ${ }^{*} \mathrm{SOC}$ concentration rate in $\mathrm{g} / \mathrm{kg} / \mathrm{yr}$ because SOC stock data is not available. 


\section{Other benefits of the practice}

\subsection{Improvement of soil properties}

Some soil properties have been reported to be improved with sludge substances. Aggregate stability, moisture retention, porosity, and decreased erosion have been reported for physical properties. With respect to the chemical properties, the addition of sewage sludge has been shown to increase $\mathrm{pH}$, electrical conductivity, nutrient concentration, organic matter, and the cation exchange capacity. Biological properties, such as microbial biomass and enzyme activities related to the $\mathrm{C}, \mathrm{N}$ and $\mathrm{P}$ cycles (e.g. urease, phosphatase, $\beta$ glucosidase, dehydrogenase) can be also improved by sewage sludge (Delibacak et al., 2020 Kumar, Chopra and Kumar, 2017).

\subsection{Minimization of threats to soil functions}

Table 55. Soil threats

\begin{tabular}{|c|c|}
\hline Soil threats & \\
\hline Soil erosion & $\begin{array}{l}\text { The addition of sewage sludge reduces erosion because it improves the } \\
\text { physical and chemical conditions of the soil and promotes plant growth } \\
\text { (Ojeda, Alcañiz and Ortiz, 2003; Ros, Hernández and García, 2003). }\end{array}$ \\
\hline $\begin{array}{l}\text { Nutrient imbalance } \\
\text { and cycles }\end{array}$ & $\begin{array}{l}\text { Increases nutrient availability and microbial activity related to nutrient } \\
\text { recycling (Delibacak et al., 2020; Makádi et al., 2012). }\end{array}$ \\
\hline Soil acidification & Improve the $\mathrm{pH}$ and buffering capacity (Delibacak et al., 2020). \\
\hline $\begin{array}{l}\text { Soil biodiversity } \\
\text { loss }\end{array}$ & $\begin{array}{l}\text { Increases biomass and activity of the microbial community (Delibacak et } \\
\text { al., 2020; Kumar et al., 2017). }\end{array}$ \\
\hline Soil compaction & $\begin{array}{l}\text { Improves soil structure and aggregate stability, thereby preventing soil } \\
\text { compaction (Delibacak et al., 2020). }\end{array}$ \\
\hline $\begin{array}{l}\text { Soil water } \\
\text { management }\end{array}$ & $\begin{array}{l}\text { Improves soil moisture retention and plant water availability to crops } \\
\text { (Delibacak et al., 2020). }\end{array}$ \\
\hline
\end{tabular}




\subsection{Increases in production (e.g. food/fuel/feed/timber/fibre)}

Sewage sludge can be used for energy generation and recovery through the production of biogas. In addition, it can improve the fertility of soils, thereby promoting growth and crop yield (Nkoa, 2014, Kumar, Chopra and Kumar, 2017).

\subsection{Mitigation of and adaptation to climate change}

The addition of sewage sludge not only increases the total amount of SOC but also the proportion of more stable SOC fractions, thereby promoting their permanence in soils against biological mineralization, contributing to mitigation and adaptation to climate change (Pitombo et al, 2015; Soriano-Disla, Navarro-Pedreño and Gómez, 2010; Tian et al., 2009).

\subsection{Socio-economic benefits}

Because they are products derived from the treatment and use of waste, it is low cost and even free for producers, which helps to replace or decrease the purchase of inorganic fertilizer (Romanos et al., 2019) and be a component of circular economy since they represent a valuation of a waste. They can be around 40 percent of the cost of inorganic fertilizers (Nkoa, 2014).

\section{Potential drawbacks to the practice}

\subsection{Tradeoffs with other threats to soil functions}

Table 56. Soil threats

\begin{tabular}{|l|l|}
\hline Soil threats & $\begin{array}{l}\text { Excess application promotes the loss of nutrients through volatilization } \\
\text { and/or lixiviation, for example increased emissions of } \mathrm{CO}_{2} \text { emissions } \\
\text { (Cattin et al., 2019; Haller et al., 2010; Kalisz et al., 2017), ammonia and } \\
\text { and cycles } \\
\text { nitrous oxide into the atmosphere (Barłóg et al., 2020; Nkoa, 2014; } \\
\text { Rigby and Smith, 2013). }\end{array}$ \\
\hline $\begin{array}{l}\text { Soil salinization and } \\
\text { alkalinization }\end{array}$ & $\begin{array}{l}\text { Sewage sludge may contain salts depending on the material and the } \\
\text { type of treatment from which they have been produced and, in case of } \\
\text { high concentrations, they can cause salinization of the soil (Hambi et al., } \\
\text { 2007) }\end{array}$
\end{tabular}




\section{Soil threats}

Soil contamination /pollution

They may contain pathogens, heavy metals, and organic pollutants depending on the material and process that originated them (Delibacak et al., 2020; Hamdi et al., 2019; Kumar, Chopra and Kumar, 2017).

Soil biodiversity loss

The presence of harmful compounds may prevent the development of certain soil organisms (Hamdi et al., 2019).

\subsection{Increases in greenhouse gas emissions}

An inadequate initial process in the treatment of sewage sludge can cause them to be a source of greenhouse gases (GHG) such as $\mathrm{CO}_{2}$ and $\mathrm{CH}_{4}$ (Yoshida et al., 2015). Once in the soils and due to their high $\mathrm{N}$ concentration, it has been observed that excess in the addition of digestate promotes ammonium oxidation (Rigby and Smith, 2013) and with this, they can be a source of nitrate ions $\left(\mathrm{NO}_{3}^{-}\right)$(Barłóg et al., 2020; Nkoa, 2014). In addition, under certain circumstances, its increased application can generate a priming effect in soils, which increases $\mathrm{CO}_{2}$ emission and can promote SOC loss (Cattin et al., 2019).

\subsection{Other conflicts}

There may be technical problems regarding the treatment, the available quantity, the transportation, and the application of these products in the field (Lukehurst, Frost and Seadi, 2014; Nkoa, 2014; Alvarenga et al., 2015).

\section{Recommendations before implementing the practice}

It is recommended to carry out physicochemical evaluations of the soil and of the sewage sludge in relation to the concentration of nutrients and SOC content, as well as possible contaminants such as heavy metals, to prevent contamination and GHG emissions. 


\section{Potential barriers for adoption}

Table 57. Potential barriers to adoption

\begin{tabular}{|l|l|l|}
\hline Barrier & YES/NO & There may be technical problems for its application related to \\
\hline Biophysical & Yes & $\begin{array}{l}\text { Then } \\
\text { infiltration capacity of soils because the high-water content when } \\
\text { applied as liquid (Alvarenga et al., 2015). }\end{array}$ \\
\hline Cultural & Yes & $\begin{array}{l}\text { Cultural aversion to handling of human waste and its application as a } \\
\text { fertilizer (Lukehurst, Frost and Seadi, 2014). }\end{array}$ \\
\hline Social & Yes & $\begin{array}{l}\text { In developing countries, the accessibility to sewage sludge will be } \\
\text { limited because of uncommonly wastewater treatment plants (Nkoa, } \\
\text { 2014). }\end{array}$ \\
\hline $\begin{array}{l}\text { Legal (Right } \\
\text { to soil) }\end{array}$ & Yes & $\begin{array}{l}\text { Sewage sludge must comply with adequate sanitary requirements for } \\
\text { its use, for example, the concentration of metals must be below the } \\
\text { permissible limits (Nkoa, 2014; Alvarenga et al., 2015). }\end{array}$ \\
\hline Knowledge & Yes & $\begin{array}{l}\text { More information needs to be generated on the effects of adding } \\
\text { sewage sludge to the soil. Its addition must be planned to avoid GHG } \\
\text { contamination or emission (Cattin et al., 2019; Nkoa, 2014; Rigby and } \\
\text { Smith, 2013). }\end{array}$ \\
\hline
\end{tabular}




\section{Photos of the practice}

a)

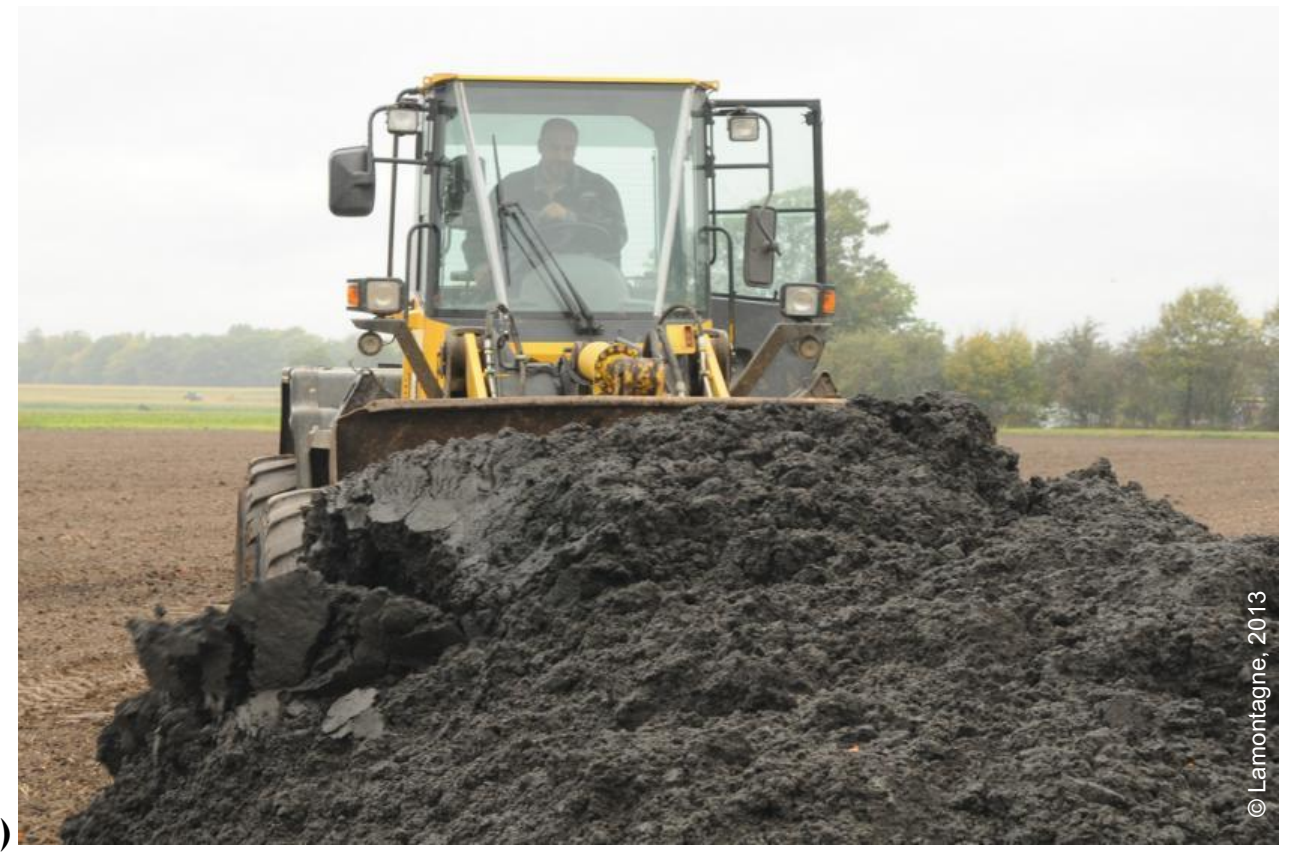

b)

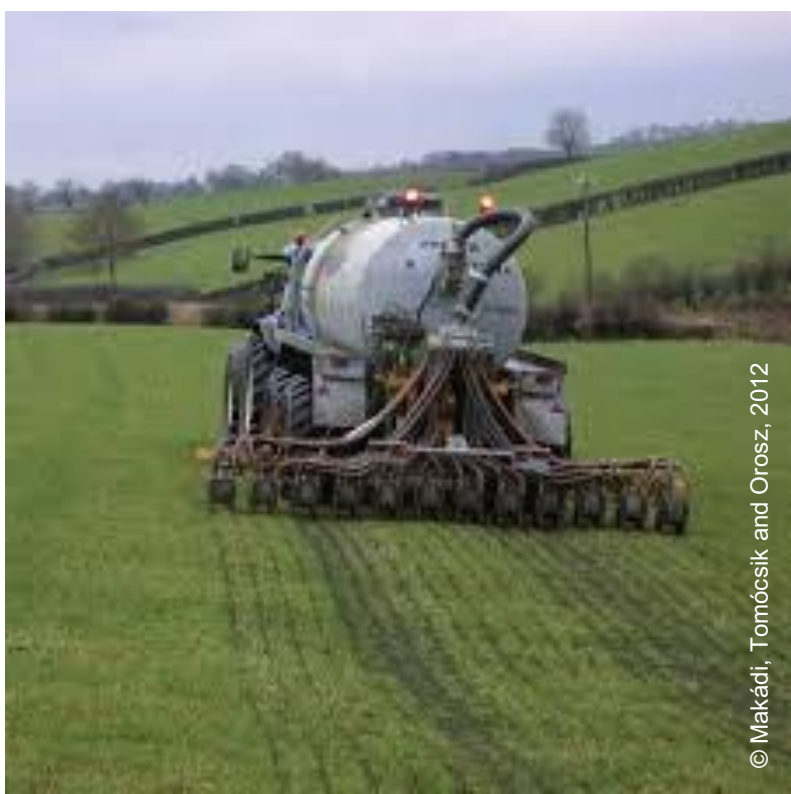

Photo 14. a) Treated sewage sludge, the solid byproduct of wastewater treatment, is often applied to farmland as a soil amendment in the United States, and b) Shallow injection of digestate into the grassland. 
Table 58. Related cases studies available in volumes 3 and 5

\begin{tabular}{|l|l|l|c|c|}
\hline Title & Region & $\begin{array}{l}\text { Duration } \\
\text { of study } \\
\text { (Years) }\end{array}$ & Volume & $\begin{array}{l}\text { Case- } \\
\text { study } \\
\text { No. }\end{array}$ \\
\hline $\begin{array}{l}\text { Cover crops, organic amendments and } \\
\text { combined management practices in } \\
\text { Mediterranean woody crops }\end{array}$ & $\begin{array}{l}\text { Europe, NENA, } \\
\text { Eurasia, North } \\
\text { America }\end{array}$ & $<30$ & 3 & 28 \\
\hline $\begin{array}{l}\text { Carbon storage in soils built from waste } \\
\text { for tree plantation in Angers, France }\end{array}$ & Europe & 3 & 5 & 22 \\
\hline $\begin{array}{l}\text { Organic amendments for soils } \\
\text { rehabilitation of open-pit mines in } \\
\text { Spain }\end{array}$ & Europe & 6,10 and \\
18 & 5 & 24 \\
\hline
\end{tabular}




\section{References}

Alvarenga, P., Mourinha, C., Farto, M., Santos, T., Palma, P., Sengo, J., Morais, M. \& Cunha-Queda, C. 2015. Sewage sludge, compost and other representative organic wastes as agricultural soil amendments: Benefits versus limiting factors. Waste Management, 40(276): 44-52.

https://doi.org/10.1016/j.wasman.2015.01.027

Barłóg, P., Hlisnikovský, L. \& Kunzová, E. 2020. Effect of Digestate on Soil Organic Carbon and PlantAvailable Nutrient Content Compared to Cattle Slurry and Mineral Fertilization. Agronomy, 10(3): 379. https://doi.org/10.3390/agronomy10030379

Börjesson, G. \& Kätterer, T. 2018. Soil fertility effects of repeated application of sewage sludge in two 30year-old field experiments. Nutrient Cycling in Agroecosystems, 112: 369-385.

https://doi.org/10.1007/s10705-018-9952-4

Cattin, M., Semple, K.T., Stutter, M., Brotons, A.L., Parry, C. \& Ben, W.J. 2019. Exploring the Impacts of Digestate Application on the Carbon Cycle in Grassland Soils. Geophysical Research Abstracts, 21. (also available at: https://meetingorganizer.copernicus.org/EGU2019/EGU2019-9579.pdf)

Delibacak, S., Voronina, L., Morachevskaya, E. \& Ongun, A.R. 2020. Use of sewage sludge in agricultural soils: Useful or harmful. Eurasian Journal of Soil Science, 9(2): 126-139.

https://doi.org/10.18393/ejss.687052

Fernández, J.M., Plaza, C., García-Gil, J.C. \& Polo, A. 2009. Biochemical properties and barley yield in a semiarid Mediterranean soil amended with two kinds of sewage sludge. Applied Soil Ecology, 42: 18-24. https://doi.org/10.1016/j.apsoil.2009.01.006

González-Ubierna, S., Jorge-Mardomingo, I., Cruz, M.T., Valverde, I. \& Casermeiro, M.A. 2013. Sewage sludge application in Mediterranean agricultural soils: Effects of dose on the soil carbon cycle. International Journal of Environmental Research, 7(4): 945-956.

https://doi.org/10.22059/ijer.2013.677

Haller, I., García-Gil, J.C., López-de-Sa, E.G., Soler-Rovira, P. \& Polo, A. 2010. Influence of soil management and thermally-dry sewage sludge amendment on soil labile $\mathrm{C}$ pools, microbial biomass $\mathrm{C}$ and $\mathrm{CO}_{2}$ fluxes. Journal of Solid Waste Technology \& Management: 1239-1248. (also available at: http://solidwaste.org/past-conferences-and-proceedings/proceedings/2011-2/)

Hamdi, H., Hechmi, S., Khelil, M.N., Zoghlami, I.R., Benzarti, S., Mokni-Tlili, S., Hassen, A. \& Jedidi, N. 2019. Repetitive land application of urban sewage sludge: Effect of amendment rates and soil texture on fertility and degradation parameters. Catena, 172: 11-20.

https://doi.org/10.1016/j.catena.2018.08.015

Lukehurst, C., Frost, P. \& Seadi, T. 2010. Utilisation of digestate from biogas plants as biofertiliser. IEA Bioenergy. Task 37. (also available at: https://www.ieabioenergy.com/wpcontent/uploads/2010/06/Digestate_Brochure_Revised_12-2010.pdf)

Kalisz, B., Lachacz, A., Głażewski, R. \& Grabowski, K. 2017. Labile organic carbon fractions after amendment of sandy soil with municipal sewage sludge and compost. Journal of Elementology, 22(3): 785797. https://doi.org/10.5601/jelem.2017.22.1.1264 
Kumar, V., Chopra, A.K. \& Kumar, A. 2017. A Review on Sewage Sludge (Biosolids) a Resource for Sustainable Agriculture. Archives of Agriculture and Environmental Science, 2(4): 340-347.

https://doi.org/10.26832/24566632.2017.020417

Makádi, M., Tomócsik, A. \& Orosz, V. 2012. Digestate: A New Nutrient Source - Review. Biogas. 295310. https://doi.org/10.5772/31355

Nkoa, R. 2014. Agricultural benefits and environmental risks of soil fertilization with anaerobic digestates: a review. Agron. Sustain. Dev., 34: 473-492. https://doi.org/10.1007/s13593-013-0196-z

Ojeda, G., Alcañiz, J.M. \& Ortiz, O. 2003. Runoff and losses by erosion in soils amended with sewage sludge. Land Degradation \& Development, 14(6): 563-573. https://doi.org/10.1002/ldr.580

Pitombo, L.M., do Carmo, J.B., de Maria, I.C., de Andrade, C.A. 2015. Carbon sequestration and greenhouse gases emissions in soil under sewage sludge residual effects. Scientia Agricola, 72(2): 147-156. https://doi.org/10.1590/0103-9016-2013-0352

Rigby, H. \& Smith, S.R. 2013. Nitrogen availability and indirect measurements of greenhouse gas emissions from aerobic and anaerobic biowaste digestates applied to agricultural soils. Waste Management, 33(12): 2641-2652. https://doi.org/10.1016/j.wasman.2013.08.005

Roig, N., Sierra, J., Martí, E., Nadal, M., Schuhmacher, M. \& Domingo, J.L. 2012. Long-term amendment of Spanish soils with sewage sludge: Effects on soil functioning. Agriculture, Ecosystems and Environment, 158: 41-48. https://doi.org/10.1016/j.agee.2012.05.016

Romanos, D., Nemer, N., Khairallah, Y., Thérèse, M. \& Saab, A. 2019. Assessing the quality of sewage sludge as an agricultural soil amendment in Mediterranean habitats. International Journal of Recycling of Organic Waste in Agriculture, 8: 8-15.

Ros, M., Hernández, M.T. \& García, C. 2003. Bioremediation of Soil Degraded by Sewage Sludge: Effects on Soil Properties and Erosion Losses. Environmental Management, 31(6): 741-747.

https://doi.org/10.1007/s00267-002-2839-8

Soriano-Disla, J.M., Navarro-Pedreño, J. \& Gómez, I. 2010. Contribution of a sewage sludge application to the short-term carbon sequestration across a wide range of agricultural soils. Environmental Earth Sciences, 61(8): 1613-1619. https://doi.org/10.1007/s12665-010-0474-x

Tian, G., Granato, T.C., Cox, A.E., Pietz, R.I., Carlson, C.R. \& Abedin, Z. 2009. Soil Carbon Sequestration Resulting from Long-Term Application of Biosolids for Land Reclamation. Journal of Environmental Quality, 38: 61-74. https://doi.org/10.2134/jeq2007.0471

Yoshida, H., Nielsen, M.P., Scheutz, C., Jensen, L.S. \& Christensen, T.H. 2015. Effects of sewage sludge stabilization on fertilizer value and greenhouse gas emissions after soil application. Soil \& Plant Science, 65(6): 506-516. 


\section{Mineral fertilization (macro, secondary and micronutrients)}

\section{James K. Mutegi', Ngonidzashe Chirinda², Hakim Boulal ${ }^{3}$, Shamie Zingore ${ }^{4}$, Thomas Oberthur ${ }^{4}$, Kaushik Majumdar ${ }^{4}$, Crispus Njeru ${ }^{5}$, Abednego Kiwia ${ }^{6}$}

${ }^{1}$ African Plant Nutrition Institute, ICIPE Compound, Nairobi, Kenya

${ }^{2}$ UM6P, Lot 660 Hay Moulay Rachid, Benguérir, Morocco

${ }^{3}$ African Plant Nutrition Institute, North Africa Program, Settat, Morocco

${ }^{4}$ African Plant Nutrition Institute, Lot 660 Hay Moulay Rachid, Benguérir, Morocco

${ }^{5}$ Mercy Corps Climate Program Kenya, Nairobi, Kenya

${ }^{6}$ AGRA, West End Towers, Waiyaki Way, Westlands, Nairobi, Kenya

\section{Description of the practice}

An important function of soil fertility in agriculture is to sustain soil health for ecosystem functions and to support crop production. In most cases the nutrient reserves in the soils are inadequate to meet the requirements of high-yielding crops and therefore external application of either mineral or organic fertilizers is necessary to supply the deficient essential nutrients. There are 14 mineral nutrients essential for crop growth, which are sourced by crop roots from the soil. These are classified as primary nutrients or macronutrients [nitrogen $(\mathrm{N})$, phosphorus $(\mathrm{P})$, potassium $(\mathrm{K})$ ], secondary nutrients; [calcium $(\mathrm{Ca})$, magnesium $(\mathrm{Mg})$ and sulfur $(\mathrm{S})$ ] and micronutrients [iron $(\mathrm{Fe})$, manganese $(\mathrm{Mn})$, zinc $(\mathrm{Zn})$, copper $(\mathrm{Cu})$, molybdenum $(\mathrm{Mo})$, boron $(\mathrm{B})$, chlorine as chloride $\left(\mathrm{Cl}^{-}\right)$and nickel $\left.(\mathrm{Ni})\right]$ (Prasad et al., 2016). Macronutrients are required in largest quantities, while secondary nutrients are required in moderate quantities and micronutrients are required in minimal amounts compared to macro and secondary nutrients.

Mineral fertilizers are produced to provide the crops with one or more of these nutrients. Organic resources can also supply these nutrients but they have a range of limitations that include: (i) their low nutrient concentration implying that bulk quantities are required to supply crop/s with sufficient nutrient rates; (ii) variable nutrient composition depending on the source and; (iii) the asynchrony of nutrient release pattern of organic resources with nutrient requirement patterns of crops at various development stages (Chivenge, Vanlauwe and Six, 2011). 
Soil and plant tissue tests are essential tools for determining the level of deficiency and potential crop nutrient requirements to guide development of fertilizer recommendations. An appropriate fertilizer recommendation ensures adequate and balanced application of fertilizer for optimal crop performance while reducing the potential negative effects of fertilizers on environmental sustainability. Whereas macro and secondary nutrients are primarily applied to the soil and taken up by crop roots, the micronutrients can be applied either to the soil with macronutrients at planting or as foliar sprays. Globally, mineral fertilizers account for more than 40 percent of crop yields (Stewart et al, 2005). Large yield increases of over 80 percent are commonly achieved in shortterm after application of fertilizers on nutrient depleted soils (Banerjee et al., 2016; Zhang et al., 2010; Njoroge et al. 2018). Balanced supply of limiting nutrients has the double benefits of enhancing both crop yield and yield quality (Prasad et al., 2016). For most countries, the main challenges affecting fertilizer use range from: limited access to knowledge, unavailability of fertilizers in remote locations and high cost of fertilizer products. There is need for appropriate dissemination of fertilizer use knowledge and fertilizer recommendation development tools to enhance benefits accruing from fertilizer use while minimizing the impact of fertilizer on the environment.

\section{Range of applicability}

Soil organic carbon (SOC) sequestration is enhanced by improved biomass production supported by various management factors, including application of inorganic fertilizers. The use of inorganic fertilizers has risen globally from 1960 to 2020. The growth of fertilizer use has particularly been high in Asia. Asia represented less than 20 percent of the world total fertilizer use in 1960 s, but between 2015 and 2018 it accounted for over 50 percent of global use of all the three macronutrients (FAO, 2020). Figure 3 provides a visual illustration of the global extent and distribution of use of N, P, K fertilizers as of 2015. The lowest fertilizer use levels of between $0.34 \mathrm{~kg}$ to $0.60 \mathrm{~kg}$ per hectare are found in sub-Saharan Africa countries, such as Niger and Central African republic. On the other hand, the highest intensity of fertilizer use of between 500 and $700 \mathrm{~kg}$ per hectare is mainly found in countries like China, Egypt and Colombia (Figure 3). The world total consumption rate of fertilizer increased from 27.4 million nutrient tons in 1960 to 208 million nutrient tons in 2020 (Bumb and Baanante, 1996; FAO, 2020). Fertilizer use grew across all the agricultural regions including Europe and North America from an average of 24.7 to 86.4 million nutrient tons over the period and from about 2.7 to 121.6 million nutrient tons in the developing countries (cumulative average for East Asia, South Asia, West Asia/North Africa, Latin America and sub-Sahara Africa). Over 80 percent of globally used fertilizers are made up of N, P and K whose 2020 cumulative demand was estimated at 115.3 million tonnes of N, 56 million tonnes of $\mathrm{P}$ and 36.7 million tonnes of $\mathrm{K}$ (FAO, 2020). 


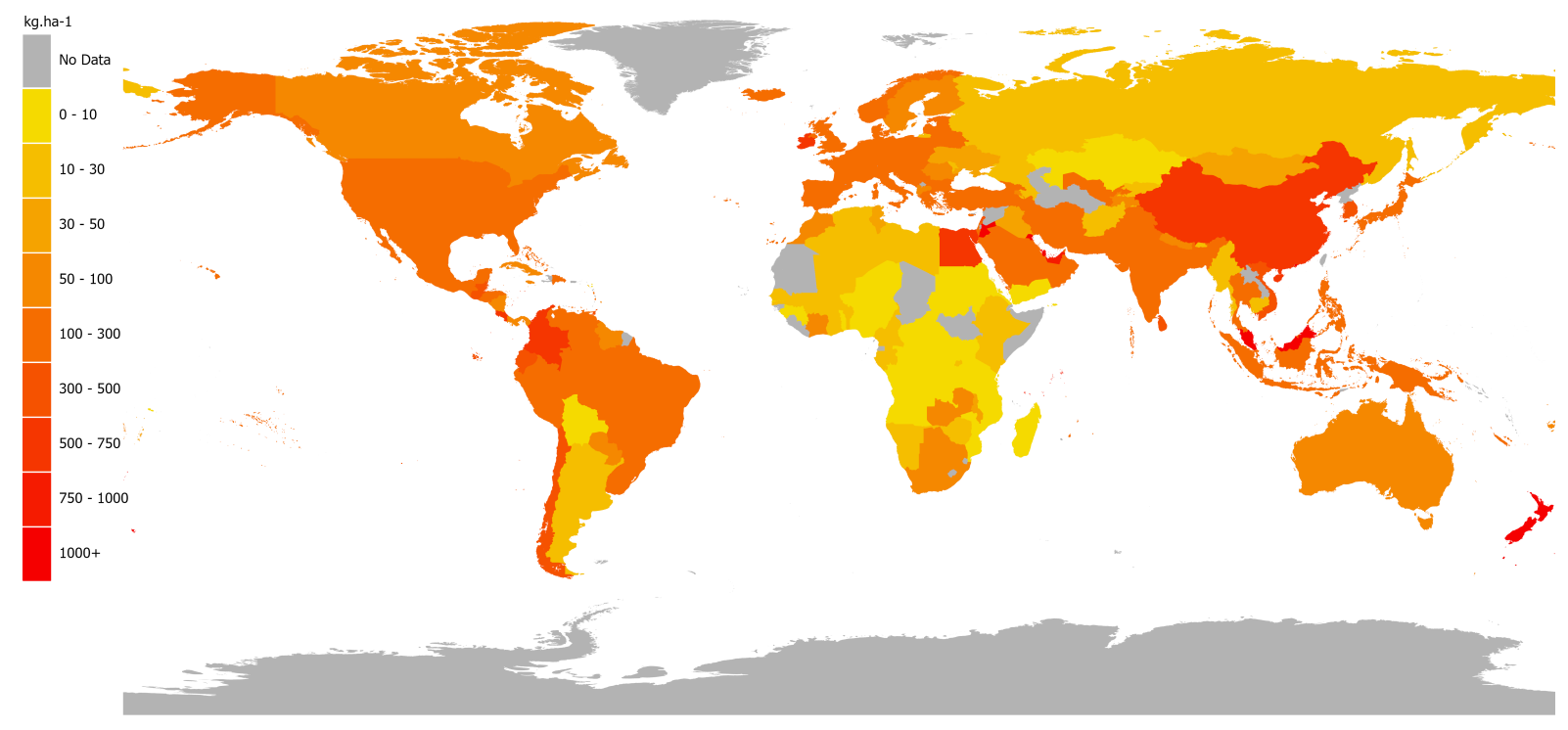

Figure 3. World Bank global estimates of quantities and the extent of fertilizer use per hectare of cropland in 2015

The fertilizer products cover nitrogenous, potash and phosphate fertilizers (including ground rock phosphate). Map adapted from: Roser and Ritchie (2016).

Although the use secondary and the micro-nutrient is considered low the amounts used have also been growing. A 2018/19 survey covering North America, Europe, Asia Pacific, South America, Middle East and Africa observed a global fertilizer micronutrient growth at a compound annual growth rate of 10.2 percent from 2018 to 2027 (Research Markets, 2020). Based on 34 crops grown under rainfed and irrigated conditions for 105 countries, Alexandratos and Bruinsma (2012) projected that the fertilizer consumption could increase from 166 million tonnes in 2005-2007 to 263 million tonnes in 2050. Cereal crops, in particular wheat, rice and maize, account at present for about 60 percent of global fertilizer use, and are expected to still account for over 50 percent of fertilizer consumption by 2050 . Fertilizer use for oilseeds (in particular to soybeans and rapeseed) is expected to grow fastest so that oilseeds by 2050 could account for over a fifth of all fertilizer consumption (Alexandratos and Bruinsma, 2012). The shares of N, P and K in total fertilizer consumption are expected to change only marginally over the projection period. 


\section{Impact on soil organic carbon stocks}

Table 59 presents SOC sequestration data from fertilizer treated plots from a range of soil type and climates for long term experiments within trial durations of between 7 and 48 years. In comparison to few studies where SOC was revealed to decrease after a long term of fertilizer application (e.g. Srinivasarao et al., 2012), a disproportionate number of empirical studies conclude that fertilizer application led to an increase in SOC sequestration (Table 59). The macro-nutrient fertilizers enhanced soil carbon sequestration by between 0.03 and $0.5 \mathrm{tC} / \mathrm{ha} / \mathrm{yr}$, with high sequestration levels associated with balanced application of NPK fertilizers as opposed to sole application of one or two macronutrients. Fertilizer application rates are an important regulator of carbon sequestration (Rudrappa et al, 2005; Brar et al., 2015). Although Zinc is an important micronutrient in crop production, Brar et al. (2015) failed to observe a difference in carbon sequestration rates between 100 percent NPK $(0.103 \mathrm{tC} / \mathrm{ha} / \mathrm{yr})$ and 100 percent NPK + Zn $(0.092 \mathrm{tC} / \mathrm{ha} / \mathrm{yr})$ over a 36 -year period. SOC sequestration from sulfur (secondary nutrient) enriched $\mathrm{N}$ and NPK fertilizers was higher than in the non-sulfur enriched N and NPK fertilizer (Malhi, Nyborg, and Soon,2010; Giweta et al., 2014). While the focus of this chapter is mineral fertilizers, it is important to note that, higher SOC sequestration (on average $>15$ percent higher) is achievable when application of mineral fertilizer/s is combined with organic resource management, such as manure or crop residue application incorporation relative to sole application of mineral fertilizers (Hu et al., 2015; Mandal et al., 2007; Brar et al., 2015). 
Table 59. Effects of soil nutrient amendments with mineral fertilizers on soil organic carbon sequestration

\begin{tabular}{|c|c|c|c|c|c|c|c|c|c|}
\hline Location & $\begin{array}{l}\text { Climate } \\
\text { zone }\end{array}$ & Soil type & Intervention & $\begin{array}{l}\text { Baseline C } \\
\text { stock } \\
\text { (tC/ha) }\end{array}$ & $\begin{array}{l}\text { Additional C } \\
\text { storage } \\
\text { (tC/ha/yr) }\end{array}$ & $\begin{array}{l}\text { Duration } \\
\text { (Years) }\end{array}$ & $\begin{array}{l}\text { Depth } \\
(\mathrm{cm})\end{array}$ & $\begin{array}{l}\text { Cropping } \\
\text { system }\end{array}$ & Reference \\
\hline \multirow{5}{*}{ Delhi, India } & \multirow{5}{*}{$\begin{array}{l}\text { Humid sub- } \\
\text { tropical }\end{array}$} & \multirow{5}{*}{$\begin{array}{l}\text { Typic Haplu- } \\
\text { stept }\end{array}$} & $\begin{array}{l}50 \text { percent NPK (130 kg } \\
\mathrm{N} / \mathrm{ha}, 35.2 \mathrm{~kg} \mathrm{P} / \mathrm{ha}, 41.5 \\
\mathrm{~kg} \mathrm{~K} / \mathrm{ha})\end{array}$ & 49 & 0.104 & \multirow{5}{*}{32} & \multirow{5}{*}{$0-45$} & \multirow{5}{*}{$\begin{array}{l}\text { Maize- } \\
\text { wheat- } \\
\text { cowpea }\end{array}$} & \multirow{5}{*}{$\begin{array}{l}\text { Rudrappa et } \\
\text { al. (2005) }\end{array}$} \\
\hline & & & $\begin{array}{l}100 \text { percent NPK ( } 260 \\
\mathrm{~kg} \mathrm{~N} / \mathrm{ha}, 70.4 \mathrm{~kg} \mathrm{P} / \mathrm{ha} \text {, } \\
83 \mathrm{~kg} \mathrm{~K} / \mathrm{ha})\end{array}$ & 49 & 0.187 & & & & \\
\hline & & & $\begin{array}{l}150 \text { percent NPK ( } 390 \\
\mathrm{~kg} \mathrm{~N} / \mathrm{ha}, 105.6 \mathrm{~kg} \mathrm{P} / \mathrm{ha} \text {, } \\
124.5 \mathrm{~kg} \mathrm{~K} / \mathrm{ha})\end{array}$ & 49 & 0.476 & & & & \\
\hline & & & $\begin{array}{l}100 \text { percent NP ( } 260 \\
\mathrm{~kg} \mathrm{~N} / \mathrm{ha}, 70.4 \mathrm{~kg} \mathrm{P} / \mathrm{ha})\end{array}$ & 49 & 0.142 & & & & \\
\hline & & & $\begin{array}{l}100 \text { percent } \mathrm{N}(260 \mathrm{~kg} \\
\mathrm{N} / \mathrm{ha})\end{array}$ & 49 & 0.106 & & & & \\
\hline \multirow{2}{*}{$\begin{array}{l}\text { Samsstaoir, } \\
\text { Iceland }\end{array}$} & \multirow{3}{*}{$\begin{array}{l}\text { Cold tempe- } \\
\text { rate }\end{array}$} & \multirow{3}{*}{$\begin{array}{l}\text { Silandic } \\
\text { Andosol }\end{array}$} & $\begin{array}{l}\text { PK treatment: } \\
\text { N: None } \\
\text { P: } 29.5 \mathrm{~kg} P / \mathrm{ha} \\
\mathrm{K}: 62.3 \mathrm{~kg} \mathrm{~K} / \mathrm{ha}\end{array}$ & 125 & 0.036 & \multirow{3}{*}{36} & \multirow{3}{*}{$0-20$} & \multirow{3}{*}{ Hay field } & \multirow{3}{*}{$\begin{array}{l}\text { Áskelsdóttir } \\
(2012)\end{array}$} \\
\hline & & & $\begin{array}{l}\text { NPK treatment } \\
\mathrm{N}: 180 \mathrm{~kg} \mathrm{~N} / \mathrm{ha} \\
\mathrm{P}: 29.5 \mathrm{~kg} \mathrm{P} / \mathrm{ha} \\
\mathrm{K}: 62.3 \mathrm{~kg} \mathrm{~K} / \mathrm{ha}\end{array}$ & 121 & 0.369 & & & & \\
\hline Akureyi, Iceland & & & PK treatment: & 110 & 0.147 & & & & \\
\hline
\end{tabular}




\begin{tabular}{|c|c|c|c|c|c|c|c|c|c|}
\hline Location & $\begin{array}{l}\text { Climate } \\
\text { zone }\end{array}$ & Soil type & Intervention & $\begin{array}{l}\text { Baseline C } \\
\text { stock } \\
\text { (tC/ha) }\end{array}$ & $\begin{array}{l}\text { Additional C } \\
\text { storage } \\
\text { (tC/ha/yr) }\end{array}$ & $\begin{array}{l}\text { Duration } \\
\text { (Years) }\end{array}$ & $\begin{array}{l}\text { Depth } \\
(\mathrm{cm})\end{array}$ & $\begin{array}{l}\text { Cropping } \\
\text { system }\end{array}$ & Reference \\
\hline & & & $\begin{array}{l}\text { N: none } \\
\mathrm{P}: 23.6 \mathrm{~kg} \mathrm{P} / \mathrm{ha} \\
\mathrm{K}: 79.7 \mathrm{~kg} \mathrm{~K} / \mathrm{ha}\end{array}$ & & & & & & \\
\hline & & & $\begin{array}{l}\text { NPK treatment: } \\
\text { N: } 55 \mathrm{~kg} \mathrm{~N} / \mathrm{ha} \\
\mathrm{P}: 23.6 \mathrm{~kg} \mathrm{P} / \mathrm{ha} \\
\mathrm{K}: 79.7 \mathrm{~kg} \mathrm{~K} / \mathrm{ha}\end{array}$ & 113 & 0.116 & & & & \\
\hline \multirow{2}{*}{$\begin{array}{l}\text { Skriðuklau-stur, } \\
\text { Iceland }\end{array}$} & & \multirow{2}{*}{ Glyic Andosol } & $\begin{array}{l}\text { PK treatment: } \\
\mathrm{N}: \text { none } \\
\mathrm{P}: 30.6 \mathrm{~kg} \mathrm{P} / \mathrm{ha} \\
\mathrm{K}: 74.7 \mathrm{~kg} \mathrm{~K} / \mathrm{ha}\end{array}$ & 86.9 & -0.167 & & & & \\
\hline & & & $\begin{array}{l}\text { NPK treatment: } \\
\text { N: } 120 \mathrm{~kg} \mathrm{~N} / \mathrm{ha} \\
\mathrm{P}: 30.6 \mathrm{~kg} \mathrm{P} / \mathrm{ha} \\
\mathrm{K}: 74.7 \mathrm{~kg} \mathrm{~K} / \mathrm{ha}\end{array}$ & 78.9 & 0.202 & & & & \\
\hline \multirow{3}{*}{ Punjab, India } & \multirow{3}{*}{$\begin{array}{l}\text { Hot-summer } \\
\text { Medite- } \\
\text { rranean }\end{array}$} & \multirow{3}{*}{$\begin{array}{l}\text { Typic } \\
\text { Ustochrepts }\end{array}$} & $\begin{array}{l}50 \text { percent NPK (75 } \\
\mathrm{kgN} / \mathrm{ha}, 16.35 \mathrm{kgP} / \mathrm{ha} \text {, } \\
15.6 \mathrm{kgK} / \mathrm{ha})\end{array}$ & 5.70 & 0.075 & \multirow{3}{*}{36} & \multirow{3}{*}{$0-15$} & \multirow{3}{*}{$\begin{array}{l}\text { Maize- } \\
\text { Wheat- } \\
\text { Cowpea }\end{array}$} & \multirow{3}{*}{$\begin{array}{l}\text { Brar et al. } \\
(2015)\end{array}$} \\
\hline & & & $\begin{array}{l}150 \text { percent NPK ( } 225 \\
\mathrm{kgN} / \mathrm{ha}, 49.05 \mathrm{kgP} / \mathrm{ha} \text {, } \\
46.8 \mathrm{kgK} / \mathrm{ha})\end{array}$ & 4.90 & 0.106 & & & & \\
\hline & & & $\begin{array}{l}100 \text { percent NPK (150 } \\
\mathrm{kg} \mathrm{N}, 32.70 \mathrm{~kg} \mathrm{P} \text { and } \\
31.20 \mathrm{~kg} \mathrm{~K} / \mathrm{ha})\end{array}$ & 5.00 & 0.103 & & & & \\
\hline
\end{tabular}




\begin{tabular}{|c|c|c|c|c|c|c|c|c|c|}
\hline Location & $\begin{array}{l}\text { Climate } \\
\text { zone }\end{array}$ & Soil type & Intervention & $\begin{array}{l}\text { Baseline C } \\
\text { stock } \\
\text { (tC/ha) }\end{array}$ & $\begin{array}{l}\text { Additional C } \\
\text { storage } \\
\text { (tC/ha/yr) }\end{array}$ & $\begin{array}{l}\text { Duration } \\
\text { (Years) }\end{array}$ & $\begin{array}{l}\text { Depth } \\
(\mathrm{cm})\end{array}$ & $\begin{array}{l}\text { Cropping } \\
\text { system }\end{array}$ & Reference \\
\hline & & & $\begin{array}{l}100 \text { percent NPK+Zn } \\
(150 \mathrm{~kg} \mathrm{~N}, 32.70 \mathrm{~kg} P \\
\text { and } 31.20 \mathrm{~kg} \mathrm{~K} / \mathrm{ha}+)\end{array}$ & 5.30 & 0.092 & & & & \\
\hline & & & $\begin{array}{l}100 \text { percent NP (150 } \\
\mathrm{kgN} / \mathrm{ha}, 32.70 \mathrm{~kg} \mathrm{P} / \mathrm{ha})\end{array}$ & 4.20 & 0.108 & & & & \\
\hline & & & $\begin{array}{l}100 \text { percent } \mathrm{N}(150 \\
\mathrm{kgN} / \mathrm{ha})\end{array}$ & 4.20 & 0.097 & & & & \\
\hline & & & Control & 6.10 & 0.033 & & & & \\
\hline \multirow{4}{*}{ Wuchang, China } & \multirow{4}{*}{$\begin{array}{l}\text { Temperate } \\
\text { humid }\end{array}$} & \multirow{4}{*}{ Albic Luvisol } & Control & \multirow{4}{*}{41.1} & 0.27 & \multirow{4}{*}{30} & \multirow{4}{*}{$0-20$} & \multirow{4}{*}{ Wheat-Rice } & \multirow{4}{*}{$\begin{array}{l}\text { Hu et al. } \\
\text { (2015) }\end{array}$} \\
\hline & & & $\mathrm{N}(60 \mathrm{~kg} \mathrm{~N} / \mathrm{ha})$ & & 0.33 & & & & \\
\hline & & & $\begin{array}{l}\text { NP (60 kg N/ha, } 13 \mathrm{~kg} \\
\mathrm{P} / \mathrm{ha})\end{array}$ & & 0.30 & & & & \\
\hline & & & $\begin{array}{l}\text { NPK (60 kg N/ha, } 13 \text { kg } \\
\text { P/ha, } 50 \mathrm{~kg} \mathrm{K/ha)}\end{array}$ & & 0.46 & & & & \\
\hline $\begin{array}{l}\text { Gayeshpur-1, } \\
\text { India }\end{array}$ & \multirow{2}{*}{$\begin{array}{l}\text { Hot Sub- } \\
\text { humid } \\
\text { Tropical }\end{array}$} & $\begin{array}{l}\text { Inceptisol } \\
\text { Typic } \\
\text { Haplustept }\end{array}$ & NPK & - & 1.91 & 7 & \multirow{2}{*}{$0-20$} & $\begin{array}{l}\text { Rice- } \\
\text { Mustard- } \\
\text { Sesame }\end{array}$ & \multirow{2}{*}{$\begin{array}{l}\text { Mandal et al. } \\
(2007)\end{array}$} \\
\hline Mohanpur, India & & $\begin{array}{l}\text { Inceptisol } \\
\text { Aeric } \\
\text { Haplaquept }\end{array}$ & NPK & - & 0.27 & 19 & & $\begin{array}{l}\text { Rice-Wheat- } \\
\text { Fallow }\end{array}$ & \\
\hline
\end{tabular}




\begin{tabular}{|c|c|c|c|c|c|c|c|c|c|}
\hline Location & $\begin{array}{l}\text { Climate } \\
\text { zone }\end{array}$ & Soil type & Intervention & $\begin{array}{l}\text { Baseline C } \\
\text { stock } \\
\text { (tC/ha) }\end{array}$ & $\begin{array}{l}\text { Additional C } \\
\text { storage } \\
\text { (tC/ha/yr) }\end{array}$ & $\begin{array}{l}\text { Duration } \\
\text { (Years) }\end{array}$ & $\begin{array}{l}\text { Depth } \\
\text { (cm) }\end{array}$ & $\begin{array}{l}\text { Cropping } \\
\text { system }\end{array}$ & Reference \\
\hline $\begin{array}{l}\text { Gayeshpur-II, } \\
\text { India }\end{array}$ & & $\begin{array}{l}\text { Inceptisol } \\
\text { Typic } \\
\text { Haplustept }\end{array}$ & NPK & - & 0.13 & 20 & & $\begin{array}{l}\text { Rice-Fallow- } \\
\text { Berseem }\end{array}$ & \\
\hline Barrakpur, India & & $\begin{array}{l}\text { Inceptisol } \\
\text { Typic } \\
\text { Haplustept }\end{array}$ & NPK & - & 0.11 & 34 & & $\begin{array}{l}\text { Rice-Wheat- } \\
\text { Jute }\end{array}$ & \\
\hline \multirow{2}{*}{ Cuttack, India } & & $\begin{array}{l}\text { Inceptisol } \\
\text { Typic } \\
\text { Endoaquept }\end{array}$ & NPK & - & 0.28 & \multirow{2}{*}{36} & & $\begin{array}{l}\text { Rice-Fallow- } \\
\text { Rice }\end{array}$ & \\
\hline & & $\begin{array}{l}\text { Hyper- } \\
\text { thermic } \\
\text { Endoaquept }\end{array}$ & NPK & - & 0.23 & & & Rice-Rice & \\
\hline Alberta, Canada & $\begin{array}{l}\text { Humid } \\
\text { continental }\end{array}$ & Gray Luvisol & NPKS vs. NPK & & $\begin{array}{c}\text { (i) Both NPKS \& } \\
\text { NPK >0 } \\
\text { (ii) NPKS = } \\
0.11 \mathrm{tC} / \mathrm{ha} / \mathrm{yr}> \\
\text { NPK }\end{array}$ & 28 & $0-15$ & $\begin{array}{l}\text { Wheat-oat- } \\
\text { barley-hay- } \\
\text { hay }\end{array}$ & $\begin{array}{l}\text { Giweta et al. } \\
(2014)\end{array}$ \\
\hline $\begin{array}{l}\text { Saskatchewan, } \\
\text { Canada }\end{array}$ & $\begin{array}{l}\text { Humid } \\
\text { continental }\end{array}$ & $\begin{array}{l}\text { Dark Grey } \\
\text { Cherno-zemic }\end{array}$ & $\begin{array}{l}\text { Nitrogen + Sulfur (NS) } \\
\text { vs. Nitrogen (N) alone } \\
\text { comparison }\end{array}$ & - & $\begin{array}{c}\mathrm{NS}=0.26 \\
\mathrm{tC} / \mathrm{ha} / \mathrm{yr}>\mathrm{N}\end{array}$ & 13 & $0-10$ & Grass forage & $\begin{array}{l}\text { Malhi, Nyborg, } \\
\text { and Soon, } \\
\text { (2010) }\end{array}$ \\
\hline \multirow{2}{*}{ Yangtze, China } & \multirow[b]{2}{*}{ Subtropical } & \multirow{2}{*}{$\begin{array}{l}\text { Eutyic } \\
\text { Cambisol }\end{array}$} & Control & - & 0.031 & \multirow[b]{2}{*}{33} & \multirow[b]{2}{*}{$0-15$} & \multirow{2}{*}{$\begin{array}{l}\text { Rice-Wheat } \\
\text { \& Bean }\end{array}$} & \multirow{2}{*}{$\begin{array}{l}\text { Wang et al. } \\
\text { (2018) }\end{array}$} \\
\hline & & & $\begin{array}{l}\text { NP (168 to } 225 \mathrm{~kg} \mathrm{~N} / \mathrm{ha} \\
\text { depending on crop in }\end{array}$ & - & 0.078 & & & & \\
\hline
\end{tabular}




\begin{tabular}{|c|c|c|c|c|c|c|c|c|c|}
\hline Location & $\begin{array}{l}\text { Climate } \\
\text { zone }\end{array}$ & Soil type & Intervention & $\begin{array}{l}\text { Baseline C } \\
\text { stock } \\
\text { (tC/ha) }\end{array}$ & $\begin{array}{l}\text { Additional C } \\
\text { storage } \\
\text { (tC/ha/yr) }\end{array}$ & $\begin{array}{l}\text { Duration } \\
\text { (Years) }\end{array}$ & $\begin{array}{l}\text { Depth } \\
(\mathrm{cm})\end{array}$ & $\begin{array}{l}\text { Cropping } \\
\text { system }\end{array}$ & Reference \\
\hline & & & $\begin{array}{l}\text { the rotation, } 49 \mathrm{~kg} \\
\mathrm{~N} / \mathrm{ha} \text { ) }\end{array}$ & & & & & $\begin{array}{l}\text { Maize } \\
\text { rotations }\end{array}$ & \\
\hline & & & $\begin{array}{l}\text { NPK (168 to } 225 \mathrm{~kg} \\
\text { N/ha depending on } \\
\text { crop in the rotation, } 49 \\
\mathrm{~kg} \mathrm{~N} / \mathrm{ha}, 155 \mathrm{~kg} \mathrm{~K} / \mathrm{ha} \text { ) }\end{array}$ & - & 0.110 & & & & \\
\hline Huang-Hai, & Sub humid & Vertisol & Control & & 0.08 & 34 & $0-20$ & Wheat- & Guo et al., \\
\hline & & & NPK & & 0.16 & & & rotation & (2019) \\
\hline \multirow{9}{*}{$\begin{array}{l}\text { Kansas, United } \\
\text { States of } \\
\text { America }\end{array}$} & \multirow{9}{*}{$\begin{array}{l}\text { Sub-tropical } \\
\text { steppe }\end{array}$} & \multirow{9}{*}{$\begin{array}{l}\text { Mesic Aridic } \\
\text { Haplustolls }\end{array}$} & Control & 36.8 & & \multirow{6}{*}{50} & \multirow{9}{*}{$0-30$} & \multirow{9}{*}{ Maize } & \multirow{9}{*}{$\begin{array}{l}\text { Blanco-Canqui } \\
\text { and Schlegel } \\
\text { (2013) }\end{array}$} \\
\hline & & & $45 \mathrm{~kg} \mathrm{~N} / \mathrm{ha}$ & 36.8 & 0.066 & & & & \\
\hline & & & $90 \mathrm{~kg} \mathrm{~N} / \mathrm{ha}$ & 36.8 & 0.079 & & & & \\
\hline & & & $134 \mathrm{~kg} \mathrm{~N} / \mathrm{ha}$ & 36.8 & 0.085 & & & & \\
\hline & & & $179 \mathrm{~kg} \mathrm{~N} / \mathrm{ha}$ & 36.8 & 0.117 & & & & \\
\hline & & & $224 \mathrm{~kg} \mathrm{~N} / \mathrm{ha}$ & 36.8 & 0.162 & & & & \\
\hline & & & O kg P/ha & 39.1 & & \multirow{3}{*}{20} & & & \\
\hline & & & $20 \mathrm{~kg} \mathrm{P} / \mathrm{ha}$ & 39.1 & 0.049 & & & & \\
\hline & & & $40 \mathrm{~kg} P / \mathrm{ha}$ & 39.1 & 0.068 & & & & \\
\hline
\end{tabular}




\section{Other benefits of the practice}

In addition to the role played by the fertilizers on soil organic carbon sequestration, there are various other benefits associated with fertilizer use. These range from increased productivity through crop biomass and harvest yields, soil fertility improvement and increased food and feed quality through grain nutrient concentration among others.

\subsection{Improvement of soil properties}

\section{Soil chemical properties}

Fertilizers play a crucial role in improving the soil chemical properties and processes. They replace deficient nutrients (macro, secondary and the micronutrients). Among other functions, the basic cations $\left(\mathrm{K}^{+}, \mathrm{Ca}^{2+}, \mathrm{Mg}^{2+}\right)$ that are added with fertilizers are crucial for managing soil acidification. High ammoniacal nitrogen and sulfate fertilizers produce acidic reactions with neutralizing effect of soil alkalinity.

\section{Soil biological properties}

Most of the biological benefits of fertilizer, including the micro-, meso- and macro-fauna activities and processes are associated with soil organic matter (Haynes and Naidu, 1998). Long-term studies have shown that application of NP and NPK fertilizers in addition to secondary and micronutrients improves soil enzymatic activities, bacteria, fungi and actinomycytes populations (Ling et al., 2014). The functional gene array-based analysis revealed for a long-term fertilization with N, NP, NK and NPK fertilizers the overall microbial functional structures could be changed by increasing the diversity and abundance of most genes involved in C, N, P and S cycling, especially for interventions with NK and NPK (Su et al., 2014). Detrimental effect of N fertilization on soil microbial functional genes can be mitigated by the addition of P fertilizer in the P-limited paddy soil they studied (Su et al., 2014), suggesting that balanced chemical fertilization is beneficial to the soil microbial community and its functions. Additionally, in $\mathrm{P}$ limited soils, external application of $\mathrm{P}$ fertilizers is an essential ingredient for Rhizobium bacteria to convert atmospheric $\mathrm{N}$ into an ammonium- $\mathrm{N}$ form that is usable by plants. Kihara et al. (2020) highlight various studies that have shown positive effects of micronutrients on soil biological properties and processes. The application of moderate amounts of boron up to $3 \mathrm{~kg} / \mathrm{ha}$ increased soil fungal and bacterial populations, and phosphatase and dehydrogenase enzyme activities by between 18 and 34 percent during different growth periods relative to no application (Bilen, Bilen and Bardhan, 2011). Application of $0.5 \mathrm{mg} / \mathrm{kg}$ of Mo increased nitrogenase enzyme activity (71 percent) and root nodule number (63 percent) (Alam et al., 2015), while application of moderate $\mathrm{Zn} \mathrm{(15} \mathrm{kg} \mathrm{Zn/ha)} \mathrm{enhanced} \mathrm{nodule} \mathrm{indices} \mathrm{of}$ cowpea by at least 38 percent (Upadhyay and Singh, 2016).

\section{Soil physical properties}

Mineral fertilizer and soil organic carbon sequestration affects soil physical properties. Increasing soil organic carbon is associated with better soil water holding capacity and soil structure. Application of $\mathrm{P}$ in combination with $\mathrm{N}$ significantly increased the hydraulic conductivity in comparison to where $\mathrm{N}$ was applied alone (Pant and Ram, 2018). In a long-term study (Hati et al., 2006), within the 0-15 cm depth, the water stable aggregates in the mineral NPK plots were 49 percent against the unfertilized control 45.4 percent. The saturated hydraulic conductivity was higher in the mineral fertilizer NPK $\left(10.5 \times 10^{-6} \mathrm{~m} / \mathrm{sec}\right)$ against absolute control $\left(8.6 \times 10^{-6}\right.$ 
$\mathrm{m} / \mathrm{sec}$ ) treatments (Hati et al., 2006) and the bulk density was higher in the unfertilized control compared to mineral fertilizer NPK. Similarly, in a 90-year experiment (Christensen, 1988), the increase in organic matter content induced by NPK applications resulted in a decrease in measured soil bulk density and a concomitant increase in total porosity with a tendency for an increase in the volume of pores in all size ranges. The root length density was also higher in the NPK than in the unfertilized control. It is important to note that in most cases inclusion of organic resources like FYM resulted in better soil physical properties than compared to mineral fertilizers.

\subsection{Minimization of threats to soil functions}

Fertilizers minimize a range of soil threats. Table 60 provides a summary of the roles played by fertilizers to minimize soil threats.

Table 60. Soil threats

\begin{tabular}{|c|c|}
\hline Soil threats & \\
\hline Soil erosion & $\begin{array}{l}\text { Balanced fertilizers improve aboveground and belowground biomass production. The } \\
\text { improved roots hold soil together, reducing soil losses by wind and rainwater. Further, } \\
\text { the improved aboveground biomass associated with better supply of nutrients provide } \\
\text { better soil cover to reduce soil losses by wind erosion. Organic matter holds soil } \\
\text { particles together reducing their breakage and transportation by water. Increasing soil } \\
\text { organic matter from } 1 \text { to } 3 \text { percent can reduce erosion by } 20 \text { to } 33 \text { percent because of } \\
\text { increased water infiltration and stable soil aggregate formation (Funderburg, 2001). }\end{array}$ \\
\hline $\begin{array}{l}\text { Nutrient } \\
\text { imbalance and } \\
\text { cycles }\end{array}$ & $\begin{array}{l}\text { Negative nutrient balances are common in many agricultural fields where nutrient } \\
\text { removal from the fields exceed nutrient inputs. In sub-Sahara Africa the negative } \\
\text { balances for } \mathrm{N}, \mathrm{P} \text { and } \mathrm{K} \text { are prevalent. There is paucity of data on nutrient balances of } \\
\text { secondary and micro-nutrient, but the crop nutrient response to their application imply } \\
\text { their deficiency in certain regions. Balanced fertilizer application and soil organic carbon } \\
\text { sequestration boost nutrient balances and cycles. The applied nutrients replace } \\
\text { nutrients that are taken up by crop or lost from croplands thus managing the soil } \\
\text { nutrient balances. Unavailability of nutrients due to fixation is common in acidic soils } \\
\text { but boosting the basic cation supply in the soil through application of fertilizers that } \\
\text { supply soils with basic cations }\left(\mathrm{K}^{+}, \mathrm{Ca}^{2+}, \mathrm{Mg}^{2+}\right) \text { reduces soil acidity, releasing most of the } \\
\text { nutrients into the soil solution for uptake by crops. In soils depleted of basic cations, } \\
\text { most of the macronutrients are held in insoluble aluminum and iron complexes that } \\
\text { render them unavailable for uptake by crops (Havlin et al., 2005). }\end{array}$ \\
\hline $\begin{array}{l}\text { Soil salinization } \\
\text { and } \\
\text { alkalinization }\end{array}$ & $\begin{array}{l}\text { Many studies emphasize the importance of limiting the amount of fertilizer salts } \\
\text { applied to prevent further salinization (Cuevas et al., 2019). Diminishing the amounts of } \\
\text { fertilizers applied as well as implementing fractional fertilization programs may } \\
\text { contribute to halting secondary salinization. Fertilizer selection is another key method } \\
\text { for limiting secondary salinization. For example, in selection of potassium fertilizers, }\end{array}$ \\
\hline
\end{tabular}




\begin{tabular}{|c|c|}
\hline \multicolumn{2}{|l|}{ Soll threats } \\
\hline & $\begin{array}{l}\text { potassium chloride }(\mathrm{KCL}) \text { has more salinization effect due to accumulation salts in the } \\
\text { soil, than potassium sulfate. The salt tolerance of the crops could be improved by the } \\
\text { addition of certain nutrients. The addition of } \mathrm{NO}_{3}, \mathrm{Ca}^{2+}, \mathrm{K}, \mathrm{P} \text {, salicylic acid, and silicon } \\
\text { (Si) to the saline medium or in foliar application improves salt tolerance of numerous } \\
\text { vegetable crops such as tomato, pepper, eggplant, melon, bean, strawberry. Soil } \\
\text { alkalization leads to high soil pH. Acidifying fertilizers have a potential for reducing soil } \\
\mathrm{pH} \text { to lower level that is more favorable for Alkali intolerant crops. The common } \\
\text { fertilizers with Alkali ameliorating effects include the ammonium fertilizers like } \\
\text { Diammonium phosphate, monoammonium phosphate, and ammonium sulfate. Other } \\
\text { fertilizers with alkaline soil ameliorating capability are the sulfur-based fertilizers like } \\
\text { the elemental sulfur }(\mathrm{S}) \text {, Aluminum sulfate }\left(\mathrm{Al}_{2}\left(\mathrm{SO}_{4}\right)_{3}\right) \text { and iron sulfate }\left(\mathrm{FeSO}_{4}\right) \text {. }\end{array}$ \\
\hline $\begin{array}{l}\text { Soil } \\
\text { contamination / } \\
\text { pollution }\end{array}$ & No studies on positive effects of fertilizers on soil contaminant. \\
\hline Soil acidification & $\begin{array}{l}\text { Continuous cropping and crop uptake of basic cations }\left(\mathrm{K}^{+}, \mathrm{Ca}^{2+}, \mathrm{Mg}^{2+}\right) \text { without } \\
\text { replacement of is one of the main causes of acidification as hydrogen }\left(\left(\mathrm{H}^{+}\right) \text {and }\right. \\
\text { aluminium }\left(\mathrm{Al}^{3+}\right) \text { ions dominate the soil solution. Balanced fertilizers, which contain the } \\
\text { basic cations are crucial for managing the rates of soil acidification }(\mathrm{Bijay}, 2 \mathrm{O} \text { ) }\end{array}$ \\
\hline $\begin{array}{l}\text { Soil biodiversity } \\
\text { loss }\end{array}$ & $\begin{array}{l}\text { Under arable cropping systems fertilizer applications increase soil microbial population } \\
\text { and biomass and the enzymatic activities in the soil. Especially when left on the soil, } \\
\text { the enhanced crop belowground and aboveground residue yield play a critical role in } \\
\text { reversing the decline of the soil fauna (Kibblewhite, Ritz and Swift, 2008; Lal, 2015). }\end{array}$ \\
\hline Soil compaction & $\begin{array}{l}\text { The resulting effect of fertilizers on soil organic matter and on microbial activities is } \\
\text { crucial for reducing soil compaction. Findings suggest that practices promoting ground } \\
\text { cover and continuous roots, both of which improve soil structure, also reduce soil } \\
\text { compaction, reduce bulk density and enhance water infiltration rates (Soane, 1990; } \\
\text { Larson et al., 1994). The increased soil organic carbon resulting from balanced } \\
\text { fertilization is linked to reduction in soil compaction. }\end{array}$ \\
\hline $\begin{array}{l}\text { Soil water } \\
\text { management }\end{array}$ & $\begin{array}{l}\text { Soil organic matter resulting from fertilizer use improves soil aggregation and structure, } \\
\text { improving soil's ability to take up and hold water. Several studies have shown a positive } \\
\text { correlation between soil nitrogen level and water use efficiency. Similarly, applying } \\
\text { phosphorus fertilizers increases root density and rooting depth and amount of water } \\
\text { available to plants is increased. Phosphorus, in a balanced soil fertility program } \\
\text { increases water use efficiency and supports crops to achieve optimal performance } \\
\text { under limited moisture conditions. The uptake of water by plant roots and transport of } \\
\text { the water to other parts of the plant are significantly influenced by potassium. Balanced } \\
\text { use of fertilizers that ensures crop access to the macronutrients, secondary nutrients } \\
\text { and the micronutrients is the best bet for optimized water use efficiency (Drechsel et } \\
\text { al., 2015). }\end{array}$ \\
\hline
\end{tabular}




\subsection{On crop production}

Globally, over 40 percent of crop yield is attributable to inorganic fertilizer nutrient inputs (Stewart et al., 2005). As shown in the Table 61 , the grain yield of most cereals could be doubled by applying the recommended rates of macronutrients as mineral fertilizers. For most soils $\mathrm{N}$ is the most limiting nutrient, followed by $\mathrm{P}$ and then $\mathrm{K}$ and in general the crop yield response is highest for these nutrients. Of the three secondary nutrients, the positive response of crop yields to sulfur is the most common. Sillanpää (1990) estimated that of the important agricultural soils of the world, 49 percent are deficient in zinc ( $\mathrm{Zn}), 31$ percent deficient in boron (B), 15 percent deficient in molybdenum (Mo), 14 percent deficient in copper (Cu), 10 percent deficient in manganese $(\mathrm{Mn})$ and 3 percent deficient in iron $(\mathrm{Fe})$. Addition of micronutrients $(\mathrm{S}, \mathrm{Zn}$ and $\mathrm{B})$ in customized fertilizer blends (combining $\mathrm{N}, \mathrm{P}$ and $\mathrm{K}$ ) resulted in 50 percent increase in yields $(2.4 \mathrm{t} / \mathrm{ha})$ over commonly recommended NPS fertilizer (81N, 14P, 6S) pointing to increased utilization of $\mathrm{N}$ and $\mathrm{P}$ at higher rates where response curve would ordinarily level off (van Vugt, 2018).

Table 61. Effect of balanced and unbalanced fertilizers on yields of various crops in China, India and Kenya

\begin{tabular}{|c|c|c|c|c|}
\hline Country & Crop & Fertilizer formulation & Yield (t/ha) & Reference \\
\hline \multirow{12}{*}{ China } & \multirow{3}{*}{ Rice } & Control & 3.3 & \multirow{3}{*}{ Zhang et al. (2010) } \\
\hline & & NP & 5.4 & \\
\hline & & NPK & 6.0 & \\
\hline & \multirow{3}{*}{ Wheat } & NP & 1.5 & \multirow{3}{*}{$\begin{array}{l}\text { Zhang et al. } \\
\text { (2008) }\end{array}$} \\
\hline & & NPK & 1.6 & \\
\hline & & NPKS & 1.8 & \\
\hline & \multirow{3}{*}{ Broad bean } & Control & 0.8 & \multirow{6}{*}{ Wang et al. (2018) } \\
\hline & & NP & 1.2 & \\
\hline & & NPK & 1.8 & \\
\hline & \multirow{3}{*}{ Cotton } & Control & 1.6 & \\
\hline & & NP & 1.9 & \\
\hline & & NPK & 2.5 & \\
\hline \multirow{7}{*}{ India } & \multirow{4}{*}{ Rice } & Control & 2.4 & \multirow{7}{*}{$\begin{array}{l}\text { Hedge and Sarkar } \\
\text { (1992) }\end{array}$} \\
\hline & & $\mathrm{N}$ & 4.2 & \\
\hline & & NP & 4.4 & \\
\hline & & NPK & 4.7 & \\
\hline & \multirow{3}{*}{ Wheat } & Control & 1.2 & \\
\hline & & $\mathrm{N}$ & 2.3 & \\
\hline & & NP & 2.9 & \\
\hline
\end{tabular}




\begin{tabular}{|c|c|c|c|c|}
\hline Country & Crop & Fertilizer formulation & Yield (t/ha) & Reference \\
\hline & & NPK & 3.4 & \\
\hline & \multirow{3}{*}{ Potato } & Control & 8.3 & \multirow{3}{*}{$\begin{array}{l}\text { Banerjee et al. } \\
(2016)\end{array}$} \\
\hline & & NP & 24.5 & \\
\hline & & NPK & 28.2 & \\
\hline \multirow{3}{*}{ Kenya } & \multirow{3}{*}{ Maize } & Control & 2.8 & \multirow{3}{*}{$\begin{array}{l}\text { Njoroge et al. } \\
(2018)\end{array}$} \\
\hline & & NP & 5.1 & \\
\hline & & NPK & 5.5 & \\
\hline
\end{tabular}

It is difficult to establish the production yield increase attributable to most of the secondary and micronutrients. However, there are a lot of research examples showing increase in yield as a result of application of certain micronutrient fertilizers. In China, Jin, Ping and Shihua (2006) reported positive cereal and legume crop responses to $\mathrm{Cu}, \mathrm{Mn}, \mathrm{Fe}, \mathrm{B}$ and $\mathrm{Zn}$ of between 2 percent and 29 percent. Based on meta-analysis of 40 studies carried out between 1969 and 2013 in 14 sub-Sahara Africa countries, (Kihara et al., 2017) concluded that crop fertilization with sulfur and micronutrients increased maize yield by 20 percent, wheat yield by 27 percent and Rice yield by 14 percent over the macronutrient only fertilizer application. A follow-up study, (Kihara et al, 2020) indicated net benefits of maize production with secondary and micronutrients for 70 percent, 85 percent, 80 percent and 75 percent of the cases for: combined secondary and micronutrients, $\mathrm{Cu}, \mathrm{Zn}$ and S, respectively.

\subsection{Impacts of fertilizer use on greenhouse gases}

The higher biomass production is related to improved absorption of carbon dioxide $\left(\mathrm{CO}_{2}\right)$ from the atmosphere, part of which is sequestered in the soil as soil organic carbon. Application $\mathrm{N}$ fertilizer is inadvertently associated with increased emission of non- $\mathrm{CO}_{2}$ greenhouse gases especially $\mathrm{N}_{2} \mathrm{O}$ (see section 5.2 ), but best nutrient management practices can improve $\mathrm{N}$ use efficiency (NUE) reducing the amount of $\mathrm{N}$ that is transformed and emitted as $\mathrm{N}_{2} \mathrm{O}$. As fertilizers are key for food production, and the increasing world population needs to be fed from finite land resource, the key objective of climate change mitigation is to enhance carbon sequestration and reduce emission of non- $\mathrm{CO}_{2}$ greenhouse gases thus reducing the carbon footprint. Producing higher yields by appropriate use of fertilizers is one way to achieve reductions in GHG emissions indirectly by sparing natural areas like forests, grasslands and peatlands. Intensifying crop production can also enable the conversion of selected lands to forests for GHG mitigation, while supplying the world's need for food, fiber, and biofuel. Estimates show that $\mathrm{CH}_{4}$ emissions from global rice fields range from $18.3 \pm 0.1 \mathrm{Tg} \mathrm{CH}_{4} / \mathrm{yr}$ under intermittent irrigation to $38.8 \pm 1.0 \mathrm{Tg} \mathrm{CH}_{4} / \mathrm{yr}$ under continuous flooding (Zhang et al, 2016). Fertilizers helps to meet the rice demand from limited areas thus reducing the potential for increased $\mathrm{CH}_{4}$ emission if land area under rice was to be expanded to meet the global rice requirements. Recent advancement in production of slow $\mathrm{N}$ release fertilizers, urease inhibitors (UIs) and nitrification inhibitors (NIs) improve nitrogen use efficiency thus reducing the amount of $\mathrm{N}_{2} \mathrm{O}$ emitted per kilogram of applied nitrogen fertilizers. 


\subsection{Socio-economic benefits}

A number of socioeconomic benefits can be attributed to fertilizers and their role on carbon sequestration and climate change. These include:

Improved farm incomes

Fertilizers improve crop yields. This is crucial for food security and improved returns on agricultural investment.

\section{Health nutritional benefits}

The nutrients provided by the fertilizers and taken up by plants support growth of nutritious food and feed for human and livestock. Ultimately, nutrients that are taken up by crops are taken up by humans and livestock through with food and feed. The mineral elements most commonly lacking in human diets are Fe, Zn, I, Se, Ca, $\mathrm{Mg}$ and $\mathrm{Cu}$ (White and Broadley, 2009). To a large extent, the low concentration of these nutrients in edible crops and low concentration of other nutritionally important minerals like $\mathrm{K}$ could be addressed through appropriate application of fertilizers (Bell and Dell, 2008). By 2001, over 3 billion people were suffering from micronutrient malnutrition globally (Welch, 2001). Deficiency of these elements is linked to various human health concerns; ranging from low immunity, bone weaknesses and less controllable hypertension among others (Bell and Dell, 2008; Prasad et al., 2016).

\section{Reduced cost due to lower crop disease incidences}

Adequate micronutrients deceases the severity of crop diseases. The number and severity of root and shoot diseases is increased by micronutrient deficiencies (Graham and Webb, 1991). For example, Zinc has been shown to suppress root rotting pathogens and root nematode infestation.

\section{Recovery of degraded land}

Land is a finite resource with a monetary and social value. Global estimates of total degraded area vary from less than 1 billion ha to over 6 billion ha (Gibbs and Salmon, 2015). The increase in soil organic matter as a result of soil nutrient replenishment by fertilizers is crucial for recovery of degraded croplands.

\section{Reduced water pollution}

Nutrient depleted soils can support only a limited level of vegetation and often the ground may become bare for a prolonged period of time. Bare soils are easily eroded by wind or rain leading to air and water pollution which can drive the air borne and water borne diseases Appropriate use of fertilizers can boost the ground cover and thus help to reduce chances of water pollution.

\section{Potential drawbacks to the practice}

The multifaceted benefits of inorganic fertilizers can be amplified when they are added to the soil in combination with organic resources like manure. Adopting good agronomy and improved seeds are also crucial for optimizing the benefits of fertilizer use. 


\title{
5.1. Tradeoffs with other threats to soil functions
}

\author{
Table 62. Soil threats
}

\begin{tabular}{|c|c|}
\hline Soil threats & \\
\hline $\begin{array}{l}\text { Soil salinization } \\
\text { and alkalinization }\end{array}$ & $\begin{array}{l}\text { Some fertilizers like potassium chloride and ammonium sulphate contain high } \\
\text { levels of potentially harmful salts (Bitew and Alemayehu, 2O17). Overuse and } \\
\text { misuse of these type of fertilizers could lead to buildup of soil salinity. Soil } \\
\text { alkalinity could be caused by accumulation of } \mathrm{Na}_{2} \mathrm{CO}_{3}, \mathrm{NaHCO}_{3}, \mathrm{CaCO}_{3} \text { or } \mathrm{MgCO}_{3} \\
\text { (Chen, Xing and Lan, 2012). }\end{array}$ \\
\hline $\begin{array}{l}\text { Soil } \\
\text { contamination } \\
\text { /pollution }\end{array}$ & 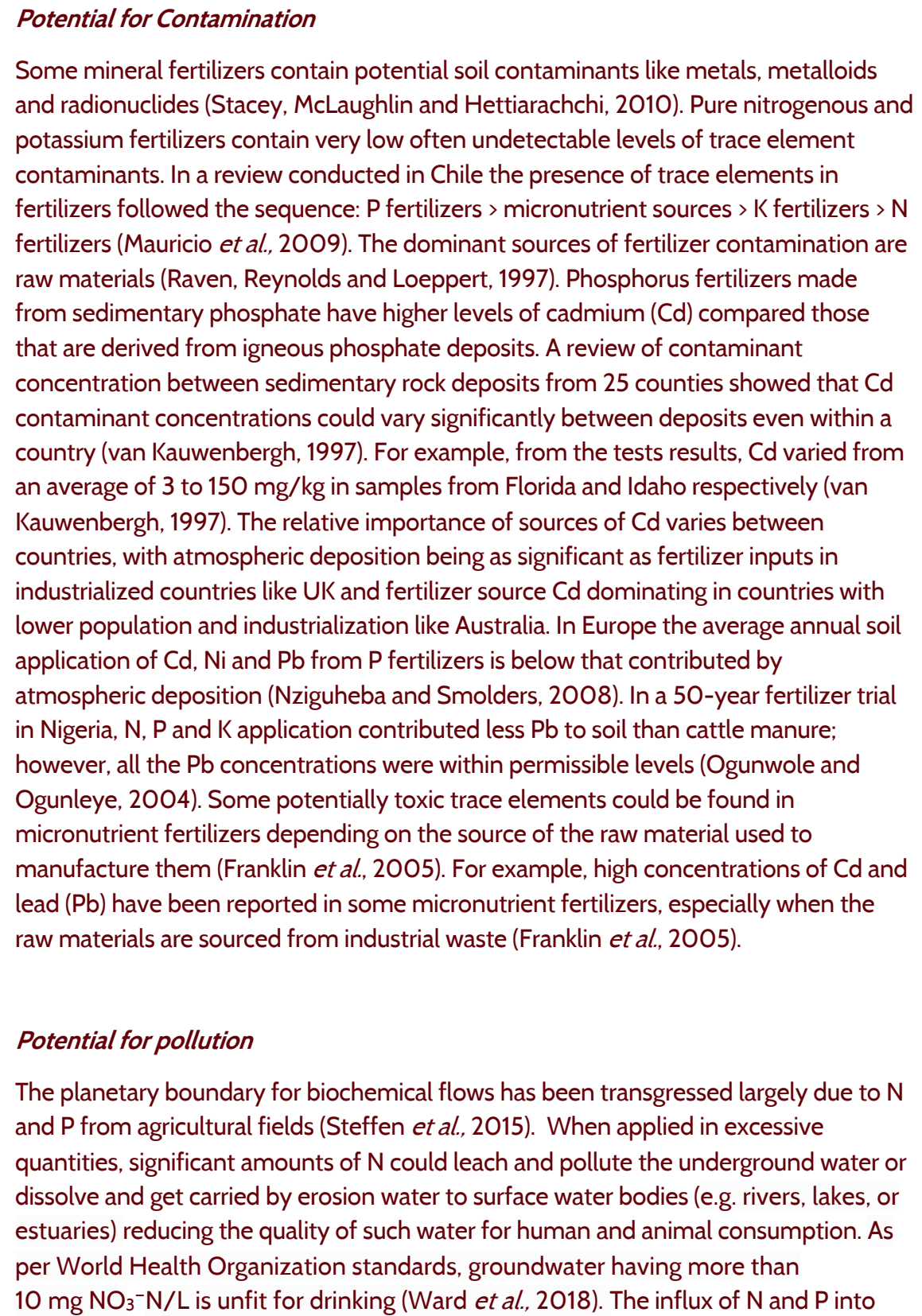 \\
\hline
\end{tabular}




\begin{tabular}{|c|c|}
\hline \multicolumn{2}{|l|}{ Soil threats } \\
\hline & $\begin{array}{l}\text { surface water bodies leads to eutrophication. One of the most conspicuous effect of } \\
\text { eutrophication is growth of harmful algal blooms, where excessive algae growth leads } \\
\text { to the production of toxins that are harmful to humans and ecosystems (Chislock et } \\
\text { al., 2013). When these dense algal blooms eventually die, microbial decomposition } \\
\text { severely depletes dissolved oxygen, creating a hypoxic or anoxic 'dead zone' lacking } \\
\text { sufficient oxygen to support most of the freshwater organisms (Chislock et al., 2013). }\end{array}$ \\
\hline Soil acidification & $\begin{array}{l}\text { Fertilizers could drive soil acidifications in various ways. Imbalanced fertilization } \\
\text { promotes enhanced crop growth and uptake of basic cations like } \mathrm{Ca}^{2+} \text { and their } \\
\text { substitution in the soil solution by protons (e.g. } \mathrm{H}^{+} \text {) causing soil acidification (Cai et al., } \\
\text { 2014). Nitrogen fertilizers, especially ammonium-based fertilizers are major } \\
\text { contributors to soil acidification (Getachew, Yirga and Erkossa, 2019). Ammonium } \\
\text { nitrogen is readily converted to nitrate and hydrogen ions in the soil. If nitrate is not } \\
\text { taken up by plants, it can leach away from the root zone leaving behind hydrogen ions } \\
\text { thereby increasing soil acidity. Examples of acidifying nitrogen fertilizer grades include: } \\
\text { urea, diammonium phosphate and ammonium nitrate (IPNI, 2016). In addition to } \\
\text { ammonium fertilizers, the sulfur enriched fertilizers like elemental sulfur, sulfur coated } \\
\text { urea, aluminums sulfate and ferric sulfate are also acidifying (IPNI, 2016). Phosphorus } \\
\text { fertilizers have less effect on soil pH than } \mathrm{N} \text { as lower rates of } \mathrm{P} \text { are applied and } \\
\text { acidification per kg phosphorus is less than for } \mathrm{N} \text {. Phosphoric acid is the most } \\
\text { acidifying of the phosphorus fertilizers (Bell and Mathesius, 2019). }\end{array}$ \\
\hline $\begin{array}{l}\text { Soil biodiversity } \\
\text { loss }\end{array}$ & $\begin{array}{l}\text { The negative effect of fertilizers on biodiversity has been observed in some studies. } \\
\text { For example, Gardi et al. (2008) reported a decline in abundance and diversity of soil } \\
\text { microarthropods following application of nitrogenous fertilizer to soil. }\end{array}$ \\
\hline
\end{tabular}

\subsection{Increases in greenhouse gas emissions}

Two key greenhouse gases associated with mineral fertilizers are $\mathrm{N}_{2} \mathrm{O}$ and $\mathrm{CO}_{2}$. Nitrogen fertilization is one of major factors contributing to anthropogenic $\mathrm{N}_{2} \mathrm{O}$ emissions from agricultural soils (Bouwman, 1996). The $\mathrm{N}_{2} \mathrm{O}$ emission increases with increasing $\mathrm{N}$ inputs and decreasing/low nitrogen use efficiency (NUE). Soil $\mathrm{pH}$, soil carbon, air filled pore spaces and water filled pore spaces are crucial controls/drivers for transformation of inorganic $\mathrm{N}$ to $\mathrm{N}_{2} \mathrm{O}$ (Bouwman, Boumans and Batjes, 2002; Mutegi et al., 2010; Wang et al., 2017). Of global concern among these factors is soil acidity (low pH soils) which is decreasing across most of the world croplands (Wang et al., 2017). $\mathrm{N}_{2} \mathrm{O}$ emission in acidic soils is more sensitive to changing $\mathrm{N}$ fertilization than that in alkaline soils. Based on 1104 measurements from 117 studies, Wang et al. (2017) observed that a 30 percent reduction of $\mathrm{N}$ fertilization rate of $200 \mathrm{kgN} / \mathrm{ha}$ to $140 \mathrm{kgN} / \mathrm{ha}$ decreases soil $\mathrm{N}_{2} \mathrm{O}$ emission by 42, 38, and 27 percent at soil $\mathrm{pH}$ of 5.0, 6.5, and 8.0 respectively. Conversely, a 30 percent increase in $\mathrm{N}$ fertilization from $200 \mathrm{kgN} /$ ha to $260 \mathrm{kgN} /$ ha will raise the $\mathrm{N}_{2} \mathrm{O}$ emission by 52,45 , and 25 percent respectively. Fertilizer use in China is a typical example of excessive application of nitrogen fertilizer with low NUE. The nitrogen use 
intensity in China is estimated at $305 \mathrm{~kg} \mathrm{~N} / \mathrm{ha} / \mathrm{yr}$ and this is more than four times the global average (Harris, 2018). The total direct $\mathrm{N}_{2} \mathrm{O}$ emissions from Chinese croplands were estimated to be $313 \times 10^{9}(\mathrm{Gg}) \mathrm{N}_{2} \mathrm{O}-\mathrm{N}$ in 2007, and the contribution to $\mathrm{N}_{2} \mathrm{O}$ emissions from croplands by synthetic $\mathrm{N}$ fertilizers was 79.4 percent (Gao et al., 2011). The 4R Nutrient Stewardship is a globally accepted framework that guides farmers and other practitioners to apply the right rate of $\mathrm{N}$ at various stages of crop growth thus minimizing $\mathrm{N}$ losses to the environment. Application of $\mathrm{N}$ at the right rate and time in accordance with the $4 \mathrm{R}$ Nutrient Stewardship framework emphasizes split application of nitrogen in smaller doses thus matching supply of nitrogen with crop requirements at various crop growth stages (Johnston and Bruulsema, 2014). This improves the NUE while also minimizing the amount of $\mathrm{N}$ available for microbial conversion to $\mathrm{N}_{2} \mathrm{O}$ at various times of the year.

In addition to $\mathrm{N}_{2} \mathrm{O}$, excessive nitrogen fertilization is linked to significant $\mathrm{CO}_{2}$ efflux through an acidification process that dissolves $\mathrm{CaCO}_{3}$ especially in calcareous soils which are prevalent in the arid and semi-arid areas. Based on a global $\mathrm{N}$ fertilization map and the distribution of soils containing $\mathrm{CaCO}_{3}$, Zemanian, Zarebanadkouki and Kuzyokov, (2018) estimated $\mathrm{CO}_{2}$ efflux emitted annually through dissolution of $\mathrm{CaCO}_{3}$ as $7.48 \times 10^{12} \mathrm{~g}(\mathrm{Pg}) \mathrm{C} /$ year. Moreover, the study, also estimated that about $273 \times 10^{12} \mathrm{~g} \mathrm{CO}_{2}-\mathrm{C}$ are released annually in the process of $\mathrm{CaCO}_{3}$ neutralization but involving liming of acidic soils. The $\mathrm{N}$ and lime $\mathrm{CO}_{2}$ source from $\mathrm{CaCO}_{3}$ correspond to 30 percent of $\mathrm{CO}_{2}$ from land use changes (Zemanian, Zarebanadkouki and Kuzyokov, 2018). While the $\mathrm{CO}_{2}$ emission from land use change lasts for 20-30 years, emissions from $\mathrm{N}$ fertilization driven $\mathrm{CaCO}_{3}$ acidification cannot reach equilibrium as long as $\mathrm{N}$ fertilizer is applied until it is completely neutralized. Hence, the rate of $\mathrm{N}$ application in calcareous soils should be applied based on plant demand to reduce $\mathrm{CO}_{2}$ release due to acidification.

Various decision support tools like the Nutrient $\operatorname{Expert}^{6}(\mathrm{NE})$ and Nutrient Manager for Rice (NMR) support implementation of site-specific fertilizer management leading to improved NUE (Rurinda et al., 2020; Sharma et al, 2019). By use of decision support tools, it is possible to significantly reduce the fertilizer application rates through improved Fertilizer Use Efficiency (FUE) while sustaining or increasing crop yield levels (Wang et al, 2020). For example, the reduced $\mathrm{N}$ fertilizer recommendation generated by NE increased yield for 64 percent of rice growers and 77 percent of wheat growers in India (Sapkota et al., 2021). Similar trends were evident when fertilizer rates were reduced by use of $\mathrm{NE}$ recommendation in the Nigeria and Ethiopia sites (Rurinda et al., 2020). Through calculations based on 1594 maize and wheat trial sites in India, Sapkota et al. (2021) concluded that adoption of NE based fertilizer recommendations in all rice and wheat fields in India would increase rice and wheat grain yield by 13.92 million tonnes, reduce $\mathrm{N}$ fertilizer consumption by 1.44 million tonnes, and reduce GHG emissions by $5.24 \times 10^{12} \mathrm{~g}$ ( $\mathrm{Tg}$ or $\mathrm{Mt}$ ) $\mathrm{CO}_{2}$ eq per year.

\subsection{Decreases in production (e.g. food/fuel/feed/timber/fibre)}

Although fertilizer is crucial for meeting the crop nutrient demands and boosting yields, in appropriate use of fertilizers can lead to negative environmental impact and soil acidification. Balanced fertilization is recommended and adoption of $4 \mathrm{R}$ Nutrient Stewardship (right source, right rate, right time and place) framework is recommended for improved social, economic and environmental sustainability. Continued use of ammonium-based $\mathrm{N}$ fertilizers and other acidifying fertilizers, without replenishment of basic cation or liming could lead to soil acidification due to accumulation of $\mathrm{H}^{+}$in the soil solution. In acidified soils, most of the nutrients present in the soil are fixed and not available in the soil solution for crop uptake. Unless, the situation

${ }^{6}$ http://software.ipni.net/article/nutrient-expert 
is addressed by liming, acidification is a major threat to crop growth and yield. For long-term sustainable management, fertilizer use must be complemented by applications of other soil ameliorant, including organic resources and lime.

\section{Recommendations before implementing the practice}

The authors of this chapter recommend the following:

- The practitioner should endeavor to boost the soil chemical, biological and physical properties for better yielding healthy soils.

- It is crucial to practice balanced nutrient management to avoid nutrient mining and soil acidification.

- Fertilizers should be used in accordance with the 4R Nutrient Stewardship framework for enhanced social, economic and environmental sustainability.

- The farmer should conduct soil test regularly to determine the level of nutrient deficiency so as to match the fertilizer application (source, rate, time) with crop needs.

\section{Potential barriers for adoption}

Table 63. Potential barriers to adoption

\begin{tabular}{|c|c|c|}
\hline Barrier & YES/NO & \\
\hline Biophysical & Yes & $\begin{array}{l}\text { Non-responsive soils characterized by either very low or no crop } \\
\text { responsive to fertilizers which are common in Africa and other } \\
\text { tropical climates }\end{array}$ \\
\hline Cultural & Yes & $\begin{array}{l}\text { Some parts of the world harbor myths that associate fertilizers } \\
\text { with negative effect on soils, crop and human health }\end{array}$ \\
\hline Social & Yes & Age, education, household size, gender \\
\hline Economic & Yes & $\begin{array}{l}\text { High fertilizer costs, poverty, lack of credit, unemployment and } \\
\text { low off-farm income, ineffective output markets, limited labor, } \\
\text { limited access to machinery requirement for fertilizer application }\end{array}$ \\
\hline
\end{tabular}




\begin{tabular}{|c|c|l|}
\hline Barrier & YES/NO & \\
\hline Institutional & Yes & $\begin{array}{l}\text { Low farmer to extension worker ratio (e.g. in certain part of Africa } \\
\text { the ratio exceeds 1:2000), limited access to credit because } \\
\text { financial institutions require collateral, poor access to information } \\
\text { and long distances from the farms to the nearest fertilizer sale } \\
\text { market especially in Africa, poor infrastructure (ports, road } \\
\text { network etc.) in developing countries }\end{array}$ \\
\hline Knowledge & Yes & $\begin{array}{l}\text { Limited knowledge on importance of fertilizer in general } \\
\text { Limited knowledge on appropriate use of fertilizers for enhanced } \\
\text { crop yields and fertilizer use efficiency } \\
\text { In adequate recognition of the importance managing soils for } \\
\text { improved soil health, soil carbon sequestration and climate } \\
\text { change mitigation }\end{array}$ \\
\hline
\end{tabular}




\section{Photos of the practice}

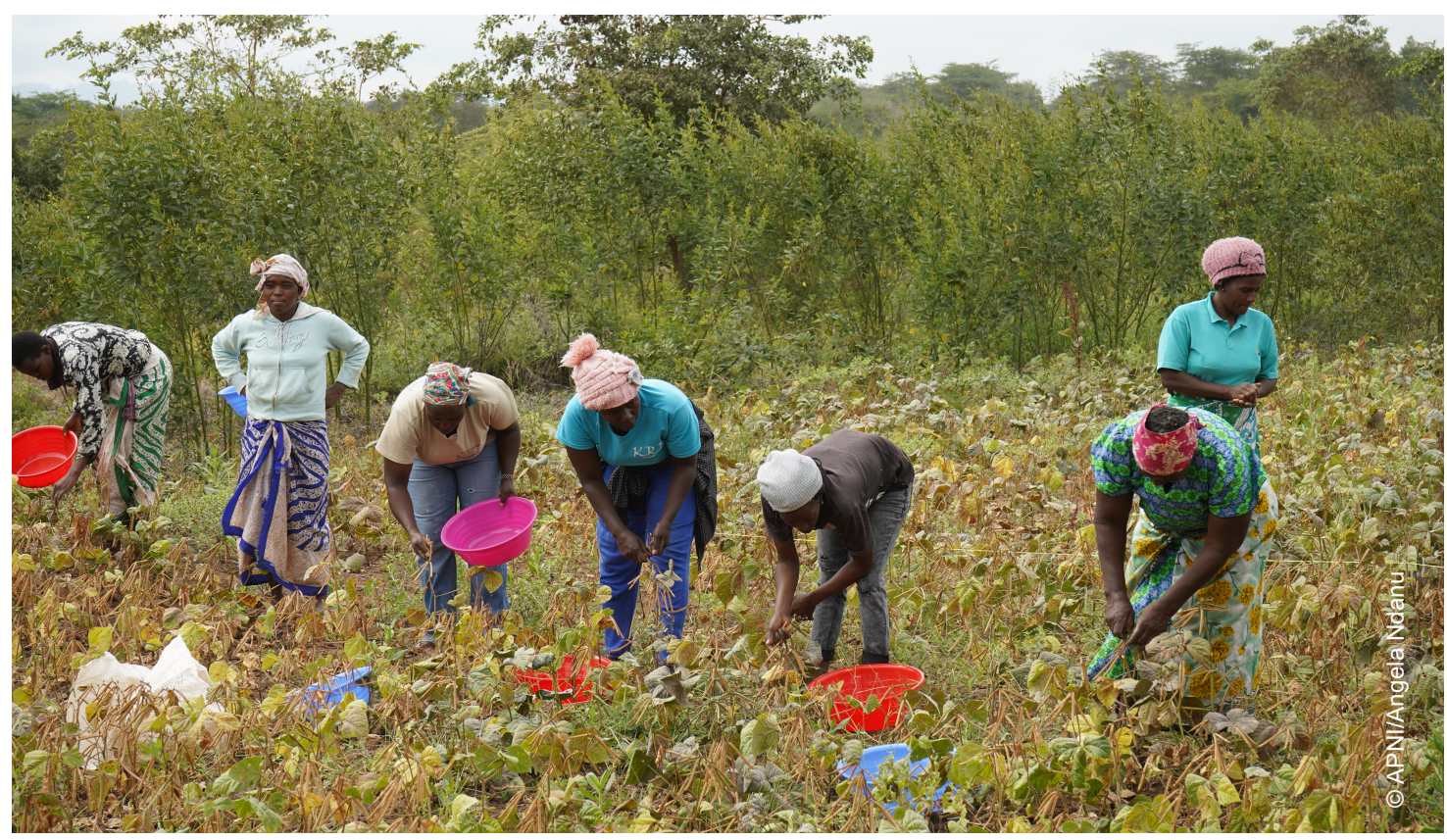

Photo 15. Farmers harvesting greengrams from APNI/AGRA Fertilizer System Project in Makueni county, Kenya in February 2019

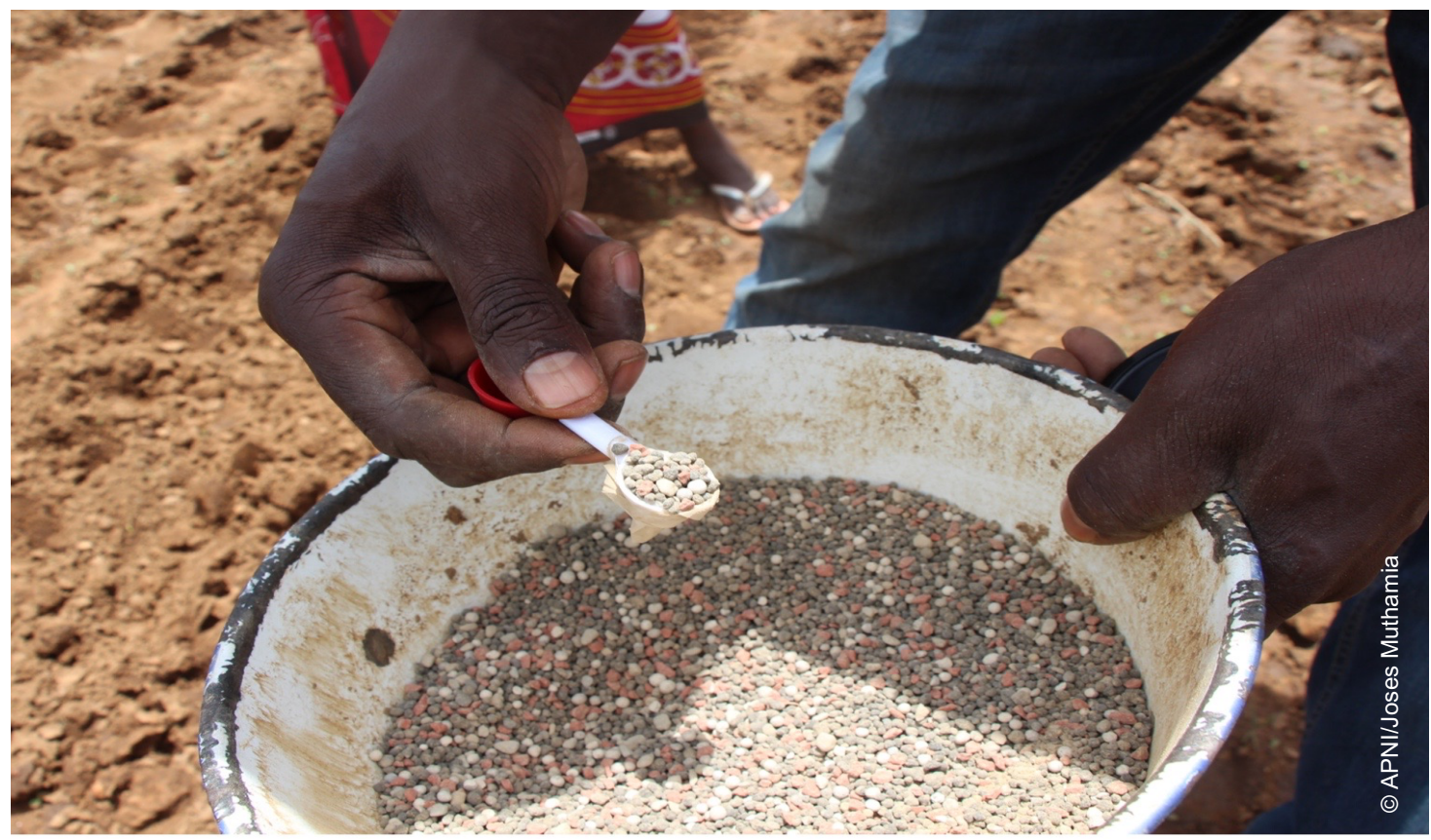

Photo 16. Farmer applying a fertilizer blend containing macronutrients, secondary nutrients and micro-nutrients in a APNI/OCP/AGRA nutrient omission trial in Meru, county Kenya in October 2019. 
Table 64. Related cases studies available in volumes 3 and 5

\begin{tabular}{|c|c|c|c|c|}
\hline Title & Region & $\begin{array}{l}\text { Duration } \\
\text { of study } \\
\text { (Years) }\end{array}$ & Volume & $\begin{array}{l}\text { Case- } \\
\text { study } \\
\text { No. }\end{array}$ \\
\hline Conservation Agriculture in Mozambique & Africa & 2 & 3 & 4 \\
\hline Conservation Agriculture in South Africa & Africa & 6 & 3 & 5 \\
\hline $\begin{array}{l}\text { Irrigated cotton cropping systems in Australian } \\
\text { Vertisols under minimum tillage }\end{array}$ & $\begin{array}{l}\text { Southwest } \\
\text { Pacific }\end{array}$ & 4 to 20 & 3 & 8 \\
\hline $\begin{array}{l}\text { Long-term experiment of manure treatments } \\
\text { on a sandy soil, Germany }\end{array}$ & Europe & 29 & 3 & 12 \\
\hline $\begin{array}{l}\text { Organo-mineral fertilization on a Ukrainian } \\
\text { black soil }\end{array}$ & Europe & 5 & 3 & 26 \\
\hline $\begin{array}{l}\text { Increasing carbon inputs in agricultural lands in } \\
\text { Argentina: fertilizer use, inclusion of cover } \\
\text { crops and integration of perennial pastures in } \\
\text { crop rotations }\end{array}$ & $\begin{array}{l}\text { Latin } \\
\text { America and } \\
\text { the } \\
\text { Caribbean }\end{array}$ & 2 to 23 & 3 & 29 \\
\hline $\begin{array}{l}\text { Increasing Yield and Carbon Sequestration in a } \\
\text { Signalgrass Pasture by Liming and Fertilization } \\
\text { in Sao Carlos (SP, Brazil) }\end{array}$ & $\begin{array}{l}\text { Latin } \\
\text { America and } \\
\text { the } \\
\text { Caribbean }\end{array}$ & 6 & 3 & 32 \\
\hline $\begin{array}{l}\text { Biochar as a Soil Amendment for Carbon } \\
\text { Sequestration in Canada }\end{array}$ & $\begin{array}{l}\text { North } \\
\text { America }\end{array}$ & 1 and 3 & 3 & 41 \\
\hline $\begin{array}{l}\text { Long term fertilization in a subtropical } \\
\text { floodplain soil in Bangladesh }\end{array}$ & Asia & 42 & 5 & 13 \\
\hline
\end{tabular}




\section{References}

Alam, F., Kim, T., Kim, Y., Alam, S., Pramanik, P., Kim, P. \& Lee, Y. 2015. Effect of molybdenum on nodulation, plant yield and nitrogen uptake in hairy vetch (Vicia villosa Roth). Soil Science and Plant Nutrition, 61(4): 664-675. https://doi.org/10.1080/00380768.2015.1030690

Alexandratos, N. \& Bruinsma, J. 2012. World agriculture towards 2030/2050: the 2012 revision. ESA Working paper No. 12-03. Rome, FAO. (also available at: http://www.fao.org/fileadmin/templates/esa/Global_persepctives/world_ag_2030_50_2012_rev.pdf)

Áskelsdóttir, S. 2012. Changes in soil organic carbon in four long-term hayfield fertilization experiments in Iceland: Monitoring and modelling. Agricultural University of Iceland. MSc. thesis.

Banerjee, H., Rana, L., Ray, K., Sarkar, S., Bhattacharyya, K. \& Dutta, S. 2016. Differential physiological response in potato (Solanum tuberosum L.) upon exposure to nutrient omissions. Indian Journal of Plant Physiol, 21(2): 129-136.

Bell, R. \& Dell, B. 2008. Micronutrients for sustainable food, feed, fibre and bioenergy production. IFA, Paris, France. (also available at: https://researchrepository.murdoch.edu.au/id/eprint/12502/1/micronutrients_in_sustainable_food.pdf)

Bell, M. \& Mathesius, K. 2019. Fertilizers and Soil pH. University of California, Agriculture and Natural Resources. (also available at: https://ucanr.edu/sites/soils/files/306283.pdf)

Bijay S. 2018. Are Nitrogen Fertilizers Deleterious to Soil Health? Agronomy, 8, 48; doi:10.3390/agronomy8040048

Bilen, S., Bilen, M. \& Bardhan, S. 2011. The effects of boron management on soil microbial population and enzyme activities. African Journal of Biotechnology, 10(27): 5311-5319.

https://doi.org/10.5897/AJB10.1376

Bitew, Y. \& Alemayehu, M. 2017. Impact of Crop Production Inputs on Soil Health: A Review. Asian journal of Plant Sciences, 16(3): 109-131. https://doi.org/10.3923/ajps.2017.109.131

Blanco-Canqui, H. \& Schlegel, A. 2013. Implications of Inorganic Fertilization of irrigated corn on soil properties: Lessons learned after 50 years. J. Environ. Qual., 42: 861-871.

https://doi.org/10.2134/jeq2012.0451

Bouwman, F. 1996. Direct emission of nitrous oxide from agricultural soils. Nutrient cycling Agroecosyst, 46: 53-70. https://doi.org/10.1007/BF00210224

Bouwman, A., Boumans, L. \& Batjes, N. 2002. Emissions of N2O and NO from fertilized fields: Summary of available measurement data. Global Biogeochem. Cycles, 16(4), 1058.

https://doi.org/10.1029/2001GB001811

Brar, S.B., Singh, J., Singh, G. \& Kaur, G. 2015. Effects of Long-Term Application of Inorganic and Organic Fertilizers on Soil Organic Carbon and Physical Properties in Maize-Wheat Rotation. Agronomy, 5: 220-238. https://doi.org/10.3390/agronomy5020220 
Bumb, L. \& Baanante, A. 1996. World trends in fertilizer use and projections to 2020. 2020 vision briefs 38, International Food Policy Research Institute (IFPRI). (also available at: http://ebrary.ifpri.org/utils/getfile/collection/p15738coll2/id/125887/filename/125918.pdf)

Cai, Z., Wang, B., Minggang, X., Zhang, H., He, X., Zhang, L \& Gao, S. 2015. Intensified soil acidification from chemical $\mathrm{N}$ fertilization and prevention by manure in an 18-year field experiment in the red soil of southern China. Journal of Soils and Sediments, 15: 260-270. https://doi.org/10.1007/s11368014-0989-y

Chen, S., Xing, J. \& Lan, H. 2012. Comparative effects of neutral salt and alkaline salt stress on seed germination, early seedling growth and physiological response of a halophyte species Chenopodium glaucum. Afr J Biotechnol, 11:9572-9581. https://doi.org/10.5897/AJB12.320

Chislock, M.F., Doster, E., Zitomer, R.A. \& Wilson, A.E. 2013. Eutrophication: Causes, Consequences, and Controls in Aquatic Ecosystems. Nature Education Knowledge, 4(4): 10.

Chivenge, P., Vanlauwe, B. \& Six, J. 2011. Does the combined application of organic and mineral nutrient sources influence maize productivity? A meta-analysis. Plant and Soil, 32: 1-30.

https://doi.org/10.1007/s11104-010-0626-5

Christensen, B. 1988. Effects of animal manure and mineral fertilizers on the total carbon and nitrogen contents of soil size fractions. Biol Fertil Soils, 5: 304-307. https://doi.org/10.1007/BF00262136

Cuevas, J., Daliakopoulos, I., Moral, F., Hueso, J. \& Tsanis, I. 2019. A Review of Soil-Improving Cropping Systems for Soil Salinization. Agronomy, 9: 295. https://doi.org/10.3390/agronomy9060295

Drechsel, P., Heffer, P., Magen, H., Mikkelsen, R. \& Wichelns, D. 2015. Managing Water and Fertilizer for Sustainable Agricultural Intensification. International Fertilizer Industry Association (IFA), International Water Management Institute (IWMI), International Plant Nutrition Institute (IPNI), and International Potash Institute (IPI). First edition, Paris, France. (also available at:

http://www.ipni.net/ipniweb/portal.nsf/0/B9C003FF28F9C9EF85257DE1007607CC/\$FILE/2015_ ifa_ipni_iwmi_ipi.pdf)

FAO. 2020. Environment Statistics. Mineral and Chemical Fertilizers: 1961-2018 [online]. [Cited 12 March 2021]. http://www.fao.org/economic/ess/environment/data/mineral-and-chemical-fertilizers/en/

Franklin, R., Duis, L., Brown, R. \& Kemp, T. 2005. Trace element content of selected fertilizers and micronutrient source materials. Commun. Soil Sci. Plant Anal., 36: 1591-1609.

https://doi.org/10.1081/CSS-200059091

Funderburg, E. 2001. What Does Organic Matter Do In Soil? [online]. Noble Research Institute [Cited 29 January 2021]. https://www.noble.org/news/publications/ag-news-and-views/2001/august/what-doesorganic-matter-do-in-soil/

Gao, B., Ju, T., Zhang, Q., Christie, P. \& Zhang, F. 2011. New estimates of direct N2O emissions from Chinese croplands from 1980 to 2007 using localized emission factors. Biogeosciences, 8: 3011-3024. https://doi.org/10.5194/bg-8-3011-2011 
Gardi, C., Menta., C., Montanarella, L. \& Cenci, R. 2008. Main threats on soil biodiversity: The case of agricultural activities impacts on soil microarthropods. In Tóth, G., Montanarella, L. \& Rusco, E. (Eds.) Threats to Soil Quality in Europe. JRC Scientific and Technical Reports, pp. 101-111. (also available at: https://esdac.jrc.ec.europa.eu/ESDB_Archive/eusoils_docs/other/EUR23438.pdf)

Getachew, A., Yirga, C. \& Erkossa, T. 2019. Soil Acidity Management. Ethiopian Institute of Agricultural Research (EIAR). Addis Ababa, Ethiopia. (also available at:

http://publication.eiar.gov.et:8080/xmlui/bitstream/handle/123456789/3151/Getachew\%20et\%20al. \%20Soil\%20Acidity\%20\%20\%20\%20\%20\%20\%20\%20\%20\%20\%20\%20Managmnet.pdf?sequence=1\&i sAllowed $=y$ )

Giweta, M., Dyck, F., Malhi, S., Puurveen, D. \& Roberson, J. 2014. Long term S-fertilization increases carbon sequestration in a sulfur deficient soil. Can J. Soil Sci, 94: 295-301.

https://doi.org/10.4141/cjss2013-022

Gibbs, H. \& Salmon, J. 2015. Mapping the world's degraded lands. Applied Geography, 57: 12-21. https://doi.org/10.1016/j.apgeog.2014.11.024

Graham, D. \& Webb, J. 1991. Micronutrients and disease resistance and tolerance in plants. In J. Mortvedt, F. Cox, L. Shuman, M. Welch (Eds.) Micronutrients in Agriculture. 2nd ed. SSSA Book Series No.4. pp. 329-37, Soil Science Society of America, Madison, Wisconsin.

Guo, Z., Zhang, Z., Zhou, H., Wang, D. \& Peng, X. 2019. The effect of 34-year continuous fertilization on the SOC physical fractions and its chemical composition in a Vertisol. Nature, 9: 2505.

https://doi.org/10.1038/s41598-019-38952-6

Harris, B. 2018. China cut fertilizer use and still increased crop yields. This is how they did it [online]. World Economic Forum. [Cited 12 March 2021]. https://www.weforum.org/agenda/2018/03/this-is-how-chinacut-fertilizer-use-and-boosted-crop-yields

Hati, K., Mandal, K., Misra, A., Ghosh, P. \& Bandyopadhyay, K. 2006. Effect of inorganic fertilizer and farmyard manure on soil physical properties, root distribution, and water-use efficiency of soybean in Vertisols of central India. Bioresource Technology, 97(16): 2182-2188.

https://doi.org/10.1016/j.biortech.2005.09.033

Havlin, J., Tisdale, S., Nelson, W. \& Beaton, J. 2005. Soil Fertility and Fertilizers: An Introduction to Nutrient Management. Pearson Education/Prentice-Hall, Inc., Upper Saddle River, New Jersey.

Haynes R \& Naidu R. 1998. Influence of lime, fertilizer and manure applications on soil organic matter. Nutrient Cycling in Agroecosystems, 51: 123-137. https://doi.org/10.1023/A:1009738307837

Hedge, M. \& Sarkar, A. Yield trends in rice-wheat system in different agro-ecological regions. In R.K. Pandey, B.S. Dwivedi \& A.K. Sharma (Eds.) Rice-Wheat Cropping System: Proceedings of the Rice-Wheat Workshop held on 15-16 October, 1990 at Modipuram, Meerut. pp. 15 - 31. Project Directorate for Cropping Systems Research, Modipuram, Meerut, India.

Hu, C., Li, S., Qiao, Y., Liu, D. \& Chen, Y. 2015. Effects of 30 years repeated fertilizer applications on soil properties, microbes and crop yields in Rice wheat cropping system. Expl Agric, 51(3): 355-369.

https://doi.org/10.1017/S0014479714000350 
IPNI. 2016. Soil Acidity Evaluation \& Management. International Plant Nutrition, 30pp.

Jin, J., Ping, H. \& Shihua, T. 2006. Micronutrient deficiencies occurrence and correction: The Chinese example. In Bell and Dell (Eds.) Micronutrients for Sustainable Food, Feed, Fibre and Bioenergy Production. Pp. 45. IFA France.

Johnston, A. \& Bruulsema, T. 2014. 4R Nutrient Stewardship for Improved Nutrient Use Efficiency. Procedia Engineering, 3: 365-370. https://doi.org/10.1016/j.proeng.2014.09.029

Kibblewhite, M.G., Ritz, K. \& Swift, M.J. 2008. Soil health in agricultural systems. Philosophical Transactions of the Royal Society B: Biological Sciences, 363(1492): 685-701.

https://doi.org/10.1098/rstb.2007.2178

Kihara, J., Sileshi, G., Nziguheba, G., Kinyua, M., Zingore, S. \& Sommer, R. 2017. Application of secondary nutrients and micronutrients increases crop yields in sub-Saharan Africa. Agron. Sustain. Dev., 37(25): 1-14. https://doi.org/10.1007/s13593-017-0431-0

Kihara, J., Bolo, P., Kinyua, M., Rurinda, J. \& Piikki, K. 2020. Micronutrient deficiencies in African soils and the human nutritional nexus: opportunities with staple crops. Environ Geochem Health, 42: 30153033. https://doi.org/10.1007/s10653-019-00499-w

Lal, R. 2015. Restoring Soil Quality to Mitigate Soil Degradation. Sustainability, 7(5): 5875-5895. https://doi.org/10.3390/su7055875

Larson, W.E., Eynard, A., Hadas, A. \& Lipiec, J. 1994. Chapter 25 - Control and Avoidance of Soil Compaction in Practice. In B.D. Soane \& C. van Ouwerkerk, eds. Developments in Agricultural Engineering, pp. 597-625. Soil Compaction in Crop Production. Elsevier. https://doi.org/10.1016/B978-0-44488286-8.50033-7

Ling, N., Sun, M., Ma, H., Guo, J., Zhu, P., Peng, C., Yu, H., Ran, W. \& Guo, W. 2014. Response of the bacterial diversity and soil enzyme activity in particle-size fractions of Mollisol after different fertilization in a long-term experiment. Biology and Fertility of Soils, 50: 901-911. https://doi.org/10.1007/s00374-0140911-1

Malhi, S., Nyborg, M. \& Soon, Y. 2010. Long term effects of balanced fertilization on grass forage yield, quality and nutrient uptake soil organic $\mathrm{C}$ and $\mathrm{N}$ and some soil quality characteristics. Nutrient cycling Agroecosyst, 86: 425-438. https://doi.org/10.1007/s10705-009-9306-3

Mandal, B., Majumder, B., Bandyopadhyay, P., Hazra, G., Gangopadhyay, A., Samantaray, R., Mishra, A., Chaudhury, J., Saha, M. \& Kundu, S. 2007. The potential of cropping systems and soil amendments for carbon sequestration in soils and long-term experiments in subtropical India. Global Change Biology, 13: 357-369. https://doi.org/10.1111/j.1365-2486.2006.01309.x

Mauricio, M., Fabián, A., Raúl, C., Marcia, C. \& Mauricio, E. 2009. Trace Element Composition of Selected Fertilizers Used in Chile: Phosphorus Fertilizers as a Source of Long-Term Soil Contamination. Soil and Sediment Contamination, 18(4): 497-511. https://doi.org/10.1080/15320380902962320

Mutegi, J., Munkholm, L., Petersen, B., Hansen, E. \& Petersen, S. 2010. Nitrous oxide emissions and controls as influenced by tillage and crop residue management strategy. Soil Biol. Biochem, 42: 1701-1711. https://doi.org/10.1016/j.soilbio.2010.06.004 
Njoroge, S., Schut, A., Giller, K. \& Zingore, S. 2018. Strong spatial-temporal patterns in maize yield response to nutrient additions in African smallholder farms. Field Crops Research, 214: 321-330. https://doi.org/10.1016/j.fcr.2017.09.026

Nziguheba, G. \& Smolders, E. 2008. Inputs of trace elements in agricultural soils via phosphate fertilizers in European countries. Sci Total Environ, 390: 53-57. https://doi.org/10.1016/j.scitotenv.2007.09.031

Ogunwole, J. \& Ogunleye, 0. 2004. Surface soil aggregation, trace, and heavy metal enrichment under long-term application of farmyard manure and mineral fertilizers. Commun. Soil Sci. Plant Anal, 35: 15051516. https://doi.org/10.1081/CSS-120038551

Pant, K. \& Ram, S. 2018. Long term manuring and fertilization effects on soil physical properties after 42 Cycles under rice-wheat system in North Indian Mollisol. International Journal of Current Microbiology and Applied Sciences, 7: 232-240. https://doi.org/10.20546/ijcmas.2018.707.028

Prasad, R., Majumdar, K., Shivay, Y. \& Kapil, U. 2016. Minerals in plant and human nutrition and health. International Plant Nutrition Institute, Peachtree Corners, GA, USA. ISBN: 978-0-9960199-5-8.

Raven, K., Reynolds, J. \& Loeppert, R. 1997. Trace element analyses of fertilizers and Soil amendments by axial-view inductively-coupled plasma atomic emission spectrophotometry. Commun. Soil Sci. Plant Anal, 28: 237-257. https://doi.org/10.1080/00103629709369788

Research Markets. 2019. Micronutrient Fertilizers - Global Market Outlook (2018-2027). Statistics Market Research Consulting Pvt Ltd. 152 pp. ID: 4844757.

Roser, M. \& Ritchie, H. 2013. Fertilizers. Our World in Data. (also available at https://ourworldindata.org/fertilizers).

Rudrappa, L., Purakayastha, T., Singh, D. \& Bhadraray, S. 2005. Long-term manuring and fertilization effects on soil organic carbon pools in a Typic Haplustept of semi-arid sub-tropical India. Soil and Tillage Research, 88: 180-192. https://doi.org/10.1016/j.still.2005.05.008

Rurinda, J., Balemi, T., Jibrin, J., Zingore, S., Masuki, K., Andersson, J., Pampolino, M., Mohammed, I., Mutegi, J., Kamara, A., Vanlauwe, B. \& Craufurd P. 2020. Science-based decision support for formulating crop fertilizer recommendations in sub-Saharan Africa. Agricultural Systems, https://doi.org/10.1016/j.agsy.2020.102790.

Sapkota, T., Jat, L., Rana, S., Khatri-Chhetri, A., Jat, S., Bijarniya, D., Sutaliya, J., Kumar, M., Singh, L., Jat, K., Kalvaniya, K., Prasad, G., Sidhu, H., Rai, M., Satyanarayana, T. \& Majumdar, K. 2021. Crop nutrient management using Nutrient Expert improves yield, increases farmers' income and reduces greenhouse gas emissions. Nature Scientific Reports, 11: 1564. https://doi.org/10.1038/s41598-02079883-X

Sharma, S., Panneerselvam, P., Castillo, R., Manohar, S., Rajendren, R., Ravi, V. \& Buresh, R. 2019. Web-based tool for calculating field-specific nutrient management for rice in India. Nutrient cycling Agroecosyst, 113: 21-33. https://doi.org/10.1007/s10705-018-9959-X

Sillanpää, M. 1990. Micronutrient assessment at country level: An international study. Soils Bulletin No. 63, FAO, Rome, 208 pp. (also available at https://www.cabdirect.org/cabdirect/abstract/19916775946). 
Soane, B.D. 1990. The role of organic matter in soil compactibility: A review of some practical aspects. Soil and Tillage Research, 16(1): 179-201. https://doi.org/10.1016/0167-1987(90)90029-D

Srinivasarao, C., Venkateswarlu, B., Lal, R., Singh, A., Vittal, K., Kundu, S., Singh, S.R. \& Singh, S.P. 2012. Long-term effects of soil fertility management on carbon sequestration in a rice-lentil cropping system of the Indo-Gangetic plains. Soil Science Society of America Journal, 76: 168-178.

https://doi.org/10.2136/sssaj2011.0184

Stacey, S., McLaughlin, M. \& Hettiarachchi, G. 2010. Fertilizer-Borne Trace Element Contaminants in Soils. In Hooda, P.S (Eds) Trace Elements in the Soil. Blackwell Publishing, Ltd.

Steffen, W., Richardson, K., Rockström, J., Cornell, S.E., Fetzer, I., Bennett, E.M., Biggs, R., Carpenter, S.R., De Vries, W., De Wit, C.A., Folke, C., Gerten, D., Heinke, J., Mace,

G.M., Persson, L.M., Ramanathan, V., Reyers, B. \& Sörlin, S. 2015. Planetary boundaries: Guiding human development on a changing planet. Science, 347(6223): 1259855.

https://doi.org/10.1126/science. 1259855

Stewart, W., Dibb, D., Johnston, A. \& Smyth, T. 2005. The Contribution of Commercial Fertilizer Nutrients to Food Production. Agronomy Journal, 97:1-6. https://doi.org/10.2134/agronj2005.0001

Su, J., Ding, L., Xue, K., Yao, H., Quensen, J., Bai, S., Wei, W., Wu, J., Zhou, J., Tiedjes, J. \& Zhu, Y. 2015. Long-term balanced fertilization increases the soil microbial functional diversity in a phosphoruslimited paddy soil. Molecular Ecology, 24: 136-150. https://doi.org/10.1111/mec.13010

Upadhyay, R.G. \& Singh, A. 2016. Effect of nitrogen and zinc on nodulation, growth and yield of cowpea Vigna unguiculata. Legume Research, 39(1): 149-151. (also available at:

https://arccjournals.com/uploads/articles/27LR3210.pdf)

van Kauwenbergh, S. 1997. Cadmium and other minor elements in world resources of phosphate rock. The International Fertilizer Society-Proceeding 400. The International Fertilizer Society, London.

van Vugt, D. 2018. Participatory approaches to diversification and intensification of crop production on smallholder farms in Malawi. Wageningen University. PhD dissertation.

Wang, J., Wang, K., Wang, X., Yuchun, A., Zhang, Y. \& Jianguang, Y. 2018. Carbon sequestration and yields with long term use of inorganic fertilizer and organic manure in a six-crop rotation system. Nutrient cycling Agroecosyst, 111: 87-98. https://doi.org/10.1007/s10705-018-9920-z

Wang, Y., Li, W., Li, Y., Zhu, L., Liu, S., Yan, L., Feng, G. \& Gao, Q. 2020. Agronomic and environmental benefits of nutrient expert on maize and rice in Northeast China. Environ Sci Pollut Res Int, 22: 28053-28065. https://doi.org/10.1007/s11356-020-09153-w

Ward, M., Jones, R., Brender, J., de Kok, T., Weyer, P., Nolan, B., Cristina, M., Villanueva, C. \& van Breda, S. 2018. Drinking Water Nitrate and Human Health: An Updated Review. Int. J. Environ. Res. Public Health, 15: 1557. https://doi.org/10.3390/ijerph15071557

Welch, M. 2001. Micronutrients, agriculture and nutrition: linkages for improved health and wellbeing. In K. Singh, S. Mori, R. Welch (Eds.) Perspectives on the Micronutrient Nutrition of Crops. p. 247-290. Scientific Publishers, India, Jodhpur. 
White, J. \& Broadley, R. 2009. Biofortification of crops with seven mineral elements often lacking in human diets - iron, zinc, copper, calcium, magnesium, selenium and iodine. New Phytologist, 182: 49-84. https://doi.org/10.1111/j.1469-8137.2008.02738.x

Zemanian, K., Zarebanadkouki, M. \& Kuzyokov, Y. 2018. Nitrogen fertilizer raises CO2 efflux from inorganic carbon. A global assessment. Global Change Biology, 24: 2810-2817.

https://doi.org/10.1111/gcb.14148

Zhang, B., Tian, H., Ren, W., Tao, B., Lu, C., Yang, J., Banger, K \& Pan, S. 2016. Methane emissions from global rice fields: Magnitude, spatiotemporal patterns, and environmental controls. Global Biogeochemical Cycles, 30: 1246-1263. https://doi.org/10.1002/2016GB005381

Zhang, H., Xu, M., Shi, X., Li, Z., Huang, Q. \& Wang, X. 2010. Rice yield, potassium uptake and apparent balance under long-term fertilization in rice-based cropping systems in southern China. Nutrient cycling Agroecosyst, 88: 341-349. https://doi.org/10.1007/s10705-010-9359-3

Zhang, W., Xu, M., Wang, B. \& Wang, X. 2008. Soil organic carbon, total nitrogen and grain yields under long term fertilizations in the upland red soil of southern China. Nutrient cycling Agroecosyst, 84(1): 59-69. https://doi.org/10.1007/s10705-008-9226-7 


\title{
CROPLAND \\ NUTRIENT MANAGEMENT \\ CHEMICAL AND MINERAL FERTILIZATION
}

\section{Fertigation}

\author{
Bijesh Maharjan, Saurav Das, Deepak Ghimire
}

Department of Agronomy \& Horticulture, University of Nebraska, Lincoln, Nebraska, United States of America

\section{Description of the practice}

Fertigation is the agronomic practice in which mineral fertilizer is dissolved in the irrigation water and delivered to the root zone via the irrigation system. Precise nutrient supply in time and space is a significant advantage of fertigation practice. Fertigation can be applied to both soil and soilless systems, and here, we focus on fertigation effects on soil organic carbon (SOC) in soil system. Optimized fertigation can reduce fertilizer application to achieve sustainable and economical crop production. It allows precise timing and rate of fertilizer application at different growth stages according to crop requirement, an option elusive to conventional fertilizer application. Precision in fertilizer application enhances crop fertilizer uptake efficiency and reduces nutrient losses via leaching, runoff, and volatilization (Gärdenäs et al., 2005). Compared to the conventional broadcast or band application of fertilizers, fertigation has several benefits: a regular supply of nutrients reducing nutrient fluctuation in soil and uniform fertilizer application throughout the irrigated soil volume (Kafkafi and Kant, 2005). Precision in nutrient application and water via fertigation increases SOC sequestration by enhanced production and retention of biomass $\mathrm{C}$ in soil (Lal, 2020).

One can employ fertigation with any existing irrigation system such as sprinkler or surface irrigation, but subsurface drip irrigation is the most adequate system for fertigation (Photo 17) since it has the following added advantages (Kafkafi and Kant, 2005):

- Precision nutrient application to subsurface and wetted soil where crop roots are more active increases crop nutrient use efficiency and reduces nutrient loss.

- Dry crop foliage, otherwise wetted by sprinkler irrigation, reduces pest or disease incidence, and avoids foliage burn.

- Nutrient distribution is more uniform and spherical around emitters.

- Depending on the depth of drip tapes, the topsoil remains dry in subsurface fertigation, reducing evaporation and runoff losses and weed germination. 
Among many crop nutrients that can be applied via fertigation, nitrogen $(\mathrm{N})$ is the primary and most used nutrient. Wastewater is also commonly applied to cropland via fertigation (Coelho et al., 2020). Fertigation is a kind of chemigation that also includes herbicides, fungicides, and insecticides, which poses severe risks to human and animal health and the environment if managed improperly. Since chemigation, including fertigation, can potentially contaminate the water supply, among many others, a backflow prevention device is a must for such systems.

\section{Range of applicability}

Fertigation practice can be applied in any climatic region and soil types where irrigation is available, and the system can be upgraded to fertigation capability. On heavy clay soil, water ponding might occur under the emitter/sprinkler outlets. Such locally developed anaerobic conditions might cause severe N losses in gaseous forms such as dinitrogen gas $\left(\mathrm{N}_{2}\right)$ or nitrous oxide $\left(\mathrm{N}_{2} \mathrm{O}\right)$ and cause crop $\mathrm{N}$ deficiency and emission of greenhouse gas emission (GHG). In such cases, the application of low $\mathrm{N}$ concentration with irrigation can reduce $\mathrm{N}$ losses and deficiency caused by denitrification. Fertigation is equally essential for arid and semi-arid regions where evaporative and leaching losses can be significant, threatening water economy. Soil and water quality are vulnerable in arid and semi-arid areas where crop productions are primarily irrigation dependent. Regular and excessive $\mathrm{N}$ (broadcast or band placement) and frequent irrigation in these regions have increased the nitrate concentration in groundwater resources (Gärdenäs et al., 2005). Fertigation as an alternative irrigation and fertilization method can strategically allocate water and fertilizer to maximize nutrient use efficiency and minimize fertilizer input and environmental losses.

\section{Impact on soil organic carbon stocks}

Fertigation enhances crop production and enhances biomass $\mathrm{C}$ in soil (Lal, 2020). Depending on crop residue and tillage management, and climatic conditions, SOC increases variably with fertigation. The lower the initial SOC, the greater the gain is (Trost et al., 2013). Sustainable agronomic management practices such as fertigation would create a positive soil $\mathrm{C}$ budget and improve the agronomic productivity and soil quality, as evidenced by increased SOC under drip fertigation (Table 65). There are few reports on fertigation management effects on SOC under sprinkler systems. 


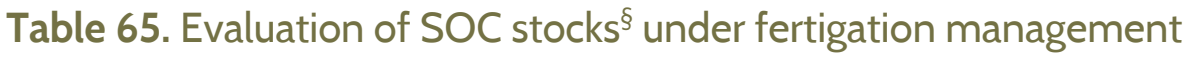

\begin{tabular}{|c|c|c|c|c|c|c|c|c|}
\hline Location & $\begin{array}{l}\text { Climate } \\
\text { zone }\end{array}$ & $\begin{array}{l}\text { Soil } \\
\text { type }\end{array}$ & $\begin{array}{l}\text { Baseline } \\
\text { C stock } \\
\text { (tC/ha) }\end{array}$ & $\begin{array}{l}\text { Additional } \\
\text { C storage } \\
\text { (tC/ha/yr) }\end{array}$ & $\begin{array}{l}\text { Duration } \\
\text { (Years) }\end{array}$ & $\begin{array}{l}\text { Depth } \\
(\mathrm{cm})\end{array}$ & $\begin{array}{l}\text { More } \\
\text { information }\end{array}$ & Reference \\
\hline $\begin{array}{l}\text { India } \\
\text { (Rajasthan) }\end{array}$ & Arid & $\begin{array}{l}\text { Loamy } \\
\text { sand }\end{array}$ & 2.07 & $\begin{array}{l}\text { a) } 0.09 \\
\text { b) } 0.18\end{array}$ & $\begin{array}{c}11 \\
(2010- \\
2011)\end{array}$ & $0-15$ & $\begin{array}{l}\text { a) weekly } \\
\text { b) daily } \\
\text { fertigation } \\
\text { compared to } \\
\text { conventional } \\
\text { fertilization } \\
\text { and irrigation in } \\
\text { tomato }\end{array}$ & $\begin{array}{l}\text { Singh et al. } \\
(2014)\end{array}$ \\
\hline $\begin{array}{l}\text { India } \\
\text { (Karnataka) }\end{array}$ & Tropical & $\begin{array}{l}\text { Sandy } \\
\text { clay } \\
\text { loam }\end{array}$ & $\begin{array}{l}\text { a) } 34.45 \\
\text { b) } 22.10\end{array}$ & $\begin{array}{l}\text { a) } 2.54 \\
\text { b) } 1.46\end{array}$ & $\begin{array}{c}10 \\
(1996- \\
2006)\end{array}$ & $\begin{array}{l}\text { a) } 0-25 \\
\text { b) } 25- \\
30\end{array}$ & $\begin{array}{l}\text { Drip NPK } \\
\text { fertigation in } \\
\text { areca nut }\end{array}$ & $\begin{array}{l}\text { Bhat and } \\
\text { Sujatha } \\
\text { (2009) }\end{array}$ \\
\hline
\end{tabular}

§SOC reported in percentage was converted to SOC stock using the equation (SOC stock=SOC concentration X depth X bulk density X 10) (Li et al., 2020).

\section{Other benefits of the Fertigation}

\subsection{Improvement of soil properties}

Fertigation in conjunction with other sustainable agricultural management practices can significantly improve soil properties. Fertigation with the addition of biochar enhances soil and crop productivity in alkaline soils by improving soil water content, available phosphorus, and soil microbes' capacity to utilize various compounds and microbial biomass carbon and by suppressing plant-parasitic nematodes (Zhang et al., 2020). Fertigation with nutritive salts $\left(\mathrm{EC}=2 \mu \mathrm{S} \mathrm{cm}^{-1}\right)$ was reported to increase soil aggregate stability (Moreira Barradas et al., 2015). However, soil accumulation can be an issue particularly in drier environments where an efficient desalinization effect of rain and snow is not available during fallow period (Moreira Barradas et al., 2015). 


\subsection{Minimization of threats to soil functions}

Table 66. Soil threats

\begin{tabular}{|c|c|}
\hline Soil threats & \\
\hline Soil erosion & A drip system reduces soil erosion. Less runoff. \\
\hline $\begin{array}{l}\text { Nutrient } \\
\text { imbalance and } \\
\text { cycles }\end{array}$ & $\begin{array}{l}\text { Better nutrient balance. It synchronizes nutrient availability and crop } \\
\text { need and improves crop nutrient use efficiency. }\end{array}$ \\
\hline $\begin{array}{l}\text { Soil } \\
\text { contamination / } \\
\text { pollution }\end{array}$ & $\begin{array}{l}\text { Fertigation reduces nutrient leaching and subsequent groundwater } \\
\text { pollution. }\end{array}$ \\
\hline Soil acidification & $\begin{array}{l}\text { Small and frequent } \mathrm{N} \text { application doses with irrigation might cause less } \\
\text { soil acidification than by conventional } \mathrm{N} \text { fertilization. }\end{array}$ \\
\hline Soil compaction & $\begin{array}{l}\text { Fertigation reduces soil compaction with reduced machinery and foot } \\
\text { traffic compared to traditional fertilizer applications. }\end{array}$ \\
\hline $\begin{array}{l}\text { Soil water } \\
\text { management }\end{array}$ & $\begin{array}{l}\text { Reduced water uses in drip fertigation compared to an overhead } \\
\text { sprinkler system. }\end{array}$ \\
\hline
\end{tabular}

\subsection{On production}

Fertigation increases nutrient uptake efficiency and crop yield compared to broadcast and band fertilization. For example, in orange and grapefruit cultivation in Florida and Israel, drip fertigation showed higher fruit yield and $\mathrm{N}$ use efficiency due to reduced nitrate leaching below the soil root volume (Dasberg et al., 1988). In the Syrian Arab Republic, drip fertigation resulted in a higher yield of potatoes than furrow irrigation (Janat, 2007). A study from Italy showed fertigation increased $\mathrm{N}$ uptake and yield in tomato (Farneselli et al., 2020). Haynes (1988) reported increased pepper yield with fertigation or a combination of broadcast $(1 / 3)$ plus fertigation $(2 / 3)$ compared to broadcast fertilization at $\mathrm{N}$ rate of $75 \mathrm{~kg} / \mathrm{ha}$. When $\mathrm{N}$ applied at $150 \mathrm{~kg} / \mathrm{ha}$, fertigation reduced pepper yields due to aluminum toxicity induced by soil acidity below the emitters. Fertigation with treated sewage effluent increased the yield and quality of palisade grass (Coelho et al., 2020). 


\subsection{Mitigation of and adaptation to climate change}

In general, irrigation may increase the emission of nitrous oxide $\left(\mathrm{N}_{2} \mathrm{O}\right)$, a potent greenhouse gas (GHG) (Horváth et al., 2010). However, soil $\mathrm{N}$ availability primarily dictates $\mathrm{N}_{2} \mathrm{O}$ emission in any system. Therefore, small doses of $\mathrm{N}$ applied at some time intervals in fertigation would effectively reduce $\mathrm{N}_{2} \mathrm{O}$ emission. The use of drip fertigation was reported to decrease methane $\left(\mathrm{CH}_{4}\right)$ emission and $\mathrm{N}$ losses $\left(\mathrm{NH}_{3}, \mathrm{~N}_{2} \mathrm{O}, \mathrm{NO}\right)$ in several studies (Badr, El-Tohamy and Zaghloul, 2012; Maris et al., 2015). In China, drip fertigation reduced $\mathrm{N}_{2} \mathrm{O}$ emissions in maize cropping systems by 19.9 percent compared to conventional flood irrigation (Zhang et al., 2019). There are few reports on fertigation management effects on GHG emissions under sprinkler systems.

\subsection{Socio-economic benefits}

Fertigation provides spoon-feeding to crops and, therefore, improves crop fertilizer use efficiency and crop production. It reduces the environmental implications of agricultural production by reducing chemical inputs and environmental nutrient losses. It also provides economic gain to producers by saving on inputs and energy and time otherwise spent separately on fertilization besides irrigation.

\subsection{Other benefits of the practice}

Integrated with the internet of things (IoT) and sensors, fertigation can be devised as a useful modern agronomic practice for sustainable agriculture. Changes in moisture in the soil profile, temperature and humidity of the surroundings, plant transpiration estimates, and crop in-season nutrient status can be collected using sensors, and fertigation can be automated with precisions depending on the crop demand for water and nutrients along with soil status. A two-year study from the University of Nebraska showed sensor-based N fertigation increased corn N use efficiency and yield (Krienke et al., 2018, 2019). Fertigation combined with improved irrigation scheduling can improve fertilizer uptake efficiency, enhance water use efficiency, increase the residence time of nutrients in the root zone, and reduce environmental implications. 


\section{Potential drawbacks to the practice}

\subsection{Tradeoffs with other threats to soil functions}

Table 67. Soil threats

\begin{tabular}{|l|l|}
\hline Soil threats & Nutrient imbalance \\
and cycles & $\begin{array}{l}\text { Rain and timing of water availability can delay fertigation and thus delay } \\
\text { fertilizer application which can be critical depending on growth stages. }\end{array}$ \\
\hline $\begin{array}{l}\text { Soil salinization and } \\
\text { alkalinization }\end{array}$ & $\begin{array}{l}\text { Due to salt content in wastewater, long-term fertilization with wastewater } \\
\text { may cause salinization and sodification of the soil profile, particularly in } \\
\text { Atier environments. } \\
\text { especially at the wetted front, between emitters where flux reaches zero } \\
\text { and at the surface where evaporation occurs. }\end{array}$ \\
\hline $\begin{array}{l}\text { Soil contamination / } \\
\text { pollution }\end{array}$ & $\begin{array}{l}\text { Depending on its composition, wastewater used in fertigation may } \\
\text { accumulate trace metals in soil. }\end{array}$ \\
\hline Soil acidification & $\begin{array}{l}\text { Fertigation at a high } \mathrm{N} \text { rate can increase soil acidification and consequent } \\
\text { increases in extractable Al, Fe, Mn, and } \mathrm{Zn} \text { compared to broadcast } \\
\text { application. }\end{array}$ \\
\hline
\end{tabular}

\subsection{Increases in greenhouse gas emissions}

Some studies have found that fertigation with similar N levels to flood irrigation levels may increase GHG emissions due to denitrification stimulated by increased water-filled pore spaces due to frequent irrigation.

\subsection{Conflict with other practice(s)}

Frequent movement of heavy farm machinery may destroy or break the irrigation line, particularly the drip tapes. 


\subsection{Decreases in production (e.g. food/fuel/feed/timber/fibre)}

- The high investment cost for installation and management of injection equipment

- Risk of insufficient nutrient supply during the rainy season

- Risk of hypoxia due to frequent irrigation (especially in clay soil)

- Risk of clogging of emitter due to insoluble salt precipitation

- Over-irrigation can lead to the leaching of $\mathrm{N}$ from an effective root-zone, making it unavailable for crops.

\subsection{Other conflicts}

For the most efficient use of drip irrigation with fertilizer, the system requires pressure regulation, which is rarely explained during purchase, and most maintenance people are not familiar with it. Easy prey for wayward mowers and other garden/machinery tools and rodents and burrowing animals.

\section{Recommendations before implementing the practice}

- Uniformity in fertilizer application depends on the uniformity of the water application. Thus, high water application uniformity is essential for fertigation.

- The fertilizer source must be water-soluble. The selection of fertilizer should be considered based on crop growth stage, irrigation system type, and water quality.

- Producers should have a general idea about the dripping system and different equipment to regularly maintain the pressure heads, injectors, emitters, and valve system from plugging. The chemical reaction between the fertilizers may result in the formation of precipitates and clog the irrigation system.

- Irrigation water $\mathrm{pH}$ should be within a $\mathrm{pH}$ range of 5.5 - 7.0. High $\mathrm{pH}$ may reduce the availability of essential nutrients like phosphorus, zinc, and iron.

- Anhydrous or aqua ammonia is not recommended for the fertigation system as they increase the $\mathrm{pH}$ of irrigation water. The most common and preferable $\mathrm{N}$ fertilizer source for fertigation is urea and ammonium nitrate due to their low plugging risk.

- Nitrogen can contribute to algal and microbial growth in the irrigation line if it remains in the pipe after a system has been shut off.

- Water sources like recycled wastewater may contain a significant amount of nitrate, salt or trace metals which should be considered while determining the fertilizer requirement. 


\section{Potential barriers for adoption}

Table 68. Potential barriers to adoption

\begin{tabular}{|l|l|l|}
\hline Barrier & YES/NO & \\
\hline Economic & Yes & $\begin{array}{l}\text { High investment cost to set up. Installation and disassembling } \\
\text { equipment in drip fertigation every season require extra labor and } \\
\text { cost. }\end{array}$ \\
\hline $\begin{array}{l}\text { Legal } \\
\text { Right to } \\
\text { soil) }\end{array}$ & Yes & $\begin{array}{l}\text { One may need permission from the regulatory authority for } \\
\text { fertigation and irrigation under water rights. }\end{array}$ \\
\hline $\begin{array}{l}\text { Knowledge } \\
\text { Yes }\end{array}$ & $\begin{array}{l}\text { It needs a skilled operator. It requires expertise in plant nutrition } \\
\text { and management of the fertigation system. }\end{array}$ \\
\hline
\end{tabular}




\section{Photos of the practice}
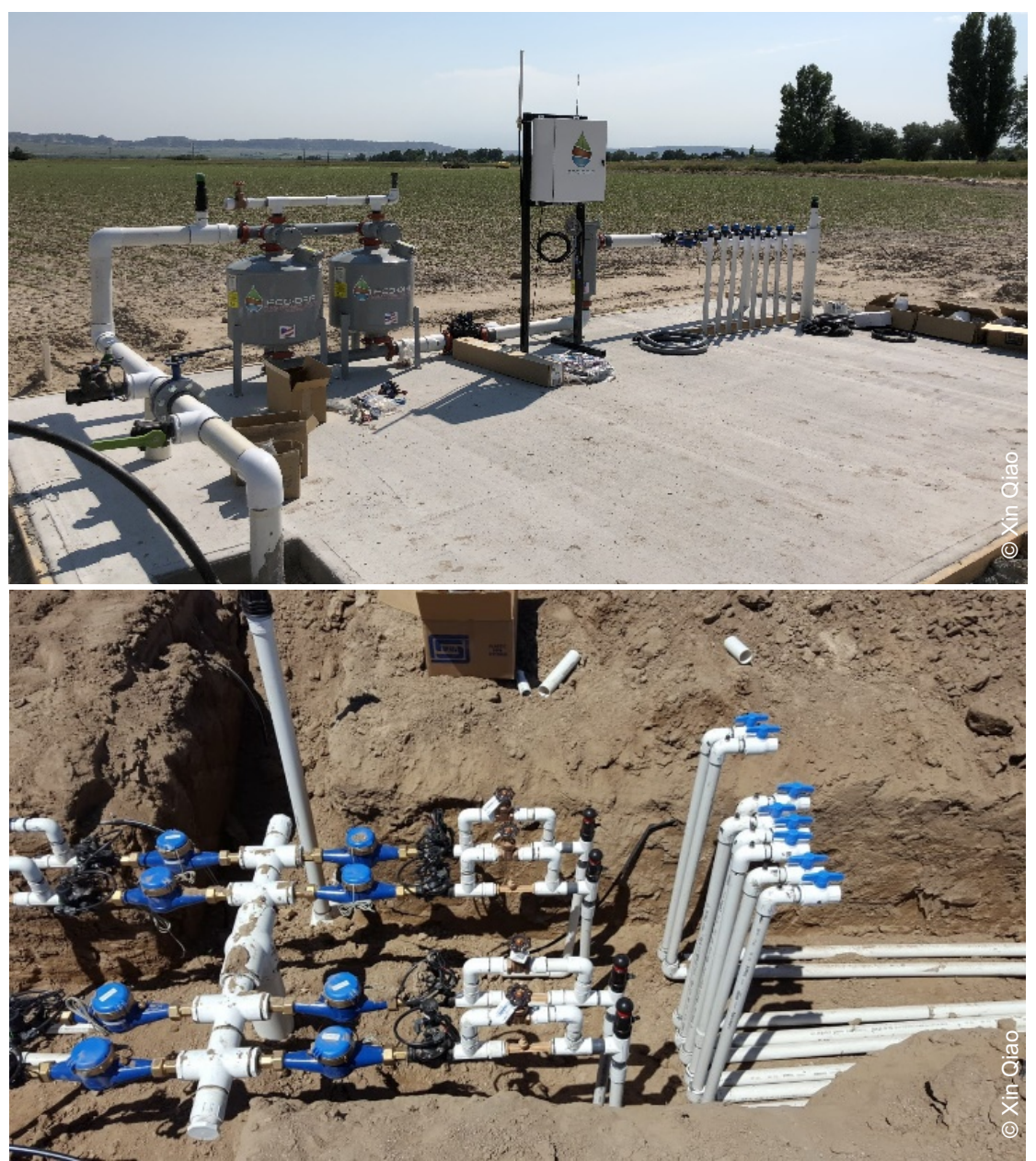

Photo 17. Subsurface drip irrigation system with the filter and control station installed on a cement pad, and manifolds, flow meters, and air injectors that supply irrigation to each management zone

Table 69. Related cases studies available in volumes 3 and 5

\begin{tabular}{|l|c|c|c|c|}
\hline Title & Region & $\begin{array}{l}\text { Duration } \\
\text { of study } \\
\text { (Years) }\end{array}$ & Volume & $\begin{array}{l}\text { Case- } \\
\text { study } \\
\text { No. }\end{array}$ \\
\hline $\begin{array}{l}\text { Mediterranean olive orchard subjected to } \\
\text { sustainable management in Matera, Basilicata, } \\
\text { Italy }\end{array}$ & Europe & 20 & 3 & 16 \\
\hline
\end{tabular}




\section{References}

Badr, M.A., El-Tohamy, W.A. \& Zaghloul, A.M. 2012. Yield and water use efficiency of potato grown under different irrigation and nitrogen levels in an arid region. Agricultural Water Management, 110: 9-15. https://doi.org/10.1016/j.agwat.2012.03.008

Bhat, R. \& Sujatha, S. 2009. Soil fertility and nutrient uptake by arecanut (Areca catechu L.) as affected by level and frequency of fertigation in a laterite soil. Agricultural Water Management, 96(3): 445-456. https://doi.org/10.1016/j.agwat.2008.09.007

Coelho, A.P., da Silva, M.F., de Faria, R.T., Fernandes, C., Dantas, G.F. \& Santos, G.O. 2020. Longterm impact of fertigation with treated sewage effluent on the physical soil quality. Environmental Pollution, 266: $1-10$.

Dasberg, S., Bar-Akiva, A., Spazisky, S. \& Cohen, A. 1988. Fertigation versus broadcasting in an orange grove. Fertilizer research, 15(2): 147-154. https://doi.org/10.1007/BF01050676

Farneselli, M., Benincasa, P., Tosti, G., Guiducci, M. \& Tei, F. 2020. Combining Green Manuring and Fertigation Maximizes Tomato Crop Yield and Minimizes Nitrogen Losses. Agronomy, 10(7): 977. https://doi.org/10.3390/agronomy10070977

Gärdenäs, A.I., Hopmans, J.W., Hanson, B.R. \& Šimůnek, J. 2005. Two-dimensional modeling of nitrate leaching for various fertigation scenarios under micro-irrigation. Agricultural Water Management, 74(3): 219-242. https://doi.org/10.1016/j.agwat.2004.11.011

Haynes, R.J. 1988. Comparison of fertigation with broadcast applications of urea-N on levels of available soil nutrients and on growth and yield of trickle-irrigated peppers. Scientia Horticulturae, 35: 189-198. https://doi.org/10.1016/0304-4238(88)90112-4

Horváth, L., Grosz, B., Machon, A., Tuba, Z., Nagy, Z., Czóbel, S.Z., Balogh, J., Péli, E., Fóti, S.Z., Weidinger, T., Pintér, K. \& Führer, E. 2010. Estimation of nitrous oxide emission from Hungarian semiarid sandy and loess grasslands; effect of soil parameters, grazing, irrigation and use of fertilizer. Agriculture, Ecosystems \& Environment, 139(1): 255-263. https://doi.org/10.1016/j.agee.2010.08.011

Janat, M. 2007. Efficiency of Nitrogen Fertilizer for Potato under Fertigation Utilizing a Nitrogen Tracer Technique. Communications in Soil Science and Plant Analysis, 38(17-18): 2401-2422. https://doi.org/10.1080/00103620701588775

Kafkafi, U. \& Kant, S. 2005. FERTIGATION. In D. Hillel (Ed.) Encyclopedia of Soils in the Environment, pp. 1-9. Oxford, Elsevier. https://doi.org/10.1016/B0-12-348530-4/00227-7

Krienke, B., Ferguson, R., Irmak, S., Rudnick, D., Shapiro, C., Shaver, T., Maharjan, B., Wortmann, C. \& Naser, M. 2019. Profit and Nitrogen Use Efficiency Gain Using Sensor-Guided Fertigation. In CropWatch [online]. [Cited 28 January 2021]. https://cropwatch.unl.edu/2019/profit-and-nitrogen-useefficiency-gain-using-sensor-guided-fertigation 
Krienke, B., Ferguson, R., Irmak, S., Rudnick, D., Shaver, T., Shapiro, C., Glewen, K. \& Naser, M. 2018. Feasibility of Sensor-Based Nitrogen Fertigation Management in Corn. In CropWatch [online]. [Cited 28 January 2021]. https:/ / cropwatch.unl.edu/2018/feasibility-sensor-based-nitrogen-fertigationmanagement-corn

Lal, R. 2020. Managing soils for negative feedback to climate change and positive impact on food and nutritional security. Soil Science and Plant Nutrition, 66(1): 1-9.

https://doi.org/10.1080/00380768.2020.1718548

Li, F., Yuan, C., Lao, D., Yao, B., Hu, X., You, Y., Wang, L., Sun, S. \& Liang, X. 2020. Drip irrigation with organic fertilizer application improved soil quality and fruit yield. Agronomy Journal, 112(1): 608-623. https://doi.org/10.1002/agj2.20052

Maris, S.C., Teira-Esmatges, M.R., Arbonés, A. \& Rufat, J. 2015. Effect of irrigation, nitrogen application, and a nitrification inhibitor on nitrous oxide, carbon dioxide and methane emissions from an olive (Olea europaea L.) orchard. Science of The Total Environment, 538: 966-978.

https://doi.org/10.1016/j.scitotenv.2015.08.040

Moreira Barradas, J.M., Abdelfattah, A., Matula, S. \& Dolezal, F. 2015. Effect of Fertigation on Soil Salinization and Aggregate Stability. Journal of Irrigation and Drainage Engineering, 141(4): 05014010. https://doi.org/10.1061/(ASCE)IR.1943-4774.0000806

Singh, A., Gulati, I.J., Chopra, R., Sharma, D. \& Gochar, R. 2014. Effect of drip-fertigation with organic manures on soil properties and tomato (Lycopersicon esculentum Mill.) yield under arid condition. Annals of Biology, 30: 345-349.

Trost, B., Prochnow, A., Drastig, K., Meyer-Aurich, A., Ellmer, F. \& Baumecker, M. 2013. Irrigation, soil organic carbon and $\mathrm{N}_{2} \mathrm{O}$ emissions. A review. Agronomy for Sustainable Development, 33(4): 733-749. https://doi.org/10.1007/s13593-013-0134-0

Zhang, X., Meng, F., Li, H., Wang, L., Wu, S., Xiao, G. \& Wu, W. 2019. Optimized fertigation maintains high yield and mitigates $\mathrm{N}_{2} \mathrm{O}$ and $\mathrm{NO}$ emissions in an intensified wheat-maize cropping system. Agricultural Water Management, 211: 26-36. https://doi.org/10.1016/j.agwat.2018.09.045

Zhang, X., Qu, J., Li, H., La, S., Tian, Y. \& Gao, L. 2020. Biochar addition combined with daily fertigation improves overall soil quality and enhances water-fertilizer productivity of cucumber in alkaline soils of a semiarid region. Geoderma, 363: 114170. https://doi.org/10.1016/j.geoderma.2019.114170 


\title{
16. Integrated soil fertility management
}

\author{
Tesfaye Bayu \\ Debre Markos University, Burie campus, Debre Markos, Ethiopia
}

\section{Description of the practice}

Integrated soil fertility management (ISFM) has been developed since the late 1990s by, among others, the Tropical Soil Biology and Fertility Institute of the International Center of Tropical Agriculture (CIAT) based in Nairobi. Besides addressing the management of organic matter, ISFM embraces social, cultural and economic processes regulating soil fertility management strategies (Bationo, Kihara and Adesina, 2012).

IFSM strives to balance the withdrawal of soil nutrients from fields, pastures and orchards by crops, livestock, and natural processes with the addition of nutrients provided by crop residues, compost, manure or commercial fertilizers. The main objective of ISFM is to optimize yields and the quality of crop production, while minimizing costs and negative environmental impacts. Failure to properly manage nutrients results in poor nutrient use efficiency and potentially harmful downstream environmental effects. Good nutrient management prevents the over-application of essential crop nutrients and sustainable nutrient management considers the full cost associated with application, including the energy embedded in added nutrients (Park et al., 2011).

ISFM is a set of soil fertility management practices that necessarily include the use of mineral fertilizers, organic inputs (e.g. animal and green manures, crop residues, urban/rural wastes, composts, bio-fertilizers) and improved germplasm combined with the knowledge on how to adapt these practices to local conditions, to optimize the agronomic efficiency of the applied nutrients and improve crop productivity (Antil, 2012; Simpson et al., 2014). All inputs need to be managed following sound agronomic and economic principles (Fairhurst, 2012). IFSM practices seek to maximize the amount of carbon stored in the soil by balancing the use of organic matter obtained on the farm or from other sources as a soil amendment with the judicious use of nutrients from mineral fertilizers; and reducing nutrient losses by synchronizing the supply of nitrogen with crop demands through sound agronomic practices, including soil and water conservation measures. Examples of IFSM practices include:

- making changes in the rates, timing and type of nitrogen fertilizer applications and using slow release fertilizers that control the formation of nitrates; 
- adding nitrification inhibitors containing ammonium to fertilizer;

- practicing no-tillage farming, while maintaining continuous soil cover and rotating cropping patterns, which provides enough structural carbohydrates (e.g. lignin) along with nitrogen to allow the nitrogen produced from decaying surface residues to be released more slowly and contribute to the growth of the following crop and minimize nutrient losses (Gál et al., 2007)

This definition focuses on maximizing the use efficiency of fertilizers and organic inputs since these are both scarce resources in the areas where agricultural intensification is needed. Agronomic efficiency (AE) is defined as the incremental return to applied inputs. The ISFM definition proposes that application of fertilizer to improved germplasm on responsive soils will boost crop yield and improve the $\mathrm{AE}$ relative to current farmer practice, characterized by traditional varieties receiving too little and insufficiently managed nutrient inputs.

In this system, all aspects of mineral and organic plant nutrient sources are integrated into the crop production system (Agegnehu and Amede, 2017). IFSM contributes to attaining agronomically feasible, economically viable, environmentally sound and sustainable high crop yields in cropping systems by enhancing nutrient use efficiency and soil fertility, increasing carbon sequestration, and reducing nitrogen losses due to nitrate leaching and emission of greenhouse gases (Milkha and Aulakh, 2010).

\section{Range of applicability}

The ISFM framework provides farming strategies for a large range of soil fertilityconditions and cropping systems. Over the last decade several ISFM interventions have been brought to scale across various agroecological zones, including i) micro-dosing of fertilizers combined with manure management and water harvesting for cereal-legume systems in dry savannas of the West African Sahel, ii) targeted fertilizer application combined with organic inputs for maize-legume intercropping and rotational systems in moist savannas of Eastern and Southern African, and iii) studies on chemical fertilizer with organic fertilizers in Nepal. Different rates of synthetic fertilizer and organic manuring was tested in Pakistan, which caused significant changes in soil physical chemical properties and carbon sequestration (Bista et al., 2010). In the last couple of years efforts have been made to tailor-make ISFM practices for crops like cassava (Pypers et al., 2012), rice and banana that are grown throughout the Tropics (Oikeh, Azoma and Saito, 2010). Because ISFM practices are designed to curb soil nutrient depletion they have great potential for reducing deforestation in slash-and-burn systems across the larger Democratic Republic of the Congo Basin. Combining organic and mineral inputs has been advocated as a sound management principle for smallholder farming in the tropics because neither of the two inputs is usually available in sufficient quantities and because positive interactions between both inputs have

often been observed (Paul et al., 2013), but as explained in this brief many of the ISFM principles are shared with other sustainable agricultural practices and thereby applicable to different cropping systems, geographies, climates and economies (Rware et al., 2014). 


\section{Impact on soil organic carbon stocks}

ISFM techniques that were designed to increase soil organic matter should be accompanied by actions that address the drivers of degradation and help preserve existing soil carbon stocks, particularly in soils with high soil organic carbon content (Smith et al., 2014,

Table 70). Changes in soil organic carbon depend on the ISFM practices associated with land use and the time since the current land-use system was established (Mohawesh, Taimeh and Ziadat, 2015).

Table 70. Soil carbon sequestration potential rates associated to ISFM

\begin{tabular}{|l|l|l|c|c|c|}
\hline & Soil type & $\begin{array}{l}\text { Cropping } \\
\text { system }\end{array}$ & Control & $\begin{array}{l}\text { NPK (200 } \\
\text { kg/ha) }\end{array}$ & $\begin{array}{l}\text { NPK + farmyard } \\
\text { manure (5t/ha) }\end{array}$ \\
\hline India & Inceptisol & Rice-Rice & 4.1 & 5.9 & 7.6 \\
\hline Ethiopia & Nitisol & Maize-wheat & 5.0 & 9.5 & 15.1 \\
\hline China & Ultisol & $\begin{array}{l}\text { Rice wheat- } \\
\text { maize }\end{array}$ & 6.0 & 9.0 & 12.3 \\
\hline Kenya & Vertisol & Cotton-cotton & 4.2 & 6.2 & 9.2 \\
\hline Pakistan & Mollisol & Rice -wheat & 6.2 & 7.2 & 14.5 \\
\hline Indonesia & Vertisol & Cassava & 5.1 & 6.1 & 10.1 \\
\hline $\begin{array}{l}\text { United } \\
\text { Republic of } \\
\text { Tanzania }\end{array}$ & Nitisol & Maize-wheat & 4.5 & 5.7 & 9.7 \\
\hline
\end{tabular}

Source: Adapted from Amanullah et al. (2019) 


\section{Other benefits of the practice}

\subsection{Improvement of soil properties}

\section{Soil chemical properties}

Application of organic manure in combination with chemical fertilizer has been reported to increase absorption of $\mathrm{N}, \mathrm{P}$ and $\mathrm{K}$ in sugarcane leaf tissue in the plant and ratoon crop, compared to chemical fertilizer alone (Bokhtiar and Sakurai, 2005). Kaur, Kapoor and Gupta (2005) compared the change of chemical and biological properties in soils receiving farmyard manure (FYM), poultry manure and sugarcane filter cake alone or in combination with chemical fertilizers for seven years under a cropping sequence of pearl millet and wheat and showed that all treatments except chemical fertilizer application improved the soil organic C, total N, P and K status. Application of ISFM improves the soil CEC due to increase in basic action in soil solution and adsorbed on clay surface ( Agegnehu, Vanbeek and Bird, 2014).

\section{Soil biological properties}

Dutta, Mishra and Dileep Kumar (2008) reported that the use of ISFM(compared to the addition of organic fertilizers alone) had a higher positive effect on microbial biomass. Application of compost and mineral fertilizer significantly affected the bacteria population found in the soil (Munyabarenzi, 2014).

\section{Soil physical properties}

Bulk density decreases with long-term application of ISFM on soil. The aggregate stability showed an increase with time on plots fertilized with ISFM. Improved aggregate stability due to ISFM facilitates water infiltration and hence increases the plant available water content and decreases runoff and erosion. Total porosity of the soil was significantly affected by ISFM (Agbede, 2010). Soil structure and aggregate stability are improved due to when the soil was treated by ISFM practices (Srinivasarao et al., 2012).

\subsection{Minimization of threats to soil functions}

Table 71. Soil threats

\begin{tabular}{|l|l|}
\hline Soil threats & \\
\hline Soil erosion & $\begin{array}{l}\text { Minimized soil erosion by increased SOM and improved soil structure } \\
\text { (Bayu, 2020). }\end{array}$ \\
\hline $\begin{array}{l}\text { Nutrient imbalance } \\
\text { and cycles }\end{array}$ & $\begin{array}{l}\text { Increased availability of macro and micronutrients and maintenance } \\
\text { of nitrogen and carbon cycle (Kaur, Kapoor and Gupta, 2005). }\end{array}$ \\
\hline $\begin{array}{l}\text { Soil salinization and } \\
\text { alkalinization }\end{array}$ & $\begin{array}{l}\text { Minimized soil salinization and alkalization by reducing cations } \\
\text { concentration on the soil surface (Amare et al., 2013). }\end{array}$ \\
\hline
\end{tabular}




\begin{tabular}{|l|l|}
\hline Soil threats & \\
\hline Soil acidification & $\begin{array}{l}\text { Minimized soil acidification by improving the soil CEC (Selassie and } \\
\text { Ayanna, 2013). }\end{array}$ \\
\hline Soil biodiversity loss & $\begin{array}{l}\text { Minimized biodiversity loss by maintaining micro-organisms } \\
\text { availability (Gadermaier et al., 2012). }\end{array}$ \\
\hline Soil compaction & $\begin{array}{l}\text { Minimized soil compaction by modification of soil structure (Vanlauwe } \\
\text { et al., 2015). }\end{array}$ \\
\hline
\end{tabular}

\subsection{Increases in production (e.g. food/fuel/feed/timber/fibre)}

The major requirements for achieving production gains agricultural land include: (i) the use of disease-resistant and improved germplasm, (ii) crop and water management practices (includes cultivation, weeding, irrigation and drainage), and (iii) application of Nutrient Stewardship ${ }^{7}$. This provides an essential basis for optimizing the use of nutrients within an ISFM framework.

Ejigu and Araya (2010) noted that use of ISFM increased yield and production of wheat, green beans, gram and rice in Ethiopia. In North-West India, Naresh, Singh and Kumar (2013) showed that grain and straw yields of rice were significantly higher when amended with compost and NPK than in no compost with NPK additions, thereby highlighting the beneficial effects of compost to increase the crop yield (Figure 4). Mukuralinda et al. (2010) in their study on P uptake and maize in Rubona, Southern Province of Rwanda showed that ISFM significantly increased maize yield from 24 to 508 percent when compared to the control which does not receive any fertilizer.

ISFM has been shown to considerably improve rice yields by minimizing nutrient losses to the environment and by managing the nutrient supply, and thereby results in high resource-use efficiency, cost reductions, and improved resistance to biotic and abiotic stresses (Parkinson, 2013). It can thus be considered an effective agricultural paradigm to ensure food security and improve environmental quality worldwide, especially in countries with rapidly developing economies (Wu and Ma, 2015)

ISFM controls $\mathrm{N}$ losses and their harmful environmental effects while achieving high crop productivity ( Gruhn, Goletti and Yudelman, 2000). The fate of $\mathrm{N}$ in field is an integrated consequence of crop $\mathrm{N}$ uptake, immobilization, and residues in the soil, and $\mathrm{N}$ losses to the environment, such as ammonia volatilization, $\mathrm{NO}_{\mathrm{X}}$ emissions, denitrification, N leaching and runoff ( Jambert, Serca and Delmas, 1997).

\footnotetext{
${ }^{7}$ Science-based framework that focuses on applying the right fertilizer source at the right rate, at the right time during the growing season, and in the right place.
} 
Table 72. Summary of effect of ISFM on crop production

\begin{tabular}{|c|c|c|c|}
\hline Reference & Common crops & Effect of ISFM & Country \\
\hline $\begin{array}{l}\text { Jha, Chaurasia and } \\
\text { Bharti (2013) }\end{array}$ & Rice & $\begin{array}{l}\text { ISFM significantly decreased } \mathrm{N} \text { application; while } \\
\text { improved rice yield and soil nutrient profile }\end{array}$ & India \\
\hline Miriti et al. (2007) & Maize & $\begin{array}{l}\text { Combination of tie-ridges with manure significantly } \\
\text { increased maize Stover by } 29 \% \text { and biomass by } 50 \\
\text { percent. } \mathrm{N} \text { application also increased cowpea stem } \\
\text { and biomass by } 57 \text { percent and } 45 \text { percent, } \\
\text { respectively. }\end{array}$ & Kenya \\
\hline Nawab et al. (2011) & Wheat & $\begin{array}{l}\text { Manures and fertilizer application (FYM, potassium } \\
\text { and zinc fertilizers) produced significantly higher } \\
\text { grain yield than the control plots. }\end{array}$ & Pakistan \\
\hline $\begin{array}{l}\text { Sharma, Bali and } \\
\text { Gupta (2001) }\end{array}$ & Summer barley & $\begin{array}{l}\text { ISFM significantly increased the grain ( } 1.1 \mathrm{t} / \mathrm{ha}) \text { and } \\
\text { straw yields ( } 2.3 \mathrm{t} / \mathrm{ha}) \text {, yield-attributing characters, } \\
\text { protein content and nutrient uptake. }\end{array}$ & India \\
\hline Chander et al. (2013) & Fava been & $\begin{array}{l}\text { ISFM significantly decreased rate of chemical } \\
\text { fertilizers by up to } 50 \text { percent; while improved S and } \\
\text { Zn uptake for grain and grain yield }\end{array}$ & $\begin{array}{l}\text { Andaman } \\
\text { Islands }\end{array}$ \\
\hline $\begin{array}{l}\text { Jagathjothi, } \\
\text { Ramamoorthy and } \\
\text { Kuttimani (2011) }\end{array}$ & Finger millet & $\begin{array}{l}\text { ISFM increased grain ( } 3.3 \mathrm{t} / \mathrm{ha}) \text { and straw yield ( } 5.9 \\
\text { t/ha) over all other treatments. }\end{array}$ & Nigeria \\
\hline Akram et al. (2007) & Sorghum & $\begin{array}{l}\text { ISFM increased the availability of nutrients and their } \\
\text { uptake resulted in improved crop growth and grain } \\
\text { yield. }\end{array}$ & Pakistan \\
\hline $\begin{array}{l}\text { Patil and } \\
\text { Sheelavantar (2013) }\end{array}$ & Chickpea & $\begin{array}{l}\text { ISFM increased number of pods per plant ( } 66.4) \text {, } \\
\text { number of seeds per pod (1.2), test weight ( } 20.91 \mathrm{~g}) \text {, } \\
\text { grain yield ( } 2.4 \mathrm{t} / \mathrm{ha} \text { ) and haulm yield ( } 3.2 \mathrm{t} / \mathrm{ha}) \text {. }\end{array}$ & India \\
\hline Thind et al. (2007) & Potato/sunflower & $\begin{array}{l}\text { Incorporation of inorganic manures improved OC, } \\
\text { available } \mathrm{K} \text { and } \mathrm{P} \text { content in soil and had significant } \\
\text { residual effect on seed yield, growth and phenology } \\
\text { of sunflower. }\end{array}$ & India \\
\hline
\end{tabular}

Source: Adapted from Wu and Ma (2015) 


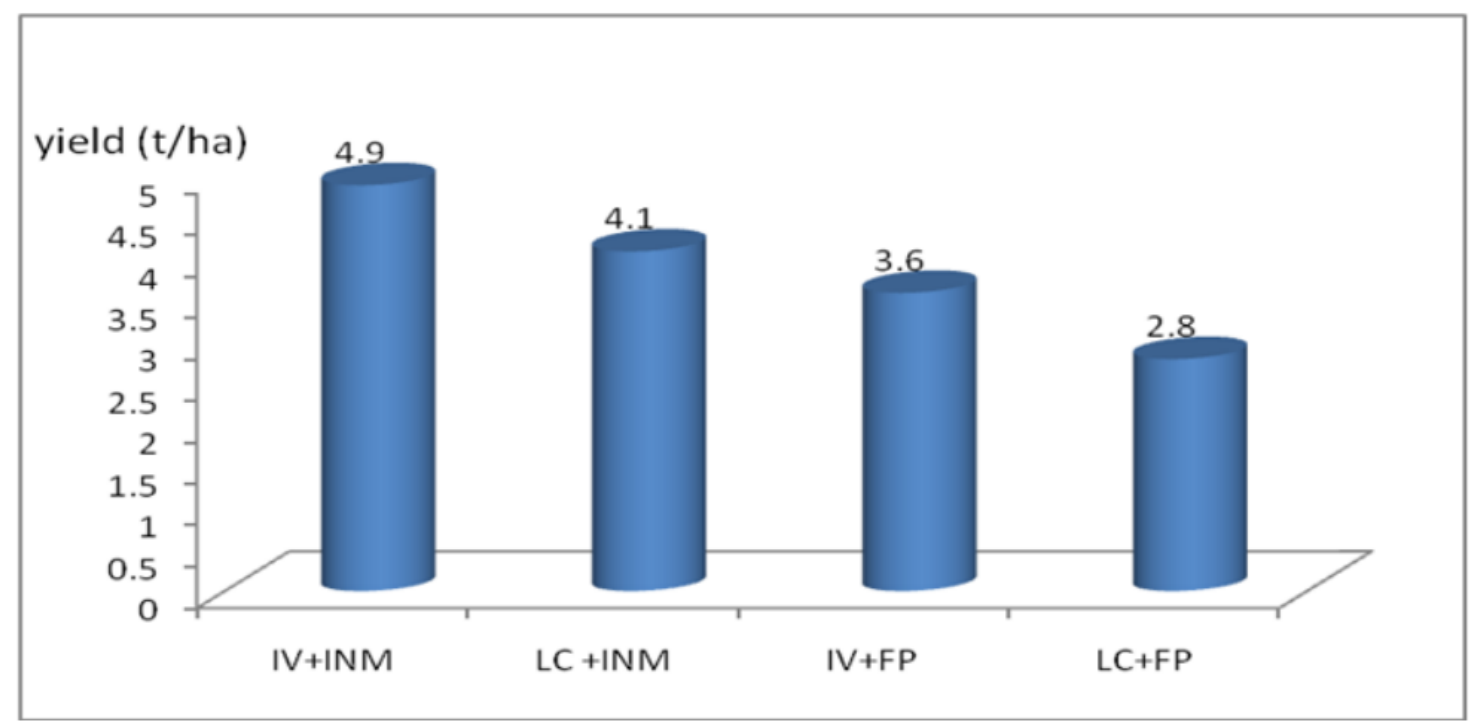

Figure 4. Effect of ISFM along with Varity different in North West India (Naresh, Singh and Kumar, 2013)

\subsection{Mitigation of and adaptation to climate change}

Climate change mitigation involves reducing the amount of greenhouse gases in the atmosphere or enhancing their sinks, for example by reducing the use of fossil fuels, planting trees, or enhancing mineralization of organic matter into soil organic carbon (Sommer et al, 2018).

ISFM advocates the deep placement of urea or ammonium bicarbonate, which can significantly increase the Nuse efficiency with a low $\mathrm{NH}_{3}$ volatilization and reduced nitrate leaching (Jambert, Serca and Delmas, 1997). The application of nitrification inhibitors can also reduce the $\mathrm{N}_{2} \mathrm{O}$ emission because $\mathrm{N}_{2} \mathrm{O}$ emissions occur mostly during the nitrification processes after fertilizer $\mathrm{N}$ application (Ma et al., 2010) and irrigation (Ju et al., 2011). In addition, ISFM favors organic regimes of fertilization, which have tremendous potential for the sustainable development of agriculture along with more direct environmental benefits. Using organic manure together with other management practices, such as incorporation of crop residues and the development of conservation tillage (e.g. no-tillage or reduced-tillage practices), also reduce GHG emissions, improve the soil quality and increase C sequestration, and are associated with high crop yields (Huang and Sun, 2006).

ISFM helps to reduce GHG emissions by:

- Using recommended rates of suitable organic and inorganic fertilizers;

- Placing the nitrogen more precisely into the root zone to make it more accessible by crops;

- If possible, using precision agriculture techniques to improve fertilizer application by helping determine exactly where to place nutrients, how much to apply, and when to apply (Lal, 2005). 
Three techniques can help achieve this objective:

- The collection of spatial data from pre-existing conditions in the field (e.g. remote sensing, canopy size, or yield measurement);

- The application of optimum fertilizer amounts to the crop when and where needed; and

- The recording of detailed $\operatorname{logs}_{\mathrm{s}}$ of all fertilizer applications for spatial and temporal mapping sequestration (Lal, 2005).

Utilization of these farmers friendly materials in rice cultivation in combination with inorganic fertilizers might contribute to higher grain productivity and subsequently increase the SOC. Integrated soil fertility management fertilizer application had significant $(\mathrm{p}<0.05)$ effects on methane emission from a rice paddy (Bharali et al., 2017).

The application of compost with crop rotation directly decreases emissions of $\mathrm{N}_{2} \mathrm{O}$ from $\mathrm{NO}_{3}$ through minimizing the leaching process. Managing grasslands with ISFM decreases methane emission from animals and $\mathrm{CO}_{2}$ emissions from the soil. At rotation level, crop residues, cattle slurry and compost substantially contribute to SOC accumulation (range 200-450 $\mathrm{kg} \mathrm{C} / \mathrm{ha} / \mathrm{yr}$ ), while contributions of pig slurry and cover crops are small (20-50 kg C/ha/yr). The use of compost and pig slurry resulted in increases of 0.61-0.73 and $3.15-3.38 \mathrm{~kg} \mathrm{~N} \mathrm{~N}_{2} \mathrm{O}-\mathrm{N}$ per $100 \mathrm{~kg}$ extra SOC accumulated, respectively, with the other fertilizers taking an intermediate position (Bos et al., 2016).

\subsection{Socio-economic benefits}

ISFM increases crop productivity, which helps farmers to satisfy household food self-sufficiency and also to improve wealth status. Net farm income increases when integrated soil fertility is used as a means of soil fertility amendment and crop production. The nutrients from application of integrated soil fertility management found in the soil are the base for the economic improvement of households because these nutrients are responsible for productivity of land which is under different economic activity (Onduru et al., 2007).

Farmers commonly set priorities in applying fertilizers in terms of crop types, market opportunities, farm locations, distance from homestead and other socio-economic conditions. In different developing countries farmers were applying by integrating different soil fertility management methods which are available locally. These locally available nutrients sources (FYM, crop rotation, fallowing, draining water etc.) decreased the cost to purchase fertilizer and the cost of transportation and were easy to apply in the soil to grow crops. Especially poor farmers in the developing world could grow crops with help of integrated application of locally available soil nutrient source ( Agegnehu, Vanbeek and Bird, 2017).

\subsection{Other benefits of the practice}

ISFM is more important in maintaining soil health which is a measure of soil quality. Ecological sustainability and function are also maintained only if ISFM is used as means of soil fertility and crop productivity enhancement option. Moreover environmental pollution is minimized if soil is rich with microorganisms which are improved by ISFM (Parikh and James, 2012). 


\section{Possible greenhouse gases emissions}

The use of ISFM can significantly reduce $\mathrm{N}_{2} \mathrm{O}$ emissions (23 percent reduction) from the soil with respect to the use of synthetic fertilizers alone. Application of ISFM may mitigate soil GHG emissions thanks to an increase in carbon sequestration, a reduction of mineral fertilizers and a decrease in the $\mathrm{N}$ losses thanks to the uptake of nitrate by catch crops both in crop and intercrop periods (Basche et al., 2014).

Application of ISFM causes greater $\mathrm{CH}_{4}$ uptake than conventional agriculture, which was attributed to the higher pore continuity and the presence of ecological niches for methanotrophic bacteria in conservation agriculture. The reduction in $\mathrm{CH}_{4}$ emissions is explained by the reduction of anaerobic decomposition of organic matter by microorganisms, which modifies the pore space and structure and affects the soil oxygen availability (Hutsch, 2001).

\section{Recommendations before implementing the practice}

- Organic fertilizers are good to improve the soil fertility so qualified organic fertilizers like compost and FYM should be used in combination with mineral fertilizer such as diammonium phosphate (DAP) and urea.

- The practice should be not restricted to only organic and inorganic nutrient inputs but rather focus on using all sources of nutrient to the soil and crop like conservation agriculture, application of physical soil and water conservation structures and other agronomic structures.

- The skill, knowledge and attitude of farmers and technicians should be improved through training to practice and apply integrated soil fertility management on their farm.

\section{Potential barriers for adoption}

The history of the world efforts to improve ISFM shows clearly the existence of negative (biophysical and socioeconomic factors) aspects that have prevented the adoption of the technology (Irungu, 2011). Other studies (e.g. Shiferaw, Okello and Reddy, 2009) reported that limited availability of affordable farm inputs like fertilizers, improved seed, poor roads and market infrastructure, locally produced farm machinery coupled with labor shortages influence adoption of ISFM technologies by the smallholder farmers. Similarly, it has been observed that lack of appropriate knowledge base to combine rainwater harvesting structures with suitable agronomic measures can contribute to low adoption of the ISFM technologies. 
Table 73. Potential barriers to adoption

\begin{tabular}{|l|l|l|}
\hline Barrier & YES/NO & \\
\hline Biophysical & Yes & $\begin{array}{l}\text { Distance to input and output markets had a negative } \\
\text { influence on use of ISFM (Nambiro and Okoth 2013). }\end{array}$ \\
\hline Social & Yes & $\begin{array}{l}\text { Availability of labour affected the adoption of ISFM (Mutoko et } \\
\text { al., 2015). }\end{array}$ \\
\hline Economic & Yes & $\begin{array}{l}\text { Inaccessibility to agricultural credit negatively influenced adoption of } \\
\text { ISFM ( Nobeji, Nie and Fang, 2011). }\end{array}$ \\
\hline Institutional & Yes & $\begin{array}{l}\text { Unavailability of relevant agricultural institutions affect adoption of } \\
\text { ISFM (Adolwa et al., 2012). }\end{array}$ \\
\hline Knowledge & Yes & $\begin{array}{l}\text { ISFM needs skilled and educated experts as well as farmers (Aura, } \\
\text { 2016). }\end{array}$ \\
\hline
\end{tabular}




\section{Photos of the practice}

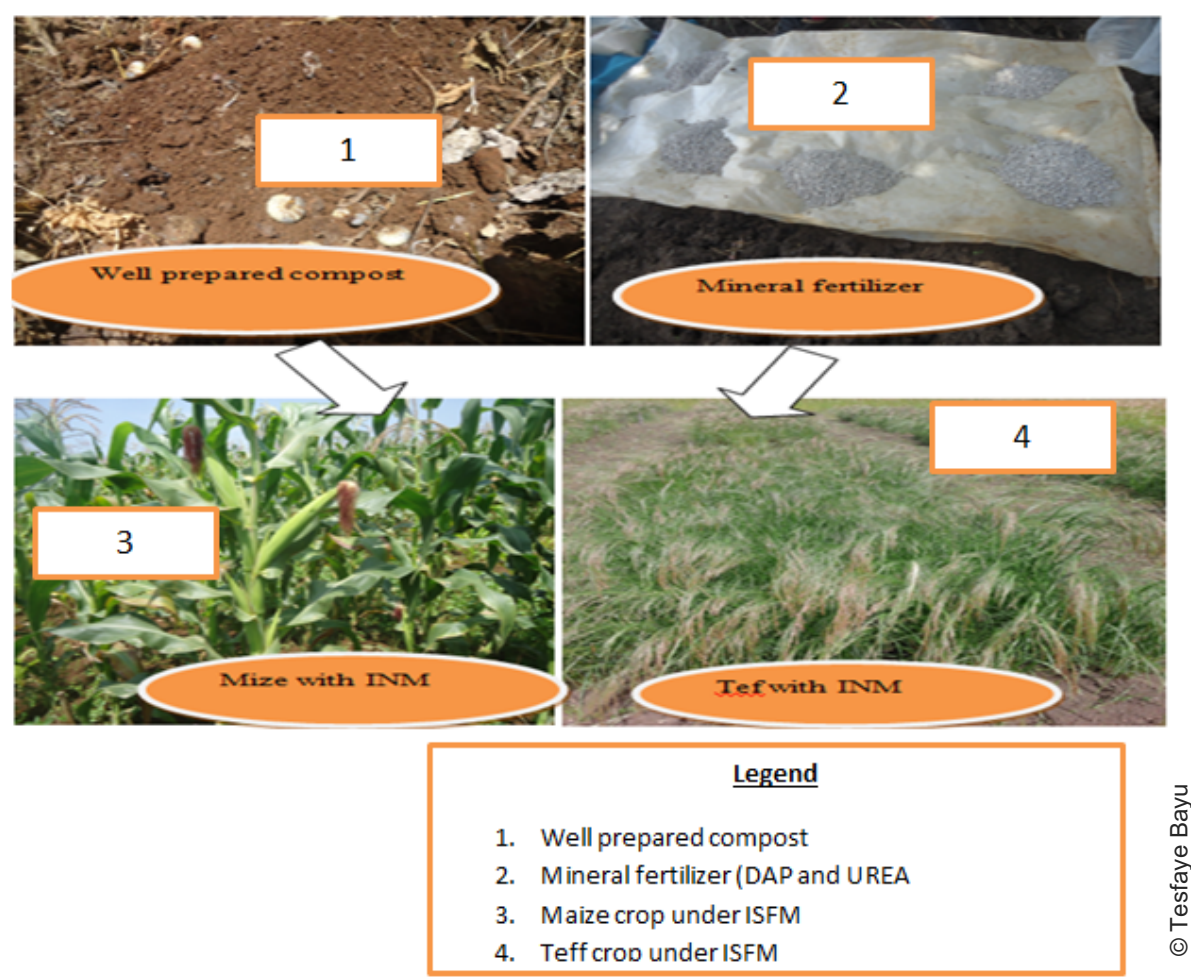

Photo 18. Components of an Integrated Soil Fertility program for Teff and Maize production

Table 74. Related cases studies available in volumes 3 and 5

\begin{tabular}{|l|c|c|c|c|}
\hline Title & Region & $\begin{array}{l}\text { Duration of } \\
\text { study (Years) }\end{array}$ & Volume & $\begin{array}{l}\text { Case- } \\
\text { study } \\
\text { No. }\end{array}$ \\
\hline $\begin{array}{l}\text { Agricultural practices for the restoration of Soil } \\
\text { Ecological Functions in Madagascar }\end{array}$ & Africa & 2 & 3 & 2 \\
\hline $\begin{array}{l}\text { Organo-mineral fertilization on a Ukrainian } \\
\text { black soil }\end{array}$ & Europe & 5 & 3 & 26 \\
\hline $\begin{array}{l}\text { Biochar as a Soil Amendment for Carbon } \\
\text { Sequestration in Canada }\end{array}$ & $\begin{array}{l}\text { North } \\
\text { America }\end{array}$ & 1 and 3 & 3 & 41 \\
\hline
\end{tabular}




\section{References}

Adolwa, I.S., Okoth, F.P., Mulwa, R.M., Esilaba, A.O., Franklin, S.M. \& Nambiro, E. 2012. Analysis of communication and dissemination channels influencing uptake of Integrated Soil Fertility Management amongst smallholder farmers in Western Kenya. The Journal of Agricultural Education and Extension, 18: 71-86. https://doi.org/10.1080/1389224X.2012.638782

Agbede, T. M. 2010. Tillage and fertilizer effects on some soil properties, leaf nutrient concentrations, growth and sweet potato yield on an Alfisol in southwestern Nigeria. Soil and Tillage Research, 110(1), 2532. https://www.sciencedirect.com/science/article/pii/S0167198710001042

Agegnehu, G. \& Amede, T. 2017. Integrated soil fertility and plant nutrient management in tropical agroecosystems: a review. Pedosphere, 27(4): 662-680. https://doi.org/10.1016/S1002-0160(17)60382-5

Agegnehu, G., Vanbeek, C., \& Bird, M. I. 2014. Influence of integrated soil fertility management in wheat and tef productivity and soil chemical properties in the highland tropical environment. Journal of soil science and plant nutrition, 14(3), 532-545.https://scielo.conicyt.cl/scielo.php?pid=S0718-

95162014005000042\&script=sci_arttext\&tlng=en

Akram, A., Fatima, M., Ali, S., Jilani, G. \& Asghar, R. 2007. Growth, yield and nutrients uptake of sorghum in response to integrated phosphorus and potassium management. Pak. J. Bot., 39: 1083-1087.

Amanullah, Khalid, S., Imran, Khan, H.A., Arif, M., Altawaha, A.R., Adnan, M., Fahad, S. \& Parmar, B. 2019. Organic Matter Management in Cereals Based System: Symbiosis for Improving Crop Productivity and Soil Health. In R. Lal \& R. Francaviglia, eds. Sustainable Agriculture Reviews 29: Sustainable Soil Management: Preventive and Ameliorative Strategies, pp. 67-92. Sustainable Agriculture Reviews. Cham, Springer International Publishing. https://doi.org/10.1007/978-3-030-26265-5_3

Amare, T., Terefe, A., Selassie, Y.G., Yitaferu, B., Wolfgramm, B. \& Hurni, H. 2013. Soil Properties and Crop Yields along the Terraces and Toposequece of Anjeni Watershed, Central Highlands of Ethiopia. Journal of Agricultural Science, 5(2): p134. https://doi.org/10.5539/jas.v5n2p134

Antil, R.S. 2012. Impact of sewage and industrial effluents on soil-plant health. Industrial Waste, 53-72.

Aura, S. 2016. Determinants of the adoption of integrated soil fertility management technologies in Mbale division, Kenya. African Journal of Food, Agriculture, Nutrition and Development, 16(1): 10697-10710. https://doi.org/10.4314/ajfand.v16i1.

Basche, A.D., Miguez, F.E., Kaspar, T.C. \& Castellano, M.J. 2014. Do cover crops increase or decrease nitrous oxide emissions? A meta-analysis. Journal of Soil and Water Conservation, 69(6): 471-482. https://doi.org/10.2489/jswc.69.6.471

Bationo, A., Kihara, J. \& Adesina, A. 2012. Beyond Biophysical Recommendations: Towards a New Paradigm. In J. Kihara, D. Fatondji, J.W. Jones, G. Hoogenboom, R. Tabo \& A. Bationo, eds. Improving Soil Fertility Recommendations in Africa using the Decision Support System for Agrotechnology Transfer (DSSAT), pp. 169-184. Dordrecht, Springer Netherlands. https://doi.org/10.1007/978-94-007-29605_11 
Bayu, T. 2020. Review on contribution of integrated soil fertility management for climate change mitigation and agricultural sustainability. Cogent Environmental Science, 6(1): 1823631.

https://doi.org/10.1080/23311843.2020.1823631

Bharali, A., Baruah, K.K., Baruah, S.G. \& Bhattacharyya, P. 2018. Impacts of integrated nutrient management on methane emission, global warming potential and carbon storage capacity in rice grown in a northeast India soil. Environmental Science and Pollution Research, 25(6): 5889-5901.

https://doi.org/10.1007/s11356-017-0879-0

Bista, P., Ghimire, R., Shah, S.C. \& Pande, K.R. 2010. Assessment of Soil Fertility Management Practices and Their Constraints in Different Geographic Locations of Nepal. Forum geografic, IX(9): 41-48.

Bokhtiar, S.M. \& Sakurai, K. 2005. Effects of organic manure and chemical fertilizer on soil fertility and productivity of plant and ratoon crops of sugarcane. Archives of Agronomy and Soil science, 51(3): 325-334. https://doi.org/10.1080/03650340500098006

Bos, J.F.F.P., ten Berge, H.F.M., Verhagen, J. \& van Ittersum, M.K. 2017. Trade-offs in soil fertility management on arable farms. Agricultural Systems, 157: 292-302.

https://doi.org/10.1016/j.agsy.2016.09.013

Chander, G., Wani, S.P., Sahrawat, K.L., Pal, C.K. \& Mathur, T.P. 2013. Integrated Plant Genetic and Balanced Nutrient Management Enhances Crop and Water Productivity of Rainfed Production Systems in Rajasthan, India. Communications in Soil Science and Plant Analysis, 44(22): 3456-3464.

https://doi.org/10.1080/00103624.2013.847450

Dutta, S., Mishra, A.K. \& Dileep Kumar, B.S. 2008. Induction of systemic resistance against fusarial wilt in pigeon pea through interaction of plant growth promoting rhizobacteria and rhizobia. Soil Biology and Biochemistry, 40(2): 452-461. https://doi.org/10.1016/j.soilbio.2007.09.009

Ejigu, F. \& Araya, H. 2010. Activity report for 2010 on Building awareness on the value of bio-slurry and its use as organic fertilizer, set up a system to record, analyze and report the impact of bio-slurry on crop yield and making cross visits. Institute For Sustainable Development (ISD) and National Biogas Program Ethiopia (NBPE).

Fairhurst, T. 2012. Handbook for Integrated Soil Fertility Management. Africa Soil Health Consortium, Nairobi. https://cgspace.cgiar.org/handle/10568/76786

Gadermaier, F., Berner, A., Fließbach, A., Friedel, J.K. \& Mäder, P. 2012. Impact of reduced tillage on soil organic carbon and nutrient budgets under organic farming. Renewable Agriculture and Food Systems, 27(1): 68-80. https://doi.org/10.1017/S1742170510000554

Gál, A., Vyn, T.J., Michéli, E., Kladivko, E.J. \& McFee, W.W. 2007. Soil carbon and nitrogen accumulation with long-term no-till versus moldboard plowing overestimated with tilled-zone sampling depths. Soil and Tillage Research, 96(1): 42-51. https://doi.org/10.1016/j.still.2007.02.007

Gruhn, P., Goletti, F. \& Yudelman, M. 2000. Integrated nutrient management, soil fertility and sustainable agriculture: current issues and future challenges. IFRPI 2020 Vision Brief. (also available at: http://ebrary.ifpri.org/utils/getfile/collection/p15738coll2/id/48020/filename/43478.pdf) 
Huang, Y. \& Sun, W. 2006. Changes in topsoil organic carbon of croplands in mainland China over the last two decades. Chinese Science Bulletin, 51(15): 1785-1803. https://doi.org/10.1007/s1 1434-006-20566

Hütsch, B.W. 2001. Methane oxidation in non-flooded soils as affected by crop production - invited paper. European Journal of Agronomy, 14(4): 237-260. https://doi.org/10.1016/S1161-0301(01)00110-1

Irungu, J.W. 2011. Food Security situation in Kenya and Horn of Africa. Presentation made during the Fourth McGill University Global Food Security Conference, 4-6 October 2011. Ministry of Agriculture, Kenya. (also available at: https:/ /www.mcgill.ca/globalfoodsecurity/files/globalfoodsecurity/kari.pdf)

Jagathjothi, N., Ramamoorthy, K. \& Kuttimani, R. 2011. Integrated nutrient management on growth and yield of rainfed direct sown finger millet. Research on Crops, 12: 79-81.

Jambert, C., Serca, D. \& Delmas, R. 1997. Quantification of N-losses as $\mathrm{NH}_{3}, \mathrm{NO}$, and $\mathrm{N}_{2} \mathrm{O}$ and $\mathrm{N}_{2}$ from fertilized maize fields in southwestern France. Nutrient Cycling Agroecosystems, 48: 91-104. https://doi.org/10.1023/A:1009786531821

Jha, M.N., Chaurasia, S.K. \& Bharti, R.C. 2013. Effect of Integrated Nutrient Management on Rice Yield, Soil Nutrient Profile, and Cyanobacterial Nitrogenase Activity under Rice-Wheat Cropping System. Communications in Soil Science and Plant Analysis, 44(13): 1961-1975.

https://doi.org/10.1080/00103624.2013.794821

Ju, X., Lu, X., Gao, Z., Chen, X., Su, F., Kogge, M., Römheld, V., Christie, P. \& Zhang, F. 2011. Processes and factors controlling $\mathrm{N} 2 \mathrm{O}$ production in an intensively managed low carbon calcareous soil under sub-humid monsoon conditions. Environmental Pollution, 159(4): 1007-1016.

https://doi.org/10.1016/j.envpol.2010.10.040

Kaur, K., Kapoor, K.K. \& Gupta, A.P. 2005. Impact of organic manures with and without mineral fertilizers on soil chemical and biological properties under tropical conditions. Journal of Plant Nutrition and Soil Science, 168(1): 117-122. https://doi.org/10.1002/jpln.200421442

Lal, R. 2005. Forest soils and carbon sequestration. Forest Ecology and Management, 220(1): 242-258. https://doi.org/10.1016/j.foreco.2005.08.015

Ma, B.L., Wu, T.Y., Tremblay, N., Deen, W., Morrison, M.J., Mclaughlin, N.B., Gregorich, E.G. \& Stewart, G. 2010. Nitrous oxide fluxes from corn fields: on-farm assessment of the amount and timing of nitrogen fertilizer. Global Change Biology, 16(1): 156-170. https://doi.org/10.1111/j.1365-

2486.2009.01932.x

Milkha A.K. \& Aulakh, M.S. 2010. Effect of long-term fertilizer management and crop rotations on accumulation and downward movement of phosphorus in semi-arid subtropical irrigated soils. Communications in soil science and plant analysis, 41(7): 848-864.

https://doi.org/10.1080/00103621003592366

Miriti, J.M., Esilaba, A.O., Bationo, A., Cheruiyot, H., Kihumba, J. \& Thuranira, E.G. 2007. Tiedridging and integrated nutrient management options for sustainable crop production in semi-arid eastern Kenya. In A. Bationo, B. Waswa, J. Kihara \& J. Kimetu (Eds.) Advances in Integrated Soil Fertility Management in sub-Saharan Africa: Challenges and Opportunities. pp. 435-442. Dordrecht. 
Mohawesh, Y., Taimeh, A. \& Ziadat, F. 2015. Effects of land use changes and soil conservation intervention on soil properties as indicators for land degradation under a Mediterranean climate. Solid Earth, 6(3): 857-868. https://doi.org/10.5194/se-6-857-2015

Mukuralinda, A., Tenywa, J.S., Verchot, L., Obua, J., Nabahungu, N.L. \& Chianu, J.N. 2010. Phosphorus uptake and maize response to organic and inorganic fertilizer inputs in Rubona, Southern Province of Rwanda. Agroforestry systems, 80(2): 211-221. https://doi.org/10.1007/s10457-010-93249

Munyabarenzi, I. 2014. Effect of farmyard manure and mineral fertilizers on maize yield and soil properties in huye and bugesera districts of Rwanda. MSc thesis, Kenyatta University.

Mutoko, M.C., Ritho, C.N., Benhin, J.K. \& Mbatia, O.L. 2015. Technical and allocative efficiency gains from integrated soil fertility management in the maize farming system of Kenya. Journal of Development and Agricultural Economics, 7(4): 143-152. https://doi.org/10.5897/JDAE2015.0633

Nambiro, E. \& Okoth, P. 2013. What factors influence the adoption of inorganic fertilizer by maize farmers? A case of Kakamega District, Western Kenya. Scientific Research and Essays, 8(5): 205-210. https://doi.org/10.5897/SRE10.1063

Naresh, R.K., Singh, S.P. \& Kumar, V. 2013. Crop establishment, tillage and water management technologies on crop and water productivity in rice-wheat cropping system of North West India. International Journal of Life Sciences Biotechnology and Pharma Research, 2(3): 237-248. (also available at: http://new.ijlbpr.com/jlbpradmin/upload/ijlbpr_51d44b872db85.pdf)

Nawab, K., Amanullah Shah, P., Rab, A., Arif, M., Khan, M.A., Mateen, A. \& Munsif, F. 2011. Impact of integrated nutrient management on growth and grain yield of wheat under irrigated cropping system. Pak. J. Bot., 43: 1943-1947.

Nobeji S.B., Nie, F. \& Fang, C. 2011. An Analysis of Factors Affecting Smallholder Rice Farmers' Level of Sales and Market Participation in Tanzania; Evidence from National Panel Survey Data 2010 -2011. Journal of Economics and Sustainable Development, 5(23): 185-204. (also available at: http:// citeseerx.ist.psu.edu/viewdoc/download?doi=10.1.1.841.7174\&rep=rep1\&type=pdf)

Oikeh, S.O Azoma, K. \&. Saito, K. 2010. Combined effects of Stylosanthes guianensis fallow and tillage management on upland rice yield, weeds and soils in southern Benin. Soil and Tillage Research, 107(2): 5763. https://doi.org/10.1016/j.still.2010.03.001

Onduru, D.D., De Jager, A., Muchena, F.N., Gachimbi, L. \& Gachini, G.N. 2007. Socio-economic Factors, Soil Fertility Management and Cropping Practices in Mixed Farming Systems of Sub-Saharan Africa: A Study in Kiambu, Central Highlands of Kenya. International Journal of Agricultural Research, 2: 426-439. https://doi.org/3923/ijar.2007.426.439

Parikh, S.J. \& James, B.R. 2012. Soil: The Foundation of Agriculture. Nature Education Knowledge, 3(10): 2.

Park, J.H., Lamb, D., Paneerselvam, P., Choppala, G., Bolan, N. \& Chung, J.-W. 2011. Role of organic amendments on enhanced bioremediation of heavy metal(loid) contaminated soils. Journal of Hazardous Materials, 185(2): 549-574. https://doi.org/10.1016/j.jhazmat.2010.09.082 
Parkinson, R. 2013. System based integrated nutrient management. Soil Use Management, 29: 608.

Patil, S.L. \& Sheelavantar, M.N. 2001. Effect of in-situ moisture conservation practices and integrated nutrient management on nutrient availability and grain yield of rabi sorghum (Sorghum bicolor) in the Vertisols of semi-arid tropics of south India. Indian J. Agric. Sci, 71: 229-233.

Paul, B.K., Vanlauwe, B., Ayuke, F., Gassner, A., Hoogmoed, M., Hurisso, T.T., Koala, S., Lelei, D., Ndabamenye, T., Six, J. \& Pulleman, M.M. 2013. Medium-term impact of tillage and residue management on soil aggregate stability, soil carbon and crop productivity. Agriculture, Ecosystems \& Environment, 164: 14-22. https://doi.org/10.1016/j.agee.2012.10.003

Pypers, P., Bimponda, W., Lodi-Lama, J.-P., Lele, B., Mulumba, R., Kachaka, C., Boeckx, P., Merckx, R. \& Vanlauwe, B. 2012. Combining Mineral Fertilizer and Green Manure for Increased, Profitable Cassava Production. Agronomy journal. (also available at https://agris.fao.org/agrissearch/search.do? recordID=US201500053912).

Rware, H., Wairegi, L., Oduor, G., Macharia, M., Romney, D., Tarfa, B.D., Maria, R. de, Ley, G., Tetteh, F., Makumba, W., Dicko, M., Nouri, M., Leon, N.N., Gondwe, B., Kaizzi, K., Negash, D., Kibunja, C., Alawy, A., Wartmann, C. \& Rutsimba, E. 2014. Assessing the Potential to Change Stakeholders Knowledge and Practices on Fertilizer Recommendations in Africa. Agricultural Sciences, 5(14): 1384-1391. https://doi.org/10.4236/as.2014.514149

Selassie, Y.G. \& Ayanna, G. 2013. Effects of different land use systems on selected physico-chemical properties of soils in Northwestern Ethiopia. Journal of agricultural science, 5(4): 112.

Sharma, M.P., Bali, S.V. \& Gupta, D.K. 2001. Soil fertility and productivity of rice (Oryza sativa)-wheat (Triticum aestivum) cropping system in an Inceptisol as influenced by integrated nutrient management. Indian Journal of Agricultural Sciences, 71: 82-86.

Shiferaw, B., Okello, J. \& Reddy, V.R. 2009. Challenges of adoption and adaptation of land and water management options in smallholder agriculture: synthesis of lessons and experiences. In S.P. Wani, J. Rockström \& T. Oweis (Eds.) Rainfed agriculture: unlocking the potential, pp. 258-275. Wallingford, CABI. (also available at http://www.cabi.org/cabebooks/ebook/20093041388).

Simpson, R.J., Richardson, A.E., Nichols, S.N. \& Crush, J.R. 2014. Pasture plants and soil fertility management to improve the efficiency of phosphorus fertiliser use in temperate grassland systems. Crop and Pasture Science, 65(6): 556-575. https://doi.org/10.1071/CP13395

Smith, J., Abegaz, A., Matthews, R.B., Subedi, M., Orskov, E.R., Tumwesige, V. \& Smith, P. 2014. What is the potential for biogas digesters to improve soil fertility and crop production in Sub-Saharan Africa? Biomass and Bioenergy, 70: 58-72. https://doi.org/10.1016/j.biombioe.2014.02.030

Sommer, R., Paul, B.K., Mukalama, J. \& Kihara, J. 2018. Reducing losses but failing to sequester carbon in soils - the case of Conservation Agriculture and Integrated Soil Fertility Management in the humid tropical agro-ecosystem of Western Kenya. Agriculture, Ecosystems \& Environment, 254: 82-91.

https://doi.org/10.1016/j.agee.2017.11.004 
Srinivasarao, C., Venkateswarlu, B., Lal, R., Singh, A.K., Vittal, K.P.R., Kundu, S., Singh, S.R. \& Singh, S.P. 2012. Long-Term Effects of Soil Fertility Management on Carbon Sequestration in a Rice-Lentil Cropping System of the Indo-Gangetic Plains. Soil Science Society of America Journal, 76(1): 168-178. https://doi.org/10.2136/sssaj2011.0184

Thind, S.S., Sidhu, A.S., Sekhon, N.K. \& Hira, G.S. 2007. Integrated Nutrient Management for Sustainable Crop Production in Potato-Sunflower Sequence. Journal of Sustainable Agriculture, 29(4): 173 188. https://doi.org/10.1300/J064v29n04_12

Vanlauwe, B., Descheemaeker, K., Giller, K.E., Huising, J., Merckx, R., Nziguheba, G., Wendt, J. \& Zingore, S. 2015. Integrated soil fertility management in sub-Saharan Africa: unravelling local adaptation. SOIL, 1(1): 491-508. https://doi.org/10.5194/soil-1-491-2015

Wu, W. \& Ma, B. 2015. Integrated nutrient management (INM) for sustaining crop productivity and reducing environmental impact: A review. Science of The Total Environment, 512-513: 415-427.

https://doi.org/10.1016/j.scitotenv.2014.12.101 
CROPLAND

NUTRIENT MANAGEMENT

BIOCHAR

\title{
17. Biochar
}

\author{
Silvia Baronti, Anita Maienza, Francesco Primo Vaccari
}

National Research Council- Institute of Bioeconomy (CNR-IBE), Florence, Italy

\section{Description of the practice}

Biochar is a relatively recent term, used to name charred organic matter when it is applied to soil in a deliberate manner, with intent to improve soil properties and long-term carbon sequestration (Lehmann and Joseph, 2015). This distinguishes biochar from charcoal that is used as fuel for cooking. Regarding its definition in production, biochar is defined by the International Biochar Initiative ${ }^{8}$ as "The solid material obtained from the thermochemical conversion of biomass in an oxygen-limited environment". Pyrolysis is the most common technology employed to produce biochar. Biochar can and should be made from biomass waste materials (Photo 19).

Biomass waste materials (feedstock) appropriate for biochar production include crop residues, as well as yard, food and forestry wastes, and animal manures. Feedstocks must not contain unacceptable levels of toxins such as heavy metals that can be found in sewage sludge and industrial or landfill waste. Pyrolysis conditions and feedstock characteristics largely control the physicochemical properties of the resulting biochar, which in turn determine the suitability for a given application, as well as define its behavior, transport and fate in the environment. Evidence suggests that components of the carbon in biochar are highly recalcitrant in soils, with reported residence times for wood biochar being in the range of 100s to $1000 \mathrm{~s}$ of years, that is approximately 10 to 1000 times longer than residence times of most soil organic matter (SOM). Therefore, biochar addition to soil can provide a potential long-term sink for C. The biochar should be primarily considered as a soil amendment and not as a fertilizer. In fact, depending on the type of soil and biochar, it can improve to different degrees the essential soil functions for long periods, but does not replace the need for nutrient additions through either inorganic or organic fertilizers. Biochar systems can reverse soil degradation and create sustainable food and fuel production in areas with severely depleted soils, scarce organic resources, and inadequate water and chemical fertilizer supplies. Commercial-scale production and use of biochar is in its infancy, and practical, affordable methods of application are still under development.

${ }^{8}$ https://biochar-international.org/ 


\section{Range of applicability}

The addition of biochar to agricultural soils is receiving much attention due to the apparent benefits to soil quality and enhanced crop yields in acidic infertile soils common to tropical regions, as well as the potential to gain carbon credits by active carbon sequestration. The diverse physical and chemical characteristics of biochar make it a compelling and useful substance in a variety of applications, from home- garden to large-scale farming. It should be noted that biochars vary widely in properties depending on the feedstock and production conditions, so suitable biochar should be chosen to address the specific soil constraints for each intended application. From a practical viewpoint, integrated biochar applications with compost, vermicompost, cattle and poultry manure are found to be effective techniques (Jien et al., 2017). However, this technology as any other must be implemented in a way that respects and supports the health of natural ecosystems. Application of biochar as a soil amendment is currently practiced in China, United States of America, Australia and New Zealand and in many countries in South East Asia. In Europe, the lack of a European Directive on biochar use in agriculture does not favor biochar application. The International Biochar Initiative provides more information (IBI https://biochar-international.org/).

\section{Impact on soil organic carbon stocks}

The molecular structure of biochar makes it more resistant to microbial decomposition compared to nonpyrolized organic matter. This allows biochar to persist in soil for thousands of years. Biochar stabilizes existing soil organic matter (SOM) by promoting aggregate stability and through its association with organo-mineral complexes (Table 75). Further, the presence of aged biochar reduces decomposition rates of crop-derived residue $\mathrm{C}$ in soil and increases crop residue stabilization in soil aggregates (Hernandez-Soriano et al., 2015).

The timing and the amount of SOC degradation are mainly linked with biochar pyrolysis temperature, the application rate and the soil texture (Yang et al., 2020). SOC increase in tropical sandy soil, clay and sandy clay soil is estimated from 19 to 69 percent following biochar application (Giagnoni et al, 2019). The increase in SOC might be attributed to the improved crop biomass, especially root biomass, after biochar application, which increased the input of fresh organic carbon. The increase in SOC could be attributed to the improvement of crop biomass, especially root biomass, after the application of biochar, which increased the fresh organic carbon input, but also the change in soil moisture content, $\mathrm{pH}$, temperature and soil enzyme activities due to biochar affect SOC turnover and degradation time. The accumulation of rhizodeposits in organo-mineral fractions promoted biochar-induced negative priming of native soil organic carbon in Ferralsol (Weng et al., 2018).

The limited number of large-scale long term field experiments and the many aspects that must be considered, make it difficult to estimate the impact on the SOC sequestration due to the biochar application at global scale (Table 75). A more useful estimate is the amount of biochar carbon remaining in the long term (i.e. 100 years). This can be estimated using the table from Volume 4/chapter 2/Appendix 4 of the 2019 refinement of the IPCC guidelines for national greenhouse gas inventories ${ }^{9}$.

$$
\text { Inc. } \mathrm{SOC}_{\mathrm{B}}=\left(\mathrm{BR}{ }^{*} \mathrm{C}_{\mathrm{B}}\right)-\mathrm{CL}
$$

\footnotetext{
${ }^{9}$ https://www.ipcc-nggip.iges.or.jp/public/2019rf/pdf/4_Volume4/19R_V4_Ch02_Ap4_Biochar.pdf
} 
Where:

- Inc. $\mathrm{SOC}_{\mathrm{B}}=$ represent the increase of $\mathrm{SOC}(\mathrm{t} / \mathrm{ha})$ due to the biochar application;

- $\mathrm{BR}=$ biochar rate application expressed as $\mathrm{t} / \mathrm{ha}$ or $\mathrm{t} / \mathrm{ha} / \mathrm{yr}$ (for multiple biochar application);

- $\mathrm{C}_{\mathrm{B}}=$ carbon content of the biochar (\%);

- $\mathrm{CL}=$ carbon loss (\%) due to biochar application (vertical and horizontal movements and priming effect), suggested values of CL are $5 \%$ or $8 \%$ (conservative estimate)

The proposed formula is a very simple and intuitive method to determine the increase of the soil carbon content due to biochar application to soil. It is normally used and applied when a rough estimate of the increase in soil carbon is necessary. To determine the exact increase in soil carbon a chemical analysis after the biochar application is the only accurate method to apply. 
Table 75. Overview of changes in soil organic carbon stocks reported with Biochar applications

\begin{tabular}{|c|c|c|c|c|c|c|c|c|c|c|}
\hline Location & $\begin{array}{l}\text { Climate } \\
\text { zone }\end{array}$ & Soil type & $\begin{array}{l}\text { Baseline C } \\
\text { stock (t } \\
\text { C/ha) }\end{array}$ & $\begin{array}{l}\text { Biochar } \\
\text { type }\end{array}$ & $\begin{array}{l}\text { Additional C } \\
\text { storage } \\
(\mathrm{tC} / \mathrm{ha} / \mathrm{yr})^{10}\end{array}$ & $\begin{array}{l}\text { Biochar } \\
\text { application } \\
\text { (t/ha) }\end{array}$ & $\begin{array}{l}\text { C content } \\
\text { of biochar } \\
(\%)\end{array}$ & $\begin{array}{l}\text { Duration of } \\
\text { experiment } \\
\text { (years) }\end{array}$ & Depth (cm) & Reference \\
\hline \multirow{3}{*}{$\begin{array}{l}\text { Hebei } \\
\text { Province, } \\
\text { China }\end{array}$} & \multirow{3}{*}{ BSk (Köppen) } & \multirow{3}{*}{ Silty loam } & \multirow{3}{*}{$\approx 26$} & \multirow{3}{*}{$\begin{array}{l}\text { Wheat } \\
\text { residues }\end{array}$} & 0.17 & 1.8 & 38.7 & 4 & $0-20$ & \multirow{3}{*}{ Kan et al. (2020) } \\
\hline & & & & & 0.34 & 3.6 & 38.7 & 4 & $0-20$ & \\
\hline & & & & & 0.70 & 7.2 & 38.7 & 4 & $0-20$ & \\
\hline Lodi, Italy & $\begin{array}{l}\text { Warm } \\
\text { Temperate } \\
\text { Moist }\end{array}$ & Loam & $\approx 46$ & Wood & 15 & 35 & 90 & 2 & $0-30$ & $\begin{array}{l}\text { Massimo Valagussa } \\
\text { INFOCHAR Project PSR } \\
\text { Regione Lombardia 2017- } \\
2019 \\
\text { Ongoing project }\end{array}$ \\
\hline $\begin{array}{l}\text { Jiangsu } \\
\text { Province, } \\
\text { China }\end{array}$ & Cfa (Köppen) & Heavy loam & $\approx 21.7$ & Rice-straw & 17 & 40 & 42.6 & 1 & $0-10$ & Yang et al. (2019) \\
\hline Parma, Italy & \multirow{3}{*}{$\begin{array}{l}\text { Warm } \\
\text { Temperate } \\
\text { Moist }\end{array}$} & Silty clay & 22,5 & $\begin{array}{l}\text { Wheat bran } \\
\text { pellets }\end{array}$ & 4,8 & 14 & 34.6 & 1 & $0-15$ & Vaccari et al. (2015) \\
\hline & & \multirow[b]{2}{*}{ Silty loam } & \multirow[b]{2}{*}{21} & \multirow[b]{2}{*}{ Wood } & 25.2 & 30 & 84 & \multirow[b]{2}{*}{2} & \multirow[b]{2}{*}{$0-15$} & \multirow[b]{2}{*}{ Vaccari et al. (2011) } \\
\hline Pistoia, Italy & & & & & 50.4 & 60 & 84 & & & \\
\hline
\end{tabular}

${ }^{10}$ Estimated: biochar application (t/ha) ${ }^{*}$ Carbon content of biochar/ duration of experiment 


\section{Other benefits of the practice}

\subsection{Improvement of soil properties}

Since biochar contains organic matter and nutrients, its addition increases in general organic carbon, total nitrogen, available phosphorus (P), and the cation-exchange capacity (CEC). The composition of biochar (for example, the amount of carbon, nitrogen, potassium, calcium) depends on the feedstock used and the duration and temperature of pyrolysis. As an example, biochar produced from feedstocks which have greater contents of potassium (such as animal litters) often have higher potassium contents than biochar made entirely from wood (which often have higher carbon contents). However, pyrolysis conditions greatly affect nutrient contents and so biochar should be tested on a batch-by-batch basis to determine specific properties. For instance, the retention of nutrients, water holding capacity, and the formation of aggregates vary depending on the texture of the soil (Ajayi and Horn, 2017). Barnes et al. (2014) reported in a lab experiment that biochar distributed at 10 percent of the total mass (equivalent to $>100 \mathrm{t} / \mathrm{ha}$ ) decreased saturated hydraulic conductivity $(\mathrm{K})$ of sandy soil by 92 percent, but increased K by 328 percent in clay-rich soil. The presence of plant nutrients and ash in the biochar and its large surface area, porous nature, and the ability to act as a medium for microorganisms have been identified as the main reasons for the improvement in soil properties and increase in the absorption of nutrients by plants in soils treated with biochar. Biochar - plant root - soil interactions affect root growth and overall plant performance via two mechanisms: a) directly as a nutrient source and b) indirectly by altering the soil nutrient availability (Prendergast-Miller, Duvall and Sohi, 2014). The beneficial effect is more pronounced for acidic soils due to the alkaline nature of many biochars (Lehmann and Joseph, 2015).

\subsection{Minimization of threats to soil functions}

\section{Table 76. Soil threats}

\begin{tabular}{|l|l|}
\hline Soil threats & \\
\hline Soil erosion & $\begin{array}{l}\text { Biochar application on temperate and tropical soils improves soil structure through } \\
\text { increasing soil water-holding capacity and porosity and consequently reduces soil erosion, } \\
\text { reduces runoff generation during high rainfall events (Sadeghi et al., 2018). Considering all } \\
\text { these beneficial effects, application of biochar in sloping upland areas can be an effective } \\
\text { strategy for minimizing crop damages due to soil erosion, especially during rainy seasons. }\end{array}$ \\
\hline $\begin{array}{l}\text { Nutrient } \\
\text { imbalance and } \\
\text { cycles }\end{array}$ & $\begin{array}{l}\text { Several studies indicated that biochar could effectively increase the amount of total and } \\
\text { plant available N, and extractable K (Vaccari et al., 2015) and available P (Giagnoni et al., }\end{array}$ \\
\hline $\begin{array}{l}\text { So19). } \\
\text { Soil salinization } \\
\text { and alkalinization }\end{array}$ & $\begin{array}{l}\text { Several studies use biochar derived from diverse source materials as a soil amendment to } \\
\text { control soil salinity. For example, impaired growth and yield performance of tomato plants } \\
\text { grown under saline irrigation conditions improve significantly following application of } \\
\text { biochar at 1-5 percent (Herath et al. 2017). }\end{array}$ \\
\hline
\end{tabular}




\begin{tabular}{|c|c|}
\hline Soil threats & \\
\hline $\begin{array}{l}\text { Soil } \\
\text { contamination / } \\
\text { pollution }\end{array}$ & $\begin{array}{l}\text { Biochar is characterized by large specific surface area, porous structure, abundant surface } \\
\text { functional groups and mineral components, which makes it useful as an adsorbent to } \\
\text { remove pollutants from aqueous solutions (Zhang et al., 2018); pesticides (Cwielag- } \\
\text { Piasecka et al., 2018); heavy metals (Liu et al., 2018). Long-term application (after } 10 \text { years) } \\
\text { on vineyards in the Mediterranean area showed no soil pollution effects (polycyclic } \\
\text { aromatic hydrocarbons-PAHs and heavy metals) (Maienza et al., 2017a). Before using it as a } \\
\text { soil amendment, the biochar must be analyzed and have the parameters relating to the } \\
\text { content of heavy metals and PHA below the limits allowed by the various "biochar quality } \\
\text { standards" }\end{array}$ \\
\hline $\begin{array}{l}\text { Soil biodiversity } \\
\text { loss }\end{array}$ & $\begin{array}{l}\text { The internal porosity of biochar particles provides a habitat for microorganisms, changing } \\
\text { the community composition and structure and it is related functions. As indirect effect of } \\
\text { liming, in acid and sub-acid soils, biochar can modify microbial diversity in soil. It can also } \\
\text { potentially improve beneficial symbiotic relationships between plants and microorganisms } \\
\text { such as fungi. Moreover, by increasing soil moisture biochar can increase richness and } \\
\text { abundance of soil macro and mesofauna (Castracani et al., 2015). }\end{array}$ \\
\hline $\begin{array}{l}\text { Soil water } \\
\text { management }\end{array}$ & $\begin{array}{l}\text { Biochar application to soils is considered as one suitable practice to increase water use } \\
\text { efficiency (Baronti et al., 2014). By increasing soil water retention and available water } \\
\text { capacity, biochar amendment has the potential for the soil to retain more water from } \\
\text { irrigation or rainfall and reduce the frequency of irrigation. }\end{array}$ \\
\hline
\end{tabular}

\subsection{Increases in production (e.g. food/fuel/feed/timber/fibre)}

To date, the lack of long-term and large-scale evidence prevents definitive conclusions on whether to implement the biochar strategy on a territorial scale. Different crops have a variable growth response to biochar application. In general, biochar has been shown to have promise for increasing crop productivity in tropical areas and in poor nutrient soil, and only a few studies have shown significant improvement in crop yield in fertile soils (Hussain et al., 2017). In addition, the type of soil plays a significant role on how biochar affects crop yields. Soils with loamy texture (sandy loam, silty loam, loamy, and fine loamy sand) show increases in crop yield from 9 percent to 101 percent, whereas clayey soils show a low yield improvement.

\subsection{Mitigation of and adaptation to climate change}

The potential of biochar to sequester atmospheric carbon after its application has been highlighted by the IPCC (2019). Producing biochar and bioenergy via pyrolysis is a carbon-negative process. In their meta-analysis, Majumder et al. (2019) determined that biochar was more effective in soil carbon sequestration than crop residue. 
The effects of biochar application on soil GHG emissions have been widely studied and found to be highly variable. In general biochar application to soil decreases emission of $\mathrm{N}_{2} \mathrm{O}$ (ca. 50 percent) across a wide range of soil types, in particular biochar derived from woody materials and crop residues are more effective that those derived from manure or processing wastes. Although biochar's effect may not be substantial in reducing $\mathrm{CH}_{4}$ emissions from upland soils, Jeffery et al. (2016) reported significant effects on reducing $\mathrm{CH}_{4}$ emission from paddy soils. Bossio et al. (2020) estimate the mitigation power from the application of the biochar in temperate zones at approximately $1.1 \mathrm{Gt} . \mathrm{CO}_{2} \mathrm{eq} / \mathrm{yr}$.

\subsection{Socio-economic benefits}

The uncertainty around investment in biochar production and the market for carbon offsets needs to be explored further through sound economic and full life-cycle analysis.

\subsection{Other}

Biochar can be used in landfill cover to promote methane oxidation and odor reduction as reported by Wong et al. (2017).

\section{Potential drawbacks to the practice}

\subsection{Tradeoffs with other threats to soil functions}

Table 77. Soil threats

\begin{tabular}{|c|c|}
\hline Soil threats & \\
\hline Soil erosion & $\begin{array}{l}\text { Biochar may be lost through wind erosion from sandy soils and from water erosion from } \\
\text { clayey and compacted soils, but no quantitative information is available. Conservation } \\
\text { agriculture methods, which help maintain a protective soil surface cover, will help reduce } \\
\text { erosion risk (Lehmann and Joseph, 2015). }\end{array}$ \\
\hline $\begin{array}{l}\text { Nutrient } \\
\text { imbalance and } \\
\text { cycles }\end{array}$ & $\begin{array}{l}\text { Pyrolysis of organic matter leads to volatilization of nitrogen, up to } 70-80 \text { percent of } \mathrm{N} \text { in } \\
\text { feedstock can be lost (Ye et al., 2020). It has been suggested that biochar may increase the } \\
\mathrm{EC} \text { of leachate, attributed to loss of } \mathrm{Na} \text { and } \mathrm{K} \text { from the biochar-soil matrix (Novak et al., } \\
2009 \text { ). It is clear that impacts on nutrients are dependent upon the properties of both soil } \\
\text { and biochar. }\end{array}$ \\
\hline $\begin{array}{l}\text { Soil salinization } \\
\text { and alkalization }\end{array}$ & $\begin{array}{l}\text { Biochar with a high } \mathrm{pH} \text { value would cause a significant } \mathrm{pH} \text { increase in soil with neutral to } \\
\text { basic properties. }\end{array}$ \\
\hline
\end{tabular}




\begin{tabular}{|c|c|}
\hline Soil threats & \\
\hline $\begin{array}{l}\text { Soil } \\
\text { contamination / } \\
\text { pollution }\end{array}$ & $\begin{array}{l}\text { Changes in soil pH can influence the bioavailability of toxic elements such as Aluminum } \\
\text { (Lehmann and Joseph, 2015). }\end{array}$ \\
\hline Soil acidification & $\begin{array}{l}\text { Biochars can have a wide range of } \mathrm{pH} \text { values ranging from slightly acidic to alkaline. Pine } \\
\text { sawdust biochar in a sandy desert soil is shown to decrease the soil } \mathrm{pH} \text { (Laghari et al., 2015). }\end{array}$ \\
\hline $\begin{array}{l}\text { Soil biodiversity } \\
\text { loss }\end{array}$ & $\begin{array}{l}\text { Biochar impact on soil microorganisms is rather diverse. Many interaction mechanisms } \\
\text { have been proposed in the literature (Palansooriya et al., 2019; Zhu et al., 2017). Some } \\
\text { authors (Awad et al., 2018; Ye et al., 2017) suggested that biochar's effect on microbial } \\
\text { communities largely depended on biochar application rates, biochar and soil types. Clearly, } \\
\text { this is an area that deserves much greater attention in future to establish the long-term } \\
\text { effect of biochar application in soil-on-soil biological health. }\end{array}$ \\
\hline Soil compaction & $\begin{array}{l}\text { A decrease in bulk density is the most consistently reported for a wide range of biochar } \\
\text { application rates (Baronti et al., 2014; Maienza et al., 2017; Giagnoni et al., 2019). }\end{array}$ \\
\hline $\begin{array}{l}\text { Soil water } \\
\text { management }\end{array}$ & $\begin{array}{l}\text { The depth and method of biochar incorporation into soils has the potential to influence soil } \\
\text { water retention. There are still several gaps in understanding the impact of biochar } \\
\text { additions on water retention and water partitioning in sandy soils (Basso et al., 2013). }\end{array}$ \\
\hline
\end{tabular}

\subsection{Increases in greenhouse gas emissions}

To understand how biochar can affect nitrous oxide $\left(\mathrm{N}_{2} \mathrm{O}\right)$ and methane emissions $\left(\mathrm{CH}_{4}\right)$, many experiments are required, because the processes influencing these emissions are complex and emissions vary markedly depending on site conditions. Cayuela et al. (2014) in a review (using 30 studies with 261 experimental treatments) found that biochar reduced $\mathrm{N}_{2} \mathrm{O}$ emissions to soil by 54 percent in laboratory and field studies. Biochar feedstock, pyrolysis conditions and $\mathrm{C} / \mathrm{N}$ ratio have been shown to be key factors affecting $\mathrm{N}_{2} \mathrm{O}$ emissions while a direct correlation was found between biochar application rate and reductions in $\mathrm{N}_{2} \mathrm{O}$ emissions. It was also found that the interactions between soil texture and biochar and the chemical form of the $\mathrm{N}$ fertilizer applied with biochar have a major influence on soil $\mathrm{N}_{2} \mathrm{O}$ emissions. While there is strong evidence that, in many cases, $\mathrm{N}_{2} \mathrm{O}$ emissions are reduced, there is still a significant lack of understanding of the key mechanisms that result in these modified emissions.

Jeffery et al. (2016) in a meta-analysis reveals that biochar use may have the potential to reduce atmospheric $\mathrm{CH}_{4}$ emissions from agricultural flooded soils on a global scale. 
In addition, some studies (Genesio et al., 2012; Bozzi et al., 2015) suggest decreased albedo of biocharamended soil could partly negate the climate change benefits of carbon stabilization in biochar but such an effect can be reduced or eliminated by incorporation, crop canopies and/or residue cover. Thus, while biochars appear to pose minimal risk of exacerbating soil $\mathrm{CO}_{2}$ emissions in the long term, more studies examining both field and lab results are needed to ascertain whether $\mathrm{CO}_{2}$ responses to biochar amendment, observed in the lab, can be used to predict $\mathrm{CO}_{2}$ responses in the field-and to accurately predict long-term $\mathrm{CO}_{2}$ emissions of biochar amended soils at the field scale.

\subsection{Conflict with other practice(s)}

Negative impacts can include binding and deactivation of agrochemicals (herbicides and nutrients) in soil, release of toxicants that may be present in biochar (e.g. heavy metals), increase in $\mathrm{EC}$ and $\mathrm{pH}$, and impacts on germination and soil biological processes (Lehmann and Joseph, 2015). These potential adverse effects can be avoided by selecting biochar with properties suited to the site of application.

\subsection{Decreases in production (e.g. food/fuel/feed/timber/fibre)}

Recent meta-analyses of yield effects of biochar show average responses of 15-25 percent (Jeffery et al, 2017; Ye et al. 2019; Dai et al., 2020) These studies show that the effect varies with feedstock, rate, soil type and climate. Nevertheless, where biochar is not matched to site conditions, nil or negative effects on yield have been observed.

For example, Vaccari et al. (2015) report that incorporation of two different biochars at $14 \mathrm{t} / \mathrm{ha}$ (obtained by slow pyrolysis and biochar obtained by fast pyrolysis) into fertile soil does not increase the tomato yield significantly. Similarly, Niu et al. (2017) report that the application of biochar had no effect on maize yield whereas in wheat, biochar increased yield when no fertilizer was applied, but decreased yield in the presence of fertilizer.

\subsection{Other conflicts}

Some biochars may be rich in metal contents and may be of concern if applied to soil without due consideration. The levels of toxic metals in biochars depend on the original content of metals in the feedstocks and processing conditions, and the potential impact on soil would depend on the soil type and ability of biochar to immobilize and detoxify the contaminants (e.g. Chen et al., 2018). Waste management is one of the attractive elements associated with biochar technology (Lehmann and Joseph, 2015), therefore, the feedstock source-especially when biochars are produced from waste material (e.g. municipal solid waste-MSW, sewage sludge, or industrial waste)-may influence the nature and extent of contaminants present in the final biochar product and/or formed during the production process.

Particulate matter (PM) released from biochar may have negative effects on human health and increase the atmospheric burden of shortwave absorbing black carbon aerosols with non-negligible effects on atmospheric radiative forcing (Maienza et al., 2017b). 


\section{Recommendations before implementing the practice}

To decide whether to use biochar in agriculture is a complex operational choice, because biochar is a soil amendment and not a fertilizer, so you need to know very well the environmental context where we want to use it and what we decide to improve in our agriculture production chain. In a nutshell, knowing the limiting factors of agricultural production and understanding whether the desired results can be obtained with biochar is critical. In fact, the scientific literature on the subject agrees in identifying that there is no biochar equal to another and that each biochar could give different answers in the same climate context. Surely apart from the concept of C-sequestration, biochar is a carbonaceous matrix, most of the time alkaline and with a hydrophobic character at the beginning, and once soaked in water it becomes hygroscopic. It certainly decreases the bulk density of the soil, increases the cation exchange capacity retains nutrients. These are the main characteristics of biochar for its agronomic use, thus it is up to the agronomist to decide whether or not to undertake the amendment with biochar, considering that it is an irreversible practice. Once distributed, it will no longer be possible to remove it from the soil. In addition to the purpose of biochar application discussed above, the choice of application methods also depends on its physical and chemical properties.

\section{Safety recommendations}

Each biochar must be assessed for its own properties and environment for handling and storage. Research and development of procedures specific to biochars for soil application are needed. It highlights a conflict between the agronomic benefits of biochar, which are maximized by powder formulation, and environmental benefits, including climate mitigation and reduced impact for human health, which are maximized in the case of pellet formulation. Pelletization of biochar and moistening practices are already recommended in the guidelines of the International Biochar Initiative (IBI) ${ }^{11}$ and the European Biochar Certificate (EBC) ${ }^{12}$.

\section{Biochar application}

Biochar should be pelletized or applied wet to avoid the emission of fine dust into the atmosphere and buried with a simple harrowing or drill. Inversion moldboard ploughing may create deep layers of biochar and may fail to mix the biochar evenly throughout the topsoil. Offset disc ploughs will generally provide better mixing. Risk of poor mixing will be reduced if the tillage system is checked for uniformity and suitability of mixing using test runs. Biochar is most effective when placed in the root zone.

\section{Biochar dose}

one kilogram of biochar per square meter should be sufficient for plants to gain most of the potential benefit. Only small improvements are likely to be gained from adding more than this amount, but as vegetable growing is relatively labor and water intensive and some biochar may be lost over time, these small improvements are likely to be worthwhile and we recommend using two/tree kilograms of biochar per square meter for vegetable gardens and fruit trees. Lower rates have been shown to be effective when biochar is combined in formulations with organic or inorganic fertilizers. However, in many instances we do not yet have a mechanistic understanding of interactions behind observed yield increase to provide universal application guidance.

\footnotetext{
${ }^{11}$ https://biochar-international.org/

12 https://www.european-biochar.org/en
} 


\section{Potential barriers for adoption}

There are some barriers that reduce the widespread use of biochar:

The technologies are currently in the initial stages of development, although a wide range of them are available to produce biochar, of variable type (pyrolysis, slow pyrolysis, gasification).

The scale of production can be very different (medium, agricultural scale, kitchens) with very variable current costs. Optimal use of new technologies is only possible if they are rapidly adopted and widely disseminated. Although the increased yields of biochar production may be a boon to the new agricultural technology, the high costs can be a deterrent (Mohammadi et al., 2020). Therefore, before the widespread promotion of biochar systems, it is important to investigate the economic implications of biochar systems compared to conventional systems. The cost of using biochar in agriculture depends on the biochar application rate, the cost associated with transporting the biochar from the production plant to the experimental field and the value that can be derived from the energy produced.

Table 78. Potential barriers for adoption

\begin{tabular}{|c|c|c|}
\hline Barrier & YES/NO & \\
\hline Biophysical & Yes & $\begin{array}{l}\text { The potential for biophysical match of biochar in the soil-crops system will } \\
\text { be modified by the effective availability of the ideal feedstock and access to } \\
\text { knowledge required for investment in the technologies optimal for biochar } \\
\text { production. }\end{array}$ \\
\hline Cultural & Yes & $\begin{array}{l}\text { The use of biochar added to soil has been commonplace in many parts of } \\
\text { the word for centuries and even millennia (Lehmann and Joseph, 2015), } \\
\text { however biochar is far from common in industrial agriculture in western } \\
\text { countries, and lack of awareness and confidence in biochar presents a } \\
\text { cultural barrier in these agricultural systems. }\end{array}$ \\
\hline Social & Yes & $\begin{array}{l}\text { Biochar systems should involve stakeholders fully and transparently in } \\
\text { planning and implementation; respect local land use rights; and should not } \\
\text { result in displacement of peoples from their ancestral lands. Biochar } \\
\text { systems should contribute to the economic development of local } \\
\text { communities, especially in regions of poverty. }\end{array}$ \\
\hline Economic & Yes & $\begin{array}{l}\text { As engineered biochar technology is relatively new, the costs and impacts } \\
\text { associated with it are starting to be explored. A potential financial obstacle to } \\
\text { the development and transfer of technology could include the high costs of } \\
\text { large-scale pyrolysis plants, infrastructure and access to start-up capital. } \\
\text { Revenue could be generated through carbon trading; farmers could have an } \\
\text { additional source of income through the collection and sale of agricultural } \\
\text { residues. } \\
\text { The practical and economic feasibility of acquiring biomass shapes the entire }\end{array}$ \\
\hline
\end{tabular}




\begin{tabular}{|c|c|c|}
\hline Barrier & YES/NO & \\
\hline & & $\begin{array}{l}\text { biochar system. The use of virgin raw materials should involve the integration } \\
\text { of the biochar system into an existing agricultural or forestry system. Due to } \\
\text { the costs of collection and transport and storage, it often makes the most } \\
\text { economic sense to use local raw materials. } \\
\text { Depending on the choice of the raw material, the drying process could take } \\
\text { place passively through careful storage or greater intervention may be } \\
\text { necessary, such as the use of a dryer (which therefore requires energy and } \\
\text { labor). }\end{array}$ \\
\hline Institutional & Yes & $\begin{array}{l}\text { According to a recent survey completed by IBI (International Biochar } \\
\text { Initiative), more than } 197 \text { biochar organizations currently exist worldwide. } \\
\text { Voluntary biochar quality standards have been formed in Europe with the } \\
\text { European Biochar Certificate (EBC) (European Biochar Foundation 2012), in } \\
\text { the United Kingdom of Great Britain and Northern Ireland with the Biochar } \\
\text { Quality Mandate (BQM) (British Biochar Foundation, 2014) and in the United } \\
\text { States with the International Biochar Initiative Biochar Standards (IBI-BS) } \\
\text { (International Biochar Initiative, 2015a). } \\
\text { In parallel to this, biochar producers and biochar users in a number of EU } \\
\text { countries were partly successful in fitting the new biochar product into the } \\
\text { existing national legislation for fertilizers, soil improvers and composts. }\end{array}$ \\
\hline Knowledge & Yes & $\begin{array}{l}\text { Despite the increase in the number of scientific studies, relevant knowledge } \\
\text { gaps may need to be addressed: long term data sets of biochar effects on } \\
\text { crop productivity, production technology and in the area of economic } \\
\text { evaluation and production technology. }\end{array}$ \\
\hline Other & & $\begin{array}{l}\text { Biochar should also be adopted by the organic grower community and be } \\
\text { allowable as a certified organic soil management where the integrity of the } \\
\text { biochar product can be guaranteed. }\end{array}$ \\
\hline
\end{tabular}




\section{Photos of the practice}

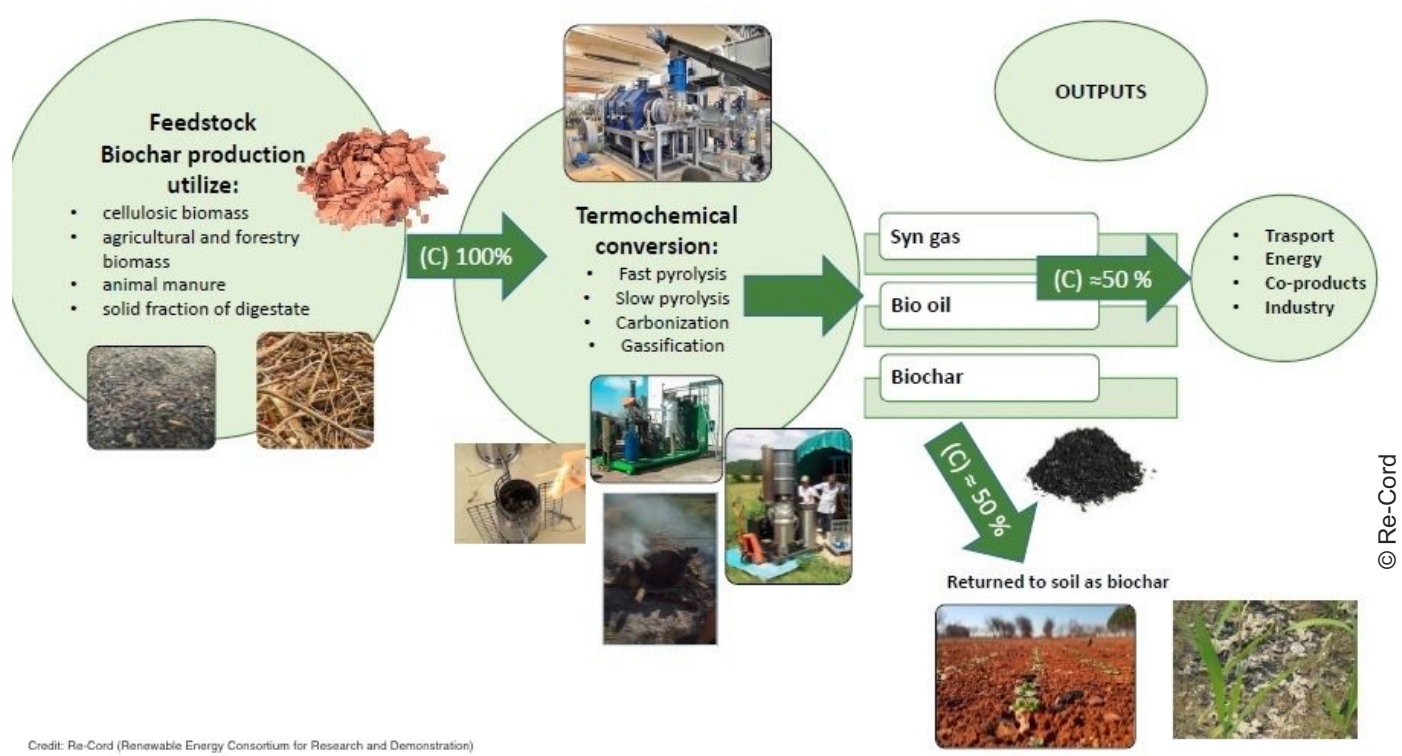

Photo 19. Thermochemical Biochar production diagram

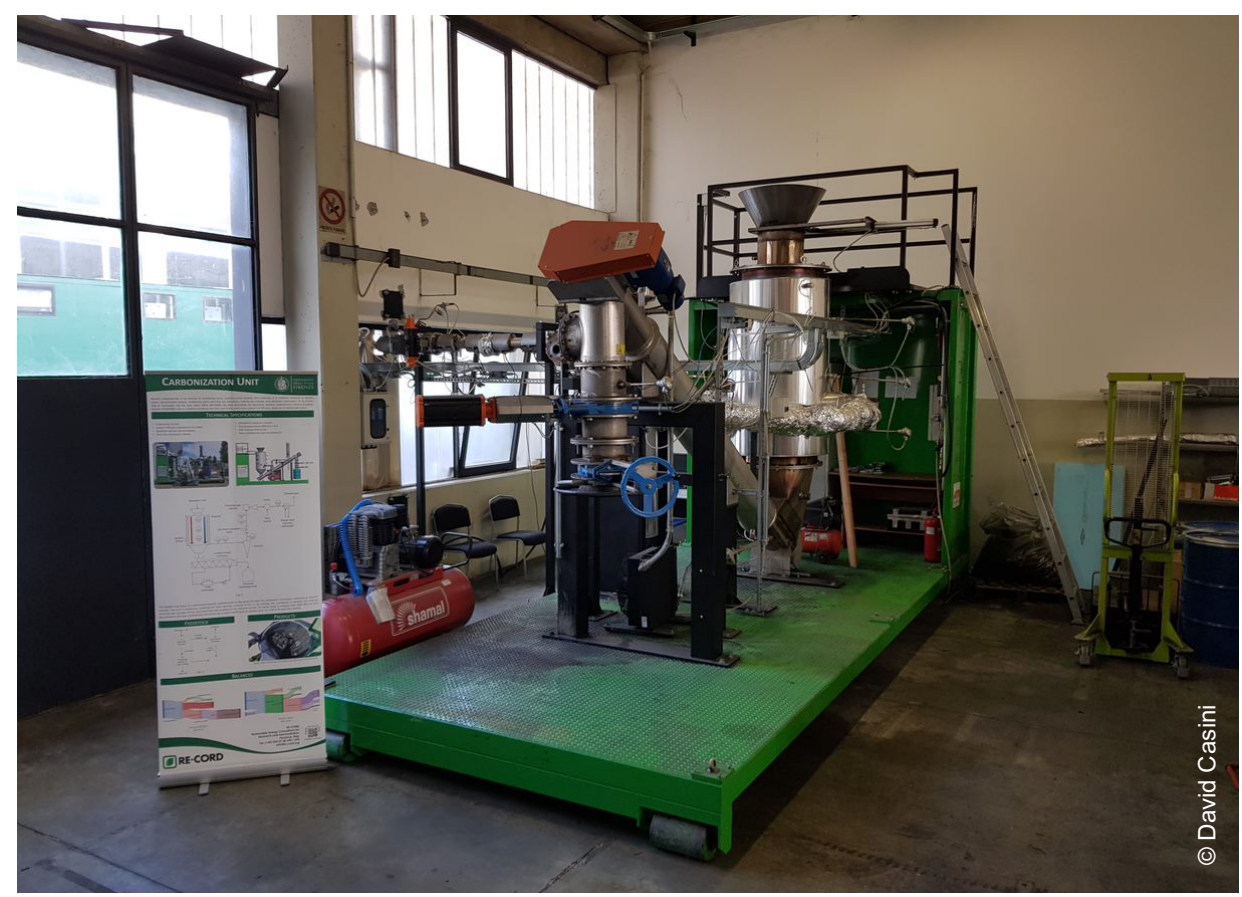

Photo 20. Carbonization pilot Plant. (“Carbon”, Pantent IT1429282) RE-CORD (Renewable Energy Consortium for Research and Demonstration), in Florence, Italy, 2019 


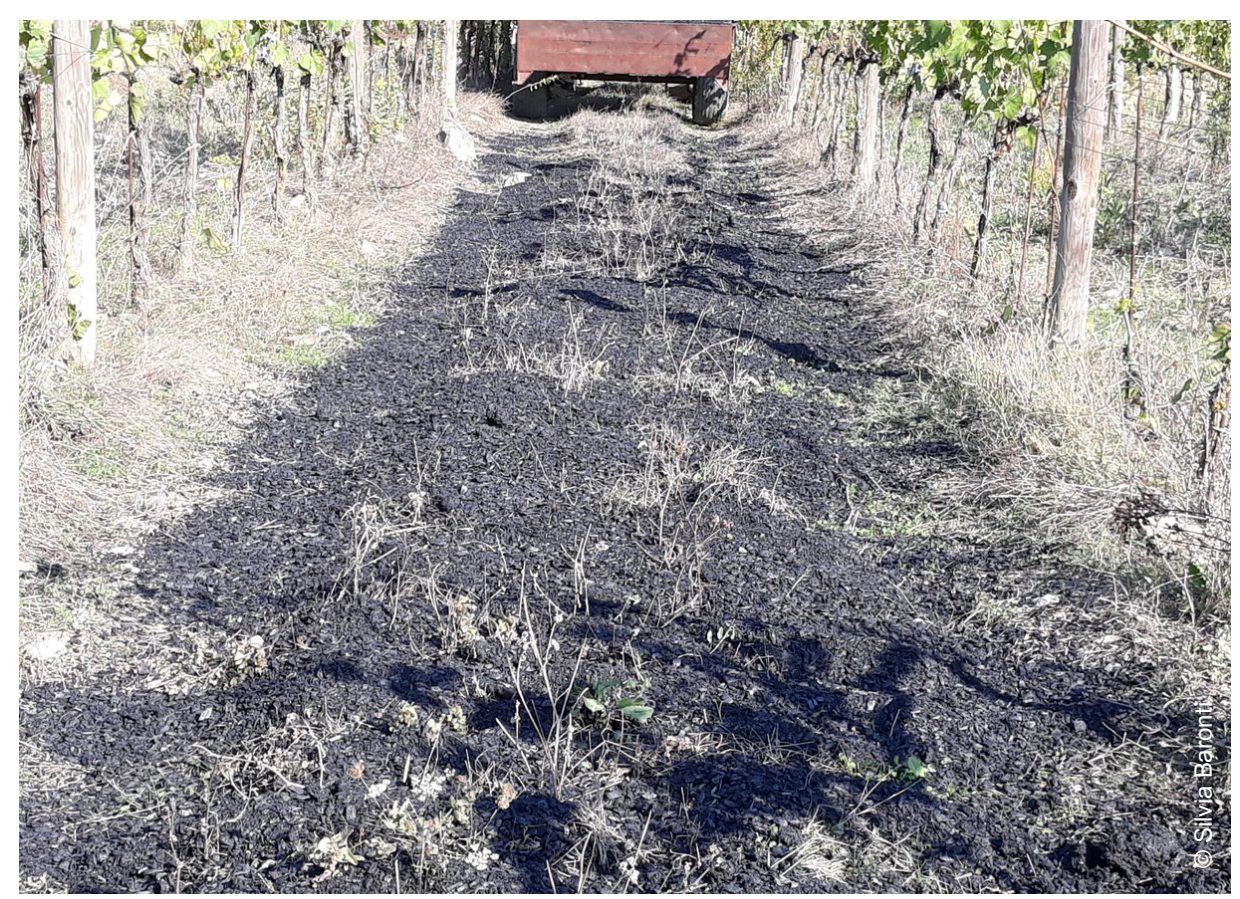

Photo 21. Biochar distribution in vineyard. Poggio Torselli Estate, Chianti Classico DOP 2018

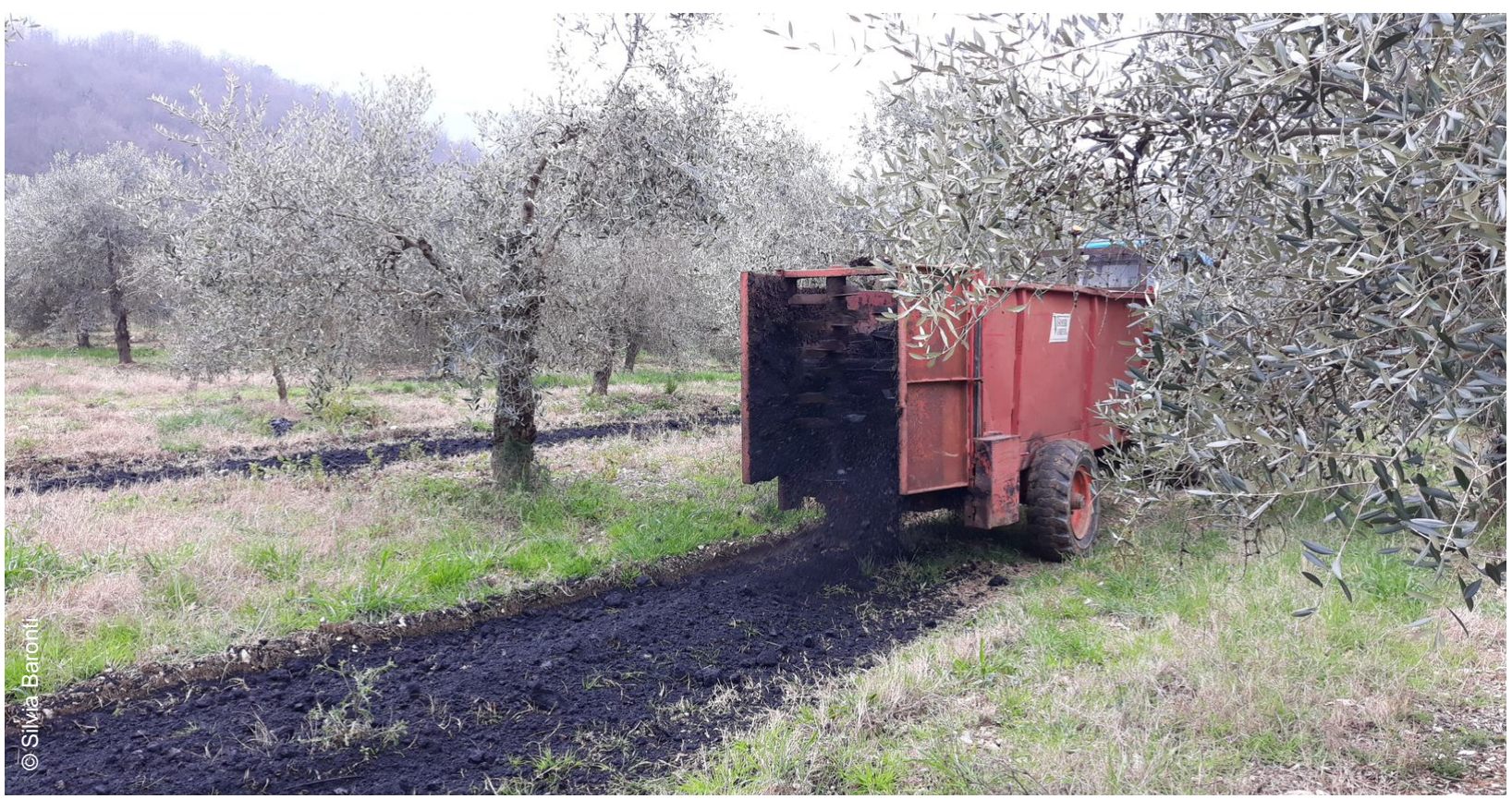

Photo 22. Biochar distribution in Orchard. Frescobaldi Estate, Le Sieci, Florence, Italy, 2020 
Table 79. Related cases studies available in volumes 3 and 5

\begin{tabular}{|l|l|c|c|c|}
\hline Title & Region & $\begin{array}{l}\text { Duration } \\
\text { of study } \\
\text { (Years) }\end{array}$ & Volume & $\begin{array}{l}\text { Case- } \\
\text { study } \\
\text { No. }\end{array}$ \\
\hline $\begin{array}{l}\text { The Biochar challenge in viticulture: long-term } \\
\text { experiment in central Italy }\end{array}$ & Europe & 1 to 10 & 3 & 14 \\
\hline $\begin{array}{l}\text { Biochar and compost application in an olive } \\
\text { orchard, Spain }\end{array}$ & Europe & 4 & 3 & 22 \\
\hline $\begin{array}{l}\text { Biochar as a Soil Amendment for Carbon } \\
\text { Sequestration in Canada }\end{array}$ & $\begin{array}{l}\text { North } \\
\text { America }\end{array}$ & 1 and 3 & 3 & 41 \\
\hline $\begin{array}{l}\text { Straw mulch and biochar application in recently } \\
\text { burned areas of Algarve (Portugal) and } \\
\text { Andalusia (Spain) }\end{array}$ & Europe & 1 & 5 & 10 \\
\hline
\end{tabular}




\section{References}

Ajayi, A.E. \& Horn, R. 2017. Biochar-induced changes in soil resilience: effects of soil texture and biochar dosage. Pedosphere, 27(2): 236-247. https://doi.org/10.1016/S1002-0160(17)60313-8

Awad, Y.M., Wang, J., Igalavithana, A.D., Tsang, D.C.W., Kim, K.-H., Lee, S.S. \& Ok, Y.S. 2018.

Chapter One - Biochar Effects on Rice Paddy: Meta-analysis. In D.L. Sparks, ed. Advances in Agronomy, pp. 1-32. Academic Press. https://doi.org/10.1016/bs.agron.2017.11.005

Barnes, R.T., Gallagher, M.E., Masiello, C.A., Liu, Z. \& Dugan, B. 2014. Biochar-induced changes in soil hydraulic conductivity and dissolved nutrient fluxes constrained by laboratory experiments. PloS one, 9(9). https://doi.org/10.1371/journal.pone.0108340

Baronti, S., Vaccari, F.P., Miglietta, F., Calzolari, C., Lugato, E., Orlandini, S., Pini, R., Zulian, C. \& Genesio, L. 2014. Impact of biochar application on plant water relations in Vitis vinifera (L.). European Journal of Agronomy, 53: 38-44. https://doi.org/10.1016/j.eja.2013.11.003

Basso, A.S., Miguez, F.E., Laird, D.A., Horton, R. \& Westgate, M. 2013. Assessing potential of biochar for increasing water-holding capacity of sandy soils. Global Change Biology and Bioenergy, 5(2): 132-143. https://doi.org/10.1111/gcbb.12026

Bossio, D.A., Cook-Patton, S.C., Ellis, P.W., Fargione, J., Sanderman, J., Smith, P., Wood, S., Zomer, R.J., von Unger, M., Emmer I.M. \& Griscom, B.W. 2020. The role of soil carbon in natural climate solutions. Nature Sustainability, 3: 391-398. https://doi.org/10.1038/s41893-020-0491-z

Bozzi, E., Genesio, L., Toscano, P., Pieri, M. \& Miglietta, F. 2015. Mimicking biochar-albedo feedback in complex Mediterranean agricultural landscapes. Environmental Research Letters, 10(8): 084014. https://doi.org/10.1088/1748-9326/10/8/084014

Castracani, C., Maienza, A., Grasso, D.A., Genesio, L., Malcevschi, A., Miglietta, F., Vaccari., F.P. \& Mori, A. 2015. Biochar-macrofauna interplay: searching for new bioindicators. Science of the Total Environment, 536: 449-456. https://doi.org/10.1016/j.scitotenv.2015.07.019

Cayuela, M.L., Van Zwieten, L., Singh, B.P., Jeffery, S., Roig, A. \& Sánchez-Monedero, M.A. 2014. Biochar's role in mitigating soil nitrous oxide emissions: A review and meta-analysis. Agriculture, Ecosystems \& Environment, 191: 5-16.

Ćwieląg-Piasecka, I., Medyńska-Juraszek, A., Jerzykiewicz, M., Dębicka, M., Bekier, J., Jamroz, E. \& Kawałko, D. 2018. Humic acid and biochar as specific sorbents of pesticides. Journal of soils and sediments, 18(8): 2692-2702. https://doi.org/10.1007/s11368-018-1976-5

Chen, D., Liu, X., Bian, R., Cheng, K., Zhang, X., Zheng, J., Joseph, S., Crowley, D., Pan, G. \& Li, L. 2018. Effects of biochar on availability and plant uptake of heavy metals - A meta-analysis. Journal of Environmental Management, 222: 76-85. https://doi.org/10.1016/j.jenvman.2018.05.004

Dai, Y., Zheng, H., Jiang, Z. \& Xing, B. 2020. Combined effects of biochar properties and soil conditions on plant growth: A meta-analysis. Science of the Total Environment, $713: 136635$. 
Genesio, L., Miglietta, F., Lugato, E., Baronti, S., Pieri, M. \& Vaccari, F.P. 2012. Surface albedo following biochar application in durum wheat. Environmental Research Letters, 7(1): 014025.

https://doi.org/10.1088/1748-9326/7/1/014025

Giagnoni, L., Maienza, A., Baronti, S., Vaccari, F.P., Genesio, L., Taiti, C., Martellini, T., Scodellini, R., Cincinelli, A., Costa, C., Mancuso, S. \& Renella, G. 2019. Long-term soil biological fertility, volatile organic compounds and chemical properties in a vineyard soil after biochar amendment. Geoderma, 344:

127-136. https://doi.org/10.1016/j.geoderma.2019.03.011

Herath, I., Iqbal, M.C.M., Al-Wabel, M.I., Abduljabbar, A., Ahmad, M., Usman, A.R.A., Sik Ok, Y. \& Vithanage, M. 2017. Bioenergy-derived waste biochar for reducing mobility, bioavailability, and phytotoxicity of chromium in anthropized tannery soil. Journal of Soils and Sediments, 17(3): 731-740. https://doi.org/10.1007/s11368-015-1332-y

Hernandez-Soriano, M.C., Kerré, B., Goos, P., Hardy, B., Dufey, J. \& Smolders, E. 2016. Long-term effect of biochar on the stabilization of recent carbon: soils with historical inputs of charcoal. GCB Bioenergy, 8(2): 371-381. https://doi.org/10.1111/gcbb.12250

Hussain, M., Farooq, M., Nawaz, A., Al-Sadi, A.M., Solaiman, Z.M., Alghamdi, S.S., Ammara U., OK, Y.S. \& Siddique, K.H. 2017. Biochar for crop production: potential benefits and risks. Journal of Soils and Sediments, 17(3): 685-716. https://doi.org/10.1007/s11368-016-1360-2

IPCC. 2019. Special Report on Climate Change, Desertification, Land Degradation, Sustainable Land Management, Food Security, and GHG fluxes in Terrestrial Ecosystems. (also available at: https://www.ipcc.ch/site/assets/uploads/2019/08/Fullreport-1.pdf)

Jeffery, S., Verheijen, F.G., Kammann, C. \& Abalos, D. 2016. Biochar effects on methane emissions from soils: a meta-analysis. Soil Biology and Biochemistry, 101: 251-258.

Jien, S.-H., Chen, W.-C., Ok, Y.S., Awad, Y.M. \& Liao, C.-S. 2018. Short-term biochar application induced variations in $\mathrm{C}$ and $\mathrm{N}$ mineralization in a compost-amended tropical soil. Environmental Science and Pollution Research, 25(26): 25715-25725. https://doi.org/10.1007/s11356-017-9234-8

Kan, Z.R., Liu, Q.Y., Wu, G., Ma, S.T., Virk, A.L., Qi, J.Y., Zhao X. \& Zhang, H.L. 2020. Temperature and moisture driven changes in soil carbon sequestration and mineralization under biochar addition. Journal of Cleaner Production, 265: 121921.

Laghari, M., Mirjat, M.S., Hu, Z., Fazal, S., Xiao, B., Hu, M., Chen, Z. \& Guo, D. 2015. Effects of biochar application rate on sandy desert soil properties and sorghum growth. Catena, 135: 313-320. https://doi.org/10.1016/j.catena.2015.08.013

Lehmann, J. \& Joseph, S. (Eds.). 2015. Biochar for environmental management: science, technology and implementation. Routledge.

Liu, H., Xu, F., Xie, Y., Wang, C., Zhang, A., Li, L. \& Xu, H. 2018. Effect of modified coconut shell biochar on availability of heavy metals and biochemical characteristics of soil in multiple heavy metals contaminated soil. Science of the Total Environment, 645: 702-709.

https://doi.org/10.1016/j.scitotenv.2018.07.115 
Maienza, A., Baronti, S., Cincinelli, A., Martellini, T., Grisolia, A., Miglietta, F., Renella, G., Stazi, S.R., Vaccari, F.P. \& Genesio, L. 2017a. Biochar improves the fertility of a Mediterranean vineyard without toxic impact on the microbial community. Agronomy for Sustainable Development, 37(5): 47.

https://doi.org/10.1007/s13593-017-0458-2

Maienza, A., Genesio, L., Acciai, M., Miglietta, F., Pusceddu, E. \& Vaccari, F.P. 2017b. Impact of Biochar Formulation on the Release of Particulate Matter and on Short-Term Agronomic Performance. Sustainability, 9(7): 1131. https://doi.org/10.3390/su9071131

Majumder, S., Neogi, S., Dutta, T., Powel, M.A. \& Banik, P. 2019. The impact of biochar on soil carbon sequestration: Meta-analytical approach to evaluating environmental and economic advantages. Journal of environmental management, 250: 109466. https://doi.org/10.1016/j.jenvman.2019.109466

Mohammadi, A., Khoshnevisan, B., Venkatesh, G. \& Eskandari, S. 2020. A Critical Review on Advancement and Challenges of Biochar Application in Paddy Fields: Environmental and Life Cycle Cost Analysis. Processes, 8(10): 1275. https://doi.org/10.3390/pr8101275

Niu, Y., Chen, Z., Müller, C., Zaman, M.M., Kim, D., Yu, H. \& Ding, W. 2017. Yield-scaled $\mathrm{N}_{2} \mathrm{O}$ emissions were effectively reduced by biochar amendment of sandy loam soil under maize-wheat rotation in the North China Plain. Atmos. Environ., 170: 58-70. https://doi.org/10.1016/j.atmosenv.2017.09.050

Novak, J.M., Lima, I., Xing, B., Gaskin, J.W., Steiner, C., Das, K.C., Ahmenda, M., Rehrah, D., Watts, D.W., Busscher, W.J. \& Schomberg, H. 2009. Characterization of designer biochar produced at different temperatures and their effects on loamy sand. Ann Environ Sci, 3:195-206.

Palansooriya, K.N., Ok, Y.S., Awad, Y.M., Lee, S.S., Sung, J.-K., Koutsospyros, A. \& Moon, D.H. 2019. Impacts of biochar application on upland agriculture: A review. Journal of Environmental Management, 234: 52-64. https://doi.org/10.1016/j.jenvman.2018.12.085

Prendergast-Miller, M.T., Duvall, M. \& Sohi, S.P. 2014. Biochar-root interactions are mediated by biochar nutrient content and impacts on soil nutrient availability. European Journal of Soil Science, 65(1): 173-185. https://doi.org/10.1111/ejss.12079

Sadeghi, S.H., Panah, M.H.G., Younesi, H. \& Kheirfam, H. 2018. Ameliorating some quality properties of an erosion-prone soil using biochar produced from dairy wastewater sludge. Catena, 171: 193-198. https://doi.org/10.1016/j.catena.2018.07.018

Thies, J. E. \& Rillig, M.C. 2009. Chapter 6: Characteristics of biochar: biological properties. In Lehmann, J. (Ed.) Biochar for environmental management. (also available at:

https://www.researchgate.net/profile/Janice-

Thies/publication/284041311_Characteristics_of_biochar_biological_properties/links/56dcc8ea08aee7 3df6d3fd94/Characteristics-of-biochar-biological-properties.pdf)

Vaccari, F. P., Maienza, A., Miglietta, F., Baronti, S., Di Lonardo, S., Giagnoni, L., Lagomarsino, A., Pozzi A., Pusceddu, E., Ranieri, R., Valboa, G. \& Genesio, L. 2015. Biochar stimulates plant growth but not fruit yield of processing tomato in a fertile soil. Agriculture, Ecosystems \& Environment, 207: 163-170. https://doi.org/10.1016/j.agee.2015.04.015 
Vaccari, F.P., Baronti, S., Lugato, E., Genesio, L., Castaldi, S., Fornasier, F. \& Miglietta, F. 2011.

Biochar as a strategy to sequester carbon and increase yield in durum wheat. Europ. J. Agronomy, 34: 231238. https://doi.org/10.1016/j.eja.2011.01.006

Weng, Z.H., Van Zwieten, L., Singh, B.P., Tavakkoli, E., Kimber, S., Morris, S.,McDonald, L.M. \&

Cowie, A. 2018. The accumulation of rhizodeposits in organo-mineral fractions promoted biochar-induced negative priming of native soil organic carbon in Ferralsol. Soil Biology and Biochemistry, 118: 91-96.

Wong, J.T.F., Chen, Z., Chen, X., Ng, C.W.W. \& Wong, M.H. 2017. Soil-water retention behavior of compacted biochar-amended clay: a novel landfill final cover material. Journal of soils and sediments, 17(3): 590-598. https://doi.org/10.1007/s11368-016-1401-x

Yang, H., Ye, S., Zeng, Z., Zeng, G., Tan, X., Xiao, R., Wang J., Song B., Du, L., Qin M., Yang, Y. \& Xu, F. 2020. Utilization of biochar for resource recovery from water: A review. Chemical Engineering Journal, 389: 125502. https://doi.org/10.1016/j.cej.2020.125502

Yang, S., Sun, X., Ding, J., Jiang, Z. \& Xu, J. 2019. Effects of biochar addition on the NEE and soil organic carbon content of paddy fields under water-saving irrigation. Environmental Science and Pollution Research, 26(8): 8303-8311. https://doi.org/10.1007/s11356-019-04326-8

Ye, J., Joseph, S.D., Ji, M., Nielsen, S., Mitchell, D.R.G., Donne, S., Horvat, J., Wang, J., Munroe, P. \& Thomas, T. 2017. Chemolithotrophic processes in the bacterial communities on the surface of mineralenriched biochars. The ISME Journal, 11(5): 1087-1101. https://doi.org/10.1038/ismej.2016.187

Ye, L., Camps-Arbestain, M., Shen, Q., Lehmann, J., Singh, B. \& Sabir, M. 2020. Biochar effects on crop yields with and without fertilizer: A meta-analysis of field studies using separate controls. Soil Use and Management, 36(1), 2-18. https://doi.org/10.1111/sum.12546

Zhang, K., Sun, P., Faye, M.C.A. \& Zhang, Y. 2018. Characterization of biochar derived from rice husks and its potential in chlorobenzene degradation. Carbon, 130: 730-740.

https://doi.org/10.1016/j.carbon.2018.01.036

Zheng-Z.R., Liu, Q.Y., Wu, G., Ma S.T., Virk, A.L., Qi, J. Y., Zhao, X. \& Zhang, H.L. 2020.

Temperature and moisture driven changes in soil carbon sequestration and mineralization under biochar addition. Journal of Cleaner Production, 265: 121921. https://doi.org/10.1016/j.jclepro.2020.121921

Zhu, X., Chen, B., Zhu, L. \& Xing, B. 2017. Effects and mechanisms of biochar-microbe interactions in soil improvement and pollution remediation: A review. Environ Pollut, 227:98-115.

https://doi.org/10.1016/j.envpol.2017.04.032 


\title{
18. Biofertilizers application
}

\author{
Evan A.N. Marks, Berta Singla, Laura Roquer, Rosa Vilaplana \\ CT BETA, Universitat de Vic - Universitat Central de Catalunya, Vic, Spain
}

\section{Description of the practice}

Biofertilizers can be defined as a substance containing live microorganisms which exhibit beneficial properties toward plant growth and development (Mącik, Gryta and Frąc, 2020). Biofertilizers are microorganisms applied to soil, seeds, or roots, where their mode/mechanisms of action is through their interaction with the soil system and where they will exert a direct influence on biological and chemical processes improving conditions for plant growth ${ }^{13}$. Biofertilizers should not be confounded with other "bio" products (e.g. from waste streams such as agri-industrial organic matter waste, derivates of manure processing; these may instead be referred to as "biobased fertilisers"), as this often leads to confusion in terminology.

Microorganisms (including but not exclusively bacteria, cyanobacteria, and fungi) mediate almost all the nutrient transformations in the soil, including degradation and decomposition of organic matter and its mineralization (transformation of organically-bound nutrients into to plant-available forms), as well as fixation of important elements such as nitrogen from the atmosphere. Biofertilizers, thus, are employed to promote these functions, positively influencing plant growth in an indirect manner. Biofertilizing organisms can be categorized into several groups, depending on taxon and the soil functions they mediate (Table 80).

\section{Table 80. Dominant biofertilizer organism classes by taxonomy or function}

Plant growthpromoting rhizobacteria (PGPR)
Functional class. PGPRs are those bacteria which are mutualistic with plants, subsisting on root exudates. As such, they carry out functions of nutrient liberation particularly relevant to plant nutrition. For biofertilizer development, they are isolated from roots of the crop of interest.

\footnotetext{
${ }^{13}$ For the purpose of this review (and in general concurrence with the literature), biofertilizers do not include organisms applied to above-ground plant parts for pathogen suppression etc.
} 
Plant growthpromoting biofertilizers (PGPB)

\begin{tabular}{|l|l|}
\hline $\begin{array}{l}\text { Functional class. A non-taxonomic classification encompassing bacteria } \\
\text { Nitrogen-fixing }\end{array}$ & $\begin{array}{l}\text { species capable of transforming atmospheric nitrogen }\left(\mathrm{N}_{2}\right) \text { into forms usable } \\
\text { by plants. Includes cyanobacteria and free-living Azobactersp. and }\end{array}$ \\
bacteria (NFB) & $\begin{array}{l}\text { Azospirillum sp., though biofertilizers are usually formulated with } \\
\text { mutualistic rhizobia (those associated with legumes). }\end{array}$
\end{tabular}

Functional class. Composed of genera distinct from PGPR, PGPBs promote plant development through the production of active substances such as siderophores and phytohormones.

Functional class. A non-taxonomic classification encompassing bacteria species capable of transforming atmospheric nitrogen $\left(\mathrm{N}_{2}\right)$ into forms usable lated with

Functional class. Can include both fungi and bacteria capable of solubilizing insoluble phosphate compounds such as tricalcium and dicalcium phosphate, hydroxyapatite, and rock phosphate. Commonly employed species belongs to the genera Aspergillus sp. and Penicillum sp.

solubilizing

biofertilizers (PSB)

Taxonomic class. Phototrophic (photosynthesizing) bacteria that may be uni- or multi-cellular, filamentous or single cells. Associated with soil crusts,

Cyanobacteria lichens, and mosses, they are important in soil nitrogen fixation. As biofertilizers, they have been employed in restoring degraded lands and also aquatic fertilization e.g. in rice paddies.

Taxonomic class. Refers to eukaryotic green algae, whereas biofertilizer applications usually employ unicellular freshwater species, with Microalgae technological applications focusing on their capacities of assimilating nutrients from waste effluents, their harvest, and soil application, promoting a circular economy approach (Photo 23).

\begin{tabular}{|l|l} 
Taxonomic class. May or may not penetrate plant roots (endomycorrhizal \\
Mycorrhizal fungi & $\begin{array}{l}\text { and ectomycorrhizal, respectively). Crucial for plant nutrition due to } \\
\text { function as P-solubilizers. Also associated with increased resistance to } \\
\text { drought stress, among others. }\end{array}$
\end{tabular}

Actinomycetes

Taxonomic class. Gram-positive bacteria which are important in the soil for influence on solubilization of potassium, oxidizing sulphur, or production of soil enzymes, among others.

Very few published studies on biofertilizers have been focused on the objective of increased soil carbon sequestration (some examples are Dębska et al, 2016; Hu et al., 2018; Srivastava et al., 2018; Shukla et al., 2017). Three postulated mechanisms promoting soil organic carbon stocks have been identified in the literature search carried out for this review: 1) by increasing plant growth, and thus increasing organic matter incorporation to the soil through roots or above-ground parts; 2) accelerated humification of organic matter, leading to less degradable organic matter constituents (protection); and 3) application of microalgal or cyanobacterial biofertilizers, which due to their photosynthetic activities can increase organic matter contents in the soil crust. 


\section{Range of applicability}

Worldwide, there has been scientific interest (I.e. published papers) in biofertilizers since at least the 1980s, if not earlier, but interest has been growing substantially in more recent years. This can be attributed to a growing preoccupation with the sustainability of agroecosystems, and lowering our dependence on mineral or fossilbased fertilization sources; biofertilizers are thought to be a promising and non-toxic alternative to synthetic agrochemicals (Mącik, Gryta and Frąc, 2020). Along those lines, such microbial inoculants are often tested as an alternative to chemical sources, or in conjunction with other organic fertilizers or soil amendments (soil improvers). That said, biofertilizers generally enhance nutrient use efficiency of crops, whereas benefits are also seen when used with synthetic fertilizers (Rubin, van Groenigen and Hungate, 2017). Biofertilizers improve soil nutrient contents (e.g. N fixation) and solubility of applied minerals, enhance nutrient acquisition through chelation and/or siderophore production, or increase cycling of nutrients in organic fertilizers.

A priori, there should not be any physical or geographical limitations to the practice. However, interest is most notable in Asian countries in terms of published scientific results (Figure 5). Biofertilizers have been tested in many different cultural and pedo-climatic contexts. However, recent meta-analyses are beginning to provide orientation about soil types or climates where biofertilizers are most effective, and there is good evidence that biofertilizers have the greatest benefits to plant productivity in dry climates (Rubin, van Groenigen and Hungate, 2017; Schmidt and Gaudin, 2018; Schütz et al., 2018). There is no limitation to the type of agricultural system in which biofertilizers may be applied. Biofertilizers are tested widely in rainfed, irrigated, or flooded croplands. Improving seedling establishment for forestry is also an application which has received attention, though much more limited. For food crops, biofertilizers may be applied both in traditional soil cultivation, greenhouse cultivation with mixed substrates, or hydroponic cultures.

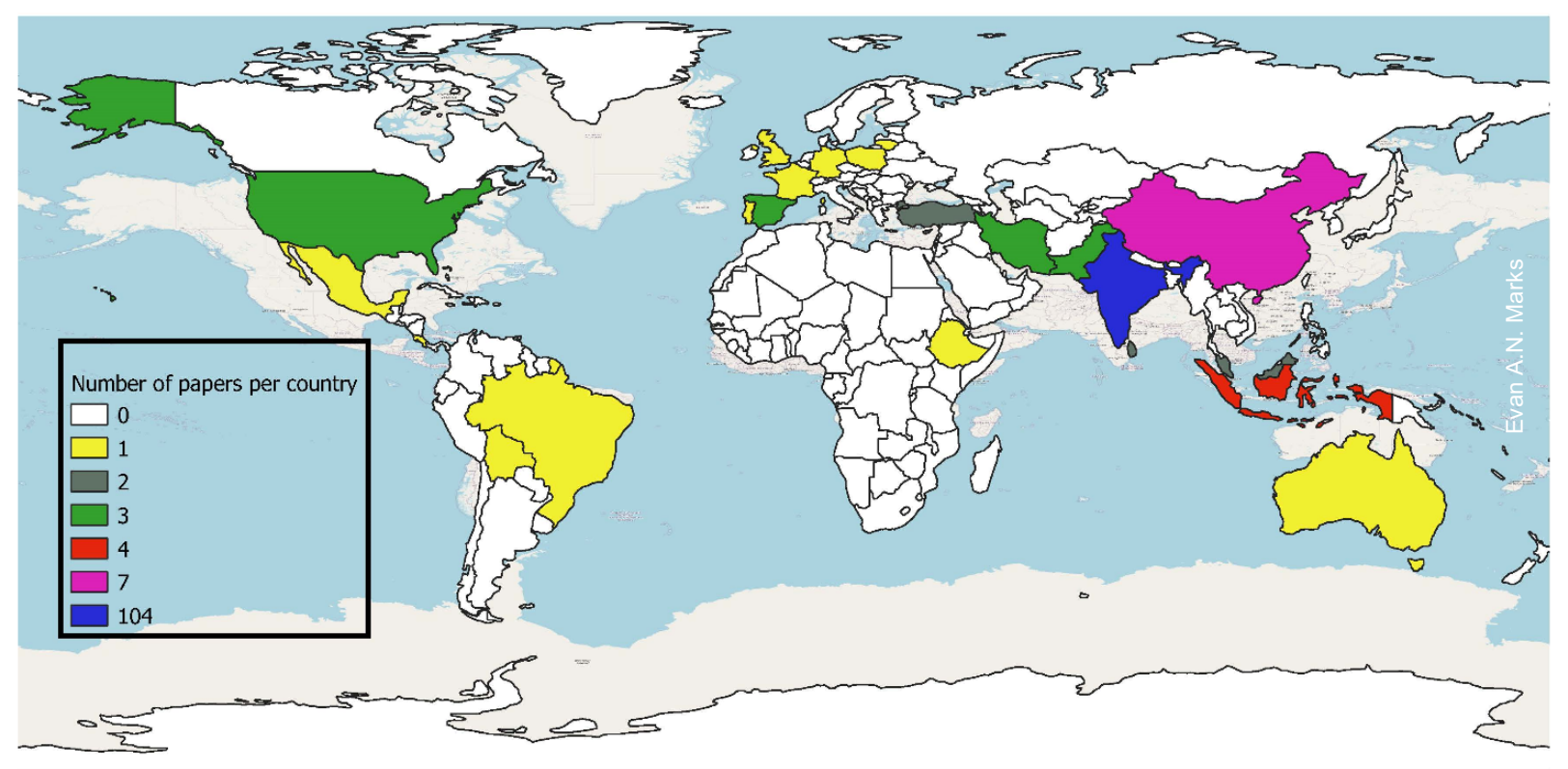

Figure 5. Worldwide origin of 145 studies reviewed for this document 
Biofertilizers are applied alone as axenic cultures (free from foreign living organisms), in mixtures of two or more organisms, or with various substrates and solid supports such as compost, biochar, fermentation products of agri-food wastes, etc. In the literature reviewed for this manuscript, the median application rate of products was $10 \mathrm{~kg} / \mathrm{ha}$ when it was the only fertilizer ( $\min =1$, $\max =20,6$ studies), and was $2 \mathrm{~kg} / \mathrm{ha}$ when applied with other fertilizers it ( $\min =0.4, \max =12,9$ studies). In more modern applications, biostimulants can be encapsulated in different biodegradable matrices - an area of high $\mathrm{R}+\mathrm{D}$ interest, crucial for product preservation and shelf life.

\section{Impact on soil organic carbon stocks}

\subsection{Meta-analysis on biofertilizers and SOC}

Due to the novelty of this research question of whether biofertilizer use can increase soil organic carbon stocks, no meta-analyses or reviews on the topic were available. As seen in Figure 6, interest in the topic has been growing quickly in recent years, and continued growth can be expected. Therefore, we conducted a new metaanalysis, the main results of which are described below.

A literature search was carried out using the ISI Web of Science during December 2020. The search was designed to probe the question of how biofertilizers may influence soil organic carbon stocks. The final validated search string was: "Soil organic carbon" OR "organic C" OR "carbon sequestration" OR "Soil organic C" OR "SOC" OR "OC" AND ("biofertili*" OR "bio-fertili*"). This search string resulted in 234 results. After reading all abstracts, 145 primary references were selected according to inclusion criteria. Among other criteria, the selected studies measured soil organic carbon with an adequate control and treatment structure and with appropriate statistical treatment. The available articles were then obtained and analysed for validation according to exclusion criteria. The final database contained 47 scientific articles from which data was extracted. Of these, 36 were field studies, 5 pot/greenhouse studies, and 6 laboratory studies.

A meta-analysis was carried out on the reported SOC concentration data from these experimental studies to ascertain the effect that biostimulants may have in increasing SOC stocks. Among all studies (47 studies, 195 comparisons) it was found that biofertilization resulted in an effective positive increase in soil carbon (raw mean difference of $0.9987 \mathrm{~g} / \mathrm{kg} \mathrm{SOC})^{14}$. It is important to mention that since the studies were carried at very different time scales, this analysis did not account for study length (time required for the measured change in SOC storage). Next steps would be to consider this and other variables for further analysis. Figure 7 below shows the differences reported by individual studies finding positive effects on SOC.

\footnotetext{
${ }^{14}$ Statistical analysis carried out using the R statistical software with escalc function of metafor package. Raw mean differences in SOC concentration were calculated for each comparison, then global effect was tested using a multi-level linear mixed-effects model, grouping by study (random variable). Confidence interval $=(0.45,1.54), \mathrm{Z}=3.61, \mathrm{p}<0.001$.
} 


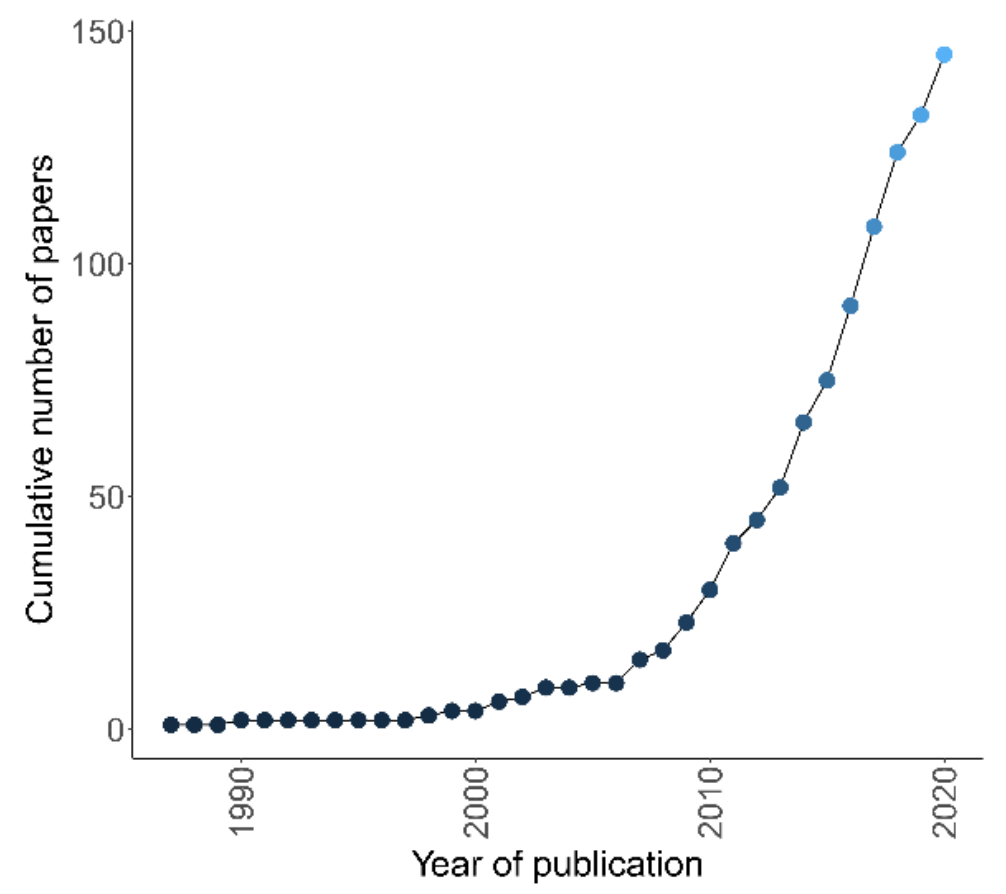

Figure 6. Evolution of rate of studies published on the topic

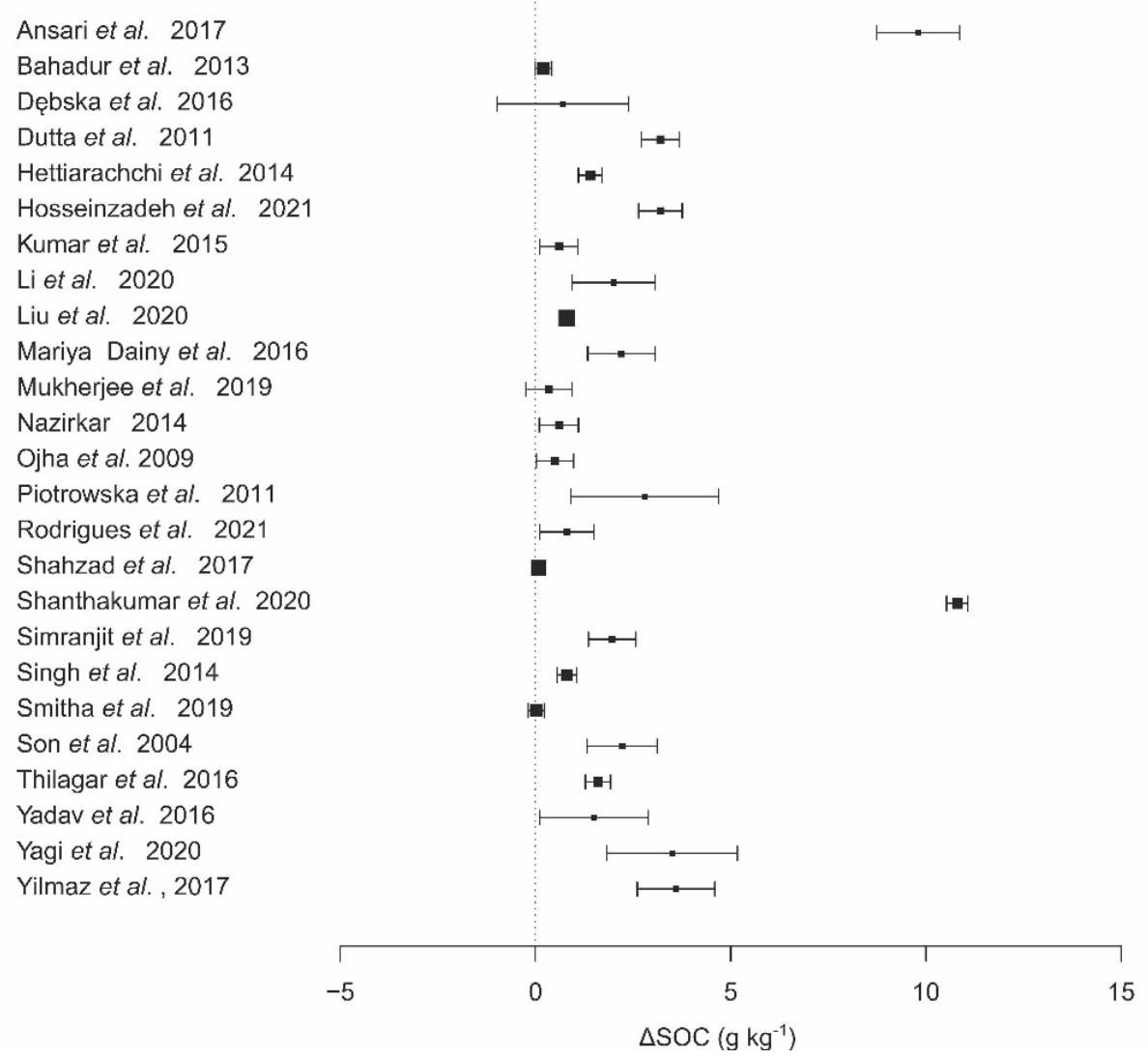

Figure 7. Calculated change (delta) in soil organic carbon found by a selection of studies (those with statistically significant positive results) 
For estimations of carbon storage, Table 81 summarizes the principal results from those studies with (statistically significant) positive effects on SOC storage ( 25 studies), and SOC storage as t/ha/yr is estimated. Also, nine studies found negative effects on SOC storage (not shown in Table 81 since no additional C storage was found). 
Table 81. Studies reporting significant positive effects on SOC storage

\begin{tabular}{|c|c|c|c|c|c|c|c|}
\hline Country & Climate zone & Soil type & $\begin{array}{l}\text { Baseline C } \\
\text { stock } \\
\text { (tC/ha) }\end{array}$ & $\begin{array}{l}\text { Additional C } \\
\text { storage } \\
\text { (tC/ ha/yr) }\end{array}$ & $\begin{array}{l}\text { Study } \\
\text { duration } \\
\text { (days) }\end{array}$ & $\begin{array}{l}\text { Depth } \\
\text { (cm) }\end{array}$ & Reference \\
\hline India & Tropical Dry & NA & 40.32 & 20.58 & 730 & 30 (est.) & Ansari and Mahmood (2017) \\
\hline India & Tropical Moist & NA & 13.86 & 0.42 & 730 & 30 (est.) & Bahadur et al. (2013) \\
\hline Poland & Cool Temperate Moist & Cambisol & 63.42 & 0.74 & 1460 & 30 (est.) & Dębska et al. (2016) \\
\hline India & Tropical Moist & NA & 21.84 & 6.72 & 730 & 30 (est.) & Dutta, Biswas and Kundu (2011) \\
\hline $\begin{array}{l}\text { Sri } \\
\text { Lanka }\end{array}$ & Tropical Moist & NA & 4.91 & 2.98 & 120 & 5 & Hettiarachchi et al. (2014) \\
\hline Iran & Warm Temperate Dry & NA & 26.04 & 13.44 & 365 & 30 & Hosseinzadeh et al. (2021) \\
\hline India & Tropical Moist & Inceptisol & 13.65 & 0.96 & 480 & 15 & Kumar et al. (2015) \\
\hline China & Warm Temperate Moist & Aridisol & 47.60 & 38.61 & 45 & 17 & Li et al. (2020) \\
\hline China & Warm Temperate Moist & NA & 23.63 & 9.08 & 90 & 20 & Liu et al. (2020) \\
\hline India & Tropical Wet & Alisol & 46.20 & 28.11 & 120 & 30 (est.) & $\begin{array}{l}\text { Mariya Dainy and Manorama } \\
\text { Thampatti (2016) }\end{array}$ \\
\hline India & Tropical Moist & NA & 22.71 & 0.48 & 730 & 20 & Mukherjee et al. (2019) \\
\hline India & Tropical Dry & Inceptisol & 28.14 & 6.15 & 150 & 30 (est.) & Nazirkar (2014) \\
\hline India & Tropical Moist & NA & 13.44 & 1.05 & 365 & 15 & Ojha et al. (2009) \\
\hline
\end{tabular}




\begin{tabular}{|c|c|c|c|c|c|c|c|}
\hline Country & Climate zone & Soil type & $\begin{array}{l}\text { Baseline C } \\
\text { stock } \\
\text { (tC/ha) }\end{array}$ & $\begin{array}{l}\text { Additional C } \\
\text { storage } \\
\text { (tC/ ha/yr) }\end{array}$ & $\begin{array}{l}\text { Study } \\
\text { duration } \\
\text { (days) }\end{array}$ & $\begin{array}{l}\text { Depth } \\
(\mathrm{cm})\end{array}$ & Reference \\
\hline Poland & Cool Temperate Moist & Cambisol & 61.74 & 2.94 & 1460 & 30 (est.) & $\begin{array}{l}\text { Piotrowska, Dlugosz and Zamorski } \\
\text { (2011) }\end{array}$ \\
\hline Portugal & Warm Temperate Moist & NA & 45.36 & 1.70 & 722 & 30 (est.) & Rodrigues et al. (2021) \\
\hline Pakistan & Warm Temperate Dry & Aridisol & 22.93 & 0.17 & 122 & 30 (est.) & Shahzad et al. (2017) \\
\hline Australia & Tropical Dry & Kurosol & 1.32 & 3.02 & 90 & 0.5 & Shanthakumar et al. (2020) \\
\hline India & Warm Temperate Moist & Cambisol & 12.52 & 18.75 & 162 & 30 (est.) & Simranjit et al. (2019) \\
\hline India & Tropical Moist & Fluvisol & 13.02 & 1.68 & 730 & 30 (est.) & Singh et al. (2014) \\
\hline India & Tropical Dry & Cambisol & 6.20 & 0.06 & 272 & 15 & Smitha et al. (2019) \\
\hline Vietnam & Tropical Moist & NA & 48.13 & 3.11 & 1095 & 30 (est.) & Son et al. (2004) \\
\hline India & Tropical Dry & NA & 12.32 & 11.68 & 140 & 20 & Thilagar, Bagyaraj and Raoca (2016) \\
\hline India & Warm Temperate Moist & NA & 65.45 & 5.47 & 350 & 25 & Yadav et al. (2016) \\
\hline Brasil & Tropical Moist & Fluvisol & 35.42 & 5.96 & 300 & 10 & Yagi et al. (2020) \\
\hline Turkey & Warm Temperate Moist & Fluvisol & 40.66 & 49.06 & 90 & 24 & Yilmaz and Sönmez (2017) \\
\hline
\end{tabular}

Source: $F A O(2017)$

When actual sampling depth was not reported, $30 \mathrm{~cm}$ is assumed (shown as "est.") to harmonize with IPCC guidelines in SOC calculations 


\subsection{Discussion of SOC storage results}

The meta-analysis of worldwide studies has shown that biofertilization practices do contribute to SOC stocks. Crop and biofertilizer type in addition to climatic zone are likely to influence the result. It is quite informative to understand which are the most common crops and biofertilizers tested, and this information can be used to identify the most promising arrangements and management schemes to promote SOC stocks. The microorganisms (biofertilizer type) and crops encountered in this study are shown below in Figure 8 and Figure 9 .

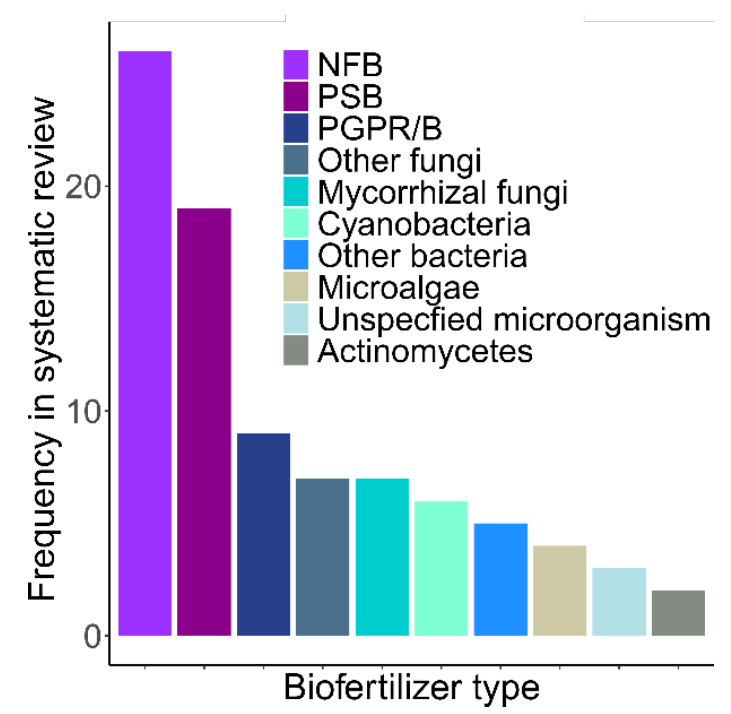

Figure 8. Sum of biofertilizer types encountered among the studies utilized in the meta-analysis above

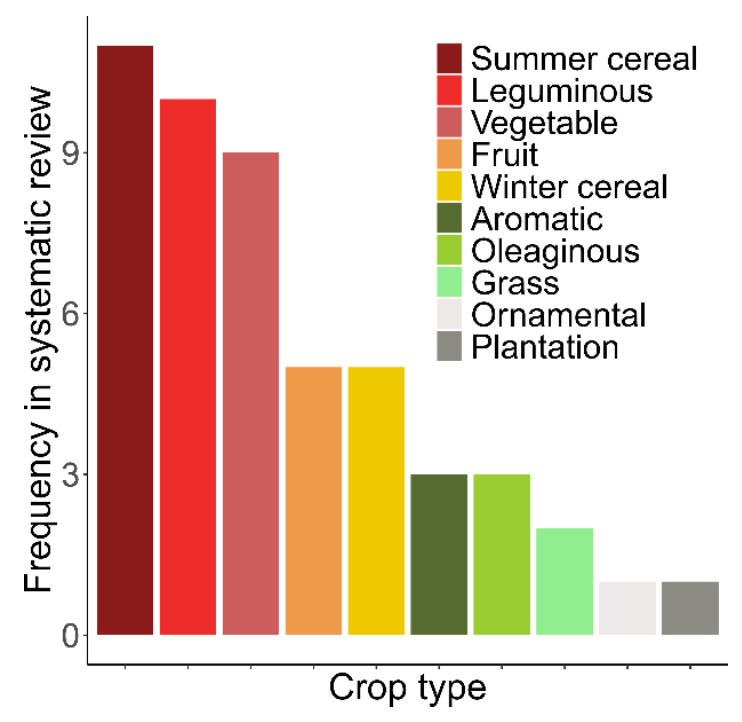

Figure 9. Sum of crop types encountered among the studies utilized in the meta-analysis above 


\section{Other benefits of the practice}

\subsection{Improvement of soil properties}

\section{Physical properties}

The role of microorganisms in soil stabilization is well documented within the field of soil science. Enmeshment with hyphal networks (fungi) or extracellular polymeric substances (EPS, produced by many microorganisms) help bind soil components. Photosynthetic biofertilizers are likely to improve soil structure due to production of high quantities of EPS, a supposition which is based on existing knowledge on ecological succession in soil biological crusts. Cyanobacteria or green algae are typically the pioneer species of bare topsoil, and for this reason cyanobacteria biostimulants have already been targeted for soil protection and desertification reversal, already applied on pilot scales (Rossi et al, 2017). However, below the soil crust, benefits of biofertilization on soil structure are not as well established. On one hand, it could be expected that increasing certain soil microorganism populations with successfully propagating strains can exert a positive influence on soil structure. However, subsoil ecological interactions are quite complex, and the dynamics of species proliferation and facilitative or competitive interactions may have the result of favouring one function (e.g. plant nutrition) over another (e.g. soil structure).

All in all, the most sure-fire way of improving soil structure is through positive effects on primary productivity (plant growth) which in addition to direct secretion of binding substances, foments microbial growth, so with application of biostimulants there can be large indirect benefits in this regard.

\section{Soil nutrient concentrations and other chemical properties}

Biofertilizers have strong and direct effects on soil nutrient contents or the bioavailable fractions of key elements, leading to improved nutrient acquisition and nutrient use efficiencies in crops. Soil nitrogen contents are increased by free-living mutualistic nitrogen-fixing bacteria (NFB), whereas effectively nodulated legume crops are a vital and indispensable aspect of sustainable agriculture, and biofertilizers contribute to this goal. The increased solubilization of mineral elements such as phosphorus will have similar positive consequences in this regard. Direct effects on chemical properties conditioned by soil mineralogy, exchangeable elements, $\mathrm{pH}$, or CEC, are of lesser relevance for biofertilizers.

\section{Biological properties}

Application of microbial inoculants to soil as biofertilizers is expected to increase key soil biological health parameters, including enzymatic activities, which are monitored in many of the surveyed studies due to their high relevance for plant growth. Also, the application of microalgal or cyanobacteria slurries to different soils results in successful colonization, and microbial activities are increased in association with the formation of a soil biofilm (Rossi et al., 2017; Marks, Montero and Rad, 2019). 


\subsection{Minimization of threats to soil functions}

Table 82. Soil threats

\begin{tabular}{|c|c|}
\hline Soil threats & \\
\hline Soil erosion & $\begin{array}{l}\text { Photosynthetic biofertilizers such as cyanobacteria and microalgae have } \\
\text { a high potential to reduce soil degradation and loss (at time of writing, } \\
\text { only demonstrated on large scale in China (Zhou et al., 202O). } \\
\text { Production of extracellular polymeric substances (EPS) leads to binding } \\
\text { of soil components, having a positive influence on soil structure (see } \\
\text { above). }\end{array}$ \\
\hline $\begin{array}{l}\text { Nutrient imbalance } \\
\text { and cycles }\end{array}$ & $\begin{array}{l}\text { Considering nitrogen-fixing potential, highly relevant for improving } \\
\text { ratios of } \mathrm{N} \text { with other elements (stoichiometries). Enhanced cycling of } \\
\text { all elements due to increased microbial activities and production of } \\
\text { bioactives. }\end{array}$ \\
\hline $\begin{array}{l}\text { Soil salinization } \\
\text { and alkalinization }\end{array}$ & $\begin{array}{l}\text { Application of microbial inoculants as biofertilizers does not directly } \\
\text { neutralize these threats, but is highly relevant for reducing plant } \\
\text { susceptibility to saline soils (Alori, Dare and Babalola, 2017). }\end{array}$ \\
\hline $\begin{array}{l}\text { Soil contamination } \\
\text { / pollution }\end{array}$ & $\begin{array}{l}\text { Microbial inoculants can improve the phytostabilization of } \\
\text { contaminated soils, and are a cost efficient alternative to incineration, } \\
\text { excavation, or landfilling (Alori, Dare and Babalola, 2017). }\end{array}$ \\
\hline Soil compaction & Potentially through positive impacts on soil structure (see above). \\
\hline $\begin{array}{l}\text { Soil water } \\
\text { management }\end{array}$ & Potentially through positive impacts on soil structure (see above). \\
\hline
\end{tabular}

\subsection{On production}

Current data suggest that biofertilizer use worldwide is quite consistently associated with productivity gains, though the magnitude of effect depends on climatic region, soil type, etc., as should be expected - separating these factors for best use recommendations is a current topic of research). Biofertilizers can be considered for use in the production of food and feed as well as forestry applications (Garía-Fraile et al., 2015). For agriculture, effective application methods often involve the inoculation of seeds or roots of annual plants, and the same approach can be used in forestry seedlings to improve rates of establishment.

Schütz et al. (2018) conducted a global meta-analysis to quantify benefits of biofertilizers (171 studies); overall, positive effects were found for all climatic regions, and the yield response for all biofertilizers and regions was found to be around +17 percent. Separating by biofertilizer type, the average benefits ranged from $\sim 12$ percent 
(phosphorus solubilizers) to $\sim 20$ percent (arbuscular mycorrhizal fungi). When considering agroclimatic zone, it was found that yield response in dry climates benefits most from biofertilizer application, in the order of dry climate +20 percent, tropical climate +14 percent, oceanic climate +10 percent, and continental climate +8 percent. In another global meta-analysis, Rubin et al. (2017) also found that productivity gains are consistently found across a wide diversity of conditions (52 studies), including whether fertilization is organic or inorganic, conducted in a greenhouse, one or multiple biofertilizer organisms used, and target crop. A more targeted meta-analysis (69 studies) on bacterial biofertilizers for maize also found that there is a large and relatively consistent benefit to productivity, between 15 percent and 18 percent (Schmidt and Gaudin, 2018).

\subsection{Mitigation of and adaptation to climate change}

\section{Climate adaptation and resilience}

Biofertilizers should quite arguably form a part of integrated agricultural practices, defined by a greater efficiency in the use of resources and sustainable management of natural processes (FAO, 2019), and integrated agriculture is a key feature of climate-smart agriculture ${ }^{15}$. Biofertilizers should be considered as part of a strategy to increase the resilience of agriculture to the multiple threats posed by global change and climate change since there is significant evidence that biofertilizers mitigate abiotic plant stress (Berruti et al., 2016; Chekwube Enebe and Oluranti Babalola, 2018). An analysis of studies testing PGPR and imposing experimental drought conditions on plant growth found that the effect size (magnitude of response) in terms of shoot biomass and reproductive yield was consistently greater under drought conditions, indicating a large opportunity for mitigating this risk (Rubin, van Groenigen and Hungate, 2017). Climate disasters cause billions in agricultural losses, with drought leading the way, responsible for 83 percent of crop productivity losses (FAO, 2018). Again, referring to the results of the different meta-analyses reported above, the evidence points to the fact that biofertilizer effects are most pronounced in environments with limitations of water, making clear the potential for these technologies to mitigate climate risks and promote climate change adaptation.

\section{Climate change mitigation}

As seen in Section 3 above, biofertilizers can have a climate mitigation effect through enhancement of the terrestrial carbon sink. However, other means of carbon sequestration must be considered for some biofertilizer classes. Microalgae and cyanobacteria are of profound biological and biochemical importance for their contribution to fixation of atmospheric carbon - with this in mind, simply the production of microalgal biofertilizers must be taken into account for its carbon-negative activity and possible links with high- $\mathrm{CO}_{2}$ concentrations in industrial applications (Perin et al., 2019).

Excessive application of nutrients in agriculture has the consequence of exacerbating GHG emissions from the sector. One of the best options for mitigating such emissions is simply the application of less fertilizer, and relying instead on sources of biological fixation, a solution provided by certain biofertilizer classes (NFBs), and long-held as a route for appropriate and innovative agricultural development (Bhattacharjee, Singh and Mukhopadhyay, 2008). Biofertilizers may have specialized uses for reducing the GHG emissions of sectors with large emissions, such as rice cropping. As an example of research aiming to solve such problems, there is very

\footnotetext{
${ }^{15}$ In the literature search for the preparation of this document, 55 of the 145 identified relevant articles included the concept of integrated nutrient management.
} 
interesting evidence that certain microbial strains, when used a biofertilizer in rice paddies, can have a double benefit of enhancing productivities while concomitantly reducing methane emissions (Kantachote et al., 2016).

\subsection{Socio-economic benefits}

Agriculture is the major economic activity of most developing countries where it engages a large share of the population, and biofertilizers are promoted as a cost-effective strategy to assure farmer incomes. One of the principal benefits of biofertilizers is enhancing nutrient use efficiency, which is fundamental for both economic and environmental reasons. Biofertilizers enhance nutrient uptake in deficient soils, or under sub-optimal fertilization regimes, thereby reducing production costs. Benefits have been quantified on a global scale for nitrogen and phosphorus use efficiency, whereas it has been found that effects are always positive for both elements, ranging from 1-8 $\mathrm{kg}$ change increase in yield per $\mathrm{kg}$ of $\mathrm{N}$ or $\mathrm{P}$ (Schütz et al., 2018). There is also ample evidence that use of biofertilizers enhances quality parameters of produce, which can translate to higher values at market (Rouphael and Colla, 2020).

Biofertilizers are also a response to correcting historical agricultural land management practices which threaten sustainability. Common problems such as low nutrient capital, weeds, and low levels of soil organic matter gave impetus to what was the Green Revolution, and the continuous and systematic use of pesticides and fertilizers has led to soil impoverishment. Microbial inoculants have the capacity to enhance productivities in a sustainable manner, which has led researchers and agriculturalists to give attention to the development of these products and practices. It has been found in a number of studies that use of biofertilizers, alone or in conjunction with organic or mineral fertilizers, lend to higher benefit:cost ratios than simple use of conventional mineral fertilizers, or provide extra benefit when making a transition to organic production.

Biofertilizers can be produced from nutrient-rich agri-food wastes and industrial by-products, opening opportunities for a circular economy approach (Rouphael and Colla, 2020), and the exploitation of suitable low-cost nutrient sources is an appropriate strategy for production of biofertilizers since multiple objectives can be achieved (e.g. wastewater treatment) while also reducing costs of transport of materials (Renuka et al., 2018). Over the past few years, work has been carried out to identify appropriate feedstocks and sources for production of biofertilizers and biostimulants (see $\mathrm{Xu}$ and Geelen, 2018).

\section{Potential drawbacks to the practice}

\section{Table 83. Soil threats}

\begin{tabular}{|l|l|}
\hline Soil threat & Microbes affect both the mobilization and immobilization of \\
\hline $\begin{array}{l}\text { Soil contamination } \\
\text { pollution }\end{array}$ & $\begin{array}{l}\text { pollunts (Gadd, 2004), so caution should be taken with attention to } \\
\text { microbe identity and particular interaction with pollutants if the } \\
\text { technique is applied for production of food or feed on contaminated } \\
\text { soils. }\end{array}$ \\
\hline
\end{tabular}




\subsection{Increases in greenhouse gas emissions}

There does not seem to be any particular current concern or risk concerning GHG emissions from biofertilizer use. As a rule, biostimulants and biofertilizers are applied in small quantities, and their actual nutrient contents are insignificant compared to chemical fertilizers. This, however, does not rule out the possibility of biostimulation of the soil microbial community, for example when algal slurries are applied, though while $\mathrm{CO}_{2}$ emissions can increase through this increased heterotrophic respiration, the total quantity of emitted carbon as $\mathrm{CO}_{2}$ is of little significance for the total soil $\mathrm{C}$ pool (Marks et al. 2017).

\subsection{Conflict with other practice(s)}

At this point there do not seem to be any particular antagonisms with other fertilization or soil management practices.

\subsection{Decreases in production (e.g. food/fuel/feed/timber/fibre)}

Since biofertilization involves the selection and application of plant growth-promoting organisms from soil or roots, unintended consequences on plant productivity have not been identified as a risk.

\subsection{Other conflicts}

As mentioned in section 4.5 above, one strategy for biofertilizer manufacture is the use of nutrient-rich waste streams. Of course, some such wastes may contain components which pose risks to human or environmental health (pathogens, heavy metals, pharmaceuticals, etc.). Therefore, any particular risks arising in product development must be properly addressed by manufacturers to assure that high-quality products are produced. Also, proper legislation is needed that addresses risks in a reasonable manner, with appropriate criteria for categorization and screening and testing methods, to assure the safety of commercially sold biostimulants (Barros-Rodríguez et al., 2020).

\section{Recommendations before implementing the practice}

As living organisms, biofertilizers are sensitive to environmental conditions just like plants and crops - as a fertilization option, this makes their effectiveness less predictable than mineral fertilizers. Much research is underway to understand which organism groups or species are most successful for any given crop, soil type, climate etc. As this potential diversity is quite large, locality is of high importance. For this reason, users interested in testing or directly applying biofertilizers should contact local or national agricultural ministries, services, extensions, or research institutions to inquire about existing knowledge, products, and practice. These actors may have already developed functional biofertilizer products, isolated strains, or have tested them with crops of economic importance to the region. 
One of the most challenging aspects of biofertilizer products is assuring adequate shelf life - these are living organisms, and quality (effectiveness) can quickly decrease when stability is not assured. Therefore, users should pay close attention to recommended handling and storage instructions so that proper benefits can be seen.

Another important aspect of biofertilizer use is its effectiveness when applied, which is highly tied to the inoculation method. Careful application to seeds or roots require relatively small quantities ${ }^{16}$ - this targeted application will reduce the amount of biofertilizer necessary to see desired effects, and is the practice applied when cultures are prepared with isolated microorganisms or consortia thereof with proper microbiological handling. On the other hand, biofertilizers used in top-dressing (inoculated organic fertilizers, algal or cyanobacterial slurries, etc.) are applied in much larger volumes of matter or liquid, similar to the application of compost, manures and fertilizers, plant protection, products, etc. In contrast to seed or root inoculation, these products can be applied using conventional methods and equipment.

\section{Potential barriers for adoption}

Table 84. Potential barriers to adoption

\begin{tabular}{|c|c|c|}
\hline Barrier & YES/NO & \\
\hline Biophysical & Yes & $\begin{array}{l}\text { Care must be taken to properly culture biofertilizers and } \\
\text { maintain them, since they are sensitive to biophysical factors } \\
\text { prior to and following application. }\end{array}$ \\
\hline Cultural & Yes & $\begin{array}{l}\text { With the green revolution and predominance of synthetic } \\
\text { chemical products, farmers have been accustomed to highly } \\
\text { predictable and reliable results from chemical fertilizers. } \\
\text { Biofertilizers incorporate a larger degree of uncertainty, which } \\
\text { can impede uptake (Herrmann and Lesueur, 2013). }\end{array}$ \\
\hline Social & Yes & $\begin{array}{l}\text { The very principle of biofertilizer function - indirect benefits } \\
\text { not tied to actual nutrient content - is not common in } \\
\text { conventional "fertilization", and this may impede uptake. }\end{array}$ \\
\hline Economic & Yes & $\begin{array}{l}\text { More } \mathrm{R}+\mathrm{D} \text { investment will be required from private and public } \\
\text { industries to help reduce uncertainties of biofertilizer use and } \\
\text { produce reliable formulations of inoculants (Herrmann and } \\
\text { Lesueur, 2013). }\end{array}$ \\
\hline
\end{tabular}

\footnotetext{
${ }^{16}$ Liquid cultures containing between $10^{8}-10^{9} \mathrm{CFU}$ per mL have been reported for inoculation of seeds. Both seeds and roots are immersed in the culture liquid for a period of time (e.g., tens of minutes) to improve the rate of impregnation/adhesion of the microbes.
} 


\begin{tabular}{|l|l|l|}
\hline Barrier & YES/NO & \\
\hline Institutional & Yes & $\begin{array}{l}\text { At the moment, legislation on biofertilizer products is } \\
\text { incipient, and even counter-productive in some cases (Barros- } \\
\text { Rodriguez et al., 2020). }\end{array}$ \\
\hline Knowledge & Yes & $\begin{array}{l}\text { There is significant work yet to be done on identifying proper } \\
\text { combinations of soil-plant-biofertilizer, and developing } \\
\text { delivery methods which assure function and product stability } \\
\text { (Herrmann and Lesueur, 2013). }\end{array}$ \\
\hline
\end{tabular}

\section{Photos of the practice}

\section{Photobioreactor, inoculated with microbial strains and fed with agro-industrial effluent or other nutrient-rich substrates}

Photo 23. An example of production and application of a biofertilizer.

In this case, a microalga-based fertilizer (though also containing other microorganisms due to scale and application in outdoor environment) is produced using recycled agri-food wastes.

Once densities approach optimum (approx. $1 \mathrm{~g} / \mathrm{L}$ dry mass), contents are transferred and applied in the field with conventional machinery. This practice can increase soil C contents and soil microbial activities. Location: Burgos, Spain, 2016 
Table 85. Related cases studies available in volumes 3 and 5

\begin{tabular}{|l|c|c|c|c|}
\hline Title & Region & $\begin{array}{l}\text { Duration } \\
\text { of study } \\
\text { (Years) }\end{array}$ & Volume & $\begin{array}{l}\text { Case- } \\
\text { study } \\
\text { No. }\end{array}$ \\
\hline $\begin{array}{l}\text { Agricultural practices for the restoration of } \\
\text { Soil Ecological Functions in Madagascar }\end{array}$ & Africa & 2 & 3 & 2 \\
\hline
\end{tabular}




\section{References}

Alori, E.T., Dare, M.O. \& Babalola, O.O. 2017. Microbial Inoculants for Soil Quality and Plant Health. In E. Lichtfouse (Ed.) Sustainable Agriculture Reviews, vol. 22, pp. 281-307. Cham, Springer International Publishing. https://doi.org/10.1007/978-3-319-48006-0_9

Ansari, R.A. \& Mahmood, I. 2017. Optimization of organic and bio-organic fertilizers on soil properties and growth of pigeon pea. Scientia Horticulturae, 226: 1-9.

https://doi.org/10.1016/j.scienta.2017.07.033

Bahadur, L., Tiwari, D.D., Mishra, J. \& Gupta, B.R. 2013. Evaluation of integrated nutrient management options in rice (Oryza sativa)- wheat (Triticum aestivum) cropping system in reclaimed sodic land. Indian Journal of Agronomy, 58(2): 137-145.

Barros-Rodríguez, A., Rangseekaew, P., Lasudee, K., Pathom-aree, W. \& Manzanera, M. 2020. Regulatory risks associated with bacteria as biostimulants and biofertilizers in the frame of the European Regulation (EU) 2019/1009. Science of the Total Environment, 740: 140239.

https://doi.org/10.1016/j.scitotenv.2020.140239

Berruti, A., Lumini, E., Balestrini, R. \& Bianciotto, V. 2016. Arbuscular mycorrhizal fungi as natural biofertilizers: Let's benefit from past successes. Frontiers in Microbiology, 6: 1-13.

https://doi.org/10.3389/fmicb.2015.01559

Bhattacharjee, R.B., Singh, A. \& Mukhopadhyay, S.N. 2008. Use of nitrogen-fixing bacteria as biofertiliser for non-legumes: Prospects and challenges. Applied Microbiology and Biotechnology, 80(2): 199-209. https://doi.org/10.1007/s00253-008-1567-2

Chekwube Enebe, M. \& Oluranti Babalola, O. 2018. The influence of plant growth-promoting rhizobacteria in plant tolerance to abiotic stress: a survival strategy. Applied microbiology and biotechnology, 102: 7821-7835. https://doi.org/10.1007/s00253-018-9214-z

Dẹbska, B., Długosz, J., Piotrowska-Długosz, A. \& Banach-Szott, M. 2016. The impact of a bio-fertilizer on the soil organic matter status and carbon sequestration-results from a field-scale study. Journal of Soils and Sediments, 16(10): 2335-2343. https://doi.org/10.1007/s11368-016-1430-5

Dutta, P., Biswas, S. \& Kundu, S. 2011. Effect of organic manures and biofertilizers on production of organic litchi. Journal of Eco-friendly Agriculture, 6(1): 13-15

FAO. 2018. Disasters causing billions in agricultural losses, with drought leading the way [online]. [Cited 24 February 2021]. http://www.fao.org/news/story/en/item/1106977/icode/

FAO. 2019. Climate-Smart Agriculture [online]. [Cited 24 February 2021]. http://www.fao.org/climatesmart-agriculture/en/

FAO. 2017. Soil Organic Carbon: the hidden potential. Rome, Italy 1-99 pp.

Gadd, G.M. 2004. Microbial influence on metal mobility and application for bioremediation. Geoderma, 122(2): 109-119. https://doi.org/https://doi.org/10.1016/j.geoderma.2004.01.002 
García-Fraile, P., Menéndez, E. \& Rivas, R. 2015. Role of bacterial biofertilizers in agriculture and forestry. AIMS Bioengineering, 2(3): 183-205. https://doi.org/10.3934/bioeng.2015.3.183

Herrmann, L. \& Lesueur, D. 2013. Challenges of formulation and quality of biofertilizers for successful inoculation. Applied Microbiology and Biotechnology, 97(20): 8859-8873.

https://doi.org/10.1007/s00253-013-5228-8

Hettiarachchi, R.P., Dharmakeerthi, R.S., Seneviratne, G., Jayakody, A.N., De Silva, E., Gunathilake, T., Thewarapperuma, A. \& Maheepala, C.K. 2014. Availability and leaching of nutrients after biofilm biofertilizer applications into a Red Yellow Podsolic soil. Journal of the Rubber Research Institute of Sri Lanka, 94: 43-53.

Hosseinzadeh, M.H., Ghalavand, A., Boojar, M.M.A., Modarres-Sanavy, S.A.M. \& Mokhtassi-Bidgoli, A. 2021. Application of manure and biofertilizer to improve soil properties and increase grain yield, essential oil and $\omega 3$ of purslane (Portulaca oleracea L.) under drought stress. Soil and Tillage Research, 205:104633. https://doi.org/10.1016/j.still.2020.104633

Hu, C., Xia, X., Chen, Y. \& Han, X. 2018. Soil carbon and nitrogen sequestration and crop growth as influenced by long-term application of effective microorganism compost. Chilean Journal of Agricultural Research, 78(1): 13-22. https://doi.org/10.4067/S0718-58392018000100013

Kantachote, D., Nunkaew, T., Kantha, T. \& Chaiprapat, S. 2016. Biofertilizers from Rhodopseudomonas palustris strains to enhance rice yields and reduce methane emissions. Applied Soil Ecology, 100: 154-161. https://doi.org/10.1016/j.apsoil.2015.12.015

Kumar, R., Deka, B.C., Kumar, M. \& Ngachan, S. V. 2015. Productivity, quality and soil health as influenced by organic, inorganic and biofertilizer on field pea in Eastern Himalaya. Journal of Plant Nutrition, 38(13): 2006-2007. http://dx.doi.org/10.1080/01904167.2014.988355

Li, H., Qiu, Y., Yao, T., Ma, Y., Zhang, H. \& Yang, X. 2020. Effects of PGPR microbial inoculants on the growth and soil properties of Avena sativa, Medicago sativa, and Cucumis sativus seedlings. Soil and Tillage Research, 199: 104577. https://doi.org/10.1016/j.still.2020.104577

Liu, Q., Meng, X., Li, T., Raza, W., Liu, D. \& Shen, Q. 2020. The growth promotion of peppers (Capsicum annuum L.) by trichoderma guizhouense NJAU4742-based biological organic fertilizer: possible role of increasing nutrient availabilities. Microorganisms, 8(9): 1-23

https://doi.org/10.3390/microorganisms8091296

Mącik, M., Gryta, A. \& Frąc, M. 2020. Chapter Two - Biofertilizers in agriculture: An overview on concepts, strategies and effects on soil microorganisms. In D.L. Sparks (Ed.) Advances in Agronomy, pp. 31-87.

Academic Press. https://doi.org/10.1016/bs.agron.2020.02.001

Mariya Dainy, M.S. \& Manorama Thampatti, K.C. 2016. Impact of biochar application on soil fertility status and yield of yard long bean in ferralitic soils. International Journal of Tropical Agriculture, 34(1): 249251.

Marks, E.A.N., Miñón, J., Pascual, A., Montero, O., Navas, L.M. \& Rad, C. 2017. Application of a microalgal slurry to soil stimulates heterotrophic activity and promotes bacterial growth. Science of the Total Environment, 605-606: 610-617. https://doi.org/10.1016/j.scitotenv.2017.06.169 
Marks, E.A.N., Montero, O. \& Rad, C. 2019. The biostimulating effects of viable microalgal cells applied to a calcareous soil: Increases in bacterial biomass, phosphorus scavenging, and precipitation of carbonates. Science of the Total Environment, 692: 784-790. https://doi.org/10.1016/j.scitotenv.2019.07.289

Mukherjee, A.K., Tripathi, S., Mukherjee, S., Mallick, R.B. \& Banerjee, A. 2019. Effect of integrated nutrient management in sunflower (Helianthus annuus L.) on alluvial soil. Current Science, 117(8): 13641368. https://doi.org/10.18520/cs/v117/i8/1364-1368

Nazirkar, R.B. 2014. Effect of PBSW and biofertilizers on the solubilization of P from RP to soybean in inceptisol. Asian Journal of Soil Science, 9(2): 240-243. https://doi.org/10.15740/has/ajss/9.2/240243

Ojha, R.K., Mandal, B.K., Pareta, D.K. \& Thomas, T. 2009. Effect of Combined Application of Inorganic, FYM and Azotobacter on Chemical Properties and N, P, K Availability after Potato Harvest. Environment \& Ecology, 27(4B): 1899-1902.

Perin, G., Yunus, I.S., Valton, M., Alobwede, E. \& Jones, P.R. 2019. Sunlight-driven recycling to increase nutrient use-efficiency in agriculture. Algal Research, 41.

https://doi.org/10.1016/j.algal.2019.101554

Piotrowska, A., Dlugosz, J. \& Zamorski, R.B. 2011. Changes in Some Biological and Chemical Properties of an Arable Soil Treated with the Microbial Biofertilizer UGmax. Polish Journal of Environmental Studies, 21(2): 453-461

Renuka, N., Guldhe, A., Prasanna, R., Singh, P. \& Bux, F. 2018. Microalgae as multi-functional options in modern agriculture: current trends, prospects and challenges. Biotechnology Advances, 36(4): 12551273. https://doi.org/10.1016/j.biotechadv.2018.04.004

Rodrigues, M.Â., Piroli, L.B., Forcelini, D., Raimundo, S., da Silva Domingues, L., Cassol, L.C., Correia, C.M. \& Arrobas, M. 2021. Use of commercial mycorrhizal fungi in stress-free growing conditions of potted olive cuttings. Scientia Horticulturae, 275: 109712

https://doi.org/10.1016/j.scienta.2020.109712

Rossi, F., Li, H., Liu, Y. \& De Philippis, R. 2017. Cyanobacterial inoculation (cyanobacterisation): Perspectives for the development of a standardized multifunctional technology for soil fertilization and desertification reversal. Earth-Science Reviews, 171: 28-43.

https://doi.org/10.1016/j.earscirev.2017.05.006

Rouphael, Y. \& Colla, G. 2020. Editorial: Biostimulants in Agriculture. Frontiers in Plant Science, 11: 1-7. https://doi.org/10.3389/fpls.2020.00040

Rubin, R.L., van Groenigen, K.J. \& Hungate, B.A. 2017. Plant growth promoting rhizobacteria are more effective under drought: a meta-analysis. Plant and Soil, 416(1-2): 309-323.

https://doi.org/10.1007/s11104-017-3199-8

Schmidt, J.E. \& Gaudin, A.C.M. 2018. What is the agronomic potential of biofertilizers for maize? A metaanalysis. FEMS Microbiology Ecology, 94(7): 1-10. https://doi.org/10.1093/femsec/fiy094 
Schütz, L., Gattinger, A., Meier, M., Müller, A., Boller, T., Mäder, P. \& Mathimaran, N. 2018. Improving Crop Yield and Nutrient Use Efficiency via Biofertilization-A Global Meta-analysis. Frontiers in Plant Science, 8. https://doi.org/10.3389/fpls.2017.02204

Shahzad, S.M., Arif, M.S., Riaz, M., Ashraf, M., Yasmeen, T., Zaheer, A., Bragazza, L., Buttler, A. \& Robroek, B.J.M. 2017. Interaction of compost additives with phosphate solubilizing rhizobacteria improved maize production and soil biochemical properties under dryland agriculture. Soil and Tillage Research, 174: 70-80. https://doi.org/10.1016/j.still.2017.06.004

Shanthakumar, S., Abinandan, S., Venkateswarlu, K., Subashchandrabose, S.R. \& Megharaj, M. 2020. Algalization of acid soils with acid-tolerant strains: Improvement in $\mathrm{pH}$, carbon content, exopolysaccharides, indole acetic acid and dehydrogenase activity. Land Degradation and Development, 31.

https://doi.org/10.1002/ldr.3849

Shukla, S.K., Shee, S., Maity, S.K., Solomon, S., Awasthi, S.K., Gaur, A., Pathak, A.D. \& Jaiswal, V.P. 2017. Soil Carbon Sequestration and Crop Yields in Rice-Wheat and Sugarcane-Ratoon-Wheat Cropping Systems Through Crop Residue Management and Inoculation of Trichoderma viride in Subtropical India. Sugar Tech, 19(4): 347-358. https://doi.org/10.1007/s12355-016-0470-х

Simranjit, K., Kanchan, A., Prasanna, R., Ranjan, K., Ramakrishnan, B., Singh, A.K. \& Shivay, Y.S. 2019. Microbial inoculants as plant growth stimulating and soil nutrient availability enhancing options for cucumber under protected cultivation. World Journal of Microbiology and Biotechnology, 35(3): 51-64. https://doi.org/10.1007/s11274-019-2623-Z

Singh, J.K., Singh, D.K. \& Singh, H.K. 2014. Studies on integrated nutrient management of vegetative growth, fruiting behaviour and nutrient status of leaves and soil of bael (Aegle marmelos Correa) orchard cv. NARENDRA BAEL-9. The Asian Journal of Horticulture, 9(2): 421-425.

https://doi.org/10.15740/has/tajh/9.2/421-425

Smitha, G.R., Basak, B.B., Thondaiman, V. \& Saha, A. 2019. Nutrient management through organics, bio-fertilizers and crop residues improves growth, yield and quality of sacred basil (Ocimum sanctum Linn). Industrial Crops and Products, 128: 599-606. https://doi.org/10.1016/j.indcrop.2018.11.058

Son, T.T.N., Thu, V., Man, L.H, Kobayashi, H. \& Yamada, R. 2004. Effect of long-term application of organic and bio-fertilizer on soil fertility under rice-soybean-rice cropping system. Omonrice, 12: 45-51

Srivastava, T., Singh, K., Singh, P., Suman, A., Singh, S., Verma, R., Singh, V. \& Singh, R. 2018. Effect of bio-manures on soil quality, cane productivity and soil carbon sequestration under long-term sugarcane (Saccharum officinarum) plant - Ratoon system in Indian sub-tropics. Indian Journal of Agricultural Sciences, 88(11): 1696-1703. (also available at

https://www.researchgate.net/publication/329034886_Effect_of_biomanures_on_soil_quality_cane_productivity_and_soil_carbon_sequestration_under_longterm_sugarcane_Saccharum_officinarum_plant_-_Ratoon_system_in_Indian_sub-tropics).

Thilagar, G., Bagyaraj, D.J. \& Raoca, M.S. 2016. Selected microbial consortia developed for chilly reduces application of chemical fertilizers by $50 \%$ under field conditions. Scientia Horticulturae, 198: 27-35. https://doi.org/10.1016/j.scienta.2015.11.021 
Xu, L. \& Geelen, D. 2018. Developing Biostimulants From Agro-Food and Industrial By-Products. Frontiers in Plant Science, 9: 1-13. https://doi.org/10.3389/fpls.2018.01567

Yadav, S.K., Khokhar, U.U., Sharma, S.D. \& Kumar, P. 2016. Response of strawberry to organic versus inorganic fertilizers. Journal of Plant Nutrition, 39(2): 194-203.

https://doi.org/10.1080/01904167.2015.1109115

Yagi, R., Quadros, T.C.F., Martins, B.H. \& Andrade, D.S. 2020. Maize yields and carbon pools in response to poultry litter, rock phosphate and P-solubilizing microorganisms. Scientia Agricola, 77(3). https://doi.org/10.1590/1678-992x-2018-0141

Yilmaz, E. \& Sönmez, M. 2017. The role of organic/bio-fertilizer amendment on aggregate stability and organic carbon content in different aggregate scales. Soil and Tillage Research, 168: 118-124. https://doi.org/10.1016/j.still.2017.01.003

Zhou, X., Zhao, Y., Belnap, J., Zhang, B., Bu, C. \& Zhang, Y. 2020. Practices of biological soil crust rehabilitation in China: experiences and challenges. Restoration Ecology, 28: S45-S55.

https://doi.org/10.1111/rec.13148 


\title{
19. Earthworm inoculation
}

\section{Onja Ratsiatosika1', Eric Blanchart²}

\author{
'Laboratoire des Radio-Isotopes, University of Antananarivo, Antananarivo, Madagascar \\ ${ }^{2}$ Eco\&Sols, Univ Montpellier, CIRAD, INRA, IRD, Montpellier SupAgro, Montpellier, France
}

\section{Description of the practice}

Earthworms are one of the most important (at least in terms of biomass) organisms in soils. They have been highly studied and their functional roles have been described making them important actors of soil functioning and provision of ecosystem services (Lavelle et al., 2006). Earthworms are widely recognized for their effects on the main soil ecological functions: formation and maintenance of soil structure (biogenic structures i.e., aggregates and porosity) (Blanchart et al., 1999), decomposition and mixing of organic matter with soil (Brown, Barois and Lavelle, 2000), nutrient cycling (especially nitrogen and phosphorus but also calcium, silicon...) (van Groenigen et al., 2019), and biological control of pathogens (Blanchart et al., 2020). These effects on soil functions have strong implications on ecosystem services such as plant production, climate mitigation and carbon sequestration, and control of erosion. Following these potentially beneficial effects on functions and services, earthworms are generally perceived as animals to be sustained in agricultural systems. It is very likely that the manipulation of the soil community is key to successful restoration of terrestrial ecosystems (Jouquet, Blanchart and Capowiez, 2014; Wubs et al., 2016).

Inoculation is here defined as the deliberate (i.e. not accidental) introduction of living organisms in a given soil with the aim that this action will result in beneficial changes in dynamics and equilibrium of the environment. Inoculation, as an active biostimulation approach, has been sometimes used for restoration programs following habitat degradation or reclamation of land (Baker et al., 2006). Nevertheless, this has barely been developed at large scales and over a long time in agricultural fields with the aim of improving yields and other ecosystem services such as carbon storage. As highlighted by Bertrand et al. (2015), this explains why it is difficult to scientifically assess the long-term benefits of increasing earthworm abundance in cropped fields. This 'in-soil' technology appears to have better effects on ecosystem functioning than 'off-soil' techniques that simply use earthworms to prepare compost (vermicomposting) (Senapati et al., 1999). 


\section{Range of applicability}

Theoretically, it seems possible to inoculate earthworms in each type of soil, for different climate, and for different land-uses. Inoculation can concern either cocoons, juveniles, adults, or micro-environments containing earthworms (sod transplantation method) (Butt et al., 1997). Before inoculation, earthworms can either be bought in a commercial unit, collected from neighboring areas or produced in large quantities in large culture beds (Senapati et al., 1999).

\section{Impact on soil organic carbon stocks}

Table 86. Measured effects of earthworm inoculation on soil organic carbon stocks

\begin{tabular}{|c|c|c|c|c|c|c|c|c|}
\hline Location & $\begin{array}{l}\text { Climate } \\
\text { zone }\end{array}$ & Soil type & $\begin{array}{l}\text { Baseline } \\
\text { C stock } \\
\text { (tC/ha) }\end{array}$ & $\begin{array}{l}\text { Additional C } \\
\text { storage } \\
\text { (tC/ha/yr) }\end{array}$ & $\begin{array}{l}\text { Duration } \\
\text { (Years) }\end{array}$ & $\begin{array}{l}\text { Depth } \\
(\mathrm{cm})\end{array}$ & More information & Reference \\
\hline $\begin{array}{l}\text { Madagascar } \\
\text { (Highlands) }\end{array}$ & $\begin{array}{l}\text { Altitude } \\
\text { tropical } \\
\text { climate }\end{array}$ & & 9.76 & 0.61 & & & $\begin{array}{l}\text { Upland rice crop. } \\
\text { Inoculation at a } \\
\text { density of } 75 \mathrm{ind} / \mathrm{m}^{2}\end{array}$ & $\begin{array}{l}\text { Ratsiatosika } \\
\text { (2018) }\end{array}$ \\
\hline $\begin{array}{l}\text { Côte d'Ivoire } \\
\text { (Lamto's } \\
\text { savanna) }\end{array}$ & $\begin{array}{l}\text { Tropical } \\
\text { savannas } \\
\text { climate }\end{array}$ & Ferralsol & 16.4 & $\begin{array}{l}0.3 \\
\text { (6\% avoided } \\
\text { loss compared } \\
\text { to the } \\
\text { decrease } \\
\text { without } \\
\text { earthworms) }\end{array}$ & 3 & $0-10$ & $\begin{array}{l}\text { Maize cultivation } \\
\text { after forest } \\
\text { deforestation }\end{array}$ & Gilot (1997) \\
\hline $\begin{array}{l}\text { Peru } \\
\text { (Yurimaguas) }\end{array}$ & Tropical & & 18.7 & No difference & & & $\begin{array}{l}\text { Maize/rice/cowpea } \\
\text { rotation after } \\
\text { deforestation }\end{array}$ & $\begin{array}{l}\text { Villenave et } \\
\text { al. (1999) }\end{array}$ \\
\hline
\end{tabular}

These few field experiments suggest that the inoculation of earthworms in agricultural plots, following deforestation, may help to reduce carbon losses due to land use change. Conversely, when earthworms are inoculated in a field with low organic matter contents, such as in Madagascar, the effect on carbon stocks seems positive. Lavelle et al. (2020) recently reminded the importance of earthworms in C storage on the medium and long-term, following soil macroaggregation whereas their effect was negative on the short-term (Table 86). 


\section{Other benefits of the practice}

\subsection{Improvement of soil properties}

Inoculation of earthworms may result in positive effects on soil properties, especially on macroaggregation (Lavelle et al., 2020) and nutrient availability (van Groenigen et al., 2019).

\subsection{Minimization of threats to soil functions}

Table 87. Soil threats

\begin{tabular}{|l|l|}
\hline Soil threats & \\
\hline Soil erosion & $\begin{array}{l}\text { Earthworms seem to reduce soil losses through the formation of } \\
\text { macropores and stable macro-aggregates (Blanchart et al., 2004). }\end{array}$ \\
\hline $\begin{array}{l}\text { Nutrient imbalance and } \\
\text { cycles }\end{array}$ & $\begin{array}{l}\text { Available N and P stocks in soil and in plants significantly increased in } \\
\text { the presence of earthworms (Ratsiatosika, 2018). }\end{array}$ \\
\hline Soil biodiversity loss & $\begin{array}{l}\text { Results on soil biodiversity show contrasting effects (i.e. either positive } \\
\text { or negative) on soil organisms. }\end{array}$ \\
\hline Soil compaction & $\begin{array}{l}\text { Bulk density higher or lower after earthworm inoculation depending on } \\
\text { the studies (Blanchart et al., 1999). }\end{array}$ \\
\hline Soil water management & $\begin{array}{l}\text { Increase in soil water retention in link with macroaggregate formation } \\
\text { (Blanchart et al., 2004). }\end{array}$ \\
\hline
\end{tabular}

\subsection{Increases in production (e.g. food/fuel/feed/timber/fibre)}

The meta-analysis by van Groenigen et al. (2014) on the effect of earthworms on plant production gives evidence of the global positive effect which is generally observed from mesocosm or microcosm experiments. Only few studies relate the effect of earthworm after inoculation in field long-term experiments. The review by Brown et al. (1999) considered such field studies and also showed a positive effect of earthworm on plant production. For instance, in India, in the conventional treatment, production was $2306 \mathrm{~kg} / \mathrm{ha} / \mathrm{yr}$; it was 3445 $\mathrm{kg} / \mathrm{ha} / \mathrm{yr}$ in treatment with prunings without earthworms, and $9534 \mathrm{~kg} / \mathrm{ha} / \mathrm{yr}$ with earthworms (Senapati et al. 1999). In Madagascar, after 3 years of earthworm inoculation, significant higher rice yields were measured in the presence of earthworms (1.20 t/ha) compared to their absence $(0.83 \mathrm{t} / \mathrm{ha})$ (Ratsiatosika, 2018). 


\subsection{Mitigation of and adaptation to climate change}

It is well recognized that earthworms have important effects on $\mathrm{C}$ and $\mathrm{N}$ cycling and their positive effects on $\mathrm{CO}_{2}$ and $\mathrm{N}_{2} \mathrm{O}$ have been demonstrated from mesocosm experiments and in the short term. Nevertheless, there are no available data from field situations, in the long term, after earthworm inoculation.

\subsection{Socio-economic benefits}

The cost of earthworm collection from natural environments is very dependent on the cost of labor: the cost to produce $1 \mathrm{~kg}$ of live earthworms ranged from a few euros in Madagascar, to $6 €$ in India, $18 €$ in Peru and 125 $€$ in Martinique (West Indies) (Ratsiatosika, 2018; Senapati et al., 1999). In culture beds, the cost of production of $1 \mathrm{~kg}$ of earthworm biomass was estimated at 3.6 (India) to $9.2 €$ (Peru). As a consequence, the cost to produce an active earthworm community with an average biomass of $400 \mathrm{~kg}$ live weight per hectare was estimated at 1400 euros. At this cost it appears that earthworm inoculation should be applied to high value crops. Earthworm inoculation thus appears a relatively expensive technology, or necessitating at least an important labor time. This may be a reason for the low utilization of this technology. Nevertheless, it should be considered that while the investment costs were higher for treatments with earthworms, the profit was much higher (7 594 USD/ha) compared to conventional system (1 997 USD/ha) in tea plantation, India (Senapati et al. 1999).

\section{Potential drawbacks to the practice}

\subsection{Tradeoffs with other threats to soil functions}

Table 88. Soil threats

\begin{tabular}{|l|l|}
\hline Soil threats & \\
\hline Soil erosion & $\begin{array}{l}\text { Earthworm inoculation may improve soil macroaggregation and favor } \\
\text { soil infiltration, reducing run-off and erosion (Blanchart } \text { et al., 2004). }\end{array}$ \\
\hline $\begin{array}{l}\text { Nutrient imbalance and } \\
\text { cycles }\end{array}$ & $\begin{array}{l}\text { Earthworm effect seems less evident in the presence of mineral } \\
\text { fertilization. }\end{array}$ \\
\hline Soil biodiversity loss & $\begin{array}{l}\text { It does not seem realistic neither beneficial to inoculate earthworms } \\
\text { from other regions or continents with the risk they become invasive } \\
\text { species with possible negative impact on non-target biota and } \\
\text { ecosystem functioning (Hendrix, 2006). }\end{array}$ \\
\hline Soil compaction & $\begin{array}{l}\text { Earthworms are very sensitive to tillage; earthworm populations are } \\
\text { generally higher in no-till than in till systems. It seems thus important to } \\
\text { reduce soil tillage to improve earthworm development (Chan, 2001). }\end{array}$ \\
\hline
\end{tabular}




\subsection{Increases in greenhouse gas emissions}

Earthworm activities are generally seen as positive for ecosystem functioning, although some issues are still under debate, namely their possible effects on greenhouse gases GHG emissions. The effects on GHG emissionsappear to increase in the short-term and decrease in the long-term, which leads to a difficulty at predicting C storage in the presence of earthworms (Zhang et al, 2013).

\subsection{Conflict with other practice(s)}

Other practices, harmful to earthworms such as tillage or the use of pesticides, should be avoided. Conversely, earthworm inoculation should be associated with superficial tillage, and high inputs of organic matter.

\subsection{Decreases in production (e.g. food/fuel/feed/timber/fibre)}

No, previous experiments following earthworm restoration showed an increase in crop production (see above (chapter 4.3).

\section{Recommendations before implementing the practice}

In addition to adapted practices, it is important to select earthworms that can thrive in cropped fields. Generally endemic species can barely support soil disturbance and it is thus better to use wide distributed, peregrine species, with large environmental tolerance. It is important that earthworms already exist in the area and can adapt to local climate, soil type and soil disturbance. The use of exotic earthworms is possible when these species are already well established in a given region or area.

For instance, in the tropics, most inoculation practices have considered the species Pontoscolex corethrurus (Rhinodrilidae), a peregrine species that can withstand most agricultural disturbances. In temperate regions, inoculation in pastures or grasslands especially considered the lumbricid species Apporectodea caliginosa (New Zealand, Baker et al, 2006) or Lumbricus terrestris (France, Forey et al, 2018) both worldwide distributed species. Top-soil dwelling, geophagous species are usually preferred because they establish better than deepburrowing species in agricultural soils. Based on the literature, it is recommended to inoculate earthworms at a minimum of $300 \mathrm{~kg} \cdot \mathrm{ha}^{-1}$ of adult worms to provide efficient earthworm activity (Spain, Lavelle and Mariotti, 1992; Senapati et al., 1999).

It is also important that earthworm environment is restored and that conditions are made favorable to their development. The main approach consists in changing practices: remove practices detrimental to earthworms (tillage, pesticides) and develop practices beneficial to earthworms (liming, organic fertilization) (Brun et al., 1987). Earthworm inoculation also needs to be associated with inputs of organic matter to feed soil macrofauna (Lavelle et al., 2001). 


\section{Potential barriers for adoption}

Table 89. Potential barriers to adoption

\begin{tabular}{|c|c|c|}
\hline Barrier & YES/NO & \\
\hline Biophysical & Yes & $\begin{array}{l}\text { Earthworm survival highly depends on rainfall pattern and organic inputs } \\
\text { (Lavelle et al., 2001) }\end{array}$ \\
\hline Cultural & Yes & $\begin{array}{l}\text { Farmers have only a poor knowledge of the potential effects of earthworms } \\
\text { on soil and plant properties; this is variable among ethnic groups (Ortiz et al., } \\
\text { 1999) }\end{array}$ \\
\hline Social & Yes & $\begin{array}{l}\text { Famers have only a poor technical knowledge on the way to inoculate } \\
\text { earthworms, or to produce vermicompost (Ortiz et al., 1999) }\end{array}$ \\
\hline Economic & Yes & $\begin{array}{l}\text { Smallholder farmers have limited access to external inputs and cannot buy } \\
\text { earthworms in commercial units }\end{array}$ \\
\hline Institutional & No & \\
\hline Legal & Yes & $\begin{array}{l}\text { The Nagoya Protocol makes difficult to relocate earthworm species from one } \\
\text { country to another }\end{array}$ \\
\hline Knowledge & Yes & $\begin{array}{l}\text { Site-specific and species-specific effects of earthworms are of great } \\
\text { importance and need deepening scientific knowledge about earthworm-plant } \\
\text { interactions (Bertrand et al., 2015) }\end{array}$ \\
\hline
\end{tabular}




\section{Photo of the practice}

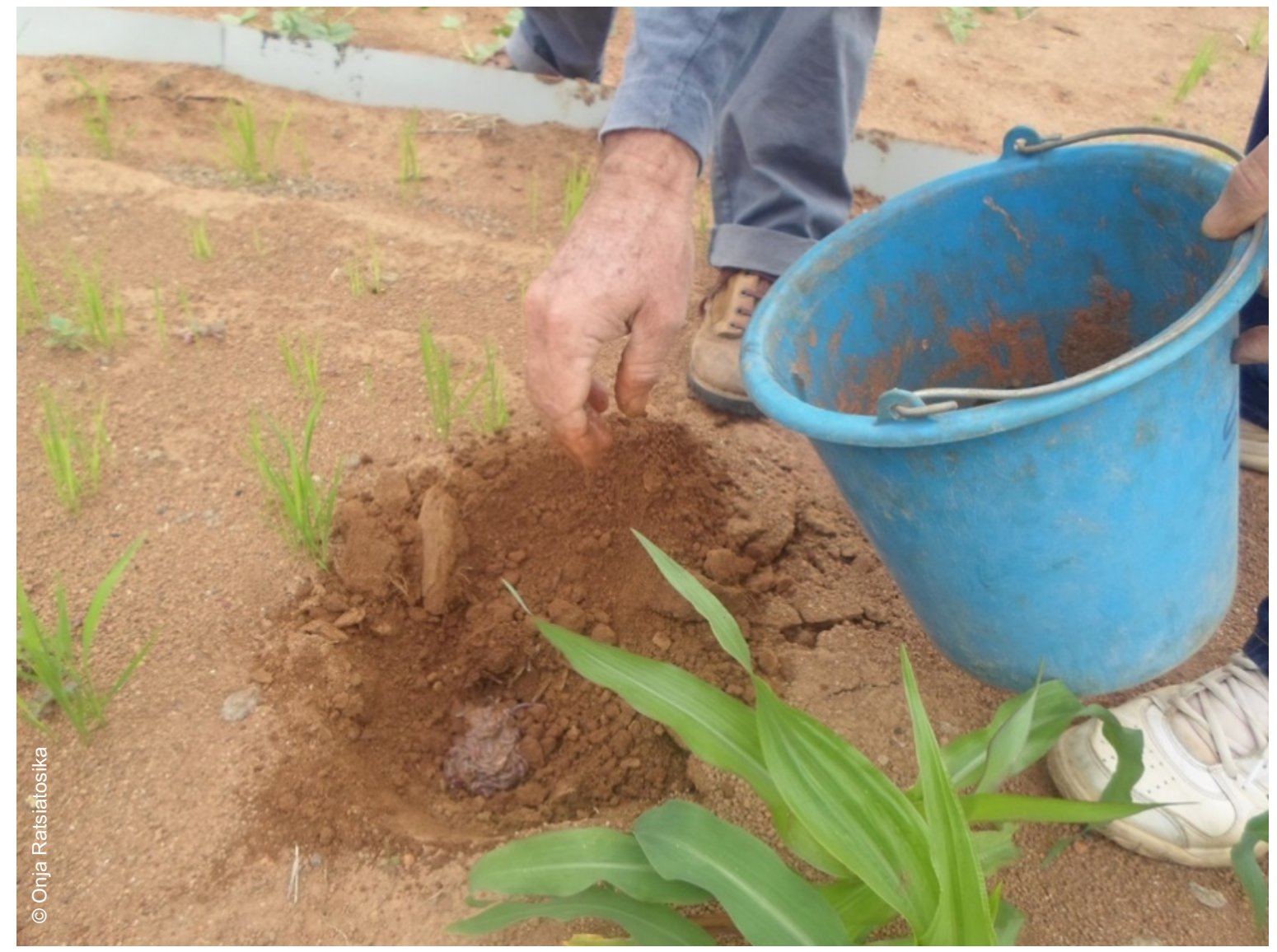

Photo 24. Inoculation of Pontoscolex corethrurus in cereal crops in Madagascar.

Handfuls of earthworms were spread at different places in the plot (one handful per m2).

Table 90. Related cases studies available in volumes 3 and 5

\begin{tabular}{|l|c|c|c|c|}
\hline Title & Region & $\begin{array}{l}\text { Duration } \\
\text { of study } \\
\text { (Years) }\end{array}$ & Volume & $\begin{array}{l}\text { Case- } \\
\text { study No. }\end{array}$ \\
\hline $\begin{array}{l}\text { Agricultural practices for the restoration of Soil } \\
\text { Ecological Functions in Madagascar }\end{array}$ & Africa & 2 & 3 & 2 \\
\hline
\end{tabular}




\section{References}

Baker, G.H., Brown, G., Butt, K., Curry, J.P. \& Scullion, J. 2006. Introduced earthworms in agricultural and reclaimed land: their ecology and influences on soil properties, plant production and other soil biota. Biological Invasions, 8(6): 1301-1316. https://doi.org/10.1007/s10530-006-9024-6

Bertrand M., BarotS., Blouin M., Whalen J., de Oliveira T. \& Roger-Estrade J. 2015. Earthworm services for cropping systems. A review. Agron. Sustain. Dev., 35: 553-567.

https://doi.org/10.1007/s13593-014-0269-7

Blanchart E., Albrecht A., Alegre J., Duboisset A., Villenave C., Pashanasi B., Lavelle P. \& Brussaard L. 1999. Effects of earthworms on soil structure and physical properties. In Lavelle P., Brussaard L. \& Hendrix P. (Eds) Earthworm management in tropical agroecosystems. CABI Publishing, 149-172.

Blanchart E., Albrecht A., Brown G.G., Decaëns T., Duboisset A., Lavelle P., Mariani L. \& Roose E. 2004. Effects of tropical endogeic earthworms on soil erosion: a review. Agriculture, Ecosystems and Environment, 104(2): 303-315. https://doi.org/10.1016/j.agee.2004.01.031

Blanchart E., Ratsiatosika O., Raveloson H., Razafimbelo T., Razafindrakoto M., Sester M., Becquer T., Bernard L. \& Trap J. 2020. Nitrogen supply reduces the earthworm-silicon control on rice blast disease in a Ferralsol. Applied Soil Ecology, 145: 103341. https://doi.org/10.1016/j.apsoil.2019.08.003

Brown G.G., Pashanasi B., Villenave C., Patron J.C., Senapati B.K., Giri S., Barois I., Lavelle P., Blanchart E., Blakemore R.J., Spain A.V. \& Boyer J. 1999. Effects of earthworms on plant production in the tropics. In Lavelle P., Brussaard L. \& Hendrix P. (Eds) Earthworm management in tropical agroecosystems. CABI Publishing, 87-148.

Brown G.G., Barois I. \& Lavelle P. 2000. Regulation of soil organic matter dynamics and microbial activity in the drilosphere and the role of interactions with other edaphic functional domains. European Journal of Soil Biology, 36: 177-198. https://doi.org/10.1016/S1164-5563(00)01062-1

Brun J.J., Cluzeau D., Tréhen P. \& Bouché M.B. 1987. Biostimulation : perspectives et limites de l'amélioration biologique des sols par stimulation ou introduction d'espèces lombriciennes. Revue d'Écologie et Biologie des Sols, 24 : 685-701.

Butt K.R., Frederickson J. \& Morris R.M. 1997. The earthworm inoculation unit technique: an integrated system for cultivation and soil inoculation of earthworms. Soil Biology and Biochemistry, 29: 251-257. https://doi.org/10.1016/S0038-0717(96)00053-3

Chan K.Y. 2001. An overview of some tillage impacts on earthworm population abundance and diversity Implications for functioning in soils. Soil and Tillage Research, 57: 179-191.

https://doi.org/10.1016/S0167-1987(00)00173-2

Forey E., Chauvat M., Coulibaly S.F.M., Langlois E., Barot S. \& Clause J. 2018. Inoculation of an ecosystem engineer (Earthworm: Lumbricus terrestris) during experimental grassland restoration: Consequences for above and belowground soil compartments. Applied Soil Ecology, 125: 148-155. https://doi.org/10.1016/j.apsoil.2017.12.021 
Gilot, C. 1997. Effects of a tropical geophageous earthworm, M. anomala (Megascolecidae), on soil characteristics and production of a yam crop in Ivory Coast. Soil Biology and Biochemistry, 29(3): 353-359. https://doi.org/10.1016/S0038-0717(96)00046-6

Hendrix P.F. 2006. Biological invasions belowground: Earthworms as invasive species. Springer, Dordrecht, The Netherlands, 129 pp.

Jouquet P., Blanchart E. \& Capowiez Y. 2014. Utilization of earthworms and termites for the restoration of ecosystem functioning. Applied Soil Ecology, 73: 34-40. https://doi.org/10.1016/j.apsoil.2013.08.004

Lavelle P., Barros E., Blanchart E., Brown G.G., Desjardins T., Mariani L. \& Rossi J.P. 2001. SOM management in the tropics: why feeding the soil macrofauna? Nutrients Cycling in Agroecosystems, 61: 53-61. https://doi.org/10.1023/A:1013368715742

Lavelle P., Decaëns T., Aubert M., Barot S., Blouin M., Bureau F., Margerie F., Mora P. \& Rossi J.P. 2006. Soil invertebrates and ecosystem services. European Journal of Soil Biology, 42: S3-S15. https://doi.org/10.1016/j.ejsobi.2006.10.002

Lavelle P., Spain A., Fonte S., Bedano J.C., Blanchart E., Galindo V., Grimaldi M., Jimenez J.J., VelasquezE. \& Zangerlé A. 2020. Soil aggregation, ecosystem engineers and the C cycle. Acta Oecologica, 105: 103561. https://doi.org/10.1016/j.actao.2020.103561

Ortiz, B., Fragoso, C., M'Boukou, I., Pashanasi, B., Senapati, B.K. \& Contreras, A. 1999 Perception and use of earthworms in tropical farming systems. In P. Lavelle, L. Brussaard \& P. Hendrix (Eds.) Earthworm management in tropical agroecosystems. CABI Publishing, 239-287.

Ratsiatosika, 0. 2018. Vers de terre et services écosystémiques en riziculture pluviale à Madagascar. Connaissances des processus et propositions d'innovations agricoles. University of Antananarivo. $\mathrm{PhD}$ dissertation.

Senapati B., Lavelle P., Giri S., Pashanasi B., Alegre J., Decaëns T., Jimenez J.J., Albrecht A., Blanchart E., Mahieu M., Rousseaux L., Thomas R., Panigrahi P.K. \& Venkatachalam M. 1999. In-soil earthworm technologies for tropical agroecosystems. In P. Lavelle, L. Brussaard \& P. Hendrix (Eds.) Earthworm management in tropical agroecosystems. CABI Publishing, 199-238.

Spain, A.V., Lavelle, P. \& Mariotti, A. 1992. Stimulation of plant growth by tropical earthworms. Soil Biology and Biochemistry, 24(12): 1629-1633. https://doi.org/10.1016/0038-0717(92)90161-P

Van Groenigen J.W., Lubbers I.M., Vos H.M.J., Brown G.G., De Deyn G.B., van Groenigen K.J. 2014. Earthworm increase plant production: a meta-analysis. Scientific Reports, 4: 6365.

https://doi.org/10.1038/srep06365

Van Groenigen J.W., van Groenigen K.J., Koopmans G.F., Stokkermans L., Vos H.M.J. \& Lubbers I.M. 2019. How fertile are earthworm casts? A meta-analysis. Geoderma, 338: 525-535.

https://doi.org/10.1016/j.geoderma.2018.11.001

Villenave C., Charpentier F., Lavelle P., Feller C., Brussaard L., Pashanasi B., Barois I., Albrecht A. \& Patron J.C. 1999. Effects of earthworms on soil organic matter and nutrient dynamics following earthworm inoculation in field experimental situations. In P. Lavelle, L. Brussaard \& P. Hendrix (Eds.) Earthworm management in tropical agroecosystems. CABI Publishing, 173-197. 
Wubs E.R.J., van der Putten W.H., Bosch M. \& Bezemer T.M. 2016. Soil inoculation steers restoration of terrestrial ecosystems. Nature Plants, 2. https://doi.org/10.1038/nplants.2016.107

Zhang W., Hendrix P.F., Dame L.E., Burke R.A., Wu J., Neher D. A., Li J., Shao Y. \& Fu S. 2013.

Earthworms facilitate carbon sequestration through unequal amplification of carbon stabilization compared with mineralization. Nature Communications, 4: 2576. https://doi.org/10.1038/ncomms3576 


\title{
20. Dung burial by beetles
}

\author{
Bernard Doube', Agasthya Thotagamuwa², Loene Doube ${ }^{1}$ \\ ${ }^{1}$ Dung Beetle Solutions International, Bridgewater, Australia \\ ${ }^{2}$ Graham Centre for Agricultural Innovation, Charles Sturt University, Bathurst, Australia
}

\section{Description of the practice}

The practice is the selection and introduction of dung beetles to beetle-depauperate regions in order to bury the dung of domestic stock and so increase levels of soil organic carbon.

This practice comprises identifying global regions with depauperate livestock dung beetle communities, identifying appropriate donor species, field collecting them, mass rearing them in quarantine facilities, releasing their progeny to dung masses in the field in target regions, monitoring their establishment and then field cropping and redistributing beetles once the field populations become abundant (100s per dung mass). Once a species is established in a suitable environment, no further introductions are required.

Dung burial by beetles provides numerous ecosystem services (including increasing soil organic carbon (SOC) levels) but pastures and rangelands in many world regions have low species diversity and a consequent minimal capacity to dispose of domestic stock dung, while other regions have dung beetle communities with high species diversity and a correspondingly higher capacity for disposal of domestic stock dung. In order to improve global dung burial, over 100 dung beetle species have been introduced to countries around the world (Hanski and Cambefort, 1991). Despite this, there is ample opportunity for further redistribution of these and additional species (Ridsdill-Smith and Edwards, 2011; Doube, 2018). The practice involves:

- identifying climatic, seasonal and geographic gaps in dung burial in beetle-depauperate regions;

- climate matching to identify suitable donor regions in countries with high species diversity;

- selection of species in donor regions to fill recognized gaps in receptor countries;

- acquiring agreement to export beetles from donor countries in compliance with the Nagoya Protocol $;{ }^{17}$

- acquiring permission to import and release beetles in receptor countries;

\footnotetext{
${ }^{17}$ The Nagoya Protocol (Secretariat of the Convention on Biological Diversity, 2011) has imposed an additional level of complexity on sourcing beetles from donor countries, which are, based on the protocol, entitled to share the economic benefits that arise from the use of the genetic resources in the recipient country.
} 
- field collection and laboratory rearing of target species;

- importing and rearing beetles in quarantine laboratories;

- mass rearing in laboratory or field nurseries;

- targeted release of mass-reared beetles;

- monitoring establishment and population increases; and

- field cropping and redistribution of beetles to other suitable regions.

\section{Range of applicability}

Dung burial by beetles has the capacity to increase levels of soil carbon in agricultural soils in tropical, subtropical, even-rainfall and temperate regions of the world (Section 3). Here we briefly consider the dung beetle communities in geographic regions that lack effective beetles and regions from which useful species might be sourced for introduction to depauperate communities.

Ball-rolling (telecoprid) and dung-burying (paracoprid) dung beetles (Figure 10) bury dung at depths that range from a few $\mathrm{cm}$ to $100+\mathrm{cm}$. These functional groups are found in a wide range of habitats, have specialized dung type preferences and are most abundant and effective in temperate, subtropical and tropical regions (Doube, 1991; Hanski and Cambefort, 1991; Davis, 1996). Large beetles commonly bury dung at a greater depth than do smaller beetles (Doube and Marshall, 2014). Here we focus on the larger species in these two functional groups that have a well-developed capacity to bury cattle dung in grassland and rangeland. Such species are well placed to promote SOC storage in soils through their dung burial activity, with large pads commonly being completely buried within a few days of production (Edwards and Aschenborn, 1987; Doube and Marshall, 2014), yet these larger species have suffered a wave of recent extinctions. 


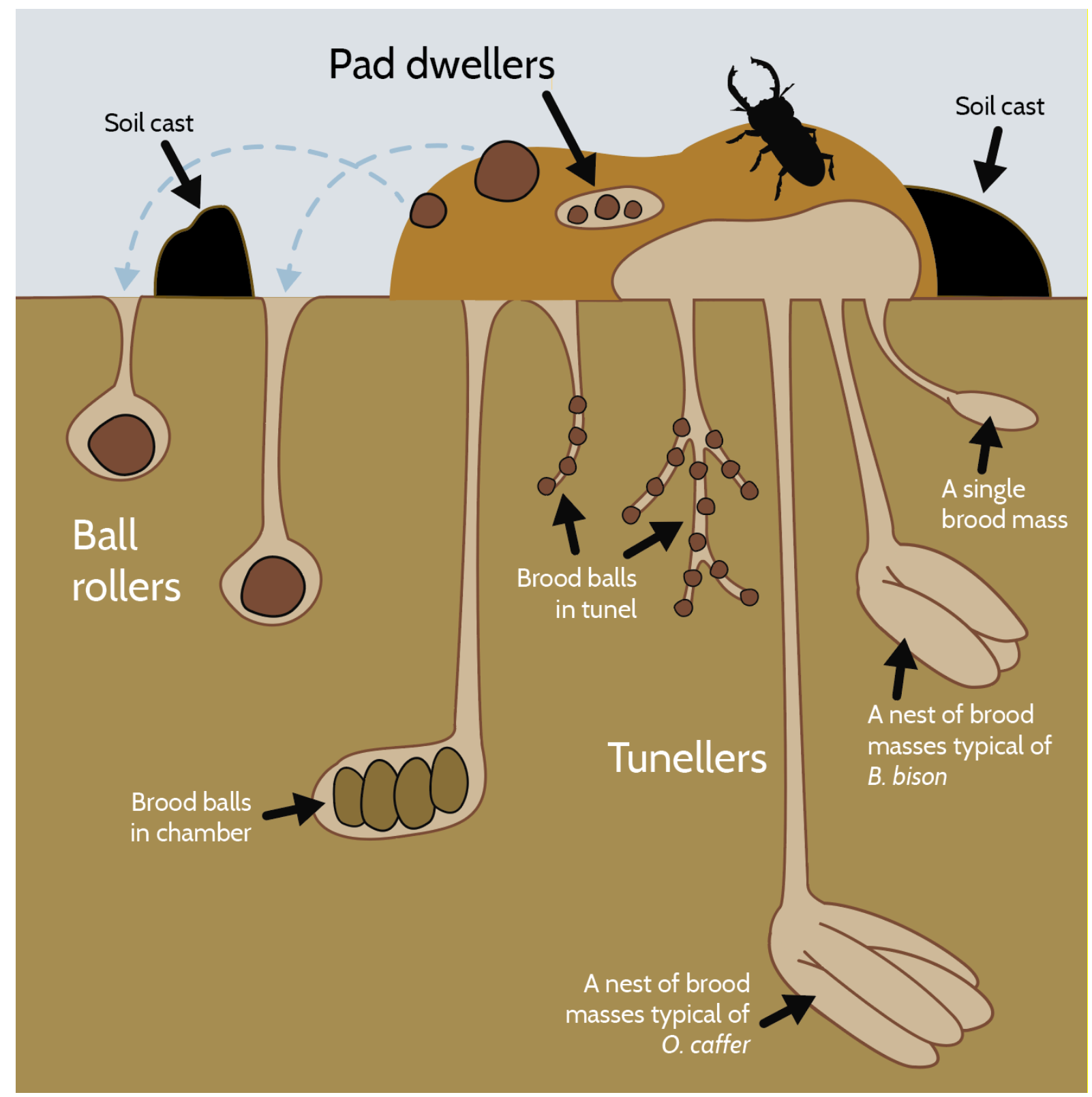

Figure 10. A functional analysis of dung burial by beetles (Adapted from Doube, 1990)

The global wave of megaherbivore extinctions during the early Holocene (Gill, 2014) is considered to have induced a corresponding extinction of many large megafauna-adapted dung beetles (Doube, 2018). Regions from which megaherbivores were exterminated (for example, Australia, the Americas, New Zealand) now have a low diversity of native beetles adapted to cattle dung (e.g. Monteith, 2015; Kadiri, Lumaret and Floate, 2014; Escobar, Halffter and Arellano, 2007; Forgie, Dymock and Tompkins, 2014) but have large populations of domestic herbivores and so present an opportunity for substantial gains in soil carbon storage if appropriate dung beetle species can be sourced and introduced. Worldwide there are about $8000 \mathrm{dung}$ beetle species but only a small number of these (perhaps 200 or so) are candidates for the applied function of burying the dung of domestic herbivores in managed grasslands. 
The most promising sources of pasture-adapted beetles to fill gaps in beetle activity are the adjacent regions of southern Europe, North Africa and the Middle East for Mediterranean climate-adapted species (for example, Lumaret and Lobo (1996) listed 547 West Palearctic species), and the tropical and subtropical regions of Africa for tropical and subtropical species, as illustrated in the appendices of Hanski and Cambefort (1991), where the environmental associations of over 800 dung beetle species are detailed.

Species niche characterization and the introduction of appropriate species to depauperate regions have been in progress for half a century, with a selection of species introduced to grasslands in Australia, Hawaii, the Americas, New Zealand and other regions (Edwards, 2007; Forgie, Dymock and Tompkins, 2014), but many promising geographic and seasonal gaps (spring, summer, autumn and winter) remain unfilled. Defining activity gaps in receptor regions and candidate species in donor regions needs to take account of climatic associations, since different species thrive in tropical, subtropical, temperate and cool temperate grasslands. Superimposed upon this are dung beetles' biophysical barriers to successful breeding (e.g. soil type preferences, also see Volume 4, case-study No.7 "Selection and introduction of dung beetles to beetle-depauperate regions in southern Australia").

\section{Impact on soil organic carbon stocks}

The published data on the effects of dung beetle activity on soil carbon levels (for example, Menendez, Webb and Orwin, 2016; Evans et al., 2019) have primarily concerned endocoprid species (which do not bury dung, Photo 25) or shallow-tunnelling species, and indicate that such species transfer only small amounts of the dung carbon into the soil (often as dissolved organic carbon (DOC)) and do little to increase persistent carbon stores in soil (Menendez, Webb and Orwin, 2016; Evans et al, 2019). In marked contrast, deep-tunnelling dung beetles bury substantial amounts of dung (Edwards and Aschenborn, 1987; Doube, 1991) but, apart from Bubas bison, none has had its capacity to increase SOC levels assessed quantitatively. However, it is to be expected that such burial produces soil carbon results similar to those of B. bison as detailed in case study No.7 (Volume 4, this manual).

Numerous publications consider the way in which dung burial transfers organic matter into the soil, and the speed and amounts buried are described as well as the depth at which the dung is lodged (e.g. Edwards and Aschenborn, 1987; Doube, 1991), but rarely do they consider the carbon content of the buried dung and none except Doube (2008) and Doube and Dale (2012) measure the amounts of carbon lodged in the soil by deeptunnelling beetles. Both studies (in a warm temperate dry Intergovernmental Panel on Climate Change (IPCC) climate zone in South Australia) demonstrated the capacity of B. bison to increase levels of carbon in pasture soils in the short (10 months) (Doube and Dale, 2012, Table 91) and the medium term (2 years) (Doube, 2008).

Further analysis of the short- and long-term impact of dung burial on SOC dynamics in different soil types in different climates in different seasons with a variety of dung beetle species is needed. 
Table 91. Evolution of SOC stocks after dung beetle burial

\begin{tabular}{|l|l|l|l|l|l|l|l|}
\hline Location & Climate zone & Soil type & $\begin{array}{l}\text { Baseline } \\
\mathrm{C} \text { stock } \\
\mathrm{(tC/ha)}\end{array}$ & $\begin{array}{l}\text { Additional C } \\
\text { storage } \\
(\mathrm{tC} / \mathrm{ha} / \mathrm{yr})\end{array}$ & Duration & $\begin{array}{l}\text { Depth } \\
(\mathrm{cm})\end{array}$ & Reference \\
\hline $\begin{array}{l}\text { South } \\
\text { Australia }\end{array}$ & $\begin{array}{l}\text { Warm } \\
\text { Temperate Dry }\end{array}$ & $\begin{array}{l}\text { Brown } \\
\text { Kurasol }^{18}\end{array}$ & 30.5 & 0.18 & 10 & $0-50$ & Doube and \\
& & 0.17 & months & $25-50$ & Dale (2012) \\
\hline
\end{tabular}

Depending upon species, dung beetles bury dung at depths ranging from a few centimeters to a meter or more, but, in pastures and rangelands, potential dung burial is confined largely to the top $50 \mathrm{~cm}$ of the soil profile. New dung beetle species can be selected so that buried dung is located throughout the soil profile; such species have the potential to transform the structure and fertility of surface soil in pastoral environments that previously lacked them. Soils in these environments have a substantial capacity to absorb and retain higher levels of SOM (Jia et al., 2019; Hoyle et al., 2013).

The fresh dung of herbivorous mammals is commonly $40-85$ percent water by weight and comprises a microbial soup mixed with partially digested plant fiber, commonly containing about 50 percent carbon. Dung beetle activity has the potential to influence the carbon dynamics both in unburied dung remains and, importantly, in association with buried dung.

Below-ground dung carbon dynamics have been considered by numerous authors in the absence and the presence of dung beetle activity. Without dung beetles, there is little movement of insoluble carbon materials and minor transfer of DOC and soluble plant nutrients into the soil: nearly all the dung carbon is lost to the atmosphere following microbial digestion (Yoshitake, Soutome and Koizumi, 2014).

The role of tunnelling dung beetles in elevating SOC levels is best appreciated in terms of their capacity to improve soil structure and water infiltration, aerate soils and increase levels of subsoil organic matter (dung and plant roots) (Doube and Marshall, 2014). One example of this is given in Table 92.

${ }^{18}$ The closest to this soil type in the UDSA Soil Taxonomy system are the Inceptisols (Hughes et al, 2018). 
Table 92. A carbon budget for soil cores (gC per soil core section) (about $40 \mathrm{~cm}$ deep) demonstrating the effects of burial of cattle dung by B. bison on mean soil carbon levels $(\mathrm{g} \pm \mathrm{SD}$ ) after 10 months in the field in a warm temperate dry IPCC climate zone in southern Australia

\begin{tabular}{|l|l|l|l|}
\hline Soil core section & Dung + beetles & Dung only & $\begin{array}{l}\text { Controls } \\
\text { (no dung) }\end{array}$ \\
\hline Surface litter & $43.6 \pm 18.6$ & $50.6 \pm 6.3$ & $3.0 \pm 1.1$ \\
\hline Upper section & $111.4 \pm 26.2$ & $91.6 \pm 20.3$ & $75.6 \pm 12.4$ \\
\hline Basal section & $78.2 \pm 34.0$ & $58.4 \pm 25.0$ & $74.2 \pm 15.3$ \\
\hline Tunnels + contents & $37.7 \pm 13.0$ & & \\
\hline Total per core & $270.8 \pm 21.6$ & $200.6 \pm 25.3$ & $152.9 \pm 19.9$ \\
\hline $\begin{array}{l}\text { Mean } \pm \text { SD dry weight of soil cores } \\
(\mathrm{kg})\end{array}$ & $28.8 \pm 3.5$ & $25.2 \pm 2.8$ & $23.6 \pm 2.9$ \\
\hline
\end{tabular}

The potential for soil carbon sequestration and storage varies considerably depending on prior and current land management approaches, soil type, resource availability, environmental conditions, microbial composition and nutrient availability, among other factors (Jia et al., 2019). Here we suggest that deep-tunnelling dung beetles offer a new and potentially substantial avenue for increasing global SOC storage in pasture and rangeland soils.

Globally, if 10 percent of the excreta of the world's 1.3 billion cattle could be subject to a dung burial regime similar to that of the two Bubas species in the Australian case, a total of about $290 \mathrm{MtCO}_{2} \mathrm{eq}$ might be sequestered annually.

\section{Other benefits of the practice}

\subsection{Improvement in soil properties}

The improvements in soil properties brought about by dung burial by dung beetles are summarized in Nichols et al. (2008), Ridsdill-Smith and Edwards (2011), Doube and Marshall (2014) and Doube (2018). Dung burial by beetles generates tunnels into the soil (1-200 cm deep) and deposits substantial amounts of organic matter through the soil profile, dramatically improving the physical, chemical and biological properties of the soil. The physical benefits include improved soil aeration, reduced bulk density and improved water infiltration. The chemical benefits include increased cation exchange capacity, improved soil $\mathrm{pH}$ and increased plant nutrients and carbon throughout the soil profile. The biological benefits include increased microbial activity, increased plant root growth and more earthworms. 


\subsection{Minimization of threats to soil functions}

Table 93. Soil threats

\section{Soil threats}

\begin{tabular}{|l|l|}
$\begin{array}{l}\text { Nutrient imbalance and } \\
\text { cycles }\end{array}$ & Dung burial adds nutrients to the soil (Doube, 2008). \\
\hline Soil acidification & Dung burial reduces soil acidification (Doube, 2008). \\
\hline Soil compaction & Dung burial decreases soil bulk density (Doube, 2018). \\
\hline Soil water management & $\begin{array}{l}\text { Dung burial removes pollutants from the soil surface, preventing } \\
\text { them from running into waterways (Doube and Marshall, 2014). }\end{array}$ \\
\hline
\end{tabular}

\subsection{Increases in production (e.g. food/fuel/feed/timber/fibre)}

Dung burial by deep-tunnelling dung beetles can increase pasture production by $20+$ percent (Doube, 2018).

\subsection{Mitigation of and adaptation to climate change}

Fresh dung can produce small amounts of nitrous oxide but aeration of the dung due to beetle activity can increase that amount while decreasing methane production (Penttila et al., 2013). Methane production occurs in anaerobic environments (such as dung) and so aeration of dung due to beetle activity reduces methane production.

\subsection{Socioeconomic benefits}

Dung disruption by introduced dung beetles during summer has dramatically reduced the numbers of pestilent bush flies in the moister regions of southern Australia (Ridsdill-Smith and Edwards, 2011). The bush fly is still a serious impediment to tourist enjoyment in arid and semi-arid regions and is a mechanical vector for the human eye disease trachoma, and pink-eye in cattle (Doube and Marshall, 2014). 


\section{Potential drawbacks to the practice}

\subsection{Tradeoffs with other threats to soil functions}

There has been little detailed analysis of the potential of introduced dung beetle species to damage local biodiversity. However, abundant populations of introduced species in Australian grasslands have been shown to decrease the abundance of native and other introduced species (Ridsdill-Smith and Edwards, 2011) but there is no evidence of local extinctions and it is likely that the abundance of native grassland species that use cattle dung was promoted by the introduction of this abundant resource. The introduced species are adapted to grassland, not bushland, and so native bushland species are largely unaffected by introduced grassland species (Doube and Marshall, 2014). The tunnels generated by dung beetle activity provide additional architectural diversity in soils, generating new habitats for soil organisms. This is not a drawback.

\subsection{Increases in greenhouse gas emissions}

Above-ground dung greenhouse gas (GHG) dynamics $\left(\mathrm{CO}_{2}+\mathrm{CH}_{4}+\mathrm{N}_{2} \mathrm{O}\right.$ emissions) have been reviewed by Fowler et al. (2020) (11 papers considered). They concluded that there was no evidence for ultimate $\mathrm{CO}_{2} \mathrm{e}$ reduction due to dung beetle activity. As dung beetle activity increases aeration of dung, anaerobic $\mathrm{N}_{2} \mathrm{O}$ production decreases while aerobic $\mathrm{CO}_{2}$ and $\mathrm{CH}_{4}$ production increases, neutralizing their joint impact (Penttila etal., 2013).

\subsection{Conflict with other practice(s)}

There is a distinct conflict with chemical control of intestinal parasites of livestock because many veterinary chemicals kill dung beetles, and hence prevent their contribution to soil carbon sequestration. Changing stock management practices are reducing the dung available to dung beetles. For example, abandoning the grazing of pasturelands in favour of feeding cut forage to shedded cattle denies beetles an important food source (Tonelli, Verdu and Zunino, 2018) and naturally the level of dung-derived soil carbon sequestered by beetles is also reduced.

\section{Recommendations before implementing the practice}

Likely donor and receptor dung beetle exchanges need to be identified. Many pastures around the world lack year-round dung beetle activity. Additional deep-tunnelling dung beetles, once abundant, could dramatically increase the amount of carbon sequestered in the soil in these regions. 
The authors recommend that potential donor dung beetle species (with an initial focus on Bubas and Onitis species) be sought in the adjacent regions of southern Europe, North Africa and the Middle East for Mediterranean climate-adapted species, and in the tropical and subtropical regions of Africa for tropical and subtropical species. The authors recommend a corresponding definition of gaps in dung beetle activity in regions with depauperate cattle pasture species (potential receptor countries). Linking these two activities can produce a list of appropriate potential donor dung beetle species for target areas including Australia, North and South America, and Mediterranean South Africa.

\section{Potential barriers for adoption}

Relatively few references exist to evidence of barriers for adoption of the practice. The information gathered in the below table is mainly conclusions made thanks to the authors' personal experiences.

Table 94. Potential barriers to adoption

\begin{tabular}{|l|l|l|}
\hline Barrier & YES/NO & \\
\hline Biophysical & Yes & $\begin{array}{l}\text { Soil type is a major determinant of the local abundance of many dung } \\
\text { beetle species. }\end{array}$ \\
\hline Cultural & Yes & $\begin{array}{l}\text { The difficulty in overcoming entrenched attitudes among landholders } \\
\text { restricts the acceptance of novel ideas such as the use of dung beetles to } \\
\text { increase levels of soil carbon. }\end{array}$ \\
\hline Social & Yes & $\begin{array}{l}\text { The economic benefits of introduced dung beetles need to be } \\
\text { demonstrated in a range of grazing environments that reflect the capacity } \\
\text { of local conditions to support the introduction of dung beetles and to } \\
\text { generate their benefits. } \\
\text { The capacity to identify sources of beetles for introduction and to } \\
\text { understand how to breed them in captivity requires considerable } \\
\text { investment. }\end{array}$ \\
\hline Institutional & Yes & $\begin{array}{l}\text { Yunding is required from relevant institutions to support beetle } \\
\text { introduction programs. This can be difficult to obtain. } \\
\text { In Australia a 5-year program, Dung Beetle Ecosystem Engineers, has } \\
\text { been funded through the Australian Government Rural Research and } \\
\text { Development for Profit Program. }\end{array}$ \\
\hline & Yes \\
\hline
\end{tabular}

${ }^{19}$ See also https://www.agriculture.gov.au/ag-farm-food/innovation/rural-research-development-forprofit/approved-projects-round3. 


\begin{tabular}{|l|l|l|}
\hline Barrier & YES/NO & \\
\hline $\begin{array}{l}\text { Legal (Right } \\
\text { to soil) }\end{array}$ & Yes & $\begin{array}{l}\text { The Nagoya Protocol (Secretariat of the Convention on Biological } \\
\text { Diversity, 2011) makes it difficult to relocate dung beetle species from one } \\
\text { country to another and also restricts the ability to relocate them within } \\
\text { countries. }\end{array}$ \\
\hline Knowledge & Yes & $\begin{array}{l}\text { Lack of knowledge on the part of landholders can be a barrier. Education } \\
\text { needs to be included in dung beetle introduction projects. } \\
\text { Many dung beetle species have complicated life histories and so learning } \\
\text { how to captive-rear species is a major hurdle. }\end{array}$ \\
\hline
\end{tabular}

\section{Photos of the practice}

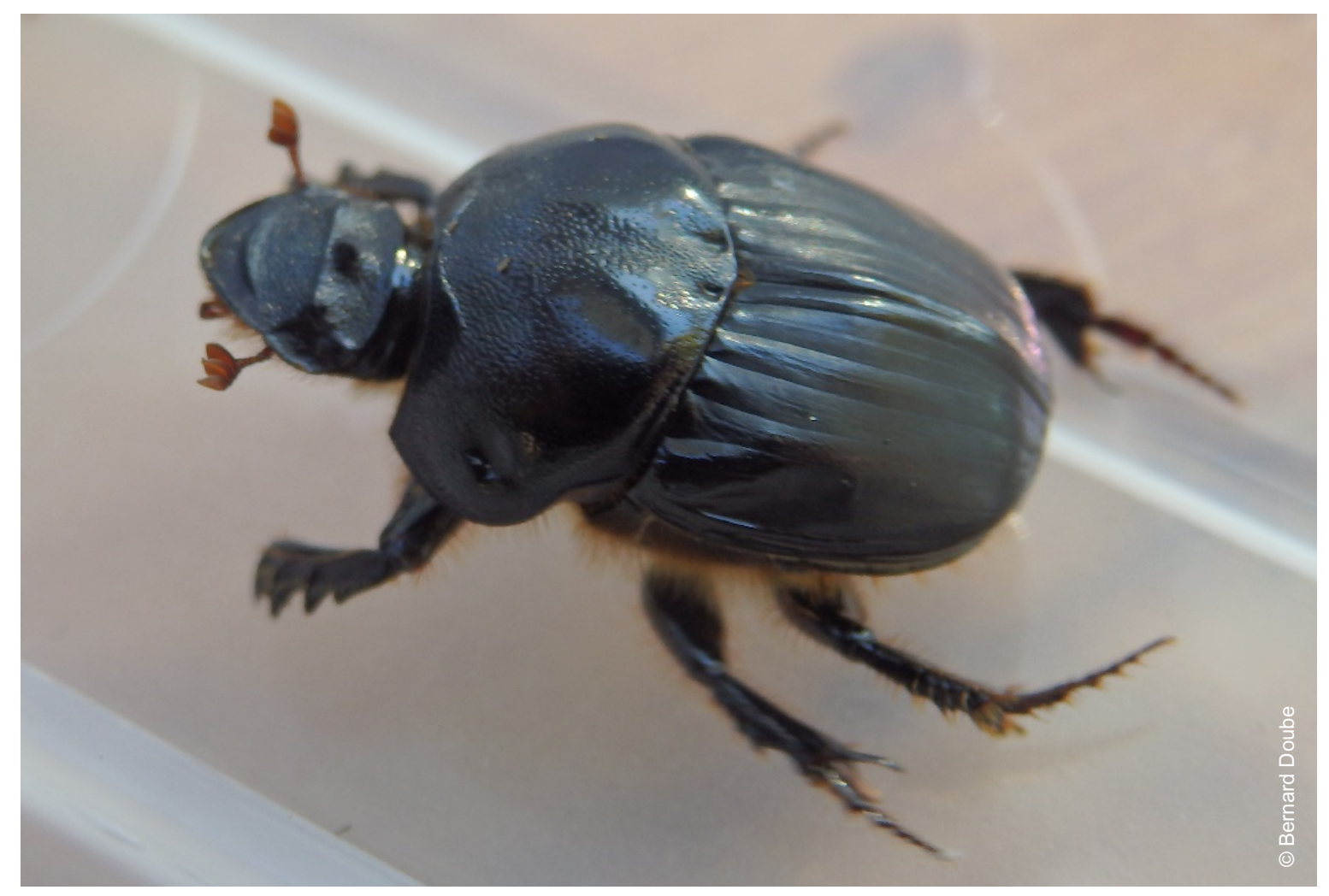

Photo 25. Bubas bison 

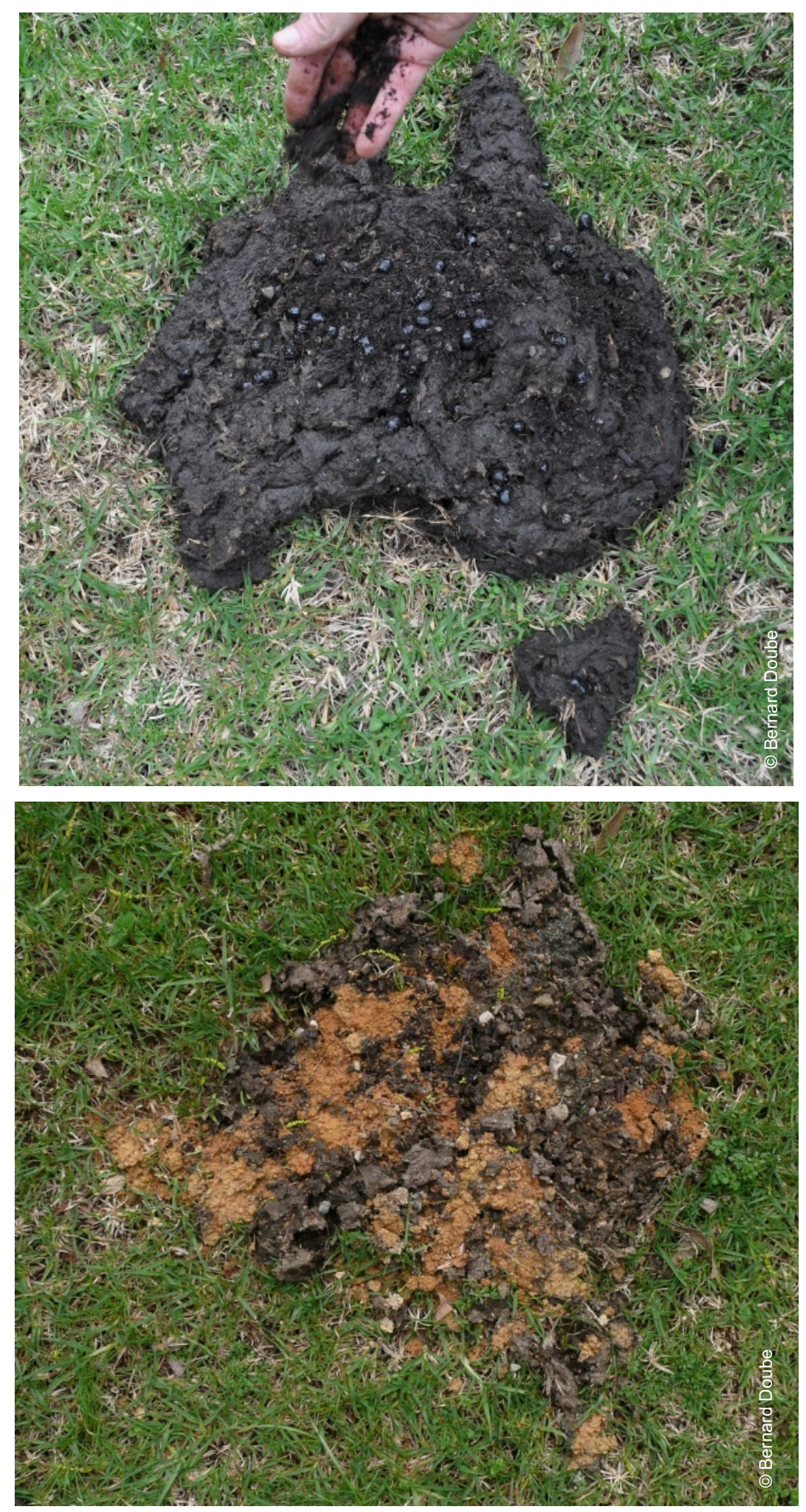

Photo 26. B. bison released onto Australia (top) and three weeks later (bottom). 

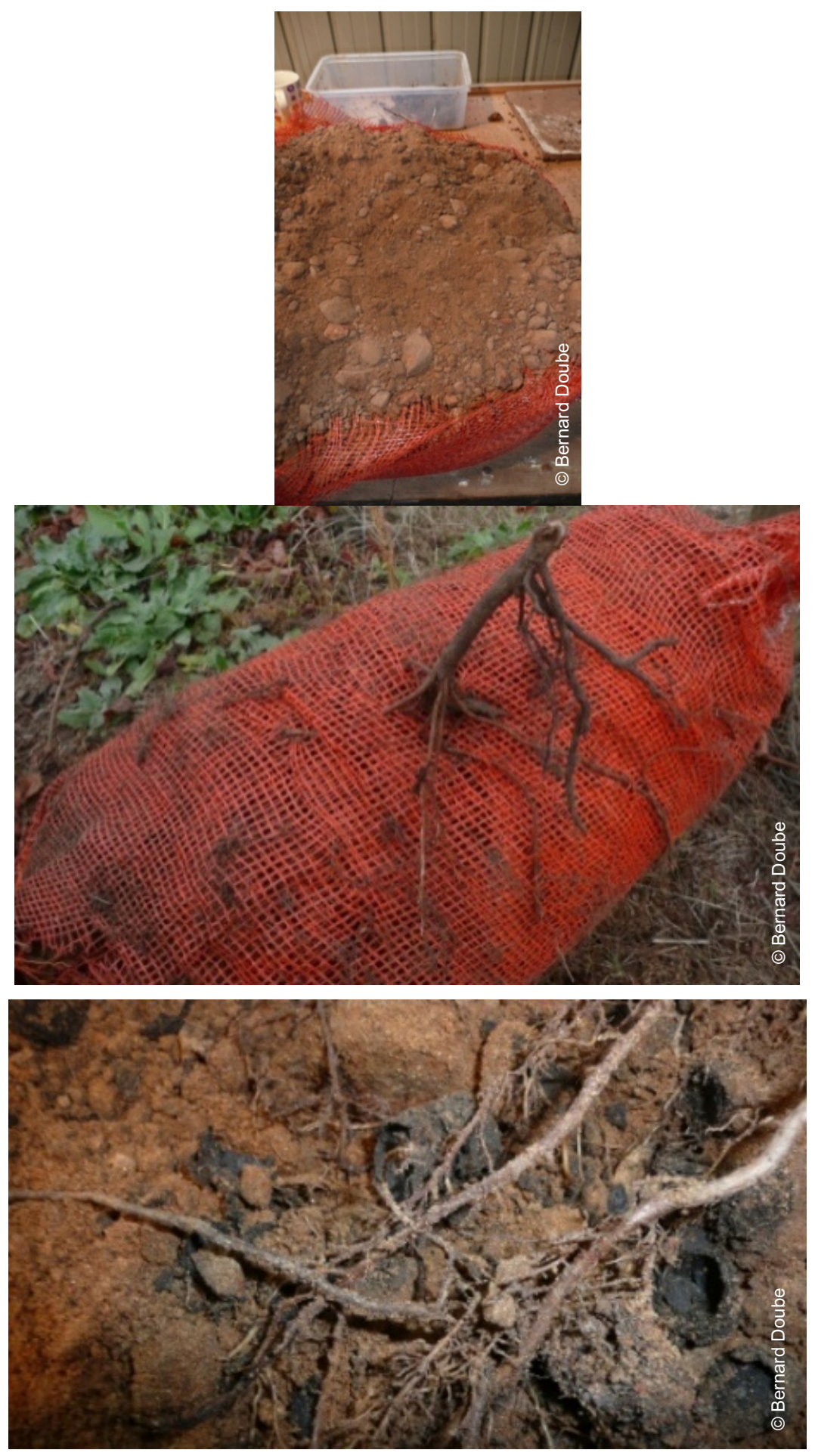

Photo 27. Soil from $25-50 \mathrm{~cm}$ deep without dung beetles (top), soil core with dung beetles showing vine roots entering soil core (after 10 months) (middle) and soil from 25-50 cm deep showing vine roots and buried dung remains (after 10 months (bottom). 


\section{References}

Davis, A.L.V. 1996. Seasonal dung beetle activity and dung dispersal in selected South African habitats: implications for pasture improvement in Australia. Agriculture, Ecosystems \& Environment, 58(2-3): 157169. https://doi.org/10.1016/0167-8809(96)01030-4.

Doube, B.M. \& Dale, M. 2012. Reconstructing vineyard soils with dung beetles: final report. Barossa Valley, Barossa Grape and Wine Association \& Eden Valley Wine Grape Growers Group.

Doube, B.M. 1990. A functional classification analysis of the structure of dung beetle assemblages. Ecological Entomology, 15(4): 371-383. https://doi.org/10.1111/j.1365-2311.1990.tb00820.x.

Doube, B.M. 1991. Dung beetles of Southern Africa. In I. Hanski \& Y. Cambefort (Eds.) Dung beetle ecology, pp. 133-155. Princeton, New Jersey, Princeton University Press.

Doube, B.M. 2008. The pasture growth and environmental benefits of dung beetles to the southern Australian cattle industry: final report for Project B.ERM.0211. North Sydney, NSW, Meat \& Livestock Australia. https://www.mla.com.au/research-and-development/search-rd-reports/final-report-details/ProductivityOn-Farm/Pasture-growth-and-environmental-benefits-of-dung-beetles-to-the-southern-Australian-cattleindustry/587\#.

Doube, B.M. 2018. Ecosystem services provided by dung beetles in Australia. Basic and Applied Ecology, 26: 35-49. https://doi.org/10.1016/j.baae.2017.09.008.

Doube, B.M., \& Marshall, T. 2014. Dung down under: dung beetles for Australia. Adelaide, Dung Beetle Solutions Australia.

Edwards, P.B. \& Aschenborn, H.H. 1987. Patterns of nesting and dung burial in Onitis dung beetles: implications for pasture productivity and fly control. Journal of Applied Ecology, 24(3): 837-851. https://www.jstor.org/stable/2403984.

Edwards, P.B. 2007. Introduced dung beetles in Australia 1967-2007: current status and future directions. Maleny, Dung Beetles for Landcare Farming Committee.

Escobar, F., Halffter, G. \& Arellano, L. 2007. From forest to pasture: an evaluation of the influence of environment and biogeography on the structure of dung beetle (Scarabaeinae) assemblages along three altitudinal gradients in the neotropical region. Ecography, 30(2):193-208.

https://doi.org/10.1111/j.0906-7590.2007.04818.x.

Evans, K.S., Mamo, M., Wingeyer, A., Schacht, W., Eskridge, K., Bradshaw, J. \& Ginting, D. 2019. Soil fauna accelerate dung pat decomposition and nutrient cycling into grassland soil. Rangeland Ecology \& Management, 72: 667-677. https://doi.org/10.1016/j.rama.2019.01.008.

Forgie, S.A., Dymock, J.J. \& Tompkins, D.M. 2014. No evidence that brushtail possums (Trichosurus vulpecula) forage on dung beetles (Coleoptera: Scarabaeidae) in New Zealand. New Zealand Journal of Zoology, 41(2): 95-102. https://doi.org/10.1080/03014223.2013.854815. 
Fowler, F., Denning, S., Hu, S. \& Watson, W. 2020. Carbon neutral: the failure of dung beetles (Coleoptera: Scarabaeidae) to affect dung-generated greenhouse gases in the pasture. Environmental Entomology, 49(5): 1-12. https://doi.org/10.1093/ee/nvaa094.

Gill, J.L. 2014. Ecological impacts of the late Quaternary megaherbivore extinctions. New Phytologist, 201: 1163-1169. https://doi.org/10.1111/nph.12576.

Hanski, I. \& Cambefort, Y. 1991. Species richness. In I. Hanski, \& Y. Cambefort, eds. Dung beetle ecology, pp. 350-365. Princeton, New Jersey: Princeton University Press.

Hoyle, F.C., D'Antuono, M., Overheu, T. \& Murphy, D.V. 2013. Capacity for increasing soil organic carbon stocks in dryland agricultural systems. Soil Research, 51: 657-667.

http://dx.doi.org/10.1071/SR12373.

Hughes, P., McBratney, A.B., Minasny, B., Huang, J., Micheli, E., Hempel, J. \& Jones, E. 2018. Comparisons between USDA soil taxonomy and the Australian soil classification system II: comparison of order, suborder and great group taxa. Geoderma, 322: 48-55.

https://doi.org/10.1016/j.geoderma.2018.02.022

Jia, G., Shevliakova, E., Artaxo, P., De Noblet-Ducoudré, N., Houghton, R., House, J., Kitajima, K., Lennard, C., Popp, A., Sirin, A., Sukumar, R. \& Verchot, L. 2019. Land-climate interactions. In P.R. Shukla, J. Skea, E. Calvo Buendia, V. Masson-Delmotte, H.-O. Pörtner, D.C. Roberts, P. Zhai, R. Slade, S. Connors, R. van Diemen, M. Ferrat, E. Haughey, S. Luz, S. Neogi, M. Pathak, J. Petzold, J. Portugal Pereira, P. Vyas, E. Huntley, K. Kissick, M, Belkacemi, J. Malley (Eds.) Climate change and land: an IPCC special report on climate change, desertification, land degradation, sustainable land management, food security, and greenhouse gas fluxes in terrestrial ecosystems. Geneva, Intergovernmental Panel on Climate Change.

Kadiri, N., Lumaret, J.-P. \& Floate, K.D. 2014. Functional diversity and seasonal activity of dung beetles (Coleoptera: Scarabaeoidea) on native grasslands in southern Alberta, Canada. The Canadian Entomologist, 146(3): 291-305. https://doi.org/10.4039/tce.2013.75

Lumaret, J.-P. \& Lobo, J.M. 1996. Geographic distribution of endemic dung beetles (Coleoptera, Scarabaeoidea) in the Western Palaearctic Region. Biodiversity Letters, 3(6): 192-199.

https://doi.org/10.2307/2999676

Mendenez, R., Webb, P. \& Orwin, K. 2016. Complementarity of dung beetle species with different functional behaviours influence dung-soil carbon cycling. Soil Biology and Biochemistry, 92: 142-148. https://doi.org/10.1016/j.soilbio.2015.10.004

Monteith, G. 2015. Australian native dung beetles. Entomological Society of Queensland, 43: 24-32.

Nichols, E., Spector, S., Louzada, J., Larsen, T., Amezquita, S. \& Favila, M.E. 2008. Ecological functions and ecosystem services provided by Scarabaeinae dung beetles. Biological Conservation, 141(6): 1461-1474. https://doi.org/10.1016/j.biocon.2008.04.011

Penttila, A., Slade, E.M., Simojoki, A., Riutta, T., Minkkinen, K. \& Roslin, T. 2013. Quantifying beetle-mediated effects on gas fluxes from dung pats. PLoS ONE, 8(8): e71454.

https://doi.org/10.1371/journal.pone.0071454 
Ridsdill-Smith, T.J. \& Edwards, P.B. 2011. Biological control: ecosystem functions provided by dung beetles. In L.W. Simmons \& T.J. Ridsdill-Smith (Eds.) Ecology and evolution of dung beetles, pp. 245-266. Chichester, John Wiley \& Sons. https://doi.org/10.1002/9781444342000.ch1

Secretariat of the Convention on Biological Diversity 2011. Nagoy a protocol on access to genetic resources and the fair and equitable sharing of benefits arising from their utilization to the Convention on Biological Diversity: text and annex. Montreal, United Nations Environmental Programme.

Tonelli, M., Verdú, J. \& Zunino, M. 2018. Effects of the progressive abandonment of grazing on dung beetle biodiversity: body size matters. Biodiversity and Conservation, 27(1): 189-204.

https://doi.org/10.1007/s10531-017-1428-3.

Yoshitake, S, Soutome, H. \& Koizumi, H. 2014. Deposition and decomposition of cattle dung and its impact on soil properties and plant growth in a cool-temperate pasture. Ecological Research, 29(4): 673684. https://doi.org/10.1007/s11284-014-1153-2. 


\title{
21. Liming Acidic Soils
}

\author{
Pedro Luiz Oliveira de Almeida Machado', Vinicius de Melo Benites², \\ Nanthi Bolan ${ }^{3,4}$ \\ ${ }^{1}$ Embrapa Rice and Beans, Ministry of Agriculture, Goias, Brazil \\ ${ }^{2}$ Embrapa Soils, Ministry of Agriculture, Rio de Janeiro, Brazil \\ ${ }^{3}$ Faculty of Science, University of Newcastle, NSW-2308, Australia \\ ${ }^{4}$ Cooperative Research Centre for High Performance Soils, Callaghan, Newcastle, NSW - 2308, Australia
}

\section{Description of the practice}

Soil acidity (indicated by values of $\mathrm{pH}_{\mathrm{w}}<5.5$ ) is a serious constraint to food production worldwide (FAO and ITPS, 2015). It occurs where hydrogen $\left(\mathrm{H}^{+}\right)$ions are produced in large amounts and interact with clay particles, releasing aluminum which in turn produces more $\mathrm{H}^{+}$ions. The production of $\mathrm{H}^{+}$ions is a natural phenomenon caused by the reaction of $\mathrm{CO}_{2}$ with water, absorption of excess cation over anion nutrients by plant roots and decomposition of organic matter, which is severe in Podzols and Histosols. Both hydrogen and aluminum are readily adsorbed by the clay minerals releasing $\mathrm{Ca}^{2+}, \mathrm{Mg}^{2+}$ and $\mathrm{K}^{+}$ions, which may subsequently be leached from the soil by percolating water, leading to their deficiencies (Blum, Shad and Nortcliff, 2018).

Loss of cations is extensive in Ferralsols, Acrisols and Lixisols which cover approx. 2185 Mha (million hectares) worldwide (IUSS-WRB, 2015). On agricultural lands, the use of ammonical fertilizers and urea are the most important cause of soil acidification, and besides plant nutrient deficiency the growth of many crop plants are impaired by the presence of high toxic levels of aluminum and low phosphorus availability as result of soil acidification (Bolan, Adriano and Curtin, 2003; Fageria and Baligar, 2008). As a result, biomass production and carbon sequestration are diminished despite fertilizer inputs.

Liming is therefore crucial and a common practice to ameliorate soil acidity in agricultural lands (Fageria and Baligar, 2008). It mostly consists of the application of ground limestone (calcium carbonate, lime or calcitic lime), dolomitic ground limestone (calcium magnesium carbonate or dolomite). Soil liming provides $\mathrm{OH}$ - ions to neutralize $\mathrm{H}^{+}$ions thereby decreasing aluminum toxicity with an increase in phosphorus availability and supply of $\mathrm{Ca}^{2+}$ and/or $\mathrm{Mg}^{2+}$ (Bolan et al., 2003). Gypsum $\left(\mathrm{CaSO}_{4} \cdot \mathrm{H}_{2} \mathrm{O}\right.$ ), which is more soluble than lime but does not change soil $\mathrm{pH}$, may also be applied after liming reducing toxic levels of aluminum and to supply calcium in the subsoil (Caires, Joris and Churka, 2011). 


\section{Range of applicability}

Worldwide, liming is the most common and effective practice to counteract soil acidification and it is a prerequisite for optimal nutrient use efficiency by crop plants growing on acid soils (FAO, 2017). The efficacy of liming in SOC sequestration is optimized if it is applied along with other management practices such as zero tillage, crop rotation with cover crops or green manure, soil mulching with crop residue retention and balanced fertilization. Combined management practices are key to increase crop biomass to offset faster $\mathrm{C}$ turnover, hence leading to G sequestration (Briedis et al., 2012; Aye, Sale and Tang, 2016; Holland et al., 2018). Reductions of soil organic carbon due to liming could be associated to increased mineralization parallel to the $\mathrm{pH}$ increase, but progressive reversion might happen due to the influence of the increased $\mathrm{C}$ inputs (Paradelo, Virto and Chenu, 2015).

\section{Impact on soil organic carbon stocks}

Liming contributes to soil organic carbon (SOC) sequestration mainly through increased biomass production resulting from improved soil health. Except for the natural ecosystems, plant growth is inhibited in acid soils due to toxicity of excessive $\mathrm{H}^{+}, \mathrm{Mn}^{2+}$ and $\mathrm{Al}^{3+}$ concentration in soil solution, low microbial activity and poor nutrient cycling. Liming mitigates these soil constraints, thereby helping to achieve climate-driven genetic yield potential of agricultural crops (Table). 
Table 95. Measured effects of liming acidic soils on soil organic carbon stocks

\begin{tabular}{|c|c|c|c|c|c|c|c|c|}
\hline Location & $\begin{array}{l}\text { Climate } \\
\text { zone }\end{array}$ & Soil type & $\begin{array}{l}\text { Baseline C stock } \\
\text { (tC/ha) }\end{array}$ & $\begin{array}{l}\text { Additional C } \\
\text { storage } \\
\text { (tC/ha/yr) }\end{array}$ & $\begin{array}{l}\text { Duration } \\
\text { (Years) }\end{array}$ & $\begin{array}{l}\text { Depth } \\
(\mathrm{cm})\end{array}$ & More information & Reference \\
\hline $\begin{array}{l}\text { South-Western } \\
\text { Sweden }\end{array}$ & \multirow{2}{*}{$\begin{array}{l}\text { Cool } \\
\text { Temperate } \\
\text { Moist }\end{array}$} & $\begin{array}{l}\text { Haplic } \\
\text { Podzol }\end{array}$ & $\begin{array}{l}\text { 116.9; } \\
\text { 35-year old Norway } \\
\text { spruce forest (BAU) }\end{array}$ & 1.60 & 10 & $0-40$ & $\begin{array}{l}\text { Surface dolomite; one } \\
\text { application of } 8.8 \mathrm{t} / \mathrm{ha} .1000 \\
\mathrm{~mm} \text { annual rainfall }\end{array}$ & $\begin{array}{l}\text { Nilsson et } \\
\text { al. (2001) }\end{array}$ \\
\hline Hertfordshire, UK & & $\begin{array}{l}\text { Chromic } \\
\text { Luvisol; } \\
\text { silty clay } \\
\text { loam }\end{array}$ & $\begin{array}{l}76 ; \\
\text { permanent } \\
\text { grassland (tO) }\end{array}$ & 0.14 & 129 & $0-23$ & $\begin{array}{l}\text { Surface calcitic lime; } 4 \text { t/ha } \\
\text { every } 4 \text { years followed by } \\
\text { different lime amounts to } \\
\text { reach pH } 7.704 \mathrm{~mm} \text { average } \\
\text { annual rainfall }\end{array}$ & $\begin{array}{l}\text { Fornara et } \\
\text { al. (2011) }\end{array}$ \\
\hline Southern Brazil & \multirow{3}{*}{$\begin{array}{l}\text { Tropical } \\
\text { Moist }\end{array}$} & $\begin{array}{l}\text { Ferralsol; } \\
\text { loamy }\end{array}$ & $\begin{array}{l}\text { 49.9; } \\
\text { Zero till rainfed } \\
\text { annual crop with } \\
\text { NPK (BAU) }\end{array}$ & 0.51 & 15 & $0-20$ & $\begin{array}{l}\text { Surface dolomite + NPK; } 6 \\
\text { t/ha in } 1993 \text { and } 3 \text { t/ha in } \\
\text { 2000; } 1545 \mathrm{~mm} \text { annual } \\
\text { rainfall }\end{array}$ & $\begin{array}{l}\text { Briedis et } \\
\text { al. (2012) }\end{array}$ \\
\hline Central Brazil & & $\begin{array}{l}\text { Ferralsol; } \\
\text { clayey }\end{array}$ & 183; (BAU) & 1.76 & 7 & $0-200$ & $\begin{array}{l}\text { Incorporated dolomite; } 7.08 \\
\text { t/ha and } 5 \mathrm{t} / \text { ha gypsum } \\
\left(\mathrm{CaSO}_{4} \cdot 2 \mathrm{H}_{2} \mathrm{O}\right)\end{array}$ & $\begin{array}{l}\text { Araújo et al. } \\
\text { (2019) }\end{array}$ \\
\hline $\begin{array}{l}\text { North-Eastern } \\
\text { India }\end{array}$ & & $\begin{array}{l}\text { Alfisol; } \\
\text { sandy } \\
\text { loam }\end{array}$ & $\begin{array}{l}\text { 15.5; } \\
\text { irrigated annual } \\
\text { crop system (tO) }\end{array}$ & 0.15 & 29 & $0-45$ & $\begin{array}{l}\text { Incorporated dolomite }+ \text { NPK } \\
\text { at } 10-\mathrm{cm} \text { depth; } 2.5 \mathrm{t} / \mathrm{ha} \\
\text { dolomite every } 4 \text { years; } 1450 \\
\text { mm average annual rainfall }\end{array}$ & $\begin{array}{l}\text { Hati et al. } \\
(2008)\end{array}$ \\
\hline
\end{tabular}




\section{Other benefits of the practice}

\subsection{Improvement of soil properties}

\section{Physical properties}

Dispersion or flocculation of soil colloid particles may be influenced by liming due to its effect on soil $\mathrm{pH}$ and Ca in the soil solution. Thus, liming weathered tropical soils such as Ferralsols, especially those cultivated under reduced tillage, leads to an improvement of soil physical properties, which in turn decrease in soil water erosion and favouring soil C sequestration (Castro and Logan, 1991; Bolan and Hedley, 2003)

\section{Chemical properties}

Adequate liming raises $\mathrm{P}$ availability and significantly reduces $\mathrm{Al}$ and $\mathrm{Mn}$ toxicity; results in more vacated sites in the soil adsorption complex to be occupied by $\mathrm{Ca}, \mathrm{Mg}$ and $\mathrm{K}$, thereby mitigating their losses by leaching. Bioavailability of micronutrients such as molybdenum and boron increases with increasing soil $\mathrm{pH}$ up to 7.5 . However, liming needs to be done judiciously to avoid nutritional imbalances due to P-Ca immobilization and low activity of Zn leading to yield reductions (Cregan, Hirth and Conyers, 1989; Bailey and Laidlaw, 1999; Fageria and Baligar, 2008).

\section{Biological properties}

Activities of microbes such as rhizobia, which are beneficial because of the atmospheric $\mathrm{N}$ fixation or plant growth-promoting rhizobacteria such as Azospirillum or Pseudomonas are improved in limed soils. Additions of lime caused an increase of mites and decrease of enchytraeids worms while collembolans were unaffected. Groups of earthworms show changes in their population numbers in soils that are limed. Also, the enzyme nitrogenase and molybdenum availability are increased with liming, therefore affecting positively the symbiotic nitrogen fixation by legumes (Bailey and Laidlaw, 1999, Bolan, Hedley and White, 1991, Holland et al., 2018).

Benefits of liming can be already observed 1 month after application, particularly on soil $\mathrm{pH}_{\mathrm{CaCl} 2}$ and exchangeable $\mathrm{Ca}+\mathrm{Mg}$ (Quaggio et al., 1995), but the positive effects can last up to 6 years and beyond depending on soil texture, nitrogen fertilizer and rainfall pattern (Pavan and Oliveira, 1997, Sime, 2001).

\subsection{Minimization of threats to soil functions}

Table 96. Soil threats

Soil threats

Losses by rainfall water in Ferralsols and Acrisols are a combination of bare soil prone to raindrop impact leading to soil crusting, reduced water infiltration and increased runoff. Soil liming, as part of soil and water conservation practices such as

Soil erosion reduced tillage with crop rotation including cover crops and contour terracing to diminish water losses, improve soil structure which facilitates infiltrability of water (Castro Filho et al., 1991; Haynes and Naidu, 1992). 


\section{Soil threats}

\begin{tabular}{|c|c|}
\hline $\begin{array}{l}\text { Nutrient imbalance } \\
\text { and cycles }\end{array}$ & $\begin{array}{l}\text { Liming increases the availability of nutrients through favouring processes such as } \\
\text { nitrification that govern } \mathrm{N} \text { availability to crop plants. Also, liming reduces } \mathrm{P} \\
\text { adsorption, resulting in an increase in P agronomic efficiency in many Sub-Saharan } \\
\text { countries (Vanlawe et al., 2015). }\end{array}$ \\
\hline $\begin{array}{l}\text { Soil salinization and } \\
\text { alkalinization }\end{array}$ & $\begin{array}{l}\text { Although liming materials are not used for ameliorating soil salinity, calcium } \\
\text { containing compounds will help to reduce sodium levels, which is a major constraint } \\
\text { in these soils (Mukhopadhyay et al., 2020). }\end{array}$ \\
\hline $\begin{array}{l}\text { Soil contamination / } \\
\text { pollution }\end{array}$ & $\begin{array}{l}\text { There are well established studies demonstrating the potential value of liming as } \\
\text { immobilizing agent in reducing the bioavailability of a range of heavy metals in soils } \\
\text { such as cadmium and arsenic (Bolan et al., 2003; Hong et al., 2014). }\end{array}$ \\
\hline Soil biodiversity loss & $\begin{array}{l}\text { Despite considerable variation in response of soil biota to acidity, liming has been } \\
\text { shown to have a positive impacts on the abundance of bacteria and earthworms, } \\
\text { important in the nutrient cycling, and of fungi in recalcitrant decomposition (C } \\
\text { storage); abundance of soil pathogens decreased through liming with subsequent } \\
\text { disease regulation (Robson and Abbott, 1989; Holland et al., 2018). }\end{array}$ \\
\hline Soil compaction & $\begin{array}{l}\text { It has been found that liming a sandy clay loam Ferralsol combined with gypsum } \\
\text { application resulted in a better organization of soil particles, increasing } \\
\text { macroporosity and reducing soil bulk density and penetration resistance (Carmeis et } \\
\text { al, 2016). } \\
\text { Soil aggregates break down during wetting cycle, then set to a hard, structureless } \\
\text { mass during drying, leading to hardsetting and soil surface sealing. Liming improves } \\
\text { soil structure, thereby helps to mitigate soil sealing and hardsetting (Roth and } \\
\text { Pavan, 1991). }\end{array}$ \\
\hline $\begin{array}{l}\text { Soil water } \\
\text { management }\end{array}$ & $\begin{array}{l}\text { The addition of lime to a sandy loam Acrisol (pH 4.1) in Brazil has increased by } 60 \\
\text { percent the gross water use efficiency in maize/cowpea intercropping system } \\
\text { compared to sole application of NPK fertilizer (Gaiser et al., 2004). In Australia, } \\
\text { application of } 2.9 \mathrm{t} / \text { ha of lime maximized the ability of pastures to utilize scarce } \\
\text { available water reserves of an acidic (pH 4.1) Ferralsol (Hayes et al., 2016). }\end{array}$ \\
\hline
\end{tabular}

\subsection{Increases in production (e.g. food/fuel/feed/timber/fibre)}

The application of an appropriate rate of lime brings several chemical and biological changes in the soil and increases root growth, which are beneficial or helpful in improving food, feed or fuel crop yields on acid soils. Optimum base saturation in soil is very critical to achieve climate-driven genetic yield potential of agricultural crops, which is both soil and crop dependent. For Ferralsols, the desired optimum base saturation for most cereals is in the range of 50 - 60 percent, and for legumes it is in the range of 60-70 percent, which, when reached, places the productivity of these crops among the highest in the world (Sanchez and Salinas, 1981; Fageria and Baligar, 2001). 
With regard to Al saturation, about 90 percent of maximum maize yield was obtained at 29 percent $\mathrm{Al}$ saturation in Ferralsols and Acrisols. In Rwandan Ferralsols, yields of wheat, beans and potatoes were significantly increased by liming (Yamoah, Burleigh and Eylands, 1992). Sandy Cambisols in Zimbabwe when limed produced large increase in groundnut (Arachis hypogaea) yield, an important component in the diet of the rural population (Murata et al., 2002). In the central highlands of Ethiopia, combined applications of $1.65 \mathrm{t} / \mathrm{ha}$ lime and $30 \mathrm{~kg} / \mathrm{ha} \mathrm{P}$ fertilizer increased barley yield of an acid $(\mathrm{pH}<5.0)$ Nitisol (Desalegn et al., 2017).

\subsection{Mitigation of and adaptation to climate change}

Liming can be a source or sink for $\mathrm{CO}_{2}$, depending on whether reaction occurs with strong acids or carbonic acid (Kunhikrishnan et al., 2016). On the other hand, particularly under zero tillage, liming provides an opportunity for $\mathrm{N}_{2} \mathrm{O}$ and $\mathrm{CH}_{4}$ mitigation (Baggs, Smales and Bateman, 2010; García-Marco et al, 2016). The strategy of soil C sequestration involves return of the biomass to the soil in excess of the mineralization capacity and to enhance formation of organo-mineral complexes or stable micro- and macroaggregates (Lal, 2003). Agricultural lands showing chemical degradation by processes such as increasing soil acidity can be restored through liming, when in combination with other best management practices. Compared to acidic soils, limed soils contain more biomass above- and belowground and therefore they have higher soil C stocks. From a perspective of adaptation to climate change, regular liming applications with zero tillage and integrated croplivestock maintain soil surface permanently covered (soil mulching) keeping soil moisture for several days whenever a dry spell of up to 20 days happens.

\subsection{Socio-economic benefits}

Unlike many fertilizers, lime has a strong carryover. In western Kenya, lime at 2, 4 and $6 \mathrm{t} /$ ha maintained $\mathrm{pH} \geq$ 5.5 of an Acrisol for 2, 3 and 4 years, respectively. It has been observed in a clayey Ferralsol in Central region of Brazil, that after 4 years with eight crop cycles (rice (Oryza spp.), common bean, maize (Zea mays), and soybean (Glycine max)), the soil $\mathrm{pH}$ and the levels of $\mathrm{Ca}$ and $\mathrm{Mg}$ were still adequate to maintain crop yields at an optimal level. From an economic perspective, liming is a capital investment rather than an operating input because of its long-term benefits (Lukin and Epplin, 2003; Fageria and Baligar, 2008; Kisinyo et al., 2014).

\subsection{Other benefits of the practice}

\section{Lime requirement}

Methods to assess lime requirement are based on soil $\mathrm{pH}$, base saturation, and aluminum saturation adjustments at appropriate levels according to the crop demands and expected yields. Care should be taken, however, on lime requirement based only on exchangeable aluminum, since it is controlled by the soil-specific relationship between exchangeable aluminum and the non-aluminum toxicity and mineralogical soil properties. For many Ferralsols and Acrisols, exchangeable aluminum accounts for a small part of the total soil acidity (Cregan, Hirth and Conyers, 1989; Cantarella, van Raij and Quaggio, 1998; Fageria and Baligar, 2008). 


\section{Lime quality}

The quality of a liming material is determined by its neutralizing value (NV) and its particle size. Fine particle limestone with an NV of 98 percent has high ability to neutralise acidity. However, coarse lime material react slower than fine particle lime and the need to repeat soil liming may be extended.

\section{Timing of application}

Soil moisture is critical to the reaction of limestone, thus rainfall patterns in the area should be monitored and used as a guide. As limestone shows lower solubility compared to some fertilizers, allow several weeks for reaction in the acidic soil, particularly Ferralsol and Acrisol before sowing time and NPK fertilizer application.

\section{Potential drawbacks to the practice}

\subsection{Tradeoffs with other threats to soil functions}

A common tradeoff is observed when lime is unevenly broadcasted, especially at large rates $(>4 \mathrm{t} / \mathrm{ha}$ ) on the soil surface (overliming) leading to $\mathrm{pH}$ higher than 6.5.

Table 97. Soil threats

\begin{tabular}{|l|l|}
\hline Soil threats & Nutrient imbalance \\
and cycles & $\begin{array}{l}\text { Overliming can cause P deficiency due to high levels of } \mathrm{Ca} \text { and also } \\
\text { significantly reduce the plant availability of micronutrients (Zn, Cu, Fe, and } \\
\text { Mn), which decreases with increasing pH, particularly over 6.5 (Bolan et al., } \\
\text { 2008) }\end{array}$ \\
\hline $\begin{array}{l}\text { Soil contamination } \\
\text { / pollution }\end{array}$ & $\begin{array}{l}\text { In some soils with high levels of molybdenum, overliming may increase Mo } \\
\text { uptake by crop plants causing Mo toxicity, especially in ruminants } \\
\text { (molybdenosis) (Bolan et al., 2003). }\end{array}$ \\
\hline Soil acidification & $\begin{array}{l}\text { Aluminum solubility is minimal in the pH range of 5.5-6.5, but when pH is } \\
\text { close to 7.O it becomes increasingly soluble as the negatively charged } \\
\text { aluminate form and can be taken up by crop plants decreasing crop yield } \\
\text { (Fageria and Baligar, 2008). }\end{array}$ \\
\hline
\end{tabular}

\subsection{Increases in greenhouse gas emissions}

Mitigation of $\mathrm{N}_{2} \mathrm{O}$ emissions from soils are optimized if the soil moisture of the limed soils is kept around the field capacity. On the other hand, liming shows variable effects on soil $\mathrm{CH}_{4}$ uptake rate (Kunhikrishnan et al., 2016). 


\subsection{Conflict with other practice(s)}

Liming is most commonly practiced to overcome the impact of soil acidification on crop yield. However, an integrated approach involving liming, management practices such as use of less-acidifying fertilizers, improved nutrient use efficiency, reduced nutrient losses by leaching or product removal by harvest, and increased plant tolerance will probably be necessary, particularly where the acidification potential is high and acidification is likely to extend into the subsoil (Bolan and Hedley, 2003).

\subsection{Decreases in production (e.g. food/fuel/feed/timber/fibre)}

Overliming can cause nutrient deficiencies, leading to a reduction in crop production (e.g, lime-induced iron chlorosis) (Bolan et al., 2008).

\subsection{Limitation of the practice}

Acid sulfate soils are a natural soil type existing as a result of the oxidation process of metal sulphides such as pyrite $\left(\mathrm{FeS}_{\mathrm{n}}\right)$ (Andriesse and van Mensvoort, 2006). These soils have a sulfuric horizon or a sulfidic material occurring in the coastal lowlands of Southeast Asia (Indonesia, Thailand, Vietnam), West Africa (Senegal, The Gambia, Guinea Bissau, Sierra Leone, Liberia) and along the north-eastern coast of South America (the Bolivarian Republic of Venezuela, the Guianas). The world extent of both coastal and inland acid sulfate soils is, however, not yet well quantified. In Australia it is estimated at $~ 22$ Mha (IUSS-WRB, 2015; Fanning, Rabenhorst and Fitzpatrick, 2017). It is generally not easy and cost-effective to neutralize acid sulfate soils using liming materials mainly because of the continuous release of sulfuric acid. Normally, acid sulfate soils are managed by keeping them submerged thereby not allowing them to get exposed to air. Reducing the oxidisation potential of metal sulfides in soils to form acid sulfate soils zones is the current preferred mitigation strategy (Gurung et al., 2017). If sufficient water and substantial investment for its management is available, rice can be cultivated on these problem soils. About 3.0 Mha of rice is cultivated on acid sulfate soils in Asia (2.9 Mha), Africa (0.5 Mha) and Americas (0.4), which are closely associated with deepwater/mangrove environments and rainfed lowlands (Haefele, Nelson and Hijmans, 2014). In Malaysia, the several steps of amelioration for rice cultivation include correct water management to prevent pyrite oxidation by maintaining water table level above the pyrite layers, liming at appropriate rate $\left(\sim 2.5\right.$ t.ha $\left.^{-1}\right)$, adequate fertilizer/organic matter application and keeping the soil submerged as long as possible before transplanting (Suswanto et al., 2007).

\section{Recommendations before implementing the practice}

Before any application of lime to acid soils, it is strongly recommended to have the soil sampled and analyzed according to recommendations provided by the local/regional extension agent or a soil expert familiar with the soil properties of the region. Also, lime specifications must comply with local/regional regulations. 


\title{
7. Potential barriers for adoption
}

\author{
Table 98. Potential barriers to adoption
}

\begin{tabular}{|c|c|c|}
\hline Barrier & YES/NO & \\
\hline Biophysical & Yes & $\begin{array}{l}\text { Lime application can lead to release of } \mathrm{CO}_{2} \text { resulting from the } \\
\text { dissolution of lime (Kunhikrishnan et al. 2016). }\end{array}$ \\
\hline Cultural & Yes & $\begin{array}{l}\text { There is still a poor understanding of the values of regular liming of soils } \\
\text { that are subjected to continuous acidification such as legume-based } \\
\text { grazed pastures (Bolan et al., 1991). }\end{array}$ \\
\hline Social & No & $\begin{array}{l}\text { In the Zambian farming districts of Mkushi and Sowezi although } \\
\text { smallholding farmers knew the benefits of using lime and that uptake } \\
\text { of agricultural lime could be encouraged, the main constraints on the } \\
\text { use of lime were the absence of soil testing and a lack of cash in the } \\
\text { rural economy (Mitchell, 2005). }\end{array}$ \\
\hline Economic & Yes & $\begin{array}{l}\text { Although lime deposits are available in most countries affected by soil } \\
\text { acidity in Sub-Saharan Africa, the cost effectiveness of liming, } \\
\text { especially in relation to transport and the commonly required high } \\
\text { application rates, is likely to negatively affect the adoption of this } \\
\text { practice (Vanlawe et al., 2015). }\end{array}$ \\
\hline Institutional & Yes & $\begin{array}{l}\text { The lack of soil testing facilities is a barrier for adoption of the practice } \\
\text { in many regions. Additionally, developing lime supply chains from } \\
\text { scratch is difficult and costly (Mitchell, 2005; Bossuet, Chamberlin and } \\
\text { Warner, 2019). }\end{array}$ \\
\hline Knowledge & Yes & $\begin{array}{l}\text { There is still a lack of knowledge on the beneficial effects of regular } \\
\text { liming on soil health and productivity, including constraints of } \\
\text { estimating lime requirement (Bolan et al., 2003). }\end{array}$ \\
\hline $\begin{array}{l}\text { Natural } \\
\text { resource }\end{array}$ & Yes & $\begin{array}{l}\text { The main source of agricultural lime is limestone deposit, whose } \\
\text { frequency varies and quality-grade dolomite may be scarce (DMRE, } \\
\text { 2003). }\end{array}$ \\
\hline
\end{tabular}




\section{Photos of the practice}

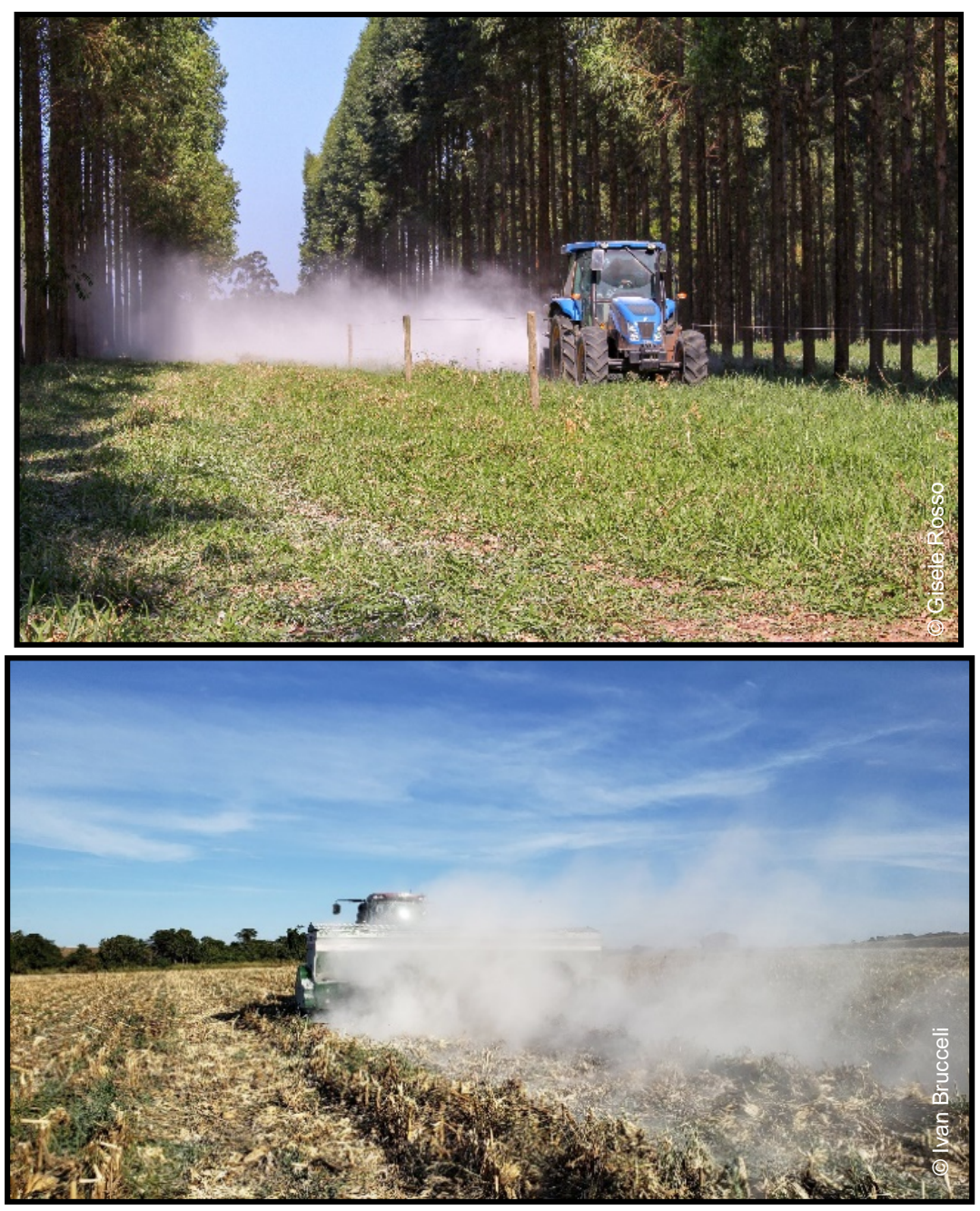

Photo 28. Liming acidic Ferralsol under grass pasture and annual crop in Brazil

Table 99. Related cases studies available in volumes 3 and 5

\begin{tabular}{|l|l|c|c|c|}
\hline Title & Region & $\begin{array}{l}\text { Duration } \\
\text { of study } \\
\text { (Years) }\end{array}$ & Volume & $\begin{array}{l}\text { Case- } \\
\text { study } \\
\text { No. }\end{array}$ \\
\hline $\begin{array}{l}\text { Increasing Yield and Carbon Sequestration } \\
\text { in a Signalgrass Pasture by Liming and } \\
\text { Fertilization in Sao Carlos (SP, Brazil) }\end{array}$ & $\begin{array}{l}\text { Latin } \\
\text { America and } \\
\text { the } \\
\text { Caribbean }\end{array}$ & 6 & 3 & 32 \\
\hline
\end{tabular}




\section{References}

Andriesse, W. \& van Mensvoort, M.E.F. 2006. Acid sulfate soils: Distribution and extent. In R. Lal (Ed.) Encyclopedia of Soil Science. pp. 14-19. New York, USA, Taylor and Francis Group.

Araújo, L.G., Sousa, D.M.G. de, Figueiredo, C.C. de, Rein, T.A., de Souza Nunes, R., Santos Júnior, J. de D.G. dos \& Malaquias, J.V. 2019. How does gypsum increase the organic carbon stock of an Oxisol profile under sugarcane? Geoderma, 343: 196-204. https://doi.org/10.1016/j.geoderma.2019.02.029

Aye, N.S., Sale, P.W.G. \& Tang, C. 2016. The impact of long-term liming on soil organic carbon and aggregate stability in low-input acid soils. Biology and Fertility of Soils, 52: 697-709.

https://doi.org/10.1007/s00374-016-1111-y

Baggs, E.M., Smales, C.L. \& Bateman, E.J. 2010. Changing pH shifts the microbial source as well as the magnitude of $\mathrm{N}_{2} \mathrm{O}$ emission from soil. Biology and Fertility of Soils, 46: 793-805.

https://doi.org/10.1007/s00374-010-0484-6

Bailey, J.S. \& Laidlaw, A.S. 1999. The interactive effects of phosphorus, potassium, lime and molybdenum on the growth and morphology of white clover (Trifolium repens L.) at establishment. Grass and Forrage Science, 54: 69-76. https://doi.org/10.1046/j.1365-2494.1999.00159.x

Blum, W.E.H., Shad, P. \& Nortcliff, S. 2018. Essentials of soil science: Soil formation, functions, use and classification (World Reference Base, WRB). Stuttgart, Borntraeger Science Publishers.

Bolan, N.S., Adriano, D.C. \& Curtin, D. 2003. Soil acidification and liming interactions with nutrient and heavy metal transformation and bioavailability. Advances in Agronomy, 78: 215-272.

https://doi.org/10.1016/S0065-2113(02)78006-1

Bolan, N.S., Hedley, M.J. \& White, R.E. 1991. Processes of soil acidification during nitrogen cycling with emphasis on legume-based pastures. Plant and Soil, 134(1): 53-63. https://doi.org/10.1007/978-94011-3438-5_18

Bolan, N.S. \& Hedley, M.J. 2003. Role of carbon, nitrogen and sulfur cycles in soil acidification. In Z. Rengel (Ed.) Handbook of Soil Acidity, pp. 29-52. New York, USA, Marcel Dekker, New York.

Bolan, N.S., Rowarth, J., de la Luz Mora, M., Adriano, D. \& Curtin, D. 2008. Biological transformation and bioavailability of nutrient elements in acid soils as affected by liming. In R. Naidu (Ed.) Chemical Bioavailability in Terrestrial Environments. pp. 413-446. Amsterdam, The Netherlands, Elsevier Science Limited.

Bossuet, J., Chamberlin, J. \& Warner, J. 2019. New tools guide interventions against acid soils in Africa using lime [online]. [Cited 19 July 2020]. https:/ /www.cimmyt.org/blogs/new-tools-guide-interventionsagainst-acid-soils-in-africa-using-lime/

Briedis, C., Sá, J.C. de M., Caires, E.F., Navarro, J. de F., Inagaki, T.M., Boer, A., Ferreira, A. de O., Neto, C.Q., Canalli, L.B. \& Santos, J.B. dos. 2012. Changes in Organic Matter Pools and Increases in Carbon Sequestration in Response to Surface Liming in an Oxisol under Long-Term No-Till. Soil Science Society of America Journal, 76(1): 151-160. https://doi.org/10.2136/sssaj2011.0128 
Caires, E.F., Joris, H.A.W. \& Churka, S. 2011. Long-term effects of lime and gypsum additions no no-till corn and soybean yield and soil chemical properties in southern Brazil. Soil Use and Management, 27:45-53. https://doi.org/10.1111/j.1475-2743.2010.00310.x

Cantarella, H., van Raij, B. \& Quaggio, J.A. 1998. Soil and plant analysis for lime and fertilizer recommendations in Brazil. Communications in Soil Science and Plant Analysis, 29: 1691-1706. https://doi.org/10.1080/00103629809370060

Carmeis Filho, A.C.A., Crusciol, C.A.C., Guimarães, T.M., Calonego, J.C. \& Mooney, S.J. 2016. Impact of amendments on the physical properties of soil under tropical long-term no till conditions. PLOS ONE, 12(2): e0173011. https://doi.org/10.1371/journal.pone.0167564

Castro, C. \& Logan, T.J. 1991. Liming Effects on the Stability and Erodibility of Some Brazilian Oxisols. Soil Science Society of America Journal, 55(5): 1407-1413. https://doi.org/10.2136/sssaj1991.03615995005500050034x

Castro Filho, C., Henklain, J.C., Vieira, M.J., Casão Jr., R. 1991. Tillage methods and soil and water conservation in southern Brazil. Soil and Tillage Research, 20: 271-283. https://doi.org/10.1016/01671987(91)90043-W

Cregan, P.D., Hirth, J.R. \& Conyers, M.K. 1989. Amelioration of soil acidity by liming and other amendments. In A.D. Robson (Ed.) Soil Acidity and Plant Growth. pp. 205-264. Sydney, Australia, Academic Press.

Desalegn, T., Alemu, G., Adella, A., Debele, T. \& Gonzalo J. 2017. Effect of lime and phosphorus fertilizer on acid soils and barley (Hordeum vulgare L.) performance in the central highlands of Ethiopia. Experimental Agriculture, 53(3): 432-444. https://doi.org/10.1017/S0014479716000491

DMRE. 2003. A Review of the Dolomite and Limestone Industry in South Africa. Report, R43. Pretoria, South Africa, Department of Mineral Resources and Energy. 11p.

Fageria, N.K. \& Baligar, V.C. 2001. Improving nutrient use efficiency of annual crops in Brazilian acid soils for sustainable crop production. Communications in Soil Science and Plant Nutrition, 32(7-8): 1303-1319. https://doi.org/10.1081/CSS-100104114

Fageria, N.K. \& Baligar, V.C. 2008. Ameliorating soil acidity of tropical Oxisols by liming for sustainable crop production. Advances in Agronomy, 99: 345-399. https://doi.org/10.1016/S00652113(08)00407-0

Fanning, D.S., Rabenhorst, M.C. \& Fitzpatrick, R.W. 2017. Historical developments in the understanding of acid sulfate soils. Geoderma, 308: 191-206.

https://doi.org/10.1016/j.geoderma.2017.07.006

FAO \& ITPS. 2015. Status of the world's soil resources (SWSR) - Main Report. Rome, Italy, Food and Agriculture Organization of the United Nations and Intergovernmental Technical Panel on Soils.

FAO. 2017. Voluntary Guidelines for Sustainable Soil Management. Rome, Italy, Food and Agriculture Organization of the United Nations. 
Fornara, D.A., Steinbeiss, S., McNamara, N.P., Gleixner, G., Oakley, S., Poulton, P.R., Macdonald, A.J. \& Bardget, R.D. 2011. Increases in soil organic carbon sequestration can reduce the global warming potential of long-term liming to permanent grassland. Global Change Biology, 17: 1925-1934.

https://doi.org/10.1111/j.1365-2486.2010.02328.x

Gaiser, T., Barros, I., Lange, F.-M. \& Williams, J.R. 2004. Water use efficiency of a maize/cowpea intercrop on a highly acidic tropical soil as affected by liming and fertilizer application. Plant and Soil, 263: 165-171. https://doi.org/10.1023/B:PLSO.0000047733.98854.9f

García-Marco, S., Abalos, D., Espejo, R., Vallejo, A. \& Mariscal-Sancho, I. 2016. No tillage and liming reduce greenhouse gas emissions from poorly drained agricultural soils in Mediterranean regions. Science of the Total Environment, 566-657: 512-520. https://doi.org/10.1016/j.scitotenv.2016.05.117

Gurung, S.R., Wijesekara, H., Seshadri, B., Stewart, R.B., Gregg, P.E.H. \& Bolan, N.S. 2017. Sources and management of acid mine drainage. In N.S. Bolan, M.B. Kirkham \& Y.S. Ok (Eds.) Spoil to Soil: Mine Site Rehabilitation and Revegetation. pp. 33-56. Boca Raton, USA, CRC Press.

Haefele, S.M., Nelson, A. \& Hijmans, R.J. 2014. Soil quality and constraints in global rice production. Geoderma, 235-236: 250-259. https://doi.org/10.1016/j.geoderma.2014.07.019

Hati, K.M., Swarup, A., Mishra, B., Manna, M.C., Wanjari, R.H., Mandal, K.G. \& Misra, A.K. 2008. Impact of long-term application of fertilizer, manure and lime under intensive cropping o physical properties and organic carbon content of an Alfisol. Geoderma, 148: 173-179.

https://doi.org/10.1016/j.geoderma.2008.09.015

Hayes, R.C., Guangdi, D.L., Conyers, M.K., Virgona, J.M. \& Dear, B.S. 2016. Lime increases productivity and the capacity of lucerne (Mendicago sativa L.) and phalaris (Phalaris aquatica L.) to utilize stored soil water on acidic soil in southern-eastern Australia. Plant and Soil, 400: 29-43.

https://doi.org/10.1007/s11104-015-2706-Z

Haynes, R.J. \& Naidu, R. 1992. Influence of lime, fertilizer and manure applications on soil organic matter content and soil physical conditions: a review. Nutrient Cycling in Agroecosystems, 51: 123-137.

https://doi.org/10.1023/A:1009738307837

Holland, J.E., Bennett, A.E., Newton, A.C., White, P.J., McKenzie, B.M., George, T.S., Pakeman, R.J., Bailey, J.S., Fornara, D.A. \& Hayes, R.C. 2018. Liming impacts on soils, crops and biodiversity in the UK: a review. Science of the Total Environment, 610-611: 316-332.

https://doi.org/10.1016/j.scitotenv.2017.08.020

Hong, C.O., Owens, V.N., Kim, Y.G., Lee, S.M., Park, H.C., Kim, K.K., Son, H.J., Suh, J.M. \& Kim, P.J. 2014. Soil pH effect on phosphate induced cadmium precipitation in arable soil. Bulletin of Environmental Contamination and Toxicology, 93: 101-105. https://doi.org/10.1007/s00128-014$1273-\mathrm{y}$

IUSS-WRB. 2015. World Reference Base for Soil Resources 2014, update 2015. International soil classification system for naming soils and creating legends for soil maps. World Soil Resources Reports, No. 106. Rome, Italy, Food and Agriculture Organization of the United Nations. 
Kisinyo, P.O., Othieno, C.O., Gudu, S.O., Okalebo, J.R., Opala, P.A., Ng'etich, W.K., Nyambati, R.O., Ouma, E.O., Agalo, J.J., Kebeney, S.J., Too, E.J., Kisinyo, J.A. \& Opile, W.R. 2014. Immediate and residual effects of lime and phosphorus fertilizer on soil acidity and maize production in western Kenya. Experimental Agriculture, 50(1): 128-143. https://doi.org/10.1017/S0014479713000318

Kunhikrishnan, A., Thangarajan, R., Bolan, N.S., Xu, Y., Mandal, S., Gleeson, D.B., Seshadri, B., Zaman, M., Barton, L, Tang, C., Luo, J., Dalal, R., Ding, W., Kirkham, M.B. \& Naidu, R. 2016. Functional relationships of soil acidification, liming, and greenhouse gas flux. Advances in Agronomy, 39. http://dx.doi.org/10.1016/bs.agron.2016.05.001

Lal, R. 2003. Offsetting global $\mathrm{CO}_{2}$ emissions by restoration of degraded soils and intensification of world agriculture and forestry. Land Degradation \& Development, 14: 309-322.

https://doi.org/10.1002/ldr.562

Lukin, V.V. \& Epplin, F.M. 2003. Optimal frequency and quantity of agricultural lime applications. Agricultural Systems, 76: 949-967. https://doi.org/10.1016/S0308-521X(02)00016-1

Mitchell, C.J. 2005. FarmLime: Low-cost lime for small-scale farming. British Geological Survey Commissioned Report CR/03/066N. Nottingham, UK, NERC, 124p.

Mukhopadhyay, R., Sarkar, B., Jat, H.S., Sharma, P.C. \& Bolan, N.S. 2020. Soil salinity under climate change: Challenges for sustainable agriculture and food security. Journal of Environmental Management: 111736. https://doi.org/10.1016/j.jenvman.2020.111736

Murata, M.R., Hammes, P.S. \& Zharares, G.E. 2002. Soil amelioration effects on nutrient availability and productivity of groundnut on acid sandy soils of Zimbabwe. Experimental Agriculture, 38: 317-331.

https://doi.org/10.1017/S0014479702003058

Nilsson, S.I., Andersson, S., Valeur, I., Persson, T., Bergholm, J. \& Wirén, A. 2001. Influence of dolomite lime on leaching and storage of $\mathrm{C}, \mathrm{N}$ and $\mathrm{S}$ in a Spodosol under Norway spruce (Picea abeis (L.) Karst.). Forest Ecology and Management, 146: 55-73. https://doi.org/10.1016/S0378-1127(00)004527

Paradelo, R., Virto, I. \& Chenu, C. 2015. Net effect of liming on soil organic carbon stocks: a review. Agriculture, Ecosystems and Environment, 202: 98-107. https://doi.org/10.1016/j.agee.2015.01.005

Pavan, M.A. \& Oliveira, E.L. 1997. Manejo da acidez do solo. Circular, 95. Londrina, Brazil, IAPAR. 86p.

Quaggio, J.A, Gallo, P.B. \& Mascarenhas, H.A.A. 1995. Agronomic efficiency of limestones with different acid-neutralizing capacity, under field condition. In R.A. Date, G.E. Grudun, G.E. Rayment \& M.E. Probert (Eds.) Plant Interactions at Low pH. pp. 491-496. Dordrecht, The Netherlands, Springer.

https://doi.org/10.1007/978-94-011-0221-6

Robson, A.D. \& Abbott, L.K. 1989. The effect of soil acidity on microbial activiti in soils. In A.D. Robson, (Ed.) Soil Acidity and Plant Growth. pp. 139-166. Sydney, Australia, Academic Press.

Roth, C.H. 1992. Soil sealing and crusting in tropical South America. In M.A. Sumner \& B.A. Stewart (Eds.) Soil Crusting: Chemical and Physical Processes. pp. 267-300. Advances in Soil Sciences, Boca Raton, USA, Lewis Publishers. 
Roth, C.H. \& Pavan, M.A. 1991. Effects of lime and gypsum on clay dispersion and infiltration in samples of a Brazilian Oxisol. Geoderma, 48, 351-361. https://doi.org/10.1016/0016-7061(91)90053-V

Sanchez, P.A. \& Salinas, J.G. 1981. Low-input technology for managing Oxisols and Ultisols in tropical America. Advances in Agronomy, 34: 279-406. https://doi.org/10.1016/S0065-2113(08)60889-5

Sime, D. 2001. Lime: The essential element in New Zealand agriculture. Kaitaia, Northland Lime Millers Association. 48p.

Suswanto, T., Shamshuddin, J., Syed Omar, S.R., Peli, M. \& Teh, C.B.S. 2007. Effects of lime and fertilizer application in combination with water management on rice (Oryza sativa) cultivated on an acid sulfate soil. Malaysian Journal of Soil Science, 11: 1-16.

Vanlawe, B., Descheenmaeker, K., Giller, K., Huising, J., Merckx, R., Nzuguheba, G., Wendt, J. \& Zingore, S. 2015. Integrated soil fertility management in sub-saharan Africa: unravelling local adaptation. Soil, 1: 491-508. https://doi.org/10.5194/soil-1-491-2015

Yamoah, C.F., Burleigh, J.R. \& Eylands, V.J. 1992. Correction of acid infertility in Rwandan Oxisols with lime from an indigenous source for sustainable cropping. Experimental Agriculture, 28: 417-424.

https://doi.org/10.1017/S0014479700020123 


\title{
22. Application of gypsum on sodic soils
}

\author{
Thiago M. Inagaki
}

Lehrstuhl für Bodenkunde, Technische Universität München, Munich, Germany.

\section{Description of the practice}

A soil is considered as "sodic" when the levels of exchangeable Na are greater than 15 percent of the cation exchange capacity (>15 percent Exchangeable Sodium Percentage (ESP)),; Abrol, Yadav and Massoud, 1988). The increase of exchangeable Na levels leads to several processes in the soil such as clay dispersion and surface crusting. Soil degradation induced by sodicity is an important environmental problem, especially in arid and semi-arid regions. This condition is present in over 75 countries in the world and it is constantly increasing mainly due to improper irrigation with water having inadequate ionic ratios (Qadir et al., 2007). The degradation process induced by the $\mathrm{Na}^{+}$ions occurs especially at the clay fraction $<2 \mu$, inducing their dispersion and consequently aggregate disruption (Qadir et al., 2001). Surface horizons are particularly sensitive to this degradation since they receive greater water input and suffer higher mechanical stress. After dispersion, the soil aggregate particles can be rearranged in densely-packed layers, creating seals which reduce infiltration capacity, which are denominated crusts upon drying (Figure 11; McIntyre, 1958; Singer and Shainberg, 2004). This sealing process is a major soil degradation mechanism affecting arid and semiarid regions with low organic matter contents and unstable soil aggregate structure, since it reduces water infiltration capacity, increases runoff and erosion potential, and prevents the emergence of seedlings when crusts become hard upon drying. 
Soil Aggregate

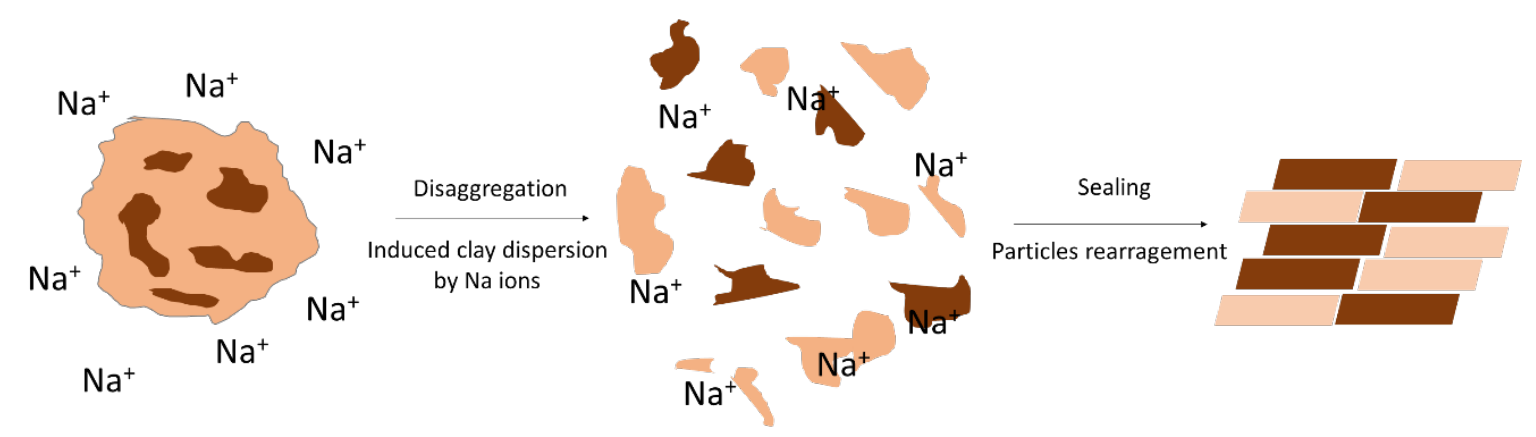

Figure 11. Schematic figure demonstrating the aggregate dispersion induced by $\mathrm{Na}$ ions and subsequent sealing and crust formation due to the rearrangement of dispersed clays

The amelioration of sodic soils is usually made by replacing the $\mathrm{Na}^{+}$ions with $\mathrm{Ca}^{2+}$ ions on the cation exchange complex. The remediation of sodic soils has been predominantly done through the application of chemical and organic amendments. This process requires a sufficient amount of water throughout the soil profile and a good drainage system (Gupta and Abrol, 1990). Products such as gypsum $\left(\mathrm{CaSO}_{4} \cdot 2 \mathrm{H}_{2} \mathrm{O}\right)$ introduce soluble sources of $\mathrm{Ca}^{2+}$ to the soil solution, replacing the excess $\mathrm{Na}^{+}$on the exchange complex. On the other hand, the amelioration of sodic soils can be also done through the introduction of plants, also called phytoremediation (Qadir et al., 2007). This remediation is based on the ability of plants to increase the dissolution of calcite, which can result in enhanced levels of $\mathrm{Ca}^{+2}$ in the system to replace $\mathrm{Na}^{+}$on the cation exchange complex. The amelioration of sodic soils through only natural leaching is considered too slow to be economically viable, due to the inherent reduced infiltration capacity of sodic soils; therefore, an external source of $\mathrm{Ca}^{+2}$ and an initial improvement of physical properties by e.g. organic amendments are necessary for soil remediation (Qadir et al., 2001).

\section{Range of applicability}

Gypsum is being applied worldwide in crop fields to ameliorate soil conditions. In sodic soils, gypsum is used to replace $\mathrm{Na}^{+}$ions with $\mathrm{Ca}^{2+}$. Using gypsum instead of lime has the advantage of a higher solubility -and therefore its effects are faster in deeper layers, and also do not cause $\mathrm{CO}_{2}$ emissions to the atmosphere. Gypsum has a greater benefit to soil health when applied together with other conservation agriculture practices such as application of organic amendments, mulching and no-till systems (Vance, Tisdall and McKenzie, 1998). Most of the gypsum amendments are applied as phosphogypsum, a by-product of the production of $\mathrm{P}$ fertilizer from phosphate rock. 


\section{Impact on soil organic carbon stocks}

As the presence of $\mathrm{Na}^{+}$leads to the abovementioned impact on soil disaggregation, it often results in increased runoff and erosion, directly affecting crop growth and yield (Wong et al. 2010). Besides, when soils are also saline, they also present low levels of organic matter due to restricted plant growth and consequent low biomassC inputs. As a result, the soil organic carbon (SOC) stocks are usually lower than adjacent non-degraded areas (Hubble, Isbell and Northcote, 1983).

Soil organic carbon stocks tend to decrease due to sodic conditions, especially due to the deterioration of physical fertility and the occurrence of erosive processes (Wong et al., 2010). Increasing erosion is considered the major driver of organic matter loss in sodic landscapes that reduces organic matter levels. Wong et al. (2008) observed that scalded and eroded soils profiles contained half the SOC stocks $(7.7 \mathrm{t} / \mathrm{ha})$ compared to uneroded unscalded profiles (19.8 t/ha) at the depth of $0-30 \mathrm{~cm}$ in a site in southern NSW, Australia. Sodic soils are also prone to waterlogging, which could restrict soil organic matter decomposition (Keiluweit et al., 2017). However, due to the adverse effects on plant growth, the net effects of sodic conditions tend to be negative for SOC stocks (Wong et al, 2010).

Studies that directly evaluate the SOC dynamics upon gypsum application in sodic soils are relatively rare in the literature since most of the research focusses on its effects on soil salinity in general and soil physical properties (Greene et al., 1988; Qadir et al., 2001; Southard, Shainberg and Singer, 1988). Since direct assessments are scarce, we can predict the impacts on SOC stocks based on properties such as clay dispersion. In this respect, the joint application of organic matter inputs and gypsum is demonstrated to be beneficial for soils as they decreased clay dispersion (Vance, Tisdall and McKenzie, 1998). Yet, the exact mechanism and factors influencing it are still unclear since the net effects of organic amendments on clay dispersion in sodic soils are contrasting in the literature, with studies demonstrating either increases (Gupta and Abrol, 1990) and decreases (Barzegar et al., 1997) of clay dispersion. Gypsum has been shown to reduce cumulative respiration rates in surface layers compared to the addition of organic matter and the addition of organic matter + gypsum (Wong, Dalal and Greene, 2009). However, evaluations of SOC stocks upon gypsum or Ca-based amendments in sodic soils are also rare in the literature. In one of the few examples, Shirale et al. (2017) observed increases of 20.4 percent in SOC stocks due to the application of dhaincha (Sesbania acculeata) as green manure $(6.97 \mathrm{t} / \mathrm{ha})$ in sodic soils, but they did not find a significant effect of gypsum applied alone. Therefore, a higher research focus on SOC stocks in response to remediation of sodic soils by gypsum could enhance our understanding of this practice.

\section{Other benefits of the practice}

\subsection{Improvement of soil properties}

Besides the benefits of reducing soil sodicity, gypsum has a series of positive effects on several soil physical properties in sodic soils. Studies have demonstrated the capacity of gypsum for increasing hydraulic conductivity (Greene et al., 1988), preventing soil crusting (Amezketa, Aragüés and Gazol, 2005), decreasing bulk density (Southard, Shainberg and Singer, 1988), and ameliorating soil water infiltration rate (Frenkel, Gerstl and Alperovitch, 1989). 


\subsection{Minimization of threats to soil functions}

Table 100. Soil threats

\begin{tabular}{|c|c|}
\hline \multicolumn{2}{|l|}{ Soil threats } \\
\hline Soil erosion & $\begin{array}{l}\text { Sodic soils often experience erosion due to higher erodibility due to clay } \\
\text { dispersion (Wong et al., 2008). Therefore, the amelioration of these soils } \\
\text { by gypsum application can contribute to reducing erosion problems. }\end{array}$ \\
\hline $\begin{array}{l}\text { Nutrient imbalance } \\
\text { and cycles }\end{array}$ & $\begin{array}{l}\text { Through the benefits of gypsum in promoting the remediation of sodic } \\
\text { soils, it can contribute to enhancing plant development and nutrient } \\
\text { cycles (Qadir et al., 2001). }\end{array}$ \\
\hline $\begin{array}{l}\text { Soil salinization and } \\
\text { alkalinization }\end{array}$ & $\begin{array}{l}\text { Gypsum is considered a major amendment in the amelioration of sodic } \\
\text { soils by replacing Na with Ca in the exchange complex (Qadir et al., 2001). }\end{array}$ \\
\hline Soil acidification & $\begin{array}{l}\text { Although gypsum has no significant effect on increasing soil } \mathrm{pH} \text { in acidic } \\
\text { soils, it has an enhanced capacity of reducing } \mathrm{Al}^{+3} \text { (potential acidity) in } \\
\text { deeper layers (Caires et al., 2002). }\end{array}$ \\
\hline Soil compaction & $\begin{array}{l}\text { Gypsum could potentially help to resolve sealing and compaction } \\
\text { problems, due to its capacity of improving soil physical properties such as } \\
\text { aggregation and porosity (Greene et al., 1988). }\end{array}$ \\
\hline $\begin{array}{l}\text { Soil water } \\
\text { management }\end{array}$ & $\begin{array}{l}\text { Gypsum significantly improves water infiltration capacity in sodic soils } \\
\text { (Frenkel, Gerstl and Alperovitch, 1989), therefore it helps improving soil } \\
\text { water management. }\end{array}$ \\
\hline
\end{tabular}

\subsection{Increases in production (e.g. food/fuel/feed/timber/fibre)}

Gypsum has been largely used to increase crop productivity by ameliorating soil conditions in a range of conditions. Reports can be found in sodic areas where the product application allows crop cultivation by reducing sodicity levels (Qadir et al., 2001).

\subsection{Mitigation of and adaptation to climate change}

As the necessity of food production rises due to population growth, future projections estimate an increase of sodic areas due to the misuse of irrigation practices (Qadir et al., 2007). Therefore, gypsum application can be a crucial practice for the remediation of these conditions in the next decades. 


\subsection{Socio-economic benefits}

Due to the benefits of gypsum application in remediating sodic areas (Qadir et al., 2007), it can be considered an important socio-economic practice to increase farmers' profits worldwide.

\section{Potential drawbacks to the practice}

\subsection{Tradeoffs with other threats to soil functions}

Table 101. Soil threats

\section{Soil threats}

\begin{tabular}{|l|l|}
$\begin{array}{l}\text { Nutrient imbalance } \\
\text { and cycles }\end{array}$ & $\begin{array}{l}\text { Gypsum can lead to leaching of Mg in the soil profile when applied at } \\
\text { higher rates (Caires et al., 2006) }\end{array}$ \\
\hline $\begin{array}{l}\text { Soil contamination / } \\
\text { pollution }\end{array}$ & $\begin{array}{l}\text { Phosphogypsum can be contaminated with over } 50 \text { elements present in } \\
\text { phosphate rock, which can lead to soil contamination if not treated } \\
\text { properly (Alcordo and Rechcigl, 1993). Moreover, the radionuclide } \\
\text { contents of gypsum make it a potential source of contamination (Hentati } \\
\text { et al., 2015). }\end{array}$ \\
\hline $\begin{array}{l}\text { Soil water } \\
\text { management }\end{array}$ & $\begin{array}{l}\text { When contaminated with heavy metals, phosphogypsum can potentially } \\
\text { contaminate groundwater. This is a problem especially in developing } \\
\text { countries, where most of the drinking water comes from surface aquifers } \\
\text { (Alcordo and Rechcigl, 1993) }\end{array}$ \\
\hline
\end{tabular}

\subsection{Increases in greenhouse gas emissions}

When changes in soil pH are not necessary, gypsum could potentially replace lime to ameliorate soil conditions, which could avoid $\mathrm{CO}_{2}$ emissions (Oster and Frenkel, 1980).

\subsection{Decreases in production (e.g. food/fuel/feed/timber/fibre)}

Some negative effects on crop tissue such as the reduction of Molybdenum in Brussel sprouts have also been reported in response to gypsum application (Alcordo and Rechcigl, 1993). 


\subsection{Other conflicts}

Transportation of the gypsum product to regions farm from the production centers can increase the costs of the application.

\section{Recommendations before implementing the practice}

A systematic analysis of soil properties (especially chemical ones) is necessary before gypsum application.

\section{Potential barriers to adoption}

Table 102. Potential barriers to adoption

\begin{tabular}{|c|c|c|}
\hline Barrier & YES/NO & \\
\hline Biophysical & Yes & $\begin{array}{l}\text { Gypsum application induces salt leaching through the soil } \\
\text { profile (Khosla, Gupta and Abrol, 1979), which could potentially } \\
\text { affect desirable nutrients such as } \mathrm{Mg} \text {. This happens mainly in } \\
\text { soils with low cation exchange capacity such as Ferralsols and } \\
\text { Acrisols since } \mathrm{Ca}^{2+} \text { and } \mathrm{Mg}^{2+} \text { compete for negative charges in } \\
\text { the soil. The high concentration of } \mathrm{Ca}^{2+} \text { in the soil caused by } \\
\text { the application of gypsum favors the displacement of } \mathrm{Mg}^{2+} \\
\text { from the exchange sites (Zambrosi et al., 2008). }\end{array}$ \\
\hline $\begin{array}{l}\text { Social and } \\
\text { Cultural }\end{array}$ & Yes & $\begin{array}{l}\text { Farmers often are wary of nutrient leaching due to gypsum } \\
\text { applications, which make them more skeptical to apply the } \\
\text { amendment especially in highly weathered soils. }\end{array}$ \\
\hline Economic & Yes & $\begin{array}{l}\text { Besides the price of the product itself, the costs of } \\
\text { transportation to areas far from the production center can } \\
\text { make the practice inviable for some farmers. }\end{array}$ \\
\hline Institutional & Yes & $\begin{array}{l}\text { Lack of professionals who understand the practice can be an } \\
\text { obstacle to its recommendation. }\end{array}$ \\
\hline Knowledge & Yes & $\begin{array}{l}\text { Understanding of the problem and interpretating soil and } \\
\text { water analysis to determine the proper gypsum and organic } \\
\text { amendment doses are necessary. }\end{array}$ \\
\hline
\end{tabular}




\section{References}

Abrol, I., Yadav, J.S.P. \& Massoud, F. 1988. Salt-affected soils and their management. FAO, Rome. (also available at: http://www.fao.org/3/x5871e/x5871e00.htm)

Alcordo, I.S. \& Rechcigl, J.E. 1993. Phosphogypsum in agriculture: A review. Advances in Agronomy, 49: 55-118. https://doi.org/10.1016/S0065-2113(08)60793-2

Amezketa, E., Aragüés, R. \& Gazol, R., 2005. Efficiency of sulfuric acid, mined gypsum, and two gypsum by-products in soil crusting prevention and sodic soil reclamation. Agronomy Journal, 97: 983-989. https://doi.org/10.2134/agronj2004.0236

Barzegar, A.R., Nelson, P.N., Oades, J.M. \& Rengasamy, P. 1997. Organic matter, sodicity, and clay type: Influence on soil aggregation. Soil Science Society of America Journal, 61: 1131-1137. https://doi.org/10.2136/sssaj1997.03615995006100040020x

Caires, E.F., Feldhaus, I.C., Barth, G. \& Garbuio, F.J. 2002. Lime and gypsum application on the wheat crop. Scientia Agricola, 59: 357-364. https://doi.org/10.1590/S0103-90162002000200023

Caires, E.F., Churka, S., Garbuio, F.J., Ferrari, R.A. \& Morgano, M.A. 2006. Soybean yield and quality a function of lime and gypsum applications. Scientia Agricola, 63(4): 370-379.

https://doi.org/10.1590/S0103-90162006000400008

Frenkel, H., Gerstl, Z. \& Alperovitch, N. 1989. Exchange-induced dissolution of gypsum and the reclamation of sodic soils. Journal of Soil Science, 40: 599-611. https://doi.org/10.1111/j.13652389.1989.tb01301.x

Greene, R., Rengasamy, P., Ford, G., Chartres, C. \& Millar, J. 1988. The effect of sodium and calcium on physical properties and micromorphology of two red-brown earth soils. Journal of Soil Science, 39: 639-648. https://doi.org/10.1111/j.1365-2389.1988.tb01246.x

Gupta, R.K. \& Abrol, I.P. 1990. Salt-Affected Soils: Their Reclamation and Management for Crop Production. In R. Lal \& B.A. Stewart (Eds.) Advances in Soil Science: Soil Degradation Volume 11, pp. 223288. Advances in Soil Science. New York, NY, Springer. https://doi.org/10.1007/978-1-4612-3322-0_7

Hentati, O., Abrantes, N., Caetano, A.L., Bouguerra, S., Gonçalves, F., Römbke, J. \& Pereira, R.

2015. Phosphogypsum as a soil fertilizer: ecotoxicity of amended soil and elutriates to bacteria, invertebrates, algae and plants. Journal of hazardous materials, 294: 80-89.

https://doi.org/10.1016/j.jhazmat.2015.03.034

Hubble, G., Isbell, R. \& Northcote, K. 1983. Features of Australian soils. In Soils: an Australian viewpoint. Division of Soils, CSIRO. CSIRO Melbourne/Academic Press, London, pp. 17-46.

Keiluweit, M., Wanzek, T., Kleber, M., Nico, P. \& Fendorf, S. 2017. Anaerobic microsites have an unaccounted role in soil carbon stabilization. Nature Communication, 8: 1771.

https://doi.org/10.1038/s41467-017-01406-6 
Khosla, B., Gupta, R. \& Abrol, I. 1979. Salt leaching and the effect of gypsum application in a saline-sodic soil. Agricultural Water Management, 2: 193-202. https://doi.org/10.1016/0378-3774(79)90002-7

McIntyre, D. 1958. Permeability measurements of soil crusts formed by raindrop impact. Soil science, 85: 185-189.

Oster, J. \& Frenkel, H. 1980. The chemistry of the reclamation of sodic soils with gypsum and lime. Soil Science Society of America Journal, 44: 41-45.

https://doi.org/10.2136/sssaj1980.03615995004400010010x

Qadir, M., Schubert, S., Ghafoor, A. \& Murtaza, G. 2001. Amelioration strategies for sodic soils: a review. Land Degradation and Development, 12: 357-386. https://doi.org/10.1002/ldr.458

Qadir, M., Oster, J., Schubert, S., Noble, A. \& Sahrawat, K. 2007. Phytoremediation of sodic and salinesodic soils. Advances in agronomy, 96: 197-247. https://doi.org/10.1016/S0065-2113(07)96006-X

Shirale, A.O., Kharche, V.K., Zadode, R.S., Meena, B.P. \& Rajendiran, S. 2017. Soil biological properties and carbon dynamics subsequent to organic amendments addition in sodic black soils. Archives of Agronomy and Soil Science, 63: 2023-2034. https://doi.org/10.1080/03650340.2017.1322194.

Singer, M.J. \& Shainberg, I. 2004. Mineral soil surface crusts and wind and water erosion. Earth Surface Processes and Landforms, 29(9): 1065-1075. https://doi.org/10.1002/esp.1102

Southard, R., Shainberg, I. \& Singer, M. 1988. Influence of electrolyte concentration on the micromorphology of artificial depositional crust. Soil Science, 145: 278-288.

Vance, W., Tisdall, J. \& McKenzie, B. 1998. Residual effects of surface applications of organic matter and calcium salts on the subsoil of a red-brown earth. Australian Journal of Experimental Agriculture, 38: 595600. https://doi.org/10.1071/EA97102

Wong, V.N.L., Dalal, R.C. \& Greene, R.S.B. 2009. Carbon dynamics of sodic and saline soils following gypsum and organic material additions: A laboratory incubation. Applied Soil Ecology, 41: 29-40. https://doi.org/10.1016/j.apsoil.2008.08.006

Wong, V.N., Greene, R., Dalal, R.C. \& Murphy, B.W. 2010. Soil carbon dynamics in saline and sodic soils: a review. Soil use and management, 26: 2-11. https://doi.org/10.1111/j.1475-2743.2009.00251.x

Wong, V.N., Murphy, B.W., Koen, T.B., Greene, R.S. \& Dalal, R.C. 2008. Soil organic carbon stocks in saline and sodic landscapes. Soil Research, 46: 378-389. https://doi.org/10.1071/SR07160

Zambrosi, F.C.B.; Alleoni, L.R.F. \& Caires, E.F. 2008. Liming and ionic speciation of an Oxisol under no-till system. Scientia Agricola., 65: 190-203. https://doi.org/10.1590/S0103-90162008000200013 
CROPLAND

SOIL AND WATER CONSERVATION TECHNIQUES

EROSION CONTROL TECHNIQUES

\title{
23. Terraces
}

\author{
John N Quinton
}

Lancaster Environment Centre, Lancaster University, Lancaster, UK

\section{Description of the practice}

Terraces are a soil and water conservation practice which are typically deployed on steep slope and found throughout the world (Wei et al., 2016). They are human-made, often aligned with or slightly off the contour and create steps in the landscape. These steps comprise of two components: a riser and a shelf. The riser can be angled or vertical, but it is always steeper than the shelf. The shelf can be flat but can also slope either towards or away from the riser (Table 103). They can be constructed gradually by allowing soil to accumulated behind stones or vegetation (progressive terraces) or by cutting and filling the slope using machines or human labour. Labour costs can be high during the construction phase and maintenance is essential further increasing labor requirements. Terraces are used to support a wide range of land uses including forestry, grazing, agroforestry, crop and vegetable production and rice paddies. Terrace failure, often due to lack of maintenance, can result in slope instability and significant erosion. 
Table 103. Styles of terracing

\begin{tabular}{l|l}
$\begin{array}{l}\text { Terrace } \\
\text { type }\end{array}$ & $\begin{array}{l}\text { Description } \\
\text { downslope used to intercept } \\
\text { overland flow and divert it to a } \\
\text { grass-waterway or drain. Can be } \\
\text { a) broad-based (15m width) or b) } \\
\text { narrow-based (3-4 m width) }\end{array}$ \\
Diversion \\
terraces
\end{tabular}

Source: Adapted from Morgan (2005) 


\section{Range of applicability}

Terraces have a long history, with some historical examples still functioning many hundreds of years after their construction. They have been adapted for a wide range of environments and cultural settings resulting a large of number of terrace designs (Photo 29). Terraces form an important part of humankind's cultural heritage with over 70 UNESCO listed world heritage sites having terraces as part of their designation (UNESCO, 2020) and have played an important role is supporting agriculture for civilizations such as the Incas and the Ancient Greeks. Today they can be found on every continent (except for Antarctica) and are still being constructed across the globe.

\section{Impact on soil organic carbon stocks}

Studies which investigate carbon stocks produced contrasting results. De Blécourt et al., (2014) investigated a chronosequence of terraces within rubber plantations in Yunnan, China and found that after 29- and 44-years stocks had significantly increased in terraced slopes, but that there was no difference in the five-year-old plantation. However, a survey of carbon densities to $60 \mathrm{~cm}$ depth in Yujiehe catchment (Xu et al., 2015) and similar work in the Wangmaogou catchment with sampling to $100 \mathrm{~cm}$ (Zhao et al., 2017), both in Shaanxi Province, China, found no significant difference between terraces, and the surrounding land uses. A similar finding was found by Garcia-Franco et al. (2014), who found no significant difference between the terraced areas and the surrounding shrub covered landscape, which they attributed to the impact of soil disturbance during terrace construction. There are a number of other studies that focus on changes in carbon concentrations (e.g. Chen et al. (2020)), which, while important for soil functioning, carbon concentrations do not allow an assessment of carbon sequestration potential.

Carbon stocks were greatest close to the terrace riser on terraces with sloping benches (De Blécourt et al, 2014), a finding supported by work showing increases in SOM concentrations in the same location, which is attributed to the deposition of organic material from upslope (Amare et al., 2013; Kagabo et al., 2013; Li and Lindstrom, 2001).

\section{Other benefits of the practice}

\subsection{Improvement of soil properties}

Terraces have the potential to influence a range of soil properties (Posthumus and Stroosnijder, 2010). As erosion and runoff are reduced (compared to non-terraced areas) soils tend to be deeper and store more water. This leads to greater crop productivity, although this is known to be variable, especially on outward sloping benches where material moves from the back to the front of the bench (Zhang, Wang and Li, 2015) in response to water and tillage erosion, with better yields found close to the riser (Amare et al, 2013). Variable crop production is likely to lead to variable returns of crop biomass to the soil leading to variable soil properties across the bench and there is some evidence that farmers are responding to this by treating areas of the bench differently (Kagabo et al., 2013). 


\subsection{Minimization of threats to soil functions}

Table 104. Soil threats

\begin{tabular}{|c|c|}
\hline Soil threats & \\
\hline Soil erosion & $\begin{array}{l}\text { The reduction of soil erosion due to terracing is well known. Terraces } \\
\text { reduce the slope angle which reduces the velocity of overland flow and } \\
\text { therefore soil detachment. In addition, the slope length is reduced, thus } \\
\text { reducing the accumulation and therefore the depth of overland flow and } \\
\text { its velocity, again reducing soil detachment. As tillage takes place on the } \\
\text { contour, tillage erosion is also reduced. }\end{array}$ \\
\hline $\begin{array}{l}\text { Nutrient } \\
\text { imbalance and } \\
\text { cycles }\end{array}$ & $\begin{array}{l}\text { Soil erosion has a significant impact on soil biogeochemical cycles } \\
\text { (Quinton et al., 2O1O). Terraces reduce erosion and the movement of N, } \\
\mathrm{P} \text { and K. Depending on terrace design and construction methods, N, P } \\
\text { and K concentrations can be variable across the shelf with highest } \\
\text { concentrations found closest to the riser. }\end{array}$ \\
\hline $\begin{array}{l}\text { Soil water } \\
\text { management }\end{array}$ & $\begin{array}{l}\text { Terraces are often designed to capture and retain water (retention } \\
\text { terraces, shown in Table 103). They hold more water than the } \\
\text { surrounding areas and improve the availability of water to plants (Rashid } \\
\text { et al., 2016) }\end{array}$ \\
\hline
\end{tabular}

\section{3. (Increases in production (e.g. food/fuel/feed/timber/fibre)}

Terraces lead to greater crop productivity (Rashid et al., 2016) attributed to greater water and nutrient conservation. Yields are known to be variable, especially on outward sloping benches where material moves from the back to the front of the bench in response to water and tillage erosion, with better yields found close to the riser (Amare et al., 2013).

\subsection{Mitigation of and adaptation to climate change}

Terraces have an important role to play in climate change mitigation. They help to protect the soil resource against extreme rainfall (see above). By reducing erosion terraces reduce decreases in soil depth thus protecting the soil's ability to store water (Rashid et al., 2016) and increase resilience to floods and droughts. 


\subsection{Socio-economic benefits}

Terraces can be expensive and labour intensive to install and maintain. The significant investment involved in building and maintaining terraces makes their adoption by farmers limited to locations where either there has been government investment in terracing or where farmers they can foresee a good rate of return from their investment (Gebremedhin and Swinton, 2003). Cheaper and less labour intensive progressive terracing is possible, for example through the planting of grass barriers on the contour.

\section{Potential drawbacks to the practice}

\subsection{Tradeoffs with other threats to soil functions}

\section{Table 105. Soil threats}

\begin{tabular}{|c|c|}
\hline Soll threats & \\
\hline Soil erosion & $\begin{array}{l}\text { If terraces are not maintained erosion can increase. Vegetation can } \\
\text { provide protection against surface erosion but terrace failure can lead to } \\
\text { significant mass movements or soil piping (Moreno-de-las-Heras et al., } \\
\text { 2019). }\end{array}$ \\
\hline $\begin{array}{l}\text { Soil salinization } \\
\text { and alkalinization }\end{array}$ & $\begin{array}{l}\text { Since the appearance of salinity is often depending on the } \\
\text { microtopography, the construction of terraces may change the spatial } \\
\text { pattern of salinity patches in salt-affected soils (e.g. Endo et al., 2012); or } \\
\text { leave salt-containing horizons or layers near the surface. }\end{array}$ \\
\hline Soil compaction & $\begin{array}{l}\text { When terraces are built by a landcut/landfill process, compacted layers } \\
\text { may appear at the surface close to the shelf, creating compacity } \\
\text { gradients from the rise to the shelf (Zhu and Li, 2000). }\end{array}$ \\
\hline
\end{tabular}

\subsection{Increases in greenhouse gas emissions}

There is very limited data on nitrous oxide or methane emissions from terraces, making it hard to draw any conclusions. 


\section{Recommendations before implementing the practice}

Terracing is primarily a practice for controlling erosion and conserving soil water. They may bring about benefits for carbon sequestration through improvements to crop yield or using other soil conservation practices in association with them. Terrace construction requires significant investment and labour and maintenance costs are high. Therefore, terracing is best suited to areas where there is a significant threat to the soil resource from erosion or where there is need to intercept surface runoff to improve the availability and where other soil and water conservation approaches are unsuitable.

\section{Potential barriers to adoption}

Following Gebremedhin and Swinton (2003) and Runezerwa (2011)

Table 106. Potential barriers to adoption

\begin{tabular}{|l|l|l|}
\hline Barrier & YES/NO & \\
\hline Biophysical & Yes & $\begin{array}{l}\text { Best suited for steep slopes vulnerable to erosion or where water } \\
\text { conservation is important. }\end{array}$ \\
\hline Cultural & No & $\begin{array}{l}\text { There are unlikely to be significant cultural barriers to terrace } \\
\text { adoption. }\end{array}$ \\
\hline Social & Yes & $\begin{array}{l}\text { Capacity to invest, influenced by family size, gender balance, } \\
\text { labour availability, off-farm income. }\end{array}$ \\
\hline Economic & Yes & $\begin{array}{l}\text { Considerable financial investment required, less for progressive } \\
\text { terraces. Financial returns important. }\end{array}$ \\
\hline Institutional & Yes & External financial involvement may be required for construction. \\
\hline $\begin{array}{l}\text { Legal (Right } \\
\text { to soil) }\end{array}$ & Yes & \begin{tabular}{l} 
Secure land tenure. \\
\hline Knowledge
\end{tabular} \\
\hline
\end{tabular}




\section{Photos of the practice}

a)
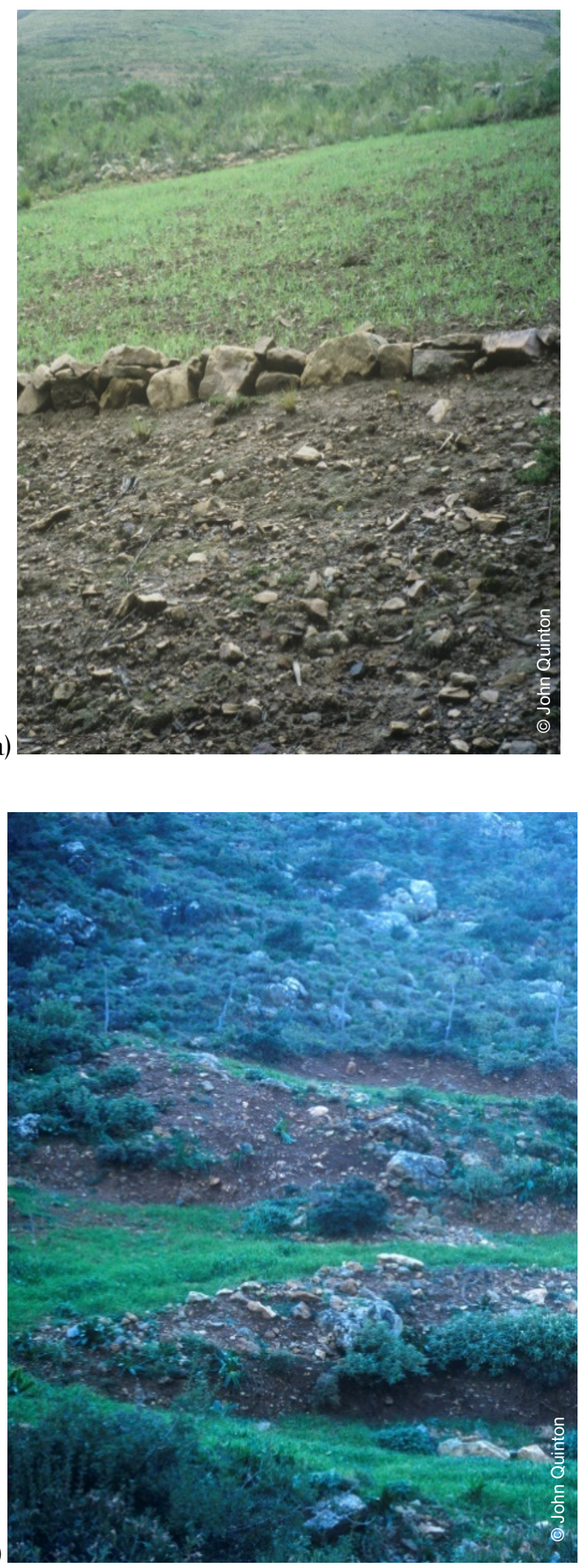

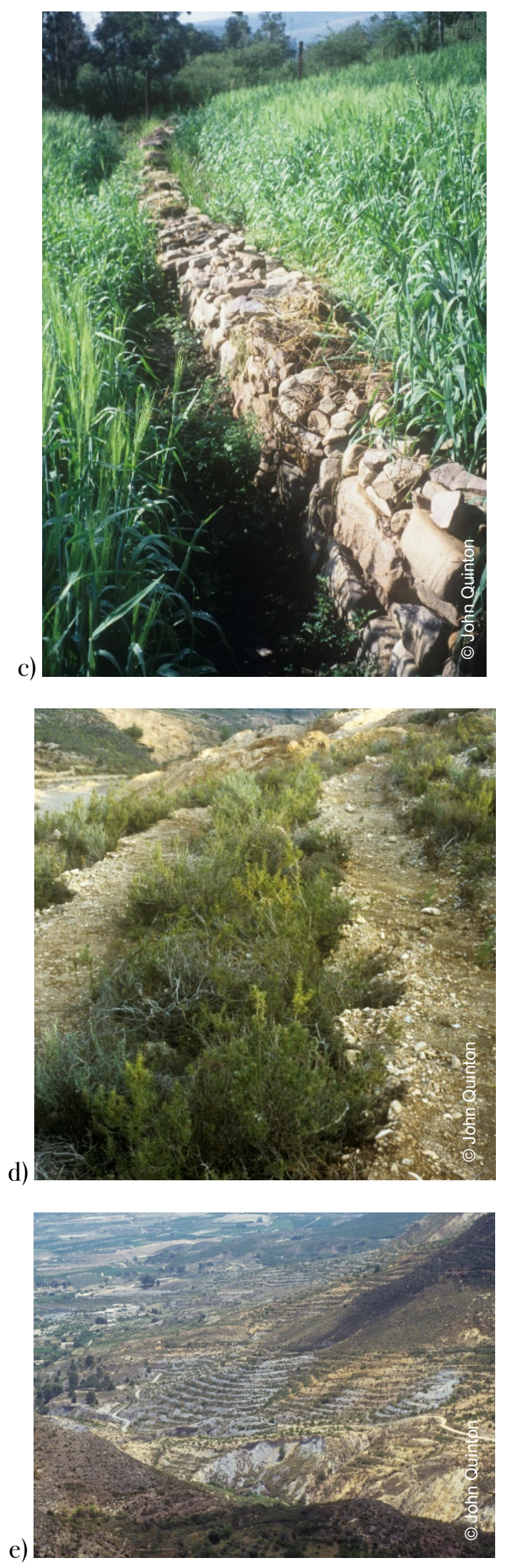

Photo 29. Examples of terraces

a) Progressive terrace (stone wall across slope accumulating soil) (the Plurinational State of Bolivia); b) zig zag horizontally sloping terraces (Greece); c) a vertical riser with an outward sloping bench (the Plurinational State of Bolivia); d) Level benches with vegetated sloping risers (Spain) e) terraced hillside (Morocco). 
Table 107. Related cases studies available in volumes 3 and 5

\begin{tabular}{|l|l|c|c|c|}
\hline Title & Region & $\begin{array}{l}\text { Duration } \\
\text { of study } \\
\text { (Years) }\end{array}$ & Volume & $\begin{array}{l}\text { Case- } \\
\text { study } \\
\text { No. }\end{array}$ \\
\hline $\begin{array}{l}\text { Application of mulching in subtropical } \\
\text { orchards in Granada, Spain }\end{array}$ & Europe & 5 & 3 & 20 \\
\hline $\begin{array}{l}\text { Afforestation by planting in bench } \\
\text { terraces: Kalimanska watershed, Grdelica } \\
\text { gorge, Southeastern Serbia }\end{array}$ & Europe & 60 & 5 & 8 \\
\hline
\end{tabular}




\section{References}

Amare, T., Terefe, A., Selassie, Y.G., Yitaferu, B., Wolfgramm, B. \& Hurni, H. 2013. Soil Properties and Crop Yields along the Terraces and Toposequece of Anjeni Watershed, Central Highlands of Ethiopia. Journal of Agricultural Science, 5(2): p134. https://doi.org/10.5539/jas.v5n2p134.

de Blécourt, M., Hänsel, V.M., Brumme, R., Corre, M.D. \& Veldkamp, E. 2014. Soil redistribution by terracing alleviates soil organic carbon losses caused by forest conversion to rubber plantation. Forest Ecology and Management, 313: 26-33. https://doi.org/10.1016/j.foreco.2013.10.043

Chen, D., Wei, W., Daryanto, S. \& Tarolli, P. 2020. Does terracing enhance soil organic carbon sequestration? A national-scale data analysis in China. Science of The Total Environment, 721: 137751. https://doi.org/10.1016/j.scitotenv.2020.137751

Endo, T., Sadahiro, Y., Haruta, T., Kitamura, Y., Li, Z., Li, P. \& Honna, T. 2012. Soil salinization related to soil morphological and physicochemical characteristics in the Luohui irrigation scheme, China. Arid Land Research and Management, 26(2): 122-136. https://doi.org/10.1080/15324982.2012.656180

Garcia-Franco, N., Wiesmeier, M., Goberna, M., Martínez-Mena, M. \& Albaladejo, J. 2014. Carbon dynamics after afforestation of semiarid shrublands: Implications of site preparation techniques. Forest Ecology and Management, 319: 107-115. https://doi.org/10.1016/j.foreco.2014.01.043

Gebremedhin, B. \& Swinton, S.M. 2003. Investment in soil conservation in northern Ethiopia: the role of land tenure security and public programs. Agricultural economics, 29(1): 69-84.

https://doi.org/10.1016/S0169-5150(03)00022-7

Kagabo, D.M., Stroosnijder, L., Visser, S.M. \& Moore, D. 2013. Soil erosion, soil fertility and crop yield on slow-forming terraces in the highlands of Buberuka, Rwanda. Soil and Tillage Research, 128: 23-29. https://doi.org/10.1016/j.still.2012.11.002

Li, Y. \& Lindstrom, M.J. 2001. Evaluating Soil Quality-Soil Redistribution Relationship on Terraces and Steep Hillslope. Soil Science Society of America Journal, 65(5): 1500-1508.

https://doi.org/10.2136/sssaj2001.6551500x

Moreno-de-las-Heras, M., Lindenberger, F., Latron, J., Lana-Renault, N., Llorens, P., Arnáez, J., Romero-Díaz, A. \& Gallart, F. 2019. Hydro-geomorphological consequences of the abandonment of agricultural terraces in the Mediterranean region: Key controlling factors and landscape stability patterns. Geomorphology, 333: 73-91. https://doi.org/10.1016/j.geomorph.2019.02.014

Morgan, R.P.C. 2005. Soil Erosion and Conservation. Hoboken, United Kingdom, John Wiley \& Sons, Incorporated. (also available at: http://ebookcentral.proquest.com/lib/lancaster/detail.action?docID=233037).

Posthumus, H. \& Stroosnijder, L. 2010. To terrace or not: the short-term impact of bench terraces on soil properties and crop response in the Peruvian Andes. Environment, Development and Sustainability, 12(2): 263-276. https://doi.org/10.1007/s10668-009-9193-4 
Quinton, J.N., Govers, G., Van Oost, K. \& Bardgett, R.D. 2010. The impact of agricultural soil erosion on biogeochemical cycling. Nature Geoscience, 3(5): 311-314. https://doi.org/10.1038/ngeo838

Rashid, M., Rehman, O. ur, Alvi, S., Kausar, R. \& Akram, M.I. 2016. The effectiveness of soil and water conservation terrace structures for improvement of crops and soil productivity in rainfed terraced system.

Pakistan Journal of Agricultural Sciences, 53(01): 241-248.

https://doi.org/10.21162/PAKJAS/16.1502

Runezerwa, A.B. 2011. Farmers, institutions and land conservation: institutional economic analysis of bench terraces in the highlands of Rwanda. Waganingen. $(\mathrm{PhD})$

UNESCO. 2020. UNESCO World Heritage Centre - World Heritage List. In: UNESCO World Heritage Centre [online]. [Cited 23 June 2020]. https://whc.unesco.org/en/list/?search=terraces\&order=country

Wei, W., Chen, D., Wang, L., Daryanto, S., Chen, L., Yu, Y., Lu, Y., Sun, G. \& Feng, T. 2016. Global synthesis of the classifications, distributions, benefits and issues of terracing. Earth-Science Reviews, 159: 388-403. https://doi.org/10.1016/j.earscirev.2016.06.010

Xu, G., Lu, K., Li, Z., Li, P., Wang, T. \& Yang, Y. 2015. Impact of soil and water conservation on soil organic carbon content in a catchment of the middle Han River, China. Environmental Earth Sciences, 74(8): 6503-6510. https://doi.org/10.1007/s12665-015-4749-0

Zhang, J.H., Wang, Y. \& Li, F.C. 2015. Soil organic carbon and nitrogen losses due to soil erosion and cropping in a sloping terrace landscape. Soil Research, 53(1): 87. https://doi.org/10.1071/SR14151

Zhao, B., Li, Z., Li, P., Xu, G., Gao, H., Cheng, Y., Chang, E., Yuan, S., Zhang, Y. \& Feng, Z. 2017. Spatial distribution of soil organic carbon and its influencing factors under the condition of ecological construction in a hilly-gully watershed of the Loess Plateau, China. Geoderma, 296: 10-17.

https://doi.org/10.1016/j.geoderma.2017.02.010

Zhu, J. \& Li, J. 2000. Experimental study on soil compaction characteristics and shearing strength when changing sloping fields into terraced land in South Shaanxi Province [China]. Transactions of the Chinese Society of Agricultural Engineering, 16(2): 36-40. https://doi.org/0.3303/CET1866085 


\title{
24. Check dams
}

\author{
John N Quinton
}

Lancaster Environment Centre, Lancaster University. Lancaster, UK

\section{Description of the practice and Range of applicability}

In this section we discuss check dams deployed as a gully erosion control measures and not small-scale dams used to dam rivers and provide water for irrigation or groundwater recharge. Gully control check dams are human-made structures built across the channel with the objective of reducing flow velocities and promoting the deposition of sediments (Photo 30Photo 30). They should consist of a dam which is concave in plan form (looking downstream), spillway and apron, although this is not always the case in practice. They range in size from a few meters in width and less than a meter high to structures which may exceed widths and heights of 10 $\mathrm{m}$. They are deployed in series along a gully with a negative slope between the spillway and the foot of the upstream dam. This creates a stepped profile as sediment is deposited behind the dam. Careful consideration of check dam spacing is required and is a function of slope and contributing area (Nyssen et al., 2004) and needs to be adjusted for local conditions (see Morgan (2005) for further details of check dam designs). Check dams are constructed using a range of materials including timber, brushwood, rock, gabions and concrete and range from simple structures designed and built by non-engineers to larger more complex construction projects requiring significant civil engineering input (Photo 30). Costs are proportional to their size and amount of external input required. In addition, all check dams require constant attention and maintenance as failure rates can be high (Nyssen et al., 2004). 


\section{Impact on soil organic carbon stocks}

Studies of carbon storage in behind check dams show that while significant amounts of carbon are trapped, the sediments are impoverished in carbon compared to the surrounding soils (Addisu and Mekonnen, 2019; BoixFayos et al., 2017). The longevity of the check dam carbon store is unknown. Although it is improbable that check dam sediments are sequestering additional carbon, it is likely that when compared to a gully system without check dams, carbon export from a catchment with check dams will be lower. Therefore, the overall carbon stocks of a gullied catchment with check dams is likely to be higher than one without. Combining check dams with revegetation of the gully may lead to an increase in catchment carbon stocks, but, to date, there is no evidence to support this in the literature.

\section{Other benefits of the practice}

\subsection{Improvement of soil properties}

Check dams to not directly impact on soil properties since they are measures directed at channels. However, by fixing the base level of the channel, they may stabilize channel side slopes, reducing the frequency of mass movements.

\subsection{Minimization of threats to soil functions}

Table 108. Soil threats

\section{Soil threats}

\begin{tabular}{|l|l|}
\hline Soil erosion & $\begin{array}{l}\text { Well-designed check dams can reduce gully erosion by reducing flow } \\
\text { velocities promoting deposition. }\end{array}$ \\
\hline $\begin{array}{l}\text { Nutrient imbalance and } \\
\text { cycles }\end{array}$ & $\begin{array}{l}\text { Check dams can trap nutrients that would otherwise end up in the fluvial } \\
\text { system. }\end{array}$ \\
\hline $\begin{array}{l}\text { Soil salinization and } \\
\text { alkalinization }\end{array}$ & $\begin{array}{l}\text { Check dams can alter the position of the local water table and there } \\
\text { may therefore be some effect on salinization and alkalinization, but } \\
\text { there is currently no research in this area. }\end{array}$ \\
\hline Soil water management & $\begin{array}{l}\text { Modelling studies indicate that check dams may increase groundwater } \\
\text { recharge and reduce runoff losses (Martin-Rosales et al., 2007). }\end{array}$ \\
\hline
\end{tabular}




\subsection{On production}

Gullies can lead to the total destruction of agricultural land; therefore, their control can have significant benefits to crop production. In addition, the author has observed farmers planting crops behind check dams, presumably due to land pressures or because greater water and nutrient availability leads to high crop yields offsetting the risk of losing the entire crop to a storm event. Check dams also have the potential to increase infiltration which may lead to greater water availability in the catchment although empirical evidence for this is hard to find.

\subsection{Mitigation of and adaptation to climate change}

Check dams can mitigate the impacts of flood events associated with increased frequency of storms. In addition, improved water infiltration behind the dam may increase catchment water availability and mitigate the impacts of droughts. Check dams can also reduce or eliminate the encroachment of gullies. As intact soils support climate related ecosystem services and help to mitigate the effects of climate change check dams have an indirect benefit for climate change mitigation and adaptation

\subsection{Socio-economic aspects}

Check dams range in cost depending on their size and complexity. Their construction has often been led by actors other than farmers, for example the large scale programmes of construction in Ethiopia and China (Bewket, 2007; Liu et al, 2017). Check dams are vulnerable to damage from storm events and need to be maintained regularly. Maintenance is a significant issue with Nyssen et al. (2004) estimating that 39 percent of 400 stone check dams had collapsed after two years in a catchment in Tigray, Norther Ethiopia. 


\section{Potential drawbacks to the practice}

\subsection{Tradeoffs with other threats to soil functions}

Table 109. Soil threats

\begin{tabular}{|l|l|}
\hline Soil threats & \\
\hline Soil erosion & $\begin{array}{l}\text { The failure of check dams can release large volumes of stored } \\
\text { sediments into the fluvial system. Cascade failures, when the } \\
\text { failure of one check dam leads to the failure of others } \\
\text { downstream are also possible, although this is not well } \\
\text { documented. }\end{array}$ \\
\hline
\end{tabular}

\subsection{Increases in greenhouse gas emissions}

There are no data on nitrous oxide or methane emissions from check dams making it hard to draw any conclusions one way or another.

\section{Recommendations before implementing the practice}

Check dams are primarily a practice for controlling gully erosion. They may bring about benefits for carbon sequestration by trapping sediments that would otherwise be lost to the fluvial system. Check dam construction and maintenance requires significant investment, careful design and labour. They are therefore best suited to areas where there is a significant threat to the soil resource from gully erosion and where other less soil and water conservation approaches are unsuitable.

\section{Potential barriers to adoption}

Following Gebremedhin and Swinton (2003) and Bewkett (2007) 
Table 110. Potential barriers to adoption

\begin{tabular}{|c|c|c|}
\hline Barrier & YES/NO & \\
\hline Biophysical & Yes & $\begin{array}{l}\text { Best suited for gullied slopes where other approaches to erosion } \\
\text { control are unsuitable. }\end{array}$ \\
\hline Cultural & Yes & $\begin{array}{l}\text { Check dams should be well designed if they are to function well. } \\
\text { Farmers may require advice to help plan their construction and } \\
\text { implementation. }\end{array}$ \\
\hline Social & Yes & $\begin{array}{l}\text { Check dams can be expensive to construct and maintain. The } \\
\text { capacity to invest in check dams is influenced by family size, } \\
\text { gender balance, labour availability, and off-farm income. }\end{array}$ \\
\hline Economic & Yes & $\begin{array}{l}\text { Considerable financial investment is required for construction } \\
\text { and ongoing maintenance costs. The financial returns to the } \\
\text { landowner are unclear. }\end{array}$ \\
\hline Institutional & Yes & $\begin{array}{l}\text { External financial involvement may be required for construction } \\
\text { meaning that external institutions become involved. It is } \\
\text { important that such Institutions do not ignore farmer views and } \\
\text { local knowledge. }\end{array}$ \\
\hline $\begin{array}{l}\text { Legal (Right } \\
\text { to soil) }\end{array}$ & Yes & $\begin{array}{l}\text { Insecure land tenure reduces opportunities for investment and } \\
\text { heightens suspicion surrounding institutional motives. }\end{array}$ \\
\hline Knowledge & Yes & $\begin{array}{l}\text { Learning opportunities from other farmers and other actors are } \\
\text { important for developing best practice. }\end{array}$ \\
\hline
\end{tabular}




\section{Photos of the practice}

a)
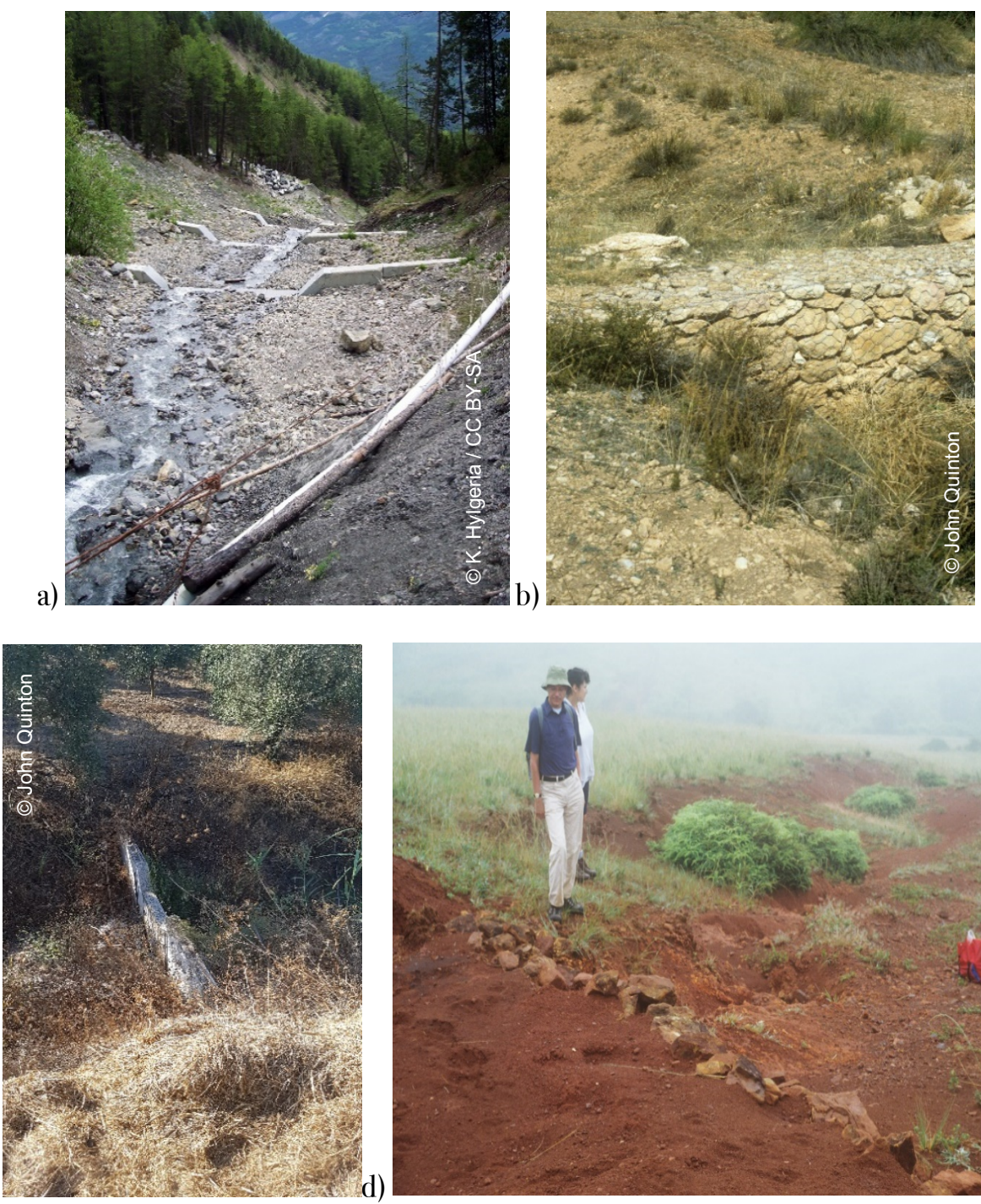

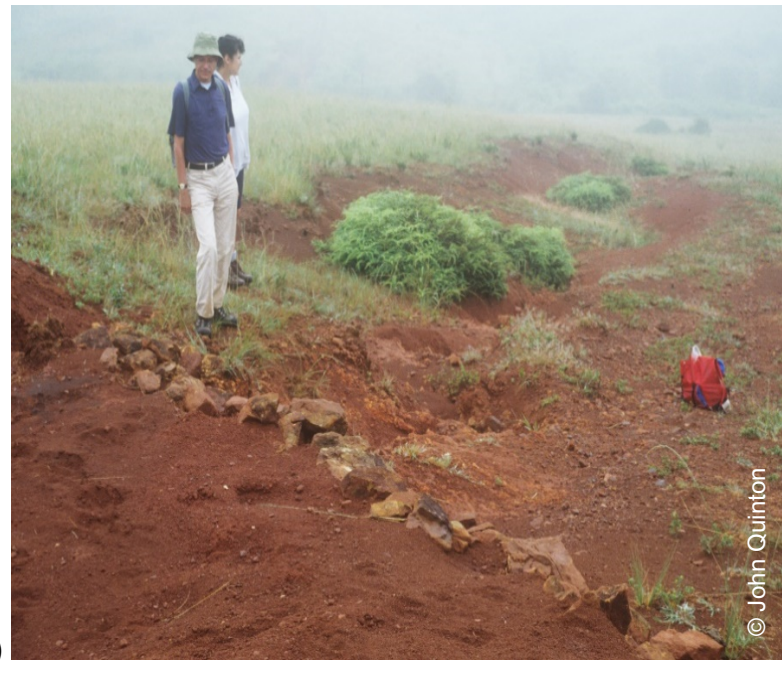

)

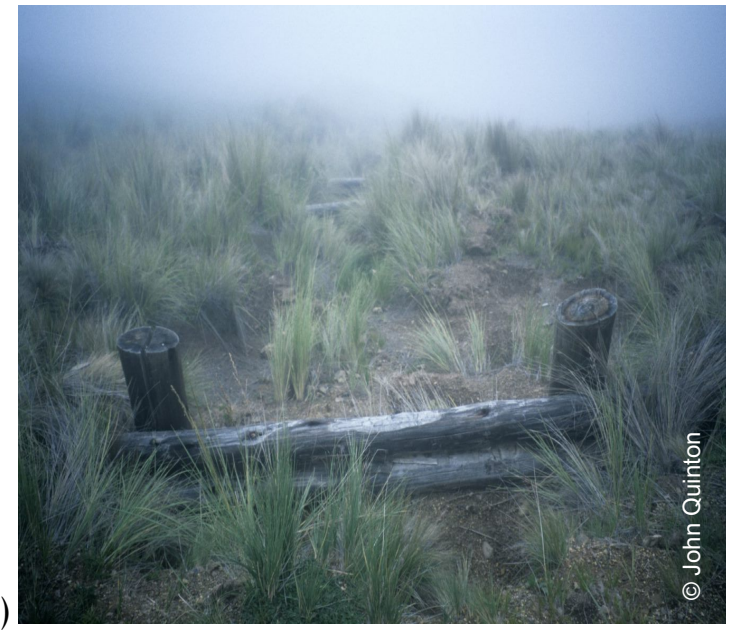

Photo 30. Examples of Check dams

a) Check dams in Barcelonette (France); b) Check dam constructed from Gabions; c) check dam with spill way (Spain); d) filled stone check dam (Swaziland) e) Series of timber check dams (the Plurinational State of Bolivia) 


\section{References}

Addisu, S. \& Mekonnen, M. 2019. Check dams and storages beyond trapping sediment, carbon sequestration for climate change mitigation, Northwest Ethiopia. Geoenvironmental Disasters, 6(1): 4. https://doi.org/10.1186/s40677-019-0120-1

Bewket, W. 2007. Soil and water conservation intervention with conventional technologies in northwestern highlands of Ethiopia: Acceptance and adoption by farmers. Land Use Policy, 24(2): 404-416.

https://doi.org/10.1016/j.landusepol.2006.05.004

Boix-Fayos, C., Martínez-Mena, M., Cutillas, P.P., de Vente, J., Barberá, G.G., Mosch, W., Navarro Cano, J.A., Gaspar, L. \& Navas, A. 2017. Carbon redistribution by erosion processes in an intensively disturbed catchment. CATENA, 149: 799-809. https://doi.org/10.1016/j.catena.2016.08.003

Gebremedhin, B. \& Swinton, S.M. 2003. Investment in soil conservation in northern Ethiopia: the role of land tenure security and public programs. Agricultural Economics, 29(1): 69-84.

https://doi.org/10.1016/S0169-5150(03)00022-7

Liu, C., Li, Z., Dong, Y., Nie, X., Liu, L., Xiao, H. \&Zeng, G. 2017. Do land use change and check-dam construction affect a real estimate of soil carbon and nitrogen stocks on the Loess Plateau of China? Ecological Engineering, 101: 220-226. https://doi.org/10.1016/j.ecoleng.2017.01.036

Martín-Rosales, W., Gisbert, J., Pulido-Bosch, A., Vallejos, A. \& Fernández-Cortés, A. 2007.

Estimating groundwater recharge induced by engineering systems in a semiarid area (southeastern Spain). Environmental Geology, 52(5): 985-995. https://doi.org/10.1007/s00254-006-0541-5

Morgan, R.P.C. 2005. Soil Erosion and Conservation. Hoboken, United Kingdom, John Wiley \& Sons, Incorporated..

Nyssen, J., Veyret-Picot, M., Poesen, J., Moeyersons, J., Haile, M., Deckers, J. \& Govers, G. 2004. The effectiveness of loose rock check dams for gully control in Tigray, northern Ethiopia. Soil Use and Management, 20(1): 55-64. https://doi.org/10.1111/j.1475-2743.2004.tb00337.x

Runezerwa, A.B. 2011. Farmers, institutions and land conservation: institutional economic analysis of bench terraces in the highlands of Rwanda. Waganingen. (PhD) 


\title{
CROPLAND
}

SOIL AND WATER CONSERVATION TECHNIQUES

EROSION CONTROL TECHNIQUES

\section{Shelterbelts}

\author{
John N Quinton
}

Lancaster Environment Centre, Lancaster University. Lancaster, UK

\section{Description of the practice and Range of applicability}

Shelterbelts or windbreaks are lines of trees or shrubs planted to form shelter for crops and pastures from winds (Photo 31 ) and are a form of agroforestry (see Factsheets No.38, 39 and 40, this volume). They are similar to hedgerows and buffer strips, which are borders of crop fields or near water courses (see factsheet No.26, this volume). They are planted perpendicular to the prevailing wind direction, or, if winds occur from more than one direction, in a crisscross pattern (Photo 31). In sloping areas shelterbelts are planted away from hill crests, where they are less effective, and sometimes on the contour, where they can also provide protection against water erosion. Typically, fast growing tree species, adapted for the local climatic conditions, are used, but it is also possible to utilize species that have additional economic value in the form of fuel, fruits or nuts. In addition to carbon sequestration, shelter belts perform a number of other services including: wind erosion control; protection of crops from winds; providing shelter for animals; and habitats for plants and animals. The literature of the role of shelterbelts in carbon sequestration is dominated by work in Canada with other contributions mainly from the United States of America, and the Russian Federation. Only limited information is available for other regions although learnings from agroforestry systems, such as alley cropping (see factsheets No.4 and 5 on intercropping, this volume), which have many of the same attributes may be applicable.

\section{Impact on soil organic carbon stocks}

Shelterbelts offer an opportunity to sequester more carbon in the soil than in cropped soils, with the greatest increase in the surface horizons of the soil (Table 111). In the largest published study to date, work on 59 sites across five soil zones in Saskatchewan, Canada demonstrated a 19 percent increase in SOC stocks under the shelterbelts compared to adjacent agricultural fields equating to a median SOC accrual rate of $0.7 \mathrm{tC} / \mathrm{ha} / \mathrm{yr}$ (Dhillon and Van Rees, 2017), although no sites younger than 15 years old showed an accrual of carbon. The amount of additional carbon depends upon the species grown in the shelterbelt (Dhillon and Van Rees, 2017) and increases with the age of the stand (Dhillon and Van Rees, 2017). It is thought that the primary factor for 
this increase is litterfall, although the structure of the tree canopy, particularly its influence on the understory vegetation, and the litter derived from it, is also important (Dhillon and Van Rees, 2017). Additionally, windblown sediment deposited due to falling wind velocities through the shelterbelt may contribute to the carbon stored (Sauer, Cambardella and Brandle, 2007).

Table 111. Evolution of SOC stocks with shelterbelts

\begin{tabular}{|c|c|c|c|c|c|c|}
\hline Location & $\begin{array}{l}\text { Climate } \\
\text { zone }\end{array}$ & Soil type & $\begin{array}{l}\text { Baseline C } \\
\text { stock } \\
\text { (tC/ha) }\end{array}$ & $\begin{array}{l}\text { Additional } \\
\text { C storage } \\
\text { (tC/ha/yr) }\end{array}$ & $\begin{array}{l}\text { Duration } \\
\text { (Years) }\end{array}$ & Reference \\
\hline $\begin{array}{l}\text { Central Russian } \\
\text { Federation } \\
\text { Upland }\end{array}$ & \multirow{3}{*}{$\begin{array}{l}\text { Cool } \\
\text { Temperate } \\
\text { Moist }\end{array}$} & Chernozems & \multirow{4}{*}{ NA } & $0.7-1.5$ & 55 & \multirow{2}{*}{$\begin{array}{l}\text { Chendev } \\
\text { et al. } \\
\text { (2015) }\end{array}$} \\
\hline $\begin{array}{l}\text { Great Plains, } \\
\text { United States } \\
\text { of America }\end{array}$ & & Haplustolls & & 1.9 & 19 & \\
\hline $\begin{array}{l}\text { Saskatchewan, } \\
\text { Canada }\end{array}$ & & $\begin{array}{l}\text { Black soils, Grey soils, } \\
\text { dark brown }\end{array}$ & & 0.7 & $4-65$ & $\begin{array}{l}\text { Dhillon } \\
\text { and Van } \\
\text { Rees } \\
\text { (2017) }\end{array}$ \\
\hline $\begin{array}{l}\text { Songnen Plain, } \\
\text { northeastern } \\
\text { China }\end{array}$ & $\begin{array}{l}\text { Continental } \\
\text { monsoon }\end{array}$ & $\begin{array}{l}\text { Black soil: Chernozem } \\
\text { (Fuyu, Lanling), } \\
\text { Phaeozem (Mingshui), } \\
\text { Cambosols (Dumeng), }\end{array}$ & & None & $\begin{array}{l}\text { Up to } \\
40 \text { years }\end{array}$ & $\begin{array}{l}\text { Wu et al. } \\
\text { (2019) }\end{array}$ \\
\hline
\end{tabular}

Baseline $\mathrm{C}$ stocks are rarely known.

\section{Other benefits of the practice}

\subsection{Improvement of soil properties and crop production}

Increased crop yields due to improved protection from the wind may lead to greater organic matter returns to the soil and increased soil sequestration, however there is little empirical evidence for this. Soil bulk density has been shown to decrease under shelter belts (Dhillon and Van Rees, 2017; Sauer, Cambardella and Brandle, 2007) and that total nitrogen may be elevated when compared with agricultural fields (Sauer, Cambardella and Brandle, 2007). 


\subsection{Minimization of threats to soil functions}

Table 112. Soil threats

\section{Soil threats}

\begin{tabular}{|l|l|}
\hline Soil erosion & $\begin{array}{l}\text { Shelterbelts can reduce wind erosion (Morgan, 2005; Sudmeyer et } \\
\text { al., 2002) and water erosion if planted on the contour. }\end{array}$ \\
\hline $\begin{array}{l}\text { Nutrient imbalance and } \\
\text { cycles }\end{array}$ & $\begin{array}{l}\text { Limited evidence, but potential to increase N store under } \\
\text { shelterbelts (Sauer, Cambardella and Brandle, 2007). }\end{array}$ \\
\hline Soil salinization and & $\begin{array}{l}\text { Possible effects due to changes in water table due to the presence } \\
\text { of trees, but no evidence. }\end{array}$ \\
\hline Soil biodiversity loss & $\begin{array}{l}\text { No evidence, but undisturbed soil and litter inputs likely to promote } \\
\text { soil biodiversity and shading provided by tree canopies can promote } \\
\text { soil biodiversity in Agroforestry systems (Martius et al., 2004). } \\
\text { Shelterbelts provide wildlife corridors (Kristensen and Caspersen, } \\
\text { 2002). }\end{array}$ \\
\hline Soil compaction & $\begin{array}{l}\text { Bulk densities tend to be lower in shelterbelts than in the } \\
\text { surrounding field (Dhillon and Van Rees, 2017). }\end{array}$ \\
\hline Soil water management & $\begin{array}{l}\text { Competition for water and nutrients is reported up to the height of } \\
\text { the shelterbelt (Kort, 1988). Windbreaks can reduce } \\
\text { evapotranspiration due to lower wind velocities and increase water } \\
\text { availability (Campi, Palumbo and Mastrorilli, 2009; Sudmeyer et al., } \\
\text { 2002). }\end{array}$ \\
\hline
\end{tabular}

\subsection{Increases in production (e.g. food/fuel/feed/timber/fibre)}

Shelterbelts increase crop production, which is attributed to snow entrapment, prevention of wind erosion and wind damage, and amelioration of microclimate (reduced evapotranspiration due to lowered wind velocities) with yield gains greatest in drier regions (Kort, 1988). The magnitude of the response depends on the design of the shelterbelt, climate, soils, crop and agronomy including planting dates. Recent estimates of yield responses range from marginal gains for cereals in Australia (Sudmeyer et al, 2002), to 6 percent for Maize in North West China (Zheng, Zhu and Xing, 2016) and between 50 and 91 percent more marketable yield for snap pea crops in Nebraska, United States of America (Hodges et al., 2004). 


\subsection{Mitigation of and adaptation to climate change}

A number of studies document the mass of carbon stored in the living biomass of shelterbelts (Kort and Turnock, 1998) and methods exist for scaling this contribution to regional or national scales using remote sensing. Czerepowicz, Case and Doscher (2012) estimate that conifer shelterbelts represent a small, yet significant carbon reservoir in New Zealand, sequestering on average $237 \pm 77 \mathrm{tC} / \mathrm{ha}$ of shelterbelt representing an additional $6 \mathrm{tC} /$ ha to the 'grassland with woody biomass' carbon pool. Shelterbelts also provide a source of fuel which may reduce net-emissions from power generation (Ballesteros-Possu, Brandle and Schoeneberger, 2017).

\subsection{Socio-economic aspects}

Socio-economic benefits derive from additional services that shelterbelts provide. By-products can provide addition income and food sources, as well as opportunities for fuel for power and heat generation.

\section{Possible greenhouse gases emissions}

There is rather limited data on GHG emissions from shelterbelts. In the only comparative study located, lower emissions of $\mathrm{N}_{2} \mathrm{O}$ and $\mathrm{CH}_{4}$ inside Canadian shelterbelts than in adjacent crop land were found by Amadi, Van Rees and Farrell (2016).

\section{Recommendations before implementing the practice}

Shelterbelts provide protection for between 12 and 17 times the height of the belt and should be at least 24 times as long as they are high (Morgan, 2005) to provide protection from the wind. To maximize the area of shelter provided tall trees should be incorporated into the belt together with a lower growing shrub. This prevents the development of open patches. A significant reduction in wind erosion is obtained with horizontal vegetation coves greater that 40 percent. 


\section{Potential barriers to adoption}

\section{Table 113. Potential barriers to adoption}

\begin{tabular}{|c|c|c|}
\hline Barrier & YES/NO & \\
\hline Biophysical & Yes & Best suited areas prone to high winds. \\
\hline Cultural & Yes & $\begin{array}{l}\text { Despite yield benefits shelterbelts are being removed in the United } \\
\text { States of America, Canada and Europe. This may be due to changes } \\
\text { from small to large farms with larger machinery (Kristensen and } \\
\text { Caspersen, 2002; Rempel, 2014). In Canada it appears to be related } \\
\text { to perceived value of the shelterbelts, with farmers seeing } \\
\text { shelterbelts as a cost related to their land area removing them, while } \\
\text { others see their benefits and do not remove them (Kulshreshtha and } \\
\text { Rempel, 2014). }\end{array}$ \\
\hline Social & Yes & $\begin{array}{l}\text { Capacity to invest, influenced by farm size: larger farms more likely } \\
\text { to adopt (Rempel, 2014). }\end{array}$ \\
\hline Economic & Yes & $\begin{array}{l}\text { Considerable financial investment required for establishment, } \\
\text { ongoing maintenance costs (Rempel, 2014). }\end{array}$ \\
\hline $\begin{array}{l}\text { Legal (Right to } \\
\text { soil) }\end{array}$ & Yes & $\begin{array}{l}\text { Insecure land tenure reduces opportunities for investment (Rempel, } \\
\text { 2014). }\end{array}$ \\
\hline Knowledge & Yes & $\begin{array}{l}\text { Learning opportunities form other farmers and other actors } \\
\text { important (Rempel, 2014). }\end{array}$ \\
\hline
\end{tabular}




\section{Photo of the practice}

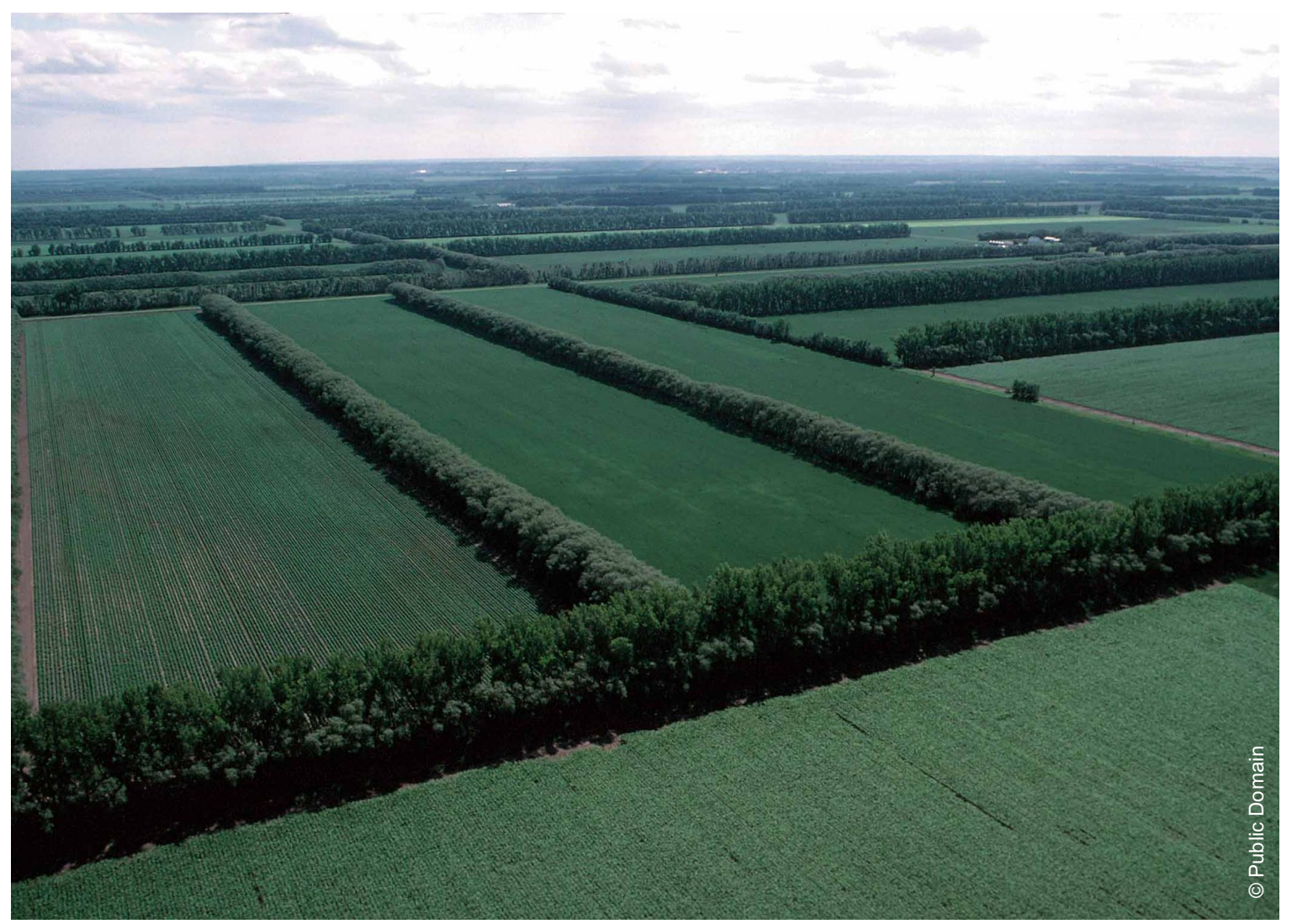

Photo 31. A criss-cross patern of shelterbelts in North Dakota, United States of America 


\section{References}

Amadi, C.C., Van Rees, K.C.J. \& Farrell, R.E. 2016. Soil-atmosphere exchange of carbon dioxide, methane and nitrous oxide in shelterbelts compared with adjacent cropped fields. Agriculture, Ecosystems \& Environment, 223: 123-134. https://doi.org/10.1016/j.agee.2016.02.026

Ballesteros-Possu, W., Brandle, J.R. \& Schoeneberger, M. 2017. Potential of Windbreak Trees to Reduce Carbon Emissions by Agricultural Operations in the US. Forests, 8(5): 138.

https://doi.org/10.3390/f8050138

Campi, P., Palumbo, A.D. \& Mastrorilli, M. 2009. Effects of tree windbreak on microclimate and wheat productivity in a Mediterranean environment. European Journal of Agronomy, 30(3): 220-227.

https://doi.org/10.1016/j.eja.2008.10.004

Chendev, Yu.G., Sauer, T.J., Gennadiev, A.N., Novykh, L.L., Petin, A.N., Petina, V.I., Zazdravnykh, E.A. \& Burras, C.L. 2015. Accumulation of organic carbon in chernozems (Mollisols) under shelterbelts in Russia and the United States. Eurasian Soil Science, 48(1): 43-53.

https://doi.org/10.1134/S1064229315010032

Czerepowicz, L., Case, B.S. \& Doscher, C. 2012. Using satellite image data to estimate aboveground shelterbelt carbon stocks across an agricultural landscape. Agriculture, Ecosystems \& Environment, 156: 142-150. https://doi.org/10.1016/j.agee.2012.05.014

Dhillon, G.S. \& Van Rees, K.C.J. 2017. Soil organic carbon sequestration by shelterbelt agroforestry systems in Saskatchewan. Canadian Journal of Soil Science: 1-16. https://doi.org/10.1139/cjss-20160094

Hodges, L., Suratman, M.N., Brandle, J.R. \& Hubbard, K.G. 2004. Growth and yield of snap beans as affected by wind protection and microclimate changes due to shelterbelts and planting dates. Hortscience, 39(5): 996-1004.

Kort, J. 1988. 9. Benefits of windbreaks to field and forage crops. Agriculture, Ecosystems \& Environment, 22-23: 165-190. https://doi.org/10.1016/0167-8809(88)90017-5

Kort, J. \& Turnock, R. 1998. Carbon reservoir and biomass in Canadian prairie shelterbelts. Agroforestry Systems, 44(2): 175-186. https://doi.org/10.1023/A:1006226006785

Kristensen, S.P. \& Caspersen, O.H. 2002. Analysis of changes in a shelterbelt network landscape in central Jutland, Denmark. Journal of Environmental Management, 66(2): 171-183.

https://doi.org/10.1006/jema.2002.0582

Kulshreshtha, S. \& Rempel, J. 2014. Shelterbelts on Saskatchewan farms: an asset or nuisance. Climate change and forest ecosystems. Nova Publishers, New York, NY, USA: 37-54.

Martius, C., Höfer, H., Garcia, M.V.B., Römbke, J., Förster, B. \& Hanagarth, W. 2004. Microclimate in agroforestry systems in central Amazonia: does canopy closure matter to soil organisms? Agroforestry Systems, 60(3): 291-304. https://doi.org/10.1023/B:AGFO.0000024419.20709.6c 
Morgan, R.P.C. 2005. Soil Erosion and Conservation. Hoboken, United Kingdom, John Wiley \& Sons, Incorporated. (also available at:

http://ebookcentral.proquest.com/lib/lancaster/detail.action?docID=233037).

Rempel, J.C. 2014. Costs, benefits and barriers to the adoption and retention of shelterbelts in prairie agriculture as identified by Saskatchewan producers. Saskatoon, University of Saskatchewan

Sauer, T.J., Cambardella, C.A. \& Brandle, J.R. 2007. Soil carbon and tree litter dynamics in a red cedarscotch pine shelterbelt. Agroforestry Systems, 71(3): 163-174. https://doi.org/10.1007/s10457-0079072-7

Sudmeyer, R.A., Adams, M.A., Eastham, J., Scott, P.R., Hawkins, W. \& Rowland, I.C. 2002. Broadacre crop yield in the lee of windbreaks in the medium and low rainfall areas of south-western Australia. Australian Journal of Experimental Agriculture, 42(6): 739-750. https://doi.org/10.1071/ea02011

Wu, Y., Wang, Q., Wang, H., Wang, W. \& Han, S. 2019. Shelterbelt Poplar Forests Induced Soil Changes in Deep Soil Profiles and Climates Contributed Their Inter-site Variations in Dryland Regions, Northeastern China. Frontiers in Plant Science, 10. https://doi.org/10.3389/fpls.2019.00220

Zheng, X., Zhu, J. \& Xing, Z. 2016. Assessment of the effects of shelterbelts on crop yields at the regional scale in Northeast China. Agricultural Systems, 143: 49-60. https://doi.org/10.1016/j.agsy.2015.12.008 


\title{
26. Hedges and Buffer Strips
}

\author{
Narendra Kumar Lenka1, Ana Patricia Fernández-Getino García² \\ ${ }^{1}$ Principal Scientist, ICAR-Indian Institute of Soil Science, Nabibagh, Bhopal, India \\ ${ }^{2}$ Instituto Nacional de Investigación y Tecnología Agraria y Alimentaria (INIA), Madrid, Spain
}

\section{Description of the practice}

Buffers strips and hedges are strips of permanent vegetation (grasses, trees or shrubs) in or around the borders of crop fields or near water courses (Photo 32, Borin et al, 2010). The width of buffer strips varies from few meters to more than 10 meters. Although both terms are used interchangeably, buffer strips are generally wider $(>5 \mathrm{~m})$ and taken up on arable lands as well as on river or stream banks. Hedge rows are narrow $(<1.5 \mathrm{~m})$ and are usually placed on arable lands (Photo 33). Buffer strips and hedges have a multi-functional role starting from soil conservation to protection of water quality, carbon sequestration, biomass production, habitat improvement and many societal services (Haddaway et al., 2018).

The primary objective of establishing hedges and buffer strips is to intercept the runoff or overland flow and hence to minimize the loss of soil, nutrients and agro-chemicals from crop lands. Thus, they contribute to reducing the non-point source pollution and improving soil quality apart from indirect effect in improving crop yield. In other words, the action of buffer strips is due to both interception and filtration of runoff water. In addition to controlling soil erosion by water, vegetated strips act like shelter belts and reduce the impact of wind erosion by breaking the speed of wind and protecting standing crops and soil. Buffer strips are also useful in preventing stream bank erosion (referred to as riparian buffers). 


\section{Range of applicability}

They are applicable to all agricultural land uses including arable lands, croplands, permanent crops and pastures. The use of buffer strips and hedges is most common in high rainfall regions, arid and semi-arid areas with high wind velocity, in sloping lands and in riverbanks. Riparian buffer strips with permanent vegetation on both banks of streams and rivers help in reducing streambank erosion and controlling flooding. The structure of the vegetation in buffer strips and hedges determine their efficacy at a given location. They can be typically designed to achieve multiple objectives depending upon the land slope, soil type, topographic and climatic conditions.

\section{Impact on soil organic carbon stocks}

Through the actions of interception and filtration, buffer strips and hedges reduce the loss of fertile soil, nutrients, suspended solids and organic matter from the up-slope crop lands. This in turn, increase the in-situ retention of soil organic carbon (SOC), enhance infiltration and percolation of rainwater by increasing the opportunity time, improves microbial activity and thus contributes to overall increase in SOC storage. In addition, leaf litter and root biomass is added, which promote $\mathrm{C}$ storage in deeper soil profile. Thus, SOC in the field margins planted with hedge rows is typically higher than the in-field areas and the SOC stock decreases with increasing distance from the hedge rows. Table 114 shows the potential of buffers on SOC sequestration / additional SOC storage as compiled from findings from across the world. 
Table 114. Effect of hedgerows and buffer strips on rate of soil carbon storage as computed from available literature

\begin{tabular}{|c|c|c|c|c|c|c|c|c|}
\hline Location & Climate zone & Soil type & $\begin{array}{l}\text { Baseline C } \\
\text { stock } \\
\text { (tC/ha) }\end{array}$ & $\begin{array}{l}\text { Additional C storage } \\
\text { (tC/ha/yr) }\end{array}$ & $\begin{array}{l}\text { Duratio } \\
\text { n (years) }\end{array}$ & $\begin{array}{l}\text { Depth } \\
\text { (cm) }\end{array}$ & More information & Reference \\
\hline Eastern India & $\begin{array}{l}\text { Sub-tropical and } \\
\text { sub-humid }\end{array}$ & $\begin{array}{l}\text { Acidic Red } \\
\text { lateritic } \\
\text { (Alfisols) }\end{array}$ & 26.62 (at to) & $\begin{array}{l}1.35 \text { (at } 1 \mathrm{~m} \text { distance } \\
\text { from hedge row) } \\
0.35 \text { (entire plot) }\end{array}$ & 5 & 60 & $\begin{array}{l}\text { Hedge row of Gliricidia } \\
\text { along with grass filter strip }\end{array}$ & $\begin{array}{l}\text { Lenka et al. } \\
\text { (2012a) }\end{array}$ \\
\hline Eastern India & $\begin{array}{l}\text { Sub-tropical and } \\
\text { sub-humid }\end{array}$ & Red lateritic & $7.65\left(\mathrm{BAU}^{\#}\right)$ & 0.67 & 5 & 15 & Barrier of Saccharum spp & Dass et al. (2011) \\
\hline Switzerland & Temperate & NA & 40.6 (BAU) & 0.10 & $\begin{array}{l}\text { Historical } \\
\odot\end{array}$ & 20 & Permanent grassland & $\begin{array}{l}\text { Leifeld Bassin and } \\
\text { Fuhrer (2005) }\end{array}$ \\
\hline North-East Italy & Sub-humid & $\begin{array}{l}\text { Fulvi-Calcaric } \\
\text { Cambisol } \\
\text { (Loamy) }\end{array}$ & 73 (to) & 2.5 & 5 & 50 & $\begin{array}{l}\text { SOC stock from buffer } \\
\text { strips }\end{array}$ & Borin et al. (2010) \\
\hline Brittany, France & Temperate & & 137.5 & 0.30 & Historical & 55 & $10 \mathrm{~m}$ from hedge row & $\begin{array}{l}\text { Follain, Powlson } \\
\text { and Smith (2007) }\end{array}$ \\
\hline France & Various & NA & 51.6 & 0.10 & 20 & 30 & Planting of hedgerows & $\begin{array}{l}\text { Arrouays et al. } \\
\text { (2002); } \\
\text { Minasny et al. } \\
\text { (2017) }\end{array}$ \\
\hline United States & Temperate & NA & NA & $0.3-0.7$ & & & Conservation buffer strips & Uri (2001) \\
\hline
\end{tabular}




\begin{tabular}{|c|c|c|c|c|c|c|c|c|}
\hline Location & Climate zone & Soil type & $\begin{array}{l}\text { Baseline C } \\
\text { stock } \\
\text { (tC/ha) }\end{array}$ & $\begin{array}{l}\text { Additional C storage } \\
\text { (tC/ha/yr) }\end{array}$ & $\begin{array}{l}\text { Duratio } \\
\text { n (years) }\end{array}$ & $\begin{array}{l}\text { Depth } \\
(\mathrm{cm})\end{array}$ & More information & Reference \\
\hline Western Nigeria & Humid tropics & Alfisol & 21.6 & $\begin{array}{l}1.8 \text { (Brachiaria) } \\
7.1 \text { (Melinis) }\end{array}$ & 2 & 20 & Grasses as cover crops & Lal (1997) \\
\hline Northern Italy & $\begin{array}{l}\text { North } \\
\text { Mediterranean }\end{array}$ & $\begin{array}{l}\text { Inceptisol- } \\
\text { Silty loam }\end{array}$ & 22.5 (BAU) & 0.97 & Historical & 15 & Hedgerow & Sitzia et al. (2014) \\
\hline United States & NA & NA & NA & $0.42-0.94$ & NA & 20 & $\begin{array}{l}\text { Vegetative barriers, filter } \\
\text { strips }\end{array}$ & $\begin{array}{l}\text { Chambers, Lal and } \\
\text { Paustian, (2016) }\end{array}$ \\
\hline Burkina Faso & Tropical & $\begin{array}{l}\text { Ferric lixisols- } \\
\text { Sandy loam }\end{array}$ & 27 (BAU) & 0.65 & 20 & 20 & $\begin{array}{l}\text { Live fence of Jatropha } \\
\text { curcas }\end{array}$ & $\begin{array}{l}\text { Baumert, } \\
\text { Khamzina and } \\
\text { Vlek (2016) }\end{array}$ \\
\hline $\begin{array}{l}\text { Nebraska, } \\
\text { United States of } \\
\text { America }\end{array}$ & NA & NA & NA & 0.85 & 15 & 15 & Switchgrass barriers & $\begin{array}{l}\text { Blanco-Canqui et } \\
\text { al. (2014) }\end{array}$ \\
\hline $\begin{array}{l}\text { Central Ohio, } \\
\text { United States of } \\
\text { America }\end{array}$ & $\begin{array}{l}\text { Humid } \\
\text { continental }\end{array}$ & $\begin{array}{l}\text { Clay loam or } \\
\text { Silty loam }\end{array}$ & $13.0(\mathrm{BAU})$ & 2.0 & 5 & 5 & $\begin{array}{l}\text { Restored/Natural } \\
\text { Riparian buffers }\end{array}$ & $\begin{array}{l}\text { Marton, Fennessy } \\
\text { and Craft (2014) }\end{array}$ \\
\hline $\begin{array}{l}\text { Nebraska- } \\
\text { Lincoln }\end{array}$ & & $\begin{array}{l}\text { Silt loam } \\
\text { (Mollisols) }\end{array}$ & 36.2 (BAU) & 0.11 & 35 & 15 & Shelter belt on crop field & $\begin{array}{l}\text { Sauer, } \\
\text { Cambardella and } \\
\text { Brandle (2007) }\end{array}$ \\
\hline
\end{tabular}

@Duration taken as 100 years, \#BAU: Crop / Agricultural field 


\section{Other benefits of the practice}

\subsection{Improvement of soil properties}

Reduced soil erosion favorably influences soil properties including aggregation, porosity, bulk density, available water capacity and soil organic matter content of field plots with buffer strips. Accumulation of soil organic matter in these plots is higher due to a mix of barrier effects and increased biomass production. Activity of soil biota, which is an important determinant of nutrient cycling, is increased. Many studies demonstrate hedges to promote higher SOM and improve chemical and physical characteristics of soil as compared to the adjacent fields (Seobi et al., 2005; Stutter and Richards, 2012). Seobi et al. (2005) reported a significantly lower bulk density, significantly higher porosity and coarse mesoporosity and significant higher hydraulic conductivity in the grass and agroforestry treatments. This also improved the potential water storage by about $10 \mathrm{~mm}$ in $30 \mathrm{~cm}$ soil profile as compared to row crop treatments. Another study reported that soils in buffer strip had significantly greater SOM (89 percent of sites), moisture content (95 percent), and water-soluble nutrient concentrations for dissolved organic $\mathrm{C}$ (80 percent), dissolved organic $\mathrm{N}$ (80 percent) and dissolved organic P (55 percent) with consistently lower bulk densities than field soils (Stutter and Richards, 2012). Aggregate stability and water retention was reported to be higher in sloping arable and non-arable lands treated with hedges (Lenka et al., 2012a, 2012b). Long-term effects are expected in soils of rural landscapes where the planting and maintenance of hedges is an ancient tradition.

\subsection{Minimization of threats to soil functions}

Table 115. Soil threats

Soil threats

\begin{tabular}{|l|l|}
\hline Soil erosion & $\begin{array}{l}\text { Soil erosion due to water and aeolian soil loss due to wind erosion are } \\
\text { lower under hedge or buffer strips. Reduced runoff up to 78 percent is } \\
\text { reported due to a } 6 \text { meters wide buffer strip in Italy (Borin et al., 2005). }\end{array}$ \\
\hline $\begin{array}{l}\text { Nutrient imbalance and } \\
\text { cycles }\end{array}$ & $\begin{array}{l}\text { Because of the retention effect, hedges promote higher soil organic } \\
\text { matter and plant available nutrients (N, P and K) near the hedge rows as } \\
\text { well as in the adjacent fields. Hedgerows reduce soil carbon and nutrients } \\
\text { loss (Lenka et al., 2012a), but retention of nutrients in narrow strips is } \\
\text { uncertain (Stutter and Richards, 2012). }\end{array}$ \\
\hline $\begin{array}{l}\text { Soil salinization and } \\
\text { alkalinization }\end{array}$ & $\begin{array}{l}\text { No direct effect, but, may reduce salinization, alkalinization indirectly by } \\
\text { promoting better soil water regime. }\end{array}$ \\
\hline
\end{tabular}




\section{Soil threats}

\begin{tabular}{|c|c|}
\hline $\begin{array}{l}\text { Soil contamination / } \\
\text { pollution }\end{array}$ & $\begin{array}{l}\text { Buffers are cost-effective in reducing agricultural non-point source } \\
\text { pollution. The extent of mitigation is } 70-80 \text { percent for suspended } \\
\text { solids, } 70-98 \text { percent for } P \text { and } 70-95 \text { percent for } N \text { (Borin et al., 2005). } \\
\text { If properly designed and maintained, they have the capacity to remove } \\
\geq 50 \text { percent of nutrients and pesticides, remove } \geq 60 \text { percent of certain } \\
\text { pathogens, and remove } \geq 75 \text { percent of sediments (USDA, 2020). }\end{array}$ \\
\hline Soil acidification & $\begin{array}{l}\text { There is no direct evidence of hedges and buffer strips regulating soil } \\
\text { acidity. Though studies are not available, buffer strips may help in } \\
\text { minimizing the soil acidification by preventing the loss of basic cations } \\
\text { through runoff. }\end{array}$ \\
\hline Soil biodiversity loss & $\begin{array}{l}\text { They help in conserving aboveground and below ground biodiversity. The } \\
\text { activity of soil flora and fauna like earthworms is promoted. }\end{array}$ \\
\hline Soil sealing & $\begin{array}{l}\text { As buffers and hedges keep the soil in well aggregated state and due to } \\
\text { higher SOC, soil sealing and crusting are minimized. }\end{array}$ \\
\hline Soil compaction & $\begin{array}{l}\text { The vegetation of buffer strips and hedges absorbs the energy of } \\
\text { raindrops, fixes the soil at its roots and reduces runoff. A higher SOC, } \\
\text { better aggregation and increase in per cent of water stable aggregates } \\
\text { (>0.25 } \mathrm{mm} \text { size aggregates) reduces the chances of soil compaction (Fan } \\
\text { et al., 2015). }\end{array}$ \\
\hline Soil water management & $\begin{array}{l}\text { Contour hedges give an added benefit in terms of in-situ soil water } \\
\text { conservation (Lenka et al., 2012a, 2012b; Fan et al., 2015). Contour hedge } \\
\text { of Gliricidia + grass filter of Sachharum sp. recorded about } 30 \mathrm{~mm} \text { of } \\
\text { higher soil water storage at } 17 \text { days of dry spell in a sandy loam soil (Lenka } \\
\text { et al., 2012a). Hedges improve water quality by reducing sediment and } \\
\text { potential contaminants in runoff. In addition, hedge rows may affect the } \\
\text { water table profile due to the higher transpiration of the trees (Thomas et } \\
\text { al., 2008), therefore improving the soil drainage conditions. }\end{array}$ \\
\hline
\end{tabular}




\section{3. (Increases in production (e.g. food/fuel/feed/timber/fibre)}

The impact of buffer strips and hedges on yield of crops in the field depends upon the type of vegetation in the buffer zones, the height of the hedge species and the distance from the hedge row. However, hedges comprising shrubs of 1-2 m height do not adversely affect crop yield (Borin et al., 2010; Lenka et al., 2012a). Van Vooren et al. (2017) showed that next to the hedgerow, until a distance of twice the hedgerow height, arable crop yield was reduced by 20 percent, but, beyond this distance, until 20 times the hedgerow height, crop yield was increased by 6 percent and the overall crop yield increased by 3 percent. Another study showed overall increase in grain yield by 49 percent due to contour hedges (Lenka et al., 2012).

Trees and shrubs in buffer zones also contribute to additional benefit in terms of periodic harvest of fodder for animals, fuel wood and timber (Borin et al., 2010). Vegetated strips can also contribute to the increase in crop yield through increase in pollinators' habitat and activity and by increasing biological control of crop pests (Wratten et al., 2012).

\subsection{Mitigation of and adaptation to climate change}

Continuous strips of hedges and buffers of perennial trees and woody plants at a regional scale contribute to climate change mitigation through carbon capture and storage in the above-ground plant biomass and in soil profile. Further, they contribute directly and indirectly to moderating the air and soil temperature. The $\mathrm{CO}_{2}$ immobilized in the wood and soil under a buffer strip can be substantial, even as high as $80 \mathrm{t} / \mathrm{ha} / \mathrm{yr}$ (Borin et al., 2010). Some studies on trace gas fluxes indicate hedgerow or tree strip significantly reduce $\mathrm{N}_{2} \mathrm{O}$ emissions (Falloon, Powlson and Smith, 2004). Increased adaptation potential of crop plants to withstand dry spells through higher soil moisture conservation due to hedge effect has also been reported. Contour hedge of Gliricidia + grass filter of Sachharum sp. recorded about $30 \mathrm{~mm}$ of higher soil water storage at 17 days of dry spell (Lenka et al, 2012a).

\subsection{Socio-economic benefits}

As an ancillary benefit, buffer strips provide fuel wood and fodder and thus save resources in the rural economy. Further, they provide a number of intangible benefits and ecosystem services such as better soil and water quality (Bentrup, 2008).

\subsection{Other benefits of the practice}

Riparian buffer strips provide a wide range of ecosystem services in agricultural landscapes with benefits extending beyond non-riparian field margins. They also contribute to enhanced aesthetic value of the landscape and protection of biodiversity (Cole, Stockan and Helliwell, 2020). Historically, hedgerow network landscapes or "bocages" are present in most parts of the Western Europe (Baudry et al., 2000; Follain et al., 2007). 


\section{Potential drawbacks to the practice}

\subsection{Tradeoffs with other threats to soil functions}

Table 116. Soil threats

\begin{tabular}{|c|c|}
\hline Soil threats & \\
\hline $\begin{array}{l}\text { Nutrient imbalance and } \\
\text { cycles }\end{array}$ & $\begin{array}{l}\text { The practice improves nutrient balance and the activity of soil biota, } \\
\text { but the effect decreases with increasing distance from hedge rows. At } \\
\text { a distance } 4 \text { times the height of hedge rows, the effect on SOC is } \\
\text { negligible, but, at a distance half of the height of the hedge row, SOC } \\
\text { stock was } 14 \text { percent higher (Van Vooren et al., 2017) }\end{array}$ \\
\hline $\begin{array}{l}\text { Soil contamination / } \\
\text { pollution }\end{array}$ & $\begin{array}{l}\text { Under high nutrient loadings, buffer strips can become saturated and } \\
\text { act as a source of pollutants (Cole, Stockan and Helliwell, 2020), } \\
\text { particularly with regard to dissolved nutrients }\end{array}$ \\
\hline
\end{tabular}

\subsection{Increases in greenhouse gas emissions}

Very little information is available on this aspect. As per the computation made using the below study, net GHG mitigation is slightly higher under shelter belt (Table 117). Some studies on trace gas fluxes indicate hedgerow or tree strip significantly reduce $\mathrm{N}_{2} \mathrm{O}$ emissions (Falloon, Powlson and Smith, 2004). However, some studies indicate riparian buffers can lead to higher $\mathrm{N}_{2} \mathrm{O}$ emissions due to continuous $\mathrm{N}$ loading from adjacent agricultural lands (Mafa-Attoye et al., 2020).

Table 117. Emission of greenhouse gases and estimated net global warming potential under shelterbelts compared with adjacent cropped fields

\begin{tabular}{|l|c|c|c|c|l|l|}
\hline Practice & $\begin{array}{l}\mathrm{CH}_{4}-\mathrm{C} \\
(\mathrm{kg} \mathrm{C} / \mathrm{ha} / \mathrm{yr})\end{array}$ & $\begin{array}{l}\mathrm{N}_{2} \mathrm{O}-\mathrm{N} \\
(\mathrm{kg} \mathrm{N} / \mathrm{ha} / \mathrm{yr})\end{array}$ & $\begin{array}{l}\Delta \mathrm{SOC} \\
(\mathrm{tC} / \mathrm{ha} / \mathrm{yr})\end{array}$ & $\begin{array}{l}\mathrm{GWP} \\
(\mathrm{t} \mathrm{CO} / 2 \mathrm{eq} / \mathrm{ha} / \mathrm{yr})\end{array}$ & $\begin{array}{l}\text { Net GHG } \\
\text { mitigation } \\
(\mathrm{t} \mathrm{CO} \text { eq/ha/yr) }\end{array}$ & Reference \\
\hline $\begin{array}{l}\text { Shelter belt } \\
\text { on crop field }\end{array}$ & -0.66 & 0.65 & 1.1 & 0.25 & 3.8 & $\begin{array}{l}\text { Amadi, } \\
\text { Rees and } \\
\text { Farrell } \\
(2016)\end{array}$ \\
\hline Cropped plot & -0.19 & 2.5 & 0 & 1.03 & 3.0 & \\
\hline
\end{tabular}




\subsection{Conflict with other practice(s)}

They may create inconvenience for the movement of people or implements from one field to another.

\subsection{Decreases in production (e.g. food/fuel/feed/timber/fibre)}

There is a loss of net cropped area due to establishment of hedge rows, which may reduce the crop production in the initial years, though, in long term, the effect may get nullified due to better soil conditions. They can harbor weeds, pests and disease-causing pathogens and thus can create a negative impact on production (Marshall and Munen, 2002). Thick grass barriers may harbor harmful insects and reptiles, but also predators.

\section{Recommendations before implementing the practice}

Width is the prominent factor deciding the efficiency of hedges and buffer strips in erosion control, besides socio-economic factors. Abuffer width of 1-3 $\mathrm{m}$ is good enough for retaining sediments, but the retention varies from 30-85 percent for dissolved nutrients such as P and dissolved N (Collins et al., 2009). In the arable field conditions, any hedge row above $0.6 \mathrm{~m}$ width can be considered as a good compromise.

Apart from width, other factors to be taken into account are ratio of source area to buffer area, soil texture and runoff intensity before making recommendations on vegetated buffer strip construction in agroecosystems (Prosser et al., 2020).

Grass species should invariably be included as a component. The species for the hedge system including grass, shrubs or trees should suit to local climate, preferably available locally and proven bio-physical growth.

Ancillary benefits in terms of fodder, fuelwood or food should be taken into account. For instance, hedges of Gliricidia sepium can be a good source of protein-rich green fodder (about $10 \mathrm{tha}^{-1}$ of dry matter) apart from Nfixing ability in soil and $\mathrm{N}$ rich leaves being used as green leaf manure.

Shading effect due to tree component may be a factor to be taken into account during design. If at all, tree component is retained in the design, tree species should preferably be tall growing, with less branching habit. 


\title{
7. Potential barriers to adoption
}

\author{
Table 118. Potential barriers to adoption
}

\begin{tabular}{|c|c|c|}
\hline Barrier & YES/NO & \\
\hline Biophysical & Yes & $\begin{array}{l}\text { Establishment during initial years ( } 2-3 \text { years) is crucial. Mortality rate of hedge } \\
\text { shrubs and grasses during initial establishment phase is higher in dry or } \\
\text { rainfed areas and where menace of stray cattle is a problem. Further, in } \\
\text { arable sloping lands, they need to be protected from erosive rain by } \\
\text { reinforcing with field bunds. In small landholding situations, there may be } \\
\text { hesitation among stakeholders to divert much land area for hedge rows } \\
\text { because farmers have to divert some land for bunds as a mark of their land } \\
\text { boundary and also use the bunds for movement from one field to other. }\end{array}$ \\
\hline Cultural & No & $\begin{array}{l}\text { Certain species are not preferred due to religious reasons, hence, species } \\
\text { selection should look into the cultural ethos of the local population. }\end{array}$ \\
\hline Social & Yes & $\begin{array}{l}\text { If the practice is followed in a large number of landholdings, then the effect } \\
\text { becomes visible at a catchment or watershed scale. In some areas such as } \\
\text { France and other parts of western Europe, they are part of the landscape and } \\
\text { therefore have been well accepted since historical time. }\end{array}$ \\
\hline Economic & Yes & $\begin{array}{l}\text { The economic constraints would mainly be associated with the cost to } \\
\text { establish the buffers and the cost to the producer of the land that remains } \\
\text { out of production (Helmers et al., 2008). Hence, incentives such as free } \\
\text { saplings or suitable subsidies may play a big role in wider adoption (Borin et } \\
\text { al., 2010). }\end{array}$ \\
\hline Institutional & Yes & $\begin{array}{l}\text { Land tenure, which broadly refers to the institutional arrangement governing } \\
\text { the control and use of farmland, should be taken into account for successful } \\
\text { planning. }\end{array}$ \\
\hline $\begin{array}{l}\text { Legal (Right } \\
\text { to soil) }\end{array}$ & Yes & $\begin{array}{l}\text { Land tenure is an issue to be sorted out. Legal right is not a problem in } \\
\text { private lands wholly used by the owner. But, in cases where land belongs to } \\
\text { the village or a society, appropriate understanding should be made. }\end{array}$ \\
\hline Knowledge & Yes & $\begin{array}{l}\text { Knowledge on soil type, climate and growth behavior of selected species is } \\
\text { required. }\end{array}$ \\
\hline
\end{tabular}




\section{Photo/Graphical representation of the practice}

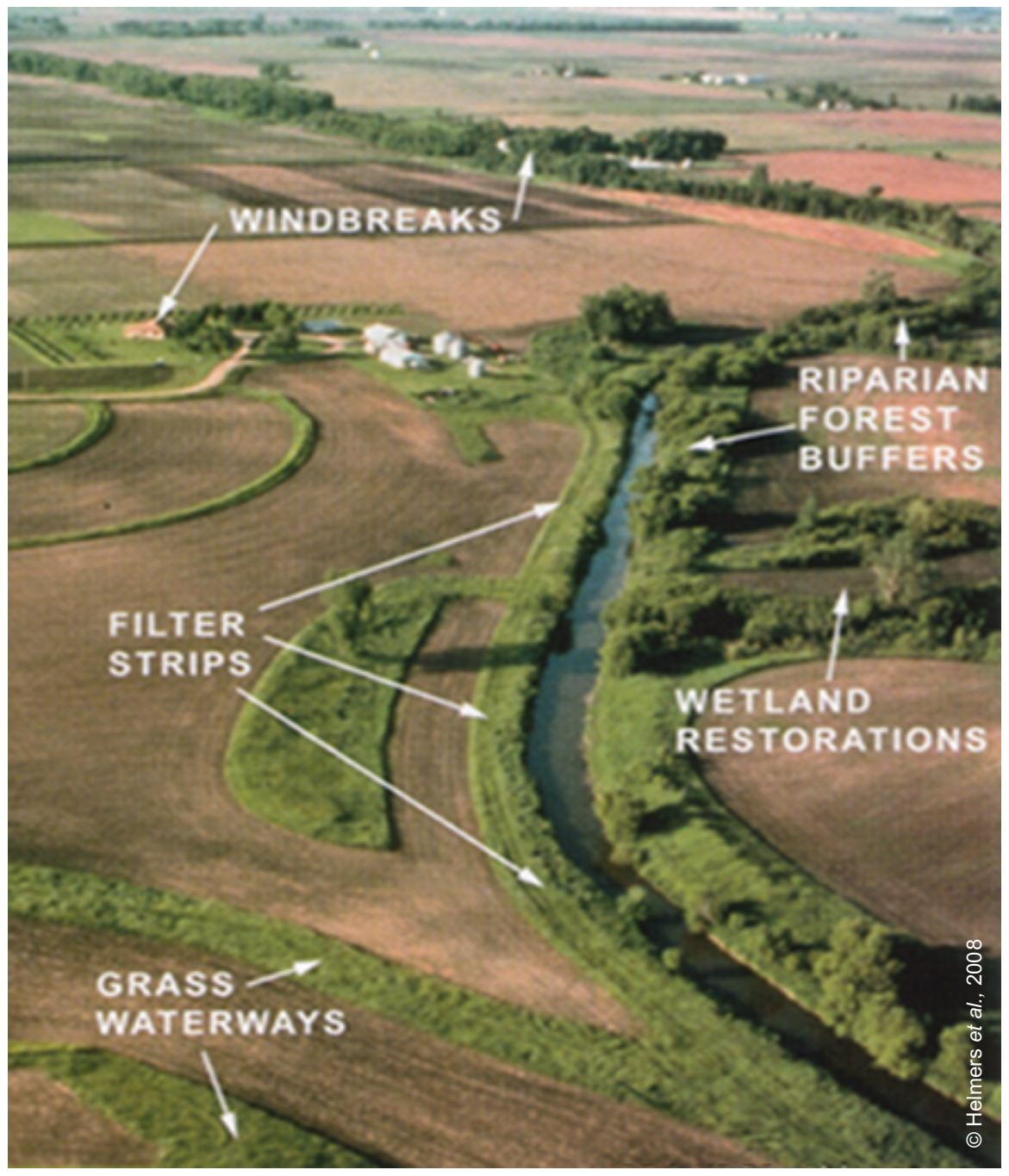

Photo 32. Aerial view of different types of hedge rows and buffer strips 

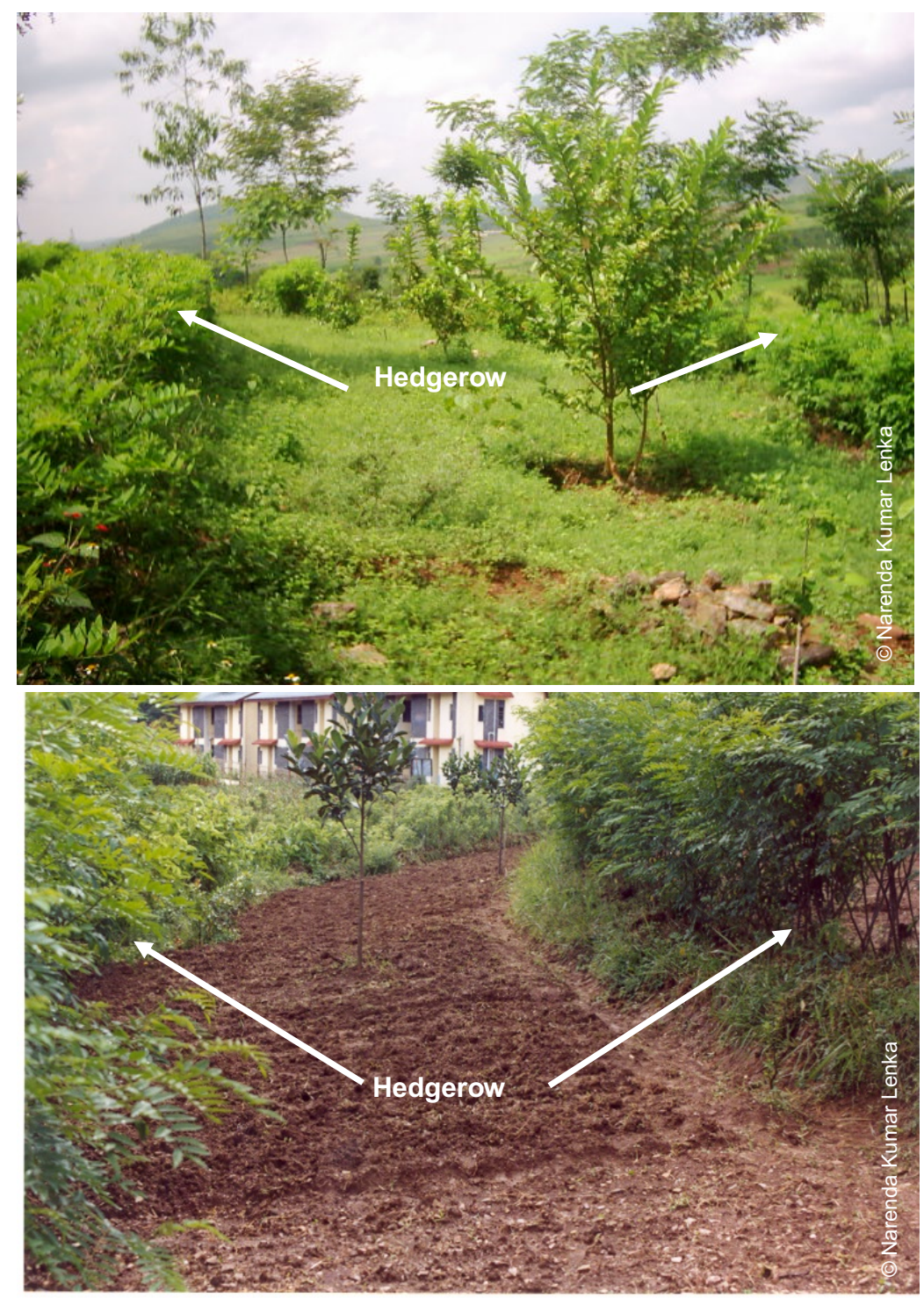

Photo 33. Hedgerows

Table 119. Related cases studies available in volumes 3 and 5

\begin{tabular}{|l|l|c|c|c|}
\hline Title & Region & $\begin{array}{l}\text { Duration of } \\
\text { study } \\
\text { (Years) }\end{array}$ & Volume & $\begin{array}{l}\text { Case- } \\
\text { study No. }\end{array}$ \\
\hline $\begin{array}{l}\text { Willow Riparian Buffer Systems for } \\
\text { Biomass Production in the Black Soils of } \\
\text { Elie, Manitoba, Canada }\end{array}$ & $\begin{array}{l}\text { North } \\
\text { America }\end{array}$ & 6 & 3 & 42 \\
\hline
\end{tabular}




\section{References}

Arrouays, D., Balesdent, J., Germon, J.C., Jayet, P.A., Soussana, J.F. \& Stengel, P. 2002. Increasing carbon stocks in French agricultural soils? Synthesis of an Assessment Report by the French Institute for Agricultural Research on Request of the French Ministry for Ecology and Sustainable Development. Scientific Assessment Unit for Expertise, INRA, Paris.

Amadi, C.C., Van Rees, K.C. \& Farrell, R.E. 2016. Soil-atmosphere exchange of carbon dioxide, methane and nitrous oxide in shelterbelts compared with adjacent cropped fields. Agriculture, Ecosystems \& Environment, 223: 123-134. https://doi.org/10.1016/j.agee.2016.02.026

Bentrup, G. 2008. Conservation buffers: design guidelines for buffers, corridors, and greenways. Gen. Tech. Rep.SRS-109. Asheville, NC: Department of Agriculture, Forest Service, Southern Research Station. 110 p. https://www.fs.usda.gov/nac/buffers/index.html

Baudry, J., Bunce, R.G.H. \& Burel, F. 2000. Hedgerows: An international perspective on their origin, function and management. Journal of Environmental Management, 60(1): 7-22. https://doi.org/10.1006/jema.2000.0358

Baumert, S., Khamzina, A. \& Vlek, P.L.G. 2016. Soil Organic Carbon Sequestration in Jatropha curcas Systems in Burkina Faso. Land degradation \& development, 27(8): 1813-1819. https://doi.org/10.1002/ldr.2310

Blanco-Canqui, H., Shapiro, C.A., Wortmann C.S., Drijber, R.A., Mamo, M., Shaver, T.M. \& Ferguson, R.B. 2013. Soil organic carbon: The value to soil properties. Journal of Soil and Water Conservation, 68: 129A-134A. https://doi.org/10.2489/jswc.68.5.129A

Borin, M., Vianello, M., Morari, F. \& Zanin, G. 2005. Effectiveness of a buffer strip in removing runoff pollutants from a cultivated field in North-East Italy. Agriculture Ecosystems \& Environment, 105(1-2): 101114. https://doi.org/10.1016/j.agee.2004.05.011

Borin M., Passoni, M. Thiene, M. \& Tempesta, T. 2010. Multiple functions of buffer strips in farming areas. European Journal of Agronomy, 32: 103-111. https://doi.org/10.1016/j.eja.2009.05.003

Chambers, A., Lal, R. \& Paustian, K. 2016 Soil carbon sequestration potential of US croplands and grasslands: Implementing the 4 per thousand initiative. Journal of Soil and Water Conservation, 71(3): 68A. https://doi.org/10.2489/jswc.71.3.68A

Cole, L.J., Stockan, J. \& Helliwell, R. 2020. Managing riparian buffer strips to optimise ecosystem services: A review. Agriculture, Ecosystems and Environment, 296: 106891. https://doi.org/10.1016/j.agee.2020.106891

Collins, A.L., Hughes, G., Zhang, Y. \& Whitehead, J. 2009. Mitigating diffuse water pollution from agriculture: Riparian buffer strip performance with width. CAB reviews: Perspectives in Agriculture, Veterinary Science, Nutrition and Natural Resources, 4: 039. 
Dass, A., Sudhishri, S., Lenka, N.K. \& Patnaik, U.S. 2011. Runoff capture through vegetative barriers and planting methodologies to reduce erosion and improve soil moisture, fertility and crop productivity in southern Orissa, India. Nutrient Cycling in Agroecosystems, 89: 45-57. https://doi.org/10.1007/s10705010-9375-3

Falloon, P., Powlson, D. \& Smith, P. 2004. Managing field margins for biodiversity and carbon sequestration: a Great Britain case study. Soil Use and Management, 20: 240-247. https://doi.org/10.1111/j.1475-2743.2004.tb00364.x

Fan, J., Lijiao, Y., Pei, Z. \& Ge, Z. 2015. Effects of grass contour hedgerow systems on controlling soil erosion in red soil hilly areas, Southeast China. International Journal of Sediment Research, 30(2): 107-116. https://doi.org/10.1016/j.ijsrc.2015.03.001

Follain, S., Walter, C., Legout, A., Lemercier, B. \& Dutin, G. 2007. Induced effects of hedgerow networks on soil organic carbon storage within an agricultural landscape. Geoderma, 142: 80-95. https://doi.org/10.1016/j.geoderma.2007.08.002

Haddaway, N.R., Brown, C., Eales, J., Eggers, S., Josefsson, J., Kronvang, B., Randall, N.P. \& UusiKämppä, J. 2018. The multifunctional roles of vegetated strips around and within agricultural fields. Environmental Evidence, 7: 14. https://doi.org/10.1186/s13750-018-0126-2

Helmers, M.J., Isenhart, T.M., Dosskey, M.G., Dabney, S.M. \& Strock, J.S. 2008. Buffers and vegetative filter strips. In Final Report: Gulf Hypoxia and Local Water Quality Concerns Workshop. St. Joseph, MI: American Society of Agricultural and Biological Engineers, pp. 43-58. https://lib.dr.iastate.edu/cgi/viewcontent.cgi?article=1580\&context=abe_eng_pubs

Lal R. 1997. Land use and soil management effects on soil organic matter dynamics on alfisol in Western Nigeria. In Lal, R. et al. (Eds.) Soil Processes and the Carbon Cycle, pp 109-126. CRC Press.

Lenka, N.K., Dass, A., Sudhishri, S. \& Patnaik, U.S. 2012a. Soil carbon sequestration and erosion control potential of hedgerows and grass filter strips in sloping agricultural lands of eastern India. Agriculture, Ecosystems \& Environment, 158: 31-40. https://doi.org/10.1016/j.agee.2012.05.017

Lenka, N.K., Choudhury, P.R., Sudhishri, S., Dass, A. \& Patnaik, U.S. 2012 b. Soil aggregation, carbon build up and root zone soil moisture in degraded sloping lands under selected agroforestry based rehabilitation systems in eastern India. Agriculture, Ecosystems \& Environment, 150: 54-62. https://doi.org/10.1016/j.agee.2012.01.003

Mafa-Attoye, T.G., Baskerville, M.A., Ofosu, E., Oelbermann, M., Thevathasan, N.V \& Dunfield, K.E. 2020. Riparian land-use systems impact soil microbial communities and nitrous oxide emissions in an agroecosystem. Science of the Total Environment, 724: 138148.

https://doi.org/10.1016/j.scitotenv.2020.138148

Marshall, E. \& Moonen A. 2002. Field margins in northern Europe: their functions and interactions with agriculture. Agriculture, Ecosystems \& Environment, 89(1):5-21. https://doi.org/10.1016/S01678809(01)00315-2 
Marton, J.M., Fennessy, M.S. \& Craft, C.B.2014. USDA Conservation Practices Increase Carbon Storage and Water Quality Improvement Functions: An Example from Ohio. Restoration Ecology, 22(1): 117-124. https://doi.org/10.1111/rec.12033

Minasny B., Malone B.P., McBratney A.B., Angers, D.A., Arrouays, D., Chambers, A., Chaplot, V., Chen, Z.S., Cheng, K., Das, B.S., Field, D.J., Gimona, A., Hedley, C.B., Hong, S.Y., Mandal, B., Marchant, B.P., Martin, M., McConkey, B.G., Mulder, V.L., O'Rourke, S., Richer-de-Forges, A.C., Odeh, I., Padarian, J., Paustian, K., Pan, G., Poggio, L., Savin, I., Stolbovoy, V., Stockmann, U., Sulaeman, Y., Tsui, C.C., Vågen, T.G., van Wesemael, B. \& Winowiecki, L. 2017. Soil carbon 4 per mille, Geoderma, 292: 59-86. https://doi.org/10.1016/j.geoderma.2017.01.002

Prosser, R.S., Hoekstra, P.F., Gene, S., Truman, C., White, M. \& Hanson, M.L. 2020. A review of the effectiveness of vegetated buffers to mitigate pesticide and nutrient transport into surface waters from agricultural areas. Journal of Environmental Management, 261: 110210.

https://doi.org/10.1016/j.jenvman.2020.110210

Sauer, T.J., Cambardella, C.A. \& Brandle, J.R. 2007. Soil carbon and tree litter dynamics in a red cedarscotch pine shelterbelt. Agroforest Systems, 71: 163-174. https://doi.org/10.1007/s10457-007-9072-7

Seobi, T., Anderson, S.H., Udawatta, R.P. \& Gantzer, C.J. 2005. Influence of Grass and Agroforestry Buffer Strips on Soil Hydraulic Properties for an Albaqualf. Soil Science Society of America Journal, 69: 893901. https://doi.org/10.2136/sssaj2004.0280

Sitzia, T., Pizzeghello, D., Dainese, M., Ertani, A., Carletti, P., Semenzato, P., Nardi, S. \& Cattaneo, D. 2014. Topsoil organic matter properties in contrasted hedgerow vegetation types. Plant Soil, 383: 337348. https://doi.org/10.1007/s11104-014-2177-7

Thomas, Z., Molénat, J., Caubel, V., Grimaldi, C. \& Mérot, P. 2008. Simulating soil-water movement under a hedgerow surrounding a bottomland reveals the importance of transpiration in water balance. Hydrological Processes: An International Journal, 22(5): 577-585.

Stutter, M.I. \& Richards, S. 2012 Relationship between soil physicochemical, microbiological properties and nutrient release in buffer soils compared to field soils. Journal of Environmental Quality, 41: 400-409. https://doi.org/10.2134/jeq2010.0456

Uri, N.D. 2001. Conservation practices in U.S. agriculture and their impact on carbon sequestration. Environmental Monitoring and Assessment, 70: 323-344. https://doi.org/10.1023/A:1010735510641

U.S. Department of Agriculture (USDA). Natural Resources Conservation Service. Buffer Strips: Common Sense Conservation [online]. [Cited 18 November 2020].

https://www.nres.usda.gov/wps/portal/nres/detail/national/newsroom/features/?cid=nres143_023568

Van Vooren, L., Reubens, B., Broekx, S., De Frenne, P., Nelissen, V., Pardon, P. \& Verheyen, K. 2017. Ecosystem service delivery of agri-environment measures: A synthesis for hedgerows and grass strips on arable land. Agriculture, Ecosystems \& Environment, 244: 32-51. https://doi.org/10.1016/j.agee.2017.04.015

Wratten, S.D., Gillespie, M., Decourtye, A., Mader, E. \& Desneux, N. 2012. Pollinator habitat enhancement: benefits to other ecosystem services. Agriculture, Ecosystems \& Environment, 159: 112-22. https://doi.org/10.1016/j.agee.2012.06.020 


\title{
27. Avoiding improper earth movements before planting tree crops
}

\author{
Edoardo A.C. Costantini', Simone Priori ${ }^{2}$ \\ 'Academy of Georgofili, Florence, National Academy of Agriculture, Bologna, Italy \\ ${ }^{2}$ Department of Agriculture and Forest Sciences, University of Tuscia, Viterbo, Italy
}

\section{Description of the practice}

Earth movement for soil preparation is a common practice before planting tree crops in intensive farming. The practice is aimed at creating smooth slopes through land leveling, to ease the mechanized management of the crop, and is accompanied by deep ploughing, backhoe delving, or ripping, to loosen soil compaction and ensure optimal conditions for root and plant growth (Saayman, 1982). Earth movements produce a deep upsetting of the original soil profile and the scalping of the surface horizons, richer in organic matter, which are lost by water and tillage erosion (Ramos, Cots-Folch and Martinez-Casanovas, 2007). Examples of earthworks resulting in significant impairment of soil chemical, physical, biological, and hydrological balances are well documented throughout the world (Brye et al., 2003; Bazzoffi et al., 2006; Costantini and Barbetti, 2008; MartínezCasasnovas and Ramos, 2009; Costantini et al., 2018b; Dazzi et al., 2019; Photo 34). On the other hand, there are few research papers reporting the benefit of avoiding scalping and improper earth movements before planting tree crops or comparing alternative deep tillage techniques (Saayman and Van Huyssteen, 1980; Coulouma et al., 2006). It is assumed that the correct choice of procedure and tool to be used for vineyard and orchards soil preparation should be dictated by the soil type and the target crop result (Van Zyl and Hoffman, 2019), but not much emphasis is given to soil functionality, and in particular, to organic carbon storage. To preserve soil organic matter, an alternative procedure has been suggested where topsoil is scraped and collected into piles to be re-spread after completion of the other earthworks (Sharp-Heward, Almond and Robinson, 2014; Costantini et al. 2018a, Photo 35). The procedure is accompanied by ripping, to loosen the soil without upsetting the deep horizons, positioning the drainage works, and a inimized land leveling, whenever there is the need to fill small depressions, or create benches. 


\section{Range of applicability}

The procedure can be applied in the sloping lands where a new tree crop is planted, either on an existing plantation or where the land use is changed. The instrument used to lose the deeper soil layers may be adapted to local soil features, for instance, the presence of cemented horizons, shallow rock, abundant stoniness, or layers with contrasted particle size (Van Zyl and Hoffman, 2019). The technique should always be complemented with the setting up of proper water regulation systems, such as subsurface drains, ditches, pipes and cut-off drains.

\section{Impact on SOC sequestration}

Minimized land leveling compared to soil scalping during pre-planting earthworks provides the preservation of more than 50 percent of the $\mathrm{C}$ storage, on average, in both topsoil and subsoil. Baseline $\mathrm{C}$ stock in Table 120 refers to the areas where soil is scalped (business as usual practice), while additional C storage shows the effect of the alternative strategy, with no scalping and adoption of minimum leveling and/or topsoil re-spreading. Statistically, the level of significant differences between C stock in scalped and non-scalped soils are reported in the column "More information" (replicates: 3 vineyards in each location).

Table 120. Evolution of SOC stocks (topsoil and subsoil) thanks to minimized land leveling operations and no scalping and/or topsoil re-spreading

\begin{tabular}{|c|c|c|c|c|c|c|}
\hline Location & Climate zone & Soil type & $\begin{array}{l}\text { Baseline C } \\
\text { stock } \\
\text { (tC/ha) }\end{array}$ & $\begin{array}{l}\text { Additional C } \\
\text { storage } \\
\text { (tC/ha) }\end{array}$ & $\begin{array}{l}\text { Depth } \\
(\mathrm{cm})\end{array}$ & $\begin{array}{l}\text { More } \\
\text { information }\end{array}$ \\
\hline \multirow{2}{*}{$\begin{array}{l}\text { Narbonne, } \\
\text { Aude, France }\end{array}$} & \multirow{2}{*}{$\begin{array}{l}\text { Warm temperate } \\
\text { dry }\end{array}$} & \multirow{2}{*}{ Haplic Calcisols } & 9.8 & +9.5 & $0-20$ & \multirow{2}{*}{$p<0.01$} \\
\hline & & & 11.1 & +1.1 & $20-60$ & \\
\hline \multirow{2}{*}{ Abalos, Spain } & \multirow{2}{*}{$\begin{array}{l}\text { Warm temperate } \\
\text { dry }\end{array}$} & \multirow{2}{*}{$\begin{array}{l}\text { Cambic Calcisols, } \\
\text { Calcaric Cambisols }\end{array}$} & 16.8 & +3.8 & $0-20$ & \multirow{2}{*}{$p<0.10$} \\
\hline & & & 31.1 & +0.3 & $20-60$ & \\
\hline \multirow{2}{*}{$\begin{array}{l}\text { Bonini, Koper, } \\
\text { Slovenia, }\end{array}$} & \multirow{3}{*}{$\begin{array}{l}\text { Warm temperate } \\
\text { moist }\end{array}$} & \multirow{2}{*}{ Calcaric Cambisols } & 10.4 & +25.6 & $0-20$ & \multirow{2}{*}{$p<0.01$} \\
\hline & & & 10.0 & +50.1 & $20-60$ & \\
\hline $\begin{array}{l}\text { Prade, Koper, } \\
\text { Slovenia }\end{array}$ & & Calcaric Cambisols & 28.3 & +12.3 & $0-20$ & $p<0.05$ \\
\hline \multirow{2}{*}{$\begin{array}{l}\text { Greve in } \\
\text { Chianti, } \\
\text { Florence, } \\
\text { Italy }\end{array}$} & \multirow{4}{*}{$\begin{array}{l}\text { Warm temperate } \\
\text { dry }\end{array}$} & \multirow{2}{*}{$\begin{array}{l}\text { Cambic Calcisols, } \\
\text { Calcaric Cambisols }\end{array}$} & 14.4 & +6.4 & $0-20$ & \multirow{2}{*}{$p<0.05$} \\
\hline & & & 21.4 & +4.0 & $20-60$ & \\
\hline \multirow{2}{*}{$\begin{array}{l}\text { Civitella } \\
\text { Marittima, } \\
\text { Grosseto, } \\
\text { Italy }\end{array}$} & & \multirow{2}{*}{$\begin{array}{l}\text { Cambic Calcisols, } \\
\text { Calcic Vertisols }\end{array}$} & 6.9 & +20.3 & $0-20$ & \multirow{2}{*}{$p<0.01$} \\
\hline & & & 17.8 & +32.7 & $20-60$ & \\
\hline
\end{tabular}




\begin{tabular}{|l|l|l|l|l|l|l|}
\hline Location & Climate zone & Soil type & $\begin{array}{l}\text { Baseline C } \\
\text { stock } \\
(\mathrm{tC} / \mathrm{ha})\end{array}$ & $\begin{array}{l}\text { Additional C } \\
\text { storage } \\
\text { (tC/ha) }\end{array}$ & $\begin{array}{l}\text { Depth } \\
(\mathrm{cm})\end{array}$ & $\begin{array}{l}\text { More } \\
\text { information }\end{array}$ \\
\hline $\begin{array}{l}\text { Tarsus, } \\
\begin{array}{l}\text { Mersin, } \\
\text { Turkey }\end{array}\end{array}$ & Petric Calcisols & 21.5 & +7.9 & $0-20$ & $\mathrm{p}<0.05$ \\
\hline
\end{tabular}

\section{Other benefits of the practice}

\subsection{Improvement of soil properties}

The comparison between scalped and non-scalped soils shows a significant difference in rooting depth, available water capacity, total nitrogen and cation exchange capacity, bulk density, and concentration of carbonates (Costantini et al., 2018b). Preserved topsoils show better biological functionality, in particular, the ability to recycle organic matter through Oribatida acari and Collembola and to decompose recalcitrant organic matter, through a more efficient enzymatic activity (Costantini et al., 2018a).

\subsection{Minimization of threats to soil functions}

Table 121. Soil threats

\begin{tabular}{|l|l|}
\hline Soil threats & \\
\hline Soil erosion & $\begin{array}{l}\text { Scalped soils are more prone to water erosion, because of the scarce } \\
\text { vegetation cover (Bazzoffi et al., 2006, Corti et al., 2011) }\end{array}$ \\
\hline $\begin{array}{l}\text { Nutrient imbalance and } \\
\text { cycles }\end{array}$ & $\begin{array}{l}\text { Preserved soils have a better nitrogen and cation exchange capacity } \\
\text { (Costantini } \text { et al., 2018a). }\end{array}$ \\
\hline $\begin{array}{l}\text { Soil salinization and } \\
\text { alkalinization }\end{array}$ & $\begin{array}{l}\text { Properly redistributed sediments may reduce soil salinization (Sharp- } \\
\text { Heward, Almond and Robinson, 2014). }\end{array}$ \\
\hline Soil biodiversity loss & $\begin{array}{l}\text { Scalped and not scalped soils differ for fungal and bacterial } \\
\text { communities (Schroers, Castaldini and Martensson, 2018). }\end{array}$ \\
\hline
\end{tabular}




\section{Soil threats}

\begin{tabular}{|l|l|}
\hline Soil compaction & $\begin{array}{l}\text { The use of ripper instead of plough reduces soil compaction } \\
\text { (Coulouma et al., 2006). }\end{array}$ \\
\hline Soil water management & $\begin{array}{l}\text { Scalped in comparison to non-scalped soils produce higher runoff } \\
\text { and increase the risk of flooding (Costantini and Barbetti, 2008). }\end{array}$ \\
\hline
\end{tabular}

\subsection{Increases in production (e.g. food/fuel/feed/timber/fibre)}

Scalped soils of rainfed vineyards show a marked decrease of grape yield and, in some worst cases, death of grapevines and/or lack of yield (Martínez-Casasnovas and Ramos, 2009). Frequently, the reduced production induces a concentration of juices in berries that leads to an excess of sugar and polyphenols accumulation, which can be detrimental to wine grape quality (Bazzoffi et al., 2007). On the other hand, irrigation could effectively counterbalance natural low water supply for table grape production (Costantini et al., 2018a; Dazzi et al., 2019).

\subsection{Mitigation of and adaptation to climate change}

No data are available about the impact on climate change mitigation and adaptation, however, non-scalped soils show better functionality than scalped, also in terms of biomass production and absorption of atmospheric $\mathrm{C}$, so it is expected that they have a positive effect on climate change mitigation. On the other hand, soils that have been scalped in the past, and are very poor in SOC, show a large potentiality to sequester carbon through proper management (Shi et al., 2009).

\subsection{Socio-economic benefits}

Mechanical levelling of the slopes and plantation of wide vineyards depreciate the visual impact of the landscape, whereas irregular morphology and small fields with heterogeneous crops increase the beauty of landscape (Costantini and Barbetti, 2008). 


\section{Potential drawbacks to the practice}

\subsection{Tradeoffs with other threats to soil functions}

Although planting a new tree crop always implies a certain disturbance of soil features, in particular soil biology, avoiding excessive earth movements before plantation is certainly less harmful than the business-as-usual practice for all soil threats.

\subsection{Increases in greenhouse gas emissions}

Reducing the amount of soil moved during the earthworks done before a new plantation implies the use of less machinery and fuel consumption, then also a lowering of the carbon footprint of the activity.

\subsection{Conflict with other practice(s)}

Farmers who prioritize the reduction of time can fear for the extra work needed with the alternative strategy, because of the more articulated mechanical operations (topsoil removing and accumulation in piles, deep ripping of subsoil, gentle levelling, re-spreading of topsoil) in comparison with the use of big bulldozers. Furthermore, the resulting slope of the vineyard or orchard may result more undulated and less uniform, thus somehow reducing the speed of mechanic operations during the ordinary crop cultivation. The relative reduced speed, however, would also decrease the risk of incident for the operators working on steep slopes.

\subsection{Decreases in production (e.g. food/fuel/feed/timber/fibre)}

Avoiding topsoil scalping has positive impact on yield. In an international trial on 17 vineyards located in Mediterranean countries, the difference of grape yield between eroded and preserved soils was on average highly significant, that is, $2 \mathrm{~kg}$ per plant instead of 0.8 (Costantini et al., 2018).

\subsection{Other conflicts}

The landowner, after obtaining permission for a new tree plantation, usually assigns the work to an earthmoving company with no expertise in soil protection and which possibly also has an interest in moving large volumes of earth to raise the requested fee (Bazzoffi and Tesi, 2011). 


\section{Recommendations before implementing the practice}

During the land preparation planning, a detailed study of the soil spatial variability, also through innovative techniques like proximal and remote sensing, allows a site-specific approach and strongly increases the success of the new plantation (Priori et al., 2013, 2018).

\section{Potential barriers to adoption}

Table 122. Potential barriers to adoption

\begin{tabular}{|c|c|c|}
\hline Barrier & YES/NO & \\
\hline Economic & Yes & $\begin{array}{l}\text { The adoption of the practice can be in some cases more } \\
\text { expensive and time consuming than the common procedure } \\
\text { of land preparation before tree plantation (Bazzoffi and Tesi, } \\
\text { 2011). }\end{array}$ \\
\hline Knowledge & Yes & $\begin{array}{l}\text { Land preparation for tree crops is usually made by third party } \\
\text { companies with little knowledge of the very local soil } \\
\text { conditions. Furthermore, the operators are not aware on the } \\
\text { importance of the topsoil and on the difficulty of restoring a } \\
\text { scalped soil (Bazzoffi and Tesi, 2011). }\end{array}$ \\
\hline
\end{tabular}




\section{Photos of the practice}

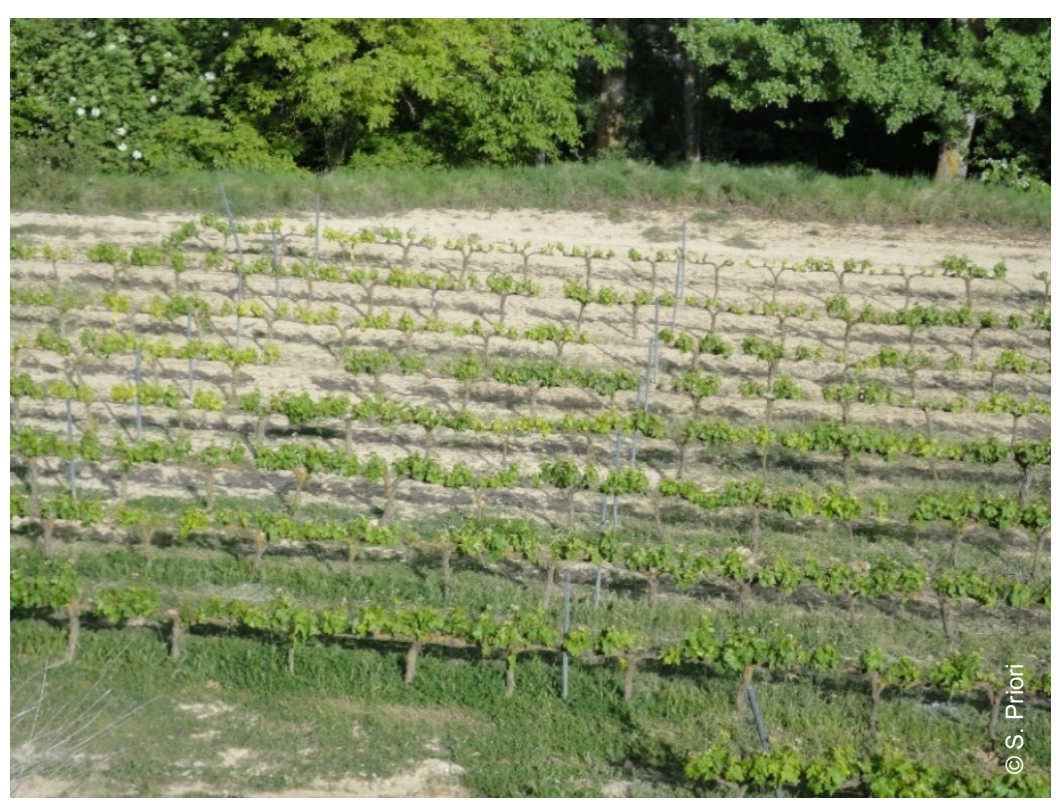

Photo 34. Effects of a business-as-usual strategy before planting a vineyard.

Scalped and non-scalped soils alternate on the slope due to improper earth movements. Note the consequencesn the topsoil organic matter content (evidenced by the pale-yellow color) and on the different vigor of the vines and grass cover

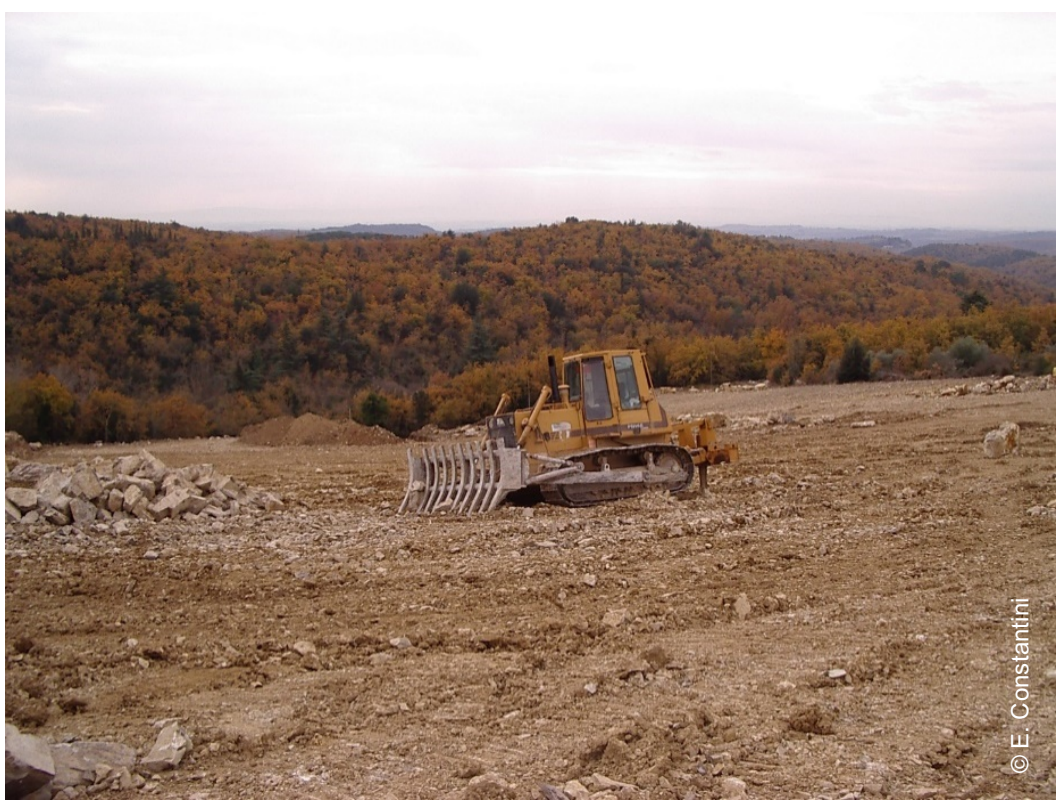

Photo 35. Alternative strategy for topsoil conservation before planting a new vineyard.

Gentle reshaping of the slope, with removal of large stones from soil surface, after soil loosening with a ripper. Note the stockpiled topsoil, in the background close to the forest, which will be spread over the surface after removing the boulders 


\section{References}

Bazzoffi, P., Abbattista, F., Vanino, S. \& Pellegrini, S. 2006. Impact of land levelling for vineyard plantation on soil degradation in Italy. Bollettino della Società Geologica Italiana. Spec., 6: 191-199.

Bazzoffi, P., Pellegrini, S., Storchi, P., Bucelli, P. \& Rocchini, A. 2007. Impact of land levelling on soil degradation, vineyard health and grape quality. In Proc. 11th GESCO International Symp., Porec, Croatia, pp. 346-357.

Bazzoffi, P. \& Tesi, P.C. 2011. Effectiveness of the GAEC standard of cross compliance Prohibition of performing unauthorized land levelling on soil erosion control. Italian Journal of Agronomy, 6: e4-e4. https://doi.org/10.4081/ija.2011.6.s1.e4

Brye, K.R., Slaton, N.A., Savin, M.C., Norman, R.J. \& Miller, D.M. 2003. Short-term effects of land leveling on soil physical properties and microbial biomass. Soil Science Society of America Journal, 67(5): 1405-1417. https://doi.org/10.2136/sssaj2003.1405

Corti, G., Cavallo, E., Cocco, S., Biddoccu, M., Brecciaroli, G. \& Agnelli, A. 2011. Evaluation of erosion intensity and some of its consequences in vineyards from two hilly environments under a Mediterranean type of climate, Italy. Soil erosion issues in agriculture. InTech, Rïeka, 113-160.

https://doi.org/10.5772/25130

Costantini, E.A. \& Barbetti, R. 2008. Environmental and visual impact analysis of viticulture and olive tree cultivation in the province of Siena (Italy). European Journal of Agronomy, 28(3): 412-426.

https://doi.org/10.1016/j.eja.2007.11.012

Costantini, E.A.C. Castaldini, M., Diago, M.P., Giffard, B., Lagomarsino, A., Schroers, H.J., Priori S., Valboa G., Agnelli A.E., Akça, E., D'Avino, L., Fulchin, E., Gagnarli, E., Kiraz, M.E., Knapič, M., Pelengić, R., Pellegrini, S., Perria, R., Puccioni, S., Simoni, S., Tangolar, S., Tardaguila, J., Vignozzi, N. \&Zombardo, A. 2018a. Effects of soil erosion on agro-ecosystem services and soil functions: A multidisciplinary study in nineteen organically farmed European and Turkish vineyards. Journal of environmental management, 223: 614-624. https://doi.org/10.1016/j.jenvman.2018.06.065

Costantini, E.A.C., Priori, S., Giffard, B., Fulchin, E., Tardaguila, J., Schroers, H.J., Pelengić, R., Akça, E., Tangolar, S. \& Valboa, G. 2018b. Causes of soil malfunctioning in degraded areas of European and Turkish vineyards. EQA-Environmental quality, 30 : 15-22. https://doi.org/10.6092/issn.2281$4485 / 7906$

Coulouma, G., Boizard, H., Trotoux, G., Lagacherie, P. \& Richard, G. 2006. Effect of deep tillage for vineyard establishment on soil structure: A case study in Southern France. Soil and Tillage Research, 88(1-2): 132143. https://doi.org/10.1016/j.still.2005.05.002

Dazzi, C., Galati, A., Crescimanno, M. \& Papa, G.L. 2019. Pedotechnique applications in large-scale farming: Economic value, soil ecosystems services and soil security. Catena, 181: 104072. https://doi.org/10.1016/j.catena.2019.104072 
Martínez-Casasnovas, J.A. \& Concepcion Ramos, M. 2009. Soil alteration due to erosion, ploughing and levelling of vineyards in north east Spain. Soil use and management, 25(2): 183-192.

https://doi.org/10.1111/j.1475-2743.2009.00215.x

Priori, S., Fantappiè, M., Magini, S. \& Costantini, E.A.C. 2013. Using the ARP-03 for high-resolution mapping of calcic horizons. International Agrophysics, 27(3). https://doi.org/10.2478/v10247-0120100-0

Priori, S., L'Abate, G., Fantappiè, M. \& Costantini, E.C. 2018. Mapping soil spatial variability at high detail by proximal sensors for a vineyard planning. EQA-Environmental quality, 30: 9-15.

https://doi.org/10.6092/issn.2281-4485/7890

Ramos, M.C., Cots-Folch, R. \& Martínez-Casasnovas, J.A. 2007. Effects of land terracing on soil properties in the Priorat region in Northeastern Spain: A multivariate analysis. Geoderma, 142(3-4): 251261. https://doi.org/10.1016/j.geoderma.2007.08.005

Saayman, D. 1982. Soil preparation studies: II. The effect of depth and method of soil preparation and of organic material on the performance of Vitis vinifera (var. Colombar) on Clovelly/Hutton soil. South African Journal of Enology and Viticulture, 3(2): 61-74.

Saayman, D. \& Van Huyssteen, L. 1980. Soil Preparation Studies: I. The Effect of Depth and Method of Soil Preparation and of Organic Material on the performance of Vi tis Vinifera (var. Chenin Blanc) on Hutton/Sterkspruit Soil. South African journal of enology and viticulture, 1(2): 107-121.

Schroers, H.J., Castaldini, M. \& Martensson, A. 2018. Inferences from inventories of microbes in ecological vineyard settings. EQA-Environmental quality, 31: 47-56. https://doi.org/10.6092/issn.2281$4485 / 7921$

Sharp-Heward, S., Almond, P. \& Robinson, B. 2014. Soil disturbance and salinisation on a vineyard affected by landscape recontouring in Marlborough, New Zealand. Catena, 122: 170-179. https://doi.org/10.1016/j.catena.2014.06.022

Shi, X.Z., Wang, H.J., Yu, D.S., Weindorf, D.C., Cheng, X.F., Pan, X.Z., Sun, W.X. \& Chen, J.M. 2009. Potential for soil carbon sequestration of eroded areas in subtropical China. Soil and Tillage Research, 105(2), 322-327. https://doi.org/10.1016/j.still.2008.12.016

Zyl, J.L. \& Hoffman J.E. 2019. Soil Preparation for Sustainable Wine and Table Grape vineyards. Ed Kindle. 


\title{
28. Adequate irrigation practices
}

\author{
Waqas Ahmad, Eva Pek, Maher Salman \\ Land and Water Division (NSL), Food and Agriculture Organization of the United Nations, Rome, Italy
}

\section{Description of the practice}

The soil-water interactions brought about by hydrological events and management practices can affect soil organic carbon (SOC) loss and/or gain. Crop irrigation using different techniques is practiced on 20 percent of the global cultivated lands and hence plays an important role in SOC sequestration. Surface or flood irrigation (where water is applied at one end of a field and is allowed to spread and infiltrate across the entire field under the action of gravity) has been the most widely used irrigation practice for thousands of years i Due to increasing competition for water, new irrigation techniques have been introduced to cope with the shortage of water and to increase water productivity such as drip, sprinkle deficit and subsurface irrigation. The type of irrigation in the presence of external factors like climate and the condition of soil is known to have variable effects on the SOC emission and sequestration (Guo et al, 2017). Although there is limited literature on the impacts of irrigation and drainage on the SOC sequestration, as a rule of thumb the irrigation water increases the capacity of the land to produce biomass (Wang et al., 2019), and therefore the accumulation of plant residues (mainly roots) are mainly responsible for the increase of SOC input. Other important changes usually occur in the SIC pool (e.g. see (Hannam et al., 2016)), but they fall out of the scope of this manual. On the other side, the higher soil moisture contents increase SOC mineralization rates, and therefore the total balance of the resulting SOC stocks can be negative or positive depending on the environment (Aguilera et al., 2018). If not well managed, surface drainage water or soil erosion takes away the stored SOC from the soil and releases it to the atmosphere through mineralization. In summary, the amount and mode of irrigation has varying impacts on the SOC sequestration potential and on the physical and biological properties of the soil in the top layer.

\section{Range of applicability}

Irrigated agriculture is being practiced worldwide on an area of about 275 million hectares (UNESCO WWAP, 2017 ) which has a large potential of SOC sequestration. Different modes of irrigation, climatic conditions, soil types, soil moisture content and soil temperature affect the degree of SOC sequestration. 


\section{Impact on soil organic carbon stocks}

Given the wide variety of irrigation types and environments, only some examples of the SOC sequestration potential of irrigated croplands (i.e. irrigation in combination with other conservation practices) is shown in Table 123. In all the reported cases the $\mathrm{C}$ sequestration potential is evaluated for different crop lands under varying irrigation amounts. Each irrigation type and the applied amount of water could perform differently in a different environmental condition. For a new environmental setting or condition it either should be initiated on a pilot scale or simulated using a mathematical model to precisely quantify the potential of irrigation type.

Table 123. SOC sequestration potential of irrigated croplands

\begin{tabular}{|c|c|c|c|c|c|c|c|}
\hline Location & $\begin{array}{l}\text { Climate } \\
\text { zone }\end{array}$ & Soil type & $\begin{array}{l}\text { Baseline } \\
\text { C stock } \\
\text { (tC/ha) }\end{array}$ & $\begin{array}{l}\text { Additional } \\
\text { C storage } \\
\text { (tC/ha/yr) }\end{array}$ & $\begin{array}{l}\text { Duration } \\
\text { (years) }\end{array}$ & More information & Reference \\
\hline Brazil & $\begin{array}{l}\text { Tropical } \\
\text { Moist }\end{array}$ & $\begin{array}{l}\text { Sandy loam, } \\
\text { sandy clay } \\
\text { loam }\end{array}$ & 40.1 & 0.27 & 20 & Sprinkler irrigation & $\begin{array}{l}\text { Campos, } \\
\text { Pires and } \\
\text { Costa } \\
\text { (2020); Dias } \\
\text { et al. (2019) }\end{array}$ \\
\hline $\begin{array}{l}\text { Kansas, } \\
\text { United } \\
\text { States of } \\
\text { America }\end{array}$ & NA & Silt loam & 11.2 & 5.2 & 1 & $\begin{array}{l}\text { Sprinkler irrigation } \\
\text { (irrigation depth } \\
\text { increased from } 66 \text { to } \\
217 \mathrm{~mm} \text { ) }\end{array}$ & $\begin{array}{l}\text { Blanco- } \\
\text { Canqui et al. } \\
\text { (2010) }\end{array}$ \\
\hline $\begin{array}{l}\text { Xuzhou, } \\
\text { China }\end{array}$ & \multirow{3}{*}{$\begin{array}{l}\text { Warm } \\
\text { temperate } \\
\text { dry }\end{array}$} & Loam & 24 & 0.43 & $\begin{array}{l}100 \\
\text { (simulation) }\end{array}$ & $\begin{array}{l}\text { In addition to irrigation, } \\
\text { optimum nitrogen } \\
\text { fertilizer treatment was } \\
\text { applied }\end{array}$ & $\begin{array}{l}\text { Wang et al. } \\
\text { (2014) }\end{array}$ \\
\hline $\begin{array}{l}\text { Nebraska, } \\
\text { United } \\
\text { States of } \\
\text { America }\end{array}$ & & Sandy & 5.6 & 0.8 & $\begin{array}{l}4 \text { to } 15 \\
\text { (composite } \\
\text { sample) }\end{array}$ & Sprinkler irrigation & $\begin{array}{l}\text { Lueking and } \\
\text { Schepers } \\
\text { (1985) }\end{array}$ \\
\hline $\begin{array}{l}\text { SW } \\
\text { Nebraska } \\
\text { United } \\
\text { States of } \\
\text { America }\end{array}$ & & $\begin{array}{l}\text { Alliance, } \\
\text { Goshen, } \\
\text { Rosebud } \\
\text { series } \\
\text { (Argiustoll) }\end{array}$ & NA & 0.19 & 33 & Center pivot, top $20 \mathrm{~cm}$ & $\begin{array}{l}\text { Gillabel et al. } \\
\text { (2007) }\end{array}$ \\
\hline
\end{tabular}




\section{Other benefits of the practice}

\subsection{Improvement of soil properties}

Irrigation has a negligible impact on the soil bulk density and particle size distribution. However, an increase in the amount of irrigation depth at selected sites have shown a significant increase in the number of water-stable aggregate (WSA $=4.75$ to $8 \mathrm{~mm}$ ) at a depth of $5-10 \mathrm{~cm}$ soil layer (Blanco-Canqui et al, 2010 ). It is also important to note that irrigation with good quality water does not affect the infiltration properties of the soil unless the water has high sodium contents (Wienhold and Trooien, 1998).

\subsection{Minimization of threats to soil functions}

\section{Table 124. Soil threats}

\begin{tabular}{|c|c|}
\hline Soil threats & \\
\hline Soil erosion & $\begin{array}{l}\text { Irrigation promotes vegetation growth which acts as a barrier to soil erosion. } \\
\text { Water erosion due to irrigation can be controlled by properly designed field slope } \\
\text { and overland flow velocity. }\end{array}$ \\
\hline $\begin{array}{l}\text { Nutrient imbalance } \\
\text { and cycles }\end{array}$ & $\begin{array}{l}\text { Irrigation water increases the availability of plant nutrients. Excess irrigation } \\
\text { (overflow or deep drainage) may leach plant nutrients from the soil. }\end{array}$ \\
\hline $\begin{array}{l}\text { Soil salinization } \\
\text { and alkalinization }\end{array}$ & $\begin{array}{l}\text { Soil salinity can be minimized by addition of water to the leaching requirements } \\
\text { in order to maintain Soil Electrical Conductivity below threshold values. The excess } \\
\text { water dissolves accumulated salts and leaches it down the root zone. In this case, } \\
\text { the groundwater table should not be shallow, or a functioning subsurface drainage } \\
\text { system must be in place to dispose of the saline drainage water. Soil alkalinization } \\
\text { risk must be assessed in advance by knowing the quality of the irrigation water (i.e. } \\
\text { Sodium Adsorption Ratio) and knowing the ionic content of the soil solution. } \\
\text { Gypsum amendments may be applied to lower the soil alkalinization risk. }\end{array}$ \\
\hline $\begin{array}{l}\text { Soil contamination } \\
\text { / pollution }\end{array}$ & $\begin{array}{l}\text { The use of contaminated irrigation water could chemically or biologically } \\
\text { contaminate soils. For example, if the irrigation water source is contaminated such } \\
\text { as a saline groundwater aquifer or reuse of municipal wastewater for irrigation. The } \\
\text { use of saline groundwater for irrigation should be managed by considering } \\
\text { appropriate leaching requirements to avoid soil contamination/salinization. }\end{array}$ \\
\hline Soil acidification & $\begin{array}{l}\text { Irrigation promotes crop/plant growth in the fields which adds organic matter to } \\
\text { the soil and hence reduces acidification. }\end{array}$ \\
\hline
\end{tabular}




\begin{tabular}{|c|c|}
\hline Soil threats & \\
\hline $\begin{array}{l}\text { Soil biodiversity } \\
\text { loss }\end{array}$ & $\begin{array}{l}\text { More organic matter is produced in irrigated lands (i.e. plants, stubble, roots, } \\
\text { leaves). This organic matter increases soil biodiversity through decomposition } \\
\text { when it comes into contact with soil and water. }\end{array}$ \\
\hline Soil sealing & $\begin{array}{l}\text { Some types of sprinkler irrigation, when drop size or velocity is too high, may } \\
\text { destroy surface aggregates and create seals and crusts. This effect is higher when } \\
\text { using water with an excess of sodium ion. }\end{array}$ \\
\hline $\begin{array}{l}\text { Soil water } \\
\text { management }\end{array}$ & $\begin{array}{l}\text { Nearly all soil water management practices depends on irrigation water, because } \\
\text { this is the primary source to add moisture to the soil. }\end{array}$ \\
\hline
\end{tabular}

\subsection{Impacts Increases in production (e.g. food/fuel/feed/timber/fibre) and socio-economic benefits}

The impact of irrigation on increase in crop production is broadly known and is well documented in the literature (Bennett and Harms, 2011; Qureshi, Ahmad and Ahmad, 2013). Periodic irrigation of food and fiber crops reduces the crop water stress during the critical growth stages and boosts their production potential. The high production capacity of irrigated agriculture system fosters many socio-economic benefits such food security, generation of enough raw material for the local industries, revival of agriculture-based industries, an elevated social status due to high monetary returns from the production, economic security (labor force engagement), attraction of migrants from the less privileged areas, expansion of irrigated lands, and increased economic activities in rural areas.

\subsection{Mitigation of and adaptation to climate change}

Climate change projections show large variations in temperature and precipitation patterns all over the world. These variations could pose a significant threat to the production capacity of the agricultural systems, mainly because the water supply will be at risk. Irrigation systems as always could play a vital role in reducing the negative impacts of variable water availability for agriculture under climate change. Appropriate modifications to the existing irrigation systems such as the conversion of flood to high efficiency irrigation system, construction of dykes for water harvesting and proper management of existing reservoirs could play a vital role in climate change mitigation and adaptations (Bhatti et al., 2019). 


\section{Potential drawbacks to the practice}

\subsection{Tradeoffs with other threats to soil functions}

Table 125. Soil threats

\begin{tabular}{|c|c|}
\hline Soil threats & \\
\hline Soil erosion & NA with a proper irrigation design. \\
\hline $\begin{array}{l}\text { Nutrient imbalance } \\
\text { and cycles }\end{array}$ & NA with a proper irrigation design. \\
\hline $\begin{array}{l}\text { Soil salinization and } \\
\text { alkalinization }\end{array}$ & $\begin{array}{l}\text { Excessive irrigation in poorly drained soils may cause water logging and } \\
\text { salinity. Water quality has to be checked for alkalinization and } \\
\text { salinization risk. Salt contained in deep layers in the soil profile, or in } \\
\text { other parts of the landscape may be mobilised and appear at the surface } \\
\text { through capillary rise. }\end{array}$ \\
\hline $\begin{array}{l}\text { Soil contamination / } \\
\text { pollution }\end{array}$ & $\begin{array}{l}\text { Soil contamination could increase if the irrigation water is contaminated } \\
\text { as in case of grey water }\end{array}$ \\
\hline Soil acidification & Excessive leaching of nitrates could cause soil acidification \\
\hline $\begin{array}{l}\text { Soil sealing } \\
\text { (crusting) }\end{array}$ & $\begin{array}{l}\text { Soil sealing may be enhanced by the interaction of irrigation water with } \\
\text { soils that are rich in sodium ion. This problem is site specific and the areas } \\
\text { with loose soil texture are less affected by soil sealing. Some types of } \\
\text { sprinkler irrigation may cause sealing and crusting by breaking down the } \\
\text { surface aggregates. }\end{array}$ \\
\hline Soil compaction & $\begin{array}{l}\text { Full irrigation cause a stronger compaction of surface soil even after deep } \\
\text { tillage (Liu et al., 2016). }\end{array}$ \\
\hline
\end{tabular}




\subsection{Increases in greenhouse gas emissions}

The amount and type of irrigation has a great impact on the contribution of soil to GHG emissions. Some field level experiments with different irrigation practices have shown the emission of GHGs from soil with varying emission rates as shown in Table 126. In all selected cases, flood irrigation caused a reduction in the $\mathrm{CO}_{2}$ emission from the soil while increased emission of $\mathrm{CH}_{4}$ and $\mathrm{N}_{2} \mathrm{O}$ was observed under the flood or high frequency irrigation. It shows that the suitability of an irrigation practice from the GHG emission point of view should be evaluated based on the total global warming potential under that particular irrigation practice rather than a single GHG emission rate.

Table 126. Greenhouse gas emission from soil under different irrigation practices

\begin{tabular}{|c|c|c|c|c|}
\hline $\begin{array}{l}\text { Irrigation } \\
\text { practice }\end{array}$ & Crop & $\begin{array}{l}\text { GHG emission } \\
\text { rate }\left(\mathrm{g} / \mathrm{m}^{2}\right)\end{array}$ & GHG & Reference \\
\hline Drip & \multirow{2}{*}{ Maize } & 1959.1 & \multirow{6}{*}{$\mathrm{CO}_{2}$} & \multirow{2}{*}{ Guo et al. (2017) } \\
\hline Flood & & 1759.1 & & \\
\hline Deficit/rainfed & \multirow{8}{*}{ Paddy } & 1795.8 & & \\
\hline Flood & & 1085.8 & & xu el al. (<U10) \\
\hline $\begin{array}{l}\text { Intermittent } \\
\text { drainage }\end{array}$ & & 460.6 & & Haque et al. \\
\hline Flood & & 386.4 & & \\
\hline $\begin{array}{l}\text { Continues } \\
\text { flooding }\end{array}$ & & 50.9 & \multirow{2}{*}{$\mathrm{CH}_{4}$} & \multirow{2}{*}{ Riya et al. (2014) } \\
\hline $\begin{array}{l}\text { Intermittent } \\
\text { flooding }\end{array}$ & & 30.6 & & \\
\hline $\begin{array}{l}\text { Continues } \\
\text { flooding }\end{array}$ & & 23.8 & \multirow[t]{2}{*}{$\mathrm{CH}_{4}$} & \multirow[t]{2}{*}{ Win et al. (2015) } \\
\hline Water saving & & 8.40 & & \\
\hline $\begin{array}{l}\text { High frequency } \\
\text { irrigation }\end{array}$ & \multirow{2}{*}{ Apple } & 0.068 & \multirow{2}{*}{$\mathrm{NO}_{2}$} & \multirow{2}{*}{$\begin{array}{l}\text { Fentabil et al. } \\
(2016)\end{array}$} \\
\hline $\begin{array}{l}\text { Low frequency } \\
\text { irrigation }\end{array}$ & & 0.049 & & \\
\hline
\end{tabular}


A different issue that has to be considered is the large $\mathrm{CO}_{2}$ emissions involved in the development of irrigation schemes, energy spent on water conveyance or water pumping, construction of the canals and manufacturing of pipes themselves, all of which should be taken into account by making a life cycle analysis of the irrigation projects.

\subsection{Other negative impacts of irrigation}

Irrigated agriculture could have negative impacts on the environment if not managed adequately. It is important to sustain the ecosystem services while developing irrigation schemes. The following are the major negative impacts of irrigation on the environment:

- Reduced flow in the downstream reaches of streams and rivers

- Waterborne diseases and malaria

- Saltwater intrusion near the river's mouth

- Reduced dilution of downstream effluents

- Human resettlement due to the development of large-scale irrigation schemes

- Destruction of valuable ecosystems (e.g. natural saline lakes)

\section{Recommendations before implementing the practice}

The impacts of irrigation on SOC sequestration is highly variable and depends upon several internal and external factors like irrigation type, irrigation amount, climate, soil type and a large number of soil management practices, which are different from place to place. Before implementing irrigation as a tool to promote SOC sequestration in a place other than the reported cases, it is recommended to evaluate its impacts under the prevailing climatic conditions and different combinations of field management practices on pilot scale field experiments. Before embarking on a large-scale adaptation, it is also useful to employ simulation studies using agricultural production simulation models such as APSIM (Wang et al., 2014) to analyze different scenarios of irrigation in combination with field management practices to optimize agriculture production and SOC sequestration at the same time. 


\section{Potential barriers to adoption}

\section{Table 127. Potential barriers to adoption}

\begin{tabular}{|c|c|c|}
\hline Barrier & YES/NO & \\
\hline Biophysical & Yes & $\begin{array}{l}\text { In some cases, there could be physical limitations on further } \\
\text { development of irrigated agriculture. This is particularly true in arid } \\
\text { regions where the water resources are already stressed. }\end{array}$ \\
\hline Cultural & Yes & $\begin{array}{l}\text { There might be difficulties in some regions to change the traditional } \\
\text { dryland crops to irrigated crops. }\end{array}$ \\
\hline Economic & Yes & $\begin{array}{l}\text { Some farmers may not adhere to irrigation if they have to pay for } \\
\text { the costs of conveying water to their fields and/or implementing an } \\
\text { irrigation system. }\end{array}$ \\
\hline Institutional & Yes & $\begin{array}{l}\text { In many parts of the world irrigation water is distributed } \\
\text { proportionally among the farmers based on the size of their land } \\
\text { holding. In this case, if there is an increased demand of water to } \\
\text { promote SOC sequestration then there could be institutional } \\
\text { limitations to accommodate this request. }\end{array}$ \\
\hline $\begin{array}{l}\text { Legal (Right } \\
\text { to soil) }\end{array}$ & Yes & In some places the farmers have no rights to water. \\
\hline Knowledge & Yes & $\begin{array}{l}\text { Good soil, plant and water knowledge is necessary to determine } \\
\text { irrigation timing and amount, as well as to avoid salinization, } \\
\text { alkalinization or erosion. }\end{array}$ \\
\hline
\end{tabular}




\section{Photo of the practice}

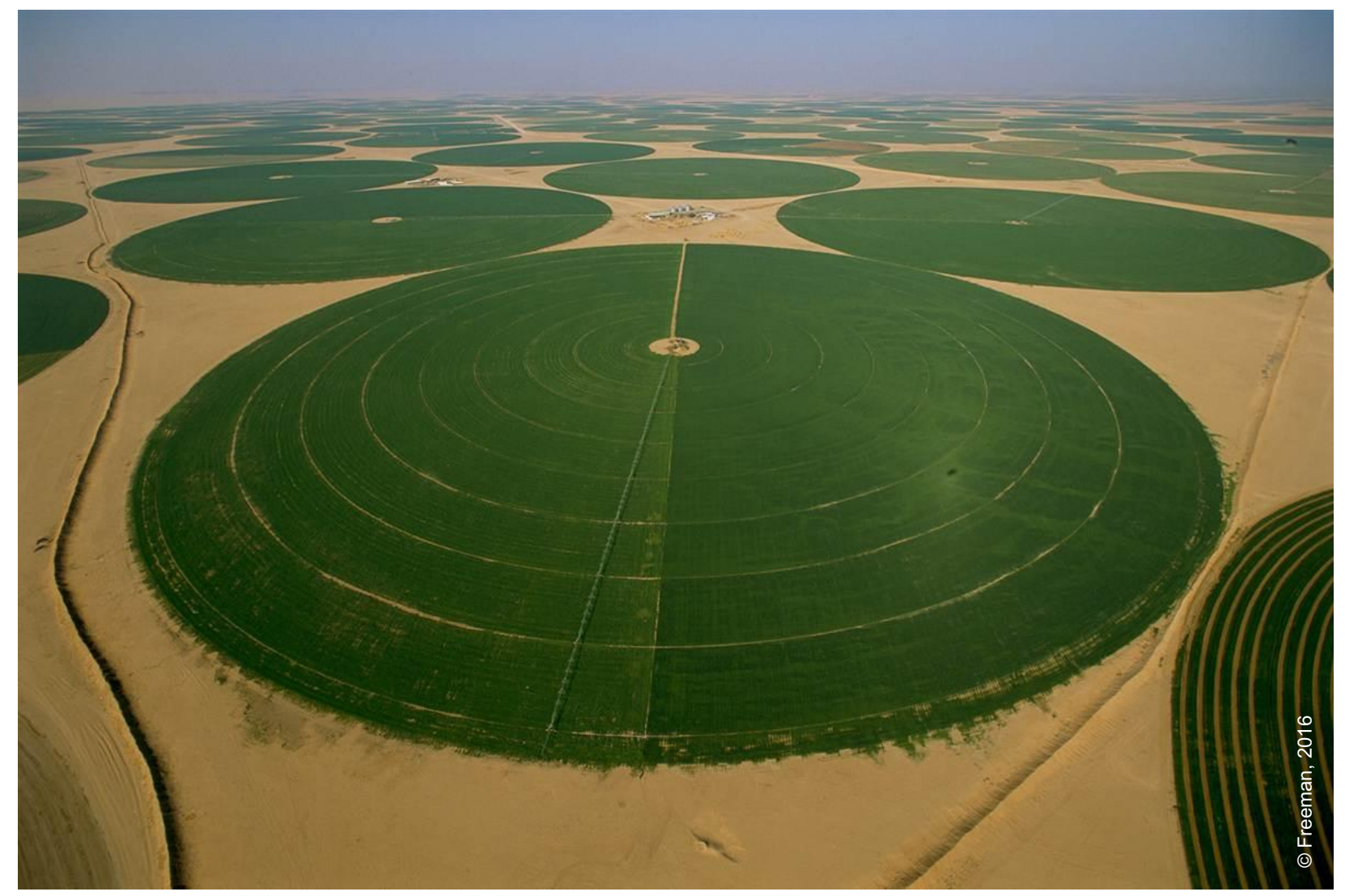

Photo 36. Center pivot sprinkler irrigation for field crops.

Table 128. Related cases studies available in volumes 3 and 5

\begin{tabular}{|l|l|l|c|c|}
\hline Title & Region & $\begin{array}{l}\text { Duration } \\
\text { of study } \\
\text { (Years) }\end{array}$ & Volume & $\begin{array}{c}\text { Case- } \\
\text { study } \\
\text { No. }\end{array}$ \\
\hline $\begin{array}{l}\text { Irrigated cotton cropping systems in } \\
\text { Australian Vertisols under minimum } \\
\text { tillage }\end{array}$ & $\begin{array}{l}\text { Southwest } \\
\text { Pacific }\end{array}$ & 4 to 20 & 3 & 8 \\
\hline $\begin{array}{l}\text { Conservation Agriculture practices in } \\
\text { north Italy }\end{array}$ & Europe & 5 to 20 & 3 & 15 \\
\hline
\end{tabular}




\begin{tabular}{|l|l|c|c|c|}
\hline Title & Region & $\begin{array}{l}\text { Duration } \\
\text { of study } \\
\text { (Years) }\end{array}$ & Volume & $\begin{array}{c}\text { Case- } \\
\text { study } \\
\text { No. }\end{array}$ \\
\hline $\begin{array}{l}\text { Mediterranean olive orchard subjected } \\
\text { to sustainable management in Matera, } \\
\text { Basilicata, Italy }\end{array}$ & Europe & 20 & 3 & 16 \\
\hline $\begin{array}{l}\text { Irrigation and SOC sequestration in the } \\
\text { region of Navarre in Spain }\end{array}$ & Europe & 6 to 20 & 3 & 19 \\
\hline $\begin{array}{l}\text { Irrigated Wheat-Maize-Cotton in the } \\
\text { Harran Plain, Southeast Turkey }\end{array}$ & Eurasia & 30 & 3 & 25 \\
\hline $\begin{array}{l}\text { Deficit irrigation scenarios using sprinkle } \\
\text { irrigation system in western Kansas, } \\
\text { United States of America }\end{array}$ & $\begin{array}{l}\text { North } \\
\text { America }\end{array}$ & 5 and 8 & 3 & 46 \\
\hline $\begin{array}{l}\text { Urban agriculture on rooftops in Paris, } \\
\text { France - the T4P research project (Pilot } \\
\text { Project of Parisian Productive Rooftops) }\end{array}$ & Europe & 5 & 5 & 23 \\
\hline $\begin{array}{l}\text { Water and residues management on a } \\
\text { golf course, Nebraska, United States }\end{array}$ & America & 4 & 5 & 30 \\
\hline
\end{tabular}




\section{References}

Aguilera, E., Guzmán, G.I., Álvaro-Fuentes, J., Infante-Amate, J., García-Ruiz, R., Carranza-Gallego, G., Soto, D. \& de Molina, M.G. 2018. A historical perspective on soil organic carbon in Mediterranean cropland (Spain, 1900-2008). Science of The Total Environment, 621: 634-648.

https://doi.org/10.1016/j.scitotenv.2017.11.243

Bennett, D.R. \& Harms, T.E. 2011. Crop Yield and Water Requirement Relationships for Major Irrigated Crops in Southern Alberta. Canadian Water Resources Journal, 36(2): 159-170.

https://doi.org/10.4296/cwrj3602853

Bhatti, M.T., Ahmad, W., Shah, M.A. \& Khattak, M.S. 2019. Climate change evidence and community level autonomous adaptation measures in a canal irrigated agriculture system of Pakistan. Climate and Development, 11(3): 203-211. https://doi.org/10.1080/17565529.2018.1442803

Blanco-Canqui, H., Klocke, N.L., Schlegel, A.J., Stone, L.R. \& Rice, C.W. 2010. Impacts of Deficit Irrigation on Carbon Sequestration and Soil Physical Properties under No-Till. Soil Science Society of America Journal, 74(4): 1301-1309. https://doi.org/10.2136/sssaj2009.0364

Campos, R., Pires, G.F. \& Costa, M.H. 2020. Soil Carbon Sequestration in Rainfed and Irrigated Production Systems in a New Brazilian Agricultural Frontier. Agriculture, 10(5): 156.

https://doi.org/10.3390/agriculture10050156

Dias, F.P.M., Hübner, R., Nunes, F. de J., Leandro, W.M. \& Xavier, F.A. da S. 2019. Effects of land-use change on chemical attributes of a Ferralsol in Brazilian Cerrado. Catena. (also available at https://agris.fao.org/agris-search/search.do? recordID=US201900209624).

Fentabil, M.M., Nichol, C.F., Jones, M.D., Neilsen, G.H., Neilsen, D. \& Hannam, K.D. 2016. Effect of drip irrigation frequency, nitrogen rate and mulching on nitrous oxide emissions in a semi-arid climate: An assessment across two years in an apple orchard. Agriculture, Ecosystems \& Environment, 235: 242-252. https://doi.org/10.1016/j.agee.2016.09.033

Freeman, F. 2016. Center Pivot Irrigation (Saudi Arabia), Reddit, https://externalpreview.redd.it/60T_Ju9i4qD4pFo8W3Acd3_Ti51e6I59LvujSd3R9k.jpg?auto=webp\&s=3340c6dcb5bab9822d6402e31c163b014bb49ff1

Guo, S., Qi, Y., Peng, Q., Dong, Y., He, Y., Yan, Z. \& Wang, L. 2017. Influences of drip and flood irrigation on soil carbon dioxide emission and soil carbon sequestration of maize cropland in the North China Plain. Journal of Arid Land, 9(2): 222-233. https://doi.org/10.1007/s40333-017-0011-9

Hannam, K.D., Kehila, D., Millard, P., Midwood, A.J., Neilsen, D., Neilsen, G.H., Forge, T.A., Nichol, C. \& Jones, M.D. 2016. Bicarbonates in irrigation water contribute to carbonate formation and CO2 production in orchard soils under drip irrigation. Geoderma, 266: 120-126.

https://doi.org/10.1016/j.geoderma.2015.12.015

Haque, M.M., Biswas, J.C., Kim, S.Y. \& Kim, P.J. 2016. Suppressing methane emission and global warming potential from rice fields through intermittent drainage and green biomass amendment. Soil Use and Management, 32(1): 72-79. https://doi.org/10.1111/sum.12229 
Liu, X., Feike, T., Shao, L., Sun, H., Chen, S. \& Zhang, X. 2016. Effects of different irrigation regimes on soil compaction in a winter wheat-summer maize cropping system in the North China Plain. CATENA, 137: 70-76. https://doi.org/10.1016/j.catena.2015.08.014

Lueking, M.A. \& Schepers, J.S. 1985. Changes in Soil Carbon and Nitrogen Due to Irrigation Development in Nebraska's Sandhill Soils. Soil Science Society of America Journal, 49(3): 626-630. https://doi.org/10.2136/sssaj1985.03615995004900030020x

Qureshi, A.S., Ahmad, W. \& Ahmad, A.-F.A. 2013. Optimum Groundwater Table Depth and Irrigation Schedules for Controlling Soil Salinity in Central Iraq. Irrigation and Drainage, 62(4): 414-424. https://doi.org/10.1002/ird.1746

Riya, S., Katayama, M., Takahashi, E., Zhou, S., Terada, A. \& Hosomi, M. 2014. Mitigation of Greenhouse Gas Emissions by Water Management in a Forage Rice Paddy Field Supplemented with DryThermophilic Anaerobic Digestion Residue. Water, Air, \& Soil Pollution, 225(9): 2118. https://doi.org/10.1007/s11270-014-2118-3

UNESCO WWAP. 2017. Fact 24: Irrigated land | United Nations Educational, Scientific and Cultural Organization. In: United Nations Educational, Scientific and Cultural Organization, World Water Assessment Programme [online]. [Cited 3 July 2020]. http://www.unesco.org/new/en/naturalsciences/environment/water/wwap/facts-and-figures/all-facts-wwdr3/fact-24-irrigated-land/

Wang, G.-C., Wang, E.-L., Huang, Y. \& Xu, J.-J. 2014. Soil Carbon Sequestration Potential as Affected by Management Practices in Northern China: A Simulation Study. Pedosphere, 24(4): 529-543. https://doi.org/10.1016/S1002-0160(14)60039-4

Wang, L., Qian, Y.L., Brummer, J., Wilhelm, S.J. \& Leach, J.E. 2019. Biomass Production and Soil Carbon Analysis of Switchgrass under Rainfed or Minimal Irrigation in a Semiarid Environment. Agronomy Journal, 111(4): 1704-1711. https://doi.org/10.2134/agronj2018.09.0568

Wienhold, B.J. \& Trooien, T.P. 1998. Irrigation water effects on infiltration rate in the northern Great Plains. Soil Science, 163(11): 853-858. https://doi.org/10.1097/00010694-199811000-00002

Win, K.T., Nonaka, R., Win, A.T., Sasada, Y., Toyota, K. \& Motobayashi, T. 2015. Effects of water saving irrigation and rice variety on greenhouse gas emissions and water use efficiency in a paddy field fertilized with anaerobically digested pig slurry. Paddy and water environment. (also available at https://agris.fao.org/agris-search/search.do?recordID=US201500159463).

Xu, Y., Zhan, M., Cao, C., Tian, S., Ge, J., Li, S., Wang, M. \& Yuan, G. 2016. Improved water management to reduce greenhouse gas emissions in no-till rapeseed-rice rotations in Central China. Agriculture, Ecosystems \& Environment, 221: 87-98. https://doi.org/10.1016/j.agee.2016.01.021 


\title{
29. Controlled traffic farming
}

\author{
Maria Josefina Masola, Carlos Agustín Alesso, María Eugenia Carrizo, \\ Silvia Imhoff
}

Institute of Agricultural Science of Litoral (ICiAgro Litoral)-National University of Litoral (UNL)/Faculty of Agricultural Sciences (FCA)-National Council for Science and Technology (CONICET), Argentina

\section{Description of the practice}

Soil compaction by machinery traffic is one of the main processes of soil degradation in agricultural lands, decreasing crop productivity (Horn et al, 1995; Imhoff et al., 2010). Degraded soils by compaction have higher microporosity, which favors the occurrence of anaerobic processes that lead to higher emissions of $\mathrm{CO}_{2}, \mathrm{CH}_{4}$ and $\mathrm{N}_{2} \mathrm{O}$. As a result, these soils may contribute to the global warming (Håkansson, 2005; Horn et al., 1995). Controlled traffic farming (CTF) is a field machinery management system that avoids soil compaction by confining the machinery traffic to permanent traffic lanes. This system generates two permanent areas: cropped or non-cropped permanent traffic lanes (PTL) and non-trafficked or permanent crop bed (PCB) (Hamza and Anderson, 2005; Tullberg, Yule and McGarry, 2007). Depending on the machinery configuration, the PTL occupy only about 10-15 percent of the total field; this percentage is generally accepted as a critical point for considering CTF as a beneficial practice. In this area soil has greater strength which results in better soil trafficability for the agricultural machinery. In contrast, the PCB occupies about 85-90 percent of the field, and it is characterized by better soil physical condition because it does not undergo compaction. This allows a better development of roots, and as a result, a higher crop productivity.

\section{Range of applicability}

The CTF system has been adopted in Australia and in many European countries but remains a novel practice for most farmers in South America. In Brazil, some sugarcane farmers use CTF because it increases sugar yield by 20 percent compared to random traffic (De Souza et al., 2014). In Argentina, this system is not applied yet despite its benefits (Antille et al., 2015a). One of the reasons is that the application of CTF requires that the width of wheel tracks of all agricultural machinery match, which is challenging for farmers because in Argentina, unlike other countries, no CTF ready machinery is offered in the market. The CTF system can be used in almost any extensive agricultural lands, with different types of crops and under any climate, which constitutes a great 
advantage. As a first step of adopting the CTF system farmers can start using the one-wheeled tramline (OWTL) method for non-matching multiple machinery widths, which reduces soil compaction to 30-40 percent of the field (Webb et al., 2004). This method acts to confine half of the compaction, mainly caused by heavy machinery, to only one common PTL without changing the wheel track width of the machinery. The round and round use of the OWTL method is easier than operating up and back; the common track is always on the same side (Figure 12).

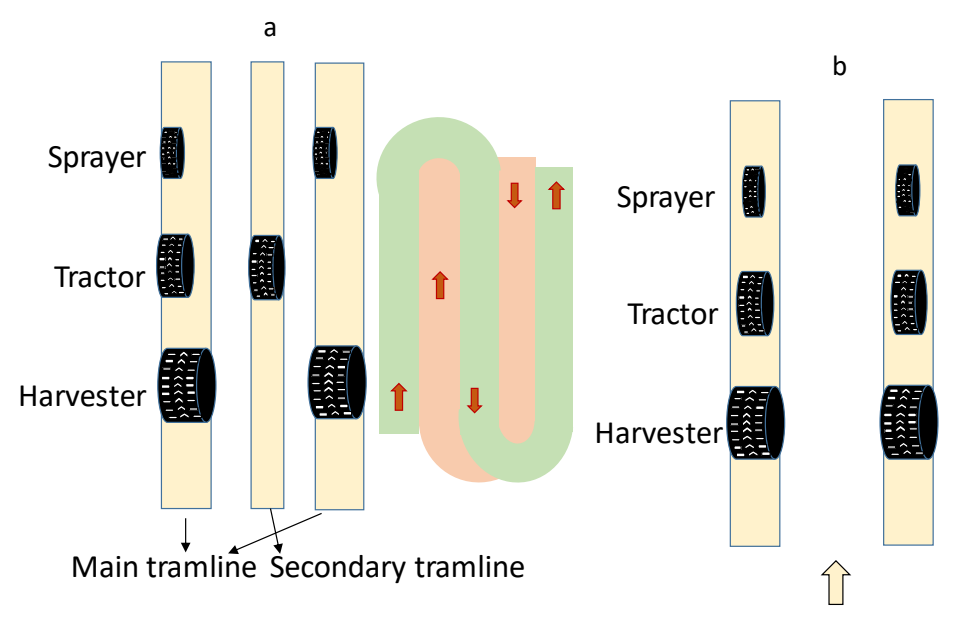

Figure 12. Schemes of alternatives to reduce soil compaction by agricultural traffic. a) One wheeled tramline (round and round), b) Controlled traffic farming.

This method allows farmers to plan to update the machinery width and tracks or to purchase of new machinery with more time, so as to finally apply the CTF system. Masola et al. (2020) reported increased yields under OWTL in Argentina, which turns this system into a promising alternative as a first step in adopting the CTF system in that country.

\section{Impact on SOC sequestration / Additional C storage}

Cid et al. (2014), in a 6-year experiment under irrigation, verified that no-tillage permanent bed planting combined with CTF system increased soil carbon sequestration while maintaining crop's yield compared with conventionally tilled bed planting. Measurements were made in the whole field. Lu et al. (2016), also in a 6-year pilot experiment, demonstrated that the combination of no-tillage and CTF system improved soil chemical properties and crop 's yield when compared with conventional tillage. Soil organic matter, available phosphorus and total nitrogen contents were especially enhanced. Measurements were made in the whole field (

Table 129). According to Antille et al. (2015b) the potential benefits of CTF system and coupling no-tillage with CTF to enhance C sequestration in arable lands have not been sufficiently studied yet. 
Table 129. Evolution of SOC stocks after application of Controlled Traffic Farming

\begin{tabular}{|l|l|l|l|l|l|l|l|}
\hline Location & $\begin{array}{l}\text { Climate } \\
\text { zone }\end{array}$ & Soil type & $\begin{array}{l}\text { Baseline } \\
\text { C stock } \\
\text { (tC/ha) }\end{array}$ & $\begin{array}{l}\text { Additional } \\
\text { C storage } \\
(\mathrm{tC} / \mathrm{ha} / \mathrm{yr})\end{array}$ & $\begin{array}{l}\text { Duration } \\
\text { (Years) }\end{array}$ & $\begin{array}{l}\text { More } \\
\text { information }\end{array}$ & Reference \\
\hline $\begin{array}{l}\text { South } \\
\text { Spain }\end{array}$ & $\begin{array}{l}\text { Warm } \\
\text { temperate } \\
\text { dry }\end{array}$ & $\begin{array}{l}\text { Typic } \\
\text { Xerofluvents }\end{array}$ & NA & $\begin{array}{l}0.95 \\
(0-0.5 \mathrm{~m} \\
\text { depth) }\end{array}$ & & $\begin{array}{l}\text { Maize/Cotton/ } \\
\text { Maize }\end{array}$ & $\begin{array}{l}\text { Cid et al. } \\
(2014)\end{array}$ \\
\hline $\begin{array}{l}\text { Daxing } \\
\text { District of } \\
\text { Beijing, } \\
\text { China }\end{array}$ & $\begin{array}{l}\text { Semi-arid } \\
\text { warm } \\
\text { temperate }\end{array}$ & Sandy loam & 5.5 & $\begin{array}{l}0.49 \\
(0-0.1 \mathrm{~m})\end{array}$ & 6 & Maize/Wheat & $\begin{array}{l}\text { Lu et al. } \\
(2016)\end{array}$ \\
\hline
\end{tabular}

\section{Other benefits of the practice}

\subsection{Improvement of soil properties}

The adoption of CTF allows soil bulk density to decrease and soil available water capacity and macropores density to increase in the PCB (McHugh, Tullberg and Freebairn, 2009). Improving the physical properties of the soil provides a range of benefits in addition to increasing crop's productivity, including higher rainfall infiltration rates and increased biological activity (Tullberg et al., 2018). Controlled traffic farming, when compared with random traffic farming (RTF), reduces water runoff (27-42 percent), and fertilizers (1-26 percent), pesticides (1-26 percent), seeds (11-36 percent) and fuels (23 percent) usage because the use of aid-navigation and auto-steering systems reduce the application overlaps that commonly occur in RTF (Gasso et al., 2013).

\subsection{Minimization of threats to soil functions}

Table 130. Soil threats

\section{Soll threats}

Soil erosion

As CTF avoids soil compaction, tillage operations are less necessary. This allows crop residues to remain on the soil surface protecting the soil from erosion (Wang et al., 2008; Antille et al., 2015b). Additionally, in cases where erodible slope gradients and lengths are present, traffic lanes should 


\begin{tabular}{|c|c|}
\hline Soll threats & \\
\hline & $\begin{array}{l}\text { follow contour lines for preventing runoff concentration and consequently } \\
\text { soil erosion. }\end{array}$ \\
\hline $\begin{array}{l}\text { Nutrient imbalance } \\
\text { and cycles }\end{array}$ & $\begin{array}{l}\text { CTF increases fertilizer use efficiency and it is absolutely compatible with } \\
\text { the application of better agricultural practices that allow maintaining an } \\
\text { adequate balance of soil nutrients (Hefner, Labouriau and Kristensen, 2019). }\end{array}$ \\
\hline $\begin{array}{l}\text { Soil contamination / } \\
\text { pollution }\end{array}$ & $\begin{array}{l}\text { CTF uses mechanical or electronic guidance systems to minimize overlap, } \\
\text { which reduces the use of pesticides, herbicide and fertilizer. Hence, CTF } \\
\text { contributes to reducing soil contamination (Jensen et al., 2012; Gasso et al., } \\
\text { 2013). }\end{array}$ \\
\hline Soil acidification & $\begin{array}{l}\text { CTF allows fertilizer use efficiency and organic matter content to increase } \\
\text { (Lu et al. 2016); thus, the effects of soil acidification are expected to } \\
\text { decrease (Gasso et al., 2013). }\end{array}$ \\
\hline Soil biodiversity loss & $\begin{array}{l}\text { The regeneration of soil structure in the PCB increases soil biodiversity } \\
\text { (Mouazen and Palmqvist, 2015). }\end{array}$ \\
\hline $\begin{array}{l}\text { Soil sealing } \\
\text { (crusting) }\end{array}$ & $\begin{array}{l}\text { CTF improves the soil physical quality in the PCB, which prevents soil } \\
\text { sealing (Masola, 2020). }\end{array}$ \\
\hline Soil compaction & $\begin{array}{l}\text { CTF avoids soil compaction by confining all machinery traffic to the PTL and } \\
\text { reducing overlap. Soil compaction is confined to the trafficked surface that } \\
\text { is less than } 15 \text { percent (Chamen et al., 2015, Etana et al., 2020). }\end{array}$ \\
\hline $\begin{array}{l}\text { Soil water } \\
\text { management }\end{array}$ & $\begin{array}{l}\text { Li et al. (2001) reported that CTF increased water storage in the PCB } \\
\text { compared with conventional tillage and random traffic. }\end{array}$ \\
\hline
\end{tabular}

\subsection{Increases in production (e.g. food/fuel/feed/timber/fibre)}

Controlled traffic farming increases crop's yield in the PCB. In Argentina Masola et al. (2020) found greater sunflower and maize yield in the PCB while the crop row located in the PTL had lower yield. Other authors found that even though 20 percent of the field was occupied by wheel tracks without planting, no yield decrease was observed in CTF when compared with conventional tillage (Li et al, 2007; Galambošová et al., 2017). Additionally, power requirements and fuel consumption in the CTF are lower than in conventional tillage because of a more efficient traffic management and easier movement of tractors and tools within the field (Qingjie et al., 2009; Jensen et al., 2012). 


\subsection{Mitigation of and adaptation to climate change}

Although CTF produces an increase in GHG emissions on the PTL, this traffic management system allows reducing total $\mathrm{N}_{2} \mathrm{O}$ and $\mathrm{CH}_{4}$ emissions between 20 and 50 percent respect to conventional systems (Tullberg et al. 2018). According to Gasso et al. (2013) CTF system reduces soil emissions of $\mathrm{N}_{2} \mathrm{O}$ from 21 to 45 percent and $\mathrm{CH}_{4}$ from 372 to 2100 percent depending on climate.

\subsection{Socio-economic benefits}

The adoption of CTF along with the use of guidance systems facilitates the local and spatial application of different inputs, such as pesticides, herbicides, fertilizers and amendments. Thus, the inputs needed for crop's production can be used more efficiently. As the input use efficiency increases, their required amount decreases. As a result, the farm profitability increases. According to Gasso et al. (2013), CTF implementation reduces soil and water eutrophication, non-renewable resource depletion, and human- and eco-toxicity. Thus, this technology is useful for better supporting environmental goals and the global green growth policy (Jensen et al, 2012)

\section{Potential drawbacks to the practice}

\subsection{Tradeoffs with other threats to soil functions}

Table 131. Soil threats

\begin{tabular}{|c|c|}
\hline Soil threats & \\
\hline Soil erosion & $\begin{array}{l}\text { Deep and rough wheel ruts can be formed when running machinery } \\
\text { during wet conditions, which can favor soil erosion (Webb et al., } \\
\text { 2004). }\end{array}$ \\
\hline $\begin{array}{l}\text { Nutrient imbalance } \\
\text { and cycles }\end{array}$ & $\begin{array}{l}\text { CTF when combined with the no-till system can produce nutrient } \\
\text { stratification in the upper centimeters of the soil (Potter and } \\
\text { Chichester, 1993). }\end{array}$ \\
\hline $\begin{array}{l}\text { Soil sealing } \\
\text { (crusting) }\end{array}$ & CTF may increase soil sealing but only in the PTL (Masola, 2020). \\
\hline Soil compaction & $\begin{array}{l}\text { CTF increases the relative soil compaction but only in the PTL (Etana } \\
\text { et al., 2020; Masola et al., 2020). }\end{array}$ \\
\hline
\end{tabular}




\subsection{Increases in greenhouse gas emissions}

Nitrous oxide $\left(\mathrm{N}_{2} \mathrm{O}\right)$ is the greatest contributor to agriculture's greenhouse gas (GHG) emissions from cropping. $\mathrm{N}_{2} \mathrm{O}$ emissions are greater when the water filled-pore space (WFPS) is higher than 60 percent and when nitrate and carbon (from crop residues) are available. Soil compaction by agricultural traffic machinery increases the WFPS and $\mathrm{N}_{2} \mathrm{O}$ emissions. As CTF improves soil structure and aeration on PCB, it reduces $\mathrm{N}_{2} \mathrm{O}$ emissions from 20 percent to 50 percent compared with non-CTF (Antille et al., 2015a). However, a higher risk of GHG emissions from the PTL exists if these lanes are not handled appropriately. In Argentina, Masola (2020) found that in a 30-day measurement period during a wet season (WFPS $=50$ percent), $\mathrm{N}_{2} \mathrm{O}$ emissions in PTL were higher $\left(0.19 \mathrm{~kg} \mathrm{~N}_{2} \mathrm{O} / \mathrm{ha}\right)$ than in PCB $\left(0.057 \mathrm{~kg} \mathrm{~N}_{2} \mathrm{O} / \mathrm{ha}\right)$. In contrast, during the dry season (WFPS=11 percent), no differences between zones were found.

\subsection{Conflict with other practice(s)}

Controlled traffic farming eliminates an important motivation for tillage and encourages the use of guidance systems of precision farming (Antille et al., 2015b). This system is compatible with the use of suitable agricultural practices; therefore, it can be used safely.

\subsection{Decreases in production (e.g. food/fuel/feed/timber/fibre)}

In CTF, trafficked lanes can be cropped or Not. In both situations the productivity of these lanes decreases. However, as the productivity in the PCB increases, the global productivity of the agricultural land also increases (Li et al., 2007; Masola et al., 2020).

\section{Recommendations before implementing the practice}

To start using the CTF system farmers firstly need to establish their farm priorities (reduce inputs, reduce compaction, improve soil quality, etc.), the agronomic opportunities that the system will allow for them, and the benefits and costs associated with the adoption of the system because these factors are very specific. Then, farmers are advised to consider the following points:

- Determine the working and track width of each agricultural machinery (tractor, planter, harvester, sprayer).

- The CTF can be easily applied by farmers if their machinery has the same or modular working and track width with guidance system; otherwise, farmers should have to adapt or buy new machinery.

- Establish the working direction and tramlines design that best suits machinery, topography and crop rotation. Consider contour tillage in case of soil erosion risk. 
- Establish the existence, degree and depth of soil compaction by observing the root development and presence of densified layers. Also, soil penetration resistance and soil bulk density can be measured. If necessary, eliminate soil compaction for allowing a better crop development.

- If matching machinery widths and tracks is unattainable, start using the one-wheeled tramline (OWTL) method for multiple machinery widths and plan the machinery update during the current changeover period of your enterprise.

\section{Potential barriers to adoption}

Table 132. Potential barriers to adoption

\begin{tabular}{|l|l|l|}
\hline Barrier & YES/NO & \\
\hline Cultural & Yes & $\begin{array}{l}\text { The data collected from eight European countries show an equal } \\
\text { distribution between the use of CTF/No-CTF among farmers } \\
\text { that are older than } 60 \text { years. Adoption among younger farmers } \\
\text { appears to be relatively low ( } 25 \text { percent) (Thomsen et al., 2018). }\end{array}$ \\
\hline Economic & Yes & $\begin{array}{l}\text { The high cost of machinery modification, the lack of } \\
\text { compatibility of equipment and also GPS systems from different } \\
\text { manufacturers can limit the adoption of CTF (Thomsen et al., } \\
\text { 2018). }\end{array}$ \\
\hline Institutional & No & $\begin{array}{l}\text { The little existing information indicates that some institutions } \\
\text { encourage the use of CTF (Chamen, 2015). }\end{array}$ \\
\hline Knowledge & Yes & $\begin{array}{l}\text { There are many studies regarding the use of CTF in many } \\
\text { countries. However, it is necessary to generate evidence of the } \\
\text { benefits of the adoption of CTF or OWTL under local conditions } \\
\text { (Antille et al., 2015a). }\end{array}$ \\
\hline
\end{tabular}




\section{Photos of the practice}

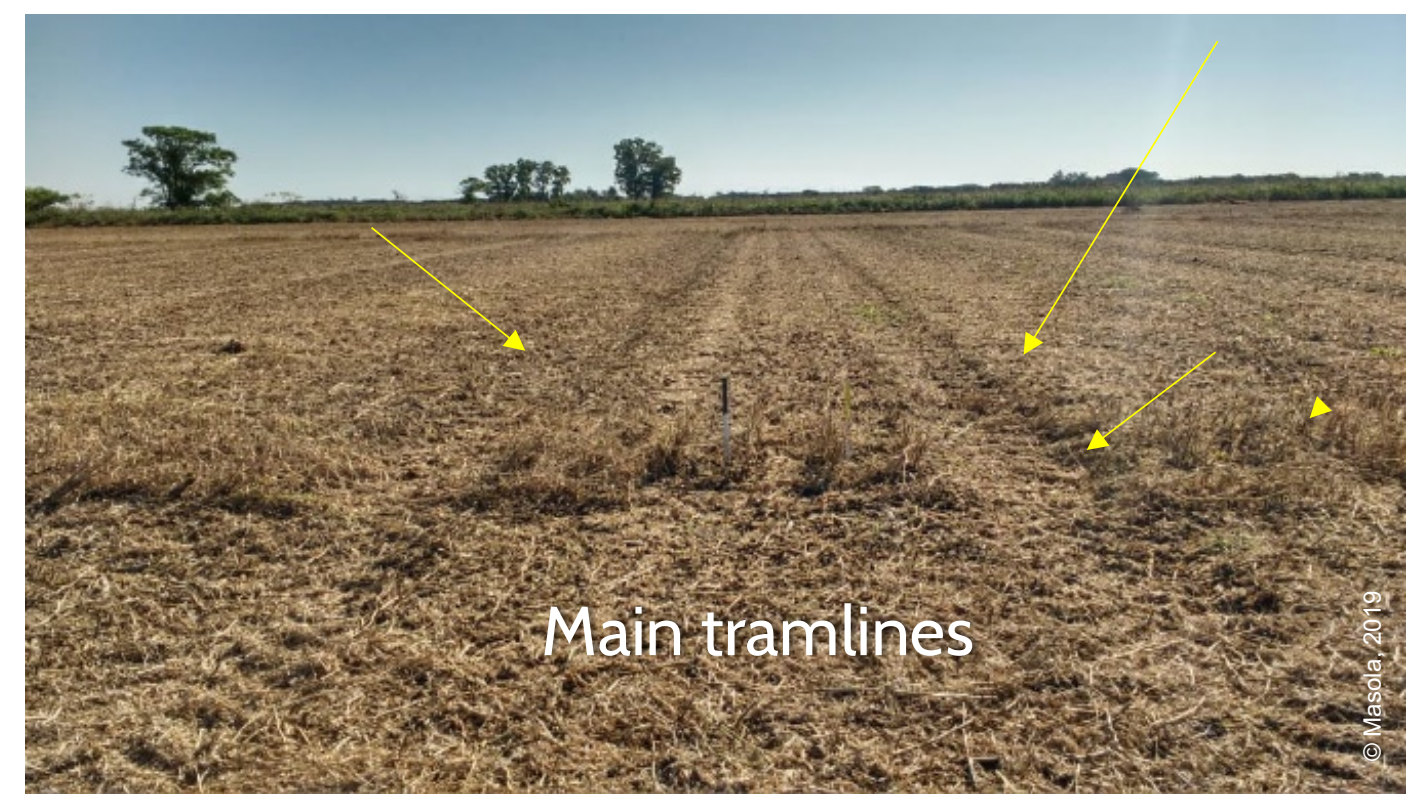

Photo 37. Controlled traffic farming in a field of Argentina

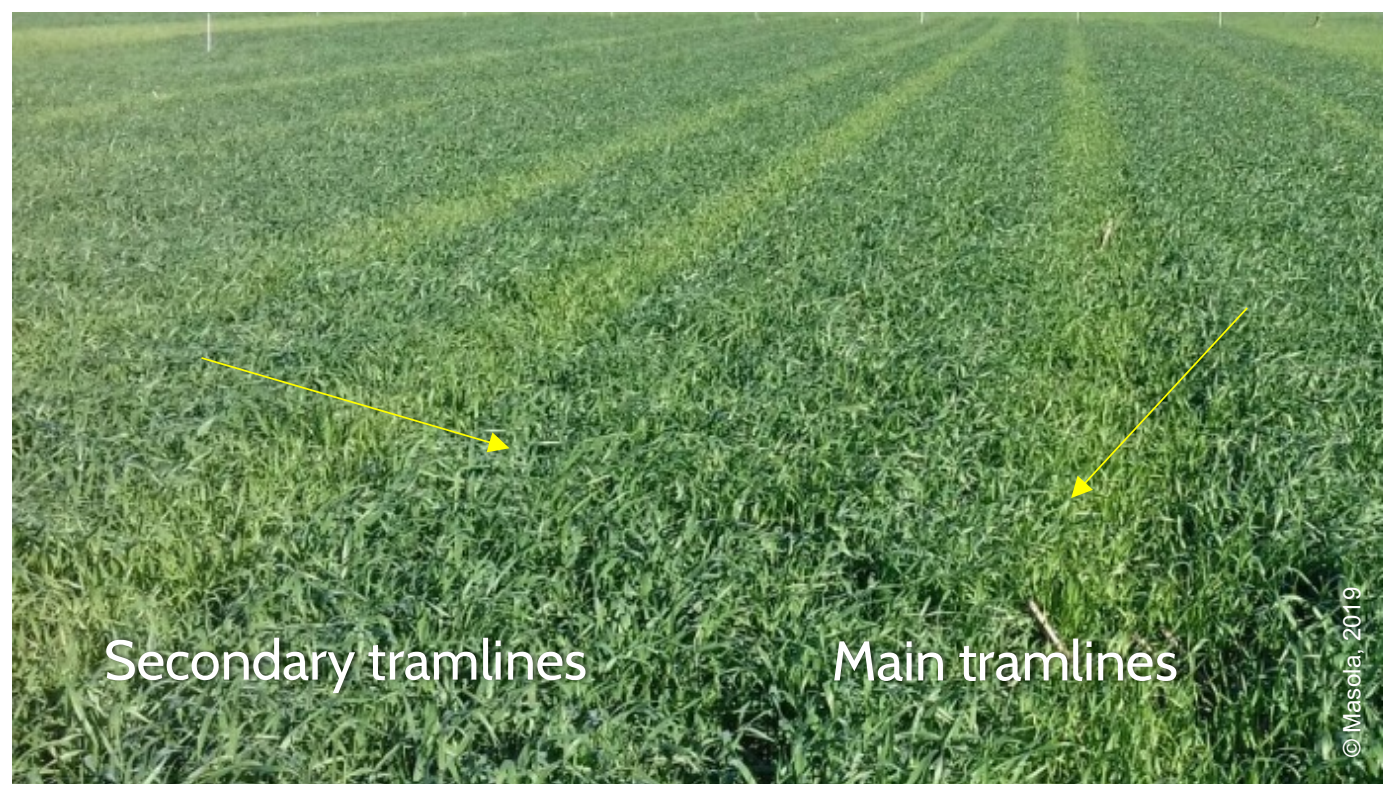

Photo 38. One-wheeled tramline for multiple machinery widths method in a field of Argentina 


\section{References}

Antille, D.L., Imhoff, S.C., Alesso, C.A., Chamen, W.C.T. \& Tullberg, J.N. 2015a. Potential to Increase Productivity and Sustainability in Argentinean Agriculture with Controlled Traffic Farming: A Short Discussion. Acta Technologica Agriculturae, 3: 83-87. https://doi.org/10.1515/ata-2015-0016

Antille, D.L., Chamen, W.C.T., Tullberg, J.N. \& Lal, R. 2015b. The potential of controlled traffic farming to mitigate greenhouse gas emissions and enhance carbon sequestration in arable land: a critical review. Transactions of ASABE, 58(3): 707-731. https://doi.org/10.13031/trans.58.11049

Chamen, T. 2015. Controlled traffic farming-from worldwide research to adoption in Europe and its future prospect. Acta Technologica Agriculturae, 3: 64-73. https://doi.org/10.1515/ata-2015-0014

Chamen, W.C.T., Moxey, A.P., Towers, W., Balana, B. \& Hallett, P.B. 2015. Mitigating arable soil compaction: A review and analysis of available cost and benefit data. Soil \& Tillage Research, 146: 10-25. https://doi.org/10.1016/j.still.2014.09.011

Cid, P., Carmona, I., Murillo, J.M. \& Gómez-Macpherson, H. 2014. No-tillage permanent bed planting and controlled traffic in a maize-cotton irrigated system under Mediterranean conditions: Effects on soil compaction, crop performance and carbon sequestration. European Journal of Agronomy, 61: 24-34. https://doi.org/10.1016/j.eja.2014.08.002

De Souza, G., De Souza, S., De Menezes, Z., Barboza da Silva, R., Sobreira Barbosa, R. \& Silva Araújo, F. 2014. Effects of traffic control on the soil physical quality and the cultivation of sugarcane. Revista Brasileira de Ciência do Solo, 38(1): 135-146. https://doi.org/10.1590/S0100-06832014000100013

Etana, A., Holma, E., Rydberg, T. \& Keller, T. 2020. Soil and crop responses to controlled traffic farming in reduced tillage and no-till: some experiences from field experiments and on-farm studies in Sweden. Acta Agriculturae Scandinavica, Section B: 1-8. https://doi.org/10.1080/09064710.2020.1728372

Galambošová, J., Macák, M., Rataj, V., Antille, D.L., Godwin, R.J., Chamen, W.C.T., Žitňák, M., Vitázková, B., Ďud'ák, J. \& Chlpík, J. 2017. Field evaluation of controlled traffic farming in central Europe using commercially available machinery. Transactions of the ASABE, 60(3): 657-669.

https://doi.org/10.13031/trans.11833

Gasso, V., Claus, A.G., Sørensen, F.W. \& Oudshoorn, O.G. 2013. Controlled traffic farming: A review of the environmental impacts. European Journal of Agronomy, 48: 66-73.

https://doi.org/10.1016/j.eja.2013.02.002

Håkansson, I. 2005. Machinery-Induced compaction of arable soils. Incidence, consequences, countermeasures. Reports from the division of soil management, NO. 109.

Hamza, M.A. \& Anderson, W.K. 2005. Soil compaction in cropping systems. Soil and Tillage Research, 82: 121-145. https://doi.org/10.1016/j.still.2004.08.009

Hefner, M., Labouriau, R. \& Kristensen, H. 2019. Controlled traffic farming increased crop yield, root growth, and nitrogen supply at two organic vegetable farms. Soil and Tillage Research, 191: 117-130. https://doi.org/10.1016/j.still.2019.03.011 
Horn, R., Domzal, H., Slowihka-Jurkiewicz, A. \& Van Ouwerkerk, C. 1995. Soil compaction processes and their effects on the structure of arable soils and the environment. Soil and Tillage Research, 35: 23-36. https://doi.org/10.1016/0167-1987(95)00479-C

Imhoff, S., Kay, B., Pires da Silva, A. \& Hajabbasi, M. 2010. Evaluating response of maize (Zea maiz, L.) to soil physical conditions using the boundary line approach. Soil and Tillage Research, 106: 303-310. https://doi.org/10.1016/j.still.2009.11.007

Jensen, H.G., Jacobsen, L., Pedersen, S.M. \& Tavella, E. 2012. Socioeconomic impact of widespread adoption of precision farming and controlled traffic systems in Denmark. Precision Agriculture, 13: 661-677. https://doi.org/10.1007/s11119-012-9276-3

Li, Y.X., Tullberg, J.N. \& Freebairn, D.M. 2007. Wheel traffic and tillage effects on runoff and crop yield. Soil and Tillage Research, 97: 282-292. https://doi.org/10.1016/j.still.2005.10.001

Li, Y., Tullberg, J.N. \& Freebairn, D.M. 2001. Traffic and residue cover effects on infiltration. Australian Journal of Soil Research, 39: 239-247. https://doi.org/10.1071/SR00017

Lu, C., Li, H., He, J., Wang, Q., Sarker, K., Li, L., Lu, Z., Rasaily, R., Li, H. \& Chen, G. 2016. Effects of controlled traffic no-till system on soil chemical properties and crop yield in annual double-cropping area of the North China Plain. Soil Research, 54(6): 760-766. https://doi.org/10.1071/SR15123

Masola, M.J. 2020. Propagación lateral de la compactación por tránsito de la maquinaria agrícola: ¿afecta la calidad del suelo, el intercambio gaseoso y la productividad de los cultivos? Facultad de Ciencias Agrarias, Universidad Nacional del Litoral (Argentina). (PhD dissertation)

Masola, M.J., Alesso, C.A., Carrizo, M.E., Berhongaray, G., Botta, G.F., Horn, R. \& Imhoff, S. 2020. Advantages of the one-wheeled tramline for multiple machinery widths method on sunflower (Helianthus annus L.) and maize (Zea mays L.) responses in the Argentinean Flat Pampas. Soil and Tillage Research, 196: 104462. https://doi.org/10.1016/j.still.2019.104462

Mchugh, A.D., Tullberg, J.N. \& Freebairn, D.M. 2009. Controlled traffic farming restores soil structure. Soil and Tillage Research, 104: 164-172. https://doi.org/10.1016/j.still.2008.10.010

Mouazen, A.M. \& Palmqvist, M. 2015. Development of a Framework for the Evaluation of the Environmental Benefits of Controlled Traffic Farming. Sustainability, 7: 8684-8708.

https://doi.org/10.3390/su7078684

Potter, K.N. \& Chichester, F.W. 1993. Physical and chemical properties of a Vertisol with continuous controlled-traffic, no-till management. Transactions of the ASAE, 36(1): 95-99.

https://doi.org/10.13031/2013.28319

Qingjie, W., Hao, C., Hongwen, L., Wenying, L., Xiaoyan, W., McHugh, A.D., Jin, H. \& Huanwen, G. 2009. Controlled traffic farming with no tillage for improved fallow water storage and crop yield on the Chinese Loess Plateau. Soil and Tillage Research, 104: 192-197.

https://doi.org/10.1016/j.still.2008.10.012 
Thomsen, M.N., Tamirat, T.W., Pedersen, S.M., Lind, K.M., Pedersen, H.H., de Bruin, S., Nuyttens, D., Vangeyte, J., Forristal, P.D. \& Sørensen, C.G. 2018. Farmers' Perception of Controlled

Traffic Farming (CTF) and Associated Technologies. Working paper, 2018/12. University of Copenhagen.

Tullberg, J.N., Yule, D.F. \& Mc Garry, D. 2007. Controlled traffic farming-From research to adoption in Australia. Soil and Tillage Research, 97: 272-281. https://doi.org/10.1016/j.still.2007.09.007

Tullberg, J.N., Antille, D.L., Bluett, C., Eberhar, J. \& Scheer, C. 2018. Controlled traffic farming effects on soil emissions of nitrous oxide and methane. Soil and Tillage Research, 176: 18-25.

https://doi.org/10.1016/j.still.2017.09.014

Wang, X.Y., Gao, H.W., Tullberg, J.N., Li, H.W., Kuhn, N., Mchugh, A.D. \& Li, Y.X. 2008. Traffic and tillage effects on runoff and soil loss on the Loess Plateau of northern China. Australian Journal of Soil Research, 46: 667-675. https://doi.org/10.1071/SR08063

Webb, B., Blackwell, P., Riethmuller, G. \& Lemon, J. 2004. Tramline farming systems: technical manual. Bulletin 4607. Government of Western Australia. 


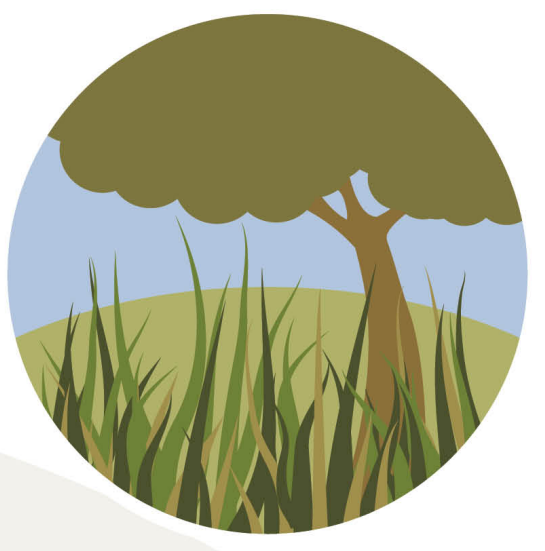

Grasslands 


\title{
Grasslands
}

\author{
Mohammad I. Khalil', Cláudia M.d.S. Cordovil², \\ Rosa Francaviglia ${ }^{3}$, Beverley Henry ${ }^{4}$, Katja Klumpp ${ }^{5}$, \\ Peter Koncz ${ }^{6}$, Mireia Llorente ${ }^{7}$, Beata E. Madari ${ }^{8}$, \\ Muñoz-Rojas Miriam ${ }^{9,10}$, Nerger Rainer ${ }^{11}$ \\ (Co-authors in alphabetical order)
}

${ }^{1}$ School of Applied Sciences \& Technology, Prudence College Dublin, Dublin 22 and School of Biology \& Environmental Science, University College Dublin, Dublin 4, Ireland

${ }^{2}$ University of Lisbon, School of Agriculture, Forest Research Center, Lisboa, Portugal

${ }^{3}$ Council for Agricultural Research and Economics, Research Centre for Agriculture and Environment (CREA-AA), Rome, Italy.

${ }^{4}$ Science and Engineering Faculty, Queensland University of Technology, Brisbane, Australia

${ }^{5}$ Grassland Ecosystem Research, INRA, Clermont-Ferrand, France

${ }^{6}$ Duna-Ipoly National Park Directorate, Budapest, Hungary and MTA-SZIE Plant Ecology Research Group, Gödöllő, Hungary

${ }^{7}$ Forest Department. University of Extremadura, Plasencia Campus. Spain

${ }^{8}$ Embrapa Rice and Beans, Santo Antônio de Goiás, GO, Brazil

${ }^{9}$ UNSW Sydney, School of Biological, Earth and Environmental Sciences, Sydney NSW, Australia

${ }^{10}$ The University of Western Australia, School of Biological Sciences, Crawley, WA, Australia

${ }^{11}$ Soil \& More Impacts GmbH, German office, Hamburg, Germany

Globally, grasslands are among the largest ecosystems and cover around 3.5 billion-hectare (ha) area, representing 26 percent of the world land area and 70 percent of the world agricultural area (FAO, 2008). These grassland soils contain about 20 percent of the world's soil organic carbon (SOC) stocks (Conant, Paustian and Elliot, 2001). There are different types of grasslands: natural grasslands, semi-natural grasslands, and agricultural grasslands. Grasslands that are self-seeded are often defined as natural or native grasslands. These grasslands are predominated by grasses, grass-like plants, forbs, or shrubs suitable for grazing or browsing. They are defined as rangelands when natural (native) or grazed, and defined as pastures when forage is managed by seeding, mowing (i.e. for hay, silage, renewable energy production), fertilization and irrigation. Agricultural grasslands can be permanent ( $>5$-years old) or temporary (i.e. included within the crop rotation, grass/arable-ley). Permanent grassland is often (semi-) natural. Grasslands are known as "Steppes" in Asia; 
"Prairies" in North America; "Pampas", "Llanos" and "Cerrados" (composed of forests, savannah-type and grassland-type formations) in South America; "Savannahs" and "Velds" in Africa; and "Rangelands" in Australia. These ecosystems support a variety of species, including wildflowers, and mostly feed various animals including 25 species of large plant-eaters. Other than natural, there are many degrees of interferences such as fire, grazing, clearing of woody vegetation, over sowing and large herds of wild herbivores that affect grassland systems.

Grasslands (pasture, silage and hay) dominate major agricultural areas and contribute 20-30 percent to the SOC pool and, by sequestering atmospheric carbon dioxide $\left(\mathrm{CO}_{2}\right)$, can contribute to climate change mitigation (e.g. Reid et al., 2004; Allard et al., 2007). Livestock is grazed mostly on grassland worldwide (pasture and meadows) (Figure 13a) (Ritchie and Roser, 2013). In Europe (with exceptions such as UK and Ireland) and South Asia grasslands typically occupies less than 20 percent of total land area whereas global coverage for most continental regions is slightly less than 50 percent. In Central Asia and nearby countries, it can reach up to 70 percent. Livestock is bred across diverse climatic and environmental regions in all continents except Antarctica, e.g. from temperate regions to hilly and semi-arid terrain. The latter ranges from low-input pastoral production systems in arid and semiarid environments to highly intensive production in more mesic environments, integrating livestock-crop-forage systems (Figure 13b). Therefore, grassland is potentially less geographically constrained than arable farming. Pastoralism refers to mobile livestock herding in the dimension of either production or livelihood (Dong, 2016).

Pastoralism occurs where resources are limited and occupies about 18-23 percent of global land area. It supports around 200 million pastoral households (Neely et al., 2009; Blench, 2001). Two essential forms of pastoralism exist all over the continents: (i) the nomadic (commonly practised in regions with little arable land) and (ii) transhumance (mostly seasonal movement of livestock between fixed summer and winter pastures) rearing of domesticated animals (Dong, 2016). 
(a)

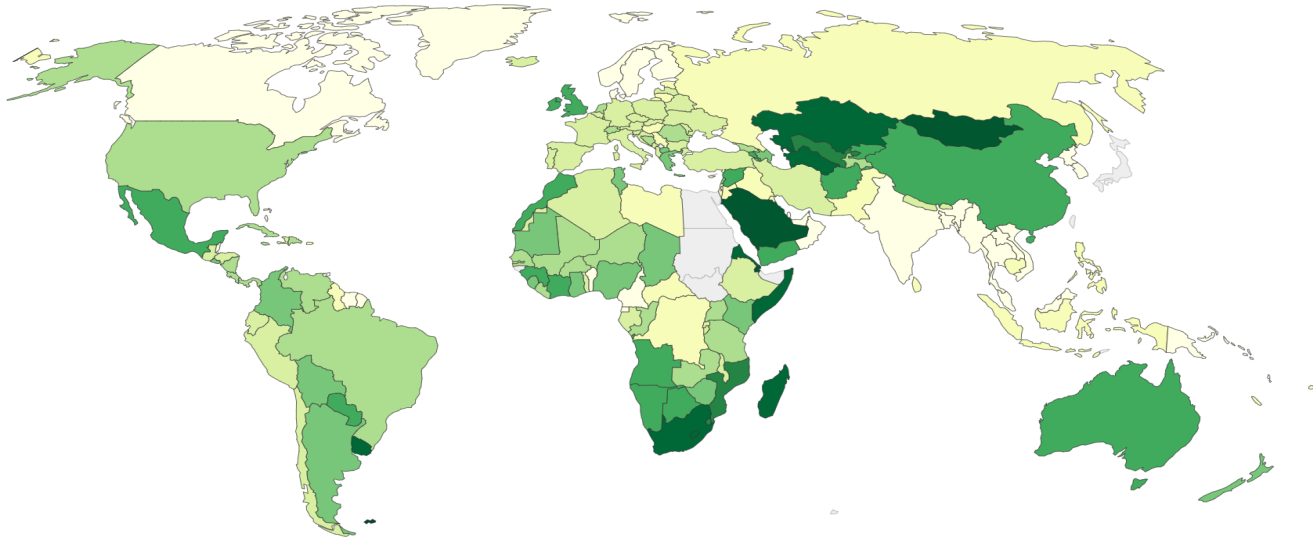

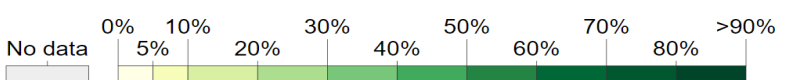

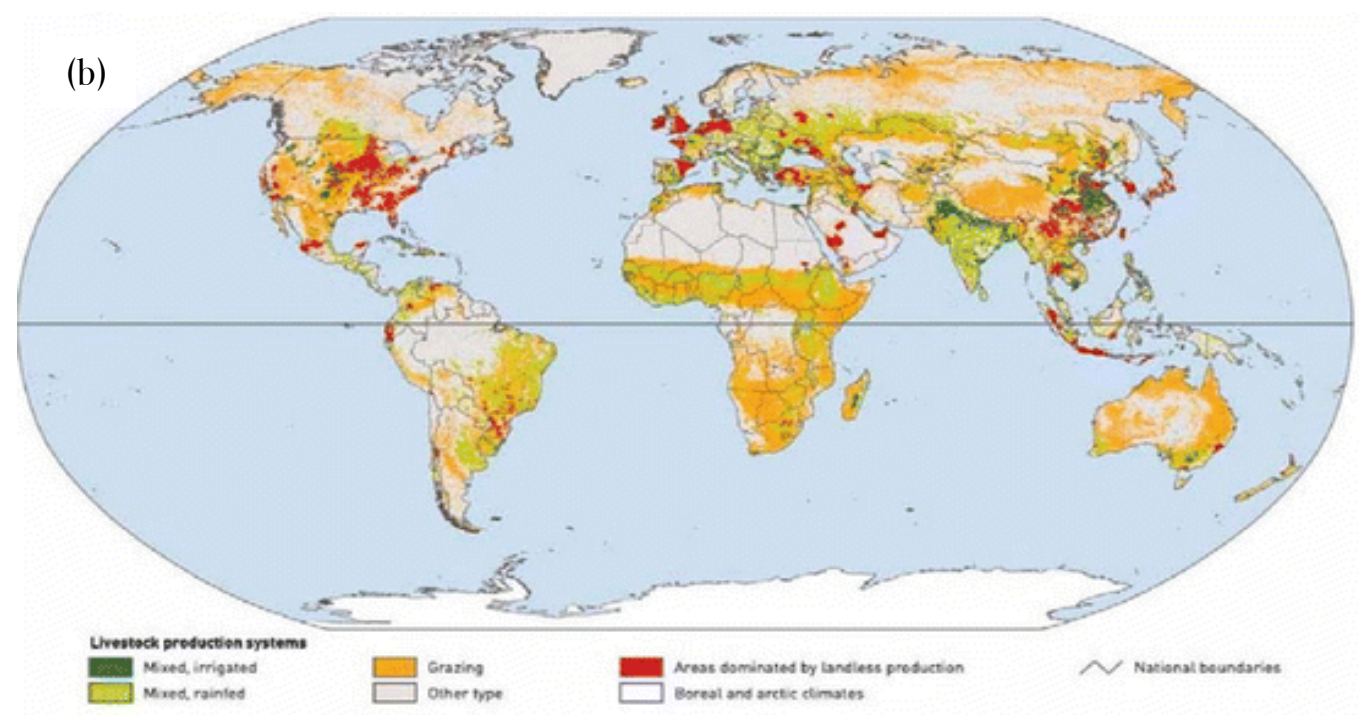

Figure 13. Global share of land area used for pastures, measured as the percentage of total land area, 1961-2014 (a), and livestock production systems (b). Source: FAO

Identifying optimum grassland management combining both profitable animal production and the delivery of ecosystem services like $\mathrm{C}$ sequestration is still a big challenge. The SOC densities/stocks are sensitive to management, re-seeding, drainage conditions, grass species and land use changes, inducing its losses or gains. Improved grazing management, inorganic and organic fertilization to pasture and silage, sowing legumes and improved grass species, irrigation, and conversion from cropland to grassland could lead to increased SOC, at rates ranging from 0.1 to more than $1 \mathrm{tC} / \mathrm{ha} / \mathrm{yr}$ (Conant et al., 2017; Khalil, Fornara and Osborne, 2020). 
Grazing is a key factor in changing soil C pools in grassland ecosystems. In general, $\mathrm{C}$ sequestration is lower in mixed cutting/grazing systems than in pure grazing systems. Soil C storage depends on C input mainly through the belowground parts of vegetation and $\mathrm{C}$ release mediated by soil processes, which are influenced by soil physical, chemical, and biological properties (Batjes, 1999). Improved grazing management can improve conditions on many degraded soils (Nordborg and Röös, 2016), and unsustainable management practices bring soils to the limit of desertification, a situation that may deteriorate further with climate change (Lal, 2009).

Manure and other organic applications have significant potential for sequestering $\mathrm{C}$ in soils, but their management depends on local climatic and edaphic conditions and the characteristics of the materials amended (Khalil, Hossain and Schmidhalter, 2005; Khalil, Fornara and Osborne, 2020). Most soils are responsive to management changes to increase SOC density, such as: (i) set aside and restoration of degraded agricultural lands, (ii) manure/bio-solid applications (Ogle, Bredit and Paustian, 2005; Hutchinson, Campbell and Desjardins, 2007; Smith et al., 2008), (iii) pasture improvement (Hutchinson, Campbell and Desjardins, 2007), (iv) adaptive grazing management systems (Bernues et al., 2011; Teague ad Barnes, 2017), (v) selective inclusion of woody species into the pasture system (Howlett et al., 2011), and (vi) conversion from cropland to pasture (e.g. Post and Kwon, 2000; McLauchlan et al., 2006).

This section reviews management-induced $\mathrm{C}$ sequestration in grassland soils, their negative and positive impacts on ecosystem functions and their adaptability and needs of protection across socio-economic and cultural settings. The objectives of this section are to: (a) improve knowledge base and understanding of management practices and technologies to increase and maintain SOC, while reducing climate change risk and achieving overall productivity and environmental services; (b) suggest adopting region/biome-specific management practices to enhance/maintain SOC, protect ecosystems and environmental degradation without sacrificing food security; and (c) outline full realization of available economic, ecological, social and policy options to accept for storing additional SOC. 


\section{References}

Allard, V., Soussana, J.-F., Falcimagne, R., Berbigier, P., Bonnefond, J.M., Ceschia, E., D'hour, P., Henault, C., Laville, P., Martin, C. \& Pinares-Patino, C. 2007. The role of grazing management for the net biome productivity and greenhouse gas budget $\left(\mathrm{CO}_{2}, \mathrm{~N}_{2} \mathrm{O}\right.$, and $\left.\mathrm{CH}_{4}\right)$ of semi-natural grassland. Agric. Ecosyst. Environ., 121: 47-58. https://doi.org/10.1016/j.agee.2006.12.004

Batjes, N.H. 1999. Management options for reducing $\mathrm{CO}_{2}$ concentrations in the atmosphere by increasing carbon sequestration in the soil. In Dutch National Research Programme on Global Air Pollution and Climate Change. Report, 410: 200-031, Technical Paper, 30, ISRIC, Wageningen.

Bernués, A., Ruiz, R., Olaizola, A., Villalba, D. \& Casasús, I. 2011. Sustainability of pasture-based livestock farming systems in the European Mediterranean context: Synergies and trade-offs. Livestock Sci, 139: 44-57. https://doi.org/10.1016/j.livsci.2011.03.018

Blench, R. 2001. 'You Can't Go Home Again': Pastoralism in the New Millennium (p. 103). London: Overseas Development Institute. http://www.fao.org/3/y2647e/y264.7e00.htm

Conant, R.T., Cerri, C.E.P., Osborne, B.B. \& Paustian, K. 2017. Grassland management impacts on soil carbon stocks: a new synthesis. Ecological Applications, 27(2): 662-668.

http://dx.doi.org/10.1002/eap.1473

Conant, R.T., Paustian, K. \& Elliot, E.T. 2001 . Grassland management and conversion into grassland: Effects on soil carbon. Ecol. Appl., 11: 343-355. https://doi.org/10.1890/1051-

0761(2001)011[0343:GMACIG]2.0.CO;2

Dong, S. 2016. Overview: Pastoralism in the World. In Dong, S., Kassam, K.A., Tourrand, J., Boone, R. (Eds.) Building Resilience of Human-Natural Systems of Pastoralism in the Developing World. Springer, Cham.

FAO. 2008. Are grasslands under threat? Brief analysis of FAO statistical data on pasture and fodder crops. 28p. http://www.fao.org/uploads/media/grass_stats_1.pdf

Howlett, D.S., Moreno, G., Losada, M.R.M., Nair, P.K.R. \& Nair, V.D. 2011. Soil carbon storage as influenced by tree cover in the Dehesa cork oak silvopasture of central-western Spain. Journal of Environmental Monitoring, 13(7): 1897-1904. https://doi.org/10.1039/C1EM10059A

Hutchinson, J.J., Campbell, C.A. \& Desjardins, R.L. 2007. Some perspectives on carbon sequestration in agriculture. Agricultural and Forest Meteorology, 142(2-4): 288-302.

https://doi.org/10.1016/j.agrformet.2006.03.030

Jobbágy, E.G. \& Jackson, R.B. 2000. The vertical distribution of soil organic carbon and its relation to climate and vegetation. Ecological Applications, 10: 323-638. https://doi.org/10.1890/10510761(2000)010[0423:TVDOSO]2.0.CO;2

Khalil, M.I., Hossain, M.B. \& Schmidhalter, U. 2005. Carbon and nitrogen mineralization in different upland soils of subtropics treated with organic materials. Soil Biol. Biochem., 37: 1507-1518.

https://doi.org/10.1016/j.soilbio.2005.01.014 
Khalil, M.I., Fornara, D. \& Osborne, B. 2020. Simulation and validation of soil organic carbon density and its changes in a long-term permanent grassland of Ireland. Geoderma, 361: 114014.

http://doi.org/10.1016/j.geoderma.2019.114014

Lal, R. 2009. Sequestering carbon in soils of arid ecosystems. Land Degrad. Dev., 20: 441-444.

https://doi.org/10.1002/1dr.934

McLauchlan, K.K., Hobbie, S.E. \& Post, W.M. 2006. Conversion from agriculture to grassland builds soil organic matter on decadal timescales. Ecological Applications, 16: 143-153. https://doi.org/10.1890/041650

Neely, C., Bunning, S. \& Wilkes, A. 2009. Review of evidence on drylands pastoral systems and climate change: implications and opportunities for mitigation and adaptation. FAO, Rome. (also available at: http://www.fao.org/uploads/media/LWdisc_paper8_temp.pdf)

Nordborg, M. \& Röös, E. 2016. Holistic Management - a critical review of Allan Savory's grazing method. SLU/EPOK - Centre for Organic Food \& Farming \& Chalmers. 47pp.

Ogle, S.M., Bredit, F.J. \& Paustian, K. 2005. Agricultural management impacts on soil organic carbon storage under moist and dry climatic conditions of temperate and tropical regions. Biogeochem., 72: 87-121. https://doi.org/10.1007/s10533-004-0360-2

Post, W.M. \& Kwon, K.C. 2000. Soil carbon sequestration and land-use change: process and potential. Global Change Biology, 6: 317-328. https://doi.org/10.1046/j.1365-2486.2000.00308.x

Reid, R.S., Thornton, P.K., McCrabb, G.J., Kruska, R.L., Atieno, F. \& Jones, P.G. 2004. Is it possible to mitigate greenhouse gas emissions in pastoral ecosystems of the tropics? Environment, Development and Sustainability, 6(1): 91. https://doi.org/10.1023/B:ENVI.0000003631.43271.6b

Ritchie, H. \& Roser, M. 2013. Crop Yields. In: Our World in Data [online]. [Cited 7 December 2020]. https://ourworldindata.org/crop-yields

Smith, P., Martino, D., Cai, Z., Gwary, D., Janzen, H., Kumar, P., McCarl, B., Ogle, S., O'Mara, F., Rice, C., Scholes, B., Sirotenko, O., Howden, M., McAllister, T., Pan, G., Romanenkov, V., Schneider, U., Towprayoon, S., Wattenbach, M. \& Smith, J. 2008. Greenhouse gas mitigation in agriculture.

Philosophical Transactions of the Royal Society B: Biological Sciences, 363(1492): 789-813.

https://doi.org/10.1098/rstb.2007.2184

Teague, R. \& Barnes, M. 2017. Grazing management that regenerates ecosystem function and grazingland livelihoods. African Journal of Range \& Forage Science, 34(2): 77-86.

https://doi.org/10.2989/10220119.2017.1334706 


\title{
30. Conservation of permanent grassland
}

\author{
Mohammad I. Khalil', Cláudia M.d.S. Cordovil², Rosa Francaviglia ${ }^{3}$, \\ Beverley Henry ${ }^{4}$, Katja Klumpp ${ }^{5}$, Peter Koncz ${ }^{6}$, Mireia Llorente ${ }^{7}$, \\ Beata E. Madari ${ }^{8}$, Muñoz-Rojas Miriam ${ }^{9,10}$, Nerger Rainer ${ }^{11}$ \\ (Co-authors in alphabetical order)
}

1School of Applied Sciences \& Technology, Prudence College Dublin, Dublin 22 and School of Biology \& Environmental Science, University College Dublin, Dublin 4, Ireland

${ }^{2}$ University of Lisbon, School of Agriculture, Forest Research Center, Lisboa, Portugal

${ }^{3}$ Council for Agricultural Research and Economics, Research Centre for Agriculture and Environment (CREA-AA), Rome, Italy.

${ }^{4}$ Science and Engineering Faculty, Queensland University of Technology, Brisbane, Australia

${ }^{5}$ Grassland Ecosystem Research, INRA, Clermont-Ferrand, France

${ }^{6}$ Duna-Ipoly National Park Directorate, Budapest, Hungary and MTA-SZIE Plant Ecology Research Group, Gödöllő, Hungary

${ }^{7}$ Forest Department. University of Extremadura, Plasencia Campus. Spain

${ }^{8}$ Embrapa Rice and Beans, Santo Antônio de Goiás, GO, Brazil

${ }^{9}$ UNSW Sydney, School of Biological, Earth and Environmental Sciences, Sydney NSW, Australia

${ }^{10}$ The University of Western Australia, School of Biological Sciences, Crawley, WA, Australia

${ }^{11}$ Soil \& More Impacts GmbH, German office, Hamburg, Germany

\section{Description of the practice}

Grasslands represent important ecosystems, providing key services to human livelihoods by producing fodder for animals, food production, the regulation of nutrients and water and the sequestration of carbon $(\mathrm{C})$ in soils (Byrnes et al., 2018). Permanent grasslands are defined as land being used for several consecutive years (normally 5 years or more) to grow grass or other herbaceous fodder, forage or energy crops, either through 
cultivation (sown/reseeded) or naturally (native/autochthone, self-seeded). Due to conversion to arable land for the production of food and animal feed crops, grasslands have declined worldwide during the last century (Queiroz et al., 2014). For example, over 90 percent of the semi-natural grasslands (i.e. grassland of natural origin, under minimum human influence vegetation dominated by grasses) in northern Europe have been lost because of agricultural intensification, abandonment, afforestation, societal changes and development pressure (Peyraud and Peeters, 2016). As permanent grasslands often contain large soil C stocks, because of higher belowground $\mathrm{C}$ inputs than croplands and year-round plant cover, management (e.g. reseeding after 5 years) and related soil and environmental factors could induce soil $\mathrm{C}$ losses faster than gains, making efforts to protect and conserve these grasslands of high priority (Smith, 2014). Likewise, in the case of temporary grasslands including arable ley, periodic/frequent tillage for re-sowing results in large losses of SOC (between 20 percent and 60 percent of C stored in surface soils (Franzluebbers, Swchik and Taboada, 2014). Even these short-term grasslands, which lay in between crops and permanent grasslands, have the potential for soil C accrual (Johnston et al., 2017). Therefore, conservation of permanent grasslands should be a global goal in order to protect existing soil C storage and to accrue more $\mathrm{C}$ in soils with high potential for $\mathrm{C}$ sequestration (e.g. Minasny et al., 2017).

\section{Range of applicability}

This sub-section is devoted to the conservation of managed "permanent grassland" (native and non-native) referring also to the "protection of native grassland". Permanent grasslands have declined considerably since the 1930s (e.g. Bullock et al., 2011). In North America, 80 percent of the central grasslands has been converted to cropland (Foley et al., 2005). Similarly, more than 43 million hectares of the Eurasian steppe have been converted into cropland. As for Europe, temporary sown grasslands have become more important in the Northern countries (i.e. 35 percent of agricultural area in Sweden, 28 percent in Finland and 24 percent in Estonia and Norway) and in several Eastern countries (circa 20 percent in Poland, Hungary, Bulgaria; Peyraud and Peeters, 2016. The area under low productive poor permanent grasslands (rangeland) has only decreased marginally between 1990 and 2007 (from 13.2 to 11.5 million ha for eight countries; Belgium, Denmark, France, Ireland, Luxemburg, Spain, Netherlands, United-Kingdom) (Peyraud and Peeters, 2016). The conversion of permanent grasslands to arable land have led to losses of large amounts of SOC ( $-36 \pm 5$ percent, Poeplau et al., 2011) due to enhanced soil organic matter decomposition due to soil disturbance, reduced C inputs from plant material (i.e. litter, roots) and increased erosion (see also 3.4 Conversion of grassland to cropland).

\section{Impacts on soil organic carbon stocks}

Across pedo-climate zones, permanent grassland soils can act either as sinks or sources for atmospheric CO2 (Table 133, also see Minasny et al., 2017). Carbon sequestration potential largely depends on grassland types, soils and environmental factors, management practices (e.g. mowing and grazing) and intensity (Abdalla et al., 2018). These authors reported that managed grassland ecosystems act as potential sinks of $\mathrm{C}$, storing on average $0.23 \pm 0.05 \mathrm{tC} / \mathrm{ha} / \mathrm{yr}$ in the $0-30 \mathrm{~cm}$ depth based on a global review and meta-analysis of 83 studies of extensive grazing, covering 164 sites across different countries and climatic zones. The observed range is, 
however, important (from -2.2 to $>1 \mathrm{tC} / \mathrm{ha} / \mathrm{yr}$ ) due to the large panoply of permanent grasslands in terms of pedo-climatic zones, vegetation cover (e.g. species composition, C3 and C4 grasses) and management intensity and type. This is close to available long-term data (i.e. soil inventory data of Belgium, the United Kingdom of Great Britain and Northern Ireland, Canada and New Zealand) showing an average storage of $0.05 \mathrm{tC} / \mathrm{ha} / \mathrm{yr}$ (e.g. Meersmans et al., 2011; Bellamy et al., 2005; Emett et al., 2010; Wang et al., 2014, Schipper et al., 2014, 2010). In contrast to temporary, permanent vegetation covers have the potential for sequestrating $\mathrm{C}$ for longer time and in deeper soil layers. After ploughing and sowing phase of temporary meadow, the $\mathrm{C}$ accumulation in the soil takes place primary in the surface layer $(0-10$ to $0-30 \mathrm{~cm})$ and then spreads gradually towards deeper horizons (Franzluebbers et al., 2014). In addition, fertilisation has a beneficial effect on $\mathrm{C}$ sequestration in permanent grasslands (Conant et al., 2017; Eze, Palmer and Chapman, 2018; Khalil et al., 2020). There is a non-linear relationship between the amount of nitrogen and $\mathrm{C}$ storage, which depends on how the grasslands are used (Poepleau et al., 2018). 
Table 133. SOC sequestration in conserved grassland at $0-30 \mathrm{~cm}$ depth

\begin{tabular}{|c|c|c|c|c|c|c|}
\hline Location & Climate zone & Soil type & $\begin{array}{l}\text { Additional C storage } \\
\text { [Range] }(\mathrm{tC} / \mathrm{ha} / \mathrm{yr})\end{array}$ & $\begin{array}{l}\text { Duration } \\
\text { (years) }\end{array}$ & More information & Reference \\
\hline \multirow{2}{*}{ Global } & \multirow{2}{*}{ Various } & \multirow{2}{*}{ Various } & 0.47 & & $\begin{array}{l}\text { Grasslands synthesis (i.e. native, permanent, } \\
\text { temporary) }\end{array}$ & $\begin{array}{l}\text { Conant et al. (2001, } \\
\text { 2017) }\end{array}$ \\
\hline & & & 0.23 & & Managed grassland ecosystems & Abdalla et al. (2018) \\
\hline Netherlands & $\begin{array}{l}\text { North } \\
\text { Atlantic } \\
\text { Climatic }\end{array}$ & Various & 0.41 & 20 & $\begin{array}{l}\text { National inventory permanent grassland (natural and } \\
>5 \text { years age) }\end{array}$ & $\begin{array}{l}\text { Hanegraaf et al., } \\
\text { (2009) }\end{array}$ \\
\hline Spain & $\begin{array}{l}\text { Mediterranean } \\
\text { basin }\end{array}$ & $\begin{array}{l}\text { Calcaric } \\
\text { Cambisol }\end{array}$ & 0.148 & 36 & $\begin{array}{l}\text { Soil sampling on permanent grassland (natural and } \\
>5 \text { years age) }\end{array}$ & $\begin{array}{l}\text { Marti-Roura, Casals } \\
\text { and Romanya (2011) }\end{array}$ \\
\hline $\begin{array}{l}\text { Northern } \\
\text { Ireland (UK) }\end{array}$ & \multirow{4}{*}{ Temperate } & $\begin{array}{l}\text { Clay loam, } \\
\text { Silurian shale, } \\
\text { greywacke }\end{array}$ & 0.48 & 45 & $\begin{array}{l}\text { Analyses of permanent grassland (natural and > } \\
5 y e a r s \text { age) under different amounts of organic } \\
\text { fertilisation }\end{array}$ & $\begin{array}{l}\text { Fornara et al. }(2016, \\
2020)\end{array}$ \\
\hline Belgium & & \multirow{5}{*}{ Various } & $0.16[0.14 ; 0.17]$ & 46 & \multirow{3}{*}{$\begin{array}{l}\text { National inventory of permanent grassland (natural } \\
\text { and > 5years age) }\end{array}$} & Meersmans et al. (2011) \\
\hline $\begin{array}{l}\text { United } \\
\text { Kingdom of } \\
\text { Great Britain } \\
\text { and Northern } \\
\text { Ireland }\end{array}$ & & & $0.05[-0.03 ; 0.14]$ & 29 & & Wang et al. (2014) \\
\hline Canada & & & $0.20[0.16 ; 0.24]$ & $4-72$ & & Wang et al. (2014) \\
\hline \multirow{2}{*}{ New Zealand } & \multirow{2}{*}{ Various } & & $0.21[-0.23 ;-0.20]$ & 30 & $\begin{array}{l}\text { National inventory of permanent grassland (native, } \\
\text { natural and > 5years age) }\end{array}$ & Schipper et al. (2014) \\
\hline & & & $-1.01[-0.7 ;-1.3]$ & 7 & $\begin{array}{l}\text { Analyses of permanent grassland (natural and > } \\
5 y e a r s \text { age) under different grazing and fertiliser } \\
\text { application }\end{array}$ & $\begin{array}{l}\text { Skinner and Dell } \\
\text { (2014) }\end{array}$ \\
\hline
\end{tabular}




\section{Other benefits of the practice}

\subsection{Improvement of soil properties}

Conservation of permanent grasslands avoids the process of ploughing which is intrinsically coupled with soil layer mixing, subsequent soil aeration, changes in soil temperate, and humidity, leading to the breakdown of soil aggregates and shifts towards more bacterial - dominated soil communities (Conant et al., 2001). Whereas, sequestering $\mathrm{C}$ in grassland soils is a win-win situation since $\mathrm{CO}_{2}$ is removed from the atmosphere, while increasing soil $\mathrm{C}$ results in many agronomic advantages including enhanced soil water retention that favours plant growth, soil health and biodiversity, increased availability of soil nutrients, improved soil structure and stability, decreased erosion and improved general soil functioning (Paustian et al., 2019).

\subsection{Minimization of threats to soil functions}

Table 134. Soil threats

\begin{tabular}{|c|c|}
\hline Soil threats & \\
\hline Soil erosion & $\begin{array}{l}\text { Minimizes surface runoff via permanent vegetation cover and } \\
\text { established root zone (Auerswald and Finer, 2018). }\end{array}$ \\
\hline $\begin{array}{l}\text { Nutrient imbalance } \\
\text { and cycles }\end{array}$ & $\begin{array}{l}\text { Species richness improves nutrient use and cycling (Cong et al., 2014, } \\
\text { De Deyn et al., 2011). }\end{array}$ \\
\hline $\begin{array}{l}\text { Soil salinization and } \\
\text { alkalinization }\end{array}$ & Depends on irrigation water quality and level. \\
\hline Soil acidification & Depends on fertilizer type and level. \\
\hline Soil biodiversity loss & $\begin{array}{l}\text { Permanent (semi) natural vegetation has likely more diversity compared } \\
\text { to sown grasslands. }\end{array}$ \\
\hline Soil compaction & $\begin{array}{l}\text { Not the practice per se but the management intensity (i.e. animal } \\
\text { stocking rate), as permanent grasslands are more likely to be grazed } \\
\text { than temporary sown grasslands. }\end{array}$ \\
\hline $\begin{array}{l}\text { Soil water } \\
\text { management }\end{array}$ & $\begin{array}{l}\text { Control on waterrunoff and water quality though permanent and } \\
\text { settled vegetation cover. }\end{array}$ \\
\hline
\end{tabular}




\subsection{Increases in production (e.g. food/fuel/feed/timber/fibre)}

Permanent grasslands are often associated with low biomass yield and forage quality. Recent studies underline plant diversity as an important production factor and independent of management intensity (e.g. Binder et al., 2018; also see Factsheet No.31/Volume 3 on Grassland diversification), because it enhances quality-adjusted yield and revenues similar to increasing fertilization and cutting frequency (Schaub et al., 2020). It also appears that grasslands with complex flora allow higher C storage than with lower species diversity (Hungate et al., 2017; Lange et al., 2015). This storage increases with the specific richness of the meadow providing a higher root biomass, often having legumes (Cong et al, 2014; Yang et al. 2019), attributing to the diversity of root systems (more or less dense and deep).

\subsection{Mitigation of and adaptation to climate change}

Permanent grasslands provide multiple services, and make an important contribution to climate change mitigation and adaptation. Conversion of permanent grasslands increases $\mathrm{CO}_{2}$ and $\mathrm{N}_{2} \mathrm{O}$ emissions due to soil disturbance through ploughing and the associated acceleration of decomposition processes, $\mathrm{N}$ and $\mathrm{C}$ availability, soil aeration and $\mathrm{pH}$ (i.e. Vellinga et al., 2004; van Kessel et al., 2012, also see Factsheet No.33/Volume 3 on Conversion of cropland to grassland). Likewise, permanent grasslands are more likely to experience low management intensities (e.g. rangelands, fertiliser inputs and animal density) than temporary sown grasslands. Low management intensities (also see Factsheets No. 34 to 36/Volume 3 on Grazing management) receive less fertiliser inputs and animal excreta resulting in less $\mathrm{N}_{2} \mathrm{O}$ emissions but increase enteric $\mathrm{CH}_{4}$ due to reduced forage digestibility (Archimède et al., 2011). Permanent grasslands are often species rich, which contributes to the temporal stability of their services, as species-rich communities tend to perform better than any individual species (Mace, Norris and Fitter, 2012).

\subsection{Socio-economic benefits}

In many parts of the world, grasslands have received less agricultural improvement (fertilizing, weed killing, ploughing or re-seeding) to become "unimproved" grasslands (e.g. rangeland, lowlands) with a (wild-) plant diversity. Agricultural intensification has led to a replacement of original or semi-natural communities in sown monocultures of cultivated varieties of grasses and clovers. Accordingly, "unimproved, natural" grasslands are among the threatened types of habitats, and thus appropriate managements are encouraged through special incentives to landowners and involvement of wildlife conservation groups. These areas are often associated with eco-tourism (e.g. Schripke et al., 2017). Likewise, flora and fauna-rich permanent grasslands and upland areas produce high quality foods/feeds, leading to rise in the number of organic farms and quality labels (PDO: protected designation of origin; PGI: protected geographical indication in the European Union). 


\subsection{Other benefits}

Conservation of permanent grasslands, naturally or as a result of human activity (i.e. long-term sown grasslands) also provides services, such as cultural heritage landscapes (Puszta, Alpes), where we perceive "natural" vs. "cultural" landscapes (Peeters, 2009; Gibon, 2005).

\section{Potential drawbacks to the practice}

\subsection{Tradeoffs with other threats to soil functions}

Conserving permanent grasslands may lead to a decline forage productivity and related $\mathrm{C}$ inputs to soil (e.g. litter and exudates), especially in nutrient-poor areas and under low $\mathrm{N}$ input management practices. Improved management actions (i.e. amendments of N, P, K, and lime) enhance $\mathrm{C}$ inputs to soil but SOC densities/stocks may eventually reach an equilibrium state over time (e.g. Smith, 2014) or may not (Khalil et al., 2020). Grasslands are highly sensitive to management and land use changes. Proper management to enhance and/or maintain SOC density/stocks to reduce atmospheric build-up of $\mathrm{CO}_{2}$, improve soil quality and fertility, reduce compaction, erosion and nutrient loss for plant growth and prevent a vegetation conversion/land use change (e.g. grazing, species composition, and mineral nutrient availability) that would decrease the soil C is important.

\subsection{Increases in greenhouse gas emissions}

Many management practices (e.g. fertilization, liming and grazing) do lead to non- $\mathrm{CO}_{2}$ emissions, in particular $\mathrm{N}_{2} \mathrm{O}$ from soil particularly due to the amount and types of $\mathrm{N}$ additions via fertilisation and biological fixation (legumes) irrespective of grassland types. However, emissions due to soil disturbance can be important (Vellinga et al., 2004; Merbold et al., 2014).

\subsection{Conflict with other practice(s)}

Although grasslands have been identified for having high conservation values and support for food production, conflicts may arise in areas of intense livestock production and competition for feed. In this area, permanent grasslands have remained under-appreciated, even though there are growing concerns for specialising agricultural systems using grass-crop-rotations and their environmental impacts (O’Mara, 2012). Besides, conserving permanent grasslands may lead to a decline in forage productivity and forage quality over time, especially in nutrient poor areas and under low $\mathrm{N}$ input management practices. 


\subsection{Other conflicts}

The conservation of grasslands associated with low management intensities induces changes in the plant community composition, and ecological succession that lead to an increased shrub abundance (Teixeira et al., 2015). Even though, this may have positive effects on $\mathrm{C}$ storage, in some areas this increases the risk of fire, and that may require the application of "prescribed fire" to maintain grasslands. Accordingly, conservation might need some flexibility to incorporate management practices such as fire into grazing systems to maintain soil fertility, avoid encroachment, and restore herbaceous productivity (Teague et al., 2010).

\section{Recommendations before implementing the practice}

Support of improved agronomical practices, sound complementary policies and good governance is imperative. Local public extension services, in collaboration with local product labels (e.g. PDO, organic farming, grassfed) and identity markers (e.g. Buffalo mozzarella, Serrano Ham, Kerrygold Irish butter), are crucial for the introduction and success of grass-based farm practices, which allow the maintenance and improvement of permanent grasslands for livestock production. Moreover, tourism and landscape heritage may provide further encouragement to preserve permanent pastures. However, identity markers and product labels are at different scales of development being either easily quantifiable (e.g. food, dishes, livestock products) or not tangible such as pastoral practices, know-how and cultural landscapes. For developing countries, the conservation of soil fertility while avoiding over grazing is most crucial. The timely uses of decision tools for early warning may help compensate drought and non-drought periods, as well as avoid overgrazing of the rangeland resources. These tools, based on advanced crop and grazing models, and empirical relationships between weather, vegetation, regrowth, can predict drought and feed shortages for livestock several weeks before grazing period. This allows them to better prepare for the incoming feed shortages and nutritional crises in a timely manner by transhumance (e.g. Wilhite and Svoboda, 2000).

\section{Potential barriers for adoption}

Even though permanent grasslands support multiple ecosystem services and C sequestration, in areas where arable land competes with grasslands possible uncertainties in grassland productivity and related required vegetation modifications (e.g. reseeding, weed and shrub control) to maintain grasslands may be a barrier for preservation. Hence, analysis of incentives (e.g. subsidies, economic policy, environmental taxes and $\mathrm{C}$ markets) to sequester carbon may help motivate farmers in adopting "good management practices" (see Rocha Correa et al., 2018). 
Table 135. Potential barriers to adoption

\begin{tabular}{|l|c|l|}
\hline Barrier & YES/NO & \\
\hline Cultural/Social & Yes & $\begin{array}{l}\text { Psychological reluctance with respect to new practices (Rocha Correa } \\
\text { et al., 2018). }\end{array}$ \\
\hline Economic & Yes & $\begin{array}{l}\text { Absence of socio-economic evaluation, markets, labels, subsidies, } \\
\text { (Rocha Correa et al., 2018). }\end{array}$ \\
\hline Institutional & Yes & Missing skills. \\
\hline $\begin{array}{l}\text { Legal (Right to } \\
\text { soil) }\end{array}$ & Yes & $\begin{array}{l}\text { Paddocks are too distant from each other to apply sustainable grazing } \\
\text { management. }\end{array}$ \\
\hline Knowledge & Yes & Lack of training, skills, advisory services, supporting tools. \\
\hline Other & Yes & Competition with other agricultural land use of economic interest. \\
\hline
\end{tabular}

\section{Photos of the practice}
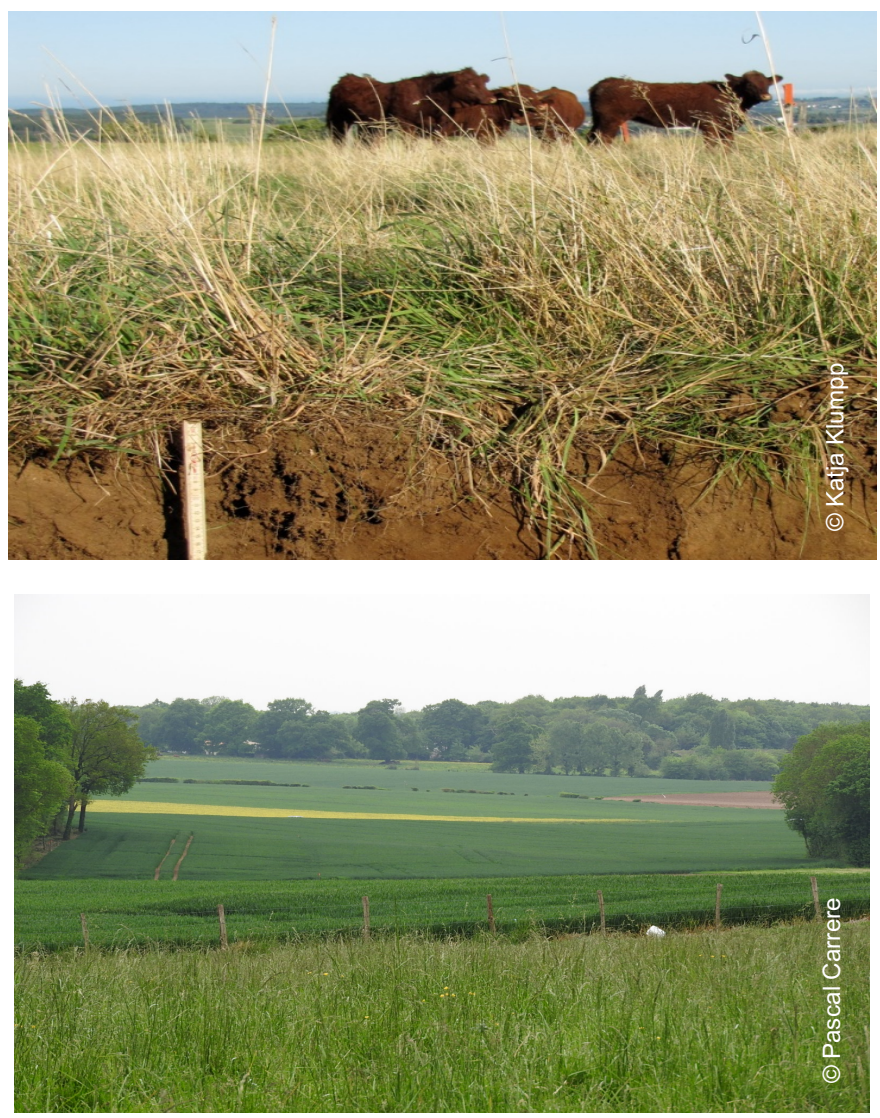

Photo 39. Permanent grassland and land mosaic with permanent grassland field in France 


\section{References}

Abdalla, M., Hastings, A., Chadwick, D.R., Jones, D.L., Evans, C.D., Jones, M.B., Rees, R.M. \& Smith, P. 2018. Critical review of the impacts of grazing intensity on soil organic carbon storage and other soil quality indicators in extensively managed grasslands. Agriculture Ecosystems \& Environment, 253: 62-81. http://dx.doi.org/10.1016/j.agee.2017.10.023

Archimède, H., Eugène, M., Magdeleine, C.M., Boval, M., Martin, C., Morgavi, D.P., Lecomte, P. \& Doreau, M. 2011. Comparison of methane production between C3 and C4 grasses and legumes. Animal Feed Science and Technology, 166-67: 59-64. https://doi.org/10.1016/j.anifeedsci.2011.04.003

Auerswald, K. \& Fiener, P. 2018. Soil organic carbon storage following conversion from cropland to grassland on sites differing in soil drainage and erosion history. Science of the Total Environment, 661: 481491. https://doi.org/10.1016/j.scitotenv.2019.01.200

Bellamy, P.H., Loveland, P.J., Bradley, R.I., Lark, R.M. \& Kirk, G.J.D. 2005. Carbon losses from all soils across England and Wales 1978-2003. Nature, 437(7056): 245-248.

https://doi.org/10.1038/nature04038

Binder, S., Isbell, F., Polasky, S., Catford, J.A. \& Tilman, D. 2018. Grassland biodiversity can pay. Proceedings of the National Academy of Sciences, 115(15): 3876-3881.

https://doi.org/10.1073/pnas.1712874115

Bullock, J., Jefferson, R.G., Blackstock, T.H., Pakeman, R.J., Emmett, B.A., Pywell, R.J., Grime, J.P., Silvertown, J. 2011. Semi-natural grasslands. In UK National Ecosystem Assessment Technical Report. UNEP-WCMC, Cambridge, pp. 161-196. (also available at: https://core.ac.uk/download/pdf/385587.pdf)

Byrnes, R.C., Eastburn, D.J., Tate, K.W. \& Roche, L.M. 2018. A Global Meta-Analysis of Grazing Impacts on Soil Health Indicators. Journal of Environmental Quality, 47(4): 758-765.

https://doi.org/10.2134/jeq2017.08.0313

Conant, R.T., Paustian, K. \& Elliott, E.T. 2001. Grassland management and conversion into grassland, effects on soil carbon. Ecological Applications, 11: 343-355. https://doi.org/10.1890/10510761(2001)011[0343:GMACIG]2.0.CO;2

Conant, R.T., Cerri, C.E.P., Osborne, B.B. \& Paustian, K. 2017. Grassland management impacts on soil carbon stocks: a new synthesis. Ecological Applications, 27(2): 662-668.

http://dx.doi.org/10.1002/eap.1473

Cong, W.F., van Ruijven, J., Mommer, L., De Deyn, G.B., Berendse, F. \& Hoffland, E. 2014. Plant species richness promotes soil carbon and nitrogen stocks in grasslands without legumes. Journal of Ecology, 102(5): 1163-1170. https://doi.org/10.1111/1365-2745.12280

Deyn, G.B.D., Shiel, R.S., Ostle, N.J., McNamara, N.P., Oakley, S., Young, I., Freeman, C., Fenner, N., Quirk, H. \& Bardgett, R.D. 2011. Additional carbon sequestration benefits of grassland diversity restoration. Journal of Applied Ecology, 48(3): 600-608. https://doi.org/10.1111/j.1365-

2664.2010.01925.x 
Emmett, B.A., Reynolds, B., Chamberlain, P.M., Rowe, E., Spurgeon, D., Brittain, S.A., Frogbrook, Z., Hughes, S., Lawlor, A.J., Poskitt, J., Potter, E., Robinson, D.A., Scott, A., Wood, C. \& Woods, C. 2010. Countryside Survey: Soils Report from 2007. Technical Report No 9/07, 0-192, NERC/Centre for Ecology \& Hydrology (CEH Project Number: C03259). http://nora.nerc.ac.uk/id/eprint/9354

Eze, S., Palmer, S.M. \& Chapman, P.J. 2018. Soil organic carbon stock in grasslands: Effects of inorganic fertilizers, liming and grazing in different climate settings. Journal of Environmental Management, 223: 7484. http://dx.doi.org/10.1016/j.jenvman.2018.06.013

Foley, J.A., Ramankutty, N., Brauman, K.A., Cassidy, E.S., Gerber, J.S., Johnston, M., Mueller, N.D., O'Connell, C., Ray, D.K., West, P.C., Balzer, C., Bennett, E.M., Carpenter, S.R., Hill, J., Monfreda, C., Polasky, S., Rockström, J., Sheehan, J., Siebert, S., Tilman, D. \& Zaks, D.P.M. 2011. Solutions for a cultivated planet. Nature, 478(7369): 337-342. https://doi.org/10.1038/nature10452

Fornara, D.A., Wasson, E.A., Christie, P. \& Watson, C.J. 2016. Long-term nutrient fertilization and the carbon balance of permanent grassland: any evidence for sustainable intensification? Biogeosciences, 13 (17): 4975-4984. http://dx.doi.org/10.5194/bg-13-4975-2016

Franzluebbers, A.J., Swchik, J. \& Taboada, M.A. 2014. Agronomic and environmental impacts of pasturecrop rotations in temperate North and South America. Agriculture, Ecosystems and Environment, 190: 1826. https://doi.org/10.1016/j.agee.2013.09.017

Franzluebbers, A.J. \& Stuedemann, J.A. 2009. Soil-profile organic carbon and total nitrogen during 12 years of pasture management in the Southern Piedmont USA. Agriculture, Ecosystems and Environment, 129: 28-36. https://doi.org/10.1016/j.agee.2008.06.013

Gibon, A. 2005. Managing grassland for production, the environment and the landscape. Challenges at the farm and the landscape level. Livestock Production Science, 96: 11-31.

https://doi.org/10.1016/j.livprodsci.2005.05.009

Hanegraaf, M.C., Hoffland, E., Kuikman, P.J. \& Brussaard, L. 2009. Trends in soil organic matter contents in Dutch grasslands and maize fields on sandy soils. European Journal of Soil Science, 60: 213-222. https://doi.org/10.1111/j.1365-2389.2008.01115.x

Hungate, B.A., Barbier, E.B., Ando, A.W., Marks, S.P., Reich, P.B., Gestel, N. van, Tilman, D., Knops, J.M.H., Hooper, D.U., Butterfield, B.J. \& Cardinale, B.J. 2017. The economic value of grassland species for carbon storage. Science Advances, 3(4): e1601880. https://doi.org/10.1126/sciadv.1601880

Johnston, A.E., Poulton, P.R., Coleman, K., Macdonald, A.J. \& White, R.P. 2017. Changes in soil organic matter over 70 years in continuous arable and ley-arable rotations on a sandy loam soil in England. European Journal of Soil Science, 68(3): 305-316. https://doi.org/10.1111/ejss. 12415

Khalil, M.I., Fornara, D.A. \& Osborne, B. 2020. Simulation and validation of long-term changes in soil organic carbon under permanent grassland using the DNDC model. Geoderma, 361: 114014.

https://doi.org/10.1016/j.geoderma.2019.114014 
Lange, M., Eisenhauer, N., Sierra, C.A., Bessler, H., Engels, C., Griffiths, R.I., Mellado-Vázquez, P.G., Malik, A.A., Roy, J., Scheu, S., Steinbeiss, S., Thomson, B.C., Trumbore, S.E. \& Gleixner, G. 2015. Plant diversity increases soil microbial activity and soil carbon storage. Nature Communications, 6(1): 6707. https://doi.org/10.1038/ncomms7707

Mace, M.M., Norris, K. \& Fitter, A.H. 2012. Biodiversity and ecosystem services: a multilayered relationship. Trends in Ecology and Evolution, 27: 19-26. https://doi.org/10.1016/j.tree.2011.08.006

Marti-Roura, M., Casals, P. \& Romanyà, J. 2011. Temporal changes in soil organic C under Mediterranean shrublands and grasslands: impact of fire and drought. Plant Soil, 338: 289-300.

https://doi.org/10.1007/s11104-010-0485-0

Meersmans, J., van Wesemael, B., Goidts, E., van Molle, M., De Baets, S. \& De Ridder, F. 2011. Spatial analysis of soil organic carbon evolution in Belgian croplands and grasslands, 1960-2006. Global Change Biology, 17: 466-479. http://dx.doi.org/10.1111/j.1365-2486.2010.02183.x

Merbold, L., Eugster, W., Stieger, J., Zahniser, M., Nelson, D. \& Buchmann, N. 2014. Greenhouse gas budget $\left(\mathrm{CO}_{2}, \mathrm{CH}_{4}\right.$ and $\left.\mathrm{N}_{2} \mathrm{O}\right)$ of intensively managed grassland following restoration. Global Change Biology, 20(6): 1913-1928. https://doi.org/10.1111/gcb.12518

Minasny, B., Malone, B.P., McBratney, A.B., Angers, D.A., Arrouays, D., Chambers, A., Chaplot, V., et al. 2017. Soil carbon 4 per mille. Geoderma, 292: 59-86.

https://doi.org/10.1016/j.geoderma.2017.01.002

O'Mara, F.P. 2012. The role of grasslands in food security and climate change. Annals of Botany, 110(6): 1263-1270. https://doi.org/10.1093/aob/mes209

Paustian, K., Larson, E., Kent, J., Marx, E. \& Swan, A. 2019. Soil C Sequestration as a Biological Negative Emission Strategy. Frontiers in Climate, 1. https://doi.org/10.3389/fclim.2019.00008

Peeters, A. 2009. Importance, evolution, environmental impact and future challenges of grasslands and grassland-based systems in Europe. Grassland Science, 55: 113-125. https://doi.org/10.1111/j.1744697X.2009.00154.x

Peyraud, J.L. \& Peeters, A. 2016. The role of grassland based production system in the protein Security. General meeting of the European Grassland Federation (EGF), Sep 2016, Trondheim, Norway.

https://hal.inrae.fr/hal-02743435/document

Poeplau, C., Don, A., Vesterdal, L., Leifeld, J., Wesemael, B.V., Schumacher, J. \& Gensior, A. 2011. Temporal dynamics of soil organic carbon after land-use change in the temperate zone - carbon response functions as a model approach. Global Change Biology, 17(7): 2415-2427.

https://doi.org/10.1111/j.1365-2486.2011.02408.x

Poeplau, C., Zopf, D., Greiner, B., Geerts, R., Korvaar, H., Thumm, U., Don, M., Heidkamp, A. \& Flessa, H. 2018. Why does mineral fertilization increase soil carbon stocks in temperate grasslands? Agriculture, Ecosystems \& Environment, 265: 144-155. http://dx.doi.org/10.1016/j.agee.2018.06.003 
Queiroz, C., Beilin, R., Folke, C. \& Lindborg, R. 2014. Farmland abandonment: threat or opportunity for biodiversity conservation? A global review. Front Ecol Environ, 12(5): 288-296.

https://doi.org/10.1890/120348

Rocha Correa, P.F., Koncz, P., Poilane, A., Schaak, H., Schönhart, M., Svoboda, P., Teixeira, R. \& van Rijn, C. 2018. EIP-AGRI Focus Group: Grazing for carbon. (also available at:

https://www.innovarurale.it/sites/default/files/2018-11/eip-

agri_fg_grazing_for_carbon_final_report_2018_en.pdf)

Schipper, L.A., Parfitt, R.L., Ross, C., Baisden, W.T., Claydon, J.J. \& Fraser, S. 2010. Gains and losses in $\mathrm{C}$ and $\mathrm{N}$ stock of New Zealand pasture soils depends on the land use. Agriculture Ecosystems \&

Environment, 139: 611-617. https://doi.org/10.1016/j.agee.2010.10.005

Schipper, L.A., Parfitt, R.L., Fraser, S., Littler, R.A., Baisden, W.T. \& Ross, C. 2014. Soil order and grazing management effects on changes in soil $\mathrm{C}$ and $\mathrm{N}$ in New Zealand pastures. Agriculture Ecosystems \& Environment, 184: 67-75. http://dx.doi.org/10.1016/j.agee.2013.11.012

Schaub, S., Finger, R., Leiber, F., Probst, S., Kreuzer, M., Weigelt, A., Buchmann, N. \& Scherer-

Lorenzen, M. 2020. Plant diversity effects on forage quality, yield and revenues of semi-natural grasslands. Nature Communications, 11(1): 768. https://doi.org/10.1038/s41467-020-14541-4

Skinner, R.H. \& Dell, C.J. 2014. Comparing pasture C sequestration estimates form eddy covariance and soil cores. Agriculture Ecosystems \& Environment, 199: 52-57.

https://doi.org/10.1016/j.agee.2014.08.020

Smith, P. 2014. Do grasslands act as a perpetual sink for carbon? Global Change Biology, 20(9) : 27082711. https://doi.org/10.1111/gcb.12561

Teague, W.R., Dowhower, S.L., Ansley, R.J., Pinchak, W.E. \& Waggoner, J.A. 2010. Integrated Grazing and Prescribed Fire Restoration Strategies in a Mesquite Savanna: I. Vegetation Responses. Rangeland Ecology \& Management, 63(3): 275-285. https://doi.org/10.2111/08-171.1

Teixeira, R.F.M, Proenca, V., Crespo, D., Valada. T. \& Domingos, T. 2015. A conceptual framework for the analyses of engineered biodiverse pastures. Ecological Engineering, 77: 85-97.

https://doi.org/10.1016/j.ecoleng.2015.01.002

Vellinga, T., van den Pol-van Dasselaar, A. \& Kuikman, P. 2004. The impact of grassland ploughing on $\mathrm{CO}_{2}$ and $\mathrm{N}_{2} \mathrm{O}$ emissions in the Netherlands. Nutrient Cycling in Agroecosystems, 70: 33-45.

https://doi.org/10.1023/B:FRES.0000045981.56547.db

van Kessel, C., Venterea, R., Six, J., Adviento-Borbe, M.A., Linquist, B. \& van Groenigen, K.J. 2012. Climate, duration, and $\mathrm{N}$ placement determine $\mathrm{N} 2 \mathrm{O}$ emissions in reduced tillage systems: a metaanalysis. Global Change Biology, 19(1): 33-44. https://doi.org/10.1111/j.1365-2486.2012.02779.x

Wang, Z., Jiao, S., Han, G., Zhao, M., Ding, H., Zhang, X., Wang, X., Ayers, E.L., Willms, W.D., Havsatad, K., A, L. \& Liu, Y. 2014. Effects of Stocking Rate on the Variability of Peak Standing Crop in a Desert Steppe of Eurasia Grassland. Environmental Management, 53(2): 266-273.

https://doi.org/10.1007/s00267-013-0186-6 
Wilhite, D.A. \& Svoboda, M.D. 2000. Drought early warning systems in the context of drought preparedness and mitigation. In Wilhite, D.A., Sivakumar, M.V.K. \& Wood D.A. Early Warning Systems for Drought Preparedness and Drought Management. WMO/TD no. 1037, 1-21. World Meteorological Organization, Geneva, Switzerland.

Yang, Y., Tilman, D., Furey, G. \& Lehman, C. 2019. Soil carbon sequestration accelerated by restoration of grassland biodiversity. Nature Communications, 10(1): 718. https://doi.org/10.1038/s41467-01908636-w 


\title{
31. Grassland diversification
}

\author{
Mohammad I. Khalil', Cláudia M.d.S. Cordovil², Rosa Francaviglia ${ }^{3}$, \\ Beverley Henry ${ }^{4}$, Katja Klumpp ${ }^{5}$, Peter Koncz ${ }^{6}$, Mireia Llorente ${ }^{7}$, \\ Beata E. Madari ${ }^{8}$, Muñoz-Rojas Miriam ${ }^{9,10}$, Nerger Rainer ${ }^{11}$ \\ (Co-authors in alphabetical order)
}

'School of Applied Sciences \& Technology, Prudence College Dublin, Dublin 22 and School of Biology \& Environmental Science, University College Dublin, Dublin 4, Ireland

${ }^{2}$ University of Lisbon, School of Agriculture, Forest Research Center, Lisboa, Portugal

${ }^{3}$ Council for Agricultural Research and Economics, Research Centre for Agriculture and Environment (CREA-AA), Rome, Italy.

${ }^{4}$ Science and Engineering Faculty, Queensland University of Technology, Brisbane, Australia

${ }^{5}$ Grassland Ecosystem Research, INRA, Clermont-Ferrand, France

${ }^{6}$ Duna-Ipoly National Park Directorate, Budapest, Hungary and MTA-SZIE Plant Ecology Research Group, Gödöllő, Hungary

${ }^{7}$ Forest Department. University of Extremadura, Plasencia Campus. Spain

${ }^{8}$ Embrapa Rice and Beans, Santo Antônio de Goiás, GO, Brazil

${ }^{9}$ UNSW Sydney, School of Biological, Earth and Environmental Sciences, Sydney NSW, Australia

${ }^{10}$ The University of Western Australia, School of Biological Sciences, Crawley, WA, Australia

${ }^{11}$ Soil \& More Impacts GmbH, German office, Hamburg, Germany

\section{Description of the practice}

Grassland diversification includes a set of practices such as the incorporation of legumes and other favourable forage grasses into grazed grasslands the planting of scattered trees in grassland or the rotation of crops with pasture. Species-rich grasslands are of high conservation value because of the diverse floral and faunal assemblages they support, and of the capacity to improve water and nutrient use. Besides creating shadow areas 
for grazing livestock, scattered trees may have local and global benefits such as the creation of microclimate, increased soil nutrient concentration and a more favourable water balance locally, increased plant species richness and habitat for animals, which will promote larger-scale ecosystem restoration. These benefits facilitate adaptive responses to climate change, particularly in modified landscapes (Manning, Gibbon and Lindenmayer, 2009). Rotating crops with pastures increases weed control and reduces greenhouse gas emissions from fields. However, as Donnison and Fraser (2016) explain, grasslands are experiencing the biggest threat to date in terms of loss of land area to other uses, including expansion of the built environment as well as from cropland, forestry and energy production (e.g. solar, biofuels).

The above-mentioned practices have two main objectives: (1) to increase the botanical diversity of species-poor grassland to restore and enhance this habitat so as to ameliorate the negative effects of isolation, fragmentation and scrub species encroachment (EC, 1992) and (2) to increase the profit-earning capacity of marginally economic or uneconomic grasslands by diversification of grass-derived products and by increasing pasture production through the incorporation of nitrogen-fixing species and perennial grassland species.

\section{Range of applicability}

The practice is applicable worldwide under a wide range of pedo-climatic conditions. The most suitable locations are those with low soil fertility and low/no weed burden.

\section{Impacts on soil organic carbon stocks}

For a given climate regime, grassland often has higher soil carbon $(\mathrm{C})$ contents than other vegetation types. Therefore, a lack of interest about their use can cause serious impacts in terms of the $\mathrm{C}$ balance. Grassland diversification, both to restore environmental values and to enhance the economic value of the land could be a good tool to ensure soil conservation and, thereby, the value of the land as a $\mathrm{C}$ sink (Table 136). A great number of studies have demonstrated that increasing plant diversity increases soil C storage, both directly and indirectly (e.g. Chen et al., 2017; Lange et al., 2015, Teixeira et al., 2011). 
Table 136. Evolution of SOC stocks with grassland diversification

\begin{tabular}{|c|c|c|c|c|c|c|c|c|}
\hline Location & Climate zone & Soil type & $\begin{array}{l}\text { Baseline } \\
\text { C stock } \\
\text { (tC/ha) }\end{array}$ & $\begin{array}{l}\text { Additional } \\
\text { C storage } \\
\text { (range) } \\
\text { (tC/ha/yr) }\end{array}$ & $\begin{array}{l}\text { Duration } \\
\text { (Years) }\end{array}$ & Depth (cm) & More information & Reference \\
\hline \multirow{2}{*}{ Global } & Various & Various & NA & 0.75 & $\begin{array}{l}\text { From } 5 \text { to } \\
9\end{array}$ & \multirow{2}{*}{$\begin{array}{l}\text { Various up to } \\
100 \mathrm{~cm}\end{array}$} & $\begin{array}{l}\mathrm{N}=6 \\
\text { Introduction of legumes. }\end{array}$ & \multirow{2}{*}{$\begin{array}{l}\text { Conant, Paustian } \\
\text { and Elliot (2001) }\end{array}$} \\
\hline & Various & Various & NA & Up to 3.04 & $\begin{array}{l}\text { From } 4 \text { to } \\
15\end{array}$ & & $\begin{array}{l}\mathrm{N}=5 \\
\text { Improved grass species. }\end{array}$ & \\
\hline France & Temperate & Luvisol/ Fluvisol & NA & $\begin{array}{l}0.24 \\
(0.09- \\
0.46)\end{array}$ & $\begin{array}{l}\text { From } 6 \text { to } \\
41\end{array}$ & $0-30$ & $\begin{array}{l}\text { Incorporation of trees } \\
\text { (Agroforestry). }\end{array}$ & $\begin{array}{l}\text { Cardinael et al. } \\
(2017)\end{array}$ \\
\hline Spain & \multirow{2}{*}{ Mediterranean } & Cambisol, Luvisol & 72.0 & 0.83 & 22 & NA & $\begin{array}{l}\text { Scattering trees-extensive } \\
\text { farming. }\end{array}$ & $\begin{array}{l}\text { Llorente et al. } \\
\text { (2019) }\end{array}$ \\
\hline $\begin{array}{l}\text { Sardinia, } \\
\text { Italy }\end{array}$ & & Cambisol & 42.9 & 1.24 & 37 & $0-20$ & $\begin{array}{l}\text { Alternating spontaneous } \\
\text { vegetation-hay crops. }\end{array}$ & $\begin{array}{l}\text { Francaviglia et al. } \\
\text { (2017) }\end{array}$ \\
\hline $\begin{array}{l}\text { Minnesota, } \\
\text { United } \\
\text { States of } \\
\text { America }\end{array}$ & $\begin{array}{l}\text { Humid } \\
\text { Continental }\end{array}$ & Sandy & NA & 0.69 & 12 & \multirow[t]{2}{*}{$0-100$} & $\begin{array}{l}\text { Combination of key C4 } \\
\text { grass-legume species on } \\
\text { degraded soils. }\end{array}$ & $\begin{array}{l}\text { Fornara and } \\
\text { Tilman (2008) }\end{array}$ \\
\hline $\begin{array}{l}\text { Mato } \\
\text { Grosso, } \\
\text { Brazil }\end{array}$ & Tropical & Kaolinitic oxisol & NA & $0.32-1.57$ & NA & & $\begin{array}{l}\text { Agrosilvopastoralism; } \\
\text { Incorporation of trees. }\end{array}$ & $\begin{array}{l}\text { Oliveira et al. } \\
(2018)\end{array}$ \\
\hline
\end{tabular}

NA: Not available. 


\section{Other benefits of the practice}

\subsection{Improvement of soil properties}

Plant interactions and feedbacks with soil biota is an important determinant of ecosystem functioning and primary productivity in terrestrial habitats. Above-ground species diversity influences below-ground diversity and regulates microbial decomposition pathways (Wagg et al., 2014). Experiments across different ecosystems indicate that soil organic matter tends to decline as local species richness in grassland decreases (Cardinael $e t$ al., 2017).

\subsection{Minimization of threats to soil functions}

Table 137. Soil threats

\section{Soil threats}

\begin{tabular}{l|l} 
Soil erosion & Soil cover control on soil surface runoff.
\end{tabular}

Nutrient imbalance and cycles

Species richness improves nitrogen fixation, use and cycling, allowing a better coupling of $\mathrm{C}$ and $\mathrm{N}$ cycles within vegetation, soil organic matter and soil microbial biomass.

\begin{tabular}{|l|l|}
\hline Soil biodiversity loss & Above-ground species diversity influences below-ground diversity. \\
\hline Soil compaction & $\begin{array}{l}\text { Improves soil structure via a greater incorporation of SOM and by } \\
\text { covering soil surface. }\end{array}$ \\
\hline $\begin{array}{l}\text { Soil water } \\
\text { management }\end{array}$ & $\begin{array}{l}\text { Control of water runoff, increasing soil water retention and infiltration } \\
\text { rates. }\end{array}$ \\
\hline
\end{tabular}

\subsection{Increases in production (e.g. food/fuel/feed/timber/fibre)}

Diversification of grassland products, including the processing and fractionation of biomass for feed, food, energy and other non-food applications, presents a positive opportunity to improve the options for grassland users and their communities. 


\subsection{Mitigation of and adaptation to climate change}

Long-term biodiversity restoration practices in grasslands increase soil $\mathrm{C}$ and therefore soil mitigation capacity. Moreover, the preservation or sowing of biodiverse pastures has proven to sequester more $\mathrm{C}$ with related cobenefits (Teixeira et al., 2011). These greater rates of $\mathrm{C}$ accumulation look to be associated with reduction of ecosystem respiration, increase of soil organic matter inputs and improvement of soil structure (De Deyn et al., 2011).

\subsection{Socio-economic benefits}

Embracing the diversification of grassland functions is in many instances an important step to ensure grassland survival and protection from the onslaught of competition for land from urbanization and from other agricultural and forestry-based land uses.

\section{Potential drawbacks to the practice}

\subsection{Tradeoffs with other threats to soil functions}

The benefits of enhancing the richness of species must be balanced with the risk of erosion during land intervention. Careful selection of plant species is important to avoid possible risks like soil desertification due to excess water extraction or unwanted $\mathrm{pH}$ modifications.

\subsection{Increases in greenhouse gas emissions}

Tillage and compaction of soil for seed incorporation could speed up organic matter mineralization with the consequent increase of soil greenhouse gas emissions. For example, Yamulki and Jarvis (2002) measured increases on $\mathrm{N}_{2} \mathrm{O}, \mathrm{NO}, \mathrm{CO}_{2}$ and $\mathrm{CH}_{4}$ fluxes after tillage and compaction of grassland.

\subsection{Conflict with other practice(s)}

Investment in diversification of grasslands can be in competition with investments in more profitable land uses like cropland, forestry, or energy production.

\subsection{Other conflicts}

A conflict can arise if introduced new botanical species become weeds and they can potentially have a negative impact on biodiversity. Seeds must be carefully chosen for each particular site. 


\section{Recommendations before implementing the practice}

Agronomy support is imperative as well as sound complementary policies and good governance. Public extension services, in collaboration with the private sector remain crucial to the success of agrarian new practices.

\section{Potential barriers for adoption}

Improving grassland through introduction of grass varieties has to be adopted together with adequate management practices. New grass and legume varieties, diversification technology options and agronomy support are needed.

\section{Photo of the practice}

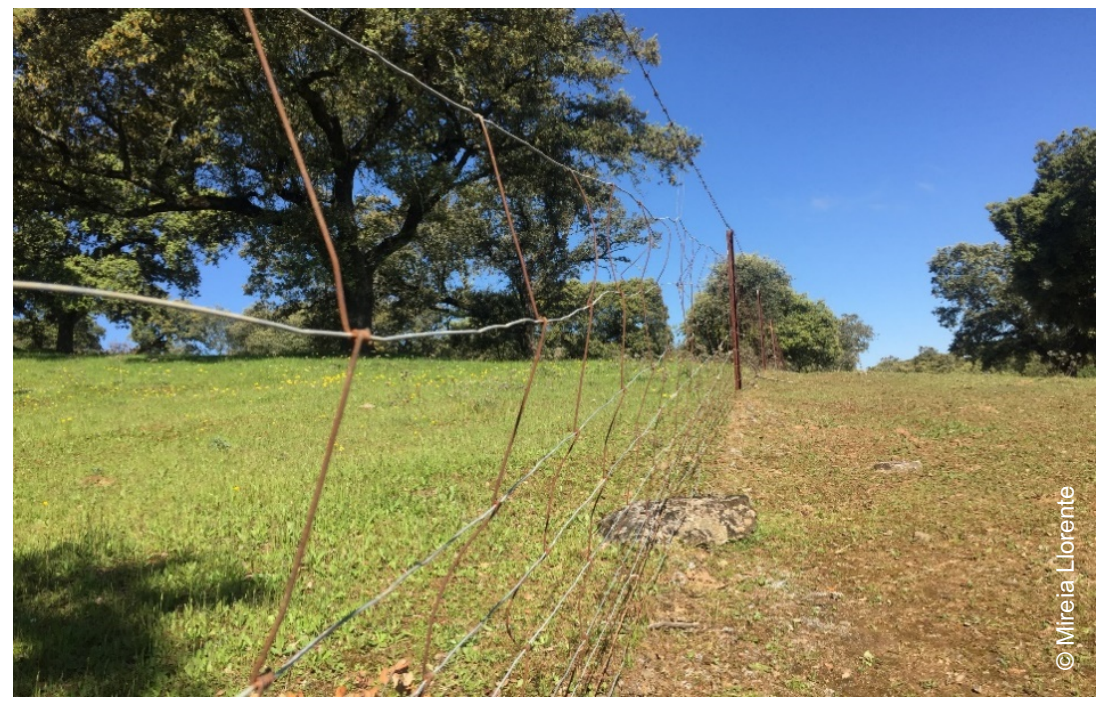

Photo 40. Showing grass growth difference between diversified (left) vs. monocultured (right) grasslands.

Table 138. Related cases studies available in volumes 3 and 5

\begin{tabular}{|l|c|c|c|c|}
\hline Title & Region & $\begin{array}{l}\text { Duration of } \\
\text { study (Years) }\end{array}$ & Volume & $\begin{array}{l}\text { Case- } \\
\text { study No. }\end{array}$ \\
\hline $\begin{array}{l}\text { Mediterranean savanna-like agrosilvopastoral } \\
\text { grassland system in Spain, Italy and Portugal }\end{array}$ & Europe & 4,22 and 37 & 3 & 17 \\
\hline
\end{tabular}




\section{References}

Cardinael, R., Chevallier, T., Cambou, A., Beral, C., Barthès, B.G., Dupraz, C., Durand, C.,

Kouakoua, E. \& Chenu, C. 2017. Increased soil organic carbon stocks under agroforestry: A survey of six different sites in France. Agriculture, Ecosystems and Environment, 236: 243-255.

https://doi.org/10.1016/j.agee.2016.12.011

Chen, H., Mommer, L., Ruijven, J. van, Kroon, H. de, Fischer, C., Gessler, A., Hildebrandt, A., Scherer-Lorenzen, M., Wirth, C. \& Weigelt, A. 2017. Plant species richness negatively affects root decomposition in grasslands. Journal of Ecology, 105(1): 209-218. https://doi.org/10.1111/13652745.12650

Conant, R.T., Paustian, K. \& Elliot, E.T. 2001 . Grassland management and conversion into grassland: Effects on soil carbon. Ecological Applications, 11: 343-355. https://doi.org/10.1890/10510761(2001)011[0343:GMACIG]2.0.CO;2

De Deyn, G.B., Shiel, R.S., Ostle, N.J., McNamara, N.P., Oakley, S., Young, I., Freeman, C., Fenner, N., Quirk, H. \& Bardgett, R.D. 2011. Additional carbon sequestration benefits of grassland diversity restoration. Journal of Applied Ecology, 48: 600-608. https://doi.org/10.1111/j.1365-

2664.2010.01925.x

Donnison, I.S. \& Fraser, M.D. 2016. Diversification and use of bioenergy to maintain future grasslands. Food and Energy security, 5: 67-75. https://doi.org/10.1002/fes3.75

EC. 1992. The Conservation of Natural Habitats and of Wild Fauna and Flora. Council Directive 92/43/EEC. The Council of European Communities, Brussels, Belgium. https://ec.europa.eu/environment/nature/legislation/habitatsdirective/index_en.htm

Fornara, A. \& Tilman, D. 2008. Plant functional composition influences rates of soil carbon and nitrogen accumulation. Journal of Ecology, 96: 314-322. https://doi.org/10.1111/j.1365-2745.2007.01345.x

Francaviglia, R., Renzi, G., Ledda, L. \& Benedetti, A. 2017. Organic carbon pools and soil biological fertility are affected by land use intensity in Mediterranean ecosystems of Sardinia, Italy. Science of the Total Environment, 599: 789-796. https://doi.org/10.1016/j.scitotenv.2017.05.021

Lange, M., Eisenhauer, N., Sierra, C.A., Bessler, H., Engels, C., Griffiths, R.I., Mellado-Vázquez, P.G., Malik, A.A., Roy, J., Scheu, S., Steinbeiss, S., Thomson, B.C., Trumbore, S.E. \& Gleixner, G. 2015. Plant diversity increases soil microbial activity and soil carbon storage. Nature Communications, 6(1): 6707. https://doi.org/10.1038/ncomms7707

Llorente, M., Moreno, G. Pulido, M. \& González-Cascón, R. 2019. Factors determining distribution and temporal changes in soil carbon in Iberian Dehesa. Geophysical Research Abstracts. EGU General Assembly 2019. (also available at: https://meetingorganizer.copernicus.org/EGU2019/EGU2019-1962.pdf)

Manning, A.D., Gibbon, P. \& Lindenmayer, D.B. 2009. Scattered trees: a complementary strategy for facilitating adaptive responses to climate change in modified landscapes? Journal of Applied Ecology, 46: 915-919. https://doi.org/10.1111/j.1365-2664.2009.01657.x 
Oliveira, J.D., Madari, B.E., Carvalho, M.T.D., Ramos, C. Rodrigues, A.L., Lima, M.L., Wruck F.J., Medeiros, J.C. \& Oliveira, P.L. 2018. Integrated farming systems for improving soil carbon balance in the southern Amazon of Brazil. Regional Environmental Change, 18: 105-116.

https://doi.org/10.1007/s10113-017-1146-0

Teixeira, R.F.M., Domingos, T., Costa, A.P.S.V., Oliveira, R., Farropas, L., Calouro, F., Barradas, A.M. \& Carneiro, J.P.B.G. 2011. Soil organic matter dynamics in Portuguese natural and sown rainfed grasslands. Ecological Modelling, 222(4): 993-1001. https://doi.org/10.1016/j.ecolmodel.2010.11.013

Yamulki, S. \& Jarvis, S.C. 2002. Short-term effects of tillage and compaction on nitrous oxide, nitric oxide, nitrogen dioxide, methane and carbon dioxide fluxes from grassland. Biology and Fertility of Soils, 36: 224231. https://doi.org/10.1007/s00374-002-0530-0

Wagg, C., Bender, S.F., Widmer, F. \& Van der Heijden, M.G.A. 2014. Soil biodiversity and soil community composition determine ecosystem multifunctionality. Proceedings of the National Academy of Sciences of the United States of America, 111: 5266-5270. https://doi.org/10.1073/pnas. 1320054111 


\title{
32. Restoration of degraded grassland
}

\author{
Mohammad I. Khalil', Cláudia M.d.S. Cordovil², Rosa Francaviglia ${ }^{3}$, \\ Beverley Henry ${ }^{4}$, Katja Klumpp ${ }^{5}$, Peter Koncz ${ }^{6}$, Mireia Llorente ${ }^{7}$, \\ Beata E. Madari ${ }^{8}$, Muñoz-Rojas Miriam ${ }^{9,10}$, Nerger Rainer ${ }^{11}$ \\ (Co-authors in alphabetical order)
}

'School of Applied Sciences \& Technology, Prudence College Dublin, Dublin 22 and School of Biology \& Environmental Science, University College Dublin, Dublin 4, Ireland

${ }^{2}$ University of Lisbon, School of Agriculture, Forest Research Center, Lisboa, Portugal

${ }^{3}$ Council for Agricultural Research and Economics, Research Centre for Agriculture and Environment (CREA-AA), Rome, Italy.

${ }^{4}$ Science and Engineering Faculty, Queensland University of Technology, Brisbane, Australia

${ }^{5}$ Grassland Ecosystem Research, INRA, Clermont-Ferrand, France

${ }^{6}$ Duna-Ipoly National Park Directorate, Budapest, Hungary and MTA-SZIE Plant Ecology Research Group, Gödöllő, Hungary

${ }^{7}$ Forest Department. University of Extremadura, Plasencia Campus. Spain

${ }^{8}$ Embrapa Rice and Beans, Santo Antônio de Goiás, GO, Brazil

${ }^{9}$ UNSW Sydney, School of Biological, Earth and Environmental Sciences, Sydney NSW, Australia

${ }^{10}$ The University of Western Australia, School of Biological Sciences, Crawley, WA, Australia

${ }^{11}$ Soil \& More Impacts GmbH, German office, Hamburg, Germany

\section{Description of the practice}

Grassland degradation is a complex concept that encompasses several aspects that relate to changes compared to a reference state. These include alterations in soil conditions, biodiversity, productivity, and socio-economic implications, and can differ at various degradation stages (Andrade et al., 2015). Degradation may be the result 
of changes of two main types of properties: biotic and abiotic (Andrade et al., 2015). Biotic changes include biodiversity alteration, species composition, and basic ecological functions such as for example pollination deviations, and these can be the consequence of land use change, overgrazing and alien species introduction. Regarding abiotic factors contributing to degradation, changes in soil chemical and physical properties are comprised mostly due to fertilization and/or soil cultivation malpractice. Additionally, degraded unmanaged grasslands in tropical regions lose carbon (C) from the system due to animal trampling (Hiltbrunner et al., 2012) that form bare steps and vegetated shoulders occurring in between the steps. Similarly, C losses occur from grasslands in Brazilian Rondônia and Mato Grosso due to burning every 5-10 years to control weeds and woody plants (Maia et al., 2009). Other direct human causes of degradation for example are soil cultivation, infrastructure construction, and pollution.

The starting point for restoration is to look at the degree of degradation and find a traditional system with autochthone species (native/pristine), with high biodiversity and under extensive management including grazing exclusions. Among restoration strategies, considering the local hotspot of native species nearby, a selfrecovery allowing re-invasion of those species in the degraded grassland may be applicable in some mild situations, or in regions lacking available techniques. Nevertheless, it is important to evaluate at what point a recovery without additional technical measures is possible. Conversely, technical assistance may be needed in more serious situations (e.g. modification of soil features, control of undesired species, and introduction of desired species). One measure is usually not enough but any change in grassland management will impact on soil properties (Andrade et al., 2015).

Grassland restoration includes actions for biotic and abiotic improvement measures for example: (i) introduction of autochthone species, (ii) elimination of invasive species, (iii) controlled fertilisation, (iv) irrigation, (v) reseeding, (vi) cutting grass, (ix) topsoil transplantation, (xi) fencing, (xii) implementation of better grazing systems, (xiii) erosion control, and (xiv) green ecological barriers (Zhou, Lee and Yue, 2020). Regardless of the measure(s) adopted, the first action is to eliminate the source of the existing pressure on the grassland.

\section{Range of applicability}

These practices are applicable worldwide for a wide range of pedo-climatic conditions, wherever there is a degraded grassland. The cause of degradation and the extent to which it affects the grassland are to be taken into consideration when planning the restoration. Tropical and sub-tropical areas are particularly sensitive to degradation, mainly due to land use change, and inappropriate livestock grazing. This also caused rapid and abrupt changes in social structure, cultural habits, and patterns of land ownership.

\section{Impact on soil organic carbon stocks}

The common impact of degraded grassland on SOC is loss of SOC rather than sequestration. For example, animal trampling on a sub-alpine pasture site used for over 150 years for summer grazing in Switzerland caused 30 percent higher SOC loss from bare shoulder than the vegetated shoulder (Hiltbrunner et al., 2012). 
Appropriate management to restore the degraded and abandoned grasslands may significantly increase C sequestration rates (Table 139).

Table 139. Evolution of Soc stocks after restoration of degraded grassland

\begin{tabular}{|l|l|l|l|l|l|l|}
\hline Location & Climate zone & $\begin{array}{l}\text { Soil type } \\
\text { (Depth) }\end{array}$ & $\begin{array}{l}\text { Additional C } \\
\text { storage } \\
(\mathrm{tC} / \mathrm{ha} / \mathrm{yr})\end{array}$ & $\begin{array}{l}\text { Duration } \\
\text { (year) }\end{array}$ & $\begin{array}{l}\text { More } \\
\text { information }\end{array}$ & Reference \\
\hline $\begin{array}{l}\text { Rondônia and } \\
\text { Mato Grosso, } \\
\text { Brazil }\end{array}$ & Humid tropics & $\begin{array}{l}\text { Oxisols } \\
(0-25 \mathrm{~cm})\end{array}$ & $\begin{array}{l}(-) 0.27- \\
0.28\end{array}$ & $5-10$ & $\begin{array}{l}\text { Degraded } \\
\text { grassland }\end{array}$ & $\begin{array}{l}\text { Maia et al. } \\
(2009)\end{array}$ \\
\hline $\begin{array}{l}\text { Minnesota, } \\
\text { United States } \\
\text { of America }\end{array}$ & $\begin{array}{l}\text { Humid } \\
\text { continental }\end{array}$ & $\begin{array}{l}\text { Glacial } \\
\text { outwash } \\
\text { sandplain } \\
(0-30 \mathrm{~cm})\end{array}$ & $0.08-0.71$ & 22 & $\begin{array}{l}\text { Managed } \\
\text { grassland } \\
\text { (i.e. grazing, } \\
\text { fertilisation) }\end{array}$ & $\begin{array}{l}\text { Yang } \text { et al. } \\
\text { (2019) }\end{array}$ \\
\hline Canada & Temperate & NA & $0.02-1.00$ & NA & $\begin{array}{l}\text { Restore } \\
\text { permanent } \\
\text { grass }\end{array}$ & $\begin{array}{l}\text { Hutchinso, } \\
\text { Campbell } \\
\text { and } \\
\text { Desjardins } \\
\text { (2007) }\end{array}$ \\
\hline
\end{tabular}

\section{Other benefits of the practice}

\subsection{Improvement of soil properties}

Developing actions to restore biodiversity in the long-term increases soil C and N storage (De Deyn et al. 2010). These are associated with the high rates of $\mathrm{C}$ and $\mathrm{N}$ accumulation and improvement of soil structure at a reduced ecosystem respiration rate. It could be further beneficial when restoration includes the increased abundance of at least one legume species in the grassland. Adequate management practices, as described above, deliver additional ecosystem benefits such as $\mathrm{N}$ storage in soil and improved soil structure (De Deyn et al., 2010). 


\subsection{Minimization of threats to soil functions}

Table 140. Soil threats

\begin{tabular}{|l|l|}
\hline Soil threats & $\begin{array}{l}\text { Restoring a grassland implies the improvement of soil quality, } \\
\text { and thereby control of erosion. }\end{array}$ \\
\hline Soil erosion & Improves nutrients cycles. \\
\hline cycles & May also be the reasons for degradation but difficult to restore. \\
\hline $\begin{array}{l}\text { Soil salinization and } \\
\text { alkalinisation }\end{array}$ & Contaminants/pollutants could be eliminated or reduced. \\
\hline $\begin{array}{l}\text { Soil contamination / } \\
\text { pollution }\end{array}$ & Reclamation of soil acidity may be feasible by means of liming. \\
\hline Soil acidification & Will improve soil biodiversity. \\
\hline Soil biodiversity loss & Increase in soil C and that can help reduce compaction. \\
\hline Soil compaction & Improved soil structure can increase water use efficiency. \\
\hline Soil water management & \\
\hline
\end{tabular}

\subsection{Increases in production (e.g. food/fuel/feed/timber/fibre)}

Restored grasslands will produce more and better pasture and silage to feed livestock.

\subsection{Mitigation of and adaptation to climate change}

In addition to improve soil quality and reduce soil $\mathrm{Closs}$, restored grassland could offset $\mathrm{N}_{2} \mathrm{O}$ and $\mathrm{CH}_{4}$ emissions through $\mathrm{C}$ sequestration while adapting to changing climatic condition.

\subsection{Socio-economic benefits}

In developing areas, grasslands are a major source of income through small farming activity and pastoralism. Therefore, grasslands restoration could have positive impacts on earning for farming communities and 
businesses. There is a strong relation between grassland-based livestock production and food security (Wilkes, Solymosi and Tennigkeit, 2012). Within this socio-economic context, improved grassland or pasture management can combat the risk of land degradation and help restore degraded sites leading to a better plant and litter cover, less vulnerability to erosion, less soil compaction and a higher biodiversity.

\subsection{Other benefits}

Landscape aesthetic benefits contribute to the wellbeing of people and facilitate tourism activities. Mixed production systems to meet up additional feed supplement for livestock products and proper use of resources to maintain biodiversity while decreasing $\mathrm{C}$ losses could be beneficial to prevent grassland degradation.

\section{Potential drawbacks to the practice}

\subsection{Tradeoffs with other threats to soil functions}

\section{Table 141. Soil threats}

\begin{tabular}{|l|l|}
\hline Soil threats & \\
\hline $\begin{array}{l}\text { Soil salinization and } \\
\text { alkalinisation }\end{array}$ & Limited or none. \\
\hline $\begin{array}{l}\text { Soil contamination / } \\
\text { pollution }\end{array}$ & Limited or none except fertilizer-induced contaminants. \\
\hline Soil acidification & Limited or none. \\
\hline Soil compaction & Limited except trampling through grazing. \\
\hline
\end{tabular}

\subsection{Increases in greenhouse gas emissions}

Although restoration of degraded grasslands increases soil C sequestration, reductions in GHGs may not always be the best indicator to evaluate improved environmental and development outcomes in grasslands (Wilkes, Solymosi and Tennigkeit, 2012). In degraded arid and semi-arid grasslands, for example, GHG emissions may be less responsive to changes in pasture and silage management than to climate variability. There are restoration practices (e.g. revegetation, grazing fallow and grassland fencing) applicable for the lightly to severely degraded grasslands. In addition to adaptive strategies, rotational grazing with moderate intensity could retain or enhance soil fertility, plant growth, carbon and nitrogen storage while reducing GHG emissions (Dong et al., 2020). 
Despite large potential for GHG mitigation in grasslands, uncertainty prevails for the implementation of good management practices. However, co-benefits of $\mathrm{C}$ sequestration and estimated emission reductions can be sufficiently attractive from an economic point of view.

\subsection{Conflict with other practice(s)}

Urbanization is a major conflicting activity for the restoration of degraded grasslands. as other activities may be prioritized in urban areas.

\subsection{Other conflicts}

Land ownership and funding could limit the restoration of degraded grasslands.

\section{Recommendations before implementing the practice}

A degraded site always needs earlier evaluation to assess the need for intervention, compared to the natural recovery capacity of the grassland. Degradation intensity and origin analysis will inform the necessary measures to implement the restoration techniques. According to Phillips-Mao (2017), this process typically includes several basic steps for example: (i) the assessment of the site to identify its characteristics and define the needs and goals for the restoration, (ii) vegetation removal to eliminate weeds and undesired vegetation that may outcompete with native species; (iii) preparation of the seed bed to ensure good seed-soil contact and promote germination, and (iv) seeding/planting of the select seed mixtures. Additionally, minimization of environmental impacts and measures to maintain or increase biodiversity are highly desirable. To avoid further land degradation, better management practices including optimal seasonal grazing, reseeding and provision of incentives and restrictions (grazing bans and reduction in livestock density) to improve grassland conditions should be adopted, depending on socio-economic and cultural conditions.

\section{Potential barriers for adoption}

Table 142. Potential barriers to adoption

\begin{tabular}{|l|c|l|}
\hline Barrier & YES/NO & \\
\hline Social & Yes & $\begin{array}{l}\text { Degraded grasslands in underdeveloped regions may be linked to } \\
\text { social issues. }\end{array}$ \\
\hline Economic & Yes & Restoration process may be costly. \\
\hline
\end{tabular}




\begin{tabular}{|l|c|l|}
\hline Barrier & YES/NO & \\
\hline $\begin{array}{l}\text { Legal (Right } \\
\text { to soil) }\end{array}$ & Yes & Depending on land ownership. \\
\hline Knowledge & Yes & $\begin{array}{l}\text { Restoration practice is a long-term process and knowledge is still } \\
\text { limited. }\end{array}$ \\
\hline
\end{tabular}

\section{Photos of the practice}
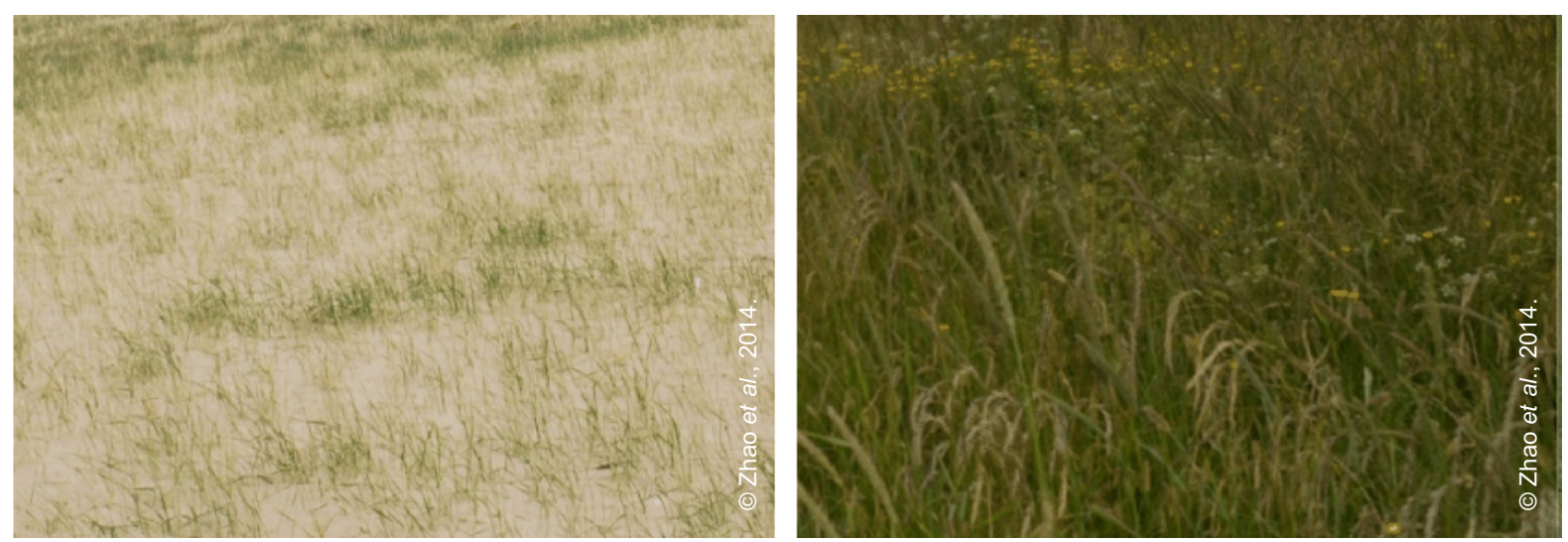

Photo 41. Showing degraded (left) and restored (Right) grasslands (Source: DOI: 10.5194/bgd-11-5613-2014).

Table 143.Related cases studies available in volumes 3 and 5

\begin{tabular}{|l|l|c|c|c|}
\hline Title & Region & $\begin{array}{l}\text { Duration } \\
\text { of study } \\
\text { (Years) }\end{array}$ & Volume & $\begin{array}{l}\text { Case- } \\
\text { study } \\
\text { No. }\end{array}$ \\
\hline $\begin{array}{l}\text { Integrated farming in tropical } \\
\text { agroecosystems of Brazil }\end{array}$ & $\begin{array}{l}\text { Latin America } \\
\text { and the } \\
\text { Caribbean }\end{array}$ & 4 to 12 & 3 & 34 \\
\hline
\end{tabular}




\section{References}

Andrade, B.O., Koch, C., Boldrini, I.I., Vélez-Martin, E., Hasenack, H., Hermann, J-M., Kollmann, J., Pillar, V.D. \& Overbeck, G.E. 2015. Grassland degradation and restoration: a conceptual framework of stages and thresholds illustrated by southern Brazilian grasslands. Natureza \& Conservação, 13(2): 95-104. https://doi.org/10.1016/j.ncon.2015.08.002

De Deyn, G.B., Shiel, R.S., Ostle, N.J., McNamara, N.P., Oakley, S., Young, I., Freeman, C., Fenner, N., Quirk, H. \& Bardgett, R.D. 2010. Additional carbon sequestration benefits of grassland diversity restoration. Journal of Applied Ecology, 48(3): 600-608. https://doi.org/10.1111/j.1365-

2664.2010.01925.x

Dong S., Shang, Z., Gao, J. \& Boone, R.B. 2020. Enhancing sustainability of grassland ecosystems through ecological restoration and grazing management in an era of climate change on Qinghai-Tibetan Plateau. Agriculture, Ecosystems \& Environment, 287: 106684.

https://doi.org/10.1016/j.agee.2019.106684.

Hiltbrunner, D., Schulze, S., Hagedorn, F., Schmidt, M.W.I. \& Zimmmermann, S. 2012. Cattle trampling alters soil properties and changes soil microbial communities in a Swiss sub-alpine pasture. Geoderma, 170: 369-377. https://doi.org/10.1016/j.geoderma.2011.11.026

Hutchinson, J.J., Campbell, C.A. \& Desjardins, R.L. 2007. Some perspectives on carbon sequestration in agriculture. Agricultural and Forest Meteorology, 142(2-4): 288-302.

https://doi.org/10.1016/j.agrformet.2006.03.030

Maia, S.M.F., Ogle, S.M., Cerri, C.E.P. \& Cerri, C.C. 2009. Effect of grassland management on soil carbon sequestration in Rondônia and Mato Grosso states, Brazil. Geoderma, 149(1-2): 84-91.

https://doi.org/10.1016/j.geoderma.2008.11.023

Phillips-Mao, L. 2017. Restoring your degraded grassland to conservation prairie. The Nature Conservancy (ed.). 12 p. (also available at:

https://www.nature.org/content/dam/tnc/nature/en/documents/Restoration-Guide-Degraded-

Grassland-to-Conservation-Prairie.pdf)

Wilkes, A., Solymosi, K. \& Tennigkeit, T. 2012. Options for Support to Grassland Restoration in the context of Climate Change Mitigation. Final Report. FAO and Unique forestry and land use. 60 p. Freiburg, Germany.

Zhao, L., Wu, W., Xu, X. \& Xu, Y. 2014. Soil organic matter dynamics under different land-use in grasslands in Inner Mongolia (northern China). Biogeosciences Discussions, 11: 5613-5637.

https://doi.org/10.5194/bgd-11-5613-2014

Zhou, W., Li, J. \& Yue, T. 2020. Grassland Degradation Restoration and Constructing Green Ecological Protective Screen. In Remote Sensing Monitoring and Evaluation of Degraded Grassland in China. Springer Geography. Springer, Singapore. https://doi.org/10.1007/978-981-32-9382-3_7 


\title{
33. Conversion of cropland to grassland
}

\author{
Mohammad I. Khalil', Cláudia M.d.S. Cordovil², Rosa Francaviglia ${ }^{3}$, \\ Beverley Henry ${ }^{4}$, Katja Klumpp ${ }^{5}$, Peter Koncz ${ }^{6}$, Mireia Llorente ${ }^{7}$, \\ Beata E. Madari ${ }^{8}$, Muñoz-Rojas Miriam ${ }^{9,10}$, Nerger Rainer ${ }^{11}$ \\ (Co-authors in alphabetical order)
}

'School of Applied Sciences \& Technology, Prudence College Dublin, Dublin 22 and School of Biology \& Environmental Science, University College Dublin, Dublin 4, Ireland

${ }^{2}$ University of Lisbon, School of Agriculture, Forest Research Center, Lisboa, Portugal

${ }^{3}$ Council for Agricultural Research and Economics, Research Centre for Agriculture and Environment (CREA-AA), Rome, Italy.

${ }^{4}$ Science and Engineering Faculty, Queensland University of Technology, Brisbane, Australia

${ }^{5}$ Grassland Ecosystem Research, INRA, Clermont-Ferrand, France

${ }^{6}$ Duna-Ipoly National Park Directorate, Budapest, Hungary and MTA-SZIE Plant Ecology Research Group, Gödöllő, Hungary

${ }^{7}$ Forest Department. University of Extremadura, Plasencia Campus. Spain

${ }^{8}$ Embrapa Rice and Beans, Santo Antônio de Goiás, GO, Brazil

${ }^{9}$ UNSW Sydney, School of Biological, Earth and Environmental Sciences, Sydney NSW, Australia

${ }^{10}$ The University of Western Australia, School of Biological Sciences, Crawley, WA, Australia

${ }^{11}$ Soil \& More Impacts GmbH, German office, Hamburg, Germany

\section{Description of the practice}

Grassland conversion is defined as converting land previously used for crop (arable) production to grassland cover. Converting cropland to grassland has been demonstrated to increase soil C content and net soil C storage worldwide (Jones, 2010; Khalil and Osborne, 2018). While SOC densities/stocks decrease significantly (and 
often rapidly) in response to the cultivation of both arable and grassland (temporary, permanent or leys), SOC accumulation is a slow and continuous process after the conversion of cropland to grassland (Popleau et al., 2011). There are different supporting mechanisms to convert land from cropland to grassland, usually in response to land degradation, such as the "Grain-for-Green" program started in China in 2000 and the US Conservation Reserve Program (CRP) initiated in 1985. In Western Europe, an arable land "set-aside" supporting mechanism started in 1988 in response to overproduction of commodity crops in EU countries. After 1992 it became compulsory and arable land was taken out of production, either for one year (rotational set-aside) or for a longer period (non-rotational set-aside) as part of the EU farm subsidy programme (Gosling et al., 2017).

\section{Range of applicability}

The practice is applicable worldwide under a wide range of pedoclimatic conditions. On average, SOC sequestration could be about $0.8 \mathrm{tC} / \mathrm{ha} /$ yr over a 50 -year period (IPCC, 2000), but with a large variability mainly due to climate conditions, soil texture, crop productivity and management intensity (Vleeshouwers and Verhagen, 2002). A study for European agricultural soils (Freibauer et al., 2004) reported that SOC could increase from 0.6 to $3.1 \mathrm{tC} / \mathrm{ha} / \mathrm{yr}$.

\section{Impact on soil organic carbon stocks}

Global literature reviews show that the lowest $\mathrm{C}$ storage ranges from 0.30 to $0.33 \mathrm{tC} / \mathrm{ha} / \mathrm{yr}$ while the highest storage may reach $1.44 \mathrm{tC} / \mathrm{ha} / \mathrm{yr}$ after 5 years in Europe under various climatic conditions (Table 144). The most common values are in the range of $0.62-0.75 \mathrm{tC} / \mathrm{ha} / \mathrm{yr}$ in cool temperate, as well as humid to semiarid climates. Interestingly, Caccrual after cropland conversion to grassland was measured at a rate of $0.92 \mathrm{tC} / \mathrm{ha} / \mathrm{yr}$ the first 20 years after conversion (i.e. the period assumed for a new equilibrium to occur after conversion) but decreased to $0.59 \mathrm{tC} / \mathrm{ha} / \mathrm{yr}$ if calculated over 100 years after conversion (Popleau et al, 2011). 
Table 144. Evolution of SOC stocks after conversion from cropland to grassland

\begin{tabular}{|c|c|c|c|c|c|c|c|}
\hline Climate zone & Soil type & $\begin{array}{l}\text { Baseline C } \\
\text { stock (tC/ha) }\end{array}$ & $\begin{array}{l}\text { Additional C storage } \\
\text { (tC/ha/yr) }\end{array}$ & $\begin{array}{l}\text { Duration } \\
\text { (Year) }\end{array}$ & $\begin{array}{l}\text { Depth } \\
(\mathrm{cm})\end{array}$ & Methodology & Reference \\
\hline \multicolumn{8}{|l|}{ Global studies } \\
\hline Temperate & \multirow{5}{*}{ Various } & $46.2 \pm 20.7$ & $\begin{array}{l}0.92 \pm 0.10 \\
0.59 \pm 0.14\end{array}$ & $\begin{array}{l}20 \\
100\end{array}$ & $0-23.5$ & CRFs & Popleau et al. (2011) \\
\hline Various & & NA & 1.01 & From 1 to 80 & $0-32.5$ & MA & $\begin{array}{l}\text { Conant, Paustian and Elliot } \\
(2001)\end{array}$ \\
\hline Various & & NA & 0.33 & NA & $0-29.5$ & LR & Post and Kwon (2000) \\
\hline Humid to semiarid & & NA & 0.75 & 14 & $0-30$ & MA & Kämpf et al. (2016) \\
\hline All & & 21.1 & 0.30 & 20 & $0-20$ & LR & Deng et al. (2016) \\
\hline \multicolumn{8}{|c|}{ Regional and national studies } \\
\hline $\begin{array}{l}\text { Warm and cool } \\
\text { temperate moist + dry }\end{array}$ & NA & NA & 1.44 & 5 & $0-30$ & MO & $\begin{array}{l}\text { Vleeshouwers and Verhagen } \\
(2002)\end{array}$ \\
\hline Cool temperate & NA & NA & 0.62 & 20 & $0-10$ & $\mathrm{FE}$ & $\begin{array}{l}\text { McLauchlan, Hobbie and Post } \\
(2006)\end{array}$ \\
\hline Cool temperate moist & $40 \%$ clay & NA & 0.71 & 28 & $0-20$ & $\mathrm{FE}$ & Miao, Qiao and Zhang (2015) \\
\hline
\end{tabular}

CRFs: carbon response functions, simple statistical models describing the relative SOC stock change with time after LUC or management change, FE: field experiment, LR: literature review, MA: meta-analysis, MO: modelling. 


\section{Other benefits of the practice}

\subsection{Improvement of soil properties}

Decrease in bulk density (Miao, Qiao and Zhang, 2015) result from the improved soil structure and porosity, which in turn, improves root penetration and water holding capacity in grassland compared with cropland. Increase of microbial biomass and activity (soil respiration) due to the greater root biomass and increased active C inputs (De et al. 2020) also stimulate the microbial population to produce more soil enzymes (Yu et al., 2017). More efficient internal $\mathrm{N}$ cycling due to the increased soil $\mathrm{C}$ levels and high $\mathrm{C}: \mathrm{N}$ ratio of inputs, linking to microbial immobilization of $\mathrm{N}$ resulting in low $\mathrm{N}$ mineralization (McLauchlan et al, 2006) and significant reduction in soil $\mathrm{NO}_{3}-\mathrm{-}$ and less excess $\mathrm{N}$ on conversion from arable to set-aside due to the lack of fertilization (Gosling et al., 2017) have been reported.

\subsection{Minimization of threats to soil functions}

\section{Table 145. Soil threats}

\begin{tabular}{|c|c|}
\hline Soil threats & \\
\hline Soil erosion & $\begin{array}{l}\text { Reduced surface runoff and increased stream flow in the dry season (Qiu et al., } \\
\text { 2011). Minimize surface erosion due to lack of tillage and continuous soil cover } \\
\text { (Lozano-García, Muñnz-Rojas and Parras-Alcantara, 2017). }\end{array}$ \\
\hline $\begin{array}{l}\text { Nutrient imbalance and } \\
\text { cycles }\end{array}$ & $\begin{array}{l}\text { Decreased potential for net } \mathrm{N} \text { mineralization, i.e. } \mathrm{N} \text { supply for plant and microbial } \\
\text { uptake (McLauchlan et al., 2006), reduction in } \mathrm{NO}_{3}^{-}-\mathrm{N} \text { losses (Gosling et al., 2017). }\end{array}$ \\
\hline $\begin{array}{l}\text { Soil salinization and } \\
\text { alkalinization }\end{array}$ & $\begin{array}{l}\text { Gradual decrease of soil salinity and sodicity in semi-arid agroecosystems (Yu et } \\
\text { al., 2018). }\end{array}$ \\
\hline $\begin{array}{l}\text { Soil } \\
\text { contamination/pollution }\end{array}$ & $\begin{array}{l}\text { Change in inputs (e.g. sewage sludges, herbicides, pesticides, etc.) in grassland } \\
\text { (Diaz et al., 2012). }\end{array}$ \\
\hline Soil acidification & $\begin{array}{l}\text { May be reduced due to the absence of acidifying fertilisers application (Schroder } \\
\text { et al., 2011). }\end{array}$ \\
\hline Soil biodiversity loss & $\begin{array}{l}\text { Increased soil respiration (Zhang et al., 2015) and enzyme activities (Yu et al., 2017) } \\
\text { foster soil fertility improvement thus increasing .autochthone plant species } \\
\text { biodiversity, which is a strong driver of the structure and functioning of soil food } \\
\text { webs (microorganisms, nematodes, microarthropods) in grasslands (Eisenhauer et } \\
\text { al., 2013). }\end{array}$ \\
\hline Soil compaction & Decreased bulk density and thereby compaction (Miao, Qiao and Zhang, 2015). \\
\hline Soil water management & Less water required compared to croplands (Qiu et al., 2011). \\
\hline
\end{tabular}




\subsection{Increases in production (e.g. food/fuel/feed/timber/fibre)}

Conversion of cropland to grazed grassland can provide meat or milk, wool, forage for stable livestock and direct feed for grazing animals (Sanderman et al., 2010).

\subsection{Mitigation of and adaptation to climate change}

Some $\mathrm{CH}_{4}\left(32 \pm 6.8 \mathrm{~g} \mathrm{CO}_{2} \mathrm{eq} / \mathrm{m}^{2} / \mathrm{yr}\right)$ and $\mathrm{N}_{2} \mathrm{O}\left(14 \pm 4.7 \mathrm{~g} \mathrm{CO}_{2} \mathrm{eq} / \mathrm{m}^{2} / \mathrm{yr}\right)$ emissions can arise during grazing (Soussana et al., 2007). However, $\mathrm{N}_{2} \mathrm{O}$ emissions are lower than those deriving from the intensive use of fertilizers in croplands (Ahlering, Fargione and Parton, 2016). The technical GHG mitigation potential of converting cropland to grassland ranges from 4.4 to $6.2 \mathrm{t} \mathrm{CO}_{2} \mathrm{eq} / \mathrm{ha} / \mathrm{yr}$ (Freibauer et al., 2004; Feliciano et al., 2013). In more detail, mean estimates for the United Kingdom of Great Britain and Northern Irelandrange from 0.53 to $5.34 \mathrm{t} \mathrm{CO}_{2} \mathrm{eq} / \mathrm{ha} / \mathrm{yr}$ for the conversion cropland to temporary $(<5 \mathrm{yr})$ and permanent $(>5 \mathrm{yr})$ grassland, respectively (Smith et al., 2010).

\subsection{Socio-economic benefits}

Grasslands provide additional valuable ecosystem services, such as biodiversity conservation, habitat for wildlife, aesthetic value and recreational opportunities.

\subsection{Other benefits of the practice}

Investment security can be achieved by selecting lands with high-carbon sequestration potential over similar lands that cannot potentially sequester as much carbon. This could result in a carbon cap-and-trade system and an economic market for carbon sequestration products. 


\section{Potential drawbacks to the practice}

\subsection{Tradeoffs with other threats to soil functions}

Table 146. Soil threats

\begin{tabular}{|l|l|}
\hline Soil threats & \\
\hline Soil erosion & $\begin{array}{l}\text { Heavy grazing decreases plant cover leaving the soil exposed and vulnerable to } \\
\text { erosion (Vanderburg et al., 2020). }\end{array}$ \\
\hline Soil compaction & $\begin{array}{l}\text { Animal trampling increases bulk density, with larger effects at higher grazing } \\
\text { densities, particularly under wet soil conditions and fine-textured soils (Hamza and } \\
\text { Anderson, 2005). On the other hand, compaction may decrease due to less } \\
\text { machine traffic in the field. }\end{array}$ \\
\hline
\end{tabular}

\subsection{Increases in greenhouse gas emissions}

The emission of GHGs per unit of land area, particularly $\mathrm{N}_{2} \mathrm{O}$, is generally higher from grassland compared to cropland. However, emissions of $\mathrm{N}_{2} \mathrm{O}$ and $\mathrm{CH}_{4}$ in European grasslands resulted in a 19 percent offset of the net ecosystem exchange of $\mathrm{CO}_{2}$ sink activity in managed grasslands that included $\mathrm{N}$ fertilization and grazing (Soussana et al., 2007) in line with values reported by Khalil and Osborne (2018).

\subsection{Conflict with other practice(s)}

Converting arable/cropland to grassland might result in a loss of financial income for farmers in some regions. For this reason, grasslands are frequently converted to cropland, including fruit orchards and vineyards (Francaviglia et al., 2012).

\subsection{Decreases in production (e.g. food/fuel/feed/timber/fibre)}

Grassland production could be low in the beginning following conversion, until the full establishment of grasses.

\subsection{Other conflicts}

In some developing countries, more cropland could be required to sustain food production due to population growth. 


\section{Recommendations before implementing the practice}

When turning arable lands to grasslands in the first-year, legumes should be used to improve soil $\mathrm{N}$ status and sustain grassland productivity; then hay-seeding is advised or sowing with a mixture of species to foster nutritional value of forages and attain a more complex grassland composition. Depending on local soil, climatic and hydrological conditions, grazing should be applied after about 10 years to allow the vegetation recovery and grassland production of biomass, and enhancing soil microbial activity, soil fertility and SOC storage, and managed to avoid over grazing.

\section{Potential barriers for adoption}

Table 147. Potential barriers to adoption

\begin{tabular}{|l|l|l|}
\hline Barrier & YES/NO & \\
\hline Biophysical & Yes & $\begin{array}{l}\text { Low farm size is a constraint for conversion to grassland that requires } \\
\text { large areas in case the final use is livestock grazing (Kuivanen et al., } \\
\text { 2016). }\end{array}$ \\
\hline Cultural & Yes & $\begin{array}{l}\text { Farmers are not experts in grassland management, and they are } \\
\text { reluctant to changes in farming activities (European Commission, 2017). }\end{array}$ \\
\hline Social & Yes & $\begin{array}{l}\text { Age of farmers, older farmers are less prone to change land uses on } \\
\text { their fields. }\end{array}$ \\
\hline Economic & Yes & $\begin{array}{l}\text { Competition with more profitable land uses is preferred for higher } \\
\text { financial benefits (Francaviglia et al., 2012). Less land for production } \\
\text { (Freibauer et al., 2004). }\end{array}$ \\
\hline Institutional & Yes & $\begin{array}{l}\text { Inefficient government policies to support the conversion, with limited } \\
\text { transfer of knowledge, training, and promotion of adoption for farmers } \\
\text { (European Commission, 2017). }\end{array}$ \\
\hline $\begin{array}{l}\text { Legal (Right } \\
\text { to soil) }\end{array}$ & Yes & $\begin{array}{l}\text { Farmers cultivating on rented lands are unlikely willing to adopt the } \\
\text { practice (Carolan et al., 2004). }\end{array}$ \\
\hline Yes & $\begin{array}{l}\text { Managing the grassland to increase financial incomes is a requisite to } \\
\text { encourage farmers to convert cropland to grassland (Freibauer } \text { et al., } \\
\text { 2004). }\end{array}$ \\
\hline
\end{tabular}




\section{Photos of the practice}
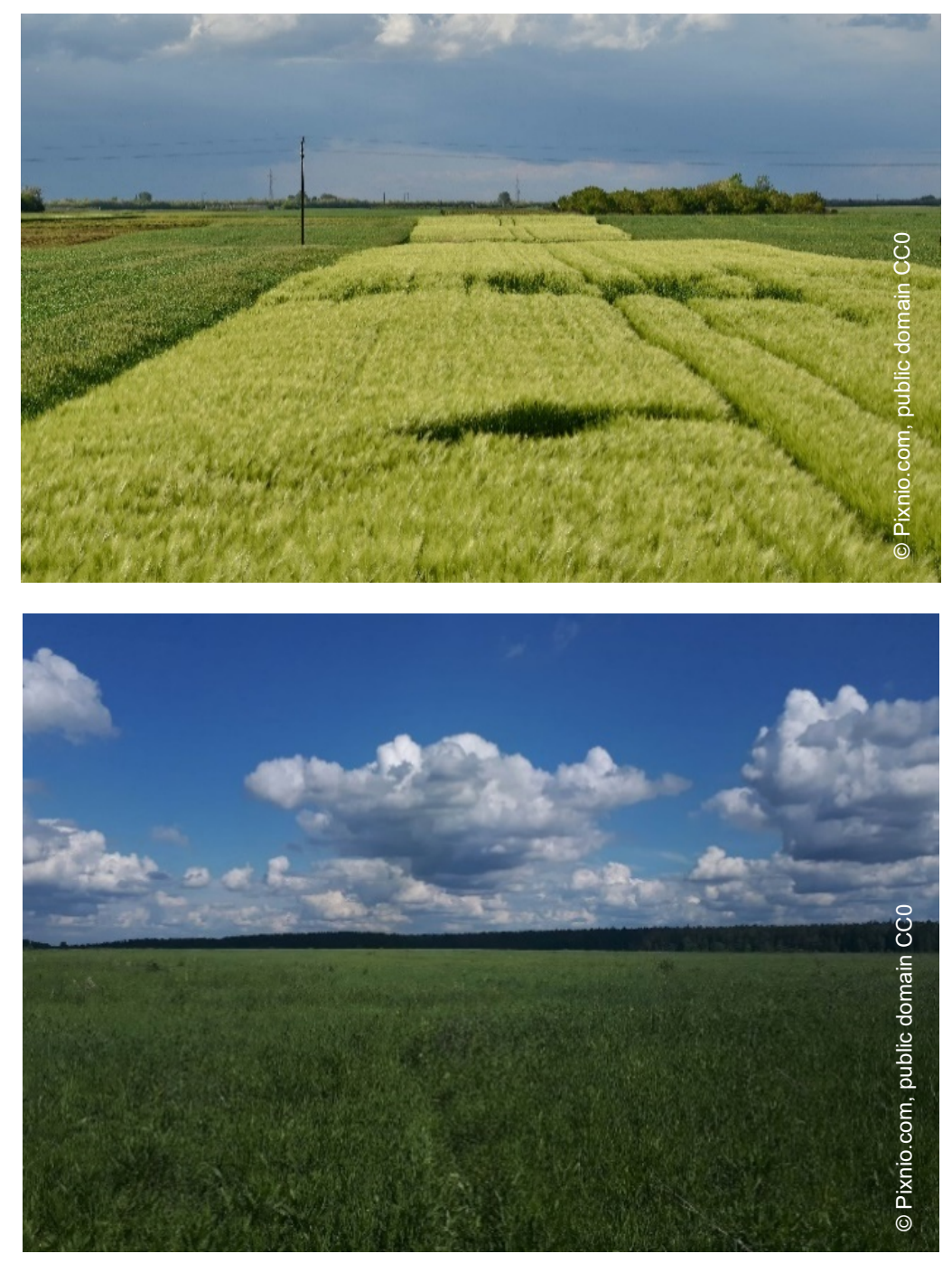

Photo 42. Conversion of cropland (top) to grassland (bottom)

Table 148. Related cases studies available in volumes 3 and 5

\begin{tabular}{|l|c|l|c|c|}
\hline Title & Region & $\begin{array}{l}\text { Duration of } \\
\text { study } \\
\text { (Years) }\end{array}$ & Volume & $\begin{array}{l}\text { Case- } \\
\text { study } \\
\text { No. }\end{array}$ \\
\hline $\begin{array}{l}\text { Avoidance of land use change (LUC) from } \\
\text { grassland to arable land, Germany }\end{array}$ & Europe & 1 to 7 & 3 & 13 \\
\hline
\end{tabular}




\section{References}

Ahlering, M., Fargione, J. \& Parton, W. 2016. Potential carbon dioxide emission reductions from avoided grassland conversion in the northern Great Plains. Ecosphere, 7(12): e01625.

https://doi.org/10.1002/ecs2.1625

Carolan, M.S., Mayerfeld, D., Bell, M.M. \& Exner, R. 2004. Rented land: Barriers to sustainable agriculture. Journal of Soil and Water Conservation, 59(4): 70A-75A.

Conant, R.T., Paustian, K. \& Elliot, E.T. 2001 . Grassland management and conversion into grassland: Effects on soil carbon. Ecological Applications, 11: 343-355. https://doi.org/10.1890/10510761(2001)011[0343:GMACIG]2.0.CO;2

De, M., Riopel, J.A., Cihacek, L.J., Lawrinenko, M., Baldwin-Kordick, R., Hall, S.J. \& McDaniel, M.D. 2020. Soil health recovery after grassland reestablishment on cropland: The effects of time and topographic position. Soil Science Society of America Journal, 84(2): 568-586. https://doi.org/10.1002/saj2.20007

Deng, L., Zhu, G.Y., Tang, Z.S. \& Shangguan, Z.P. 2016. Global patterns of the effects of land-use changes on soil carbon stocks. Global Ecology and Conservation, 5: 127-138.

https://doi.org/10.1016/j.gecco.2015.12.004

Diaz, D., Rashford, B., De Gryze, S., Zakreski, S., Dell, R. \& Niles, M.T. 2012. Evaluation of avoided grassland conversion and cropland conversion to grassland as potential carbon offset project types. The Climate Trust. Portland, Oregon. (also available at: https://climatetrust.org/wpcontent/uploads/2014/07/Evaluation-of-Avoided-Grassland-Conversion-and-Cropland-Conversion-toGrassland-as-Potential-Carbon-Offset-Project-Types-.pdf)

Eisenhauer, N., Dobies, T., Cesarz, S., Hobbie, S.E., Meyer, R.J., Worm, K. \& Reich, P.B. 2013. Plant diversity effects on soil food webs are stronger than those of elevated $\mathrm{CO}_{2}$ and $\mathrm{N}$ deposition in a long-term grassland experiment. Proceedings of the National Academy of Sciences, 110(17): 6889-6894.

https://doi.org/10.1073/pnas.1217382110

European Commission 2017. Modernising and simplifying the CAP. Background document. SocioEconomic challenges facing EU agriculture and rural areas. (also available at:

https://ec.europa.eu/info/sites/info/files/food-farming-

fisheries/key_policies/documents/soc_background_final_en.pdf)

Feliciano, D., Hunter, C., Slee, B. \& Smith, P. 2013. Selecting land-based mitigation practices to reduce GHG emissions from the rural land use sector: A case study of North East Scotland. Journal of environmental management, 120: 93-104. https://doi.org/10.1016/j.jenvman.2013.02.010

Francaviglia, R., Coleman, K., Whitmore, A.P., Doro, L., Urracci, G., Rubino, M. \& Ledda, L. 2012. Changes in soil organic carbon and climate change-Application of the RothC model in agro-silvo-pastoral Mediterranean systems. Agricultural Systems, 112: 48-54. https://doi.org/10.1016/j.agsy.2012.07.001

Freibauer, A., Rounsevell, M.D., Smith, P. \& Verhagen, J. 2004. Carbon sequestration in the agricultural soils of Europe. Geoderma, 122: 1-23. https://doi.org/10.1016/j.geoderma.2004.01.021 
Gosling, P., van der Gast, C. \& Bending, G.D. 2017. Converting highly productive arable cropland in Europe to grassland: -a poor candidate for carbon sequestration. Scientific Reports, 7(1).

https://doi.org/10.1038/s41598-017-11083-6

Hamza, M.A. \& Anderson, W.K. 2005. Soil compaction in cropping systems: A review of the nature, causes and possible solutions. Soil and tillage research, 82: 121-145.

https://doi.org/10.1016/j.still.2004.08.009

Jones, M.B. 2010. Potential for carbon sequestration in temperate grassland soils. In Abberton, M., Conant, R. \& Batello, C. (Eds.) Grassland carbon sequestration: management, policy and economics. Proceedings of the Workshop on the role of grassland carbon sequestration in the mitigation of climate change. Food and Agriculture Organization of the United Nations, Rome, 15-17 April 2009. (also available at: http://www.fao.org/fileadmin/templates/agphome/scpi/cgwg/Jones.pdf)

Kämpf, I., Hölzel, N., Störrle, M., Broll, G. \& Kiehl, K. 2016. Potential of temperate agricultural soils for carbon sequestration: A meta-analysis of land-use effects. Science of The Total Environment, 566-567: 428435. https://doi.org/10.1016/j.scitotenv.2016.05.067

Khalil, M.I. \& Osborne, B. 2018. Improving estimates of soil organic carbon (SOC) stocks and their longterm temporal changes in agricultural soils in Ireland. Geoderma, 322: 172-183.

https://doi.org/10.1016/j.geoderma.2018.02.038

Kuivanen, K.S., Alvarez, S., Michalscheck, M., Adjei-Nsiah, S., Descheemaeker, K., Mellon-Bedi, S. \& Groot, J.C. 2016. Characterising the diversity of smallholder farming systems and their constraints and opportunities for innovation: A case study from the Northern Region, Ghana. NJAS-Wageningen Journal of Life Sciences, 78: 153-166. https://doi.org/10.1016/j.njas.2016.04.003

Lozano-García, B., Muñoz-Rojas, M. \& Parras-Alcantara, L. 2017. Climate and land use changes effects on soil organic carbon stocks in a Mediterranean semi-natural area. Science of the Total Environment, 579: 1249-1259. https://doi.org/10.1016/j.scitotenv.2016.11.111

McLauchlan, K.K., Hobbie, S.E. \& Post, W.M. 2006. Conversion from agriculture to grassland builds soil organic matter on decadal timescales. Ecological Applications, 16: 143-153. https://doi.org/10.1890/041650

Miao, S., Qiao, Y. \& Zhang, F. 2015. Conversion of Cropland to Grassland and Forest Mitigates Global Warming Potential in Northeast China. Polish Journal of Environmental Studies, 24(3): 1195-1203. https://doi.org/10.15244/pjoes/33928

Poeplau, C., Don, A., Vesterdal, L., Leifeld, J., Van Wesemael, B. A.S., Schumacher, J. \& Gensior, A. 2011. Temporal dynamics of soil organic carbon after land-use change in the temperate zone-carbon response functions as a model approach. Global Change Biology, 17(7): 2415-2427.

https://doi.org/10.1111/j.1365-2486.2011.02408.x

Post, W.M. \& Kwon, K.C. 2000. Soil carbon sequestration and land-use change: process and potential. Global Change Biology, 6: 317-328. https://doi.org/10.1046/j.1365-2486.2000.00308.x 
Qiu, G.Y., Xie, F., Feng, Y.C. \& Tian, F. 2011. Experimental studies on the effects of the "Conversion of Cropland to Grassland Program" on the water budget and evapotranspiration in a semi-arid steppe in Inner Mongolia, China. Journal of Hydrology, 411(1-2): 120-129.

https://doi.org/10.1016/j.jhydrol.2011.09.040

Sanderman, J., Farquharson, R. \& Baldock, J. 2010. Soil carbon sequestration potential: A review for Australian agriculture. CSIRO, Canberra, Australia. 81 pp. (also available at: https://publications.csiro.au/rpr/download?pid=ecsiro:EP10121\&dsid=DS8)

Schroder, J.L., Zhang, H., Girma, K., Raun, W.R., Penn, C.J. \& Payton, M.E. 2011. Soil acidification from long-term use of nitrogen fertilizers on winter wheat. Soil Science Society of America Journal, 75(3): 957-964. https://doi.org/10.2136/sssaj2010.0187

Smith, P., Bhogal, A., Edgington, P., Black, H., Lilly, A., Barraclough, D., Worral, F., Hillier, J. \& Merrington, G. 2010. Consequences of feasible future agricultural land-use change on soil organic carbon stocks and greenhouse gas emissions in Great Britain. Soil use and management, 26(4): 381-398. https://doi.org/10.1111/j.1475-2743.2010.00283.x

Soussana, J.F., Allard, V., Pilegaard, K., Ambus, P., Amman, C., Campbell, C., Ceschia, E., CliftonBrown, J., Czobel, S., Domingues, R., Flechard, C., Fuhrer, J., Hensen, A., Horvath, L., Jones, M., Kasper, G., Martin, C., Nagy, Z., Neftel, A., Raschi, A., Baronti, S., Rees, R.M., Skiba, U., Stefani, P., Manca, G., Sutton, M., Tuba, Z. \& Valentini, R. 2007. Full accounting of the greenhouse gas $\left(\mathrm{CO}_{2}, \mathrm{~N}_{2} \mathrm{O}\right.$, $\mathrm{CH}_{4}$ ) budget of nine European grassland sites. Agriculture, Ecosystems \& Environment, 121: 121-134.

https://doi.org/10.1016/j.agee.2006.12.022

Vanderburg, K.L., Steffens, T.J., Lust, D.G., Rhoades, M.B., Blaser, B.C., Peters, K. \& Ham, M.J. 2020. Trampling and Cover Effects on Soil Compaction and Seedling Establishment in Reseeded Pasturelands Over Time. Rangeland Ecology \& Management, 73: 452-461.

https://doi.org/10.1016/j.rama.2020.01.001

Vleeshouwers, L.M. \& Verhagen, A. 2002. Carbon emission and sequestration by agricultural land use: a model study for Europe. Global Change Biology, 8: 519-530. https://doi.org/10.1046/j.1365-

2486.2002.00485.x

Yu, P., Liu, S., Han, K., Guan, S. \& Zhou, D. 2017. Conversion of cropland to forage land and grassland increases soil labile carbon and enzyme activities in northeastern China. Agriculture, Ecosystems \& Environment, 245: 83-91. https://doi.org/10.1016/j.agee.2017.05.013

Yu, P., Liu, S., Yang, H., Fan, G. \& Zhou, D. 2018. Short-term land use conversions influence the profile distribution of soil salinity and sodicity in northeastern China. Ecological Indicators, 88: 79-87.

https://doi.org/10.1016/j.ecolind.2018.01.017

Zhang, Y., Guo, S., Liu, Q., Jiang, J., Wang, R. \& Li, N. 2015. Responses of soil respiration to land use conversions in degraded ecosystem of the semi-arid Loess Plateau. Ecological engineering, 74: 196-205. https://doi.org/10.1016/j.ecoleng.2014.10.003 


\title{
34. Improved pasture management
}

\author{
Mohammad I. Khalil1', Cláudia M.d.S. Cordovil², Rosa Francaviglia ${ }^{3}$, \\ Beverley Henry ${ }^{4}$, Katja Klumpp ${ }^{5}$, Peter Koncz ${ }^{6}$, Mireia Llorente ${ }^{7}$, \\ Beata E. Madari ${ }^{8}$, Muñoz-Rojas Miriam ${ }^{9,10}$, Nerger Rainer ${ }^{11}$ \\ (Co-authors in alphabetical order)
}

'School of Applied Sciences \& Technology, Prudence College Dublin, Dublin 22 and School of Biology \& Environmental Science, University College Dublin, Dublin 4, Ireland

${ }^{2}$ University of Lisbon, School of Agriculture, Forest Research Center, Lisboa, Portugal

${ }^{3}$ Council for Agricultural Research and Economics, Research Centre for Agriculture and Environment (CREA-AA), Rome, Italy.

${ }^{4}$ Science and Engineering Faculty, Queensland University of Technology, Brisbane, Australia

${ }^{5}$ Grassland Ecosystem Research, INRA, Clermont-Ferrand, France

${ }^{6}$ Duna-Ipoly National Park Directorate, Budapest, Hungary and MTA-SZIE Plant Ecology Research Group,

Gödöllő, Hungary

${ }^{7}$ Forest Department. University of Extremadura, Plasencia Campus. Spain

${ }^{8}$ Embrapa Rice and Beans, Santo Antônio de Goiás, GO, Brazil

${ }^{9}$ UNSW Sydney, School of Biological, Earth and Environmental Sciences, Sydney NSW, Australia

${ }^{10}$ The University of Western Australia, School of Biological Sciences, Crawley, WA, Australia

${ }^{11}$ Soil \& More Impacts GmbH, German office, Hamburg, Germany

\section{Description of the practice}

Grazing systems and related pasture management have different forms, ranging from simple to complex systems and involving single species swards to multispecies swards that occur across a range of soil types and climatic conditions. Grazing animals mostly refer to grazing herbivores, both domestic and wild, that feed mainly or only on vegetation. Pastures refer to areas fenced or with other barriers that are devoted to the production of forage 
primarily for grazing. Optimisation of soil $\mathrm{C}$ by grazing management is mainly associated with biomass production, involving grass regrowth intervals, non-grazing season management and sward persistency. These changes in biomass production are achieved by stocking methods that define how, when, what and how much animals graze (Allen et al., 2011). Grazing strategies aim to allocate nutrition uptake among varying classes of livestock (i.e. creep grazing), improve efficiency of forage use (i.e. frontal grazing, mixed grazing), reduce negative effects on soils or plants (rotational grazing, deferred grazing), and extend seasons (i.e. sequence grazing). Accordingly, grazing (land) management refers to the manipulation of the soil-plant-animal complex in pursuit of a desired result. Grazing management may be extensive, meaning that a relatively large areas per animal is used at a relatively low level of labour or intensive, which is defined by relative increase of stocking rates, grazing pressure and forage. Importantly, rotational grazing is defined by repeated periods of grazing and rest among a number of paddocks throughout the time when grazing is allowed ${ }^{20}$, and contrasts with continuous grazing where animals have unrestricted and uninterrupted access throughout the time grazing is allowed (Allen et al., 2011). Irrespective of grazing management, defoliation affects photosynthesis and subsequent $\mathrm{C}$ allocation to root and shoot (Chen et al., 2015, Zhou et al., 2017), but also $\mathrm{C}$ and $\mathrm{N}$ returns to the pasture (25 to 40 percent of the intake, depending on the digestibility of diet), as well as the nature of remainders (e.g. litter, and ungrazed leaves and roots). So, timing and duration of grazing events, as well as their frequency and intensity play an important role on $\mathrm{C}$ sequestration through increase in biomass production by replacing aging or dead plant tissues with active photosynthetic younger plant tissues, and by recycling of $\mathrm{N}$ though animal ingestion and urine distribution (Tälle et al., 2016). Accordingly, adapted good management practices (grazing strategies) may significantly influence soil function (Teague et al., 2013; Hennessy et al., 2018) and thereby C storage.

\section{Range of applicability}

Grasslands occupy up to half the earth's terrestrial surface (3.4 billion ha; FAO, 2015) and are often marginally productive compared to intensively managed agricultural areas. About 60 percent of the world's agricultural land is covered by grazing systems. Distributed between arid, semi-arid and sub-humid, humid rainforest, and temperate and tropical highlands, grazing systems support about 360 million cattle and over 600 million sheep and goats. With regard to climate zones, grazing systems range from areas with verdant pastures in north western Europe or New Zealand, to humid areas, with ranch encroachment and deforestation of tropical forest (i.e. replacement of palatable species and by less palatable, herbaceous plants or bushes), as well as arid zones with extent of land degradation (e.g. moderately or severely degraded). In arid ecosystems, the periodicity of rain becomes the single most important factor affecting the quantity of feed available and excessive, prolonged grazing can leads to the disappearance of palatable species and degradation. In other areas (e.g. Kenya, western United States and Guinea) livestock improve soil and vegetation cover through biomass removal and dejections (i.e. nutrient recycling) while interacting with land, water, plant, and animal biodiversity. The way pasture systems are managed explains, to a large extent, their resilience. In arid rangelands, livestock is often moved in search of pasture according to season (e.g. after the wet season grazing animals are moved to "higher-potential" areas (e.g. valleys, mountain meadows). This continuous dis-equilibrium may conserve soil and vegetation in arid areas.

${ }^{20}$ Also see Factsheet No. 35 “Grazing exclusion and rotational grazing” 
Grazing practices, which vary in nature, frequency, and intensity of biomass removals (Allen et al., 2011), affect soil structure and soil functions (Cui et al., 2005) and thus, C cycling and C balance of grasslands. The environmental challenge is thus to identify pasture management which will maintain the positive and ease the negative effects of grazing.

\section{Impact on soil organic carbon stocks}

Grazing strategies have received increasing national and global interest as potential "climate-smart" pathways for sequestering $\mathrm{C}$ and improving soil health (e.g. Derner, Stanley and Chad, 2016). Grazing of grasslands can act either as potential sinks or source of $\mathrm{C}$, ranging from -1.3 to more than $1 \mathrm{tC} / \mathrm{ha} / \mathrm{yr}$ storing on average 0.26 $\pm 0.07 \mathrm{tC} / \mathrm{ha} / \mathrm{yr}$ (mean of 11 literature references e.g. Conant et al, 2017 , Sandermann et al., 2015, Abdalla

et al., 2017, Franzluebbers and Stuedemann 2009; Table 149). Carbon sequestration potential depends on climate, soil characteristics, vegetation (i.e. species composition, presence/absence of $\mathrm{C}_{3}$ or $\mathrm{C}_{4}$ grasses, etc.) and intensity of biomass removal, as well as animal stocking densities and the ingested amount of biomass produced. A comparison between grazing and mowing regimes shows that under comparable biomass exports grazing systems tend to sequester more C, in particular when moderately fertilised (Liu et al., 2014), and in biodiverse pastures (Teixeira et al., 2011).

Grazing intensity seems to increase SOC stocks under a medium warm climate $(+7.6$ percent), but decreases $\mathrm{C}$ sequestration potential under moist cool climate (-19.5 percent, Abdalla et al, 2017$)$. For dry wet and dry cool climates, grazing intensity may lead to C increase in soils when combined with low to medium grazing intensities (e.g. Byrnes et al., 2018, Conant and Paustian, 2002). Overall, it appears that optimal use (biomass removed to biomass produced ratio) of grasslands has the potential to significantly increase $\mathrm{C}$ sequestration while reducing $\mathrm{N}$ losses. Several studies showed a low to moderate biomass removal (30 percent to 70 percent of biomass produced), indicating a potential to sequester 0.2 to $0.5 \mathrm{tC} / \mathrm{ha} / \mathrm{yr}$, whereas biomass removals of above 80 percent led to either no or some $\mathrm{C}$ losses. In light to moderate grazing systems, less biomass intake and lengthy growth period and reduced animal disturbance can promote photosynthetic activity (i.e. plant growth and increase pasture production, Hennessy et al., 2018), nutrient cycling through animal ingestion and distribution of urine (Chen et al., 2015), resulting in increased soil C storage (Zhou et al., 2017). Meta-analyses suggest that rotational grazing strategies (e.g. high-intensity, short-duration grazing) can improve SOC and bulk density over continuous grazing (Byrnes et al, 2018). Grazing systems tend to sequester more C, particularly when fertilised (e.g. Franzluebbers and Stuedemann, 2009), than mowing regimes linked to the biomass removal differences. 
Table 149. Evolution of SOC stocks with improved pasture management at $0-30 \mathrm{~cm}$ depth

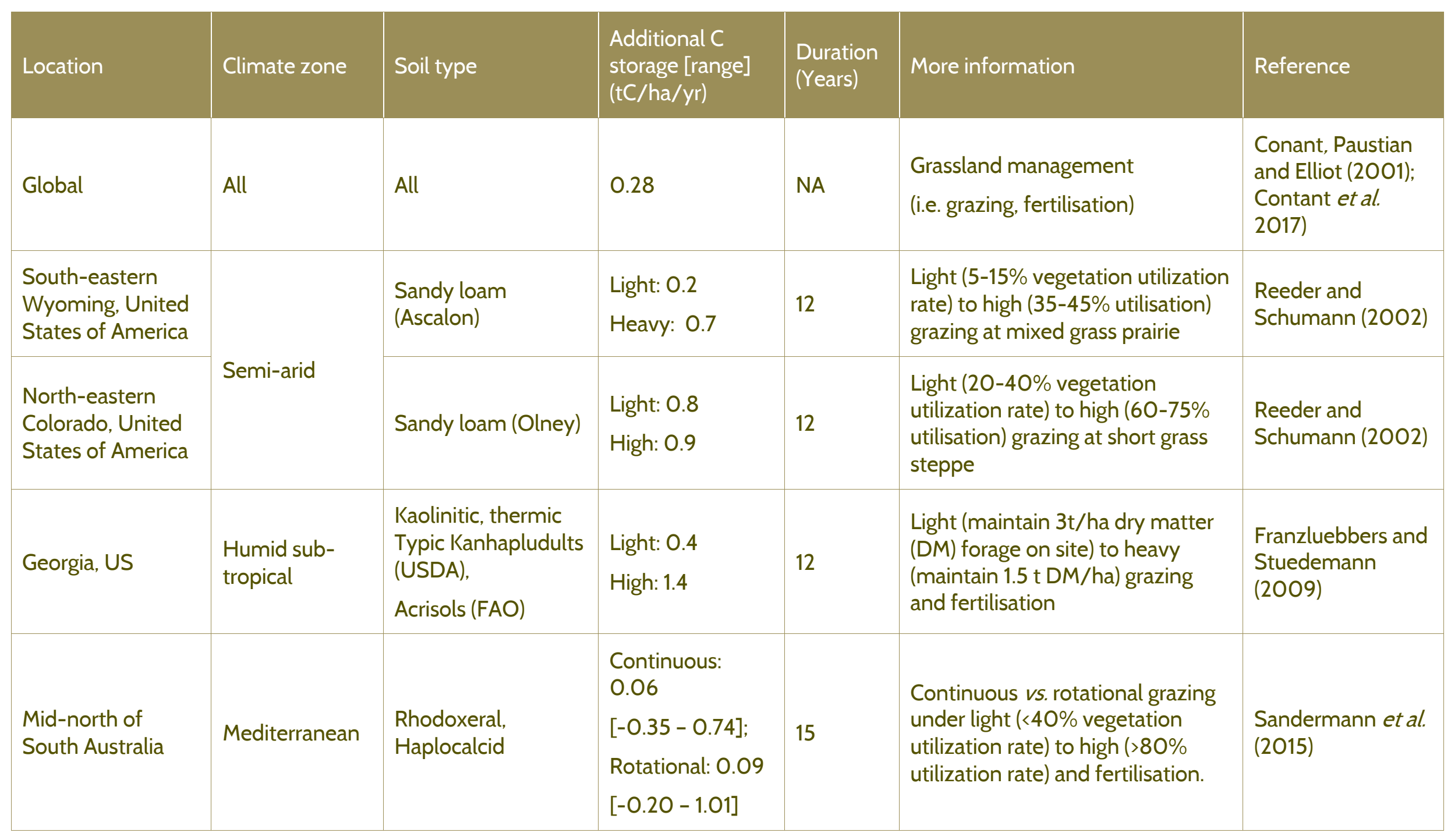




\begin{tabular}{|c|c|c|c|c|c|c|}
\hline Location & Climate zone & Soil type & $\begin{array}{l}\text { Additional C } \\
\text { storage [range] } \\
\text { (tC/ha/yr) }\end{array}$ & $\begin{array}{l}\text { Duration } \\
\text { (Years) }\end{array}$ & More information & Reference \\
\hline $\begin{array}{l}\text { UK, Northern } \\
\text { Ireland }\end{array}$ & \multirow{3}{*}{ Tempe-rate } & $\begin{array}{l}\text { Brown clay-loam } \\
\text { and gley }\end{array}$ & $\begin{array}{l}\text { Light: }-0.90 \\
\text { [-4.6 - 2.3]; } \\
\text { Moderate: } 0.88 \\
\text { [-1.01 - 3.55]; } \\
\text { High: } 0.58 \\
\text { [-3.85 - 3.24] }\end{array}$ & $>20$ & $\begin{array}{l}\text { Light (0.2 LU/ha) to high ( } 2 \\
\text { LU/ha) livestock density, } \\
\text { fertilisation, with different time } \\
\text { span since reseeding events. }\end{array}$ & $\begin{array}{l}\text { Carolan and } \\
\text { Fornara (2016) }\end{array}$ \\
\hline $\begin{array}{l}\text { Temperate steppe, } \\
\text { Guyuan County, } \\
\text { China }\end{array}$ & & Loamy sand & $\begin{array}{l}\text { Light: } 0.37 \\
\text { Moderate: } 0.62 \\
\text { High: } 0.12\end{array}$ & - & $\begin{array}{l}\text { Light ( } 30 \% \text { vegetation utilization } \\
\text { rate) to high ( } 64 \% \text { utilization rate) } \\
\text { by sheep grazing and fertilisation }\end{array}$ & Chen et al. (2015) \\
\hline $\begin{array}{l}\text { Eastern Cape, } \\
\text { South Africa }\end{array}$ & & $\begin{array}{l}\text { Sandy loam } \\
\text { (Aridosol) }\end{array}$ & $\begin{array}{l}\text { Light: } 0.093 \\
\text { High: } 0.097\end{array}$ & 75 & $\begin{array}{l}\text { Light ( } 0.8 \text { sheep/ha) to high } 1.2 \\
\text { sheep/ha) grazing by sheep }\end{array}$ & Talore et al. (2015) \\
\hline $\begin{array}{l}\text { East Africa } \\
\text { (Burundi, Ethiopia, } \\
\text { Kenya, Rwanda, } \\
\text { United Republic of } \\
\text { Tanzania and } \\
\text { Uganda) }\end{array}$ & Various & & Mean $1.07 \pm 0.26$ & NA & $\begin{array}{l}\text { Review of different practices } \\
\text { (enclosure, fencing, light and } \\
\text { heavy grazing) }\end{array}$ & $\begin{array}{l}\text { Tessema et al. } \\
(2020)\end{array}$ \\
\hline Portugal & Mediterranean & Various & $0.71-1.91$ & 42 & $\begin{array}{l}\text { Agrosilvopastoral } \\
\text { systems/biodiverse pastures }\end{array}$ & $\begin{array}{l}\text { Teixeira et al. } \\
\text { (2011) ; Cordovil et } \\
\text { al. }(2020)\end{array}$ \\
\hline
\end{tabular}

LU = Livestock Unit 


\section{Other benefits of the practice}

\subsection{Improvement of soil properties}

Effects of grazing on soil properties are driven by plant tissue removal including defoliation, excretion (urine and dung deposits), but also by trampling. These exert mechanical pressure on soil pore space (Oenema et al., 1997, van Klink et al., 2015), and cause physical damage to the vegetation due to repeated passes of animals (Tate et al., 2004). It implies that less intense management is thus a way to avoid soil degradation, in particular SOC depletion, and thereby attaining sustainable production. For instance, light to moderate grazing has been shown to significantly increase soil $\mathrm{C}$ and improve soil structure compared to heavy grazing (e.g. Reeder and Schuman 2002, Zhou et al., 2017, Abdalla et al., 2017, Byrnes et al., 2018).

\subsection{Minimization of threats to soil functions}

Table 150. Soil threats

\begin{tabular}{|c|c|}
\hline Soil threats & \\
\hline Soil erosion & $\begin{array}{l}\text { Depends on livestock density and frequency of use; carrying capacity } \\
\text { should be respected to avoid degradation (i.e. low, moderate, and avoid } \\
\text { high). }\end{array}$ \\
\hline $\begin{array}{l}\text { Nutrient imbalance } \\
\text { and cycles }\end{array}$ & $\begin{array}{l}\text { Grazing improves nutrient use and (re-) cycling under low and moderate } \\
\text { livestock density and frequency of use, high animal density may lead to } \\
\text { large nutrient inputs and decouple NPK Cycle (Rumpel et al., 2014). }\end{array}$ \\
\hline Soil biodiversity loss & $\begin{array}{l}\text { Grazing fosters both above- and below-ground species diversity under } \\
\text { moderate and low livestock density (Tälle et al., 2016). }\end{array}$ \\
\hline Soil compaction & $\begin{array}{l}\text { Grazing animals lead to soil compaction under high livestock densities, } \\
\text { whereas adapting to the carrying capacity prevents soil compaction (van } \\
\text { Klink et al., 2015). }\end{array}$ \\
\hline $\begin{array}{l}\text { Soil water } \\
\text { management }\end{array}$ & $\begin{array}{l}\text { High livestock densities and frequent grassland use decline water quality } \\
\text { due to excess in animal dejections. Adapting pasture management to the } \\
\text { carrying capacity prevents excess (Vertes et al., 2012). }\end{array}$ \\
\hline
\end{tabular}




\subsection{Increases in production (e.g. food/fuel/feed/timber/fibre)}

At low grazing intensities, animals may favour $\mathrm{N}$ recycling through ingestion and re-distribution of $\mathrm{N}$ via dejections (Soussana and Lemaire, 2014; Rumple et al., 2015). Both promote net primary productivity of vegetation and thus increase litter production (Chen et al., 2015; Zhou et al., 2017). Too intense and frequent grazing decreases the number of living plants and produces less litter and that reduce $\mathrm{C}$ sequestration. In between these two extreme situations, several grazing systems may promote not only soil $\mathrm{C}$ but also improve grassland quality. For example, managed grasslands with high plant diversity enhance SOC at least in low to moderate input/output grasslands (Sebastià et al., 2018). Light to moderate grazing enhanced SOC by increasing plant productivity, and recycling of $\mathrm{N}$ (e.g. Chen et al 2015). There is evidence that strategic management of grazing can positively affect production and might even reverse negative impacts of poorly managed grasslands through the enhancement of $\mathrm{N}$ cycling. For instance, under medium to high grazing pressure, fast-growing palatable species typical of nutrient-rich, managed grasslands show a high above-ground productivity and quality (lower $\mathrm{C} / \mathrm{N}$ ) promoting higher $\mathrm{C}$ inputs to soil and a rapid degradation by bacteria (Cotrufo et al., 2013). Accordingly, grazing has the capacity to change vegetation by modifying plant community composition (presence of legumes in particular) (Zhou et al., 2017) which play a key role in supplying aerial and root plant biomass into soil systems.

\subsection{Mitigation of and adaptation to climate change}

There are improved techniques to reduce livestock GHG emissions while increasing livestock productivity and resilience. Strategic grazing that sequesters C could contribute to trade-off/offsetting of GHGs emitted from the grassland systems. There is controversy on grass-fed ruminants that could mitigate livestock and agricultural GHG production. However, a reduction of emissions from manure storage (barn) and manure spreading, as well as the reduction in fertiliser use (i.e. urine and promotion of biological $\mathrm{N}$ fixing plants) was reported (Hirstov et al. 2013). Strategic feeding (e.g. use of inhibitors, seaweed and balanced nutrition) could reduce enteric $\mathrm{CH}_{4}$ and $\mathrm{NH}_{3}$, in particular, substantially.

\subsection{Socio-economic benefits}

In many parts of the world, grazing grasslands under low to moderate livestock density are found to be sustainable in areas that are rich in flora and fauna. Ruminants fed by these grasslands are likely to produce tastier meat that is more nutritious and healthy feeds, which may be applied in organic farming and other quality farms (for example, PDO: protected designation of origin or PGI: protected geographical indication). In addition, diverse grassland systems grazed by ruminants often receive recognition as cultural landscapes to attract tourists and beautify areas. 


\subsection{Other benefits}

Good grazing practices are a win-win situation since these allow a better coupling of $\mathrm{C}$ and $\mathrm{N}$ cycles within vegetation, soil organic matter and soil microbial biomass (Lemaire et al., 2015), that favours plant growth, soil health and biodiversity. Environment-friendly grazing constitutes a compromise among biomass production, C sequestration and emissions (Soussana and Lemaire, 2014) and that may enhance product quality and revenues similar to increasing fertilization and cutting frequency (Schaub et al., 2020).

\section{Potential drawbacks to the practice}

\subsection{Tradeoffs with other threats to soil functions}

Grazing affects grasslands via plant tissue removal (defoliation), excretion (urine and dung deposits), but also by trampling, which exerts mechanical pressure on soil pore space (Oenema et al., 1997; Houlbrooke et al., 2009), and causes physical damage to the vegetation where animals pass repeatedly (Tate et al, 2004). There are "bad effects" (risk of erosion, leaching) of grassland management due to high animal density in combination with unfavourable climate conditions (i.e. dry and wet, respectively) and exposure (i.e. hilly lands). Accordingly, there are trade-offs between production (biomass and livestock) and environmental services such as $\mathrm{C}$ sequestration, soil health and water quality (Soussana and Lemaire, 2014).

Table 151. Soil threats

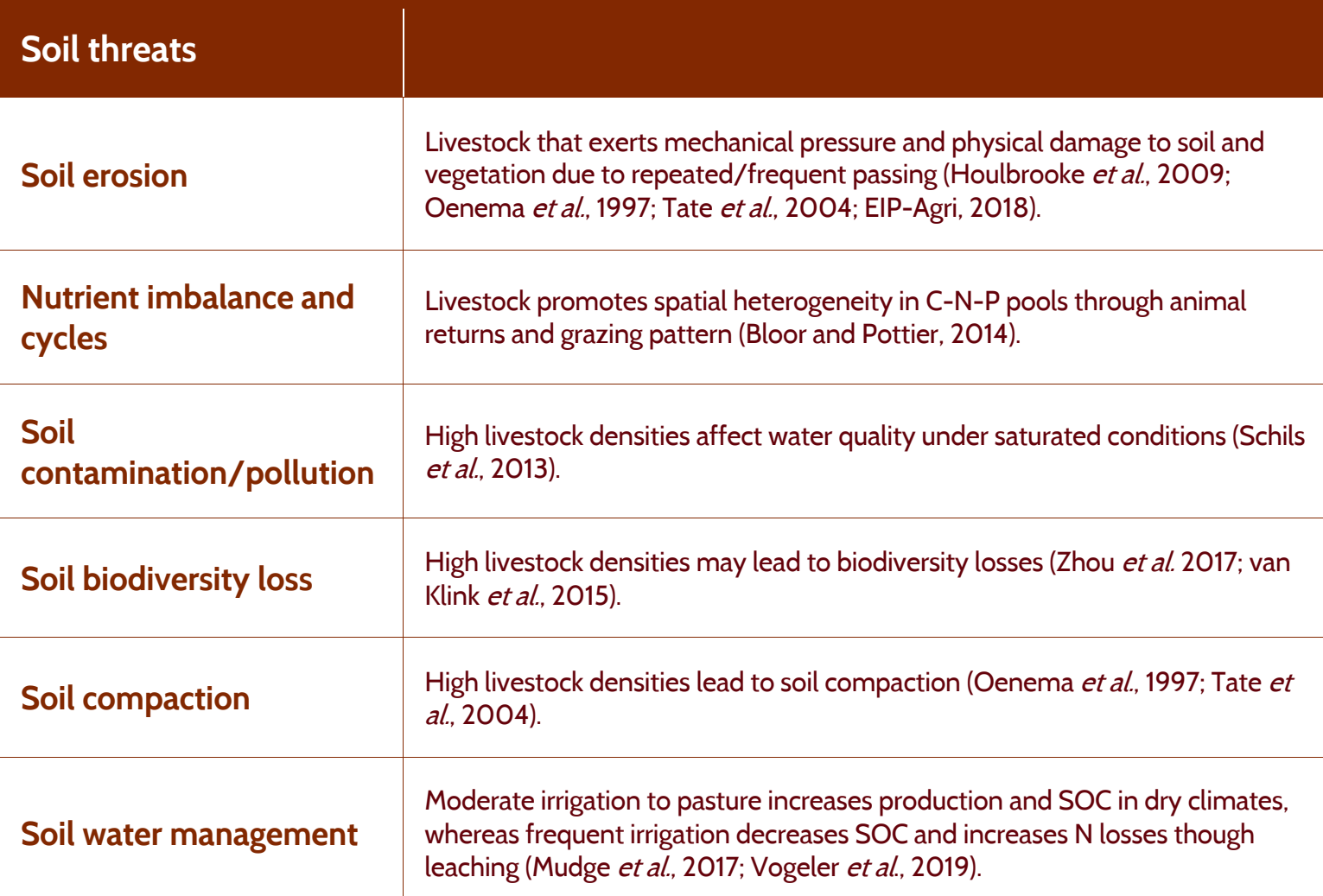




\subsection{Increases in greenhouse gas emissions}

There is evidence that grazing management strategies can affect $\mathrm{N}$ cycling, where intense livestock grazing and excreta inputs may lead to an increase in $\mathrm{N}_{2} \mathrm{O}$ emissions and $\mathrm{N}$ losses from urine hotspots, which may become greater when grazed under saturated soil water conditions (Schils et al., 2013). Rotational grazing events can cause the same but for a short term under high stock densities (e.g. more than 80 percent of biomass is removed by grazing). Likewise, animal diet either grass-fed and/or mixed (barn and occasional intense grazing) could lead to a decoupling of N, P and C cycles in grassland systems (see Rumple et al., 2015). Similarly, grazing management has the capacity to modify enteric fermentation via changes in vegetation, plant community composition, presence of legumes, leaf-to-stem ratio, and thus forage quality. For example, forage digestibility declines with an increase of stem in biomass (i.e. reduction in leaf-to-stem ratio) and across growth stages (vegetative, bud, flower); poor forage quality increases enteric $\mathrm{CH}_{4}$ emissions.

\subsection{Conflict with other practice(s)}

Pasture management including fertilisation has to take into consideration a compromise between biomass production to promote animal production and increasing $\mathrm{C}$ sequestration (Soussana and Lemaire, 2014) (i.e. intensity of biomass export, and $\mathrm{C}$ inputs to soil via litter and roots). Besides, promotion of animal production may lead to possible conflicts with (i) a life span extension of temporary grasslands in order to ensure forage quantity and quality, (ii) the introduction of annual legumes (e.g. clover and alfalfa) into temporary grasslands, leading to less complex grassland composition and (iii) the reduction of intensive systems to improve farm management towards a sustainable use of permanent and upland grassland areas.

\section{Recommendations before implementing the practice}

Carbon sequestration via grazing management needs to consider sustainable practices to preserve and improve present soil quality. Appropriate timing and duration of grazing could help achieve the goals for example by (i) identifying ideal period of rotation that allows the grassland to regrow and renew following defoliation and (ii) preventing grasslands from overgrazing that cause deterioration of pasture structure. Grazing events can be adjusted with the leaf stage of the perennial grass. The optimal time to graze perennial ryegrass pastures is between the 2- and 3-leaf stages because grazing before the 2-leaf stage reduces pasture growth and $\mathrm{C}$ inputs to soil. Both C storage and forage use reach an optimum beyond which C storage decreases (a threshold of 0.5 to 0.7 for the ratio between biomass produced and biomass removed by grazing was reported (Klumpp and Graux, 2020). As for livestock, information on the nutritive value of forage across phenological stages help to select suitable grazing times and stocking rates, in order to achieve optimum animal performance without damaging vegetation, reducing $\mathrm{C}$ sequestration potential, and increasing soil $\mathrm{N}_{2} \mathrm{O}$ and enteric $\mathrm{CH}_{4}$ emissions (Hennessy et al., 2018), 


\section{Potential barriers for adoption}

Increasing demand for livestock products often results in competition for natural resources, and between food and feed, leading to a carbon-constrained economy, poor human health and a change in socio-cultural values. In general, intensification of livestock farming is accompanied by decreasing use of open range feeding and local resources, and increasing use of concentrated feeds, mainly feed grains. Therefore, it is necessary to consider the total agricultural systems. There are potential risks for ecosystem services and maintaining diverse grazing grasslands due to the numerous episodes of land degradation associated with drought and overgrazing. Other possible barriers that limit the adoption of the beneficial pasture management practices for example are natural degradation; scarce socio-economic evaluation; lack of training, skills, advisory services, supporting tools, inputs; and psychological reluctance in some instances.

Table 152. Potential barriers to adoption

\begin{tabular}{|c|c|c|}
\hline Barrier & YES/NO & \\
\hline Biophysical & Yes & $\begin{array}{l}\text { Limits to sustainably manage grasslands due to competition } \\
\text { between food and feed. }\end{array}$ \\
\hline Cultural & Yes & $\begin{array}{l}\text { Psychological and structural reluctance with regard to changes in } \\
\text { practices, due to difficulties in separating local behaviour (e.g. } \\
\text { farmer traditions) from practices. }\end{array}$ \\
\hline Social & Yes & $\begin{array}{l}\text { Social and cultural barriers are often related. Inherent identities } \\
\text { which include, sense of identity, occupation, control, and status } \\
\text { in the community, as well as social and cultural capital, influence } \\
\text { on decisions to adopt climate-friendly practices (Gruère and } \\
\text { Wreford, 2017). }\end{array}$ \\
\hline Economic & Yes & $\begin{array}{l}\text { There is reluctance to competition in food, feed and markets } \\
\text { due to missing socio-economic evaluation, markets and labels. }\end{array}$ \\
\hline Institutional & Yes & $\begin{array}{l}\text { Lack of funding or programmes and networks to support skill } \\
\text { development of ranchers. }\end{array}$ \\
\hline $\begin{array}{l}\text { Legal (Right } \\
\text { to soil) }\end{array}$ & Yes & $\begin{array}{l}\text { Paddocks may be far away from each other to apply sustainable } \\
\text { grazing management. }\end{array}$ \\
\hline Knowledge & Yes & Lack of training, skills, advisory services, supporting tools. \\
\hline Other & Yes & Natural degradation \\
\hline
\end{tabular}




\section{Photos of the practice}
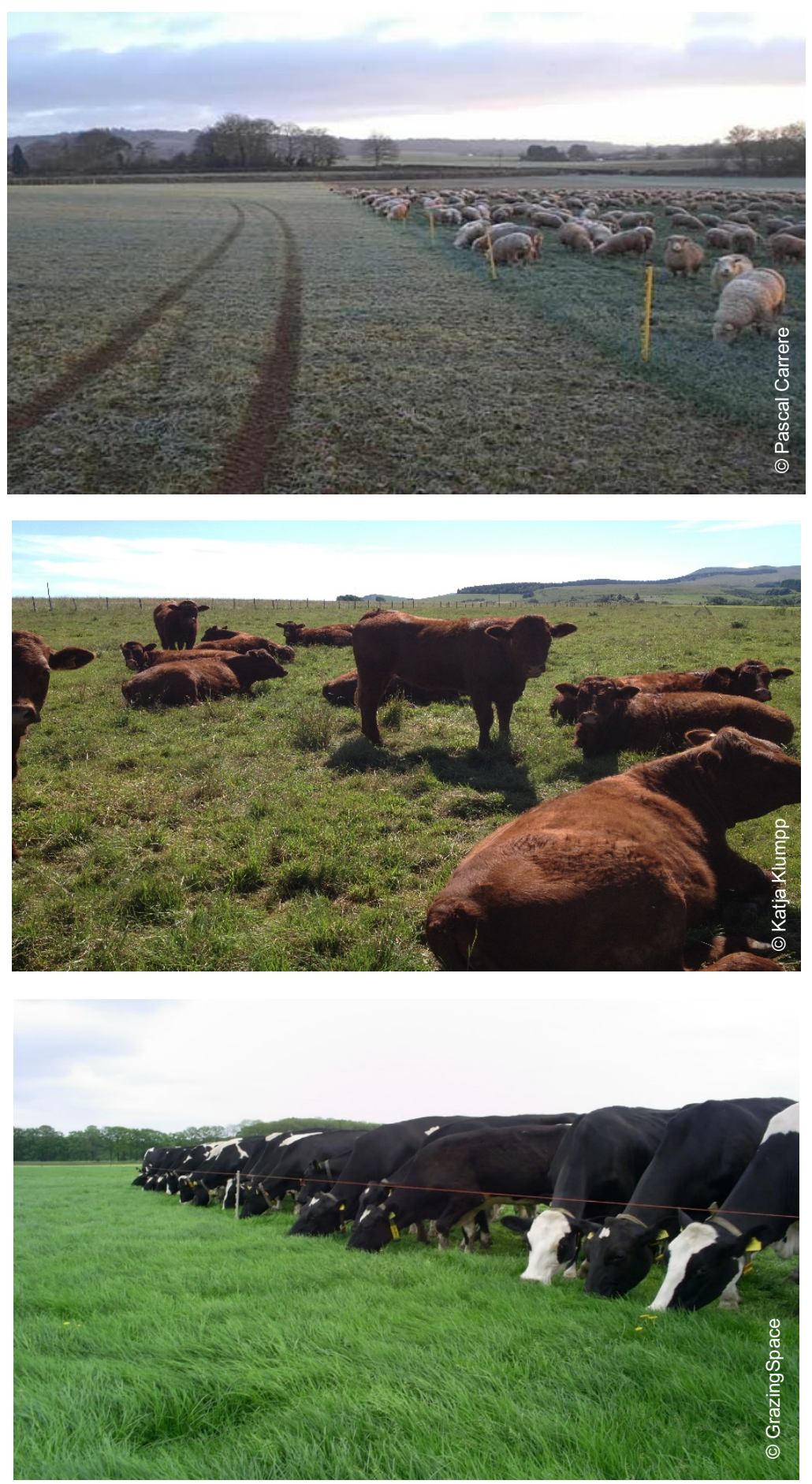

Photo 43. Example of different grazing management practices and intensities when grazed by sheep grazing (top), continuously grazing by cattle (middle) and strip-grazed by cattle (bottom). 
Table 153. Related cases studies available in volumes 3 and 5

\begin{tabular}{|l|l|l|c|c|}
\hline Title & Region & $\begin{array}{l}\text { Duration } \\
\text { of study } \\
\text { (Years) }\end{array}$ & Volume & $\begin{array}{l}\text { Case- } \\
\text { study } \\
\text { No. }\end{array}$ \\
\hline $\begin{array}{l}\text { Mitigation of SOC losses due to the } \\
\text { conversion of dry forests to pastures in the } \\
\text { plains of Venezuela (Bolivarian Republic of) }\end{array}$ & $\begin{array}{l}\text { Latin } \\
\text { America } \\
\text { and the } \\
\text { Caribbean }\end{array}$ & 5 and 18 & 3 & 40 \\
\hline
\end{tabular}




\section{References}

Abdalla, M., Hastings, A., Chadwick, D.R., Jones, D.L., Evans, C.D., Jones, M.B., Rees, R.M. \& Smith, P. 2018. Critical review of the impacts of grazing intensity on soil organic carbon storage and other soil quality indicators in extensively managed grasslands. Agriculture Ecosystems \& Environment, 253: 62-81. http://dx.doi.org/10.1016/j.agee.2017.10.023

Allen, V.G., Batello, C., Berretta, E.J., Hodgson, J., Kothmann, M., Li, X., McIvor, J., Milne, J., Morris, C., Peeters, A. \& Sanderson, M. 2011. An international terminology for grazing lands and grazing animals. Grass and Forage Science, 66(1): 2-28. https://doi.org/10.1111/j.1365-2494.2010.00780.x

Bloor, J.M.G. \& Pottier, J. 2014. Grazing and spatial heterogeneity: implications for grassland structure and function. In Mariotte, P. \& Kardol, P. (Eds.) Grassland Biodiversity and Conservation in a Changing World. Nova Science Publishers, Inc., Hauppage, New York, USA, pp 135-162.

Byrnes, R.C., Eastburn, D.J., Tate, K.W. \& Roche, L.M. 2018. A Global Meta-Analysis of Grazing Impacts on Soil Health Indicators. Journal of Environmental Quality, 47(4): 758-765.

http://dx.doi.org/10.2134/jeq2017.08.0313

Carolan, R. \& Fornara, D.A. 2016. Soil carbon cycling and storage along a chrono sequence of re-seeded grasslands: Do soil carbon stock increase with grassland age? Agriculture, Ecosystems and Environment, 218: 126-132. https://doi.org/10.1016/j.agee.2015.11.021

Chen, W., Huang, D., Liu, N., Zhang, Y., Badgery, W.B., Wang, X. \& Shen, Y. 2015. Improved grazing management may increase soil carbon sequestration in temperate steppe. Scientific Reports, 5(1): 10892. https://doi.org/10.1038/srep10892

Conant, R.T. \& Paustian, K. 2002. Potential soil carbon sequestration in overgrazed grassland ecosystems. Global Biogeochemical Cycles, 16(4): 90-1-90-9. https://doi.org/10.1029/2001GB001661

Conant, R.T., Paustian, K. \& Elliot, E.T. 2001 . Grassland management and conversion into grassland: Effects on soil carbon. Ecological Applications, 11: 343-355. https://doi.org/10.1890/10510761(2001)011[0343:GMACIG]2.0.CO;2

Conant, R.T., Cerri, C.E.P., Osborne, B.B. \& Paustian, K. 2017. Grassland management impacts on soil carbon stocks: a new synthesis. Ecological Applications, 27 (2): 662-668.

http://dx.doi.org/10.1002/eap.1473

Cordovil, C.M.d.S., Farto, A., Mendes-Jorge, L. \& Lucas, V. 2020. A importância da exploração pecuária na sustentabilidade ambiental: um estudo de caso na ganadaria brava. XII Jornadas Hospital Veterinário Muralha de Évora.https://www.hvetmuralha.pt/evento/xii-jornadas-programa-ruminantes/

Cotrufo, F., Wallenstein, M.D., Boot, C.M., Denef, K. \& Paul, E. 2013. The Microbial Efficiency-Matrix Stabilization (MEMS) framework integrates plant litter decomposition with soil organic matter stabilization: do labile plant inputs form stable soil organic matter? Global Change Biology, 19: 988-995.

https://doi.org/10.1111/gcb.12113 
Cui, X., Wang, Y., Niu, H., Wu, J., Wang, S., Schnug, E., Rogasik, J., Fleckenstein, J. \& Tang, Y. 2005. Effect of long-term grazing on soil organic carbon content in semiarid steppes in Inner Mongolia. Ecological Research, 20(5): 519-527. https://doi.org/10.1007/s11284-005-0063-8

Derner, J.D., Stanley, C. \& Chad, E. 2016. Usable science: Soil health. Rangelands, 38:64-67. https://doi.org/10.1016/j.rala.2015.10.010

FAO, IFAD \& WFP. 2015. The State of Food Insecurity in the World 2015. Meeting the 2015 international hunger targets: taking stock of uneven progress. Rome, FAO. http://www.fao.org/3/i4646e/i4646e.pdf

Franzluebbers, A.J. \& Stuedemann, J.A. 2009. Soil-profile organic carbon and total nitrogen during 12 years of pasture management in the Southern Piedmont USA. Agriculture, Ecosystems and Environment, 129(1-3): 28-36. https://doi.org/10.1016/j.agee.2008.06.013

Gruère, G. \& Wreford, A. 2017. Overcoming barriers to the adoption of climate-friendly practices in agriculture. OECD Food, Agriculture and Fisheries Papers, No. 101, OECD Publishing, Paris. http://dx.doi.org/10.1787/97767de8-en

Hennessy, D., De Vliegher, A., Die Dean, M., Klumpp, K., Poilane, A., Richmond, R., Schönhart, M., Svoboda, P. \& van Rijn, C. 2018. EIP-AGRI Focus Group - Grazing for carbon Mini-paper - Guidelines. (also available at: https://ec.europa.eu/eip/agriculture/sites/agrieip/files/fg25_03_minipaper_guidelines.pdf)

Houlbrooke, D.J., Drewry, J.J., Monaghan, R.M., Paton, R.J., Smith, L.C. \& Littlejohn, R.P. 2009. Grazing strategies to protect soil physical properties and maximise pasture yield on a Southland dairy farm. New Zealand Journal of Agricultural Research, 52(3): 323-336. https://doi.org/10.1080/00288230909510517

Klumpp, K. \& Graux, AI. 2020. Etat des connaissances sur les pratiques réputées « stockantes » en prairies permanentes. In Pellerin et al. (Eds.) Stocker du carbone dans les sols français, Quel potentiel au regard de l'objectif 4 pour 1000 et à quel coût? Synthèse du rapport d'étude, INRA (France). https:/ / hal.archivesouvertes.fr/hal-02284521/document

Lemaire, G., Gastal, F., Franzluebbers, A. \& Chabbi, A. 2015. Grassland-Cropping Rotations: An Avenue for Agricultural Diversification to Reconcile High Production with Environmental Quality. Environmental Management, 56(5): 1065-1077. https://doi.org/10.1007/s00267-015-0561-6

Liu, M., Liu, G., Gong, L., Wang, D. \& Sun, J. 2014. Relationships of Biomass with Environmental Factors in the Grassland Area of Hulunbuir, China. PLoS ONE, 9: e102344.

https://doi.org/10.1371/journal.pone.0102344

Mudge, P.L., Kelliher, F.M., Knight T.L., O'Connell, E., Fraser, S., \& Schipper, .. 2017. Irrigating grazed pasture decreases soil carbon and nitrogen stocks. Global Change Biology, 23: 945-954.

https://doi.org/10.1111/gcb.13448

Oenema, O., Velthof, G.L., Yamulki, S. \& Jarvis, S.C. 1997. Nitrous oxide emissions from grazed grassland. Soil Use Manage., 13: 288-295. https://doi.org/10.1111/j.1475-2743.1997.tb00600.x 
Reeder, J.D. \& Schumann, G.E. 2002. Influence of livestock grazing on C sequestration in semi-arid mixed-grass and short-grass rangelands. Environmental Pollution, 116: 457-463.

https://doi.org/10.1016/S0269-7491(01)00223-8

Rumpel, C., Crème, A., Ngao, P.T., Velaquez, G., Mora, A. \& Chabbi, A. 2015. The impact of grassland management on biogeochemical cycles involving carbon, nitrogen and phosphorus. Journal of Soil Science and Plant Nutrition, 15: 353-371. http://dx.doi.org/10.4067/S0718-95162015005000034

Sebastià M.-T., Chabbi A., Cordovil C., De Vliegher A., Hennessy D., Kramberger B., Newell Price P., Richmond R. \& van Rijn C. 2018. Grazing for carbon: Mixtures of species. Mini paper. Agriculture \& Innovation (EIP-AGRI) (also available at https://ec.europa.eu/eip/agriculture/sites/default/files/fg25_02_minipaper_mixtures_of_species_0.pdf)

Sandermann, J., Reseigh, J., Wurst, M., Young, M-A. \& Austin, J. 2015. Impacts of Rotational Grazing on Soil Carbon in Native Grass-Based Pastures in Southern Australia. PLoS ONE, 10(8): e0136157. https://doi.org/10.1371/journal.pone.0136157

Schils, R.L.M., Eriksen, J., Ledgard, S.F., Vellinga, T.V., Kuikman, P.J., Luo, J., Petersen, S.O. \& Velthof, G.L. 2013. Strategies to mitigate nitrous oxide emissions from herbivore production systems. Animal, 7(s1): 29-40. https://doi.org/10.1017/S175173111100187X

Schrama, M., Heijning, P., Bakker, J.P., Berg, M.P. \& Olff, H. 2013. Herbivore trampling as an alternative pathway for explaining differences in nitrogen mineralization in moist grasslands. Oecologia, 172 : 231-43. https://doi.org/10.1007/s00442-012-2484-8

Soussana, J.-F. \& Lemaire, G. 2014. Coupling carbon and nitrogen cycles for environmentally sustainable intensification of grasslands and crop-livestock systems. Agr. Ecosyst. Environ., 190: 9-17.

https://doi.org/10.1016/j.agee.2013.10.012

Schaub, S., Finger, R., Leiber, F., Probst, S., Kreuzer, M., Weigelt, A., Buchmann, N. \& SchererLorenzen, M. 2020. Plant diversity effects on forage quality, yield and revenues of semi-natural grasslands. Nature Communications, 11(1). https://doi.org/10.1038/s41467-020-14541-4

Tälle, M., Deák, B., Poschlod, P., Valkó, O., Westerberg, L. \& Milberg, P. 2016. Grazing vs. mowing: A meta-analysis of biodiversity benefits for grassland management. Agriculture, Ecosystems \& Environment, 222: 200-212. https://doi.org/10.1016/j.agee.2016.02.008

Talore, D.G., Tesfamariam, E.H., Hassen, A., Toit, J.D., Klampp, K. \& Soussana, J-F. 2016. Longterm impacts of grazing intensity on soil carbon sequestration and selected soil properties in the arid Eastern Cape, South Africa. Journal of the Science of Food and Agriculture, 96(6): 1945-1952.

https://doi.org/10.1002/jsfa.7302

Tate, K.W., Dudley, D.M., McDougald, N.K. \& George, M.R. 2004. Effect of canopy and grazing on soil bulk density. Journal of Range Management Archives, 57(4): 411-417.

Teague, W.R., Provenza, F., Kreuter, U.P., Steffens, T. \& Barnes, M. 2013. Multi-paddock grazing on rangelands: Why the perceptual dichotomy between research results and rancher experience? Journal of Environmental Management, 128: 699-717. https://doi.org/10.1016/j.jenvman.2013.05.064 
Teixeira, R.F.M., Domingos, T., Costa, A.P.S.V., Oliveira, R., Farropas, L., Calouro, F., Barradas, A.M. \& Carneiro, J.P.B.G. 2011. Soil organic matter dynamics in Portuguese natural and sown rainfed grasslands. Ecological Modelling, 222(4): 993-1001.https://doi.org/10.1016/j.ecolmodel.2010.11.013

Tessema, B., Sommer, R., Piikki, K., Söderström, M., Namirembe, S., Notenbaert, A., Tamene, L., Nyawira, S. \& Paul, B. 2020. Potential for soil organic carbon sequestration in grasslands in East African countries: A review. Grassland Science, 66(3): 135-144. https://doi.org/10.1111/grs.12267

van Klink, R., Schrama, M., Nolte, S., Bakker, J.P., WallisDeVries, M.F. \& Berg, M.P. 2015.

Defoliation and Soil Compaction Jointly Drive Large-Herbivore Grazing Effects on Plants and Soil Arthropods on Clay Soil. Ecosystems, 18(4): 671-685. https://doi.org/10.1007/s10021-015-9855-Z

Vogeler, I., Thomas S., Van der Weerden, T., 2019. Effect of irrigation management on pasture yield and nitrogen losses. Agricultural Water Management, 216, 60-69.

https://doi.org/10.1016/j.agwat.2019.01.022

Vertes, F., Guiet, S., Morvan, T. \& Gascuel-Odoux, C. 2012. De la parcelle à la succession de cultures et au territoire: exemple de l'outil Territ' eau (module azote). Journée de synthèse scientifique 30 ans de références pour comprendre et limiter les fuites d'azote à la parcelle. 63-67.

Zhou, G., Zhou, X., He, Y., Shao, J., Hu, Z., Liu, R., Zhou, H. \& Hosseinibai, S. 201 7. Grazing intensity significantly affects belowground carbon and nitrogen cycling in grassland ecosystems: a meta-analysis. Global Change Biology, 23(3): 1167-1179. https://doi.org/10.1111/gcb.13431 


\title{
35. Grazing exclusion and rotational grazing
}

\author{
Mohammad I. Khalil', Cláudia M.d.S. Cordovil², Rosa Francaviglia ${ }^{3}$, \\ Beverley Henry ${ }^{4}$, Katja Klumpp ${ }^{5}$, Peter Koncz ${ }^{6}$, Mireia Llorente ${ }^{7}$, \\ Beata E. Madari ${ }^{8}$, Muñoz-Rojas Miriam, ${ }^{9}$, , Nerger Rainer $^{11}$ \\ (Co-authors in alphabetical order)
}

'School of Applied Sciences \& Technology, Prudence College Dublin, Dublin 22 and School of Biology \& Environmental Science, University College Dublin, Dublin 4, Ireland

${ }^{2}$ University of Lisbon, School of Agriculture, Forest Research Center, Lisboa, Portugal

${ }^{3}$ Council for Agricultural Research and Economics, Research Centre for Agriculture and Environment (CREA-AA), Rome, Italy.

${ }^{4}$ Science and Engineering Faculty, Queensland University of Technology, Brisbane, Australia

${ }^{5}$ Grassland Ecosystem Research, INRA, Clermont-Ferrand, France

${ }^{6}$ Duna-Ipoly National Park Directorate, Budapest, Hungary and MTA-SZIE Plant Ecology Research Group, Gödöllő, Hungary

${ }^{7}$ Forest Department. University of Extremadura, Plasencia Campus. Spain

${ }^{8}$ Embrapa Rice and Beans, Santo Antônio de Goiás, GO, Brazil

${ }^{9}$ UNSW Sydney, School of Biological, Earth and Environmental Sciences, Sydney NSW, Australia

${ }^{10}$ The University of Western Australia, School of Biological Sciences, Crawley, WA, Australia

${ }^{11}$ Soil \& More Impacts GmbH, German office, Hamburg, Germany

\section{Description of the practice}

Overgrazing in poorly managed agricultural areas is a debateable concept based on the equilibrium between biomass production and livestock demand, and degradation of grassland. A strong indicator of overgrazing is the necessity for additional feeds to be brought in from outside the farm to support livestock. Overgrazed, often 
degraded, areas commonly show a reduction in plant species diversity (and sometimes spread of invasive species of non-native plants and of weeds), productivity, canopy cover, soil structure and soil nutrients. Sustainable grassland management can restore these degraded areas, and thus is an issue to maintaining animal production and the health of the grassland ecosystem (Conant, Paustian and Elliott, 2001; Dong et al, 2015). Low- to medium stocking rates generally contribute to maintaining sufficient vegetation cover to feed livestock and help limit soil degradation processes (e.g. C depletion, nutrient losses, erosion). Hence, regulating grazing intensity and periodic grazing exclusion with fenced paddocks are effective practices for grassland restoration (McSherry and Ritchie 2013; Davies et al., 2014). Previous studies have indicated that periodical grazing exclusion is an effective way to stimulate soil nutrient content through aboveground biomass and root biomass, which act as primary input sources to the soil (Liu, 2016; Sun et al., 2014).

Grazing exclusion can have various durations, from short-term (1 year) to several years depending on the severity of degradation, vegetation type and pedo-climatic zone (Li et al., 2018, Wang et al., 2018). Short- to medium-term exclusion strengthens above- and belowground biomass only, while longer periods of grazing exclusion lead to greater improvements in soil properties, as a new equilibrium can be reached after a few decades (Wang et al., 2018, Dong et al., 2020). Exclusion can be managed by "shifting" livestock systematically at desirable intervals to different subunits of a range area or fenced subdivisions, where some are unmanaged when excluded from grazing. This is called rotational grazing (i.e. adaptive multi paddock grazing) which, under low- to medium-intensity, has been proposed as an alternative to severe grazing exclusion in order to enhance grassland SOC stocks, maintain ecosystem sustainability (Davies et al., 2014; Sanjari et al., 2008), and encourage vegetation (self-)recovery (e.g. seed recolonization through seed maturity) (Briske et al., 2011). Exclusion through set aside (unmanaged) may be combined with restoration to improve plant species pool and diversity (Andrade et al., 2015)

\section{Range of applicability}

Overgrazing is a key factor in soil and plant degradation, particularly in semi-arid and arid rangelands where plant communities are often composed of a majority of grazing resistant shrubs. About 20 percent of global pasture and 73 percent of the rangelands in the drylands have been degraded (Steinfeld, Wassenaar and Jutzi, 2006 ) in last decades. Due to variability in rainfall and the short growing periods these areas are not suitable for intensive agriculture and the main land use is grazing based mostly on native vegetation cover. Analyses in several dry countries showed that, livestock numbers increased by 20 percent (Steinfeld et al., 2006) without changes in areas under grazing. As a result, the increase in stock numbers has been one of the main causes of land degradation in these low productive lands, for example Central Asian countries, Mongolia, Africa, Australia and US, affecting high percentages of arid lands (Jafari et al, 2008, Zhao, Li and Qi, 2007). Besides the degradation of vegetation in dry areas, exclusion may be used for the protection of water quality in other sensitive areas such as streambanks, riparian areas, uplands and wetlands order to achieve environmental improvements (EPA, 2003). Depending on severity of degradation, attained area, vegetation type and pedo-climatic conditions, grazing exclusion can have different forms; short to long-term exclusion, combined with low rotational grazing and restoration actions (e.g. Tessema et al., 2020). 


\section{Effects on soil organic carbon stocks}

Globally, grazing exclusion is an effective management practice to restore degraded grasslands and improve soil quality (i.e. C density and soil C stocks). However, soil restoration on degraded soils, in particular sandy soil, is a slow process, although vegetation can recover rapidly after removal of livestock disturbance (Wang et al., 2018). Soil $\mathrm{N}$ is a key factor in the regulation of soil $\mathrm{C}$ sequestration in long term grazing exclusion ( $>20$ years), with higher $\mathrm{C}$ accrual in soils with higher $\mathrm{N}$ availability. For that reason, in nutrient poor and long-term grazing degraded areas, the recovery of already weakened SOC is very slow (e.g. semi-arid typical steppes, Cui et al., 2005). Table 154 provides some figures of SOC sequestration enabled by grazing exclusion or rotational grazing. 
Table 154. Evolution of SOC stocks with grazing exclusion of rotational grazing

\begin{tabular}{|c|c|c|c|c|c|c|c|c|}
\hline Location & $\begin{array}{l}\text { Climate } \\
\text { zone }\end{array}$ & Soil type & $\begin{array}{l}\text { Baseline C } \\
\text { stock } \\
\text { (tC/ha) }\end{array}$ & $\begin{array}{l}\text { Additional C } \\
\text { storage } \\
\text { (tC/ha/yr) }\end{array}$ & $\begin{array}{l}\text { Depth } \\
(\mathrm{cm})\end{array}$ & $\begin{array}{l}\text { Duration } \\
\text { (Year) }\end{array}$ & More information & Reference \\
\hline Global & $\begin{array}{l}\text { wet, mesic, } \\
\text { dry } \\
\text { temperate, } \\
\text { tropical, } \\
\text { subtropical } \\
\text { regions }\end{array}$ & Various & NA & 0.3 to 0.7 & NA & NA & $\begin{array}{l}\text { Strong relation to rainfall: } \\
\text { annual rainfall from } 333 \\
\text { to }<1800 \mathrm{~mm}\end{array}$ & $\begin{array}{l}\text { Conant and } \\
\text { Paustian (2002) }\end{array}$ \\
\hline China & Various & Various & NA & $\begin{array}{l}0.27 \\
0.23 \\
0.18\end{array}$ & $\begin{array}{l}0-10 \\
10-20 \\
20-30\end{array}$ & 27 & $\begin{array}{l}\text { Grazing exclusion; } \\
\text { Strong relation to rainfall }\end{array}$ & $\begin{array}{l}\text { Deng et al. (2017), } \\
\text { Wang et al. (2018) }\end{array}$ \\
\hline \multirow[t]{2}{*}{$\begin{array}{l}\text { Inner } \\
\text { Mongolia, } \\
\text { China }\end{array}$} & \multirow{2}{*}{$\begin{array}{l}\text { Continental } \\
\text { semi-arid } \\
\text { monsoon } \\
\text { temperate }\end{array}$} & $\begin{array}{l}\text { Cambic } \\
\text { Arenosol } \\
\text { (sandy) }\end{array}$ & $\begin{array}{l}7.3 \\
(0-20 \mathrm{~cm}) \\
56 \\
(0-100 \mathrm{~cm})\end{array}$ & 0.14 & NA & 25 & $\begin{array}{l}\text { Grazing exclusion; } \\
\text { Analyses for } 3 \text { periods: } 7 \text {, } \\
12 \text { and } 25 \text { years }\end{array}$ & Li et al. (2012) \\
\hline & & Kastanozem & NA & 0.4 to 0.37 & $0-10$ & $21-35$ & $\begin{array}{l}\text { Grazing enclosure vs. } \\
\text { rotational grazing (labile } \\
\text { SOC stocks) }\end{array}$ & Dong et al. (2020) \\
\hline $\begin{array}{l}\text { Chepareria, } \\
\text { Kenya }\end{array}$ & $\begin{array}{l}\text { Semi-arid } \\
\text { sub-Saharan }\end{array}$ & NA & 26.1 & 3.1 & $0-100$ & 10 & $\begin{array}{l}\text { Live fences for rotational } \\
\text { grazing }\end{array}$ & $\begin{array}{l}\text { Svanlund (2014) in } \\
\text { Tessema et al. } \\
(2020)\end{array}$ \\
\hline
\end{tabular}




\section{Other benefits of the practice}

\subsection{Improvement of soil properties}

Changes in ground cover characteristics also improve soil properties such as bulk density, SOC and total N concentration, which are usually good indicators of good grazing management on soils (Wang et al., 2018; Dong et al., 2020; Mchunu and Chaplot, 2012).

\subsection{Minimization of threats to soil functions}

Table 155. Soil threats

\begin{tabular}{|c|c|}
\hline Soil threats & \\
\hline Soil erosion & $\begin{array}{l}\text { Overgrazing reduces coarseness in surface soil, therefore } \\
\text { increasing soil losses by wind and water erosion (Golluscio et al., } \\
\text { 2009). Restoration of permanent vegetation cover improves soil } \\
\text { structure and prevents soil surface runoff (Petz et al., 2014). }\end{array}$ \\
\hline $\begin{array}{l}\text { Nutrient imbalance } \\
\text { and cycles }\end{array}$ & $\begin{array}{l}\text { Improved nutrient use and cycling trough vegetation recovery (Pei } \\
\text { et al., 2008; Su et al., 2005). }\end{array}$ \\
\hline $\begin{array}{l}\text { Soil salinization and } \\
\text { alkalinization }\end{array}$ & $\begin{array}{l}\text { Conservation of vegetation cover without nutrient inputs avoids } \\
\text { alkalinization. }\end{array}$ \\
\hline Soil biodiversity loss & $\begin{array}{l}\text { Above-ground species diversity influences below-ground diversity } \\
\text { (Golluscio et al., 2009). The increased level of soil fertility from } \\
\text { grazing exclusion promotes biodiversity restoration and plant } \\
\text { growth. }\end{array}$ \\
\hline Soil compaction & Cessation of grazing avoid damages by trampling. \\
\hline $\begin{array}{l}\text { Soil water } \\
\text { management }\end{array}$ & $\begin{array}{l}\text { Conservation of vegetation cover fosters a better water retention } \\
\text { capacity and infiltration rate and prevents from water runoff and } \\
\text { preserves water quality. }\end{array}$ \\
\hline
\end{tabular}




\subsection{Increases in production (e.g. food/fuel/feed/timber/fibre)}

Grazing exclusion often leads to encroachment of unpalatable shrubs into grasslands, which can decrease the livestock carrying capacity over time. However, adaptive multi-paddock grazing can provide the flexibility needed to incorporate management practices such as fire into grazing systems (Teague et al., 2010). Even so, native or selected introduction of shrubs, including drought-tolerant species and nitrogen-fixing legumes, can provide valuable browse for cattle, sheep and goats in arid and semi-arid grazing lands once exclusion is levered.

\subsection{Mitigation of and adaptation to climate change}

Grazing restrictions and exclusions are practices commonly associated with combating soil degradation and SOC losses mostly to overgrazing coupled with drought or wet soils. Restoration of groundcover and nutrient buildup (i.e. in dry soils) can improve soil carbon stocks and water quality (i.e. wet soils), mitigate the risk of erosion (e.g. Petz et al. 2014) and limit potential water pollution (EPA, 2003).

\subsection{Socio-economic benefits}

Appropriate grazing management can be a viable solution where the abiotic function of the degraded grazing land has not been irreversibly damaged (Papanastasis, 2009). Implementation of short- to medium- grazing exclusion can be economically interesting as grasslands regain productivity as well as aesthetics, especially when coupled with restoration techniques.

\subsection{Other benefits}

Based on the livestock types and land capacity, grazing exclusion may help to improve species composition and restore biodiversity through regrowth of perennial species (Briske et al., 2011). Also arranging alternative feeds, relocating animals to other areas, and adoption of low-performance animal breeds may restore grassland functions. Accordingly, a combination of several measures such as managing grazing intensity and timing, increasing productivity, management of nutrients, and finding alternatives to the use of shrubs and dung for energy, have implications for socio-economic conditions.

\section{Potential drawbacks to the practice}

\subsection{Tradeoffs with other threats to soil functions}

No trade-offs recorded. 


\subsection{Increases in greenhouse gas emissions}

In nutrient poor and long-term grazing degraded areas, the recovery of already weakened SOC is very slow (e.g. semi-arid typical steppes, Cui et al., 2005). In these cases, grazing exclusion coupled with increased soil N supply to grasslands may enhance ecosystem $\mathrm{C}$ sequestration during the recovery stage (Deng et al., 2017), but may increase $\mathrm{N}_{2} \mathrm{O}$ emission (Schönbach et al., 2012).

\subsection{Conflict with other practice(s)}

The increasing demand for livestock products, results in competition for natural resources, and between food and feed, might lead to priorities that omit arranging alternative feeds and relocation of feed areas.

\subsection{Other conflicts}

Grazing exclusion and possible uncontrolled encroachment of woody species is not desirable, as is the infestation of invasive species. Despite that, introduction of native or selected shrubs can provide valuable feed for livestock once exclusion is levered. Accordingly, more detail analyses are needed to adapt grazing management to regional conditions. For instance, smaller paddocks can improve distribution of animals across a landscape, which can increase or decrease vegetation diversity and soil quality, depending on how animals were previously distributed (see Teague et al., 2013).

\section{Recommendations before implementing the practice}

The effectiveness of grazing management to restore degraded areas is often limited. There is a strong need for adopting sustainable practices at lower intensification management to prevent further soil degradation (Pereira et al., 2017; Davis et al, 2014). Accordingly, better analyses are needed to adapt grazing management to regional conditions. For instance, smaller paddocks can improve distribution of animals across a landscape, which can increase or decrease vegetation groundcover, depending on how animals were previously distributed (see Teague et al, 2013). Also arranging alternative feeds, relocation to other areas, adopt to low-performance animal breeds may allow to restore grassland function. Hence, a combination of several measures in managing grazing, alternatives to the use of shrubs and dung for energy are needed before implementation. These analyses may include diverse knowledge sources for managers, agency professionals, and researchers, to replace the narrow technological approach to grazing systems (see Briske et al., 2011). 


\section{Potential barriers for adoption}

Contrasting effects of grazing cessation have been reported when studying similar areas, showing either ameliorating effects or not under short-term exclusion (<5years, e.g. Li et al., 2012). In view of that, implementation is hampered by the absence of generalizable evidence. Furthermore, for low-productivity rangelands (grasslands), the cost of fencing and water systems to enable rotational grazing systems (i.e. including parcels of exclusion) can be prohibitive. For this, an effective implementation of adaptive management would require overcoming institutional barriers and with help of meaningful incentives to promote adoption. To wit, institutional cultures influence behavioural patterns of personnel, approaches to solve problems, and the establishment of goals and priorities. In addition, the decrease in grassland areas due to grazing exclusion may require an increase in concentrated feeds (e.g. feed grains), to supplement fodder. Therefore, it is necessary to consider the total agricultural system and not to restrict the analysis to animal husbandry only (Table 156).

Table 156. Potential barriers to adoption

\begin{tabular}{|l|c|l|}
\hline Barrier & YES/NO & \\
\hline Social & Yes & $\begin{array}{l}\text { Psychological reluctance related consequences of exclusion } \\
\text { such e.g. relocation of feed areas, adoption of low- } \\
\text { performance animals. }\end{array}$ \\
\hline Economic & Yes & $\begin{array}{l}\text { Contrasting effects of grazing cessation are only detected in } \\
\text { the long term (> 12years, Wang et al. 2018, Li } \text { et al. 2012). }\end{array}$ \\
\hline $\begin{array}{l}\text { Legal (Right } \\
\text { to soil) }\end{array}$ & Yes & Lack of supplementary grassland area. \\
\hline Knowledge & Yes & Lack of training, skills, advisory services, supporting tools. \\
\hline
\end{tabular}




\section{Photos of the practice}
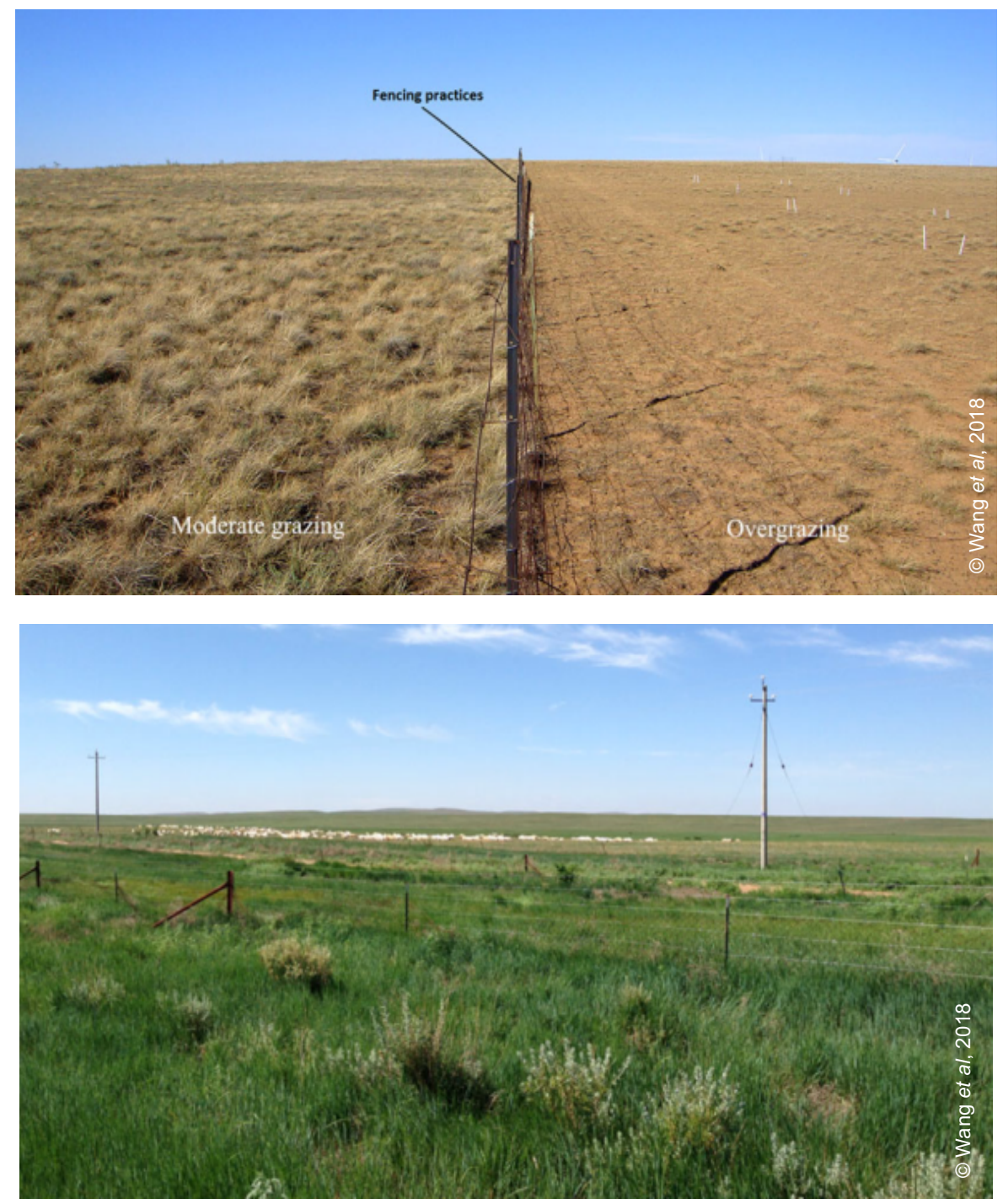

Photo 44. Example of moderate and overgrazed area (left) and grazing exclusion (right)

Table 157. Related cases studies available in volumes 3 and 5

\begin{tabular}{|l|l|c|c|c|}
\hline Title & Region & $\begin{array}{l}\text { Duration of } \\
\text { study (Years) }\end{array}$ & Volume & $\begin{array}{l}\text { Case- } \\
\text { study No. }\end{array}$ \\
\hline $\begin{array}{l}\text { Grazing management in rangeland } \\
\text { grassland systems in South and East } \\
\text { Australia }\end{array}$ & $\begin{array}{l}\text { Southeast } \\
\text { Pacific }\end{array}$ & 4 to 10 & 3 & 9 \\
\hline
\end{tabular}




\section{References}

Andrade, B.O., Koch, C., Boldrini, I.I., Vélez-Martin, E., Hasenack, H., Hermann, J.-M., Kollmann, J., Pillar, V.D. \& Overbeck, G.E. 2015. Grassland degradation and restoration: a conceptual framework of stages and thresholds illustrated by southern Brazilian grasslands. Natureza \& Conservação, 13(2): 95-104. https://doi.org/10.1016/j.ncon.2015.08.002

Briske, D.D., Sayre, N.F., Huntsinger, L., Fernandez-Gimenez, M., Budd, B. \& Derner, J.D. 2011. Origin, persistence, and resolution of the rotational grazing debate: integrating human dimensions into rangeland research. Rangeland Ecology and Management, 64(4): 325-334.

https://doi.org/10.2111/REM-D-10-00084.1

Conant, R.T. \& Paustian, K. 2002. Potential soil carbon sequestration in overgrazed grassland ecosystems. Global Biogeochemical Cycles, 16(4): 90-1--90-9. https://doi.org/10.1029/2001GB001661

Conant, R.T., Paustian, K. \& Elliott, E.T. 2001. Grassland management and conversion into grassland effects on soil carbon. Ecological Applications, 11: 343-355. https://doi.org/10.1890/10510761(2001)011[0343:GMACIG]2.0.CO;2

Cui, X., Wang, Y., Niu, H., Wu, J., Wang, S., Schnug, E., Rogasik, J., Fleckenstein, J. \& Tang, Y. 2005. Effect of long-term grazing on soil organic carbon content in semiarid steppes in Inner Mongolia. Ecological Research, 20(5): 519-527. https://doi.org/10.1007/s11284-005-0063-8

Davies, K.W., Vavra, M., Schultz, B. \& Rimbey, N. 2014. Implications of Longer Term Rest from Grazing in the Sagebrush Steppe. Journal of Rangeland Applications, 1: 14-34.

Deng, L., Shangguan, Z-P., Wu, G-L. \& Chang, X-F. 2017. Effects of grazing exclusion on carbon sequestration in China's grassland. Earth-Science Reviews, 173: 84-95.

https://doi.org/10.1016/j.earscirev.2017.08.008

Dong, L., Martinsen, V., Wu, Y., Zheng, Y., Liang, C., Liu, Z. \& Mulder, J. 2020. Effect of grazing exclusion and rotational grazing on labile soil organic carbon in north China. European Journal of Soil Science. https://doi.org/10.1111/ejss.12952

Dong, W., Gao-Lina, W., Yua, L., Zhenga, Y. \& Hong-Mina, H. 2015. Effects of grazing exclusion on CO2 fluxes in a steppe grassland on the Loess Plateau (China). Ecological Engineering, 83: 169-175. https://doi.org/10.1016/j.ecoleng.2015.06.017

EPA. 2003. Chapter 4E: Grazing Management. In National Management Measures to Control Nonpoint Source Pollution from Agriculture. EPA 841-B-03-004. pp. 129-156. (also available at: https://www.epa.gov/sites/production/files/2015-10/documents/chap4e.pdf)

Golluscio, R.A., Austin, A.T., Garcia Martinez, E.G., Gaonzalez-Polo, M., Sala, E.E. \& Jackson R.B. 2009. Sheep Grazing Decreases Organic Carbon and Nitrogen Pools in the Patagonian Steppe: Combination of Direct and Indirect Effects. Ecosystems, 12: 686-697. https://doi.org/10.1007/s10021-009-9252-6 
Jafari, R., Lewis, M.M. \& Ostendorf, B. 2008. An image-based diversity index for assessing land degradation in an arid environment in South Australia. Journal of Arid Environments, 72: 1282-1293. https://doi.org/10.1016/j.jaridenv.2008.02.011

Li, Y., Zhou, X., Brandle, J.R., Zhang, T., Chen, Y. \& Han, J. 2012. Temporal progress in improving carbon and nitrogen storage by grazing exclosure practice in a degraded land area of China's Horqin Sandy Grassland. Agriculture, Ecosystems \& Environment, 159: 55-61.

https://doi.org/10.1016/j.agee.2012.06.024

Li, W., Liu, Y., Wang, J., Shi, S. \& Cao, W. 2018. Six years of grazing exclusion is the optimum duration in the alpine meadow-steppe of the north-eastern Qinghai-Tibetan Plateau. Nature Scientific Reports, 8: 17269. https://doi.org/10.1038/s41598-018-35273-y

Liu, J. 2016. Research on nitrogen forms and microbial community structure of grassland soil in Inner Mongolia (in Chinese with English abstract). Hangzhou, China: Zhejiang University.

Mchunu, C. \& Chaplot, V. 2012. Land degradation impact on soil carbon losses through water erosion and $\mathrm{CO}_{2}$ emissions. Geoderma, 177-178: 72-79. https://doi.org/10.1016/j.geoderma.2012.01.038

McSherry, M.E. \& Ritchie, M.E. 2013. Effects of grazing on grassland soil carbon: a global review. Global Change Biology, 19: 1347-1357. https://doi.org/10.1111/gcb.12144

Papanastasis, V.P. 2009. Restoration of Degraded Grazing Lands through Grazing Management: Can It Work? Restoration Ecology, 17(4): 441-445. https://doi.org/10.1111/j.1526-100X.2009.00567.x

Pei, S., Fu, H. \& Wan, C. 2008. Changes in soil properties and vegetation following exclosure and grazing in degraded Alxa desert steppe of Inner Mongolia, China. Agriculture, Ecosystems and Environment, 124: 33 - 39. https://doi.org/10.1016/j.agee.2007.08.008

Pereira, P., Brevik, E.C., Muñoz-Rojas, M., Miller, B.A., Smetanova, A., Depellegrin, D., Misiune, I., Novara, A. \& Cerdà, A. 2017. Chapter 2 - Soil Mapping and Processes Modeling for Sustainable Land Management. In Pereira, P., Brevik, E.C., Muñoz-Rojas, M.\& Miller, B.A. (Eds.) Soil Mapping and Process Modeling for Sustainable Land Use Management, pp. 29-60. Elsevier. https://doi.org/10.1016/B978-012-805200-6.00002-5

Petz, K., Alkemade, R., Bakkenes, M., Schulp, C.J.E., van der Velde, M. \& Leemans, R. 2014. Mapping and modelling trade-offs and synergies between grazing intensity and ecosystem services in rangelands using global-scale datasets and models. Global Environmental Change, 29: 223-234.

https://doi.org/10.1016/j.gloenvcha.2014.08.007

Sanjari, G., Ghadiri, H., Ciesiolka, C.A.A., \& Yu, B. 2008. Comparing the effects of continuous and timecontrolled grazing systems on soil characteristics in Southeast Queensland. Australian Journal of Soil Research, 46: 348 - 358. https://doi.org/10.1071/Sr07220

Schönbach, P., Wolf, B., Dickhöfer, U., Wiesmeier, M., Chen, W., Wan, H., Gierus, M., ButterbachBahl, K., Kögel-Knabner, I., Susenbeth, A., Zheng, X. \& Taube, F. 2012. Grazing effects on the greenhouse gas balance of a temperate steppe ecosystem. Nutrient Cycling in Agroecosystems, 93(3): 357371. https://doi.org/10.1007/s10705-012-9521-1 
Steinfeld, H., Gerber, P., Wassenaar, T., Castel, V., Rosales, M., \& DeHaan, C. 2006a. Livestock's long shadow: Environmental issues and options. Food and Agriculture Organization of the United Nations, Rome, Italy. http://www.fao.org/3/a0701e/a0701e00.htm

Steinfeld, H., Wassenaar, T. \& Jutzi, S. 2006b. Livestock production systems in developing countries: status, drivers, trends. Revue Scientifique Et Technique (International Office of Epizootics), 25(2): 505-516. https://doi.org/10.20506/rst.25.2.1677

Su, Y.Z., Li, Y.L., Cui, H.Y. \& Zhao, W.Z. 2005. Influences of continuous grazing and livestock exclusion on soil properties in a degraded sandy grassland, Inner Mongolia, northern China. Catena, 59: 267-278. https://doi.org/10.1016/j.catena.2004.09.001

Sun, J., Wang, X., Cheng, G., Wu, J., Hong, J. \& Niu, S. 2014. Effects of grazing regimes on plant traits and soil nutrients in an alpine steppe, northern Tibetan Plateau. PLoS One, 9: e108821.

https://doi.org/10.1371/journal.pone.0108821

Svanlund, S. 2014. Carbon sequestration in the pastoral area of Chepareria, western Kenya: A comparison between open-grazing, fenced pastures and maize cultivations. Faculty of Forest Sciences, SLU: Thesis at the Department of Forest Ecology and Management. (also available at:

https://stud.epsilon.slu.se/6602/7/svanlund_s_140424.pdf)

Teague, W.R., Dowhower, S.L., Ansley, R.J., Pinchak, W.E. \& Waggoner, J.A. 2010. Integrated Grazing and Prescribed Fire Restoration Strategies in a Mesquite Savanna: I. Vegetation Responses. Rangeland Ecology \& Management, 63(3): 275-285. https://doi.org/10.2111/08-171.1

Teague, W.R., Provenza, F., Kreuter, U.P., Steffens, T. \& Barnes, M. 2013. Multi-paddock grazing on rangelands: Why the perceptual dichotomy between research results and rancher experience? Journal of Environmental Management, 128:699-717. https://doi.org/10.1016/j.jenvman.2013.05.064

Tessema, B., Sommer, R., Piikki, K., Söderström, M., Namirembe, S., Notenbaert, A., Tamene, L., Nyawira, S. \& Paul, B. 2020. Potential for soil organic carbon sequestration in grasslands in East African countries: A review. Grassland Science, 66(3): 135-144. https://doi.org/10.1111/grs.12267

Wang, L., Gan, Y., Wiesmeier, M., Zhao, G., Zhang, R., Han, G., Siddique, K.H.M. \& Hou, F. 2018. Grazing exclusion-An effective approach for naturally restoring degraded grasslands in Northern China. Land Degradation \& Development, 29(12): 4439-4456. https://doi.org/10.1002/ldr.3191

Wang, Z., Johnson, D.A., Rong, Y. \& Wang, K. 2016. Grazing effects on soil characteristics and vegetation of grassland in northern China. Solid Earth, 7: 55-65. https://doi.org/10.5194/se-7-55-2016

Zhao, W.Y., Li, J.L. \& Qi, J.G. 2007. Changes in vegetation diversity and structure in response to heavy grazing pressure in the northern Tianshan Mountains, China. Journal of Arid Environments, 68: 465-479. https://doi.org/10.1016/j.jaridenv.2006.06.007 


\title{
36. Pastoralism
}

\author{
Mohammad I. Khalil', Cláudia M.d.S. Cordovil², Rosa Francaviglia ${ }^{3}$, \\ Beverley Henry ${ }^{4}$, Katja Klumpp ${ }^{5}$, Peter Koncz ${ }^{6}$, Mireia Llorente ${ }^{7}$, \\ Beata E. Madari ${ }^{8}$, Muñoz-Rojas Miriam ${ }^{9,10}$, Nerger Rainer ${ }^{11}$ \\ (Co-authors in alphabetical order)
}

'School of Applied Sciences \& Technology, Prudence College Dublin, Dublin 22 and School of Biology \& Environmental Science, University College Dublin, Dublin 4, Ireland

${ }^{2}$ University of Lisbon, School of Agriculture, Forest Research Center, Lisboa, Portugal

${ }^{3}$ Council for Agricultural Research and Economics, Research Centre for Agriculture and Environment (CREA-AA), Rome, Italy.

${ }^{4}$ Science and Engineering Faculty, Queensland University of Technology, Brisbane, Australia

${ }^{5}$ Grassland Ecosystem Research, INRA, Clermont-Ferrand, France

${ }^{6}$ Duna-Ipoly National Park Directorate, Budapest, Hungary and MTA-SZIE Plant Ecology Research Group, Gödöllő, Hungary

${ }^{7}$ Forest Department. University of Extremadura, Plasencia Campus. Spain

${ }^{8}$ Embrapa Rice and Beans, Santo Antônio de Goiás, GO, Brazil

${ }^{9}$ UNSW Sydney, School of Biological, Earth and Environmental Sciences, Sydney NSW, Australia

${ }^{10}$ The University of Western Australia, School of Biological Sciences, Crawley, WA, Australia

${ }^{11}$ Soil \& More Impacts GmbH, German office, Hamburg, Germany

\section{Description of the livelihood}

Pastoralism refers to mobile livestock herding for either production or livelihood (Dong, 2016). Pastoralism occurs on about 18-23 percent of global land area and it supports around 200 million pastoral households (Neely, Bunning and Wilkes, 2009; Blench, 2001). It usually occurs where resources are limited, and thus 
movement to pasturage places provides enough biomass and water for the animals (cattle, camels, goats, yaks, llamas, reindeer, horses and sheep).

Mobility is also a key strategy to manage the quality of pastures and livestock (increase gene pool, provide a variety of food resources, occasionally include residuals of croplands), access market and increase socialcultural interaction including transboundary integration. The two essential forms of pastoralism are the nomadic and transhumance rearing of domesticated animals (Dong, 2016). The nomads migrate with their families according to the changing seasons from one area to another to meet the needs of their animals. On the other hand, transhumance is a movement of livestock (typically seasonal) by usually hired herders between fixed summer and winter pastures (often with stables). Besides, food production pastoralism is important to preserve traditional knowledge, provided that the grazing intensity is optimum under the local circumstances to maintain high biodiversity, prevent the spread of invasive species, maintain soil fertility, protect soil from erosion, and increase soil C sequestration (McGahey et al., 2014).

Other pastoral systems (e.g. enclosed systems, ranching or agropastoralists) do not belong here because these are settled pastoral system and/or associated with the cultivation or uses of crops.

\section{Range of applicability}

Pastoralism simultaneously secures livelihoods, conserves ecosystem services, promotes wildlife conservation, and honours cultural values and traditions especially in dryland and semi-arid landscapes (Neely, Bunning and Wilkes, 2009), but in general occurs in places where feed resources are limited. Nomadic pastoralism is commonly practised in regions with little arable land, especially in the drylands of Africa, in the highlands of Asia and Latin America (Dong, 2016), and in the steppe lands of Eurasia. Transhumance pastoralism can be found on all continents.

\section{Impact on soil organic carbon stocks}

Dry and semi-arid rangelands are vulnerable to overgrazing and climate change, but these areas still capture and store large amount of carbon $(\mathrm{C})$. Rangeland soils are considered to be far from saturation (McGahey et al. 2014). Literature with measured C stocks is scarce (Table 158). Movement of livestock could lead to increase or maintain soil $\mathrm{C}$ sequestration. 
Table 158. Evolution of SOC stocks with pastoralism

\begin{tabular}{|c|c|c|c|c|c|c|c|}
\hline Location & Climate zone & Soil type & $\begin{array}{l}\text { Baseline C } \\
\text { stock } \pm \text { SD } \\
\text { (tC/ha) }\end{array}$ & $\begin{array}{l}\text { Additional C } \\
\text { storage (tC/ha/yr) }\end{array}$ & $\begin{array}{l}\text { Duration } \\
\text { (years) }\end{array}$ & More information & Reference \\
\hline $\begin{array}{l}\text { Guinan } \\
\text { county, } \\
\text { China }\end{array}$ & \multirow{2}{*}{ Alpine-cold } & \multirow{3}{*}{ NA } & \multirow{3}{*}{ NA } & 0.18 & NA & \multirow{2}{*}{$\begin{array}{l}\text { Seasonal } \\
\text { movement of } \\
\text { sheep and yak }\end{array}$} & $\begin{array}{l}\text { Zhuang and Li } \\
\text { (2017) }\end{array}$ \\
\hline $\begin{array}{l}\text { Ruoergai } \\
\text { county, } \\
\text { China }\end{array}$ & & & & 0.4 & 3 & & $\begin{array}{l}\text { Zhuang et al. } \\
\text { (2019) }\end{array}$ \\
\hline Senegal & Hot steppe & & & 0.04 & 1 & $\begin{array}{l}\text { Landscape level C } \\
\text { sequestration }\end{array}$ & $\begin{array}{l}\text { Assouma et al. } \\
\text { (2019) }\end{array}$ \\
\hline Botswana & $\begin{array}{l}\text { Warm-semi } \\
\text { arid }\end{array}$ & Entisols & $39.4 \pm 4.1$ & $\begin{array}{l}\text { Light grazing had } \\
\text { no effect on SOC, } \\
\text { but heavy grazing } \\
\text { decreased SOC }\end{array}$ & 2 & $\begin{array}{l}\text { Pastoral farming is } \\
\text { the principal } \\
\text { livelihood activity } \\
\text { across most of the } \\
\text { Kalahari }\end{array}$ & $\begin{array}{l}\text { Thomas et al. } \\
\text { (2015) }\end{array}$ \\
\hline
\end{tabular}




\section{Other benefits of the practice}

\subsection{Improvement of soil properties}

In general, pastoralism improves soil properties (Zhuang et al., 2019). When comparing pastoralism and sedentary livestock systems it was found that in general pastoralism improves soil properties (Zhuang et al., 2019; Table 159). However, grazing management and sedentary livestock production systems with high stocking rate could lead to soil erosion, degradation of vegetation and encroachment by unpalatable shrubs, $\mathrm{C}$ release from soil organic matter decomposition, loss of biodiversity due to habitat changes, and adverse impacts on soil hydrological function and water cycles (McGahey et al., 2014).

\subsection{Minimization of threats to soil functions}

Table 159. Soil threats

\begin{tabular}{|c|c|}
\hline Soil threats & \\
\hline Soil erosion & $\begin{array}{l}\text { Enhances soil structural formation (manure production, litter } \\
\text { accumulation) and compositional diversity (Zhuang et al., 2019). }\end{array}$ \\
\hline $\begin{array}{l}\text { Nutrient imbalance and } \\
\text { cycles }\end{array}$ & Improves nutrient cycling (Zhuang et al., 2019). \\
\hline $\begin{array}{l}\text { Soil } \\
\text { contamination/pollution }\end{array}$ & $\begin{array}{l}\text { Slow release of } \mathrm{N} \text { and other nutrients, preventing water } \\
\text { pollution by leaching (Yilmaz et al. 2019). }\end{array}$ \\
\hline Soil biodiversity loss & $\begin{array}{l}\text { Improved plant diversity, nutrient cycling influences below- } \\
\text { ground diversity (Zhuang et al., 2019). }\end{array}$ \\
\hline Soil compaction & Reduced trampling (Zhuang et al., 2019). \\
\hline Soil water management & $\begin{array}{l}\text { Reduces pressure on water resources; the herds consume water } \\
\text { on the move where it is available (Mekonnen and Hoekstra } \\
\text { 2012). }\end{array}$ \\
\hline
\end{tabular}

\subsection{Increases in production (e.g. food/fuel/feed/timber/fibre)}

Products mainly include food (meat, milk, and dairy products), but it could also include wool, hay, medical plants, dung pellet or timber (in case of wooded pastures). 


\subsection{Mitigation of and adaptation to climate change}

Pastoralism preserves soil C storage and increases $\mathrm{C}$ sequestration; therefore, it enhances mitigation capacity (Reid et al., 2004). Extensive farming systems have been found to be climate friendly (Koncz et al., 2017). However, climate change (drought) and desertification from livestock overgrazing (locally and depending on rangeland management, vegetation condition overgrazing could occur even under extensive management) emits globally as much as 100 million tonnes of $\mathrm{CO}_{2}$ equivalent per year (McGahey et al., 2014).

\subsection{Socio-economic benefits}

Pastoralism means the survival of many people especially for those with low incomes. This system is likely to be more resilient than sedentary livelihoods while preserving traditional knowledge (Neely, Bunning and Wilkes, 2009). However, higher economic valuation of the products and services provided by pastoralism and higher access to the markets could be a good tool to secure the benefits and C sink potential.

\subsection{Other benefits}

Despite increasing vulnerability of pastoralism (climate change, drought, marginalized market, etc.), pastoral systems provide a win-win scenario for preserving ecosystem services, sequestering $\mathrm{C}$, reversing environmental degradation and improving the health, well-being and long-term sustainability of livestock based livelihoods (Neely, Bunning and Wilkes, 2009).

\section{Potential drawbacks to the practice}

\subsection{Tradeoffs with other threats to soil functions}

In rangelands, most $\mathrm{C}$ is stored below ground and up to 70 percent of dryland soil $\mathrm{C}$ can be lost through conversion to agricultural use (McGahey et al., 2014). There is a trade-off between agricultural (cropland) and rangeland because cropland provides vegetable-based food production but lowers soil $\mathrm{C}$ and limits space for rangeland-based food production.

\subsection{Increases in greenhouse gas emissions}

Due to very low external inputs (lack of fertilization, irrigation, sowing, tillage, low use of electricity and industrialised equipment), pastoralism was shown to have very low GHG emission $\left(0.59 \mathrm{t} \mathrm{CO}_{2} \mathrm{eq} / \mathrm{ha}\right)$, which were lower than intensive system $\left(1.07 \mathrm{t} \mathrm{CO}_{2} \mathrm{eq} / \mathrm{ha}\right)$ when soil $\mathrm{C}$ sequestration was taken into account (Zhuang and $\mathrm{Li}, 2017)$. In another study in Senegal, the annual $\mathrm{C}$ balance of the pastoral ecosystem was $0.04 \pm 0.01 \mathrm{tC}$ eq/ha/year (sink), showing that total GHG emissions were mitigated by $\mathrm{C}$ accumulation in trees, soil and livestock (Assouma et al., 2019). 


\subsection{Conflict with other practice(s)}

Investment in intensified grassland management, sedentary livestock farming, land use change to cropland management, afforestation, protected areas, and industrial developments (extractive industries) and urbanization is in competition with pastoralism (Dong, 2016). Transboundary movements of people and animals could also be a source of conflict. Security of livestock is challenged due to movements of animals.

\subsection{Other conflicts}

Pastoralism requires low external inputs but it is labour intensive. Many people are employed in this sector. However, in many cases pastoralism is not a choice (job opportunity) but rather a fate (heritage). Many of those who can choose switch to other job possibilities (Galvin, 2009)

\section{Recommendations before implementing the practice}

Suitable agricultural policy and additional support could strengthen pastoralism. Loss of C could occur if pastoralism is applied inappropriately (e.g. during transformation of semiarid thicket by goat pastoralism (Mills et al., 2005)), or during introduction of domestic grazing on watersheds grazed by native herbivores (Bagchi and Ritchie, 2010). Grazing exclusion should be occasionally applied to restore $\mathrm{C}$ sink capacity of rangeland (Schönbach et al., 2012).

\section{Potential barriers for adoption}

Table 160. Potential barriers to adoption

\begin{tabular}{|l|l|l|}
\hline Barrier & YES/NO & \\
\hline Biophysical & Yes & $\begin{array}{l}\text { Desertification and land degradation in the drylands are reducing } \\
\text { the capacity of the land to sustain livelihoods (Neely, Bunning and } \\
\text { Wilkes, 2009). }\end{array}$ \\
\hline Cultural & Yes & $\begin{array}{l}\text { Pastoralism is less appealing and stereotyped. Declining prestige } \\
\text { (Blench, 2001) }\end{array}$ \\
\hline Social & Yes & $\begin{array}{l}\text { Pastoralists are often socially marginalized (Neely, Bunning and } \\
\text { Wilkes, 2009). }\end{array}$ \\
\hline Economic & Yes & Pastoralism is associated with low benefits. \\
\hline Institutional & Yes & $\begin{array}{l}\text { National instead of regional policy is not favouring pastoralism, } \\
\text { targeted subsidies are needed (Blench, 2001). }\end{array}$ \\
\hline
\end{tabular}




\begin{tabular}{|l|l|l|}
\hline Barrier & YES/NO & \\
\hline Legal (Right to soil) & Yes & $\begin{array}{l}\text { Pastoralists have often insecure land tenure rights (Neely, Bunning } \\
\text { and Wilkes, 2009). }\end{array}$ \\
\hline Knowledge & Yes & Requires indigenous knowledge (Blench 2001). \\
\hline
\end{tabular}

\section{Photos of the practice}

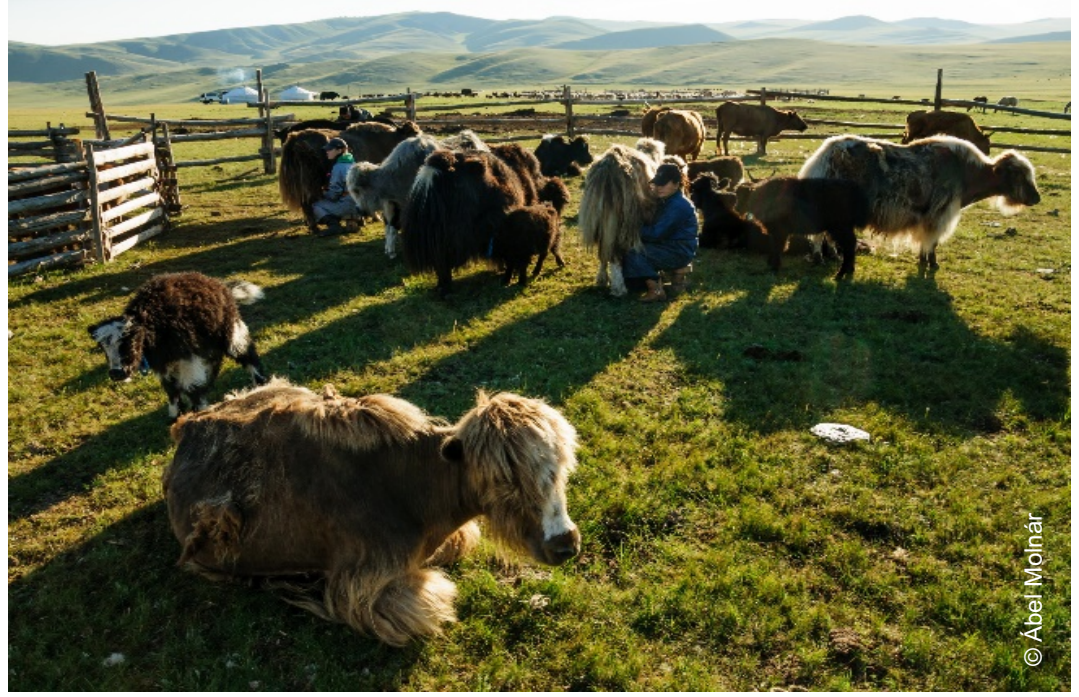

Photo 45. Nomadic pastoralism in Mongolia (Khövsgöl Province), milking of yaks (2017).

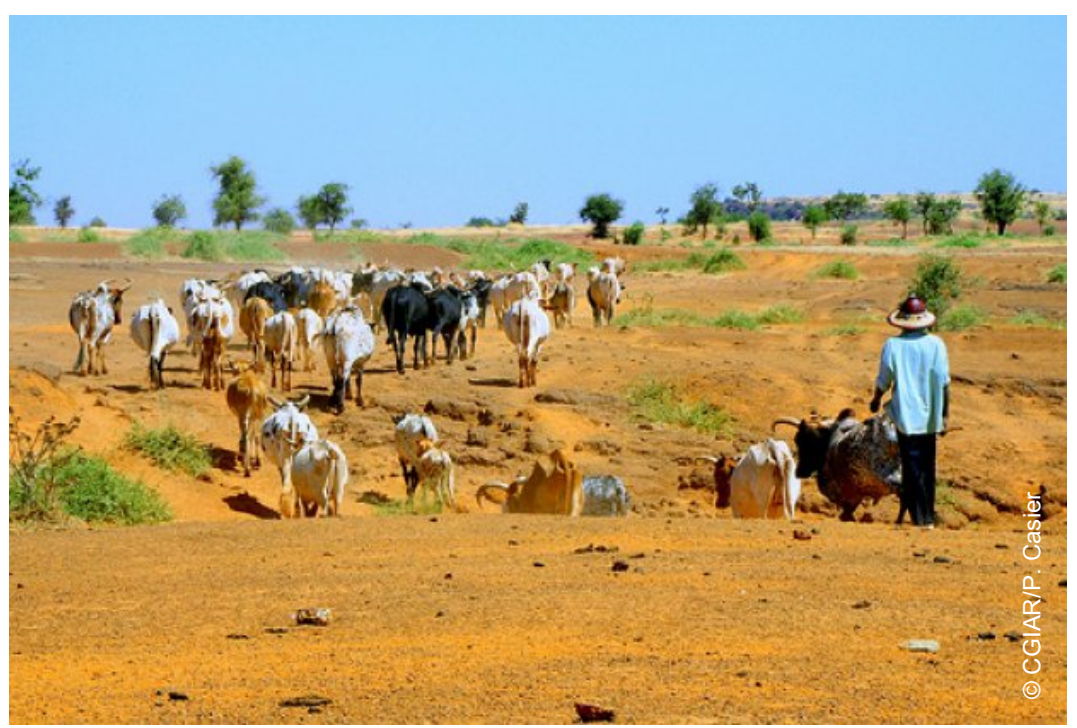

Photo 46. Free livestock movements in Africa. 


\section{References}

Assouma, M.H., Hiernaux, P., Lecomte, P., Ickowicz, A., Bernoux, M. \& Vayssières, J. 2019.

Contrasted seasonal balances in a Sahelian pastoral ecosystem result in a neutral annual carbon balance. Journal of Arid Environments, 162: 62-73. https://doi.org/10.1016/j.jaridenv.2018.11.013

Blench, R. 2001. 'You Can't Go Home Again': Pastoralism in the New Millennium (p. 103). London: Overseas Development Institute. http://www.fao.org/3/y2647e/y2647e00.htm

Bagchi, S. \& Ritchie, M.E. 2010. Introduced grazers can restrict potential soil carbon sequestration through impacts on plant community composition. Ecology Letters, 13: 959-968. https://doi.org/10.1111/j.14610248.2010.01486.x

Dong, S. 2016. Overview: Pastoralism in the World. In Dong, S., Kassam, K.A., Tourrand, J., Boone, R. (Eds.) Building Resilience of Human-Natural Systems of Pastoralism in the Developing World. Springer, Cham.

Galvin, K. A. 2009. Transitions: pastoralists living with change. Annual review of anthropology, 38: 185198. https://doi.org/10.1146/annurev-anthro-091908-164442

Koncz, P., Pintér, K., Balogh, J., Papp, M., Hidy, D., Csintalan, Z., Molnár, E., Szaniszló, A., Kampfl, G., Horváth, L. \& Nagy, Z. 2017. Extensive grazing in contrast to mowing is climate-friendly based on the farm-scale greenhouse gas balance. Agriculture, Ecosystems \& Environment, 240: 121-134.

https://doi.org/10.1016/j.agee.2017.02.022

Mekonnen, M.M. \& Hoekstra, A.Y. 2012. A global assessment of the water footprint of farm animal products. Ecosystems, 15: 401-415. https://doi.org/10.1007/s10021-011-9517-8

McGahey, D., Davies, J., Hagelberg, N. \& Ouedraogo, R. 2014. Pastoralism and the Green Economy-a natural nexus? Status, challenges and policy implications. IUCN and UNEP, Nairobi, Kenya. 58p. (also available at: https://portals.iucn.org/library/sites/library/files/documents/2014-034.pdf)

Mills, A.J., Cowling, R.M., Fey, M.V., Kerley, G.I.H., Donaldson, J.S., Lechmere-Oertel, R.G., Sigwela, A.M., Skowno, A.L. \& Rundel, P. 2005. Effects of goat pastoralism on ecosystem carbon storage in semiarid thicket, Eastern Cape, South Africa. Austral Ecology, 30(7): 797-804.

https://doi.org/10.1111/j.1442-9993.2005.01523.x

Neely, C., Bunning, S. \& Wilkes, A. 2009. Review of evidence on drylands pastoral systems and climate change: implications and opportunities for mitigation and adaptation. FAO, Rome. (also available at: http://www.fao.org/uploads/media/LWdisc_paper8_temp.pdf)

Reid, R.S., Thornton, P.K., McCrabb, G.J., Kruska, R.L., Atieno, F. \& Jones, P.G. 2004. Is it possible to mitigate greenhouse gas emissions in pastoral ecosystems of the tropics? Environment, Development and Sustainability, 6(1): 91. https://doi.org/10.1023/B:ENVI.0000003631.43271.6b

Schönbach, P., Wolf, B., Dickhöfer, U., Wiesmeier, M., Chen, W., Wan, H., Gierus, M., ButterbachBahl, K., Kögel-Knabner, I., Susenbeth, A., Zheng, X. \& Taube, F. 2012. Grazing effects on the 
greenhouse gas balance of a temperate steppe ecosystem. Nutrient Cycling in Agroecosystems, 93(3): 357371. https://doi.org/10.1007/s10705-012-9521-1

Thomas, A.D., Elliott, D.R., Griffith, T.N. \& Mairs, H. 2015. Chapter 11: Pastoralism and Kalahari Rangeland Soils. In Brearley, F.Q. \& Thomas, A.D. (Eds.) Land-Use Change Impacts on Soil Processes: Tropical and Savannah Ecosystems. ISBN: 9781780642109

Yılmaz, E., Zogib, L., Urivelarrea, P. \& Demirbaş, S. 2019. Mobile pastoralism and protected areas: conflict, collaboration and connectivity. Parks, 25: 6 .

Zhuang, M. \& Li, W. 2017. Greenhouse gas emission of pastoralism is lower than combined extensive/intensive livestock husbandry: A case study on the Qinghai-Tibet Plateau of China. Journal of Cleaner Production, 147: 514-522. https://doi.org/10.1016/j.jclepro.2017.01.126

Zhuang, M., Gongbuzeren, Zhang, J. \& Li, W. 2019. Community-based seasonal movement grazing maintains lower greenhouse gas emission intensity on Qinghai-Tibet Plateau of China. Land Use Policy, 85: 155-160. https://doi.org/10.1016/j.landusepol.2019.03.032 


\section{General Conclusions}

Grasslands (pasture, hay and silage with or without integrated livestock farming, arable cropping and forestry) represent 70 percent of the world agricultural area and contain 20-30 percent of the global SOC pool. Overall, they are a net $\mathrm{C}$ sink, thus contributing to climate change mitigation. Identifying optimum grassland management combining both profitable animal production and the delivery of ecosystem services, like $\mathrm{C}$ sequestration, is still a big challenge. Depending on the type of managements, there are negative and positive impacts on grassland ecosystem functions and C sequestration, as well as their adaptability and needs of protection across socio-economic and cultural settings. Understanding the potential impacts of grassland management practices, in particular with regards to sequester $\mathrm{C}$ in soils, is essential. Improvements in livestock and resource management are imperative to prevent overgrazing that decreases productivity, feeding efficiency and $\mathrm{C}$ sequestration, and increases GHG emissions.

Measures comprise the implementation of policies and practices for restoring and maintaining environmentally sustainable SOC density/stocks, while preserving grasslands, improving soil cover strategies, maintaining plant diversity/biomass, adopting appropriate grazing management, controlling stocking rates and trampling, improving manure management, keeping soil moisture favourable and improving livestock quality and productivity. Recommended practices include: (i) optimization of stocking rates to reduce land degradation, (ii) introduction of improved pasture species and legumes to increase above- and below-ground biomass production and SOC accumulation, (iii) application of recommended fertilization including manure to stimulate biomass production, and (iv) bringing degraded land under pasture to reduce erodibility while making the system a $\mathrm{C}$ sink to offset the potential for increased emissions of GHGs from grazing.

It is vital to understand the spatial pattern of SOC sequestration potentials and soil biodiversity impacting grassland ecosystem functions while taking conservation measures across grassland settings, restoring degraded ones, and improving converted lands and other pastoral systems. These could address the range of natural resources and social dimensions, encourage holistic approaches and partnership processes to achieve a vibrant and sustainable pastoral sector at national, regional and international levels. Recommended guidelines are provided in the summary table to take appropriate measures for grassland managements.

However, other generalized opportunities include supporting climate change mitigation and adaptation among people engaged in agricultural and livestock sectors and promoting technically advanced management options. Further research should be targeted to value natural grasslands and livestock-based ecosystems, develop methods for SOC measurement over time, as well as strategic monitoring and verification of C sequestration related to different management practices. This is to ensure full GHG accounting and balance while generating improved understanding of the socio-economic aspects of $\mathrm{C}$ sequestration involving people engaged in grassland management systems. Besides, tailored interventions (e.g. providing knowledge, technical know-how and innovative ideas in land use planning; building management capacity; improving communication among stakeholders; and offering financial support) in rural areas and developing countries are essential to address local issues by designing appropriate management practices that would improve soil $\mathrm{C}$ sequestration without compromising food security. 
Integrated

systems

and farming

approaches 


\section{Integrated crop-livestock systems}

\section{Alan J. Franzluebbers' ${ }^{1}$ Paulo C.F. Carvalho² ${ }^{2}$ Carlos A.C. Crusciol ${ }^{3}$, Fernando Garcia-Prechac ${ }^{4}$}

'United States Department of Agriculture, Agricultural Research Service, Raleigh, North Carolina, United States of America

${ }^{2}$ Federal University of Rio Grande do Sul, Faculty of Agronomy, Porto Alegre, Rio Grande do Sul, Brazil

${ }^{3}$ São Paulo State University, Department of Crop Science, Botucatu, São Paulo, Brazil

${ }^{4}$ Faculty of Agronomy, Republic University of Uruguay, Uruguay

\section{Description of the practice/concept}

Integrated crop-livestock systems (ICLS) vary widely depending on environmental, social, and cultural conditions, but the common thread is the intertwining of crop and livestock enterprises at some spatial and/or temporal levels (Franzluebbers, Sulc and Russelle, 2011). Integrated crop-livestock systems may occur on a single farm to gain synergies and efficiencies at the whole-farm level or can be on separate farms with the sharing/purchasing of resources from one enterprise and utilization on another (Russelle, Entz and Franzluebbers, 2007). Wide variations exist in ICLS based on type of livestock and crop species, climatic and edaphic factors within a region, and socio-economic conditions within a region (Photo 47). Some specific cases of ICLS include sod-based crop rotations, spreading of animal manure on croplands, dual-purpose grain crops, grazing of crop residues, grazing of cover crops, and integration of crops, livestock, and forestry (Photo 48, Photo 49, Photo 50 and Photo 51). At the simplest form of spreading animal manure onto cropland and feeding forage or grain to livestock, it becomes clear that two disparate agricultural operations simply share the resources, products, or by-products from one enterprise with another. This may often occur within the same farm, but it may also be conducted from mutual agreement among farms within a region or more distantly through shipping and purchasing arrangements on an open market. In more complex systems with livestock and crops intimately intertwined on the same parcels of land, the trampling and feeding actions of livestock can impart either negative or positive impacts on plant production and/or soil. Field-specific crop and grazing management is essential to gain the most benefit from integrated systems since success will depend on management of the entire system. 


\section{Range of applicability}

Integrated crop-livestock systems have applicability throughout the world. The most appropriate choice of a specific type of ICLS will be determined by climate and edaphic factors, as seasonal temperature, precipitation, and the degree to which soil (such as slope and hydric condition) may be able to support such management interactions influence the types of crops that can be grown, magnitude of forage production, and ability of soil to withstand trampling effects of livestock. Most suitable environments for the widest range of ICLS could be considered sub-humid and humid regions from temperate to tropical zones. Soils that remain continuously wet are not well suited for grazing ruminant livestock but may be ok for pasture-raised poultry. Arid regions have particular limitations due to the ability of land to sustain sufficient ground cover, so the extent of grazing has to be carefully managed to avoid permanent degradation. Irrigation of croplands in arid regions may provide necessary fodder for livestock during particular periods. Developing well-designed protocols for geospatial and temporal considerations are important to avoid economic catastrophes and long-term environmental damage. Integration of timber with crops and livestock can provide shade for livestock, fodder from mast, leaf droppings, or prunings, and alternative economic opportunities from multiple agricultural operations on the same farm, which ultimately provides multifunctionality of the agroecosystem.

\section{Impact on soil organic C sequestration / potential of additional storage}

Significant soil organic C sequestration is possible with ICLS when perennial forages are rotated with annual crops (Franzluebbers, Sawchik and Taboada, 2014). Several studies have reported high rates of soil organic C sequestration $(>0.75 \mathrm{t} \mathrm{C} / \mathrm{ha} / \mathrm{yr})$ with multiple years of perennial forages established on previously cropped agricultural land (Franzluebbers, 2010; Senapati et al., 2014). Additionally, grazing of seasonal annual forages in rotation with grain and/or fiber crops appears to have some potential to either stabilize soil organic C or lead to small increases in soil organic C (Table 161).

With digestibility of forages ranging from 40 to 80 percent, grazing allows return of a large portion of accumulated forage biomass back to the soil as either dung or trampled/uneaten forage. Other reasons for high soil organic $\mathrm{C}$ accumulation rates with forages are the intensive root systems that not only explore shallow surface soil layers with fine roots, but also penetrate deep into the profile, particularly for perennial forages. Root exploration is associated with a high degree of rhizodeposition (i.e. the process of leaving behind sloughed roots and secreting exudates that attract soil microorganism to decompose and process these $\mathrm{C}$ compounds into organic matter). Perennial forages also have the advantage of a long growth cycle, which allows $\mathrm{C}$ fixation and soil water extraction over a long period leading to greater $\mathrm{C}$ input and potentially reduced decomposition. These same factors of root exploration, promotion of organic $\mathrm{C}$ and $\mathrm{N}$ inputs to soil, and extending the growing season are present in grazed cover crop systems, particularly when farming systems are managed with conservation agricultural approaches, such as with minimum or no tillage (Assmann et al., 2014b). 
Table 161. Estimates of soil organic C sequestration with integrated crop-livestock systems around the world

\begin{tabular}{|c|c|c|c|c|c|c|c|c|}
\hline Location & Climate zone & Soil type & $\begin{array}{l}\text { Baseline } \\
\text { C stock } \\
\text { (tC/ha) }\end{array}$ & $\begin{array}{l}\text { Additional C } \\
\text { storage } \\
\text { (tC/ha/yr) }\end{array}$ & Duration & $\begin{array}{l}\text { Depth } \\
\text { (cm) }\end{array}$ & More information & Reference \\
\hline Argentina & Temperate & Typic Argiudoll & 55.1 & $0.63(t=0)$ & $7 \mathrm{yr}$ & $0-15$ & $\begin{array}{l}\text { Pasture-crop rotation sequence with different } \\
\text { years of pasture and cropping }\end{array}$ & $\begin{array}{l}\text { Studdert, Echeverria } \\
\text { and Casanovas (1997) }\end{array}$ \\
\hline Australia & Subtropical & $\begin{array}{l}\text { Typic } \\
\text { Chromustert }\end{array}$ & 24.7 & $0.65(\mathrm{BAU})$ & $4 \mathrm{yr}$ & $0-30$ & $\begin{array}{l}\text { Following 4-yr perennial pasture compared with } \\
\text { continuous cropping }\end{array}$ & Dalal et al. (1995) \\
\hline Brazil & $\begin{array}{l}\text { Subtropical with } \\
\text { warm, humid } \\
\text { summer }\end{array}$ & Rhodic Hapludox & 50.8 & $0.96(\mathrm{BAU})$ & $9 \mathrm{yr}$ & $0-20$ & $\begin{array}{l}\text { Soybean rotated with annual ryegrass/black oat } \\
\text { pasture; Moderate grazing intensity (only } 0.1 \mathrm{t} \\
\mathrm{C} / \mathrm{ha} \text { /yr with highest grazing intensity) }\end{array}$ & $\begin{array}{l}\text { Assmann et al. } \\
\text { (2014a) }\end{array}$ \\
\hline Brazil & Tropical & Typic Acrustox & 61 & $-0.62(t=0)$ & 13 & $0-30$ & & $\begin{array}{l}\text { Marchao et al. } \\
\text { (2009) }\end{array}$ \\
\hline Brazil & Tropical & Typic Acrustox & 66.9 & $-0.11(t=0)$ & 22 & $0-30$ & $\begin{array}{l}\text { ICLS compared with native vegetation condition; } \\
\text { ICLS had } 2.9 \mathrm{t} \text { C/ha more than no-till cropping } \\
\text { alone }\end{array}$ & $\begin{array}{l}\text { Sant-Anna et al. } \\
\text { (2017) }\end{array}$ \\
\hline China & Temperate & NA & 49.1 & $2.04(\mathrm{BAU})$ & $9 \mathrm{yr}$ & $0-100$ & $\begin{array}{l}\text { Following 9-yr lucerne crop compared with } \\
\text { continuous cropping }\end{array}$ & Hou et al. (2008) \\
\hline $\begin{array}{l}\text { France, } \\
\text { Denmark, } \\
\text { Sweden }\end{array}$ & $\begin{array}{l}\text { Mediterranean, } \\
\text { temperate, and } \\
\text { nordic }\end{array}$ & $\begin{array}{l}\text { Luvisol, Arenosol, } \\
\text { Cambisol (FAO) }\end{array}$ & & $\begin{array}{l}\text { Mediterranean }= \\
0.26 \pm 0.09(\mathrm{t}=0) \\
\text { Temperate }=0.32 \pm \\
0.11(\mathrm{t}=0) \\
\text { Nordic }=0.43 \pm \\
0.15(\mathrm{t}=0)\end{array}$ & $20 \mathrm{yr}$ & $0-30$ & $\begin{array}{l}\text { Simulations based on exogenous organic matter } \\
\text { inputs of } 1 \mathrm{tC} / \mathrm{ha} / \mathrm{yr}\end{array}$ & Peltre et al. (2012) \\
\hline Uruguay & Subtropical & Abruptic Argiudoll & 32.9 & $0.52(t=0)$ & $6 \mathrm{yr}$ & $0-20$ & $\begin{array}{l}\text { Pasture-crop rotation compared with continuous } \\
\text { cropping under no tillage }\end{array}$ & $\begin{array}{l}\text { Garcia-Prechac et al. } \\
(2004)\end{array}$ \\
\hline $\begin{array}{l}\text { United } \\
\text { States of } \\
\text { America }\end{array}$ & Temperate & $\begin{array}{l}\text { Typic } \\
\text { Kanhapludult }\end{array}$ & 43.3 & $0.89-1.31(t=0)$ & $7 \mathrm{yr}$ & $0-30$ & $\begin{array}{l}\text { Corn, sorghum, wheat grown with winter and } \\
\text { annual summer crops for grazing (marginally } \\
\text { greater rates of organic } C \text { sequestration rates } \\
\text { occurred without grazing) }\end{array}$ & $\begin{array}{l}\text { Franzluebbers and } \\
\text { Stuedemann (2014) }\end{array}$ \\
\hline
\end{tabular}




\section{Other benefits of the practice}

Forages grown in rotation with crops are known to affect a wide range of soil, environmental, socio-economic, landscape-scale, and cultural attributes, that collectively can be called ecosystem services (Franzluebbers, 2012). Grass leys, pastures, hayland, and conservation buffers are used extensively around the world, but have become less prevalent in more industrialized countries with the focus on specialized agricultural systems that rely on synthetic nutrient inputs. The value of ICLS to maintain a productive agricultural enterprise may not always be appreciated with the contemporary thrust to focus on high yield of specialized crops and livestock enterprises. However, stabilization of the whole-farm system in terms of production, environmental quality, and economic risk may be one of the most important attributes of ICLS in its widest distribution.

\subsection{Improvement of soil properties}

Perennial pastures rotated with crops can improve soil organic matter near the surface, which leads to a long list of other positive changes in soil physical, chemical, and biological properties (Franzluebbers, Sawchik and Taboada, 2014). Water infiltration increases due to the development of water-stable aggregates and abundant surface residue cover during and after pasture phases of the rotation, particularly when pastures are terminated without extensive soil disturbance. Although compaction can be a concern in intensively grazed pastures or with frequent hay cutting, the accumulation of soil organic matter at the surface often leads to lower soil bulk density (Franzluebbers, 2010). Cation exchange capacity increases with increasing soil organic matter. Soil microbial biomass and activity increase when total organic $\mathrm{C}$ increases (Franzluebbers and Stuedemann, 2008a). Changes in soil biological activity can lead to changes in nutrient cycling with greater amounts of $\mathrm{N}$ cycled internally within soil (Franzluebbers and Pershing, 2020).

Undersowing of corn silage with annual forages has been shown to increase soil organic matter, soil-extractable $\mathrm{P}, \mathrm{K}$, and $\mathrm{Mg}$, and base saturation, as well as reduce penetration resistance when silage is cut at $0.45-\mathrm{m}$ height rather than a traditional 0.2-m height (Pariz et al., 2017). Grazing of annual forages planted during or after summer grain crops can have positive (Deiss et al., 2020) or neutral effects (Franzluebbers and Stuedemann, 2008a) on soil biochemical properties, but often can lead to some degree of soil compaction if livestock graze during wet periods and forage resources diminish in mass toward the end of the growing season (Franzluebbers and Stuedemann, 2008b). Research in southern Brazil suggests that a moderate grazing intensity will optimize positive benefits with grazing of cover crops (Carvalho et al., 2010). This moderate grazing intensity can also stabilize yield variations and optimize economic returns (Nunes et al., 2021).

\subsection{Minimization of threats to soil functions}

Synergies between crop, timber, and livestock operations can lead to improved agroecological outcomes of ICLS on individual farms and when sharing of resources occurs in relatively proximity within watersheds (Ryschawy et al., 2017). Several ecosystem threats from conventional agricultural approaches can be minimized or prevented with adoption of ICLS: 


\begin{tabular}{|c|c|}
\hline Soil threats & \\
\hline Soil erosion & $\begin{array}{l}\text { Perennial forages rotated with food crops greatly reduce soil erosion [several studies } \\
\text { summarized in Singer, Franzluebbers and Karlen (2009)]. Although evidence for } \\
\text { erosion control in grazed cover crops or spreading of animal manure is scant, } \\
\text { maintaining greater soil cover with these annual forages likely reduces soil erosion if } \\
\text { trampling effects in heavy-use areas can be minimized. }\end{array}$ \\
\hline $\begin{array}{l}\text { Nutrient } \\
\text { imbalance and } \\
\text { cycles }\end{array}$ & $\begin{array}{l}\text { Abundant evidence suggests that balanced recycling of nutrients is possible with } \\
\text { dissolution and decomposition mediated by organic C inputs via the diversity of forages } \\
\text { and crops (Franzluebbers and Stuedemann, 2008a; Carvalho et al., 2010; Assmann et } \\
\text { al., 2014a; Deiss et al., 2016; Pariz et al., 2016, 2017; Denardin et al., 2020). Forage and } \\
\text { grain legumes in crop rotations offer the advantage of fixing atmospheric N via } \\
\text { biological N fixation and adding N to soil. }\end{array}$ \\
\hline $\begin{array}{l}\text { Soil salinization } \\
\text { and alkalinization }\end{array}$ & $\begin{array}{l}\text { Depending on adaptability of forages and crops, combinations of plant species may be } \\
\text { able to improve productivity of salt- and alkaline-affected soils. Avoiding salinization } \\
\text { with more continuous soil water uptake with mixed farming systems is likely. }\end{array}$ \\
\hline $\begin{array}{l}\text { Soil } \\
\text { contamination / } \\
\text { pollution }\end{array}$ & $\begin{array}{l}\text { Enriched soil organic matter cycling with a diversity of crops and forages consumed by } \\
\text { livestock will limit the need for inorganic amendments, and therefore, avoid } \\
\text { introduction of contaminants. Heavy metals that may be intrinsic in some animal } \\
\text { manures from confined livestock production facilities can be rendered less bio-available } \\
\text { with greater soil organic matter (Düring, Hoß } 3 \text { and Gäth, 2002). }\end{array}$ \\
\hline Soil acidification & $\begin{array}{l}\text { Integrated crop-livestock systems may not be able to prevent soil acidification, as } \\
\text { organic matter turnover through decomposition results in increasingly acidic soil, but } \\
\text { ICLS may lead to organo-Al complexation that can avoid negative impacts on crop } \\
\text { growth (Martins et al., 2020. However, animal manure amendments can sometimes } \\
\text { contain significant liming agents and could reduce acidification in some cases, } \\
\text { particularly from layer hen manure with high Ca excretion from the diet. }\end{array}$ \\
\hline $\begin{array}{l}\text { Soil biodiversity } \\
\text { loss }\end{array}$ & $\begin{array}{l}\text { Soil biodiversity can be greatly improved with greater diversity in crop rotations and } \\
\text { intercropping, leading to the accumulation of surface residues and soil organic matter in } \\
\text { ICLS (Moraes et al., 2014). Earthworms, dung beetles, nematodes, and springtails are } \\
\text { visible evidence, but large increases in soil microbial diversity and biological activity are } \\
\text { also apparent (Salton et al.,. 2014). }\end{array}$ \\
\hline Soil compaction & $\begin{array}{l}\text { Soil compaction is generally not significant under optimized ICLS condition, particularly } \\
\text { when using a no-tillage system, but it can be an issue when grazing occurs during wet } \\
\text { periods and when available forage is reduced below a critical threshold of } 1-2 \mathrm{t} / \text { ha. This } \\
\text { effect may be dependent on tillage system (Franzluebbers and Stuedemann, 2008b) } \\
\text { and soil texture. Overgrazing of forage crops can lead to excessive trampling that can } \\
\text { cause crusting and sealing of the surface. Heavy-use areas can limit water infiltration } \\
\text { (Pietola, Horn and Yli-Halla, 2005) }\end{array}$ \\
\hline
\end{tabular}




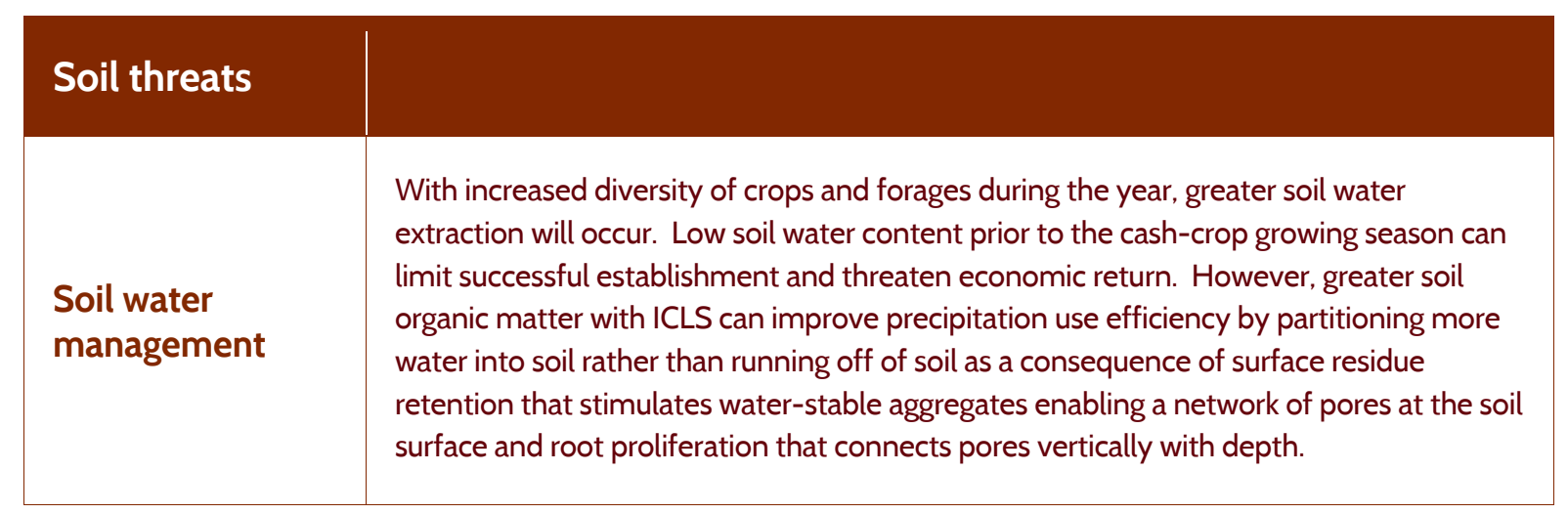

\subsection{On production}

Across almost all ICLS scenarios, productive capacity of a farm will increase if sufficient nutrients can be supplied. Compared with contemporary industrialized production of grains, ICLS will be able to match or improve crop yield potential but only in the year with that crop in the rotation. Monoculture production of a crop is typically not possible with ICLS. However, continuous annual production of soybean is being practiced in Brazil, and annual soybean production is not harmed when growing cover crops and grazing livestock are introduced (Moraes et al., 2014; Nunes et al., 2021). If viewed on balance across a region, ICLS offers greater stability and equal productive capacity of food, fuel, feed, and timber. Integrated crop-livestock-forestry has become a sustainable intensification strategy in Brazil (Alves, Madari and Boddey, 2017).

\subsection{Mitigation of and adaptation to climate change}

Greenhouse gas emissions may be both reduced and increased with ICLS, depending on the management approach and species of greenhouse gas of interest. Compared with specialized crop and livestock system without integration, any increase in soil organic $\mathrm{C}$ with ICLS would effectively reduce $\mathrm{CO}_{2}$ emissions to the atmosphere. This assumes that the lifespan for livestock would not be altered whether from specialized or ICLS. If specialized livestock production leads to reduced lifespan, then $\mathrm{CO}_{2}$ emissions could be lower than in ICLS.

Enteric $\mathrm{CH}_{4}$ emission from ruminant livestock is generally greater when forages are consumed via grazing than when fed as total mixed ration, assuming that forages are of moderate or low nutritive value (Harper et al, 1999). However, forages in ICLS can be of high nutritive value, particularly with annual forages from small grains and/or mixed grass-legume species. Higher digestibility and lower fiber concentration of forages leads to lower $\mathrm{CH}_{4}$ emission. Moderate grazing intensity in ICLS was able to reduce $\mathrm{CH}_{4}$ emission intensity compared with more intensive grazing intensity (Souza Filho et al., 2019).

The impact of ICLS on $\mathrm{N}_{2} \mathrm{O}$ emission depends on soil $\mathrm{N}$ availability and the need for $\mathrm{N}$ amendments to supplement the supply of $\mathrm{N}$ from mineralization of organic matter. Some studies indicate lower $\mathrm{N}_{2} \mathrm{O}$ emissions with ICLS (Sato et al., 2017), while other studies indicate greater $\mathrm{N}_{2} \mathrm{O}$ emissions with ICLS than conventional cropping systems (Piva et al., 2014). Limiting large pulses of inorganic $\mathrm{N}$ in soil solution will generally suppress $\mathrm{N}_{2} \mathrm{O}$ emissions. 


\subsection{Socio-economic benefits}

Integrated crop-livestock systems can have positive socio-economic benefits from the diversity of crops and livestock produced that offers risk abatement and opportunities for family members to lead a particular aspect of a whole-farm approach. Rural communities may be considered more vitally connected with diverse farming operations than those focused on large, specialized operations, in which vertical integration sidesteps the need for community organization and cooperation. If ICLS can reduce economic risk, increase productivity, and enhance resilience (Nunes et al., 2021), then rural landscapes will maintain popularity and vigor.

\section{Conflict with other practices, possible drawbacks}

\subsection{Tradeoffs with other threats to soil functions}

Adoption of any complex agricultural system may cause undesired consequences. Introducing livestock to a cash grain or horticultural farm will change nutrient and energy flows, and therefore the consequences of these interactions need to be accounted before adoption so that threats can be averted. Livestock trampling may be the most serious threat to soil, but this can be mitigated with rotational stocking and allowing sufficient forage mass. Some threats to soil from adoption of ICLS are listed below:

\section{Table 163. Soil threats}

\begin{tabular}{|c|c|}
\hline Soil threat & \\
\hline Soil erosion & $\begin{array}{l}\text { Soil erosion can still be a concern in ICLS on highly sloping landscapes that are marginal } \\
\text { for crop production. Treading by livestock can temporarily impede water infiltration and } \\
\text { lead to various forms of water erosion. }\end{array}$ \\
\hline $\begin{array}{l}\text { Nutrient } \\
\text { imbalance and } \\
\text { cycles }\end{array}$ & $\begin{array}{l}\text { Repeated application of animal manures with imbalanced NPK loads specific to crop } \\
\text { demands can lead to inorganic accumulation. An example of this has been the } \\
\text { elevation of soil P with repeated poultry litter application to target the N requirement of } \\
\text { crops. }\end{array}$ \\
\hline $\begin{array}{l}\text { Soil } \\
\text { contamination / } \\
\text { pollution }\end{array}$ & $\begin{array}{l}\text { Repeated application of animal manures with high metal concentrations to specific crop } \\
\text { fields can lead to contaminant concern. Application of P fertilizers can increase soil Cd } \\
\text { content, which can be taken up by forages and consumed by livestock to be deposited } \\
\text { in liver and kidneys. Concentration of Cd in P fertilizer depends on the source of } \\
\text { phosphoric rock. }\end{array}$ \\
\hline Soil compaction & $\begin{array}{l}\text { Repeated animal traffic with high stock density on tilled cropland or in heavy-use areas } \\
\text { of seasonal or perennial pastures can lead to compaction. }\end{array}$ \\
\hline $\begin{array}{l}\text { Soil water } \\
\text { management }\end{array}$ & $\begin{array}{l}\text { Careful attention must be given to termination of cover crops in anticipation of } \\
\text { following cash crops to avoid excessive moisture depletion and subsequent crop failure. }\end{array}$ \\
\hline
\end{tabular}




\subsection{Possible greenhouse gas emissions}

Few comprehensive evaluations have been made of net greenhouse gas emissions to estimate total global warming potential under ICLS (Peterson et al., 2020). Such studies are likely to occur in the near future with several long-term studies underway, particularly in Brazil with integrated crop-livestock-forestry systems. One closely related study in Brazilian beef cattle production found that the $\mathrm{C}$ footprint of improved pasture management scenarios was about half (8.1-8.9 $\mathrm{kg} \mathrm{CO}_{2}-\mathrm{C}$ equivalent per $\mathrm{kg}$ carcass) of that from degraded pastures ( $15.9 \mathrm{~kg} \mathrm{CO}_{2}$-C equivalent per $\mathrm{kg}$ carcass), while moderate improvement in pasture was intermediate (Cardoso et al., 2016). Moderately grazed native pastures were net sinks for greenhouse gases (computed as $\mathrm{CO}_{2}$ equivalence from soil organic $\mathrm{C}$ stock change, ruminant $\mathrm{CH}_{4}$ emission, and soil $\mathrm{N}_{2} \mathrm{O}$ emission) in North Dakota, while an introduced pasture that was heavily grazed and N-fertilized was a net source of global warming potential (Liebig et al., 2010).

\subsection{Conflictwith other practices}

No obvious conflicts occur with other good agricultural practices. However, sufficient hygiene precautions are needed when ICLS involves vegetable crops in the rotation with animal manure application or grazing. The risk of transmission of fecal-borne pathogens can be eliminated with appropriate timing of operations and sufficient soil biological activity to de-activate pathogens.

It should be noted that failures can occur between management components within a farm or when sharing resources across farms. When there is no spatio-temporal planning, the transition between crop and pasture phases can cause conflicts from different expectations with sowing dates and grazing periods. Poor grazing management that generates low biomass for the no-till cropping phase can also result in undesired outcomes.

\subsection{Negative impacts on production}

The only concern with production problems will be if the focus is to produce maximum quantity of a particular product on a parcel of land over time. Otherwise, the diversity of production and establishment of an agricultural system to foster environmental quality and biodiversity should have no negative impacts on production. Integrating livestock with crops in a diverse rotation sequence is a potential way of increasing soil biodiversity and, consequently, long-term sustainability.

\subsection{Other conflicts}

Spreading animal manure and having livestock grazing on land can be considered a nuisance, if a region has not had livestock present for some time. However, historically agriculture has always been associated with animal husbandry. 


\section{Recommendations before implementing the practice}

Management is the key to success in ICLS. Although there are a variety of different ICLS, some are more complex and difficult to implement than others. The first step is to plan ahead for each operation to take place at the right time. Grazing livestock may not be suitable for all landforms (e.g. highly dissected interfluvial parcels that would require excessive fencing or without sufficient water availability for livestock) or soil types (e.g. persistently wet soils that are prone to pugging). Crop production may not be suitable for highly sloping landscapes or regions without sufficient precipitation. Understanding the ecological limitations of a region are important for success. Market structures, storage and processing facilities, and timing of product availability are important considerations for success since farmers have little scope for improvising.

Depending on the need for hay and/or grazed perennial pasture, at least 3 years and preferably 5 years of sod helps to improve soil organic matter and other soil properties. Fewer years in sod means that annualized cost of seed and infrastructure (i.e. fencing and water) will be greater. More than 5 years may not provide as large a benefit to soil properties, as typically there is a non-linear effect.

Access to water and constructing temporary fencing in grazing of annual forages in rotation with cash crops are important considerations. Type of forage species as cover should fit the scheme of operations for cash crops, as well as consider cost and potential benefits to soil, the environment, and the agricultural enterprise. Machinery and labor necessary to manage both crops and livestock in an efficient and ecological manner are important.

\section{Potential barriers for adoption}

Social and ecological constraints to adoption of ICLS were recently reviewed following synthesis of what is known and unknown from research conducted mostly since the turn of the millennium (Garrett et al, 2017). Compared with more conventional management systems of specialized cropping or specialized livestock production, ICLS can (1) provide greater ecological integrity, (2) be more profitable when input costs are high and labor cost is low, and (3) reduce farm-level risk based on a diversity of enterprises. Integrated crop-livestock systems require a high level of management, may require a social network for support, and suitable policy incentives to be successful. Some key unknowns are (1) landscape-level economic resilience of ICLS and (2) the role of knowledge systems and social networks in influencing farmers' access to information and perceptions about costs and benefits. Agricultural research and extension agencies, civil society groups, and farmer-tofarmer knowledge transfers can all influence the adoption of best management and sustainable practices (Garrett et al., 2017). Additional barriers to adoption of ICLS are described below: 
Table 164. Potential barriers to adoption

\begin{tabular}{|c|c|c|}
\hline Barrier & YES/NO & \\
\hline Biophysical & Yes & $\begin{array}{l}\text { Adequate climatic conditions to meet ICLS goals should be } \\
\text { present to avoid failure. }\end{array}$ \\
\hline Cultural & Maybe & $\begin{array}{l}\text { Farm families must be willing to produce crops and raise } \\
\text { livestock or they have to cooperate with willing } \\
\text { partners/neighbors. }\end{array}$ \\
\hline Social & Maybe & $\begin{array}{l}\text { Markets and processing infrastructure have to be present for } \\
\text { commercial scale, but subsistence scale should not limit } \\
\text { adoption. Farmer age, willingness to engage in agroecological } \\
\text { concepts, and availability of supportive agricultural advisors may } \\
\text { be limitations for more widespread adoption. }\end{array}$ \\
\hline Economic & No & $\begin{array}{l}\text { Few external resources needed to take advantage of both crops } \\
\text { and livestock. }\end{array}$ \\
\hline Institutional & No & $\begin{array}{l}\text { No known barriers, unless there are specific ordinances against } \\
\text { raising livestock in a region. }\end{array}$ \\
\hline $\begin{array}{l}\text { Legal (Right } \\
\text { to soil) }\end{array}$ & No & No known barriers. \\
\hline Knowledge & Yes & $\begin{array}{l}\text { Well-rounded agricultural background will need to be studied or } \\
\text { experienced through an apprenticeship to be immediately } \\
\text { successful. }\end{array}$ \\
\hline
\end{tabular}




\section{Photos and graphical representation of the practice}

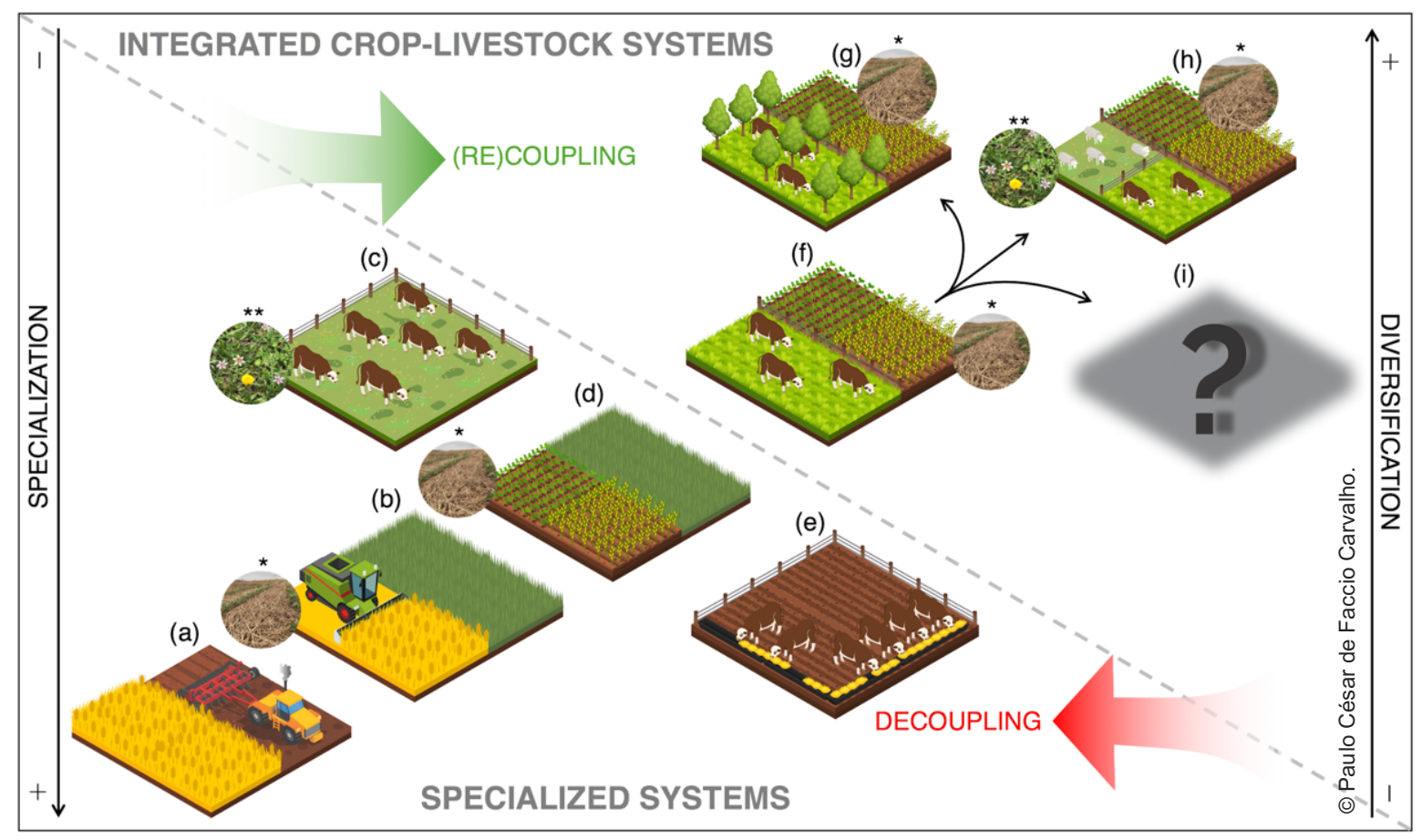

Photo 47. Conceptual model for decoupling and (re)coupling of crop and livestock production across a range of possible specialization/diversification scenarios.

Coupling of crop and livestock production can be done at any system level and at different spatio-temporal scales (i.e., at field, farm or landscape scale). The probability of synergies and complementarities occurring between system components is higher as system diversification increases. The same applies to the complexity and magnitude of biogeochemical cycles. System representations correspond to: (a) monocropping system under multi-pass tillage, (b) specialized cash crop production under no-till ( $\left.{ }^{*}\right)$ plus cover crops, (c) extensive livestock production on native grasslands $\left({ }^{* *}\right)$, (d) specialized cash crop production under no-till $\left({ }^{*}\right)$ plus cover crops plus crop rotation, (e) intensive livestock production in feedlots, (f) integrated system with livestock grazing cover crops plus cash crops under no-till $\left({ }^{*}\right)$ plus crop rotation, $(\mathrm{g})$ integrated system with livestock grazing cover crops plus cash crops under no-till $\left({ }^{*}\right)$ plus crop rotation plus trees, (h) integrated system with different livestock species grazing cover crops and native grasslands $\left({ }^{* *}\right)$ plus cash crops under no-till $\left({ }^{*}\right)$ plus crop rotation, and (i) any other crop-livestock combination not represented previously (could include silvopastoral systems with native grassland species and trees, livestock integration into perennial systems such as orchards and vineyards, or even mixed grazing). 


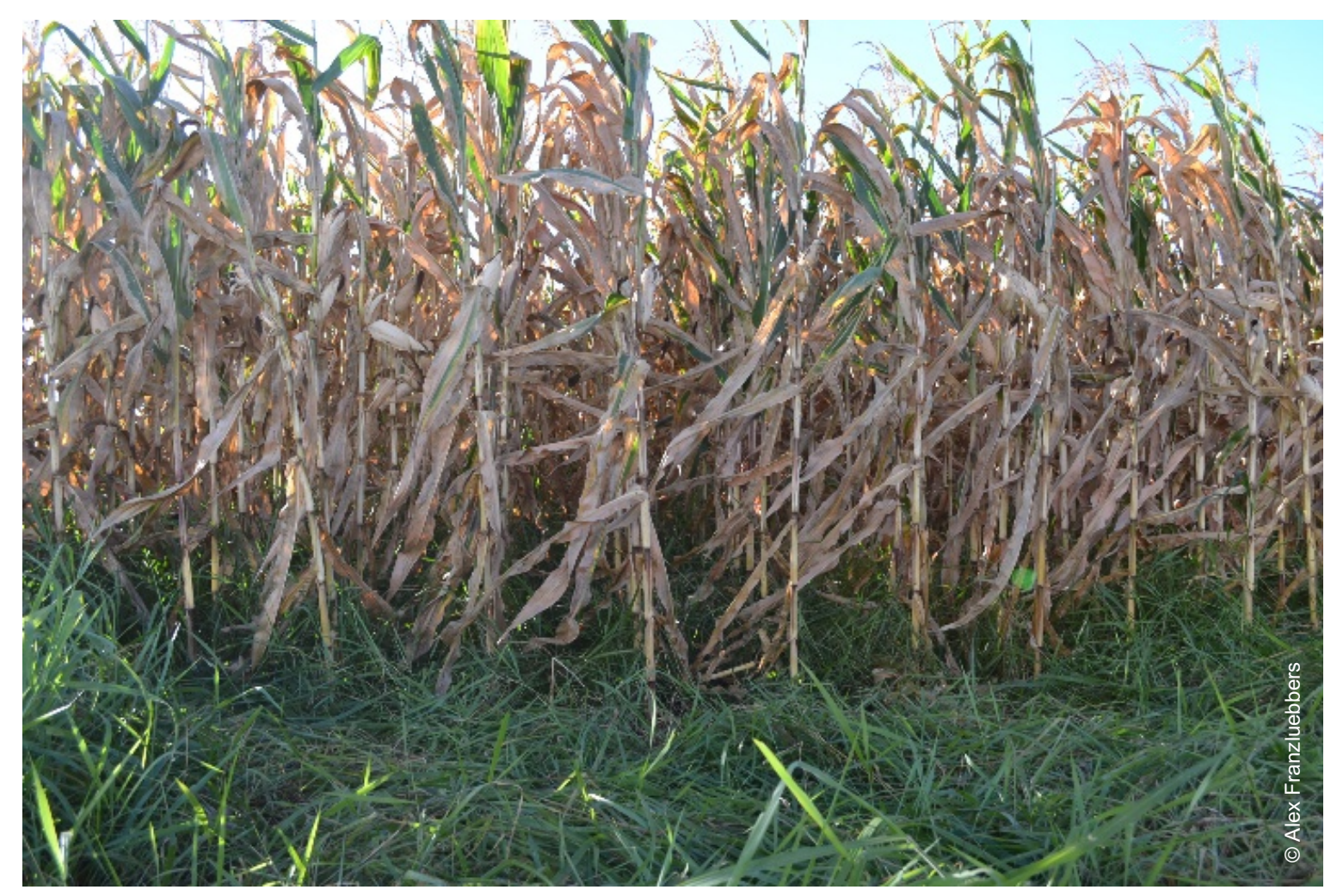

Photo 48. Urochloa forage intercropped with maize in the Federal District of Brazil, July 2015.

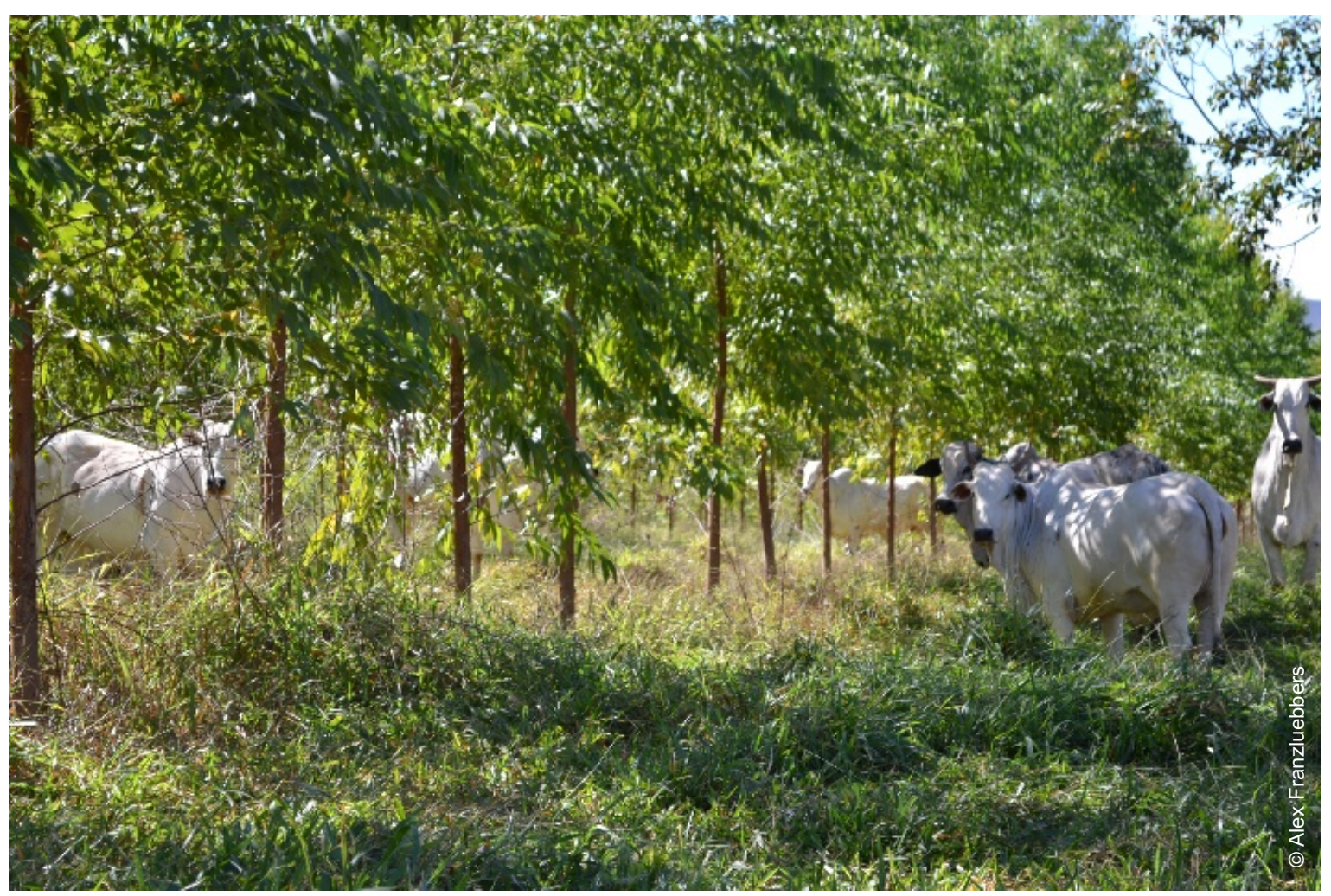

Photo 49. Nelore cattle grazing Urochloa forage under Eucalyptus silvopasture in the Federal District of Brazil, July 2015. 


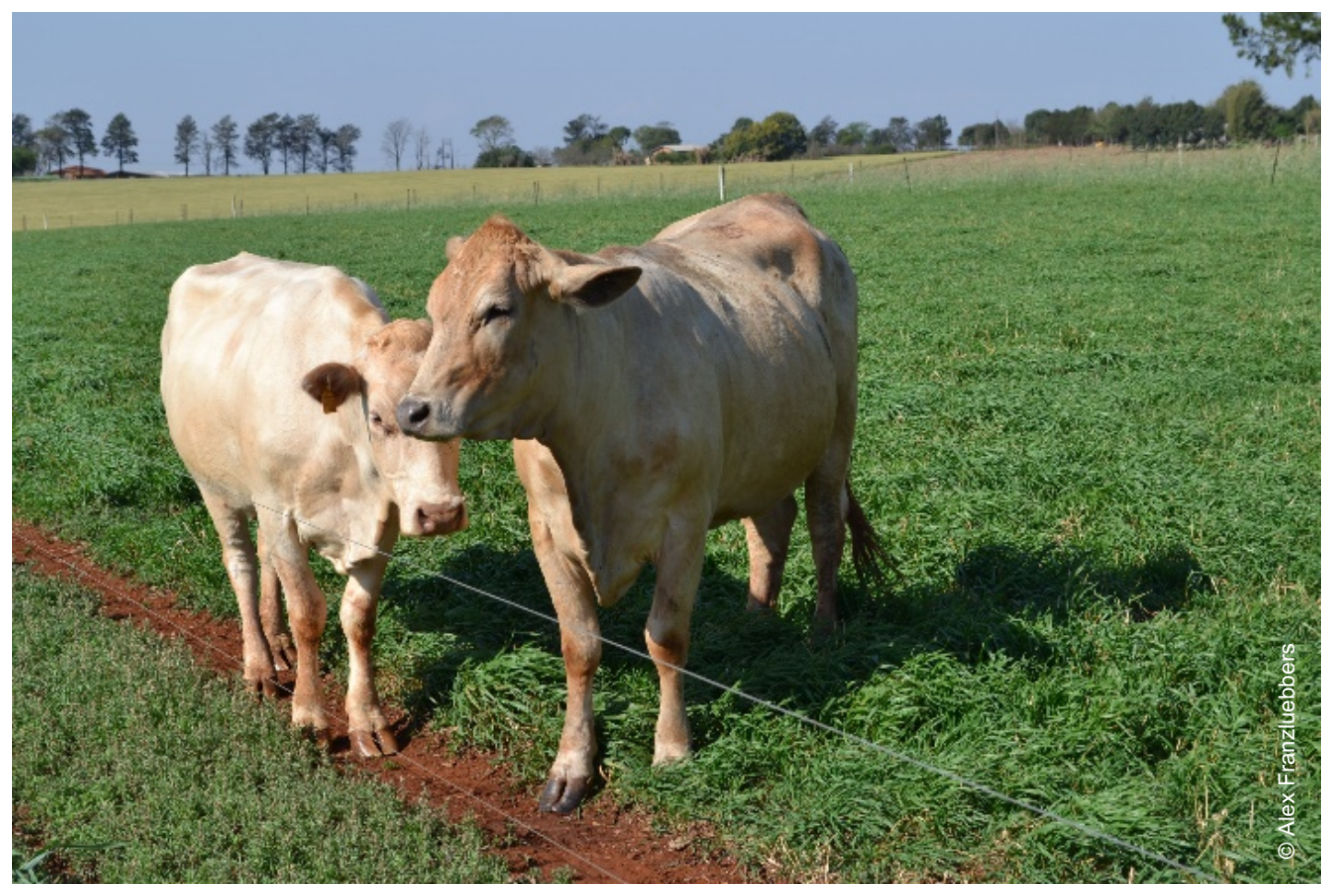

Photo 50. Cattle grazing winter annual forages prior to maize production in Parana, Brazil, August 2017.

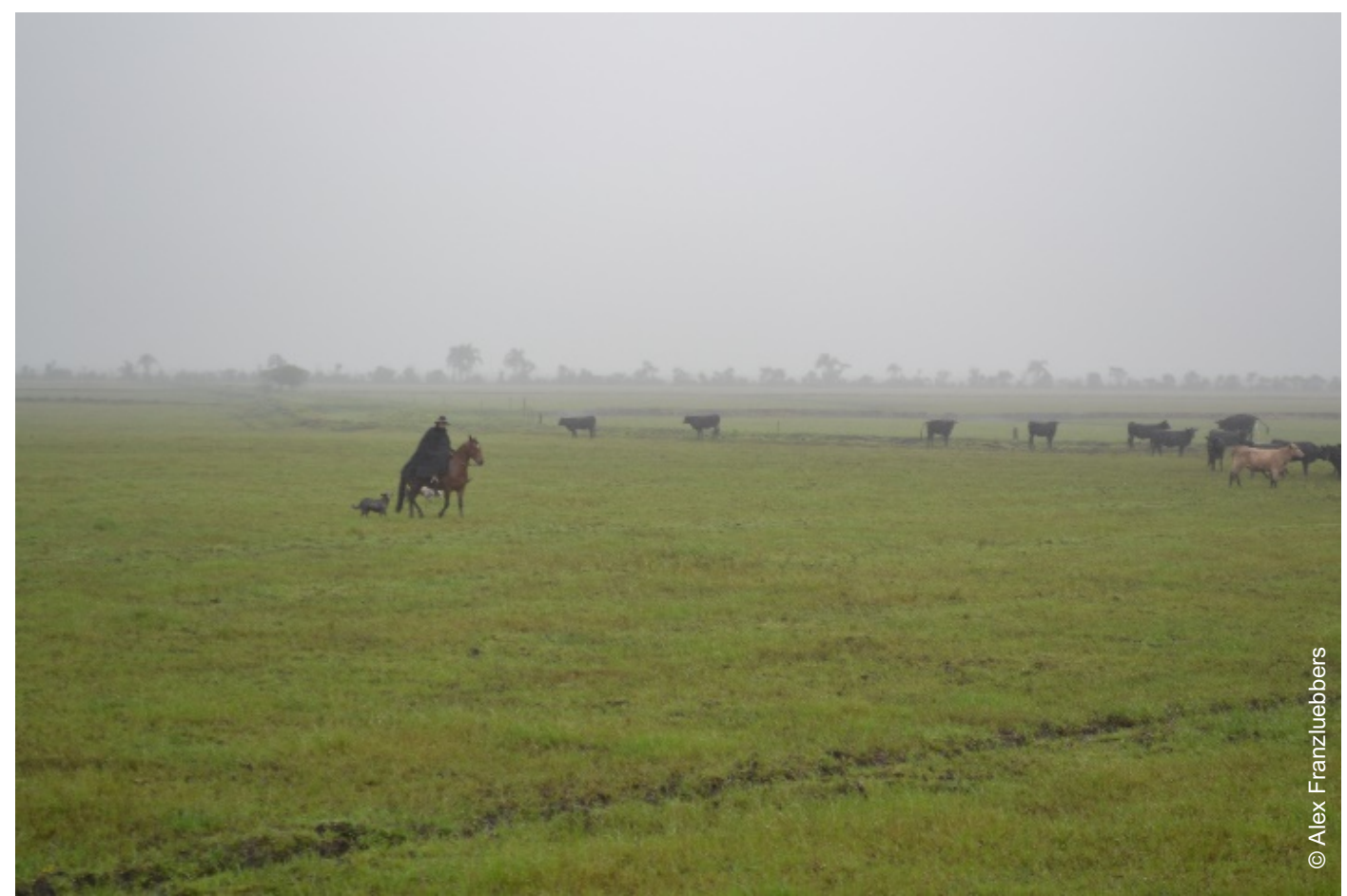

Photo 51. Cattle grazing winter annual forage prior to rice cultivation in Rio Grande do Sul, Brazil, September 2012. 
Table 165. Related cases studies available in volumes 3 and 5

\begin{tabular}{|c|c|c|c|c|}
\hline Title & Region & $\begin{array}{l}\text { Duration } \\
\text { of study } \\
\text { (Years) }\end{array}$ & Volume & $\begin{array}{l}\text { Case- } \\
\text { study No. }\end{array}$ \\
\hline $\begin{array}{l}\text { Pickle Melon (Cucumis melo) production in } \\
\text { Karapınar, Central Turkey }\end{array}$ & Eurasia & 60 & 3 & 24 \\
\hline $\begin{array}{l}\text { Increasing carbon inputs in agricultural } \\
\text { lands in Argentina: fertilizer use, inclusion } \\
\text { of cover crops and integration of perennial } \\
\text { pastures in crop rotations }\end{array}$ & $\begin{array}{l}\text { Latin } \\
\text { America } \\
\text { and the } \\
\text { Caribbean }\end{array}$ & 2 to 23 & 3 & 29 \\
\hline $\begin{array}{l}\text { Conservation agriculture in lowlands - an } \\
\text { experience from South America }\end{array}$ & $\begin{array}{l}\text { Latin } \\
\text { America } \\
\text { and the } \\
\text { Caribbean }\end{array}$ & 9 & 3 & 33 \\
\hline $\begin{array}{l}\text { Integrated farming in tropical } \\
\text { agroecosystems of Brazil }\end{array}$ & $\begin{array}{l}\text { Latin } \\
\text { America } \\
\text { and the } \\
\text { Caribbean }\end{array}$ & 4 to 12 & 3 & 34 \\
\hline $\begin{array}{l}\text { Integrated crop-livestock systems on SOC } \\
\text { sequestration in subtropical Brazil }\end{array}$ & $\begin{array}{l}\text { Latin } \\
\text { America } \\
\text { and the } \\
\text { Caribbean }\end{array}$ & 1.5 to 9 & 3 & 35 \\
\hline $\begin{array}{l}\text { Crop-pasture rotation on Black Soils of } \\
\text { Uruguay and Argentine }\end{array}$ & $\begin{array}{l}\text { Latin } \\
\text { America } \\
\text { and the } \\
\text { Caribbean }\end{array}$ & 10 to 48 & 3 & 39 \\
\hline
\end{tabular}




\section{References}

Alves, B.J.R., Madari, B.E. \& Boddey R.M. 2017. Integrated crop-livestock-forestry systems: Prospects for a sustainable agricultural intensification. Nutrient Cycling in Agroecosystems, 108: 1-4.

https://doi.org/10.1007/s10705-017-9851-0

Assmann, J.M., Anghinoni, I., Martins, A.P., Costa, S.E.V.G.A., Cecagno, D., Carlos, F.S. \&

Carvalho, P.C.F. 2014a. Soil carbon and nitrogen stocks and fractions in a long-term integrated croplivestock system under no-tillage in southern Brazil. Agriculture, Ecosystems and Environment, 190: 52-59. https://doi.org/10.1016/j.agee.2013.12.003

Assmann, T.S., Bortolli, M.A., Assmann, A.L., Soares, A.B., Pitta, C.S.R., Franzluebbers, A.J., Glienke, C.L. \& Assmann, J.M. 2014b. Does cattle grazing of dual-purpose wheat accelerate the rate of stubble decomposition and nutrients released? Agriculture, Ecosystems and Environment, 190: 37-42. https://doi.org/10.1016/j.agee.2014.01.011

Cardoso, A.S., Berndt, R., Leytem, A., Alves, B.J.R., Carvalho, I.N.O., Soares, L.H.B., Urquiaga, S. \& Boddey, R.M. 2016. Impact of the intensification of beef production in Brazil on greenhouse gas emissions and land use. Agricultural Systems, 143: 86-96. https://doi.org/10.1016/j.agsy.2015.12.007

Carvalho, P.C.F., Anghinoni, I., Moraes, A., Souza, E.D., Sulc, R.M., Lang, C.R., Flores, J.P.C., Lopes, M.L.T., Silva, J.L.S., Conte, O., Wesp, C.L., Levien, R., Fontanelli, R.S. \& Bayer, C. 2010. Managing grazing animals to achieve nutrient cycling and soil improvement in no-till integrated systems. Nutrient Cycling in Agroecosystems, 88: 259-273. https://doi.org/10.1007/s10705-010-9360-х

Dalal, R.C., Strong, W.M., Weston, E.J., Cooper, J.E., Lehane, K.J., King, A.J. \& Chicken, C.J. 1995. Sustaining productivity of a Vertisol at Warra, Queensland, with fertilisers, no-tillage, or legumes. 1. Organic matter status. Australian Journal of Experimental Agriculture, 35: 903-913.

Deiss, L., Kleina, G.B., Moraes, A., Franzluebbers, A.J., Motta, A.C.V., Dieckow, J., Sandini, I.E., Anghinoni, I. \& Carvalho, P.C.F. 2020. Soil chemical properties under no-tillage as affected by agricultural trophic complexity. European Journal of Soil Science, 71: 1090-1105.

https://doi.org/10.1111/ejss. 12869

Deiss, L., Moraes, A., Dieckow, J., Franzluebbers, A.J., Gatiboni, L.C., Sassaki, G.L. \& Carvalho, P.C.F. 2016. Soil phosphorus compounds in integrated crop-livestock systems of subtropical Brazil. Geoderma, 274: 88-96. https://doi.org/10.1016/j.geoderma.2016.03.028

Denardin, L.G.O., Martins, A.P., Carmona F.C., Veloso, M.G., Carmona G.I., Carvalho, P.C.F. \& Anghinoni, I. 2020. Integrated crop-livestock systems in paddy fields: New strategies for flooded rice nutrition. Agronomy Journal, 112: 2219-2229. https://doi.org/10.1002/agj2.20148

Düring, R.-A., Hoß, T. \& Gäth, S. 2002. Depth distribution and bioavailability of pollutants in long-term differently tilled soils. Soil and Tillage Research, 66: 183-195. https://doi.org/10.1016/S0167-

1987(02)00026-0 
Franzluebbers, A.J. 2010. Achieving soil organic carbon sequestration with conservation agricultural systems in the southeastern United States. Soil Science Society of America Journal, 74: 347-357.

https://doi.org/10.2136/sssaj2009.0079

Franzluebbers, A.J. 2012. Grass roots of soil carbon sequestration. Carbon Management, 3: 9-11. https://doi.org/10.4155/cmt.11.73

Franzluebbers, A.J. \& Pershing, M.R. 2020. Soil-test biological activity with the flush of $\mathrm{CO}_{2}$ : VIII. Soil type and management diversity. Soil Science Society of America Journal, 84: 1658-1674.

https://doi.org/10.1002/saj2.20128

Franzluebbers, A.J., Sawchik, J. \& Taboada, M.A. 2014. Agronomic and environmental impacts of pasture-crop rotations in temperate North and South America. Agriculture, Ecosystems and Environment, 190: 18-26. https://doi.org/10.1016/j.agee.2013.09.017

Franzluebbers, A.J. \& Stuedemann, J.A. 2008a. Early response of soil organic fraction to tillage and integrated crop-livestock production. Soil Science Society of America Journal, 72: 613-625.

https://doi.org/10.2136/sssaj2007.0121

Franzluebbers, A.J. \& Stuedemann, J.A. 2008b. Soil physical responses to cattle grazing cover crops under conventional and no tillage in the Southern Piedmont USA. Soil and Tillage Research, 100: 141-153. https://doi.org/10.1016/j.still.2008.05.011

Franzluebbers, A.J. \& Stuedemann, J.A. 2014. Temporal dynamics of total and particulate organic carbon and nitrogen in cover crop grazed cropping systems. Soil Science Society of America Journal, 78: 1404-1413. https://doi.org/10.2136/sssaj2014.01.0042

Franzluebbers, A.J., Sulc, R.M. \& Russelle, M.P. 2011. Opportunities and challenges for integrating North-American crop and livestock systems. In G. Lemaire, J. Hodgson \& A. Chabbi (Eds.) Grassland productivity and ecosystem services. pp. 208-218. CAB International.

https://doi.org/10.1079/9781845938093.0208

Garcia-Prechac, F., Ernst, O., Siri-Prieto, G. \& Terra, J.A. 2004. Integrating no-till into crop-pasture rotations in Uruguay. Soil and Tillage Research, 77(1): 1-13. https://doi.org/10.1016/j.still.2003.12.002

Garrett, R.D., Niles, M.T., Gil, J.D.B., Gaudin, A., Chaplin-Kramer, R., Assmann, A., Assmann, T.S., Brewer, K., Carvalho, P.C.F., Cortner, O., Dynes, R., Garbach, K., Kebreab, E., Mueller, N., Peterson, C., Reis, J.C., Snow, V. \& Valentim, J. 2017. Social and ecological analysis of commercial integrated crop livestock systems: Current knowledge and remaining uncertainty. Agricultural Systems, 155: 136-146. https://doi.org/10.1016/j.agsy.2017.05.003

Harper, L.A., Denmead, O.T., Freney, J.R. \& Byers, F.M. 1999. Direct measurements of methane emissions from grazing and feedlot cattle. Journal of Animal Science, 77: 1392-1401.

https://doi.org/10.2527/1999.7761392x

Hou, F.J., Nan, Z.B., Xie, Y.Z., Li, X.L., Lin, H.L. \& Ren, J.Z. 2008. Integrated crop-livestock production systems in China. The Rangeland Journal, 30: 221-231. https://doi.org/10.1071/RJ08018 
Liebig, M.A., Gross, J.R., Kronberg, S.L. \& Phillips, R.L. 2010. Grazing management contributions to net global warming potential: A long-term evaluation in the northern Great Plains. Journal of Environmental Quality, 39: 799-809. https://doi.org/10.2134/jeq2009.0272

Marchao, R.L., Becquer, T., Brunet, D., Balbino, L.C., Vilela, L. \& Brossard, M. 2009. Carbon and nitrogen stocks in a Brazilian clayey Oxisol: 13-year effects of integrated crop-livestock management systems. Soil and Tillage Research, 103: 442-450. https://doi.org/10.1016/j.still.2008.11.002

Martins, A.P., Denardin, L.G. de O., Tiecher, T., Borin, J.B.M., Schaidhauer, W., Anghinoni, I., Carvalho, P.C. de F. \& Kumar, S. 2020. Nine-year impact of grazing management on soil acidity and aluminum speciation and fractionation in a long-term no-till integrated crop-livestock system in the subtropics. Geoderma, 359: 113986. https://doi.org/10.1016/j.geoderma.2019.113986

Moraes, A., Carvalho, P.C.F., Anghinoni, I., Lustosa, S.B.C., Costa, S.E.V.G.A. \& Kunrath, T.R. 2014. Integrated crop-livestock systems in the Brazilian subtropics. European Journal of Agronomy, 57:4-9.

Nunes, P.A.A., Laca, E.A., Carvalho, P.C.F., Li, M., Filho, W.S., Kunrath, T.R., Martins, A.P. \& Gaudin, A. 2021. Livestock integration into soybean systems improves long-term system stability and profits without compromising crop yields. Scientific Reports, 11:1649. https://doi.org/10.1038/s41598-02181270-Z.

Pariz, C.M., Costa, C., Crusciol, C.A.C., Meirelles, P.R.L., Castilhos, A.M., Andreotti, M., Costa, N.R., Martello, J.M., Souza, D.M., Protes, V.M., Longhini, V.Z. \& Franzluebbers, A.J. 2017.

Production, nutrient cycling and soil compaction to grazing of grass companion cropping with corn and soybean. Nutrient Cycling in Agroecosystems, 108(1): 35-54. https://doi.org/10.1007/s10705-016$9821-y$

Pariz, C.M., Costa, C., Crusciol, C.A.C., Meirelles, P.R.L., Castilhos, A.M., Andreotti, M., Costa, N.R., Martello, J.M., Souza, D.M., Sarto, J.R.W. \& Franzluebbers, A.J. 2016. Production and Soil Responses to Intercropping of Forage Grasses with Corn and Soybean Silage. Agronomy Journal, 108(6): 2541-2553. https://doi.org/10.2134/agronj2016.02.0082

Peltre, C., Christensen, B.T., Dragon, S., Icard, C., Katterer, T. \& Houot, S. 2012. RothC simulation of carbon accumulation in soil after repeated application of widely different organic amendments. Soil Biology and Biochemistry, 52: 49-60. https://doi.org/10.1016/j.soilbio.2012.03.023

Peterson, C.A., Bell, L.W., Carvalho, P.C.F. \& Gaudin, A.C.M. 2020. Resilience of an integrated croplivestock system to climate change: A simulation analysis of cover crop grazing in southern Brazil. Frontiers in Sustainable Food Systems, 4: 604099. https://doi.org/10.3389/fsufs.2020.604099

Pietola, L., Horn, R. \& Yli-Halla, M. 2005. Effect of trampling by cattle on the hydraulic and mechanical properties of soil. Soil and Tillage Research, 82: 99-108. https://doi.org/10.1016/j.still.2004.08.004

Piva, J.T., Dieckow, J., Bayer, C., Zanatta, J.A., Moraes, A., Tomazi, M., Pauletti, V., Barth, G. \& Piccolo, M.C. 2014. Soil gaseous $\mathrm{N}_{2} \mathrm{O}$ and $\mathrm{CH}_{4}$ emissions and carbon pool due to integrated crop-livestock in a subtropical Ferralsol. Agriculture, Ecosystems and Environment, 190: 87-93.

https://doi.org/10.1016/j.agee.2013.09.008 
Russelle, M.P., Entz, M.H. \& Franzluebbers, A.J. 2007. Reconsidering integrated crop-livestock systems in North America. Agronomy Journal, 99: 325-334. https://doi.org/10.2134/agronj2006.0139

Ryschawy, J., Martin, G., Moraine, M., Duru, M. \& Therond, O. 2017. Designing crop-livestock integration at different levels: Toward new agroecological models? Nutrient Cycling in Agroecosystems, 108: 5-20. https://doi.org/10.1007/s10705-016-9815-9

Salton, J.C., Mercante, F.M., Tomazi, M., Zanatta, J.A., Concenco, G., Silva, W.M. \& Retore, M. 2014. Integrated crop-livestock system in tropical Brazil: Toward a sustainable production system. Agriculture, Ecosystems and Environment, 190: 70-79. https://doi.org/10.1016/j.agee.2013.09.023

Sant-Anna, S.A.C., Jantalia, C.P., Sa, J.M., Vilela, L., Marchao, R.L., Alves, B.J.R., Urquiaga, S. \& Boddey, R.M. 2017. Changes in soil organic carbon during 22 years of pastures, cropping or integrated crop/livestock systems in the Brazilian Cerrado. Nutrient Cycling in Agroecosystems, 108: 101-120. https://doi.org/10.1007/s10705-016-9812-Z

Sato, J.H., Carvalho, A.M., Figueiredo, C.C., Coser, T.R., Sousa, T.R., Vilela, L. \& Marchao, R.L. 2017. Nitrous oxide fluxes in a Brazilian clayey oxisol after 24 years of integrated crop-livestock management. Nutrient Cycling in Agroecosystems, 108: 55-68. https://doi.org/10.1007/s10705-017-9822-5

Senapati, N., Chabbi, A., Gastal, F., Smith, P., Mascher, N., Loubet, B., Cellier, P. \& Naisse, C. 2014. Net carbon storage measured in a mowed and grazed temperate sown grassland shows potential for carbon sequestration under grazed system. Carbon Management, 5(2): 131.

https://doi.org/10.1080/17583004.2014.912863

Singer, J.W., Franzluebbers, A.J. \& Karlen, D.L. 2009. Grass-based farming systems: Soil conservation and environmental quality. In W.F. Wedin \& S.L. Fales (Eds.) Grassland: Quietness and strength for a new American agriculture. pp. 121-136. American Society of Agronomy, Crop Science Society of America, Soil Science Society of America, Madison, WI.

Souza Filho, W., Nunes, P.A.A., Barro, R.S., Kunrath, T.R., Almeida, G.M., Genro, T.C.M., Bayer, C. \& Carvalho, P.C.F. 2019. Mitigation of enteric methane emissions through pasture management in integrated crop-livestock systems: Trade-offs between animal performance and environmental impacts. Journal of Cleaner Production, 213: 968-975. https://doi.org/10.1016/j.jclepro.2018.12.245

Studdert, G.A., Echeverria, H.E. \& Casanovas, E.M. 1997. Crop-pasture rotation for sustaining the quality and productivity of a Typic Argiudoll. Soil Science Society of America Journal, 61: 1466-1472. https://doi.org/10.2136/sssaj1997.03615995006100050026x 


\section{Agroforestry 1: Agrisilvicultural systems}

\section{Bernard Fungo ${ }^{1}$, Martin Wiesmeier ${ }^{2,3}$, Rémi Cardinael $4,5,6$}

1Agroforestry Research Program, National Agricultural Research Organization (NARO), Kampala, Uganda

${ }^{2}$ Chair of Soil Sciences, TUM School of Life Sciences Weihenstephan, Technical University of Munich, Freising, Germany

${ }^{3}$ Bavarian State Research Center for Agriculture, Institute for Organic Farming, Soil and Resource Management, Freising, Germany

${ }^{4}$ CIRAD, UPR AIDA, Harare, Zimbabwe

${ }^{5}$ AIDA, Université Montpellier, CIRAD, Montpellier, France

${ }^{6}$ University of Zimbabwe, Crop Science Department, Harare, Zimbabwe

\section{Description of the practice}

Agrisilviculture is an agroforestry practice in which trees are associated with crops on the same piece of land, either simultaneously (each component occupying a separate space but both existing at the same time) or sequentially (one component replacing another one in rotation) (Nair, 1985). An example of simultaneous practice is hedgerow intercropping/alley cropping (e.g. maize between rows of nitrogen-fixing trees such as Sesbania sp. or Gliricidia sp) while a sequential practice could be improved fallows (for instance, legume trees such as Calliandra sp in rotation with maize). Some of the benefits targeted by farmers include improved soil fertility, shade for understory crops by trees, trees acting as stakes for climbing plants, soil erosion control, improved microclimate and increased yield stability.

Agrisilvicultural practices affect soil organic carbon (SOC) primarily through litterfall, root turnover and exudates, and by increasing overall net primary productivity of the system (Cardinael et al., 2018a). The key processes leading to additional SOC carbon sequestration in agrisilvicultural systems compared to treeless systems include the increased input of organic matter both in the top- and subsoil and increased physical stabilization of organic matter due to improved soil aggregation. 


\section{Range of applicability}

Agrisilviculture is practiced in various cropping systems and in a variety of climatic zones and is of particular importance in small-scale and low input farming systems, especially in systems with limited access to mineral fertilizers. This is the case for many farmers in developing countries in sub Saharan Africa, South East Asia and Latin America, where agroforestry plays an important role of supplementing limited fertilizer inputs with nitrogen-fixing trees and controlling soil erosion in mountainous areas (Zomer et al., 2014). Trees are also frequently integrated in large-scaled farming systems in form of windbreaks, particularly in areas prone to wind erosion such as Eastern Europe and North America.

\section{Potential for $\mathrm{C}$ sequestration}

Several recent studies have shown that the conversion of croplands to agrisilvicultural systems has a positive effect on SOC stocks (Cardinael et al., 2018b; de Stefano and Jacobson, 2018; Feliciano et al., 2018; Shi et al., 2018). Some examples of carbon sequestration rates by agrisilvicultural systems are shown in Table 166. The rate of carbon sequestration varies widely depending on the climate zone, site conditions, tree species and management practices. Generally, under humid climate conditions higher annual rates of SOC accumulation are observed compared to arid/semi-arid environments. This may be due to the higher net primary productivity. 
Table 166. Examples of observed soil organic carbon (SOC) sequestration rates of selected agrisilvicultural systems

Where applicable, errors are standard errors of the mean.

\begin{tabular}{|c|c|c|c|c|c|c|c|c|c|}
\hline Location & Soil type & $\begin{array}{l}\text { Climate } \\
\text { zone }\end{array}$ & Agrisilvicultural system & $\begin{array}{l}\text { Baseline } \\
\text { stock } \\
\text { (tC/ha) }\end{array}$ & $\begin{array}{l}\mathrm{SOC} \\
\text { sequestration } \\
\text { rate }(\mathrm{tC} / \mathrm{ha} / \mathrm{yr})\end{array}$ & $\begin{array}{l}\text { More } \\
\text { information }\end{array}$ & $\begin{array}{l}\text { Depth } \\
(\mathrm{cm})\end{array}$ & $\begin{array}{l}\text { Duration of } \\
\text { experiment } \\
\text { (years) }\end{array}$ & Reference \\
\hline \multirow{2}{*}{ Canada } & $\begin{array}{l}\text { Grey-Brown } \\
\text { Luvisol }\end{array}$ & & $\begin{array}{l}\text { Populus deltoides } \times \text { Populus } \\
\text { nigra, maize/ wheat/ soybean } \\
\text { /barley }\end{array}$ & 81.0 & 0.30 & $\begin{array}{l}21 \text { years old, } 111 \\
\text { trees/ha }\end{array}$ & $0-30$ & 8 & $\begin{array}{l}\text { Bambrick et al. } \\
\text { (2010) }\end{array}$ \\
\hline & NA & & $\begin{array}{l}\text { Walnut, } \\
\text { wheat/soybean/barley }\end{array}$ & NA & 0.05 & $\begin{array}{l}25 \text { years old, } 111 \\
\text { trees/ha }\end{array}$ & $0-40$ & 25 & $\begin{array}{l}\text { Wotherspoon } \\
\text { et al. (2014) }\end{array}$ \\
\hline England & NA & & $\begin{array}{l}\text { Populus trichocarpa } \times \text { Populus } \\
\text { deltoids, wheat/barley }\end{array}$ & 4.1 & 0.46 & 19 years old & $0-150$ & 19 & $\begin{array}{l}\text { Upson and } \\
\text { Burgess (2013) }\end{array}$ \\
\hline \multirow{2}{*}{ France } & \multirow{2}{*}{ Fluvisol } & & $\begin{array}{l}\text { Hybrid walnut trees + durum } \\
\text { wheat }\end{array}$ & 31.5 & 0.35 & $\begin{array}{l}18 \text { years old, } 110 \\
\text { trees/ha }\end{array}$ & $0-100$ & 18 & $\begin{array}{l}\text { Cardinael et al. } \\
(2015)\end{array}$ \\
\hline & & & $\begin{array}{l}\text { Mixed tree species and arable } \\
\text { crops }\end{array}$ & 21.3 & 0.24 & 17.8 years old & $0-30$ & 17.8 & $\begin{array}{l}\text { Cardinael et al. } \\
(2017)\end{array}$ \\
\hline $\begin{array}{l}\text { Republic } \\
\text { of } \\
\text { Moldova }\end{array}$ & Chernozems & $\begin{array}{l}\text { Temperate } \\
\text { continental }\end{array}$ & $\begin{array}{l}\text { Windbreaks with Populus nigra, } \\
\text { Juglans regia, Quercus robur }\end{array}$ & 55.9 & 0.9 & $40-68$ years old & $0-30$ & 68 & $\begin{array}{l}\text { Wiesmeier et al. } \\
(2018)\end{array}$ \\
\hline Peru & $\begin{array}{l}\text { Typic Paleudult } \\
\text { soil }\end{array}$ & - & $\begin{array}{l}\text { Intercropping of annual crops } \\
\text { between Inga edulis contour } \\
\text { hedgerows } 4 \mathrm{~m} \text { apart }\end{array}$ & - & 0.14 & 5 years & $0-15$ & 5 & $\begin{array}{l}\text { Alegre and Rao } \\
(1996)\end{array}$ \\
\hline Senegal & $\begin{array}{l}\text { Three distinct soil } \\
\text { types used } \\
\text { (Arenosols, Ferric } \\
\text { Luvisols, and } \\
\text { chromic Vertisols) }\end{array}$ & Semi- arid & $\begin{array}{l}\text { Cultivated fields with scattered } \\
\text { trees, mainly Faidherbia albida } \\
\text { and Acacia sp }\end{array}$ & 59.3 & 0.43 & 25 years & $0-40$ & 5 & $\begin{array}{l}\text { Tschakert } \\
(2004)\end{array}$ \\
\hline
\end{tabular}




\section{Other benefits of the practice}

\subsection{Improvement of soil properties}

Agrisilviculture is associated with a large set of benefits for soil properties, including physical, chemical and biological improvements (Bayala et al., 2015: Cardinael et al., 2020). Increased amount of SOC is associated with enhanced water storage capacity and availability of nutrients for crops. Tree roots increase water infiltration and air movement, which improve crop performance. Integration of leguminous trees into agricultural systems increases the nitrogen supply through biological nitrogen fixation. Estimates of nitrogen fixation of Laucaena leucocephala ranged from $13 \mathrm{~kg} \mathrm{~N} / \mathrm{ha} / \mathrm{yr}$ to $500 \mathrm{~kg}$ N/ha/yr (Sanginga, Vanlauwe and Danso, 1995). Deeprooted trees can also retrieve nitrogen from the layer below to the crop rooting zone, and reduced nitrogen loss through leaching (Bergeron et al., 2011).

\subsection{Minimizing soil threats}

\section{Table 167. Soil threats}

\begin{tabular}{|c|c|}
\hline Soil threats & \\
\hline Soil erosion & $\begin{array}{l}\text { The litter layer produced by trees has an effect in reducing both runoff and } \\
\text { soil erosion. Reduction of wind and water erosion due to trees planted on } \\
\text { contour bands and in form of windbreaks (Zhu et al., 2019). }\end{array}$ \\
\hline $\begin{array}{l}\text { Nutrient } \\
\text { imbalance and } \\
\text { cycles }\end{array}$ & $\begin{array}{l}\text { Deep tree roots can capture leached nutrients below the crop rooting zone, a } \\
\text { process described as the "root safety net" (Rowe et al., 1998). Deep tree roots } \\
\text { are able to absorb nutrients in the subsoil and transfer it to the surface } \\
\text { (Vanlauwe et al., 2005), making them available for crops. }\end{array}$ \\
\hline $\begin{array}{l}\text { Soil salinization } \\
\text { and alkalinization }\end{array}$ & $\begin{array}{l}\text { Trees are often the first vegetation that is able to grow on salinized lands, as } \\
\text { some tree species, like eucalyptus, can withstand fairly high salt } \\
\text { concentration. }\end{array}$ \\
\hline $\begin{array}{l}\text { Soil contamination } \\
\text { / pollution }\end{array}$ & $\begin{array}{l}\text { Reduction of pesticides' transfer from soil to water by tree roots (Pavlidis et } \\
\text { al., 2018; Zhu et al., 2020). }\end{array}$ \\
\hline Soil acidification & $\begin{array}{l}\text { A study in West Africa showed Senna siamea trees could reduce soil } \\
\text { acidification (Vanlauwe et al., 2005). The observed increase in topsoil pH was } \\
\text { caused by the decomposition of calcium-rich residuesthat trees had absorbed } \\
\text { in the subsoil. }\end{array}$ \\
\hline
\end{tabular}




\begin{tabular}{|c|c|}
\hline Soil threats & \\
\hline $\begin{array}{l}\text { Soil biodiversity } \\
\text { loss }\end{array}$ & $\begin{array}{l}\text { Earthworm abundance, biomass and diversity are enhanced in agrisilvicultural } \\
\text { systems (Cardinael et al., 2019). In general, these systems have a positive } \\
\text { effect on soil fauna (Marsden et al., 2020). }\end{array}$ \\
\hline Soil compaction & $\begin{array}{l}\text { Tree roots open bio-pores for their growth, and through exudates and } \\
\text { association with mycorrhizal fungi improve particle aggregation, resulting in } \\
\text { improved soil structure (Coder, 2000). }\end{array}$ \\
\hline $\begin{array}{l}\text { Soil water } \\
\text { management }\end{array}$ & $\begin{array}{l}\text { Increased infiltration and reduced runoff and erosion as roots proliferate into } \\
\text { the soil (Zhu et al., 2019). }\end{array}$ \\
\hline
\end{tabular}

\subsection{Increased productivity (e.g. food/fuel/feed/timber)}

Hydraulic lift is defined as the passive movement of water through the roots of trees, from deeper and wetter soil layers to shallower and drier horizons, along a gradient of soil water potential (Bayala and Prieto, 2020). This process of water redistribution, together with increased soil fertility and better microclimate can increase crop yields, especially under arid conditions (Félix et al., 2018). A meta-analysis has shown that soil inorganic nitrogen $\left(\mathrm{NH}_{4}{ }^{+}\right.$and $\left.\mathrm{NO}_{3}{ }^{-}\right)$under agroforestry was 46 percent higher than in crop monocultures (Muchane $e t$ $a l ., 2020)$. In temperate regions, yield of annual crops per unit area in the agroforestry system is usually reduced (Van Vooren et al., 2017; Cardinael et al., 2018a; Pardon et al., 2018), mainly due to competition for light. However, the total productivity (trees + crops) of the system is often increased compared to a system with crops and trees grown separately (Graves et al., 2010).

\subsection{Climate change mitigation and adaptation}

Agrisilvicultural practices contribute to yield stability due to improved water retention and redistribution, as well as improved microclimate reducing heat stresses of crops (Takács et al., 2016). Income diversification through the diversification of agricultural productions (honey, timber, fruits, etc.) is also an adaptation measure because it provides alternatives when one crop fails as a result of unfavorable weather events, such as erratic rainfall or drought.

In addition to increased SOC stocks, agrisilvicultural systems have the potential to reduce direct $\mathrm{N}_{2} \mathrm{O}$ and $\mathrm{CH}_{4}$ emissions by up to 2.0 times (Kwak et al., 2019), and by the fixation of a part of nitrogen with legume tree species instead of the use of mineral nitrogen by up to 30 percent (Rosenstock et al., 2014), or by a reduction of cropped area (tree rows) and therefore associated reduction in mineral fertilizer use (Kim, Kirschbaum and Beedy, 2016). Indirect $\mathrm{N}_{2} \mathrm{O}$ emissions are also reduced due to a reduction in nitrogen leaching and runoff. 


\subsection{Socio-economic benefits}

Diversification of the income by tree-crop integration reduces food insecurity. Some trees such as Prunus africana, Wabugia ugandensis and Carisa edulis are medicinal and provide supplemental healthcare for resource-constrained farmer households (Galabuzi et al., 2010).

\subsection{Other benefits of the practice}

Increased habitat and species diversity, and improved aesthetics of agricultural landscapes (Jose, 2012).

\section{Conflict with other practice(s), Drawbacks}

\subsection{Tradeoffs with other threats to soil functions}

Table 168. Soil threats

\section{Soil threats}

Soil water

Agroforestry systems have an optimal tree density for water recharge. management

\subsection{Increases in greenhouse gas emissions}

Little evidence exists concerning the role of biological nitrogen fixation on emission of nitrous oxide but some studies suggested potential increased emissions when nitrogen-fixing trees are grown (Rosenstock et al., 2014).

\subsection{Conflict with other practice(s)}

Some crops such as cereals are strictly light demander and integration of trees significantly affects yield (Artru etal., 2017). 


\subsection{Decreases in production (e.g. food/fuel/feed/timber/fibre)}

After several years, a decrease in crop yield can be observed in mature and highly dense plantations, especially close to the trees, due to competition between crops and trees for light, water, and nutrients. Practicing agrisilviculture reduces land area available to annual crops. In some cases, trees can harbor pests of crops or provide nesting habitats to birds and rodents that damage crops. Some agroforestry species have negative allelopathic effects on food and fodder crops (Rizvi et al., 1999).

\subsection{Other conflicts}

Some trees harbor pests for crops. For example, Sesbania sesban has been blamed for supporting nematode growth in banana plantations in Uganda and hence reducing their productivity (Van der Veken et al., 2008).

\section{Practical recommendations}

- During land preparation, avoid clearing all vegetation completely (e.g. burning, forest conversion) or intensive land preparation that causes net loss of biomass or soil carbon

- Select tree species with deep rooting systems to reduce competition with crops but also to recycle nutrients leached below that crop roots (Sendzimir, Reij and Magnuszewski, 2011). Nitrogen-fixing species are preferable in poor soils and in low input systems. But a mix of species is recommended to reduce risks of failure (for example, from pests and storms) and generate income both on the short and long-term.

- Optimize the spacing and structure of the agroforestry system to optimize the use of nutrients, water and sunlight in order to ensure high productivity, but also to allow mechanization when available.

- Establish stabilizing structures before planting seedlings for soil erosion control in hilly landscapes.

- Retain prunings and other residues as well as organic fertilizers to the soil to increase soil nutrient stocks.

\section{Potential barriers for adoption}

Table 169. Potential barriers to adoption

\begin{tabular}{|l|c|l|}
\hline Barrier & YES/NO & \\
\hline Biophysical & Yes & $\begin{array}{l}\text { Higher altitudes, and lower temperature and sunlight intensity can be limiting } \\
\text { factors in the adoption of agroforestry as a source of shade for crops (Sood and } \\
\text { Mitchell, 2009). }\end{array}$ \\
\hline
\end{tabular}




\begin{tabular}{|c|c|c|}
\hline Barrier & YES/NO & \\
\hline Cultural & Yes & $\begin{array}{l}\text { Farmers' negative perception of the impact of trees can discourage them from } \\
\text { planting certain species (Etshekapea, Atangana and Khasa, 2018). }\end{array}$ \\
\hline Social & Yes & $\begin{array}{l}\text { Social learning through a network of peers, and lack of access to appropriate } \\
\text { extension services. Agroforestry systems are sometimes considered } \\
\text { subsistence practices. This negative connotation may prevent wider adoption. }\end{array}$ \\
\hline Economic & Yes & $\begin{array}{l}\text { Lack of capital to purchase required planting materials (seeds/seedlings). } \\
\text { Limited access to market of inputs and products of tree-based resources, and } \\
\text { limited access to infrastructure such as radio signals, saving and credit facilities, } \\
\text { central storage facilities for grain crops. The cost of maintaining trees together } \\
\text { with crops is higher compared to maintaining the components separately } \\
\text { because of the initial investment required, such as tree seedlings or fencing. } \\
\text { This can translate into negative cash flows in the first years. Limited access to } \\
\text { capital and deficits in farm management capacity can be major barriers for } \\
\text { adoption (Janssen at al., 2004). } \\
\text { Agrisilvicultural systems take a long time to show results due to slow } \\
\text { establishment rate of most trees compared with herbaceous components. This } \\
\text { demotivates farmers from adopting such systems. Maintaining an income } \\
\text { stream is a challenge when initially introducing or maintaining trees in a system. }\end{array}$ \\
\hline Institutional & Yes & $\begin{array}{l}\text { Absence of specific regulations on tree planting and and institutions or } \\
\text { inadequate enforcement reduces adoption rates. }\end{array}$ \\
\hline $\begin{array}{l}\text { Legal (Right } \\
\text { to soil) }\end{array}$ & Yes & $\begin{array}{l}\text { Farmers are more likely to adopt the technology if there is security of land } \\
\text { tenure. For example, communal land is considered less secure than freehold } \\
\text { land }\end{array}$ \\
\hline Knowledge & Yes & $\begin{array}{l}\text { Inadequate knowledge of the agronomic and economic benefits of tree-crop } \\
\text { interactions leads to the perception that trees occupy space that would have } \\
\text { been used for crop growth resulting in economic loss. Inadequate technical } \\
\text { skills to manage the tree-crop interactions, both from extension services and } \\
\text { farmers. } \\
\text { The knowledge required to manage trees is much different from that needed to } \\
\text { manage crops. Hence farmers may not easily manage both effectively. A } \\
\text { balance among the various components while extracting multiple products } \\
\text { (plant and animal) requires far greater understanding, skills, and experience to } \\
\text { maintain. }\end{array}$ \\
\hline Other & & $\begin{array}{l}\text { In open-grazed systems, livestock graze freely in the system, potentially } \\
\text { destroying crops and creating conflicts with farmers. }\end{array}$ \\
\hline
\end{tabular}




\section{Photo of the practice}

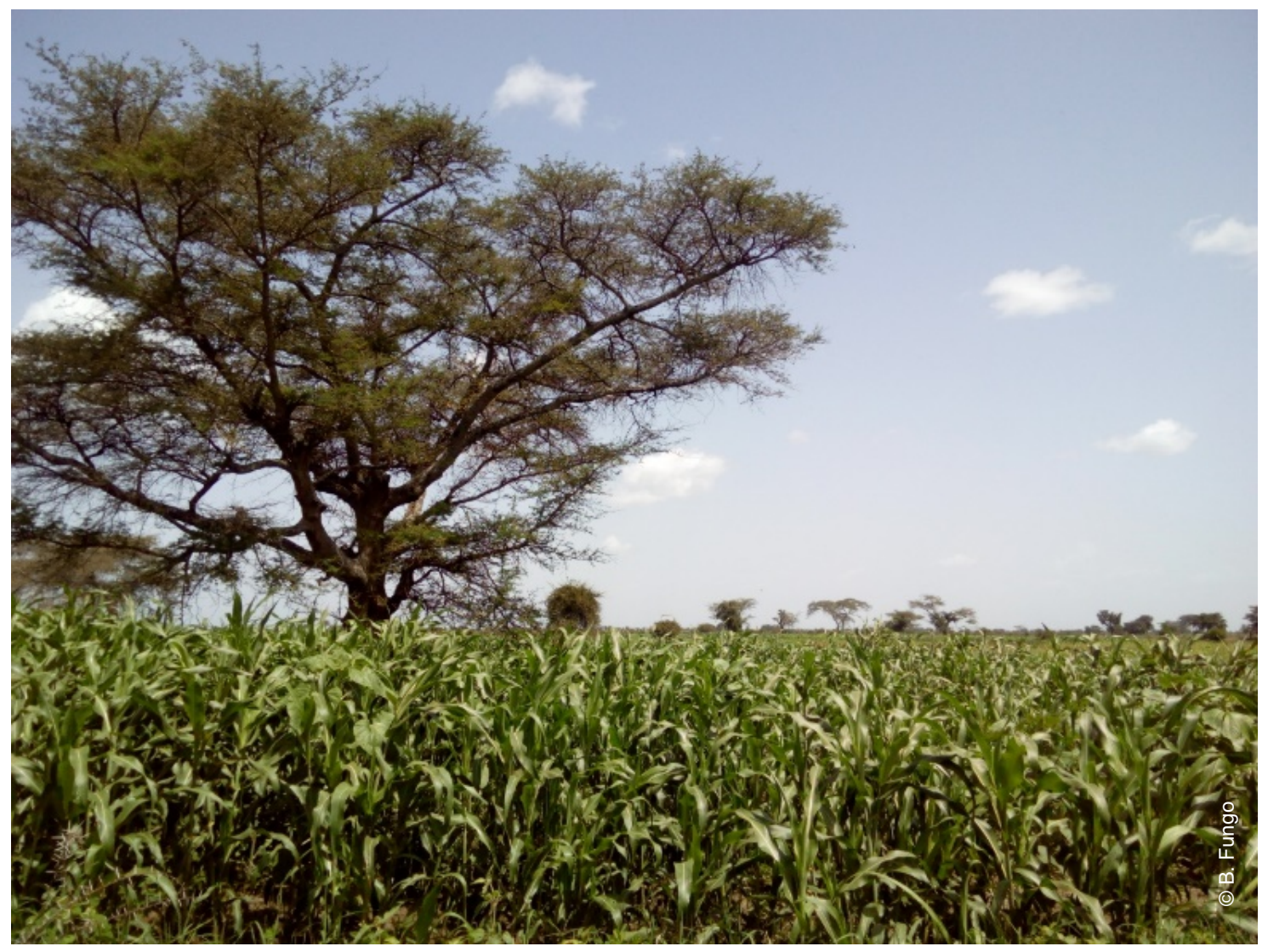

Photo 52. Sorghum under Faidherbia albida in Napak district, North-Eastern Uganda.

The good performance of sorghum under the tree canopy is due to increased soil fertility.

Table 170. Related cases studies available in volumes 3 and 5

\begin{tabular}{|l|l|l|c|c|}
\hline Title & Region & $\begin{array}{l}\text { Duration } \\
\text { of study } \\
\text { (Years) }\end{array}$ & Volume & $\begin{array}{l}\text { Case- } \\
\text { study } \\
\text { No. }\end{array}$ \\
\hline $\begin{array}{l}\text { Agroforestry, silvopastoral systems and } \\
\text { water funds initiatives contribute to } \\
\text { improve soil capacity to remove and } \\
\text { store carbon in Colombia }\end{array}$ & $\begin{array}{l}\text { Latin America } \\
\text { and the } \\
\text { Caribbean }\end{array}$ & $\begin{array}{c}9,20 \text { and } \\
40\end{array}$ & 3 & 36 \\
\hline
\end{tabular}




\section{References}

Alegre, J.C. \& Rao, M.R. 1996. Soil and water conservation by contour hedging in the humid tropics of Peru. Agriculture, Ecosystems \& Environment, 57(1): 17-25. https://doi.org/10.1016/01678809(95)01012-2

Artru, S., Garré, S., Dupraz, C., Hiel, M.-P., Blitz-Frayret, C. \& Lassois, L. 2017. Impact of spatiotemporal shade dynamics on wheat growth and yield, perspectives for temperate agroforestry. European Journal of Agronomy, 82: 60-70. https://doi.org/10.1016/j.eja.2016.10.004

Bambrick, A.D., Whalen, J.K., Bradley, R.L., Cogliastro, A., Gordon, A.M., Olivier, A. \&

Thevathasan, N. V. 2010. Spatial heterogeneity of soil organic carbon in tree-based intercropping systems in Quebec and Ontario, Canada. Agrofor. Syst., 79: 343-353. https://doi.org/10.1007/s10457-010-9305-Z

Bayala, J., Sanou, J., Teklehaimanot, Z., Ouedraogo, S.J., Kalinganire, A., Coe, R. \& van Noordwijk, M. 2015. Advances in knowledge of processes in soil-tree-crop interactions in parkland systems in the West African Sahel: A review. Agric. Ecosyst. Environ., 205: 25-35.

https://doi.org/10.1016/j.agee.2015.02.018

Bayala, J. \& Prieto, I. 2020. Water acquisition, sharing and redistribution by roots: applications to agroforestry systems. Plant Soil, 453: 17-28. https://doi.org/10.1007/s11104-019-04173-Z

Bergeron, M., Lacombe, S., Bradley, R. L., Whalen, J., Cogliastro, A., Jutras, M.-F. \& Arp, P. 2011. Reduced soil nutrient leaching following the establishment of tree-based intercropping systems in eastern Canada. Agrofor Syst, 83:321-330. https://doi.org/10.1007/s10457-011-9402-7

Cardinael, R., Chevallier, T., Barthès, B.G., Saby, N.P.A., Parent, T., Dupraz, C., Bernoux, M. \& Chenu, C. 2015. Impact of alley cropping agroforestry on stocks, forms and spatial distribution of soil organic carbon - A case study in a Mediterranean context. Geoderma, 259-260: 288-299. https://doi.org/10.1016/j.geoderma.2015.06.015

Cardinael, R., Chevallier, T., Cambou, A., Béral, C., Barthès, B.G., Dupraz, C., Durand, C., Kouakoua, E. \& Chenu, C. 2017. Increased soil organic carbon stocks under agroforestry: A survey of six different sites in France. Agric. Ecosyst. Environ., 236 : 243-255.

https://doi.org/10.1016/j.agee.2016.12.011

Cardinael, R., Guenet, B., Chevallier, T., Dupraz, C., Cozzi, T. \& Chenu, C. 2018a. High organic inputs explain shallow and deep SOC storage in a long-term agroforestry system - Combining experimental and modeling approaches. Biogeosciences, 15: 297-317. https://doi.org/10.5194/bg-2017-125

Cardinael, R., Umulisa, V., Toudert, A., Olivier, A., Bockel, L. \& Bernoux, M. 2018b. Revisiting IPCC Tier 1 coefficients for soil organic and biomass carbon storage in agroforestry systems. Environ. Res. Lett., 13: 1-20. https://doi.org/10.1088/1748-9326/aaeb5f 
Cardinael, R., Hoeffner, K., Chenu, C., Chevallier, T., Béral, C., Dewisme, A., \& Cluzeau, D. 2019. Spatial variation of earthworm communities and soil organic carbon in temperate agroforestry. Biol Fertil Soils, 55:171-183. https://doi.org/10.1007/s00374-018-1332-3

Cardinael, R., Mao, Z., Chenu, C., Hinsinger, P. 2020. Belowground functioning of agroforestry systems: recent advances and perspectives. Plant Soil 453, 1-13. https://doi:10.1007/s11104-020-04633-X

Coder, K.D. 2000. Trees and Soil Compaction: A Selected Bibliography. University of Georgia School of Forest Resources Extension Publication FOR00-1. 2pp.

de Stefano, A. \& Jacobson, M.G. 2018. Soil carbon sequestration in agroforestry systems: a meta-analysis. Agrofor. Syst., 92: 285-299. https://doi.org/10.1007/s10457-017-0147-9

Etshekapea P.G., Atangana A.R. \& Khasa D.P. 2018. Tree planting in urban and peri-urban of Kinshasa: Survey of factors facilitating agroforestry adoption. Urban Forestry \& Urban Greening, 30: 12-23.

https://doi.org/10.1016/j.ufug.2017.12.015

Feliciano, D., Ledo, A., Hillier, J. \& Nayak, D.R. 2018. Which agroforestry options give the greatest soil and above ground carbon benefits in different world regions? Agric. Ecosyst. Environ., 254: 117-129. https://doi.org/10.1016/j.agee.2017.11.032

Félix, G.F., Scholberg, J.M.S., Clermont-Dauphin, C., Cournac, L. \& Tittonell, P. 2018. Enhancing agroecosystem productivity with woody perennials in semi-arid West Africa. A meta-analysis. Agron Sustain Dev, 38:57. https://doi.org/10.1007/s13593-018-0533-3

Galabuzi, C., Agea, G.J., Fungo, B. \& Kamoga, R.M.N. 2010. Traditional Medicine as an alternative form of health care system: A preliminary case study of Nangabo Sub-County, Central Uganda. African Journal of Traditional Complementary and Alternative Medicine, 7(1): 11-16.

Graves, A.R., Burgess, P.J., Palma, J., Keesman, K.J., van der Werf, W., Dupraz, C., van Keulen, H., Herzog, F. \& Mayus, M. 2010. Implementation and calibration of the parameter-sparse Yield-SAFE model to predict production and land equivalent ratio in mixed tree and crop systems under two contrasting production situations in Europe. Ecological Modelling, 221(13): 1744-1756.

https://doi.org/10.1016/j.ecolmodel.2010.03.008

Ilstedt, U., Bargués Tobella, A., Bazié, H.R., Bayala, J., Verbeeten, E., Nyberg, G., Sanou, J., Benegas, L., Murdiyarso, D., Laudon, H., Sheil, D. \& Malmer, A. 2016. Intermediate tree cover can maximize groundwater recharge in the seasonally dry tropics. Scientific Reports, 6(1): 21930.

https://doi.org/10.1038/srep21930

Jose, S. 2012. Agroforestry for conserving and enhancing biodiversity. Agroforestry Systems, 85: 1-8. https://doi.org/10.1007/s10457-012-9517-5

Kim, D.G., Kirschbaum, M.U.F. \& Beedy, T.L. 2016. Carbon sequestration and net emissions of $\mathrm{CH}_{4}$ and $\mathrm{N}_{2} \mathrm{O}$ under agroforestry: Synthesizing available data and suggestions for future studies. Agric Ecosyst Environ, 226: 65-78. https://doi.org/10.1016/j.agee.2016.04.011 
Kwak, J.-H., Lim, S.-S., Baah-Acheamfour, M., Choi, W.-J., Fatemi, F., Carlyle, C.N., Bork, E.W. \& Chang, S.X. 2019. Introducing trees to agricultural lands increases greenhouse gas emission during spring thaw in Canadian agroforestry systems. The Science of the Total Environment, 652: 800-809.

https://doi.org/10.1016/j.scitotenv.2018.10.241

Marsden, C., Martin-Chave, A., Cortet, J., Hedde, M. \& Capowiez, Y. 2020. How agroforestry systems influence soil fauna and their functions - a review. Plant Soil, 453: 29-44.

https://doi.org/10.1007/s11104-019-04322-4

Muchane, M.N., Sileshi, G.W., Gripenberg, S., Jonsson, M., Pumariño, L. \& Barrios, E. 2020.

Agroforestry boosts soil health in the humid and sub-humid tropics: A meta-analysis. Agriculture, Ecosystems \& Environment, 295: 106899. https://doi.org/10.1016/j.agee.2020.106899

Nair, P.K.R. 1985. Classification of agroforestry systems. Agrofor Syst, 3: 97-128.

https://doi.org/10.1007/BF00122638

Pardon, P., Reubens, B., Mertens, J., Verheyen, K., De Frenne, P., DeSmet, G., Van Waes, C. \&

Reheul, D. 2018. Effects of temperate agroforestry on yield and quality of different arable intercrops.

Agricultural Systems, 166: 135-151. https://doi.org/10.1016/j.agsy.2018.08.008

Pavlidis, G. \& Tsihrintzis, V.A. 2018. Environmental Benefits and Control of Pollution to Surface Water and Groundwater by Agroforestry Systems: a Review. Water Resources Management, 32: 1-29.

https://doi.org/10.1007/s11269-017-1805-4

Rizvi, S.J.H., Tahir, M., Rizvi, V., Kohli, R.K. \& Ansari, A. 1999. Allelopathic Interactions in Agroforestry Systems. Critical Reviews in Plant Sciences, 18(6): 773-796.

https://doi.org/10.1080/07352689991309487

Rosenstock, T., Tully, K., Arias-Navarro, C., Neufeldt, H., Butterbach-Bahl, K. \& Verchot, L. 2014. Agroforestry with N2-fixing trees: sustainable development's friend or foe? Current Opinion in Environmental Sustainability, 6: 15-21. https://doi.org/10.1016/j.cosust.2013.09.001

Rowe, E.C., Hairiah, K., Giller, K.E., Van Noordwijk, M. \& Cadisch, G. 1998. Testing the safety-net role of hedgerow tree roots by 15N placement at different soil depths. Agroforestry Systems, 43(1): 81-93. https://doi.org/10.1023/A:1022123020738

Sanginga, N., Vanlauwe, B. \& Danso, S.K.A. 1995. Management of biological $\mathrm{N}_{2}$ fixation in alley cropping systems: Estimation and contribution to N balance. Plant Soil, 174: 119-141.

Shi, L., Feng, W., Xu, J. \& Kuzyakov, Y. 2018. Agroforestry systems: Meta-analysis of soil carbon stocks, sequestration processes, and future potentials. Land Degradation and Development, 29: 3886-3897. https://doi.org/10.1002/ldr.3136

Sendzimir, J., Reij, C. \& Magnuszewski, P. 2011. Rebuilding Resilience in the Sahel: Regreening in the Maradi and Zinder Regions of Niger. Ecology and Society, 16(3). https://doi.org/10.5751/ES-04198160301 
Sood, K.K., \& Mitchell, C.P. 2009. Identifying important biophysical and social determinants of on-farm tree growing in subsistence-based traditional agroforestry systems. Agroforestry Systems, 75: 175-187. https://doi.org/10.1007/s10457-008-9180-Z

Takács, Á., Kiss M., Hof A., Tanács E., Gulyás Á. \& Kántor, N. 2016. Microclimate modification by urban shade trees - An Integrated Approach to Aid Ecosystem Service Based Decision-making. Procedia Environmental Sciences, 32: 97-109. https://doi.org/10.1016/j.proenv.2016.03.015

Tschakert, P. 2004. Carbon for farmers: Assessing the potential for soil carbon sequestration in the old peanut basin of Senegal. Climatic Change, 67(2-3): 273-290. https://doi.org/10.1007/s10584-004$1821-2$

Upson, M.A. \& Burgess, P.J. 2013. Soil organic carbon and root distribution in a temperate arable agroforestry system. Plant Soil, 373: 43-58. https://doi.org/10.1007/s11104-013-1733-x

Van der Veken, L., Win, P.P., Elsen, A., Swennen, R. \& De Waele, D. 2008. Susceptibility of banana intercrops for rhizobacteria, arbuscular mycorrhizal fungi and the burrowing nematode Radopholus similis. Applied Soil Ecology, 40(2): 283-290. https://doi.org/10.1016/j.apsoil.2008.05.003

Van Vooren, L., Reubens, B., Broekx, S., De Frenne, P., Nelissen, V., Pardon, P. \& Verheyen, K. 2017. Ecosystem service delivery of agri-environment measures: A synthesis for hedgerows and grass strips on arable land. Agriculture, Ecosystems \& Environment, 244: 32-51. https://doi.org/10.1016/j.agee.2017.04.015

Vanlauwe, B., Aihou, K., Tossah, B.K., Diels, J., Sanginga, N. \& Merckx, R. 2005. Senna siamea trees recycle Ca from a Ca-rich subsoil and increase the topsoil $\mathrm{pH}$ in agroforestry systems in the West African derived savanna zone. Plant and Soil, 269(1): 285-296. https://doi.org/10.1007/s11104-004-0599-3

Wiesmeier, M., Lungu, M., Cerbari, V., Boincean, B., Hübner, R. \& Kögel-Knabner, I. 2018. Rebuilding soil carbon in degraded steppe soils of Eastern Europe: The importance of windbreaks and improved cropland management. Land Degradation \& Development, 29(4): 875-883.

https://doi.org/10.1002/ldr.2902

Wotherspoon, A., Thevathasan, N.V., Gordon, A.M. \& Voroney, R.P. 2014. Carbon sequestration potential of five tree species in a 25-year-old temperate tree-based intercropping system in southern Ontario, Canada. Agrofor Syst, 88: 631-643. https://doi.org/10.1007/s10457-014-9719-0

Zhu, X., Liu, W., Chen, J., Bruijnzeel, L.A., Mao, Z., Yang, X., Cardinael, R., Meng, F.-R., Sidle, R.C., Seitz, S., Nair, V.D., Nanko, K., Zou, X., Chen, C. \& Jiang, X.J. 2019. Reductions in water, soil and nutrient losses and pesticide pollution in agroforestry practices: a review of evidence and processes. Plant and Soil, 453: 45-86. https://doi.org/10.1007/s11104-019-04377-3

Zomer, R.J., Trabucco, A., Coe, R., Place, F., van Noordwijk, M. \& Xu, J.C. 2014. Trees on farms: an update and reanalysis of agroforestry's global extent and socio-ecological characteristics. Working Paper 179. Bogor, Indonesia, World Agroforestry Centre (ICRAF) Southeast Asia Regional Program. (also available at: http://apps.worldagroforestry.org/downloads/Publications/PDFS/WP14064.pdf) 


\section{Agroforestry 2: Sylvopastoral systems}

\section{Bernard Fungo', Martin Wiesmeier ${ }^{2,3}$, Rémi Cardinael ${ }^{4,5,6}$}

${ }^{1}$ Agroforestry Research Program, National Agricultural Research Organization (NARO), Kampala, Uganda

${ }^{2}$ Chair of Soil Sciences, TUM School of Life Sciences Weihenstephan, Technical University of Munich, Freising, Germany

${ }^{3}$ Bavarian State Research Center for Agriculture, Institute for Organic Farming, Soil and Resource Management, Freising, Germany

${ }^{4}$ CIRAD, UPR AIDA, Harare, Zimbabwe

${ }^{5}$ AIDA, Université Montpellier, CIRAD, Montpellier, France

${ }^{6}$ University of Zimbabwe, Crop Science Department, Harare, Zimbabwe

\section{Description of the practice}

Silvopastoralism is the practice of integrating trees with pastures and livestock husbandry. Trees may be planted or retained in a grazing system to supplement animal feed or to provide proteins during the dry season when grass is scarce and nutritive content is poor. In addition, these trees also provide shade and cooler temperature to animals and grasses. Silvopastoral systems include a diversity of systems, either intensive systems, for example fodder banks, intensive feed gardens, or extensively managed systems (open grazing) (Nair, 1985). Furthermore, silvopastoralism may involve cut-and-carry from systems in which fodder trees planted in one part of the farm are cut and transferred to feed livestock.

Several grazing management practices are known to have an impact on soil organic carbon sequestration: (i) stocking rate management (Mcsherry and Ritchie 2013; Zhou et al., 2017), (ii) rotational, planned or adaptive grazing (DeLonge and Basche, 2018), (iii) enclosure of livestock from grassland (Aynekulu et al., 2017; Wang et al. 2018), and (iv) pasture management (sowing, mixed leguminous/gramineous species, fertilization) (Fornara, Olave and Higgins, 2020).

In silvopastures, direct carbon inputs to the soil can be increased by (a) branches and trunks returning to the ground and decomposing (b) dung of grazing livestock, (c) belowground biomass input by woody species in both topsoil and subsoil. 


\section{Range of applicability}

Silvopastoral systems can be practiced where pastures and trees reveal suitable conditions. In temperate environments such as Europe and North America, integration of livestock (sheep and goats) within high value tree systems (e.g., apple orchards, olive groves, chestnut woodlands, and walnut plantations) is increasingly being practiced with the primary objective of maintaining the value of tree products like apples, olives, oranges, or nuts, or particularly high value timber (den Herder et al., 2017). In the dry lands of Sahel in West Africa and Eastern Africa (Kenya and Somalia, Uganda), silvopastoralism exists largely in the form of trees such as Acacia spp. and Faidherbia albida scattered on grazing lands (Bayala et al, 2014). Both large (cows, camels, donkeys) and small ruminant (goats, sheep) livestock are common. Trees are also being integrated in large-scale plantations in form of windbreaks in areas where strong winds cause dust storms and also damage livestock housing (Kort, 1988). In Eastern Africa, biomass transfer from fodder trees to livestock is a common practice using species such as Calliandra and Leucaena (Nair, 2014).

\section{Potential for $\mathrm{C}$ sequestration}

Trees planted on degraded grassland or unproductive pasture can lead to increased soil organic carbon (SOC) stocks. However, on productive pastures, especially in temperate regions, results are more contrasted, probably because SOC stocks are already very high (SOC saturation) in the treeless pastures (Upson, Burgess and Morison, 2016; Fornara, Olave and Burgess, 2018; Cardinael et al, 2018). A selection of evidence from different systems around the world shows that soil carbon sequestration or loss rates by silvopastoralism ranges from -0.72 to $2.2 \mathrm{tC} / \mathrm{ha} / \mathrm{yr}$ depending on the climate and soil conditions (Table 171). 
Table 171. Examples of soil organic carbon sequestration or loss rates of selected silvopastoral systems

\begin{tabular}{|c|c|c|c|c|c|c|c|c|}
\hline Location & Climate & Soil type & Silvopastoral system & $\begin{array}{l}\text { Baseline } \\
\text { stock } \\
\text { (tC/ha) }\end{array}$ & $\begin{array}{l}\text { Cseq rate } \\
\text { (tC/ha/yr) }\end{array}$ & $\begin{array}{l}\text { Depth } \\
\text { (cm) }\end{array}$ & More information* & Reference \\
\hline $\begin{array}{l}\text { Florida, } \\
\text { United } \\
\text { States of } \\
\text { America }\end{array}$ & & & $\begin{array}{l}\text { Slash pine (Pinus elliottii) + } \\
\text { bahiagrass (Paspalum notatum) }\end{array}$ & 30.3 & 2.2 & $0-125$ & $\begin{array}{l}\text { Conversion of open pastures to } \\
\text { silvopastoral systems with trees } \\
\text { ages ranging from age } 8,12,14 \\
\text { and } 40 \text { years. Tree density } \\
\text { rangesd from } 70-120 / \text { ha }\end{array}$ & $\begin{array}{l}\text { Haile, Nair } \\
\text { and Nair } \\
(2010)\end{array}$ \\
\hline $\begin{array}{l}\text { United } \\
\text { States of } \\
\text { America }\end{array}$ & $\begin{array}{l}\text { Mediterranean } \\
\text { with cool moist } \\
\text { winters and } \\
\text { warm dry } \\
\text { summers }\end{array}$ & $\begin{array}{l}\text { Vertic } \\
\text { Hyploxeroll }\end{array}$ & $\begin{array}{l}\text { Douglas-fir + perennial ryegrass + } \\
\text { subclover }\end{array}$ & 28.3 & 0.8 & $0-45$ & 11 years old, 571 trees/ha & $\begin{array}{l}\text { Sharrow and } \\
\text { Ismail (2004) }\end{array}$ \\
\hline $\begin{array}{l}\text { United } \\
\text { Republic of } \\
\text { Tanzania }\end{array}$ & $\begin{array}{l}\text { Tropical semi- } \\
\text { arid }\end{array}$ & Entisol & $\begin{array}{l}\text { Rotational woodlot of Acacia spp., } \\
\text { Gliricidia sepium and Leucaena } \\
\text { diversifolia }\end{array}$ & 6.1 & 2.2 & $0-15$ & $\begin{array}{l}5 \text {-year tree fallow in rotation } \\
\text { with annual crops, } 1406 \text { trees/ha }\end{array}$ & $\begin{array}{l}\text { Kimaro, Isaac } \\
\text { and } \\
\text { Chamshama } \\
\text { (2011) }\end{array}$ \\
\hline France & & & Wild cherry + ryegrass, fescue & 21.3 & $\begin{array}{l}-0.16 \\
0.49\end{array}$ & $\begin{array}{l}0-30 \\
0-50\end{array}$ & 26 years old, 200 trees/ha & $\begin{array}{l}\text { Cardinael et } \\
\text { al. (2017) }\end{array}$ \\
\hline Ireland & & & Fraxinus excelsior, Lolium perenne & 43.9 & -0.27 & $0-20$ & 26 years old, 400 trees/ha & $\begin{array}{l}\text { Fornara, } \\
\text { Olave and } \\
\text { Burgess } \\
(2018)\end{array}$ \\
\hline England & & & Ash trees, grassland & 27.5 & -0.72 & $0-40$ & 14 years old & $\begin{array}{l}\text { Upson, } \\
\text { Burgess and } \\
\text { Morison,. } \\
\text { (2016) }\end{array}$ \\
\hline Scotland & & & $\begin{array}{l}\text { Acer pseudoplantanus, Lolium } \\
\text { perenne }\end{array}$ & 75 & 0.32 & $0-50$ & 24 years old trial, 400 trees/ha & $\begin{array}{l}\text { Beckert et al. } \\
(2016)\end{array}$ \\
\hline
\end{tabular}

* The indicated age is always the age of the trees, i.e. age since planting 


\section{Other benefits of the practice}

\subsection{Improvement of soil properties}

Litterfall from trees and dung from livestock all supply organic matter that improve the soil quality for pasture growth. Roots of trees support an assemblage of soil microbes that promote nutrient cycling. Tree litter decomposition and incorporation with soil fauna together with tree roots biomass mitigate increased bulk density due to livestock compaction (Ford et al., 2019), therefore contributing to better water infiltration.

\subsection{Minimizing soil threats}

Table 172. Soil threats

\begin{tabular}{|c|c|}
\hline Soil threats & \\
\hline $\begin{array}{l}\text { Nutrient } \\
\text { imbalance and } \\
\text { cycles }\end{array}$ & $\begin{array}{l}\text { Trees absorb nutrients either located or leached below pasture root systems } \\
\text { and bring them at the surface through litter fall (Brakas and Aune, 2011). }\end{array}$ \\
\hline $\begin{array}{l}\text { Soil biodiversity } \\
\text { loss }\end{array}$ & $\begin{array}{l}\text { Substantial and preferably complex and multi-layered canopies formed by } \\
\text { native tree species, reduced levels of disturbance, and high levels of litter and } \\
\text { soil organic matter - are also basic ingredients of land use systems that } \\
\text { harbor elevated levels of biodiversity in vegetation, litter, and soil (Negasa et } \\
\text { al. 2017; Pinto-Correia et al., 2018). }\end{array}$ \\
\hline Soil compaction & $\begin{array}{l}\text { Tree litter decomposition and incorporation with soil fauna together with tree } \\
\text { roots biomass mitigate increased bulk density due to livestock compaction } \\
\text { (Ford et al., 2019), therefore contributing to better water infiltration. }\end{array}$ \\
\hline $\begin{array}{l}\text { Soil water } \\
\text { management }\end{array}$ & $\begin{array}{l}\text { Soil moisture availability and mild temperature under trees create better } \\
\text { condition for mineralization of nitrogen (N) which contributes to improving } \\
\text { and extending the forage quality in the dry season (Nair et al., 2011). }\end{array}$ \\
\hline
\end{tabular}

\subsection{Productivity (e.g. Food/Fuel/Feed/Timber)}

Silvopastoral systems provide fodder for livestock, in addition to providing shade. This improves overall productivity of the farming system through tolerance to low soil fertility (by efficient nutrient acquisition and utilization); formation of symbiotic associations with mycorrhizae and rhizobia that enhance their access to immobile phosphorus and atmospheric $\mathrm{N}_{2}$, respectively (García-Tejero and Taboada, 2016); and contributing to efficient use of nutrients for biomass production (Suarez et al., 2018). Food products such as nuts or fruits harvested from the trees are additional of silvopastoral systems (Luedeling et al., 2011). 


\subsection{Mitigation of and adaptation to climate change}

Trees such as Faidherbia albida in pasture lands provide seeds and foliage that can be used as supplemental feed during the dry season. Shade provided by the trees minimize the impact of high temperature on livestock and helps maintaining milk production (Paterson, Kiruiro and Arimi, 1999; Magalhães et al., 2020). Use of nitrogen-fixing trees can improve soil nitrogen supply and reduce the need for mineral fertilizer for pasture production and hence contribute to avoided greenhouse gas emissions (Sierra and Nygren, 2005). Increased tree cover, improved pasture production and protecting livestock from hot weather are all indicative of climate mitigation and adaptation measures. The use of Brachiaria species in silvopastures could also inhibit biological nitrification and reduce $\mathrm{N}_{2} \mathrm{O}$ emissions (Byrnes et al., 2017). $\mathrm{NH}_{3}$ volatilization could also be reduced in silvopastoral systems compared to treeless pastures where fertilizers are usually broadcast on the surface without incorporation into the ground (Bretas et al., 2020).

\subsection{Socio-economic benefits}

Trees in pasture lands such as Vitellaria paradoxa and Balanites aegyptiaca provide products such as firewood, stakes, flowers for bee hives, and seeds.

\subsection{Other benefits of the practice}

Trees in silvopastoral systems contribute to an increased connectivity between forest fragments to ensure conservation of forest fragments (Haggar et al., 2019). The integration of trees and shrubs in silvopastoral systems reduces air temperature and wind speed, increases relative humidity (Coble et al., 2020), thereby creating a pleasant environment for livestock (Magalhães et al., 2020).

\section{Conflict with other practice(s), Drawbacks}

\subsection{Tradeoffs with other threats to soil functions}

\section{Table 173. Soil threats}

\section{Soil threats}

Soil erosion

Soil erosion in a possible consequence of overgrazed parts in the vicinity of trees where livestock stays during hot weather. Average stocking density can range from 0.1 in drylands to 2.5 livestock units per hectare in temperate regions depending on the carrying capacity of the system as determined by the climate, soil and landscape characteristics (FAO, 2011). 


\begin{tabular}{|l|l|}
\hline Soil threats & Soil \\
$\begin{array}{l}\text { contamination / } \\
\text { pollution }\end{array}$ & $\begin{array}{l}\text { Overgrazing can reduce vegetation cover and structure, which may increase the } \\
\text { sediment deposition in water bodies (Dumont et al., 2007). }\end{array}$ \\
\hline $\begin{array}{l}\text { Soil biodiversity } \\
\text { loss }\end{array}$ & $\begin{array}{l}\text { Overgrazing due to an intensive land use has also been related to reduced soil C } \\
\text { content, which could be related, at least in part, to changes in the abundance, } \\
\text { composition and activity of soil microbial communities (Eldridge et al., 2017). }\end{array}$ \\
\hline Soil compaction & Livestock grazing increases soil compaction through trampling. \\
\hline
\end{tabular}

\subsection{Increases in greenhouse gas emissions}

Livestock tends to concentrate under trees during hot weather, heaping the manure in one place. The heaps of manure under the trees can increase emissions of $\mathrm{CH}_{4}$ and $\mathrm{N}_{2} \mathrm{O}$ compared to areas where livestock is scattered uniformly in the pastureland or in an organized kraal. Total greenhouse gas emissions from the manure (composting, turning) were 2.7 times higher than those of the stockpiled manure (Bai et al, 2020). However, on a carbon equivalent basis, emissions from manure due to tree integration can be offset by the carbon sequestered in the soil and tree biomass (Lorenz and Lal, 2014).

\subsection{Conflict with other practice(s)}

Mechanized operations such as silage harvest are complicated by the presence of trees in the pasture.

\subsection{Decreases in production (e.g. food/fuel/feed/timber/fibre)}

Trees in pastures, if not appropriately spaced, may significantly reduce the biomass production of pastures due to excessive shading (Qin, Wu and Zhang, 2010). In Brazil, an average of 50 Eucalyptus trees ha ${ }^{-1}$ are appropriate for silvopastoral systems (Lira Junior et al., 2020). Legumes such as those used in parkland agroforestry, contain high content of condensed tannins. These are reported to reduce forage digestibility in livestock and to induce low milk yield. Baldassini et al. (2018) found that tree presence reduced Gatton panic aboveground primary production by nearly $50 \%$. 


\subsection{Other conflicts}

In dryland systems dominated by thorny Acacias, identification of alternative species is difficult because few species are adapted to arid environments.

\section{Recommendations}

- When implementing silvopastoral practices the following should be considered in order to optimize SOC sequestration:

- Select tree species which are not harmful to livestock (e.g. thorny, poisonous trees/shrubs) but are productive enough to provide both fodder and litter to the ground. Suitable examples of species may include short-rotation legumes Calliandra, and Gliricidia), Eucalyptus, Acacia spp., among others.

- A spacing of about 10-15 meters between trees is recommended to allow free movement of livestock

- Trees should be pruned to a height above $2 \mathrm{~m}$ for ease of movement of the livestock, and the prunings, possibly chipped, retained on the ground to decompose and add organic matter to the soil

- Paddocking is highly recommended to enable rotational grazing, which allows time for forage to regrow and reduce overgrazing, which subsequently causes soil erosion.

\section{Potential barriers for adoption}

Table 174. Potential barriers to adoption

\begin{tabular}{|c|c|c|}
\hline Barrier & YES/NO & \\
\hline Biophysical & Yes & $\begin{array}{l}\text { Higher altitudes, and lower temperature and sunlight intensity can be limiting } \\
\text { factors in the adoption of agroforestry as a source of shade for cattle or to } \\
\text { pastures (Sood and Mitchell, 2009). }\end{array}$ \\
\hline Cultural & Yes & $\begin{array}{l}\text { Farmers' negative perception of the impact of trees can discourage them from } \\
\text { planting certain species (Meijer et al., 2015). }\end{array}$ \\
\hline Social & Yes & $\begin{array}{l}\text { Social learning through a network of peers, and lack of access to appropriate } \\
\text { extension services. } \\
\text { Silvipastoral systems are sometimes considered subsistence practices. This } \\
\text { negative connotation may prevent wider adoption. }\end{array}$ \\
\hline Economic & Yes & $\begin{array}{l}\text { Lack of capital to purchase required planting materials (seeds/seedlings). } \\
\text { Limited access to market of inputs and products of tree-based resources, and } \\
\text { limited access to infrastructure such as radio signals, saving and credit facilities, } \\
\text { central storage facilities for grain crops. }\end{array}$ \\
\hline
\end{tabular}




\begin{tabular}{|c|c|c|}
\hline Barrier & YES/NO & \\
\hline & & $\begin{array}{l}\text { The cost of maintaining trees together with livestock is higher compared to } \\
\text { maintaining the components separately because of the initial investment } \\
\text { required, such as tree seedlings or fencing. This can translate into negative cash } \\
\text { flows in the first years. Limited access to capital and deficits in farm } \\
\text { management capacity can be major barriers for adoption (Janssen, Anderies } \\
\text { and Walker, 2004). } \\
\text { Silvopastoral systems take long to show results due to slow establishment rate } \\
\text { of most trees compared with herbaceous components. This demotivates } \\
\text { farmers from adopting such systems. Maintaining an income stream is a } \\
\text { challenge when initially introducing or maintaining trees in a system. }\end{array}$ \\
\hline Institutional & Yes & $\begin{array}{l}\text { Absence of laws and institutions or inadequate enforcement reduces adoption } \\
\text { rates. }\end{array}$ \\
\hline $\begin{array}{l}\text { Legal (Right } \\
\text { to soil) }\end{array}$ & Yes & $\begin{array}{l}\text { Farmers are more likely to adopt the technology if there is security of land } \\
\text { tenure. For example, communal land is considered less secure than freehold } \\
\text { land }\end{array}$ \\
\hline Knowledge & Yes & $\begin{array}{l}\text { Inadequate knowledge of the agronomic and economic benefits of tree- } \\
\text { livestock interactions leads to the perception that trees occupy space that } \\
\text { would have been used for crop growth resulting in economic loss. Inadequate } \\
\text { technical skills to manage the tree-crop interactions, both from extension } \\
\text { services and farmers. } \\
\text { The knowledge required to manage trees is much different from that needed to } \\
\text { manage livestock. Hence farmers may not easily manage both effectively. A } \\
\text { balance among the various components while extracting multiple products } \\
\text { (plant and animal) requires far greater understanding, skills, and experience to } \\
\text { maintain. }\end{array}$ \\
\hline Other & & $\begin{array}{l}\text { In open-grazed systems, livestock graze freely in the system, potentially } \\
\text { destroying crops and creating conflicts with farmers. }\end{array}$ \\
\hline
\end{tabular}




\section{Photo of the practice}

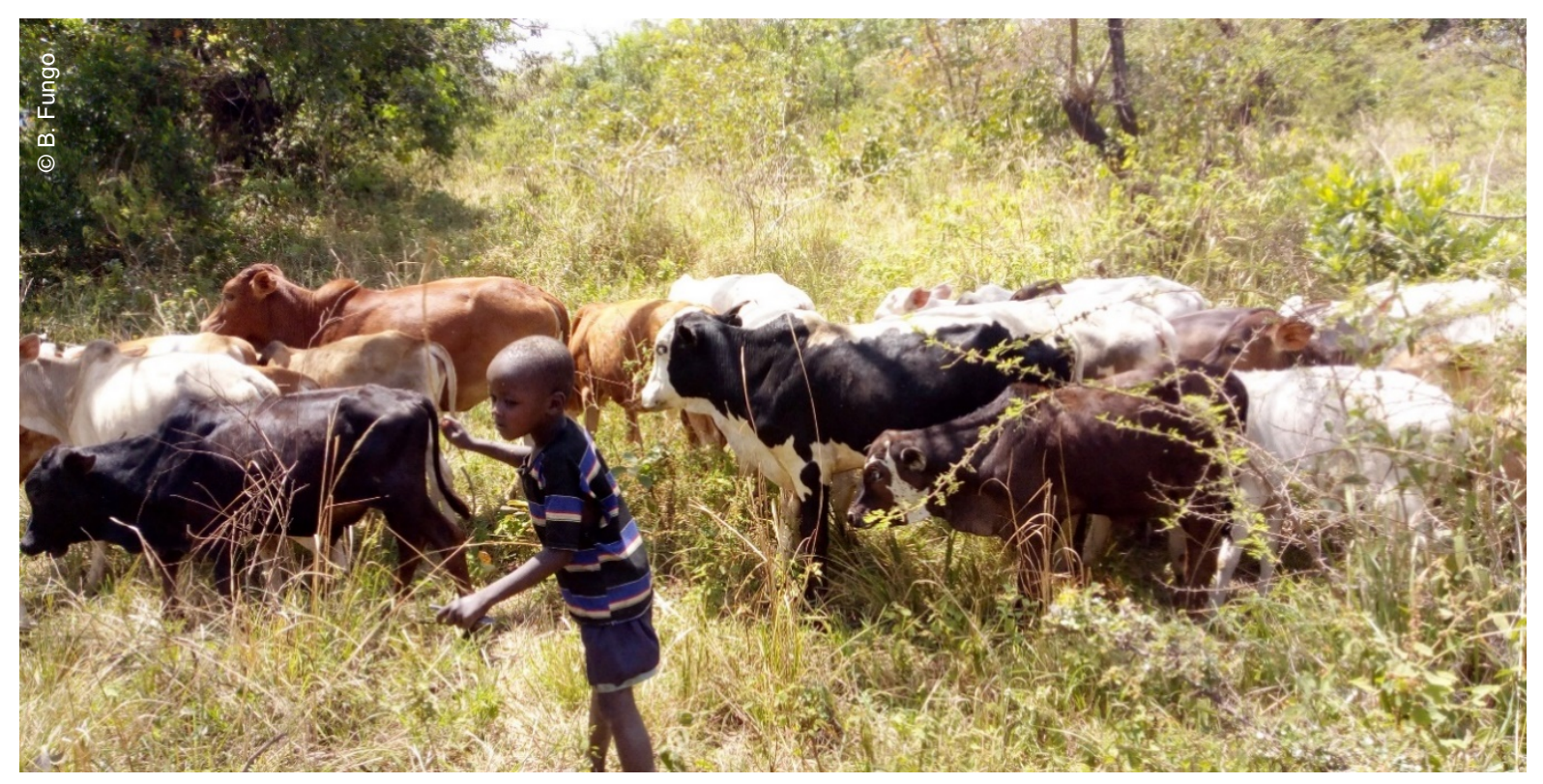

Photo 53. Silvopastoral system with Acacia hokii in Karamoja, Uganda.

Table 175. Related cases studies available in volumes 3 and 5

\begin{tabular}{|l|l|l|c|c|}
\hline Title & Region & $\begin{array}{l}\text { Duration } \\
\text { of study } \\
\text { (Years) }\end{array}$ & Volume & $\begin{array}{l}\text { Case- } \\
\text { study No. }\end{array}$ \\
\hline $\begin{array}{l}\text { Integrated farming in tropical } \\
\text { agroecosystems of Brazil }\end{array}$ & $\begin{array}{l}\text { Latin } \\
\text { America } \\
\text { and the } \\
\text { Caribbean }\end{array}$ & 4 to 12 & 3 & 34 \\
\hline $\begin{array}{l}\text { Agroforestry, silvopastoral systems and } \\
\text { water funds initiatives contribute to } \\
\text { improve soil capacity to remove and } \\
\text { store carbon in Colombia }\end{array}$ & $\begin{array}{l}\text { Latin } \\
\text { America } \\
\text { and the } \\
\text { Caribbean }\end{array}$ & $\begin{array}{c}9,20 \text { and } \\
40\end{array}$ & 3 & 36 \\
\hline $\begin{array}{l}\text { Conservation of degraded forests of } \\
\text { central and western Spain }\end{array}$ & Europe & 22 to 80 & 5 & 9 \\
\hline
\end{tabular}




\section{References}

Aynekulu, E., Mekuria, W., Tsegaye, D., Feyissa, K., Angassa, A., de Leeuw, J. \& Shepherd, K. 2017. Long-term livestock exclosure did not affect soil carbon in southern Ethiopian rangelands. Geoderma 307: 17. https://doi.org/10.1016/j.geoderma.2017.07.030

Bai, M., Flesch, T., Trouvé, R., Coates, T., Butterly, C., Bhatta, B., Hill, J. \& Chen D. 2020. Gas emissions during cattle manure composting and stockpiling. Journal of Environ. Quality, 49: 228-235. https://doi.org/10.1002/jeq2.20029

Baldassini, P., Despósito, C., Piñeiro, G. \& Paruelo, J.M. 2018. Silvopastoral systems of the Chaco forests: Effects of trees on grass growth. Journal of Arid Environments, 156: 87-95.

https://doi.org/10.1016/j.jaridenv.2018.05.008

Bayala, J., Sanou, J., Teklehaimanot, Z., Kalinganire, A. \& Ouédraogo, S.J. 2014. Parklands for buffering climate risk and sustaining agricultural production in the Sahel of West Africa. Curr. Opin. Environ. Sustain., 6: 28-34. https://doi.org/10.1016/j.cosust.2013.10.004

Beckert, M., Smith, P., Lilly, A. \& Chapman, S.J. 2016. Soil and tree biomass carbon sequestration potential of silvopastoral and woodland-pasture systems in North East Scotland. Agroforestry Systems, 90: 371-383. https://doi.org/10.1007/s10457-015-9860-4

Brakas S.G. \& Aune J.B. 2011. Biomass and carbon accumulation in land use systems of Claveria, the Phillipines. In Kumar \& Nair (Eds) Carbon Sequestration Potential of Agroforestry Systems; opportunities and challenges. Springer, New York. Pp. 163-178

Bretas, I.L., Paciullo, D.S.C., Alves, B.J.R., Martins, M.R., Cardoso, A.S., Lima, M.A., Rodrigues, R.A.R., Silva, F.F. \& Chizzotti, F.H.M. 2020. Nitrous oxide, methane, and ammonia emissions from cattle excreta on Brachiaria decumbens growing in monoculture or silvopasture with Acacia mangium and Eucalyptus grandis. Agriculture, Ecosystems \& Environment, 295: 106896.

https://doi.org/10.1016/j.agee.2020.106896

Byrnes, R.C., Nùnez, J., Arenas, L., Rao, I., Trujillo, C., Alvarez, C., Arango, J., Rasche, F. \& Chirinda, N. 2017. Biological nitrification inhibition by Brachiaria grasses mitigates soil nitrous oxide emissions from bovine urine patches. Soil Biology and Biochemistry, 107: 156-163.

https://doi.org/10.1016/j.soilbio.2016.12.029

Cardinael, R., Chevallier, T., Cambou, A., Béral, C., Barthès, B.G., Dupraz, C., Durand, C., Kouakoua, E. \& Chenu, C. 2017. Increased soil organic carbon stocks under agroforestry: A survey of six different sites in France. Agriculture, Ecosystems \& Environment, 236: 243-255.

https://doi.org/10.1016/j.agee.2016.12.011

Cardinael, R., Umulisa, V., Toudert, A., Olivier, A., Bockel, L. \& Bernoux, M. 2018. Revisiting IPCC Tier 1 coefficients for soil organic and biomass carbon storage in agroforestry systems. Environmental Research Letters, 13(12). https://doi.org/10.1088/1748-9326/aaeb5f 
Coble, A.P., Contosta, A.R., Smith, R.G., Siegert, N.W., Vadeboncoeur, M., Jennings, K.A., Stewart, A.J. \& Asbjornsen, H. 2020. Influence of forest-to-silvopasture conversion and drought on components of evapotranspiration. Agric. Ecosyst. Environ., 295: 106916. https://doi.org/10.1016/j.agee.2020.106916

DeLonge, M. \& Basche, A. 2018. Managing grazing lands to improve soils and promote climate change adaptation and mitigation: A global synthesis. Renew. Agric. Food Syst., 33: 267-278.

https://doi.org/10.1017/S1742170517000588

den Herder, M., Moreno, G., Mosquera-Losada, R.M., Palma, J.H.N., Sidiropoulou, A., Santiago Freijanes, J.J., Crous-Duran, J., Paulo, J.A., Tomé, M., Pantera, A., Papanastasis, V.P., Mantzanas, K., Pachana, P., Papadopoulos, A., Plieninger, T. \& Burgess, P.J. 2017. Current extent and stratification of agroforestry in the European Union. Agric. Ecosyst. Environ. 241: 121-132.

https://doi.org/10.1016/j.agee.2017.03.005

Dumont, B., Rook, A.J., Coran, C. \& Röver, K.-U. 2007. Effects of livestock breed and grazing intensity on biodiversity and production in grazing systems. 2. Diet selection. Grass and Forage Science, 62 (2): 159171. https://doi.org/10.1111/j.1365-2494.2007.00572.x

Eldridge, D.J., Delgado-Baquerizo, M., Travers, S.K., Val, J., Oliver, I., Hamonts, K. \& Singh, B.K. 2017. Competition drives the response of soil microbial diversity to in-creased grazing by vertebrate herbivores. Ecology, 98: 1922-1931. https://doi.org/10.1002/ecy.1879

FAO. 2011. Guidelines for the preparation of livestock sector reviews. Animal Production and Health Guidelines. No. 5. Rome

Ford, H., Healey, J.R., Pagella, T.F. \& Smith, A.R. 2019. How do hedgerows influence soil organic carbon stock in livestock-grazed pasture? Soil Use Management, 1-9. https://doi.org/10.1111/sum.12517

Fornara, D.A., Olave, R. \& Burgess, P. 2018. Land use change and soil carbon pools: evidence from a longterm silvopastoral experiment. Agroforestry Systems, 92: 1035-1046. https://doi.org/10.1007/s10457017-0124-3

Fornara, D., Olave, R., Higgins, A. 2020. Evidence of low response of soil carbon stocks to grassland intensification. Agric. Ecosyst. Environ., 287: 106705. https://doi.org/10.1016/j.agee.2019.106705

García-Tejero, S. \& Taboada, Á. 2016. Microhabitat heterogeneity promotes soil fertility and grounddwelling arthropod diversity in Mediterranean wood-pastures. Agriculture, Ecosystems \& Environment, 233: 192-201. https://doi.org/10.1016/j.agee.2016.09.004

Haggar, J., Pons, D., Saenz, L. \& Vides, M. 2019. Contribution of agroforestry systems to sustaining biodiversity in fragmented forest landscapes. Agriculture, Ecosystems \& Environment, 283: 106567. https://doi.org/10.1016/j.agee.2019.06.006

Haile, S.G., Nair, V.D. \& Nair, P.K.R. 2010. Contribution of trees to carbon storage in soils of silvopastoral systems in Florida, USA. Global Change Biology, 16(1): 427-438. https://doi.org/10.1111/j.1365-

2486.2009.01981.x 
Janssen, M.A., Anderies, J.M. \& Walker, B.H. 2004. Robust strategies for managing rangelands with multiple stable attractors. Journal of Environmental Economics and Management, 47(2): 140-62. https://doi.org/10.1016/S0095-0696(03)00069-X

Kimaro, A.A., Isaac, M.E. \& Chamshama, S.A.O. 2011. Carbon Pools in Tree Biomass and Soils Under Rotational Woodlot Systems in Eastern Tanzania. In B.M. Kumar \& P.K.R. Nair (Eds.) Carbon Sequestration Potential of Agroforestry Systems. Springer, Netherlands, pp. 129-143.

Kort, J. 1988. Benefits of windbreaks to field and forage crops. Agriculture, Ecosystems \& Environment, 2223: 165-190. https://doi.org/10.1016/0167-8809(88)90017-5

Lira Junior, M.A., Fracetto, F.J.C., Ferreira, J. da S., Silva, M.B. \& Fracetto, G.G.M. 2020. Legumebased silvopastoral systems drive $\mathrm{C}$ and $\mathrm{N}$ soil stocks in a subhumid tropical environment. CATENA, 189: 104508. https://doi.org/10.1016/j.catena.2020.104508

Lorenz, K. \& Lal, R. 2014. Soil organic carbon sequestration in agroforestry systems. A review. Agron. Sustain. Dev. 34: 443-454. https://doi.org/10.1007/s13593-014-0212-y

Luedeling, E., Sileshi, G., Beedy, T. \& Dietz, J. 2011. Carbon Sequestration Potential of Agroforestry Systems in Africa. In B.M. Kumar and P.K.R. Nair (Eds.) Carbon Sequestration Potential of Agroforestry Systems: Opportunities and Challenges. Advances in Agroforestry, 8. https://doi.org/10.1007/978-94007-1630-8_4

Magalhães, C.A.S., Zolin, C.A., Lulu, J., Lopes, L.B., Furtini, I.V., Vendrusculo, L.G., Zaiatz, A.P.S.R., Pedreira, B.C. \& Pezzopane, J.R.M. 2020. Improvement of thermal comfort indices in agroforestry systems in the southern Brazilian Amazon. Journal of Thermal Biology, 91: 102636. https://doi.org/10.1016/j.jtherbio.2020.102636

Mcsherry, M.E. \& Ritchie, M.E. 2013. Effects of grazing om grassland soil carbon: a global review. Global Change Biology, 19: 1347-1357.

Meijer, S.S., Catacutan, D., Sileshi, G.W. \& Nieuwenhuis, M. 2015. Tree planting by smallholder farmers in Malawi: Using the theory of planned behaviour to examine the relationship between attitudes and behaviour. Journal of Environmental Psychology, 43: 1-12.

https://doi.org/10.1016/j.jenvp.2015.05.008

Nair, P.K.R. 1985. Classification of agroforestry systems. Agroforestry Systems, 3: 97-128. https://doi.org/10.1007/BF00122638

Nair, P.K.R., Tonucci, R.G., Garcia, R. \& Nair, V.D. 2011. Silvopasture and Carbon Sequestration with Special Reference to the Brazilian Savanna (Cerrado). In B.M. Kumar \& P.K.R. Nair (Eds.) Carbon Sequestration Potential of Agroforestry Systems: Opportunities and Challenges, pp. 145-162. Advances in Agroforestry. Dordrecht, Springer Netherlands. https://doi.org/10.1007/978-94-007-1630-8_8

Nair, P.K.R. 2014. Agroforestry: Practices and Systems. In N.K. Van Alfen, ed. Encyclopedia of Agriculture and Food Systems, pp. 270-282. Oxford, Academic Press. https://doi.org/10.1016/B978-0-444-52512$3.00021-8$ 
Negasa, T., Ketema, H., Legesse, A., Sisay, M. \& Temesgen, H. 2017. Variation in soil properties under different land use types managed by smallholder farmers along the toposequence in southern Ethiopia.

Geoderma, 290: 40-50. https://doi.org/10.1016/j.geoderma.2016.11.021

Paterson, R.T., Kiruiro, E. \& Arimi, H. 1999. Calliandra calothyrsus as a supplement for milk production in the Kenya highlands. Tropical Animal Health and Production, 31: 115-126.

https://doi.org/10.1023/A:1005119808090

Pinto-Correia, T., Guiomar, N., Ferraz-de-Oliveira, M.I., Sales-Baptista, E., Rabaça, J., Godinho, C., Ribeiro, N., Sousa, P.S., Santos, P., Santos-Silva, C., Simões, M.P., Belo, A.D.F., Catarino, L., Costa, P., Fonseca, E., Godinho, S., Azeda, C., Almeida, M., Gomes, L., Castro, J.L. de, Louro, R., Silvestre, M. \& Vaz, M. 2018. Progress in Identifying High Nature Value Montados: Impacts of Grazing on Hardwood Rangeland Biodiversity. Rangeland Ecology and Management, 71(5): 612-625.

https://doi.org/10.1016/j.rama.2018.01.004

Qin, S., Wu, B. \& Zhang, Y. 2010. A review of above-ground interactions in silvopasture systems. Acta Ecol. Sin., 30(13): 3616-3627

Sharrow, S.H. \& Ismail, S. 2004. Carbon and nitrogen storage in agroforests, tree plantations, and pastures in western Oregon, USA. Agroforestry Systems, 60(2): 123-130.

https://doi.org/10.1023/B:AGFO.0000013267.87896.41

Sierra, J. \& Nygren, P. 2005. Role of root inputs from a dinitrogen-fixing tree in soil carbon and nitrogen sequestration in a tropical agroforestry system. Soil Research, 43(5): 667-675.

https://doi.org/10.1071/SR04167

Suarez, L.R., Paladines Josa, Y.T., Astudillo Samboni, E.J., Lopez Cifuentes, K.D., Durán Bautista, E.H. \& Salazar, J.C.S. 2018. Soil macrofauna under different land uses in the Colombian Amazon. Pesqui Agropecu Bras, 53: 1383-1391.

Upson, M.A., Burgess, P.J. \& Morison, J.I.L. 2016. Soil carbon changes after establishing woodland and agroforestry trees in a grazed pasture. Geoderma, 283: 10-20.

https://doi.org/10.1016/j.geoderma.2016.07.002

Wang, L., Gan, Y., Wiesmeier, M., Zhao, G., Zhang, R., Han, G., Siddique, K.H. \& Hou, F. 2018.

Grazing Exclusion - An Effective Approach for Naturally Restoring Degraded Grasslands in Northern China. Land Degradation and Development, 120: 126-133. https://doi.org/10.1002/ldr.3191

Zhou, G., Zhou, X., He, Y., Shao, J., Hu, Z., Liu, R., Zhou, H. \& Hosseinibai, S. 2017. Grazing intensity significantly affects belowground carbon and nitrogen cycling in grassland ecosystems: a meta-analysis. Glob. Chang. Biol., 23: 1167-1179. https://doi.org/10.1111/gcb.13431 


\section{Agroforestry 3: Agrosilvopastoral systems}

\section{Bernard Fungo ${ }^{1}$, Martin Wiesmeier ${ }^{2,3}$, Rémi Cardinael $4,5,6$}

'Agroforestry Research Program, National Agricultural Research Organization (NARO), Kampala, Uganda

${ }^{2}$ Chair of Soil Sciences, TUM School of Life Sciences Weihenstephan, Technical University of Munich, Freising, Germany

${ }^{3}$ Bavarian State Research Center for Agriculture, Institute for Organic Farming, Soil and Resource Management, Freising, Germany

${ }^{4}$ CIRAD, UPR AIDA, Harare, Zimbabwe

${ }^{5}$ AIDA, Université Montpellier, CIRAD, Montpellier, France

${ }^{6}$ University of Zimbabwe, Crop Science Department, Harare, Zimbabwe

\section{Description of the practice}

Agrosilvopastoralism is the integration of crops, pastures, livestock and woody perennials into the same farming system (Nair, 1989). The integration of these components can take various forms, depending on the objectives of the farmer, and is also determined by ecological conditions. There are several forms of agrosilvopastoralism: (i) A mixture of pure cultivated and grazing areas near or distant from homesteads where animals may be herded or tethered, (ii) Animals allowed to graze in previously cultivated land to eat crop stubble or fallen grain, and (iii) Permanently cultivated land with zero-grazing through cut-and-carry for dairy cattle, sheep or goats. Typical examples of agrosilvopastoral systems include home gardens and woody hedges for livestock browsing, green manuring, mulching and soil conservation.

In agrosilvopastoral systems, trees provide shade, forage and protection to animals. Trees also provide shade for crops and pastures. Litter fall enriches soil in organic matter with beneficial effects to the crops in terms of nutrient furniture. Livestock also add manure to the soil through deposition of dung and urine. The improvement in soil fertility resulting from animal manure enhances crop growth, some of crops being fed on by livestock. Famous examples of agrosilvopastoral systems are "integrated crop-livestock-forestry systems (ICLF)" or "Mixed Tree Crop-Livestock System" in Brazil (Oliveira et al., 2018). 


\section{Range of applicability}

Globally, agrosilvopastoral systems are found predominantly in the highlands of Eastern Africa, in Asia and South East Asia, and in Latin America. Although not widely spread in Europe, these systems are observed in the Spanish region of Catalonia and in Austria. These systems are widely distributed mostly in areas with high rainfall and where population density is generally high (Nair, 2014). These mixed systems are a valuable option for circular food systems as they recycle more nutrients through the integration of livestock, pastures and crops.

\section{Potential for $\mathrm{C}$ sequestration}

Evidence of the carbon sequestration potential of agroforestry systems has been documented in previous studies (Cardinael et al., 2018; Feliciano et al., 2018; Shi et al., 2018). Most of the studies usually focus either on the effect of trees, of livestock or pastures on soil organic carbon (SOC) stocks, Agrosilvopastoral systems in humid climates sequester more SOC compared to systems in dry climates. Tree density is also generally higher under more humid environments. The combined effect of litterfall from trees, integration of pastures, and dung from livestock increases SOC stocks probably faster than other agroforestry systems (Table 176). 
Table 176. Examples of SOC sequestration rates of selected agrosilvopastoral systems

\begin{tabular}{|c|c|c|c|c|c|c|c|}
\hline Location & Climate & Soil type & $\begin{array}{l}\text { Baseline } \\
\text { stock } \\
\text { (tC/ha) }\end{array}$ & $\begin{array}{l}\mathrm{SOC} \\
\text { sequestration } \\
\text { rate } \\
(\mathrm{tC} / \mathrm{ha} / \mathrm{yr})\end{array}$ & $\begin{array}{l}\text { Depth } \\
(\mathrm{cm})\end{array}$ & More information & Reference \\
\hline Brazil & $\begin{array}{l}\text { Humid } \\
\text { tropical } \\
\text { climate }\end{array}$ & Ferralsol & $\begin{array}{l}55.05 \\
110.66\end{array}$ & $\begin{array}{l}0.58 \\
1.47\end{array}$ & $0-30$ & $\begin{array}{l}\text { System consisted of growing forestry species } \\
\text { (Eucalyptus urograndis) simultaneously with } \\
\text { soybean (Glycine max) and aerobic rice (Oryza } \\
\text { sativa) for } 2 \text { years when graincrops were } \\
\text { followed by palisade grass (Urochloa bryzan- } \\
\text { tha). Baseline degraded pasture. Age of the } \\
\text { system is } 12 \text { years. }\end{array}$ & $\begin{array}{l}\text { Oliveira et al. } \\
(2018)\end{array}$ \\
\hline Haiti & $\begin{array}{l}\text { Humid } \\
\text { tropical } \\
\text { climate }\end{array}$ & Typic Hapludalf & 22.7 & $1.21 \pm 1.04$ & $0-15$ & $\begin{array}{l}\text { Site was unimproved pasture prior to the } \\
\text { establishment of hedgerows for } 5 \text { years. }\end{array}$ & $\begin{array}{l}\text { Isaac et al. } \\
(2005)\end{array}$ \\
\hline India & $\begin{array}{l}\text { Humid } \\
\text { tropical } \\
\text { climate }\end{array}$ & $\begin{array}{l}\text { Fluventic } \\
\text { Dystropepts }\end{array}$ & $\begin{array}{l}28.5 \\
31.2 \\
25.7 \\
16.1\end{array}$ & $\begin{array}{l}0.26 \\
0.30 \\
0.29 \\
0.12\end{array}$ & $\begin{array}{l}0-20 \\
20-50 \\
50-80 \\
80- \\
100\end{array}$ & Home gardens of $\sim 30$ years & $\begin{array}{l}\text { Saha et al. } \\
(2009)\end{array}$ \\
\hline Nigeria & $\begin{array}{l}\text { Dry tropical } \\
\text { climate }\end{array}$ & Alfisol & 82.0 & $0.20 \pm 0.01$ & $0-15$ & Home garden monitored for 12 years & $\begin{array}{l}\text { Kang et al. } \\
\text { (1999) }\end{array}$ \\
\hline
\end{tabular}

Where applicable, errors are standard errors of the mean. 


\section{Other benefits of the practice}

\subsection{Improvement of soil properties}

Litterfall from trees and dung from livestock all supply organic matter that improves the soil quality for pasture growth. Roots of trees support an assemblage of soil microbes that promote nutrient cycling. Tree components in agrosilvopastoral systems contribute to litter fall, which increases decomposition and subsequent supply of soil nutrients and organic matter and reduces bulk density (Issac and Borden, 2019), therefore contributing to better water infiltration. Increased amount of SOC is associated with enhanced water storage capacity and availability of nutrients for crops. Tree roots increase water infiltration and air movement, which improve crop performance. Integration of leguminous trees into agricultural systems increases the nitrogen supply through biological nitrogen fixation.

\subsection{Minimizing soil threats}

Grazing of understory vegetation reduces fire risks because it reduces the amount of grass which is the fuel for bush fires.

Table 177. Soil threats

\begin{tabular}{|c|c|}
\hline \multicolumn{2}{|l|}{ Soil threats } \\
\hline Soil erosion & $\begin{array}{l}\text { Fodder trees planted in hedgerows on contours bands reduce the risk of soil erosion } \\
\text { (Atangana et al., 2014). }\end{array}$ \\
\hline $\begin{array}{l}\text { Nutrient imbalance } \\
\text { and cycles }\end{array}$ & $\begin{array}{l}\text { Prunings of several tree species contain sufficient nutrients to meet crop demand } \\
\text { (Palm, 1995). Legume tree/shrub species can contribute to biological nitrogen } \\
\text { fixation. }\end{array}$ \\
\hline $\begin{array}{l}\text { Soil contamination / } \\
\text { pollution }\end{array}$ & $\begin{array}{l}\text { Tree roots in agroforestry systems are able to reduce nitrogen and phosphorus } \\
\text { residues in soils from } 20 \text { percent up to } 100 \text { percent, have the potential to reduce } \\
\text { pesticides leaching and runoff in considerable amounts (up to } 90 \% \text { for runoff) } \\
\text { (Pavlidis and Tsihrintzis, 2017; Zhu et al., 2019). }\end{array}$ \\
\hline Soil acidification & $\begin{array}{l}\text { The use of nitrogen-fixing trees can lead to soil acidification (Bolan, Hedley and } \\
\text { White, 1991). }\end{array}$ \\
\hline Soil biodiversity loss & $\begin{array}{l}\text { Soils with tree roots harbour greater diversity of microbial biomass compared to soils } \\
\text { with monocrops (Sridhar and Bagyaraj, 2017). }\end{array}$ \\
\hline Soil compaction & $\begin{array}{l}\text { Rotation of pastures with crops mitigates soil compaction caused by livestock during } \\
\text { the grazing period. }\end{array}$ \\
\hline
\end{tabular}




\begin{tabular}{|l|l|}
\hline Soil threats & \\
\hline $\begin{array}{l}\text { Soil water } \\
\text { management }\end{array}$ & $\begin{array}{l}\text { The complex vegetation without bare soil during the year reduces evapotranspiration } \\
\text { and reduces surface flow and soil erosion, and increase infiltration. }\end{array}$ \\
\hline
\end{tabular}

\subsection{Productivity (e.g. Food/Fuel/Feed/Timber)}

Crop yields are sustained with manure application from livestock. Shade-tolerant species of perennial are likely to have higher yields due to an improved microclimate created by trees (Schroth et al., 2001; Brandt et al., 2015). A large-scale deployment of agroforestry over seven countries in West Africa can sequester up to 135 $\mathrm{Mt} \mathrm{CO}_{2}$ /year over two decades, corresponding to about $166 \%$ of the carbon emissions from fossil fuels and deforestation in the region (Tschora and Cherubini, 2020).

\subsection{Climate change mitigation and adaptation}

Agroforestry practices also offer climate change adaptation by buffering temperature, ameliorating microclimate (Magalhães et al., 2020), maintaining long-term soil health (Arevalo et al., 2015), and minimizing the incidence of insect and pests. Trees sequester significant amount of carbon in the above- and belowground parts. In the tropics, trees have been estimated to accumulate large amounts of carbon (C) from 12 to $228 \mathrm{t} \mathrm{ha}^{-1}$ with an average of $95 \mathrm{tha}^{-1}$ (Swamy and Tewari, 2017).

\subsection{Socio-economic benefits}

Smallholder farmers may use the trees to produce fodder for livestock without reducing areas dedicated to crops. In addition to direct advantages, farmers may also obtain economic benefits from fuelwood, timber, poles, and forage, which are used eventually on the farm for cattle management. Leguminous shrubs also provide protein supplement when their edible parts are utilized as forage. Trees provide diversification of products and services within the farm, which reduce risk of economic disasters and improved resilience and adaptation to climate change (Palsaniya and Ghosh, 2016).

\subsection{Other benefits of the practice}

The associations of cattle with crops is advantageous because 60 to 70 percent of tree biomass can be used as feed for cattle (Franzel et al., 2014), reducing competition with crops for human consumption. 


\section{Conflict with other practice(s), Drawbacks}

\subsection{Tradeoffs with other threats to soil functions}

Table 178. Soil threats

\begin{tabular}{|l|l|}
\hline Soil threats & \multicolumn{1}{|l|}{$\begin{array}{l}\text { Soil erosion } \\
\text { Livestock can provide manure to croplands, but they can also cause soil } \\
\text { compaction, potentially restricting water infiltration, and causing runoff and } \\
\text { soil erosion. Therefore, stocking rate should be managed to avoid this effect. }\end{array}$} \\
\hline Soil compaction & $\begin{array}{l}\text { Livestock can compact the soil, reduce infiltration and increase the risk of soil } \\
\text { erosion. }\end{array}$ \\
\hline $\begin{array}{l}\text { Soil water } \\
\text { management }\end{array}$ & $\begin{array}{l}\text { Trees contribute to higher infiltration (Dollinger et al., 2019). These cause } \\
\text { more leaching of soil nitrogen from the root zone of annual crop (Cuttle and } \\
\text { Gill, 1991). }\end{array}$ \\
\hline
\end{tabular}

\subsection{Increases in greenhouse gas emissions}

Tree-based systems can mitigate climate change by offsetting greenhouse gas (GHG) emission from livestock (CH4 from enteric fermentation and $\mathrm{N} 2 \mathrm{O}$ emission from dung and urine) through carbon sequestration in tree biomass, with an average estimated carbon sequestration and mitigation potential of the $0.26 \mathrm{tC} / \mathrm{ha} / \mathrm{yr}$ and $0.95 \mathrm{t} \mathrm{CO} 2 \mathrm{eq} / \mathrm{ha} / \mathrm{yr}$ in India (Chavan et al., 2020).

\subsection{Conflict with other practice(s)}

Trees in croplands also reduce land area available to crops, especially for crops that cannot tolerate shade such as rice and maize.

\subsection{Decreases in production (e.g. food/fuel/feed/timber/fibre)}

Integrating livestock with crops increases the risk of crop damage by livestock. Allowing livestock to graze in croplands after harvesting may result in soil compaction (Cherubin, Chavarro-Bermeo and Silva-Olaya, 2019). Thorny trees such as Acacias in pasture lands hurt and ruin the hides of animals. Some trees harbour pests for crops. After several years, a decrease in crop yield can be observed in mature and highly dense plantations, especially close to the trees, due to competition between crops and trees for light, water, and nutrients (Dilla et al., 2019). 


\subsection{Other conflicts}

It is more expensive to manage all the three components on the same farming unit due to labor requirements to carry manure into croplands, transfer of fodder from parks to livestock among other activities. Trees on farm also make mechanization more difficult and may increase the cost of labor for land preparation.

\section{Recommendations}

- Avoid land clearing (e.g. burning, forest conversion) or land preparation (e.g. tillage) that causes net loss of biomass or soil carbon.

- Select species with high leaf litter or root biomass production to increase SOC inputs to the soil and which may have other additional benefits such as timber, medicine and fruits for humans and livestock.

- Select species with deep rooting systems to reduce competition with crops.

- Select N2-fixing trees to improve soil fertility.

- Optimize the spacing and structure of the system to make best use of nutrients, water and sunlight to ensure high productivity of the system.

- A proper design is necessary to allow for mechanization, but also to reduce transport of manure and fodder within the system.

- On sloppy land, plant trees perpendicularly to the slope to reduce erosion.

- Retain pruning and other residues and manure on site.

- Avoid tree species that are poisonous to livestock.

\section{Potential barriers for adoption}

Smallholder farmers in rural areas usually have limited access to appropriate plant material due to high cost and availability. Using farmer-managed natural regeneration may be a suitable option for farmers.

Table 179. Potential barriers to adoption

\begin{tabular}{|l|l|l|}
\hline Barrier & YES/NO & \\
\hline Biophysical & Yes & $\begin{array}{l}\text { Higher altitudes, and lower temperature and sunlight intensity can be limiting } \\
\text { factors in the adoption of agroforestry as a source of shade for crops, cattle or } \\
\text { to pastures (Sood and Mitchell, 2009). }\end{array}$ \\
\hline Cultural & Yes & $\begin{array}{l}\text { Farmers' negative perception of the impact of trees can discourage them from } \\
\text { planting certain species (Meijer et al., 2018). }\end{array}$ \\
\hline
\end{tabular}




\begin{tabular}{|c|c|c|}
\hline Barrier & YES/NO & \\
\hline Social & Yes & $\begin{array}{l}\text { Social learning through a network of peers, and lack of access to appropriate } \\
\text { extension services. } \\
\text { Agroforestry systems are sometimes considered subsistence practices. This } \\
\text { negative connotation may prevent wider adoption. }\end{array}$ \\
\hline Economic & Yes & $\begin{array}{l}\text { Lack of capital to purchase required planting materials (seeds/seedlings). } \\
\text { Limited access to market of inputs and products of tree-based resources, and } \\
\text { limited access to infrastructure such as radio signals, saving and credit facilities, } \\
\text { central storage facilities for grain crops. } \\
\text { The cost of maintaining trees together with livestock is higher compared to } \\
\text { maintaining the components separately because of the initial investment } \\
\text { required, such as tree seedlings or fencing. This can translate into negative cash } \\
\text { flows in the first years. Limited access to capital and deficits in farm } \\
\text { management capacity can be major barriers for adoption (Chará et al., 2004). } \\
\text { Silvopastoral systems take long to show results due to slow establishment rate } \\
\text { of most trees compared with herbaceous components. This demotivates } \\
\text { farmers from adopting such systems. Maintaining an income stream is a } \\
\text { challenge when initially introducing or maintaining trees in a system. }\end{array}$ \\
\hline Institutional & Yes & $\begin{array}{l}\text { Absence of laws and institutions or inadequate enforcement reduces adoption } \\
\text { rates. }\end{array}$ \\
\hline $\begin{array}{l}\text { Legal (Right } \\
\text { to soil) }\end{array}$ & Yes & $\begin{array}{l}\text { Farmers are more likely to adopt the technology if there is security of land } \\
\text { tenure. For example, communal land is considered less secure than freehold } \\
\text { land }\end{array}$ \\
\hline Knowledge & Yes & $\begin{array}{l}\text { Inadequate knowledge of the agronomic and economic benefits of tree-crop } \\
\text { interactions leads to the perception that trees occupy space that would have } \\
\text { been used for crop growth resulting in economic loss. Inadequate technical } \\
\text { skills to manage the tree-crop interactions, both from extension services and } \\
\text { farmers. } \\
\text { The knowledge required to manage trees is much different from that needed to } \\
\text { manage livestock. Hence farmers may not easily manage both effectively. A } \\
\text { balance among the various components while extracting multiple products } \\
\text { (plant and animal) requires far greater understanding, skills, and experience to } \\
\text { maintain. }\end{array}$ \\
\hline Other & & $\begin{array}{l}\text { In open-grazed systems, livestock graze freely in the system, potentially } \\
\text { destroying crops and creating conflicts with farmers. }\end{array}$ \\
\hline
\end{tabular}




\section{Photo of the practice}

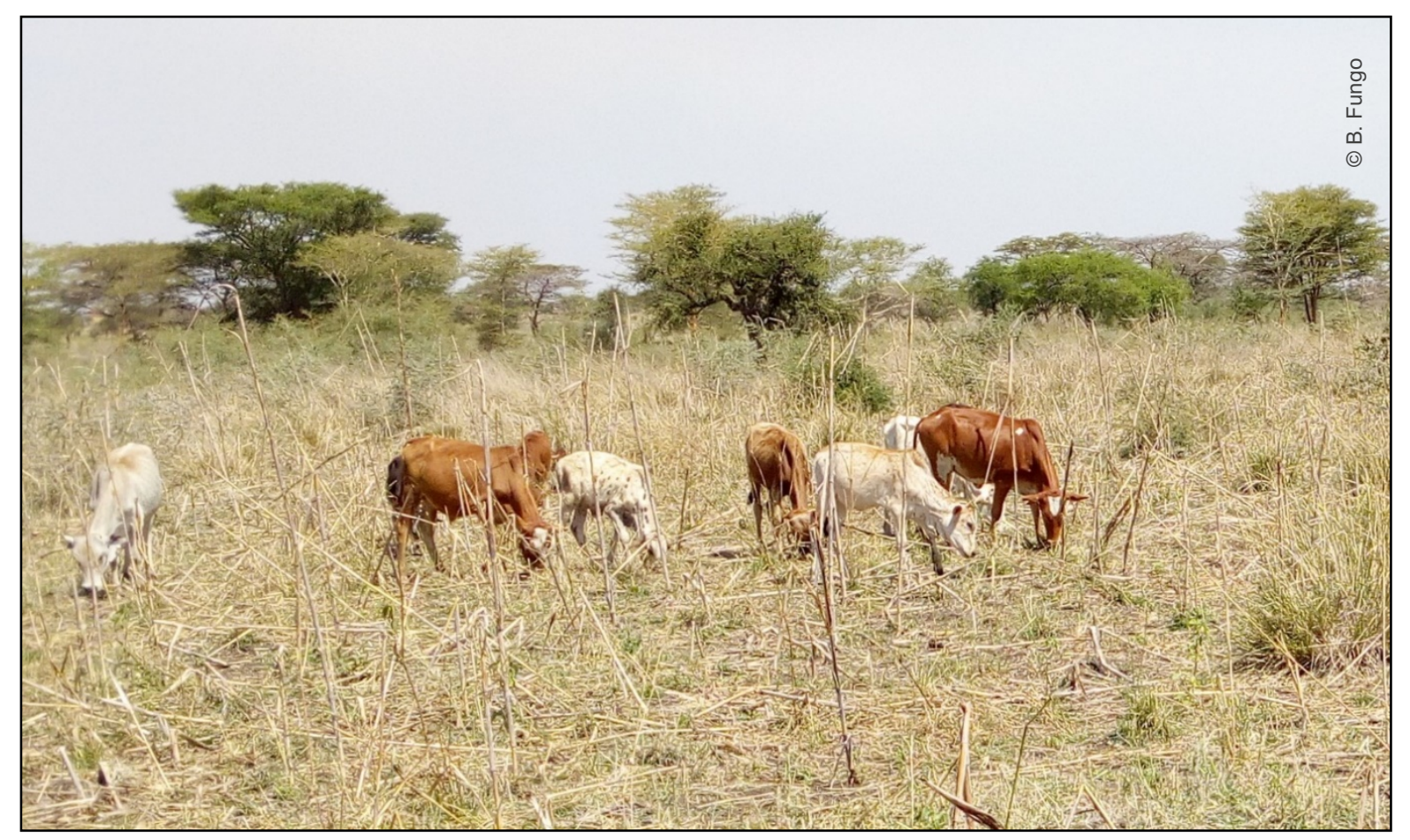

Photo 54. Grazing of livestock in a field after the harvest of sorghum in an Acacia-dominated parkland in Karamoja, northeastern Uganda.

Table 180. Related cases studies available in volumes 3 and 5

\begin{tabular}{|l|l|l|l|c|}
\hline Title & Region & $\begin{array}{l}\text { Duration of } \\
\text { study (Years) }\end{array}$ & $\begin{array}{l}\text { Volu } \\
\text { me }\end{array}$ & $\begin{array}{l}\text { Case- } \\
\text { study No. }\end{array}$ \\
\hline $\begin{array}{l}\text { Mediterranean savanna-like } \\
\text { agrosilvopastoral grassland system in } \\
\text { Spain, Italy and Portugal }\end{array}$ & Europe & 4,22 and 37 & 3 & 17 \\
\hline $\begin{array}{l}\text { Integrated farming in tropical } \\
\text { agroecosystems of Brazil }\end{array}$ & $\begin{array}{l}\text { Latin } \\
\text { America } \\
\text { and the } \\
\text { Caribbean }\end{array}$ & 4 to 12 & 3 & 34 \\
\hline
\end{tabular}




\section{References}

Arevalo, E., Canto, M., Alegre J., Oscar L., Julca O. \& Alberto B.V. 2015. Changes in Soil Physical and Chemical Properties in Long Term Improved Natural and Traditional Agroforestry Management Systems of Cacao Genotypes in Peruvian Amazon. PLoS ONE, 10. 10.1371/journal.pone.0136784.

Atangana, A., Khasa, D., Chang, S. \& Degrande, A. 2014. Agroforestry for Soil Conservation. In A. Atangana, D. Khasa, S. Chang \& A. Degrande (Eds.) Tropical Agroforestry, pp. 203-216. Dordrecht, Springer Netherlands https://doi.org/10.1007/978-94-007-7723-1_9

Bolan, N.S., Hedley, M.J. \& White, R.E. 1991. Processes of soil acidification during nitrogen cycling with emphasis on legume based pastures. Plant and Soil, 134(1): 53-63.

https://doi.org/10.1007/BF00010717

Brandt, M., Mbow, C., Diouf, A.A., Verger, A., Samimi, C. \& Fensholt, R. 2015. Groundand satellitebased evidence of the biophysical mechanisms behind the greening Sahel. Global Change Biology, 21(4). https://doi.org/10.1111/gcb.12807

Cardinael, R., Umulisa, V., Toudert, A., Olivier, A., Bockel, L. \& Bernoux, M. 2018. Revisiting IPCC Tier 1 coefficients for soil organic and biomass carbon storage in agroforestry systems. Environ. Res. Lett. 13 : 1-20. https://doi.org/10.1088/1748-9326/aaeb5f

Chará J., Reyes E., Peri P., Otte J., Arce E. \& Schneider F. 2019. Silvopastoral Systems and their Contribution to Improved Resource Use and Sustainable Development Goals: Evidence from Latin America. FAO, CIPAV and Agri Benchmark, Cali, 60 pp.

Chavan, S.B., Newaj, R., Rizvi, R.H., Ajit, Prasad, R., Alam, B., Handa, A.K., Dhyani, S.K., Jain, A. \& Tripathi, D. 2020. Reduction of global warming potential vis-à-vis greenhouse gases through traditional agroforestry systems in Rajasthan, India. Environment, Development and Sustainability.

https://doi.org/10.1007/s10668-020-00788-w

Cherubin, M.R., Chavarro-Bermeo, J.P. \& Silva-Olaya, A.M., 2019. Agroforestry systems improve soil physical quality in northwestern Colombian Amazon. Agroforest Syst, 93: 1741-1753

https://doi.org/10.1007/s10457-018-0282-y

Cuttle, S.P. \& Gill, E.K. 1991. Concentrations of nitrate in soil water following herbicide treatment of tree planting positions in an upland agroforestry system. Agroforest Syst, 13:225-234.

https://doi.org/10.1007/BF00053580

Dilla, A.M., Smethurst, P.J., Barry, K., Parsons, D. \& Denboba, M. A. 2019. Tree pruning, zone and fertiliser interactions determine maize productivity in the Faidherbia albida (Delile) A. Chev parkland agroforestry system of Ethiopia. Agroforest Syst, 93: 1897-1907 https://doi.org/10.1007/s10457-0180304-9

Dollinger, J., Lin, C., Udawatta, R.P., Pot, V., Benoit, P. \& Jose, S. 2019. Influence of agroforestry plant species on the infiltration of S-Metolachlor inbuffer soils. Journal of Contaminant Hydrology, 225: 103498. https://doi.org/10.1016/j.jconhyd.2019.103498 
Feliciano, D., Ledo, A., Hillier, J. \& Nayak, D.R., 2018. Which agroforestry options give the greatest soil and above ground carbon benefits in different world regions? Agric. Ecosyst. Environ. 254: 117-129. https://doi.org/10.1016/j.agee.2017.11.032

Franzel, S., Carsan, S., Lukuyu, B., Sinja, J. \& Wambugu, C. 2014. Fodder trees for improving livestock productivity and smallholder livelihoods in Africa. Current Opinion in Environmental Sustainability, 6: 98103. https://doi.org/10.1016/j.cosust.2013.11.008

Isaac, M.E. \& Borden, K.A. 2019. Nutrient acquisition strategies in agroforestry systems. Plant Soil, 444: 1-19. https://doi.org/10.1007/s11104-019-04232-5

Isaac, M.E, Gordon, A.M, Thevathasan, N., Oppong, S.K. \& Quashie-Sam, J. 2005. Temporal changes in soil carbon and nitrogen in west African multistrata agroforestry systems: A chronosequence of pools and fluxes Agroforestry Systems, 65: 23-31. https://doi.org/10.1007/s10457-004-4187-6

Kang, B.T., Caveness, F.E., Tian, G. \& Kolawole, G.O. 1999. Long-term alley cropping with four hedgerow species on an Alfisol in southwestern Nigeria - effect on crop performance, soil chemical properties and nematode population. Nutrient Cycling in Agroecosystems, 54: 145-155.

https://doi.org/10.1023/A:1009757830508

Magalhães, C.A.S., Zolin, C.A., Lulu, J., Lopes, L.B., Furtini, I. V., Vendrusculo, L.G., Zaiatz, A.P.S.R., Pedreira, B.C. \& Macedo Pezzopane, J.R. 2020. Improvement of thermal comfort indices in agroforestry systems in the southern Brazilian Amazon. Journal Therm. Biol., 91: 102636.

https://doi.org/10.1016/j.jtherbio.2020.102636

Meijer, S.S., Catacutan, D., Sileshi, G.W. \& Nieuwenhuis, M. 2015. Tree planting by smallholder farmers in Malawi: Using the theory of planned behaviour to examine the relationship between attitudes and behaviour. Journal of Environmental Psychology, 43: 1-12.

https://doi.org/10.1016/j.jenvp.2015.05.008

Nair, P.K.R. 1989. Classification of agroforestry systems. In P.K.R. Nair (Ed.) Agroforestry systems in the tropics. Dordretch, The Netherlands. Klwer Academic Press ICRAF. pp. 39-52.

Nair, P.K.R. 2014. Agroforestry: Practices and Systems. In N.K. Van Alfen (Ed.) Encyclopedia of Agriculture and Food Systems, pp. 270-282. Oxford, Academic Press. https://doi.org/10.1016/B978-0-444-525123.00021-8

Oliveira, J. de M., Madari, B.E., Carvalho, M.T. deM., Assis, P.C.R., Silveira, A.L.R., de Leles Lima, M., Wruck, F.J., Medeiros, J.C. \& Machado, P.L.O. de A., 2018. Integrated farming systems for improving soil carbon balance in the southern Amazon of Brazil. Reg. Environ. Chang., 18: 105-116. https://doi.org/10.1007/s10113-017-1146-0

Palm, C.A. 1995. Contribution of agroforestry trees to nutrient requirements of intercropped plants. Agroforestry Systems, 30: 105-124. https://doi.org/10.1007/BF00708916 
Palsaniya, D.R. \& Ghosh, P.K. 2016. Agroforestry for Natural Resource Conservation, Livelihood Security and Climate Change Mitigation in Himalayan Agroecosystems. In J.K. Bisht, V.S. Meena, P.K. Mishra \& A. Pattanayak (Eds.) Conservation Agriculture: An Approach to Combat Climate Change in Indian Himalaya, pp. 203-223. Singapore, Springer. https://doi.org/10.1007/978-981-10-2558-7_8

Pavlidis, G. \& Tsihrintzis, V.A. 2017. Environmental Benefits and Control of Pollution to Surface Water and Groundwater by Agroforestry Systems: a Review. Water Resources Management, 32(1): 1-29. https://doi.org/10.1007/s11269-017-1805-4

Saha, S.K., Nair, P.K.R., Nair, V.D. \& Kumar, B.M. 2009. Soil carbon stock in relation to plant diversity of homegardens in Kerala, India. Agroforestry Systems, 76(1): 53-65. 10.1007/s10457-009-9228-8

Schroth, G., Lehmann, J., Rodrigues, M.R.L., Barros, E. \& Macèdo, J.L.V., 2001. Plant-soil interactions in multistrata agroforestry in the humid tropics. Agroforestry Systems, 53(2): 85-102.

https://doi.org/10.1023/a:1013360000633

Shi, L., Feng, W., Xu, J. \& Kuzyakov, Y. 2018. Agroforestry systems: Meta-analysis of soil carbon stocks, sequestration processes, and future potentials. Land Degradation \& Development, 29: 3886-3897. https://doi.org/10.1002/ldr.3136

Sood, K.K. \& Mitchell, C.P. 2009. Identifying important biophysical and social determinants of on-farm tree growing in subsistence-based traditional agroforestry systems. Agroforestry Systems, 75(2): 175-187. https://doi.org/10.1007/s10457-008-9180-Z

Sridhar, K.R. \& Bagyaraj, D.J. 2017. Microbial Biodiversity in Agroforestry Systems. In J.C. Dagar \& V.P. Tewari, eds. Agroforestry: Anecdotal to Modern Science, pp. 645-667. Singapore, Springer. https://doi.org/10.1007/978-981-10-7650-3_25

Swamy, S.L. \& Tewari, V.P. 2017. Mitigation and Adaptation Strategies to Climate Change Through Agroforestry Practices in the Tropics. Agroforestry, 725-738. https://doi.org/10.1007/978-981-107650-3_29

Tschora, H. \& Cherubini. 2020. Co-benefits and trade-offs of agroforestry for climate changemitigation and other sustainability goals in West Africa. Global Ecology and Conservation, 22: e00919.

https://doi.org/10.1016/j.gecco.2020.e00919

Zhu, X., Liu, W., Chen, J., Bruijnzeel, L.A., Mao, Z., Yang, X., Cardinael, R., Meng, F.-R., Sidle, R.C., Seitz, S., Nair, V.D., Nanko, K., Zou, X., Chen, C. \& Jiang, X.J. 2019. Reductions in water, soil and nutrient losses and pesticide pollution in agroforestry practices: a review of evidence and processes. Plant and Soil. https://doi.org/10.1007/s11104-019-04377-3 


\title{
41. Syntropic Agriculture
}

\author{
Felipe S. Pasini', Dayana V. Andrade1, Fabio Rubio Scarano ${ }^{1,2}$ \\ 'Universidade Federal do Rio de Janeiro, PPG Ciências Ambientais e Conservação, Macaé, Brasil \\ ${ }^{2}$ Universidade Federal do Rio de Janeiro, Dept. Ecologia, Rio de Janeiro, Brasil
}

\section{Description of the practice}

Syntropic Agriculture (SA), also known as Successional Agroforestry21, is a set of principles and practices created by the Swiss geneticist and farmer Ernst Götsch, who has lived in Brazil since 1982. It conciliates food production and ecosystem regeneration by incorporating ecological succession and plant stratification into planning and management of croplands. As a result, layers of vegetation are harmonized with their life cycle, one after another, respecting the timeline of successional consortia: placenta (annual and biannual species), secondary (trees and shrubs of short and medium lifecycle), climax (long lifecycle), and transitional (very long lifecycle), according to Ernst Götsch's classification (Figure 14). All designs and techniques aim to optimize photosynthesis and biomass production, by placing each cultivated plant in its "just right" position in space (strata) and in time (succession).

Each consortium of each successional step is divided into vertical layers of occupancy based on plants' relative height and sunlight demands. In general, the degree of occupation in each layer follows the pattern of 20 percent of emergent species, 40 percent of canopy species, 60 percent of medium strata, 80 percent of lower layer, and 15-20 percent of ground cover species, considering overlaps between different strata (Figure 15). The constant pruning and positioning of the vegetation are key practices to guarantee enough biomass production to keep the ground covered all year, which feeds the soil's fauna and protects it from direct rain, overheating, and erosion. It also replaces the need for herbicides, since the optimum occupation of all strata and the mulch provided by their pruning leave no niche for non-desired plants.

\footnotetext{
${ }^{21}$ According to ICRAF's definition, agroforestry is a land-use system in which woody perennials are integrated with crops and/or animals, simultaneously or not (Nair, 1993). An approach based, therefore, essentially on consortia and crop rotation. Syntropic Agriculture (SA) differs from agroforestry because its main pillars are (1) succession, (2) stratification, and (3) the notion of syntropy applied to ecosystem dynamics. Although SA embraces tree-species succession in most designs, its approach can also be applied to non-forest environments.
} 
SA is a process-based rather than an input-based approach. Organisms behave as open systems that overcome the tendency to increase entropy by converting environmental resources (food, oxygen, water) into growth, reproduction and differentiation. This capacity (that is present in biological systems) results in hierarchically broader organizational levels throughout succession, which culminates in the emergence of increasingly complex biological structures. In short, while entropy governs thermodynamic transformations that release energy at the expense of complexity, syntropy governs life, which accumulates and organizes energy, for example, in organic molecules, resulting in progressively more complex forms (Andrade, Pasini and Scarano, 2020). Life processes are cumulative; therefore, each successional step "uses" the accumulated resources of previous cycles to grow, and in turn, delivers a more complex environment to the next one. Just like in natural ecosystems, each assemblage within a successional stage is an inseparable entity of biotic and abiotic elements, arranged and distributed to favor synergistic relationships that result in higher accumulation of energy by the system, which translates into fertility for cultivated plants.

\section{Range of applicability}

Since 1993, Ernst Götsch's approach began to spread among Brazilian farmers mainly through practical courses and with specific channels on the internet. Estimates are that at least 5000 family farms have adopted this practice (or some aspects of it) all across the country (Andrade, 2019), under different terminologies such as Successional Agroforestry, Dynamic Agroforestry, Analog Regenerative Agroforestry, and since 2013, as Syntropic Agriculture (SA). It has also been exported to other countries in Latin America (the Plurinational State of Bolivia, Colombia, Chile, Mexico), The Caribbean (Martinique, Curacao Islands), Europe (Portugal, Spain, France, Germany, Italy, Greece), Africa (Mozambique), and Oceania (Australia) ( Andrade, Pasini and Scarano, 2020).

\section{Impact on soil organic carbon stocks}

Given the heterogeneity of SA and its application, it remains challenging to state its overall potential to stock carbon. It is possible though to infer that highly diverse tree-based and successional designs promote carbon sequestration both aboveground (embedded in the biomass of trees) and belowground (with the increase of organic matter and biologic activity in the soil). Despite the increasing number of adopters in the past three decades, SA (or Successional Agroforestry) received little consideration from formal investigation institutes and universities in its early years. The practice has only recently attracted academic communities' attention, given the escalation of agriculture-related environmental impacts and the general recognition that innovative approaches can also emerge from farmers' experiences. As SA spreads to other countries, such as Germany, Spain, and Portugal, we expect to see more research relating the practice with specific environmental benefits. 


\section{Other benefits of the practice}

\subsection{Improvement of soil properties and benefits to soil threats}

One of the premises of SA is the permanence of soil cover with living plants and mulch all year long. To achieve that without using external inputs, the system must produce high quantities of biomass yearly.

This practice has proven beneficial for the following reasons:

(A) It prevents humidity loss and soil erosion. It increases soil permeability and its capacity to retain water (Primavesi, 2002);

(B) It protects the soil from direct rain and sun exposure, favoring the proliferation of organisms responsible for soil structuring and humus formation;

(C) The diversity of biomass sources increases nutrient availability. A soil sample from a 12-year old system on Ernst Götsch's farm (Bahia, Brazil) contained seven times more phosphorus available than an adjacent unmanaged site (Peneireiro, 1999);

(D) The same study showed that the constant pruning and mulching increased nutrient flow when compared to a natural forest. Furthermore, both vegetation and soil macrofauna were in more advanced stages of succession when compared to a natural regeneration site of the same age;

(E) The high quantity of wood decomposition favors the proliferation of beneficial fungi, which helps create more stable forms of carbon in the soil, enhancing structure and fertility (Tugel, Lewandowski and Happe-vonArb, 2000);

(F) Multilayer vegetation works as a windbreak and prevents soil drying. Multistrata root occupation holds soil together and avoids erosion (Primavesi, 2002);

(G) Layers of vegetation mean layers of photosynthesis. Since photosynthesis is an endothermal process, the gradual difference in a layer's occupation - denser at the bottom and sparser in the upper strata works as a heat sink. It creates a temperature gradient that helps maintain moisture in the soil (Coats, 2001);

(H) Multicrop designs based on species succession associated with a year-long soil cover also triggers the succession of soil organisms (Tugel, Lewandowski and Happe-vonArb, 2000);

(I) Since the system is mainly occupied by perennial species and the soil is constantly covered by mulch, there is no need to use herbicides;

(J) Biomass cycling combined with a multilevel root occupancy favor water infiltration and soil structure, which allows the occupation of deeper layers of soil by beneficial microorganisms;

(K) Having plants performing vegetative growth in different development stages guarantees a constant food supply via exudates, favoring beneficial soil organisms;

Table 181 shows how each of the soil properties above are related to soil threats. 


\begin{tabular}{|l|l|}
\hline Soil threats & Related soil property \\
\hline Soil erosion & $(\mathrm{A}),(\mathrm{B}),(\mathrm{F}),(\mathrm{I})$ \\
\hline Nutrient imbalance and cycles & $(\mathrm{C})$ \\
\hline Soil salinization and alkalization & $(\mathrm{B}),(\mathrm{E})$ \\
\hline Soil contamination/pollution & $(\mathrm{I})$ \\
\hline Soil acidification & $(\mathrm{B}),(\mathrm{E})$ \\
\hline Soil biodiversity loss & $(\mathrm{B}),(\mathrm{E}),(\mathrm{H}),(\mathrm{J}),(\mathrm{K})$ \\
\hline Soil sealing & $\mathrm{NA}$ \\
\hline Soil compaction & $(\mathrm{A}),(\mathrm{J})$ \\
\hline Soil water management & (A), (G) \\
\hline
\end{tabular}

\subsection{Increases in production (e.g. food/fuel/feed/timber/fibre)}

One advantage of successional models is their potential to generate multiple harvests as the system evolves, which improve farmer's resilience to market fluctuations, environmental variables, and self-sufficiency (Miccolis et al., 2016). This can be a key factor when establishing tree-based plantations (fruits, timber, oil), providing the farmer with short-term yields until the main crops enter production. An experiment of successional agroforestry associated with palm-oil plantation in the Brazilian Amazon has shown that small hold farmers can benefit from adding short-cycle crops in the first years, before the palm trees start producing (Kato et al., 2011). The same experiment also included slow-growing timber and nut trees to become the main products after the decline of palm-oil yields ( 25 years).

In Brazilian semiarid zones, it was a common practice even for small hold farmers to grow castor-oil plant (Ricinus communis) in monoculture designs. When the project "Policultura no Semi-Árido" (Del Arco Sanches, 2009) introduced the successional agroforestry approach in the 1990's, 750 families started combining castoroil with other plants, both with shorter cycles (beans, corn, watermelon, sesame) and longer cycles (fruit trees, prickly pear and timber). In addition to diversified harvests, some farmers also saw an increase in castor-oil production. Previously, the average in the region was $800 \mathrm{~kg} / \mathrm{ha}$ of castor seeds. Within the project, some farmers harvested as much as $2100 \mathrm{~kg} / \mathrm{ha}$, with the benefit of inheriting a fruit and timber plantation after the castor-oil yield, instead of empty and exposed soil. 
Hoffmann (2013) compared economic data from eight agroforestry systems in Brazil and found that the average yields projected for 25 years in two SA sites were 16 and $21 \mathrm{t} / \mathrm{ha} / \mathrm{yr}$. Other agroforestry systems produced between 2 and $13 \mathrm{t} / \mathrm{ha} / \mathrm{yr}$. Successional systems were an advantageous alternative in southeast of Brazil when compared to conventional agriculture. Rebeschini (2008), by collecting data from four family farms that implemented succession-based agroforestry designs at Ribeira valley, showed that these systems produce more and for longer in less space. They concluded that in order to achieve the same economic productivity, the conventional grains (soybean and corn) and milk production would require at least 10 times more land.

\subsection{Mitigation of and adaptation to climate change}

Multi-layer successional tree-based designs aim to mimic natural forest dynamics and therefore deliver all environmental services associated with that (see also 4.1). The higher the metabolism and photosynthetic rate, the greater the carbon absorption by plants (Ramachandran Nair et al., 2010; Miccolis et al., 2016). Therefore, there is great potential for carbon sequestration in SA practices, although we are unaware of studies in this respect. Similarly, SA has a large potential for climate-change adaptation. Since it both mimics successional processes and fosters ecosystem regeneration, it seems it can be framed as a type of ecosystem-based adaptation to climate change (EbA). The United Nations Convention of Biological Diversity (CBD, 2009) broadly defines this adaptation practice as "the use of biodiversity and ecosystem services as part of an overall adaptation strategy to help people to adapt to the adverse effects of climate change". It refers to the use of natural capital by people to adapt to climate change impacts, which can also have multiple co-benefits for mitigation, protection of livelihoods and poverty alleviation (Munang et al., 2013). It has also been argued that practices that fit within the EbA framework can be important in the transition to sustainability (Scarano, 2017). In this sense, SA as a practice can simultaneously address multiple Sustainable Development Goals of the 2030 Agenda, such as poverty reduction (SDG1), ending hunger and malnutrition (2), promoting health and wellbeing (3), climate action (13), and land biodiversity conservation and restoration (15).

\subsection{Socio-economic benefits}

A set of interviews reported in Andrade, 2019 allows an understanding of the socio-economic implications derived from the adoption of SA's framework. Different profiles of practitioners mentioned: (1) new kinds of engagement in innovative ways of commerce and service exchange, favoring community rather than individualistic values; (2) establishment of a horizontal network of mutual support, where practitioners can exchange knowledge and resources; (3) changed perception of natural processes related to farming, which creates another level of attachment and intimacy with land, now seen as an organism; (4) changes towards healthier eating habits of the family, based on their own production; and (5) "joy" and "pride", in the personal dimension, as a result of implementing the new practices and seeing the result in coping with extreme weather conditions such as hurricanes and drought.

Luz (2015) assessed the economic viability of a successional system in central Brazil focused on horticulture for later fruit and coffee production. Payback happened after 1.1 month. In one year, benefits surpassed costs in 82 percent. 


\section{Potential drawbacks to the practice}

\subsection{Conflict with other practice(s)}

SA relies on ecological succession and stratification as a replacement for fertilizers and defensives. Therefore, it conflicts with all practices that interrupt succession, being it conventional or organic.

\subsection{Decreases in production (e.g. food/fuel/feed/timber/fibre)}

Despite several studies show that successional tree-based designs do not impact production (Luz, 2015; Hoffmann, 2013; Rebeschini, 2008; Schneider et al., 2017; Schulz, Becker and Götsch, 1994), it is challenging to scale it up because of the constrictions listed next, in item 7.

\section{Recommendations before implementing the practice}

An ideal syntropic design includes a stratified consortium of plants for each successional step. Therefore, farmers should identify the species suitable to fill all gaps in space and time based on their behavior and life cycle. All consortia - be it placenta, secondary, or climax - must have species occupying most of their layers: lower, medium, canopy, and emergent, in a distribution ratio described in Figure 15. The strata are related to plants' sunlight demand and their shade tolerance, not their height. For example, corn is emergent from placenta II consortia, eucalypt is emergent from secondary I consortia, and cork oak tree is also emergent but from climax consortia. Ideally, all species from all strata and succession steps are planted together to cause minimum soil disturbance and enhance synergistic relationships, and each consortium is succeeded by the next according to their growing speed. For example, a placenta consortium of arugula or black beans (lower-medium layer), lettuce (medium), broccoli (canopy), and crotalaria (emergent) can be succeeded by a longer cycle consortium of watermelon (lower), carrot (medium), tomato (canopy) and corn or sunflower (emergent). It is still possible to go further in the placenta stage with ginger or pineapple (lower), garlic, taro, green pepper, (medium), manioc (canopy), castor-oil, and/or papaya (emergent). After the placenta stage, which can take up to 24 months, the secondary plants take over the area, following the same stratification pattern, for example, rosemary (lower), pomegranate (medium), avocado (canopy), and eucalypt (emergent), and so on until reaching the next longer lifecycle consortium. In some cases, technical pruning might be necessary to synchronize plant's growth and production. In each step, there should be plants to produce biomass enough to keep the soil covered all year through pruning and mulching. In deciduous and semideciduous environments, it is possible to include placenta species every year as the trees drop their leaves. In evergreen forests, the repetition of placenta cycles (annuals and biannuals) is possible (though not always recommended) by promoting severe pruning in the trees. 


\section{Potential barriers for adoption}

Despite the environmental, social and economic advantages described above, farmers face several challenges in adopting SA practices (Table 182).

Since there is no machinery suitable for managing stratified and biodiverse designs, most of SA operations are still performed manually. This increases labor costs and turns it inviable for large-scale enterprises.

SA does not have a toolkit easily applicable to all conditions. Diagnosing and decision-making processes require transdisciplinary education articulated with SA's particularities. The required knowledge, that is regarding the management of multiple species, goes against the trend towards specialization normally imposed by the market and educational institutions.

The establishment of a biodiverse system requires specific planning and logistics. Farmers need forestry and agricultural materials, of all successional stages at their disposal at the moment of implementation.

Table 182. Barriers to adoption

\begin{tabular}{|c|c|c|}
\hline Barrier & YES/NO & \\
\hline Cultural & Yes & $\begin{array}{l}\text { Diversified forest-based systems are culturally detached from } \\
\text { current monoculture paradigm (Andrade, 2019; Pasini, 2017). }\end{array}$ \\
\hline Social & No & $\begin{array}{l}\text { In the household level, the adoption of innovative agricultural } \\
\text { practices is perceived as a risk (Valdivia, Barbieri and Gold, 2012). } \\
\text { Transitions in the scale of a territory require governance with } \\
\text { participatory approaches. }\end{array}$ \\
\hline Economic & Yes & $\begin{array}{l}\text { Difficulty to access credit and subsidies. High cost to implement a } \\
\text { new system (Hoffmann, 2013). }\end{array}$ \\
\hline Institutional & Yes & $\begin{array}{l}\text { In general, Institutions responsible for rural extension, technical and } \\
\text { theoretical education, and related to access to credit are still not } \\
\text { prepared to assist farmers in complex agroforestry designs } \\
\text { (Rebeschini, 2008). }\end{array}$ \\
\hline $\begin{array}{l}\text { Legal } \\
\text { (Right to } \\
\text { soil) }\end{array}$ & Yes & $\begin{array}{l}\text { Long-term cultivation designs can be difficult when farmers do not } \\
\text { have safe and legal access to the land. }\end{array}$ \\
\hline Knowledge & Yes & Lack of educational material and accessible training facilities. \\
\hline Technology & Yes & $\begin{array}{l}\text { Scaling up is difficult because specialized machinery is not available } \\
\text { in the market (Andrade, 2019). }\end{array}$ \\
\hline
\end{tabular}




\section{Representations of the practice}

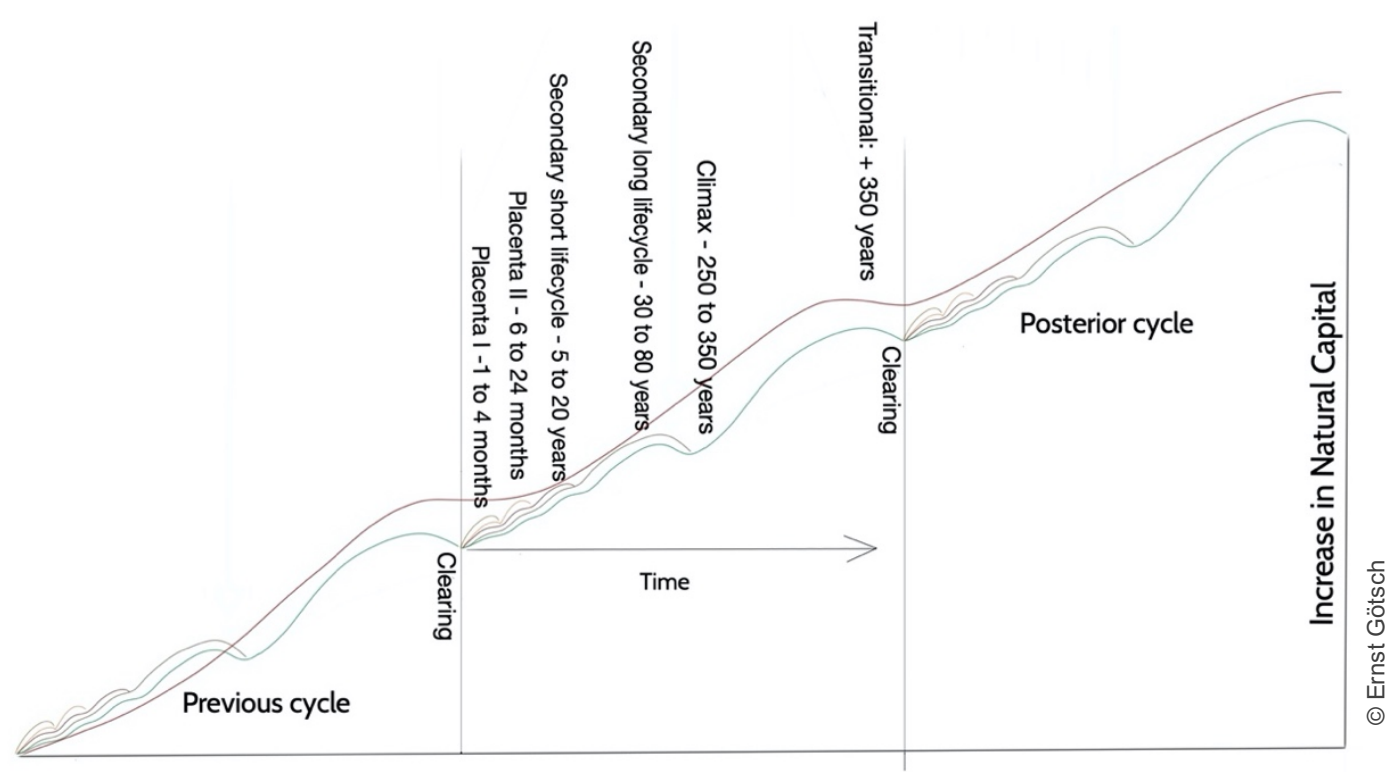

Figure 14. Succession scheme proposed by Ernst Götsch that illustrates the intervals of successional consortia occupation (placenta, secondary, climax and transitional) between disturbances (clearings) under natural conditions

In managed systems, it's possible to accelerate succession through pruning and removal of aged vegetation

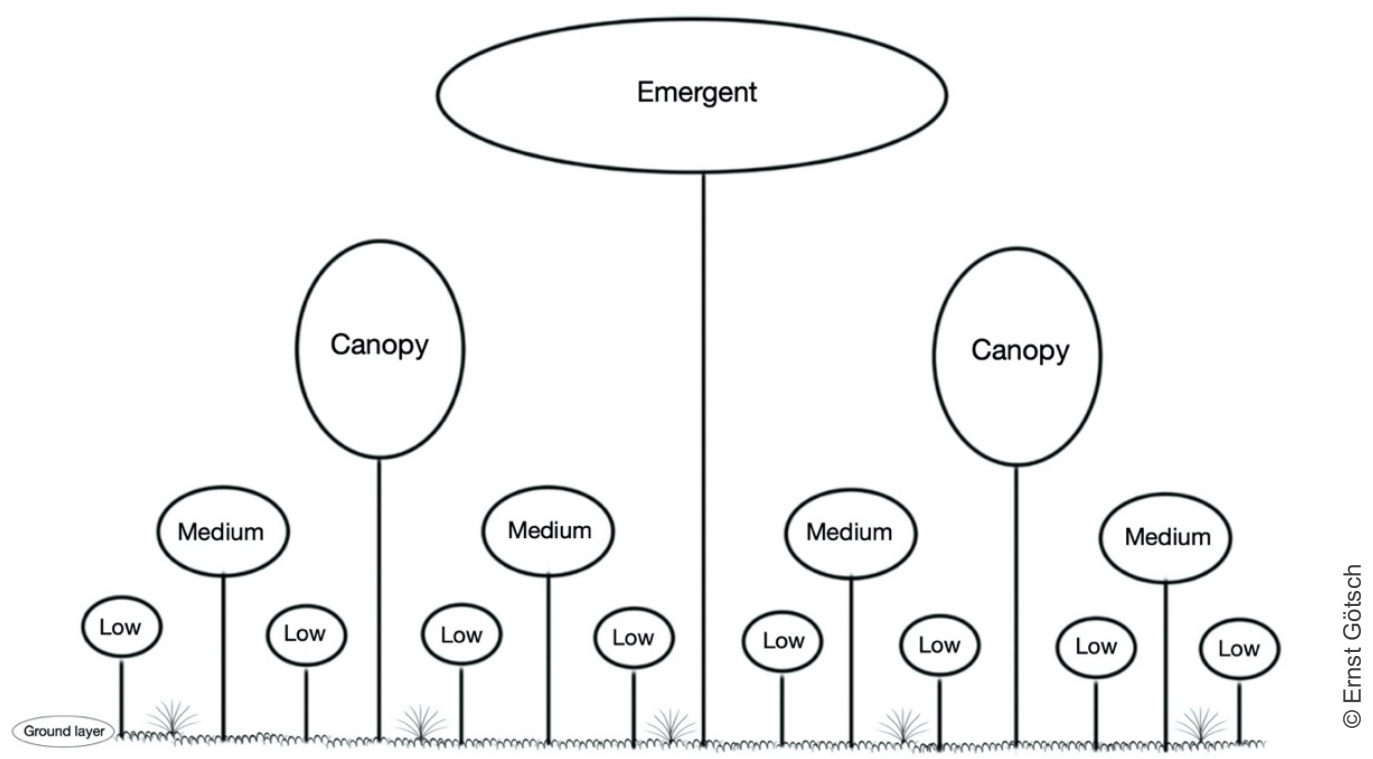

Figure 15. Strata occupation proposed by Ernst Götsch with approximately 20 percent cover of emergent layer, 40 percent canopy, 60 percent medium, 80 percent low and 15-20 percent ground layer

Such distribution increases the photosynthesis rate per area and facilitates cooling-down thermodynamic processes and water retention 


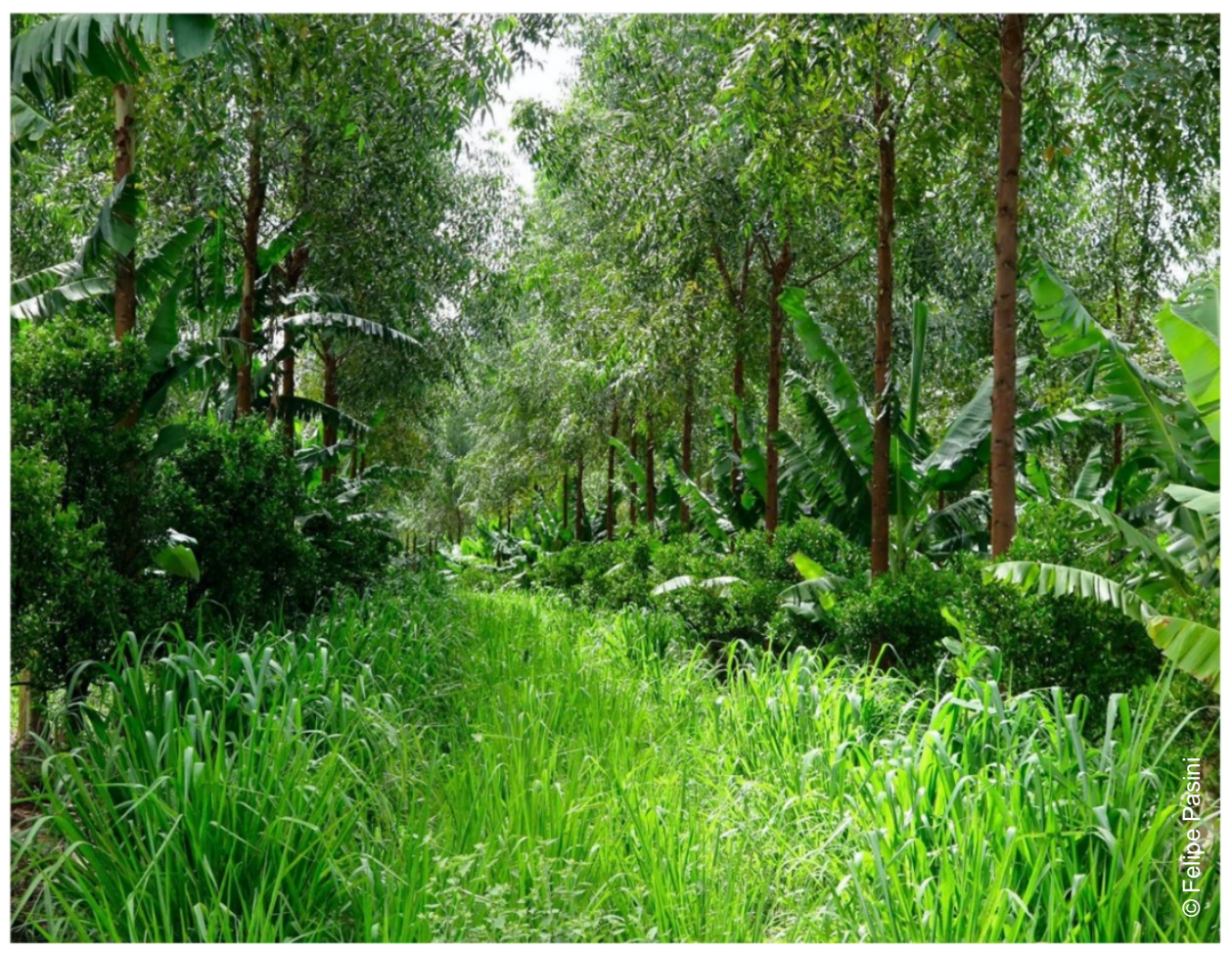

Photo 55. Stratified system at Fazenda da Toca, Brazil. Eucalypts, banana, citrus and grass.

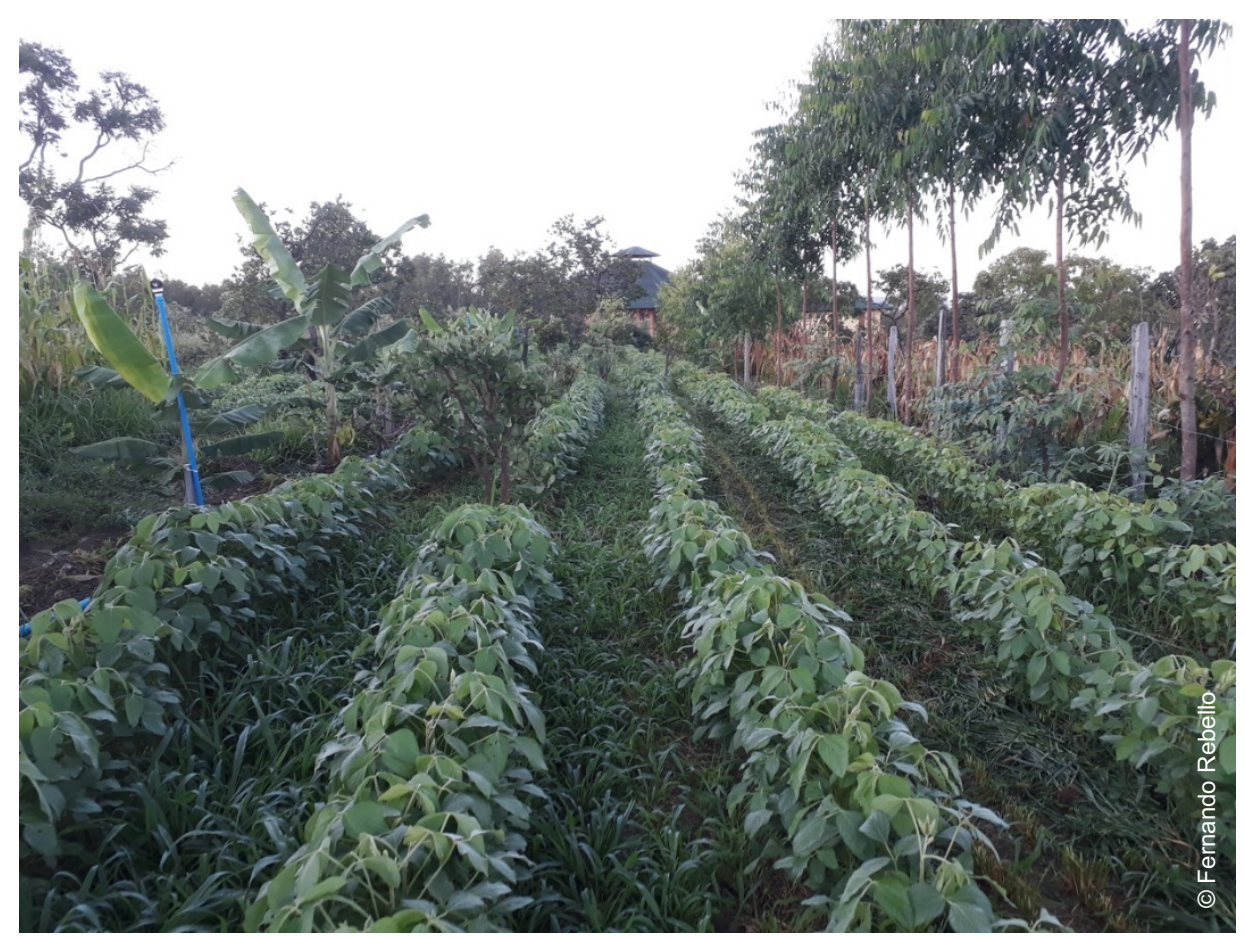

Photo 56. Scheme for grains/vegetables cultivation between tree-lines.

The area in the photo (CEPEAS, Brazil, 2019) aims to grow soybeans in single lines amongst grass stripes (Panicum maximum), which maintain the soil covered all year and provide mulch for soybeans. The trees are heavily pruned before seeding the grains, mimicking the dynamics of a forest clearing to allow the addition of placenta species. 
Table 183. Related cases studies available in volumes 3 and 5

\begin{tabular}{|l|c|c|c|c|}
\hline Title & Region & $\begin{array}{l}\text { Duration } \\
\text { of study } \\
\text { (Years) }\end{array}$ & Volume & $\begin{array}{l}\text { Case- } \\
\text { study } \\
\text { No. }\end{array}$ \\
\hline $\begin{array}{l}\text { Syntropic Agriculture in a Mediterranean } \\
\text { Context }\end{array}$ & Europe & 2 & 3 & 23 \\
\hline
\end{tabular}




\section{References}

Andrade, D.V.P. 2019. Agricultura, meio ambiente e sociedade: um estudo sobre a adotabilidade da agricultura sintrópica. Rio de Janeiro, Brazil. Núcleo em Ecologia em Desenvolvimento Sócio-Ambiental, Universidade Federal do Rio de Janeiro. (PhD Thesis)

Andrade, D., Pasini, F. \& Scarano, F.R. 2020. Syntropy and innovation in agriculture. Current Opinion in Environmental Sustainability, 45: 20-24. https://doi.org/10.1016/j.cosust.2020.08.003

Convention of Biological Diversity (CBD). 2009. Connecting Biodiversity and Climate Change Mitigation and Adaptation: Report of the Second Ad Hoc Technical Expert Group on Biodiversity and Climate Change. CBD Technical Series 41, Montreal, Canada. (also available at: https://www.cbd.int/doc/publications/cbdts-41-en.pdf)

Coats, C. 2001. Living Energies. Dublin, Gateway Press.

Del Arco Sanches, C. 2009. Projeto Policultura no Semi-Árido. Revista Brasileira de Agroecologia, 4(2): 2348-2352.

FAO. 2017. Agroforestry for landscape restoration. [online]. [Cited 15 June 2020]. http://www.fao.org/3/b-i7374e.pdf

Hoffmann, M. 2013. Sistemas Agroflorestais para Agricultura Familiar: análise econômica. Departamento Agronegócios, Universidade de Brasília (MSc. Dissertation).

Kato, O.R., Vasconcelos, S.S., Capela, C.J., Miranda, I.S., Lemos, W.P, Maues, M.M., Azevedo, R., Castellani, D.C. \& Thom, G. 2011. Projeto Dendê em sistemas agroflorestais na agricultura familiar. In Porro, R., Kanashiro, M., Ferreira, M.S.G., Sampaio, L.S., Sousa, G.F. (Eds.) Congresso Brasileiro de Sistemas Agroflorestais. Anais. Pará, Brasil, Embrapa Amazônia oriental, UFRA, CEPLAC, EMATER, ICRAF.

Luz, I.S.B. 2015. Sistemas agroflorestais sucessionais: viabilidade financeira para a agricultura familiar. Departamento de Engenharia Florestal, Universidade de Brasília. (MSc. Dissertation).

Miccolis, A., Peneireiro, F.M., Marques, H.R., Vieira, D.L.M., Arco-Verde, M.F., Hoffman, M.R., Rehder, T. \& Pereira, A.V.B.. 2016. Restauração ecológica com sistemas agroflorestais: como conciliar conservação com produção. Opções para Cerrado e Caatinga. Instituto Sociedade, População e Natureza ISPN/Centro Internacional de Pesquisa Agroflorestal. Brasília, Brasil. ICRAF.

Munang, R., Thiaw, I., Alverson, K., Mumba, M., Liu, J. \& Rivington, M. 2013. Climate change and Ecosystem-based Adaptation: a new pragmatic approach to buffering climate change impacts. Current Opinion in Environmental Sustainability, 5(1): 67-71.

Nair, P.K.R. 1993. An introduction to agroforestry. Kluwer Academic Publishv cers. Dordrecht, Netherlands.

Pasini, F.S. 2017. A Agricultura Sintrópica de Ernst Götsch: história, fundamentos e seu nicho no universo da Agricultura Sustentável. Núcleo em Ecologia em Desenvolvimento Sócio-Ambiental, Universidade Federal do Rio de Janeiro. (MSc. Dissertation). 
Peneireiro, F.M. 1999. Sistemas Agroflorestais Dirigidos Pela Sucessão Natural: Um Estudo De Caso. Escola superior de Agricultura, Area Ciências Florestais, Universidade de São Paulo. (MSc. Dissertation).

Primavesi, A. 2002. Manejo ecológico do solo: a agricultura em regiões tropicais. São Paulo, Nobel.

Ramachandran Nair, P.K., Nair, V., Kumar, B.M. \& Showalter, J.M. 2010. Carbon sequestration in agroforestry systems. In Sparks, D.L. (Ed.) Advances in Agronomy. San Diego, USA, Academic Press of Elsevier, 108: 237-307.

Rebeschini, A.P. 2008. Construção participativa de Indicadores de Sustentabilidade em Sistemas Agroflorestais no Vale do Ribeira. Registro, Brasil, Porter.

Sanches, C.D.A. 2011. A contribuição da sistematização de experiências para of fortalecimento do campo agroecológico e da agricultura familiar no Brasil. Departamento Ciências Agrárias, Universidade Federal de São Carlos. (MSc. Dissertation).

Scarano, F.R. 2017. Ecosystem-based adaptation to climate change: concept, scalability and a role for conservation science. Perspectives in Ecology and Conservation, 15(2): 65-73.

Schneider, M., Andres, C., Trujillo, G., Alcon, F., Amurrio, P., Perez, E. \& Milz, J. 2017. Cocoa and total system yields of organic and conventional agroforestry vs. monoculture systems in a long-term field trial in Bolivia. Experimental Agriculture, 53(3): 351-374.

Schulz, B., Becker, B. \& Götsch, E. 1994. Indigenous knowledge in a “modern” sustainable agroforestry system - a case study from eastern Brazil. Agroforestry Systems, 25(1): 59-69.

Tugel, A.J., Lewandowski, A.M. \& Happe-vonArb, D. 2000. Soil Biology Primer. Iowa, USA, Ankeny.

Valdivia, C., Barbieri, C. \& Gold, M.A. 2012. Between Forestry and Farming: policy and environmental implications of the barriers to agroforestry adoption. Canadian Journal of Agricultural Economics/Revue Canadienne d'Agroeconomie, 60(2) : 155-175. https://doi.org/10.1111/j.1744-7976.2012.01248.x 


\section{Conservation agriculture}

\section{Stéphane Boulakia',2, Hoà Tran Quoc ${ }^{3,4}$, Olivier Husson ${ }^{1,2}$, Pascal Lienhard ${ }^{11,2}$, Oumarou Balarabé ${ }^{1,2}$, Florent Tivet ${ }^{1,2}$, Jean-Pierre Sarthou ${ }^{5}$}

${ }^{1}$ CIRAD, UPR AIDA, Montpellier, France

${ }^{2}$ AIDA, Univ Montpellier, CIRAD, Montpellier, France

${ }^{3}$ CIRAD, UPR GECO, Montpellier, France

${ }^{4} \mathrm{GECO}$, Univ Montpellier, CIRAD, Montpellier, France

${ }^{5}$ University of Toulouse, INRA/INPT UMR AGIR., Castanet-Tolosan, France

\section{Description of the practice}

Conservation Agriculture (CA) regroups a vast diversity of agroecological cropping systems characterized by the simultaneous and continuous application of 3 practical principle ${ }^{22}$ :

i. No-Tillage, i.e. minimum mechanical soil disturbance with direct seeding, no-till weeding and minimum soil disturbance with other operations, including harvest. The FAO definition specifies that "the disturbed area must be less than $15 \mathrm{~cm}$ wide or less than 25 percent of the cropped area (whichever is lower). Mechanical disturbance should be limited to the purpose of placing seed or fertilizer";

ii. Permanent soil organic cover with crop residues and/or cover crops to the extent allowed by water availability. Ground cover measured immediately after the seeding operation should have over 30 percent cover;

iii. Species diversification through varied crop sequences and associations involving at least three different crops.

\footnotetext{
${ }^{22}$ after FAO (http://www.fao.org/conservation-agriculture/overview/principles-of-ca/en/2
} 
However, the widespread and generic use of the term CA, for diverse soils, agroecosystems, and without consideration to the differences among CA-based cropping systems (principally the annual input of biomass (i.e. crop sequence, intensity, frequency and quality of dry matter inputs)) can lead to ambiguous and unexplained interpretation regarding the effects of $\mathrm{CA}$ on the rate and magnitude of soil resilience, increase in grain yield and soil ecosystem services. For the sake of accuracy, when assessing the soil organic carbon (SOC) accumulation, enhancement of soil ecosystem functions and services, it is key to properly describe the cropping systems as reminded by Derpsch (2014) and Séguy (2009).

Through the application of these 3 practical principles, the restoration of soil functioning is driven by: (1) a high and continuous production of above- (litter system) and belowground (roots and roots exudates) biomass, even in poor soils and across the dry season, through the use of a large functional diversity of plants enhancing ecosystem services (e.g. soil protection; enhancing water and nutrient-use efficiency, exploring a large volume of soil, restructuring the soil); (2) keeping the soil permanently covered, maintaining through the litter system a continuous flow of fresh soil organic matter (FOM) enhancing the dynamics of water and nutrients; and (3) sustaining biological regulations by and of macro- and microorganisms thanks to plant biomass and organic matter as energy sources, hence resulting in the enhancement of various functions (i.e. bioturbation, chemical transformation, aggregation, biological nitrogen fixation, ecological balance of the different food webs in the soil including deleterious ones for crops...). These three functional principles (high functional diversity of plants, continuous flow of fresh SOM, high and diverse biological activity) are mutually reinforcing, allow CAbased cropping systems to fulfill complementary ecosystem services, common to all CA systems and key for forthcoming agricultural and environmental challenges. Finally, they make it possible to reach two complementary objectives which should always be targeted as they represent the core explanatory processes: retaining most of the nutrients into the biomass and minimizing nutrient loss; both of these occur thanks to spatial and temporal arrangement of main crops and cover/relay crops, which optimizes nutrient recycling (Séguy, 2009).

CA cropping systems can be designed for field crops, horticultural productions and even integrate trees. Through the use of cover crop species of high fodder value, they open multiple pathways for strengthened synergies between crop and livestock productions.

CA-based cropping systems can be split into two groups of practices:

- CA on dead soil cover in which crops are sown in residues/stubble and/or a terminated cover crop biomass. The termination of the cover crop can be done physically (mechanically with a roller, or naturally thanks to frost), with or without using complementary application of herbicide;

- CA on living cover in which the vigor of the cover crop is restrained, but plants are not terminated, prior to the sowing of the main crop but cover crops are not terminated and will grow back after harvest of the main crops . The control of the cover crop is made using mechanical implement like e.g. a roller crimper, possibly associated with moderate herbicide application. 
Some recent trends in CA-based system design, developed and adopted by advanced practitioners, include:

- mix species and multifunctional cover; a mix of 3-4 species is common and more elaborated mixes can blend up to more than 10 species to aggregate with organic matter fluxes multiple ecosystemic functions like symbiotic and non-symbiotic N-fixation, pest regulation, soil macroporosity and drainage capacity ,

- green planting (Duiker, 2015) consists of planting the main crop into a green and standing cover crop; it tends to replace practices where cover crops used to be terminated several weeks prior to crop sowing, easing the planning and decision for sowing in face of more unpredictable weather,

- broadcast seeding of the following crop (either cash- or cover crop) in the still living and standing crop (either cover- or cash crop), followed by the cover rolling when the previous one was the cover crop, and

- preference given to living mulch rather than dead one, when possible, offering more resilient options in the face of climate change.

\section{Range of applicability}

There are no biophysical limits to CA extension among existing agro-ecosystems. However, the intensification of CA-based systems will vary depending on context elements like the agro-ecosystems, initial soil status, rainwater regime and on the implemented cropping systems itself with its quality and amount of biomass produced and returned to the soil.

We can consider that CA was first initiated with first significant no-till development in the 60's in United States of America (Farooq and Siddique, 2015). It then reached subtropical regions in South America (Brazil, Argentina) in early-mid 70's through the key involvement of pioneers farmers (Herbert Bartz, Frank Dijkstra, Manoel Pereira) and first research studies (Kemper and Derpsch, 1981; Borges, 1993). Later on, from the mid 80's started adaptation to humid tropical regions of Central Brazil with the necessity to insert cover crops to strengthen FOM inputs (Landers, 1994; Séguy et al., 1998). In parallel or later on, CA has been developed under hot semi-arid (Australia), continental (Canada, Northern United States of America, Central Asia), oceanic (western Europe), mediterranean (Southern Europe and North Africa) pedoclimatic conditions (Kassam, 2019).

All types of field crops can be grown under CA: systems have been developed and successfully promoted for cereals, pulse, oilseed, cotton, roots and tubers crops, both under rainfed and irrigated conditions.

However, CA extension can be strongly questioned by socio-economic conditions at different levels, farms (production systems), community and/or region (agrarian system) that call for dynamic and participatory process to support adaptation and adoption of CA. Those processes have to consider adaptation of CA practices to farm types, relationships between farmers or communities and market actors, and agrarian environment. They may also have to consider organizational arrangements at levels above farms that are needed for a possible and efficient development of CA-based systems.

For instance, in Savanna regions of west Africa, fierce competition for biomass may exist between farmers and cattle breeders who can be sedentary or pastoralist (Palm et al., 2014). In these contexts, CA is more challenged by the capacity to engage coordination at community level on fire control, open grazing regulation, land 
development and small farmers' equipment with adapted machines and technology (Giller et al., 2015), than by biophysical conditions even under limited rainfall of 700-800 mm. Nevertheless, those complex coordinations appear to be prerequisites to allow the production of high biomass-C inputs, FOM return to soil and adjust biomass fluxes for livestock from plot to farm and community levels. In those contexts, the main issue is not to produce biomass but to set collective organizations that allow to decide and implement trade-off between biomass kept for soil fertility management and biomass exported to feed animals.

CA extension when newly introduced in a region or country is rapidly confronted by the lack of cover crops seeds in local markets with challenges related to the quantity, quality, and regularity at affordable price. Other constraints are still linked to the access to specific implements with no-till planters, seeders, roller crimpers, among other implements that match with the principles of CA. In some contexts (Africa, Asia), the availability of services to farm dedicated to CA is also one of the main bottlenecks (Giller et al. 2015; Vernet et al., 2020). It is thus necessary to rapidly consider and address these issues through adapted arrangements involving farmers' organizations and market actors.

Whatever the farming context, when introduced, CA represents a disruption compared to conventional tillagebased practices. From a practical and conceptual point of view, CA represents radical changes with habits and instituted references of all stakeholders that call for continuous awareness raising and support. CA adoption often induces varying transformations of the farmers' mindset and their communities including views about the desirable future for agriculture by both public and private sectors.

A large number of studies emphasized that the biomass-C input is the main determinant of SOC accumulation (Sá et al., 2013a; Tivet et al., 2013; Paustian et al, 2019), improvement of main soil properties (porosity, water holding capacity, nutrient cycling ...), productivity and reduction of costs (Verhulst et al., 2010). That means that science-based evidence should guide the implementation of collective decision at local territory scale along with agricultural policies (financial mechanisms rewarding farmers, facilitating access to plant diversity, machinery) that contribute to a higher production and/or retention of biomass at the field and territory scale.

\section{Impact on SOC sequestration / Potential of additional}

\section{storage}

Efforts to increase SOC stocks should focus on management practices, diversified cropping sequence including cover crops, increasing biomass-C inputs which is the main driver of an accumulation of SOC (Séguy, Bouzinac and Husson, 2006; Powlson et al., 2016). Several studies, including those by Luo et al. (2010) and Fujisaki et al. (2018), emphasized that soil and climate characteristics had lower impact on SOC accumulation when compared with management practices and thus $\mathrm{C}$ inputs.

Under tropical conditions, biomass inputs (quantity and quality) and their distribution both during the wet and dry seasons are keys to increase SOC accumulation. Living cover crops during the dry season are needed to produce above and belowground biomass during extended periods, improving systems' attainable biomass yield compared to conventional systems.

Legumes, which contributed a net input of biologically fixed N, played an important role in promoting soil C accumulation. Crop rotation, and the use of deep root systems of cover crops, provide larger $\mathrm{C}$ inputs below ground (root biomass and rhizodeposition). It is largely emphasized that most accumulated $\mathrm{C}$ is derived from 
crop roots (Balesdent and Balabane, 1996) and that different crop species contribute to different soil C pools (Rossi et al., 2020). Agricultural machinery is also key to increase the residence time of the biomass on the top soil (e.g. use of roller crimpers in front of the sowing line, green sowing practice, broadcasting of cover crops minimizing soil disturbance by planters , to minimize soil disturbance, to increase SOC accumulation through increased soil aggregation with slower turnover rates in untilled soils.

We also need to consider that the positive impacts of CA on SOC accumulation, especially on topsoil horizons, are reversible and $\mathrm{SOC}$ can be released as $\mathrm{CO}_{2}$ if soil and crop management are reverted to conventional or temporary mechanical soil disturbance. It can be also offset during the transition phase by priming effect in deeper horizons where addition of labile carbon through strengthened roots exploration enhances microbial activities, increasing stable OM decomposition rate in deeper horizons (Kuzyakov, Friedel and Stahr, 2000).

Methodological considerations need also to be emphasized. First, biomass-C inputs should be recorded or estimated under medium to long-term assessment (experiments and on-farm). Second, deep sampling should be considered at least or below $30-\mathrm{cm}$ as several studies emphasized a substantially increase in SOC accumulation at deeper soil layers (Sisti et al., 2004; Boddey et al., 2010, Veloso et al., 2018) although controversies continue in this area (Luo et al., 2012). Third, Neto et al. (2010) emphasized how cautious synchronic approach, where farm fields of different years under CA are sampled simultaneously, can be used to assess changes in SOC stocks over time. Younger NT fields tended to exhibit a higher increase in soil C stocks when compared with older fields for which a steady state is reached (Brazilian Cerrado; Corbeels et al., 2016). Soil C stocks under NT cropping systems and high biomass-C inputs can reach the levels of those under the native vegetation (example of the Brazilian Cerrado; Sá et al., 2013; de Oliveira Ferreira et al., 2016) emphasizing the soil restoration potential of NT cropping systems. Finally, we have to keep in mind that SOC accumulation is only one component of GHG emissions budget and that fuel-associated $\mathrm{CO}_{2}$ emissions saving (fuel, pesticides, inorganic fertilizers) have also to be considered for a global assessment (Table 184). 
Table 184. Evolution of SOC stocks under Conservation Agriculture

\begin{tabular}{|c|c|c|c|c|c|c|}
\hline Location & $\begin{array}{l}\text { Climate } \\
\text { zone }\end{array}$ & Soil Depth & $\begin{array}{l}\text { Additional C storage } \\
(\mathrm{tC} / \mathrm{ha} / \mathrm{yr}) \pm \text { Standard Error }\end{array}$ & $\begin{array}{l}\text { Duration } \\
\text { (Years) }\end{array}$ & More information & Reference \\
\hline $\begin{array}{l}\text { Sub- } \\
\text { Saharan Africa }\end{array}$ & Tropical dry & $\begin{array}{l}\text { Between } 0.15 \text { and } 0.3 \mathrm{~m} \text {, few } \\
\text { cases } 0.4 \mathrm{~m} .3 \text { sites at } 0.6 \mathrm{~m} \\
\text { depth }\end{array}$ & $\begin{array}{l}\text { All treatments } 0.54 \pm 0.213(n=47) \\
\text { RT: } 0.96 \pm 0.226(n=9) \\
\text { RT + RR: } 0.45 \pm 0.118(n=33) \\
\text { RT + RR + D: } 1.01 \pm 0.39(n=3)\end{array}$ & 2 to 16 & $\begin{array}{l}\text { Meta analysis } \\
\text { Annual rate of increase of SOC under } \\
\text { CA compared to conventional practice } \\
\text { Reduced tillage (RT), Residue retention } \\
\text { (RR), or Crop diversification (D) }\end{array}$ & Powlson et al. (2016) \\
\hline $\begin{array}{l}\text { China } \\
\text { NE, Jinlin } \\
\text { Province } \\
\text { N, Shandong } \\
\text { Province } \\
\text { N, Hebei } \\
\text { Province } \\
\text { NW, Gansu } \\
\text { Province }\end{array}$ & & $\begin{array}{l}0-20 \mathrm{~cm} \\
0-30 \mathrm{~cm} \\
0-20 \mathrm{~cm} \\
0-40 \mathrm{~cm} \\
0-20 \mathrm{~cm} \\
0-30 \mathrm{~cm} \\
0-30 \mathrm{~cm}\end{array}$ & $\begin{array}{l}-0,10 \\
-0,46 \\
1,45 \\
0,26 \\
0,22 \\
-0,44 \\
0,32\end{array}$ & 10 & $\begin{array}{l}\text { Synthesis of various studies } \\
\text { Annual rate of increase of SOC under } \\
\text { CA compared to conventional practice }\end{array}$ & Zhang et al. (2014) \\
\hline $\begin{array}{l}\text { India } \\
\text { (Indo-Gangetic } \\
\text { Plain) }\end{array}$ & & $\begin{array}{l}\text { Between } 0.15 \text { and } 0.3 \mathrm{~m} \text {, few } \\
\text { cases } 0.4 \mathrm{~m} .4 \text { experiments at } \\
0.6 \mathrm{~m} \text { and one } 1.05 \mathrm{~m} \text { depth }\end{array}$ & $\begin{array}{l}\text { All treatments: } 0.37 \pm 0.045(n=29) \\
\text { RT: } 0.49 \pm 0.081(n=6) \\
\text { RR: } 0.16 \pm 0.046(n=19) \\
\text { D: } 0.47 \pm 0.099(n=4)\end{array}$ & 2 to 25 & $\begin{array}{l}\text { Meta analysis } \\
\text { Annual rate of increase of SOC under } \\
\text { CA compared to conventional practice } \\
\text { Reduced tillage (RT), Residue retention } \\
\text { (RR), or Crop diversification (D) }\end{array}$ & Powlson et al. (2016) \\
\hline
\end{tabular}




\begin{tabular}{|c|c|c|c|c|c|c|}
\hline Location & $\begin{array}{l}\text { Climate } \\
\text { zone }\end{array}$ & Soil Depth & $\begin{array}{l}\text { Additional C storage } \\
\text { (tC/ha/yr) } \pm \text { Standard Error }\end{array}$ & $\begin{array}{l}\text { Duration } \\
\text { (Years) }\end{array}$ & More information & Reference \\
\hline \multirow{4}{*}{$\begin{array}{l}\text { South America, } \\
\text { Brazil }\end{array}$} & Tropical humid & $\begin{array}{l}0-20 \mathrm{~cm} \\
0-40 \mathrm{~cm} \\
0-100 \mathrm{~cm}\end{array}$ & $\begin{array}{l}0.48-1.30 \\
0.73-1.98 \\
0.79\end{array}$ & $>10$ & $\begin{array}{l}\text { Complex crop rotations and cover } \\
\text { crops }\end{array}$ & Sá et al. (2013b) \\
\hline & Sub-tropical & $0-40 \mathrm{~cm}$ & $0.99 \pm 0.11$ & 22 & $\begin{array}{l}\text { Brazilian Oxisol under no-tillage } \\
\text { chronosequence, Paraná State }\end{array}$ & Sá et al. (2001) \\
\hline & Tropical humid & $0-30 \mathrm{~cm}$ & 1.90 & 12 & $\begin{array}{l}\text { Double cropping system legume - } \\
\text { cereal }\end{array}$ & Neto et al. (2010) \\
\hline & Sub-tropical & $\begin{array}{l}0-30 \mathrm{~cm} \\
0-100 \mathrm{~cm}\end{array}$ & $\begin{array}{l}0.04-0.88 \\
0.48-1.53\end{array}$ & $15-26$ & $\begin{array}{l}\text { Range of SOC accumulation from } \\
\text { experiments on free-draining } \\
\text { Ferralsol, rotations with intercropped } \\
\text { or cover crop legumes }\end{array}$ & Boddey et al. (2010) \\
\hline $\begin{array}{l}\text { United States } \\
\text { of America, } \\
\text { Michigan }\end{array}$ & $\begin{array}{l}\text { Continental } \\
\text { humid }\end{array}$ & $0-100 \mathrm{~cm}$ & $0.33-0.50$ & 12 years & & $\begin{array}{l}\text { Syswerda et al. (2011) in } \\
\text { Lal (2015) }\end{array}$ \\
\hline $\begin{array}{l}\text { United States } \\
\text { of America } 8 \\
\text { S-East states }\end{array}$ & Oceanic & $0-20 \pm 1 \mathrm{~cm}$ & $0.45 \pm 0.04$ & $11 \pm 1$ & review & Franzluebbers (2010) \\
\hline SE Australia & Multiple & $0-100 \mathrm{~cm}$ & $0.18-0.31$ & & & $\begin{array}{l}\text { Grace et al. (2010) in Lal } \\
(2015)\end{array}$ \\
\hline EU, Spain & Mediterranean & $0-30 \mathrm{~cm}$ & 0.29 & & & $\begin{array}{l}\text { Alvaro-Fuentes et al. } \\
\text { (2014) }\end{array}$ \\
\hline
\end{tabular}




\section{Other benefits of the practice}

\subsection{Improvement of soil properties}

CA is a major pathway to implement an Integrated Fertility Management based on continuous organic matter recycling at plot level. FOM fluxes feed and activate an intense biological activity that drives and improves physical (soil aggregation and its stability, temperature, porosity, bulk density, water holding capacity...), chemical (OM, pH and Eh buffering, cation exchange capacity, saturation rate, nutrients availability ...) and biological soil properties (enhancement of both species and functional biodiversity as well as biological regulation of soil pests and diseases) (Verhulst et al., 2010; Henneron et al., 2014; Basche et al., 2015). All these soil property improvements end in bringing the soil to a healthier state, leading to both improved plant growth performances and a better health of them (Reeve et al., 2016).

\subsection{Minimization of threats to soil functions}

\section{Table 185. Soil threats}

\begin{tabular}{|c|c|}
\hline Soil threats & \\
\hline Soil erosion & $\begin{array}{l}\text { No-tillage combined with soil cover have well known effect on any type of } \\
\text { soil erosion (Lal, Wilson and Okigbo, 1979). }\end{array}$ \\
\hline $\begin{array}{l}\text { Nutrient imbalance } \\
\text { and cycles }\end{array}$ & $\begin{array}{l}\text { CA improves nutrients availability for crops through multiple pathways } \\
\text { through FOM and SOM mineralization, enhancement of symbiotic and non } \\
\text { symbiotic } \mathrm{N} \text {-fixation, bio-mediated access to soil and subsoil nutrient pools } \\
\text { (Boulakia et al., 2020). }\end{array}$ \\
\hline Soil acidification & $\begin{array}{l}\text { FOM amendments and SOM act as pH buffer; regular OM inputs } \\
\text { orchestrated by CA leads to progressive soil acidity and Al toxicity } \\
\text { neutralization (Vieira et al., 2008). }\end{array}$ \\
\hline $\begin{array}{l}\text { Disruption of } \mathrm{pH}- \\
\text { Eh-EC balance }\end{array}$ & $\begin{array}{l}\text { Regulation of the relationship between } \mathrm{pH}-\mathrm{Eh}-\mathrm{EC} \text {. CA alters soil electrical } \\
\text { activity and conductivity. Fields under CA or conventional practices } \\
\text { displayed reversed soil profiles for electrical activity with more oxidized } \\
\text { surface soil layer }(0-5 \mathrm{~cm}) \text { under conventional systems, while the lower } \\
\text { horizon ( } 15-25 \mathrm{~cm}) \text { was more oxidized in CA systems. Decreased electrical } \\
\text { conductivity is observed under CA soils when it was initially high, and } \\
\text { increased it when it was low, especially in the surface horizon }(0-5 \mathrm{~cm}) \\
\text { (Husson et al., 2018). }\end{array}$ \\
\hline
\end{tabular}




\begin{tabular}{|c|c|}
\hline Soll threats & \\
\hline $\begin{array}{l}\text { Soil salinization and } \\
\text { alkalinization }\end{array}$ & $\begin{array}{l}\text { Regarding soil salinization and alkalinization, CA can alleviate or remediate it } \\
\text { through different ways, depending on stressors origin. For instance, FOM } \\
\text { inputs act as pH buffer and neutralize basic (as well acid) soil pH; improved } \\
\text { soil (macro)porosity and/or presence of a mulch may reduce salinization } \\
\text { process by capillary ascent of brackish water. }\end{array}$ \\
\hline $\begin{array}{l}\text { Soil contamination } \\
\text { /pollution }\end{array}$ & $\begin{array}{l}\text { The FOM amendment and SOC pool increase enables the sorption of both } \\
\text { organic and inorganic contaminants; they reduce their mobility and keep } \\
\text { them exposed to microbial activity. }\end{array}$ \\
\hline $\begin{array}{l}\text { Soil biodiversity } \\
\text { loss }\end{array}$ & $\begin{array}{l}\text { Regular FOM inputs from diversified plant species, abandon of soil tillage, } \\
\text { moisture and temperature regulation enhance a rapid restauration of macro, } \\
\text { meso and micro soil biodiversity (Lienhard et al., 2013). }\end{array}$ \\
\hline Soil compaction & $\begin{array}{l}\text { No-till has been mentioned to induce progressive soil compaction by } \\
\text { machinery traffic and rains; however, this type of problem can be addressed } \\
\text { through cover crop species chosen for their capacity to (re)structure and } \\
\text { harness soil against soil compaction. In context where climate conditions } \\
\text { limit possibility to insert cover crop, damage to soil structure from } \\
\text { machinery can be addressed through control traffic farming. } \\
\text { Permanent soil cover combined with the abandon of any surface tillage } \\
\text { prevent soil crusting after heavy rains (Lal and Shukla, 2004). }\end{array}$ \\
\hline $\begin{array}{l}\text { Soil water } \\
\text { management }\end{array}$ & $\begin{array}{l}\text { Under short-term perspective, some competition between crops and cover } \\
\text { crops has been mentioned in case of dry spell or drought. } \\
\text { However, in the medium and long term, CA improves rain and water-use } \\
\text { efficiency through multiple and combined effects: reduction of run-off and } \\
\text { better infiltration rate; reduction of evaporation by mulching; improvement } \\
\text { of soil water holding capacity through porosity and SOM restauration; } \\
\text { deeper crops roots development (Hobbs, 2007; Blanco-Canqui and Lal } \\
2007 \text {; Verhulst et al., 2010). }\end{array}$ \\
\hline
\end{tabular}

\section{3. (Increases in production (e.g. food/fuel/feed/timber/fibre)}

In the initial stages of adoption, CA can result in decreased crops yield; however, initial soil fertility status and biomass-C inputs are the main drivers to enhance soil functions and services, and thus productivity. Because of the high competition for organic resources in the tropics (Africa, Asia), $\mathrm{C}$ inputs available to smallholders is generally insufficient to raise SOC stocks at an early stage when farmers and practitioners are still on the learning mode. 
Once this initial learning phase passed and degraded soil progressively regenerated, CA usually gets at least equivalent or better technical results than conventional systems; Pittelkow et al. (2014) show that yields under CA progress with years of practice; however, on degraded soil conditions, moderate to high cover crop biomass inputs induce immediate beneficial effect on crops productivity. CA also opens ways for diversification of farming activities, notably with the integration of legumes species as primary or secondary crops; through insertion of fodder / cover crop species in the annual succession and rotations, CA eases integration with livestock productions. All these diversification options are, in fact, more subject to limitation in terms of markets access than technical possibilities.

\subsection{Mitigation of and adaptation to climate change}

CA has proven positive effects on soil carbon accumulation and GHG emissions budget (see above). However, due to the vast diversity of practices and contexts of application embraced by the term CA, there are still uncertainties in the GHG budget after a management change under CA.

Regarding $\mathrm{CH}_{4}$ emissions, CA-based systems applied to rainfed and irrigated lowland rice pair with a change in water management and soil-plant-water relations, notably with the adoption of Alternate Wetting and Drying ${ }^{23}$ to pilot irrigation (Richards and Sander, 2014).

No clear trends of any effect of CA on nitrous oxide $\left(\mathrm{N}_{2} \mathrm{O}\right)$ emissions can be drawn from literature; however $\mathrm{N}_{2} \mathrm{O}$ emissions are stimulated by two factors, mineral $\mathrm{N}$ fertilizers applications and excessive moisture (Metay et al, 2007), that are progressively alleviated under CA.

CA helps to strengthen production systems against climate change (CC) through different levers. First, by its mentioned effects on soil temperature and water retention; second, by a higher flexibility in crops implementation and management, easing rapid intervention within good weather windows (Duiker, 2015); and third, CA opens possibilities of choices not only for crop management but also in the decision of crops succession and rotation. This is the case, for instance, with systems based on living cover in which farmers can decide, according to cover crop state or weather trend forecast (Niño / Niña) to sow or not in order to produce biomass from the cover/fodder crop species.

Increases in SOC concentration in near-surface soil (Hok et al., 2015) cause improvements in soil physical and biological conditions (Pheap et al, 2019), contributing to higher adaptability to climate change, providing larger opportunities of crop diversification. To scale-up CA, agricultural policies should first target these factors contributing to a larger adaptability. Climate change mitigation has to be looked as an additional benefit for States to fulfil international obligations as long as no financial mechanisms are put in place to reward farmers and rural communities engaged in CA management as a mitigation strategy to address national and global warming.

${ }^{23}$ Also see Volume 5/Factsheet No.16 «Water level management » 


\subsection{Socio-economic benefits}

Properly implemented, CA progressively harnesses agroecosystems with ecosystemic functions. This ecological intensification sustains cropping and farming systems and enables reduction of external inputs for fertility and pest management.

Through soil tillage cancellation it allows to reduce labour and costs. Under mechanized systems, this pair with a marked reduction of the farm equipment, reduction of the draught power and related fixed costs.

$\mathrm{CA}$ in mechanized farming leads to rapid land and labour productivity improvement, gross and net profit margins. In non or partially mechanized farming systems, some controversial views exist on CA adaptation to resource poor farmers (Bouwman Andersson and Giller, 2020; Tittonell et al., 2012; Lestrelin et al., 2012) while complementary studies are needed to keep exploring how CA impacts gender relations and women livelihood (Wekesah, Mutua and Izugbara, 2019).

However, CA adoption usually represents a rupture with conventional practices and a challenge in many farming contexts. For instance, in the case of degraded soil conditions, switching to CA may require prior field works like soil decompaction, land leveling ... and benefits from CA could be postponed by a primary phase of soil restoration (Daujanov et al., 2016). Pittelkow et al. (2014) conclude that only the combined application of the 3 CA principles provide noticeable effect on crops performance. They thus wonder about the relevance to promote $\mathrm{CA}$ in context where maintenance of soil cover and diversification of the cropping systems are challenged by agrarian system.

In fact, CA, beyond sustainable crops performances, is often promoted to restore degraded natural resources (soil, water and biodiversity) through activation and provision of ecosystem functions and services. In this perspective, accountability of CA should integrate and give a value to these positive externalities on natural and other livelihood assets in order to finance the investment.

\subsection{Other benefits of the practice}

CA is not only offering sustainable and integrated fertility management options based on an intensification of biomass cycling first at field level (before to be though on farm or upper territory level). It also triggers, through biomass inputs supply and progressive biodiversity restoration, multiple functions of integrated pest manangement (weeds, diseases and phytophagous invertebrates). This leads, in contexts where CA is properly implemented (high and regular FOM inputs, high crops and cover crops diversity in the rotation), to the progressive substitution of chemical inputs (mineral fertilizers, pesticides) by agroecological functions, opening pathways toward "bio-intensive" organic farming. 


\section{Potential drawbacks to the practice}

\subsection{Tradeoffs with other threats to soil functions}

Table 186. Soil threats

\begin{tabular}{|c|c|}
\hline Soil threats & \\
\hline $\begin{array}{l}\text { Nutrient } \\
\text { imbalance and } \\
\text { cycles }\end{array}$ & $\begin{array}{l}\text { Competition between biomass uses (combustible, construction, forage, bioenergy) and } \\
\text { restitution to soil. A complex accountability to adjust these types of trade-off between } \\
\text { direct and ES-mediated value of FOM. }\end{array}$ \\
\hline Soil compaction & $\begin{array}{l}\text { CA with insufficient biomass input and soil cover may pair with increase in soil bulk density. } \\
\text { This may call for a redesign of the system to improve the macroaggregation and } \\
\text { macroporosity maintenance functions through roots systems and macrofauna (earthworms } \\
\text { notably) activity. }\end{array}$ \\
\hline $\begin{array}{l}\text { Soil water } \\
\text { management }\end{array}$ & $\begin{array}{l}\text { In scarce water context, water consumption by cover and or associated crops / cover crops } \\
\text { may call for the adjustment of decision rules. These decision rules evolve with the soil } \\
\text { transformation and restoration of its water holding capacity. However, Basche et al. (2016) } \\
\text { shows winter cover crop improves soil water reserve available for spring crop sown in } \\
\text { succession. }\end{array}$ \\
\hline
\end{tabular}

\subsection{Increases in greenhouse gas emissions}

Regarding $\mathrm{CO}_{2}$, $\mathrm{CA}$ introduces acknowledged net $\mathrm{SOC}$ accumulation. However, this progressive $\mathrm{C}$ accumulation can be reverted and SOC lost in case of return to soil tillage, even shallow one. Priming effects in lower horizons, during transition phase, may also limit $\mathrm{C}$ sequestration balance.

Potential of $\mathrm{CH}_{4}$ emission reduction through CA introduction in irrigated rice production is depending on the adoption of AWD-based management of water and abandoned of permanent flooding. However, this association of CA with the drainage of excessive moisture cannot be practiced in all rice agroecosystems, and net effect of $\mathrm{CA}$ adoption on $\mathrm{CH}_{4}$ emission in some rainfed lowland conditions (e.g. deep water rice in flooded plains) needs further investigation. 
There are no known specific threats of increasing $\mathrm{N}_{2} \mathrm{O}$ emissions through CA adoption. Pathways to counteract $\mathrm{N}_{2} \mathrm{O}$ emissions drivers within CA is strongly depending on context (systems types, biophysical conditions) and should call for specific studies to adjust the practices. However, in a 30-year-old CA system in the United States of America, it has been observed that $\mathrm{N}_{2} \mathrm{O}$ emissions were 40 to 57 percent lower than when soil was plowed or chiseled (Omonode et al. 2011). Such decrease in $\mathrm{N}_{2} \mathrm{O}$ emissions in CA systems could be linked to more abundant and diversified microbiological communities in such soils (Palm et al. 2014; Mangalassery et al. 2014). It is worth noting that even when $\mathrm{N}_{2} \mathrm{O}$ emissions are higher in CA systems than in soil tillage systems, global GHG budget can lead to a global warming potential lower in CA systems thanks to their systematic lower $\mathrm{CO}_{2}$ emissions and very likely lower $\mathrm{CH}_{4}$ emissions (Ahmad et al. 2009; Dendooven et al. 2012; Mangalassery et al. 2014).

Improved management of $\mathrm{N}$ fertilizer, in regions such as Asia, where $\mathrm{N}$ fertilization is high, suggests that this is a more effective target of mitigating climate change than considering SOC accumulation as a solo activity (Powlson et al., 2016).

\subsection{Conflict with other practice(s)}

CA adoption may require some trade-off adjustments between the progressive development of ecosystem functions (and attached economic benefits on short to long term) and immediate profits generated by the exportation and valorization of the biomass (forage, bioenergy...). Those trade-offs are particularly difficult to adjust to resource poor farming contexts where competition for biomass between different uses and multiples stakeholders is important.

CA adoption could be challenged by slash and burn or residue burning practices; this type of practices are characterized by their simplicity, low investment request and high labour productivity which make them particularly attractive for smallholders as long as soil fertility is maintained and weeds pressure controlled.

\subsection{Decreases in production (e.g. food/fuel/feed/timber/fibre)}

During transition phase where farmers are learning new practices and restore the organo-biological fertility of their soils through the combination of No-Till and intensified FOM inputs (which can usually last to 2 to 5 years), CA may generate lower profit margins than conventional systems. There is a need to identify financial mechanisms to reward smallholder farmers who invest into soil restoration management. 


\section{Recommendations before implementing the practice}

i. At plot level, start CA-based systems after alleviating major physical (e.g. soil compaction, presence of hardpan, unevenness of soil surface...) and/or chemical (e.g. marked desaturation in exchangeable bases) limitations. Then, whenever possible start crops sequence by the production of mixed-species based biomass to prime the "biological pump" (Séguy et al., 2006).

ii. Think the integration of CA-based cropping systems within farm and/or community's territory management, in terms of in and out FOM fluxes. This complex participatory design of FOM fluxes aims at articulating and reconciling sustainable and less conflictual space and resource management, from plot to upper scale levels.

iii. Anticipate, in order to alleviate them in R\&D and/or D phases, key technical constrains like regular access to diversified and open-pollinated cover crop species and appropriate-scale mechanization (notill planter, rollers and related services to farm). Along with the access to plant diversity, appropriatescale mechanization is a key determinant to develop at the early stage efficient CA cropping systems specifically for smallholder farmers.

iv. Realize a baseline survey to assess SOC stock prior to CA implementation, in an anticipation of coming payment for ecosystem services.

Finally, the safest and most reliable indicators to assess the efficiency of CA-based cropping systems are (Séguy, 2009):

- Total soil cover, during and until the end of the dry season regardless of its intensity and length.

- This significant and permanent cover of the soil, either dead or alive, effectively allows less expensive and easier control of weeds.

- The use of the functional plant biodiversity for the production process (productivity and stability, diversification and quality), requires compulsory use of rotations and/or diversified successions of crops and cover/relay crops.

- Increased productivity compared to existing systems with the use of fewer chemical inputs.

- The regular increase in soil fertility and its capacity, with less chemical inputs, to maintain high yield thanks to the significant improvement of its organic status and the gradually and significantly decrease of production costs.

- Resulting impact on the quality of the productions (which should be acknowledged by new standard and receive compensatory prices), in particular free of agrotoxic residues and endowed with improved food quality. 


\section{Potential barriers to adoption}

Table 187. Potential barriers to adoption

\begin{tabular}{|c|c|c|}
\hline Barrier & YES/NO & \\
\hline Biophysical & No & $\begin{array}{l}\text { CA can be practiced in any agroecosystems dedicated to field crops } \\
\text { production. }\end{array}$ \\
\hline Cultural & Yes & Traditional burning of fields for 'soil and native pasture' regeneration. \\
\hline Social & Yes & $\begin{array}{l}\text { Many CA pioneer farmers have to face the gaze of neighbors, which is } \\
\text { sometimes hard to stand in a period of trials and uncertainty. } \\
\text { CA extension can be confronted to collective community-based } \\
\text { custom related to agropastoral resources uses (e.g. collective open } \\
\text { grazing during dry season). }\end{array}$ \\
\hline Economic & Yes & $\begin{array}{l}\text { Adopting CA induces extra costs (even if reduces some others) like } \\
\text { cover crop seeds and may need some specific investments in land } \\
\text { development (hedge, decompaction, leveling...) and/or machinery } \\
\text { (e.g. planter/seeder). }\end{array}$ \\
\hline $\begin{array}{l}\text { Institutional, } \\
\text { organizational }\end{array}$ & Yes & $\begin{array}{l}\text { Linkages between actors, e.g. local service providers offering services } \\
\text { to farm (sowing, rolling) are key actors to scale-up CA under several } \\
\text { conditions (South-East Asia, Africa). }\end{array}$ \\
\hline $\begin{array}{l}\text { Legal (Right to } \\
\text { soil) }\end{array}$ & Yes & $\begin{array}{l}\text { CA adoption by farmers cannot occur without a medium- to long- } \\
\text { term vision on landuse right. }\end{array}$ \\
\hline Knowledge & Yes & $\begin{array}{l}\text { CA is knowledge intensive and counter intuitive from a conventional } \\
\text { point of view. The progressive adaptation and adoption of CA at farm } \\
\text { level go hand in hand with a progressive transformation of farmer's } \\
\text { mindset and an abandon of CT-based knowledge landmarks. }\end{array}$ \\
\hline
\end{tabular}




\section{Photos and representations of the practice}

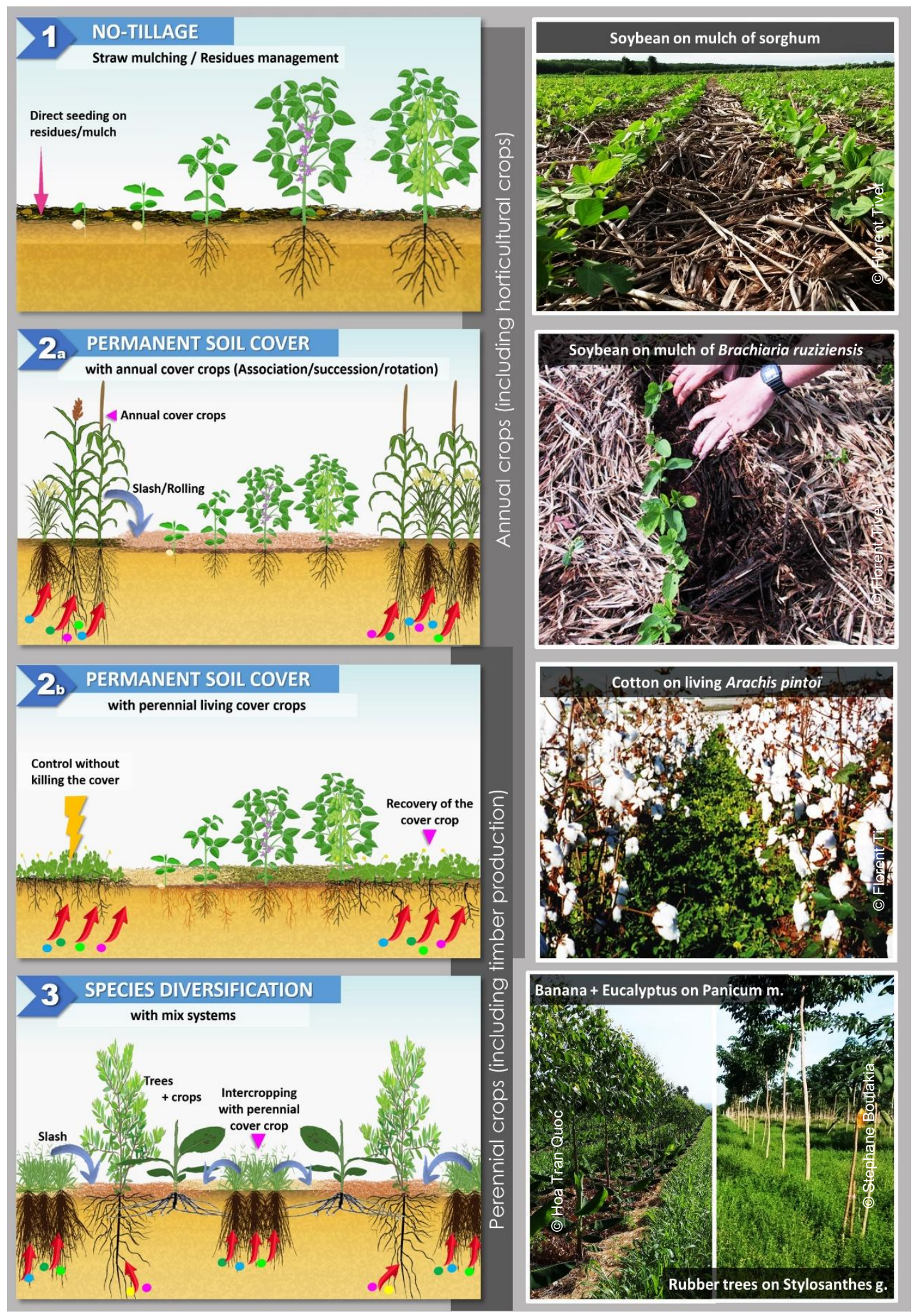

Photo 57. Typology of CA-based systems. 


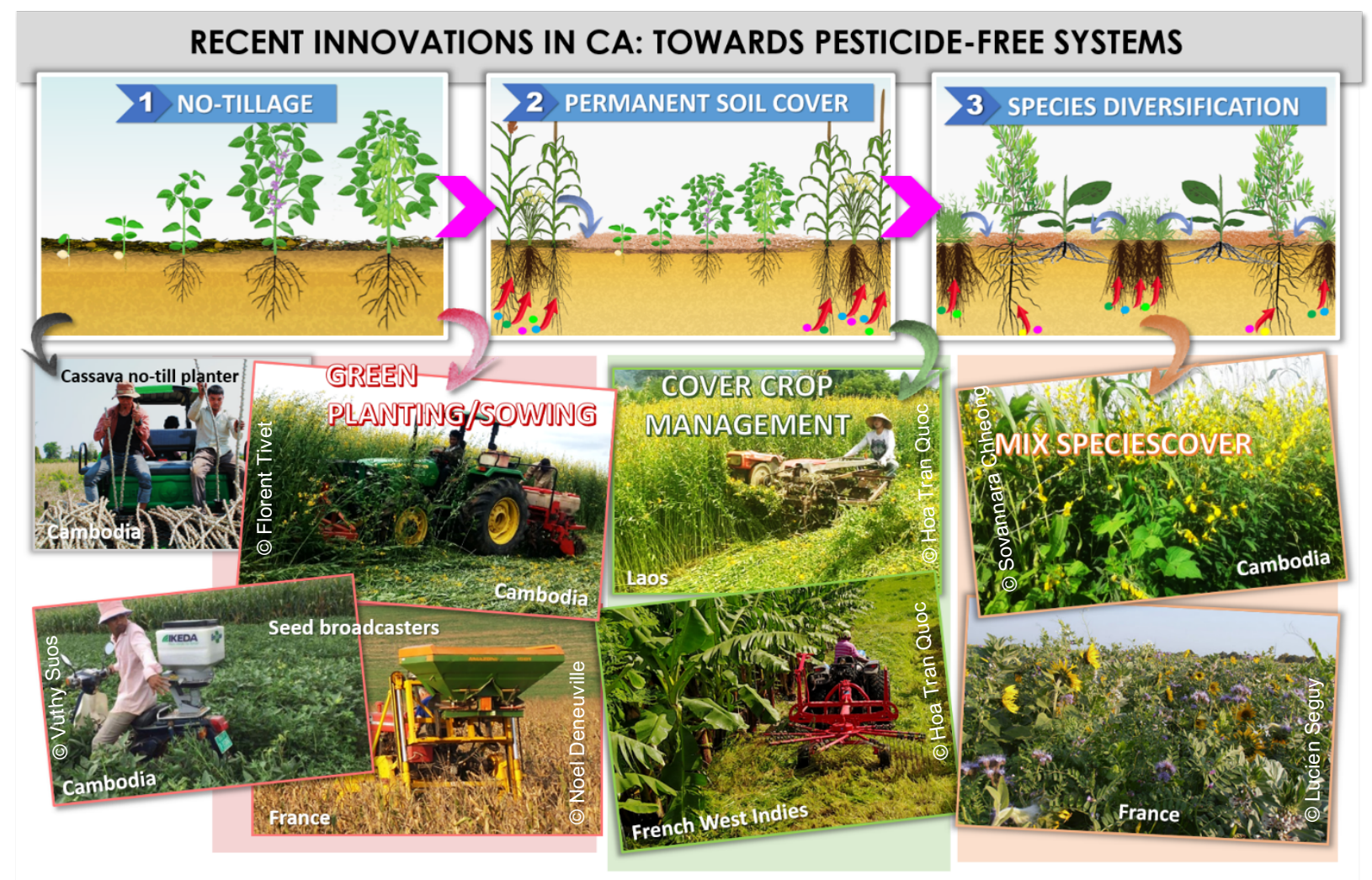

Photo 58. Some recent trends incorporated in advanced CA-based systems design.

Table 188. Related cases studies available in volumes 3 and 5

\begin{tabular}{|l|l|c|c|c|}
\hline Title & Region & $\begin{array}{l}\text { Duration } \\
\text { of study } \\
\text { (Years) }\end{array}$ & Volume & $\begin{array}{l}\text { Case- } \\
\text { study } \\
\text { No. }\end{array}$ \\
\hline $\begin{array}{l}\text { Conservation Agriculture in } \\
\text { Mozambique }\end{array}$ & Africa & 2 & 3 & 4 \\
\hline Conservation Agriculture in South Africa & Africa & 6 & 3 & 5 \\
\hline $\begin{array}{l}\text { Conservation Agriculture practices in } \\
\text { north Italy }\end{array}$ & Europe & 5 to 20 & 3 & 15 \\
\hline $\begin{array}{l}\text { Conservation agriculture in lowlands - } \\
\text { an experience from South America }\end{array}$ & $\begin{array}{l}\text { Latin America } \\
\text { and the } \\
\text { Caribbean }\end{array}$ & 9 & 3 & 33 \\
\hline
\end{tabular}




\begin{tabular}{|l|l|c|c|c|}
\hline Title & Region & $\begin{array}{l}\text { Duration } \\
\text { of study } \\
\text { (Years) }\end{array}$ & Volume & $\begin{array}{l}\text { Case- } \\
\text { study } \\
\text { No. }\end{array}$ \\
\hline $\begin{array}{l}30 \text { years of conservation agriculture } \\
\text { practices on Vertisols in central Mexico }\end{array}$ & $\begin{array}{l}\text { Latin America } \\
\text { and the } \\
\text { Caribbean }\end{array}$ & 30 & 3 & 37 \\
\hline $\begin{array}{l}\text { Conservation Agriculture in intensive } \\
\text { rice-based cropping systems in the } \\
\text { Eastern Gangetic Plain }\end{array}$ & Asia & 5 & 5 & 12 \\
\hline $\begin{array}{l}\text { Conservation tillage to tackle smog } \\
\text { issue and improve carbon sequestration } \\
\text { in rice-wheat cropping system in } \\
\text { Pakistan }\end{array}$ & Asia & 1 & 5 & 15 \\
\hline
\end{tabular}




\section{References}

Ahmad, S., Li, C., Dai, G., Zhan, M., Wang, J., Pan, S. \& Cao, C. 2009. Greenhouse gas emission from direct seeding paddy field under different rice tillage systems in central China. Soil and Tillage Research, 1061, 54-61. https://doi.org/10.1016/j.still.2009.09.005

Álvaro-Fuentes, J., Plaza-Bonilla, D., Arrúe, J.L., Lampurlanés, J. \& Cantero-Martínez, C. 2014. Soil organic carbon storage in a no-tillage chronosequence under Mediterranean conditions. Plant and Soil, 376(1): 31-41. https://doi.org/10.1007/s11104-012-1167-x

Basche, A.D., Kaspar, T.C., Archontoulis, S.V., Jaynes, D. B., Sauer, T.J., Parkin, T.B. \& Miguez, F.E. 2016. Soil water improvements with the long-term use of a winter rye cover crop. Agricultural Water Management, 172: 40-50. https://doi.org/10.1016/j.agwat.2016.04.006

Balesdent, J. \& Balabane, M. 1996. Major contribution of roots to soil carbon storage inferred from maize cultivated soils. Soil Biol. Biochem., 28: 1261-1263. http://dx.doi.org/10.1016/0038-0717(96)00112-5

Blanco-Canqui, H. \& Lal, R. 2007. Impacts of Long-Term Wheat Straw Management on Soil Hydraulic Properties under No-Tillage. Soil Sci. Soc. Am. J., 71: 1166-1173.

https://doi.org/10.2136/sssaj2006.0411

Boddey, R.M., Jantalia, C.P., Conceicao, P.C., Zanatta, J.A., Bayer, C., Mielniczuk, J., Dieckow, J., Dos Santos, H.P., Denardin, J.E., Aita, C., Giacomini, S.J., Alves, B.J.R. \& Urquiaga, S. 2010. Carbon accumulation at depth in Ferralsols under zero-till subtropical agriculture. Global Change Biology, 16: 784795

Borges, G.O. 1993. Resumo histórico do plantio direto no Brasil. In Plantio Direto no Brasil, Passo FundoRS, Brasil, EMBRAPA-CNPT, Fundacep Fecotrigo, Fundação ABC

Boulakia, S., Tivet, F., Husson, O. \& Séguy, L. 2020. Nutrient management practices and benefits in Conservation Agriculture systems. In Kassam, A. (Ed.). Advances in Conservation Agriculture, Volume 2: Practice and Benefits. Cambridge, U.K.: Burleigh Dodds Science Publishing. ISBN 978-1-78676-264-1

Bouwman, T.I., Andersson, J.A. \& Giller, K.E. 2020. Herbicide Induced Hunger? Conservation Agriculture, Ganyu Labour and Rural Poverty in Central Malawi. The Journal of Development Studies. https://doi.org/10.1080/00220388.2020.1786062

Corbeels, M., Marchão, R.L., Neto, M.S., Ferreira, E.G., Madari, B.E., Scopel, E. \& Brito, O.R. 2016. Evidence of limited carbon sequestration in soils under no-tillage systems in the Cerrado of Brazil. Scientific Reports, 6: 21450. https://doi.org/10.1038/srep21450

Daujanov, A., Groeneveld, R., Pulatov, A., \& Heijman, W.J.M. 2016. Cost-benefit analysis of Conservation Agriculture implementation in Syrdarya province of Uzbekistan. Visegrad Journal on Bioeconomy and Sustainable Development, 5: 48-52. https://doi.org/10.1515/vjbsd-2016-0009

de Oliveira Ferreira, A., Amado, T., Rice, C. W., Ruiz Diaz, D. A., Keller, C. \& Massao Inagaki, T. 2016. Can no-till grain production restore soil organic carbon to levels natural grass in a subtropical Oxisol? Agriculture, Ecosystems \& Environment, 229: 13-20. https://doi.org/10.1016/j.agee.2016.05.016 
Dendooven, L., Patiño-Zúñiga, L., Verhulst, N., Luna-Guido, M., Marsch, R. \& Govaerts, B. 2012. Global warming potential of agricultural systems with contrasting tillage and residue management in the central highlands of Mexico. Agriculture, Ecosystems \& Environment, 152: 50-58.

https://doi.org/10.1016/j.agee.2012.02.010

Derpsch, R., Franzluebbers, A.J., Duiker, S. W., Reicosky, D.C., Koeller, K., Friedrich, T., Sturny, W.G., Sá, J.C.M. \& Weiss, K. 2014. Why do we need to standardize no-tillage research? Soil and Tillage Research, 137: 16-22. https://doi.org/10.1016/j.still.2013.10.002

Duiker, S.W. 2015. Planting Green - A New Cover Crop Management Technique. In: Penn State Extension [online]. [Cited 8 September 2020]. https://extension.psu.edu/planting-green-a-new-cover-cropmanagement-technique

Farooq, M. \& Siddique, K. 2015. Conservation Agriculture: Concepts, Brief History, and Impacts on Agricultural Systems. In Farooq, M., Siddique, K. (Eds) Conservation Agriculture. Springer, Cham. https://doi.org/10.1007/978-3-319-11620-4_1

Franzluebbers, A.J. 2010. Achieving Soil Organic Carbon Sequestration with Conservation Agricultural Systems in the Southeastern United States. Soil Sci. Soc. Am. J., 74: 347-357.

https://doi.org/10.2136/sssaj2009.0079

Fujisaki, K., Chevallier, T., Chapuis-Lardy, L., Albrecht, A., Razafimbelo, T., Masse, D., Ndour, Y. B., \& Chotte, J-L. 2018. Soil carbon stock changes in tropical croplands are mainly driven by carbon inputs: A synthesis. Agriculture, Ecosystems and Environment, 259: 147-158.

https://doi.org/10.1016/j.agee.2017.12.008

Giller, K., Andersson, J., Corbeels, M., Kirkegaard, J., Mortensen, D., Erenstein, O. \& Vanlauwe, B. 2015. Beyond conservation agriculture. Frontiers in Plant Science, 6.

https://doi.org/10.3389/fpls.2015.00870

Henneron, L., Bernard, L., Hedde, M., Pelosi, C., Villenave, C., Chenu, C., Bertrand, M., Girardin, C. \& Blanchart, E. 2015. Fourteen years of evidence for positive effects of conservation agriculture and organic farming on soil life. Agronomy for Sustainable Development, 35(1): 169-181.

https://doi.org/10.1007/s13593-014-0215-8

Hobbs, P.R. 2007. Conservation agriculture: what is it and why is it important for future sustainable food production? Journal of Agricultural Science, 145: 127-137.

https://doi.org/10.1017/S0021859607006892

Hok, L., de Moraes Sá, J.C., Boulakia, S., Reyes, M., Leng, V., Kong, R., Tivet, F.E., Briedis, C., Hartman, D., Ferreira, L.A., Magno, T. \& Pheav, S. 2015. Short-term conservation agriculture and biomass-C input impacts on soil C dynamics in a savanna ecosystem in Cambodia. Agriculture, Ecosystems \& Environment, 214: 54-67. https://doi.org/10.1016/j.agee.2015.08.013

Husson, O., Brunet, A., Babre, D., Charpentier, H., Durand, M. \& Sarthou, J.P. 2018. Conservation Agriculture systems alter the electrical characteristics (Eh, pH and EC) of four soil types in France. Soil \& Tillage Research, 176: 57-68. https://doi.org/10.1016/j.still.2017.11.005 
Kemper, B. \& Derpsch, R. 1981. Results of studies made in 1978 and 1979 to control erosion by cover crops and no-tillage techniques in Parana, Brazil. Soil and Tillage Research, 1(3): 253-267.

https://doi.org/10.1016/0167-1987(80)90028-8

Kassam, A., Friedrich, T. \& Derpsch, R. 2019. Global spread of Conservation Agriculture. International Journal of Environmental Studies, 76(1): 29-51. https://doi.org/10.1080/00207233.2018.1494927

Kuzyakov, Y., Friedel, J.K. \& Stahr, K. 2000. Review of mechanisms and quantification of priming effects. Soil Biology and Biochemistry, 32(11-12): 1485-1498. https://doi.org/10.1016/S0038-

0717(00)00084-5

Lal, R., Wilson, G. \& Okigbo, B. 1979. Changes in properties of an Alfisol produced by various crop covers. Soil Science, 127. https://doi.org/10.1097/00010694-197906000-00009

Lal, R. 1979. Influence of Six Years of No-tillage and Conventional Plowing on Fertilizer Response of Maize (Zea mays L.) on an Alfisol in the Tropics. Soil Science Society of America Journal-SSSAJ, 43. https://doi.org/10.2136/sssaj1979.03615995004300020033x.

Lal, R. \& Shukla, M. 2004. Principles of Soil Physics. Boca Raton: CRC Press, https://doi.org/10.4324/9780203021231

Lal, R. 2015. Sequestering carbon and increasing productivity by conservation agriculture. Journal of Soil and Water Conservation, 70(3): 55A-62A. doi:10.2489/jswc.70.3.55A

Landers, J.N. 1994. Fasciculo de Experiencias de Plantio Direto no Cerrado. Goiânia, Brazil, APDC: 254.

Lestrelin, G., Tran Quoc, H., Jullien, F., Rattanatray, B., Khamxaykhay, C. \& Tivet, F. 2012.

Conservation agriculture in Laos: Diffusion and determinants for adoption of direct seeding mulch-based cropping systems in smallholder agriculture. Renewable Agriculture and Food Systems, 27 (1): 81-92. https://dx.doi.org/10.1017/S174217051100055X

Lienhard, P., Terrat, S., Mathieu, O., Levêque, J., Chemidlin Prévost-Bouré, N., Nowak, V., Régnier, T., Faivre, C., Sayphoummie, S., Panyasiri, K., Tivet, F., Ranjard, L. \& Maron P.A. 2013. Soil microbial diversity and $\mathrm{C}$ turnover modified by tillage and cropping in Laos tropical grassland. Environmental Chemistry Letters, 11(4): 391-398. http://dx.doi.org/10.1007/s10311-013-0420-8

LuoZ., WangE., \& Sun O.J. 2010. Can no-tillage stimulate carbon sequestration in agricultural soils? A meta-analysis of paired experiments. Agriculture, Ecosystems and Environment, 139: 224231.

Mangalassery, S., Sjögersten, S., Sparkes, D.L., Sturrock, C.J., Craigon, J. \& Mooney, S.J. 2014. To what extent can zero tillage lead to a reduction in greenhouse gas emissions from temperate soils? Scientific Reports, 4(1): 4586. https://doi.org/10.1038/srep04586

Metay, A., Oliver, R., Scopel, E., Douzet, J.-M., Moreira, J. A. A., Maraux, F., Feigl, B. J. \& Feller, C. 2007. $\mathrm{N}_{2} \mathrm{O}$ and $\mathrm{CH}_{4}$ emissions from soils under conventional and no-till management practices in Goiânia (Cerrados, Brazil). Geoderma, 141(1-2): 78-88. https://doi.org/10.1016/j.geoderma.2007.05.010. 
Neto, M.S., Scopel, E., Corbeels, M., Cardoso, A.N., Douzet, J-M., Feller, C., Piccolo, M.d.C., Cerri, C.C. \& Bernoux, M. 2010. Soil carbon stocks under no-tillage mulch-based cropping systems in the Brazilian Cerrado: An on-farm synchronic assessment. Soil and Tillage Research, 110: 187-195. https://doi.org/10.1016/j.still.2010.07.010

Omonode, R.A., Smith, D.R., Gal, A. \& Vyn, T.J. 2011. Soil nitrous oxide emissions in corn following three decades of tillage and rotation treatments. Soil Science Society of America Journal, 75(1): 152-63. https://doi.org/10.2136/sssaj2009.0147

Palm, C., Blanco-Canqui, H., DeClerck, F., Gatere, L. \& Grace, P. 2014. Conservation agriculture and ecosystem services: An overview. Agriculture, Ecosystems \& Environment, 187: 87-105. https://doi.org/10.1016/j.agee.2013.10.010

Paustian, K., Larson, E., Kent, J., Marx, E. \& Swan, A. 2019. Soil C Sequestration as a Biological Negative Emission Strategy. Front. in Clim. 1: 8. https://doi.org/10.3389/fclim.2019.00008

Pheap, S., Lefêvre, C., Thoumazeau, A., Leng, V., Boulakia, S., Koy, R., Hok, L., Lienhard, P., Brauman, A. \& Tivet, F. 2019. Multi-functional assessment of soil health under Conservation Agriculture in Cambodia. Soil and Tillage Research, 194: 104349. https://doi.org/10.1016/j.still.2019.104349

Pittelkow, C.M., Liang, X., Linquist, B.A., van Groenigen, K.J., Lee, J., Lundy, M.E., van Gestel, N., Six, J., Venterea, R.T. \& van Kessel, C. 2015. Productivity limits and potentials of the principles of conservation agriculture. Nature, 517(7534): 365-368. https://doi.org/10.1038/nature13809

Powlson, D.S., Stirling, C.M., Thierfelder, C., White, R.P. \& Jat, M.L. 2016. Does conservation agriculture deliver climate change mitigation through soil carbon sequestration in tropical agro-ecosystems? Agric. Ecosyst. Environ, 220: 164-174. https://doi.org/10.1016/j.agee.2016.01.005

Reeve, J.R., Hoagland, L.A., Villalba, J.J., Carr, P.M., Atucha, A., Cambardella, C., Davis, D.R. \& Delate, K. 2016. Chapter Six - Organic Farming, Soil Health, and Food Quality: Considering Possible Links. In D.L. Sparks, ed. Advances in Agronomy, pp. 319-367. Advances in Agronomy. Academic Press. (also available at http://www.sciencedirect.com/science/article/pii/S0065211315300031).

Richards, M. \& Sander, B.O. 2014. Alternate wetting and drying in irrigated rice: Implementation guidance for policymakers and investors. p. 6. (also available at https://www.researchgate.net/publication/306372935_Alternate_wetting_and_drying_in_irrigated_rice _Implementation_guidance_for_policymakers_and_investors).

Rossi, L.M.W., Mao, Z., Merino-Martín, L., Roumet, C., Fort, F., Taugourdeau, O., Boukcim, H., Fourtier, S., Del Rey-Granado, M., Chevallier, T., Cardinael, R., Fromin, N. \& Stokes, A., 2020. Pathways to persistence: plant root traits alter carbon accumulation in different soil carbon pools. Plant and Soil, https://doi.org/10.1007/s11104-020-04469-5

Sá, J.C.D., Cerri, C.C., Dick, W.A., Lal, R., Venske, S.P., Piccolo, M.C. \& Feigl, B.E. 2001. Organic matter dynamics and carbon sequestration rates for a tillage chronosequence in a Brazilian Oxisol. Soil Science Society of America Journal, 65(5): 1486-1499. https://doi.org/10.2136/sssaj2001.6551486x 
Sá, J.C.M., Bürkner dos Santos, J., Lal, R., de Moraes, A., Tivet, F., Machado Sá, M.F., Briedis, C., de Oliveira Ferreira, A., Eurich, G., Farias, A. \& Friedrich T. 2013a. Soil-specific inventories of landscape carbon and nitrogen stocks under no-till and native vegetation to estimate carbon offset in a subtropical ecosystem. Soil Science Society of America Journal, 77(6): 2094-2110.

http://dx.doi.org/10.2136/sssaj2013.01.0007

Sá, J.C.d.M., Séguy, L., Tivet, F., Lal, R., Bouzinac, S., Borszowskei, P.R., Briedis, C., dos Santos, J.B., da Cruz Hartman, D., Bertoloni, C.G., Rosa, J. \& Friedrich, T. 2013b. Carbon depletion by plowing and its restoration by no-till cropping systems in Oxisols of subtropical and tropical agro-ecoregions in Brazil.

Land Degradation and Development, 26(6): 531-43. https://doi.org/10.1002/ldr.2218

Séguy, L., Bouzinac, S., Trentini, A. \& Côrtes, N.A. 1998. Brazilian frontier agriculture. Agriculture et Développement (Special Issue).

Séguy, L., Bouzinac, S. \& Husson, O. 2006. Direct-seeded tropical soil systems with permanent soil cover: Learning from Brazilian experience. In Uphoff N, Ball AS, Fernandes E et al. (Eds.). Biological approach to sustainable soil systems, pp 323-342. CRC Press, Taylor and Francis.

Séguy, L. 2009. A propos des SCV: justifications pour une définition plus précise et propositions; les voies du futur SCV. (also available at: http://open-library.cirad.fr/files/2/88__note_a_propos_des_SCV_2.pdf)

Sisti, C.P.J., dos Santos, H.P., Kohhann, R., Alves, B.J.R., Urquiaga, S., Boddey, R.M. 2004. Change in carbon and nitrogen stocks in soil under 13 years of conventional or zero tillage in southern Brazil. Soil and Tillage Research, 76: 39-58. https://doi.org/10.1016/j.still.2003.08.007

Tittonell, P., Scopel, E., Andrieu, N., Posthumus, H. , Mapfumo, P., Corbeels, M., van Halsema, G.E., Lahmar, R., Lugandu, S., Rakotoarisoa, J., Mtambanengwe, F., Pound, B., Chikowo, R., Naudin, K., Triomphe, B. \& Mkomwa, S. 2012. Agroecology-based aggradation-conservation agriculture (ABACO): Targeting innovations to combat soil degradation and food insecurity in semi-arid Africa. Field Crops Research, 132: 168-174. https://doi.org/10.1016/j.fcr.2011.12.011

Tivet, F., de Moraes Sá, J. C., Lal, R., Briedis, C., Borszowskei, P. R., dos Santos, J. B., Farias, A., Eurich, G., Hartman, DdC., Nadolny Junior, M., Bouzinac, S. \& Séguy, L. 2013. Aggregate C depletion by plowing and its restoration by diverse biomass-C inputs under no-till in sub-tropical and tropical regions of Brazil. Soil and Tillage Research, 126: 203-18. https://doi.org/10.1016/j.still.2012.09.004

Veloso, M.G., Angers, D. A., Tiecher, T., Giacomini, S., Dieckow, J. \& Bayer, C. 2018. High carbon storage in a previously degraded subtropical soil under no-tillage with legume cover crops. Agriculture, Ecosystems \& Environment, 268: 15-23. https://doi.org/10.1016/j.agee.2018.08.024

Verhulst, N., Govaerts, B., Verachtert, E., Castellanos-Navarrete, A., Mezzalama, M., Wall, P., Deckers, J. \& Sayre, K.D. 2010. Conservation Agriculture, Improving Soil Quality for Sustainable Production Systems? In Lal, R., Stewart, B.A. (Eds.), Advances in Soil Science: Food Security and Soil Quality. CRCPress, Boca Raton, FL, USA, pp. 137-208

Vernet, P.A., Faysse, N., Suos, V., Oung, N., Son, S., Leng, V., Theng, D., Rendall, T., Lor, L., Reyes, R., Chan, S., Pradhan, R., Vang, S. \& Tivet, F. 2020. Investing in a no-till planter in Cambodia: A promising opportunity for various types of service providers? Asian Journal of Agricultural and Environmental Safety, forthcoming 
Vieira, F.C.B., He, Z.L., Bayer, C., Stoffella, P.J. \& Baligar, V.C. 2008. Organic amendment effects on the transformation and fractionation of aluminum in acidic sandy soil. Communications in Soil Science and Plant Analysis, 39(17-18): 2678-94. https://doi.org/10.1080/00103620802358813

Wekesah, F.M., Mutua, E.N. \& Izugbara, C.O. 2019. Gender and conservation agriculture in sub-Saharan Africa: a systematic review. International Journal of Agricultural Sustainability, 17(1): 78-91.

https://doi.org/10.1080/14735903.2019.1567245

Zhang, H-L., Lal, R., Zhao, X., Xue, J-F. \& Chen, F. 2014. Opportunities and Challenges of Soil Carbon Sequestration by Conservation Agriculture in China. Advances in Agronomy, 124: 1-36.

http://dx.doi.org/10.1016/B978-0-12-800138-7.00001-2 


\title{
43. Permaculture
}

\author{
Kristof J. Nordin
}

Never Ending Food (NEF), Malawi, Africa

\section{Description of the practice}

Permaculture is a term coined in Australia in the 1970s from the combination of the two words permanent and agriculture. It is an agroecological-based philosophy (Holmgren, 2002), which uses consciously designed landscapes to mimic the diversity, stability, and resilience of natural ecosystems. Through a sustainable integration between landscapes and people, Permaculture serves to fulfill human requirements for food, energy, shelter, and other material and non-material needs (Mollison, 1988). Unique to Permaculture is the fact that it is based upon three ethics: Earth Care (care of all the earth's biodiversity); People Care (ranging from individual health to the designing of sustainable urban cities); and Fair Share (an ethical approach to economics, the return of surplus, and the equitable use of natural resources) (FoodTank, 2018).

Specific to soil management and carbon sequestration, Permaculture focuses on the four areas where soils are conserved or increased: forest systems; under the water of lakes and ponds; in permanent planting systems; and where agriculture occurs under mulched or non-tillage practices (Mollison, 1988). As a holistic design system, Permaculture borrows best-practices from a wide range of traditional and modern approaches, so it is common to see a combination of many beneficial technologies being employed on a single site. In terms of soil management, this includes concepts such as: mulch, compost, green manure, liquid manure, ecological succession, vermiculture (worm farming), crop rotation, diversified polyculture, agroforestry, biochar, covercrops, low-to-no till soil preparation, aquaculture, food forests, woodlot management, and intercropping (especially with legumes) (Horvath, 2015).

Two of the main tools used by Permaculture practitioners are guilds and zones. Guilds are groupings of living and non-living elements which serve multiple functions (Guilds, 2020). In terms of soil management, a functioning guild requires the use of groundcovers and things which feed the soil. This may include a diverse range of mulching materials, cover crops, the intercropping of legumes, various composting technologies, fungi, vermiculture, ecological sanitation (composting toilets), etc. Along with food for the soil and groundcovers, guilds also include: attractors/protectors, climbers/supporters, and miners/diggers. The task of the Permaculture designer is to choose the most advantageous and multi-functional resources that are bestsuited to the conditions of the site. This promotion of functions, rather than specific species, is one of the aspects of Permaculture that helps to make it highly adaptable to any situation, site, or region. 
Zones are a tool that enables designers to consider factors such as soil, water, energy, patterns, ecological biodiversity, human needs, and external influences (e.g. climate, wind, sun/shade, noise, fire). Zone 0 is generally the starting point (a house, structure, water source, etc.) where there is an accumulation of energy and resources; Zone 1 is a horticulturally higher-maintenance area, often irrigated; Zone 2 generally contains orchard-type production and smaller animals; Zone 3 is often reserved for larger animals and rain-fed agricultural systems; Zone 4 tends to be manage woodlot systems; and Zone 5 is natural forest (OSU and Millison, 2020). Zones are determined by available energy and labor constrictions, plot size, and the needs being met by the design. Zones can be scaled up or down in size to accommodate small urban households or large commercial farms.

\section{Range of applicability}

Permaculture offers practitioners tools that may be universally adapted to any living situation, on any sized site, in any climate, and in any part of the world. Sites are laid out according to a 3 -step process: observation (allows practitioners to identify a range of factors, such as soil type, water sources, existing and future structures, natural resources, and needs analysis); mapping (plots existing resources and helps to identify areas for improvement); and design (a well-thought-out plan for the future sustainability and productivity of each unique site). Once observation and mapping have been completed, guilds and zones are overlaid onto the design to help ensure the beneficial integration of resources and conservation of energy.

\section{Impact on soil organic carbon stocks}

As a design system, Permaculture is not limited to one specific technique, climate, or location, but rather promotes a compendium of tools for practitioners to assess and determine what is most suitable, beneficial, and productive for each unique situation. For instance, Permaculture encourages the emulation of natural forest patterns (food forests, multistrata forest systems, agroforestry, silvopasture, etc.) to reap the benefits of carbon sequestration, perennial stability, diversified natural resources, increased biodiversity, and more. Species selection will be dependent upon an analysis of the inputs required, the outputs yielded, and the characteristics for each element in relation to a needs assessment of the site. The patterning and placement of elements are determined by factors such as climate, soil type, growing conditions, energy/labor requirements, functionality within guilds, and relevance to zones. Project Drawdown has estimated that a single Permaculture toolmultistrata agroforestry-has the potential to sequester 4.5 tons of carbon per hectare per year. If this practice were to be scaled up from the currently existing 100 million hectares, to an additional 39-66 million hectares, 11.3-20.4 gigatons of carbon dioxide could be sequestered. Similar estimations are made for Permaculture tools such as abandoned farmland restoration, biochar production, coastal wetland restoration, composting, managed grazing, perennial biomass production, regenerative annual cropping, renewable energies, silvopasture, tropical forest restoration, and many others. A table of solutions and specific analysis of carbon sequestration rates may be found on the Project Drawdown website (Drawdown, 2020). 


\section{Other benefits of the practice}

\subsection{Improvement of soil properties}

Permaculture practices enhance soil organic matter storage, while significantly improving nutrient bioavailability. Comparisons between conventional agricultural plots and Permaculture practices (forest gardens, mounds, and beds) have shown organic carbon stocks 5 to 6 times higher in the Permaculture plots, and up to 1.5 times higher than in pasture land (Tombeur et al., 2018).

\subsection{Minimization of threats to soil functions}

Table 189. Soil threats

\begin{tabular}{|c|c|}
\hline Soil threats & \\
\hline Soil erosion & $\begin{array}{l}\text { Permanent crops, forestry, mulching, groundcovers, green manure, } \\
\text { hedgerows, and minimal tillage are all imperative to reducing erosion } \\
\text { (Mollison, 1988). }\end{array}$ \\
\hline $\begin{array}{l}\text { Nutrient imbalance and } \\
\text { cycles }\end{array}$ & $\begin{array}{l}\text { Compared to conventional agriculture, Permaculture soils were over } 5 \\
\text { times higher in (N), } 12 \text { times higher in (P), and over } 6 \text { times higher in (K) } \\
\text { (Tombeur et al., 2018). }\end{array}$ \\
\hline $\begin{array}{l}\text { Soil salinization and } \\
\text { alkalization }\end{array}$ & $\begin{array}{l}\text { Fungal networks and decomposition help to render salt inert (Kamel, } \\
\text { 2013). }\end{array}$ \\
\hline $\begin{array}{l}\text { Soil } \\
\text { contamination/pollution }\end{array}$ & $\begin{array}{l}\text { Permaculture promotes anti-contamination practices, including: } \\
\text { organics, reforestation, bioremediation, and biodegradables (Bethany, } \\
\text { 2017). }\end{array}$ \\
\hline Soil acidification & $\begin{array}{l}\text { Permaculture soils maintained healthy neutral } \mathrm{pH} \text { levels between } 6.5- \\
7.5 \text {, compared to conventional plots which fell below the } 6.5 \text { acidic } \\
\text { level (Tombeur et al., 2018). }\end{array}$ \\
\hline Soil biodiversity loss & $\begin{array}{l}\text { Permaculture safeguards biodiversity, but asserts that stability within } \\
\text { ecosystems is dependent upon the number of beneficial connections } \\
\text { between diverse elements (Mollison, 1988). }\end{array}$ \\
\hline Soil sealing & $\begin{array}{l}\text { Urban Permaculture strategies eliminate or mitigate the effects of soil } \\
\text { sealing, using practices such as permeable asphalt, rain gardens, raised } \\
\text { beds, living (green) roofs, solar platforms, and more (Danks, 2010). }\end{array}$ \\
\hline
\end{tabular}




\begin{tabular}{|l|l|}
\hline Soil threats & \\
\hline Soil compaction & $\begin{array}{l}\text { Strategies for dealing with compacted soil include: soil conditioning, } \\
\text { water management, plant species selection, bioremediation and } \\
\text { mycoremediation (Mollison, 1988). }\end{array}$ \\
\hline Soil water management & $\begin{array}{l}\text { Analysis has shown that vegetated water infiltration basins contained } \\
\text { twice as much soil moisture and soil organic matter (containing 58 } \\
\text { percent carbon) when compared to rock or gravel soak-away pits } \\
\text { (Lancaster, 2019). }\end{array}$ \\
\hline
\end{tabular}

\subsection{Increases in production (e.g. food/fuel/feed/timber/fibre)}

Production is often synonymous with yield. In Permaculture, yields are grouped into product yield (primary products available from, or in surplus to, the system), and energy yield (the sum of conserved, stored, or generated energy in surplus to the system). The total yield for a system then is the sum total of surplus energy remaining after the products of the system have been able to meet their needs for growth, reproduction, and maintenance. Yields within a well-functioning Permaculture design system become a year-round and continuous process, based upon natural laws of perpetual growth and return. Yields are increased by spreading them throughout space (vertical, horizontal, and underground), as well as throughout time (using perennial, seasonal, and preservation strategies) (Mollison, 1988).

\subsection{Mitigation of and adaptation to climate change}

Permaculture applies systems thinking to both ecological as well as social solutions to climate change. These solutions comprise a wide range of interrelated approaches, including: increasing soil organic carbon, bioremediation, renewable energies, reforestation, biochar, water harvesting, scientific and information exchange, sustainable diets, community-based economic models, regenerative agricultural practices, rotational grazing, grassland restoration, agroforestry, restoration of oceanic ecosystems, ecovillages, transition towns, and mitigation strategies (ClimateChange, 2020).

\subsection{Socio-economic benefits}

Analysis has shown that Permaculture practitioners experience agricultural, environmental, livelihood, and food and nutrition security benefits in comparison to farmers who solely used conventional agriculture. Practitioners experienced multifaceted benefits from using practices that addressed specific household constraints and expanded their adaptive capacity (Conrad, 2014). Reducing external inputs, while simultaneously increasing the diversity of overall yields, are also advantages associated with Permaculture (Didarali and Gambiza, 2019). 


\subsection{Other benefits of the practice}

Along with benefits of having increased access to seasonal and diversified nutrition, perennial food forests (like those found in Permaculture design) can offer further value through the use and marketing of non-timber forest products (e.g. nuts, honey, mushrooms, thatch grass, fuel, etc.). Tangible income from non-timber forest products varies between communities and regions, but has been estimated to range from just a few percent to over 50 percent (Mahonya, Shackleton and Schreckenberg, 2019).

\section{Potential drawbacks to the practice}

\subsection{Conflict with other practice(s)}

Permaculture advocates for food and seed sovereignty. At times, this may be in conflict with conventional agriculture approaches, often supported by the economic interests of companies at the expense of environmental sustainability and smallholder farmers' economic and food sovereignty (Conrad, 2014).

\subsection{Decreases in production (e.g. food/fuel/feed/timber/fibre)}

Yield of a single crop under Permaculture production may be lower when compared to a monocropped system, but total system yield (harvests, surplus energy, add-on benefits) under Permaculture polyculture is higher when the diversity of production is added together-referred to as additive yielding (Jacke and Toensmeier, 2005).

\section{Recommendations before implementing the practice}

Prior to the implementation of a Permaculture design, practitioners are encouraged to enroll in an internationally-recognized 72-hour Permaculture Design Course. These courses are offered worldwide and follow an established curriculum covering core topics, including: design methods and process, soil and water management, climates and microclimates, ecosystemic patterns, energy, aquaculture, animal management, appropriate technologies, forest systems and cultivated ecologies, waste and bioremediation, and social systems (information exchange, urban strategies, and economics) (Solkinson and Chi, 2018). Courses are often conducted over 12 days, with food, lodging, and travel reimbursements provided for participants. If inexpensive accommodation is sourced, a complete 12-day course can be delivered for approximately U.S.\$35/participant/day (Greenblot and Nordin, 2012). 


\section{Potential barriers to adoption}

\section{Table 190. Potential barriers to adoption}

\begin{tabular}{|c|c|c|}
\hline Barrier & YES/NO & \\
\hline Cultural & Yes & $\begin{array}{l}\text { There are often cultural norms, beliefs, and stigma which create barriers } \\
\text { to the implementation of Permaculture activities (Greenblot and Nordin, } \\
\text { 2012). }\end{array}$ \\
\hline Social & Yes/No & $\begin{array}{l}\text { Gender roles, age, and social norms were all indicated to create various } \\
\text { challenges for the implementation of Permaculture practices (Thornton, } \\
\text { 2008). Permaculture, as social learning, can help build community } \\
\text { capacity and expand knowledge sharing (Conrad, 2014). }\end{array}$ \\
\hline Economic & Yes/No & $\begin{array}{l}\text { The fact that Permaculture is not dependent on access to money created } \\
\text { options for farmers who learned about and used Permaculture, but land } \\
\text { ownership has been associated higher adoption rates of Permaculture } \\
\text { practices (Conrad, 2014). }\end{array}$ \\
\hline Institutional & Yes & $\begin{array}{l}\text { The benefits of Permaculture are important in the context of structural } \\
\text { violence where farmers face systemic risk to impoverishment, food } \\
\text { insecurity, and malnutrition. However, the adoption of Permaculture is } \\
\text { constrained by the broader agrofood system, resource entitlements, and } \\
\text { other structural constraints (Conrad, 2014). }\end{array}$ \\
\hline Legal & Yes & $\begin{array}{l}\text { Large-scale privatization of land and seeds has become a hallmark of } \\
\text { corporate and industrialized agriculture, creating barriers for the } \\
\text { affordable, equitable, and sustainable use of resources (Grain, 2015). }\end{array}$ \\
\hline Knowledge & Yes & $\begin{array}{l}\text { Information and resources regarding Permaculture may be limited and } \\
\text { sometimes contradictory to conventional agricultural messages (Conrad, } \\
\text { 2014). }\end{array}$ \\
\hline
\end{tabular}




\section{Photos of the practice}

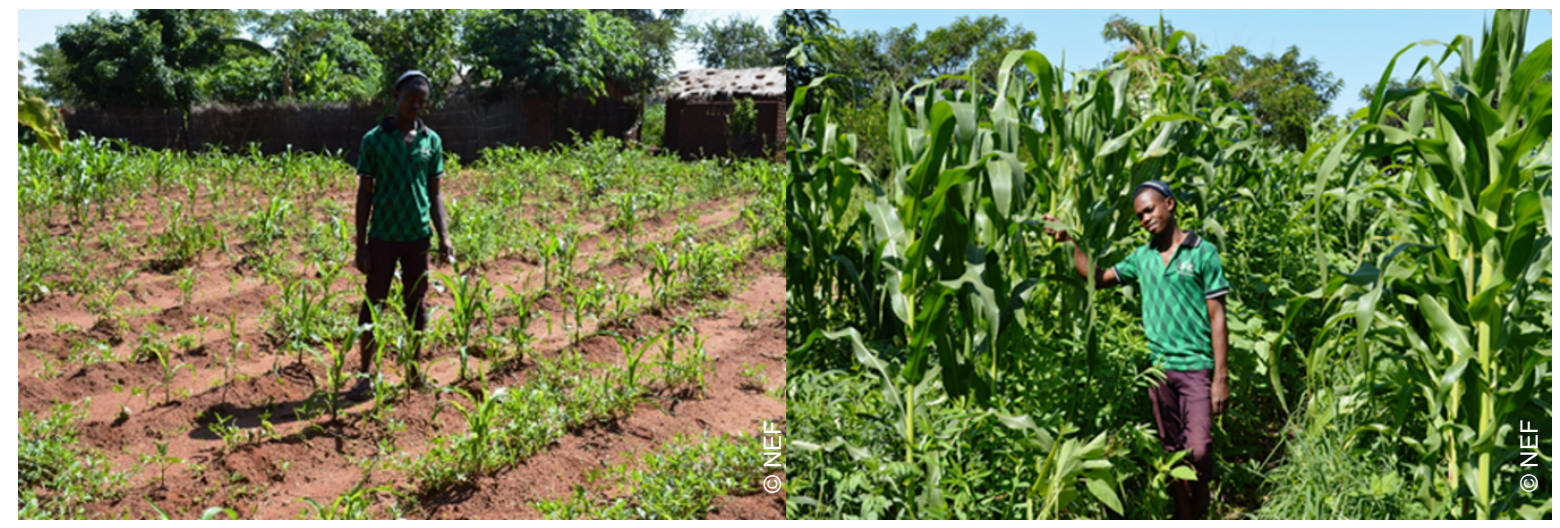

Photo 59. Two pictures taken on the same day (Feb. 10, 2016) during the midst of a drought in Malawi, Africa.

The two fields were about 50 meters apart from each other and both received the same amount of rainfall. The field on the left was conventionally monocropped with hybridized maize, treated with synthetic fertilizer, and all crop residue from the previous season was gathered up and burned. The Zone 3 Permaculture field on the right was organically intercropped with a diversity of highly-nutritious foods, intecropped with legumes, mulched with the previous season's crop residue, under-storied with groundcovers, and planted using open-pollinated seeds.

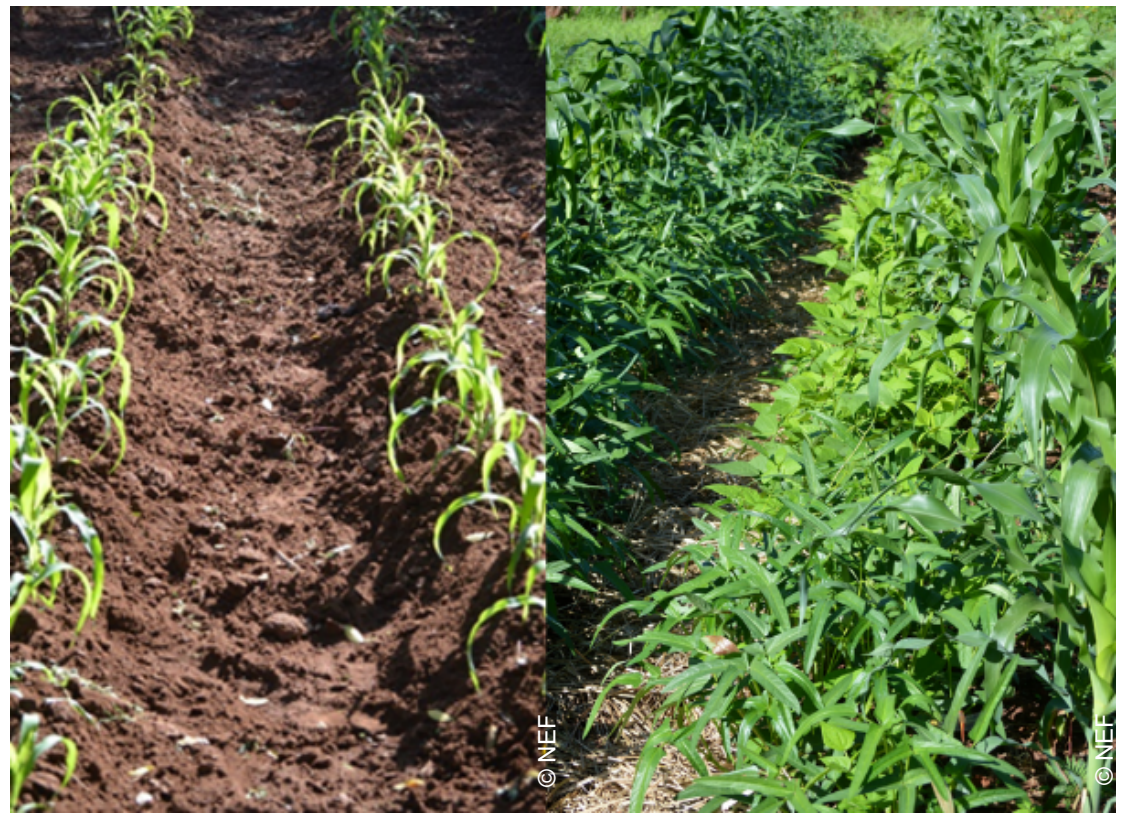

Photo 60. (Left) Both pictures taken on the same day (Jan 21, 2016) from fields about 50 meters apart. 
The 'conventional' ridged maize rows, on the left, using about 50 percent for cultivation and 50 percent for pathway; organic matter cleared and burned. The permanent, intercropped, one-meter Permaculture beds, on the right, using about 70 percent for cultivation and 30 percent for pathway; organic matter returned as mulch.

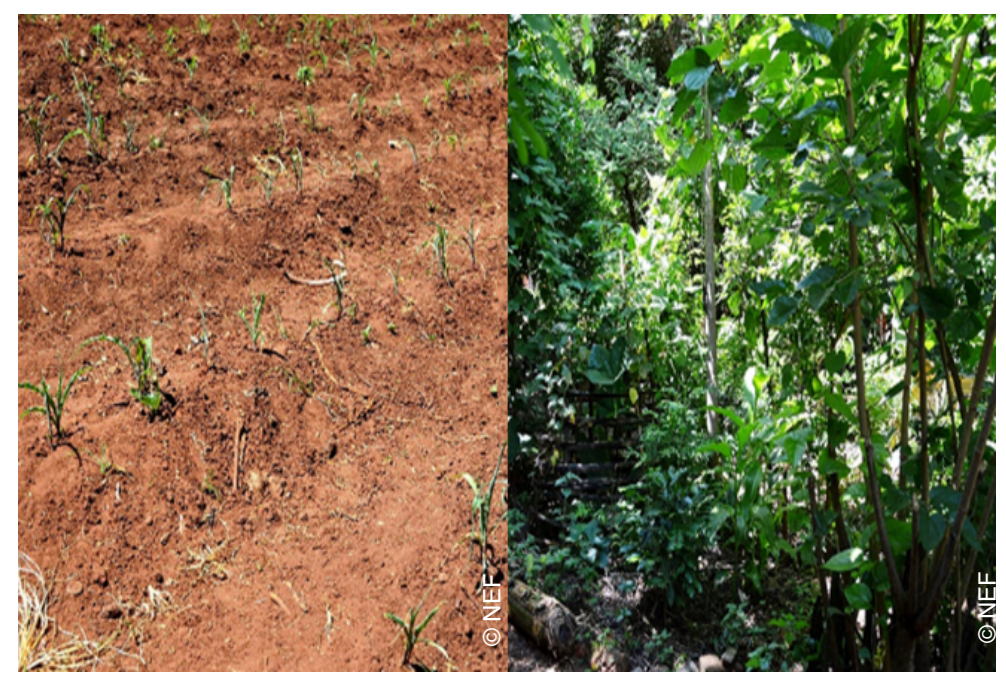

Photo 61. (Right) Two contrasting agricultural systems photographed on the same day (Jan. 08, 2018) about 100 meters apart from each other during Malawi's 2018 drought.

$\mathrm{T}$

he Permaculture food-forest 'guild' system on the right was planted with free open-pollinated annual and perennial plants; all organic matter returned as mulch.

Both systems received the same amount of rainfall, but the maize on the left was suffering the effects of drought, while the Permaculture system on the right was thriving (containing: mulberries (Morus spp.), sweet potatoes, taro, papaya (Carica papaya), local lima beans (Phaseolus lunatus), turmeric (Curcuma longa), air potatoes (Dioscorea spp.), local vegetables (blackjack (Bidens pilosa), amaranth (Amaranthus spp.), etc.), and agroforestry species (senna, acacia, etc.).

Table 191. Related cases studies available in volumes 3 and 5

\begin{tabular}{|l|l|l|c|c|}
\hline Title & Region & $\begin{array}{l}\text { Duration } \\
\text { of study } \\
\text { (Years) }\end{array}$ & Volume & $\begin{array}{l}\text { Case- } \\
\text { study } \\
\text { No. }\end{array}$ \\
\hline $\begin{array}{l}\text { Never Ending Food (NEF) } \\
\text { Permaculture Initiative in Malawi }\end{array}$ & Africa & $\begin{array}{l}\text { Not } \\
\text { available }\end{array}$ & 3 & 3 \\
\hline
\end{tabular}




\section{References}

Bethany, L. 2017. Soil Contamination: Its Causes, Effects, and Solutions. In: Permaculture News [online]. [Cited 7 June 2020]. https://www.permaculturenews.org/2017/04/03/38249/

ClimateChange. 2020. Permaculture Solutions for Climate Change [online]. [Cited 7 June 2020]. http://www.permacultureclimatechange.org/

Conrad, A. 2014. We are farmers: Agriculture, food security, and adaptive capacity among permaculture and conventional farmers in central Malawi. Washington, D.C., American University. (also available at https://dra.american.edu/islandora/object/auislandora\%3A10431).

Danks, S.G. 2010. Asphalt to ecosystems: Design ideas for schoolyard transformation. Oakland, CA, New Village Press.

Didarali, Z. \& Gambiza, J. 2019. Permaculture: Challenges and Benefits in Improving Rural Livelihoods in South Africa and Zimbabwe. Grahamstown, South Africa, Department of Environmental Sciences, Rhodes University.

Drawdown, P. 2020. Table of Solutions. In: Project Drawdown [online]. [Cited 8 June 2020]. https://www.drawdown.org/solutions/table-of-solutions

FoodTank. 2018. Food Tank Lists [online]. [Cited 7 June 2020].

https://foodtank.com/news/2018/11/earth-care-people-care-fair-share-19-approaches-to-permaculturearound-the-world/

Grain. 2015. Land and Seed Laws under Attack: Who is Pushing Changes in Africa? Grain. (also available at https://www.grain.org/en/article/5121-land-and-seed-laws-under-attack-who-is-pushing-changes-inafrica).

Greenblot, K. \& Nordin, K. 2012. Permaculture Design for Orphans and Vulnerable Children Programming: Low-Cost, Sustainable Solutions for Food and Nutrition Insecure Communities. Arlington, VA, AIDSTAR-One; United States Agency for International Development (USAID).

Guilds. 2020. Plant Guilds. In: Directory of Permaculture [online]. [Cited 8 June 2020]. http://directoryofpermaculture.com/permaculture-concepts/plant-guilds/

Holmgren, D. 2002. Principles and Pathways beyond Sustainability. Victoria, Australia, Holmgren Design Services.

Horvath, W. 2015. The Definitive Guide to Building Soils by Imitating Nature. In: Permaculture Apprentice [online]. [Cited 6 June 2020]. https://permacultureapprentice.com/building-soil/

Jacke, D. \& Toensmeier, E. 2005. Edible forest gardens: Ecological design and practice for temperate climate permaculture. White River Junction, Vt., Chelsea Green Publishing Company.

Kamel, N. 2013. Greywater and Food Forests for Jordan's Future: an Examination of Water and Food Sustainability in Rural Jordanian Villages. Jordan, Academia. 
Lancaster, B. 2019. Rainwater harvesting for drylands and beyond. Third edition edition. Tucson, Arizona, Rainsource Press.

Mahonya, S., Shackleton, C.M. \& Schreckenberg, K. 2019. Non-timber Forest Product Use and Market Chains Along a Deforestation Gradient in Southwest Malawi. Frontiers in Forests and Global Change, 2: 365. https://doi.org/10.3389/ffgc.2019.00071

Mollison, B. 1988. Permaculture: A designer’s manual. Tyalgum NSW, Tagari Publications.

OSU, O.S.U. \& Millison, A. 2020. Zones. In: Oregon State University [online]. [Cited 7 June 2020]. https://open.oregonstate.education/permaculture/chapter/zones/

Solkinson, D. \& Chi, K. 2018. Permaculture Design Core Curriculum Notes. In: Permaculture Design [online]. [Cited 7 June 2020]. www.permaculturedesign.ca

Thornton, H. 2008. Permaculture adoption among Malawian farmers: A positive deviance inquiry. Brattleboro, Vermont, Capstone Paper, Master of Intercultural Service, Leadership, and Management at the School for International Training.

Tombeur, F. de, Sohy, V., Chenu, C., Colinet, G. \& Cornelis, J.-T. 2018. Physicochemical Properties and Organic Matter Distribution in Aggregates: A Case Study of the Bec-Hellouin Farm (France). France. 


\section{Zero Budget Natural Farming}

\section{Jo Smith', Zofie Hobzikova', Jagadeesh Yeluripati², Pete Smith', Mark Sutton³, Ute Skiba ${ }^{3}$, Dali Nayak ${ }^{1}$}

'School of Biological Science, University of Aberdeen, Aberdeen, UK

${ }^{2}$ Information \& Computational Sciences, James Hutton Institute, Aberdeen, UK

${ }^{3}$ UK Centre for Ecology \& Hydrology, Bush Estate, Penicuik, Midlothian, UK

\section{Description of the practice}

Zero Budget Natural Farming (ZBNF) is a farming system that is spreading across India, initially without any formal organization, but more recently with financial and practical support from Government and state-run Farmers' Associations (RySS, 2020). The ZBNF practices were developed by Subhash Palekhar in the 1990s (RySS, 2020). The system relies on easily available ingredients to produce crop treatments on-farm, using microorganisms to build soil fertility (Khadse and Rosset, 2019). Strict ZBNF differs from traditional organic farming in that it does not provide nutrients for crop growth, but instead aims to change the functioning of the soil so that nutrients are made available to crops without external inputs. It uses zero inputs of synthetic fertilizers to avoid reliance on purchased inputs, and only small inputs of animal manures, so also avoiding limitations in available manure. Fertility is instead built on four wheels (RySS, 2020; Palekhar, 2019): (1) stimulation of microbial activity using a dung-based microbial inoculum, jüwamrita, to make nutrients available to plants and protect against pathogens; (2) protection of young roots from fungal and soil-borne diseases using another microbial culture, beejamrita ; (3) mulching ( acchadana) to produce stabilized soil organic matter and conserve topsoil; and (4) improvement of soil aeration (whapahasa) by reduced tillage and building soil organic matter. Mulching includes "living mulches", especially using nitrogen fixing plants. Manure from the local "humped-cow" (Bos indicus) is preferred as it is considered to contain more micro-organisms than non-local breeds. Vermicompost is avoided as the aim is to increase local species of earthworms through increasing soil organic matter. Contours and bunds are used to retain rainwater and reduce soil losses by erosion. The ZBNF system also attempts to use crop and tree associations (the "five-layer" model) to make nutrient and water savings, while compensating for any reductions in yield (Khadse and Rosset, 2019). 


\section{Range of applicability}

As a grassroots agroecological movement, ZBNF originated in Karnataka, India, during 2002 (Khadse and Rosset, 2019). It inspired a high level of commitment from its supporters, with the movement initially being self-organized at a local level, running in an informal way through both organized and spontaneous farmer-tofarmer exchange and all activities being done by volunteers. Early adoption of the system was in part a response to the high rates of farmer suicide in India, which have been linked to extreme financial pressures due to high production costs, high interest rates and volatile market prices (Kennedy and King, 2014). Therefore, ZBNF aimed to avoid borrowing and purchases from large corporations and to instead support economies of local communities by maintaining the cycle of production within the villages. It is now practiced throughout India, but with a focus in the southeastern state of Andhra Pradesh, where the official website of the ZBNF Programme of Rythu Sadhikara Samstha (RySS, 2020) stated that, by June 2020, 580000 farmers had already converted to ZBNF in 3011 villages across 260000 ha. This is equivalent to nearly 17 percent of the area of the state under productive agriculture (Smith et al., 2020a). The long-term aim of the government of Andhra Pradesh is to roll-out ZBNF to all 6 million farmers in the state by 2024 (UNEP, 2019). Nationally, the number of farmers practicing ZBNF is likely to increase over the next few years after Prime Minister Narendra Modi announced India's focus on ZBNF at the United Nations conference on desertification in 2019, and Finance Minister, Nirmala Sitharaman, called in her 2019 budget speech for a "back to basics" approach with an emphasis on ZBNF (Sitharaman, 2019).

\section{Impact on soil organic carbon}

The focus of ZBNF on increasing soil micro-organisms and fauna with emphasis on soil protection and mulching has the potential to greatly increase soil organic carbon (SOC), and so also increase efficiency of nutrient and water use. However, the extremely low nutrient inputs used have sparked criticism of ZBNF from many scientists as unscientific and unproven (Ramakumar and Arjun, 2019; Raghuram, 2020). As of June 2020, this remained a reasonable criticism, as no independent, replicated, fully randomized and peer-reviewed trials had yet established its efficacy. However, despite the lack of published evidence, ZBNF is already being widely practiced across India. A controversial claim made by practitioners of ZBNF is that the nutrients required by crops do not need to be added in applied treatments but are instead provided "by the soil itself" (RySS, 2020). If the $\mathrm{N}$ needed for crop growth was indeed only supplied by release from organic matter (the main form of $\mathrm{N}$ in the soil), there would be an associated loss of SOC of approximately $1 \mathrm{t} / \mathrm{ha} / \mathrm{yr}$, which would result in complete mining of organic matter from Indian soils in as little as 20 years (Smith et al., 2020a). Therefore, for ZBNF to be sustainable, other parts of the system must provide a net input of organic carbon (C) and N, and/or biological $\mathrm{N}$ fixation must be increased by the inocula and intercropping practices used. Smith et al. (2020a) used wider agroecological literature to examine possible sources of $\mathrm{N}$ in $\mathrm{ZBNF}$ and concluded that the $\mathrm{N}$ available in the system does have potential to achieve improved yields, although this is only likely on farms previously with below-average N inputs. Similar issues over sustainability of sources of other nutrients remain unanswered. Smith et al (2020a) also demonstrated that ZBNF is likely to increase SOC compared to conventional farming, rather than decrease it. Because no trials have yet been published that establish either the short- or long-term impacts of ZBNF, estimates of changes in SOC are necessarily derived from examination of trials that include the components of ZBNF, but within other agroecological systems, and rely on extrapolation of results using simulation modelling to determine the longer-term impacts. 
The main impact on SOC of the different inocula used in ZBNF is not through direct addition of $\mathrm{C}$ but via stimulation of microbial growth and enhanced $\mathrm{N}$ fixation; as shown in Table 192, at the rates recommended in ZBNF systems for application of dhrava jüwamrita $\left(225 \mathrm{dm}^{3}\right.$ per acre), ghana jüwamrita (100 kg per acre) and beejamrita $\left(37 \mathrm{dm}^{3}\right.$ per acre) (RySS, 2020) only $\sim 0.14 \mathrm{t} / \mathrm{ha} / \mathrm{yr} \mathrm{C}$ would be provided. This compares to $\sim 5.2 \mathrm{t} / \mathrm{ha} / \mathrm{yr} \mathrm{C}$ if all the $\mathrm{N}$ required was added as organic manure (Smith et al., 2020a).

Table 192. Calculation of approximate carbon (C) content of jiwamrita and beejamrita

\begin{tabular}{|c|c|c|c|c|c|c|c|c|c|c|c|c|}
\hline \multirow{3}{*}{$\begin{array}{l}\text { Ingredient } \\
\text { Water }\end{array}$} & \multirow{2}{*}{\multicolumn{3}{|c|}{ C concentration }} & \multicolumn{3}{|c|}{ Dhrava jiwamrita } & \multicolumn{3}{|c|}{ Ghana jiwamrita } & \multicolumn{3}{|c|}{ Beejamrita } \\
\hline & & & & \multicolumn{2}{|c|}{ Quantity } & \multirow{2}{*}{$\begin{array}{c}\mathrm{C} \\
\text { added } \\
(\mathrm{g}) \\
0\end{array}$} & \multicolumn{2}{|c|}{ Quantity } & \multirow{2}{*}{$\begin{array}{c}\mathrm{C} \\
\text { added } \\
\text { (g) }\end{array}$} & \multicolumn{2}{|c|}{ Quantity } & \multirow{2}{*}{$\begin{array}{c}\mathrm{C} \\
\text { added } \\
\text { (g) } \\
0 \\
0\end{array}$} \\
\hline & 0 & $\mathrm{~g} / \mathrm{dm}$ & & 200 & $\mathrm{dm}^{3}$ & & & & & 21 & $\mathrm{dm}^{3}$ & \\
\hline Fresh cow dung & 117 & $\mathrm{~g} / \mathrm{kg}$ & (1) & 10 & $\mathrm{~kg}$ & 1168 & 100 & $\mathrm{~kg}$ & 11680 & 5 & $\mathrm{~kg}$ & 584 \\
\hline Aged cow urine & 21 & $\mathrm{~g} / \mathrm{dm}^{3}$ & (2) & $5-10$ & $\mathrm{dm}^{3}$ & 213 & 5 & $\mathrm{dm}^{3}$ & 107 & 10 & $\mathrm{dm}^{3}$ & 213 \\
\hline Jaggary & 84 & $\mathrm{~g} / \mathrm{kg}$ & (3) & 2 & $\mathrm{~kg}$ & 167 & 2 & $\mathrm{~kg}$ & 167 & & & \\
\hline Pulse flour & 605 & $\mathrm{~g} / \mathrm{kg}$ & (4) & $1-2$ & $\mathrm{~kg}$ & 1210 & 1 & kg & 605 & & & \\
\hline Lime & & & & & & & & & & 0.5 & $\mathrm{~kg}$ & 0 \\
\hline \multicolumn{4}{|c|}{ Amount applied per acre per application } & \multicolumn{2}{|c|}{$\sim 225 \mathrm{dm}^{3}$} & 2758 & \multicolumn{2}{|c|}{$\sim 100 \mathrm{~kg}$} & 12559 & \multicolumn{2}{|c|}{$\sim 37 \mathrm{dm}^{3}$} & 797 \\
\hline \multicolumn{4}{|c|}{ Total applications per year } & \multicolumn{2}{|c|}{$8 \times 2$ crops } & 44128 & \multicolumn{2}{|c|}{1} & 12559 & \multicolumn{2}{|c|}{$1 \times 2$ crops } & 1594 \\
\hline TOTAL C input & & 6 t per & cre pe & year & & $=0.14$ & ha/y & & & & & \\
\hline
\end{tabular}

C concentration of ingredients estimated from (1) Pelster et al. (2016), (2) Sorensen (1998), (3) Singh, Solomon and Kumar, (2013), (4) Smith et al. (2020a).

Jiwamrita is likely to speed up the decomposition of applied organic mulches by adding to the microbial population in the soil (Smith et al., 2020a). Significant increases in microbial populations have been observed in jüwamrita by Ram, Singha and Vaish, (2018) presumably increasing the rate of decomposition of organic materials, so making nutrients contained in the mulches available to the crop and stimulating the incorporation of organic matter into the soil structure (Liao et al., 2019). This microbial culture could reduce problems with immobilization of $\mathrm{N}$ and build-up of a hydrophobic and acidic undecomposed organic matter layer from crop residues with a low $\mathrm{N}$ content (Goswami, Mondal and Mandi, 2019). For beejamrita, the main impact on SOC is likely to be through increased plant inputs associated with improved plant growth. Inoculation of soybean seed with bacterial isolates from beejamrita were observed by Sreenivasa et al. (2009) to improve germination and increase seedling vigor; these bacteria fix N, solubilize phosphorus, induce cell elongation and division, and suppress Sclerotium fungi which cause disease in a wide range of crops (Sreenivasa et al., 2009). 
Direct impacts on $\mathrm{C}$ are achieved through the three mulching practices (acchadana) recommended in ZBNF systems. These are soil mulching, mulching with dried biomass and live mulching (Table 193). Soil mulching involves limiting tillage to the top $10-15 \mathrm{~cm}$ of soil. Reduced tillage in other agroecological systems has been observed to reduce breakdown of the soil aggregates which protect organic matter from decomposition, so retaining more C in the soil (Rasmussen and Collins, 1991; Tripathi, Sharma and Singh, 2006). Some authors argue these measured increases do not reflect increased SOC in the whole profile, being due instead to decreased mixing of the surface and sub-surface layers. However, in Haryana, India, increases in SOC were observed to below the plough layer $(30 \mathrm{~cm})$ of $0.15 \mathrm{t} / \mathrm{ha} / \mathrm{yr}$ over 8 years (Patra et al., 2019), and 0.18 to 0.47 t/ha/yr over 15 years (Singh et al., 2014) (Table 193). This is consistent with regional estimates for C sequestration with conversion to no-till of $0.4( \pm 0.05) \mathrm{t} / \mathrm{ha} / \mathrm{yr}$ in the top $30 \mathrm{~cm}$ soil (Sun et al., 2020). Mulching with dried biomass uses residues from previous crops, which are intended to rapidly decompose and become incorporated into the soil under the action of the increased micro-organisms provided by jiwamrita. Given the proportion of crops, yields and harvest indices typical in India, this would increase C inputs to the soil, on average, by $1.2 \mathrm{t} / \mathrm{ha} / \mathrm{yr}$ (Smith et al, 2020a). Simulations for the main agroecological zone / soil type combinations of India provided by the $\mathrm{C}$ sub-model of ORATOR (Smith et al, 2020b) suggest that this could increase SOC in the top $30 \mathrm{~cm}$ by 0.15 to $0.32 \mathrm{t} / \mathrm{ha} / \mathrm{yr}$ over 10 years, with $\mathrm{C}$ sequestration at steady state of 2.7 to $13.7 \mathrm{t} / \mathrm{ha} / \mathrm{yr}$ (Table 193). Live mulching mainly involves intercropping with legumes and monocots (such as rice and wheat), combinations with trees (agroforestry), and propagation of a $\mathrm{N}$ fixing water fern (Azolla pinnata-Photo 62). The impact on SOC depends on the soil, climate and choice of species combinations, but could be very high, especially for agroforestry; for a range of multi-tier agroforestry combinations with finger millet, Jakhar et al. (2017) observed initial increases in SOC to $30 \mathrm{~cm}$ at a rate of 1.5 to $2.8 \mathrm{t} / \mathrm{ha} / \mathrm{yr}$ over 4 years (Table 193). This is consistent with regional estimates by Feliciano et al. (2018) of $3.83( \pm 2.36) \mathrm{t} / \mathrm{ha} / \mathrm{yr}$ for home gardens (which represent an example of a multi-tiered system). Bringing together the above observations, conversion of conventional farming to ZBNF has potential to increase SOC over at least the first 4 years at a rate of 0.3 to $0.8 \mathrm{t} / \mathrm{ha} / \mathrm{yr}$ in fields practicing reduced tillage and mulching, and up to $2.8 \mathrm{t} / \mathrm{ha} / \mathrm{yr}$ in fields practicing a five-layer model of multi-tier agroforestry. 


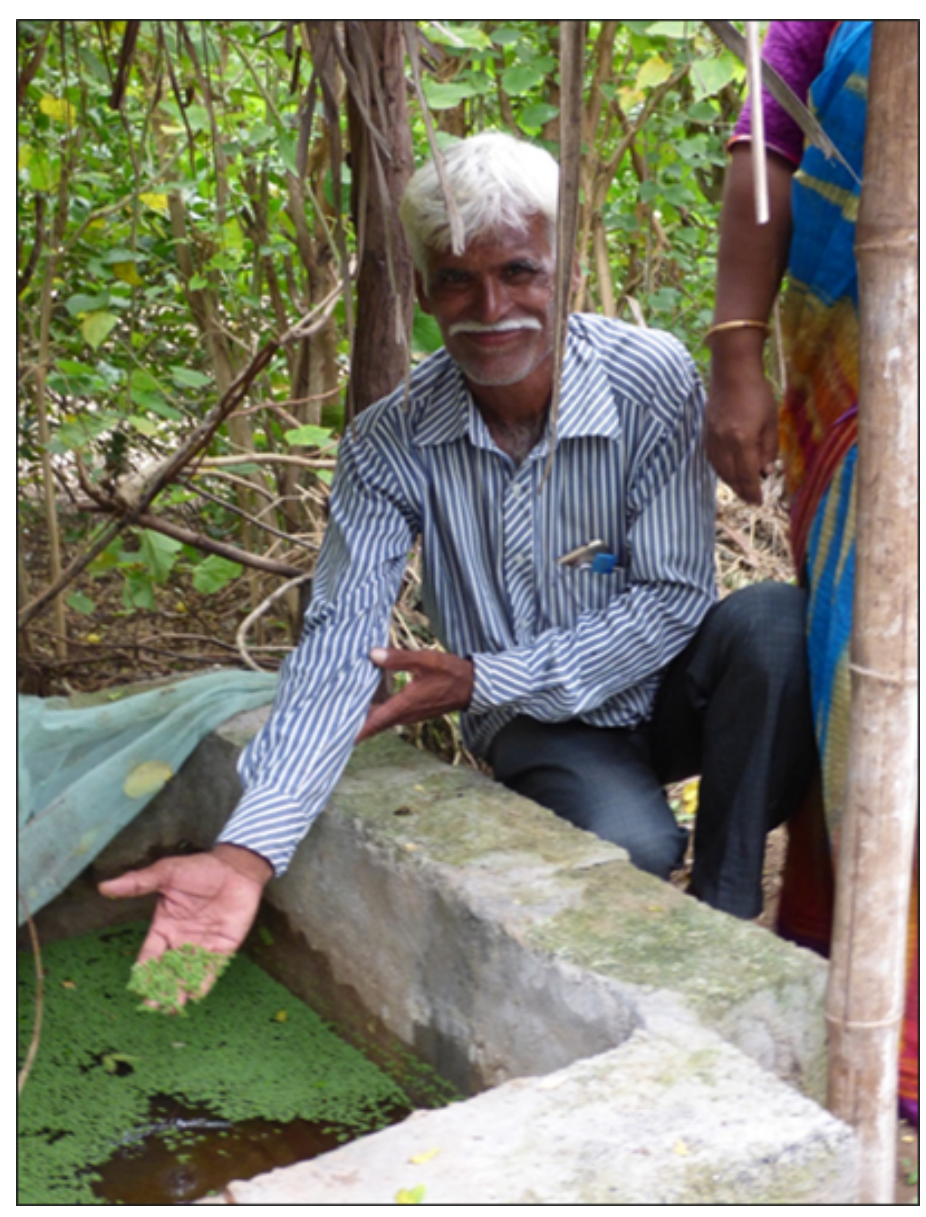

Photo 62. Production of Azolla pinnata for live mulching with rice. 
Table 193. Impact of Acchadana (mulching) on soil carbon (C) content in India

\begin{tabular}{|c|c|c|c|c|c|c|c|c|}
\hline Location & Climate zone & Soil type & $\begin{array}{l}\text { Baseline C stock } \\
\text { (t/ha) }\end{array}$ & $\begin{array}{l}\text { Additional C } \\
\text { storage } \\
\text { (t/ha/yr) }\end{array}$ & $\begin{array}{l}\text { Duration } \\
\text { (Years) }\end{array}$ & $\begin{array}{l}\text { Depth } \\
(\mathrm{cm})\end{array}$ & $\begin{array}{l}\text { More } \\
\text { informa- } \\
\text { tion }\end{array}$ & Reference \\
\hline \multicolumn{9}{|c|}{ Reduced cultivation (“soil mulching”) } \\
\hline \multirow{4}{*}{ Haryana, India } & \multirow{4}{*}{$\begin{array}{l}\text { Temperate dry semi- } \\
\text { arid }\end{array}$} & Sandy loam & 18.75 & 0.18 & \multirow{3}{*}{15} & \multirow{3}{*}{$0-30$} & \multirow{3}{*}{$\begin{array}{l}\text { Zero till - } \\
\text { measured } \\
\text { to } 40 \mathrm{~cm} \text { so } \\
\text { multiplied } \\
\text { by } 30 / 40\end{array}$} & \multirow{3}{*}{ Singh et al. (2014) } \\
\hline & & Loam & 19.84 & 0.35 & & & & \\
\hline & & Clay loam & 23.83 & 0.47 & & & & \\
\hline & & Loam & 20.83 & 0.15 & 8 & $0-30$ & Reduced till & Patra et al. (2019) \\
\hline \multicolumn{9}{|c|}{ Mulching with dried biomass } \\
\hline $\begin{array}{l}\text { Central / West } \\
\text { India }\end{array}$ & $\begin{array}{l}\text { Tropical moist semi- } \\
\text { arid }\end{array}$ & Chromic Vertisols & 37.1 & 0.15 & \multirow{5}{*}{10} & \multirow{5}{*}{$0-30$} & $\begin{array}{l}2.7 \mathrm{t} / \mathrm{ha} 50 \\
\text { years }\end{array}$ & \multirow{5}{*}{$\begin{array}{l}\text { Duration extrapolated to } \\
\text { steady state using } \\
\text { simulation modelling. } \\
\text { Assumes } \mathrm{C} \text { inputs of } \\
1.2 \mathrm{t} / \mathrm{ha} / \mathrm{yr} \text { (after Smith et } \\
\text { al., 202Oa); } \\
5 \text { major climate zones } \\
\text { covering } 92 \text { percent of India } \\
\text { (Monfreda Ramankutty and } \\
\text { Hertel, 2O08); Simulations } \\
\text { for the major soil type in } \\
\text { each zone (HWSD, 2O2O) } \\
\text { after Smith et al. (2O2Ob) }\end{array}$} \\
\hline South East India & Tropical sub-humid & Eutric Nitisols & 24.0 & 0.22 & & & $\begin{array}{l}7.0 \mathrm{t} / \mathrm{ha} \text { in } \\
150 \text { years }\end{array}$ & \\
\hline South West India & $\begin{array}{l}\text { Temperate dry semi- } \\
\text { arid }\end{array}$ & Eutric Cambisols & 41.1 & 0.30 & & & $\begin{array}{l}11.5 \mathrm{t} / \mathrm{ha} \text { in } \\
150 \text { years }\end{array}$ & \\
\hline $\begin{array}{l}\text { Central South } \\
\text { India }\end{array}$ & $\begin{array}{l}\text { Temperate moist } \\
\text { semi-arid }\end{array}$ & Chromic Luvisols & 21.0 & 0.20 & & & $\begin{array}{l}5.9 \mathrm{t} / \mathrm{ha} \text { in } \\
150 \text { years }\end{array}$ & \\
\hline Northern India & Tropical humid & Orthic Acrisols & 42.0 & 0.32 & & & $\begin{array}{l}13.7 \mathrm{t} / \text { ha in } \\
200 \text { years }\end{array}$ & \\
\hline \multicolumn{9}{|l|}{ Live mulching } \\
\hline India & Tropical sub-humid & Silty clay loam & 16.7 & $1.5-2.8$ & 4 & $0-30$ & $\begin{array}{l}\text { Multi-tier } \\
\text { finger millet }\end{array}$ & Jakhar et al. (2017) \\
\hline
\end{tabular}




\section{Other benefits of the practice}

\subsection{Improvement of soil properties}

The reduced tillage and increased soil organic matter associated with the different mulching practices included in ZBNF are likely to reduce bulk density and retain soil aggregates (Liao et al, 2019). Soil pH and cation exchange capacity are expected to increase due to increased mineralization of $\mathrm{N}$ (Liao et al., 2019). Because ZBNF relies only on preparations from natural sources, such as the neem tree, soil biological activity is likely to increase with the reduced use of synthetic herbicides and pesticides. Application of soil inocula rich in micro-organisms (Ram, Singha and Vaish, 2018; Ram, 2019; Sreenivasa et al., 2009) and retention of earthworms through reduced tillage (Orzech and Zaluski, 2019) are also likely to increase biological activity.

\subsection{Minimization of threats to soil functions}

Most soil threats listed in Table 194 are reduced by ZBNF, but there are potential negative impacts on soil nutrient balance as it is more difficult to adjust nutrient ratios without using chemical fertilizers.

\section{Table 194. Soil threats}

\begin{tabular}{|c|c|}
\hline Soil threats & \\
\hline Soil erosion & $\begin{array}{l}\text { Reduced due to increased soil organic matter, reduced tillage and } \\
\text { reduced exposure of bare soil associated with mulching with crop } \\
\text { residues, intercropping and agroforestry (Liao et al., 2019). }\end{array}$ \\
\hline $\begin{array}{l}\text { Nutrient imbalance and } \\
\text { cycles }\end{array}$ & $\begin{array}{l}\text { Potential for nutrients to become unbalanced (Loke, Kotzé and Du } \\
\text { Preez, 2013). }\end{array}$ \\
\hline $\begin{array}{l}\text { Soil salinization and } \\
\text { alkalinization }\end{array}$ & $\begin{array}{l}\text { Reduced due to reduced need for irrigation associated with increase soil } \\
\text { organic matter (Liao et al., 2019). }\end{array}$ \\
\hline $\begin{array}{l}\text { Soil } \\
\text { contamination/pollution }\end{array}$ & $\begin{array}{l}\text { Reduced due to not using manufactured pesticides and herbicides and } \\
\text { no input of chemical fertilizers (RySS, 2020). }\end{array}$ \\
\hline Soil acidification & $\begin{array}{l}\text { Increased pH due to net mineralization of nitrogen from residue input } \\
\text { plus jiwamrita (Liao et al., 2019; Loke, Kotzé and Du Preez, 2013). }\end{array}$ \\
\hline
\end{tabular}




\begin{tabular}{|c|c|}
\hline Soil threats & \\
\hline Soil biodiversity loss & $\begin{array}{l}\text { Improved due to reduced use of toxic chemicals, use of microbial } \\
\text { inocula and increased diversity of food-web structure associated with } \\
\text { avoidance of mono-cropping (Liao et al., 2019; Ram, Singha and Vaish, } \\
\text { 2018; Sreenivasa et al., 2009; Venter, Jacobs and Hawkins, 2016). }\end{array}$ \\
\hline Soil sealing & $\begin{array}{l}\text { Decreased potential for soil sealing due to mulching (da Silva et al., } \\
\text { 2019). }\end{array}$ \\
\hline Soil compaction & $\begin{array}{l}\text { Reduced compaction (lower bulk density) due to increased organic } \\
\text { inputs and reduced tillage (Liao et al., 2019). }\end{array}$ \\
\hline Soil water management & $\begin{array}{l}\text { Improved water management through mulching, and use of contours } \\
\text { and bunds (Maetens, Poesen and Vanmaercke, 2012; Singh et al., } \\
\text { 2014). }\end{array}$ \\
\hline
\end{tabular}

\subsection{On production}

The impact of ZBNF on food production depends on the practices used before its implementation. The $\mathrm{N}$ available to crops in a ZBNF system is likely to be less than 80 percent of the national average fertilizer application rate in India (Smith et al., 2020a). Therefore, if $\mathrm{N}$ is the limiting nutrient and overall $\mathrm{N}$ losses remain unchanged, conversion to ZBNF is expected to improve crop yields only in farming systems that use below the national average rate of $\mathrm{N}$ fertilizer (Smith et al, 2020a). Significant benefits could be achieved if ZBNF enables higher nitrogen use efficiency thus generating smaller nitrogen losses, but this remains to be demonstrated.

\subsection{Mitigation of and adaptation to climate change, and air pollution}

A number of the practices used in ZBNF are listed by Sapkota et al. (2019) as having high potential for cost-effective mitigation of climate change in India. These are reduction in carbon dioxide equivalents due to tillage (519 to 1996 $\mathrm{kg} / \mathrm{ha} / \mathrm{yr}$ ), residue burning (-3 to $522 \mathrm{~kg} / \mathrm{ha} / \mathrm{y}$ ), fertilizer production (57 to $529 \mathrm{~kg} / \mathrm{ha} / \mathrm{yr}$ ) and fertilizer consumption (48 to $199 \mathrm{~kg} / \mathrm{ha} / \mathrm{yr}$ ), giving a total mitigation potential of 563 to $2517 \mathrm{~kg} / \mathrm{ha} / \mathrm{yr}$. In addition, potential for reduced cattle numbers associated with reduced requirement for manure could further mitigate climate change by approximately 952 to $988 \mathrm{~kg} / \mathrm{ha} / \mathrm{yr}$ in carbon dioxide equivalents (Smith et al, 2020a). Reduced use of urea fertilizers and covered storage of excreted urine is likely to reduce ammonia emissions, improving air quality (Bittman etal., 2014). 


\subsection{Socio-economic benefits}

The success of ZBNF as a social movement is undeniable having become one of the largest agroecological movements worldwide since it was first popularized (Khadse et al., 2018). Conversion to ZBNF is encouraged by economic benefits perceived by low income farmers through reduced financial outlay, improved yields, reduced labor and increased farm income (Khadse et al., 2018; Bharucha, Mitjans and Pretty, 2020).

\section{Potential drawbacks to the practice}

\subsection{Tradeoffs with other threats to soil functions}

Tradeoffs of ZBNF with soil threats include potential loss of nutrient balance (4.2) and formation of a hard pan due to reduced tillage (Singh et al., 2014). Other soil threats are reduced as discussed in Table 194.

\subsection{Possible greenhouse gas emissions}

A significant contribution to global greenhouse gas emissions is from $\mathrm{N}$ fertilizers, manures, ruminants and rice production (Carlson et al., 2017; Dangal et al., 2017). Because of the reduced reliance of ZBNF systems on inputs of both inorganic and organic fertilizers, they have high potential to reduce greenhouse gas emissions. Smith et al. (2020a) estimated that implementation of ZBNF across all of India would require dung and urine from only 18 - 21 percent of the cows reported in the 2012 Livestock Census, which if reflected in a corresponding reduction in cattle numbers would be equivalent to reduced greenhouse gas emissions of $1.71-1.78 \times 10^{8} \mathrm{t} /$ year $\mathrm{CO}_{2} \mathrm{eq}$ (Smith $e t$ al. 2020a). Adding to this reduced emissions due to zero use of synthetic fertilizers ( $\sim 1 \times 10^{8} \mathrm{t} / \mathrm{yr} \mathrm{CO}_{2}$ eq.; Tirado et al., 2010), implementing ZBNF across all of India could reduce greenhouse gas emissions compared to business as usual by a total of $2.71-2.78 \times 10^{8} \mathrm{t} / \mathrm{yr} \mathrm{CO}_{2} \mathrm{eq}$, equivalent to $76-78$ percent of reported agricultural emissions and 21 percent of reported net national emissions (Smith et al., 2020a). The potential C sequestration of $0.3-2.8$ t/ha/yr (section 3) across the total agricultural land area of India (1.797 x $10^{8}$ ha https://data.worldbank.org/) could add a further $1.98-18.45 \mathrm{t} / \mathrm{yr} \mathrm{CO}_{2} \mathrm{eq}$ during the first 4 years after conversion, although this may be countered by increased nitrous oxide emissions associated with incorporation of crop residues from legumes (Jensen et al., 2012). While there remains uncertainty in the impact of ZBNF systems on greenhouse gas emissions, it is clear that they have high potential for emission reduction.

\subsection{Conflict with other practice(s)}

A detailed analysis of potential conflicts of ZBNF with other practices has not yet been completed. 


\subsection{Decreases in production (e.g. food/fuel/feed/timber/fibre)}

Where low input farms may see yield benefits on conversion to ZBNF, the limited supply of $\mathrm{N}$ could result in yield penalties in more-intensive systems normally receiving higher inputs (Smith, 2020a) (4.3).

\section{Recommendations before implementing the practice}

This paper has highlighted the important role of mulching in ZBNF systems to maintaining SOC. The impacts of ZBNF should be demonstrated using fully replicated and randomized field trials but these practices are already being very successfully implemented at scale. Therefore, scientists should urgently work together with practitioners to bring scientific rigor to the choice of practices. High input farms are more likely to see yield penalties on conversion to ZBNF, so more work is urgently needed to develop methods to ensure high production rates can be maintained on these farms.

\section{Potential barriers to adoption}

Potential barriers to adoption have been largely overcome in ZBNF through mobilization of volunteers and resources in group activities, self-organization of training, farmer-to-farmer knowledge dissemination by "Champion farmers", distribution of equipment through village shops, focus on marginal, poor, female and young farmers, and involvement of non-governmental organizations and international funders (Khadse et al, 2018). 
Table 195. Potential barriers to adoption

\begin{tabular}{|c|c|c|}
\hline Barrier & YES/NO & \\
\hline Biophysical & No & All resources are available on farm (Palekhar, 2019). \\
\hline Cultural & No & $\begin{array}{l}\text { Works within Vedic principles to encourage uptake by farmers } \\
\text { (Palekhar, 2019). }\end{array}$ \\
\hline Social & No & $\begin{array}{l}\text { Works with communities, with champions located in villages } \\
\text { (Khadse et al., 2018). }\end{array}$ \\
\hline Economic & No & Minimizes requirement for off-farm purchases (Palekhar, 2019). \\
\hline Institutional & Yes & $\begin{array}{l}\text { Widespread opposition to ZBNF amongst scientists (Ramakumar } \\
\text { and Arjun, 2019; Raghuram, 2020). }\end{array}$ \\
\hline $\begin{array}{l}\text { Legal (Right } \\
\text { to soil) }\end{array}$ & No & Members are mainly land-owning peasantry (Khadse et al., 2018). \\
\hline Knowledge & No & $\begin{array}{l}\text { Training of farmers in mass training camps is an integral part of the } \\
\text { ZBNF system (Khadse and Rosset, 2019). }\end{array}$ \\
\hline
\end{tabular}




\section{References}

Bharucha, Z.P., Mitjans, S.B. \& Pretty, J. 2020. Towards redesign at scale through zero budget natural farming in Andhra Pradesh, India. International Journal of Agricultural Sustainability, 18(1): 1-20.

https://doi.org/10.1080/14735903.2019.1694465

Bittman, S., Dedina, M., Howard C.M., Oenema, O. \& Sutton, M.A (Eds.). 2014. Options for ammonia mitigation: Guidance from the UNECE Task Force on Reactive Nitrogen. Centre for Ecology and Hydrology, Edinburgh, UK. (also available at: http://www.clrtap-tfrn.org/sites/clrtap-

tfrn.org/files/documents/AGD_final_file.pdf)

Carlson, K.M., Gerber, J.S., Mueller, N.D., Herrero, M., MacDonald, G.K., Brauman, K.A., Havlik, P., O'Connell, C.S., Johnson, J.A., Saatchi, S. \& West, P.C. 2017. Greenhouse gas emissions intensity of global croplands. Nature Climate Change, 7(1): 63-68. https://doi.org/10.1038/nclimate3158

Dangal, S.R.S., Tian, H., Zhang, B., Pan, S., Lu, C. \& Yang, J. 2017. Methane emission from global livestock sector during 1890-2014: Magnitude, trends and spatiotemporal patterns. Global Change Biology, 23(10): 4147-4161. https://doi.org/10.1111/gcb.13709

da Silva, F.F., Souza, T.E.M. dos S., de Souza, E.R., Correa, M.M. \& Rolim, M.M. 2019. Surface sealing and water erosion of soils with mulching in the semi-arid region of Brazil. Revista Brasileira de Engenharia Agrícola e Ambiental, 23(4): 277-284. https://doi.org/10.1590/1807-1929/agriambi.v23n4p277-284

Feliciano, D., Ledo, A., Hillier, J. \& Nayak, D.R. 2018. Which agroforestry options give the greatest soil and above ground carbon benefits in different world regions? Agriculture, Ecosystems \& Environment, 254: 117129. https://doi.org/10.1016/j.agee.2017.11.032

Goswami, S.B., Mondal, R. \& Mandi, S.K. 2019. Crop residue management options in rice-rice system: a review. Archives of Agronomy and Soil Science, 66(9): 1218-1234.

https://doi.org/10.1080/03650340.2019.1661994

HWSD. 2020. Harmonized World Soil Database v1.2. [online]. [Cited 29 June 2020].

http://www.fao.org/soils-portal/soil-survey/soil-maps-and-databases/harmonized-world-soil-databasev12/en/

Jakhar, P., Dass, A., Adhikary, P.P., Sudhishri, S., Naik, B.S., Hombegowda, H.C., Madhu, M., Lenka, N.K., Chaudhary, P.R. \& Panda, R.K. 2017. Multitier agroforestry system for integrated resource conservation on uplands of Eastern Ghats region in India. Agroforestry Systems, 91: 697-712.

https://doi.org/10.1007/s10457-016-9976-1

Jensen, E.S., Peoples, M.B., Boddey, R.M., Gresshoff, P.M., Hauggaard-Nielsen, H., J.R. Alves, B. \& Morrison, M.J. 2012. Legumes for mitigation of climate change and the provision of feedstock for biofuels and biorefineries. A review. Agronomy for Sustainable Development, 32(2): 329-364.

https://doi.org/10.1007/s13593-011-0056-7 
Kennedy, J. \& King, L. 2014. The political economy of farmers' suicides in India: indebted cash-crop farmers with marginal landholdings explain state-level variation in suicide rates. Globalization and Health, 10: 16. https://doi.org/10.1186/1744-8603-10-16

Khadse, A. \& Rosset, P.M. 2019. Zero budget natural farming in India - from inception to institutionalization. Agroecology and Sustainable Food Systems, 43(7-8): 848-871. https://doi.org/10.1080/21683565.2019.1608349

Khadse, A., Rosset, P.M., Morales, H. \& Ferguson, B.G. 2018. Taking agroecology to scale: the Zero Budget Natural Farming peasant movement in Karnataka, India. The Journal of Peasant Studies, 45: 192-219. https://doi.org/10.1080/03066150.2016.1276450

Liao, J., Xu, Q., Xu, H. \& Huang, D. 2019. Natural farming improves soil quality and alters microbial diversity in a cabbage field in Japan. Sustainability, 11: 3131. https://doi.org/10.3390/su11113131

Loke, P.F., Kotzé, E. \& Du Preez, C.C. 2013. Impact of long-term wheat production management practices on soil acidity, phosphorus and some micronutrients in a semi-arid Plinthosol. Soil Research, 51(5): 415-426. https://doi.org/10.1071/SR12359

Maetens, W., Poesen, J. \& Vanmaercke, M. 2012. How effective are soil conservation techniques in reducing plot runoff and soil loss in Europe and the Mediterranean? Earth-Science Reviews, 115(1-2): 21-36.

https://doi.org/10.1016/j.earscirev.2012.08.003

Monfreda, C., Ramankutty, N. \& Hertel, T.W. 2008. Global agricultural land use data for climate change analysis. GTAP Working Paper No. 40. Purdue University, Center for Global Trade Analysis, Global Trade Analysis Project. (also available at: http://ageconsearch.umn.edu/record/283458/files/40.pdf)

Orzech, K. \& Zaluski, D. 2019. Effect of soil compaction and different soil tillage systems on chemical properties of soil and presence of earthworms in winter oilseed rape fields. Journal of Elementology, 25(2): 413429. https://doi.org/10.5601/jelem.2019.24.2.1874

Patra, S., Julich, S., Feger, K.-H., Jat, M.L., Sharma, P.C. \& Schwärzel, K. 2019. Effect of conservation agriculture on stratification of soil organic matter under cereal-based cropping systems. Archives of Agronomy and Soil Science, 65(14): 2013-2028. https://doi.org/10.1080/03650340.2019.1588462

Pelster, D.E., Gisore, B., Koske, J. K., Goopy, J., Korir, D., Rufino, M.C. \& Butterbach-Bahl, K. 2016. Methane and nitrous oxide emissions from cattle excreta on an East African grassland. Journal of Environmental Quality, 45: 1531-1539. https://doi.org/10.2134/jeq2016.02.0050

Raghuram, N. 2020. Zeroing in on farm budgets or zero budget natural farming? UNEP Perspective Series, Issue No. 37.

https://wedocs.unep.org/bitstream/handle/20.500.11822/31512/Perspective_37\%20LR.pdf?sequence=1 \&isAllowed $=\mathrm{y}$

Ram, A.R. 2019. Innovations in organic production of fruits and vegetables. Shodh Chintan, 11: 85-98. https://www.researchgate.net/publication/333507700 
Ram, R.A., Singha, A. \& Vaish, S. 2018. Microbial characterization of on-farm produced bio-enhancers used in organic farming. Indian Journal of Agricultural Science, 88: 35-40.

Ramakumar, R. \& Arjun, S.V, 2019. Stirring up the truth about Zero Budget Natural Farming. The Hindu, 9 October 2019. (also available at https:/ / www.thehindu.com/opinion/lead/stirring-up-the-truth-aboutzbnf/article29620843.ece).

Rasmussen, P.E. \& Collins, H.P. 1991. Long-term impacts of tillage, fertilizer and crop residue on soil organic matter in temperate semiarid regions. Advances in Agronomy, 45: 93-134. https://doi.org/10.1016/S00652113(08)60039-5

RySS. 2020. Rythu Sadhikara Samstha. Zero budget natural farming [online] [Cited 6 June 2020]. http://apzbnf.in

Sapkota, T.B., Vetter, S.H., Jat, M.L., Sirohi, S., Shirsath, P.B., Singh, R., Jat, H.S., Smith, P., Hillier, J. \& Stirling, C.M. 2019. Cost-effective opportunities for climate change mitigation in Indian agriculture. Science of the Total Environment, 655: 1342-1354. https://doi.org/10.1016/j.scitotenv.2018.11.225

Singh, J., Solomon, S. \& Kumar, D. 2013. Manufacturing jaggery, a product of sugarcane, as health food. Agrotechnology, S11. https://doi.org/10.4172/2168-9881.1000S11-007

Singh, A., Phogat, V.K., Dahiya, R., Batra, S.D. 2014. Impact of long-term zero till wheat on soil physical properties and wheat productivity under rice-wheat cropping system. Soil and Tillage Research, 140: 98-105. https://doi.org/10.1016/j.still.2014.03.002

Sitharaman, N. 2019. Budget 2019-2020 Speech of Nirmala Sitharaman, Minister of Finance July 5, 2019 [online] [Cited 6 June 2020]. https://www.amazon.co.uk/Budget-2019-2020-Nirmala-Sitharaman-Ministerebook/dp/B07TZ63GPG

Smith, J., Yeluripati, J., Smith, P. \& Nayak, D.R. 2020a. Potential yield challenges to scale-up of zero budget natural farming. Nature Sustainability, 3(3): 247-252. https://doi.org/10.1038/s41893-019-0469-X

Smith, J., Nayak, D., Datta, A., Narkhede, W.N., Albanito, F., Balana, B., Bandyopadhyay, S.K., Black, H., Boke, S., Brand, A., Byg, A., Dinato, M., Habte, M., Hallett, P.D., Lemma, T., Mekuria, W., Moges, A., Muluneh, A., Novo, P., Rivington, M., Tefera, T., Vanni, E.M., Yakob, G. \& Phimister, E. 2020b. A systems model describing the impact of organic resource use on farming households in low to middle income countries. Agricultural Systems, 184: 102895. https://doi.org/10.1016/j.agsy.2020.102895

Sorensen, P. 1998. Effects of storage time and straw content of cattle slurry on the mineralization of nitrogen and carbon in soil. Biology and Fertility of Soils, 27: 85-91. https://doi.org/10.1007/s003740050404

Sreenivasa, M.N., Naik, N. \& Bhat, S.N. 2009. Beejamrutha: A source of beneficial bacteria. Karnataka Journal of Agricultural Science, 22: 1038-1040. 
Sun, W., Canadell, J.G., Yu, L., Yu, L., Zhang, W., Smith, P., Fischer, T. \& Huang, Y. 2020. Climate drives global soil carbon sequestration and crop yield changes under conservation agriculture. Global Change Biology, 26(6): 3325-3335. https://doi.org/10.1111/gcb.15001

Tirado, R., Gopikrishna, S.R., Krishnan, R. \& Smith, P. 2010. Greenhouse gas emissions and mitigation potential from fertilizer manufacture and application in India. International Journal of Agricultural Sustainability, 8: 176-185. https://doi.org/10.3763/ijas.2009.0422

Tripathi, R.P., Sharma, P. \& Singh, S. 2006. Soil physical response to multi-year rice wheat production in India. International Journal of Soil Science, 1(2): 91-107. https://dx.doi.org/10.3923/ijss.2006.91.107

UNEP 2019. Zero Budget Natural Farming as a nature-based solution for climate action .

https://wedocs.unep.org/handle/20.500.11822/28895?show=full

Venter, Z.S., Jacobs, K. \& Hawkins, H.J. 2016. The impact of crop rotation on soil microbial diversity: A metaanalysis. Pedobiologia, 59: 215. https://doi.org/10.1016/j.pedobi.2016.04.001 


\title{
45. Agroecological farming
}

\author{
Edmundo Barrios, Haekoo Kim, Teodardo Calles
}

Plant Production and Protection Division, FAO, Rome, Italy

\section{Description of the concept}

Agroecology is an integrated farming approach that simultaneously applies ecological and social concepts and principles to the design and management of sustainable food and agricultural systems (FAO, 2018a). The historical evolution of Agroecology since early last century (Figure 16) shows its development as a science, as a practice and as a social movement (Wezel et al. 2020). Recognizing that the inherent complexity of achieving sustainability is commonly seen as a deterrent to decision-making, FAO has approved the 10 Elements of Agroecology as an analytical framework to support the design of differentiated paths for agriculture and food systems transformation that embrace a holistic approach and long-term perspective (Barrios et al. 2020). The 10 Elements of Agroecology are interlinked and interdependent, and include: diversity; co-creation and sharing of knowledge and practices, science and innovation; synergies; efficiency; resilience; recycling; human and social values; culture and food traditions; responsible governance; and circular and solidarity economy (FAO, 2019). This framework emanated from a four-year multi-stakeholder consultative process, including global and regional dialogues, expert reviews, and based on review of the scientific literature, intending to facilitate transformative change and social-ecological transitions towards sustainable agriculture and food systems (Barrios et al., 2020). Agroecological farming seeks to optimize the interactions between agroecosystem components (i.e. plants, animals, humans) and the environment while taking into consideration the social aspects that need addressing for sustainable food and agricultural systems (FAO, 2018a). Diversification is central to agroecological farming as it can promote multiple ecosystem services without necessarily compromising yield (Tamburini et al., 2020). Adistinct feature of agroecological farming is that system optimization decisions embrace co-creation processes that encourage and facilitate the blending of local and scientific knowledge, experience and innovations, which enhances adaptability to context variation ( Barrios, Coutinho and Medeiros, 2012a; FAO 2018b; Wartenberg et al., 2018). A number of management practices described in earlier factsheets of this volume are usually part of agroecological farming. Here, we consider agroforestry, crop-residue retention, cover-cropping, integration of legumes and no-tillage management which are often found individually or combined in agroecological farming all contributing to enhance soil carbon (C) storage. The contribution of agroecological farming to soil C status through agroforestry practices is reported here through 
two recent complementary meta-analyses (Chatterjee et al., 2018; Muchane et al., 2020), where the latter also quantified other soil-mediated ecosystem services, specifically, regulation of soil erosion, storage of soil nitrogen $(\mathrm{N})$, availability of soil $\mathrm{N}$, availability of soil phosphorus $(\mathrm{P})$, and alleviation of soil acidity. In addition, the contribution of practices involving the retention of crop residues, cover cropping, legume integration and no-tillage management to soil C storage are reported through recent review studies and meta-analyses (Six et al., 2002; Haddaway et al., 2017; Kumar et al., 2018; Bolinder et al., 2020).

\section{Range of applicability}

There are multiple farming systems that can be considered using a holistic approach. Agroecological farming provides a unique set of strategies to counter climate change linking adaptation to local context with mitigation measures (Leippert et al., 2020). Optimizing the interactions of the different sources of organic matter (i.e. crop residue, cover crop, soil) for crop production are considered within the framework of environmental sustainability, and C storage is an essential component to build the resilience of the system. Here, we build our argument on recent meta-analyses and reviews evidencing that agroecological farming offers a large set of adaptable practices to tackle climate change impact in different contexts from arid to humid tropics in various production systems.

\section{Impact on soil organic carbon stocks}

The different meta-analyses presented in Table 196 were global in nature and provide a quantitative synthesis of paired comparisons (e.g. conventional agriculture vs. agroecological farming practice) conducted under the same condition of climate and soil type, and using the same soil sampling depth and lab analysis protocols. Our focus on existing meta-analyses relies on the capacity of this statistical procedure to compare and synthesize results from different studies in order to find common patterns, discrepancies, or other interesting relationships that may not be detectable from individual studies.

The Chatterjee et al. (2018) study reported differences in SOC stocks up to $100 \mathrm{~cm}$ soil depth under agroforestry systems in comparison with other land-use systems (i.e. agriculture, forestry, pasture, or uncultivated land) ranging from 5 to 50 years of age in different regions across the world. Increases in mean soil C stocks ranged from 27 percent (Arid and semiarid region), 26 percent (Lowland humid tropical region), 5.8 percent (Mediterranean region) and a 5.3 percent decrease in soil C stocks was found in temperate regions. The Muchane et al., (2020) study compared plots representing one or more agroforestry practices with plots of cropland without trees (i.e. control plots) ranging from 3 to 50 yrs of age in humid and sub-humid regions across the planet and found a mean 21 percent increase in soil $\mathrm{C}$ stocks up to $30 \mathrm{~cm}$ soil depth. These findings provide strong evidence that agroecological farming relying on agroforestry has its greatest potential contribution to additional soil $\mathrm{C}$ storage ranging between 21-27 percent in tropical regions. Nevertheless, a literature review on SOC storage under agroforestry systems in sub-Saharan Africa highlights that only improved fallow and multistrata agroforestry systems are able to achieve storage rates significantly higher than 4\%o per year (Corbeels et al., 2019). Concerning crop 
residue retention and cover-cropping, the synthesis of reviews by Bolinder et al. (2020) analyzed the contributions of such practices to soil C storage through the analysis of long term experiments data by summarizing mean response ratios (RRs) and stock change rate (SCR) effect size indices from twenty existing reviews. Overall, crop residue retention increased soil $\mathrm{C}$ stocks by 9 percent with accrual rates of $0.12 \mathrm{t} \mathrm{C} / \mathrm{ha} / \mathrm{yr}$, while cover-cropping increased soil C stocks by 10 percent with accrual rates of $0.33 \mathrm{t} \mathrm{C/ha/yr.} \mathrm{The} \mathrm{review} \mathrm{by} \mathrm{Kumar} \mathrm{et} \mathrm{al.} \mathrm{(2018)} \mathrm{synthesized}$ findings in the literature on the effect of integration of legumes into agricultural systems and estimated that the cumulative impact on soil C storage is expected to be $33 \mathrm{t} \mathrm{C} /$ ha in Asia and $35.1 \mathrm{t} \mathrm{C} /$ ha in Africa by 2030. The review by Six et al., (2002) compared the effects of no-tillage practices on soil C dynamics and additional storage in tropical and temperate soils finding a common soil C storage rate of $33 \mathrm{t} \mathrm{C} / \mathrm{ha} / \mathrm{yr}$ in the top $10 \mathrm{~cm}$ thus contributing to an accrual of $7 \mathrm{tC} / \mathrm{ha}$ in $20 \mathrm{yrs}$. The meta-analysis by Haddaway et al., (2017) on the impact of no-tillage management used as a basis evidence identified within a recently completed systematic map on the impacts of farming on SOC and found a mean soil C stock increase under no-tillage of $4.6 \mathrm{t} / \mathrm{ha}$ in the top $30 \mathrm{~cm}$ after more than 10 yrs using this practice 
Table 196. Evolution of soil organic carbon (SOC) stocks under different agroecological systems over time

\begin{tabular}{|c|c|c|c|c|c|c|c|}
\hline Methodology & Climate zone & Baseline C stock & Additional C storage & $\begin{array}{l}\text { Duration } \\
\text { (Years) }\end{array}$ & Depth $(\mathrm{cm})$ & Practice & Reference \\
\hline $\begin{array}{l}\text { Meta-analysis } \\
\text { (858 data points) }\end{array}$ & $\begin{array}{l}4 \text { agroecological zones: arid and } \\
\text { semiarid (ASA), lowland humid } \\
\text { tropics (LHT), Mediterranean } \\
\text { (MED), and temperate (TEM) }\end{array}$ & Pasture or uncultivated land & $\begin{array}{l}+27 \% \text { in ASA, } \\
+26 \% \text { in LHT, } \\
+5.8 \% \text { in MED } \\
-5.3 \% \text { in TEM }\end{array}$ & $5-50$ & $0-100$ & Agroforestry & $\begin{array}{l}\text { Chatterjee et al. } \\
\text { (2018) }\end{array}$ \\
\hline $\begin{array}{l}\text { Meta-analysis } \\
\text { (152 data points) }\end{array}$ & $\begin{array}{l}\text { Humid and subhumid tropics: } \\
\text { Tropical, montane, tropical wet, } \\
\text { tropical moist }\end{array}$ & Cropland without trees & $+21 \%$ & $3-50$ & $<30$ & Agroforestry & $\begin{array}{l}\text { Muchane et al. } \\
(2020)\end{array}$ \\
\hline $\begin{array}{l}\text { Synthesis of } \\
\text { Reviews } \\
\text { ( } 279 \text { data points) }\end{array}$ & \multirow{3}{*}{ Multiple climates } & $\begin{array}{l}\text { Cropland with removal of } \\
\text { crop residues }\end{array}$ & $\begin{array}{l}+9 \% \\
0.12 \text { tC/ha/yr }\end{array}$ & $<56$ & $<30$ & \multirow[t]{2}{*}{$\begin{array}{l}\text { Retention of crop } \\
\text { residues }\end{array}$} & \multirow{2}{*}{$\begin{array}{l}\text { Bolinder et al. } \\
\text { (2020) }\end{array}$} \\
\hline $\begin{array}{l}\text { Synthesis of } \\
\text { Reviews } \\
\text { (176 data points) }\end{array}$ & & $\begin{array}{l}\text { Cropland without cover- } \\
\text { crops }\end{array}$ & $\begin{array}{l}+10 \% \\
0.33 \mathrm{tC} / \mathrm{ha} / \mathrm{yr}\end{array}$ & $<98$ & $<30$ & & \\
\hline $\begin{array}{l}\text { Review } \\
\text { (Roth C model } \\
\text { prediction) }\end{array}$ & & $\begin{array}{l}\text { Cropland without } \\
\text { leguminous cover }\end{array}$ & $\begin{array}{l}+33 \mathrm{tC} / \text { ha (Asia) }+35.1 \mathrm{tC} / \mathrm{ha} \\
\text { (Africa) }\end{array}$ & By 2030 & $<200$ & Pulses & $\begin{array}{l}\text { Kumar et al. } \\
\text { (2018) }\end{array}$ \\
\hline Review & Tropical and temperate & \multirow{2}{*}{$\begin{array}{l}\text { Cropland under } \\
\text { conventional tillage }\end{array}$} & $0.33 \mathrm{tC} / \mathrm{ha} / \mathrm{yr}$ & 20 & $0-30$ & No-till & $\begin{array}{l}\text { Six et al. } \\
(2002)\end{array}$ \\
\hline $\begin{array}{l}\text { Meta-analysis } \\
\text { (351 studies) }\end{array}$ & Warm-temperate & & $4.6 \mathrm{tC} / \mathrm{ha}$ & $>10$ & $0-30$ & $\begin{array}{l}\text { No-till and } \\
\text { conservation } \\
\text { tillage }\end{array}$ & $\begin{array}{l}\text { Haddaway et } \\
\text { al. (2017) }\end{array}$ \\
\hline
\end{tabular}

Soil types are various and therefore not reported in the table. 


\section{Other benefits of the practice}

\subsection{Improvement of soil properties}

The Muchane et al. (2020) meta-analysis also provides quantitative evidence that agroforestry reduced soil erosion rates by 50 percent compared to cropland without trees. This finding is supported by higher infiltration rates, lower runoff, higher proportion of soil macroggregates, and greater stability of soil structure under agroforestry. Furthermore, $\mathrm{N}$ storage increased by 13 percent, available $\mathrm{N}$ by 46 percent and available $\mathrm{P}$ by 11 percent while soil $\mathrm{pH}$ increased by 2 percent under agroforestry compared to cropland without trees. An earlier review by Barrios $e t$ al.(2012b) showed that mean densities of beneficial soil biota (i.e. soil macrofauna, mesofauna and microfauna) significantly increased under agroforestry. The retention of crop residues, cover cropping and no-tillage practices also contribute to reduced soil erosion, lower soil temperature and enhanced water-holding capacity (Paustian et al., 2019). Integration of legumes into agricultural systems (e.g. legume-nonlegume rotations) generates an increased turnover rate of plant $\mathrm{C}$ and soil $\mathrm{C}$ when compared with continuous non-legume cropping; thus, leading to an increase on soil organic carbon (Kumar et al, 2018). Furthermore, Bowles et al. (2017) highlight in their metaanalysis that cover cropping and reduced tillage management, when combined with other practices like crop diversification and organic matter management, further enhance populations of arbuscular mycorrhiza fungi and thus show the greatest promise to increase soil C storage and nutrient cycling while reducing soil erosion and nutrient losses. This suggests that agroecological farming practices considered here can make significant contributions to soil health through enhanced biological activity underpinning the provision of soil-mediated ecosystem services.

\subsection{Minimization of threats to soil functions}

\section{Table 197. Soil threats}

\begin{tabular}{|l|l|}
\hline Soil threats & $\begin{array}{l}\text { Increased soil macroaggregates and mean weight diameter, consistent with } \\
\text { increased stability of soil structure and reduced soil erosion rates under } \\
\text { agroecological farming (Six et al. 2002; Fonte et al. 2010; Bowles et al. 2017; Kumar } \\
\text { et al. 2018; Paustian et al., 2019; Muchane et al., 2020). }\end{array}$ \\
\hline $\begin{array}{l}\text { Nutrient } \\
\text { imbalance and } \\
\text { cycles }\end{array}$ & $\begin{array}{l}\text { Improved nutrient N storage and recycling (Fonte, Barrios and Six, 2010; Bowles et } \\
\text { al., 2017 ; Duchene et al. 2017; Kumar et al., 2018; Muchane et al., 2020). }\end{array}$ \\
\hline
\end{tabular}




\begin{tabular}{|l|l|}
\hline \multicolumn{1}{|c|}{ Soil threats } & Soil biodiversity \\
loss & $\begin{array}{l}\text { Enhanced abundance, diversity and activity of soil biota under agroecological } \\
\text { farming (Six et al. 2002; Pauli et al., 2011; Barrios et al., 2012b; Bowles et al. 2017; } \\
\text { Duchene, Vian and Celette, 2017; Kamau et al., 2017; Kumar et al., 2018). }\end{array}$ \\
\hline $\begin{array}{l}\text { Soil compaction } \\
\text { Enhanced abundance and activity of decompacting soil macrofauna species } \\
\text { Soil water } \\
\text { management }\end{array}$ & $\begin{array}{l}\text { Enhanced infiltration and soil water holding capacity (Paustian et al., 2019; Muchane } \\
\text { et al., 2020). }\end{array}$ \\
\hline
\end{tabular}

\subsection{Increases in production (e.g. food/fuel/feed/timber/fibre)}

In their meta-analysis study, Tamburini et al. (2020) highlight that variation in crop yield response is largely benefited by diversification practices supporting agroecological farming; however, it is significantly affected by context. Therefore, there are conditions and practices that need to be avoided in certain contexts but there are numerous opportunities for production to benefit from diversification. For instance, results from a quantitative review using meta-analysis by Sileshi et al. (2008) showed that the mean increase in maize yield promoted by agroforestry ranged between 1.3 and 1.6 t/ha compared with unfertilized maize for non-coppicing and coppicingand-mulching management of woody legumes respectively. However, it was common to find doubling and tripling of yields compared to the control, particularly for coppicing woody legumes, in sites of low or medium inherent productivity (e.g. low fertility soils). Furthermore, a mean yield increase of 20 percent resulting from crop rotations was reported in a recent meta-analysis (Zhao et al., 2020), where residual effects lasted about 2-3 years and greatest benefits resulted from legume-based rotations.

\subsection{Mitigation of and adaptation to climate change}

Agroecological farming, involving agroforestry systems, rotations with legumes, crop residue retention and cover crops can play a significant role in mitigating GHG emissions to the atmosphere while helping smallholder farmers to adapt to an increasingly changing climate through the improvement and diversification of output per unit area of tree/crop/livestock and new products which adds economic flexibility to farming communities (Mbow et al., 2014; Kumar et al., 2018; Paustian et al., 2019). It is important to note that unlike other management systems reported here, old agroforestry systems have been shown to increase deep SOC stocks thus enhancing the potential for longer-term storage (Cardinael et al., 2017). 


\subsection{Socio-economic benefits}

While agroecological farming may not lead to the greatest rates of C storage in soil, it embraces an integrated and holistic perspective that can enhance farmer communities' capacity to adapt to local contexts as well as facilitating transformative change and social-ecological transitions towards sustainable agriculture and food systems (Barrios et al., 2020). Agroecological farming can substantially contribute to the food and nutrition security of local communities through increasing crop diversity including fruits, nuts and seeds as well as starchy tree parts, which complement and diversify staple-based diets given that tree foods often have high contents of micronutrients (minerals and vitamins), macronutrients (protein, fatty acids, carbohydrates) and beneficial phytochemicals (e.g. antioxidants) (Prabhu et al., 2015). Furthermore, given that trees often exhibit higher resilience during droughts and have different harvest times than annual crops, tree foods can play an important role in circumventing hunger gaps and also can provide year-round food for home consumption or income generation which often benefit particularly women (Vinceti et al., 2013).

\section{Potential drawbacks to the practice}

\subsection{Tradeoffs with other threats to soil functions}

Table 198. Soil threats

\section{Soil threats}

Soil biodiversity

Enhanced abundance of specific soil macrofauna species found associated with loss particular tree species can result in C loss. Therefore, selection of tree species for
an agroforestry system can be critical in shaping the soil aggregation process and
long-term C storage in the soil (Kamau et al., 2020).

\subsection{Increases in greenhouse gas emissions}

Selected examples from the literature summarized by Ogle et al. (2014) show that agroecological farming involving agroforestry can lead to GHG mitigation effects ranging from -3.5 to -10.8 $\mathrm{tCO}_{2} \mathrm{eq} / \mathrm{ha} / \mathrm{yr}$. Furthermore, the metaanalysis by Sainju (2016) highlights that improved combined management including no-till, crop rotation/perennial crop, and reduced $\mathrm{N}$ rate than the traditional combined management that including conventional till, monocropping/annual crop, and recommended $\mathrm{N}$ rate reduced GHG mitigation effects by 87 percent. 


\subsection{Decreases in production (e.g. food/fuel/feed/timber/fibre)}

Optimal tree cover needs to be optimized to minimize possible crop production trade-offs in agroforestry (Blaser $e t$ al., 2018).

\section{Recommendations before implementing the practice}

Soil C storage relies on the adoption of management practices that increase the amount of carbon in agricultural land as soil organic matter. The contributions of agroecological farming to soil C storage have focused here on management practices enhancing diversification of system components (i.e. agroforestry, rotations and legume integration), together with those protecting soil structure (i.e. no-tillage management) or improving resource use efficiency (i.e. crop residue retention) that results in enhanced soil C storage. Systems approaches like agroecological farming encourage the integrated use of these practices aiming at maximizing synergies and complementarities, and minimizing trade-offs, guided by knowledge co-creation and sharing processes. For instance, local knowledge and experience would be critical during the selection of system components that enhance soil C storage while contributing to building resilience and improving food and nutrition security. Furthermore, the effective involvement of local communities in the co-development of improved management options has been shown to contribute to increased adoption as part of scaling up processes.

\section{Potential barriers for adoption}

Table 199. Potential barriers to adoption

\begin{tabular}{|c|c|l|}
\hline Barrier & YES/NO & $\begin{array}{l}\text { Agroecological farming is by nature designed to be adapted to context } \\
\text { variation. However, trade-offs resulting from agroforestry tree shading } \\
\text { reducing crop yields demands adjustment and optimization for } \\
\text { different crops (Blaser et al., 2018). Furthermore, the predominant } \\
\text { model of agriculture does not properly consider environmental } \\
\text { externalities and thus limiting their contribute to decisions shaping the } \\
\text { development of food systems (HLPE, 2019). }\end{array}$ \\
\hline Yultural & Yes & $\begin{array}{l}\text { The predominant model of agriculture and food systems is supported } \\
\text { by the slow changing public policies, existing corporate structures, } \\
\text { education systems, consumer habits and investment in research, all of } \\
\text { which represent blockages to agroecological farming (HLPE, 2019). }\end{array}$ \\
\hline
\end{tabular}




\begin{tabular}{|c|c|c|}
\hline Barrier & YES/NO & \\
\hline & & $\begin{array}{l}\text { In addition, the slash and burn tradition which dominates a large } \\
\text { proportion of tropical agricultural landscapes likely represents an } \\
\text { important cultural barrier to some practices in agroecological farming } \\
\text { (Sanchez et al., 2019). }\end{array}$ \\
\hline Social & Yes & $\begin{array}{l}\text { The process of urbanization, coupled with dietary changes, can prevent } \\
\text { some forms of system diversification which are essential for } \\
\text { agroecological farming (Vicenti et al., 2013). Furthermore, the } \\
\text { predominant model of agriculture does not properly consider social } \\
\text { externalities and thus limiting their contribute to decisions shaping the } \\
\text { development of food systems (HLPE, 2019). }\end{array}$ \\
\hline Economic & Yes & $\begin{array}{l}\text { Initial costs of transitioning to agroecological farming can be limiting, } \\
\text { but there is increasing evidence of significant improvements in farm } \\
\text { incomes in addition to allowing more sustainable production of } \\
\text { healthier food (van der Ploeg et al., 2019). }\end{array}$ \\
\hline Institutional & Yes & $\begin{array}{l}\text { Agroecological farming implementation programs often face a } \\
\text { mandate challenge between national agricultural and environment } \\
\text { institutions in many countries. }\end{array}$ \\
\hline $\begin{array}{l}\text { Legal (Right } \\
\text { to soil) }\end{array}$ & Yes & $\begin{array}{l}\text { Lack or limited tenure rights to land and trees can constitute important } \\
\text { disincentives to adoption of agroecological farming (Otsuka and Place, } \\
\text { 2014). }\end{array}$ \\
\hline Knowledge & Yes & $\begin{array}{l}\text { Agroecological farming is knowledge-intensive and demands holistic } \\
\text { thinking skills to be able to adequately manage and optimize trade-offs } \\
\text { (Barrios et al., 2020). }\end{array}$ \\
\hline
\end{tabular}




\section{Photos of the practice}

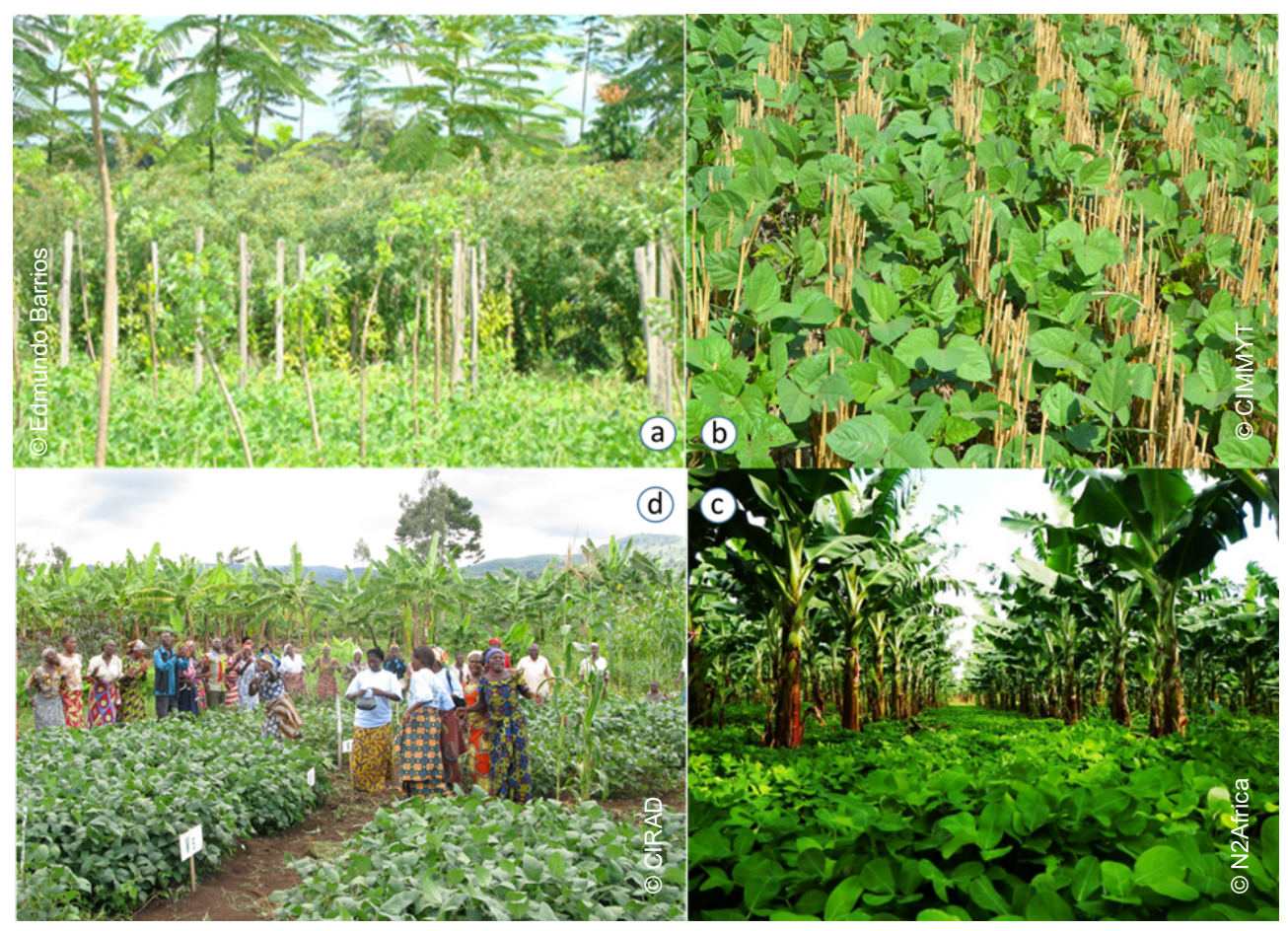

Photo 63. Agroecological farming:

a) Multistrata agroforestry systems in Brazil, b) No-tillage wheat-rice-bean rotations in India c) Bananas grown with a cover crop of Arachis pintoi in Guadeloupe, d) Participatory evaluation of legume-rhizobium inoculation options in Nigeria.

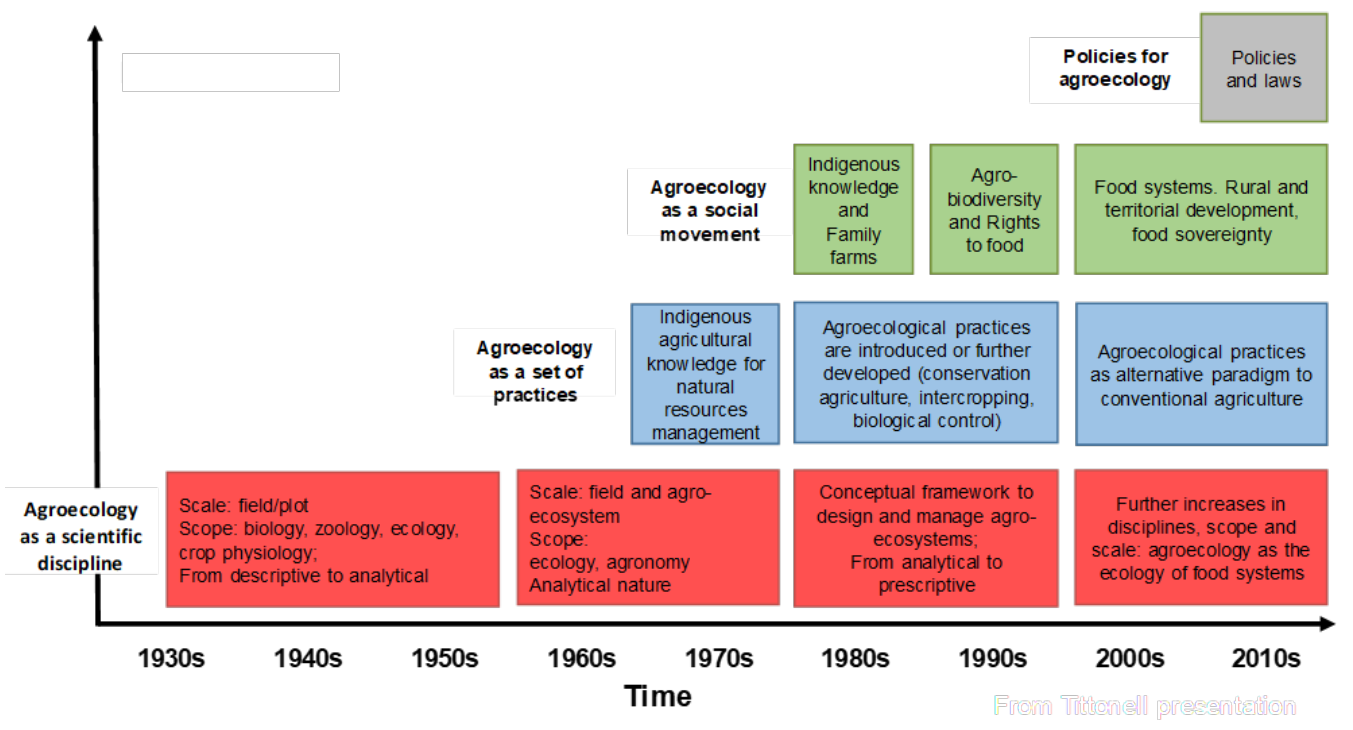

Figure 16. Historical development of Agroecology (Adapted from Wezel et al. 2020) 


\section{References}

Barrios, E., Coutinho, H.L. \& Medeiros C.A. 2012a. InPaC-S: Participatory Knowledge Integration on Indicators of Soil Quality - Methodological Guide. ICRAF, Embrapa, CIAT. Nairobi, 178 p. (also available at: http://www.worldagroforestry.org/downloads/publications/PDFs/B17459.PDF)

Barrios, E., Sileshi, G.W., Shepherd, K. \& Sinclair, F. 2012b. Agroforestry and soil health: Linking trees, soil biota and ecosystem services, pp. 315-330. In Wall, D.H., Bardgett, R.D., Behan-Pelletier, V., Herrick, J.E., Jones, T.H., Ritz, K., Six, J., Strong, D.R., van der Putten, W. (Eds.) Soil Ecology and Ecosystem Services. Oxford University Press, Oxford.

https://www.researchgate.net/publication/232053107_Agroforestry_and_Soil_Health_Linking_Trees_Soil_ Biota_and_Ecosystem_Services

Barrios, E., Gemmill-Herren, B., Bicksler A., Siliprandi, E., Brathwaite, R., Moller, S., Batello, C. \& Tittonell, P. 2020. The 10 Elements of Agroecology: Enabling transitions towards sustainable agriculture and food systems through visual narratives. Ecosystems and People, 16(1): 230-247.

https://doi.org/10.1080/26395916.2020.1808705

Blaser, W., Oppong, J., Hart, S., Landolt, J., Yeboah, E. \& Six, J. 2018. Climate-smart sustainable agriculture in low-to-intermediate shade agroforests. Nature Sustainability, 1: 234.

https://doi.org/10.1038/s41893-018-0062-8

Bolinder M.A., Crotty F., Elsen A., Frac M., KismányokyT., Lipiec J., Tits M., Tóth Z. \& Kätterer T. 2020. The effect of crop residues, cover crops, manures and nitrogen fertilization on soil organic carbon changes in agroecosystems: a synthesis of reviews. Mitigation and Adaptation Strategies for Global Change, 25: 929-952. https://doi.org/10.1007/s11027-020-09916-3

Bowles, T.M., Jackson, L.E., Loeher, M. \& Cavagnaro, T.R. 2017. Ecological intensification and arbuscular mycorrhizas: a meta-analysis of tillage and cover crop effects. Journal of Applied Ecology, 54: 1785-1793. https://doi.org/10.1111/1365-2664.12815

Cardinael, R., Chevallier, T., Cambou, A., Beral, C., Barthes, B.G., Dupraz, C., Durand, C., Kouakoua, E. \& Chenu, C. 2017. Increased soil organic carbon stocks under agroforestry: A survey of six different sites in France. Agriculture, Ecosystems \& Environment, 236: 243-255.

https://doi.org/10.1016/j.agee.2016.12.011

Chatterjee, N., Nair, P.K.R., Chakraborty, S. \& Nair, V.D. 2018. Changes in soil carbon stocks across the Forest-Agroforest-Agriculture/Pasture continuum in various agroecological regions: A meta-analysis. Agriculture, Ecosystems and Environment, 266: 55-67. https://doi.org/10.1016/j.agee.2018.07.014

Corbeels, M., Cardinael, R., Naudin, K., Guibert, H. \& Torquebiau, E. 2019. The 4 per 1000 goal and soil carbon storage under agroforestry and conservation agriculture systems in sub-Saharan Africa. Soil Tillage Research, 188: 16-26. https://doi.org/10.1016/j.still.2018.02.015 
Duchene, O., Vian, J.F. \& Celette, F. 2017. Intercropping with legume for agroecological cropping systems: Complementarity and facilitation processes and the importance of soil microorganisms. A review. Agriculture, Ecosystems \& Environment, 240: 148-161. https://doi.org/10.1016/j.agee.2017.02.019

FAO. 2018a. The 10 Elements of Agroecology: Guiding the transition to sustainable food and agricultural systems. Food and Agriculture Organization of the United Nations, Rome, 12 pp. (also available at: http://www.fao.org/3/i9037en/i9037en.pdf)

FAO. 2018b. Global review of the Farmer Field School. Wageningen University-FAO Working Paper. 116 p.

FAO. 2019. The Ten Elements of Agroecology. FAO Council, CL 163/13 Rev.1. Food and Agriculture Organization of the United Nations, Rome. (also available at: http://www.fao.org/3/ca7173en/ca7173en.pdf)

Fonte, S.J., Barrios, E. \& Six, J. 2010. Earthworms, soil fertility and aggregate-associated soil organic matter dynamics in the Quesungual agroforestry system. Geoderma, 155: 320-328.

https://doi.org/10.1016/j.geoderma.2009.12.016

Haddaway N.R., Hedlund K., Jackson L.E., Katterer T., Lugato E., Thomsen I.K., Jorgensen H.B. \& Isberg P-E. 2017. How does tillage intensity affect soil organic carbon? A systematic review. Environmental Evidence, 6: 30. https://doi.org/10.1186/s13750-017-0108-9

HLPE. 2019. Agroecological and other innovative approaches for sustainable agriculture and food systems that enhance food security and nutrition. A report by the High Level Panel of Experts on Food Security and Nutrition of the Committee on World Food Security, Rome. (also available at: http://www.fao.org/3/ca5602en/ca5602en.pdf)

Kamau, S., Barrios, E., Karanja, N., Ayuke, F. \& Lehmann, J. 2017. Soil macrofauna under dominant tree species increases along a soil degradation gradient. Soil Biology and Biochemistry, 112: 35-46.

https://doi.org/10.1016/j.soilbio.2017.04.016

Kamau, S., Barrios, E., Karanja, N., Ayuke, F. \& Lehmann, J. 2020. Dominant tree species and soil earthworms affect soil aggregation and carbon content along a soil degradation gradient in an agricultural landscape. Geoderma, 359. https://doi.org/10.1016/j.geoderma.2019.113983

Kumar S., Meena R.S., Lal R., Yadav G., Mitran T., Meena B.L., Dotaniya M.L. \& EL-Sabagh A. 2018. The role of legumes in soil carbon sequestration. In Meena R., Das A., Yadav G., Lal R. (Eds.) Legumes for Soil Health and Sustainable Management. Springer, Singapore. https://doi.org/10.1007/978-981-13-0253-4_4.

Leippert, F., Damaun, M., Bernoux, M. \& Mpheshea, M. 2020. The potential of Agroecology to build climateresilient livelihoods and food systems. Rome. FAO and Biovision. https://doi.org/10.4060/cb0438en

Mbow, C., Smith, P., Skole, D., Duguma, L. \& Bustamante, M. 2014. Achieving mitigation and adaptation to climate change through sustainable agroforestry practices. Current Opinion on Environmental Sustainability, 6 :

8-14. https://doi.org/10.1016/j.cosust.2013.09.002 
Muchane, M.N., Sileshi, G.W., Gripenberg, S., Jonsson, M., Pumariño, L. \& Barrios, E. 2020. Agroforestry boosts soil health in the humid and sub-humid tropics: A meta-analysis. Agriculture, Ecosystems and Environment, 295: 106899. https://doi.org/10.1016/j.agee.2020.106899

Ogle, S.M., Olander, L., Wollenberg, L., Rosenstock, T., Tubiello, F., Paustian, K., Buuendia, L., Nihart. A. \& Smith, P. 2014. Reducing greenhouse gas emissions and adapting agricultural management for climate change in developing countries: providing the basis for action. Global Change Biology, 20(1-6): 1-6.

https://doi.org/10.1111/gcb.12361

Otsuka, K. \& Place, P. 2014. Changes in land tenure and agricultural intensification in sub-Saharan Africa. WIDER Working Paper 2014/051, pp. 18. World Institute for Development Economics Research (WIDER) at United Nations University (UNU). https://www.wider.unu.edu/sites/default/files/wp2014-051.pdf

Pauli, N., Barrios, E., Conacher, A.J. \& Oberthur, T. 2011. Soil macrofauna in agricultural landscapes dominated by the Quesungual Slash-and-Mulch Agroforestry System, western Honduras. Applied Soil Ecology, 47: 119-132. https://doi.org/10.1016/j.apsoil.2010.11.005

Paustian K., Larson E., Kent J., Marx E. \& Swan A. 2019. Soil C sequestration as a biological negative emission strategy. Frontiers in Climate, 1: 8. https://doi.org/10.3389/fclim.2019.00008

Prabhu, R., Barrios, E., Bayala, J., Diby, L., Donovan, J., Gyau, A., Graudal, L., Jamnadass, R., Kahia, J., Kehlenbeck, K., Kindt, R., Kouame, C., McMullin, S., van Noordwijk, M., Shepherd, K., Sinclair, F., Vaast, P., Vågen, T.-G. \& Xu, J. 2015. Agroforestry: realizing the promise of an agroecological approach. In Agroecology for Food Security and Nutrition: Proceedings of the FAO International Symposium, pp. 201-224, Rome. http://www.fao.org/3/i4729e/i4729e.pdf

Sainju, U.M. 2016. A global meta-analysis on the impact of management practices on net global warming potential and greenhouse gas intensity from cropland soils. PLoS ONE, 11(2): e0148527.

https://doi.org/10.1371/journal.pone.0148527

Sanchez, P.A. 2019. Properties and Management of Soils in the Tropics, Second edition. Cambridge University Press, UK. 666 p.

Sileshi, G., Akinnifesi F.K., Ayayi, O.C. \& Place, F. 2008. Meta-analysis of maize yield response to woody and herbaceous legumes in sub-Saharan Africa. Plant and Soil, 307: 1-19. https://doi.org/10.1007/s11104-008$9547-y$

Six J., Feller C., Denef K., Ogle S.M., Moraes Sa J.C. \& Albrecht A. 2002. Soil organic matter, biota and aggregation in temperate and tropical soils - Effects of no-tillage. Agronomy, 22: 755-775.

https://doi.org/10.1051/agro:2002043

Tamburini, G., Bommarco, R., Wanger, T.C., Kremen, C., Heijden, M.G.A. van der, Liebman, M. \& Hallin, S. 2020 . Agricultural diversification promotes multiple ecosystem services without compromising yield. Science Advances, 6(45): eaba1715. https://doi.org/10.1126/sciadv.aba1715

van der Ploeg, J.D., Barjolle, D., Bruil, J., Brunori, G., Costa Madureira, L.M., Dessein, J., Drąg, Z., FinkKessler, A., Gasselin, P., Gonzalez de Molina, M., Gorlach, K., Jürgens, K., Kinsella, J., Kirwan, J., 
Knickel, K., Lucas, V., Marsden, T., Maye, D., Migliorini, P., Milone, P., Noe, E., Nowak, P., Parrott, N., Peeters, A., Rossi, A., Schermer, M., Ventura, F., Visser, M. \& Wezel, A. 2019. The economic potential of agroecology: Empirical evidence from Europe. Journal of Rural Studies, 71: 46-61.

https://doi.org/10.1016/j.jrurstud.2019.09.003

Vinceti, B., Termote, C., Ickowitz, A., Powell, B., Kehlenbeck, K. \& Hunter, D. 2013. Strengthening the contribution of forests and trees to sustainable diets: challenges and opportunities. Sustainability, 5: 4797-4824. https://doi.org/10.3390/su5114797

Wartenberg, A.C., Blaser, W.J., Janudianto, K.N., Roshetko, J.M., van Noordwijk, M. \& Six, J. 2018. Farmer perceptions of plant-soil interactions can affect adoption of sustainable management practices in cocoa agroforests: a case study from Southeast Sulawesi. Ecology and Society, 23(1): 18 https://doi.org/10.5751/ES09921-230118

Wezel, A., Gemmill-Herren, B., Bezner-Kerr, R., Barrios, E., Gonçalves, A.L.R. \& Sinclair, F. 2020. Agroecological principles and elements and their implications for transitioning to sustainable food systems. A review. Agronomy for Sustainable Development, 40: 40. https://doi.org/10.1007/s13593-020-00646-Z

Zhao, J., Yang, Y., Zhang, K., Jeong, J., Zeng, Z. \& Zang, H. 2020. Does crop rotation yield more in China? A meta-analysis. Field Crops Research, 245: 107659. https://doi.org/10.1016/j.fcr.2019.107659 


\title{
46. Climate-smart agriculture
}

\author{
Wei Ren, Yawen Huang, Stefani Daryanto, Bo Tao \\ Department of Plant \& Soil Sciences, College of Agriculture, Food, and Environment, University of Kentucky, \\ Lexington, United States of America.
}

\section{Description}

Climate-smart agriculture (CSA) is defined as agricultural systems that aim to increase agricultural productivity and food security in the face of climate change, enhance adaptive capacity at multiple levels, and mitigate climate change impacts where possible (Campbell et al., 2014). Broadly, CSA is not a new set of practices but rather an integrated approach aiming to 1) increase crop productivity, 2) build climate-resilient food production systems, 3) reduce environmental impacts such as greenhouse gas (GHG) emissions and nutrient leaching, and 4) improve soil carbon sequestration. Locally accepted solutions are expected to consider trade-offs between these CSA objectives at multiple scales from local to global, and over various time horizons from short-term to long-term (Lipper et al., 2014).

CSA practices may involve one or more systems described in this volume (e.g. the combination of integrated crop, livestock, and agroforestry). CSA practices also include improved pest, water, and nutrient management; landscape approaches; improved pasture management (Factsheet No.34, this volume) and forestry management (Volume 5); practices such as reduced tillage and use of diverse varieties and breeds; integrating trees into agricultural systems (Factsheets 38 to 40, this volume); restoring degraded lands (e.g. grassland, Factsheet No.32, this volume; forests, Factsheet No.6, volume 5; peatland, Factsheet No.12, volume 5); improving the efficiency of water and nitrogen fertilizer use (Section 1.4.7); and manure management, including the use of anaerobic bio-digesters (Factsheet No. 11, this volume), etc. (Lipper et al., 2014).

The effectiveness of CSA practices for enhancing soil carbon sequestration weighs markedly on its ability to simultaneously increase carbon inputs and reduce carbon outputs. For example, no-tillage, conservation tillage, reduced or minimum tillage, and superficial tillage all lower the extent of soil disturbance and provides soil cover, and therefore minimize soil erosion and soil organic matter decomposition rate compared to conventional tillage. Cover crops offer biomass inputs, promote soil aggregation and structure, increase carbon sources, and reduce potential carbon loss due to soil erosion. Biochar amendment also improves soil aggregation and physical protection 
of aggregate-associated soil organic carbon (SOC) against microbial attack. More importantly, biochar directly increases the recalcitrant organic carbon pool and further reduces the SOC decomposition rate.

\section{Range of applicability}

Of the different CSA practices, limited tillage (i.e. no-tillage, conservation tillage, reduced or minimum tillage, superficial tillage), cover crop, and biochar amendment are considered the most beneficial for SOC accumulation (Bai et al., 2019). The reduction in frequency and depth of tillage has been applied to preserve soil moisture and prevent soil erosion and degradation. The use of cover crops is not a new concept, and there is a long history of research documenting its benefit for agriculture and the environment. Cover crop is generally applicable for all croplands requiring seasonal vegetative cover for natural resource protection or improvement. Similarly, the application of biochar is potentially suitable for all farmlands. However, the suitability of biochar application for sloping cropland is not apparent, considering the preferential erosion of biochar due to its fine size and light mass (Kuppusamy et al., 2016). More information on reduced tillage practices, cover crops, and biochar can be found in Chapter 1 of this manual.

\section{Impact on carbon sequestration / Potential of additional}

\section{storage}

Biochar applications can sequester about $6.39 \pm 4.54 \mathrm{tC} / \mathrm{ha} / \mathrm{yr}(0-15 \mathrm{~cm})$ and $14.01 \pm 20.37 \mathrm{tC} / \mathrm{ha} / \mathrm{yr}(0-20 \mathrm{~cm})$ of SOC, respectively, compared to no-biochar application (Table 200). Compared to conservation tillage and cover crop, biochar sequestered most carbon, mainly due to the nature of biochar amendments as a direct source of recalcitrant carbon input. The sequestration rate largely depends on the amount of biochar applied, which typically ranges from $2 \mathrm{t} / \mathrm{hato} 97 \mathrm{t} / \mathrm{h}$. Different sources of biochar could also affect the carbon sequestration rate. However, the long-term effect of biochar application on soil carbon sequestration is unclear given the experiments' short-term nature.

The addition of cover crop to the non-cover crop system can provide an additional SOC of about $0.06 \pm 0.52 \mathrm{tC} / \mathrm{ha} / \mathrm{yr}(0-10-\mathrm{cm})$ and $0.68 \pm 0.87 \mathrm{tC} / \mathrm{ha} / \mathrm{yr}(0-30-\mathrm{cm})$ (Table 200$)$. The sequestration rate tends to increase as the duration of cover crop increases, consistent with a report showing that soil carbon sequestration increases with time even up to 54 years after cover crop introduction (Poeplau and Don, 2015). Because a large

portion of the $\mathrm{C}$ inputs from the cover crop is added as roots, they contribute more effectively to the relatively stable C pool than other organic amendments or above ground C inputs (Kätterer et al., 2011). 
Limited tillage practices can sequester about $0.54 \pm 1.19 \mathrm{tC} / \mathrm{ha} / \mathrm{yr}, 0.62 \pm 2.46 \mathrm{tC} / \mathrm{ha} / \mathrm{yr}$ and $0.5 \pm 1.69 \mathrm{tC} / \mathrm{ha} / \mathrm{yr}$ of $\mathrm{C}$ to the $10-\mathrm{cm}, 30-\mathrm{cm}$, and $60-\mathrm{cm}$ soil depth, respectively, compared to conventional tillage (Table 200). The higher sequestration potential of the top $30 \mathrm{~cm}$ soil than the deeper soil reflects a surface stratification effect of the practice (Skaalsveen, Ingram and Clarke, 2019).

Table 200. Global evolution of SOC stocks after application of reduced tillage, cover crop, and biochar as an example of CSA Practices

\begin{tabular}{|c|c|c|c|c|c|c|}
\hline Location & Practice & $\begin{array}{l}\text { Climate } \\
\text { zone }\end{array}$ & $\begin{array}{l}\text { Soil } \\
\text { depth }\end{array}$ & $\begin{array}{l}\text { Additional C } \\
\text { storage } \\
(\mathrm{tC} / \mathrm{ha} / \mathrm{yr}) \pm \\
\text { Standard } \\
\text { Deviation }\end{array}$ & Duration & More information \\
\hline \multirow{17}{*}{ Global } & \multirow{17}{*}{$\begin{array}{l}\text { Limited } \\
\text { tillage } \\
\text { practices }\end{array}$} & Overall & \multirow{6}{*}{$0 \sim 10 \mathrm{~cm}$} & $0.54 \pm 1.19$ & Overall & \multirow{17}{*}{$\begin{array}{l}\text { A baseline is considered } \\
\text { the business as usual } \\
\text { practice, which is } \\
\text { conventional tillage. }\end{array}$} \\
\hline & & Arid & & $0.65 \pm 1.53$ & & \\
\hline & & Humid & & $0.48 \pm 1.02$ & & \\
\hline & & & & $1.31 \pm 2.12$ & Short & \\
\hline & & & & $0.34 \pm 0.53$ & Medium & \\
\hline & & & & $0.21 \pm 0.25$ & Long & \\
\hline & & Overall & \multirow{6}{*}{$0 \sim 30 \mathrm{~cm}$} & $0.62 \pm 2.46$ & Overall & \\
\hline & & Arid & & $0.61 \pm 1.83$ & & \\
\hline & & Humid & & $0.53 \pm 2.56$ & & \\
\hline & & & & $1.26 \pm 4.55$ & Short & \\
\hline & & & & $0.47 \pm 1$ & Medium & \\
\hline & & & & $0.2 \pm 0.46$ & Long & \\
\hline & & Overall & \multirow{5}{*}{$0 \sim 60 \mathrm{~cm}$} & $0.5 \pm 1.69$ & Overall & \\
\hline & & Arid & & $0.63 \pm 1.01$ & & \\
\hline & & Humid & & $0.46 \pm 1.87$ & & \\
\hline & & & & $0.06 \pm 2.6$ & Short & \\
\hline & & & & $0.83 \pm 1.43$ & Medium & \\
\hline
\end{tabular}




\begin{tabular}{|c|c|c|c|c|c|c|}
\hline Location & Practice & $\begin{array}{l}\text { Climate } \\
\text { zone }\end{array}$ & $\begin{array}{l}\text { Soil } \\
\text { depth }\end{array}$ & $\begin{array}{l}\text { Additional C } \\
\text { storage } \\
(\mathrm{tC} / \mathrm{ha} / \mathrm{yr}) \pm \\
\text { Standard } \\
\text { Deviation }\end{array}$ & Duration & More information \\
\hline & & & & $0.11 \pm 0.4$ & Long & \\
\hline & \multirow{8}{*}{ Cover crop } & Humid & \multirow{3}{*}{$0 \sim 10 \mathrm{~cm}$} & $0.06 \pm 0.52$ & Overall & \multirow{8}{*}{$\begin{array}{l}\text { A baseline is considered } \\
\text { the business as usual } \\
\text { practice, which does no } \\
\text { include cover cropping. }\end{array}$} \\
\hline & & & & $0.18 \pm 0.37$ & Medium & \\
\hline & & & & $0.01 \pm 0.57$ & Short & \\
\hline & & Overall & \multirow{5}{*}{$0 \sim 30 \mathrm{~cm}$} & $0.68 \pm 0.87$ & Overall & \\
\hline & & Arid & & $1 \pm 0.87$ & & \\
\hline & & Humid & & $0.57 \pm 0.79$ & & \\
\hline & & & & $0.98 \pm 1.07$ & Medium & \\
\hline & & & & $0.6 \pm 0.79$ & Short & \\
\hline & \multirow{6}{*}{ Biochar } & Overall & \multirow{3}{*}{$0 \sim 15 \mathrm{~cm}$} & $6.39 \pm 4.54$ & Short & \multirow{6}{*}{$\begin{array}{l}\text { A baseline is considered } \\
\text { the business as usual } \\
\text { practice, which does not } \\
\text { include biochar } \\
\text { application }\end{array}$} \\
\hline & & Arid & & $4.19 \pm 1.78$ & Short & \\
\hline & & Humid & & $6.92 \pm 4.76$ & Short & \\
\hline & & Overall & \multirow{3}{*}{$0 \sim 20 \mathrm{~cm}$} & $14.01 \pm 20.37$ & Short & \\
\hline & & Arid & & $3.19 \pm 1.5$ & Short & \\
\hline & & Humid & & $3.06 \pm 2.06$ & Short & \\
\hline
\end{tabular}

The experiment durations were grouped into short-term ( $\leq 5$ years), medium-term (6-20 years), and long-term ( $\geq 20$ years). "Overall" means all the available data for analysis without considering their climate type or duration.

When the dataset in a climate zone has mixed durations, the duration column is left blank.

When the dataset in a duration category contains both climate types, the climate column is left blank.

Data were adapted from Bai et al. (2019) and re-calculated where SOC mass value is available.

Only observations of SOC stock to the same soil depths were selected. 


\title{
4. Other benefits of the practice
}

\subsection{Minimization of threats to soil functions}

\author{
Table 201. Soil threats
}

\begin{tabular}{|c|c|}
\hline \multicolumn{2}{|l|}{ Soil threats } \\
\hline Soil erosion & $\begin{array}{l}\text { In clay-rich soils and soils prone to surface crusting, cover crops can provide } \\
\text { immediate reduction of soil and water loss, compared to no-tillage alone } \\
\text { (Lanzanova et al., 2013). }\end{array}$ \\
\hline $\begin{array}{l}\text { Nutrient } \\
\text { imbalance and } \\
\text { cycles }\end{array}$ & $\begin{array}{l}\text { As conservation tillage, cover crop, and biochar add organic matter to the soil, } \\
\text { nutrient cycles are usually supported with all practices. }\end{array}$ \\
\hline $\begin{array}{l}\text { Soil salinization } \\
\text { and alkalinization }\end{array}$ & $\begin{array}{l}\text { Biochar produced at low temperatures }\left(300^{\circ} \mathrm{C} \text { ) might correct the alkalinity }\right. \\
\text { problems (Kuppusamy et al., 2016). }\end{array}$ \\
\hline $\begin{array}{l}\text { Soil } \\
\text { contamination } \\
\text { /pollution }\end{array}$ & $\begin{array}{l}\text { The amendment of contaminated soils with biochar enables the sorption of both } \\
\text { organic and inorganic contaminants and reduces their mobility. }\end{array}$ \\
\hline Soil acidification & $\begin{array}{l}\text { The use of lime is common to minimize acidification due to conservation tillage } \\
\text { (Caires et al., 2008). }\end{array}$ \\
\hline $\begin{array}{l}\text { Soil biodiversity } \\
\text { loss }\end{array}$ & $\begin{array}{l}\text { Residue accumulation associated with CSA practices prevents soil biodiversity loss } \\
\text { by forming the base of the food chain (microbial diversity), and cascading upward } \\
\text { to higher organisms such as soil mesofauna (e.g. collembola) and macrofauna (e.g. } \\
\text { earthworm) (Holland, 2004). }\end{array}$ \\
\hline Soil sealing & $\begin{array}{l}\text { Crop residues reduce surface sealing. Some cover crop species (i.e. those with large } \\
\text { taproot diameter) allow the development of macropores, act as 'natural' tillage, } \\
\text { and improve aggregate stability (Chen and Weil, 2010). }\end{array}$ \\
\hline Soil compaction & $\begin{array}{l}\text { Higher soil moisture content combined with lower near-surface bulk density } \\
\text { indicated that cover crops could reduce the susceptibility of near-surface soils to } \\
\text { compaction (Daryanto et al., 2018). }\end{array}$ \\
\hline $\begin{array}{l}\text { Soil water } \\
\text { management }\end{array}$ & $\begin{array}{l}\text { The porous, low-density carbon-rich biochar and the dead biomass accumulation } \\
\text { with conservation tillage or cover crop practices generally retain and/or replenish } \\
\text { topsoil moisture (Mukherjee and Lal, 2013; Daryanto et al., 2018). }\end{array}$ \\
\hline
\end{tabular}




\section{2 (Increases in production (e.g. food/fuel/feed/timber/fibre)}

The capacity to increase crop yield through cover crops (compared to without cover crops) depends on cover crop species and the level of nutrient input made to the subsequent cash crop. Leguminous cover crops generate higher yield increase than non-leguminous cover crops. Cash crops with zero $\mathrm{N}$ have a higher yield increase than those with fertilizer N added (Daryanto et al., 2018). Legume cover crops could, therefore, be a promising strategy for lowinput agriculture (i.e. reducing the amount of synthetic fertilizer and replacing it with cover crop biomass).

No-tillage systems consistently perform better than conventional tillage for rain-fed crops in a dry climate (Huang et al., 2018; Pittelkow et al., 2015), mainly due to surface residues that help prevent erosion and increase water use efficiency. Although in humid climate/conditions, the differences are more variable, the positive effects of no-tillage on soil properties (e.g. bulk density, penetration resistance, water aggregate stability) can accumulate with notillage duration (Blanco-Canqui and Ruis, 2018). Thus, we can expect a sustained yield over time (Pittelkow et al., 2015).

\subsection{Mitigation of and adaptation to climate change}

The emissions of $\mathrm{CO}_{2}, \mathrm{CH}_{4}$, and $\mathrm{N}_{2} \mathrm{O}$ from biochar-amended soil can be inconsistent as influenced by soil physical properties (e.g. moisture content, aeration, porosity, organic matter content) and biotic (microbial response) or abiotic (mineralization or decomposition of soil organic matter) processes. However, we can expect lower GHGs suppression over time, despite an initial increase in their flux (Mukherjee and Lal, 2013). Similarly, large spatiotemporal variations exist in the capacity of conservation tillage in reducing GHG emissions. For example, notillage can significantly decrease $\mathrm{CO}_{2}$ emissions in dry climates and reduce $\mathrm{CH}_{4}$ emissions in humid climates (Huang et al., 2018).

\subsection{Socio-economic benefits}

Economic (productivity) benefits of conservation agriculture are the determining factors of its adoption. Generally, practices associated with big investments and long-term returns have relatively lower adoption rates in both developed and developing countries (Giller et al., 2011; Rochecouste et al., 2015). 


\section{Potential drawbacks to the practice}

\subsection{Tradeoffs with other threats to soil functions}

\section{Table 202. Soil threats}

\begin{tabular}{|c|c|}
\hline Soil threats & \\
\hline $\begin{array}{l}\text { Nutrient imbalance and } \\
\text { cycles }\end{array}$ & $\begin{array}{l}\text { Biochar can alter the bioavailability of soil nutrients due to its ability to } \\
\text { change soil pH and its absorbent nature (Kuppusamy et al., 2016). }\end{array}$ \\
\hline $\begin{array}{l}\text { Soil salinization and } \\
\text { alkalinization }\end{array}$ & $\begin{array}{l}\text { Biochar produced at high temperatures }\left(700^{\circ} \mathrm{C}\right) \text { is alkaline (Kuppusamy et } \\
\text { al., 2016). }\end{array}$ \\
\hline $\begin{array}{l}\text { Soil } \\
\text { contamination/pollution }\end{array}$ & $\begin{array}{l}\text { Acidified soil that results from long-term conservation tillage can mobilize } \\
\text { some heavy metals such as } \mathrm{Al}^{3+} \text { (Caires et al., 2008). }\end{array}$ \\
\hline Soil acidification & $\begin{array}{l}\text { Under conservation tillage, acidification can occur with the lack of soil } \\
\text { mixing and can be exacerbated with heavy fertilizer use. }\end{array}$ \\
\hline Soil biodiversity loss & $\begin{array}{l}\text { Shifts of soil biodiversity may occur (e.g. with changes in } \mathrm{pH} \text { or other soil } \\
\text { properties) rather than loss. }\end{array}$ \\
\hline Soil compaction & $\begin{array}{l}\text { Soil compaction is a widespread and common problem associated with } \\
\text { conservation tillage at initial years of adoption. }\end{array}$ \\
\hline Soil water management & $\begin{array}{l}\text { Elevated evapotranspiration with cover crops, particularly in the drylands, } \\
\text { can decrease water content in the deep soil }(>60 \mathrm{~cm} \text { ) (Daryanto et al., } \\
\text { 2018). }\end{array}$ \\
\hline
\end{tabular}

\subsection{Increases in greenhouse gas emissions}

Previous studies indicated that GHG emission increases with no-tillage and cover crop practice, although the total GHG emissions per unit yield might be lower if there is a yield increase (Holland, 2004; Daryanto et al., 2018). An exception will be the total GHG emissions of submerged rice cultivation, which increases by $400 \%$ on an annual basis if the cover crop is used (Haque et al, 2015). No-tillage can increase $\mathrm{N}_{2} \mathrm{O}$ emission in humid conditions but may reduce $\mathrm{N}_{2} \mathrm{O}$ emission if we apply subsurface $\mathrm{N}$ fertilizer (Huang et al., 2018). There is a substantial decrease in 
$\mathrm{CO}_{2}$ emissions in no-tillage systems, especially in dry climates. But such reduction could diminish with time as the no-tillage systems reach new equilibrium status of the soil carbon cycle (Huang et al., 2018).

\subsection{Conflict with other practice(s)}

A combination of tillage and freshly-killed plant material during pulses of high rainfall may accelerate the oxidation of the more stable, old SOC compounds (Poeplau and Don, 2015), a process commonly known as "priming effect". Materials producing biochar may have other uses or fates, and the biochar-making processes may also generate $\mathrm{CO}_{2}$.

\subsection{Decreases in production (e.g. food/fuel/feed/timber/fibre)}

A global meta-analysis study suggested that no-tillage reduces the yield of most crops during the first two years after its implementation. During the next 3-10 years, the yield of no-tilled crops can gradually match that of conventionally tilled, except for wheat and maize in the humid climate. The extent of yield reduction with no-tillage can also be minimized with the addition of $\mathrm{N}$ fertilizer (Pittelkow et al., 2015).

\subsection{Other conflicts}

Resource-poor farmers often must face several competing uses for their crop residues (e.g., mulch, livestock feed, fuel, or construction) (Giller et al., 2011).

\section{Recommendations before implementing the practice}

With the adoption of CSA practices such as biochar, cover crop, and conservation tillage, croplands could sequester more carbon. However, the capacity of each practice to sequester carbon varies. Local environment (e.g. climate and soil conditions) and the combination with other management practices should be considered when identifying the appropriate CSA practices for mitigating GHG emissions and ensuring crop productivity (Bai et al., 2019). For example, no-tillage in humid regions prefers well-drained soil conditions (Skaalsveen, Ingram and Clarke, 2019). Cover crops prevent topsoil compaction and provide continuing surface cover as living biomass and dead residues, therefore serve as a good option in a conservation tillage system. In the regions where soil moisture is a concern, early cover crop termination (i.e. one month before senescence) is recommended to offset potential deep soil moisture loss without losing cover crop biomass (Islam et al, 2006). Using cover crops with low $\mathrm{C} / \mathrm{N}$ ratios (i.e. legume) and practicing intermittent, instead of continuous flooding, is recommended to reduce methane emission with minimum impact on yield (Haque et al., 2017). When it comes to biochar application, one should carefully consider the appropriate rate and method (e.g. topsoil incorporation, deep use, top-dressing) to minimize cost and to avoid loss from water and wind (El-Naggar et al., 2019). 


\section{Potential barriers to adoption}

Table 203. Potential barriers to adoption

\begin{tabular}{|c|c|c|}
\hline Barrier & YES/NO & \\
\hline Biophysical & Yes & $\begin{array}{l}\text { Despite the ability of CSA practices to reduce soil erosion, it should be noted } \\
\text { that their effects remain limited in areas with steep declivity (>7 percent) } \\
\text { (Edwards and Burney, 2007) }\end{array}$ \\
\hline Cultural & Yes & $\begin{array}{l}\text { Conservation agriculture is a knowledge-intensive enterprise. Promoting its } \\
\text { adoption requires the incorporation of short-term successes to meet farmers' } \\
\text { immediate needs while contributing to long-term ecological sustainability } \\
\text { (Halbrendt et al., 2014). }\end{array}$ \\
\hline Social & Yes & $\begin{array}{l}\text { Most consumers are likely unaware of the environmental benefits of } \\
\text { conservation farming practices and unlikely to challenge traditional models } \\
\text { of food consumption (Klinglmayr et al., 2017). }\end{array}$ \\
\hline Economic & Yes & $\begin{array}{l}\text { The inclusion of cover crops and biochar adds to the production costs } \\
\text { (Kuppusamy et al., 2016; Daryanto et al., 2019). }\end{array}$ \\
\hline Knowledge & Yes & $\begin{array}{l}\text { There are knowledge gaps that need to be addressed, and uncertainties } \\
\text { associated with biochar and cover crop practices can hinder their adoption } \\
\text { (Kuppusamy et al., 2016; Daryanto et al., 2019). }\end{array}$ \\
\hline
\end{tabular}




\section{References}

Bai, X., Huang, Y., Ren, W., Coyne, M., Jacinthe, P.A., Tao, B., Hui, D., Yang, J. \& Matocha, C. 2019. Responses of soil carbon sequestration to climate-smart agriculture practices: A meta-analysis. Global Change Biology, 25(8): 2591-2606. https://doi.org/10.1111/gcb.14658

Blanco-Canqui, H. \& Ruis, S.J. 2018. No-tillage and soil physical environment. Geoderma, 326: 164-200. https://doi.org/10.1016/j.geoderma.2018.03.011

Campbell, B.M., Thornton, P., Zougmoré, R., Asten, P.V. \& Lipper, L. 2014. Sustainable intensification: What is its role in climate smart agriculture? Current Opinion in Environmental Sustainability, 8(8): 39-43. https://doi.org/10.1016/j.cosust.2014.07.002

Caires, E.F., Garbuio, F.J., Churka, S., Barth, G. \& Corrèa, J.C.L. 2008. Effects of soil acidity amelioration by surface liming on no-till corn, soybean, and wheat root growth and yield. European Journal of Agronomy, 28(1): 57-64. https://doi.org/10.1016/j.eja.2007.05.002

Chen, G. \& Weil, R.R. 2010. Penetration of cover crop roots through compacted soils. Plant and Soil, 331(12): 31-43. https://doi.org/10.1007/s11104-009-0223-7

Daryanto, S., Fu, B., Wang, L., Jacinthe, P.A. \& Zhao, W. 2018. Quantitative synthesis on the ecosystem services of cover crops. Earth-Science Reviews, 185: 357-373.

https://doi.org/10.1016/j.earscirev.2018.06.013

Daryanto, S., Jacinthe, P.A., Fu, B., Zhao, W. \& Wang, L. 2019. Valuing the ecosystem services of cover crops: barriers and pathways forward. Agriculture, ecosystems \& environment, 270: 76-78.

https://doi.org/10.1016/j.agee.2018.10.021

Edwards, L. \& Burney, J. 2007. Effect of cover crop seeding rate and date on cool period soil erosion control in potato production in PEI, Canada. Canadian Journal of Plant Science, 87(4): 851-854.

https://doi.org/10.4141/CJPS06039

El-Naggar, A., Lee, S.S., Rinklebe, J., Farooq, M., Song, H., Sarmah, A.K., Zimmerman, A.R., Ahmad, M., Shaheen, S.M. \& Ok, Y.S. 2019. Biochar application to low fertility soils: a review of current status, and future prospects. Geoderma, 337: 536-554. https://doi.org/10.1016/j.geoderma.2018.09.034

Giller, K.E., Corbeels, M., Nyamangara, J., Triomphe, B., Affholder, F., Scopel, E. \& Tittonell, P. 2011. A research agenda to explore the role of conservation agriculture in African smallholder farming systems. Field Crops Research, 124(3): 468-472. https://doi.org/10.1016/j.fcr.2011.04.010

Halbrendt, J., Gray, S.A., Crow, S., Radovich, T., Kimura, A.H. \& Tamang, B.B. 2014. Differences in farmer and expert beliefs and the perceived impacts of conservation agriculture. Global Environmental Change, 28: 50-62. https://doi.org/10.1016/j.gloenvcha.2014.05.001 
Haque, M.M., Kim, S.Y., Ali, M.A. \& Kim, P.J. 2015. Contribution of greenhouse gas emissions during cropping and fallow seasons on total global warming potential in mono-rice paddy soils. Plant and Soil, 387(1-2): 251-264. https://doi.org/10.1007/s11104-014-2287-2

Haque, M.M., Biswas, J.C., Kim, S.Y. \& Kim, P.J. 2017. Intermittent drainage in paddy soil: ecosystem carbon budget and global warming potential. Paddy and water environment, 15(2): 403-411.

https://doi.org/10.1007/s10333-016-0558-7

Holland, J.M. 2004. The environmental consequences of adopting conservation tillage in Europe: reviewing the evidence. Agriculture, Ecosystems \& Environment, 103(1): 1-25.

https://doi.org/10.1016/j.agee.2003.12.018

Huang, Y., Ren, W., Wang, L., Hui, D., Grove, J.H., Yang, X., Tao, B. \& Goff, B., 2018. Greenhouse gas emissions and crop yield in no-tillage systems: A meta-analysis. Agriculture, Ecosystems \& Environment, 268 : 144-153. https://doi.org/10.1016/j.agee.2018.09.002

Islam, N., Wallender, W.W., Mitchell, J., Wicks, S. \& Howitt, R.E. 2006. A comprehensive experimental study with mathematical modeling to investigate the effects of cropping practices on water balance variables. Agriculural Water Management, 82(1-2): 129-147. https://doi.org/10.1016/j.agwat.2005.07.029

Kätterer, T., Bolinder, M.A., Andrén, O., Kirchmann, H. \& Menichetti, L. 2011. Roots contribute more to refractory soil organic matter than above-ground crop residues, as revealed by a long-term field experiment. Agriculture, Ecosystems \& Environment, 141(1-2): 184-192. https://doi.org/10.1016/j.agee.2011.02.029

Klingłmayr, J., Bergmair, B., Klaffenböck, M.A., Hörmann, L. \& Pournaras, E. 2017. Sustainable consumerism via context-aware shopping. International Journal of Distributed Systems and Technologies, 8(4): 54-72.

Kuppusamy, S., Thavamani, P., Megharaj, M., Venkateswarlu, K. \& Naidu, R. 2016. Agronomic and remedial benefits and risks of applying biochar to soil: Current knowledge and future research directions. Environment International, 87: 1-12. https://doi.org/10.1016/j.envint.2015.10.018

Lanzanova, M.E., Eltz, F.L.F., Nicoloso, R.D.S., Cassol, E.A., Bertol, I., Amado, T.J.C. \& Girardello, V.C. 2013. Residual effect of soil tillage on water erosion from a Typic Paleudalf under long-term no-tillage and cropping systems. Revista Brasileira de Ciência do Solo, 37(6): 1689-1698. https://doi.org/10.1590/S010006832013000600025

Lipper, L., Thornton, P., Campbell, B.M., Baedeker, T., Braimoh, A., Bwalya, M., Caron, P., Cattaneo, A., Garrity, D., Henry, K., Hottle, R., Jackson, L., Jarvis, A., Kossam, F., Mann, W., McCarthy, N., Meybeck, A., Neufeldt, H., Remington, T., Sen, P.T., Sessa, R., Shula, R., Tibu, A. \& Torquebiau, E.F. 2014. Climate-smart agriculture for food security. Nature Climate Change, 4(12): 1068-1072.

https://doi.org/10.1038/nclimate2437

Mukherjee, A. \& Lal, R. 2013. Biochar impacts on soil physical properties and greenhouse gas emissions. Agronomy, 3(2): 313-339. https://doi.org/10.3390/agronomy3020313 
Pittelkow, C.M., Linquist, B.A., Lundy, M.E., Liang, X., van Groenigen, K.J., Lee, J., van Gestel, N., Six, J., Venterea, R.T. \& van Kessel, C. 2015. When does no-till yield more? A global meta-analysis. Field Crops Research, 183: 156-168. https://doi.org/10.1016/j.fcr.2015.07.020

Poeplau, C. \& Don, A. 2015. Carbon sequestration in agricultural soils via cultivation of cover crops - A metaanalysis. Agriculture, Ecosystems \& Environment, 200: 33-41. https://doi.org/10.1016/j.agee.2014.10.024

Rochecouste, J.F., Dargusch, P., Cameron, D. \& Smith, C. 2015. An analysis of the socio-economic factors influencing the adoption of conservation agriculture as a climate change mitigation activity in Australian dryland grain production. Agricultural Systems, 135: 20-30. https://doi.org/10.1016/j.agsy.2014.12.002

Skaalsveen, K., Ingram, J. \& Clarke, L.E. 2019. The effect of no-till farming on the soil functions of water purification and retention in north-western Europe: A literature review. Soil and Tillage Research, 189: 98-109. https://doi.org/10.1016/j.still.2019.01.004 


\title{
47. Regenerative agriculture
}

\author{
Antonious Petro', Reginaldo Haslett-Marroquín² \\ ${ }^{1}$ Régénération Canada, Université du Québec en Abitibi-Témiscamingue \\ ${ }^{2}$ Regenerative Agriculture Alliance
}

\section{Description of the concept}

\subsection{Concept}

Regenerative agriculture as a concept comes from the ancestral and long-held principles practiced by Indigenous communities around the world (Regenerative Agriculture Alliance, 2020; Dwiartama, 2020) and backed up by modern science. It is an indigenous way of thinking, one that reflects an understanding that the earth is a fully functioning ecosystem, and that because of its capacity to regenerate, evolve and find a biological, physical and chemical balance life was able to emerge and thrive. At the centre of this regeneration is a continuous process of energy transformation which over billions of years has given birth to millions of energy expressions that are reflected in the organisms that inhabit the planet, both known and unknown. Regenerative agriculture from an indigenous perspective is a way of seeing and working with the ecosystems (Fikret, Colding and Folke, 2000) on which life and its continued evolution depends, one where humans are but one of those life forms. Regenerative agriculture in modern days has to be understood at the higher level first if we are to keep it from being reduced to a set of practices or focused primarily on soil health, which negates the origin and full potential of the concept of regeneration.

\subsection{Definition}

Because of the large scope of activities and practices that regenerative agriculture implies, there is no universal definition internationally agreed upon but rather somewhat different definitions (Elevitch, Mazaroli and Ragone, 2018; Newton et al., 2020; Schreefel et al., 2020). For example, Terra Genesis (2017) proposes that it is a process of regeneration of the health, vitality, and evolutionary capability of whole living systems and that it is multi-layered: functional, integrative, systemic, and evolutionary. According to Jones (2003), regenerative practices utilise natural ecological services to replenish and reactivate the resource base. When agriculture is regenerative, soils, water, 
vegetation and productivity continually improve rather than staying the same or slowly getting worse. Other authors and organizations, such as the 4p1000 initiative (2020) ${ }^{24}$, Hawken (2017), Toensmeier (2016) and The Carbon Underground (2017), have focused on farming practices that lead to regenerative outcomes.

A regenerative agriculture system delivers soil health, carbon sequestration, improved water cycles and an endless list of other ecological regeneration benefits (Rhodes, 2017). It also incorporates a vast array of practices, such as cover crops, no-till, reduced tillage, agroforestry, etc. When implemented and adapted to the needs of the ecosystem, these practices lead to outcomes that support ecological, social, economic, and spiritual regeneration (Table 204). Soil regeneration practices may or may not include organic practices and go beyond the reduction of negative impacts, to rather ensure that agriculture has positive environmental impacts (Burgess et al., 2019). But fundamentally, regenerative agriculture engineering starts by questioning the very need for planting crops that need those practices in the first place; instead it starts by evaluating the original ecological blueprint of a region and then designing a process by which food, fiber, and other outputs can be generated while restoring the original ecology of a region.

\subsection{Principles and practices}

Because Regenerative Agriculture goes beyond on farm practices, the following principles are important to integrate before moving to the engineered specific practices:

Fair: The system is structured to balance the distribution of benefits and burdens and incorporates ecological, social, economic, and spiritual factors central to the development of criteria, indicators, and verifiers of how fairly the system works for everyone involved. It should adopt holistic management that considers the inter-relatedness of all parts of a farming system, including the farmer (Francis, Harwood and Parr, 1986).

Resilient: The system is structured to reduce risks and safeguard the geo-evolutionary genetic integrity of the plants and animals, the integrity of the ecology, the foundation of healthy social relations, and the economic commonsbased appreciation of the system resources so that the system can effectively respond to social, economic and ecological shock.

Sustainable: The system is structured to perennially sustain the ecology, economy, and social fabric on which it depends.

Healthy: The system results in a healthy working environment, a healthy economy for everyone involved, healthy ecology and nutritious foods that support the health and wellbeing of consumers.

Transparent: The system is structured to be socially, ecologically, and economically accountable to all involved.

\footnotetext{
${ }^{24}$ The Scientific and Technical Committee of the Initiative " 4 per 1000" considers that Regenerative agriculture is a system of farming principles and practices that seeks to rehabilitate and enhance the entire ecosystem of the farm. It is a method of farming that places a heavy premium on soil health and improves the resources (soil, water, biodiversity, etc.) it uses.
} 
Table 204. Examples of practices that lead to incremental regenerative outcomes ${ }^{25}$

\begin{tabular}{|c|c|c|c|}
\hline Goals & Practices & Systemic outcomes & Reference \\
\hline \multirow{10}{*}{$\begin{array}{l}\text { Perennial and } \\
\text { annual } \\
\text { soil cover and } \\
\text { minimal soil } \\
\text { disturbance }\end{array}$} & No-till & \multirow{10}{*}{$\begin{array}{l}\text { - Microbial diversity conservation } \\
\text { - Improved soil structure } \\
\text { (SOM) } \\
\text { - } \quad \text { Decreased soil erosion } \\
\text { - } \quad \text { Soil carbon sequestration } \\
\text { Increased aboveground and root } \\
\text { biomass }\end{array}$} & Zuber and Villamil (2016) \\
\hline & Conservation Tillage & & Thomas et al. (2019) \\
\hline & Strip tilling & & Li et al. (2019) \\
\hline & Direct seeding & & Poeplau and Don (2015) \\
\hline & \multirow{2}{*}{$\begin{array}{l}\text { Cover crops and green } \\
\text { manure }\end{array}$} & & Blanco-Canqui et al. (2015) \\
\hline & & & Lal, (2004) \\
\hline & Perennial cropping & & Ferchaud, Vitte and Mary (2016) \\
\hline & Agroforestry & & Fortier et al. (2015) \\
\hline & Riparian buffers & & Vidon et al. (2010) \\
\hline & Alley cropping & & Shi et al. (2018) \\
\hline \multirow{2}{*}{$\begin{array}{l}\text { Integration } \\
\text { of livestock }\end{array}$} & Silvopasture & \multirow{2}{*}{$\begin{array}{l}\text { - Increased SOM } \\
\text { - Improved water infiltration } \\
\text { Improved plant growth }\end{array}$} & Howlett et al. (2011) \\
\hline & Holistic planned grazing & & $\begin{array}{l}\text { Savory (1983); Teague and } \\
\text { Barnes (2017) }\end{array}$ \\
\hline \multirow{4}{*}{$\begin{array}{l}\text { Optimisation } \\
\text { of inputs }\end{array}$} & $\begin{array}{l}\text { Compost and other } \\
\text { organic amendments }\end{array}$ & \multirow{4}{*}{$\begin{array}{l}\text { - Increased SOM } \\
\text { - } \quad \begin{array}{l}\text { Reduced } \\
\text { dependency on synthetic inputs }\end{array} \\
\text { - Enhanced microbial diversity }\end{array}$} & Diacono and Montemurro (2010) \\
\hline & Green manure & & \multirow{3}{*}{ Talgre et al. (2012) } \\
\hline & $\begin{array}{l}\text { Micro-organisms } \\
\text { inoculation }\end{array}$ & & \\
\hline & $\begin{array}{l}\text { Minimizing synthetic } \\
\text { fertilizers and pesticides } \\
\text { usage }\end{array}$ & & \\
\hline \multirow{2}{*}{$\begin{array}{l}\text { Increase } \\
\text { biodiversity }\end{array}$} & Crop rotation & \multirow{2}{*}{$\begin{array}{ll}\text { - } & \text { Enhanced soil fertility } \\
\text { Increased soil organic carbon }\end{array}$} & $\begin{array}{l}\text { Venter, Jacobs and Hawkins } \\
\text { (2016) }\end{array}$ \\
\hline & $\begin{array}{l}\text { Polyculture / } \\
\text { Intercropping }\end{array}$ & & $\begin{array}{l}\text { Cong et al. (2015; Finney and } \\
\text { Kaye (2017) }\end{array}$ \\
\hline
\end{tabular}

${ }^{25}$ These are some examples of regenerative practices but there are many more developed in other parts of this book. 


\section{Range of applicability}

While principles of regenerative agriculture stay the same for different regions and climatic zones of the world, practices are often subject to adaptation (Lal, 2020). As demonstrated in Table 204, different practices result in different ecological services (LaCanne and Lundgren, 2018; Luján Soto, Cuéllar Padilla and de Vente, 2020; Newton et al., 2020) and thus, a profound assessment of the farm and regional needs is imperative before implementation. Furthermore, the soil type, the local ancestral knowledge and the availability of resources are some of the important factors to take in consideration (Schreefel et al, 2020). Regenerative land management often implies the establishment of several regenerative practices at the same time to achieve the desired goal. Soil regeneration is a complex process (Luján Soto, Cuéllar Padilla and de Vente, 2020) and an estimated period of 3-5 years of transition is usually expected, depending on the original state of soil. For most Indigenous communities, regenerative agriculture means restoring ancient management systems such as salmon routes, forests, wild animals and traditions and relates very little to the production of commodities (corn, cocoa, coffee, soybeans, beef, chicken, etc.) yet it is precisely these factors that dominate the market-driven discussion which tends to focus on brands and corporate positioning, on securing some sort of differentiation and competitive advantage and on gaining a leg-up in the already confused marketplace filled with labels, claims and certification schemes.

There are many similarities between regenerative agriculture and other ecological farming movements or practices, such as permaculture, agroecology, or climate smart agriculture (Burgess et al., 2019; Gosnell, Gill and Voyer, 2019; Newton et al, 2020). It is not surprising to notice that many regenerative agriculture practices are applied under different names or different movements. Permaculture and regenerative agriculture share a holistic approach that goes beyond farming practices and looks at the agricultural system as a complex ecosystem that should include environmental, economic, social, and especially spiritual components (Rhodes, 2017; Schreefel et al., 2020).

Regenerative agriculture is a process of continued improvement where practices have a wide spectrum for application with one goal: to regenerate the agricultural ecosystem. Therefore, similarly to climate-smart agriculture and carbon farming, regenerative agriculture helps mitigate climate change and sequester carbon in soils (Lal, 2020). As does permaculture, it sustains a just and healthy food system. Moreover, in the same way agroecology does, regenerative agriculture adopts an ecosystems approach that lead to multiple ecological outcomes.

Regeneration is our last opportunity to truly change the systems and structures that are degenerating the planet - it is a transformative and revolutionary approach but it only delivers the desired results if applied with integrity. 


\section{Potential barriers for adoption of soil regenerative practices}

Table 205. Potential barriers to adoption

\begin{tabular}{|c|c|c|}
\hline Barrier & Yes/No & \\
\hline Biophysical & Yes & $\begin{array}{l}\text { - Short growing seasons add extra challenges for the application of certain } \\
\text { practices (Carlisle, 2016) such as cover cropping or intercropping. } \\
\text { - } \\
\text { Physical compatibility (land shape, topography) and land availability can } \\
\text { be challenging (Ranjan et al., 2019). }\end{array}$ \\
\hline Cultural & Yes & $\begin{array}{l}\text { Cultural norms and emotional barriers (Gosnell, Charnley and Stanley, } \\
\text { 202O; Gosnell, Gill and Voyer, 2019; O'Connor, 2020) cause resistance } \\
\text { to change therefore sustaining the classic conventional system } \\
\text { (Rodriguez et al., 2009). } \\
\text { - Integrating regenerative practices might result in yield reduction } \\
\text { particularly during the first years. It is therefore imperative to shift the } \\
\text { focus from yield to profit to take into consideration the reduction of } \\
\text { external inputs such as machinery, fuel and fertilizers (Gosnell, Charnley } \\
\text { and Stanley, 2020). }\end{array}$ \\
\hline Social & Yes & $\begin{array}{l}\text { - Peer, family and social pressure and social isolation (Gosnell, Charnley } \\
\text { and Stanley, 2020): many regenerative farmers report that they feel } \\
\text { isolated and go under a great social pressure from family members or } \\
\text { from their peers because they farm "differently". } \\
\text { - Many farmers also report a fear of the unknown ( O'Connor, 2020). }\end{array}$ \\
\hline Economic & Yes & $\begin{array}{l}\text { The initial investment of time and money for learning and redesigning } \\
\text { the whole ecosystem on their land while transitioning to regenerative } \\
\text { agriculture is an important financial obstacle for many farmers (Gosnell, } \\
\text { Charnley and Stanley, 2020). } \\
\text { - Costly equipment changes or modifications are still necessary } \\
\text { (O'Connor, 2020; Rodriguez et al., 2009). Although shifting to } \\
\text { regenerative practices result in reducing dependance on some machines } \\
\text { (like tilling machines), some adjustments and modifications need to be } \\
\text { made to other machines (like seeders and roller-crimpers). } \\
\text { - Important ongoing investments include seeds, labor and management } \\
\text { (Carlisle, 2016). }\end{array}$ \\
\hline
\end{tabular}




\begin{tabular}{|c|c|c|}
\hline Barrier & Yes/No & \\
\hline Institutional & Yes & $\begin{array}{l}\text { - There is a lack of bank and insurances programs supporting transition } \\
\text { and trial (Rodriguez et al., 2009). Traditional programs don't leave space } \\
\text { for innovation or farmer-led experience in situ. } \\
\text { - There is a lack of institutional support (Rodriguez et al., 2009) to provide } \\
\text { education materials. } \\
\text { - } \quad \text { As there is no global certification or recognition, there is a lack of market } \\
\text { for products issued from regenerative farming, which don't get sufficient } \\
\text { monetary value. } \\
\text { - Lack of supportive policies to facilitate transition to regenerative } \\
\text { agriculture (Carlisle, 2016; Lal, 2020). } \\
\text { - There aren't enough governmental programs that incentivize or } \\
\text { promote transition to regenerative agriculture (ex: financing transition, } \\
\text { cost-share programs, etc.) (Ranjan et al., 2019). } \\
\text { - Land tenure restrictions often apply (Carlisle, 2016; O'Connor, 2020; } \\
\text { Ranjan et al., 2019). }\end{array}$ \\
\hline Knowledge & Yes & $\begin{array}{l}\text { There is a need for agricultural services providers (agronomists, } \\
\text { agriculture advisors, etc.) to get exhaustive training in soil health and } \\
\text { regenerative practices and there is a lack of accessible knowledge } \\
\text { transfer channels (Rodriguez et al., 2009). Other gaps are identified in } \\
\text { knowledge of risk management on the farm. } \\
\text { - There is a disconnect between the scientific community and } \\
\text { farmer/practitioner led trials (Lunn-Rockliffe et al., 2020). Easy and } \\
\text { accessible scientific data and materials on regenerative agriculture are } \\
\text { crucial to ensure a modern science-based transition. }\end{array}$ \\
\hline
\end{tabular}




\section{References}

Blanco-Canqui, H., Shaver, T.M., Lindquist, J.L., Shapiro, C.A., Elmore, R.W., Francis, C.A. \& Hergert, G.W. 2015. Cover crops and ecosystem services: Insights from studies in temperate soils. Agronomy Journal, 107(6): 2449-2474. https://doi.org/10.2134/agronj15.0086

Burgess, P., Harris, J., Graves, A. \& Deeks, L. 2019. Regenerative Agriculture: Identifying the impact; enabling the potential. Report for SYSTEMIQ. Cranfield University, UK. (also available at:

https://www.foodandlandusecoalition.org/wp-content/uploads/2019/09/Regenerative-Agriculture-final.pdf)

Carlisle, L. 2016. Factors influencing farmer adoption of soil health practices in the United States: a narrative review. Agroecology and Sustainable Food Systems, 40(6): 583-613.

https://doi.org/10.1080/21683565.2016.1156596

Cong, W.F., Hoffland, E., Li, L., Six, J., Sun, J.H., Bao, X.G., Zhang, F.S. \& van der Werf, W. 2015. Intercropping enhances soil carbon and nitrogen. Global Change Biology, 21(4): 1715-1726.

https://doi.org/10.1111/gcb.12738

Diacono, M. \& Montemurro, F. 2010. Long-term effects of organic amendments on soil fertility. A review. Agronomy for Sustainable Development, 30(2): 401-422. https://doi.org/10.1051/agro/2009040

Dwiartama, A. 2020. Indigenous livelihood. In Duncan, J., Carolan, M. \& Wiskerke, J.S. (Eds.). Routledge Handbook of Sustainable and Regenerative Food Systems. Routledge. Pp. 26-37.

Elevitch, C.R., Mazaroli, N.D. \& Ragone, D. 2018. Agroforestry standards for regenerative agriculture. Sustainability (Switzerland), 10(9): 1-21. https://doi.org/10.3390/su10093337

Ferchaud, F., Vitte, G. \& Mary, B. 2016. Changes in soil carbon stocks under perennial and annual bioenergy crops. GCB Bioenergy, 8(2): 290-306. https://doi.org/10.1111/gcbb.12249

Fikret, B., Colding, J. \& Folke. C. 2000. Rediscovery of traditional ecological knowledge as adaptive management. Ecological applications, 10(2): 1251-1262. https://doi.org/10.1890/1051-

0761(2000)010[1251:ROTEKA]2.0.CO;2

Finney, D.M. \& Kaye, J.P. 2017. Functional diversity in cover crop polycultures increases multifunctionality of an agricultural system. Journal of Applied Ecology, 54(2): 509-517. https://doi.org/10.1111/1365-

2664.12765

Fortier, J., Truax, B., Gagnon, D. \& Lambert, F. 2015. Biomass carbon, nitrogen and phosphorus stocks in hybrid poplar buffers, herbaceous buffers and natural woodlots in the riparian zone on agricultural land. Journal of Environmental Management, 154: 333-345. https://doi.org/10.1016/j.jenvman.2015.02.039

Francis, C.A., Harwood, R.R. \& Parr, J.F. 1986. The potential for regenerative agriculture in the developing world. American Journal of Environmental Management, 1: 65-74. https://www.jstor.org/stable/44506928 
Gosnell, H., Charnley, S. \& Stanley, P. 2020. Climate change mitigation as a co-benefit of regenerative ranching: insights from Australia and the United States. Interface Focus, 10(5): 20200027.

https://doi.org/10.1098/rsfs.2020.0027

Gosnell, H., Gill, N. \& Voyer, M. 2019. Transformational adaptation on the farm: Processes of change and persistence in transitions to 'climate-smart' regenerative agriculture. Global Environmental Change, 59. https://doi.org/10.1016/j.gloenvcha.2019.101965

Hawken, P. 2017. Drawdown the most comprehensive plan ever proposed to reverse global warming. New York, USA, Penguin

Howlett, D.S., Moreno, G., Mosquera Losada, M.R., Nair, P.K.R. \& Nair, V.D. 2011. Soil carbon storage as influenced by tree cover in the Dehesa cork oak silvopasture of central-western Spain. Journal of Environmental Monitoring, 13(7): 1897-1904. https://doi.org/10.1039/c1em10059a

O'Connor, J. 2020. Barriers For Farmers \& Ranchers To Adopt Regenerative Ag Practices In The US. (also available at https://forainitiative.org/wp-content/uploads/Barriers-to-Adopt-Regnerative-AgricultureInteractive.pdf).

Jones, C. 2003. Recognise, Relate, Innovate. Pp. 29. Department of Land \& Water Conservation, New South Wales Government. (also available at http://www.amazingcarbon.com/PDF/JONESRecogniseRelateInnovate.pdf).

LaCanne, C.E. \& Lundgren, J.G. 2018. Regenerative agriculture: Merging farming and natural resource conservation profitably. PeerJ, (2): 1-12. https://doi.org/10.7717/peerj.4428

Lal, R. 2004. Soil Carbon Sequestration Impacts on Global Climate Change and Food Security. Science, 304(5677): 1623-1627. https://doi.org/10.1126/science.1097396

Lal, R. 2020. Regenerative agriculture for food and climate. Journal of Soil and Water Conservation, 75(5): 123A-124A. https://doi.org/10.2489/jswc.2020.0620a

Li, Y., Li, Z., Cui, S., Jagadamma, S. \& Zhang, Q. 2019. Residue retention and minimum tillage improve physical environment of the soil in croplands: A global meta-analysis. Soil and Tillage Research, 194: 104292. https://doi.org/10.1016/j.still.2019.06.009

Luján Soto, R., Cuéllar Padilla, M. \& de Vente, J. 2020. Participatory selection of soil quality indicators for monitoring the impacts of regenerative agriculture on ecosystem services. Ecosystem Services, 45: 101157. https://doi.org/10.1016/j.ecoser.2020.101157

Lunn-Rockliffe, S., Davies, M.I., Willman, A., Moore, H.L., McGlade, J.M. \& Bent, D. 2020. FARMER LED REGENERATIVE Farmer Led Regenerative. London.

Newton, P., Civita, N., Frankel-Goldwater, L., Bartel, K. \& Johns, C. 2020. What Is Regenerative Agriculture? A Review of Scholar and Practitioner Definitions Based on Processes and Outcomes. Frontiers in Sustainable Food Systems, 4: 1-11. https://doi.org/10.3389/fsufs.2020.577723 
Poeplau, C. \& Don, A. 2015. Carbon sequestration in agricultural soils via cultivation of cover crops - A metaanalysis. Agriculture, Ecosystems and Environment, 200: 33-41.

https://doi.org/10.1016/j.agee.2014.10.024

Ranjan, P., Church, S.P., Floress, K. \& Prokopy, L.S. 2019. Synthesizing Conservation Motivations and Barriers: What Have We Learned from Qualitative Studies of Farmers' Behaviors in the United States? Society and Natural Resources, 32(11): 1171-1199. https://doi.org/10.1080/08941920.2019.1648710

Regenerative Agriculture Alliance. 2019. Regenerative Agriculture Alliance [online]. Minneapolis, Minnesota. USA. [Cited 19 November 2020]. https://www.regenagalliance.org/RegenerativeAgriculture

Rhodes, C.J. 2017. The imperative for regenerative agriculture. Science Progress, 100(1): 80-129. https://doi.org/10.3184/003685017X14876775256165

Rodriguez, J.M., Molnar, J.J., Fazio, R.A., Sydnor, E. \& Lowe, M.J. 2009. Barriers to adoption of sustainable agriculture practices: Change agent perspectives. Renewable Agriculture and Food Systems, 24(1): 60-71. https://doi.org/10.1017/S1742170508002421

Savory, A. 1983. The Savory Grazing Method or Holistic Resource Management. Rangelands, 5(4): 155-159. https://doi.org/10.2307/3900847

Schreefel, L., Schulte, R.P.O., de Boer, I.J.M., Schrijver, A.P. \& van Zanten, H.H.E. 2020. Regenerative agriculture - the soil is the base. Global Food Security, 26. https://doi.org/10.1016/j.gfs.2020.100404

Shi, L., Feng, W., Xu, J. \& Kuzyakov, Y. 2018. Agroforestry systems: Meta-analysis of soil carbon stocks, sequestration processes, and future potentials. Land Degradation and Development, 29(11): 3886-3897. https://doi.org/10.1002/ldr.3136

Talgre, L., Lauringson, E., Roostalu, H., Astover, A. \& Makke, A. 2012. Green manure as a nutrient source for succeeding crops. Plant, Soil and Environment, 58(6): 275-281. https://doi.org/10.17221/22/2012-pse

Teague, R. \& Barnes, M. 2017. Grazing management that regenerates ecosystem function and grazing land livelihoods. African Journal of Range and Forage Science, 34(2): 77-86.

https://doi.org/10.2989/10220119.2017.1334706

Terra Genesis. 2016. Levels of Regenerative Agriculture [online]. [Cited 15 July 2020]. http://www.terragenesis.com/wp-content/uploads/2017/03/Levels-of-Regenerative-Agriculture-1.pdf

The Carbon Underground. 2017. What is Regenerative Agriculture? [online]. [Cited 23 July 2020]. https://secureservercdn.net/50.62.174.113/02f.e55.myftpupload.com/wpcontent/uploads/2017/02/Regen-Ag-Definition-7.27.17-1.pdf

Thomas, B.W., Hunt, D., Bittman, S., Hannam, K.D., Messiga, A.J., Haak, D., Sharifi, M. \& Hao, X. 2019. Soil health indicators after 21 yr of no-tillage in South Coastal British Columbia. Canadian Journal of Soil Science, 99(2): 222-225. https://doi.org/10.1139/cjss-2018-0146 
Toensmeier, E. 2016. The carbon farming solution. A global toolkit of perennial crops and regenerative agriculture practices for climate change mitigation and food security. Vermont, USA, Chelsea Green Publishing.

Venter, Z.S., Jacobs, K. \& Hawkins, H.J. 2016. The impact of crop rotation on soil microbial diversity: A metaanalysis. Pedobiologia, 59(4): 215-223. https://doi.org/10.1016/j.pedobi.2016.04.001

Vidon, P., Allan, C., Burns, D., Duval, T.P., Gurwick, N., Inamdar, S., Lowrance, R., Okay, J., Scott, D. \& Sebestyen, S. 2010. Hot spots and hot moments in riparian zones: Potential for improved water quality management. Journal of the American Water Resources Association, 46(2): 278-298.

https://doi.org/10.1111/j.1752-1688.2010.00420.x

Zuber, S.M. \& Villamil, M.B. 2016. Meta-analysis approach to assess effect of tillage on microbial biomass and enzyme activities. Soil Biology and Biochemistry, 97: 176-187.

https://doi.org/10.1016/j.soilbio.2016.03.011 


\title{
48. Precision agriculture
}

\author{
Iria Soto', Athanasios T. Balafoutis² \\ 'European Commission, Joint Research Centre (JRC), Directorate Sustainable Resources, Economics of \\ Agriculture, Seville, Spain \\ ${ }^{2}$ Institute of Bioeconomy \& Agro-Technology, Centre of Research \& Technology Hellas, Volos, Greece
}

\section{Description of the practice}

Precision Agriculture (PA) is a farming management concept based on observing, measuring and responding to spatial and temporal field variability and crop requirements with the use of modern information and communication technologies (ICT) into agriculture (Soto et al., 2019; Bacco et al., 2019). The approach of this concept is based on precise and resource-efficient applications in farming practices that attempt to increase efficiency and quality of agricultural production without jeopardizing sustainability (Balafoutis et al., 2017a).

According to Balafoutis et al. (2017b), PA Technologies are consisted of: (i) Guidance systems including all forms of automatic steering/guidance for agricultural machinery that offer precise machinery movement in all agricultural practices to reduce overlapping that increases input use and machinery fuel consumption; (ii) Recording technologies that map soil properties, canopy characteristics, yield, etc. and provide data from the field which are converted to information after processing, so that PA applications can be achieved; and (iii) Reacting technologies that use the information derived by recording field conditions and assist in variable rate input application regarding seeding or transplanting, fertilization, irrigation, crop protection and weeding. This is the last step of PA and it focuses on minimizing all inputs in the optimum quantity required by the crop to grow. All three categories of PATs require the use of Global Navigation Satellite Systems (GNSSs) that provide localization of all actions and assist on keeping positioning records of the development of all field parameters. In closing the loop of PA actions, the integration of farm management information systems (FMIS) is significant as all farm operations have to be inventoried and analyzed in a digital form so that future actions can be planned (Fountas et al., 2015). The right combination of the above PA technologies is expected to increase or at least maintain yield increasing product quality and reducing environmental impact. 


\section{Range of applicability}

The application of PA is possible worldwide in all types of crops, soils, terrains and climates. However, the socioeconomic and environmental benefits of its application are greater for larger farms farm and where field heterogeneity is significant.

PA is a farming practice not intended to affect SOC balance directly. PA aims to optimize agricultural inputs to increase (or at least maintain) crop yields. The application of fewer inputs can result in less GHG emissions. Crops biomass can increase simultaneously with the yield, resulting in higher carbon stock incorporation in the transition phase until the next growing season (Balafoutis et al, 2017b). In addition, the reduction of the movements of the machinery prevents over-compaction and reduces soil degradation (Soto et al., 2019; CTF- Europe, 2020). However, is difficult to provide global estimations of the impacts of PA in SOC increase as the practice is normally applied to heterogeneous soil types. 


\section{Impact on soil organic carbon stocks}

Table 206. Impact on soil organic carbon stocks

\begin{tabular}{|c|c|c|c|c|c|c|c|c|}
\hline Location & $\begin{array}{l}\text { Climate } \\
\text { zone }\end{array}$ & $\begin{array}{l}\text { Soil } \\
\text { type }\end{array}$ & $\begin{array}{l}\text { Soil } \\
\text { depth } \\
(\mathrm{cm})\end{array}$ & $\begin{array}{l}\text { Baseline } \\
\text { C stock } \\
\text { (tC/ha) }\end{array}$ & Additional C storage (tC/ha/yr) & $\begin{array}{l}\text { Duration } \\
\text { (Years) }\end{array}$ & More information & Reference \\
\hline Regional (Italy) & $\begin{array}{l}\text { Warm } \\
\text { temperate } \\
\text { moist }\end{array}$ & $\begin{array}{l}\text { Sandy- } \\
\text { loam }\end{array}$ & $0-10$ & $\begin{array}{l}15,68 \\
\text { (average } \\
\text { for } 0,1 \mathrm{~m} \\
\text { depth) }\end{array}$ & $\begin{array}{l}\text { In this work, carbon stock have been shown } \\
\text { to be slightly reduced }\left(0,77 \mathrm{tC} \text {.ha-1-1. } \mathrm{r}^{-1}\right) \text { after } \\
3 \text { years of consecutive cultivation. This } \\
\text { reduction was lower using minimum and no } \\
\text { tillage techniques. The carbon stock in fields } \\
\text { where minimum tillage was applied was } 0.6 \\
\mathrm{tC} / \mathrm{ha} / \mathrm{yr} \text { ( } 22 \text { percent less), while in fields of } \\
\text { no tillage carbon stock was } 0.25 \mathrm{tC} / \mathrm{ha} / \mathrm{yr} \\
\text { ( } 67 \text { percent less). }\end{array}$ & 15 & $\begin{array}{l}\text { This work is the combination of } \\
\text { CA and PA practices. The carbon } \\
\text { stock change is mainly due to CA } \\
\text { and PA assists in reducing GHG } \\
\text { emissions. }\end{array}$ & $\begin{array}{l}\text { Cillis et al. } \\
(2018)\end{array}$ \\
\hline $\begin{array}{l}\text { Regional } \\
\text { (central } \\
\text { Queensland, } \\
\text { Australia) }\end{array}$ & Tropical dry & NA & NA & \multicolumn{2}{|c|}{$\begin{array}{l}\text { PA (i.e. Controlled Traffic Farming, CTF } 26 \text { ), coupled with no- } \\
\text { tillage might increase SOC stocks by increasing the rainfall } \\
\text { use efficiency and crops yields from } 0.7 \text { to } 1.2 \text {. } \\
\text { It might also increase SOM and long-term C sequestration by } \\
\text { providing a more C-rich residue and reducing stimulation of } \\
\text { oxidation. }\end{array}$} & NA & $\begin{array}{l}\text { PA increases soil porosity in the } \\
\text { range of } 5 \text { percent to } 70 \text { percent, } \\
\text { water infiltration by a factor of } 4 \text {, } \\
\text { and saturated hydraulic } \\
\text { conductivity by a factor of } 2 \text {. The } \\
\text { use of CTF can also reduce } \mathrm{N}_{2} \mathrm{O} \\
\text { emissions by } 2 \mathrm{O} \text { percent to } 50 \\
\text { percent }\end{array}$ & $\begin{array}{l}\text { Antille et al. } \\
\text { (2015) }\end{array}$ \\
\hline $\begin{array}{l}\text { Regional } \\
\text { (United } \\
\text { Kingdom of } \\
\text { Great Britain } \\
\text { and Northern } \\
\text { Ireland) }\end{array}$ & $\begin{array}{l}\text { Cool } \\
\text { temperate } \\
\text { moist }\end{array}$ & $\begin{array}{l}\text { sandy } \\
\text { loam }\end{array}$ & $10-25$ & \multicolumn{2}{|c|}{$\begin{array}{l}\text { PA (i.e. Controlled Traffic Farming') with reduced tillage can } \\
\text { increase SOC stock by increasing harvestable wheat grain } \\
\text { yield by } 9 \text { percent. }\end{array}$} & 1 & $\begin{array}{l}\text { PA enables less yield reduction in } \\
\text { the early years of reduced tillage } \\
\text { conversion. }\end{array}$ & $\begin{array}{l}\text { Smith et al. } \\
\text { (2014) }\end{array}$ \\
\hline
\end{tabular}

${ }^{26}$ Controlled Traffic Farming (CTF) is a PA system that confines all machinery loads to the least possible area of permanent traffic lanes. It is based on machine guidance, but it keeps record of each field and application to follow the same route every year. CTF allows optimised driving patterns, more efficient operations (i.e. reduced overlaps) and targeted input applications (Balafoutis et al. (2017b). For more information look at factsheet No.29 "Controlled Traffic Farming" of this volume. 


\section{Other benefits of the practice}

\subsection{Improvement of soil properties}

PA has numerous effects on crop performance that are mainly related to input application optimization based on crop needs for the specific soil type of the field under study. However, indirectly, PA is related to soil maintenance and enhancement. The most important PA application of this kind comes from all guidance systems and CTF systems that reduce significantly the overlapping on self-propelled machinery. CTF can reduce tracking surface, and thus compaction, to just 15 percent, even over several years (Gasso et al., 2013). Reducing soil compaction makes soils porosity larger and therefore water infiltration is increased and soil penetration resistance is reduced, allowing crop plants to grow their roots and absorb nutrients easier. Another indirect impact on soil properties is the application of soil mapping systems that then can be used for soil conditioners application to improve its properties. Such technology is the VERIS systems ${ }^{27}$ that give the ability to the end-use to measure with one field pass the soil electrical conductivity, while measuring simultaneously soil organic matter and $\mathrm{pH}$ (Balafoutis et al., 2017b).

\subsection{Minimization of threats to soil functions}

\section{Table 207. Soil threats}

\begin{tabular}{|c|c|}
\hline Soil threats & \\
\hline Soil erosion & $\begin{array}{l}\text { Using guidance systems, tillage can be executed following the contours in hilly } \\
\text { fields and soil erosion can be reduced mainly from water (McBratney et al., } \\
\text { 2005). }\end{array}$ \\
\hline $\begin{array}{l}\text { Nutrient imbalance and } \\
\text { cycles }\end{array}$ & $\begin{array}{l}\text { Precision fertilization nutrient availability efficiency reducing the nutrients that } \\
\text { are not used by the crop plants (Balafoutis et al., 2017b). }\end{array}$ \\
\hline $\begin{array}{l}\text { Soil } \\
\text { contamination/pollution }\end{array}$ & $\begin{array}{l}\text { Precision fertilization can reduce nutrient leaching in the soil. Precision spraying } \\
\text { can reduce over-application of pesticides that can potentially end up in the soil. } \\
\text { In addition, precise mechanical weeding that replaces herbicides assist in } \\
\text { preventing soil contamination (Balafoutis et al., 2017b). }\end{array}$ \\
\hline Soil biodiversity loss & $\begin{array}{l}\text { Impacts on biodiversity and ecosystem services are positive because of the } \\
\text { reduction in application of inputs, especially chemicals like fertilizers and } \\
\text { pesticides (Balafoutis et al., 2017a and b). Controlled Traffic Farming might have } \\
\text { a positive effect on soil structure and soil organisms (Jensen et al., 2012; CTF } \\
\text { Europe, 202O) }\end{array}$ \\
\hline
\end{tabular}

${ }^{27}$ https://www.veristech.com/ 


\begin{tabular}{|l|l|}
\hline Soil threats & \\
\hline Soil compaction & $\begin{array}{l}\text { Confining all machinery loads to the smallest possible area of permanent traffic } \\
\text { lanes using guidance systems and especially CTF systems reduces soil } \\
\text { compaction in a high extent (Jensen et al, 2012; CTF Europe, 2020). }\end{array}$ \\
\hline Soil water management & $\begin{array}{l}\text { Site-specific and efficient fertilizer and pesticide application might decrease the } \\
\text { risk of ground- and surface water contamination. }\end{array}$ \\
\hline
\end{tabular}

\subsection{Increases in production (e.g. food/fuel/feed/timber/fibre)}

PA has the capacity to increase or maintain crop production. Its impacts are diverse depending of the type of PA technology. Lambert and Lowenberg-DeBoer (2000) reported yield increase (in corn production) using variable rate irrigation. Soto et al. (2019) indicate that farmers that adopted variable rate nutrient technology, on average, report increases of production of 4.1 percent compared with traditional farming practices. Variable seeding rate can offer an increase in winter wheat yield from 3 percent (Decisive Farming, 2011) to 6 percent in corn yield ( $\mathrm{AgPhD}, 2020)$. Control Traffic Farming can increase winter wheat yields by 7 percent due to an improved water storage and a higher mean soil moisture while (Qingjie et al., 2009).

\subsection{Mitigation of and adaptation to climate change}

The adoption of PA practices has a positive impact on GHG emission reduction due to: i) the reduction of input (mainly nitrogen-fertilizers) for the agricultural field operations; ii) the reduction of fuel consumption through less in-field operations with the tractor and; iii) the enhancement of the ability of soils to operate as carbon stock reserve by reducing tillage and increasing yield (Balafoutis et al., 2017b).

PA technologies are mainly based on ICT and are adapted on existing machinery. Therefore, their negative impact on climate change is negligible and associated with the GHG emissions generating during their production.

\subsection{Socio-economic benefits}

The economic benefits of using PA are associated mainly with the costs savings of the optimization of inputs such as fuel or fertilizers and time (Soto et al., 2019). Bora (2009) identified a cost reduction of $\$ 138$ per hectare when using PA (i.e. variable rate application of urea) in citrus groves in Florida. Control traffic farming adopters in Australia report savings of 15 percent in seed and spray, 25 percent on labour and 33 percent of fuel costs (Yule and Radford 2003). Other social benefits are time saving, accuracy at work, optimization of the logistics/managerial improvements, reduction of fatigue and possibility to work extended hours (e.g. dusk and down) (van der Wal, 2014). However, PA might not be beneficial in all farming situations as the purchase of new technologies can be capital-intensive (Lawson et al., 2011). 


\section{Potential drawbacks to the practice}

\subsection{Increases in greenhouse gas emissions}

The GHG emission reduction associated with the use of PA comes directly from the fuel consumption decrease during field operations and indirect GHG emission $\left(\mathrm{CO}_{2}, \mathrm{~N}_{2} \mathrm{O}, \mathrm{CH}_{4}\right)$ derived during the production of all inputs (seeds, fertilizers, pesticides). Compared to traditional farming practices when machinery is used, PA optimizes the necessary passes of machinery and avoids overlapping, decreasing fuel consumption and the related GHG emissions. In addition, by variable rate application of inputs, their applied quantities are reduced together with the respective GHG emissions from their production.

\subsection{Conflict with other practice(s)}

In developing countries, where conventional mechanized agricultural practices might not be widely applied, the direct replacement of manual practices by PA would not affect positively soil compaction. However, current technological and information access levels for those countries might not provide the appropriate space for PA development. It might also be difficult for those countries to pass directly from manual to precision agriculture without passing first through conventional mechanization.

\subsection{Decreases in production (e.g. food/fuel/feed/timber/fibre)}

PA is expected to maintain or increase production rate. However, when inputs are not correctly applied, PA might have a negative impact on yield. Examples of such improper PA application would be the misplaced delineation of management zones or the use of imprecise algorithms for variable rate fertilization, crop protection, weeding or irrigation.

\section{Recommendations before implementing the practice}

Upfront investments in PA tend to be high. Thus, in-depth ex-ante cost-benefit assessments are needed before its adoption. Two features are key determinants of the economic profitability of the system: the size of the agricultural holding and the variability of the soil properties. PA are information intensive technologies that require previous technical knowledge and skills from the adopter farmers. Thus, promotion policies should be coupled with a well-designed participatory research strategy in which the government, researchers, extension agents and farmers support, share and generate knowledge and skills on PA. 


\section{Potential barriers for adoption}

Until now, due to increased cost of transition between conventional practices to PA, the application of PA is mainly directed to large acreage farms (Matese et al., 2015), of high income due to high added value of the cultivated crops (Blackmore et al., 2006), high labor cost (Lowenberg-Deboer, 1998) and increased educational level of the farmer (Lawson et al., 2011).

Table 208. Potential barriers to adoption

\begin{tabular}{|c|c|c|}
\hline Barrier & YES/NO & \\
\hline Biophysical & Yes & $\begin{array}{l}\text { Potential benefits depend on farm size and farm biophysical heterogeneity (Soto } \\
\text { et al., 2019). Until now, the application of PA is mainly directed to large farms in } \\
\text { acreage (Matese et al., 2015) due to increased cost of transition between } \\
\text { conventional practices to PA. }\end{array}$ \\
\hline Cultural & Yes & $\begin{array}{l}\text { Traditional farmers, with low levels of trust in the technology, low information } \\
\text { seeking and innovative behaviors are reluctant to uptake the technology (Eidt, } \\
\text { Hickey and Curtis, 2012; Barnes et al., 2019) } \\
\text { Non-cooperative behavior limits uptake of the technology as cooperatives and } \\
\text { machinery rings provide support services (e.g. data analysis) and transfer } \\
\text { information to farmers (Barnes et al., 2019; Chen, Wachenheim and Zheng, } \\
\text { 2020). }\end{array}$ \\
\hline Social & Yes & $\begin{array}{l}\text { Age and education of the farmer limits adoption. Older or less educated farmers } \\
\text { with less years of farming experience are less likely to adopt PA (Barnes et al., } \\
\text { 2019). They tend to have less capability to decode new information and tend to } \\
\text { be less skilled in dealing with new technologies (Soto et al., 2019). }\end{array}$ \\
\hline Economic & Yes & $\begin{array}{l}\text { A mayor concern towards the adoption of PAT is the high initial investment costs } \\
\text { of the technologies, which generate uncertainty around the possibility of } \\
\text { recovering this investment (Soto et al., 2019). Farmer income determines the } \\
\text { capacity to bear financial risks, to handle the uncertainty towards the income of } \\
\text { the technology (Barnes et al., 2019). Therefore, adoption is greater on high- } \\
\text { income farms (Blackmore et al., 2006). }\end{array}$ \\
\hline Institutional & Yes & $\begin{array}{l}\text { Limited institutional support on planning (e.g. integrating new knowledge on } \\
\text { their farming practices) and follow-up discourages trust and adoption of newly } \\
\text { introduced technologies ( Eidt, Hickey and Curtis, 2012). }\end{array}$ \\
\hline $\begin{array}{l}\text { Legal (Right } \\
\text { to soil) }\end{array}$ & Yes & $\begin{array}{l}\text { Owner-occupied farms are more likely to adopt PATs, due to access to capital to } \\
\text { enable investment in machinery (Paustian and Theuvsen, 2016). }\end{array}$ \\
\hline Knowledge & Yes & $\begin{array}{l}\text { The technical complexity and information-gathering characteristics of the } \\
\text { technology limits uptake among less skilled and knowledge farmers (Soto et al., }\end{array}$ \\
\hline
\end{tabular}




\begin{tabular}{|l|l|l|}
\hline Barrier & YES/NO & \\
\hline & & $\begin{array}{l}\text { 2019). } \\
\text { Peer to peer learning, farmer extension, advisors and field demonstration are } \\
\text { important factors in the diffusion of this innovation (Soto et al., 2019; Barnes et } \\
\text { al., 2018). }\end{array}$ \\
\hline
\end{tabular}

\section{Photos of the practice}
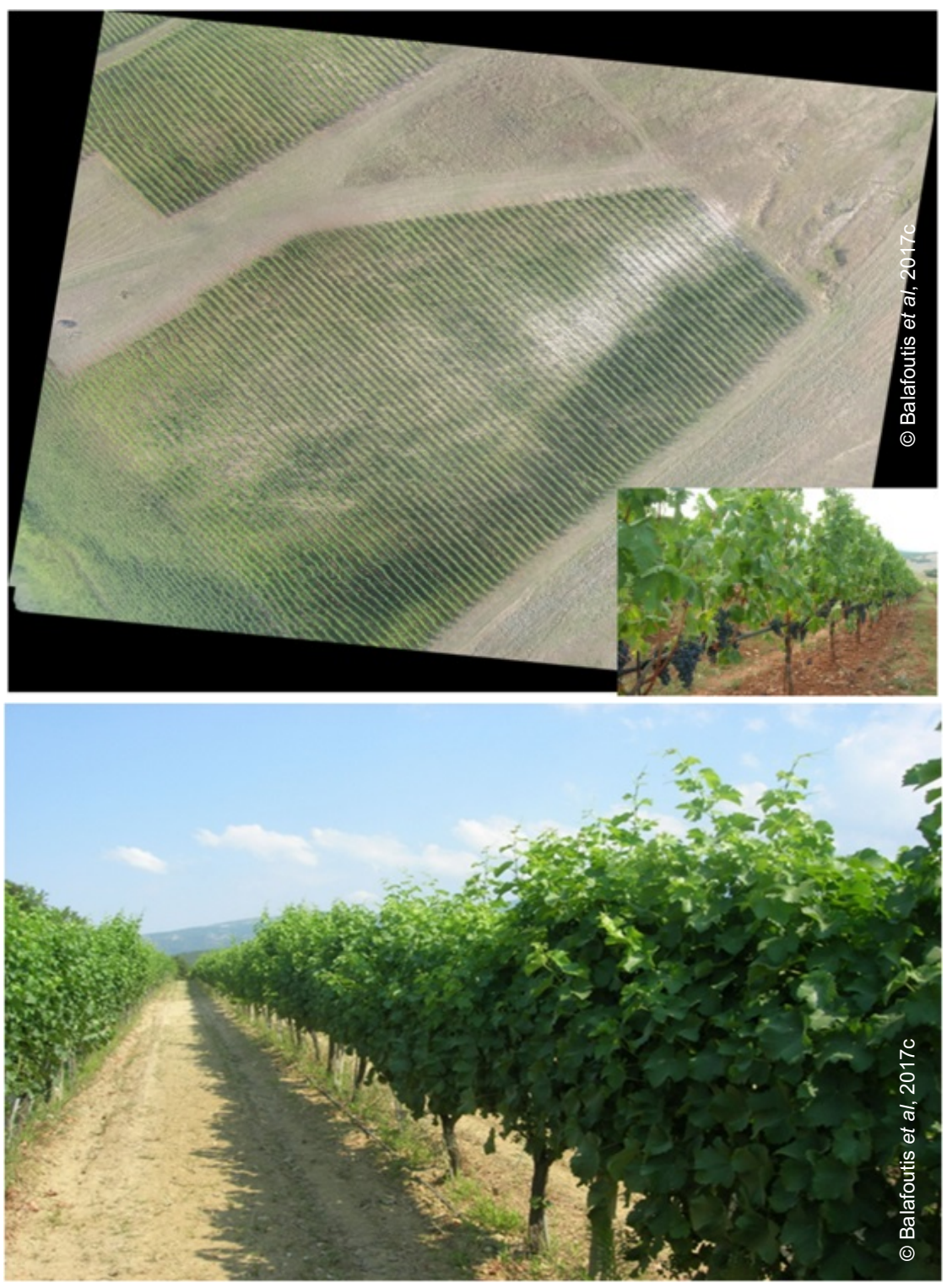

Photo 64. Syrah Vineyard in Drama, Greece 
(a)
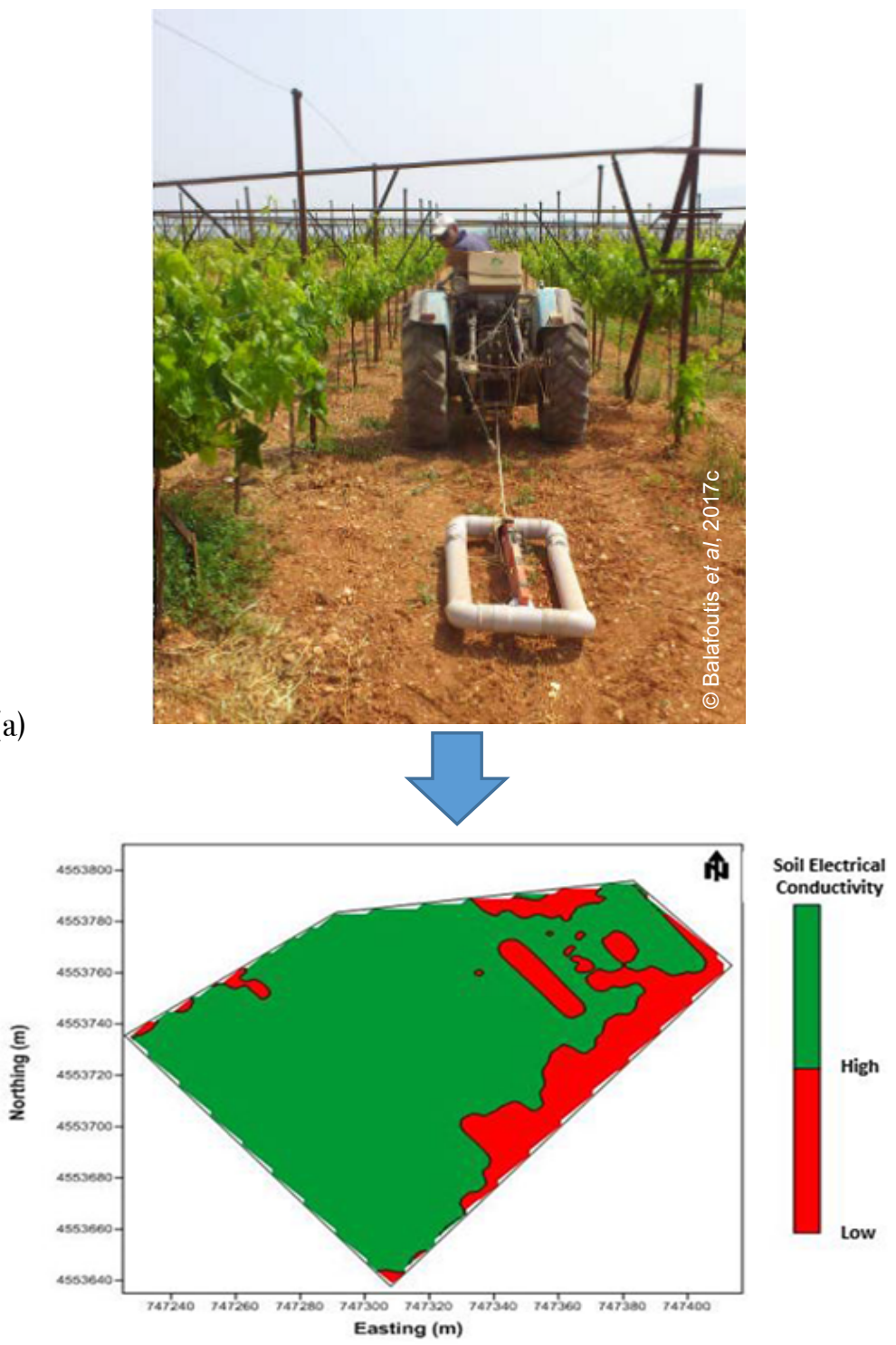

(b)
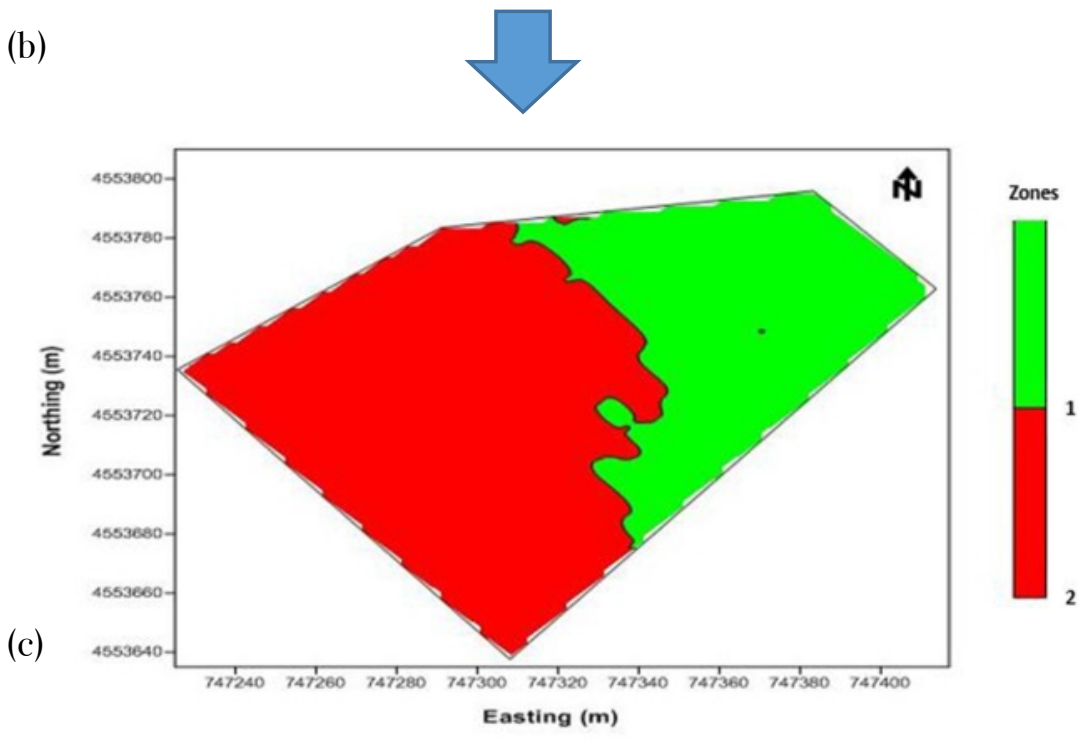

Photo 65. Measurement of soil electrical conductivity (a) to produce a map (b) and based on this information delineate management zones (c) in a Syrah Vineyard in Drama, Greece. 

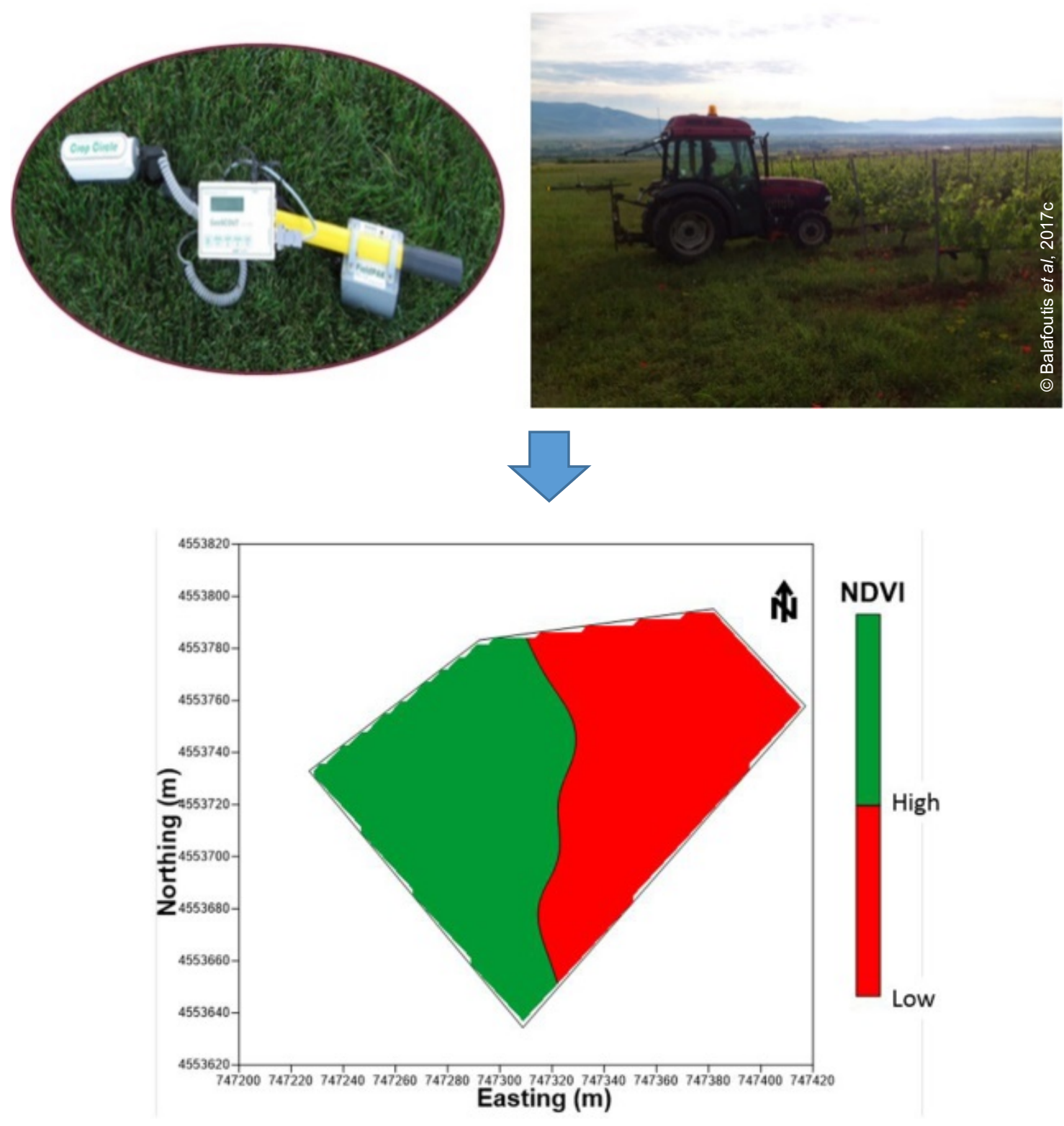

Photo 66. Measurement of vineyard side canopy to map their vigor using the Normalized Difference Vegetation Index (NDVI) in a Syrah Vineyard in Drama, Greece 


\section{References}

Antille, D.L., Chamen, W.C., Tullberg, J.N. \& Lal, R. 2015. The potential of controlled traffic farming to mitigate greenhouse gas emissions and enhance carbon sequestration in arable land: a critical review.

Transactions of the ASABE, 58(3): 707-731. https://doi.org/10.13031/trans.58.11049

AgPhD. 2020. Variable Rate \& Variety Planting in Wheat and Soybeans [online]. [Cited 20 July 2020].

http://www.agphd.com/ag-phd-newsletter/2014/03/21/variable-rate-variety-planting-in-wheat-andsoybeans/

Bacco, M., Barsocchi, P., Ferro, E., Gotta, A. \& Ruggeri, M. 2019. The Digitisation of Agriculture: a Survey of Research Activities on Smart Farming. Array, 3-4: 100009.

https://doi.org/10.1016/j.array.2019.100009

Balafoutis, A., Beck, B., Fountas, S., Vangeyte, J., Wal, T. V. D., Soto, I. \& Eory, V. 2017a. Precision agriculture technologies positively contributing to GHG emissions mitigation, farm productivity and economics. Sustainability, 9(8): 1339. https://doi.org/10.3390/su9081339

Balafoutis, A.T., Koundouras, S., Anastasiou, E., Fountas, S. \& Arvanitis, K. 2017b. Life Cycle Assessment of Two Vineyards after the Application of Precision Viticulture Techniques: A Case Study. Sustainability, 9(11): 1997. http://dx.doi.org/10.3390/su9111997

Balafoutis, A.T., Beck, B., Fountas, S., Tsiropoulos, Z., Vangeyte, J., van der Wal, T., Soto, I., GómezBarbero, M. \& Pedersen, S.M. 2017c. Smart Farming Technologies-Description, Taxonomy and Economic Impact. In Pedersen, S.M., Lind, K.M. (Eds.) Precision Agriculture: Technology and Economic Perspectives. Springer International Publishing: Cham, Switzerland, pp. 21-77.

Barnes, A.P., Soto, I., Eory, V., Beck, B., Balafoutis, A.T., Sánchez, B. \& Gómez-Barbero, M. 2018. Influencing factors and incentives on the intention to adopt precision agricultural technologies within arable farming systems. Environmental Science and Policy, 93: 66-74.

https://doi.org/10.1016/j.envsci.2018.12.014

Barnes, A.P., Soto, I., Eory, V., Beck, B., Balafoutis, A., Sánchez, B. \& Gómez-Barbero, M. 2019.

Exploring the adoption of precision agricultural technologies: A cross regional study of EU farmers. Land use policy, 80: 163-174. https://doi.org/10.1016/j.landusepol.2018.10.004

Bora, G.C. 2009. Economics of variable rate nitrogen application in Florida citrus grove. Tree and Forestry Science and Biotechnology, special issue 1: 164- 168.

Blackmore, S., Griepentrog, H.W., Pedersen, S.M. \& Fountas, S. 2006. Precision Agriculture in Europe. In Srinivasan A. (Ed.) Handbook of Precision Agriculture. Principles and Applications. pp.567-613.

Cillis, D., Maestrini, B., Pezzuolo, A., Marinello, F. \& Sartori, L. 2018. Modeling soil organic carbon and carbon dioxide emissions in different tillage systems supported by precision agriculture technologies under current climatic conditions. Soil and Tillage Research, 183: 51-59.

https://doi.org/10.1016/j.still.2018.06.001

CTF_Europe. 2020. Benefits of Controlled Traffic Farming [online]. [Cited 22 July 2020]

http://www.controlledtrafficfarming.com/WhatIs/Benefits-Of-CTF.aspx 
Chen, Q., Wachenheim, C., \& Zheng, S. 2020. Land scale, Cooperative Membership and Benefits Information: Unmanned Aerial Vehicle Adoption in China. Sustainable Futures, 2: 100025. https://doi.org/10.1016/j.sftr.2020.100025

Decisive farming. 2011. Variable Rate Seeding Presented at Master Seeders Conference [online]. [Cited 20 July 2020]. https://decisivefarming.com/variable-rate-seeding-benefits

Eidt, C.M., Hickey, G.M., \& Curtis, M.A. 2012. Knowledge integration and the adoption of new agricultural technologies: Kenyan perspectives. Food Security, 4(3): 355-367.

https://doi.org/10.1007/s12571-012-0175-2

Fountas, S., Carli, G., Sørensen, C.G., Tsiropoulos, Z., Cavalaris, C., Vatsanidou, A., Liakos, B., Canavari, M., Wiebensohn, J. \& Tisserye, B. 2015. Farm management information systems: Current situation and future perspectives. Comput. Electron. Agric., 115: 40-50.

https://doi.org/10.1016/j.compag.2015.05.011

Gasso, V., Sørensen, C.A.G., Oudshoorn, F.W. \& Green, O. 2013. Controlled traffic farming: A review of the environmental impacts. European Journal of Agronomy, 48: 66-73.

https://doi.org/10.1016/j.eja.2013.02.002

Jensen, H.G., Jacobsen, L.-B., Pedersen, S.M. \& Tavella, E. 2012. Socioeconomic impact of widespread adoption of precision farming and controlled traffic systems in Denmark. Precis. Agric., 13: 661-677. https://doi.org/10.1007/s11119-012-9276-3

Lambert, D. \& Lowenberg-De Boer, J. 2000. Precision agriculture profitability review. Working paper. Site-Specific Management Center, School of Agriculture, Purdue University. (also available at: https://agriculture.purdue.edu/SSMC/Frames/newsoilsX.pdf)

Lawson, L.G., Pedersen, S.M., Sørensen, C.G., Pesonen, L., Fountas, S., Werner, A., Oudshoorn, F.W., Herold, L., Chatzinikos, T., Kirketerp, I.M. \& Blackmore, S. 2011. A four nation survey of farm information management and advanced farming systems: A descriptive analysis of survey responses. Computers and Electronics in Agriculture, 77(1): 7-20. https://doi.org/10.1016/j.compag.2011.03.002

Matese, A., Toscano, P., Di Gennaro, S.F., Genesio, L., Vaccari, F.P., Primicerio, J., Belli, C., Zaldei, A., Bianconi, R. \& Gioli, B. 2015. Intercomparison of UAV, Aircraft and Satellite Remote Sensing Platforms for Precision Viticulture. Remote Sensing, 7(3): 2971-2990. https://doi.org/10.3390/rs70302971

McBratney, A., Whelan, B., Ancev, T. \& Bouma, J. 2005. Future Directions of Precision Agriculture. Precis. Agric., 6: 7-23. https://doi.org/10.1007/s11119-005-0681-8

Paustian, M. \& Theuvsen, L. 2017. Adoption of precision agriculture technologies by German crop farmers. Precision agriculture, 18(5): 701-716. https://doi.org/10.1007/s11119-016-9482-5

Qingjie, W., Hao, C., Hongwen, L., Li, W., Xiaoyan, W., McHugh, A. D., Jin, H. \& Huanwen, G. 2009. Controlled traffic farming with no tillage for improved fallow water storage and crop yield on the Chinese Loess Plateau. Soil \& Tillage Research, 104(1): 192-197. https://doi.org/10.1016/j.still.2008.10.012

Smith, E.K., Misiewicz, P.A., Girardello, V., Arslan, S., Chaney, K., White, D.R. \& Godwin, R.J. 2014. Effects of traffic and tillage on crop yield (winter wheat Triticum aestivum) and the physical properties of a sandy loam soil. American Society of Agricultural and Biological Engineers. Montreal, Quebec. 
Soto, I., Barnes, A., Balafoutis, A., Beck, B., Sanchez, B., Vangeyte, J., Fountas, S., Van der Wal, T., Eory, V. \& Gómez-Barbero, M. 2019. The contribution of Precision Agriculture Technologies to farm productivity and the mitigation of greenhouse gas emissions in the EU. Technical Report. Publications Office of the European Union, Luxembourg.

Van der Wal. 2014. Harvesting GNSS potential [online]. [Cited 17 December 2020] Geospatial world, 29. https://www.geospatialworld.net/article/harvesting-gnss-potential/

Yule, D.F. \& Radford, B. 2003. Case study 9: Controlled traffic farming. Summary of QPI14 Project. Rockhampton, Queensland, Australia: Queensland Department of Primary Industries. 


\title{
49. Organic agriculture
}

\author{
Rainer Nerger, Inka Sachse
}

Soil \& More Impacts, Hamburg, Germany

\section{Description}

The definition of organic agriculture (OA) from IFOAM - Organics International is: "Organic Agriculture is a production system that sustains the health of soils, ecosystems, and people. It relies on ecological processes, biodiversity and cycles adapted to local conditions, rather than the use of inputs with adverse effects. Organic Agriculture combines tradition, innovation, and science to benefit the shared environment and promote fair relationships and good quality of life for all involved" (IFOAM, 2020).

However, there are many different standards, labels, and certifiers (BIOC, 2020) of organic agriculture worldwide. Each standard but also each country has its own certification system (e.g. Seufert, Ramankutty and Mayerhofer, 2017). Among the most used organic standards worldwide are the European Union (EU) organic standard (Council Regulation 834/2007; EUR-Lex, 2007), the US NOP (USDA, 2020) and the Japanese JAS (Ecolabelindex, 2020). A specific private standard is the biodynamic agriculture with its trademark Demeter. The biodynamic agriculture has higher requirements compared to the EU or the NOP standard (i.e. composting, circular economy, set-aside land), but also features measures as the biodynamic preparations, which are not scientifically proven (Chalker-Scott, 2013; Carpenter-Boggs, Reganold and Kennedy, 2000).

The least common denominator is the avoidance of chemical fertilizers and of synthetic pesticides and herbicides. On top, different organic standards (private and governmental ones) include different recommended soil management (e.g. composting, cover cropping, conservation tillage, appropriate use of crop residues, agroforestry, alley cropping, etc.) (Knapp and van der Heijden, 2018; Seufert, Ramankutty and Mayerhofer, 2017; SMI, 2019). The greater number of such practices that are included the higher the standard and the higher the price of the sold products. The inclusion of recommended soil management practices can enable the increase of soil organic carbon/matter and soil biodiversity and as well resilience against soil erosion, drought, and other forms of degradation. 


\section{Range of applicability}

There is a worldwide applicability as the pedo-climatic limitations are identical to all forms of agriculture.

\section{Impact on soil organic carbon stocks}

Often, organic agriculture has been described to enhance soil organic carbon (SOC) sequestration (e.g. Gattinger et al., 2012, Table 209). However, there are also contrary findings, as of Leifeld and Fuhrer (2010) who state that often only SOC concentration increases were reported instead of SOC stock increases, or that some conditions were not fully comparable in evaluations.

Table 209. Evolution of SOC stocks in organic agriculture

\begin{tabular}{|l|l|l|l|l|}
\hline Location & Context & C additional storage & More information & Reference \\
\hline $\begin{array}{l}\text { Mainly temperate } \\
\text { zone, subtropical, } \\
\text { some tropical sites }\end{array}$ & $\begin{array}{l}\text { Large review } \\
\text { study using } \\
\text { topsoil data }(0- \\
30 \mathrm{~cm})\end{array}$ & $\begin{array}{l}3.50 \pm 1.08 \mathrm{tC} / \mathrm{ha} \text { (stocks } \\
\text { compared to conventional } \\
\text { agriculture. } \\
\text { O.45 } \pm 0.21 \mathrm{tC} / \mathrm{ha} / \mathrm{y} \text { (annual } \\
\text { sequestration rate) }\end{array}$ & $\begin{array}{l}\mathrm{n}=29 \text { different } \\
\text { studies; Significant } \\
\text { difference compared } \\
\text { to C stocks of } \\
\text { conventional } \\
\text { agriculture }\end{array}$ & $\begin{array}{l}\text { Gattinger } \text { et } \\
\text { al. }\end{array}$ \\
\hline
\end{tabular}

\section{Other benefits of the practice}

\subsection{Improvement of soil properties}

Organic agriculture is known to increase soil organic matter and beneficial soil microbes, build up soil fertility, improve soil physical properties (soil structure, porosity, water availability and aeration) (Reeve et al., 2016). However, the simple avoidance of chemical fertilizers and pesticides is not responsible for this but rather the usage of recommended soil management practices, such as those listed in this manual (use of compost, manure, cover crops, crop residues, etc.; Knapp and van der Heijden, 2018; Seufert, Ramankutty and Mayerhofer, 2017; SMI, 2019). 


\subsection{Minimization of threats to soil functions}

\section{Table 210. Soil threats}

\begin{tabular}{|c|c|}
\hline Soil threats & \\
\hline Soil erosion & $\begin{array}{l}\text { Organic agriculture was associated with higher weed biomass density and } \\
\text { cover at the end of the growing season and a higher amount of crop } \\
\text { residues added to the soil. This resulted in } 18-25 \text { percent less water erosion } \\
\text { (Arnhold et al., 2014). }\end{array}$ \\
\hline $\begin{array}{l}\text { Nutrient imbalance } \\
\text { and cycles }\end{array}$ & $\begin{array}{l}\text { Organic agriculture means independence from chemical fertilizers. Instead, } \\
\text { organic fertilizers are used which have a much longer nutrient release phase } \\
\text { Also, crop residues are often left on the field or incorporated. These } \\
\text { practices can provide sufficient nutrients for the plant and often greater } \\
\text { independence from external sources; thus, fostering nutrient cycling } \\
\text { (Maheshwari, 2014). }\end{array}$ \\
\hline Soil acidification & $\begin{array}{l}\text { Using a higher amount of organic fertilization has been shown to reduce soil } \\
\text { acidification (Cai et al., 2015). }\end{array}$ \\
\hline Soil biodiversity loss & $\begin{array}{l}\text { Organic agriculture is associated with higher organic inputs into the soil, } \\
\text { sometimes also with conservation tillage. Both measures increase soil } \\
\text { biodiversity (Krauss et al., 2020). }\end{array}$ \\
\hline $\begin{array}{l}\text { Soil water } \\
\text { management }\end{array}$ & $\begin{array}{l}\text { Organic agriculture builds up more organic matter compared to } \\
\text { conventional farming. More organic matter means a higher water-holding } \\
\text { capacity in the soil (Huntington, 2007). }\end{array}$ \\
\hline
\end{tabular}

\subsection{Increases in production (e.g. food/fuel/feed/timber/fibre)}

Organic agriculture is typically associated with a lower yield ( $\sim 20$ percent; De Ponti, Rijk and Van Ittersum, 2012), but nearly matches conventional yields when using good management practices (appropriate crop species, crop rotation, fertilization practices, diversification, intercropping, etc.) (Seufert, Ramankutty and Foley, 2012; Davis et al., 2012). Although yields are often lower, the productivity, as the input/output ratio, can be higher in organic agriculture (Adamtey et al., 2016; Forster et al., 2013).

\subsection{Mitigation of and adaptation to climate change}

On the example of a long-term trial in Switzerland, Skinner et al. (2019) showed that organically managed fields can generate 40 percent lower $\mathrm{N}_{2} \mathrm{O}$ emissions compared to conventionally managed fields. 


\subsection{Socio-economic benefits}

At farms practicing organic agriculture usually more jobs will be created compared to conventional farms, as in organic agriculture more labor force is needed (Finley et al., 2018). Especially some Demeter farms as the Polish Juchowo farm (Juchowo, 2019) practicing biodynamic agriculture are organized in a very diversified manner (uniting field crops, vegetables, animal husbandry, direct marketing, and processing units on the same farm) and offer integrative working concepts employing people with special needs for care, guidance or education.

Further, there are health benefits for those working in organic agriculture as they are less exposed to possibly harmful pesticides or herbicides, as these are not allowed in OA.

\section{Potential drawbacks to the practice}

\subsection{Tradeoffs with other threats to soil functions}

Table 211. Soil threats

\begin{tabular}{|c|c|}
\hline Soil threats & \\
\hline $\begin{array}{l}\text { Nutrient imbalance } \\
\text { and cycles }\end{array}$ & $\begin{array}{l}\text { Just avoiding the application of chemical fertilizers would result in draining } \\
\text { the soil of nutrients and there are suggestions to consider new plant } \\
\text { nutrient sources (Röös et al., 2018). However, most organic farmers use } \\
\text { organic fertilizers and/or cover crops, intercropping, crop residue } \\
\text { incorporation and other recommended soil management practices which } \\
\text { can foster the nutrient balance (e.g. Beck et al., 2016). }\end{array}$ \\
\hline $\begin{array}{l}\text { Soil contamination / } \\
\text { pollution }\end{array}$ & $\begin{array}{l}\text { Depending on the sources of origin, it is possible that organic fertilizers as } \\
\text { sewage sludge, compost, slurry, manure or crop residues may contain } \\
\text { contaminants, e.g. heavy metals, which incidentally can also be found in } \\
\text { mineral fertilizers (Rashmi et al., 2020). However, in many organic } \\
\text { standards sewage sludge is not allowed (e.g. EU Organic Standard, EUR- } \\
\text { Lex, 2007) and organic fertilizers from conventional production potentially } \\
\text { containing more heavy metals and drug residues, are not allowed, e.g. in the } \\
\text { Bioland standard (Bioland, 2019). The final regulation depends on the } \\
\text { organic standard. }\end{array}$ \\
\hline Soil compaction & $\begin{array}{l}\text { In organic agriculture there is no use of synthetic herbicides allowed, thus in } \\
\text { OA without practicing conservation tillage comparably more machine use is } \\
\text { necessary in order to compensate their function. Potentially, this might } \\
\text { cause more soil compaction (Röös et al., 2018). However, it depends on the } \\
\text { type of soils and machinery, especially vehicles with heavy load working on } \\
\text { wet soils are responsible for soil compaction (FAO, 202Oc). }\end{array}$ \\
\hline
\end{tabular}




\subsection{Increases in greenhouse gas emissions}

Using closed GHG sampling chambers, Skinner et al. (2019) measured $\mathrm{N}_{2} \mathrm{O}-\mathrm{N}$ and $\mathrm{CH}_{4}$-C emissions of organic and conventional managed areas in Switzerland using the long-term DOK trial ${ }^{28}$. Combined and expressed in tonnes of carbon dioxide equivalents per hectare and year $\left(\mathrm{tCO}_{2} \mathrm{e} / \mathrm{ha} / \mathrm{yr}\right.$ using the Global Warming Potential 100 values (IPCC, 2014) their results were 1.23 at the organic experiment and 0.90 in the biodynamic experiment field. Compared to the conventional experiment fields $\left(1.88 \mathrm{tCO}_{2} \mathrm{eq} / \mathrm{ha} / \mathrm{yr}\right)$, the results for the organic varieties were lower. These values exclude any emissions from organic or chemical fertilizer production, livestock, fuel, electricity, processing, or transport emissions.

Subtracting the above-mentioned values found by Skinner et al. (2019) (organic: $1.65 \mathrm{tCO}_{2} \mathrm{eq} / \mathrm{ha} / \mathrm{yr}$; biodynamic: $1.65 \mathrm{tCO}_{2} \mathrm{eq} / \mathrm{ha} / \mathrm{yr}$ ) from sequestration values of Gattinger et al. (2012), et al. $\left(1.65 \mathrm{tCO}_{2} \mathrm{e} / \mathrm{ha} / \mathrm{yr}\right)$, this results in a positive net balance for organic $\left(-0.42 \mathrm{tCO}_{2} \mathrm{eq} / \mathrm{ha} / \mathrm{yr}\right)$ and biodynamic ($\left.0.75 \mathrm{tCO}_{2} \mathrm{eq} / \mathrm{ha} / \mathrm{yr}\right)$ treatments, considering the limitations mentioned above.

\subsection{Conflict with other practice(s)}

There can be an overlap (no conflict) with the practices of regenerative agriculture, agroecology and climatesmart agriculture (CSA) as some requirements are identical for all these practices.

\subsection{Decreases in production (e.g. food/fuel/feed/timber/fibre)}

See sub-section 4.3.

\subsection{Other conflicts}

Occasionally, there are conflicts between farmers who believe that $\mathrm{OA}$ is the best solution, and traditional farmers who feel that the good agricultural practices they apply are devaluated and not recognized by the organic farming movement. However, there are also many cases where OA or regenerative practices are adopted by conventional farmers (LaRose and Myers, 2019) - especially where the effects of climate change become strongly noticeable.

\footnotetext{
${ }^{28}$ DOK: biologisch-dynamisch (in english: Biologic-dynamic) (D), organisch-biologisch (in english: organic-biologic) (O), konventionell (in English: conventional) (K)
} 


\section{Recommendations before implementing the practice}

One possible recommendation is a partial conversion to organic agriculture as a first step. This can be related to just one crop of several or to a specific area of the total farm. This is recommended because there is financial risk during the 2-3-year conversion period when converting the farming system to organic agriculture, especially when there is no financial support of the government, unlike in the European Union or the United States (FAO, 2020b).

A further recommendation is the exchange of ideas and experiences among farmers. It is very common that there are knowledge gaps, and it is recommended not to repeat mistakes made by other farmers who adopted the practice earlier.

Also taking relevant courses and trainings, or requesting expert consultations, is highly recommended before starting the conversion.

A high risk for the farmer adopting organic agriculture exists when neighboring farms use synthetic pesticides and/or GMO (Genetically modified organisms) crop varieties on their fields. These pesticides can reach the organic fields via wind transport and in case of GMO via pollinators. This possible contamination of the presumed organic products can result in a loss of the organic certification; thus, products cannot be sold as organic any longer. This means a significant income loss, as organic products are often sold at higher prices (FAO, 2020a).

\section{Potential barriers to adoption}

\section{Table 212. Potential barriers to adoption}

\begin{tabular}{|c|c|c|}
\hline Barrier & YES/NO & \\
\hline Cultural & Yes & $\begin{array}{l}\text { A cultural barrier can exist in a region where practically no organic farming has } \\
\text { existed before. Farmers might not know the viability of a concept as organic } \\
\text { agriculture and consumers lack of awareness for organic products (Altarawneh, } \\
\text { 2016). }\end{array}$ \\
\hline Economic & Yes & $\begin{array}{l}\text { There is an economic risk in the transition period from conventional to organic } \\
\text { agriculture. Sometimes there is no financial support by the government, but the } \\
\text { German government, for example, provides such financial support } \\
\text { (Ökolandbau.de, 2018). Furthermore, labeling organic field products as organic } \\
\text { requires certification to be paid by the producer. This also represents a } \\
\text { significant cost factor. }\end{array}$ \\
\hline Institutional & Yes & $\begin{array}{l}\text { If there is no national law for organic agriculture, then this can be a barrier for } \\
\text { the adoption, as legal backup and guidance are lacking. In the European Union } \\
\text { there is the Council Regulation on Organic Agriculture (EUR-Lex, 2007). Such a } \\
\text { law regulates the production, trade and labeling of organic products. }\end{array}$ \\
\hline
\end{tabular}




\begin{tabular}{|l|l|l|}
\hline Barrier & YES/NO & Legal (Right \\
to soil) & Yes & $\begin{array}{l}\text { Planning security for farmers is of high importance as the conversion to organic } \\
\text { farming is an investment. For example, if the lease contract expires or the legal } \\
\text { framework changes, then it is more likely that there is no investment into a } \\
\text { conversion (Schneeberger, Darnhofer and Eder, 2002). }\end{array}$ \\
\hline Knowledge & Yes & $\begin{array}{l}\text { Specific knowledge is necessary for the adoption of OA, this means a lack of } \\
\text { knowledge can be a barrier to the adoption of OA ( Schneeberger, Darnhofer } \\
\text { and Eder, 2002). There is government advice or semi-governmental institutes. } \\
\text { The latter is characterized to be funded by third parties and the quality can be } \\
\text { questionable sometimes. Private consultancy, on the other hand, is good but is } \\
\text { associated with costs and farmers are not always prepared to pay for these } \\
\text { services. It also plays a major role whether it is possible to study organic farming } \\
\text { or whether it is otherwise integrated into education (e.g. integration into } \\
\text { curricula of agricultural technical schools). }\end{array}$ \\
\hline
\end{tabular}

\section{Photos of the practice}

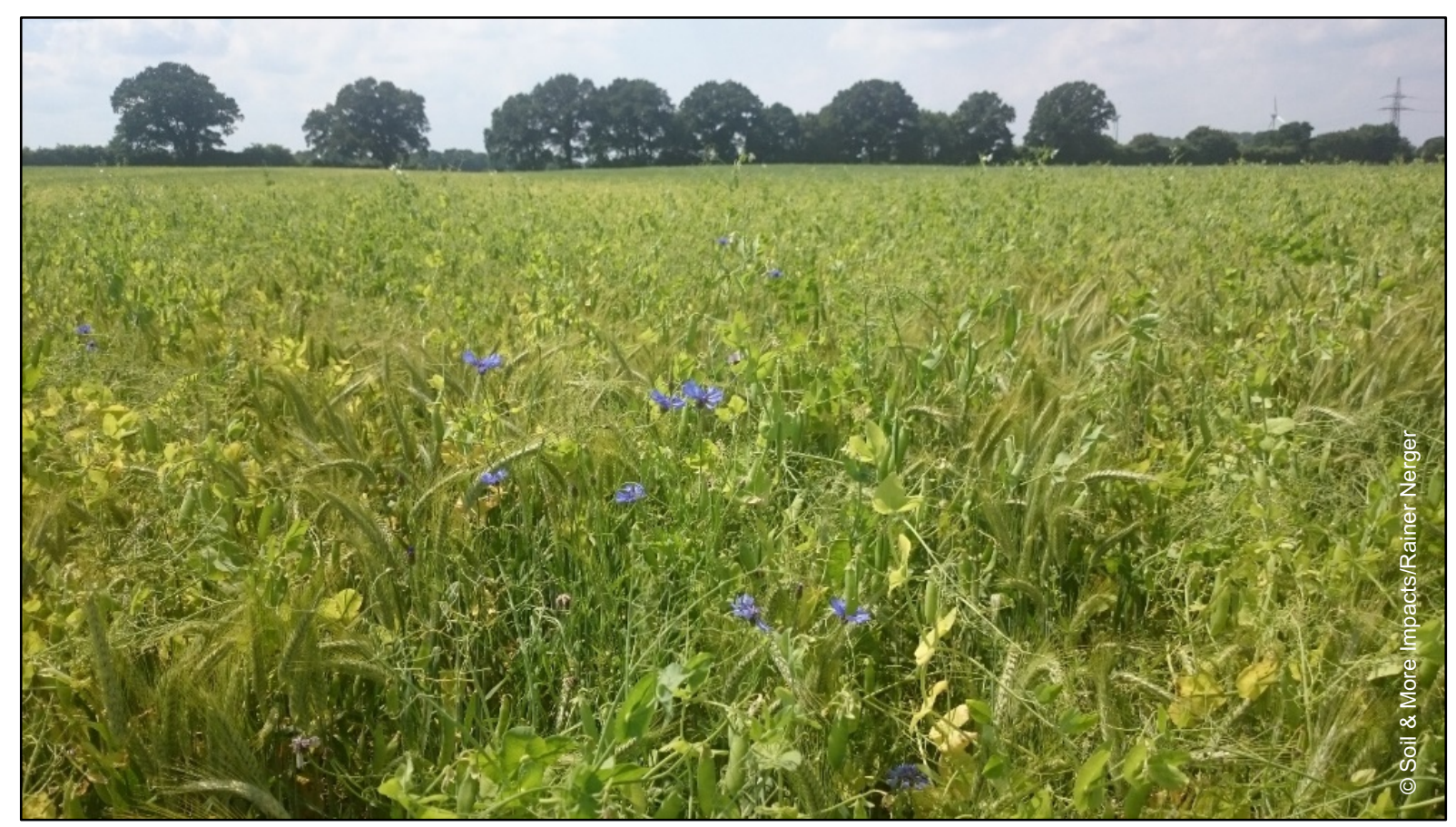

Photo 67. Organic cereals intercropped with legumes and grass; Northern Germany (Schleswig-Holstein), June 2017. 


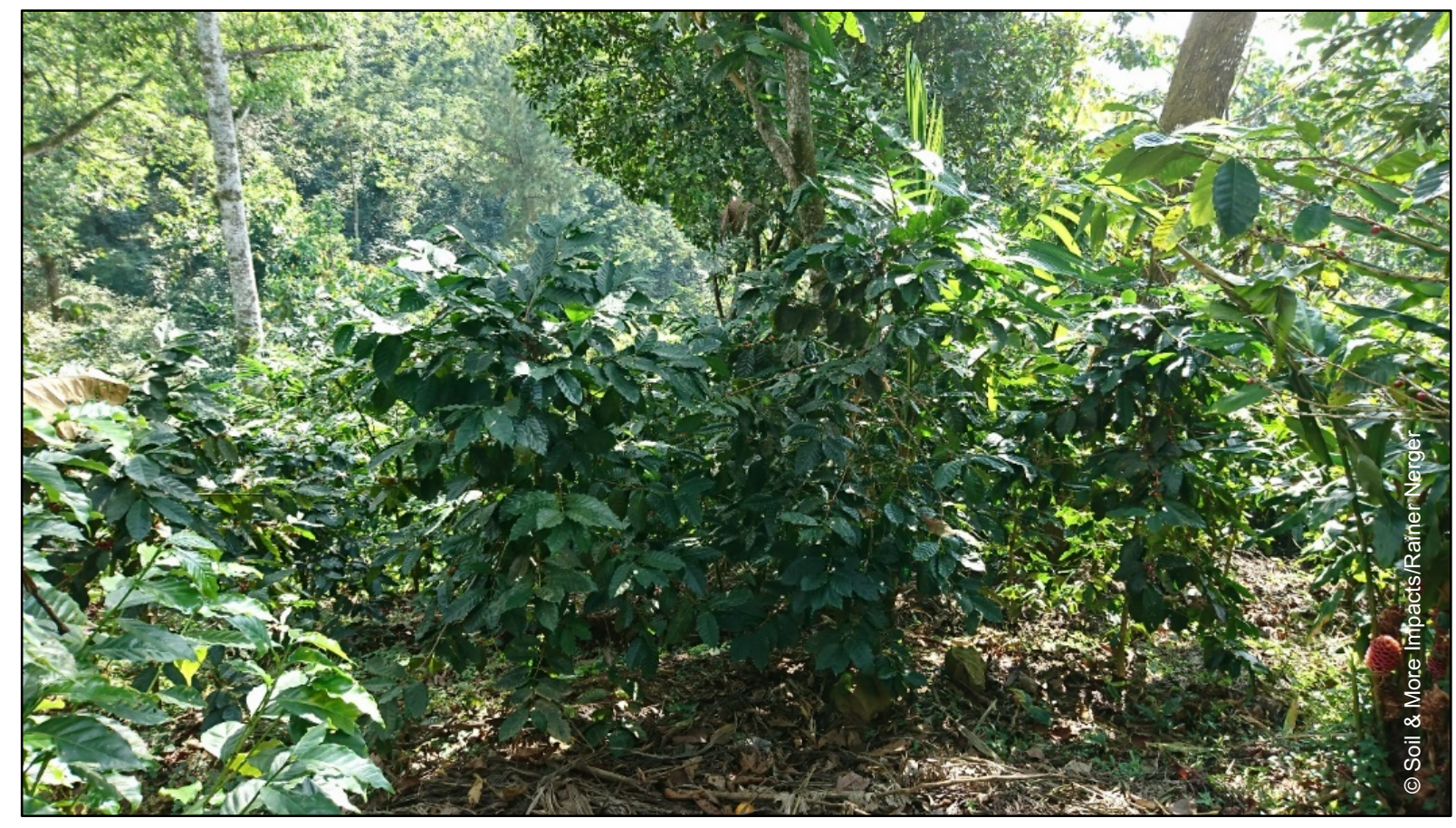

Photo 68. Shaded organic coffee, with soil cover and intercropped; South Mexico (Chiapas), December 2018

Table 213. Related cases studies available in volumes 3 and 5

\begin{tabular}{|l|l|l|c|c|}
\hline Title & Region & $\begin{array}{l}\text { Duration } \\
\text { of study } \\
\text { (Years) }\end{array}$ & Volume & $\begin{array}{l}\text { Case- } \\
\text { study No. }\end{array}$ \\
\hline $\begin{array}{l}\text { Irrigation and SOC sequestration in the } \\
\text { region of Navarre in Spain }\end{array}$ & Europe & 6 to 20 & 3 & 19 \\
\hline $\begin{array}{l}\text { Reduced tillage frequency and no-till to } \\
\text { allow ground covers and seeding cover crops } \\
\text { in rainfed almond fields, Spain }\end{array}$ & Europe & 10 & 3 & 21 \\
\hline $\begin{array}{l}\text { Biochar and compost application in an olive } \\
\text { orchard, Spain }\end{array}$ & Europe & 4 & 3 & 22 \\
\hline $\begin{array}{l}\text { Organic rice cultivation with internal nutrient } \\
\text { cycling in Japanese Andosols }\end{array}$ & Asia & $\begin{array}{c}4,8 \text { and } \\
12\end{array}$ & 5 & 14 \\
\hline
\end{tabular}




\section{References}

Adamtey, N., Musyoka, M.W., Zundel, C., Cobo, J.G., Karanja, E., Fiaboe, K.K., Muriuki, A., Mucheru-Muna, M., Vanlauwe, B., Berset, E., Messmer, M.M., Gattinger, A., Bhullar, G.S., Cadisch, G., Fliessbach, A., Mäder, P., Niggli, U. \& Forster, D. 2016. Productivity, profitability and partial nutrient balance in maize-based conventional and organic farming systems in Kenya. Agriculture, Ecosystems \& Environment, 235: 61-79. https://doi.org/10.1016/j.agee.2016.10.001

Altarawneh, M. 2016. Determine the barriers of organic agriculture implementation in Jordan. Bulgarian Journal of Agricultural Science, 22(1): 10-15.

Arnhold, S., Lindner, S., Lee, B., Martin, E., Kettering, J., Nguyen, T.T., Koellner, T., Sik Ok, Y. \& Huwe, B. 2014. Conventional and organic farming: Soil erosion and conservation potential for row crop cultivation. Geoderma, 219: 89-105. https://doi.org/10.1016/j.geoderma.2013.12.023

Beck, J.E., Schroeder-Moreno, M.S., Fernandez, G.E., Grossman, J.M. \& Creamer, N.G. 2016. Effects of cover crops, compost, and vermicompost on strawberry yields and nitrogen availability in North Carolina. HortTechnology, 26(5): 604-613. https://doi.org/10.21273/HORTTECH03447-16

BIOC. 2020. List of certification bodies in Germany. [online]. [Cited 22 June 2020]. https://www.bioc.info/standard/controlbodylist?country=DE\&\%40format=html

Bioland. 2019. Bioland Standards as of November 25th, 2019. [online]. [Cited 23 October 2020]. https://www.bioland.de/fileadmin/user_upload/Verband/Dokumente/Richtlinien_fuer_Erzeuger_und_H ersteller/Bioland_Standards_2019-11-25.pdf

Cai, Z., Wang, B., Xu, M., Zhang, H., He, X., Zhang, L. \& Gao, S. 2015. Intensified soil acidification from chemical $\mathrm{N}$ fertilization and prevention by manure in an 18-year field experiment in the red soil of southern China. Journal of Soils and Sediments, 15(2): 260-270. https://doi.org/10.1007/s11368-014-0989-y

Carpenter-Boggs, L., Reganold, J.P. \& Kennedy, A.C. 2000. Biodynamic preparations: Short-term effects on crops, soils, and weed populations. American Journal of Alternative Agriculture, 15(3): 110-118. https://doi.org/10.1017/S0889189300008614

Chalker-Scott, L. 2013. The science behind biodynamic preparations: A literature review. HortTechnology, 23(6): 814-819.

Davis, A.S., Hill, J.D., Chase, C.A., Johanns, A.M. \& Liebman, M. 2012. Increasing cropping system diversity balances productivity, profitability and environmental health. PloS One, 7(10). https://doi.org/10.1371/journal.pone.0047149

DePonti, T., Rijk, B., \& Van Ittersum, M.K. 2012. The crop yield gap between organic and conventional agriculture. Agricultural systems, 108: 1-9. https://doi.org/10.1016/j.agsy.2011.12.004

Ecolabelindex. 2020. Japanese Agricultural Organic Standard (JAS). [online]. [Cited 23 June 2020]. http://www.ecolabelindex.com/ecolabel/japanese-agricultural-organic-standard

EUR-Lex. 2007. Council Regulation (EC) No 834/2007. [online]. [Cited 22 June 2020]. https://eurlex.europa.eu/legal-content/EN/TXT/?uri=celex\%3A32007R0834 
FAO. 2020a. Why is organic food more expensive than conventional food? [online]. [Cited 24 June 2020]. http://www.fao.org/organicag/oa-faq/oa-faq5/en/

FAO. 2020b. Is there any kind of economic help for conversion into organic agriculture? [online]. [Cited 24 June 2020]. http://www.fao.org/organicag/oa-faq/oa-faq9/en/

FAO. 2020c. Manual for the implementation of the Soil Doctors Global Programme at the country level-A farmer-to-farmer training programme. Rome. Educational material: Poster "How to prevent and remediate soil compaction”. http://www.fao.org/global-soil-partnership/pillars-action/2-awareness-raising/soildoctor/en/

Finley, L., Chappell, M.J., Thiers, P. \& Moore, J.R. 2018. Does organic farming present greater opportunities for employment and community development than conventional farming? A survey-based investigation in California and Washington. Agroecology and Sustainable Food Systems, 42(5): 552-572. https://doi.org/10.1080/21683565.2017.1394416

Forster, D., Andres, C., Verma, R., Zundel, C., Messmer, M.M. \& Mäder, P. 2013. Yield and economic performance of organic and conventional cotton-based farming systems-results from a field trial in India. PloS One, 8(12). https://doi.org/10.1371/journal.pone.0081039

Gattinger, A., Muller, A., Haeni, M., Skinner, C., Fliessbach, A., Buchmann, N., Mäder, P., Stolze, M., Smith, P., El-Hage Scialabba, N. \& Niggli, U. 2012. Enhanced topsoil carbon stocks under organic farming. Proceedings of the National Academy of Sciences, 109(44): 18226-18231.

https://doi.org/10.1073/pnas.1209429109

Huntington, T.G. 2007. Available Water Capacity and Soil Organic Matter. In Encyclopedia of Soil Science, Second Edition. Taylor and Francis: New York; 139-143.

IFOAM. 2020. Definition of Organic Agriculture [online]. [Cited 22 June 2020]. https://www.ifoam.bio/why-organic/organic-landmarks/definition-organic

IPCC. 2014. Climate Change 2014: Synthesis Report. In Core Writing Team, Pachauri, R.K. and Meyer, L.A. (Eds.). Contribution of Working Groups I, II and III to the Fifth Assessment Report of the Intergovernmental Panel on Climate Change. IPCC, Geneva, Switzerland, 151 pp.

Juchowo. 2019. Activities for disabled people [online]. [Cited 24 June 2020]. https://www.juchowo.org/en/activity/activities-for-disabled-people.html

Knapp, S. \& van der Heijden, M.G. 2018. A global meta-analysis of yield stability in organic and conservation agriculture. Nature Communications, 9(1): 1-9. https://doi.org/10.1038/s41467-01805956-1

Krauss, M., Berner, A., Perrochet, F., Frei, R., Niggli, U. \& Mäder, P. 2020. Enhanced soil quality with reduced tillage and solid manures in organic farming-a synthesis of 15 years. Scientific Reports, 10(1): 1-12. https://doi.org/10.1038/s41598-020-61320-8

LaRose, J. \& Myers, R. 2019. PROGRESS REPORT: Adoption of Soil Health Systems Based on Data from the 2017 U.S. Census of Agriculture. Report. Soil Health Institute. 
Leifeld, J. \& Fuhrer, J. 2010. Organic farming and soil carbon sequestration: what do we really know about the benefits? Ambio, 39(8): 585-599. https://doi.org/10.1007/s13280-010-0082-8

Maheshwari, D.K. (Ed.). 2014. Organic farming - Feed the soil to feed the plant. In Composting for sustainable agriculture (Vol. 3). Springer.

Ökolandbau.de. 2018. Förderung des Ökolandbaus in Deutschland [online]. [Cited 24 June 2020]. https://www.oekolandbau.de/landwirtschaft/umstellung/ablauf-und-planung/foerdermittel/

Rashmi, I., Roy, T., Kartika, K. S., Pal, R., Coumar, V., Kala, S. \& Shinoji, K.C. 2020. Organic and Inorganic Fertilizer Contaminants in Agriculture: Impact on Soil and Water Resources. In Contaminants in Agriculture (pp. 3-41). Springer, Cham.

Reeve, J.R., Hoagland, L.A., Villalba, J.J., Carr, P.M., Atucha, A., Cambardella, C., Davis, D.R. \& Delate, K. 2016. Organic farming, soil health, and food quality: considering possible links. Advances in Agronomy, 137: 319-367. https://doi.org/10.1016/bs.agron.2015.12.003

Röös, E., Mie, A., Wivstad, M., Salomon, E., Johansson, B., Gunnarsson, S., Wallenbeck, A., Hoffmann, R., Nilsson, U., Sundberg, C. \& Watson, C.A. 2018. Risks and opportunities of increasing yields in organic farming. A review. Agronomy for Sustainable Development, 38(2): 14.

https://doi.org/10.1007/s13593-018-0489-3

Seufert, V., Ramankutty, N. \& Foley, J.A. 2012. Comparing the yields of organic and conventional agriculture. Nature, 485(7397): 229-232. https://doi.org/10.1038/nature1 1069

Seufert, V., Ramankutty, N. \& Mayerhofer, T. 2017. What is this thing called organic? - How organic farming is codified in regulations. Food Policy, 68: 10-20.

https://doi.org/10.1016/j.foodpol.2016.12.009

Skinner, C., Gattinger, A., Krauss, M., Krause, H.M., Mayer, J., van der Heijden, M.G. \& Mäder, P. 2019. The impact of long-term organic farming on soil-derived greenhouse gas emissions. Scientific Reports, 9(1): 1-10. https://doi.org/10.1038/s41598-018-38207-w

Schneeberger, W., Darnhofer, I. \& Eder, M. 2002. Barriers to the adoption of organic farming by cash-crop producers in Austria. American Journal of Alternative Agriculture, 24-31.

https://doi.org/10.1079/AJAA20017

SMI (Soil \& More Impacts). 2019. Best practices in farming - The way to a 'more'. [online]. [Cited 22 June 2020]. https://www.soilandmore.com/en/news/best-practices-in-farming-the-way-to-a-more

USDA. 2020. Organic regulations. [online]. [Cited 23 June 2020]. https://www.ams.usda.gov/rulesregulations/organic 


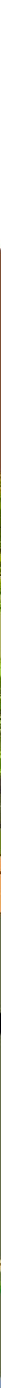




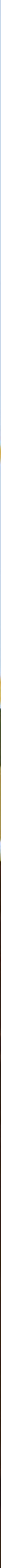


The Global Soil Partnership (GSP) is a globally recognized mechanism established in 2012. Our mission is to position soils in the Global Agenda through collective action. Our key objectives are to promote Sustainable Soil Management (SSM) and improve soil governance to guarantee healthy and productive soils, and support the provision of essential ecosystem services towards food security and improved nutrition, climate change adaptation and mitigation, and sustainable development.

Thanks to the financial support of
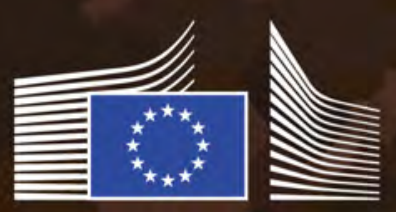

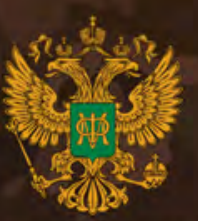

Ministry of Finance of the Russian Federation

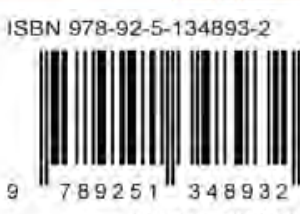

CB6595EN/1/09 21 